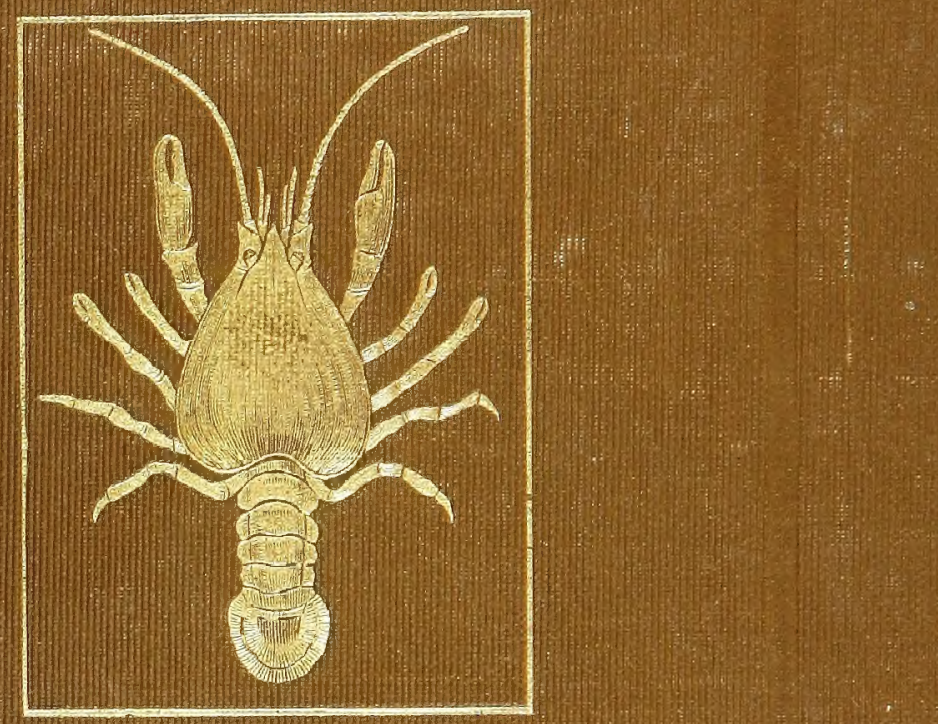

a:

19 


\section{Curnell älniwersity diluraty}

BOUGHT WITH THE INCOME FROM THE

SAGE ENDOWMENT FUND

THE GIFT OF

Henrg W. Sage

$$
\text { I89x }
$$

$524+794$

$26 / \pi / 1.0$ 


\section{Date Due}

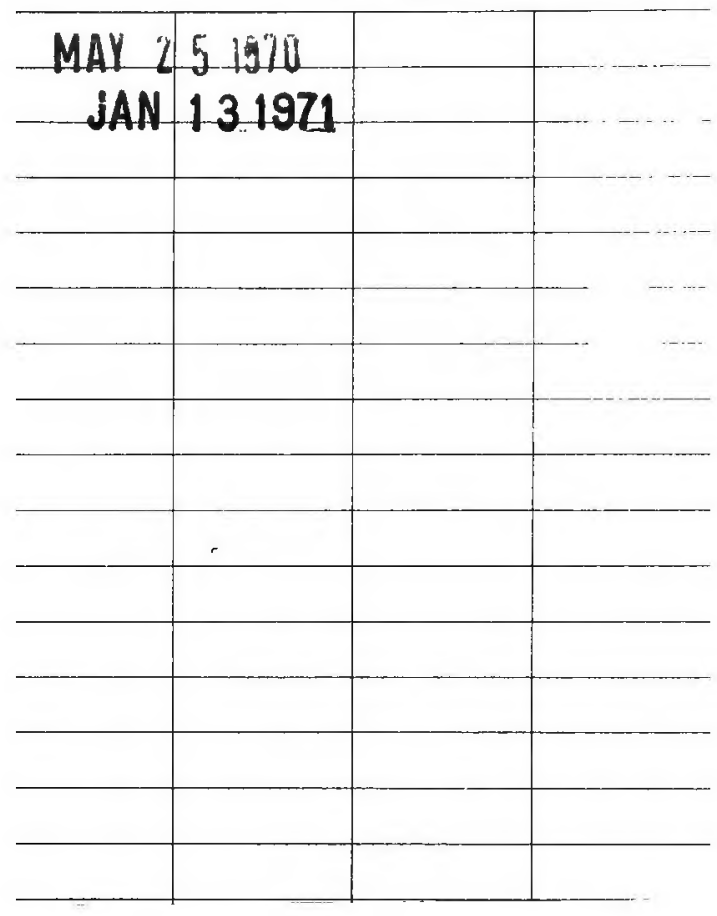

RETURN TO

\section{ALBERT R. MANN LIBRARY}

ITHACA, N. Y.

\section{Cornell University Library
47.866 1896}

Text book of zoology

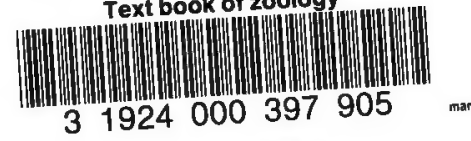




\section{Cornell University Library}

The original of this book is in the Cornell University Library.

There are no known copyright restrictions in the United States on the use of the text. 


\title{
TEXT BOOK OF ZOOLOGY
}

BY

\section{DR. J. E. V. BOAS}

Lecturer in Zoology in the Royal College of Agriculture, etc.,

Copenhagen.

\author{
Transtated BY \\ J. W. KIRKALDY
}

Tutor in Natural Science to the Association for the Education of Women, Oxford.

AND

E. C. POLLiARD, B.Sc., Lond.,

Assistant Lecturer in Biology, University Extension College, Reading.

WITH 427 FIGURES

LONDON

SAMPSON LOW, MARSTON \& COMPANY

Limited

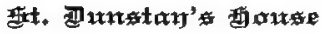

FETTER LANE, FLEET STREET, E.C.

1896. 


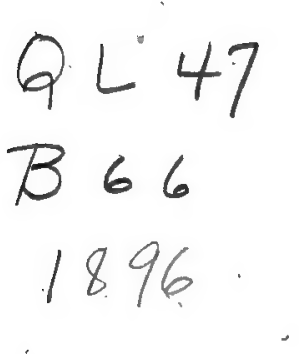

A. 244.794 LONDON :

PRINTED BY HORACE COX, WINDSOR HOUSE, BREAM's BUILDINGS, H.C. 


\section{P REF A CE.}

An English translation of Dr. Boas's " Lehrbuch der Zoologie," which has already appeared in two Danish and two German editions, has been arranged in the hope that it may prove useful to yet a larger public.

The translation is designed, in the first place, to assist beginners in the study of Zoology ; but the needs of students of Medicine, of Veterinary Surgery, and of Forestry have also been kept in view. It will be noticed that Dr. Boas gives prominence to facts rather than to theories, and of these, such as should be of use to one or other of the classes of readers just enumerated, or as should be most convenient for verification.

In the German edition lists of the more important forms belonging to the German fauna are appended to the descriptions of the several groups : these have been replaced here by species met with in the British Isles, and it is hoped that they will be of special service to Naturalists. For this amount of the subject-matter, and for this alone, are the Translators responsible : in all other respects they have merely endeavoured to give a faithful rendering of the German text. Certain differences appear between the English and the German, as the book has undergone a thorough revision at the Author's hands, and certain portions have been deleted, whilst new matter has been introduced. 
The Translators desire to express their thanks to Mr. W. E. Hoyle for suggesting that they should undertake the work, and also for his valuable help throughout, especially for his kindness in looking over many proof-sheets : to Prof. Newton, for kind assistance with terms relating to the migrations of Birds : to Prof. E. B. Poulton, Mr. Barclay Thompson, and Dr. W. B. Benham, for many helpful suggestions. Finally, they desire to express their indebtedness to the Radcliffe Library, where they have not only had complete access to recent Zoological literature, but also every assistance in tracing references and in procuring records for the faunistic sections.
J. W. KIRKALDY.
E. C. POLLARD.

Oxford, 1896. 


\section{0 N T E N T S.}

\section{GENERAL PART.}

I. Cells and Tissues (Histology) 3

II. Organs . 13

1. Skin 14

2. Skeleton 15

3. Musculature 16

4. Nervous system 17

$\begin{array}{ll}\text { 5. Sense organs . } & 18\end{array}$

6. Alimentary canal $\quad 23$

7. Vascular system 25

8. Respiratory organs . . . . 27

9. Excretory or urinary organs . 30

10. Reproduction and reproductive organs . . 31

11. The relations of the organs to one another.-The body cavity . 39

12. Rudimentary organs. . . 40

III. Fundamental Form and External Configuration of the Body . 40

IV. Embryology or Ontogeny . • • • • • . . . 42

V. Affinities of Animals-Classification-The Doctrine of Descent . 53

$\begin{array}{lll}\text { VI. Biology . } & 58\end{array}$

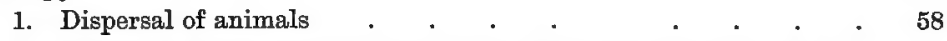

2. Different kinds of food and their effect on the form of the body.-Parasitism . . . . . . . . . 62

3. Different kinds of locomotion.-Their effects upon the structures of animals.-Sessile forms .

4. The transforming effects of the environment. : : $\quad 66$

5. Stages of life.-Duration of life ; ; 68

6. Protective adaptations . . . 70

7. The power of resisting unfavourable conditions 72

VII. Geographical Distribution . . . . 73

VIII. Geological Distribution. $\quad 76$

$\begin{array}{ll}\text { Appendix.-Resemblances and differences between plants and animals } & 80\end{array}$ 


\section{SPECIAL PART.}

Protozoa.

Class 1. Gymnomyxa (Sarcodina)

Order 1. Rhizopoda . $\quad 87$

$\begin{array}{ll}\text { Oxder 2. Radiolaria } & 89\end{array}$

Class 2. Infusoria (Ciliata) . . 91

Class 3. Gregarinida . . 95

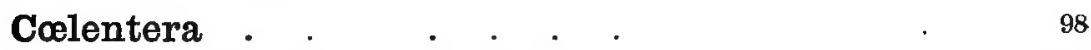

Class 1. , Hydrozoa . . . . . . . 102

Order 1. Hydromedusæ (Craspedota) . . 103

Order 2. Siphonophora . . . . . . 107

Order 3. Acalepha (Scyphomedusæ, Acraspeda) . . 109

Class 2. Anthozoa . . . . . 111

Order 1. Alcyonaria (Octactinia) .. 113

Order 2. Zoantharia (Polyactinia)) . 115

Class 3. Ctenophora. . . . . . . . $\quad 118$

Appendix to the Ceelentera, Spongiæ or Porifera. $\quad 118$

Echinoderma . . . . . . . 122

Class 1. Crinoidea (Sea-Lilies). . $\quad 127$

Class 2. Asteroidea. . . 130

Order 1. Asterida 131

Order 2. Ophiurida . . . . . . . . . $\quad 133$

Class 3, Echinoidea. . . . . 134.

Order 1. Echinoidea Regularia. $\quad 138$

Oxder 2. Eichinoidea Irregularia . 138

Class 4. Holothuroidea. . . . . . . 138

Platyhelminthia . . . . . . 142

Class 1. Turbellaria . . . . . . . . $\quad 143$

Class 2. Trematoda..$\quad$. $\quad$. . . . 145

Order 1. Polystomeæ (Monogenetic Trematodes) 146

Order 2. Distomeæ (Digenetic Trematodes) . 147

Class 3. Cestoda . . . . . . . . . . 149

Class 4. Nemertinea (Rhynchocola) . . . 153

Appendix to the Platyhelminthia : Rotifera 156

Nemathelminthia. . . . . . . 158

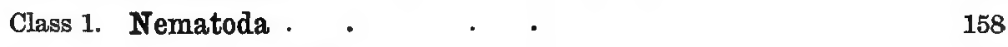

Class 2. Acanthocephala $\quad$ - . . . . 163

Annelida. • • • • . . . $\quad 165$

Class 1. Chætopoda. • . . . 167

Order 1. Polychæta . . . . 172

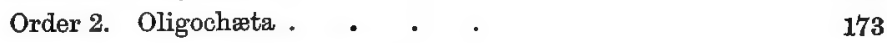

Appendix to the Chætopoda: Gephyrea . 174,

Class 2. Discophora. . . . 175

Class 3. Onychophora . . $\quad$. 177 
Polyzoa

Brachiopoda

\section{Arthropoda}

Class 1. Crustacea.

Sub-class 1. Entomostraca. . . . . . . . . 193

Order 1. Phyllopoda . . . . . . . . . 193

Order 2. Cladocera . . 195

Order 3. Xiphura . . 196

Order 4. Trilobita . . . . . . . . . 198

Order 5. Ostracoda $\quad$. $\quad . \quad . \quad 199$

Order 6. Copepoda $\quad$. . 200

Order 7. Cirripedia . . . . . . . . . 203

Sub-class 2. Malacostraca . . $\quad$. . . $\quad$. $\quad . \quad . \quad 207$

Order 1. Euphausiacea . . . . . . . 210

Order 2. Mysidacea . . . . . . . . . 211

Order 3. Cumacea . . . . . . . . . 213

Order 4. Isopoda . . . . . . . . . 214

Order 5. Amphipoda. . . . . . . $\quad$. 217

Order 6. Decapoda . $\quad$. . . . 219

Order 7. Stomatopoda . . . . . . . . 227

Class 2. Myriapoda. . . . . . . . . 227

Order 1. Chilopoda . . . . . . . . . 229

Order 2. Chilognatha. . . . . . . . . . 230

Class 3. Insecta . . . . . . . . . . . 231

Order 1. Orthoptera . . . . . . . . . 252

Order 2. Rhynchota . . . . . . . . . 256

Order 3. Neuroptera . . . . . . . . 260

Order 4. Coleoptera . . . . . . . . 263

Order 5. Hymenoptera . . . . 267

Order 6. Lepidoptera . . . . 272

Order 7. Diptera . . 275

Class 4. Arachnida . . . . . . . . . 278

Order 1. Arthrogastra . . 281

Order 2. Araneina . . . . . . . 283

Order 3. Acarina . . . . 284

Appendix to the Arachnida:

Pentastomum $\quad . \quad 285$

Pycnogonidæ . . . 286

Tardigrada . . . . $\quad$. $\quad$. 286

Mollusca

Class 1. Placophora. . . . . . . . . 290

Class 2. Gastropoda. . . . . . . . $\quad$. . 291

Order 1. Prosobranchiata . .. . . . . . . 302

Order 2. Opisthobranchiata . . . . . . 303

Order 3. Pulmonata . . . . . . . . 305

Class 3. Acephala (Lamellibranchs) . $\quad$. . . . . 306

Class 4. Cephalopoda . . . . . . . . . . 315

Order 1. Tetrabranchiata. . . . . 322

Order 2. Dibranchiata . $\quad . \quad . \quad . \quad . \quad . \quad .323$ 
Vertebrata

Class 1. Leptocardii . . . . . . $\quad$. $\quad . \quad$. 354

Class 2. Pisces . . . . . . . . . . . $\quad$. 356

Order 1. Cyclostomi. . . . . . 382

Order 2. Selachii .

Order 3. Ganoidei . . . . . 384

$\begin{array}{llll}\text { Order 4. Dipnoi. } & \text {. . . } & 386\end{array}$

Order 5. Teleostei . . . . . 387

Class 3. Amphibia . . . . . . 391

Order 1. Urodela . . . . 404

Order 2. Anura . . . . . . . 406

Order 3. Gymnophiona . . . $\quad 407$

Class 4. Reptilia . . . . . . . . 407

Order 1. Lacertilia . $\quad 421$

Order 2. Ophidia . . . . . . . . 422

Order 3. Chelonia . . . . . 425

Order 4. Crocodilia . . . . . . . . . 426

Extinct orders of Reptiles $\quad$. 427

Class 5. Aves (Birds) . . . . . $\quad$. 430

Order 1. Saururæ . $\quad$. $\quad . \quad$. $\quad . \quad . \quad 452$

Order 2. Odontornithes . . . . . . 453

Order 3. Ratitæ. . . 453

Order 4. Rasores . . . 455

Order 5. Natatores $\quad \mathbf{4 5 6}$

Order 6. Grallatores . . . . . 458

Order 7. Accipitres . . . . 460

Order 8. Oscines . . . . . 461

Order 9. Clamatores. $\quad 464$

Order 10. Scansores . . . 465

Class 6. Mammalia . . $\quad 466$

Order 1. Monotremata . . 495

Order 2. Marsupialia . . . . $\quad 496$

Order 3. Insectirora . 499

Order 4. Chiroptera . 500

Order 5. Ungulata . . . . . . 501

Order 6. Proboscidea. . . 510

Order 7. Sirenia. . . . 512

Order 8. Carnivora . . 513

Order 9. Pinnipedia . . . . $\quad . \quad 517$

Order 10. Cetacea . . . 520

Order 11. Bruta (Edentata) - 525

$\begin{array}{llll}\text { Order 12. Rodentia . . . . } \quad ~ & 527\end{array}$

Order 13. Prosimiæ . . . $\quad 531$

Order 14. Primates . . . . 532

Appendix to the Vertebrata: Tunicata (Ascidia) $\quad . \quad 537$

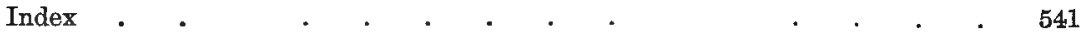




\section{LIST OF ILLUSTRATIONS.}

Fig.

PAGE

1. An Amoba at two different moments

3. Diagram of indirect nuclear division . . $\quad$. 6

4. Simple epithelium : $A, B$ squamous, $C$ columnar 7

5. Stratified epithelium : $A$ squamous, $B$ columnar 7

6. $A$ ciliated cells, $B$ columnar cells . $\quad$. $8^{-}$

7. Simple epithelium with a cuticle . 8

8. A columnar epithelium with goblet cells, $B$ other gland cells 8

9. Diagrams of different glands $\quad$. . 9

10. Hyaline cartilage . . . . . . 10

11. Bone . . 10

12. Fat cells . . . . . 10

13. Muscle cells and fibre 11

14. Connective tissue and smooth muscle-cells 12

15. Various ganglion cells . . . . 13

16. Diagrams of a sucker 14

17. Diagram of a nervous system . . . . . . 17

18. Section through a small piece of the antenna of an Insect 19

19. Auditory capsule of a Gastropod . $\quad 20$

20. Different forms of optic organs . . . $\quad 21$

21. Different kinds of Arthropod eyes . 22

22. Diagrams of hearts . . . . . . . . 26

23. Diagram illustrating the usual relations of the respiratory organs to the vascular system.

24. Human ovum . . . . . . . $\quad 33$

25. Diagram of an ovum with many yolk spherules . . . 33

26. Spermatozoa of different animals . . . . 33

27. Diagram of the formation of polar bodies . . 34

28. Diagram of fertilisation . . . . . 35

29. Stages in the development of an ovum . 42

30. Stages in the development of the orum of a Water-snail . . . 43

31. Section through the egg of a Chætopod at different stages of development 43

32. Gastrula of a marine Gastropod . . . . 44

33. Diagrammatic figures of the formation of the epibolic gastrula . . . 44. 
Frg.

34. Formation of the gastrula in Amphibia . . . . . . . . 45

35. Diagram of gastrula-formation in Vertebrata with partial segmentation . 46

36. Development of the ovum of a Crustacean . . . . . . . 47

37. Diagrammatic sections explaining the formation of the gastrula in certain Hydrozoa . . . . . . . . . . . 47

38. Diagrammatic figures of the formation of the mesoblast in the Vertebrata .

39. Diagrammatic transverse section showing the formation of the notochord and of the nervous system in the Vertebrata. . . . 49

40. Embryo of the Dog-fish with yolk sac . . . 49

41. Diagram of a young embryo with yolk sae . . 49

42. Dimorphism of a Heteropteran . .

43. Two specimens of a Kallima . . . . . 71

44. Two Looper Caterpillars . . . . . 71

45. An Infusorian in the free state and encysted. . . . . 86

46. An encysted Infusorian which has broken up into a number of spores $\quad 86$

47. Diffugia, Euglypha, and Rotalia . 87

48. Nummulites distans . $\quad$. $\quad 89$

49. Radiolarian and skeleton . . 89

50. Actinosphærium Eichornii . $\quad$. 91

51. Two Infusoria in various stages of conjugation $\quad 93$

52. Paramacium . . . . 94.

53. Vorticella. .

54. Various Monadidæ . . . . . . $\quad 95$

55. Diagrammatic figure of a Gregarine . 95

56. Coccidium oviforme . . $\quad . \quad . \quad . \quad$. $\quad .97$

57. Raineyan sac in a muscle fibre . . $\quad 97$

58. Diagrammatic figures of the chief types of Colentera 98

59. Cells of a Coelenterate . . . . 100

60. Hydroid colonies and polyps . . . . . 104.

61. Various forms of the sexual generation of Hydromedusoids . 105

62. Diagram of Physophora . . . . 108

63. Diagram of Porpita . . 108

64. Section through a Scyphomedusan . . 109

65. Development of Aurelia . . . 110

66. Longitudinal section through a solitary Anthozoon 111

67. Transverse section of an Alcyonarian . . . 112

68. Sections through various young Alcyonaria . 114 .

69. Longitudinal and transverse sections of a Madreporarian 116

70. Portion of a massive Madreporarian 116

71. Portion of an arborescent Madreporarian . . . 117

72. Small portion of Heliastræa . . . . 117

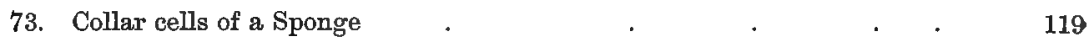

74. Various forms of Sponges $\quad . \quad$. . . . . . . 119

75. Diagrammatic figures explaining the radial structure of the Echinoderma. 122

76. Pedicellarix of a Sea-urchin . . . . 124

77. Diagrammatic sketch of the water-vascular system of a Star-fish . 124 .

78 and 79. Diagrammatic longitudinal sections of a Star-fish and of a Seaurchin . . . . . . . . . 125

80. Diagrammatic figures of Echinoderm larvæ $\quad$. . . . . 126 
FIt.

81. Larvæ of : Star-fish, Ophiurid, Sea-urchin, Holothurian . 127

82. Rhizocrinus lofotensis . . . . . . . . . . . 128

83. Antedon. . . . . . . . . . . 128

84. Ventral surface of Antedon . . . . . . . . . . . . $\quad$. 128

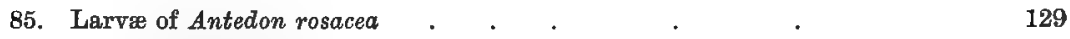

86. Antedon Eschrichtii . . . . $\quad$. . . . . . . . 129

87. Diagrammatic figures explaining the structure of a Star-fish . . 132

88. Diagram explaining the structure of an Ophiurid . 133

89. Shell of a Regular Sea-urchin . . . . . . . 135

90. Shell of an Irregular Sea-urchin . . 135

91. Diagrammatic longitudinal section of the spine of a Sea-urchin . 137

92. Diagram of a Holothurian . . . . . . . . . 139

93. Transverse section of the body-wall of a Holothurian . $\quad 139$

94. Transverse section through a radius of the body-wall of a Holothurian . 139

95. Nervous system of Distomum . . . . . 142

96. Part of the excretory system of a Flatworm $\quad 142$

97. Longitudinal section through Cycloporus papillosus . . 143

98. Sketch of Mesostomum splendidum. . 144.

99. Sketch of Provortex affinis . . . . . $\quad$. 144

100. Planaria lactea . . . . . . . . 145

101. Larva of a marine Turbellarian . . . . . $\quad 145$

102. Sketch of Liver-fluke . . . . . . . . . . . 146

103. Diplozoon paradoxum . . . . . . . . . . 147

104. Distomum hepaticum . . . 148

105. Polystomum integerrimum . . . . . . . . $\quad$.

106. Snail with Leucochloridium in both tentacles . 149

107. Tænia mediocanellata . . . . . . . 149

108. Six hooked larva of T. solium . . . . 150

109. Proscolex of the same . . . . . . . 150

110. Ciliated larva of Bothriocephalus latus . . . . . $\quad 150$

111. Proscolex of the same . . . . . 150

112. Head of Tcenia solium (A), of T. mediocanellata (B), and of Bothrio-

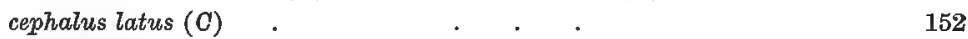

113. Tænia echinococcus . . . . $\quad$. $\quad$. 152

114. Bothriocephalus latus . 153

115. Sketches of a Nemertine . . . . 154

116. Diagrammatic section of a Nemertine . 155

117. Three larval stages of a Nemertine . . . . . 155

118. $A$, diagram of a female Rotifer : $B$, the male . . . . $\quad 156$

119. Transverse section of a Nematode . . . 158

120. Hind end of a male Nematode . . . . . . 159

121. Intestinal Trichina . . . . 161

122. Muscle Trichina . . $\quad$. 161

123. Dochmius duodenalis . 161

124. Filaria medinensis . . 161

125. Heterodera Schachtii $\quad . \quad$. . $\quad . \quad 163$

126. Echinorhynchus . 164

127. Larva of an Acanthocephalon . . . 164

128. An Annelid seen from the side . . 165 
FIG.

129. Nervous system in different Chætopods . 166

130. Anterior end of an Annelid . . . . . . . . 166

131. Diagrammatic section through the skin of a Chætopod 168

132. Anterior end of a Chætopod . . . . . . . 168

133. Diagrammatic sections of different Chætopods . . . 168

134. Diagram of the reproductive apparatus of an Earthworm. 171

135. Larva of Nereis . 171

136. Myrianida fasciata . . 172

137. Digestive tract, etc., of a Leech 175

138. Genital apparatus of a Leech $\quad$. 176

139. Peripatus . . . . . . . . . . . 177

140. A, B, diagrammatic longitudinal sections of a Polyzoon ; C A vicularia . 179

141. Plumatella polymorpha . . . . . 180

142. Fresh-water Polyzoon . . . . . . 181

143. Diagrammatic longitudinal section of a Brachiopod . 182

144. and 145. Larvæ of Brachiopods . . . . 183

146. Section through a seta and the adjacent skin of an Arthropod 185

147. The last four joints of an Arthropod limb . . . . 186

148. Longitudinal section through a joint of an Arthropod 186

149. Nervous system of Gammarus . . . 187

150. Examples of typical Crustacean limbs 189

151. Limbs of different Crustacea. $\quad 190$

152. Vascular system of the Lobster 191

153. Nauplius of Penæus 192

154. Branchipus vernalis. 194

155. Apus productus 194

156. Sida crystallina . 195

157. Limulus polyphemus . 196

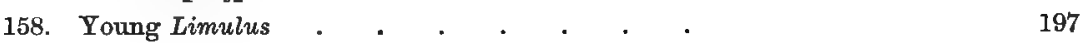

159. A Limulus, B Belinurus, $C$ Eurypterus, $D$ Dalmanites 198

160. Stages in the derelopment of Sao hirsuta . 198

161. Cypris . . . . . . . 199

162. Nauplius of an Ostracode . 199

163. Cyclops . . . . . 200

164. Various parasitic Copepoda 202

165. A, B, Penella sagitta, C Herpyllobius arcticus 202

166. Lepas . . . . . . . 203

167. Balanus. . . . . . . . . . . 203

168. Diagrammatic figures showing the transition from Lepas to Balanus. 204

169. Sacculina on the ventral side of the abdomen of a Shore-crab 207

170. The appendages of a Lobster. . 208

171. Nebalia Geofroyi . . 210

172. Thysanopus tricuspidatus 211

173. Boreomysis megalops . 212

174. Mysis-nauplius . . 212

175. Diastylis neapolitana . $\quad 213$

176. 1 Arga, 2-3 Cymothoa . . . . . . 214

177. 1 Cepon elegans, 2-3 Portunion Kossmanni . 215

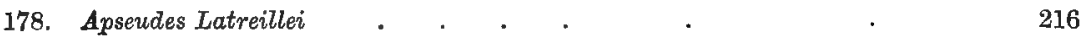


Fig.

179. An Amphipod. . . . . . . 217

180. Transverse section of the thorax of Gammarus 218.

181. 1, 2 Caprella acutifrons, 3 Cyamus mysticeti 218.

182. Palæmon. . . 220

183. Zoæa of a Prawn . . . 221

184. Mysis-stage of Penæus . 222

185. A young Lobster-larva . 224

186. Newly-hatched Crayfish . 224

187. Phyllosoma 225

188. Larvæ of a Crab 226 .

189. Squilla . . . 227

190. Digestive tract of Lithobius . 228.

191. Newly-hatched larva of a Diplopod . . . 228 .

192. Head and anterior body segments of a Scolopendra . 229

193. Transverse sections : $A$ of a Chilopod, $B, D$ of different Diplopods . $\quad 230$

194. Antennæ of various Insects 231

195. Diagram of the mouth parts of various Insects 232

196. Diagrammatic transverse sections of the proboscis of various Insects 233

197. Transverse section through the thorax of a Beetle . . . 235 .

198. Diagram of the principal anatomical points in an Insect . . 237

199. Nervous system of various Insects . . . 237

200. Diagrammatic longitudinal section of the head of an Insect with sucking mouth-parts

201. Diagram of the chief trunks of the tracheal system of an Insect. 239 .

202. Portion of a trachea from a Gall-fly larva. . 239

203. Apparatus for closing the trachea of a Beetle 240 .

204. Portion of the heart of an Insect . 242

205. Ovariole of an Insect $24: 2$

206. Female genital organs of the Cockchafer 243

207. Male genital organs of the same . 243

208. Females of three allied species of Geometridæ 244.

209. Blastophaga grossorum . $24 \Sigma$

210. Larva, pupa, and imago of a Sphinx 248

211. Larva and pupa of a Wasp 250 .

212. Termes lucifugus 254 .

213. Lepisma. . 255

214. Abdomen of Machilis 256 .

215. Phylloxera vastatrix 258 .

216. 1 Cimex lectularius, 2 Pediculus capitis, 3 Phthirius pubis 260 .

217. Chrysopa. . . . . . . 261

218. Panorpa communis. $\quad . \quad$. . . 261

219. Boreus hiemalis . . . . . 261

220. 1-4 Xenos Rossii, 5 X. Peckii . . . 262

221. A Abdomen with ovipositor of one of the Uroceridæ, $B$ Transverse section of the spine and the lobes. . . . 268

222. Heads of Honey-bees . 271

223. Psyche. . . 274.

224. Culex . . . . . 276

225. Gastrus . . . . 277 . 
FIG.

226. Pulex irritans .

227. Diagram of the anatomy of a Spider . . . . . . . . 279

228. Alimentary canal of a Spider . . . . . . . 280

229. Sexual apparatus of one of the Phalangiidæ . . . . . . . 281

230. A Scorpion . . . . . . . . . . . . 282

231. Diagram of the anatomy of a Tyroglyphus . . . . . . . 284

232. Female of Pentastomum tænioïdes . . . . . . . . . . . 285

233. Pycnogonum . . . . . . . . . . . . 286

234. Diagrammatic figure of a Tardigrade . $\quad . \quad$. . . 286

235. Portion of the radula of a Cephalopod . . . . . . . 288

236. Diagrammatic longitudinal section of the mouth of a Gastropod $\quad 288$

237. Diagram of the central nervous system in various Mollusca . . 289

238. Chiton . . . . . . . . . . . . . 291

239. Diagrammatic figure of a Gastropod . . . . . . . 292

240. Diagrams of various forms of Gastropod shells . . . . . . $\quad 292$

241. The shells of two examples of a tropical land Snail . . . . . . 293

242. Shell of a Snail (Paludina) . . . . . . . . . . 293

243. Diagram of the nervous system in relation to the alimentary canal . . 296

244. Male Periwinkle . . . . . . . . . . 298

245. Female Periwinkle. . . . . . . . . . . 299

246. Diagram of the genitalia of various Gastropods . . . . . 300

247. Larva of a Gastropod . . . . . . . . . . 301

248. Carinaria . . . . . . . . . . . 303

249. Cleodora. . . . . . . . . . . . . 304

250. Cleodora. . . . . . . . . . . . 304

251. Pneumodermon . . . . . . . . . . . 304

252. A transverse section through a Lamellibranch . . . . . 306

253. Transverse sections through two Lamellibranchs. . . . . 307

254. Lamellibranch removed from the shell. . . . . . . . 308

255. Tapes decussatus . . . . . . . . . . . . 308

256. Right shell valves of two different Lamellibranchs . . . . . . $\quad 309$

257. Diagrammatic transverse sections through the shell of Lamellibranchs with internal $\left(A, A^{\prime}\right)$ and external $\left(B, B^{\prime}\right)$ ligaments . . . . . 311

258. Diagrammatic longitudinal section of a Lamellibranch . . 313

259. Larvæ of Cardium . . . . . . $\quad 313$

260. Mya arenaria. . . . . . . . . . . 314

261. Teredo navalis . $\quad$. $\quad . \quad$. $\quad$. 315

262. Diagram of a decapodous Cephalopod . $\quad . \quad . \quad . \quad$. $\quad 316$

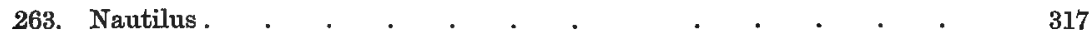

264. Diagrammatic figures of various Cephalopod shells . . . . $\quad$. $\quad 318$

265. Diagrammatic transverse sections through the eyes of various Cephalopoda $\quad 319$

266. Diagram of the heart, etc., of a Cephalopod . . . . . . 320

267. An octopodous Decapod in which the hectocotylised portion of the right arm is very well developed

268. Diagrammatic figures to illustrate the relations between the Chitons and the Cephalopods . . . . . . . . . 322

269. Diagram of a vertebra and the parts connected with it . . . . 326

270. Diagram of the skeleton of the fore-limb of a higher Vertebrate . . 328

271. Longitudinal section of a joint . . . . . . . . 329 
272. Diagrammatic vertical longitudinal section through the brain of a Vertebrate . . . . . . . . . . . . 330

273. Horizontal longitudinal section through the brain of a Vertebrate . . 331

274. Central nervous system of a Tortoise . . . . . . . . 332

275. Diagrammatic representation of the development of the vertebrate eye . 334

276. Diagrammatic section through the orbit, $A$ of a Fish, $B$ of a Mammal $\quad 335$

277. Pineal eye of a Lizard . . . . . . . 337

278. Diagram of the auditory organ of a Vertebrate . . . . $\quad 338$

279. Diagram of various developing teeth . . . . . . $\quad . \quad 339$

280. Section through a tooth to show the structure of the dentine . . . 340

281. Portion of the upper jaw of a Lizard . . . . . . $\quad 340$

282. Diagram to explain the structure of the mesentery . . . . . 342

283. Anterior portion of Chick embryo . . . . . . 343

284. Heart of an Amphibian. . . . . . . . . . 344

285. Diagrammatic longitudinal section through the head and front end of the body to show the position of the heart and pericardium . $\quad 345$

286. Diagrams of the arterial arches of various Vertebrata . . 347

287. Diagram of the end of a urinary tubule of a Vertebrate . . . . 348

288. Testis, kidney, etc., of an Amphibian . . . . . 349

289. Testis, embryonic kidney, etc., of the embryo of a higher Vertebrate . $\quad 349$

290. Diagram of a pronephros . . . . . . . . 350

291. Section through the ovary of a Mammal . . . . 351

292. Dlustrating the development of the embryonic membranes in a Bird embryo . . . . . . . . . . . . . . 353

293. Diagrammatio longitudinal section of Amphioxus . . . . . 355

294. Transverse section through the anterior region of the body of Amphioxus 355

295. Diagrammatic section of the skin of a Teleostean . . . . . . 356

296. Portion of a fin with spiny rays and soft rays _ . . . . . $\quad .358$

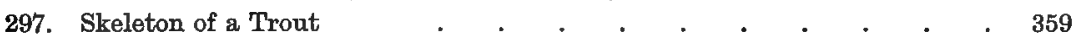

298. Longitudinal sections through the vertebral column of various Fish . $\quad 360$

299. End of the tail of various Fish $\quad$. . . . . . 361

300. Skull of a Perch . . . . . . . . . . . . . . . 363

301. Skull and risceral arches of $A$ a Shark, $B$ a Pike. . . . . 364

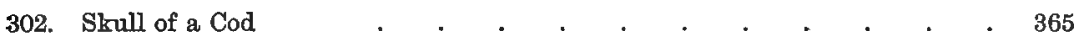

303. Shoulder-girdle and fore-limb of a Perch . . . . . $\quad$. 366

304. Skeleton of fore-limb of $A$ Shark, $B$ Polypterus, $C$ Amia, $D$ Cod. $\quad . \quad$. 367

305. Sensory papilla of a young Teleostean . . . . . . . . 369

306. Sternarchus curvirostris . . . . . . . . . . 371

307. Horizontal section through the head of Acanthias . . . . . 372

308. Horizontal section through the head of a Cod . . . . . . 372

309. Transverse section of a gill arch in various Fish . . . . . . 373

310. Longitudinal section of the heart of different Fish . . . . . 376

311. Young Pike at different stages . . . . . . . . . 380

312. Larva of Trachypterus . . . . . . . . . . . . 380

313. A Ray embryo, $B$ Shark embryo . . . . . . . . . 381

314. Chimsara monstrosa . . . . . . 384

315. Lepidosteus . . . . . . . . . • . . 385

316. Ceratodus . . . . . . . . . . . . . . . . 386

317. Protoptemus annectens . $\quad . \quad$. $\quad . \quad$. 386 
Fia.

318. Skeleton of a Urodele . . . . . . . . 392

319. Visceral arches of a Salamander . . . . . . . . . . . . 393

320. Skull of a Frog . . . . . . . . . . 394

321. Sternum and shoulder-girdle of a Salamander . . . . . . . 395

322. Sternum and shoulder-girdle of a Frog . . . . . . . . 395

323. Pelvis and last vertebræ of a Frog . . . . 396

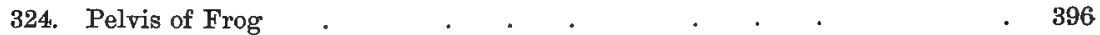

325. Transverse section of head of Frog . . 396

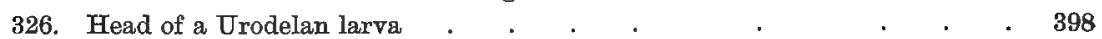

327. Tadpoles. . . . 390.39

328. Arterial arches of the Urodela . . . . . . . . . 400

329. Larvæ of the large Triton . . . . . 403

330. Skull of a Stegocephalon . . . . . . . . 405

331. Embryo of Epicrium glutinosum . . . 407

332. Sections through scales of Reptiles . . . . . . 408

333. Transverse section of the atlas of a Vertebrate 409

334. Diagram of axis . . . . . . 409

335. Skull of a Lizard . . . $\quad$. . . . . . . . 411

336. Hyoid of a Lizard . . . . . 411

337. Skull of a Boa constrictor . . . . . . . . . . . . 412

338. Skall of a Crotalus. . . . . $\quad 412$

339. Sternum and shoulder girdle of Lacerta 4,13

340. Carpus of a Turtle. . . . . . . . . . 414

341. Carpus of a Lizard . . . . 4 4i14.

342. Pelvis of a Lizard . . . . . . . 414

343. Brain of a Lizard . . . . . 4115

344. Vertical section of eye of Lizard and of Snake . . . 415

$\begin{array}{llll}\text { 345. Diagrams of various lungs . . . . . . . . . } 417 & 417\end{array}$

346. Head and neck of an Alligator . . . . $\quad 418$

347. Diagram of the heart and arterial arches of a Crocodile . . 4419

348. Poison tooth of a Crotalus . . . . . 423

349. Poison gland and poison fang of a Snake . . . 424

350. Tail end of a Crotalus . . . . . . . . 425.

351. Skull of a Gavial . . . . . 427

352. An Ichthyosaurian . . . . . 427

353. A Plesiosaurian $\quad$. 428

354. A Pterodactyle $\quad$. $\quad$. . . $\quad$. 428

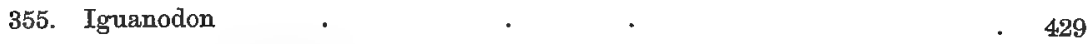

356. Fore and hind limbs of a Dinosaurian . . . 430

357. Portion of a feather . . . 431

358. Diagrams of feathers with aftershafts . . . . 432

359. Down and feather of young Bird . . . 432

360. Tails of various Birds . . . . 433

361. Skeleton of a Raven $\quad 436$

$\begin{array}{lllll}\text { 362. Skull of a Raven } & \text {. . . . . } & 438\end{array}$

363. Diagrams illustrating movement of beak in Birds . $\quad 438$

364. Skull of young Chick . . . . . . 439

365. "Hyoid of the Fowl . . . . . . . . . . . 439

366. Sternium and shoulder girdle of the Raven . 440 
FIA.

367. Manus of a young Ostrich . . . 440

368. Foot of a young Chick . . . . . 440

369. Pelvis of a young American Ostrich. 441

370. Brain of a Pigeon . . . . 442

371. Eye of a Bird. . . . . . 443

372. Lungs of a Chick embryo 446

373. Lungs of a Pigeon . . 446

374. Section through the trachea and bronchus of a Bird . . . 447

375. Diagram of the heart and arterial arches of Crocodile and Bird . 448

376. Reproductive organs of a Hen . . 449

377. Reproductive organs of a Cock 449

378. Archæopteryx . . . 452

379. Longitudinal section of a hair . 467

380. A claw, $B$ nail, $C$ hoof . . 4,69

381. Longitudinal section of the tip of a Mammalian digit. ․ . $\quad 470$

382. Tips of toes seen from below . . 470

383. Axis vertebra of a young Platypus . . . 472

384. Sternum and clavicle of a Kangaroo . 473

385. Skull of a Dog . . . . . . . . . 4774

386. Skull of an old Pig . . . . . . . $\quad 475$

387. Right half of the shoulder girdle of a young Platypus 477

388. Right half of the shoulder girdle of a young Ape 4777

389. Left half of the pelvis of a young Ornithorynchus 478

390. Left half of the pelvis of a new-born Calf . . 478

391. Tibia of a one-year-old Horse . . . . . 479

392. Diagrammatic transverse section of the head of a Mammal to show the relations of the auditory organ. . . 481

393. Dentition of a Mole . . . . . 483

394. The teeth of a Pig, showing the replacement 484

395. $A$, incisor of a Dog shortly after it has come into use; $B$, the same tooth in an old Dog

396. Longitudinal section through the head and neck of a Dog 486

397. Small portion of a mammalian lung . . . . 488

398. Heart and arterial arches of Mammalia . . . . 489

399. The terminal portions of the gut, of the urinary and genital apparatus in the females of various Mammalia . . . . 490

400. The Müllerian ducts and urinogenital sinus of various Mammalia 491

401. Diagrammatic longitudinal section of the cloaca (or rectum) and copulatory organs, $A$ of a Crocodile, $B$ of a Monotreme, $C, D$ of various other Mammalia .

402. Placenta of a Mammal . . . . . Pight hind foot of : A Phalangista, B a Kangaroo, C Chorcepus 494

404. Fore-foot of : $A$ Tapir, $B$ Rhinoceros, $C$ Horse.

405. Left fore-foot of Anchitherium, Hipparion, and Horse . 504

406. Manus of: $A$ Pig, $B$ Stag, $C$ Camel . 505

407. Diagrammatic longitudinal section of the stomach : $A$ of a Camel, $B$ of an ordinary Ruminant, $C$ of a Tragulus $\quad 507$

408. Skeleton of a Mastodon . . . . 510 
Fig.

409. Longitudinal sections of molar teeth, $A-B$, various species of Mastodon, $C$ Elephant

410. Skull of Dinotherium . . .

411. The teeth of the permanent dentition of the left half of the skull of a Dog, and the milk dentition of the same . . . . . . . 513

412. The same of a Cat . . . . . . . . . . 513

413. The teeth of the left half of the upper jaws of : $A$ Dog, B Bear, $C$ Marten, $D$ Badger, $E$ Viverid, $F$ Hyæna, $G$ Lion . . . . . . . 514

414. Pes of a young Sea Elephant . . . . . 518

415. Upper teeth of the Sea Elephant . . . . . $\quad 518$

416. Right anterior appendage of a Pilot Whale . $\quad 520$

417. Skull of a Dolphin. . . . . . . . 521

418. Skull of a Mystacocete . . . . . . . . 521

419. Diagrammatic transverse section of the anterior portion of the head of a

- Balmopterid . . . . . . . . . . 522

420. Skull of a Pilot Whale . . . . . . . . 524

421. A Manus of the Great Anteater, $B$ of the Two-toed Anteater 526

422. Right ramus of the mandible : $A$ of a Rabbit, $B$ of an Agouti 527

423. Transverse sections of molars of various Rodents . . . 528

424. Left hind foot of a Maki . . . . 531

425. A diagram of Appendicularia, $B$ diagram of an Ascidian larva , $\quad 537$

426. Diagrammatic longitudinal section of an Ascidian 538

427. Diagram of a Salpa . 540 


\section{G E N E R A L P A R T.}

\section{Cells and Tissues (Histology).}

The name Protozoa is given to a group of animals of very simple organisation, which form the lowest grade in the animal kingdom. It is well to begin a study of zoology by considering the $\mathrm{Am} \propto \mathrm{b} \mathrm{a}$, one of the many animals belonging to this group, because, in order to appreciate animal organisms in general, it is extremely important to understand thoroughly a simple creature such as this.

The Amœba is a microscopic organism which is frequently found in fresh water. Its shape is irregular and indefinite. It consists of a substance called protoplasm, a finely granular, viscid substance, which, on chemical analysis, is found to consist of a number of different constituents, albumen being one of the most important. Protoplasm also contains a considerable quantity of water and of various other materials. In the protoplasm is a rounded or oval body, the nucleus, and in this again is a smaller spherical body, the $\mathrm{nu}$ cleolus. The Amcba possesses a number of qualities, the most obvious of which is its power of movement: small processes, called p s e ud o podia, are thrust out from the surface, by the streaming of part of the general substance of the animal towards certain
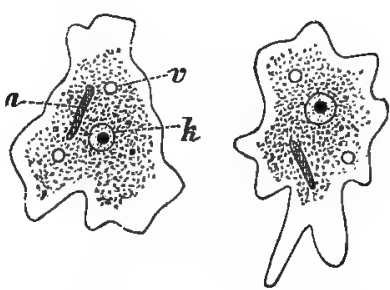

Fig. 1. An Amoebr at two different moments. $k$ nucleus, $v$ vacuole, $n$ ingested food.-After Gegenbaur. points; the pseudopodia then disappear, and new ones are formed; but apart from this the protoplasm is in constant motion, as is shown by the way the granules move about. The mobility of the protoplasm gives the Amœba the power of locomotion, whereby it can glide through the water, past any given object, with greater or less rapidity. Movements ma y take place without an external stimulus, they are then said to be spontaneous: in other cases there is such a stimulus; a movement, often the drawing-in of the pseudopodia, follows contact with some object, but the movement proceeds from the Amoba itself, it is not directly caused by the 
external stimulus, this simply gives the organism an occasion for moving. The power of reacting to stimuli is called ir ritability. The Amœba is further characterised by building up its own substance from materials which it has taken from the environment, i.e., it feeds: it does this by surrounding other little organisms, and inanimate particles, with its pseudopodia, and absorbing them into its protoplasm, expelling, after a time, that part of the food which cannot be assimilated. Besides these solid particles, the Amœba absorbs water, and also free oxy g e $\mathrm{n}$, which is present in all natural waters, and which is absolutely necessary for its existence, since it cannot live in water which does not contain this gas, even if all other necessary conditions are fulfilled. The oxygen unites with some of the carbon present in the protoplasm, forming carbon dioxide, which is got rid of; the Amœba is, as it were, a little machine, in which, just as in a steam-engine, carbon is burnt; a certain amount of energy is liberated by this combustion, and is manifest as motion. So much is clear and certain, but there are many unknown, steps in the activity of the little machine: by the combination of the oxygen and carbon, the complex constituents of protoplasm are broken down, and simultaneously, new compounds, principally water and nitrogenous matter (uric acid), are formed. "The latter is of no use to the Amœba, but must be got rid of; it collects, as waste material dissolved in water, in little cavities in the protoplasm, called vacuoles, and by their contraction (or rather, the contraction of the protoplasm round them) the contents are expelled. It is evident, therefore, that a partial destruction of the complicated materials of the Amcba is constantly going on, so that its mass is diminished, but the food taken in makes good this loss, and even produces a surplus, so that the Amoba grows-it actually increases in bulk. In close relation with this is the last important quality of the Amoeba, its power of multiplying by fission. In this process the nucleus first breaks in two, a constriction of the protoplasm follows, and finally, it is separated into two nearly equal pieces, each with its own nucleus.

All the characters just mentioned distinguish the Amoba as living, as an organism (though of the simplest kind), in contrast to the lifeless particles which occur near it in the water. Death, which is caused by a change in the external conditions (e.g. by too great heat), deprives the Amœba of all these qualities.

In their principal features the rest of the Protozoa are all essentially similar to the Amoeba. A few variations may arise by the formation of a hard, protecting part (skeleton) of lime or silica, in, or round, the protoplasm : or its outer layer may be of a firmer consistency than the rest, so that, although it retains its mobility (the movements of the granules and rough alterations of shape, affecting the whole body, may still be observed), 
there is no protrusion of true pseudopodia. Oil-globules and like matters may be differentiated in the protoplasm: the surface of the body may be covered with fine, hair-like protoplasmic structures, called cilia, which exhibit constant waving movements, etc. (For details, see special part-"Protozoa," p. 85).

All other animals, the Metazoa, begin life as ova, small corpuscles of living protoplasm provided with a nucleus, and at this stage agree, in all essential respects, with the Amœeba. Unlike Protozoa, however, they do not remain in this state: the ovum or egg divides into a number of segments, each with its own nucleus, but, instead of separating from one another, they remain in connection; such segments are called cells; the essential properties of the ovum, as also of the Amceba, are possessed by each one, and the body of the Metazoon attains its definite form by repeated division and differentiation of the cells. The perfect Metazoon, then, is an intimate association of cells like an Amœba, but showing greater or smaller modifications.

Cells consist of protoplasm, like that of the Amoba, either throughout their existence, or only in the young stages. There is, in the protoplasm, a vesicular nucleus containing a watery liquid (achromatin), and a network of delicate threads (the chromatin). One or more rounded nucleoli are also frequently present, often appearing as thickened spots in the network. The cells are capable of $\mathrm{di}$ viding; division is preceded by peculiar changes in the network, then the nucleus divides, and finally the protoplasm separates into two pieces. The cell exhibits all the other essential properties of the Amœba; it absorbs oxygen, takes in food, and so on.

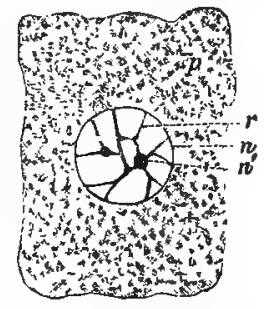

Fig. 2. A cell. $n$ nucleus, $n^{\prime}$ nucleolus, $r$ nuclear thread, $p$ protoplasm.--Orig.

It has recently been shown for many cells that the protoplasm consists of a homogeneous matrix, and of numerous tine threads besides the granules just mentioned. Sometimes many nuclei are present in a cell, which is then considered to be an incompletely divided cell-mass the nucleus has divided, but no division of the cell body has resulted.

The division of the nucleus is, in a few cases, direct; it simply constricts in one place, and then separates into two pieces. As a rule, however, nuclear division is indirect, and then it takes place in a complicated way, as follows: First, the whole nuclear network forms a long coiled thread (Fig. 3, 1), which breaks up into a number of rod-like or curved pieces, the chromosomes; next, the nuclear membrane disappears (Fig. 3,2), and each chromosome splits lengthways in two (Fig. 3,3); but before this, two little bodies, the centrosomes, from which fine threads radiate to the chromosomes, may be noticed in the protoplasm.* Then these threads shorten,

* One or two centrosomes may be noticed lying near the nucleus in many resting cells (i.e., cells which are not ready for division). Possibly they are as constant a constituent of the cell as is the nucleus. 
and the halves of the chromosomes, apparently, are (Fig. 3,4) thereby drawn away from each other, so as to form two separate groups (Fig. 3,5). Each group is the beginning of a new nucleus, for its chromosomes unite again to form a new network, and a nuclear membrane arises round it (Fig, 3, 6). Before this happens, however, the protoplasm begins to divide into two parts corresponding to the new nuclei.

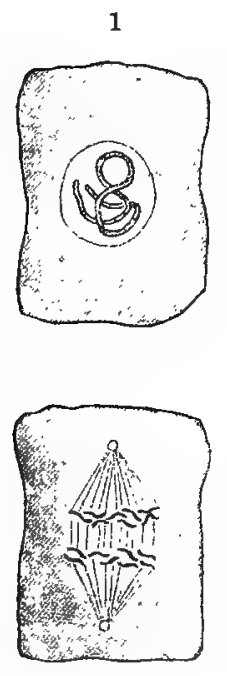

4
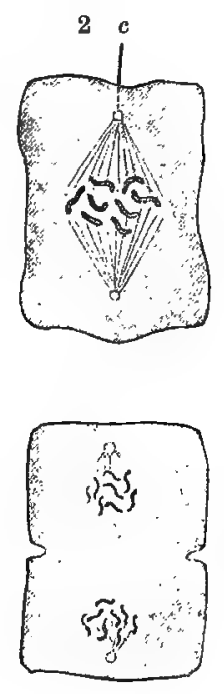

5
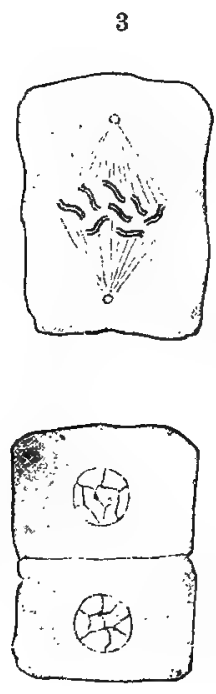

6
Fig. 3. Diagram of indirect nuclear division. $c$ centrosome. See text. -Orig.

Each cell of the animal body has an entity of its own, but it differs from the Amceba, in that it is a member of, and to a certain extent subordinate to, a great whole. The degree of its independence varies, however, very considerably. Some cells, called wandering cells, retain throughout life a very considerable freedom, and, in nearly all respects, remain on a level with the Amœba. They have the power of protruding pseudopodia, and each moves about by itself, freely, in the spaces of the body: moreover, whilst most other cells (see the section on the alimentary canal) can only absorb food in a liquid form, the wandering cells can also take up hard particles and dissolve them. Sometimes this peculiarity seems to be exerted even against the organism itself : for instance, in those cases where (as in the metamorphosis of Insects) some organs atrophywithout the death of the creature itself-the wandering cells eat up the dead parts and digest them. They devour also foreign bodies, such as Bacteria, which have got into the body. The blood corpuscles of most invertebrate animals, ${ }^{*}$ and the white blood corpuscles of the Vertebrata, are wandering cells. There are still other free cells, viz., the red blood corpuscles of Vertebrates, which

* All the Metazoa, with the exception of the Vertebrata, belong to this group. 
resemble wandering cells in their isolation, but differ from them in that they have not the power of independent amceboid movement; they are just carried along passively by the blood.

Most of the cells of the animal body are, however, fixed, they are immovably united to one another and, as they cannot protrude pseudopodia, are fairly constant in shape. These fixed cells are variously modified in the adult: they are specialised, in correlation with differentiation of function. Usually, cells which are modified in the same or in similar ways, are arranged in groups, and such groups are called tissues. Four principal kinds of tissue may be recognised: epithelial, skeletal, muscular, and nervous.

1. The name epithelium is used to designate those tissues which form a thicker or thinner covering to the outer or inner surface of the body, and which consist simply of a number of closely apposed cells. Epithelial cells generally consist of protoplasm, in which there may be excretions such as pigment granules; oil globules, etc. : they vary in shape; some are squamous, the height

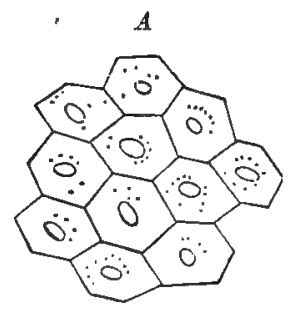

Fig. 4. A Simple squamous epithelium, surface view; $B$ the same in section. $C$ Section of simple colnmnar epithelium.-After Gegenbaur. less than the breadth; others columnar, the height greater than the breadth; or the breadth and the length may be approximately the same.

The epithelial cells are joined together by small quantities of intercellular cement-substance: often, too, there are delicate strands of protoplasm passing from cell to cell.

A

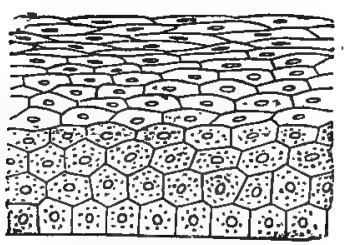

B

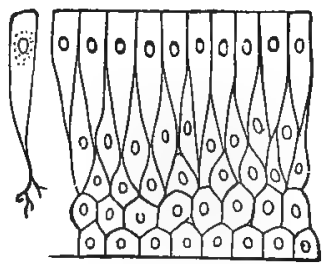

Fig. 5. A Stratified squamous epithelium. After Gegenbaur.

Epithelia may be simple or stratified. Simple epithelium consists of a single layer of closely adherent cells, which may either be flattened (simple squamous epithelium) or cylindrical (columnar epithelium), or about equal in height and breadth (cubical epithelium). Ștratified epithelium consists of several layers of cells, or, to 
be more precise, it is many cells deep, for the cells are generally not arranged in layers. Whilst the deeper-lying cells are usually undifferentiated, the most superficial, or several of the outer layers, exhibit many modifications. Sometimes they are flattened, and the epithelium is termed stratified squamous epithelium; or the outermost layer consists of cylindrical cells, when it is known as stratified columnar epithelium.

The free surfaces of the cells, whether of simple or of stratified epithelium, may be provided with cilia, delicate hair-like processes, which are in constant motion. These are called ciliate cells, or if only one large hair, a flagellum, is present, flagellate cells. Sometimes isolated groups of such cells are found in simple, or in the superficial layer of stratified epithelium; or, again, they may compose the whole, or almost the whole, layer (of course, the outer layer only in stratified epithelium). This is commonly called ciliate epithelium.

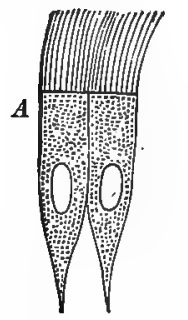

Fig. 6. A Ciliated cells. $B$ Columnar cells, with a outioular plate (c).-Orig.

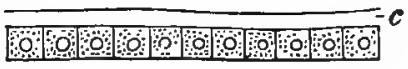

Fig. 7. Simple epithelium, with a cuticle (c). Orig.

Not infrequently the epithelial cells secrete, at their free edge, a firmer substance, a cuticular plate (often called the cuticular border); the cuticular deposits of neighbouring cells usually become closely connected, so as to form a continuous covering or cuticle, which occasionally attains a considerable thickness and hardness.

Fig. 8. A Columnar epithelium, with goblet cells, from one of these mucus is escaping. $B$ Other gland célls.-Orig.
$A$

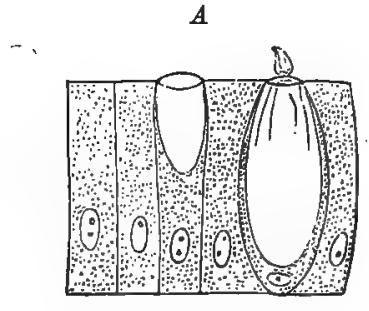

$B$

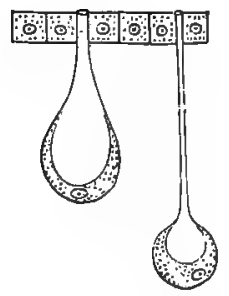

The primary function of the epithelium is to form a protecting covering for the other tissues, but it often performs a second, viz., that of secreting: materials, usually fluids, which are either of great importance to the body, or else need to be discharged from it (urea). Isolated gland cells, usually of a peculiar form, are often met with in epithelia, such are the goblet cells of many animals. They have a 
central cavity, wherein is contained the substance separated from the protoplasm (e.g., mucus), and this escapes to the exterior through an aperture. Secreting cells commonly consist of a long thin neck, wedged between the adjacent epithelial cells, and a wide sac-shaped inner part, lying in the deeper tissues. All secreting cells derived from the general epithelium are called glandular cells, for any part of the animal body which forms a secretion is considered to be a gland. Sometimes glandular activity is distributed over a large, continuous area of the epithelium, which is usually invaginated into the subjacent connective tissue; such are true glands. In its simplest form the gland is a flat patch of cells, a pit, a small sac, or a longer tube; but the tube itself may form evaginations, and these on their part may possess branches, and so

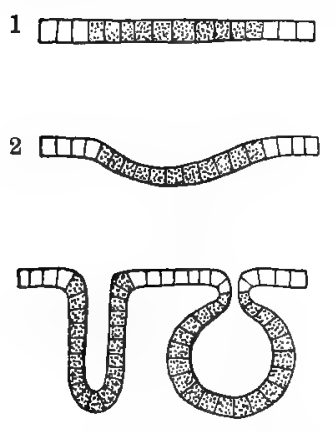

3

4

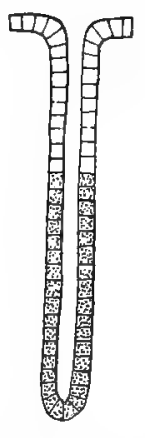

5

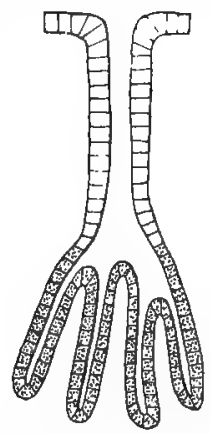

6

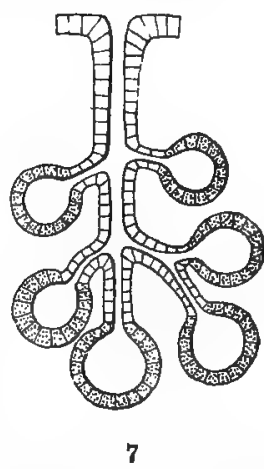

Fig. 9. Diagrams of different true glands. The secreting cells are dotted. 1 The 'simplest form; the secreting cells are not invaginated. 2-5 Different kinds of simple glands. 6 Branched tubular gland. 7 Racemose gland.-Orig.

on; so that a compound gland is made up of a complex system of canals (the walls of which consist of a single layer of epithelium), held together and supported by connective tissue (see below). In these large glands, the ends of the branches only secrete, whilst the rest of the system serves as a reservoir and as a means of exit. Such a distinction into secreting portion and duct, may be noticed even in very simple glands. Sometimes the ends of the canals exhibit a rounded enlargement, when the gland is called $\mathrm{racemose}$, to distinguish it from the $\mathrm{tubular}$ gland, which is without this expansion.

For the development of sensory epithelium, see "Sense-organs."

2. Skeletal tissue is characterized by the great prominence of the intercellular substance. In an early stage of development it consists, like epithelia, of cells only; but later, the cells, though they always remain simple and undifferentiated themselves, secrete some form of intercellular substance which constitutes the chief part of the hard structures, and gives them their great importance in the animal body. Of skeletal tissues, connective 
tissue, cartilage, and bone are recognised. In the first of these the intercellular substance is more or less soft, and the cells are of various shapes, fusiform, stellate, flat. There are three kinds of connective tissue:-cellular, in which the intercellular substance is but slightly developed, often forming only a membranous partition between the large vesicular cells: mucous or gelatinous, with a homogeneous and jelly-like intercellular substance (cells rounded or stellate): and fibrillar connective tissue, where the intercellular substance is composed of delicate threads, among which are often branched elastic fibres; when these are very numerous, the tissue is called elastic tissue. The intercellular substance of $c$ artilage is firmer, it is usually homogeneous (hyaline cartilage), or it may contain elastic fibres (elastic cartilage). In this kind of tissue, which is principally found in the Vertebrata, the cells are, as a rule, rounded. Bon e is

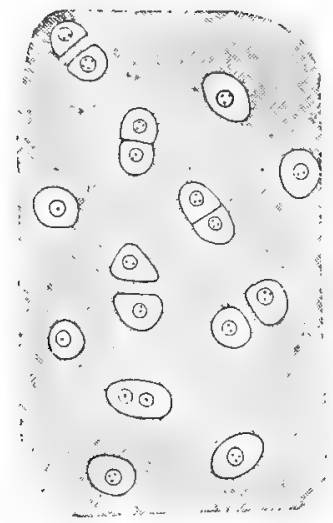

Fig. 10. Hyaline cartilage.-Adapted from Gegenbaur.

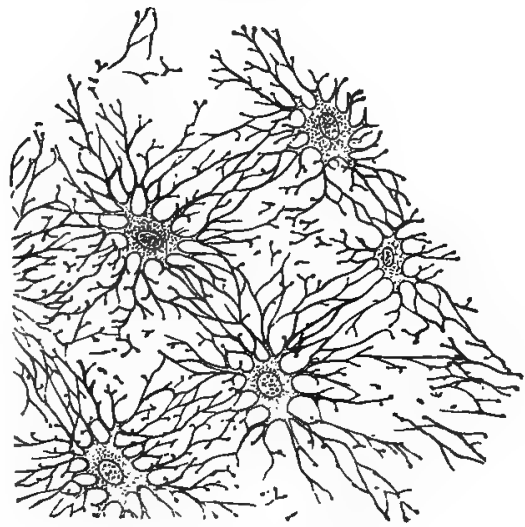

Fig. 11. Bone. - After Gegenbaur.

characterized by a yet greater hardness, on account of the lime salts (especially phosphate of lime), which are deposited in the intercellular substance.* The cells are stellate, with anastomosing processes. This tissue occurs only in the Vertebrata.

Fat cells are sometimes present in the connective tissue; when they are very abundant, the tissue is termed a dipose. The

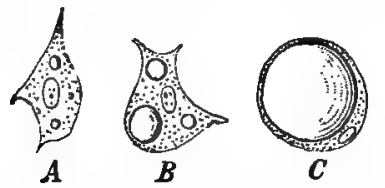

Fig. 12. $A-B$ Young fat cells. $C$ Older fat cell, with a very large oil-drop.-Orig. cells contain drops of fat or oil, which may increase in number to such an extent that they run together to form one large drop. In this case the cell comes to consist of only a delicate layer of protoplasm surrounding a large drop of oil. Connective tissue cells may also contain pigment (usually dark); these are known as pigment cells (chromatophores).

* Sometimes lime salts are deposited in cartilage (calcified cartilage). 
3. In muscular tissue the cell-protoplasm is partially or entirely modified into a peculiar contractile substance, which does not move spontaneously, but only contracts in response to some stimulus.* It differs in another respect also from other protoplasm: the movement of the muscle cell always occurs in a definite way: first, there is a shortening and thickening of the cell followed by a lengthening and narrowing, so that it has assumed its original condition by the end of the movement. The streaming movements seen in the Amœba, which are characteristic of protoplasm in general, are never found in the contractile substance of muscle cells.

The simplest form of muscular tissue is composed of smooth muscle cells, which are spindle-shaped, elongate or ribbon-like, and pointed or occasionally forked at both ends. A nucleus is present in each, lying either in the middle of the cell, surrounded by a small quantity of protoplasm, or on one side of the contractile substance, enveloped in a varying amount. The contractile substance is homogeneous and shining, and is destitute of granules or transverse striation, though it may sometimes be longitudinally striped. The muscles of most of the Invertebrata, with the exception of the Arthropoda, consist of this tissue, and it is found also in Vertebrates, in the walls of the digestive tract. To the smooth are nearly allied the striped muscle cells, the only essential difference being that the contractile substance of the latter has a transversely striated appearance, due to its division into regularly alternating discs of different refrangibility: it occurs, for example, in the Vertebrate heart.

The transversely striated muscle fibres differ from the striped muscle cells in that they are multinucleate. A striped muscle fibre originates as a simple cell with on e nucleus, which divides repeatedly without corresponding division of the cell, and in the perfect fibre, the nuclei lie either in or upon the surface of the contractile substance. The fibre is not only transversely striped, but a more or less distinct longitudinal striation, depending on the very delicate fibrillæ of which the fibre is composed, may also be noticed. The whole muscle fibre is

* As to the source of the stimulus, see below. 
enveloped by a thin sheath, the sarcole mma, which is wanting in smooth as well as in striped muscle cells. The fibres are usually cylindrical, and are rounded, rarely branched or forked, at the ends; they are often of considerable length. In the Arthropoda the whole,

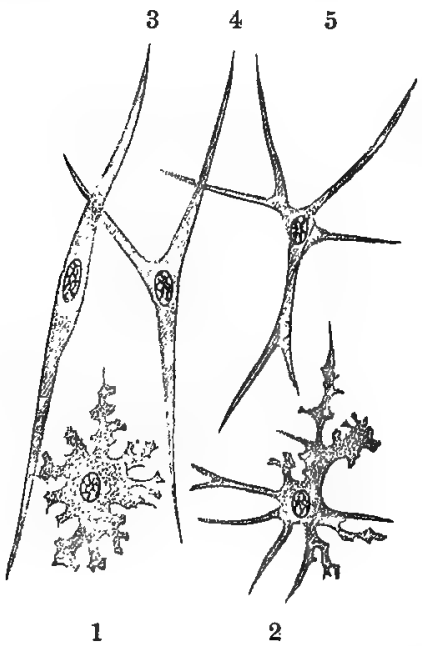

Fig. 14. Conneotive tissue cell (1), smooth muscle cells ( $3-5)$, and a cell (2) which is undergoing transformation into a muscle cell. From the urinary bladder of the Salamander. - After Flemming. and in the Vertebrata the greater part, of the musculature consists of striated muscle fibres, which like the striped muscle cells, contract with greater rapidity, and with greater force, than the smooth muscle cells.

Muscle cells and fibres may not only form large tracts of tissue, but they may also occur as isolated secondary constituents of the connective tissue; where these are very numerous and the connective tissue is scanty, an appearance of muscular tissue is produced. This shows the intimate relation between connective tissue cells and muscle cells, which is further demonstrated, in the case of the scattered muscle elements, by the occurrence of connective tissue cells which have been partially modified into muscle cells. Sometimes, like epithelial cells, muscle cells are held together by cement substance.

4. Nervous tissue. The contraction of muscle cells * is brought about by stimuli received from ganglion cells, each of which has a thread-like prolongation often of considerable length (Fig. 15, 2). At its free end each of these processes breaks into a tuft of branches which lie closely upon the muscle cell; sometimes a process gives off branches on its course, and these are attached to muscle cells. Besides these long processes, the ganglion cell may also give origin to numerous shorter branching offshoots, which do not pass to muscle cells.

Ganglion cells of this description are called motor: there is another kind, the sensory (Fig. 15, 4), which are, externally, just like the motor ones, but receive, by their long processes, impressions from the outer world. The process may, for instance, go to the epithelium covering the surface of the body, and branch between its cells. (See the section on Sense Organs, p. 18).

The ganglion cells occur in groups, comprising both kinds. They are attached to one another by some of the prolongations; those of one cell do not as a rule, however, pass directly to another, but

* Under muscle cells, smooth and striped muscle cells and also muscle fibres are included. 
ramify over it or its processes (Fig. 15, 3). There is yet a third kind of ganglion cell connected with neither epithelium nor muscle, but only with other ganglion cells (Fig. 15, 1).

It may be noticed here that many gla $\mathrm{nd} \mathrm{cells,} \mathrm{like} \mathrm{muscle} \mathrm{cells,} \mathrm{only}$ become active when they receive a stimulus from a ganglion cell; in glands, therefore, terminal branches of ganglion processes are present in great numbers.

1

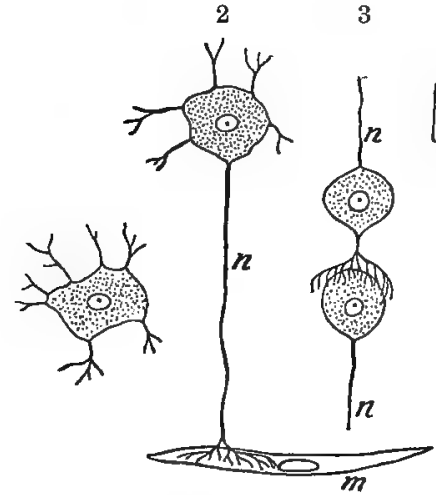

4

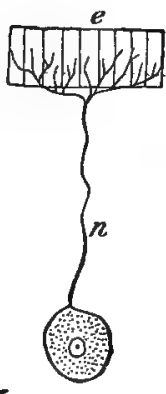

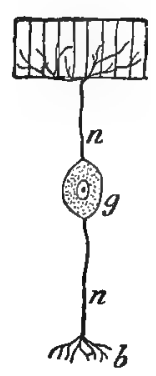

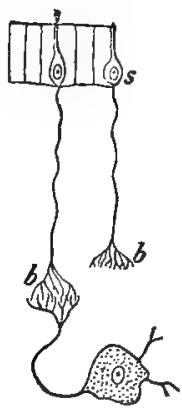

Fig. 15. Varions ganglion cells, etc. 1 Ganglion cell, with dendritic, but without the longer provesses. 2 Motor ganglion cell connected to a muscle cell $m$ by a nerve fibre $n$. 3 Two ganglion cells connected with one another. 4 Sensory ganglion cell, with its nerve fibre branching in the epithelium e. 5 Sensory ganglion cell $g$, with two long processes; $b$ the terminal tuft of the process. 6 Sense cells $s ; b$ the terminal tuft of an efferent nerve fibre; a ganglion cell below.-Orig.

The long processes of ganglion cells are called nerve fibres, and according to their connection with muscle or with sense cells, they are called motor or sensory: the short processes are termed dendrites. Sometimes nerve fibres originate not in ganglion, but in sensory, cells (Fig. 15, 6): the epithelial cells are, in this case, usually tall, narrow cells, with a tuft or fringe of cilia at the free end; they pass, on the inner side, into long delicate processes, which are intimately connected with ganglion cells, for the end breaks up into a fine anastomosis, which is closely apposed to a ganglion cell or its processes. Such epithelial cells are called sensory cells, and the prolongations, sensory nerve fibres.

The nerve fibres are frequently enveloped in sheaths of specially developed connective tissue. Most of those in Vertebrates have a double sheath, a strongly refringent fatty medulla within, and an outer neurilemma or sheath of $\mathrm{Schwann}$. Some vertebrate nerve fibres are covered with a neurilemma only.

\section{Organs.}

Although the animal body forms a connected whole, yet in most Metazoa a varying number of more or less independent o r g a $\mathrm{n}$ s may be distinguished, each composed of one or more of the tissues already 
described. The more general characteristics of these organs will now be studied.

There is a very slight differentiation of organs in the lowest Metazoa, the Coelentera; but this group is peculiar in many repects (see Special Part).

\section{The Skin.}

The skin which forms the external limit of the body consists, in the simplest cases, of an epithelium only, the epidermis. It frequently includes, however, a layer of connective tissue also, the dermis. In most Metazoa the epidermis is a simple epithelium, consisting of squamous or columnar cells, and often ciliated; in the Vertebrata alone, it is a stratified epithelium, of which the outer cells are horny and thus form a protective covering for those beneath: this is represented in many other Metazoa by the cuticle (Chrtopoda, Insecta, and others). The dermis is a layer of connective tissue of varying thickness, and of a firm consistency, lying beneath the epidermis; it is not generally sharply marked off from the neighbouring structures, in most Vertebrates it passes gradually into the loose sub-cutaneous connective tissue. In the lower Metazoa calcareous deposits of different sizes and shapes are frequent in the dermis (Echinoderms) and among the Vertebrata there are often bony plates (scales of Fish). Muscle cells also are very common.

Glands, both unicellular and true glands, of diverse function, are often found in the skin, such as mucous glands, stink glands, oil or sweat glands. Various appendages, such as hairs or bristles, may be present, but under these names are comprised structures of very different kinds. The $\mathrm{ch}$ ætæ of Chætopoda, for example, are solid cuticular processes, arising as a secretion from certain epidermal cells ; the setæ of Arthropods are also cuticular, but they are hollow, and contain an epidermal core. Mammalian hairs, on the other hand, consist of horny epidermal cells.

Suckers are specially modified muscular portions of the skin, serving in many animals as organs of adhesion. They usually stand out from the surface

$A$

$h$

$u$

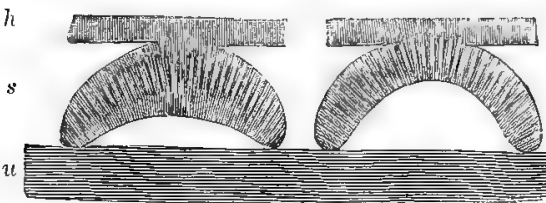

Fig. 16. Diagram of $\mathrm{sucker}$ in two different conditions; transverse sections. $h$ skin, s sucker, $u$ foreign body. See text.Orig.

in the form of small thick-walled cups with smooth edges, the convex side towards the skin, and the concave side free. The sucker generally works somewhat in the following way: its edge is pressed against a foreign object (Fig. 16, A), and by the contraction of the muscles abundant in its walls, the space between the two increases (Fig. 16, $B$ ), so that a chamber is formed wherein the pressure of the air or water is sub-normal, while the external pressure on the outer wall of the sucker holds it firmly to the object.

Suckers of this description occur in Flat-worms, Leeches, Cuttle-fish, some Mammals, etc. . but other a dhesive organs are also found. Some animals 
have areas of sticky skin by which they can adhere to other bodies (Amphibian larvæ), others can hold fast by simple a dh e si on,* applying a surface which is smooth and damp, but not sticky, to the foreign object (Tree-frog); others again can fix themselves by means of hooks, \&c.

The colour of the skin depends, in many cases, upon cells containing pigment granules, present either in the dermis or the epidermis (or in both). The pigment varies in chemical composition. Sometimes the blood in the dermal blood-vessels shows through the skin (cocks' combs). The colour of the skin may, however, depend on totally different circumstances: for instance, in ter fere n ce colours* are known, which are due to the peculiar structure of the skin, e.g., the stratification of cuticle or dermis; the well-known metallic colours are often caused thus. The skin, or its derivatives, is sometimes white (e.g.,. hair of Mammalia); this occasionally depends upon the presence of tiny airvesicles.

Moulting is frequent in animals; the outer layer of the epidermis, either the cuticle (Insecta, Crustacea), or the stratum corneum (Vertebrata) becomes loosened from the rest of the skin, and is thrown off all at once, or more rarely in pieces. Such a moult is always accompanied by a new formation of cuticle or of stratum corneum, which has always begun before the animal casts the old one.

Mucous membrane is the name given to the skin-like lining of the cavities of the body which open directly on to the outer surface (e.g. the digestive tract); it consists of an epithelium supported by a layer of connective: tissue, corresponding with the epidermis and dermis of the skin.

\section{Skeleton.}

The protective structures found upon, or the calcifications and ossifications present in, the skin, which have just been referred to, frequently attain a considerable thickness, hardness, and coherence, and then form the supporting organ of the body, the skeleton, under which name all the hard parts are included. The skeletal elements of the skin, the exoskeleton, owes its origin either. to the epidermis, as, e.g., in the Lobster, where the well-developed thick calcareous cuticle forms the supporting structure, or, as in the Snail, whose shell is the secretion of certain epidermal cells; or to the dermis, e.g. the numerous calcareous plates forming the shell of the Sea-urchin, or the bony plates which compose the carapace of the Turtle. In many animals, however, especially in the Vertebrata there is also a firm supporting framework, the endoskeleton which lies within the body and is quite independent of the skin: it consists chiefly of cartilage and bone, and is often present in addition to the exoskeleton, with some portions of which it may be intimately connected (Tortoise).

The exoskeleton comes to have, in most cases, a protective as well as a supporting function, often indeed, this is its principal duty, e.g., Molluscs and

* See Text-book of Physics. 
Chelonians. This is true, also, although in a more limited sense, for the endoskeleton, which usually not only supports the body but also protects certain of the organs, e.g., the skull and vertebral column of the Vertebrata protecting the central nervous system.

\section{Muscular System.}

Muscular tissue occurs as a subordinate component of many organs, e.g., the skin, the alimentary canal. It is, however, the principal and essential constituent of $\mathrm{muscles}$, those organs which cause movements of the body as a whole, of individual portions of it, or of its appendages, and which, taken together, constitute the muscular system. In many of the lower animals which have neither exo- nor endo-skeleton, the musculature is closely adherent to the skin, and forms a continuous layer beneath it. In many worms, there is such a body-wall, causing movements of the animal by its contractions. The formation of an exoskeleton has a great influence upon the development of the musculature, especially when the former is divided into a number of movable pieces (as in Crustacea, etc.) ; the continuous coat is then separated into a number of more or less independent strands, the muscles, extending between adjacent portions of the skeleton and causing them to move upon one another. The muscles are still, however, connected with the skin, of which the skeleton is indeed, in this case, only a part. The connection ceases, where, as in the Vertebrata, an en doskeleton is developed, for the muscles are now attached to this, and movements of the body are chiefly caused by movements of its different parts.

It has already been stated that the essential part of a muscle is muscular tissue, but this is not its only constituent, a certain amount of connective tissue is usually present, surrounding and holding together the muscle elements, and often forming te $\mathrm{ndons}$ at the ends of the organ. The tendons are thinner than the muscle proper, often narrower, and are composed exclusively of fibrous connective tissue. They make it possible for the contractile, thicker part of the muscle, to be at a considerable distance from the spot at which the force is applied. The name tendon has been used to designate not only these connective tissue organs, but also others of a like significance, but of a different structure, as will be seen in the detailed account of the Arthropoda.

In the lower Metazoa movement of the body is caused at least in part, by the cilia of the epidermis (or of some portion of it). This is especially the case in many minute free swimming larvæ (Cœlentera, Echinoderma, Chætopoda, Mollusca), which are driven about by ciliary movements. In these forms there is an actual locomotor apparatus consisting of cilia, which either cover the whole body uniformly, or are restricted to definite lines or rings. In the adult, on the other hand, the ciliated cells are but seldom of locomotor importance (Platyhelminths, Rotifers). 


\section{Nervous System.}

Ganglion cells and nerve-fibres are usually aggregated, the groups of ganglion cells are known as ganglia, the bundles of nerve-fibres as nerves. It generally happens that most of the ganglia are connected to form a central nervous system, from which spring the nerves supplying muscles, sense organs, and so on, all these nerves are included in the peripheral nervous system; nerve fibres are, of course, present also in the central nervous system, and especially in the strands connecting the ganglia. Similarly the ganglia do not occur exclusively in the central nervous system, although they are connected with it, even when situated in remote parts of the body.

Nerves are called motor or sensory, consisting respectively of motor or sensory fibres*; many are, however, mixed, and contain both kinds of fibres. Nerves usually branch during their course, dividing

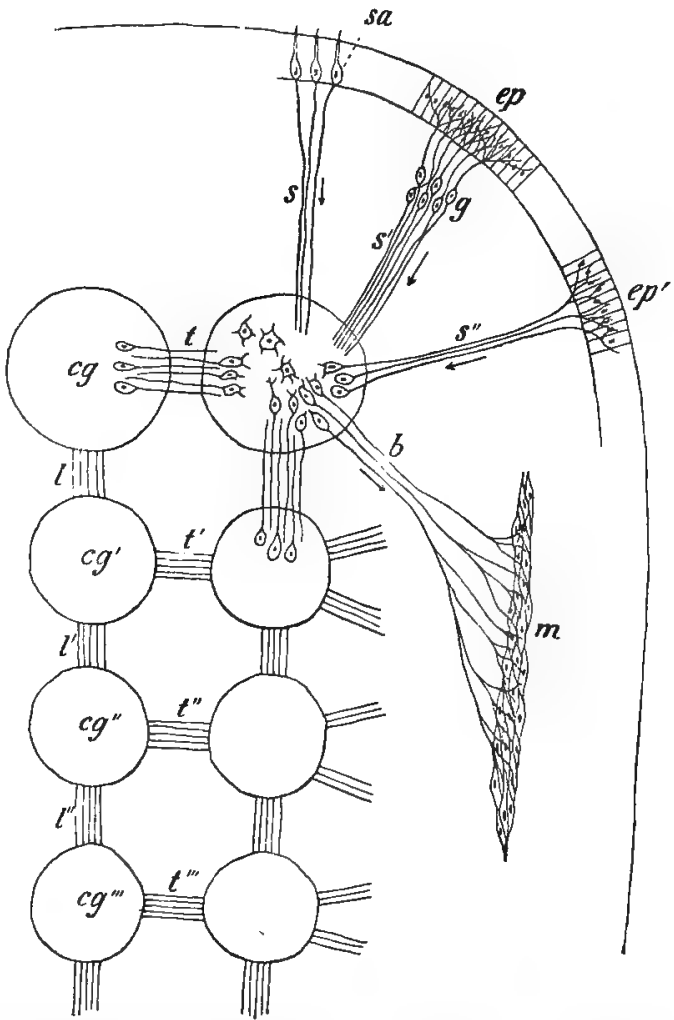

Fig. 17. Diagram of a nervous system. $c g-c g^{\prime \prime \prime}$ ganglia of the central nervous system, $l-l^{\prime \prime}$ and $t-t^{\prime \prime \prime}$ longitudinal and transverse connective fibres. $s a$ sensory cells. $s$ nerve fibres, springing from them. $g$ peripheral ganglion, each of whose cells gives off two fibres, one branching in the epidermis ep, the others going to $\mathrm{cg}$. $\mathrm{s}^{\prime \prime}$ sensory fibre, which arises in cells lying in $c g$, and branches in $e p^{\prime}$. $b$ motor fibre, which goes to a muscle $m$.-Orig. gradually into thinner and thinner strands, consisting of fewer and fewer fibres.

The central nervous system is, as it were, the "Exchange" of the body; by the motor nerves it transmits impulses to the muscular elements and thus controls their movements; by the sensory nerves it receives impressions sent from the different sense organs. sight.

* Sensory nerves are further classified as nerves of touch, taste, smell, hearing, or 
The sympathetic nervous system, present in many animals, consists of a series of small ganglia and nerve trunks, from which nerves are given off to the digestive tract (in the Vertebrata to other viscera also and to the vascular. system). The sympathetic system is comparatively independent of the central nervous system, although it is connected with it; the muscle cells of an organ supplied by it, contract without impulse from the central system, they are involuntary muscles, and receive the necessary stimulus from the sympathetic ganglion cells.

\section{Sense Organs.}

An animal receives impressions from the outer world by means of the sense organs, which are usually modified tracts of epidermis. They are always closely connected with the nervous system either by nerve fibres springing from ganglion cells (within or external to the central nervous system) and ramifying amongst the cells of the sense organ (Fig. 17, $e p$, and $e p^{\prime}$ ); or by fibres originating in the sense cells of such an organ (p. 17) and passing to the ganglia (Fig. 17, sa).

Sense organs may be classified as simple or complex; to the former class the organs of touch, of $\mathrm{smell}$, and of $\mathrm{taste}$ are usually referred, since they are of a simpler structure than the organs of $h$ earing and sight, which are often very complicated.

The sense of touch contrasts with the other senses in that it is distributed over the whole, or the greater part, of the surface of the body; the entire skin, especially the epidermis, is, therefore, a sense organ. In some cases, e.g., in the Vertebrata, nerve fibres pass to these portions of the skin and ramify amongst the epidermal cells; in others, e.g., amongst Annelida and Mollusca, special tactile cells, each furnished at its free surface with one or more hair-like processes, ${ }^{*}$ and continued at the other end into a nerve fibre, $\dagger$ occur amongst the ordinary epidermal cells. In some animals the skin is furnished with warty or filamentous processes, provided with numerous tactile cells, or with a rich nerve supply, and, therefore, important as tactile organs.

Amongst Vertebrates, certain tactile nerve fibres, as already mentioned, innervate the epidermis, but others terminate in the dermis. The latter are sometimes enveloped in concentric laminæ of connective tissue, pacinian bodies, + or the nerve endings may be of a different form.

Olfactory Organs are acted upon by gaseous matters in a peculiar way; they can be ascribed with perfect certainty to only a small proportion of forms, viz., existing terrestrial animals. They are formed of epithelium, derived from the epidermis, in which there are tall, thin, sensory cells, each with a tuft or tufts of sensory hairs at

* Sometimes these tactile, and other such, sensory cells are very like ciliate epithelium; the hairs are, however, not actively, but merely passively movable.

$\dagger$ Sometimes only a narrow external part of the sense cell is situated between the other epidermal cells, whilst the thicker part, with the nucleus, lies in the subjacent connective tissue.

† These may also occur internally-e.g. in the mesentery of the cat. 
the tip, and continued at the other end into a nerve fibre, which passes to the front part of the brain. Amongst Insects, which have been proved by many experiments to possess a very acute sense of smell, the olfactory organs are situated upon the anteanæ. These are provided with delicate hairs, which, like arthropod hairs in general, are evaginations of the cuticle; they are very thin walled, generally seated in small depressions, and into each, a thread-like process extends from one or more underlying sense cells (Fig. 18).

Whilst these insectan structures are probably true olfactory organs, there are numerous contrivances to which, even in this book, the same name is applied, but with doubtful right. This is the case with all the "olfactory organs" of aquatic animals, which seldom or never come in contact with air. The olfactory pits of Fish, which, from their position, \&c., are homologous* with the olfactory organs of higher Vertebrates, can, for instance, hardly be really olfactory. This is also true of the delicate "olfactory hairs" present on the antennules of many Crustacea; like the above-mentioned structures in Fish they are undoubtedly sense organs, but their special function is unknown: they are, perhaps, organs of taste.

Gustatory Organs are affected

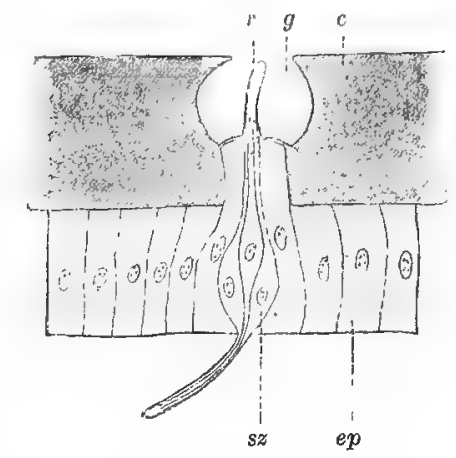

Fig. 18. Section through a small piece of the antenna of an Insect, diagrammatio ; $c$ cuticle; $g$ pit, with $r$ the "olfactory hair," arising from the thin part of the cuticle ; ep epidermis, $s z$ sense-cell. - Orig. only by substances which occur in a liquid form. In the Vertebrata they are represented by the so-called taste-buds of the tongue and the walls of the mouth, specially modified tracts of the buccal epithelium, consisting of groups of cells, amongst which are long thin cells with a delicate point projecting from the free end; these are, probably, the true organs of taste. The buds are supplied with nerve fibres which branch between the cells. In Fish, taste-buds may occur on the external surface of the body; similar struetures are found also in many Chætopods and Molluscs (e.g., Gastropods), not only in the buccal cavity but also externally upon the anterior end of the body. Of a different type are the organs which appear to bring about sensations of taste in Insects; they are short hairs placed on the underlip, jaws, etc., and are in all essential respects like the olfactory hairs just mentioned.

Yet other structures are included under the simple sense organs, but of their special function nothing is definitely known. Such are the lateral line organs of Fish (See Pisces) and Amphibia, and the similar structures in Annelids and Molluses.

Auditory Organs always occur as vesicles filled with fluid. The vesicles (otocysts) are formed by epidermic invaginations, and 
either remain as sacs, opening freely on the surface: or more often are entirely cut off from the epidermis, and are therefore closed

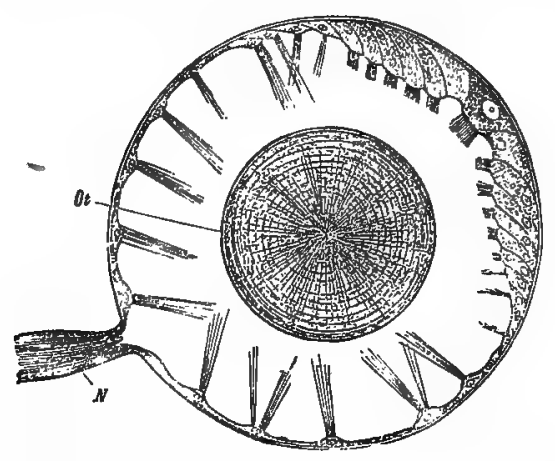

Fig. 19. Auditory capsule of a Gastropod. $O t$ otolith, $N$ auditory nerve.-After Clans. sacs and frequently lie deep in the body. The auditory capsules are usually almost spherical, but in the Vertebrata they are more complicated, as will be seen later. The wall into which the nerve fibres (auditory nerve) pass, consists of a simple epithelium, whose cells-all, or only some-are provided with fine hair-like processes projecting into the liquid. These hairs are set in vibration by sound waves, and the sensations thus caused are conducted by the auditory nerve to the central nervous system. One or more hard calcareous corpuscles, the otoliths, are usually suspended in the liquid. Amongst the higher Vertebrates, various kinds of accessory apparatus function as resonators, etc.

The auditory organ of A rth ropods differs essentially from the usual type. In Crustacea this sense is closely connected with specially developed hairs, which are free on the surface of the body, or contained in cup-shaped invaginations. In Insects again, there is quite a different arrangement, as will be seen in the Special Part.

It has been proved, experimentally, that the auditory capsules of many animals have yet an other f unction than that of bringing about impressions of sound. They (or in Vertebrata, their semicircular canals) are of significance in the maintenance of the normal equilibrium in an animal. If they are destroyed, movement becomes unsteady, the animal falls on one side, etc.* In what manner, however, the auditory organs exercise this control is not yet understood.

Optic Organs of most animals are, like the preceding sense organs, specially developed parts of the epithelium. In its simplest form (Fig. 20, 1, ) the organ of sight is a little pigmented patch of the epidermis, innervated by a nerve, the optic nerve (some Medusæ, a few Lamellibranchs). In other cases (Fig. 20,2) the pigmented epidermis forms a small open pit (some Gastropoda, Cœlentera); this arrangement may be complicated by having the cuticle, which covers the skin in many lower animals, thickened over the cells forming the depression, so that a lens, a refractile body, is formed (Fig. 20, 3, certain Cœlentera). Or the cavity is deepened to form a sac with a small external opening, which may contain a gelatinous secretion of the epithelium (Fig. 20, 4, some Gastropods). Again, by the contraction of the aperture, and by the loss of all connection with the skin,

* In some animals, these occurrences only take place when eyes as well as ears are destroyed, whilst the destruction of eyes alone, or of otocyst alone, has no effect. 
the optic organ may come to be a closed capsule lying beneath it. The side of the capsule away from the surface is thickened and pigmented, and forms an organ for the perception of light, the retina,* whilst the opposite side is thin and transparent, as is also the skin above it (the cornea); the cavity of the visual organ then contains a jelly-like mass, the vitreous-body (Fig. 2u, 5, most Gastropods, Chætopods). In others again, the le $\mathrm{ns}$, secreted by the
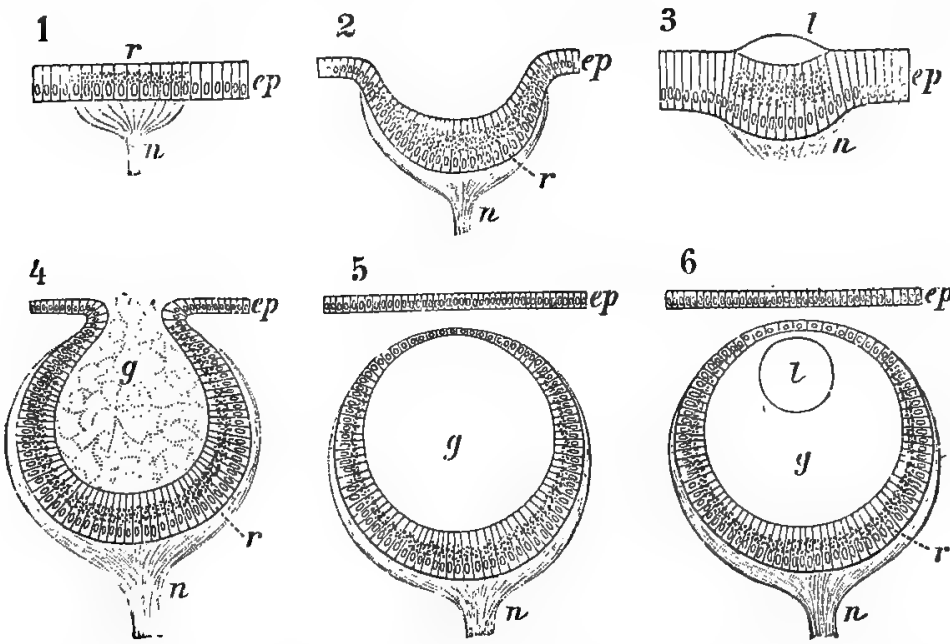

5

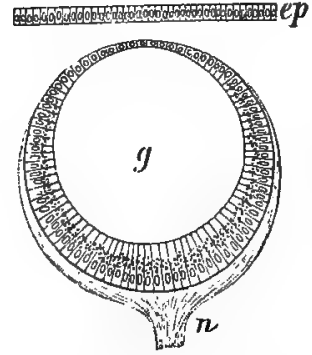

6

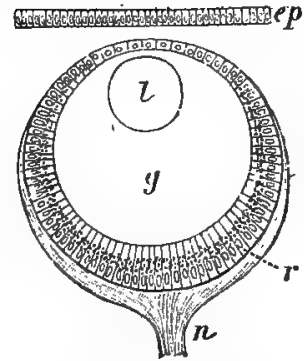

Fig. 20. Different forms of optic organ, diagrammatic. $n$ optic nerve, $r$ retina, ep epidermis, $g$ vitreous body, $l$ lens.-Orig.

front part of the capsule, floats in the vitreous humour, and may be regarded as a specially modified part of that body (Fig. 20, 6, some Chætopods and Gastropods). With the development of this lens the optic capsule has become a true eye; and as in the human eye a real image may be formed upon the retina, whilst the simpler forms can at most merely differentiate between light and darkness.

In such eyes as are described above, the retina consists partly of pigmented cells, which appear to be the true perceptive cells, and partly of colourless supporting cells, which apparently secrete the vitreous body. The former frequently bear, on the ends towards the vitreous body, unpigmented, transparent rods.

The optic organs of Arthropods exhibit a peculiar structure. In its simplest form (Fig. 21, A), peculiar to certain insect-larvæ, the arthropod eye appears as an invaginate area of the skin, passing: directly into the general epidermis: so far, therefore, it resembles that represented in Fig. 20,2. The retinal or perceptive cells are, however, always distinguished by being removed from the surface and covered by neighbouring epidermal cells, the outer portions

* This name is used to denote that part of the optic organ which is actually sensitive to light. 
of which are transparent, whilst they are strongly pigmented within, as is the case also with the retinal cells, their outer rod-like ends excepted. The cuticle covering the eye is thickened like a lens. The next stage is represented by those eyes, in which the retinal cells have lost all direct connection with the skin (Fig. 21, $B$, ocelli of Insects, Insect-larvæ and Spiders). Below the lens there is, therefore, a layer of transparent cells, continuous with the adjacent epidermal cells, and corresponding with those which in the simple type are pushed in above the retina. The compound ey e (Fig. 21, C, D), occurring in most Insecta and Crustacea, consists of a large number (as many as several thousands) of simple eyes, closely packed together, and each
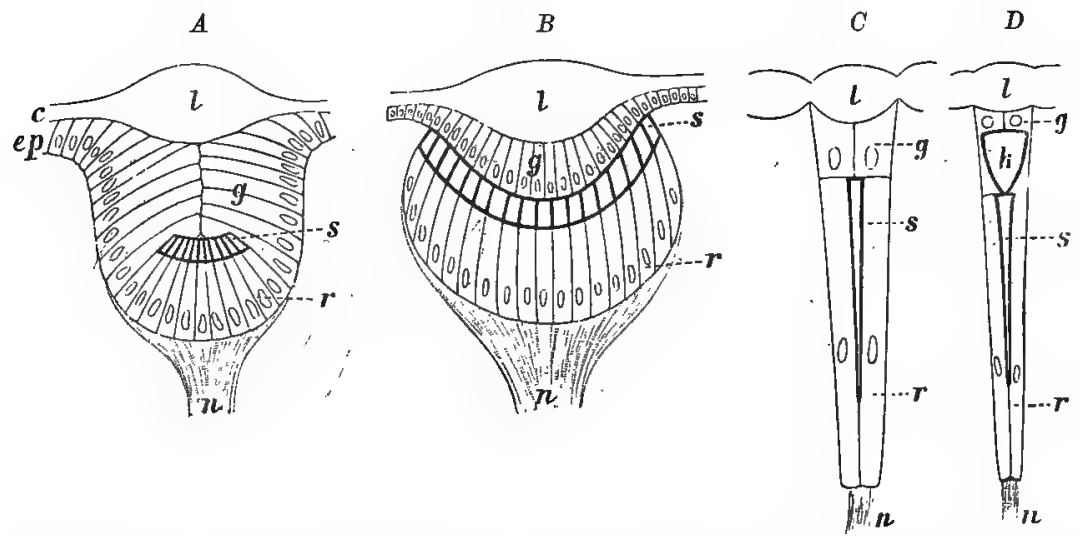

Fig. 21. Different kinds of Arthropod eyes, diagrammatic. $A, B$ ocelli, $C, D$ ommatidia from a compuund eye. $n$ optic nerve, $r$ retina, $s$ rod of retinal cell, $g$ vitreous body, $k$ crystalline cone, $l$ lens, $c$ limit of the general cuticle, ep epidermal cells.-Orig.

possessing a structure similar to that just described. The compound eye is, however, peculiar, in that the eye-elements (ommatidia) of which it consists are very narrow and elongate, and each one consists of only a few retinal $(6-8)$ and vitreous cells. As in the ocellus, each retinal cell ends above or within in a rod-like transparent piece; there is frequently, also, in each vitreous cell, a peculiar refractile body, which is closely united with its fellows of the neighbouring cells to form the so-called crystalline cone (D, $k)$.

Essentially different from the forms of eye already described, where the retina invariably represents a specially developed area of the epidermis, is the visual organ of Vertebrata, whose retina is not epidermic, but a specially modified portion of the brain. These eyes will be described in the section Vertebrata.

Besides the types of optic organ mentioned here, there are still others found in certain animals, but they are simply specialised portions of the epidermis (Platyhelminths, Lamellibranchs, etc.).

It should be noticed here, that not a few animals which have no special visual organs, are, nevertheless, sensitive to light; this is true, 
for example, of Earthworms, which withdraw with the greatest rapidity into their holes if a strong light is thrown upon their anterior ends, where the cerebral ganglion is situated.

\section{Alimentary Canal.}

Like the Amoba, Metazoan cells undergo constant metabolic change with consequent loss of material, and this is a necessary condition for the continuance of the vital processes. In order to make good this loss, they must feed. Unlike the Amœba, however, the individual cells are unable to obtain food direct from the environment: there must be some special arrangements for nutrition: hence the development of an a limentary canal. Food is taken into this canal and there digested, i.e., reduced to a fluid state, so that it can be absorbed by the wall of the digestive tract, and carried to the various tissues of the body. Those portions which are not digested and absorbed pass out again (excreta).

Whilst in most animals, secretions, by means of which the food is digested, are poured into the alimentary canal, there is another arrangement amongst Coelentera and Sponges. No such digestive juices are found here, but the food comes in contact with epithelial cells, and is digested and absorbed directly by them. Smaller organisms, e.g., Diatoms, may even be entirely engulfed by the epithelial cells. This also frequently occurs among Flat-worms.

In its simplest form the digestive tract is a sac or a canal, which communicates with the exterior by a single aperture only. This opening then serves both as an entrance or mouth, and also as an exit for the undigested portions of the food (Cœlentera, Flat-worms).

In most animals, however, the digestive tract has $t$ w o openings, a mouth and an anus. It is then usually a long tube, with the mouth at one end, and the anus at the other. It is frequently divided into several regions, with different functions. In the simplest cases, only three regions can be distinguished, the fore-gut (stomodæum), which is often very muscular, and serves in different ways for the ingestion of food: the mid-gut (mesenteron), which is usually long, and in which digestion and absorption go on; and the hind-gut (proctodæum), which serves as an excurrent canal, and as a reservoir for undigested materials (Nematodes, Annelids). In other cases, the fore-gut is again divided into a large buccal cavity, often provided with organs for the prehension and retention of prey, or for the comminution of food (teeth); and a narrow œsophagus. The mid-gut is frequently, as in Vertebrata, divided into a capacious anterior portion, the stomach, and a long narrow hinder part, the true gut or in testine (small intestine of Vertebrata). The stomach is digestive, its walls are beset with glands, which secrete a digestive fluid; whilst the intestine is absorptive, though digestion goes on here also. More rarely the hind-gut is also divided into several sections. Occasionally some 
portion of the canal is modified to form a gizzard for the trituration of food. In some animals, e.g., the higher Crustacea, the hind part of the œsophagus widens out, becomes muscular, and is furnished within with hard denticles; in other cases, as in Birds, it is the posterior region of the stomach.

Very frequently diverticula, blind sacs of various kinds, are found at different parts of the alimentary canal. Their function varies: those belonging to the fore-gut serve only as provisional reservoirs for the food which has just been taken in (crop in Birds and Insects); those of other regions usually serve to enlarge the canal, or to increase the digestive and absorptive surface (diverticula of Leeches, cæca of Mammalia), but this may be effected in other ways-by the elongation of the intestine, by delicate foldings of the intestinal wall, or by some similar contrivance (Vertebrata).

The digestive fluids are secreted partly by the epithelium of the digestive tract; partly by minute glands imbedded in the wall, especially of the stomach and intestine; and partly also by larger glands, lying outside the alimentary canal, and communicating with it only by means of their ducts. In many lower animals the glandular activity is associated with the epithelium of the digestive tract alone, whilst among more highly organised forms, the Vertebrata, for example, it may be essentially or exclusively possessed by special glands. The larger glands lying without the canal are variously named, according to the region into which their ducts open; those for instance, which open into the buccal cavity, and which are often of little direct use in digestion, serving only to moisten the food and to facilitate its onward course, are called salivary glands; whilst those which open into the intestine are usually termed liver. The secretions of these glands differ in different animals; the effect of the "liver" secretion for example, is by no means always the same.

As to its minute structure: in its simplest form, the alimentary canal consists only of a layer of epithelium, but connective tissue and muscle may come to surround this, so that in a more highly specialised gut, the wall is composed of several layers: within, a layer of epithelium coated by a connective tissue sheath, often including numerous little glands, the two being closely adherent, and constituting the mucous membrane; outside this there is a sheath of muscle cells (the muscular coat) whose contractions are of great importance for the passage of nutriment through the canal.

In only a few Metazoa is the alimentary canal wanting. In such cases, either there is a functional mouth to allow of the entrance of food into the soft tissues of the body, and these perform the digestion: or, there is no mouth, and the food passes through the skin of the animal by endosmosis. This, however, only occurs in parasitic animals, especially in such as live in the gut of other animals, where 
they are always surrounded by food in a fluid or semi-fluid condition (Cestoda, Acanthocephala).

Unlike plants, animals cannot feed upon inorganic substances. In addition to water, their food consists of other organisms, animals and plants; or of substances derived from them.

\section{Vascular System.}

In many of the lower animals, the digested food stuffs, after traversing the walls of the alimentary canal, make their way through the various tissues by a kind of endosmosis.* In most, the arrangement is, however, somewhat more complicated; there is a special system of branching canals which conveys the nutritive material derived from the gut all over the body, to be absorbed in part by the tissues, after having undergone certain changes within the vessels. This arrangement of tubes is termed the vascular system.

The vascular network is sometimes very complete, the ultimate branches ramifying throughout almost all the organs and tissues: usually, epithelia alone are non-vascular, deriving their nutriment from the adjacent tissues. The vascular system may, on the other hand, consist of relatively few definite channels which communicate directly with the spaces of the body. In a well-developed vascular system, some of the vessels are distinguished by their greater width, and from these main trunks, others are given off to supply the various regions of the body. These latter, again, give off branches dividing into smaller and smaller twigs, and finally terminating in a network of the finest vessels, traversing the organs. The fluid flows from the larger into the smaller trunks, and thence into the smallest of all, from which it is conducted back into the large trunks by another set of vessels which are also in communication with these minute ones. In most cases there is, therefore, a circulation of the vascular fluid or blood plasma, which is really to be regarded as the digested food, although it must be noticed that it has also received certain waste products of metabolism from the various tissues. It is usually clear and colourless, more rarely coloured red or green. Floating in it are numerous free cells, the blood corpuscles, which are usually amoboid and colourless; $\dagger$ less frequently some of the corpuscles are of definite form (unable to throw out pseudopodia), and red in colour. These red corpuscles, which have the form of circular or oval discs, occur especially

\footnotetext{
* In many the distribution of the products of digestion is facilitated by the branching of the alimentary canal, which is furnished with numerous diverticula of considerable extent (e.g., Medusæ, Liver-flukes).
}

† Their protoplasm may, however, contain coloured granules. 
in the Vertebrata, where they are far more numerous than the white ones. They are found also in many of the lower animals (e.g., certain Chætopods and Molluscs), but in these are less regular in form. The plasma and corpuscles together constitute the blood, the colour of which is usually dependent upon the colour of the fluid portion, but in the Vertebrata upon that of the corpuscles.

The blood corpuscles usually originate in cellulàr connective tissue, which may constitute specialised organs, the lymph glands. They are therefore connective tissue cells which have broken away from their point of origin and entered the blood stream.

Since it is of importance that the blood should be in constant movement, hearts are formed, i.e., the vascular walls become very muscular in certain definite regions and can therefore pulsate, or contract rhythmically, and thus drive the blood along. There may be several hearts in the same animal: usually, however, there is only one: or if there are more, one is characterised by its size and strength, and is known as the heart; it is always in direct connection with some of the largest vessels of the body, forming, as it were, the centre of the whole system. The openings into the heart are frequently guarded by peculiar folds, the $\mathrm{v}$ a l ves, whose function it is to regulate the blood stream by permitting a flow in one direction only, blocking up the way if the blood tends to stream in the opposite direction (see Fig. 22, 1). Very often the heart. consists of several

1

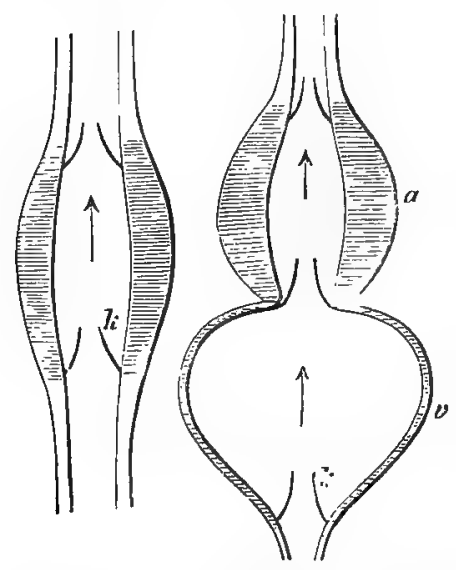

Fig. 22. 1 Diagram of a simple heart; 2 of one consisting of an auricle and ventricle. $a$ ventricle, $v$ auricle, $k$ valve.-Orig. independent divisions, which are frequently regarded as so many aggregated hearts. The blood then usually flows first into a thin walled chamber, the auricle, and from this passes into a thicker walled, more muscular portion, the ven tricle, which is, apparently, the more important of the two. Sometimes it happens that several auricles open into the ventricle (many Molluses). The vessels in which the blood flows towards the heart are termed veins, those in which it flows in the opposite direction, arteries. The finest vessels of all, which form a network uniting the ultimate branches of the arteries and veins, are termed capillaries. They, are often altogether wanting, when they are to some extent replaced by portions of the body cavity, with which the veins and arteries then communicate directly, the blood flowing from the arteries into the sinuses and thence into the veins. 
The minute structure of the vessels varies considerably; the lining consists of a single layer of flattened cells, and these constitute the entire wall of the capillaries; in other vessels the endothelial lining is usually surrounded by connective tissue, smooth muscle fibres, etc., so that the walls of the large vessels may be fairly thick.

For the lymphatic vessels peculiar to the Vertebrata, see that group.

\section{Respiratory Organs.}

The cells of the metazoan body, like the Amœba, must have oxy gen in order to live: it must therefore be continually taken into the body, and all the cells of the different organs must be supplied. Further, the waste-products, resulting from the constant combustion going on in the cells, must be got rid of : one of these waste-products is carbon-dioxide, a combination of carbon and oxygen $\left(\mathrm{CO}_{2}\right)$ whose excretion alternates regularly with the absorption of oxygen, whilst other waste matters are got rid of in other ways (see excretory organs). The taking in of oxygen, with the giving out of carbondioxide, is known as rospiration, and the organs performing this function are called respiratory organs. Some animals (airbreathing) obtain oxygen from atmospheric air, of which it forms approximately one-fifth. Others, on the other hand, make use of the free (dissolved) oxygen which occurs in all natural waters.

The vascular system, when present, plays an important part in connection with the introduction of oxygen and the removal of carbon-dioxide. 'The blood in the vessels of the skin absorbs the former, carries it through the body, giving it up on the way; receives the latter and returns with it to the skin, where it is expired and the oxygen inspired again (cutaneous respiration). In many animals, with no respiratory organs, the digestive tract performs the respiratory function. Air, or water containing air, is always swallowed with the food, and the oxygen is absorbed during its passage through the digestive organs (intestinal respiration). Many of the lower animals, chiefly aquatic, but a few terrestrial (e.g., Earthworms), are destitute of special breathing apparatus, and respiration is effected by endosmosis, which goes on over the whole surface of the body. These forms are almost always thin-skinned, i.e., they are without a hard, thick cuticle, or any other covering difficult for oxygen to penetrate, and are almost invariably of small size--a small body has, of course, a relatively larger surface than a large body of the same shape.

Most animals are, however, provided with special organs of respiration. The universally observed principle is that water containing air, or atmospheric air itself, is brought into relation with a large thin-skinned surface, through which oxygen is taken in in large quantities, whilst carbon-dioxide is given off; generally a capillary net-work is distributed immediately beneath this skin. 
Those animals which are specially adapted for obtaining the oxygen dissolved in water, usually breathe by means of gills, thinskinned appendages with a relatively large surface, which either project freely from the body, or are situated in a cavity which is in direct communication with the exterior (branchial chamber). An increase of surface may be obtained by simple extension, by folding or branching of the gill. When the gills are enclosed in a cavity, there is generally some special contrivance for causing a stream of water to pass continuously, or at least frequently, through it (many Crustacea, e.g., the Lobster, and Pisces); and thus it is ensured that fresh currents of water, and therefore fresh oxygen should constantly come in contact with the gills.

Amongst air-breathing animals, the respiratory organs are, as a rule, thin-skinned, saccular ingrowths, the lungs, communicating with the exterior by a larger or smaller aperture. The respiratory surface is frequently increased by the presence of small outgrowths, which may themselves be sacculated, until the lung becomes much branched, and its inner surface is extraordinarily enlarged (especially in Vertebrata). In the wall of the sac there is usually, just as in the gills, a delicate vascular network.

A peculiar kind of lung, the system of $\operatorname{trachex,}$ is present in many Arthropods, and will be described in detail for the Insects.

Just as it is necessary for the gills to come constantly in contact with fresh supplies of water, so the air in air-breathing organs must be continually renewed. If the air remained stationary, the lung would cease to act as a respiratory organ, for the oxygen would be gradually consumed, and the air loaded with carbon dioxide. The expulsion of air always takes place by the contraction of the organ of respiration: the inception, as a rule, by its expanding again, so that the air remaining is rarified, and the outer air rushes in to equalise the pressure. The special contrivances differ to a very great extent.

The structure of the respiratory apparatus has a very great influence upon the vascular system, whose disposition is largely determined by these organs. The following circumstance is of special significance: it is advantageous for the organism to have the blood, on leaving the respiratory apparatus for the other organs, as rich in oxygen and as free from carbon-dioxide as possible, whilst, on the other hand, it is important that blood flowing towards the respiratory organ should take along with it as much carbon-dioxide as possible, in order to complete the gaseous exchange. This is always effected by the accumulation of the so-called ven o u s blood, which has flowed through the organs and is rich in carbon-dioxide, in a large c o $\mathrm{m} \mathrm{m}$ on reservoir, whence it is passed on to the gills or lungs. After the carbon-dioxide has been exchanged for oxygen, the blood, which is now distinguished as arterial, goes into a second large blood 
reservoir, whence it flows all over the body. This is the usual arrangement in known animals with a respiratory apparatus and a complete vascular system, but great variations occur within these limits. Among many Invertebrates (Mollusca, Crustacea), the arterial blood spaces are represented by the heart, which, as it receives blood from the gills, is an arterial heart, and drives it all over the body; the impure blood is carried into a large non-contractile reservoir, a venous blood sinus, whence again it returns to the gills.

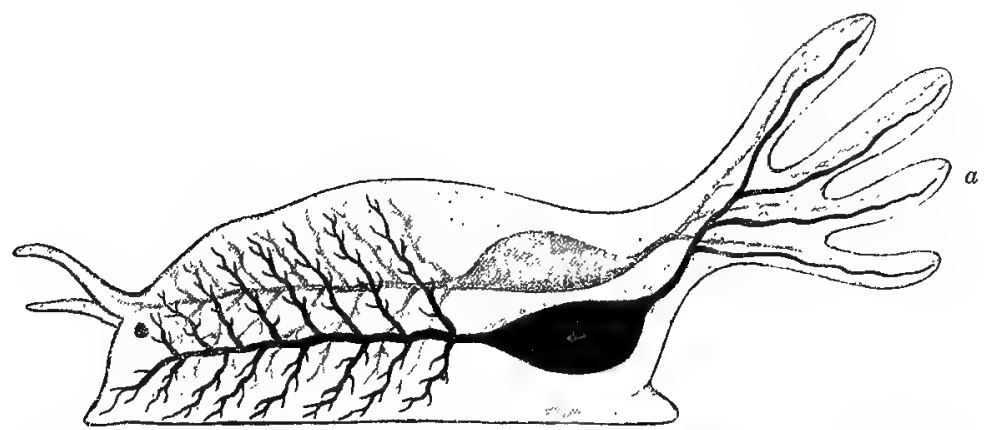

Fig 23. Diagram illustrating the usual relations of the respiratory organs $a$ to the vascular system. Arterial blood light, renous blood dask. See text.-Orig.

In Pisces it is entirely different: here the heart is represented by a venous reservoir, which receives blood from the body and sends it to the gills; it is, therefore, a venous heart. Blood goes from the gills to the aorta, a large non-pulsatile vessel corresponding to the arterial reservoir, and passes thence to the body. In Mammalia and Aves, again, there is another arrangement, for, functionally, t wo hearts are present, of which one, the right side of the organ (right auricle and right ventricle) represents the venous reservoir, and receives blood from the body and sends tt to the lungs; whilst the other (left auricle and left ventricle) replaces the arterial reservoir, receives blood from the respiratory apparatus and distributes it all over the organism.

It must be noticed here that the formation of special respiratory organs is not accompanied by cessation of cutaneous and intestinal respiration. These are, it is true, of very slight significance in many animals, e.g., Mammalia ; in others, however, they play an important part, especially cutaneous respiration.

A red substance, hæmoglobin, present in the coloured corpuseles of Vertebrates, is of great respiratory importance, for most of the oxygen absorbed is not simply dissolved in the blood-plasma, but enters into a loose chemical combination with the hæmoglobin, from which it is easily separated again, and is seized upon by the tissues as the blood travels through the capillaries. When the hæmoglobin is combined with oxygen, the blood is of a bright red colour, and is called arterial blood; when the oxygen has been given up, the blood looks dark red, and is known as venous blood. The same, or a similar, substance is found in the red 
blood-corpuscies of lower animals, and is present in the red blood-plasma of Chrtopods and others. In the light-blue blood of Cephalopods, there is an allied substance, hæmocyanin, with the same function as hæmoglobin, whilst the same, or a similar, pigment also occurs in the blood of other Molluscs and in many Arthropods.

The constant oxidation (combustion) in the cell not only liberates the energy manifested in the vital processes (protoplasmic movements, muscle contractions, the peculiar behaviour of nerve-cells and fibres), but also results in the production of heat. This heat is dissipated rapidly from the surface of the body by radiation, and in other ways, so that the temperature is but little higher in most animals than that of the surrounding medium. The production of heat is of paramount importance only in the so-called warmblooded Vertebrates (Aves and Mammalia), and here contrivances for its better retention are present, so that the body is kept at a constant, tolerably high temperature, which may be very different from that of the environment. The body requires, moreover, a certain temperature of its own (differing in different animals), in order that its vital processes can be normally carried on.

Sound-producing Organs.-Many animals are able to produce sounds of different kinds. This faculty is mentioned here, not because it is related to the special respiratory process, but because the sound-producing organs of air-breathing animals (which are specially endowed with this power), are usually connected with the respiratory apparatus. Thin laminæ or folds of skin (vocal cords), are often present at the entrance of the air passages, and can be set in vibration by the expired air. Not only is the voice of most Vertebrates produced in this way, but also many of the sounds made by Insects. The utterance of sound may, however, be wholly independent of the respiratory organs. Certain shrill and rattling noises in Insects, Crustaceans, Fish, etc., are caused by hard surfaces being rubbed against one another. The buzzing sounds of Bees and other flying Insects are produced by movements of the wings. These various sounds serve partly as a means of communication with individuals of the same species, partly to terrify enemies.

Phosphorescent Organs.-Luminosity which is met with in many animals, especially among the Invertebrates, is closely connected with the respiratory process. The production of light is usually confined to certain cells, in whose protoplasm a fatty substance is present: oxygen unites with this substance by a kind of combustion, producing light, but not necessarily heat. Very many animals of different groups exhibit this phenomenon (though of course the great majority are non-luminous), such as Protozoa, Coelenterates, Echinoderms, Chætopods, Crustaceans, Insects, Lamellibranchs, Cephalopods, Ascidians, Fish. This luminosity has no connection with that exhibited by dead animals, e.g., Pisces, which is caused by certain Bacteria: leaving it a question whether the light emanates from the decomposing tissues or from the Bacteria themselves.

\section{Excretory and Urinary Organs.}

By the chemical processes in the cell, certain other matters, besides carbon-dioxide, are formed, which cannot be further employed by the organism; the most important are the nitrogenous waste products, urea and uric acid. For their removal, special glandular organs, the 
urinary or excretory organs (kidneys), are present in most animals. They are usually tubular or sac-shaped glands opening on to the surface of the body or into the hinder part of the alimentary canal. The secretion (the urine) is either entirely liquid or it contains hard, granular, or crystalline concretions. Sometimes a vesicle (a urinary bladder) is formed, which serves as a reservoir for urine; it may either be a widened region of the canal of a single gland, or of an excurrent canal common to several. In many animals, however, are other organs also excretory in function; amongst some of the Chrtopoda, the cells of a certain part of the alimentary canal secrete hard concretions, * which are, undoubtedly, urea; such occur also in the hind gut of the Rotifers, and in some of the liver-cells of the Gastropoda; also in the epithelium lining the body-cavity of certain Chætopods, and of Sharks, urea is formed, and is eliminated in different ways.

The waste products are, however, not always removed from the body; in some, probably in many, cases, they are stored up in cells which have no connection with the exterior. This occurs, e.g., in certain Fly-larvæ where a mass of cells containing excretory deposits surrounds the heart; in a Slug, whose true kidney is degenerate, cells containing concretions of uric acid are scattered throughout the body (See also Tunicata.)

The pigments so abundant in most animals, may also, in part, represent waste products, which have accumulated in cells in the way just mentioned. In other cases large masses of pigment are regularly removed from the body: the pigment present in the cuticle, particularly in the hair and feathers, $\uparrow$ is got rid of by ecdysis in many animals, by the shedding of hair in Mammals, by moulting in Birds.

\section{Reproduction and Reproductive Organs.}

Reproduction, the formation of new individuals, occurs in the Animal Kingdom in two quite different ways, sexually and asexually. Asexual reproduction will be first considered in its two forms of $f$ is sion and gemmation.

In $f$ is sion, a longitudinal or transverse furrow appears on the individual concerned, and gradually deepens, until finally, the organism divides into two approximately equal pieces, which grow whilst the process is going on, or after it is complete, until each has attained the size of the parent. Less frequently division occurs without the preceding constriction, the animal breaking suddenly into two pieces. Gemmation differs from fission in that only a small part of the body of the original individual develops (by rapid growth) into a new animal, so that it is possible to distinguish between the

\footnotetext{
* Small bodies lying in the protoplasm.

+ The pigment present in the epidermis and hair of Mammalia is, at any rate for the most part, not formed in situ, but is brought there by wandering cells which migrate into the epidermis from the subjacent connective tissue.
} 
parent and bud; in fission, the two individuals are exactly alike. These methods of reproduction are, however, not sharply defined, so that it is often quite impossible to say whether fission or budding has taken place. In the course of the special part, various instances of asexual reproduction will be met with, especially in Colentera, Platyhelminths, Chætopoda, and more rarely in Echinoderms, in addition to the Protozoa.

Often in gemmation or fission the new individual does not separate completely from the other, but remains in more or less intimate connection with it. In this case, if division is repeated, the result is a colony or stock consisting of a varying number of animals, produced by asexual reproduction from one original individual. The members of the colony have lost their independence to an extent corresponding with the closeness of their connection with one another (see special part). Stocks or colonies occur especially in Corals, Hydroids, Tape-worms, Polyzoa, and Tunicates.

Regeneration.-Nearly akin to asexual reproduction is the power of replacing parts of the body which have been lost by some accident, by new formations resulting from growth of the tissue nearest to the wound: the edges of the epidermis form new epidermis; the connective tissue, new connective tissue, and so on. Different animals display this faculty in very different degrees. In Mammalia, for instance, it hardly appears at all; they can, indeed, repair injured epidermis and the like, but the loss of larger portions of the organism (e.g., tail, limbs) is not made good. It is more evident in certain lower Vertebrates, e.g., Lizards, where the tail may be replaced, or in Newts, which can not only form a new tail, but also new limbs. Amongst Invertebrates, even in so complex an organism as the Earthworm, large tracts of the body can be regenerated; indeed, in some animals, the power of repair is so great that, when cut into two or more parts, they grow out into as many new animals; the best known example of this is the Fresh-water Polyp (Hydra).

Whilst asexual reproduction occurs in some animals and is wanting in others, all Metazoa exhibit sexual reproduction, which consists essentially in the development of a single cell, after luberation from the parent, into a new individual. Every cell in the Metazoan body has not this capacity, but only certain peculiar ones called ova. As a rule, the ovum cannot develop by itself into a new individual; it must first be fertilised, i.e., it must unite with another cell, usually of a smaller size, and always with special properties, a spermatozoon.

The ovum is generally rounded, often spherical. Like other cells it consists of protoplasm with a nucleus, the germinal vesicle, which often encloses a large nucleolus (or several), the germinal spot. The ovum is often surrounded with a covering of varying thickness, the vitelline membrane, in which may be one or more openings for the entrance of the spermatozoon. In the protoplasm, there are usually numerous fatty or albuminous particles, the yolk granules, spherules, or discs, which are all called yolk granules, or in contrast to the protoplasm in which they lie, the dentoplasm. 
Compared with other cells the ovum is almost always large: in many cases,,.$g$. , the Mammalia, it is, however, microscopically small; but in others where there is a large quantity of deutoplasm, it attains

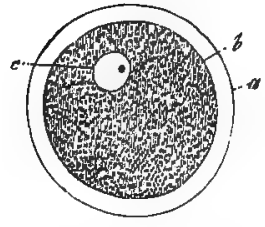

Fig. 24. Human ovum. $a$ vitelline membrane, $b$ protoplasm, $c$ nucleus.After Kölliker.

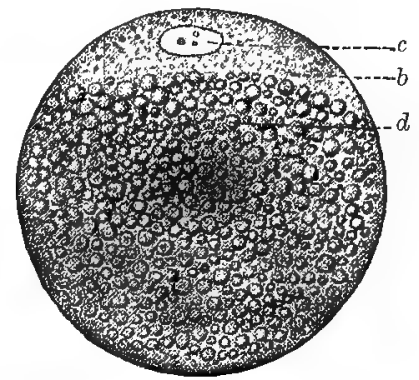

Fig. 25. Diagram of an ovum, with many yolk-spherules. $d$ protoplasm. $b$, with the nucleus, $c$, chietly accumulated at one pole. - After Hertwig.

a colossal size (Squalidæ, Aves). In this case it frequently happens that most of the protoplasm, with the nucleus, accumulates at one pole, whilst the chief mass of the egg consists of yolk held together by the rest: or the protoplasm forms a thin layer over the whole surface, whilst the inside consists principally of deutoplasm (Insecta).

The spermatozoon is, at first, a single small cell consisting of protoplasm with a nucleus, and it may remain in this condition (Fig. 26, 1) ; but as a rule, when mature, it is not so simple: very frequently it consists of a thickened part, the head, and a long, thin, whiplike tail, by the lashing of which it moves actively along. The head consists of the modified nucleus; the protoplasm merely forms the tail, which may be regarded as a giant flagellum. This

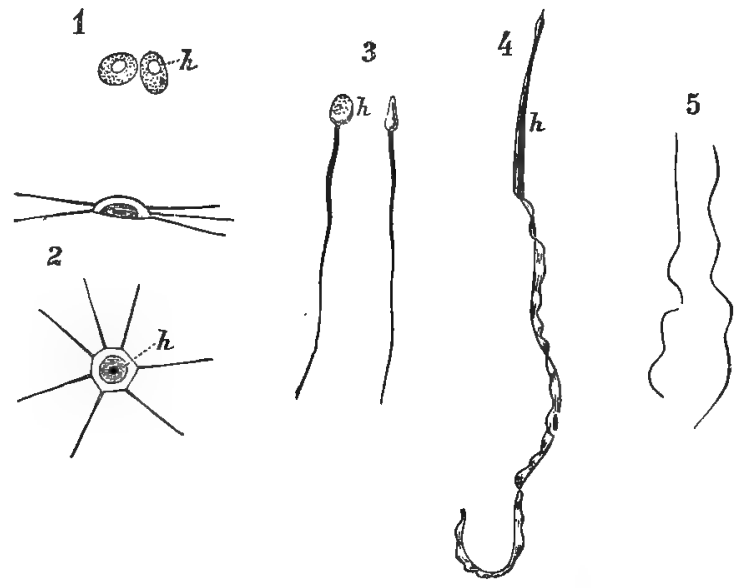

Fig. 26. Spermatozoa of different animals, 1 Crustacean (Thysanopus), 2 Crab, 3 Man, 4 Salamander (with a flattenedout tail), 5 Beetle, $h$ head (nucleus).-After different authors.

kind of spermatozoon is met with in many animals belonging to very different groups, but it may assume a variety of other forms.

Genital Organs.-The formation of ova and spermatozoa is usually confined to special regions of the body, and as a rule, but not always, to definite organs. The organ in which the ova are formed is 
called the ovary. Its structure differs largely in different animals; it always, however, contains egg-cells in different stages of develop ment, held together by connective tissue. Frequently the ovary is a hollow, glandular organ which is continued into a tubular oviduct. The ripe ova break loose, fall into the cavity of the ovary, and reach the exterior through the oviduct. In other cases it is a compact organ, attached to the wall of the body cavity; the ova fall into the body cavity and pass out by a tube opening into it at one end and to the exterior at the other. Before leaving the body of the parent, the ovum is often enveloped in different hard or soft coverings (albumen, shell), secreted by glands opening into the oviduct. Saccular evaginations are frequently present for storing the spermatozoa received during coitus (receptacula seminis). Further, part of the oviduct is often expanded to form a uterus in which the eggs may be retained for some time, often until they have undergone a part of their development.

When the ovum has attained its full size, the nucleus moves to the surface, and divides into two. The protoplasm undergoes corresponding division, but into two unequal parts, so that before separation, one part looks like a little bud of the larger egg-cell: a second small cell is constricted off in
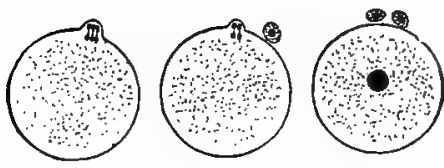

Fig. 27. Diagram of the formation of polar bodies. In the left figure the first, in the middle the second, polar body is shown in process of formation; the right figure shows the ripe ovum with both.

the same way; and as soon as these polar or directive bodies are formed, the ovum is mature and ready for fertilisation. The first polar body may sometimes divide again, so that altogether three are present. They are formed whilst the ovum is still in the ovary, or soon after its liberation; later, they atrophy.

The spermatozoa are formed in the testis, which contains sperm-cells in different stages of development. The testis even more often than the ovary has a glandular structure, and the spermatozoa usually pass out by a duct, the seminal duct or vas deferens, with which it is in direct connection. Organs for the transmission of the spermatozoa into the female apparatus are often present at the external opening of the duct (copulatory organ, penis). Sometimes the genital products which pass from the male to the female are enveloped in a sheath formed by the hardened secretion of certain glands opening into the vas deferens. Such a mass is called a spermatophore (Cephalopoda).

In most species, some individuals give rise to ova only (females), and others to spermatozoa (males). There are, however, many animals, e.g., many Gastropods, which produce both: these are known as hermaphrodites. In this case there is either a specialised ovary (or more than one) and a specialised testis in the 
same animal, or the ova and spermatozoa are both formed in a common hermaphrodite gland.

In many hermaphrodites, ripe ova and spermatozoa are produced at the same time. In others, however, either the ova are formed first and the spermatozoa later, so that the animal functions first as a female, then as a male (protogynous hermaphrodites, e.g., Salpa; or they first produce spermatozoa, later ova (protandrous hermaphrodites, e.g., certain Nematodes),

Secondary Sexual Characters.-Distinctions between males and females other than those resulting from the different nature of the genital organs (primary sexual characters) are of frequent occurrence. The male is provided with special organs, or parts of the body are specially modified for holding the female during copulation (Water-beetles); or they possess special weapons for fighting with other males during the breeding season (Stags); or they are specially ornamented with brilliant colours, peculiar excrescences, etc. (many Birds). On the other hand, the female may be provided with organs of importance in rearing the young ones (mammary glands of Mammalia), whilst the male is more rarely specialised in this way (Pipe-fish). There is often a perceptible, or even considerable, difference in the size of the sexes; sometimes the male is larger than the female (many Mammals, Birds, and Insects), in other cases (Birds of Prey, Round Worms) the female is the larger: the difference is sometimes extraordinarily great, as in many parasitic Crustacea, where the males only attain a fraction of the size of the female; or again in an Annelid (Bonellia), where the males are microscopic, and entirely different in appearance from the fairly large female; indeed, they were at one time regarded as parasites of a distinct species, since they live within the oviducts.

Fertilisation is effected by the entrance of the spermatozoon into the ovum, and their ultimate fusion. There is evidently a peculiar mutual attraction between ova and spermatozoa; not only

1

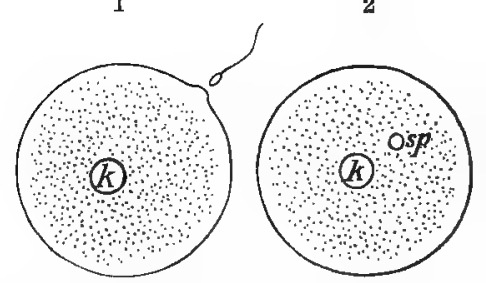

Fig. 28. Diagram of $f$ ertilisation.
3

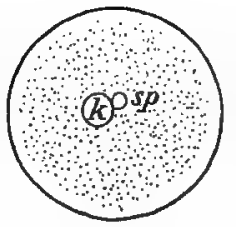

$\hbar$ female pronucleus, $s p$ male pronucleus. In 4 , union has taken place. See Text.--Orig.

does the ovum attract the spermatozoon, but at the approach of the latter its protoplasm may rise up to form a small papilla. After entrance, the spermatozoon loses its tail or protoplasm, if it possess any, and its head (nucleus), which is now called the male pronucleus, enlarges, gradually approaches the nucleus of the ovum (female pronucleus), the two come to lie side by side, and finally fuse. This union of the nuclei is evidently the essential part of fertilisation; when it has taken place the egg is able to segment and thus to give rise to a new organism. (See Sect. IV._- Ontogeny.")

The ovum is fertilised by one spermatozoon only, and as a rule only one enters even though thousands may be swarming round. This is due to the fact that when a spermatozoon has entered, the ovum undergoes certain changes which 
prevent the entrance of others; in Echinoderms, for instance, a delicate membrane is secreted over the surface. In some cases, however, several sperm-cells. penetrate the ovum (e.g., Squalidx), but only a single one unites with the female pronucleus, the rest move about in the protoplasm for some time but eventually disappear.

For the phenomena in Protozoa corresponding to the fertilisation of Metozoa see Special Part.

In many cases, most Fish, Frogs, etc., fertilisation occurs in the water outside the body of the parent. The female lays the eggs and at the same time, or soon after, the male deposits the spermatozoa. The genital products are mingled together, and the spermatozoa have an opportunity of making their way into the ova. In other cases fertilisation takes place in the oviducts of the female, into which the spermatozoa are introduced by the male copulatory organs. (copulation).

Copulation is not always followed immediately by fertilisation. In many animals spermatozoa may be retained within the body of the female for a long time without losing their efficacy. (In Hens, two to three weeks; Bats, as many months; Queen-bees, three years.) Frequently the ova are very immature when it occurs.

As regards hermaphrodites, it is very seldom that self-fertilisation obtains, i.r., that an ovum and a spermatozoon from the same individual unite; hermaphrodites almost always copulate, and then fertilisation is either reciprocal, or one individual only, gives. spermatozoa to the other.

Hybridisation.-An ovum under ordinary circumstances is. fertilised by a spermatozoon which has been formed in an animal of the same species. In most cases, however, it is possible for ova to unite with spermatozoa of a nearly allied species; indeed, copulation between two more distantly connected species may sometimes occur.* As a rule, such a union results in a new organism, a hybrid, which is more or less imperfect. For instance, hybrids, even of nearly related species are very often sterile, i.e., they are unable to produce ripe ova or spermatozoa, although they may be otherwise strong and well-developed animals, e.g., the mule of the Horse and Ass. In other cases the hybrid is feeble and dies young, or even does not outlive the embryonic period (hybrid of pheasant and Domestic Fowl). On the other hand, however, there are hybrids quite as well developed as the parent species, and also perfectly fertile. This holds for the crosses between certain species of Stags, different species of Pheasants, the hybrid of the Chinese and European Goose, \&c.

* It has been recently found by experiment, that Frog's spawn can be fertilised by the spermatozoa of the Newt, and the eggs of Regular Sea-urchins by the spermatozoa of Irregular species; but development is usually irregular and soon ceases. On the other hand, there are nearly allied forms whose genital products will not unite; and there are species which give remarkable results in that ova from one, $A$, can be fertilised by spermatozoa from another, B, but not ova from B by spermatozoa. from $\mathbf{A}$. 
Under natural conditions, hybrids occur relatively seldom. On the borderland between the ranges of two species (see section V.), hybrids are, indeed, often found, and in some groups (Fresh-watex Trout), seem fairly common. The interbreeding of species is hindered, amongst other things, by the fact that the individuals of different species, at least in a state of nature, are generally disinclined to pair.

Just as the offspring of a union between remote species is unhealthy, so also is that produced by too close in-breeding; if the offspring of the same parents, or if other near relations are mated, and this close breeding continues for several generations, the descendants gradually show a marked deterioration of one kind or another.

Parthenogenesis.-The development of an ovum into a new individual is generally dependent, as already stated, on its being fertilised: but in not a few animals, it has been observed that the ovum can develop without fertilisation. This peculiar modification of sexual reproduction, which occurs in Platyhelminths, Insects, and Crustacea, is called parthenogenesis, and although in some forms where the unfertilised ova usually atrophy, it may be exceptional (Silk-worms), yet in other cases it is quite an ordinary phenomenon. In many species there are whole generations which consist exclusively of females, so that the eggs cannot possibly be fertilised, and yet they develop nevertheless (Aphides). Amongst other forms the male seldom or never appears. In Bees and other Hymenoptera, all the fertilised eggs develop in a remarkable way into females, whilst the unfertilised ova become males (see also the Special Part, Trematoda, Aphides, Daphnidæ, and Insecta).

In some animals, although parthenogenesis does not occur regularly, it is yet suggested. It has been found that development begins in an unfertilized Hen's egg for instance, but does not go beyond the very first stages (segmentation), which are followed by degeneration. The same thing has been observed in the ovum of the Rabbit.

Ova, which are, as it were, predestined not to be fertilised; which, for example, are produced from generations consisting only of females, are peculiar in giving origin to o ne polar body only (summer eggs of Daphnia). It is a question whether the eggs of Bees, which sometimes are fertilised and sometimes are not, throw off two polar bodies as is customary. It has been observed in some ova, which are usually fertilised, but which can develop parthenogenetically to a certain point, that the nucleus for the second polar body always forms; but if fertilisation does not take place, it unites again with the egg nucleus, and subsequently plays the part of the missing male pronucleus (Round-worms, Star-fish).

Alternation of Generations.--Some animals reproduce both sexually and asexually: many Corals can produce new individuals by budding as well as by ova and spermatozoa, and this is true also of certain Annelids and Ascidians. In other cases, however, those individuals which give rise to buds, do not produce sexual cells; asexual reproduction is restricted to some members of the species, sexual to others, and there is a more or less regular alternation of sexual and asexual broods or generations; asexual individuals produce, by gemmation or fission, sexual individuals whose fertilised 
ova again become asexual animals : or, two or more asexual generations are followed by a sexual brood, these again by more asexual generations. Such a regular alternation of sexual and asexual broods is known as alternation of generations, the generations may resemble one another, but usually they differ, often very considerably (Hydroids, Tapeworms).

Heterogony.-The alternation just mentioned occurs between sexual and asexual generations, but in many animals a generation consisting exclusively of females, which reproduce parthenogenetically, alternates with another which consists of males and females giving rise to fertilised ova. There is generally some difference between the virgin and the sexual generations. A simple form of such alternation occurs in certain species of Gall-wasps, which cause oak-galls; a. female generation here alternates regularly with a generation consisting of males and females. The process is more complicated in the Aphides, where, during the summer, several generations of females appear in succession until, finally, a sexual generation is produced; the fertilised eggs rest through the winter and become the first virgin brood of the next year; there are, therefore, several parthenogenetic generations to each sexual generation. In the Vine-louse (Phylloxera) there is a further complication, not only is the sexual very different from the parthenogenetic generation, but of these one differs considerably from the rest. Such an alternation of virgin and sexual (or hermaphrodite) generations. occurs not only among Insects but also in various Crustacea and Platyhelminthia.

Amongst those animals which reproduce only by fertilised ova, successive generations are almost always alike; a regular alternation of generations of different appearance is found only exceptionally, and in consequence of special conditions. In certain buttertlies, for example, two generations, consisting of both males and females, occur annually. The winter-brood, having wintered as pupæ, appears in the spring as perfect insects : their eggs give rise to the individuals of the other generation, the summer-brood, from which they differ considerably in coloration (seasonal dimorphism). A hermaphrodite Nematode (Rhabdonema nigrovenosum), is parasitic in the lungs of Frogs and Toads; its embryos develop into another generation which is free-living and of separate sexes, and which is essentially different in appearance from the hermaphrodite generation. The embryos of the free-living generation re-enter the Amphibia, and become hermaphrodite worms like their grandparents. Some other Nematodes exhibit the same peculiarity: an alternation of a hermaphrodite generation, which leads a parasitic life, with a diœcious, free-living generation.

* Instead of on e summer-brood, there may be $\mathrm{t} w 0$ (i.e., three generations annually). 
Under heterogony, are included all cases of regular alternation between sexual generations, whether these differ in appearance or in their mode of propagation, therefore alternations of parthenogenetic and dicecious generations, as well as seasonal dimorphism come under this heading.

Heredity.-The offspring produced by an animal or by a pair of animals is, generally, when fully developed, just like its parents. This similarity extends not only to specific characters (see Section V.), but, in great measure, to the individual peculiarities of the progenitors. But such features are not always inherited, some may be passed over : frequently, also, the offspring exhibits a greater resemblance either to the male or to the female parent; or again, the young ones may, under the influence of the environment differ in small points from the parents.

Sometimes characters which are present in an animal do not appear in its offspring, but skipping that generation, are seen again in the next. An animal may thus possess certain peculiarities which are present, not in the parents, but in the grandparents; indeed, characters from still further back in the line of ancestors may reappear: this peculiarity is called atavis $\mathrm{m}$.

The phenomena referred to above (metagenesis and heterogony) are not opposed to the fundamental principles of heredity. Even if the offspring is unlike the parent, and sometimes very unlike, yet it always bears some resemblance to an earlier generation; no persistent deviation is produced in these cases which may perhaps all be regarded as regular atavisms.

\section{The Relations of the Organs to One Another.- The Body Cavity.}

The organs mentioned above form the metazoan body, and are usually held together by connective tissue. This often fills up the interspaces, so that the animal forms a compact mass (Platyhelminthia); it is not the case, however, in most animals, where there is a large bod ycavity, in which some of the organs, viz., the digestive tract, the excretory and genital organs are enclosed, being generally fastened to the walls by threads, or thin sheets (mesenteries) of connective tissue.

The body cavity is often divided into compartments by septa: in Mammalia, for example, by the diaphragm, into thorax and abdomen: in Chætopoda by transverse septa into many compartments. The body-cavity is usually more or less completely filled with organs known as the "viscera," the remaining space containing a fluid, which is sometimes blood, since the vessels are frequently in free communication with it. Besides the body cavity, other spaces may be present, of various forms, sizes, and significance, and filled with a 
fluid, which is for the most part to be regarded as exuded blood plasma. Such are the synovial cavities of Vertebrata.

\section{Rudimentary Organs.}

Besides the great majority of organs which evidently perform definite functions, structures are occasionally found which are of no importance to the animal, the so-called $\mathrm{rudimentary}$ organs.

The hind leg of the Greenland Whale may be cited as an example of such an organ. It consists of a femur and a tibia, both of which are concealed beneath the skin, and have entirely lost their function. The "wolf tooth" of the Horse affords another example of a rudimentary organ; that in the lower jaw especially, is an instance of extensive reduction, for, though it is formed in the customary way, it is very seldom cut. The eyes of Myxine (the hag-fish), Proteus, and many other blind animals; the wings of Apteryx (the kiwi) and other Struthious Birds; the minute wings of many female Butterflies are examples of rudimentary organs.

It may seem strange that these rudimentary and functionless organs are so generally present, but further consideration renders their existence less incomprehensible. Rudimentary organs which are now useless, were, in ancestral forms (see Section V.), functional and useful parts. The adaptation of the animal to a new and peculiar environment rendered them useless, and during the development of the existing form, they became reduced and functionless. It must, for example, be admitted that the Whale is descended from a Mammal, which, like most other Mammals, was provided with well-developed hind limbs, but these were gradually reduced by the adaptation of the animal to an aquatic life, while the tail came to function as the essential locomotor apparatus. A similar explanation can be given for the other cases cited above.

This explanation does not, however, hold for every rudimentary structure. Such parts as the rudimentary mammary glands of male Mammals, the rudimentary oviducts of male Amphibia, the rudiment of a copulatory organ in many female animals, etc., are to be accounted for in another way. Those parts which are always present in one sex in a well-developed and functional condition have probably been transmitted by this sex to the other. The mammary glands, for example, were probably, first of all, present only in the female, and have been inherited by the male; the copulatory organs, conversely, usually present at first only in the male, have then been transmitted to the female in an incipient state.

\section{Fundamental Form and External Configuration of the Body.}

In a small number of animals, Echinoderma and Colentera, the body is so constructed that it may be divided into a number of nearly congruent radially-arranged segments, a n timeres (rays): 
such animals exhibit a radial symmetry. Their individual organs must naturally either have a radiate structure also, or be equal in number to the antimeres.

Most Metozoa are, however, bilateral in plan: the body is capable of division into two nearly equal parts, which are similar to one another, looking-glass-wise, but are not congruent; the parts of the body are here symmetrically disposed in relation to a median plane.

One of these types governs the structure of all Metazoa. Neither is, of course, ever carried out with quite mathematical precision, but in many cases it is clearly manifested throughout the whole body. In other cases, variations are obvious; in many Echinoderms, for instance, the body can be cut into five parts, which, indeed, shew many points of accordance, but are yet far from identical. This is true also for many bilateral animals: amongst Vertebrates, for example, most of the organs are usually symmetrical in form and arrangement, but as a rule, the greater part of the digestive tract in the adult is an exception, though in the youngest embryos it is symmetrical; still more aberrant is the condition in others, for the symmetrical type is clear only in certain parts of the body, whilst in most regions it is difficult to make out (Gastropoda).

In some groups the bilaterally symmetrical body is divided into a series of similar seginents (metameres). The segmental arrangement of many Chætopoda is representative of the simplest form of this metamerism. The body is composed of a number of $\mathrm{se} g \mathrm{~m}$ e $\mathrm{nts}$ or rings, which, from the first to the last, are essentially alike, both externally and internally : each ring contains a pair of excretory organs, a pair of nerve ganglia, is provided with a pair of parapodia, etc. Amongst other metameric animals, e.g., the Arthropoda, the segments are not as a rule so uniform in structure.

Amongst bilateral animals, a dorsal and a ventral side may be distinguished, and further, an anterior and a posterior end. The ventral side is that part of the body which is turned downwards when the animal is moving; the other is the dorsal side: or more exactly, that side of the body which, in the majority of animals belonging to a large natural group, is turned downwards, is known in all the members as the ventral surface; the overlying surface is dorsal. In all Gastropods, for example, the ventral side is that which is provided with the so-called foot, and this holds good for all the group, even when, as in pelagic forms, the foot is turned upwards. The anterior end is usually characterised by the presence of the mouth, of certain sense organs, and of a large portion of the central nervous system (the brain); and by being usually directed forwards during locomotion : it is often marked off from the rest of the body, or in some way developed in contrast to it, and then it is known as the head. The opposite, posterior end is often also peculiar in construction, e.g., it is thinner than the rest of the body, or specially muscular, and then is called the tail. 
Movable appendages, which subserve locomotion, are called limbs. They are usually elongate, and often jointed. Amcng the lower animals they play only a small part, whilst in the Arthropods and higher Vertebrates they become important as locomotor organs. Of other important appendages may be mentioned the tentacles, antennæ, etc., of different animals, employed as tactile or prehensile organs (see also under skin). The end of an outstretched appendage, farthest from the body, is called the distal, that which is nearest, the proximal, end.

\section{Embryology or Ontogeny.}

Embryology treats of development from the egg to the mature organism, that is, the changes which the individual undergoes until it reaches the adult form. As a matter of fact, the animal changes continually during its entire existence, and the developmental history should really, therefore, embrace the whole life. Practically, however, it is restricted to the earlier periods, when changes. are more obvious than they are later.

A special interest attaches to the earliest stages in most Metazoa, for they exhibit striking conformity, a characteristic common type, in spite of numerous individual modifications.

1. In the simplest cases (Fig. 29), development begins with the division of the ovum into two nearly equal cells, each of which divides again into two, in a radial plane, so that the result of the segmentation, as this process of division is called, is a number of

1

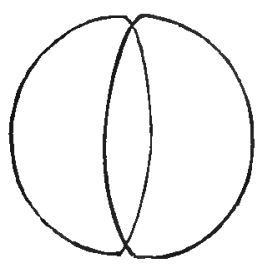

4

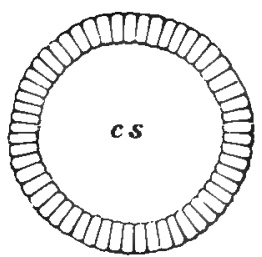

2

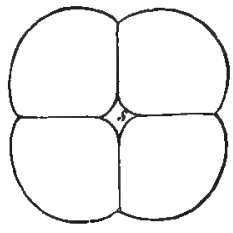

5

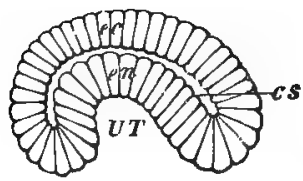

3

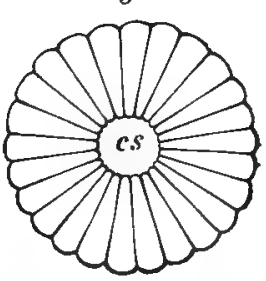

6

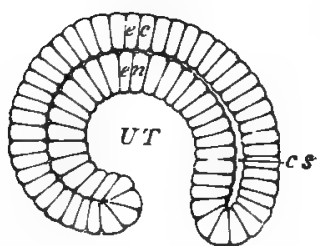

Fig. 29. Stages in the development of the ovum of a N e mertine (Lineus). 1 The: egg divided into two cells. 2 Young blastala with small segmentation cavity. $3-4$ Later stage of segmentation with larger segmentation cavity. 5-6 Younger and older gastrulæ. 1 seen from the surface, the rest are sections. cs segmentation cavity. ec: epiblast, en hypoblast. UT Archenteron.-After Barrois. 
nearly equal radial cells composing a sphere. These cells gradually move away from one another, so as to surround a central cavity filled with a liquid, the segmentation cavity; thus the wall of the sphere, the so-called blastula, consists of a single layer of cells. When this stage is reached, an invagination of half the sphere in to the other half takes place, so that it forms a cup or sac with a double wall. The layers of this wall are separated from one another by the segmentation cavity; when, however, as often occurs, it is completely obliterated by the invagination, they lie close together. This stage is known as the gastrula stage. The outer layer of the gastrula is called the epiblast or outer germ-layer; the inner, the hypoblast or inner germ-layer. The cavity of the sac is called the archenteron; its opening the blastopore or gastrula-mouth.

2. In other cases, of frequent occurrence (Fig. 30), the cells of the blastula are not of equal size, but those which form the hypoblast are large $r$ (richer in yolk) than the rest. Segmentation, invagination, etc., proceed as in case (1).

1

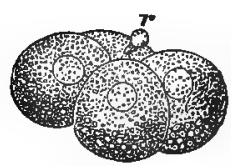

2

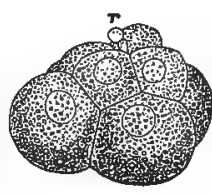

3

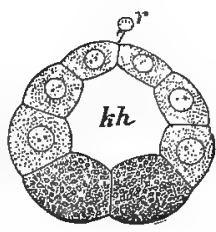

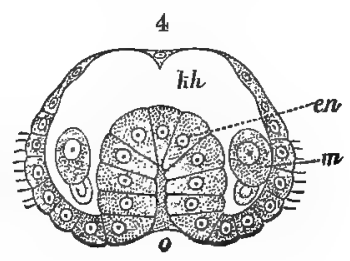

Fig. 30. Different stages in the development of the ovum of a Water-snail (Planorbis). 1-2 Stages in the segmentation, 3 section of a blastula (the large hypoblast cells are seen below), 4 gastrula, en hypoblast, $k h$ segmentation cavity, $m$ mesoblast, o blastopore, $r$ polar-bodies,-After Rabl.

The early development of a large number of animals takes place in one of these ways; Colentera (for these see also p. 45), Porifera, Echinoderma, many Worms, Molluscs, one Vertebrate (Amphioxus), Tunicata.

3. In some cases (Fig. 31) which otherwise adhere to the order described under (2), the cells which form the hypoblast are very
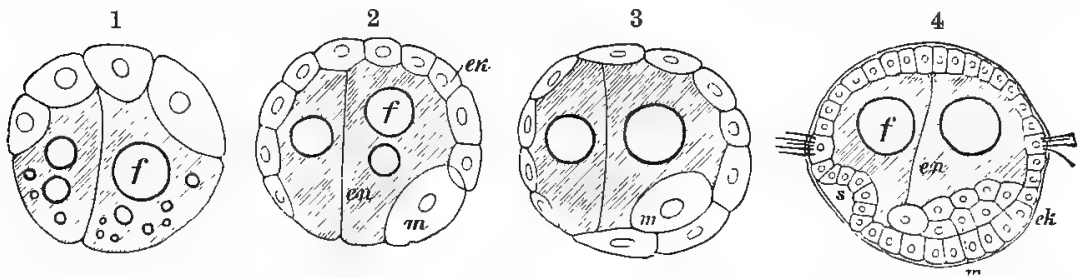

Fig. 31. Section through the egg of a Chætopod (Nereis) at different atages of development. In 3 , the gastrula is fully formed, but the blastopore (below, to the left) is still open; in 4 it is olosed, and the formation of the mouth (s) has begun. ek epiblast, en hypoblast, $f$ oil globules, $m$ mesoblast, $s$ developing mouth. The vitelline membrane is removed in the first three figures, present in the last. - After Götte. 
large, and the segmentation cavity is wanting, or is very small. In such cases the gastrula appears to be formed in a manner different from the foregoing: the epiblast cells, which originally form a cap upon the hypoblast, gradually g r ow round it, and the hypoblast itself often encloses no archenteron, but forms a compact mass.

In the first and second types of gastrula formation described above, the blastula grows directly into the gastrula by a modification of some cells to form

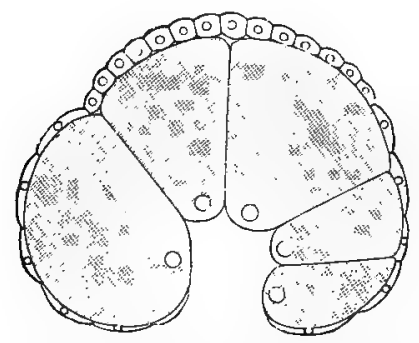

Fig. 32. Gastrula of a marine Gastropod (Natica). formed in the same way as the gastrula in Fig. 31, from which it differs in the possession of an archenteron.After Bobretzsky. the hypoblast (Fig. 29, 4, 5). In the blastula, the outer end of these cells is the wider, but they gradually alter in shape until the inner ends are wider than the others, which results simply from a different arrangement of their protoplasm (protoplasmic movements). This change in the shape of the cells must necessarily lead to a flattening of one side of the blastula and then to its invagination. Simultaneously an alteration occurs in the epiblast cells: these become shorter and, wider, so that they can extend over a large area. Whilst the invagination remains, the original relations of hypoblast and epiblast are retained. The epibolic gastrula (gastrula by overgrowth) described under (3), is probably formed in the same way.

There is no need to assume that the epiblast cells get loose from the hypoblast and grow over it. It seems that the wide outer ends of the hypoblast cells (Fig. 33, 1) gradually become narrower, and the inner ends broader, whilst the epiblast cells at the same time spread out, so that, by the same process as in the invagination of the typical gastrula, they gradually enclose the hypoblast, without its being possible to speak of an active migration of cells, and without the contiguous surfaces of the epiblast and hypoblast cells being altered.

1

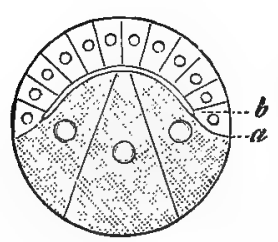

2

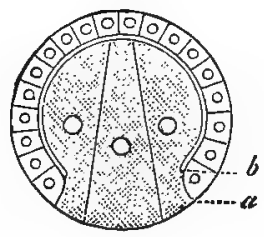

3

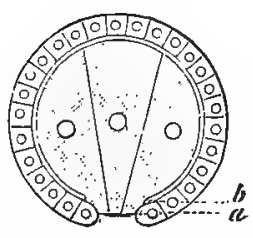

Fig. 33. Diagrammatic figure of the formation of the epibolic gastrula (See Text). 1 youngest, 3 oldest stage. 0 hypoblast. The letters $a$ and $b$ indicate the same points in all three figures - Orig.

4. In many lower Vertebrata (Cyclostomi, Sturgeon, some other Pisces, Amphibia) the blastula wall does not consist of a single layer as in the cases mentioned above, but is many cells thick (Fig. 34, 1); the cells are larger on one side of the sphere than on the other, and contain much yolk. This part of the blastula is invaginated, and a gastrula is formed as before (Fig. 34, 4) ; but part of the hypoblast is very thick, so that it stands up into the archenteron as a large boss. 
The cells composing this mass are destined to degenerate later on, and to serve as food for the rest (food yolk). The invagination too, occurs in a somewhat peculiar way: first of all, a fold is formed on one side of the blastula (Fig. 34, 2), and gradually surrounds the ovum; then it grows down over the lower portion of the egg, so that this comes to project into the archenteron.
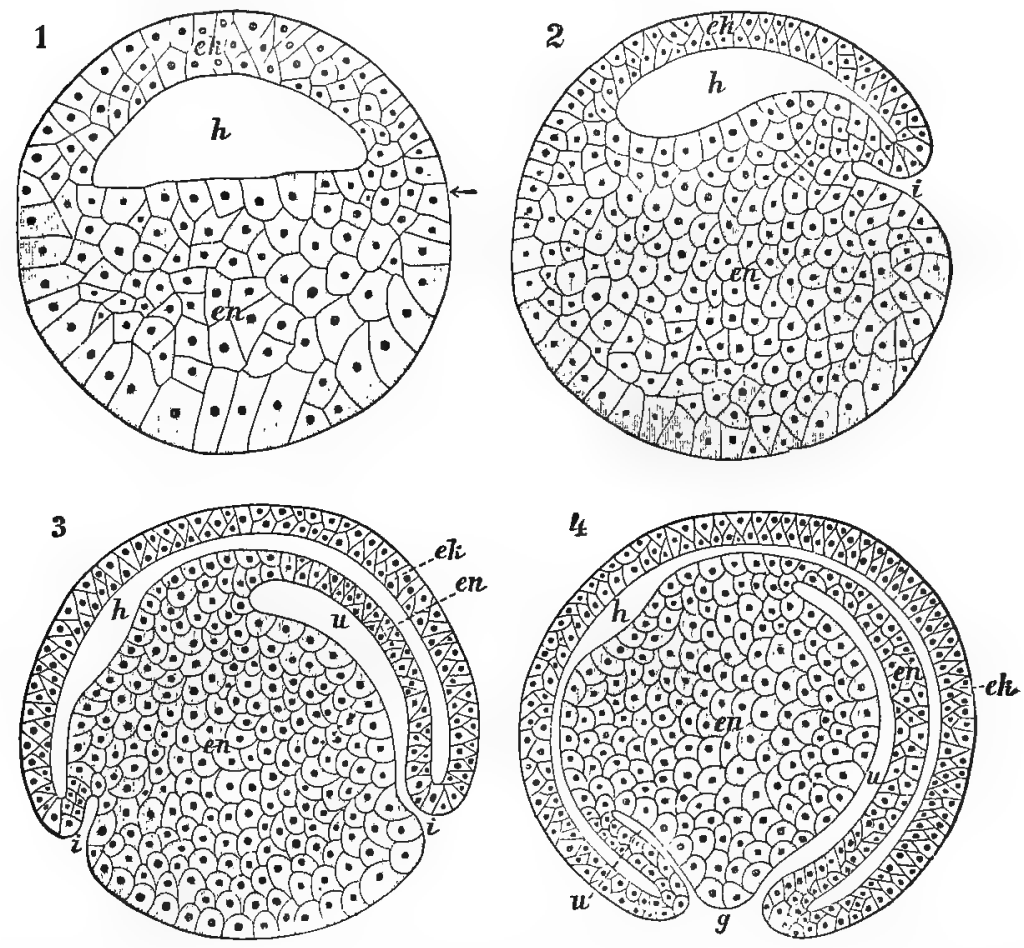

Fig. 34.-Formation of the gastrula in Amphibia, diagrammatic, longitudinal section. 1 Blastula. 2 The invingination has begun at $i$ (the corresponding place in 1 is indicated by an arrow); the invagination is in the form of a furrow, but does not yet surround the egg. 3 The invagination is proceeding. 4 Perfect gastrula; the archenteron is almost filled with a projecting part of the hypoblest, which is later dissolved and absorbed by the embryo, ek epiblast (light), en hypoblast (shaded), $g$ gastrula mouth, $h$ segmentation cavity, $i$ invagination furrow, $u$ archenteron.-Orig.

This mode of gastrula formation is readily derived from the typical one; and indeed, simply results from the excessive thickening of the hypoblast cells. If to the blastula figured in Fig. 294 , there were added a large mass of hypoblast cells, which took no active part in invagination, this would occur as in Fig. 34 .

5. This is the kind of development which occurs in many Fish, in Reptiles and in Birds. The egg-cell contains a large amount of yolk, practically all the protoplasm is accumulated at one pole of the egg, and it alone segments, while the greater part of the cell remains unsegmented; this is known as partial segmentation. 
The unsegmented portion corresponds to that part in the forms mentioned under (4), which does segment, but which unites later, and is invaginated with some of the adjacent cells into the remaining cellmass, the process taking place as in the way last mentioned (Figs. 34, 35). An enormous mass of unsegmented substance, the food
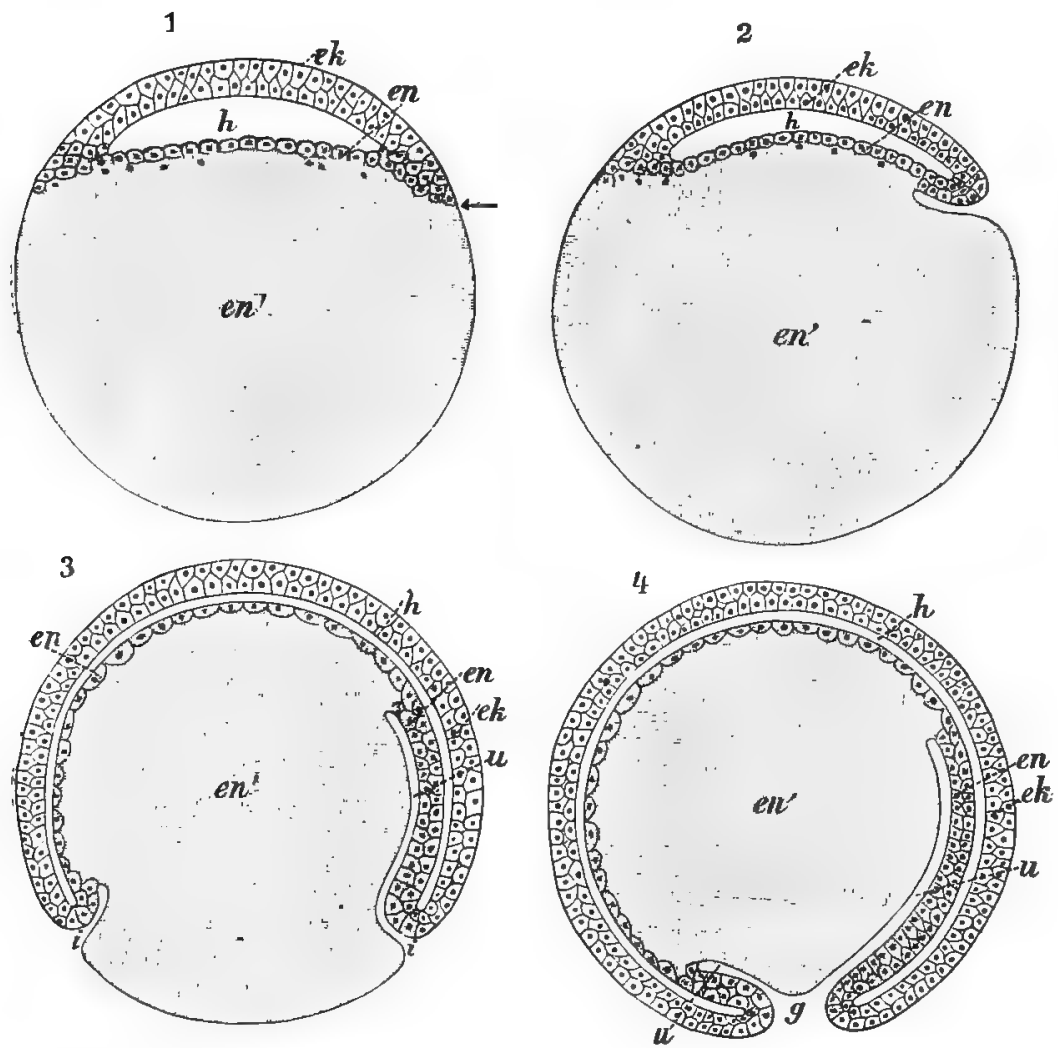

Fig. 35. Diagrammatic representation of gastrula-formation in Vertebrata, with partial segmentation (Selachians, Teleostians, Reptilia, Aves); compare preceding figures; the letters are the same as in these, with the exception of $e n^{\prime}$ the unsegmented part of the hypoblast (the yolk); en the segmented hypoblast. It must be noticed that the yolk is usually, relatively much greater than is figured here.-Orig.

yolk, becomes enclosed by the archenteron; is gradually absorbed by the cells; and thus serves as nutriment during development. These animals are, moreover, peculiar, in that invagination proceeds very slowly. An advanced stage of development is reached by the time that gastrulation is complete. No notice has been taken of this in the figures.

6. In most of the Arthropoda, as in the animals just referred to, partial segmentation obtains, but the food yolk lies centrally; surrounded by the segmentation spheres. The segmentation cavity is 
wanting; its place is occupied to a certain extent by food-yolk. Invagination takes place as in the typical gastrula $(1$, p. 40) and the food-yolk is gradually absorbed by the cells.

Fig. 36. Development of the ovum of a Crustacean (sections). $B l$ Yolk, ek epiblast, en hypoblast, $m$ mesoblast, o blastopore. - After Hæckel.
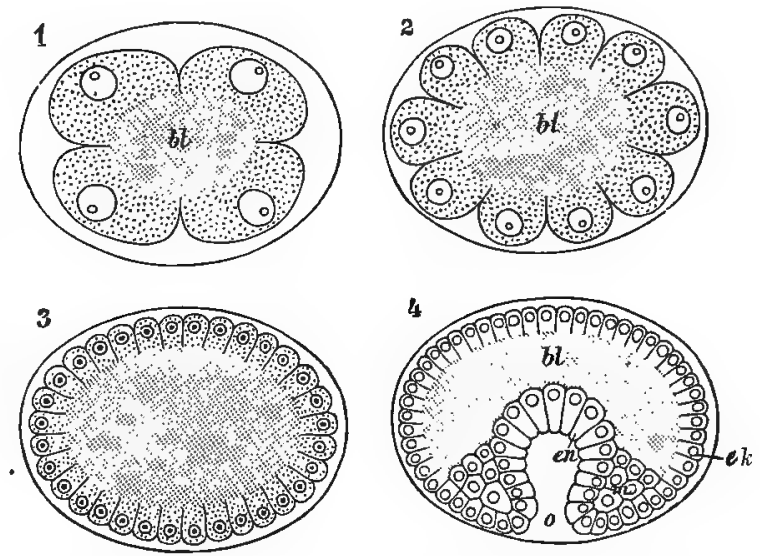

From the above it follows that a gastrula, always formed in essentially the same way, is found almost universally (when the examination has been sufficiently thorough), as a result of the earliest development of the egg.

An aberrant gastrula-formation occurs in many Hydrozoa. No invagination takes place here, but at different points in the blastula, cells break away from the rest and wander into the segmentation cavity to constitute the hypoblast (Fig. $37 \mathrm{~A}$ ). Sometimes the proliferation of cells is limited to one side of the blastula (B). Possibly the mode of hypoblast formation figured in $\mathrm{A}$ is primitive: from this $\mathrm{B}$ can easily be derived, and it may be a transition to the typical gastrula, which also occurs in the Hydrozoa.

Between the two primary germlayers, of which the gastrula consists, a third layer, the meso-

A

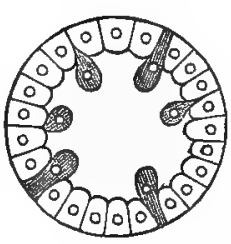

Fig. 37. Diagrammatic sections explaining the formation of the gastrula in certain Hydrozoa.

blast or middle germ-layer, is formed in most animals (Colentera excepted), very soon after, or even during, the formation of the gastrula. There is no general type of formation recognisable for this, as there is for the gastrula. In some cases it is formed, for example, by some few cells lying at the blastopore, at the boundary between the epiblast and the hypoblast, breaking from their connection with the rest, pushing between the two sheets, and after repeated division, extending between them as an independent layer (Figs. 30, 31). In other cases it originates as a double fold or sac of hypoblast, which 
becomes nipped off from the rest of the layer, and takes up a position between this and the epiblast (Fig. 38).

1

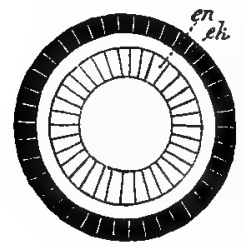

2

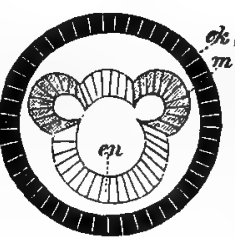

3

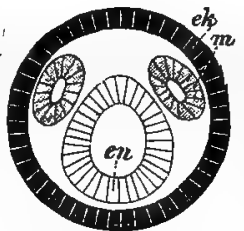

4

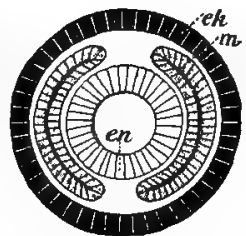

Fig. 38. Diagrammatic figures in explanation of the formation of the mesoblast in the Vertebrata (transverse sections); 1 youngest, 4 oldest stage. ek, en, $m$, epi-, hypo-, meso-blast.-Orig.

After the origin of the mesoblast, the organism consists of three germ-layers, epiblast, mesoblast, and hypoblast. From these three layers the different organs of the animal are formed; from the mesoblast all the connective tissue, the skeleton* (in so far as it is not a cuticular structure), the muscular tissue, the vascular system, the excretory and genital organs; from the epiblast, the epidermis, the nervous system, most of the sense organs; from the hypoblast, the epithelial lining of the whole or of the greater part of the alimentary canal, and its glands. I he mesoblastic structures, therefore, constitute, at least in the higher animals, the main mass of the body of the adult.

Of the different systems of organs, only the development of the nervous system and digestive tract is dealt with here, and quite briefly.

The central nervous system usually arises as a folding, invagination or thickening of the epiblast, from which it separates later.
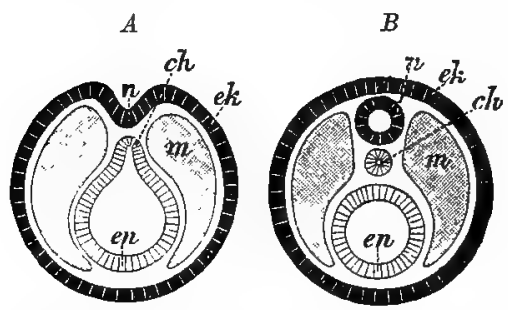

Fig. 39. Diagrammatic transverse section, showing the formation of the nervous system and the notochord in the Vertebrata. $A$ young, $B$ older stage. ch Notuchord, ek, en, $m$ epi., hypo-, mesoblast, $n$ nervous system.-Orig.
Among the Vertebrata, for instance, a long furrow-like fold is formed along the dorsal side of the animal, and is the incipient central nervous system (brain and spinal cord); it loses its attachment later, and lies beneath the skin, as a tube. In some cases (e.g., Echinoderms and Chætopods) the primitive connection of the derivatives of the epiblast (i.e., the nervous system and the epidermis) is retained throughout life, either over a large area, or at least at certain points.

* The chorda dorsalis of the Vertebrata, however, develops from the hypoblast, as a fold, which is later constricted off to form a cord, dorsal to the gut. 
As to the development of the alimentary canal: the primitive opening, the blastopore, usually closes, so that the hypoblastic tube is represented for a long time by a closed sac. Later, the epiblast at each end of the animal invaginates to form, respectively, the stomodæum and the proctodæum (Fig. 41). The epiblastic invaginations and the hypoblastic tube, break into communication at their points of contact, so that the complete alimentary canal consists consequently, partly of the primitive hypoblastic tube, partly of these epiblastic invaginations, and to these are added, in many animals, elements from the mesoblast lying outside the canal, and forming its connective tissue and the muscular layers.

When the digestive tract contains much food-yolk, the young animal or embryo is much distended: a yolk sac, an outgrowth of the alimentary canal containing food-yolk, and surrounded by a corresponding outgrowth of the body-

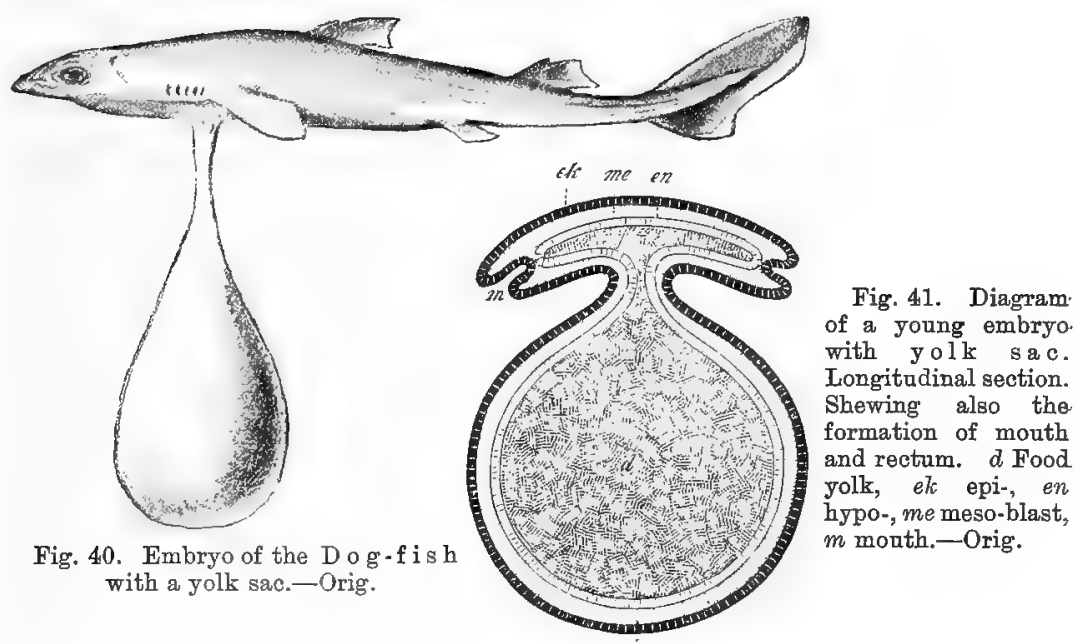

wall, is then often formed. It is frequently much constricted, so that its cavity is connected with the rest of the alimentary canal only by a narrow opening. Its size is often relatively immense, so that the young animal looks more like an appendage of the yolk sac, than the yolk sac of the young animal. As development advances, the sac gradually becomes smaller, and finally disappears altogether.

Most animals are oviparous, i.e., the egg is laid, surrounded by the egg-membranes, whether fertilised or not. Sometimes even after it is laid, it is still a single cell, but in other cases segmentation has already taken place, or development may be even further advanced. Other animals are viviparous, i.e., the ovum stays so long in the body of the parent, that the egg-membranes or shell are burst before the young organism leaves it. The distinction between oviparous and viviparous animals goes no deeper than this, and some forms are transitional between the two, for development 
is occasionally so far advanced among oviparous animals, that the young one hatches almost as soon as the egg is laid; whilst there are others, whose offspring break the shell at the moment of birth.

Those eggs which are laid without other covering than a vitellinemembrane or egg-shell, develop without receiving any nutriment from without. Oxygen is absorbed from the environment, and carbon-dioxide is given off, for the egg breathes just as does the perfect animal, and cannot develop in an atmosphere devoid of oxygen. Water also is sometimes absorbed. When the egg is laid, surrounded by albumen (as in Birds), this is gradually used up during the development of the young organism*, so that whilst it is still enclosed in the shell, it receives an addition to the material originally present in the egg-cell. In many, but by no means all, viviparous animals, the developing organism receives food from the parent; either certain glands opening into the uterus secrete a nutritive liquid which is absorbed by the offspring, or part of the embryo forms an absorptive organ which comes into intimate relation with the uterine wall, and the young animal lives like a parasite on the blood of the parent (Mammalia).

So long as the animal remains enclosed in the egg-membranes, or in the body of the parent, it is called an embryo (fœetus). After birth, i.e., when it breaks through the egg-membranes or leaves the parent, as a rule, it begins instantly to lead an independent life, feeding on its own account. $\dagger$ The new-born animal differs more or less from the perfect organism. In some cases, the distinction is relatively unimportant, as when it consists essentially in smaller size and immature genitalia, as in many Mammalia. In other cases the difference is greater, e.g., among Birds; fledglings, as is well known, differ considerably in plumage from the adult. Still more important is this difference in many other animals which are said to undergo a metamorphosis before they attain the perfect form. The changes undergone by the young animals, in such cases called la r v æ, are often very considerable, and frequently the larva has but the slightest resemblance to the adult.

The causes and also the nature of differences between larva and adult are of various kinds. In many cases the most obvious is that the egg is too small for a creature like the adult to be formed from it. The imperfect state in which many Fish are hatched is fully accounted for by the small size of the eggs (see the paragraph on the development of the Pike in the special part, "Pisces"): in allied forms with

* This absorption goes on usually through the whole surface, or by only a part of it. Occasionally the albumen is talsen up into the digestive tract of the young organism.

+ Sometimes there is a greater or less quantity of yolk within the body of the newly born animal, and then it does not feed for a long time. (See also, remarks, p. 52, on "Parental instinct.") 
large ova, the young ones, when they leave the embryonic membranes, are very similar to, or almost identical in appearance with, the adult. This holds, also, for many Crustacea, which are hatched in a very immature condition, with only a small number of appendages (three pairs). Here the egg is evidently too small to form an animal with so large a number of legs as is displayed by the adult Crustacean. It seems as if there were a minimum, differing for different animals, for the size of the limbs, and so on. In the larger eggs found in other Crustacea, the animals hatch at a more advanced stage of development.

The differences in the conditions of life must be regarded as another important factor in determining the differences between adult and larval forms; they are often intimately related to the cause just mentioned. Many marine animals, which live as adults upon the sea-bottom, are pelagic as larvæ, and this has a great effect upon the whole structure (Chætopoda, Mollusca, Crustacea). Sometimes, for instance, among the Amphibia, the larvæ are aquatic, the adults terrestrial, which also entails great differences. The metamorphosis of Insects seems to be connected with the difference of the functions of the larva and of the perfect animal, for the latter lives almost exclusively for reproduction, whilst the larva devotes itself to feeding and growth.

In consequence of diversity in mode of life, parts are frequently wanting in the larvæ which are present in the adult. On the other hand, they often possess special organs, the so-called p r ovis i o n al larval organs, which are absent from the perfect animal (velum of molluscan larvæ, gills of Tadpoles, pro-legs of Caterpillars). These larval organs may be so large that only a small part of the original larval body develops into the adult, whilst the greater part atrophies (Echinoderma).

Compared with the whole life, the larval period is usually short: as a rule the animal attains the adult form, in most respects, long before it reaches its full size and is sexually mature. Insects are a noteworthy exception to this rule, for the state of perfect insect is usually assumed only when the organism has attained its full size.

The transition from the larval state to that of the mature animal is never sudden, as might be supposed from the actual metamorphosis, but is always gradual. The transformation often, however, takes place in relatively short spaces of time; if a larva has been for a long time in the same condition, it may pass through great changes quickly, so that, to a certain extent, it assumes the adult form at a bound. This is specially marked in the Arthropoda, since all external changes are connected with the moults; sometimes before an ecdysis, the living part of the skin is loosened from the cuticle and more or less altered, so that the larva, when it strips off the old cuticle, suddenly appears in a new and changed form. 
As a matter of fact, these changes also take some time, and the development only appears to be a sudden one.

It is frequently found that an animal, during its development, whether in. the egg or in the larval state, shows certain characters, wanting in the adult, which are proper to a lower type. Tadpoles, for instance, possess gills, and a piscine arrangement of the greater part of the blood vascular system. Many larval Decapoda have an exopod upon the last pair of thoracic legs, which atrophies later, but is permanent in many lower Crustacea. Among the higher Vertebrates, gill-slits, etc., are present in the embryo. Frequently, as in the last mentioned, these structures are functionless, and of a rudimentary nature. These facts are only intelligible when it is admitted that the forms in question were derived from the lower type, or from one near it, and that these particular characters are retained in an early stage of development, though lost in the adult (see next Section).

Parental Instinct.-In many cases the egg or the embryo after leaving the body of the parent is specially cared for by the mother, or more rarely, by the father. The object of this is usually two-fold, firstly, to protect the egg or young animal from dangers of all sorts; secondly, to supply it with food. Less frequently some special means is resorted to, in order to keep the egg or young animal at the temperature most favourable to development (Birds). In the simplest. cases the mother is satisfied with depositing the egg in a safe place, (e.q., by burying it), or parental solicitude is confined to the careful selection of the place in which the eggs are to be laid, so that the young ones may at once find suitable food. In other cases, the mother sits upon the eggs until the young animals hatch out, or even after this, in order to protect and warm them: or the eggs and youngones are carried about by her. The parental instinct is still more actively manifested in cases where the young ones are able. to take in and digest food, but are still unable to procure it for themselves, and are therefore fed for some time by the mother. In many instances, the care of the brood not only dominates the whole life of the parent, but may even lead to the formation of special organs, or to the modification of those already existing, (brood sac, mammary glands, etc.) ; in many cases, too, it closely affects the mode of development both of the eggs and young animals, many features of which appear to be largely determined by this factor. (See, for example, the Mysidæ, the Marsupials.)

Careful consideration leads to the belief that the viviparous method of retaining the egg within the body of the mother is only a form of parental care. The difference between providing for the embryo within the oviduct (as in all viviparous animals), or in a pouch of the slin (Marsupials), appears tobe a purely superficial one; the aim is in both cases identical, and in both cases. the results are the same for the parent as well as for the young animal; the formation of some special arrangement in the former, some peculiarities in the development of the latter. 


\section{The Affinities of Animals.-Classification.- The Doctrine of Descent.}

The innumerable animals inhabiting the earth have been separated into many principal groups, and these again into smaller and smaller sections. This graduated arrangement is not arbitrary, but is based upon the degree of conformity of animals to one another, so that the most subordinate orders comprised in a division present the greatest conformity. The theory of species will now be discussed in some detail.

In the first place, all those individuals are ranked as the same species which show a close correspondence in all details of their structure at the same age: further, all those individuals which $p$ a ir with one another, under normal conditions, and produce fertile offspring, whether they resemble each other or not: finally, also, the offspring, which may or may not be like the parents. All the female Hares in England, for instance, belong to one species, for they exhibit at the same age a striking similarity in all parts of the body: to the same species, also, belong all the male English Hares, in spite of the difference in the genitalia, since they readily copulate with the females, and produce fertile offspring: finally, the young ones, which, in this case, do not differ from the progenitors. In cases of metagenesis and heterogony, on the other hand, the offspring differ considerably from the parents; every second, or third, or fourth generation is alike, but differs from the intermediate ones; all, however, belong to the same species.

The conception of species depends, consequently, upon three points: 1. similarity of structure; 2. possibility of interbreeding; 3. genetic connection (descent). The similarity of different specimens of the same species is, however, not absolute, even if those peculiarities which result from differences of sex, of age, or of generations (in alternation of generations, and heterogony) are overlooked. Identical individuals are never seen; a careful examination always brings out differences, excepting in the very smallest creatures, where they cannot be seen: species vary, as it is said, in a greater or a less degree. The variation is usually not striking; externally it is generally restricted to slight differences of colour, shape, to relative size of the same part, or to the absolute size or weight of the whole animal. Internally, there are corresponding small differences to be observed. Each large mammalian species presents obvious examples of these ordinary variations. But sometimes, variations become more conspicuous: for instance, Foxes are usually white underneath, but specimens have been found with black undersides, or with black cross- 
markings on the shoulders, or even entirely black; and similar variations are found in many other Mammals and in Birds; male Stagbeetles have, as a rule, very large mandibles, but individuals have. been found with mandibles whose length is only a fraction of the usual size. Foxes, again, are sometimes destitute of the hindmost. molar in the lower jaw, etc. All these points in which an animal differs from the usual type, are called individual variations.

In some species two or more forms may occur, which are in some respects. distinct from one another, and generally not connected by those transitional
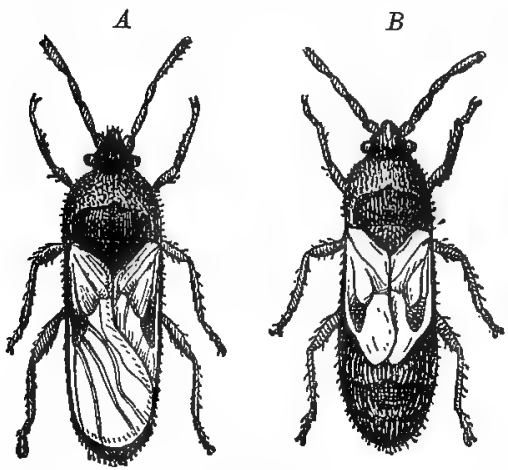

Fig. 42. Dimorphism of a Heteropteran (Blissus leucopterus). A Winged form with fore and hind wings; $B$ wing. less form with reduced fore wings and no hind wings.-After Riley. forms usual with the individual variations just mentioned. This phenomenon is called dimorphism, if the species occur in two different forms; polymorphism, if there are more than two. Dimorphism is found, for example, in the Heteroptera, in different. species of which, winged, as well as wingless forms occur. This and similar cases are easily derived from the ordinary individual variations, as the result of great individual variation, the transitional forms having disappeared. Dimorphism and polymorphism are fairly common amongst colonial animals; e.g., Craspedota, Siphonophora, Octactinia, Polyzoa; and here it is evidently the result of the common life of the individuals composing the colony: since they are in direct organic communication with one another, some have one special function, some another, and a. natural result of this division of labour, is a differentiation of the individuals. The dimorphism or polymorphism occurring among the Sosial Insects (Bees, Ants, Termites) is due to similar causes.

When a species has a wide distribution, it frequently happens that the individuals living in one locality differ, as a $\mathrm{rul} \theta$, in some respects, from the inhabitants of another: the individuals of each region are then said to form a special variety (race, sub-species). Hares, for example, which are distributed over the greater part of Europe, all belong to a single species, but form three groups: a south European, whose individuals are distinguished by short, loose fur, long ears and rust-coloured back ; a Mid-European, with long and close fur; and a North-East European (in North Russia), with a coat, longer and thicker than either of the others, and very white. These three groups are different $\mathrm{v}$ arieties, for though they are in general agreement, still they usually differ from one another in the points just noticed; but they are not different species, for each comprises specimens which approach the type of one of the other groups, so that they are not sharply defined. What has been said of Hares holds good also for various species of Mammalia and Aves. which are distributed through Europe and North Asia. The Siberian 
forms constitute a special variety; for, as a rule, they differ in some respects from the European. On the other hand, if a group of individuals found in a larger or smaller tract of land or water, differs in certain characters with o ut $\theta \mathrm{xc} \theta \mathrm{pti}$ on, from similar individuals of another locality, then it forms a distinct but nearly related species. The Wapiti Deer (Cervus canadensis) of North America, for instance, is a different species from the European Red-Deer, but is nearly allied to it. So also the North American Beaver is a different species from the nearly related European form, since some individuals of the first display peculiar characters (specially in the skull) which are never present in the European, and conversely. Often it is very difficult to determine whether a group of animals forms a variety or an independent species; it is difficult, in proportion as the particular variations are constant or not, as they are present occasionally (variety), or without exception (species), and of this it is not easy to be perfectly certain. Practically, a group of individuals is regarded as a definite species when transitional forms from it to another are unknown, i.e., when no specimens exhibit even a partial obliteration of these characters, which separate them frpm a closely allied species. So soon as such links are found, the group is recognised as a variety of the other. There is no sharp limit in Nature between a variety and a species.

The different behaviour of species and varieties in crossing also, as was mentioned earlier, cannot be employed as an absolute specific test, for, although hybrids of different varieties are almost always perfectly fertile, this ma y also be the case with hybrids of species (see above, p. 36).

Similar species are grouped as a genus; e.g., the lion, tiger, jaguar, cat, and others as species of one genus. Each genus of the Animal Kingdom is designated, according to a fixed rule, by its Latin or latinised name, which is always a single word; e.g., the genus which includes the animals just mentioned is called Felis. A species is known by the name of the genus with a subjoined distinguishing name; the latter is usually an adjective, sometimes a substantive in apposition to the name of the genus, or a substantive in the genitive case; the domestic cat, for instance, is called Felis domestica, the lion, Felis leo, the cochineal insect, Coccus cacti. Several similar genera are collected together as a family. For example, the badger-genus (Meles), the otter-genus (Lutra), the marten-genus (Mustela), and others, all belong to the marten-family, Mustelidx; the family name is usually formed by appending the termination $i d x$ to the name of one of the genera. Several families, again, are united to form an order, the cat-family, marten-family, bear-farmily, etc., form the order Carnivora. Orders, again, constitute classes, e.g., the Carnivora and a number of other orders belong to the class Mammalia. Classes form phy la, e.g., the classes Mammalia, Aves, Reptilia, etc., form the phylum Vertebrata. 
This grouping of animals in grades is, as noticed above, not arbitrary but founded on Nature itself. The animals are connected by some degree of relationship, i.e., by a more or less exact correspondence of structure: this connection finds an incomplete expression in the classification of the Animal Kingdom. When, for instance, Fish, Amphibia, Reptiles, Birds, and Mammals are comprised in one group, it is indeed implied, with sufficient clearness, that all these divisions agree in certain important points of structure, but not that they are united together like the links of a $\mathrm{chain}$. Such is, however, the case, and Amphibia follow Pisces, Reptilia, Amphibia; Aves and Mammalia, the Reptilia. Such a connection of forms, more intimate than the system expresses, exists in the Animal Kingdom; a true linking together, such as that just set forth for the principal groups of the Vertebrata is everywhere more or less easily recognisable.

What remains now is to ask the reason of this remarkable connection of different animals. Until a few years ago, the answer always was that the whole situation is one of the enigmas of Nature, incomprehensible to the mind of man. Now it is universally acknowledged that this affinity, this similarity, of different animals is an effect of those laws which govern the resemblance between parent and child, brother and sister, or more distant relations, viz., the laws of heredity. The lion, the tiger, the wildcat and other species, in virtue of a close accordance in most points of structure, all belong to one genus, implying that they all originally sprang from one species, which has gradually broken up into several. If bears, martens, cats, etc., are all united in one order, it is because they have all sprung from a common ancestor; so also for the larger groups, Mammalia, Vertebrata. Two such groups as Reptiles and Birds are linked together, because the latter have arisen from the former: by gradual change, a branch from the Reptile-stem is modified towards a Bird-form, from which the rest have sprung.

The completion of the conception leads to the conclusion that all animals have originated in one common primitive ancestor, which was probably similar to the Amoba. This is the purport of the Theory of Descent (Darwinism), according to which all the immense multiplicity of plants and animals have gradually evolved, in the course of enormous periods of time, from the same progenitor.

That this theory is correct is evident on the one hand, because it not only elucidates the long-noticed "conformity to type," in a natural way, but also renders intelligible an endless number of other phenomena of the organic world: and on the other, because in spite of strenuous efforts, facts irreconcilable with the theory have not been found. For the most important points supporting the theory of descent, without the acceptance of which, indeed, they are incompre- 
hensible, see p. 40, "Rudimentary Organs"; p. 52, some phenomena in Embryology; and also the Sections following, VI. to VIII.

Whilst the doctrine of descent has been talked about for a long time, it is only in the latter part of the present century that it has gained general acceptance, and this is primarily due to Charles Darwin (1809-1882), especially to his work, "The Origin of Species," which first :appeared in 1859. The chief value of Darwin's work appears to consist in his having brought together facts from all sides, demonstrating the necessity for the acceptance of the theory. He has proved that a large number of facts of Zoo-geography, of Geology, of Embryology : that rudimentary organs : that the whole theory of the relations of animals remain unintelligible if a doctrine of "Special Creations" is accepted. He has also, and this is of the greatest importance, disproved the generally received dogma of the immutability of species, by an examination of the variations of animals in a domesticated as well as in the wild state. His theory as to the means by which the modifications have been brought about ("Theory of Selection") appears to be of relatively less importance, although this was a side of his work upon which he bestowed the greatest energy and the most extensive studies. Darwin considered that in Nature, as in the breeding of domestic animals, a selection takes place: for those individuals are best fitted for the struggle for existence, which are distinguished by small favourable variations: and in this way development advances, for the less well equipped animal goes to the wall, whilst the better survives (Natural Selection). Whether this always occurs in Nature seems by no means certain, and in any case there are many characters in animals which are useless, and cannot therefore be explained by an appeal to Natural Selection. Besides this factor, Dar'win acknowledged the more direct influence of the -environment as a cause of variation; but the whole question seems still too far from solution to be treated more elaborately in a Text Book such as this.

The way in which evolution has proceeded and is proceeding, is probably as follows : the formation of va rieties is the first step; when a species has spread over a large area, it develops differently at different places, the varieties* formed become more sharply separated under favourable conditions and become distinct species. A species develops to constitute a new genus if the difference between it and the other species of the same genus becomes clearly defined; if it adapts itself, for example, to special conditions : by adaptation to a herbivorons and digging life, the badger genus has developed from the genus marten. A genus differentiated in this way may afterwards divide into several species. By continuous progressive development, and by the accumulation of variations, new families, new orders, etc., may arise. Each new type is, originally, only a specially differentiated species, but this species may split, later, into several, each of which again may be the starting-point for new genera. From this account, it may be supposed that the limits of the different genera, families, etc., are never fixed : and in many cases this is so: in many instances it is quite arbitrary whether one or more distinct species is reckoned as a separate genus or not, or whether a genus is considered as the type of a separate family or not. In other cases, on the other hand, the limit is sharper, for many species and genera, which might have obscured the boundary line have become extinct in course of time (Section VIII.).

Homology, Analogy.-By the gradual modification of animals, the individual parts of organisms have often altered very much in course of time, not infrequently indeed an organ has lost its original

* In many cases new varieties have actually been produced by the transportal of species to new localities. 
function and acquired a new one. Organs or parts of the body, which have had a common origin, may perform the same, or different, functions; such are called homologous: the arm of a man is homologous with the fore-leg of a dog and the wing of a bird, although the function in each case is different. On the other hand, it frequently happens that the same function is performed by different organs in different animals. Those which, differing in origin, are functionally equivalent, are said to be analogous: the eye of a Vertebrate is, for instance, analogous with that of a Gastropod. That branch of science, whose special task is to trace homologies and to notice changes in organs, is called Comparative Anatomy: together with Embryology, it forms the Morphology of Animals. In contrast to this, it is the task of Physiology to study the functions of organs.

\section{Biology.}

Biology, in the narrow sense,* treats of the habits of animals, their relations to the environment, and so forth. Various biological questions have already been touched upon in the sections on Organs and on Embryology: others of more general interest will now be considered.

\section{Dispersal of Animals.}

Animals are adapted to their environment in various ways; some forms flourish in one set of conditions, others in another; members of the same group, occurring in diverse situations, are differentiated in correspondence with the environment. On the other hand, the same environment often induces, in many respects, a similar type in members of different groups (Fish, Whales).

Mammalia, Aves, Reptilia, Insecta, Arachnida, and Myripoda, are emphatically terrestrial; the Amphibia, which are partly aquatic, are less distinctive in type. There are also many forms which, although belonging to essentially marine groups, have become adapted to a terrestial life, such as the great division, Pulmonata, not a few Crustacea, some Annelids. (Earthworm, Leech), a few Platyhelminths. The land animals have one characteristic in common; they are provided with a special respiratory apparatus, and almost always breathe by means of $\mathrm{lungs}$, or lung-like organs. The $f a u n a$ of a place varies in correspondence with the character of the district, of the soil and of

* Biology in the wider sense comprises the whole science of organisms, the whole of Zoology and Botany. 
the flora so largely dependent upon the latter; species, genera and even families found in forests, are in some respects different from those met with in deserts, so that a forest-fauna, steppe-fauna, or mountain-fauna, may be spoken of.

The animal life of fresh water has a relatively non-distinctive stamp; of the larger groups, there is scarcely one which can be called a special fresh water type. This fauna is composed largely of forms which are partly undoubted land types, partly marine: it is a peculiarly borrowed and mongrel collection. The numerous Pulmonata, Insects and Spiders, living in fresh water are derived from the terrestrial fauna, which, however, gives absolutely no Bird or Mammal as a constant inhabitant, although not a few of these creatures spend a part, or even the most, of their time there. A fair number of forms is furnished by Reptiles, most of which however, come on land now and then (Crocodiles, Turtles). A m phibia are almost all fresh water in the larval form; in the adult state many are, for the most part or entirely so. The freshwater fauna has drawn upon the sea also. Numorous Fish, whole families of which belong almost exclusively to rivers and lakes, a number of branchiate Gastropods, Lamellibranchs, Crustacea, Chrtopods, Polyzoa, Platyhelminths, some $\mathrm{C} œ \mathrm{lentera,} \mathrm{not} \mathrm{a} \mathrm{few} \mathrm{Rhizopoda,} \mathrm{etc.} \mathrm{A} \mathrm{very} \mathrm{few} \mathrm{of}$ a mammalian group modified for a sea life, the Whales, live in fresh water. Leeches, Rotifers, Infusorians, groups which are fresh water as well as marine, are so common in the former that they should perhaps be regarded as fresh-water types. The modifications which terrestrial or marine forms have undergone by the transition, are usually not very important. The land animals generally remain air-breathing, and the modification is practically limited to that necessitated by the difference in mode of locomotion; the alterations in the marine forms are also non-essential.*

The class Pisces, the phylum Mollusca, the Crustacea, Chrtopoda, the Polyzoa, Brachiopoda, Platyhelminthia, Echinoderma, Cœlentera, Porifera, Rhizopoda and Radiolaria are obviously marine groups. Of these the Brachiopoda, Echinoderma, and Radiolaria exclusively, and the Cœlentera and Porifera with few exceptions, belong to the sea. The sea has received a large contribution from the terrestrial fauna, especially as regards the Vertebrata. Two orders of Mammalia the Whales and the Sirenia, the former with many genera and species, have adapted themselves entirely to a marine life, and their structure has undergone correspondingly important modifications; a third Mammalian order, the Seals, are also distinctly marine, though they

* It is very noteworthy that many fresh-water forms do not pass through a freeswimming larval stage, whilst their relatives in the sea do (Astacus, Lamellibranchs, Oligoohæts). 
repair to the land for reproductive purposes. Reptiles have also contributed to the animal life of the sea (Sea-snakes, Sea-turtles). Amongst $\mathrm{Birds}$ there are none which have become entirely marine, although many are connected more or less closely with the sea, of those most so, the Penguin and many other Swimming Birds. Of living Amphibia there are no entirely marine forms. Only a few Spiders live in the sea, and hardly any Insects. Like the terrestrial, the marine fauna is modified according to the very different conditions existing in different parts of the ocean, the dissimilar character of the bottom, the depth, etc. The littoral fauna is different from that at greater depths, which again varies according to the bottom. The salinity is also of the greatest importance, for greater salinity is universally favourable to animal life; it suits a greater number of species, though less salt water may also be rich in individuals.

This is very clearly illustrated by comparing the state of the salt Kattegat with that of the less salt Western Baltic, and the almost brackish East Baltic. In the Kattegat there is a tolerably rich fauna, but already the north end of the Sound, where the salinity is less, contains a scantier collection. Most species which live in the Kattegat are represented here, but for the most part by smaller specimens and in fewer numbers. Southwards in the Sound, as in the entire western part of the Baltic (south of Denmark), very many of the Kattegat forms have disappeared; others are indeed present, but dwarfed, or in the case of the Molluses, thin shelled. Lastly, only a fraction of the fauna of the West Baltic is found in the fresher East Baltic, and this is also the case in that part of the latter (south of Sweden), which, as regards climate, does not materially differ from the West.

Some fresh water animals (pike, perch) may also occur on the coast in water of slight salinity; and on the other hand, some marine animals may be present in fresh water, the flounder (Pleuronectes flesus). Also, some Fish go to spawn either from fresh water into the sea (eels), or conversely (salmon, trout, sturgeon). Sudden immersion in sea water is fatal to most fresh-water animals, and most marine forms are similarly affected by fresh water. On the other hand, many endure, to a certain extent, a gradual salting or freshening of the water.

Temperature has often a great influence upon the dispersal of animals; this is especially striking in the terrestial fauna which, other conditions being the same, is much richer in hot than in cold districts; in the very coldest, it is almost entirely absent, or at least, reduced to a minimum. This is due not only to a higher temperature being universally favourable to animals, but also to the dependence of animals upon plant life, which is much affected by temperature. Since the temperature of sea water does not sink as low as that of the atmosphere, the sea in the coldest regions may harbour a rich fauna, although it cannot compare with that of tropical seas.

The more general features of the dispersal of animals have now been touched upon; in what follows, some special adaptations will be considered. 
Cavernicolous Fauna.-The entirely dark, subterranean caves: which occur in mountains of different countries, and which are also present beneath the water, harbour a small but peculiar fauna. Most of the animals living in these places, in contrast to their relatives of the daylight, exhibit very degenerate optic organs, or these are even entirely wanting. Frequently the skin is destitute of pigment: the blind, pale Proteus of the Carniola caves may be taken as a typical cave animal, and some Fish, certain Crustacea, Insecta, Spiders, also belong to this fauna. All cave animals, however, are not blind; some have retained their eyes, and are consequently less suited to life in the dark.

Those animals, which burrow in the earth, and which come to the surface seldom or only on dark nights, are like the cave forms; their eyes, too, may be more or less degenerate (Moles).

The rich animal life of the deep sea, where no daylight penetrates, resembles the cave fauna. The deep sea animals are often almost destitute of pigment, and have, frequently, very degenerate eyes, or indeed none, even when belonging to groups, the other members of which have these organs well-developed (some deep sea Crustacea and Fish). Other deep sea forms are, however, furnished with well-developed eyes, e.g., the majority of the fish, which are considered abysmal.* Many of these Fish are phosphorescent. Among the abysmal animals there are many which have only remote relations elsewhere-Stalked Crinoids, peculiar Echinoids and Crustacea, Hexactinellids, and others, so that this fauna is strikingly peculiar.

Pelagic Fauna.-Still more extraordinary are the animals living in the open sea, at a considerable distance from land, the socalled Pelagic Fauna. This comprises first, a number of groups, which either never, or only exceptionally come close to shore, and which generally do not occur elsewhere: the Radiolaria and other groups of the Protozoa, the Siphonophora, Euphausidæ, Pteropoda, Heteropoda, and Salpidæ: secondly, a multitude of forms whose near relatives live closer to land: lastly, an immense number of larvæ, belonging to forms which live in the adult state at the bottom of the sea. But what specially characterises the pelagic fauna is not so much the number of groups peculiar to it, as the fact that in virtue of their life in the surface waters, all the members of these different groups have certain features in common which are, however, less well marked in some than in others. There is, in particular, an erident tendency towards such a modification of the animal as may enable it to float with the greatest facility. This is attained, in some cases, by the tissues absorbing such considerable quantities of water that the specific gravity is only slightly greater than that of the sea; such animals have a jelly-like appearance (Medusæ, many Pteropoda and

* It is possible, however, that many of these live nearer the surface, and were caught in the net as it was drawn up from the bottom. 
Heteropoda, Salpidæ). In other cases, the surface is increased, either by the flattening of the body, by the lengthening of the limbs, or by the formation of long spines, as in many Crustacea and their larvæ, and in young Fish: in both cases it is found that the muscles causing the movement of the animal are reduced. Sometimes the atrophy goes so far that they become exceedingly weak, and the animal is only capable of feeble movements. In other cases, in spite of the weakened muscles, they are still good or even excellent swimmers, since the body floats in the water almost without muscular exertion. The majority of pelagic forms are further characterised by a great transparency. Many possess as perfect optic or gans as most of their relatives, as in the case of certain pelagic Chrtopods: others, on the contrary, exhibit degenerate eyes, which is explained by the fact that many members of the pelagic fauna appear on the surface of the sea only at night, remaining in greater depths by day.

Many animals remain for their whole life near the place where they were born: others stray about in search of food: others, again, undertake further migrations, usually in great numbers.

These wanderings have often a casual and irregular character. Unfavourable conditions, for example, scarcity of food or of water, will cause an animal to leave the usual habitat of the species, and to seek a new home. The migrations of Locusts, of Prairie-hens, of the Lemming, result from such causes. These wanderings but seldom increase the range of a species: after a short time it disappears again from the new place.

The migrations may, however, have a more regular character, such as those undertaken annually by Fish and Birds: at one time of year they inhabit one place, at another time another place, from which they then return to the first, and so on. Among many Fish, the migrations are due to a search, at a fixed time of year, for a suitable breeding-ground; whilst the flight of Northern Birds towards the South is really explained by -their not finding enough food for the winter in their summer home. (For details see these groups.)

\section{Different kinds of Food and their effect on the form of the Body.-Parasitism.}

The food of animals is of very different kinds. It may be vegetable, consisting of living or dead plants; or animal, consisting of living animals or carrion. Some animals eat a variety of things both animal and vegetable, others are, however, much restricted, e.g., to a few species of plants. Some feed on organisms of relatively very small size, others consume animals larger than themselves. 
In many cases it is evident that the kind of food exercises a very great influence on the structure of the animal. This influence manifests itself primarily in the form of the digestive tract. In allied animals, its length is generally greater in herbivorous than in carnivorous individuals; among the Mammalia, for example, it is much longer in Ruminants than in Carnivora. Many other variations in the alimentary canal result from the differences in food; this is specially marked in the buccal cavity, in the hard structures serving for the prehension and comminution of the food, e.g., the teeth of Vertebrata. Among the Mammalia this is very apparent; the teeth of a Cat, for instance, may be compared with those of a Horse, or again, the teeth of different Carnivora, Cat, Dog, Bear, etc., with one another. Since the modification and insertion of the teeth again affects, for example, the structure of the skull, the influence of the food extends indirectly to other systems.

The nature of the food, further, often affects the locomotor organs, and the entire external conformation. This is very evident in the Insecta, where the slender, active larva of the Carabus, with its elongate limbs, running about after its food, may be contrasted with the maggot-like larvæ of the Curculionidæ and Longicornia, which either have only rudimentary legs or are entirely apodous, and live in the midst of plenty. Frequently, again, the limbs of predaceous animals function secondarily as prehensile organs and are correspondingly modified. As the limbs are affected, so also are the sense organs, especially the eyes, by the nature, and particularly by the greater or less activity of the prey. Carnivores often possess large, well developed eyes, whilst herbivores, whose food is plentiful, have smaller or even degenerate optic organs. Insects also afford characteristic illustrations of this.

This does not mean that the locomotor or sensory organs of herbivorous animals, whose food is plentiful, are always poorly developed. Amongst the Mammalia there are numerous herbivores rivalling Carnivora in this respect, as Stags, Antelopes. In these cases the high state of development of these parts affords a means of escaping the pursuit of the Carnivora.

A discussion of the direct and indirect influence of food leads on to a consideration of Parasites. Those animals are called parasites which live upon or in other living animals known as the hosts, and feed at their expense; either upon some part of the body (e.g., its blood), or upon food taken in and digested by it, as in the Tapeworms. They may be temporary or stationary. The former do not stay continuously on the host, but seek it out at intervals simply for the sake of food, whilst the stationary parasites remain constantly upon or within it. Parasites have, indeed, been classified according as they live on the outer surface or in the interior of the host, as ecto- or endo-parasitic. A sharp line cannot be drawn between these two groups, since the 
boundary between the external and internal organs is not clearly defined. Most parasites do not spend their whole lives as such, but lead an independent existence at one time or another. Some are, for example, parasitic when young, but free-living as adults. (the Gadflies), whilst others, conversely, are first free-living and later on become parasitic (parasitic Crustacea). This mode of life has a marked effect on the structure of the animal: it is often relatively slight in the temporary parasites, and in those stationary ectoparasites which can move about freely on the body of the host; but very great in most stationary ecto- or endo-parasites. The fact that nutriment is plentiful and immediately accessible exercises its usual effect: the power of locomotion is decreased; a more or less complete reduction of the limbs is seen: the sense organs, further, e.g., the eyes, are much affected: most stationary forms, especially the endoparasites, are blind. Organs of adhesion, on the other hand, are frequently well-developed, as suckers, hooks; or certain limbs are modified for this purpose. Parasitism, moreover, exerts a definite influence upon the whole life. A natural result is, for instance, that the animal must generally undertake $\mathrm{migr}$ at i o $\mathrm{s}$, i.e., that it must not spend the whole of its life, from the ovum to sexual maturity, in the same host, but must be actively or passively transported at some period into another. Besides this one migration, there are often others. Many parasites regularly produced as eggs in one host, or in the free state, live for some time in another, the intermediate host, and finally attain sexual maturity in a third.

Parasites belong to various sections of the Animal Kingdom. There are, nevertheless, certain large groups of which none or only a few individuals are modified in this direction. Among the Vertebrata, only a single Fish : so also only a few Molluses, Chætopods, Ccelenterates, and absolutely no Echinoderms live in this way. The Arthropoda, especially the Crustacea, furnish a large contingent, which are for the most part, ect o p a r as ites, the Crustacea, exclusively upon aquatic animals, the others almost as exclusively upon land animals. Further, amongst Annelids, a great many Leeches are ecto-parasites : most of the Nemathelminths are endoparasitic: whilst of the numerous parasitic Platyhelminths, some are endo- and some ecto-parasitic. The parasitic Round-and Flat-worms are frequently called intestinal (Entozoa). Many Protozoa lead this life, Gregarines, many Infusoria, etc. Many of the members of all the chief divisions of animals must serve as hosts, especially the Vertebrata, which principally on account of their large size, complicated structure, and relativelylong life, afford an excellent scene of action for parasites, both internal and external. Many of these creatures are limited as regards the choice of a host; some live, for instance, always in one species, never in any other; others are confined to a few allied forms; others, again, have a larger, but always a restricted choice. The same parasite, for instance, cannot live indiscriminately in a Fish and in a Mammal. On the other hand, it frequently happens that the same form lives, at different periods of development, in animals of a very different systematic position; Echinorhynchus, for example, is found, when young, in Arthropods, as an adult, in Vertebrates.

Some animals, e.g., some Leeches, form a transition from the predatory to the temporary parasitic condition, for sometimes they eat small animals, and 
sometimes suck the blood of larger ones. Other animals, as certain Fly-larvæ, may be both carrion feeders and parasites. Again, there are forms which are a transition to the parasites, in so far as they live in other animals, but do not feed at their expense; at most they take a moderate share at meal-times (commensalism).

\section{Different Kinds of Locomotion.-Their Effects upon the Structure of Animals.-Sessile Forms.}

The movements of animals are known to be very diverse. Many, especially lower animals (Worms, etc.), $\mathrm{cr}$ a w l by means of contractions of the muscles of the body-wall, or by movements of the cilia upon the surface. Others swim: this is often accomplished by movements of the whole body, or by the greater part of it; or it may be effected by means of limbs. Walking and running, on the other hand, are absolutely dependent on the presence of limbs. S pring ing movements are of various kinds: amongst aquatic animals, some part of the body strikes upon the water (decapodous Crustacea); amongst terrestrial forms, a jump generally results from violently jerking the limbs off the ground. Flying is always effected by means of specially developed limbs, just as burrowing and climbing, well-known forms of locomotion.

Each of the kinds of movement mentioned, which may be combined, to a certain extent, in the same individual, may influence the structure of the animal more or less deeply. This is clearly manifest when allied groups, typically distinguished from one another by different modes of locomotion, are compared. A great many of the specially marked differences between Fish and the higher Vertebrates, are to be ascribed to diversities of movement. In Pisces, limbs are but slightly developed, the body muscles, and those of the powerful tail, much more so, in accordance with the needs of an aquatic life. In the most decided walking types of Vertebrata, the Mammalia, the tail is reduced, the dorsal and caudal muscles also, but the limbs are well developed. That all this is a result of a difference in locomotion is borne out by the fact that if Mammalia are adapted, as an exception, to a swimming life, they again come to resemble Fish. This is the case in Whales, which are derived from terrestrial Mammalia: their limbs have atrophied under the influence of an aquatic life, whilst the tail is developed as a powerful locomotor organ. In like manner the difference between a Shrimp and a Crab depends principally upon the former being a springing and swimming animal, whilst the latter is distinctly an ambulatory form. The different burrowing types (Mole, Mole-cricket), may also be compared with their nearest relations. The kind of movement does not, however, always leave so clear a mark upon the structure as in the cases mentioned. The adaptation is not always so exact, the modification not always so complete as in these: other swimming Mammals may 
be compared, for example, with the Whale; Seals, Otters ; or other digging forms with the Mole; the Mole-cricket and Rabbits, Dung-Beetles.

Sessile Animals.-In spite of the fact that the power of freelocomotion is specially characteristic of the Animal Kingdom, there are yet many forms which are fixed to one spot for at least the greater part of their lives. The fixation usually results from a certain part of the surface of the animal coming into connection inseparably with a foreign object, a stone, a dead Mollusc-shell, or the surface of another animal; the connection is often effected by a cuticular secretion. Frequently, as in many Chrtopods, the connection is less intimate, for the shell only, with which the animal is not in direct union, from which, it may escape, is fixed to the foreign object. The transition from the free-living to the sessile form, is given by those animals which, though capable of locomotion, yet generally remain for some time (days or years) in the same place (some Gastropods, Lamellibranchs, Fresh-water Polyps, and others).

The immediate and very natural result of a sessile life is the atrophy of the locomotor organs. The mouth in sessile forms (Corals, Hydroids, Tubiculous-worms, Polyzoa, Brachiopoda) is very often surrounded with long, prehensile arms or tentacles, which are adapted either for seizing those animals which happen to come near, or, by means of their ciliated border, for driving small organisms into it. In other sessile animals (Vorticella, Sponges, Oysters), the $e_{s}$ tentacles are wanting, but small organic particles are conveyed to the digestive organs by ciliary currents. This mode of life is further obviously favourable to the formation of colonies : most colonies are sessile, especially those which are arborescent.

\section{The Transforming Effects of the Environment.}

In the foregoing paragraphs, cases of adaptation to the environment have been examined: some facts illustrating its $d i r e c t$ in $f l u$ ence upon organisms will now be considered.*

It is a matter of universal observation that organs, at least in many cases, improve with use, whilst they degenerate with disuse. This may be seen, on the one hand, in the great development of the arm, in people employed in manual labour: on the other, in the feebler condition of this part, in those whose work is entirely mental. Similar facts are also known for domestic animals.

It has further been proved in various instances that a special food may have a modifying effect upon the colour of certain birds. A reddish-yellow colour may be quickly produced in

* There is still much to be done in this connection so that the following remarks must be taken somewhat tentatively. 
canaries by feeding them with cayenne pepper. Pigeons fed on meat have the gizzard modified like that of a bird of prey (thin-walled), whilst, conversely, the stomach of an ordinary flesh-eating bird (Gull) is so modified by a diet of corn that it becomes like that of a graineating bird.

Interesting experiments have been made upon the $\mathrm{tr}$ a s f o $\mathrm{r} \mathrm{m}$ in $\mathrm{g}$ influence of an alteration in salinity. A Crustacean living in salt lakes, Artemia salina, nearly related to the fresh water Brannchipus, is modified, by gradual increase in salinity, into another form, which has been described as a peculiar and different species, A. milhausenii. The modification does not arise immediately in one and the same individual; it is gradual, extending through many generations. Conversely if $A$. milhausenii is placed in slightly salt water it becomes $A$. salina. Further, if the water is freshened by degrees, until it is finally quite fresh, $A$. salina is gradually modified in the other direction, so that at last it assumes the characters of the genus Branchipus.

Animals may also be affected by variations in $1 \mathrm{ight}$; the cave form Proteus, whitish under normal conditions, becomes speckled or brownish if exposed to the light; for the pigment, usually absent, now develops in the skin. If Sticklebacks are put in a glass with a white bottom, they turn quite pale in the course of a few days, and if they are kept five or six weeks under these conditions, the dark colour does not return, even when they are put in a glass with a dark ground.* Similar observations have been made upon other fish. It has been found in not a few species of Butterflies, that the colour of the pupa really depends upon the colour of the background upon which the larva rests during the days immediately preceding pupation. If the larva has lived on a light background (e.g. a light wall), the pupa will be light, and vice-versâ. In this, as in the other cases mentioned, the action of light upon the skin of the animal is evidently the primary cause.

Among Butterflies, noteworthy observations have also been made as to the influence of temperature. If certain pupæ are kept at a low temperature, the colour of the Butterfly varies from the normal.

The seasonal dimorphism already mentioned, is probably originally called forth by the effect of a different temperature upon the pupæ: and as a matter of fact, when the summer pupæ are exposed to a lower temperature, specimens may appear which are like the winter-brood.

In many cases animals are curiously affected by settling in a new locality, no further cause being ascertainable. A few examples will illustrate this. In 1870, a few wild Turkeys were

* The change depends upon the fact that the pigment-cells (chromatophores) of the skin have almost entirely atrophied. This d efinite change in the colour of the skin must not be confused with the colour change occurring in many animals (Cephalopoda, Chameleon), which depends on the alternate contraction and relaxation of the chromatophores. 
taken to a small island near California. They multiplied very rapidly, and ten years later numerous descendants inhabited the island, but the weight of the specimens had fallen to one-third of that of the birds introduced; in the course of a few generations they had assumed a pigmy form. The effect of island life generally appears to result in the formation of dwarfs. Changes in other respects have resulted from the removal of animals into new surroundings. A peculiar form of Wild Rabbit has been produced in course of time in the island of Porto Santo; it seems to be a distinct species, possessing special colour-markings, etc. This has sprung from Spanish Rabbits, which were imported 400 or 500 years ago. Trout were introduced into New Zealand about twenty years ago; their progeny varies now in certain points in the operculum, from the European species.

The influence of different external conditions upon the organism is very clearly exhibited in domestic animals. Many of the peculiarities exhibited by domestic races are simply the product of local conditions, special food, etc.

It should be pointed out that the nature of the above explanations is unintelligible. Why Artemia should undergo modifications, as a result of migrating into fresh water: why pigment should be developed in Proteus when it is exposed to the light: is not as yet exactly understood. These facts are, however, of great importance, since they demonstrate that the peculiarities of different faunas, the adaptation to different kinds of food, the modification and degeneration of parasites, mentioned in the preceding paragraphs, are in all probability, at least to a great extent, the effects of external causes.

\section{The Stages of Life.-Duration of Life.}

A number of stages may easily be distinguished in the life of most animals. The first stage is the embryonic period; a second, the ensuing time of youth; a third, the period of complete development; and this, finally, may be followed by a state of retrogression.

The embryonic period has already (p. 50) been sufficiently described. The time of youth extends from birth until the animal becomes sexually mature (i.e., produces ripe ova or spermatozoa), and therewith has almost attained its ultimate size and form. During the adult period development is almost stationary, and the organism passes quite gradually into the senile period, when the organs suffer partial degeneration, and are functionally less active. The whole strength of the organism, moreover, fails, so that it readily falls a victim to adverse influences. This state is, however, only clearly seen amongst the higher Vertebrata (Mammalia and Aves). It need scarcely be said that the different phases are by no means sharply demarcated. 
In each year and in each stage of life there are, often, regularly recurring periods. For many animals, there is, daily, a period of activity and a period of rest. Many sleep during the latter, i.e., they fall into a peculiar state of unconsciousness, in which the activity of the organs is actually lessened (Mammals, Birds). The time of rest usually occurs in the night, the period of activity in the day; but it is well known that some rest in the day, whilst they become energetic in the evening or at night (nocturnal animals).

The year is similarly divided for very many animals into two great periods, one of which is devoted to active life, during which the daily activity and rest may naturally alternate: the other to repose. This is specially the case with animals of the temperate and frigid zones, which are, for the most part, rendered entirely passive by the cold of winter, when their vitality is reduced to a minimum (e.g., hibernating insects). Many Mammals (Bears, Dormice) fall during this time into "winter sleep" (hibernation), a state resembling ordinary sleep, but of an intensified kind. The unconsciousness is deeper, the activity of the organs decidedly less, and the temperature of the body may fall several degrees. Similarly the resting period of tropical forms may occur during the dry season, and in certain English animals the hottest summertime may occasion a summer sleep (Earthworms, Reptiles, Amphibia).

Many animals, especially those of cold climates, have an annual breeding season, at which time the ova and spermatozoa are ripe, and when pairing takes place. At other times the ovaries and testes are in a state of relative inactivity, and the sexual impulse is extinguished. In warm countries, at least in most cases, such a period does not occur. Many animals, whose nearest relatives in the temperate zone have a limited period for breeding, reproduce all the year round. It must also be noticed that the periodicity of marine forms is less pronounced than that of land animals; ripe ova are found in some marine animals throughout the year in cold regions. This diversity naturally depends on the fact that the variations of temperature are much less in sea-water than in air.

Death, i.e, the final cessation of all manifestations of life, usually occurs in consequence of the functional passivity of a vital organ: If, for instance, the heart of a Vertebrate ceases to contract, the body is deprived of a necessary condition of existence, viz., the supply of blood laden with oxygen, and all the organs and tissues gradually die. An exact moment of death cannot be spoken of: if a Mammal be killed by a violent blow on the head, movements of the heart and respiratory organs, of course, cease almost immediately, and the animal is said to be dead: but many of the 
tissues continue to live for many hours the muscles are capable of contraction, etc.

Death may occur in any period of life: in forms which produce very numerous ova, the majority of specimens invariably die in the embryonic or larval stages, only a small proportion attaining sexual maturity. This results almost always from detrimental influences in the environment. The existence of most animals terminates because they are killed or eaten by others: many fall victims to pathogenic parasites, principally Bacteria and Fungi; others, again, succumb to the effects of climatic changes. Death, conditioned by the normal internal state of the body, occurs rarely: even when an animal appears to die of old age, or when, as is frequently the case, it dies, necessarily, after a single production and discharge of ova or spermatozoa, external conditions may still be an agent.

The duration of life in animals, the time over which life can extend under favourable circumstances, varies enormously for different forms: in some, it may be limited to a few weeks or less, in others it may extend to a hundred years or more. The rule may be generally laid down that in a natural group, the larger species live longer than the smaller, just as their developmental period is longer: the Elephant lives more than a hundred years, the Horse, seldom more than thirty; the Mouse, only a few; Large Insects often live several (the Cockchafer, e.g., four), smaller ones, a year, or even a few months only. In some, the duration of life is very definite (e.g., most Insects die soon after laying their eggs), amongst others it is quite unlimited.

\section{Protective Adaptations.}

It is well-known that most animals are preyed upon by others, and, as a rule, they do not confront the enemy defenceless, but are protected in different ways.

The means of protection are sometimes offensive: the prey itself practically attacks the aggressor. The weapons used in defence are sometimes such as are used in other cases for attack: a carnivore, for instance, tears its prey to death with its teeth, and also uses them as defensive weapons when it is attacked. As a rule, animals resist their enemies with any means at their disposal; Mammals with hard hoofs, kick; if provided with strong claws, they scratch; many insect larvæ pour out a malodorous or sticky secretion. The caudal spines of some Rays; the stink-glands of many Insects and of the Skunk (Mephitis), whose disagreeable secretion is ejected to drive away the enemy, are special weapons of defence.

Other animals try to frighten the enemy by violent cries, by terrifying movements, by bristling the feathers or hair. 
Other means of protection are of a purely defensive nature, and here there is a great diversity. Many animals try to escape danger by flight, and many weak and defenceless forms have remarkable powers in this direction (e.g. Antelopes). Others save themselves by hiding. Many offer resistance in the shape of a specialised protective covering (a prickly skin, a stiff coat of mail). Not a few Insects are characterised by a repulsive taste or odour, and by this keep the enemy at a distance; many animals are protected, whether in motion or at rest, by their resemblance to their surroundings (protective resemblance) ; many, on account of their green colour, are difficult to distinguish from their food-plant; many, again, are concealed by the leaf-like appearance of the wings or other parts: the most delusive resemblance is perhaps found in some Indian diurnal Butterflies (Kallima) which can scarcely be distinguished

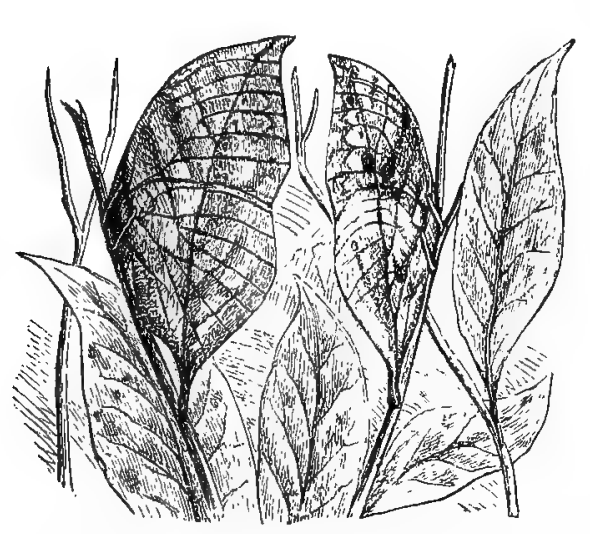

Fig. 43. Two spevimens of a Kallima settled, with closed wings, between withered leaves.-After Wallace.

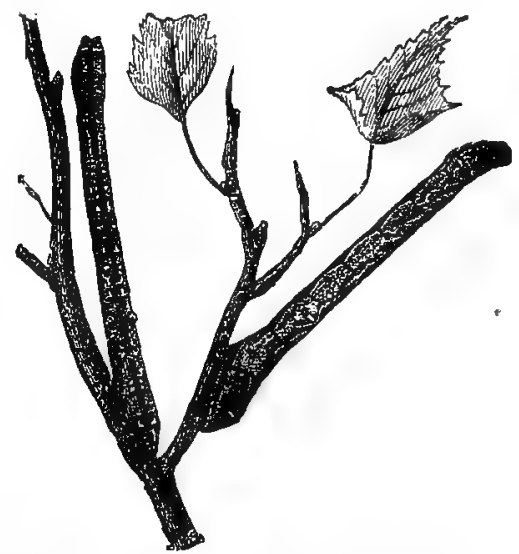

Fig. 44. Two looper caterpillers (Geometra betularia).

from the dry leaves of the trees or shrubs whereon they rest with folded wings. Among European Lepidoptera, a Bombyx (Gastropacha quercifolia) also exhibits a remarkable similarity to dry leaves. Other Insects are like withered twigs, e.g. some Looper-caterpillars (Fig. 44), which extend motionless from a branch, attached only by the hinder end.

It seems specially remarkable that some animals which are protectively modified have come to resemble, externally, others which are from some cause unpleasant to their enemies, and therefore, are comparatively unmolested (mimicry). In South America, for instance, there lives a group of Butterflies (the Heliconidæ), poor fliers, with conspicuous colouring but with so unpleasant a smell, that they are rejected by Birds; certain other Butterties, living with these, but not possessing their odour, are remarkably like 
them in shape and colour and are thereby protected from the attacks of Birds. Not a few moths (Clearwings) of which specimens live in England are, in external appearance, extraordinarily similar to Hymenoptera, which are protected by the possession of stings.

\section{The Power of Resisting Unfavourable Conditions.}

Most cold-blooded animals can remain alive in spite of a considerable $d$ ecrease of temperature, so long as the fluids in the body do not freeze; and in many the water in the tissues does not freeze even if the temperature of the body sinks several degrees below zero. Some animals are so constituted that they revive after being frozen through and through; although as a rule, the injury occasioned is so great that death ensues. In a dry state, some animals can withstand a very great fall of temperature, even to many degrees below zero; for instance, small dried Nematodes have been exposed to a temperature of $-19^{\circ}$, and have revived afterwards. Not a few forms are still active at freezing point; others, however, are exhausted and stupified, and many, long before it has sunk to this. The warmblooded Vertebrates, also, whose temperature under normal conditions is fairly constant, may endure a considerable decrease of heat, but die long before freezing point is reached: Rabbits, whose normal heat is $31^{\circ}$ or $32^{\circ}$ C., die if it sinks to $15^{\circ}$. Hibernation forms an exception, for here the temperature falls to within a few degrees of freezing, without danger to life.

Very often the outer layers of the body are of such a nature that they serve to protect the inner part against cold: fur, in many Mammals; panniculus adiposus (blubber) of Seals and Whales. To escape the winter cold, many animals betake themselves underground (Earthworms), or, if aquatic, to the warmer waters below the surface or to the mud at the bottom.

Warm-blooded animals suffer more from a $\mathrm{r}$ is e in the temperature than from a fall. As soon as there is a rise of a few degrees above the normal they die. Protoplasm coagulates at $40^{\circ}-50^{\circ} \mathrm{C}$., and therefore they cannot endure a higher temperature than this. Possibly dried animals alone form an exception.

When small pools dry up, their inhabitants seem to disappear; but when they fill again with water, the same animals usually reappear very soon. This depends, principally, upon the fact that many eggs have a hard covering which resists desiccation. Many Protozoa also can secrete a similar capsule; more rarely the creature can endure a real drying-up, a withdrawal of much water from the tissues. This has been demonstrated for some forms: a Nematode (Tylenchus tritici) may, after remaining for a long time in a completely desiccated and shrivelled state, revive, when it is put in water again, absorbing it into the tissues; this is true also of many other small Nematodes, and for Rotifers and Tardigrada, 
which live in earth or on plants (moss). Most animals, however, die when water is withdrawn to any considerable extent, whilst many (e.g., Gastropods) sustain no injury from a slight deprivation.

Whilst some animals can scarcely live a day without food, others can endure $\mathrm{hunger}$ for a considerable time without injury: Frogs, snakes, and many others can live several months without food. Sometimes animals can hold out for a very long time if they are supplied with water alone, although they soon die if this cannot be obtained. Many fast periodically; the periods of rest in particular, which so many undergo, are simultaneous with periods of starvation. It has been shown for some Fish (Salmon) that before, or during, the breeding season they take no food for many weeks, and the digestive tract becomes quite shrunken up : the same thing, occurs also in certain other forms. Moreover, many Insects, in the perfect state (which is here the breeding time also), can take no food on account of the rudimentary condition of their mouth-parts. During the period of starvation the body naturally loses in weight, for oxidation of the tissues still continues. Often when such a period approaches, masses of fat are stored up in the body, and are consumed whilst it lasts (Bears, Insect-larvæ before pupation); or reserves may be provided in other ways (crystalline style of Lamellibranchs).

Whilst many animals, if deprived of oxygen, die instantaneously, or after a few minutes, there are others which can dispense with it for a considerable time, evell for many days. Such animals as the Frog, indeed, can live in an atmosphere destitute of oxygen: only after several hours does a certain dulness occur, followed by an apparent death, from which the frog can recover if it has not continued too long. Many Insects, Worms, etc., exhibit a similar peculiarity. Since the production of carbondioxide continues, combustion must be carried on by means of the oxygen stored up in the cells.

\section{The Geographical Distribution of Animals.}

WHEN the great divisions of the surface of the earth are compared with one another, it is found that animal life is possessed of a considerable diversity of character, and this is true for different land faunas (including fresh-water), as well as for marine. The animals which live in South America are different from those inhabiting Europe; the fauna of the East Indian coasts differs from that occurring round Europe, and so on. 
As regards terrestrial and fresh water faunas, it is found that the surface of the earth may be divided into a number of large zoo-geographical regions, each of which harbours a fauna, distinguishing it, in certain respects, from the rest. Of such regions, the following have been established:

1. The Palæarctic region: Europe, Temperate Asia, Africa north of the Atlas Mountains.

2. The Nearctic region Greenland and North America, to North Mexico.

3. The Ethiopian region: Africa south of the Atlas Mountains, Madagascar, South Arabia.

4. The Oriental region: India and Further India, with the adjacent islands.

5. The Australian region: Australia, with some islands which belong, geographically, to Asia.

6. The Neotropical region: South America, the Antilles, South Mexico and Central America.

Each of these regions is distinguished by the possession of a number of animals not occurring in the others, and giving it its special characteristics, which may be more or less definite. The regions are subdivided: the Palæarctic region is separated into four sub-regions: the European, including Europe, with the exception of the South European peninsulas; the Mediterranean, districts around the Mediterranean Sea; the Siberian, the greater part of Northern Asia; the Manchurian, the eastern part of the Chinese Empire, and Japan; each of these divisions is characterized by lesser peculiarities.

This diversity of animal life results from many causes. Temperature plays a great part, and explains, for example, why tropical regions present a richer and more varied fauna than cold countries, and it is further evident that certain groups and species are suited to a warm, others to a cold climate. By no means all the peculiarities of the large areas can be explained in this way: that, for instance, a large number of species occurs in the Palrarctic region, but not in the Nearctic, cannot be ascribed entirely to the influence of temperature or other climatic conditions; for large tracts of both regions agree in these respects ; and many of the animals which are characteristic of the one can flourish abundantly in the other. This experiment has been made with several species, among them the Common Sparrow, which was introduced into North America, and has so increased there that it has become a perfect pest. The same is true also of other regions: with a single exception, the only Mammals in Australia are Monotremes and Marsupials, and the reason for this cannot be that others are unsuited to the country, for large numbers of European animals are established there, and thrive exceedingly; the Rabbit, for instance, has run wild, and is now counted by millions. There must, consequently, be other causes. 
On a closer inquiry into these causes, it is found that large regions are generally separated from one another by natural barriers of different kinds-large seas, high mountains, extensive wildernesses-difficult to surmount. That each region retains its own peculiar fauna, is primarily to be ascribed to the fact that the animals have lived for a long time as a relatively circumscribed group, and during this separation have been modified in on e direction, whilst their relations elsewhere have developed in others. The differentiation of two regions, which were originally continuous and in conformity, but now are separated, has been explained as follows: on the one hand a number of peculiar forms has originated in each; on the other, some of the original forms have survived in one region and become extinct in the other. In this way it is easy to understand, for example, the great faunistic differences of the Neotropical and Ethiopian regions, which present similar physical conditions, but are divided by extensive seas, so that for immensely long periods of time they have probably had no connection. If some of the regions in sharp zoo-geographical contrast are less clearly separated geographically (e.g., India and Australia) it is probably due to their having been more definitely divided at an earlier period. On the other hand, the circumstance that the Palæarctic and the Nearctic regions, for instance, which are now absolutely distinct, exhibit in many points a considerable similaritya number of Mammals is common to both regions, and other types are represented in both by nearly allied species-is plainly explicable, on the ground that in early times, and even relatively late, these regions were more closely connected than they are now. The diversities and similarities of the zoo-geographical regions may consequently be regarded to a great extent, as the result of alterations in the conditions of the earth's surface.

Regions similar to these may also be established for marine animals, especially for littoral forms. They do not, of course, coincide with the terrestrial divisions; the fauna of the east coast of South America, for instance, belongs to one region, that of the west coast to another, and so on. The abysmal fauna, on the other hand, has, for the most part, the same characters in all seas. Many abysmal species have the widest geographical distribution; this is quite intelligible since the physical conditions, the temperature, etc., are relatively uniform, and there seem to be no impassible barriers. This holds also for the pelagic fauna, which, throughout the warm zones, exhibits a very uniform character though in the cold seas, both north and south, it is of a somewhat different type : since other natural barriers are wanting, temperature is here of paramount importance. 


\section{The Geological Distribution of Animals.}

Geology, the history of the evolution of the earth, shows that animal life has existed during immeasurable periods of time; and further that the fauna through these ages has by no means been always the same as at present, but has been subject to a series of extraordinarily great changes. The animal remains of different periods, enclosed in the earth's crust, are the source of this knowledge.

The presence of such traces in the earth's crust is intelligible from the following considerations. In all natural waters, but especially in the sea, there is a constant deposition of finer or coarser particles, which may be either in solution or in suspension in the water: the waves, assisted by atmospheric action, tear off portions of the coast, and the débris is laid down again, the coarser sand or gravel at once, in the shallower places; the very fine, which is set in motion by the slightest ripple, only at greater depths, where the waves do not penetrate. Rivers carry down to the sea great quantities of alluvium, into which sink the dead shells of numberless minute marine organisms: in this way the sand, gravel, or mud of the ocean bed, forms compressed deposits, which, in course of time, often harden into solid masses of shale, limestone or sandstone. The deposits are stratified: the end of one stratum and the beginning of another are important as indicating either an interruption in the sedimentation or the beginning of a different formation. The same thing occurs on a small scale in great inland seas.

Such a formation of strata has taken place from the earliest times, and in so far as animals have lived in water, or have chanced to fall in after death, their remains are imbedded in the deposits. These stores of animal remains would, however, avail little, were they inaccessible; but the great changes which have occurred in the earth's surface in the course of ages and which are always taking place, have put the beds, even of oceans and of inland seas, within reach. Parts of the surface, which in earlier times were covered by the sea, are now, in consequence of elevations, dry land, and therefore may be investigated, and with great facility, for at many places and in many ways natural sections of the strata have been produced, so that remains buried in the bed of the sea at different epochs have been brought to light. The same thing has often occurred in fresh-water beds.

The animal remains which have been found in the earth's strata are, as a rule, grouped under the general term of petrifactions or fossils. The former name is, however, not absolutely correct, for "petrified" remains are only exceptionally found. As a rule, only the hardest of the calcified parts have been preserved; traces of the soft parts occur quite rarely, and then usually as impressions in the stone, which must in these cases be of a very fine grain: impressions of Hydrozoa, for example, are sometimes found in the Bavarian lithographic slate, originally a calcareous silt. The hard parts occurring in the strata are in many cases relatively unaltered; the 
shells of Molluscs, Echinoderms, etc., are often but little changed, so also are the bones of Vertebrata, except that the organic substances disappear, and only calcareous matters remain, so that fossil bones are lighter than fresh ones. In other cases, the bones or shells have been infiltrated with other matters, such as the silica dissolved in the water. For instance, Echinoid shells of the Cretaceous period are found, filled with silicates (flint); or silicated bones, as hard as stone, penetrated throughont with silica. To such cases, the term "petrified" may be correctly applied. Often the hard parts themselves are not found, but merely their impressions in the stone in which they were imbedded. Sometimes the shells, originally filled with silica, have later been dissolved away, so that only the cast remains, a mass of flint with an impression of the interior of the shell on its surface.

It is evident that the conception of the fauna of early times to be formed from the study of fossils (Palæontology), must be very incomplete. Only an extremely small proportion of the animals of a given period are preserved in deposits; by far the greater number disappear without a trace. Of these there are, in the first place, all those which possess no skeleton, so that they vanish entirely with few exceptions. Of the rest, the terrestrial forms are preserved only in favourable cases, for, all those which are left after death, on dry land, soon disappear entirely. Aquatic animals, especially marine forms, with hard structures in or round them, stand, on the other hand, a better chance of being preserved; but of these, by far the greater number vanish, even when they are not devoured by other animals, for by no means every part of the ocean bed is fit for their permanent preservation. As for the parts which, in earlier times, were really imbedded in a suitable place, and so were preserved, many have been lost again, for the strata have been disturbed by volcanic action, for example, which destroys all traces of life. Finally, it must be noticed that only an extremely small proportion of the materials contained in the strata now, is accessible to the investigator: most of it is only too well preserved. All this must be taken into consideration in estimating the importance of the conception of the farna of byegone ages, supplied by the examination of fossils.

Now it is clear, from this inquiry, that animal life has gradually undergone great changes from the oldest times until now; the different periods (see p. 79), into which the history of the earth may be divided, were characterized by different floras and faunas whose remains are preserved in the strata, where they have been ever since. The more remote the time, the more different is the animal (and plant) life from that now existing. The animals found in the older formations may, for the most part, be classified without difficulty to-day, in the phyla and classes arranged for existing forms. 
They belong, however, without exception, to different genera and species, often, also, to families and orders not occurring in this age, and, moreover, large divisions, forming the most conspicuous part of the present fauna, did not then exist. So, for instance, the Vertebrata, from the oldest formation to the Devonian, are represented only by Fish, whilst Amphibia, Reptiles, Birds and Mammals are entirely wanting. From what has been said above it will be seen that these observations must be interpreted with great caution, for the absence of certain animals from a given formation does not necessarily mean that they did not live in that period. This much, however, can be said: had the group been largely represented in that early time, some remains at least would probably have been found. The nearer the period in which the formation was laid down to the present day, the more closely does the fauna resemble that now existing.

This agrees with what must be expected on the theory of descent. An opinion is expressed by the opponents of this theory that, were it correct, the contents of the strata should furnish a far more complete phylogeny: and they say further that there is, even in the oldest fossiliferous formation, the Cambrian, a small fauna, which, though poor, is still far more specialised than the original fauna could be, according to this hypothesis. The first objection is already disposed of by the foregoing remarks, from which it is evident that our knowledge of primæval times must necessarily be extremely incomplete; and as for the second objection, it must be pointed out that the oldest $\mathrm{known}$ animals are not necessarily the earliest to have existed; it is quite possible that they had a long line of unknown ancestors. Geology can show below this formation others still older, which probably were also formed under water, but were so modified (metamorphosed) in course of time that it would not be extraordinary if the then existing fauna, which may have consisted chiefly of soft forms, had left no trace.*

When the appearance, in course of time, of individual groups is observed, a similar impression of the small number of facts against the doctrine of descent, is gained. This is, for example, the case with the classes of the Vertebrata: if a Vertebrate pedigree based on a consideration of the structure of the different Vertebrate groups is designed, Amphioxus being excluded, the following arrangement results. The Pisces are the most primitive and from them arose the Amphibia, from the Amphibia again, the Reptilia, and from these, on one side, Aves, on the other, Mammalia. The story from the earth's strata corresponds closely with this. Fish are the only Vertebrates of the Silurian and Devonian. The earliest Amphibia are found in the Carboniferous. In the great formation following,

* From the nature of some of these old strata it can with certainty be asserted that organisms must have assisted in their formation; this is especially indicated by the presence of chalk, graphite, and anthracite. 
the Permian, are the first Reptiles; in the Trias, the first Mammals; and in the Jurassic, the earliest Birds. A study of special examples gives a similar result. Amongst living Mammalia the Horse, as is well-known, is in some respects, e.g., the structure of the foot, a very peculiar and aberrant form. The horse itself appears late; the true horse, Equus, with a single toe on each foot, as in the living form, first occurs in the upper Pliocene, but its near relative, Hipparion, having the middle toe, and also the second and fourth in a reduced form, lived in the early Miocene and on into the Pliocene. In the lowest strata of the Viocene, before the appearance of Hipparion, a third genus, Anchitherium, is found, more remotely related to the horse, from which the structure of its teeth shows a considerable removal: it has, however, the same toes as Hippari n, but better developed, and in this respect it approaches the genus Palæotherium, of the Eocene. In attempting to reconstruct this phylogeny from anatomical considerations, it is certain that Equus must be derived from Hipparion; Hipparion from Anchitherium; and the latter from Palæotherium, or some nearly related form: and with this the geological succession agrees closely.

A similar genealogy may be drawn up for many other groups, although the imperfect knowledge of extinct forms often renders it impossible.

TABIE OF Fosiliferous Formations.

\begin{tabular}{|c|c|}
\hline Quaternary & Pleistocene. \\
\hline $\begin{array}{l}\text { Tertiary } \\
\text { or } \\
\text { Cainozoic }\end{array}$ & $\begin{array}{l}\text { Pliocene. } \\
\text { Miocene. } \\
\text { Eocene. }\end{array}$ \\
\hline $\begin{array}{c}\text { Secondary } \\
\text { or } \\
\text { Mesozoic }\end{array}$ & $\begin{array}{l}\text { Cretaceous. } \\
\text { Jurassic. } \\
\text { Triassic. }\end{array}$ \\
\hline $\begin{array}{l}\text { Primary } \\
\text { or } \\
\text { Palæozoic }\end{array}$ & $\begin{array}{l}\text { Permian. } \\
\text { Carboniferous. } \\
\text { Devonian. } \\
\text { Silurian. } \\
\text { Cambrian. }\end{array}$ \\
\hline
\end{tabular}




\section{Appendix.}

\section{Resemblances and Differences between Plants and Animals.}

Now that the most important features in the structure of animals in general have been studied, a consideration of the relations of the Animal Kingdom to the other great division of the organic world, the Vegetable Kingdom, may not be out of place.

Both plants and animals are composed of cells; in the simplest forms of a single cell only, but usually of a great number. The cells consist, at least when young, of protoplasm, and generally (perhaps always) in plants as well as animals, contain a nucleus. The protoplasm of plants displays the same essential character's as that of animals (see pp. 1-4); it exhibits the power of movement; it possesses irritability; it feeds, by taking up materials from the environment; it absorbs oxygen and gives off carbon-dioxide. The cells grow, and multiply by fission. Sexual reproduction, i.e., the formation of a new organism from a single cell after ats union with one from another individual of the same species, occurs in most plants as well as in most animals.

In considering the differences between the two kingdoms, the lowest unicellular plants and animals, the Protophyta and the Protozoa will, at first, be disregarded, whilst the Metazoa and the multicellular plants are contrasted.

In general construction there are important differences. Animals, with very few exceptions, possess an alimentary canal, a cavity into which food is taken, and in which it is digested and absorbed; it is specially characteristic of this system that it appears at a very early stage of development. Anything homologous, or even only analogous with, the digestive canal is entirely wanting in plants, which take in their food through the surface in a liquid or gaseous form. This is not, however, an absolute distinction, for an alimentary canal is absent from some animals (e.g., the Tape-worms). Further, a muscular and a nervou s system are wanting in all plants, whilst these organs apparently occur in all Metazoa: sense organs, which in their simplest form, as sensecells, are probably represented in all Metazoa, are never present in plants : a vascularsystem and special excretory organs are also peculiar to the Animal Kingdom, although they do not occur in every member of it. On the whole, it may be said that plants exhibit in internal organisation, only a very slight indication of that specialisation of organs which is, comparatively, so pronounced in animals. The so-called organs of plants are only special regions and appendages of the body.

Quite as significant is the contrast in the tis sues composing the body. In $a \mathrm{nimals}$, the cells, originally undifferentiated, develop in very different ways. Some remain in their primitive condition, others secrete an intercellular substance, varying in structure and chemical composition; in others, the protoplasm becomes modified into a peculiar contractile substance. In plants, the cells are almost always surrounded with cell-walls, consisting of c e $11 \mathrm{u}$ lo se, and the internal differentiation of the body, as far as its tissues are concerned, depends, principally, upon a variation in the form of the cell or upon the thickness, toughness, etc., of the cellulose wall, and less upon modifications of the protoplasm, which always remains protoplasm, unless, as in many adult cells, it has disappeared entirely. 
With regard to foo $d$, there is a wide difference between animals and the great majority of plants. A $\mathrm{n}$ i mals feed upon other organisms, or on organic substances; they cannot build up new tissues, nor repair the wiste occasioned by vital activity, with inorganic materials alone, although, of course, these occur as constituents of the food (water, calcareous salts). Plants, on the other hand, can feed exclusively upon inorganic substances, and in correlation with this, some of their cells are provided with a peculiar green substance, chlorophyll, which enables them, in the presence of light, to separate and assimilate the carbon from the carbon-dioxide in the air. This power is not possessed by animal cells, which are always destitute of chlorophyll*: on the other hand, there are not a few plants (e.g. all the Fungi), from which it is also absent. These cannot, therefore, assimilate carbon from the air, but, like animals, must obtain it from organic substances.

Finally, in contrast to plants, animals possess sensibility and the power of voluntary movement, which seems to be a mark of absolute distinction between plants and the Metazoa.

From the foregoing remarks, there can never be any doubt as to whether a given organism is a multicellular plant or a Metazoon, at all events, if a careful examination has been made; but it is otherwise with the unicellular organisms. They cannot be considered in the light of the differences characteristic of multicellular plants and animals, just enumerated: indeed, it is impossible to speak of alimentary canal, of musculature, of nervous system, or of systems of organs at all, in unicellular animals; for the most part it is: difficult to say whether sensation and voluntary motion are exbibited or not; and the mode of feeding is no absolute test ( $c f$., above). To divide the unicellular organisms between the two kingdonas is, to a certain extent arbitrary, but since chlorophyll is peculiar to plants, and never present in the Metazoa, it is justifiable to regard all organisms containing this colouring matter as plants: on the other hand, the numerous unicellular organisms which are destitute of chlorophyll must not be regarded as animals without some further demonstration : for it is undoubtedly wanting in many plants, and its absence is not therefore adequate proof that the organism is an animal. Further, it must be taken for granted that those organisms with a cellulose cell-wall belong to the vegetable kingdom; whilst, on the other hand, those which exhibit in their protoplasm a differentiation recalling the cell-modification of the Metazoa (the muscle-like protoplasm in the Infusoria), should be classed as. animals. All those, moreover, which ingest solid food are considered to be animals; but for many "animals" even this does not hold, and their location is purely a matter of custom.

* It has been stated that chlorophyll is present in some animals, but this supposed chlorophyll has been proved either to be some other green colouring matter, or to belong to Algr parasitic in the animal. 



\section{S PECIAL PART.}

\section{Systematic View.}

THє Animal Kingdom may be classified as follows:-

\section{Sub-Kingdom I.: Protozoa.}

Unicellular animals: they may be colonial, but then the members of the colony are essentially alike.

\section{Sub-Kingdom II. : Metazoa.}

Multicellular animals: the cells are differentiated (division of abour); alimentary canal, nervous and muscular systems, etc. The Metazoa are subdivided into the following Phyla:-

Phylum 1. Colentera.-Radiate animals of a very simple structure; body saccular, composed of three layers enclosing a digestive cavity. No anus. No body-cavity. The different organs present elsewhere in the Metazoa are but slightly indicated here. -Appendix: Sponges.

Phylum 2. Echinoderma.-Radial symmetry. Body-cavity. Specialised systems of organs. Calcifications in the body-wall. A vascular system. A special water vascular system in connection with tube-feet. Larva bilateral.

Phylum 3. Platyhelminthia. - Bilaterally symmetrical, unsegmented animals, without body-cavity; usually without vascular system or anus. A branched excretory apparatus with peculiar terminations.-Appendix: Rotife ra.

Phylum 4. Nemathelminthia. - Bilaterally symmetrical, unsegmented animals of a cylindrical shape, with body-cavity and anus.

Phylum 5. Annelida.-Bilaterally symmetrical, segmented animals, with relatively similar segments. Limbs, when present, unjointed. Thin cuticle. Body-cavity, vascular system (usually), and anus present. Segmented ventral nerve cords, connected with a pair of ganglia lying above the pharynx. Eyes feeble. A pair of tubular excretory organs in most segments (segmental organs). -Appendix: Polyzoa, Brachiopoda.

Phylum 6. Arthropoda.-Bilaterally symmetrical segmented animals, with variously modified somites. Limbs jointed. Dermal skeleton formed from a well-developed cuticle. Body-cavity. Heart 
on the dorsal side. Nervous system as in Annelida. Highly specialized optic organs (compound eyes). Segmental organs always much reduced in number or entirely wanting.

Phylum 7. Mollusca.-Bilaterally symmetrical unsegmented animals. A ventral muscular foot. A fold of skin, the mantle, covering part of the body. No continuous cuticle. A part of the integument secretes a shell which is closely adherent to the animal at certain points. Body-cavity. Dorsal heart. A pair of ganglia above and beneath the pharynx, no ventral nerve cord. Usually a. lingual ribbon armed with rows of chitinous teeth. Segmental organs reduced in number.

Phylum 8. Vertebrata,-Bilaterally symmetrical animals; certain parts of the body (skeleton, musculature) segmentally arranged. Usually two pairs of limbs, never more. Body-cavity. Ventral heart. Central nervous system in the form of a continuous thick-walled tube along the dorsal side, generally enlarged anteriorly. Beneath this an elongate rod, the chorda dorsalis (notochord), which forms the basis of the usually highly specialised endoskeleton.-Appendix: Tun icata. 


\section{Sub-Kingdom $I$.}

\section{Protozoa.}

IT has already been noticed in the general part that the animals included in the group Protozoa are simple cells, each individual consisting of a single cell only. In certain cases, however, several individuals may be united to form colonies, approaching the metazoan condition, where each individual consists of groups of cells. There is, however, an essential difference between a protozoan colony and a metazoan individual, since the former consists of cells which are identical in the more important respects, whilst the cells of the latter vary in structure and in function, division of labour having occurred.

In all the Protozoa, the body consists of protoplasm with a nucleus; in many there are numerous nuclei. The nucleus is usually spherical or oval, but sometimes more elongate. The protoplasm frequently contains vacuoles, small cavities filled with a fluid; some of these are contractile, i.e., they contract and enlarge alternately as the fluid is expelled or fresh supplies are obtained from the cell substance. Their contraction is, of course, dependent upon protoplasmic movements, since they have no definite walls. Apparently their function is essentially excretory, and in various forms uric acid has been found in their contents. Not infrequently different substances are secreted by the protoplasm, such as oil globules, pigment granules, and so forth. Very often, too, it secretes skeletal structures usually consisting of lime or silica; these will be considered in greater detail under the Rhizopods and Radiolarians.

Many Protozoa, like the Amœba, can protrude pseudopodia from any part of the body, so that its external form is constantly changing. In others this power of amœboid movement is entirely wanting, the outer layer of protoplasm being firmer than the inner softer portion, from which, however, it is often not sharply marked 
off (Infusuria): or the outer layer may constitute a definite and well-defined, although flexible, coat, as in the ectoplasm of Gregarines. In both cases the body is, however, usually capable of considerable change of shape. Where the form is fairly constant, the body is often beset with cilia or flagella, varying in size and number. Almost all the Protozoa are microscopic in size.

Reproduction, like multiplication in all other cells, occurs by fission; the nucleus dividing first and then the protoplasm. Usually the fission is binary, but very often the animal breaks up simultaneously into a large number of small individuals (spores). Sometimes division occurs by a process of gemmation, a small portion being constricted off to form a new individual.

A great many forms, of various divisions, have the power of encysting if the conditions are unfarourable, emerging again when they improve. The body becomes spherical and secretes a membrane of varying thickness over its whole surface, and in this state it is able to survive complete desiccation: thus encystation occurs if the water in which the organism is living begins to dry up: or, again, if it is too stagnant, or when the food is'exhausted, or, after the animal has ingested a large supply of food, when it remains at rest until digestion is completed.

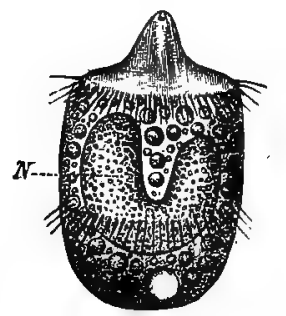

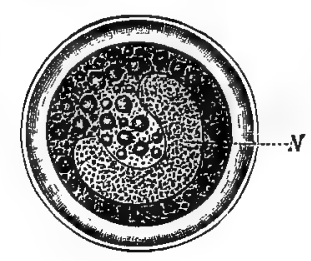

Fig. 45 .

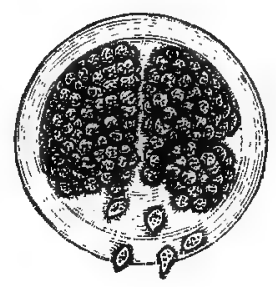

Fig. 46.

Fig 45. An Infusorian in the free state (left), and encysted (right). $N$ nucleus (macronucleus).-After Balbiani.

Fig. 46. An encysted Infusorian which has broken up into a number of spores, now in the act of leaving the cyst.-After Fourquet.

Lastly, it may encyst before undergoing fision. The protoplasm then divides into spores (frequently a very large number), which later on leave the cyst ( $c f$. , the Gregarines; the same thing also occurs in other forms, e.g., in the Infusoria).

In many Protozoa, conjugation may sometimes be observed: two individuals of the same species approach one another and fuse, a fusion of the nuclei also occurring. This conjugation recalls the fertilisation of the Metazoa, which also consists in a fusion of two cells and their nuclei. Very often it is followed by reperited 
division (cf. the Gregarines), just as in the Metazoa, fertilisation is succeeded by active cell division. For the conjugation of the Infusoria, see that group.

Gymnomyxa. . . . . . With pseudopodia.

$\left.\begin{array}{l}\text { Infusoria. Body covered with cilia } \\ \text { Gregarina. Without cilia }\end{array}\right\}$ Without pseudopodia.

\section{Class 1. Gymnomyxa. (Sarcodina.)}

The numerous forms belonging to this class are all possessed of the power of protruding pseudopodia, by means of which they move about, and ingest their food. Most of them are provided with a skeleton, harder portions within or around the protoplasm, varying in form and in chemical composition. They are for the most part marine.

\section{Order 1. Rhizopoda.}

In some Rhizopoda, for instance, in the Amœba, which has already been mentioned, there is no skeleton. Most of them, however, are furnished with a protective structure in the form of a shell, which surrounds the greater part of the body. In the simplest cases the shell is cap-shaped, with a single large opening through which the protoplasm projects. In others it is complicated by being

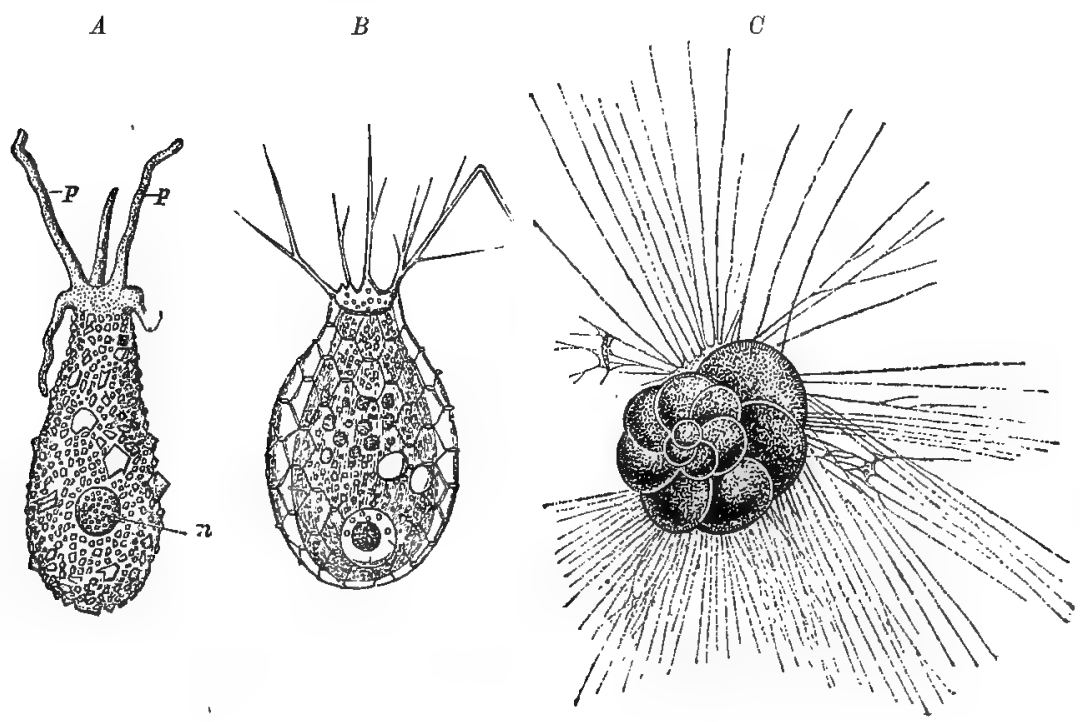

Fig. 47. A (Diflugia) and B (Euglypha), two imperforate fresh-water Rhizopods, with chitinous shells ; in $A$ small foreign bodies are fastened to the shell. $n$ nucleus, $p$ psendopodia. $C$ Marine Rhizopod (Rotalia).- $A$ after Stein, $B$ after Hertwig and Leaser, $C$ after M. Schultze. 
multilocular, i.e., divided by transverse septa into several small cavities, which, however, communicate by small pores in the septa, so that the protoplasm is continuous. Such shells are either straight or spirally coiled, a character which may also obtain in the unilocular shells; and they often closely resemble a nautilus shell in miniature. Both unilocular and multilocular shells may either be perforate, i.e., having besides the larger opening, numerous fine pores, through which the protoplasm may extend; or they way be imperforate, without such apertures. In the Perforata and in some Imperforata the shell lies actually within the protoplasm, for its surface is covered by the overflow of protoplasm. The shells either consist of a chitinous material, to which grains of sand or other foreign bodies are often firmly cemented; or they are composed entirely of calcium carbonate as in most marine forms (the calcareous shell may also be strengthened by the incorporation of foreign bodies).

The protoplasm is usually homogeneous throughout, but sometimes there is a superficial layer which differs from the rest in its hyaline appearance and in the absence of granules, although not sharply separated off from the inner granular portion. Pigment granules frequently occur in the protoplasm; there are often vacuoles, which are as a rule non-contractile. The nucleus is of simple spherical form; occasionally there are several, sometimes many, present. The pseudopodia are either broad lobes (Fig. 1, Fig $47 \mathrm{~A}$ ), or delicate threads which then radiate out in great numbers, and frequently anastomose (Fig. $47 \mathrm{C}$ ) ; these thin pseudopodia are often of considerable length, and may be even as much as ten times as long as the shell. The animal moves by means of its pseudopodia, crawling about at the bottom of the sea, over plants, etc.; by means of them also, it surrounds microscopic organisms or decayed organic fragments, and thus feeds upon them.

Comparatively little is known as to their reproduction. Simple binary fission has been observed in various Rhizopods; in the forms with shells, one of the newly-developed individuals usually constructs a new shell, whilst the other remains in the old one; or both may leave the original shell, and form new ones. Sometimes, also, a large number of smaller individuals may arise within an older organism.

Some of the Rhizopods inhabit freshwater; some are found in moss or damp earth, a few upon dung, a few are parasitic (e.g. Amoeba coli, in the human intestine). The majority, however, are marine, they are usually found crawling about on marine plants, colonial animals, or on the ground, usually at no great depth. A few forms are Pelagic, swimming about in the open sea, often in great numbers; as they die their shells sink to the bottom, where they are met with in the extonsive deposits composed of their remains 
(Globigerina). Similar beds of Rhizopod shells have also been deposited in earlier times, and these, though in a more or less fragmentary condition, form a considerable part of important geological strata (chalk).

A m ce b æ with lobate pseudopodia and without shells (ef., p. 3.5) occur both in salt and fresh water. Several genera. with simple unilocular. chitinons shells (sometimes covered with foreign bodies) are found in fresh water (Fig. $47 A, B$ ). One of the numerous marine shell-bearing forms, which are often extremely fragile, is drawn in Fig. if $\mathrm{C}$. Among the very numerous fossil forms may be mentioned the genus Nummulites (Fig. 48), distinguished by its size, which is, for a Protozoon,

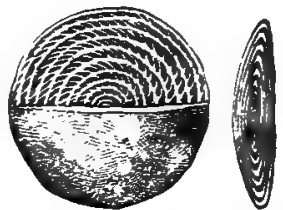

Fig. 48. Summulites distans, Datural size; the figare on the left represents an example which has been longitudinally dissected; that on the right, a transverse section.- - After Archiac and Haime. enormous.

\section{Order :. Radiolaria.}

The Radiolaria differ from the Rhizopoda, in possessing a central capsule, a porons membrane enclosing the greater portion of their protoplasm. Outside the central capsule there is a thin protoplasmic sheath, and outside this again, a thinner or thicker sheath of gelatinous material. The body is typically

$A$

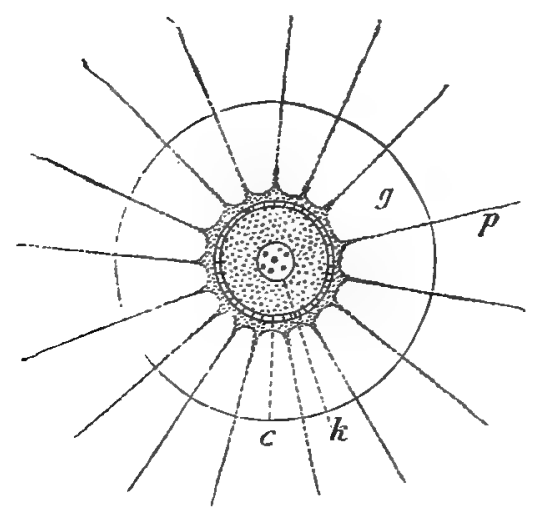

$B$

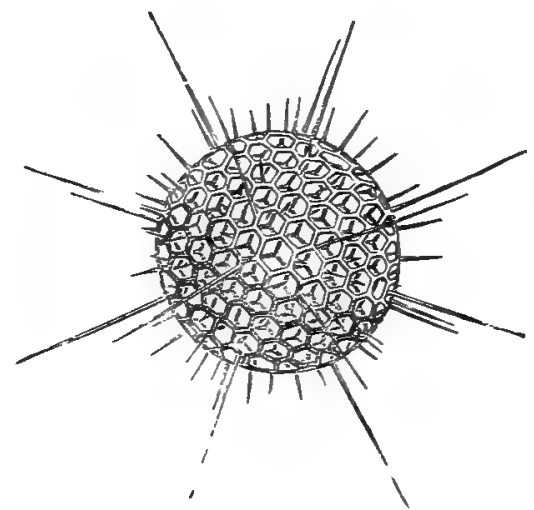

Fig. 49 A. Diagrammatic figure of a Radiolarian from which the skeleton has been r moved. $c$ central capsule, $g$ jelly veil, $k$ noclens, $p$ pseadopodia_-Orig.

Fig. 49 B. Skeleton of a Radiolarian.-After Haeckel.

spherical; but the deviations from this type are numerous. In addition to the capsule, there is usually a well-developed skeleton, which generally consists of silica uccasionally of an organic substance); it varies considerably in the different forms. In some it consists of a number of isolated spines which radiate from the 
centre of the animal, perforate the c'intral capsule and the varions soft layers and project all over the surfence. In others it forms a latticed sphere perforated by many large upertures whilst spius sometimes radiate from it to the surface (lig. $49, B)$. In otheres again, there are several such spheres, enclosed one within the of heiv' and connected by radial spines cressing from one sphere to sturther'; if there are two such spheres one lies within, the other outwide the central capsule; if there are three, the innermost may lio within the central nucleus. In other cases the shell is more discoid or dommshaped; altogether the skeletons of this division offer the prentest variety of beautiful forms.

Within the protoplasm of the central capsule there is a $\mathrm{nu}$ (' le $11 \mathrm{~s}$ (sometimes several); in that extrmal to the capsule there are noncontractile vacuoles; white, red, and yollow oil globules; and red, yellow, and brown pigmerit. J) Jicute pNeudopodia usually radiate on all sides from the thin layen of protoplatwin surrounding the central capsule; they often mastomose. They porforate the transparent jelly veil and project into the water as long filamertis. In the protoplasm external to the capsuld there are numerous $v$ ace uolos which may also occur in the portions of the psourlopodia lying in the jelly veil, wo that the whole of the jelly has a verwirular, foamy appearance.* Food, consisting of unicellular plunts and animals, is caught by the jeseudopodia and drawn inte the protoplasm.

The reproductive processes are imperfectly known. In mome forms the contents of the contral capsule have been observend to break uy into a number of smaller c'ells (ach furnished with trom one to three long flagella, "swarm nprores," whose ultimate fate is unknown; it is only certain that the ceupsule bursts and the spores become free-swimming.t Some Radiolaria, in consequence of repreatesd division, form colonies, the individuals of which are connected by a common jelly veil.

Small yellow cells are frequently found in the Riadioliaria; their existence used often to be cited as onc of a number of points indicating the multicellular structure of this group). Recent rescinsehes have, however, demonstrated that thery are really independent organisms, small Alga, which are parasitic in the Radiolaria, or more correctly, commetisal with them, for the orgmism sec:me rather to profit than' to suffer by their presence, since like othor plants they

- The vacuoles, at least in some of the Radiolaria, contain a watery fluid of a less specific gravity than sea watur. The protoplasm contracts in respons: 1 , surno external stimulus (e.g., the violent movements of the water), sorne of the vacuoles wro burst and the animal sinks; after a storm, therefore, there are no Kadiolaria to bo. found at the surface. They rise again later on, in conserquence of the reappearances of the vacuoles.

+ Sometimes in one and the same central capsule: two kinds of spores arise, lurker and smaller, macro- and micro-spores. 
give out free oxygen which is required by the hosts for respiratory purposes (symbiosis).

The Radiolaria are exclusively $\mathrm{mar}$ in $\mathrm{e}$; they are frequent in the open sea, and live at very various depths, though chiefly near the surface, where, especially in warmer zones, they occur in enormous numbers and in a great variety of forms. Like the shells of the pelagic Rhizopods, the silicious skeletons of the Radiolaria also sink to the bottom, where they form the chief constituent of the extensive deep sea deposits found in some regions.

Fig. 50. Actinosphorium Eichornii. $k$ nucleus, $n$ food par. ticles, $v$ vacuoles. (After K. Hertwig).

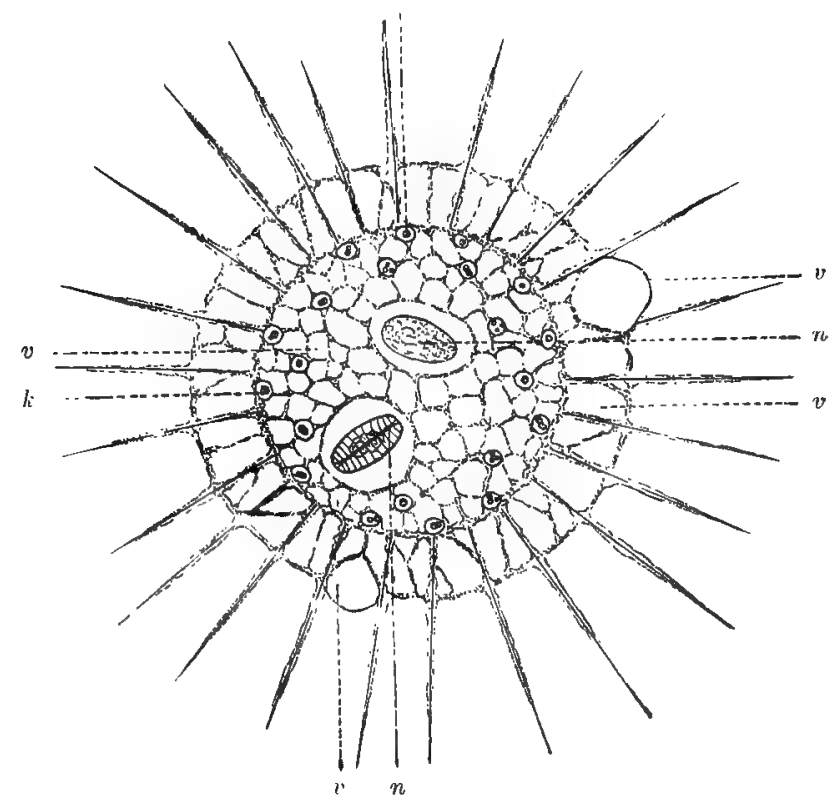

A small group of Protozoa, the Heliozoa is found in fresh water, rarely in the sea. They differ from the Radiolaria in the absence of a central capsule, but in most other respects agree with this group. When a skeleton is present, it is silicious (trellissed spheres or spines); the pseudopodia are radially disposed. Of the freshwater forms, Actinospharvium Eichhornii, which attains the size of a pin's head, may be mentioned. The protoplasm is very vesicular, and contains numerous nuclei. There is no silicious skeleton, but the pseudopodia are supported by firm axial threads of organic material.

\section{Class :. Infusoria (Ciliata).}

The Infusoria possess a thin extermal layer (ectosarc) of a firmer consistency than the rest of the protoplasm (endosarc), and in consequence cannot protrude pseudopodia. The body, which is usually round, oval, or somewhat more elongate, is, however, 
flexible to a certain extent, and may alter in form. The ectosarc is absent from two spots on the surface; one of these serves for the ingestion of food, and is termed the mouth, whilst through the other, the anus, undigested material is ejected. The two openings are usually situated at opposite extremities of the body; the end where the mouth lies is termed anterior, the other posterior. The mouth usually opens at the base of a funnel, which is often fairly deep, whilst the anus is only visible as a slit, when excreta are ejected. The Infusoria are all provided with cilia, which constitute the chief locomotor apparatus. In some forms they are distributed over the whole surface, and are then often arranged in longitudinal rows; in others, some of the cilia are specially developed as spines or hooks, or there may be one or several rows of these between the other cilia; specially common is the presence of a spiral row of powerful cilia, at the anterior end of the body, by means of which the food is driven into the mouth. In others, again, the whole covering, with the exception of this row, or this row and one other, is completely lost.* Hard skeletal structures like those of the Gymnomyxa do not occur here; but some of the Infusoria secrete round the body a gelatinous or membranous shell, beaker-shaped or tubular, into which their protoplasm may be withdrawn: the relation between the animal and its shell is the same as that between a tubicolous worm and its tube. The shell is generally attached to a foreign object, but it is carried about by some marine Infusoria.

In the protoplasm there is a large macronucleus, which may be round, sausage-shaped, ribbon-like, or moniliform (Fig. 52), and one or more smaller micronuclei; rarely there are several macronuclei. Near the surface are the contractile vacuoles, which excrete the water they contain through one or more fine pores, and then take up a fresh supply from the protoplasm. In the outer portion of the protoplasm there are frequently delicate threads of a contractile substance, muscle fibrillæ: fat globules and pigment granules also occur in the endosarc.

The Infusoria reproduce by fission (Fig. 52), which usually occurs at right angles to the long axis, and is thus transverse; it is preceded by a division of both macronucleus and micronucleus.

Whilst permanent conjugation is rarely met with here, a temporary fusion of two individuals, which separate again later, occurs very frequently. They are adherent over a limited area, and during

* In some Infusoria there are the so-called membranellæ, vibrating, laminate structures, each of which is regarded as a short row of fused cilia. The "undulating membranes," long, vibratile ribbons attached by one edge, are also to be regarded as membranellæ, which have arisen from long rows of cilia. 
(and after) the union, a series of changes occurs in the nucleus, with a partial exchange of nuclear material.*

The details of the process are as follows : the macronncleus becomes irregular, breaks into several fragments, and is completely absorbed into the protoplasm. At the same time, the micronucleus divides into several pieces (Fig. 51, 2), which with one exception, disappear (Fig. 51, 3), the remaining portion divides into two parts (Fig. 51 4), one of which migrates into the other animal (Fig. 51, $\therefore$, 6), so that a mutual exchange of micronuclei occurs. The organisms then

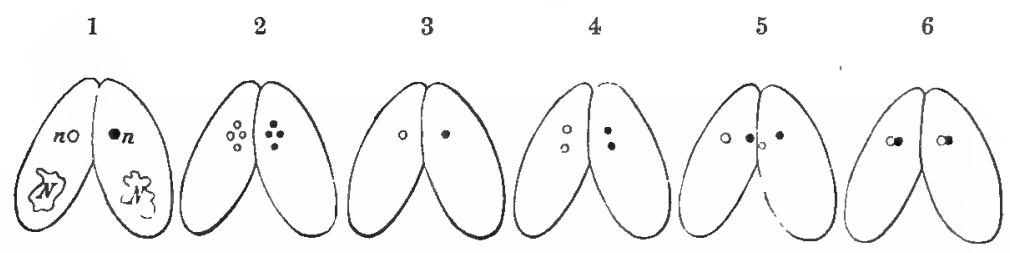

Fig. 51. Two Infusoria in various stages of conjugation. Diagrammatic. $n$ micronuclens, $N$ macronucleus. The micronucleus of the one individual is left clear, that. of the other, dark. See the text.-Orig.

separate. The two micronuclei, one of which is derived from each of the conjugates, fuse, and by the division of the nucleus thus formed, a new macronucleus, and a new micronucleus arise.

The food of the Infusoria consists principally of unicellular plants. and animals-Bacteria, Diatoms, Flagellata, other Infusoria, etc. In many the food is brought to the mouth by ciliary currents, as described above; in others, the edges of the mouth (which are often supported by tiny rod-like structures) effect the prehension of prey. The Infusoria are for the most part extremely active little organisms, swimming by means of their covering of cilia, or by the contractions. of the body: or gliding about over foreign bodies. Not a few are temporarily or permanently fixed, and sereral of these form colonies by incomplete fission or budding. Both as regards individuals and species, they are abundantly represented in freshwater . they congregate principally about decaying plants or animals. A proportionately smaller number occur in the sea; one division of shell-bearing Infusoria is pelagic, its members swimming in the open sea together with Radiolaria and pelagic Rhizopods. Some live as. parasites on the skin of fish or other aquatic animals; and it is by no means rare to find them in the alimentary canal of different Vertebrata. When the ponds in which they live become dried up, great numbers in the encysted condition may be carried away by

* Conjugation usually occurs after the animals have been for some time rapidly increasing by division, and have exhausted the food supply. When there is a constant and abundant supply of nutrition, conjugation does not take place, and fission continues; the consequence of this, however, is the degeneration and finally the death of the individual. 
winds, together with other dust, and thus they appear quickly wherever it is possible for them to exist.

1. Of the innumerable fres $\mathrm{h}$ - wa te r Infusoria, the following common forms may be mentioned: Paramoecium, body oval, covered with uniform rows of cilia Stylonychia, oval, with a series of cilia, anteriorly, leading to the mouth, and

Fig. 52.

Fig. 53.

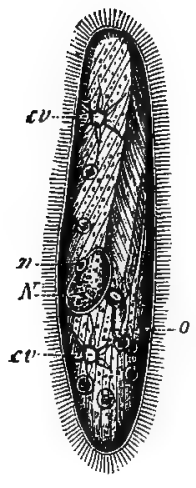

1
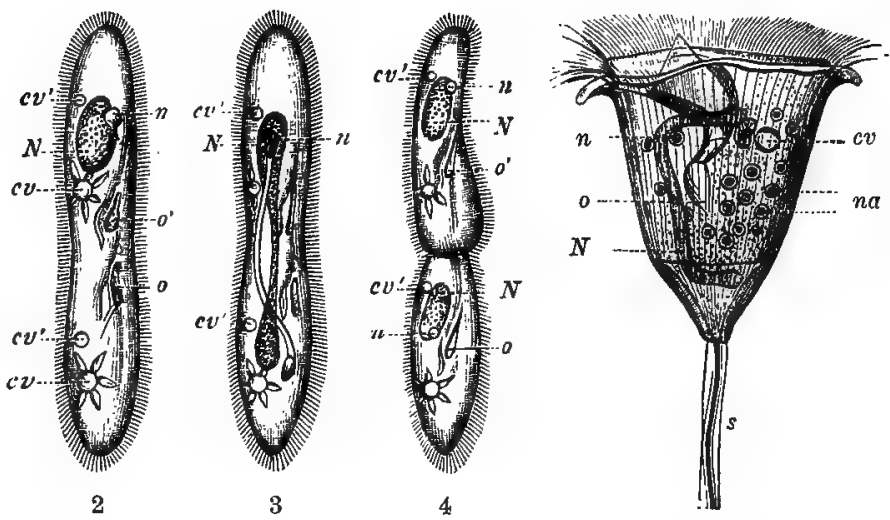

Fig. 52. 1 An Infusorian (Paramacium). 2-4 The same in various stages of division; in 2 the mouth and contractile vacuole are re-duplicated; in 3 the macro- and micro- nuclei are much elongated and constricted, in 4 they have divided, $c v$ contractile vacuole, $c v^{\prime}$ newly-formed vacuole, $o$ mouth, $o^{\prime}$ newly-formed mouth, $N$ macro-, $n$ micronucleus.- After Bütschli.

Fig. 53. Vorticella; lower portion of the stalk not drawn. na food particles, $s$ stalk. Other letters as in Fig. 52.-After Bütschli.

with strong spiny or hook-like cilia ventrally; Vorticella (Bell-animalcule), a stalked Infusorian furnished with spirally arranged cilia at the anterior end, but otherwise naked; mouth and anus situated in a common groove, anteriorly : at the opposite end arises a stalk, through which runs a muscular thread. By means of the stalk, which is frequently very long, the animal attaches itself to various foreign bodies. The Vorticellæ may sometimes break free from their stalks, and become free-swimming.* Many form branched colonies.

2. Many species belonging to the genus Tintinnus, etc., and bearing very beautiful chitinous shells, are sometimes found in great numbers in the open sea.

3. As an example of parasitic Infusoria, Balantidium coli may be mentioned; it is always present in the large intestine of the Pig, and more rarely in Man. The body is egg-shaped, uniformly covered with cilia, but with a somewhat stronger series near the mouth. Other Infusoria occur, e.g., in the paunch of Ruminants, and in the large intestine of the Horse.

* The conjugation of the Vorticellids is interesting. It occurs between a larger sessile individual or macrozoid, and a smaller free-swimming microzoid. The latter attaches itself to the larger one, and conjugation occurs in the ordinary way; after the exchange of the micronucleus, however, the protoplasm of the microzoid is absorbed into that of the macrozoid, and the empty cuticle of the former drops off. 
Under the term Flagellata is included a large number of diverse unicellular animals, which are all, characterised by the possession of a single powerful flagellum. These organisms do not, however, constitute a natural division: some of them seem to be most elosely allied to the Infusoria, and in any case undoubtedly belong to the Animal Kingdom. Others are certainly vegetable organisms, provided with chlorophyll (cf. p. 81), whilst yet others are of very doubtful position. Among the Flagellates possessing animal characters may be mentioned the Monadinidæ, small organisms provided with one or several flagella at the anterior end, occurring in vast numbers in decaying substances and in the alimentary canal of various animals; some

$A$

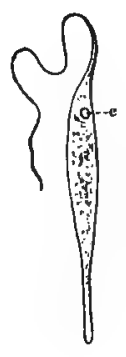

$B$

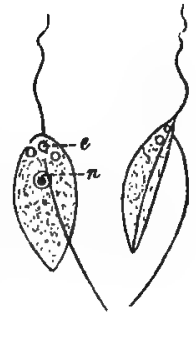

C

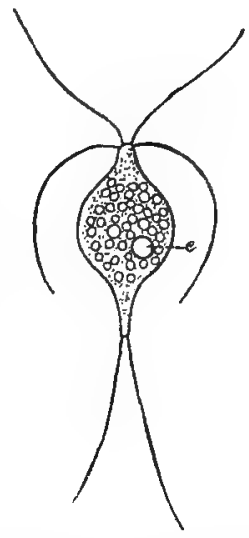

Fig. 54. Varions Monadidæ. A Cercomonas muscee (from the dnodenum of the honse-fly). $B$ Bodo ovatus, $C$ Hexamita rostrata. c contractile vacuole, $n$ nuoleus.-After Stein. species are invariably pre-

sent in the stomach of the Ruminants, the cæcum of the Pig, the rectum of Frogs and Toads; whilst some have been found in the alimentary canal of Man.

\section{Class 3. Gregarinida.}

The Gregarines which are, without exception, parasitic, are, like the Infusoria, unable to protrude pseudopodia, but are distinguished from these, amongst other things, by the absence of cilia. The unicellular body is usually, although not always, surrounded by a definite coat: the protoplasm is usually divided into two layers, the inner granular and the outer clearer, not, however, sharply
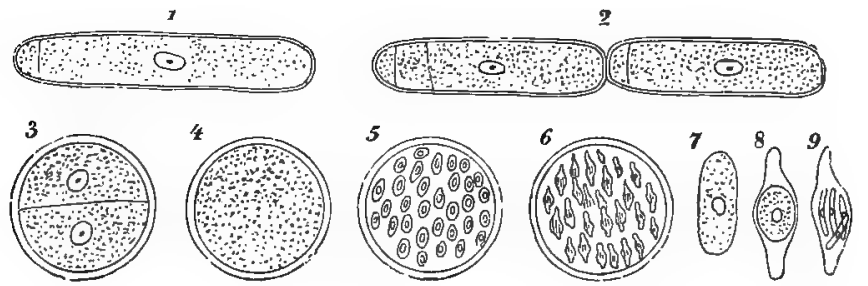

Fig. 55. Diagrammatic figure of a Gregarine. 1 a gingle individual, 2 two conjugating individuals, 3 two such encysted, 4 the same, completely fused, 5 they have divided into spores, 6 within the epores falciform young are formed, $7-9$ a spore at various stages of development strongly magnified.-Orig. 
separated. There is almost invariably only a single round $\mathrm{nucleus.}$ The Gregarines are mostly elongate ; in many the cell is divided into an anterior smaller, and a posterior larger, section (the nucleus being in the latter) separated by a thin septum; in others, on the contrary, the cell is quite simple. Sometimes at the anterior end, there is a proboscis-like process which is often armed with hooks, and forms an organ for attachment to the intestinal wall of the host. The Gregarines move about within the host by contraction, expansion, or flexure of the body, movements dependent on the streaming of the protoplasm. Food is absorbed over the whole surface by endosmosis, but no solid material is taken in.

Reproduction is very characteristic. It begins by the rounding of the body and the secretion of a cyst; sometimes the actual firm wall of the cyst is surrounded by a gelatinous envelope. This encystation is usually preceded by the conjugation of two individuals, which come into connection without, however, fusing at first; true fusion only occurs after encysting. A single individual may, however, also encyst. The contents of the cyst divide into a varying number of smaller cells, spores, each of which becomes surrounded with a separate coat. Lastly, the contents of each spore divide to form a small number of most minute cells, usually of elongate form, the falciform young (pseudonavicellæ). These are liberated by the bursting of the coat; their further development has not been followed, but it is probable that each develops into a Gregarine. In this group, therefore, reproduction consists essentially in a repeated division of the original cell, usually preceded by conjugation.

The Gregarines occur as parasites in a large number of Metazoa belonging to the most widely separated groups: Echinoderms, Flatworms, Chætopods, Arthropods, Molluscs, Vertebrates; the Myriapoda and Insecta may be mentioned as groups which are specially liable to infection. They occur both in the various cavities of the body (e.g., in the alimentary canal) and also in the tissues.

Of numerous forms recorded the following may be cited as examples :

1. Porospora gigantea, very common in the alimentary canal of the Lobster, a very long narrow Gregarine, which attains the length (enormous for a Protozoon) of $16 \mathrm{~m} / \mathrm{m}$.

2. Coccidium oviforme, small $(.035 \mathrm{~m} / \mathrm{m}$. long), egg-shaped forms which frequently occur in the bile ducts of the Rabbit, occasionally in Man (where they may cause fatal disease). Whilst young, the Coccidia are naked cells, but later encyst. In this condition they resemble the eggs of certain parasitic worms for which they have been sometimes mistaken. After encysting they pass into the intestine with the bile, and thence escape with the excreta. Development then proceeds to a further stage: the contents of the cyst divide into four spores, within each of which two falciform young develop. If such a spore-containing coccidian cyst is taken in by a rabbit with its 
food, the cyst wall apparently dissolves and the young wander through the bile ducts into the liver and there develop into Coccidia.

3. Embedded in the transverse muscle fibres of many Mammalia, e.g., very frequently in the Pig, there occur small bodies, the so-called $R$ ainey's or Miescher's corpuseles (Sarcocystis). They are cylindrical or spindle-shaped bodies, varying in length (up to several $\mathrm{m} / \mathrm{m}$.), each enclosed in a single musclefibre. Eachis surrounded by a coat, and consists of a

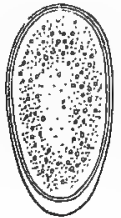

A

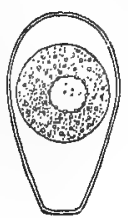

$\mathrm{B}$

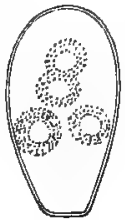

C

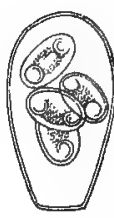

D
Fig. 56. Coccidium oviforme, $A-B$ encysted, $C-D$ formation of spores and falciform young.After Leuckart. protoplasmic mass containing a large number of small falciform young; the latter are arranged in groups. each surrounded by a thin membrane. Rainey's sacs are now usually regarded as organisms allied to the Gregarines; the groups of falciform young are considered comparable to the spores of a Gregarine. If this comparison prove correct it would still be a peculiarity of these organisms that the formation of the spores and falciform young begins very early, before the growth of the sac is complete (the falciform young occurring even in very small forms), and goes on quite gradually, so that fresh portions of protoplasm are constantly forming spores and falciform young. The method of infection is unknown: they do not usually appear to be very injurious; and so far they have not been found in Man.
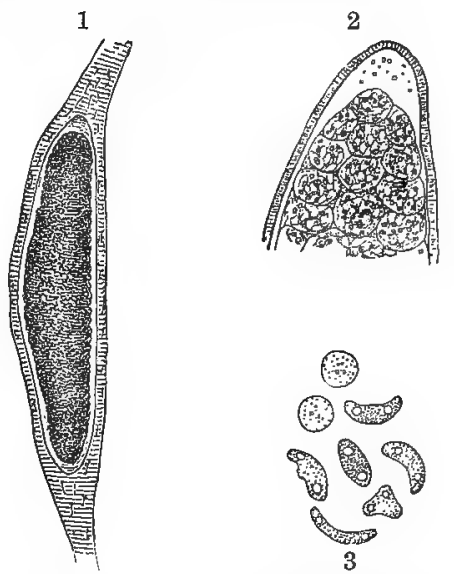

Fig. 57. 1 Raineyian sac in a muscle fibre, 2 the tip of one highly magnified, 3 various falciform young.-After Leuckart. 


\section{Sub-Kingdom II.}

\section{Metazoa.}

\section{Phylum 1. Cœlentera.}

T표 Cœlentera are chiefly distinguished by the extreme simplicity of their structure, and by the slight degree of differentiation in their organisation. They occupy a more primitive position than any other Metazoa known.

Various types of Cœlentera are met with. In the simplest cas $\theta$, the body consists of a longer or shorter sac, one end of which

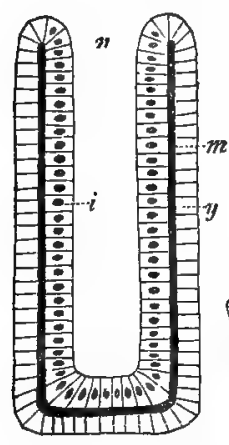

A

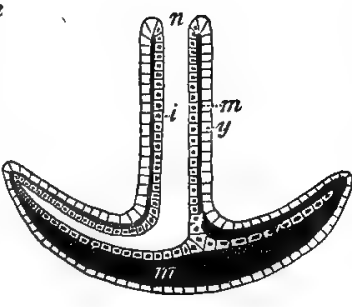

$B$

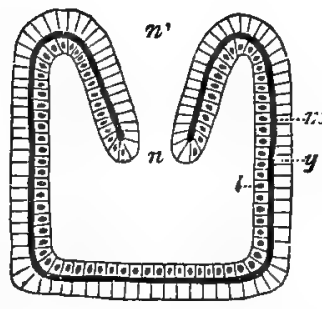

o

Fig. 58. Diagrammatic figures of the chief types of Ccelentera. $A$ the simplest form, $B$ medusa, $C$ coral type. $i$ endoderm, $m$ mesoglæa, $y$ ectoderm, $n$ mouth, $n^{\prime}$ external aperture of the stomadrum of a coral polyp.-Orig.

is open, the other closed (Fig. $58 \mathrm{~A}$ ). The body-wall consists of an external layer of cells, the ectoderm; within this a thin sheath of structureless jelly, the mesoglæa, and most internally another cellular layer, the end od erm, lining the cavity of the sac ; the ectoderm and endoderm are continuous at the open end of the tube, the mouth. It is evident, therefore, that such a Coelenterate differs but little from a gastrula, the difference 
consisting in the presence of a gelatinous layer between the inner and outer layers of cells, the two latter corresponding with the hypoblast and epiblast of the gastrula. In addition to this, however, as will be seen more fully later on, the cells of each layer are not all similar as in the gastrula, but have been developed in different ways, some as muscle cells, others as nerve cells, etc.

Allied to this simpler form, and capable of being derived from it are other types of somewhat more complicated structure. Very often (Fig. 58, B) the lower closed end of the sac is broadened out to form a convex disc, so that the animal resembles an old-fashioned candlestick. The discoid portion consists of the same layers as the rest of the body; the mesoglæa is, however, especially well developed on the convex side, and the two laminæ of the endoderm, which cover respectively the upper and the lower walls of the cavity in this portion, are fused in certain regions, so that instead of a flat simple cavity there is a regular system of canals; at the lines of fusion the endoderm shrinks to a thin membrane. Whilst Coelentera of the first and simplest type are usually sessile, those just described, the medusoid forms, are generally free-swimming; the disc is turned upwards in swimming, the opening of the sac downwards.

A third type occurs in the coral polyps (Fig. 58, C). Here the cavity of the sac is very wide, and its upper portion is invaginated, so that the true mouth is situated at the base of the turned in portion or stomodæum.

The Colentera are usually provided with soft appendages, the tentacles, which are outgrowths or evaginations of the body-wall and consist of the same layers; they are usually found near the mouth, but in the medusæ are carried out on to the edge of the disc.

In the whole structure of the body, e.g., in the arrangement of the appendages just mentioned, and in the distribution of the canals in the disc of medusæ, a more or less sharply marked radial symmetry is evinced, such that the chief axis, about which the radial structures are arranged, corresponds with the central axis of the sac. The number of rays differs in the various C'œlenterate groups; most frequently the body is divided into four, or a multiple of four rays, in others into six, or a multiple of six.

The ectoderm, which forms the outer covering of the body (analogous with the epidermis of Metazoa), is a peculiar epithelial layer, the cells of which are developed in very various ways. Some are simple epithelial cells, either flat or cylindrical, and provided with one or more cilia; such cells are quite comparable with the epidermal cells of other Metazoa, and on this account they are termed true epidermal cells. Others, like these and occurring among them, differ in that their inner ends are modified to form contractile fibrils, so that their outer protoplasmic portions perform the function of epithelial cells, their inner portions of muscle 
fibres; such cells, which only occur among Cœlentera, are termed epithelio-muscle cells.* They may, however, lose their close connection with the rest of the epithelium, and may lie within this as simple $\mathrm{muscle}$ cells; then the non-contractile portion of the protoplasm, together with the nucleus, lies as in the muscle cells of many other animals on one side (the outer) of the contractile fibril. Further between the true epidermal cells, or

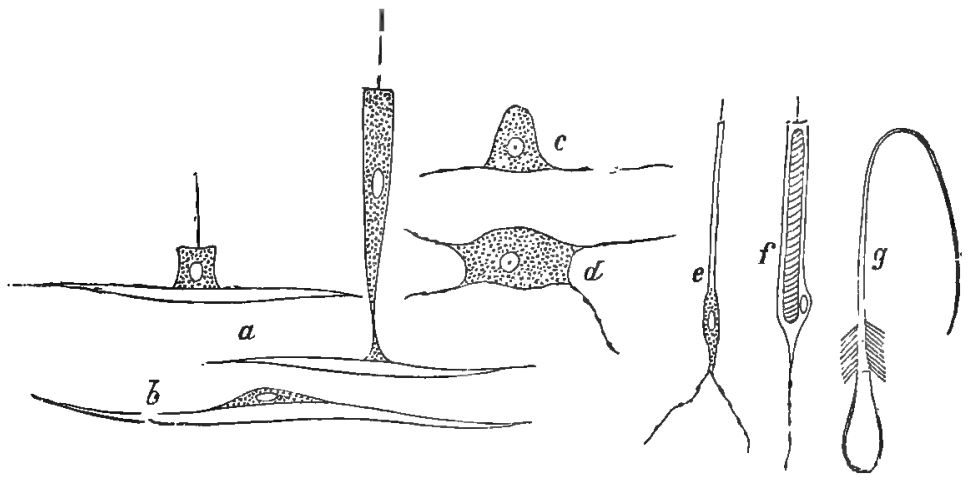

Fig. 59. Cells of a Coelenterate (Actinian). $a$ epithelio-muscle cell, $b$ muscle cell, $c-\lambda$ ganglion cells, $e$ sensory cells, $f$ nettle cell, $g$ nematocyst with everted head.-Partly after Hertwig, partly Orig.

between the epithelio-muscle cells, s en s or y cells may be present. Each of these bears at its outer free end, a single delicate sensory hair, whilst its inner end is produced into one or several fine threads. Between the inner ends of the other cells, or entirely below these, lie certain cells which from their general appearance must be regarded as ganglion cells; from each, several delicate fibrils run out in various directions, and ramify among the similar fibrils of the sensory cells. In the ectoderm, there are, moreover, a number of nettle cells, each of which bears a fine hair at its outer end, and encloses a so-called $\mathrm{n}$ e $\mathrm{m}$ at oc yst, a tiny bladder-like structure, containing a coiled thread, which under certain conditions, notably if the animal is irritated, can be shot out with great force; within the threads is an irritative fluid, which may cause a burning sensation in the skin of man, more powerful in some forms than in others, whilst it paralyses or kills smaller animals. Lastly, there are beaker-shaped cells which secrete the mucus with which these forms are so often covered ( $\mathrm{gland}$ cells). It must be noticed, further, that the cells which have been described as constituting the ectoderm, are not uniformly distributed over the whole body, but on the contrary, occur very unequally in different regions;

* Quite recently it has been stated that epithelio-muscle cells occur also in. Echinoderma and some Chotopoda. 
some portions being much richer in muscle cells, ganglion cells, etc., than others. It may also be mentioned here that, in some Coelentera, part of the body is surrounded by a cuticle, secreted by the ectoderm; further, certain portions of the ectoderm may be modified to form simple optic or auditory organs.

The endoderm, corresponding with the epithelium lining the alimentary canal of other Metazoa, and originating from the endoderm of the gastrula, is, structurally, very like the ectoderm, since besides simple epithelial cells, epithelio-muscle cells, muscle cells, sensory cells, ganglion cells, thread cells, and gland cells may also be present. As regards details, e.g., the form of the cells, the differences between the two layers are by no.means insignificant.*

The mesoglæa in some forms is a thin structureless sheath without cells. In others it is better developed, and cells from the ectoderm and endoderm migrate into it, so that it becomes like a connective tissue. Occasionally, in the Ctenophora, some of the cells which have wandered in, develop into muscle and ganglion cells. In the mesoglæa of some Cœlentera hard structures may develop, but these will be described later.

$\mathrm{Ora}$ and spermatozoa develop in the ectoderm in some cases, in the endoderm in others, arising as the modification of the ordinary cells of the layer. In general they are developed in definite regions of the body, which may then be termed, by analogy, ovaries and testes, but in some forms they are irregularly distributed.

From the foregoing description it will be seen that there is extraordinary simplicity of structure among the Colentera. For the most part the animal consists throughout life of the two layers of the gastrula, the only further development being the various modifications of the constituent cells. An actual mesoblast, arising in other Metazoa as a special mass or masses of cells from which large portions of the body are developed, is absent. The muscle elements and the sexual cells (ova and spermatozoa), which are elsewhere formed from the mesoblast, develop here from the ectoderm and endoderm. For the most part it is almost impossible to speak of organs as in other Metazoa; in any case there is very little specialisation. Thus, a central nervous system which, even in very simple Metazoa, is usually well-developed, can hardly be distinguished; at most, the ganglion cells are more closely collected in some places than in others. Excretory, vascular, and respiratory organs there are none; and a body-cavity is never developed.

* In the inner layer of various Colentera (Actinia, Medusæ, Hydroids), great numbers of green or yellow cells are frequently found, each surrounded by a definite cellulose cell-wall. These were formerly regarded as a part of the animal, but as a matter of fact they are unicellular plants (Algr), which have taken up their abode within the organism ( $c f$. Radiolaria). 
On the other hand, reproduction is often fairly complex. A metamorphosis is very general, the animal leaving the egg as a very simple organism, ciliated and free-swimming, but without tentacles; whilst later, usually after it has become sessile, it is modified to a greater or less extent. Moreover, a sexual reprod u ction by fission or budding often attains considerable importance; and colonies are frequently formed. In many species a regular alternation of generations occurs.

The Colentera are almost all marine, only quite a few inhabit fresh water. Their nettle cells enable them to prey upon even relatively large and powerful animals, which are digested by the endoderm cells of the gastric cavity, the indigestible portions being thrown out through the mouth.

\section{Class 1. Hydrozoa.}

This group is characterised by the general occurrence of an alternation of generations, and by the great dissimilarity of the sexual and asexual generations.

The asexual generation (the polypoid individuals), presents the simplest coelenterate form, which consists of a simple sac with the mouth at one end and the body-wall of the usual layers ( $c f$. Fig. 58, $A$ ); at the upper end of the animal there is a varying number of tentacles, which are usually arranged some little distance from the centre of the disc. The polyps are usually attached (often immovably) by their free ends to some foreign object. Most of them form colonies by budding.

The sexual generation, the medusoid individuals, are characterised by the broadening out of that portion of the body which corresponds to the lower end of the polyp, to form a circular convex disc, the umbrella (cf. Fig. $58, B$ ) in which the middle layer is specially well-developed on the convex side; radial processes of the alimentary canal extend into the disc as the radial canals, the ends of which are generally united by a ring canal running round the umbrella close to its edge. The medusa is typically freeswimming (for exceptions see the Hydromedusæ and Siphonophora) with. the disc turned upwards; from the centre of the umbrella depends a tubular portion corresponding to the upper extremity of the polyp, and forming a longer or shorter manubrium perforated by the mouth. From the edge of the bell hang contractile marginal tentacles which are richly supplied with nettle cells; simple auditory and optic organs are present on the margin, and below the epidermis (at any rate, in the Hydromedusæ), numerous nerve cells are distributed all round the disc, forming a $n$ erve $r i n g$ with their processes. On the concave lower side, there is a layer of 
circular muscle fibres, often tranversely striped, which make the umbrella extremely concave when they contract, and thus move the whole animal. As a rule, the medusæ are of separate sexes; the formation of ova and spermatozoa will be considered in the individual orders.

A ciliated larva develops from the fertilised egg, fixes itself, and develops tentacles; polyps which have originated in this way may form colonies, but some remain solitary. The medusoid form arises again as a bud from the polyp, or by its transverse fission. More rarely the egg of the medusa develops direct into a new medusa, in which case the polypoid form (and with it, alternation of generations) does not occur.

In structure, the Hydrozoa generally display a markedly radial symmetry; the number of rays is usually four or some multiple of four, more rarely six.

\section{Order 1. Hydromedusæ. (Craspedota).}

The polyp generation consists of hydroids, which usually form colonies, but are occasionally solitary. The tubular, often extremely elongated, body of the polyp is almost always surrounded by a cuticle, which forms a chitinous case, usually thin, more rarely thick and calcified. This does not surround the whole body; a portion of the upper part remains uncovered. Sometimes the tube has a cup-shaped expansion above, into which the naked, broader, tentacle-bearing portion of the polyp can be withdrawn. The tentacles are arranged in one or more circles below the mouth, or more irregularly at the upper end of the animal (Fig. 60.) They are not generally hollow as in Corals, but possess a solid axis formed of a single row of endoderm cells; outside this there is a continuation of mesoglæa, and most externally ectoderm with abundant nettle cells. The colonies formed by the polyps are generally small compared with those of the corals ( $c f$. below), of which the solitary individuals are similarly very much larger. Sometimes the colonies are formed on a definite system of branching: the polyp developed from the egg continually increases in length, whilst at the same time it forms lateral buds, of which the oldest are at the base; these develop into new individuals (lateral polyps), which again branch in the same way as the main stem. In other cases the growth of the first polyp ceases; only one or two lateral branches arise from it, each of which stops growing after forming one or two buds ( $c f$. the cymose branch-system of plants). The lower end of the colony is provided with hollow, root-like outgrowths of the body-wall, covered by the cuticle; by means of these stolons, which are sometimes connected to form a 
network, occasionally giring rise to several stems, the colonies may be closely attached to the substratum.

On account of the feeble skeleton (cuticle) the colonies are frequently supported by various bodies occurring in the water, winding round or creeping over them.

Very frequently the polyps of one colony are not all alike; for instance, those persons which bear the medusa-buds are often somewhat different from the rest, having smaller tentacles or none

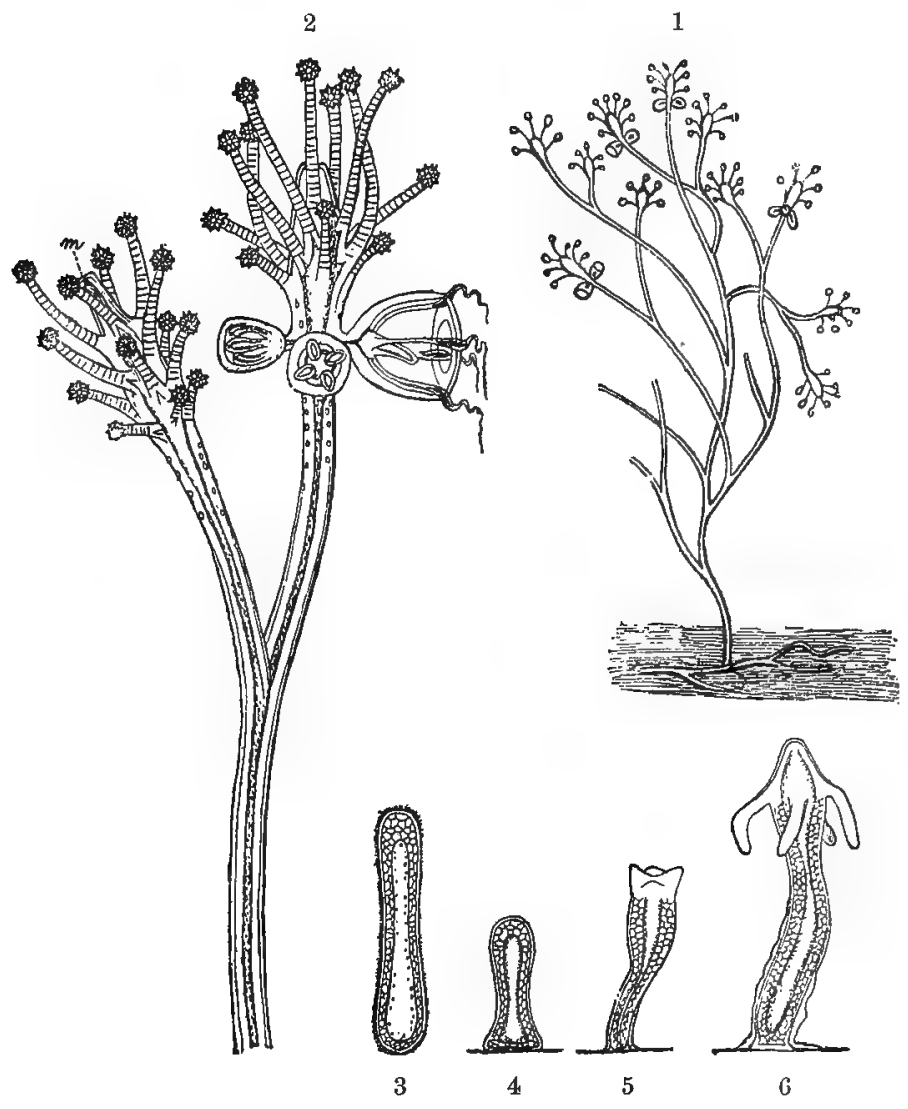

Fig. 60. $1 \mathrm{Hydroid}$ colony (Syncoryne fructicosa). Natural size. 2 two polyps of the same, one with medusoid bods, one of which is about to break free. 3 larva of another hydroid (Cordylophora lacustris). 4-6 the same after attachment.-After Allman.

at all, or even possessing no mouth: in this case the cuticle covers the whole body, and certain individuals (nutritive polyps), which do not produce such buds, perform the function of nutrition for the whole colony.

In some Hydroids there is yet a third kind of individual, thin, mouthless, and very short, and bearing tentacles provided with a great number of thread- 
cells. If the colony be disturbed, these individuals roll themselves up in a spiral fashion and then uncoil again. The tentacles are absent from others, which seem then to function only as the tactile apparatus, whilst in the former case they may perhaps be regarded as defensive polyps (dactylozooids),

The medusoid generation formed by budding from the polypoid generation, usually attains only a small size in this Order. Round the edge of the disc runs a thin collar-like, horizontal inturned ledge, the velum, which narrows the opening of the bell (hence the name Craspedotal.* The edge of the umbrella is entire and bears the naked sense organs, either auditory or optic (rarely
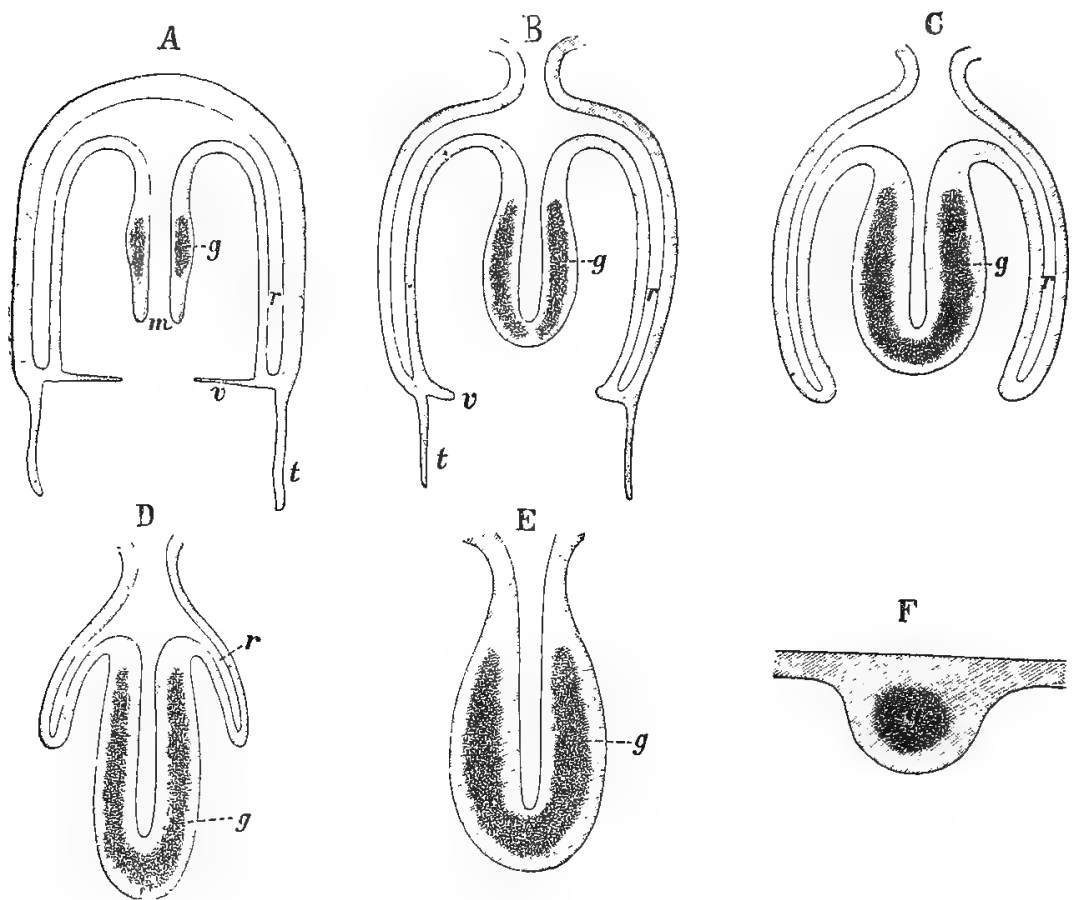

Fig. 61. Vaxious forms of the sexual generation of Hydromedusoids. Diagrammatic longitudinal sections. $A$ free living medusa. $B$ sessile, relatively little modified medusa. $C-D$ more modified forms. $E-F$ the most degenerate form. $E$ without umbrella. $F$ a simple knob-like outgrowth from the polyp, $g$ sexual cells, $m$ mouth, $r$ radial canal, $t$ tentacle, $n$ velum.-Orig.

both in the same individual). There is usually a small number of simple radial canals $(4-8)$ in the umbrella, connected by a marginal ring canal. Ora and spermatozoa develop in these canals, or on the outer wall of the manubrium; they arise in some forms from the ectoderm, in others from the endoderm; in some species, the ova appear in the endoderm, the spermatozoa in the ectoderm, or vice versâ. 
The sexual generation, however, is by no means invariably so well developed. In very many Hydromedusæ, the medusoid buds do not separate from the hydroids, but remain firmly attached. Such sessile medusæ (Fig. 61) remain in a more or less incomplete condition; in some cases, an umbrella with marginal tentacles develops, but the tentacles are small; in others, the latter are altogether absent, although the umbrella is well developed; in others, again, the umbrella itself is degenerate; and lastly, it also is wanting, so that the medusa consists simply of a mouthless manubrium with, in the most degenerate forms, no cavity (Fig. 61, $F$; $H y d r a)$. Without knowledge of the various transitional stages, these small medusoid buds, which are never more than incipient, would be regarded simply as organs of the polyp. The medusoid bud, like the free-swimming medusa, always contains the sexual cells, ova or spermatozoa, and new polyps or colonies arise from these.

In the Hydromedusæ an alternation of generations as just described, generally occurs; there are, however, not a few exceptions. In various forms the ova do not develop into polyps, but give rise directly to medusæ ; from these a hydroid generation is altogether absent. Other deviations from the typical mode of reproduction may be noticed, such, for instance, as the occurrence, in some forms, of an asexual multiplication of the medusæ, which produce other medusa like themselves, as buds from the manubrium or the margin of the disc. These species also possess a hydroid generation, and the medusæ formed by budding propagate sexually.

The majority of Hydromedusæ are marine, numerous examples occurring in the northern seas. A considerable number both of hydriform and medusiform persons are phosphorescent. Quite a few, of which the genus Bydra is the best known, occur in fresh water.

1. Of the marine forms the Milleporidæ deserve special consideration. It has been mentioned above that stolons, surrounded by a continuation of the chitinous covering of the polyp. usually arise from the lower end of the hydroid stock, and often anastomose to form networks. These are sometimes of considerable extent, and gives rise to a large number of small polypoid stocks or isolated polyps. In the genus Millepora and allied forms the chitinous case is calcified, and since new stolons are constantly being formed above the older ones, the soft parts of which die away, these animals build regular coral-like colonies, sometimes of considerable size. The outer layer is made up of living stolons, from which the polyps arise, whilst the inner portion of the coral consists of the calcified walls of dead stolons. The Milleporidæ, which occur exclusively in warm seas, play a not unimportant part in the formation of coral reefs.

2. The Fresh-water Polyp (Hydra), a small, elongate, solitary form, without a chitinous cuticle; round the mouth, is a circlet of long tentacles (4-10), which, like the whole body, contract vigorously when irritated. The animal often remains for a long time in one place, e.g., with the lower end attached just below the surface of the water; but it is able to crawl about like a leech. If an animalcule comesin to the vicinity of an attached Hydra, it is seized by the tentacles, paralysed by the nettle-cells, and conveyed to the mouth. Hydra has 
the power of budding, but never forms a colony, since the new individuals soon separate from the parent. The medusoid generation is represented by wart-like outgrowths from the body-wall in which ova or spermatozoa develop. Hydra is noted for its enormous power of regeneration; an individual may be cut into several pieces, each of which can develop into a complete animal.

\section{Order 2. Siphonophora.}

The Siphonophora are closely allied to the Hydromedusæ, differing from them, primarily, in that the colonies are free-swimming. The colonies correspond with those of the Hydroids, and like them consist of variously modified polyps. Further, they also bear medusæor medusa-buds, which again may derelop in various ways. These diverse individuals are borne upon a common stem (hydrosoma), which is usually either a long tube or a flat disc; at the upper end it is furnished with an air sac (pneumatocyst), or if it is discoid, it encloses several small air spaces, which enable the colony to float. The hydrosoma is to be regarded either as a much elongated, or a much flattened polyp; of which the air sacs are invaginations, each communicating by a fine aperture with the exterior.

The polypoid generation occurs in the following principal forms:-1. Nutritive persons (gastrozooids), saccular polyps, with a mouth, and with only a single tentacle* arising close to its base; which, however, attains a considerable length, and is provided with lateral branches and numerous "batteries" of nettle cells; this tentacle may be absent. The gastrozooids of the same stock may sometimes vary considerably in size. 2. Feelers (hydrocysts), with tentacles like the gastrozooids, but without a mouth. 3. Tentacle-like persons (dactylozooids), which arise independently from the stem, and must not be confused with tentacles; they have no mouth and are furnished with nettle cells.†

The medusoid generation arises either from the hydrosoma of the colony at the base of the hydrocysts, or of the gastrozooids; it appears in the following forms: (1) Fertile medusæ (gonozooids), or medusoid buds with sexual cells, corresponding exactly to the medusoid generation of the Hydromedusæ; if the medusæ are set free-a rare occurrence among the Siphonophora-they look exactly like the free Medusæ of the Hydromedusæ (they are provided with a velum, etc.); as a rule however, they remain in situ throughout life, and then resemble the sessile medusæ of the

* In certain Hydroids also each polyp possesses only a single tentacle.

+ Such tentacle-like individuals occur at the margin of the discoid stem in the genus Porpita (Fig. 63). 
latter. (2) Swimming bells (nectocalyces), sessile and sterile medusæ, without manubrium or mouth, but with well-developed umbrella and velum, by the contractions of which the colony moves about. (3) Covering pieces (hydrophyllia), like the nectocalyces, but with the umbrella reduced to a stiff plate; they serve as coverings for other individuals.

Fig. 62.

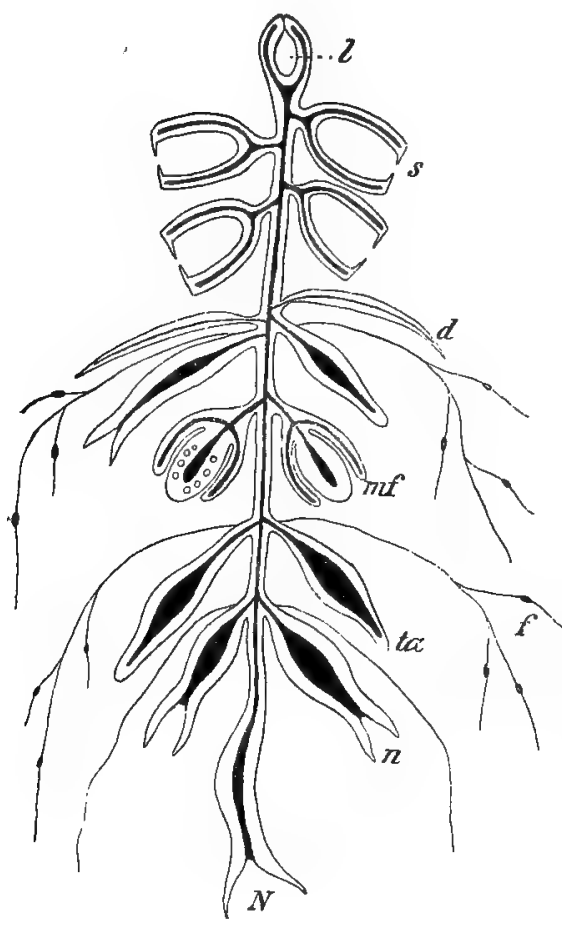

Fig. 63.

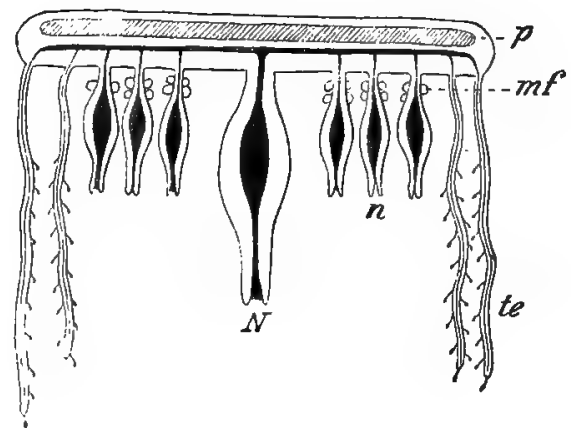

Fig. 62. Diagram of a Siphonophoran with an elongated hydrosoma (Physophora); the digestive cavities are drawn black.-Orig.

Fig. 63. Diagram of a Siphonophoran with a disc-like bydrosoma (Porpita).-Orig.

General lettering : $d$ covering piece, $f$ tentacle, $l$ air sac, $m f$ fertile Medusæ, $N$ large Gastrozooid, $n$, small ditto, $p$ disc with small air cavities, s swimming bell, $t a$ feeler, te teutacle-like person.

A colony of the Siphonophora consists of these individuals, but they are not always all present; the swimming bells may for instance be absent, in which case the colony drifts passively; the covering pieces may also be wanting; the number and arrangement of the individuals, and consequently the form of the colony, is extremely varied.

The Siphonophora are true pelagic animals, are almost exclusively limited to tropical and warm seas (e.g., frequent in the Mediterranean).

As examples may be mentioned: Physophora and its allies (Fig. 62), with long hydrosoma, terminating in a small pneumatophore, and with numerous nectocalyces on the upper part of the stem; Physalia with huge pneumatocyst and bearing gastrozooids and hydrocysts (with long tentacles), without hydrophyllia or nectocalyces; Porpita (Fig. 63) with disc-like circular hydrosoma 
enclosing numerous pnetmatocysts, and bearing the various individuals on the underside (a large gastrozooid centrally, dactylozooids at the edge, no nectocalyces or hydrophyllia); Velella like the previous forms, but the disc is. elliptical with an upright keel. All met with in the Mediterranean.

\section{Order 3. Acalepha (Scyphomedusce, Acraspeda).}

The medusoid generation is usually represented by transparent animals of fair size. The edge of the mouth is prolonged into four oral tentacles with which the prey is caught. Similar processes, but as a rule less well developed, may occur in the Hydromedusæ. The canal in the manubrium widens out in the umbrella to form the gastric cavity, which may extend into a number of large radial outgrowths (gastric pouches) : and the radial canals, often branched, also. arise from it. It contains a number of tentacular threads, the gastral filaments, which are absent from the Hydromedusæ; also the gonads, ovaries and testes, usually in the form of four folded bands;

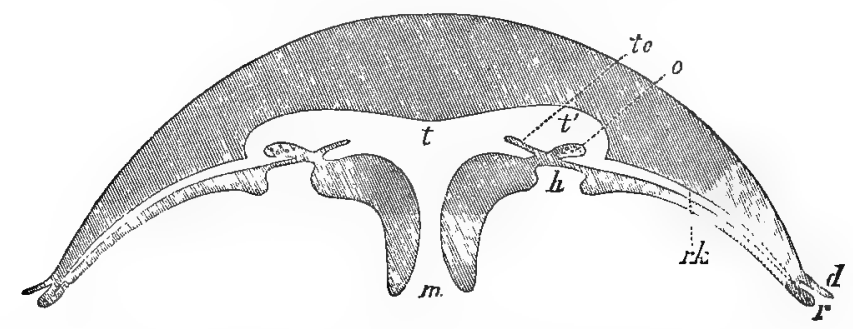

Fig. 64. Section through a Scyphomedusa passing between two oral tentacles. $d$ hood covering one of the tentaculocysts, $h$ sub-genital pit, $m$ mouth, o ovary, $r$ tentaculocyst, $r k$ radial canal, $t$ gastric portion of enteric cavity, $t^{\prime}$ radial portion of ditto, te gastral flament.-Orig.

beneath each of these, on the under side of the umbrella, there is a cavity separated from the enteric cavity by a thin septum ( $h$ Fig. 64); this is pushed out when the genitalia are well-developed, so that they appear to depend from the oral side of the umbrella. The ova and spermatozoa which usually occur in different individuals, fall into the enteric-cavity, and escape through the mouth. The cells from which they originate, are endodermal. The umbrella in which the mesoglæa attains a considerable thickness, has eight notches in its margin; in each notch, covered by a lappet of the umbrella, there is a small round marginal body which bears an auditory, often also an optic organ. Along the edge of the umbrella, there is often a considerable number of marginal tentacles, usually of a considerable length; a velum, on the other hand, is wanting. The ova of Aurelia develop, as. 
far as the larval stage, in saccular evaginations of the oral arms of the parent; others pass through the early stages in similar outgrowths of the radial canals.

The polypoid generation. When the ciliated larva, developed from the egg, leaves the parent, it attaches itself to some foreign body, and grows into a small polyp with a circlet of tentacles.

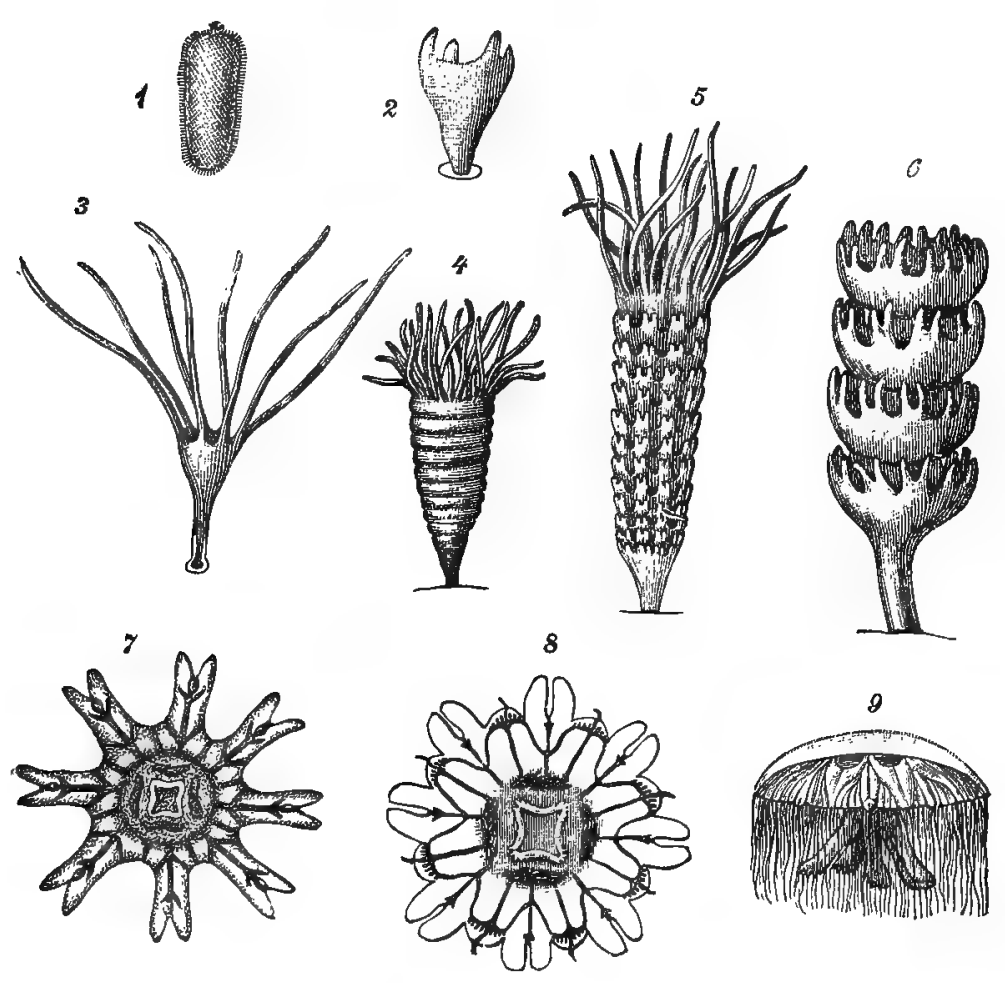

Fig. 65. The development of Aurelia. 1 free larva. 2 the polyp, a short time after attachment. 3 the same somewhat later. 4 transverse fission. 5 later stage. -6 the polyp after the separation of a number of young Medusæ. $7-9$ the young Medusæ at various stages of development. $7-8$ viewed from below. 9 from the side.-After M. Sars.

Like Hydra, this polyp may form buds, which separate off and develop into adults like the parent, but no true colony is formed. The polyp, which is at first relatively short, gradually grows to a considerable length, becoming cylindrical, with a conical base. Next, a number of annular constrictions appear, dividing the main portion of the body into a series of discs, which separate from each other, and, breaking away from the polyp, form small $\mathrm{Medusæ.} \mathrm{In}$ exceptional cases the polyp generation is absent, the medusa-egg developing direct into a new medusa. 
The Acalepha, several of which are phosphorescent, occur only in the sea. The following may be cited as examples:-

1. The Common Jelly-fish (Aurelia aurita), with dise only slightly convex, bearing at its margin numerous short tentacles; the marginal bodies include both auditory and optic organs (tentaculocyst). The enteric cavity is produced into four short radial sacs, containing the four gonads, each shaped like the edge of a human ear. The mouth is provided with four large oral tentacles. Like its allies, this animal contains a large amount of water (95-96 per cent. water, 4-5 per cent. dry substance). Very abundant in N. European seas.

2. Cyanea capillata, a large and beautiful medusa, which is specially characterised by the arrangement of the extraordinarily long marginal tentacles in eight groups, on the under surface of the much lobed disc. The nematocysts cause an intensely burning sensation in thin-skinned portions of the human body. Commonly found with Aurelia.

\section{Class 2. Anthozoa.}

The body is cylindrical, and contains a large internal digestive cavity, into which the upper region of the body-wall is invaginated to form the stomodæum, as already explained. The external opening of the stomodæum is usually termed the mouth, but it must be remembered that the true mouth, the entrance into the digestive cavity (homologous, with the mouth of a medusa) is situated at the lower end of the stomadæum. The former may be termed the external mouth, the latter, the internal mouth. Within the enteric cavity there are perpendicular radial mesenteries, reaching from the stomodæum to the body-wall above, ${ }^{*}$ whilst below, the inner edge is free (something like the dissepiments of a poppyhead). The number of mesenteries varies $(8,12$, etc. $)$. At the upper end of the animal there is a circle of tentacles (in exceptional cases several circles) the number corresponding with that

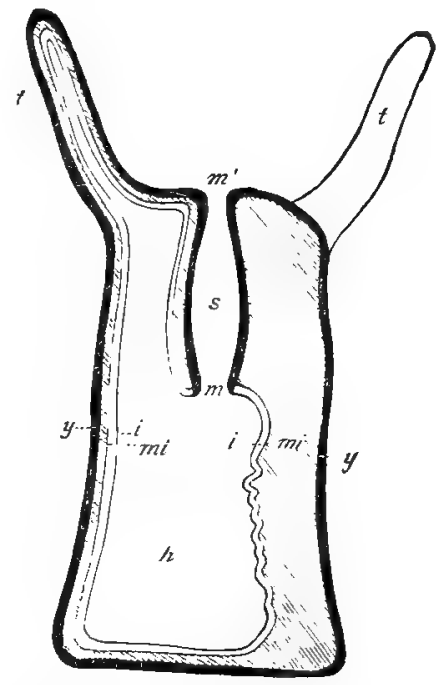

Fig. 66. Longitadinal section through a solitary Anthozoon, diagrammatic; the section passes through a mesentery on the right side, but between two on the left. $i$ endoderm, $m i$ mesoglrea, $y$ ectoderm, $m$ internal, $m^{\prime}$ external mouth, $s$ stomodæum, $t$ tentacle.-Orig.

* In most Actinia, which possess a large number of mesenteries, only some reach from the body-wall to the stomodæum, in the others, the inner border is free for its whole length. 
of the mesenteries. These tentacles are hollow evaginations of the body-wall and correspond in position with the spaces between the mesenteries; they are richly provided with nematocysts. The somewhat discoid expanse within the circle, in the centre of which is the mouth, is termed the oral disc, the lower flattened extremity of the body the pedal disc.

Each mesentery is a fold of endoderm, covering a lamina of mesoglæa, which arises from the mesoglæa of the body-wall (See Fig. 67). Along the inner edge are cord-like thickenings,
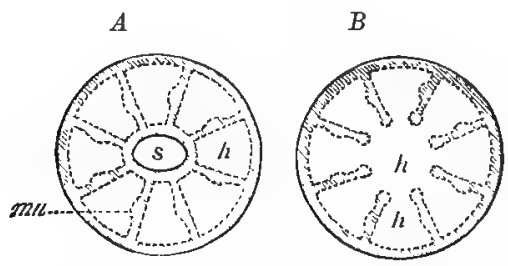

Fig. 67. Transverse sections of an Alcyonarian. Diagrammatic. $A$ through the stomodæum. $B$ lower down. s stomodæum, $h$ gastric cavity, mu muscle. The ectoderm is represented by an entire line; the endoderm dotted; the mesoglæa shaded. - After $\mathrm{T}$. Koch.

ovary or testis. The Anthozoa are usually of separate sexes, only in exceptional cases hermaphrodite. They generally develop hard parts, which will be considered under the different orders.

The Anthozoa, like other Colentera, exhibit a radial symmetry, which is, however, rarely completely carried out. In transverse section, the stomodæum is almost always oval, the external mouth slit-like, and thus a median plane is defined. Each end of the oval corresponds with a tentacle; moreover, the muscles in the mesenteries usually form a thickening on the one side only; the thickenings may occur on either side, but are always arranged s $\mathrm{ym}$ metrically with regard to the median plane noticed above (see Fig. 67, which shows the arrangement in an Alcyonarian).

In many of the $\mathrm{Z}$ oantharia (at all events, in most of the Actinians), the stomodæum is furnished with two thickly-ciliated g r o o ves, corresponding with the two ends of the oval; when the stomodæum is elsewhere pressed together these two grooves remain open, and probably serve to maintain the current of water necessary for respiration. In many of the Alcyonaria there is a similar groove, characterised by deep epithelium and long cilia at one side only; the ciliary current here sets from without inwards, in other regions of the stomodæum in the opposite direction.

In most corals asexual reproduction occurs by budding or fission. The young animals thus formed separate from the

* From the lower end of the mesenteries in some Actinia, there arise peculiar free threads, similar in structure to the filaments and very rich in thread cells; they may be shot out through the body-wall, and serve for attack or defence (acontia). 
parent ouly in exceptional cases; as a rule they remain connected and thus colonies arise. These usually consist of a great number of indiriduals, and frequently attain a very considerable size. An alternation of generations occurs but rarely, since the same indiridual can reproduce both sexually and asexually.

The Anthozoon leaves the egg as a Iarva without tentacles, swimming about by means of cilia. Later it attaches itself and attains the adult form. Only a few (e.g., the Actinians) retain a capacity for locomotion throughout life, and even then to a very limited extent.

The Anthozoa, all of which are marine, are predaceous animals. By means of their tentacles they seize their prey, paralyse it, and pass it through the stomodæum into the digestive cavity. When undisturbed, they remain with their tentacles quietly extended; if irritated, the tentacles and all the soft portions of the body are instantly contracted.

\section{Order 1. Alcyonaria (Octactinia).}

The members of this group possess only eight mesenteries, and corresponding with these, eight $\mathrm{branched}$ pinnate tentacles. In the mesoglæa there is a number of almost microscopic calcareous spicules. These are provided with knobs or sharp points, are of various colours, and are less numerous in the upper than in the lower region of the body, so that the former can be retracted into the lower firmer portion. The spicules, which are for the most part not very closely connected, arise in cells which have wandered in from the ectoderm.

Only a rery few species are solitary; most of them form colonies. Occasionally the individuals of a colony are united by thin stolons containing canals which are in communication with the gastric carities. More often, the lower, harder portions of the polyps are united br large connecting regions (cœenenchyme), consisting chiefly of mesoglæa, which may be perforated by numerous canals. These canals are lined with endoderm, and put the individuals of the colony in communication with each other (for the connection of indiriduals in the Organ-pipe Coral, see below). The external form of the colony is rerr raried; not infrequently it is arboriform; and then there is usually an axial skeleton in the stem and branches, formed in the Red Coral by the fusion of innumerable calcareous spicules, and consequently situated in the mesoglæa. The axial skeleton of the Gorgonidæ is quite different in structure: the roung colony secretes a horny mass on its pedal surface: between it and the foreign body to which it is attached, 
this becomes gradually more convex, growing up with the colony; it is secreted by the ectoderm and is a true cuticular structure (cf. Fig. $68 \mathrm{C}$ ). The axial skeleton of $I$ sis which is to a great extent calcified, is similar in its relations.

$A$

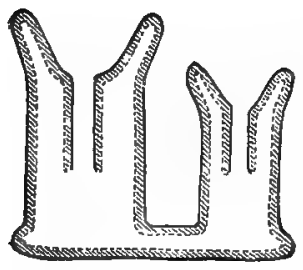

$B$

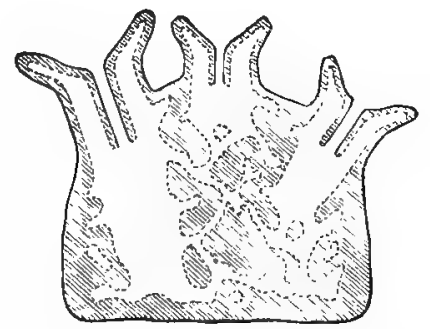

$C$

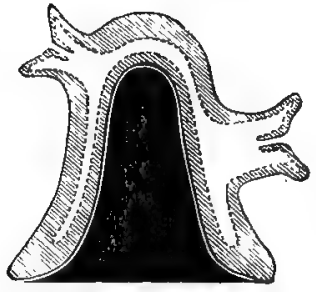

Fig. 68. Sections through young colonies of various Alcyonaria (diagrammatic). The layers of the body indicated as in Fig. 67. A simplest mode of communication between the individuals. $B$ young colony of Alcyonium. $C$ ditto of a Gorgonian axial skeloton, figured black.-After v. Koch.

It is of interest to notice the constant presence, in various Alcyonarians, of arrested individuals (siphonozooids) amongst the normal persons (autozooids). In the most extreme cases, they have no tentacles and differ in other respects from autozooids; in other cases the difference is less pronounced. Their chief function appears to be the reception and ejection of water, and is thus respiratory. They are very common among the $\mathrm{Pennatulids,} \mathrm{but} \mathrm{are} \mathrm{also}$ found in Alcyonium and Corallium rubrum, although not in the Gorgonidæ.

Of the forms belonging to this group, the following may be mentioned:

1. Dead Men's Fingers (Alcyonium digitatum) form yellow or whitish leathery colonies of an irregular bulky shape, with short thick branches. The gastric-cavities are continued back from the free, upper, soft portions of the individuals, as slightly sinuous tubes reaching far down into the stem, and are connected by fine canals. No axial skeleton. Common on English coasts.

2. Organ-pipe Coral (Tubipora) forms massive colonies, consisting of long tubular zooids, arranged almost parallel to one another; the individuals of the colony are not connected by coenenchyme, but by transverse platforms, containing a network of tubes communicating with the gastric cavities. In each individual the spicules (with the exception of those in the upper soft portions of the body) are united to form a hard tubular mass : in the transverse platforms they are fused into calcareous plates connected with the tubes. In the Indian and Pacific Oceans.

3. The Gorgonidæ (genus Gorgonia and others) form arboriform colonies with hard, dark, horny axes both in the main stem and branches; the rest of the colony covering the horny axis is termed the "cortex," contains numerous spicules, and may be perforated by canals. On the surface of the dried colony 
may be seen small pits, in which the free, soft portions of the individuals were situated. In some forms (Rhipidigorgia) the branches of the colony all lie in the same plane and partially coalesce, so as to constitute a perforated plate. Chiefly in warmer seas, some species in the Mediterranean. Isis approaches the Gorgonidæ, but the axis consists of a series of calcareous joints limited by horny transverse septa, One species in the Mediterranean.

4. The Precious Coral (Corallium rubrum). Branching colonies, with hard, calcareous axis. Both axis and cortex are of a beautiful red colour, the free portions of the individuals are white. In the Mediterranean.

5. Sea-feathers (genus Pennatula and others). The colonies consist of a lower naked stalk and an upper, broader, often feather-like region, from which the individuals project. Within the axis of the colony there is a calcareous unbranched rod. They are found at the bottom of the sea, with the stalk sticking into the ground, and if disturbed, they bore their way deep down. The phosphorescent, red, feather-like Pennatula phosphorea occurs in the North Sea.

\section{Order 2. Zoantharia (Polyactinia).}

The number of mesenteries is either a multiple of six, or a large indefinite number. The tentacles, which are simple, almost always correspond in number with the mesenteries, and in most forms, as in the Alcyonarians, there is a skelet o $\mathrm{n}$ which is, however, very different in character. It is limited exclusively to the lower region of the body, and consists of a continuous, very porous or thick mass of calcium carbonate, whilst the upper portion is entirely without skeletal structures. The skeleton corresponds closely with the general form of the animal, and usually consists of the following parts: a discoid basal-plate, bearing a tubular theca and a number-12, 24, 48-of radial septa which ar ${ }^{3}$ fused with the theca and basal-plate, and often with each other also.

From this description it might be supposed that the theca represented a calcification occurring in the outer body-wall; the septa, similar hardenings in the mesenteries and the basal plate in the lower discoid portion of the animal. As a matter of fact, however, this is not the case. In the first place, it must be noticed that the skeleton is not developed in the mesoglæa as was formerly believed, but is an external secretion of the ectoderm. As soon as the tiny free-swimming larva settles, it secretes, upon its attached surface, a thin calcareous disc, which becomes, later, the basal-plate. Next, the septa appear upon this plate, gradually growing taller and more plate-like, until, covered by the soft tissues of the pedal disc, they project into the gastric cavity, between the twelve soft mesenteries which have already been formed. A further secretion from the attached surface now appears. as a ring-like ridge upon the basal plate, the beginning of the the ca; the ridge grows into a tall cylinder, covered like the septa by a fold of the basal disc. and also projecting into the celenteron; it lies some little distance within the soft body-wall. Other septa may develop later between those of the original set, and these again have nothing to do with the mesenteries. It may also be remarker that, in some Corals, the basal-plate may project up on to the lateral wall of the 
animal (as in Fig. $69 \mathrm{~A}$ ), so as to form a cylindrical calcareous deposit external to the body-wall (and of course external to the theca); this is the epitheca. The skeleton is thus a purely external, cuticular structure, secreted by the ectoderm.

$A$

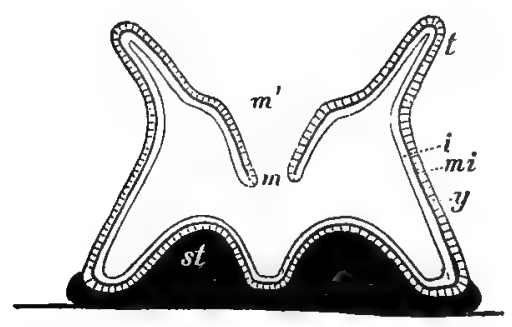

$B$

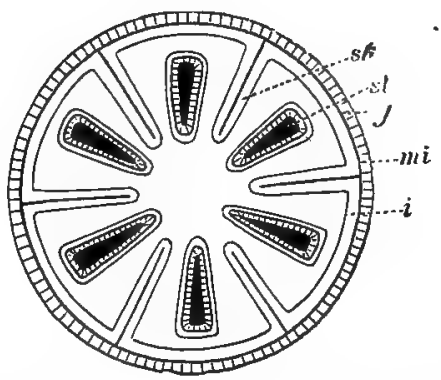

Fig. 69. A diagrammatic longitudinal section of a Madre orarian which has only just attached itself, passing between two septa. $B$ transverse section of the lower end of the same. For the sake of simplicity, only 6 septa and 6 mesenteries are drawn, instead of 12. $i$ eudoderm, $m$ internal mouth, $m^{\prime}$ external month, $m i$ mesoglæa, sk mesentery; st septum, $t$ tentacle, $y$ ectoderm. The skeleton is drawn black.-Orig.

Between the septa in the lower portion of the animal, small, calcareous transverse beams or platforms often develop, passing from one septum to another (s ynapticulæ). In the lower region, the septa often fuse in the middle, and from the point of fusion there usually arises one or more vertical columella e. All the septa are not equally well-developed; those last formed do not reach so' firr in as the older ones, with which they alternate regularly. In proportion as the animal grows in height, the lower portions of the septa and the theca thicken so that the lower part of the skeleton becomes more solid and compact than the upper. In older animals the soft parts withdraw from the lower portions, leaving this region of the skeleton naked.

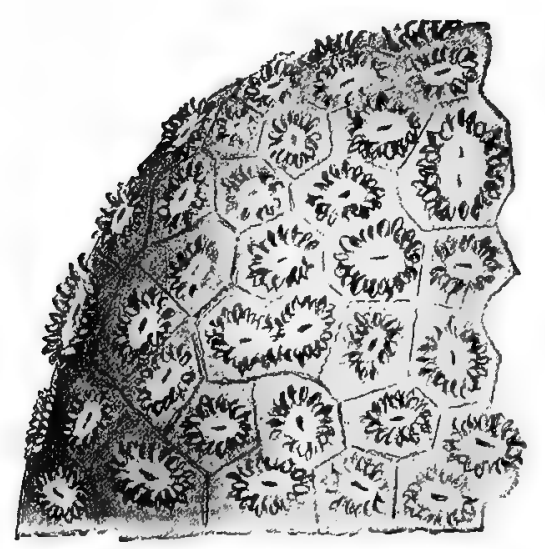

Fig. 70. Portion of a massive Madrepora $\mathrm{r}$ i an; two individuals (one on the right above, one centrally) in the act of dividiog longitudinally.-After Dana.

The majority of Zoantharia, especially those which are furnished with a skeleton, form colonies by budding or by longitudinal fission; the colonies usually consist of a great number of individuals. In budding, a lateral evagination arises from the body-wall and gradually develops into a new individual; longitudinal fission occurs by the formation of a new mouth on the oral disc, the latter becoming oval, and finally dividing to form two (see Fig. 70), whilst a corresponding division of the whole animal occurs. Just as in the Alcyonaria, the 
gastric cavities are connected by a system of fine canals. 'The outer form of the colony corresponds with that of the skeleton, and is very varied. Some forms are arborescent, others massive, the individuals situated close together like the cells of honeycomb; the upper portions into which the skeleton does not extend are for the most part independent, whilst the portions surrounded by the skeleton are fused with the neighbouring individuals, either throughout their whole length or in the lower region only. Sometimes, but this is only exceptionally the case, the connection between adjacent polyps is still more intimate; the oral openings are indeed separated, but the gastric cavities communicate freely, and in the dry skeleton, each individual is not, as in other cases, indicated by a star separated from others by its theca; but a whole row of individuals is indicated by a groove, from septa project: in correspondence with tentacles are not situated in circles round the mouths, but in a double row along the groove (Fig. 72).

Only in somewhat rare instances, do the new in. dividuals. formed by budding or fission, separate off from the parent. This happens, for instance, in the Actinia without skeletons, in which budding as well as fission, both transverse and longitudinal, may occur. In some forms with a calcareous skeleton, the buds or individuals formed by fission may, in rare cases, separate, taking with them a portion of the calcareous skeleton. The Mushroom Coral (Fungia), a solitary form, which lies freely on the bottom of the sea, and attains

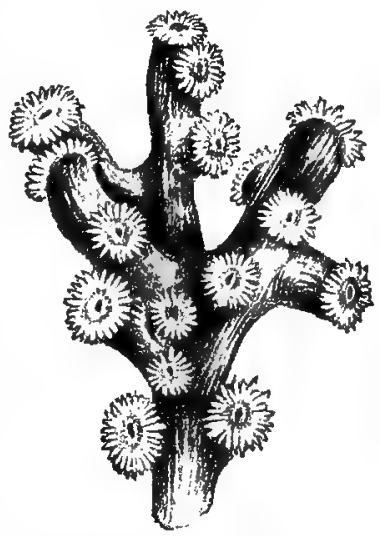

Fig. 71. Portion of an a rborescent Madreporarian, which increases by budding.After L. Agassiz.

the sides of which the this arrangement, the

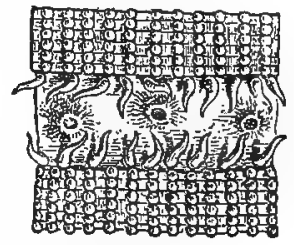

Fig. 72. Small portion of the surface of a Coral in which the individuals are incompletely separated (Heliastraa). Thre oral apertures may be noticed; tentacles in two rows.-After M. Edwards and Haime. a considerable size, develops in this way by the transverse fission of a small attached solitary coral (or from a colony consisting of quite a few individuals). After fission, the individuals separate and undergo further growth.

Coral reefs, which are so frequently met with in the seas of the Torrid Zone, and which may be even miles in length, owe their origin chiefly to different calcareous Zoantharia. Besides these, certain other animals, notably certain Hydroids (Millepura, p. 106) also assist in the formation of the reef. The reef consist: in part of skeletons of dead colonies, in part of living colonies, which have attached themselves to the latter. Upon and within the reef there live a great number of other animals, which are to some extent 
adapted to this peculiar mode of life, so that it is possible to speak of a special reef fauna. Coral reefs are a very characteristic feature of tropical seas.

1. Almost the only representatives of the order occurring in northern seas, are the Actiniaria (Sea-anemones, Sea-roses); solitary forms without skeletons, mostly of relatively large size, and usually with several whorls of tentacles. They have a broad pedal dise below, by which they attach themselves to foreign objects ; they are capable of slow locomotion. Several species on English coasts.

2. The Madreporaria, forms provided with a calcareous skeleton, belong almost exclusively to warm seas, where they occur in great abundance, usually in colonies, but occasionally solitary; a few species are met with in the Mediterranean.

\section{Class 3. Ctenophora.}

The Ctenophora may be regarded as medusæ from which the manubrium is absent, and the umbrella so convex and so much narrowed that the sub-umbrella cavity forms a tube of varying width, at the base of which lies the $m o u t h$, the entrance into the alimentary canal. Eight narrow streaks may be seen passing round the surface of the body like the meridians of a globe; each of these lines or $\mathrm{ribs}$, as they are called, is composed of a series of tiny laminæ, consisting of transverse rows of cilia fused together; these plates constitute the chief locomotor apparatus of the animal. Many of the Ctenophora possess two long branched tentacles, which, arise from opposite sides of the body, and can be withdrawn into special pouches. There are no other appendages. The mouth leads into a small digestive cavity, the so-called in f un dibulum, from which arise other canals running along the ribs. At the upper pole of the body there is an auditory organ. Thread cells are absent.

An eight-rayed plan is to a certain extent indicated, but is not followed throughout. As a matter of fact, the body can only be divided into two symmetrical portions : is two-rayed arrangement is seen, e.g. in the disposition of the tentacles, the branches of the alimentary canal, etc.

The Ctenophora, of whose structure only certain important points have been noticed above, whilst others have been altogether ignored, are $\mathrm{h}$ e $\mathrm{rmaph} \mathrm{rod}$ i te without an alternation of generations, and occupy a somewhat isolated position among the Colentera. They mostly occur in warm seas; all are pelagic.

It is specially interesting to note that the larvæ of some forms often attain sexual maturity quite soon after leaving the egg, and may lay fertile ova; but this only occurs during the warm seasons: the sexual organs then degenerate, and the larvæ develop into normal individuals, which again become sexually mature ( $c f$. pædogenesis of Insects).

Of the various forms may be mentioned Beroë, barrel-shaped, with a wide subumbrella cavity, without tentacles; Cydippe, spherical, with a narrow cavity and long tentacles; Cestus veneris (Venus's girdle), with body much compressed and elongated to a ligamentous form structure. All these occur in the Mediterranean, the first two also on English coasts.

\section{APPENDIX TO THE CoELENTRA:}

\section{Spongiæ or Porifera (Sponges).}

The Sponges form a very peculiar group of animals of low organisation, which is usually placed near to the Colentera, but whose systematic position is not yet determined. 
In the simplest form (Fig. $74 A$ ), the body, which is always attached to some foreign object, consists of a sac open at the upper end, and composed of three layers. Of these, the ectoderm consists of flattened cells, each furnished with a flagellum; the middle layer, of a mass of connective tissue ; and the endoderm, again, of a special form of flagellate cell (Fig. 73), peculiar in bearing at the free end a thin drainpipe-like tube, within which is the flagellum (collar cells). The cavity of the sponge (exhalent callal) not only communicates with the exterior by the large opening (osculum), butalso by in halent canals, which perforate the wall and open by pores. The inhalent current of water passes through the pores into the large cavity, and out through the osculum; the

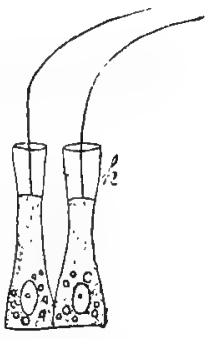

Fig. 73. Collar cells of a Sponge. $k$ collar. movement is kept up by the flagella.

The simplest of these types occurs only in a minority of the Sponges (in certain calcareous forms). In others, it is complicated by

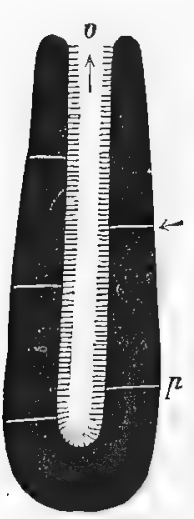

$A$

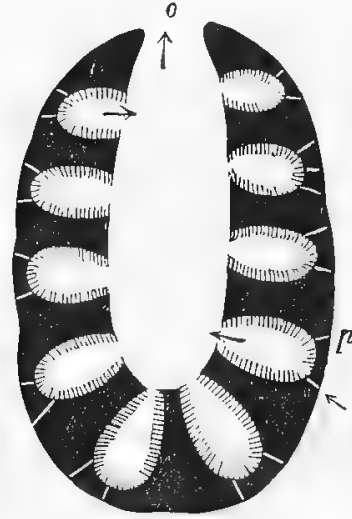

B

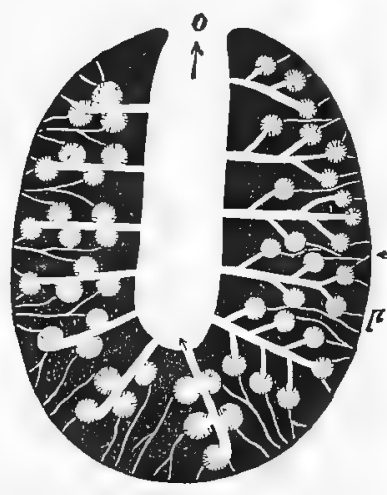

$c$

Fig. 74. Various forms of S p onges ; diagrammatic longitudinal sections. o oscalum, $p$ pores.-Orig.

saccular evaginations of the exhalent canal (Fig. 74, B). The lining of collar cells is limited to these chambers, whilst the main cavity is lined by a flat epithelium; the inhalent canals open into the evaginations. In others (Fig. 74 C, the left side of the figure), the evaginations are, again, prorided with smaller pouches, and in these alone, collar cells are found; they are, therefore, termed the flagellate chambers; they communicate with the exterior by branching inhalent canals. Finally, the chambers may be racemose, that is, they may be connected with the main branches by longer or shorter stalks (Fig. 74 C, the right side of the figure). In all cases, 


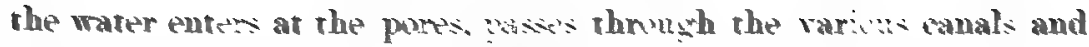

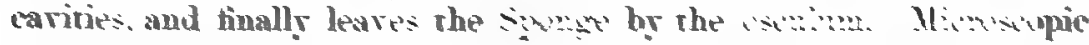
particles, which swe tow the nourishment of the animal, enter with the water: the collar "r"."s take these up. and later, eject the

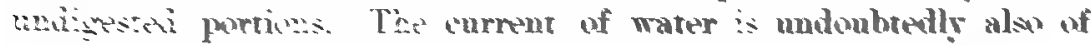
the greatest imporrance for: sespration.

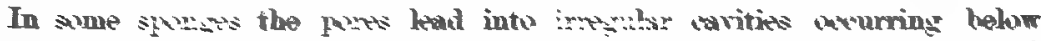

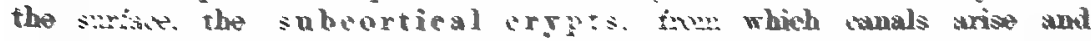
run so the thagellat chambers

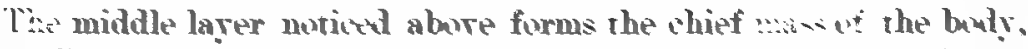

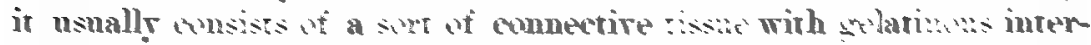

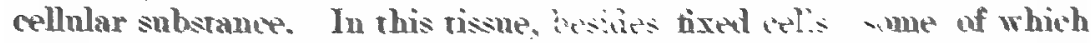

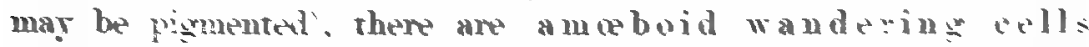
which more alunt in the mass of jelly. Hen sibs hard parts ane

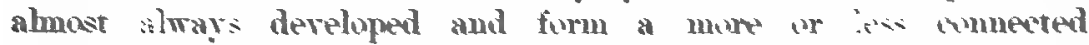
steleton. This cunsists eirher of a metwerk of horuy tibres: or of fine calcareous soicules, which may be simp'se, ar pascessed at three or four bramches. radiating ont in vitferent directions: or thewe is a silicions skeleton of a very different

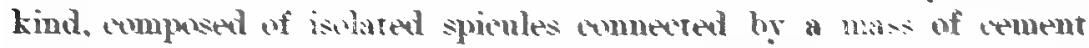
substance. or of silicions fibres. The siojons spacules arr

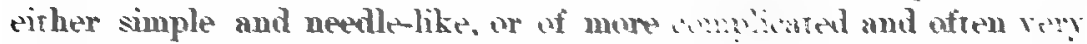
beauriful forms anchors, asio's, ete. Cive infrequently the

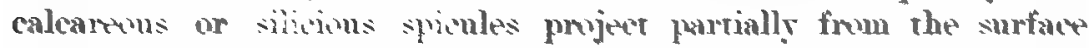

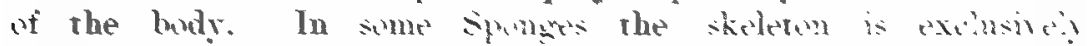

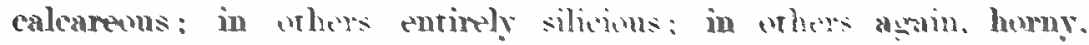
In many forms. however, it is burh horny and silicious, rhmonh

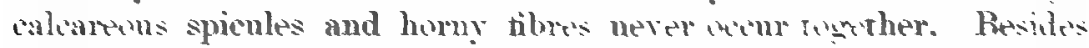
the strucrumes alneads mentiomed muscle cells are present in the middle larer: and it is swid that nerre cells are alse found there. In sume smonges smerticial semsorp cells are provent in certain reximas.

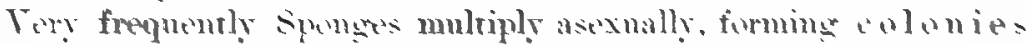
of various kinds, the individuals of which are only in a tew eases clearly distmünishathe, whilst they are, for the most parr. so intimately comnected with their neighbours that, extemally, the number of uscula alone prowes that there is move than one indiridual. In some forms, howerer, after eremmation, the new indivituals separate off and derelop independently. In kirsh-water spungers there is a peculiar mode of ascixal repmoluction: poutions of the animal encys in small capsules formed within it, and after a resting stave develop into new individuals arimmulat.

With these axeptions. Spumges reprodnce in the nsual way by ora and spermarozoa, which are formed either in the sume indiridual or colemy, or in different ones. The orum is naked and capable of amoeboid movement: it dereleps within the haty of the 
parent inta a cillated Iarra, which atraches is olf after a short

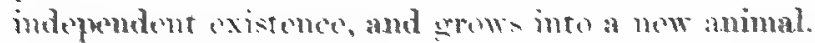

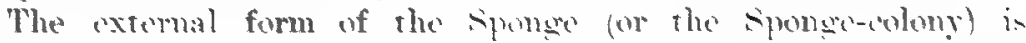

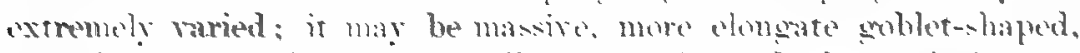

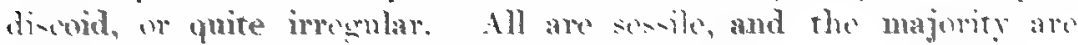
marine; only a few acour in frosh water.

Of mumeinus forms only a few will be mentioned.

1. The Buth spouse (Euspongin), of which diverse sprecies and marieties are the ohjert of important fisheries in the Meditedramenu. possessess an

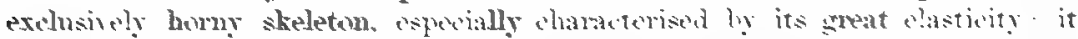

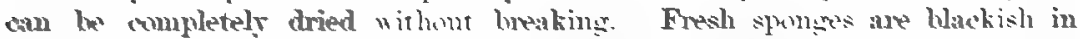
whour. and ouly when the soft parts are removed do they become lishter.

$\therefore$ Vitreous s pou ses (Hzactimellider) are silicions forms characterised by the striking beanty of the skeletom, which is like spun-ylass. A well-kmown form of this smup is the beantiful Philiphine Tenus's Flower-hasket, Enplectella (spergillnm), which like soversl of its allies, lives at considerable depths.

3. Boring sponges (1) sma). small silicions forms which cam eat their

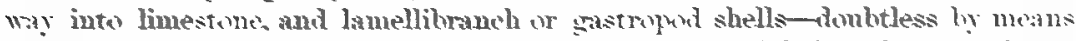
of a chemional secretion. In stomes or shells which they inhahit (they attank not

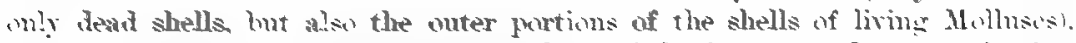

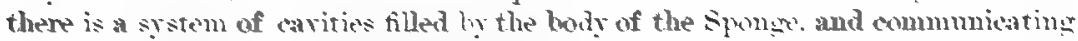
with the citerion by fue perforations of the surfan of the shell or stome. The

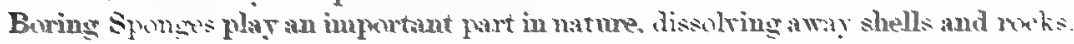
Alundant in all Euriferan se:as.

4 Fresh Water Sponses (spongilla finrintilis, and wher sprecies). athudant in fresh water in England, form colonies of varous forms thamehing. massive), wttached to mater plants or pilcs. The exterual form of the olnuy is Actermined by the substanow upan which it is smoming. It is a

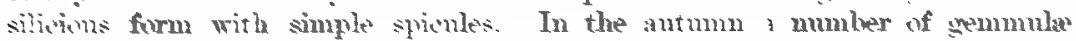

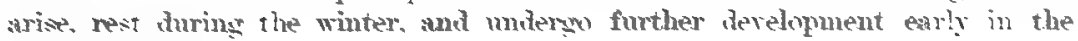
following vear. 


\section{Phylum 3. Echinoderma.}

The Echinoderma were formerly grouped with tho Coolenter"a as Radiata, on account of their both exhibiting a radial sy motry and in spite of their differing much in other respects; in the Echinoderms, there is an early development of masoblast; " bodycavity, a circulatory and a water-vascular system are present.

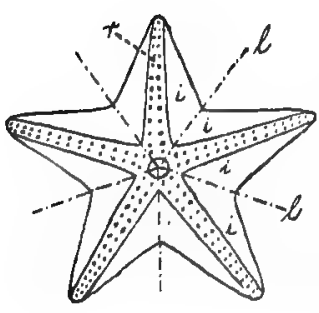

1

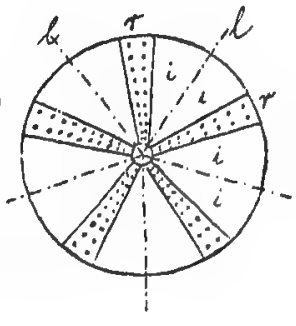

2

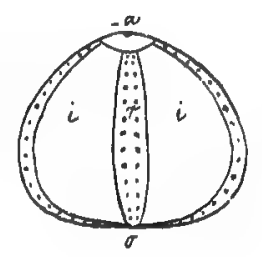

3

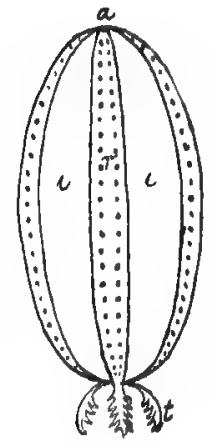

4

Fig. 75. Diagrammatic figures explaining the radial struture of the Echinoderma. 1 Star-fish from beneath. 2 Sea-urchin from beneath, 8 Sea-urchin. lateral viow. 4. Holothurian from the side. $a$ anus, o mouth, $r$ radius, $i$ inter-radius, $l$ lines indicating the scissions by which the animal may be divided into rays, $t$ tentaole.-Orig.

The characteristics of the $f u n d a m e n t a l$ form of a $r \in g u l a r$ Echinoderm are as follows: the body is usually pentamerous, i.e., it may be divided into five approximately identical ray $*$ (antimu( by five scissions meeting in the principal or median axis. The external form of the body varies in accordance with the l'ngth of this axis; when it is longer than the transverse, the body is elongate; when the axes are equal, or the transverse is slightly long' r', it is almost spherical; if the principal axis is much shorter, the busly becomes discoid. All these various types are connected by transitional forms. The mouth lies at one pole of the principal axis, the oral pole. The surface of the body may be divided by meridians into ten segments, five of which, termed $r a d i i$, bear the $t u b \in-f \in e t$ 
(to be described in detail below), and alternate regularls with the other five, called inter-radii.

The radial trpe of structure is not onl indicated externally, but is al-o conspicnous in most of the internal organs we below), although it is nerer completely carried out; there are alwars deriations, in some srstems at least, and these are rerr considerable in mans forms (for details, see the rarious gronps, especially the Echinoids.

It is characteristic of the Echinoderm: that, in almost all, calcifications of rarsing size and form occur in the connectire tissue of the bodr-mall. Sumetimes ther are small, almost microscupic deposits, often rerr beantiful in form: small perforate plates, wheels or anchors; sometimes larger laminæ murabls or immorably cumnected together. With the exception of sime quite small depusits, all the calcifications are porous and spongr. In most cases ther are present in such numbers that ther form a considerable portion of the whole mass ; occasionalls (Holothurians) the are more subordinate.* C'alcifications may occur not onls in the bodr-wall, bat also in other regions, e.g., in the wall of the stune-canal (see below) and in the pharynx of the Holuthurians.

The skin is, as a rule, ciliated and often brightly coloured. In connection with it, there are rarious a p pendages, many of which are calcified like the bodr-wall. This is the case for instance in the morable spinest usually present, part of which is calcareous matter, although connectire tissue and epidermis are br no means wanting. A peculiar form of appendage, the so-called pedicellariæ, also occurs in starfish and Sea-urchins. Each consists of two or three short calcified ralres connected at one end. Whilst the free ends are often prorided with curred tips capable of snapping together like pincers; the pedicellariæ are usuall t borne upon a longer or shorter morable stalk, supported prosimalls br a calcareous rud. They serre as defensire organs; small animals are seized and held fast until ther die; excreta and foreign particles are also remored from the surface of the animal br their agencr.

Among the appendages of the Echinoderms, the tube-feet are of special interest; ther are soft, delicate, usuall cylindrical structures, the free ends of which are either furnished with suckers or rounded; only in the former case do thes serre as organs of attachment. Thes mar stretch out to a considerable length, and then hare the appearance of rerr long thin threads, whilst in the contracted condition, the shrink up to a small fraction of their former

* The calcifications lie in connective tissue covered by the epidermis. In regions where the body is exposed to friction, the suft corering may, however, be rubbed off, so that they become partially bare (tips of the spines of Sea-urchins, portions of the surface of the Ophiurids, etc.).

+ Frequently the spines are not altogether simple in structure, bat are forked and so on. Such $e g$. are the paxill of some Starfish, bearing a rosette of fine points at the end of the shaft. 
size. The tube-feet which end in suckers, may serve as locomotor organs, since they can stretch out and attach themselves to foreign objects, and

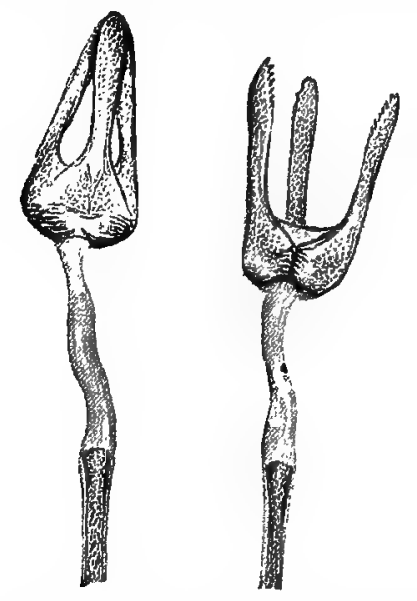

Fig. 76. Pedicellarig of a Sea-urchin closed and open. Proximal portion of the stalk not drawn.-Orig. then contract so as to draw the body after them; when they are rounded at the ends, they usually have a tactile function. Within the feet there are cavities connected with the water-vascular system peculiar to the Echinoderms.

The water-vascular system, a series of tubes, containing a fluid, and lined with a ciliated epithelium, consists of a ring-canal surrounding the alimentary canal close to the mouth, and of five branched radial canals in connection with it. The ring canal, which is usually beset with a number of vesicular outgrowths (polian vesicles), communicates with the exterior by the socalled stone-canal, * which is attached to a perforated plate, the madreporite, lying in the body-wall, and allowing of the passage of water. The radial canals lie along the middle of each radius

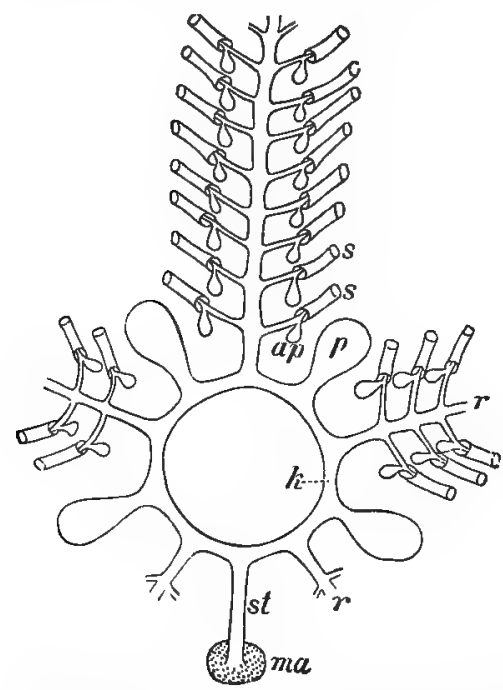

Fiv. 77. "Diagrammatic sketch of the water-vascular s y tem of a Starfish. ap ampalla, $k$ ring - canal, $m a$ madreporite, $p$ polian vesicle, $r$ radial canal, $s$ tube feet, st stone canal.-Modified from Gegenbaur. giving off a tiny vessel, provided with a small swelling or a $\mathrm{m} \mathrm{p} \mathrm{u} 11 \mathrm{a}$, to each tube-foot. Water is driven into the tube-feet by the contraction of the water-vessels and ampullæ, and causes their elongation; when, however, they contract, it is driven back into the canals. $\dagger$ In most Holothurians, and in Crinoids, the stone-canal (or canals, for there may be several) is not connected with the surface, but opens into the body-cavity by one or more apertures, through which the fluid is taken into the watervascular system. In the Crinoids, the body-wall is perforated by fine pores, through which the sea-water passes into the body-cavity.

The water-vascular system of the larva is always in direct communication with the sea-water by a stone-canal opening at the surface. Here, too, there is always only a single stone-canal.

* This name is derived from the fact that the wall of the canal often contains calcareous deposits.

+ Amoboid cells, like those of blood, float in the water-vascular fluid. 
The water-vascular system has no connection with the true bloodvascular system; so that the Lichinoderms possess two separate sets of vessels containing fluid. In the blood-vascular system there is a circular vessel round the mouth, from which arise numerous branches, amongst them, the radial vessels. In Starfish and Ophiurids, there is a second circular vessel, lying farther from the mouth, and connected with the former by a vascular plexus. There is no heart.

The alimentary canal differs considerably in the various. groups. It may be noticed here, that whilst the mouth is always situated at one pole, the anus (which is usually present) lies in one of the inter-radii, although in some forms it is quite near the aboral pole.

Special respiratory organs are usually little developed, or. entirely absent. They are of various forms: "respiratory trees" in Holothuria, dermal-branchiæ in Starfish, and tufted gills at the mouth of Sea-urchins; these will be considered in greater detail under the different groups.

Excretory organs are as yet unknown in this group. A glandular organ lying along the stone-canal, which was formerly regarded as a heart, has more recently been considered to be excretory, but in reality it appears to be a. "lymphatic" organ, in which the blood corpuscles are developed.

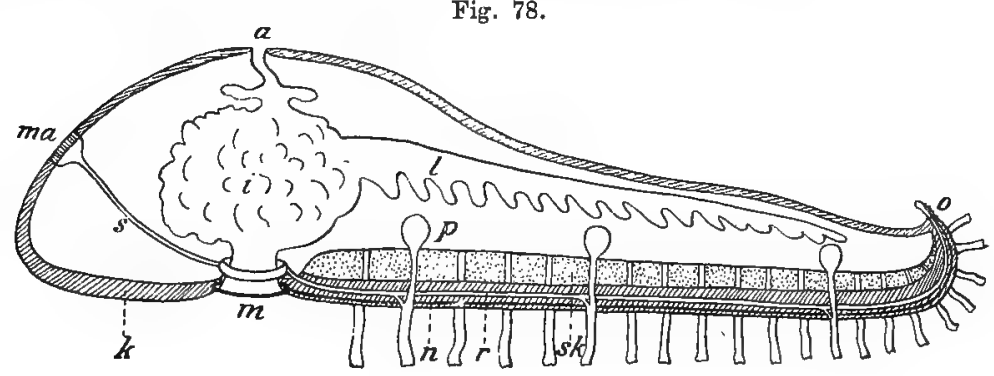

Fig. 79.

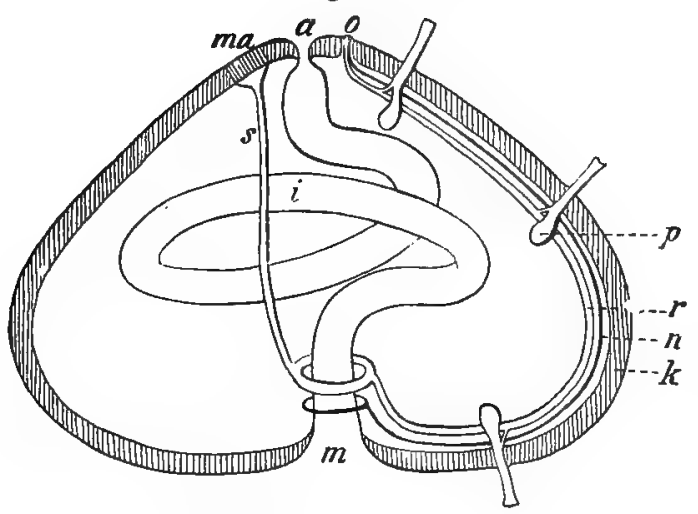

Fig. 78 and 79. Diagrammatic longitudinal section of a Star-fish and of a Seaurchin, fassing through a radius on the right, an interradius on the left. $a$ anus, $i$ gut, $k$ body-wall, $l$ cæcum of the gut, $m$ mouth, $m a$ madreporite, $n$ radial nerve, $o$ in Fig. 78 eye, in Fig. 79 sensory spot, $p$ ampulla, $r$ radial canal, $s$ stone canal, sk skeletal plate. The Polian vesicles, eic. are left out.Orig.

The nervous system in all Echinoderms consists of a nerve ring round the mouth, from which nerve cords pass off to the radii. In Starfish and Crinoids both the ring and the radial nerve cords lie- 
in the epidermis, whilst in other forms they have sunk farther in. As to sense organs, the small eyes present at the tips of the arms of Starfish must be mentioned. Small eyes are also present in certain Holothurians (Synapta), at the base of the tentacles; and lastly, optic organs have been described in some of the Sea-urchins where they are distributed in larger numbers over the whole surface of the body. Vesicular a uditory organs are known only in some of the Holothurians.

Reproduction, with a few exceptions, is sexual, and the Echinoderms are almost all of separate sexes. The male and female organs are usually very similar in form, but they may generally be distinguished without microscopic examination, since the ovary is yellow or red, the testis white. As a rule they are radially arranged, in each inter-radius an ovary or testis, or a small group of these; sometimes they are absent from one or more inter-radii, as in Irregular Sea-urchins; or they may be present in one only, as in the Holothurians. They may be saccular or branched, and each opens by a pore upon its inter-radius; in some forms near to the aboral pole, in others at some distance from this; or again, quite close to the mouth.

Fertilisation generally occurs after the deposition of the ova, which are usually small. Some few Echinoderms are, however, viviparous, and in these, of cour'se, fertilisation takes place within the body of the parent. In some forms the ova are carried about by the parent, either protected by the spines or in special pits in the surface of the body; some Starfish form a kind of brood-pouch by bending the arms down over the eggs.

The development of the Echinoderms is of special interest, for a complicated metamorphosis often occurs: the larval
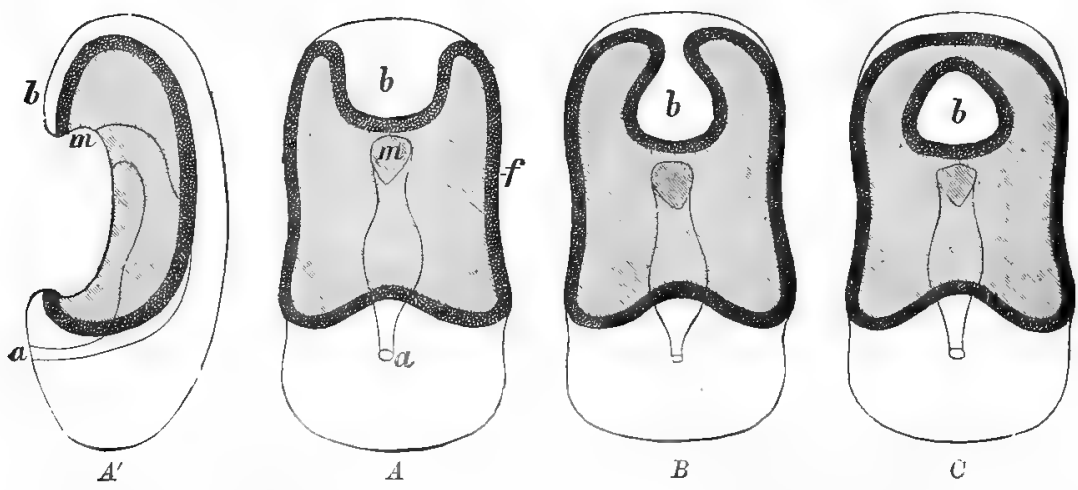

Fig. 80. Diagrammatic figures of the prineipal forms of young Echinoderm Iarvæ. $A, B, C$ seen from below; $A^{\prime}$ is $A$ from the left side. $a$ anus, $f$ ciliated ridge, $m$ moutb. The saddle-shaped concave region is shaded. For the rest, see the text.-Orig.

f or $m$, unlike the adult, shows no trace of a radial structure, but on the contrary is bilaterally symmetrical, and all groups conform to a common type, excepting the Crinoids and a few others. The simplest form (see Fig. 80), seen in young larvæ, 
is almost spherical, somewhat longer than broad, with a saddleshaped depression on the rentral surface. The edge of the raddle is a thickened ridge corered with cilia, which enables the animal ru swim. The mouth is situated anteriorls in the depression, the anus is poisterior to the hinder edge of the ciliated ridge. In front, the ridge bounds a projecting lappet $(b$ Fig. $s 11 A)$, which in sume caves is only connected br a narrow bridge with the rest of the conrex surface (Fig. $\$ 0 \quad B$, Holuthorian), or again, mar even be completels cut off from it, forming a special island in the concare region, surrounded br a small ciliated ridge (Fig. sl) C. starti-ln'. In older larræ, the ciliated rim is more or less lobed, usualls formin y long processes or arms, which are then frequently supported by delicate internal calcareous rods. as in the Ophiurids and Sea-urchins. After some time, a portion of the larra undergoes complicated modifications to form the bodr of the adult, whilst the rest gradualls shrivels up. The final product of the metamorphosis is a small animal possessing the chief features of the adult, although differing from it in man! respects. e.g., in the small number of tube-feet. The adult is thus produced br a remodelling of the larral bodr. large portions degenerating whilst others become further developed and modified. In some Echinoderms, especially in thise
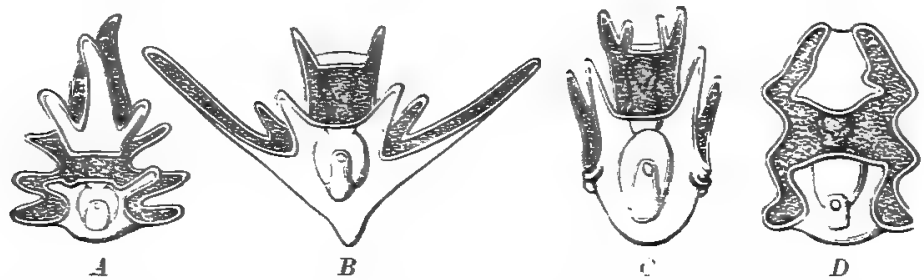

Fig. 51. Larræ of: A Starfizh. B Ophimid, C Seg-trehin. $D$ Holotharian-After J. Mǖller.

which are ririparons, a metamorphosis does not occor; or it is not obrions; or it is modified in rarious wars.

Asexual reproduction occurs in only a ferr forms; see Asiruidea and Holothuroidea. All the Echinoderma are marine, liring at rarious depths; ther crawl about at the bottom, от are sessile: onl exceptionally are they capable of smimming. The group is represented in the uldest fusiliferwus snrata, and on account of its abnondance. and the frequent presence of a rell-dereloped dermal skeleton, fossils are rerr numerous.

\section{Class 1. Crinoidea sta-Lities?.}

The Crinoids are primarily distinguished frum other Echizoderms. in that, either in the adolt condition, or at least in the early -tagere: following free larval existence, ther are attached to the sea-bottom, 
or to some foreign object, by a stalk arising from the middle of the dorsal surface. The actual body is small in comparison with the whole size of the animal, usually soft and flat on the up-turned

Fig. 83.

Fig. 82.
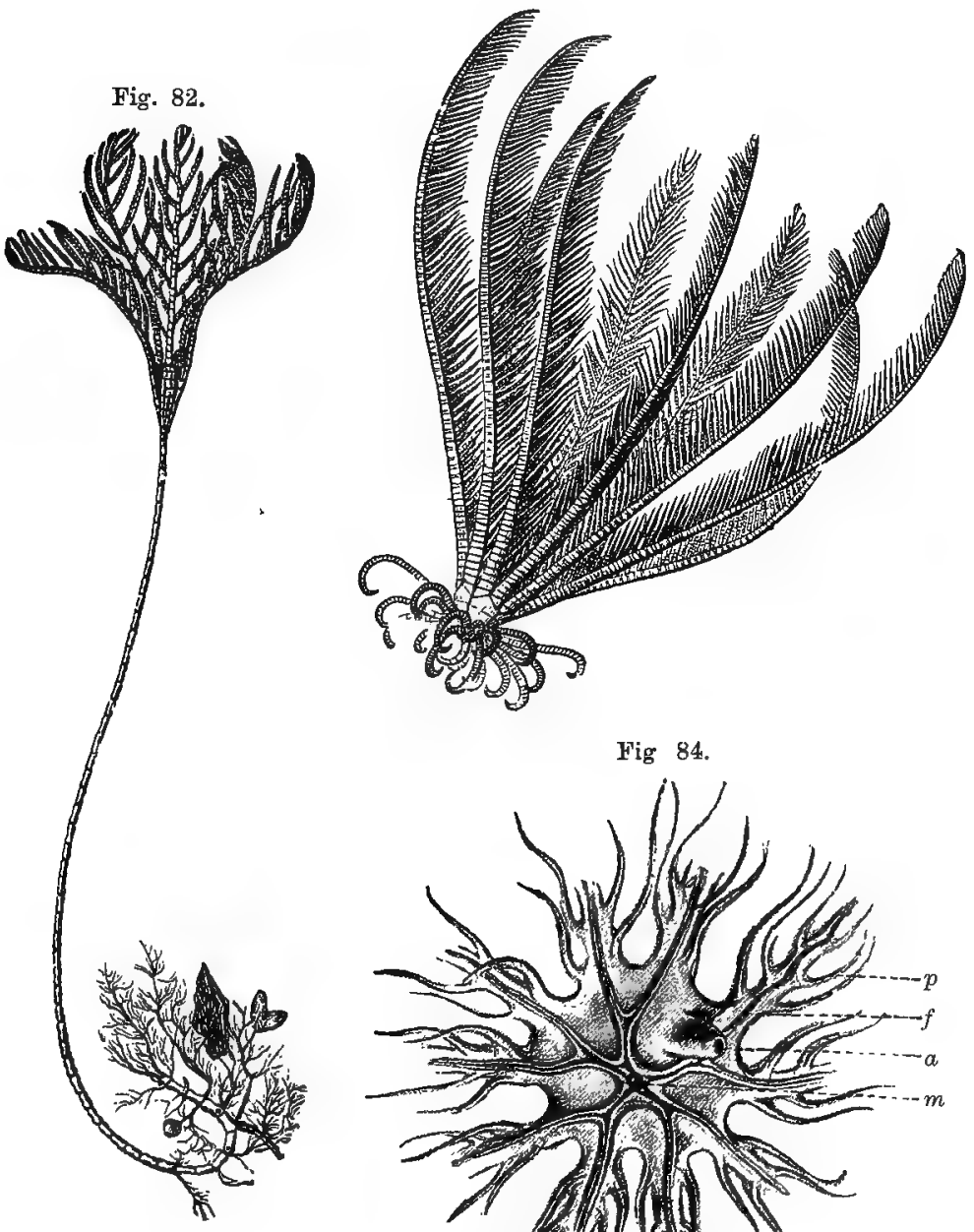

Fig 84.

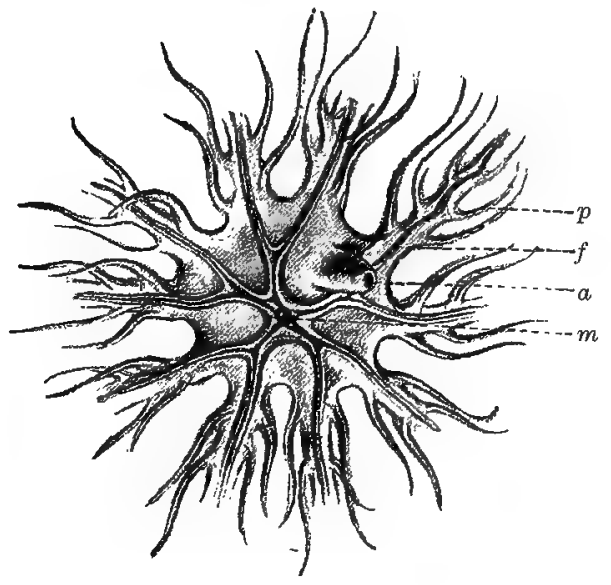

Fig. 82. Rhizocrinus lofotensis.

Fig. 83. Antedon.

Fig. 84. The up-turned surface (ventral side) of Antedon; the ten arms are cut away not far from their bases. $x$ anus, at the tip of a papilla; $f$ furrow, $m$ mouth, $p$ pinnule. -Orig.

ventral surface (or oral pole), firm and arched on the dorsal side. From the margin of the body arises a number of arms, usually five or ten, which divide frequently, sometimes repeatedly. A series of lateral branches or pinnules arises on either side of the arms, 
like the barbs of a feather. The dorsal surface is covered all over, even to the pinnules, with large, thick, calcareous plates, closely set together, forming in each arm a series of vertebra-like joints, and upon the body a calyx, in which the viscera are disposed. All these

Fig. 85 .

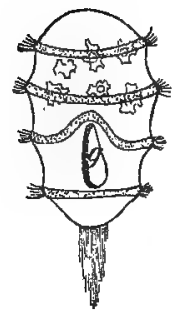

1

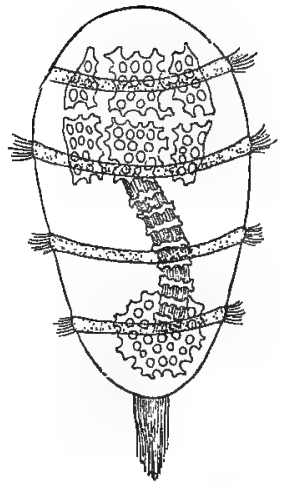

2

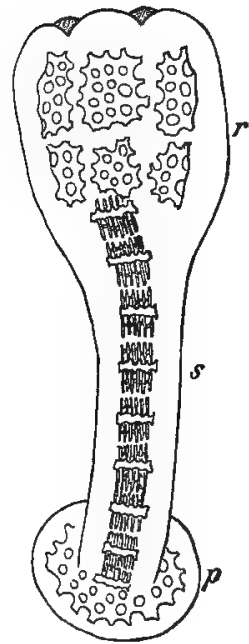

3

Fig. 85. I.arvæ of Antellon rosacea at various stages of development. 1 and 2 young free-swimming larvæ; in the latter, considerable portions of the adult skeleton are already deposited. 3 larva shortly after fixation. $g$ gastrula-mouth, $p$ pedal disc, $r$ body, $s$ stalk.-After Wyville Thompson.

Fig. 86. Another species of Antedon (Antedon Eschrichtii) $\times 4$; in the sessile condition.-After Levinson.

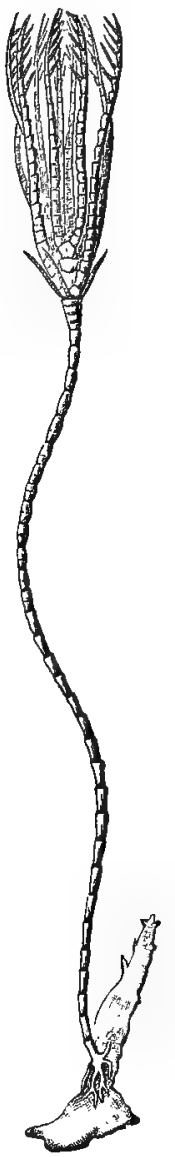

ossicles, which make up a considerable portion of the body, are calcifications of the dorsal wall. The stalk, too, is chiefly composed of a series of calcareous joints; the cirrhi, filiform, or rarely rootlike or branched processes, frequently arise from it, and contain similar calcifications. In contradistinction to the dorsal, the ventral surface of both body and arms is usually soft and little calcified. The mouth lies centrally (rarely excentrically) on the ventral surface, and a short distance off is the anus at the tip of a small conical tube in one of the inter-radii. Five ciliated furrows, ambulacral grooves, radiate from the mouth, and if only 
five arms are present, one is produced along each; whilst, if there are ten, each groove forks just as do the arms, and sends a small furrow to each pinnule. Along each edge of the furrows both of the arms and of the pinnules there is a row of small, soft feet or suckers (so-called tentacles); a water vessel runs below it, and gives off branches to the feet. (For the stone-canal, see p. 124). The genitalia, which are similar in both sexes, extend as long tubes through the arms and give off branches to the pinnules; these branches alone produce ripe reproductive cells, whilst the main trunk is sterile: the ova and spermatozoa escape by small openings in the pinnules, the latter having become much swollen by the ripe genital products.

Development is known only for Antedon, which as an adult has no stalk. The ovate body of the newly-hatched larva is provided with four ciliated girdles and with a tuft of cilia at the hinder pole. After swimming freely for some time it attaches itself by one end, which elongates, to form a stalk, whilst the arms bud out at the other pole. Later on, the body with the arms breaks from the stalk, and the animal is free-swimming for the rest of its life.

The stalked Crinoids are almost exclusively abysmal, whilst those which are free-swimming usually occur in shallow water near the coast. Crinoids $f$ e e $d$ upon microscopic organisms which are driven to the mouth by ciliary movements in the furrows already mentioned. In earlier geological periods, especially in the Silurian and Cretaceous, they were as common as they are now scarce, and were principally represented by stalked forms, in fact entirely so down to the Jurassic. Genera, species, and individuals, were all abundant.

The following may be mentioned as examples of present-day forms :-

1. Rhizocrinus lofotensis, a small, long-stalked (to 8-c/m) form, with five (occasionally 4,6, or 7) simple arms; the end of the stalk is provided with branching root-like cirrhi, by which it attaches itself to objeets at the bottom of the sea, whilst elsewhere there are none. The animal was first met with off the Lofoden Islands, at depths of 100-300 fathoms, but afterwards, also at great depths in various other localities.

2. Sea $\mathrm{Palms}$ (Pentacrinus) are large animals with ten arms, which may divide repeatedly; the strong stalk is beset with whorls of jointed cirrhi down its whole length. At great depths in warm seas.

3. Antedon or Comatula, a stalk-less Crinoid, with ten or more arms. In the young stalked condition, cirrhi occur only at the junction of the stalk and the body; these cirrhi persist after the animal has broken away, and by means of them, Antedon can climb upon various foreign objects, whilst it can swim with its arms. A. rosacea occurs in the Mediterranean and the Atlantic.

\section{Class 2. Asteroidea.}

In this class the body is always $d$ iscoid (the principal axis short) and drawn out into a number of arms (usually five), for the radii are better developed than the inter-radii. The tube-feet 
are developed only on the ventral (or a l) surface, which, unlike that of the Crinoids, is considerably more calcified than the dorsal side. The alimentary canal is very short and almost radially symmetrical. The Asteroidea are divisible into two fairly dissimilar orders, the Star fish, and the Brittle-stars.

In some of the Starfish and Brittle-stars with six arms or more, reproduction by fission has been observed; the disc divides transversely, so that two individuals are formed, each consisting of half a disc and half the number of arms; they attain the perfect form later by regeneration. Other Starfish divide by throwing off the arms; a new individual develops from eacb, whilst the disc buds out new ones at the old scars. Whilst such an asexual multiplication occurs in a few forms only, a great capacity for regeneration

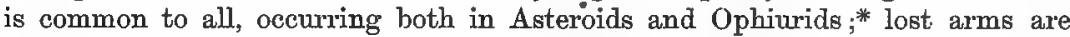
easily renewed, even if several are destroyed at the same time, and, especially among the Ophinids, there are individuals which are almost perpetually engaged in regenerating lost parts.

\section{Order 1. Asterida (Starfish).}

The flattened body consists of a disc with five or more a r m s, broadest at the base near the point of origin, and narrowing towards the tip. The disc and arms pass directly into each other without any distinct limit. The length of the latter varies very much, for in some they are many times longer than the breadth of the disc, whilst in others they are only just indicated, so that the whole animal looks like a pentagonal plate, and there are all possible intermediate forms between these two extremes.

The mouth which is without armature, lies in the middle of the oral surface; it leads into a spacious gastric cavity, circular in form, and with much-folded walls, the so-called stomach. This gives off (sometimes in pairs) ten $\dagger$ long-branched cæca, two extending into each arm; they are glandular and pour their secretion into the stomach. A circle of short, and also glandular, cæca arises from the stomach above the large ones, close to the anus; this is a small aperture almost in the centre of the dorsal surface, lying in an interradius, close to, but not at, the aboral pole: it is wanting in some forms. The madreporite, which is perforated like a sieve, also lies aborally in one of the inter-radii. Along the ventral side of each arm runs the ambulacral groove, which is continued on to the ventral surface of the oral disc as far as the mouth. The tube-feet are situated in this groove, generally arranged in two, occasionally in four, rows; each is usually provided with a sucker at

* Also in the Crinoids.

$\dagger$ In forms with five arms; with a larger number of arms there is a corresponding increase in the number of cæca. 
its tip. At the distal end of each groove, there is an unpaired filiform structure, which bears on its lower surface close to the base, several small red eye-spots: since the tips of the arms are curved upwards, the eyes look up in spite of their position on the ventral surface. The genital apertures usually occur on the dorsal surface of the disc; two or more tiny openings in each interradius. The body-wall is much calcified, especially on the ventral surface; a series of yoke-like calcareous plates movably jointed

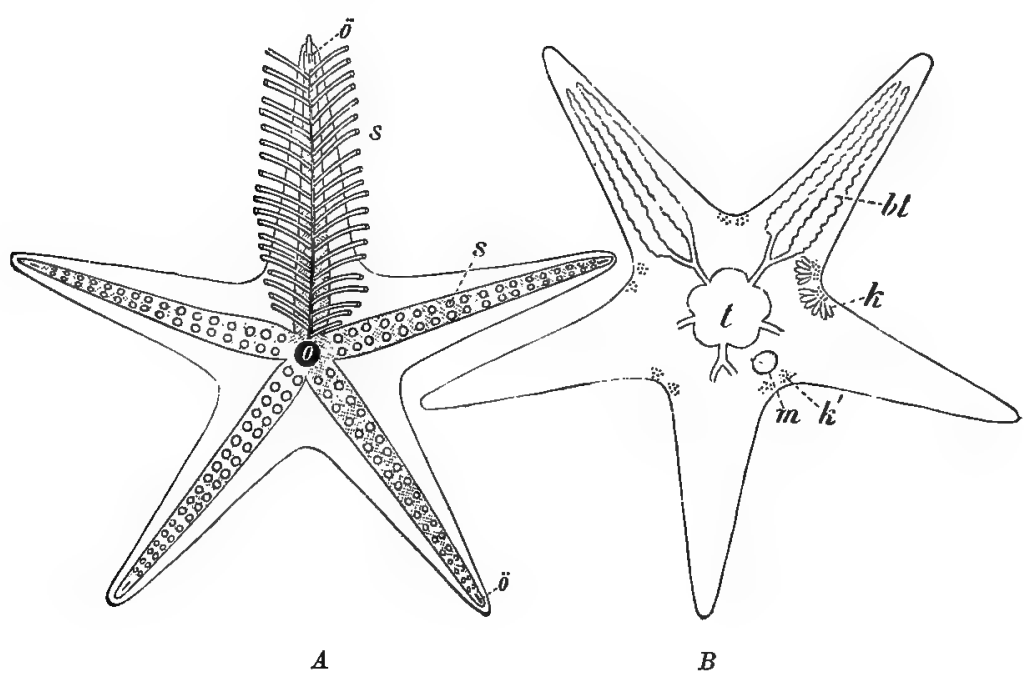

Fig. 87. Diagrammatic figures explaining the structare of a Starfish. A oral, $B$ aboral view; in $B$ some of the internal organs are figured. $b l$ cæcum of the stomach, $k$ genital gland, $k^{\prime}$ genital pore, $m$ madreporite, $o$ mouth, $s$ tube-feet, $t$ stomach, $\ddot{o}$ eyespots.-Orig.

together, and each consisting of a pair of closely-connected calcifications, roofs in the ambulacral groove: the radial water-vessel and the radial nerve lie ventral to these ossicles. The upper side of the body is less strongly calcified; numerous delicate thin-walled outgrowths of the body-wall, which may be regarded as gills, project from it: they are not connected with the vascular system, nor do they contain blood-ressels. Dorsally, at the edges of the arms, and ventrally, as far as the margins of the ambulacral grooves, numerous movable or fixed spines may occur together with pedicellariæ, which are sessile, or provided with short stalks.

In order to ingest large animals, Lamellibranchs, Sea-urchins, and the like, the Starfish evert the stomach through the mouth, so as to cover the prey, which is killed by the action of the digestive juices, and its soft parts dissolved and absorbed. Smaller animals are received entire into the non-everted stomach, the indigestible portions 
being thrown out again from the mouth, for the anus plays only a subordinate part.

The species of Starfish are numerous, and occur in all seas. The following may be cited as examples:

1. Asterias rubens, a five-rayed form, with the tube-feet arranged in four rows, and each foot furnished with a sucker. Very common in North European seas, occurring on the shores and to considerable depths. It is inimical to Oyster beds, also causing havoc by devouring Fish caught in nets or on hooks. Those from deeper water attain a breadth of $50 \mathrm{c} / \mathrm{m}$., the littoral ones are much smaller.

2. Solaster, Starfish of considerable size, with a large number of arms (about ten), tube-feet with suckers, in two rows. In North European seas.

\section{Order 2. Ophiurida (Brittle Stars).}

The arms, usually five in number, are long and narrow, and do not meet at their bases; the edge of the disc between each two arms is usually straight, or somewhat bulging. In addition, the dorsal walls of the arms, by a different arrangement of the ossicles, generally differ somewhat in appearance from that of the disc; so that they seem to be well marked off from this, especially when examined from above. They differ, further, from those of the Starfish, in the absence of ambulacral grooves; the ventral surface is flat, and usually covered with calcareous ossicles which lie ventral to the radial water-vessel ; dorsal to it are vertebralike ossicles, which constitute the chief part of the arm, and are similar to those of the Starfish, although developed somewhat differently. The tube-feet, which are without suckers, are arranged in two series on the ventral surface, near the margin of the arms; and on the disc, near to the mouth. The aboral surface of

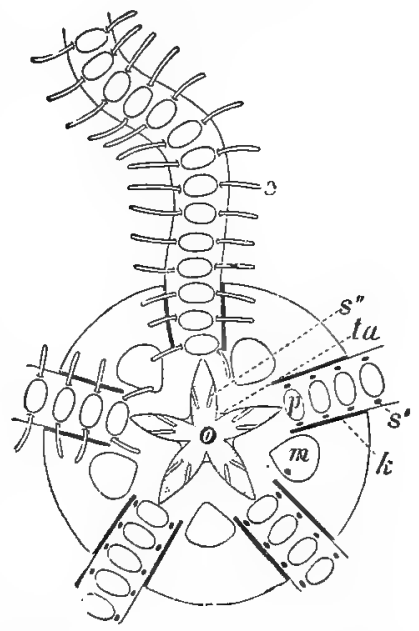

Fig. 88. Diagram explaining the structure of an Ophiurid, seen from below. $k$ slit-like aperture of a bursa, $m$ madreporite, $o$ month, $p$ one of the ossicles from the ventral surface of the arms, $s$ tube-feet, $s^{\prime}$ point of origin of one of them, $s^{\prime \prime}$ tube-feet round the mouth, $t a$ denticles.-Orig.

the arms is usually covered with large calcareous plates, that of the dise is generally soft, with larger or smaller calcifications. The arms are very flexible, and are capable of a serpentine movement. The circular mouth lies in a stellate depression, the corners of which are in the inter-radii, and are furnished with denticles. The stomach is a wide sac, occupying the greater portion of the 
body; large cæca are wanting, there are only short pouches, which do not extend into the arms. There is no anus. The opening or openings of the stone canal are in the madreporite, which lies close to the mouth. On the lower side of the disc, close to the bases of the arms, there are ten narrow slits, leading into the same number of sacs,* which have a respiratory significance (bursæ). On their walls are the genital glands; ova and spermatozoa escape into the sacs, and leave the body through the slits. Eyes, pedicellariæ, and gills are absent, but longer or shorter spines, which are important in locomotion, occur especially along the edges of the arms.

Those Ophiurids which are unable to evert the stomach, feed upon dead animals, or upon such as are not capable of resistance; they gnaw their food with the denticles mentioned above.

1. True Brittle-stars (genus Ophiura, etc.). With five (rarely a larger number) of simple arms; occurring in all seas, and represented in those of northern Europe by a number of nearly allied species. Some are spiny, others smooth. They may often be found climbing over foreign objects with the aid of their arms.

2. Astrophyton, distinguished from the true Ophiurids by the fact that the five arms, which can be rolled up towards the mouth, are much branched. The dermal skeleton is somewhat less developed than in the true Ophiurids, and they can swim like the Antedons. They attain a considerable size. Species of this genus occur in northern seas, but are much less abundant than the former.

\section{Class 3. Echinoidea (Sea-urchins).}

In some Sea-urchins the body is almost spherical, but in most, on account of the shorter main axis, it is flattened or occasionally discoid; arms are completely absent. The greater portion of the body-wall is furnished with immovably connected calcareous plates. In the so-called Regular-urchins, with spherical body (the transverse axes being of about equal lengths), there are twenty rows of these plates extending meridionally from one end of the principal axis to the other. Ten of the rows bear fine pores, each plate having one or several pairs; $\dagger$ each pair of pores corresponds with a tube-foot. In each radius there are two series of these, pore or ambulacral plates, whilst in each interradius there are two interambulacral plates. The latter are often broader than the pore plates, and like these are covered with larger or smaller, nearly hemispherical $\mathrm{knobs}$, each of which bears

\footnotetext{
* Sometimes twice this number of slits is present, each of the original ones being divided by a transverse bridge; but the number of sacs remains the same.

+ Each pore plate bears primitively only a single pair of pores; in consequence of the fusion of several plates, however, there are several pairs in most of the Regularurchins.
} 
a small, smooth, wart-like elevation; the largest knobs occur on the imperforate interambulacral plates. The upper ends of the twenty rows of plates touch upon a circle of ten apical plates, five

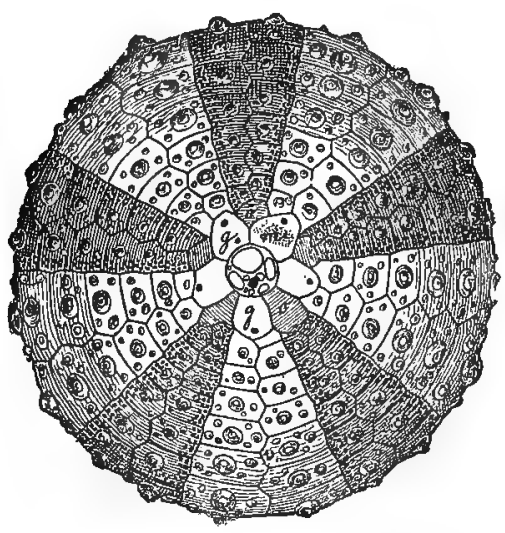

A

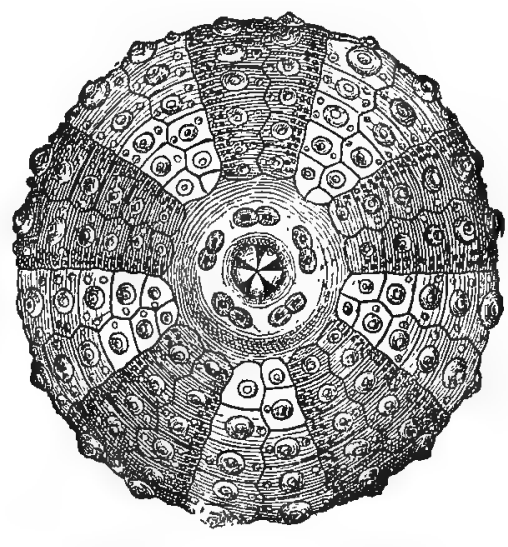

$B$

Fig. 89. Shell of a Regular Sea-urchin, Toxopneustes Droebachiensis (young specimen, enlarged), from above $(A)$ and from below $(B)$. The radii are dark. $g$ genital plate, $m$ madreporite, o ocular plate. In the middle of $A$ is the anal area with the anus.Orig.

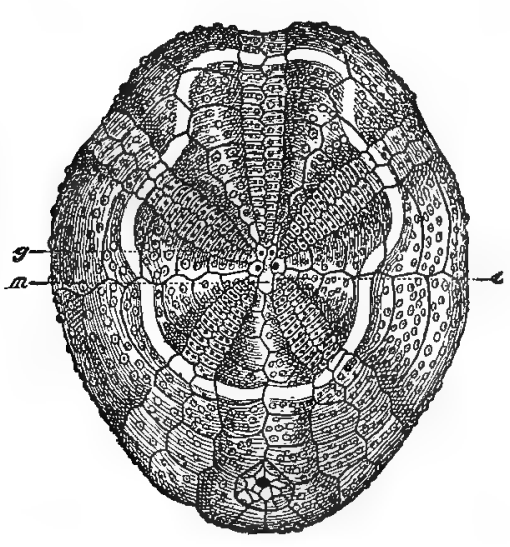

A

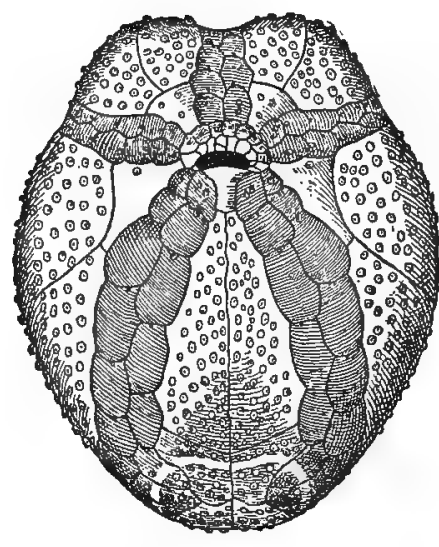

$B$

Fig 90. Shell of an Irregular Sea-urchin, Brissopsis lyrifera (young specimen, enlarged), from above $(A)$ and below $(B)$. (The radii in $A$ are not dark enough). Posteriorly in $A$ the anal area may be seen. The white bands are areæ with verJ small spines.-Orig.

large and five small, with which they are immovably connected; in each inter-radius lies one of the larger, in each radius one of the smaller plates. Each of the larger plates is pierced by the opening of a genital gland, and they are therefore termed the genita 
plates. One of them is larger than the others, and is the madreporite, for it exhibits, besides the sexual aperture, n number of other delicate pores, allowing of the entranes of watur into the stone canal which is attached at this point. Each of the tive smaller plates is similarly perforated by an opening, smaller than the genitnl pore, through which a nerve passes, to be distributed to the skin in the region of the aperture; this spot is particularly sensitive; the plates are termed ooular plates, because it wis formerly believed that each bore an eye. Tho apical plates surround " smull membranous region, the anal area, in which the anus opens, usually somewhat excentrically; it is provided with small, movablyconnected, calcareous plates. The lower culs of the ambulacral and interambulacral series of ossicles surround in linge space, the peristome, on which also there is no connected skildem, though it is furnished with a certain number of larerer or smmller calcareous plates: the mouth is central.

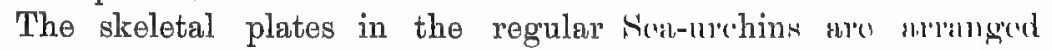
on this plan; other less regular typos may be derived from it. A simple departure occurs in certain Sica-urchins, which ure yet considered as "regular"; for although the wholl is ovate, instead of round, the general structure conforms to this type

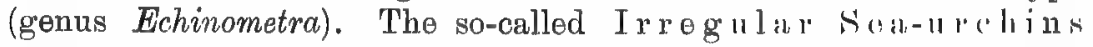
are more aberrant; the whole of the mal arcil his moverl from the circle of apical plates, into one of the inter-radii, and lic's between two rows of interambulacral plates at some distance firom the apex, occasionally even close to the oral aren; the aprial pluter draw together dorsally, and the regular structure of the shcll nay be almost completely retainod, even the spherical l'orm. 'T'le intur'radius, in which the anal area licis, is termed postcinisl. The loss of radial symmetry is greater if, as in malny Irrcumular. Sea-urchins (Fig. 90 B), the mouth no longer licis in the c'int re of the ventral surface, but more antoriorly; this has a mentkerl affecet. upon the whole structure of the animal, nince the mouth lials not moved into a radius, but retains its position at the lower pole of tho principal axis, where it is still the meeting-place of all the radii

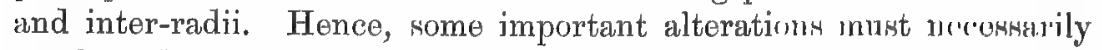
result; the development of both radii and inforremelii is assontially; changed (see Fig. 90 B). Twenty rows of pliten mily, lowwerver',

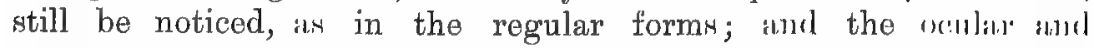
genital plates remain as before, excepting that the lidter ine usually only four, or even fewer, in number. Many of the l'regulur ficlinoirls (see Fig. 90), are also peculiar in that the ambulacral platis are differently developed on the dorsal and ventral sides of the sholl, in correspondence with differences in the tube-f(et, to be dencribed; often, too, the ambulacral plates of the anterior radius diffor l'rom the rest. 
Movable calcareous spines articulate with the smooth prominences of the numerous tubercles mentioned above, and are attached to the shell by muscle fibres. In the Regular Urchins the spines are usually of considerable importance; in some, they are very long and thick, and serve as locomotor organs, accessory to the tube-feet; in irregular forms, on the contrary, they remain small and thin, or even bristle-like. All the spines in the same individual, however, are by no means of equal size; those with the large kind also exhibit smaller, or even quite tiny, ones. The spines are straight, and rounded in section; but some are curved and flattened. Like the shell, the spines are calcifications of the body-wall, and like the shell also, they are covered with a soft superficial layer, which is, however, often worn away from the tip.* Stalked or sessile pedicellariæ are also articulated with the shells ( $c f . p .124)$.

In the Regular Sea-urchins the tubefeet are usually all alike; at the end of each is a sucker, supported by a perforated calcareous plate; occasionally those on the dorsal surface are

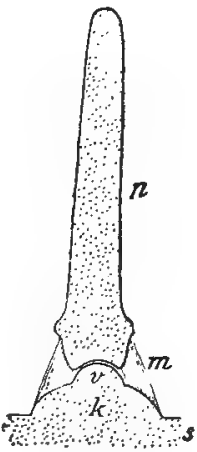

Fig. 91.

Diagrammat $c$ longitudinal section of the $\mathrm{spine}$ of a Sea-urehin. $s$ shell, $k$ tubercle, $v$ wartlike process of the same, $p$ spine, $m$ muscles.-Orig. pointed and compressed. In many Irregular Urchins, however, several forms occur: 1. true tube-feet with suckers; 2. a similar kind, but with rounded ends; 3. a brush-like form, with numerous threads at the ends, modified as tactile organs, and occurring near the mouth; 4. dermal branchiæ, i.e., leaf-like appendages, notched at the edges, present on the dorsal surface.

The mouth in the regular and in some of the irregular forms, is armed with a circle of five very powerful calcareousteeth, supported on a somewhat complicated framework of calcareous pieces, the so-called "Aristotle's lantern"; in most of the irregular forms, however, there are no teeth. The alimentarycanal is a long, cylindrical, much-coiled tube, occupying the greater portion of the shell cavity. The position of the anus has been already described.

In the Regular Urchins the lantern is again surrounded and supported by a calcareous ring provided with five upwardly directed processes, and connected to the lower rim of the shell. In these forms the masticatory apparatus occupies a considerable part of the whole cavity. The so-called siphon, or accessory intestine, is a very peculiar structure occurring in most Echinoids. It consists of a fine canal, running parallel to the gut for part of its length, and opening into it at either end; in some forms this canal is wanting, and instead there is a groove on the inner side of the gut. It is conceivable that the accessory intestine arose by the constriction of a groove such as this.

* In very large spines the soft covering may be present only at the base. 
The peristome, in most of the regular forms, bears ten dendritic outgrowths of the body-wall, the oral branchiæ, close to the edge of the shell; in others, however, they are wanting.

On the ventral side of the shell, close to the peristome, there are attached to the pore-plates of most Echinoids tiny globular structures, with short stalks and a glassy, calcareous skeleton. These so-called spheridia are probably senseorgans, possibly gustatory or olfactory.

Urchins occur in all seas, and are abundant both in genera and species. They were also well represented in earlier geological periods.

\section{Order 1. Echinoidea regularia (Regular}

Sea-urchins).

The anal area lies at the upper pole. The body is usually cpproximately spherical. Spines strong. Teeth present. Dermal branchiæe usually present.

The Regular Sea-urchins feed partly upon other animals, e.g., large Crustacea, which they catch by means of their tube-feet; partly upon the Polyzoan and Hydroid colonies attached to them, and also to a certain extent upon Algæ. Some of them use their teeth to form small cavities in the rocks in which they live.

As examples may be given : Cidaris, with long strong spines, without dermal branchiæ; Echinus, with smaller spines, to which Toxopneustes (Fig. 89) is closely allied; Echinometra, with an oval shell. The genus Asthenosoma differs from other Urchins, in that the skeletal plates, which are imbricate, are movably connected.

\section{Order 2. Echinoidea irregularia (Irregular}

Sea-urchins).

The anal area has moved into an inter-radius. The body is round or, more often, ovate. Spines small, often bristle-like. Usually toothless. Dermal branchiæ absent.

1. Shield-urchins or Clypeastridæ (genus Clypeaster, and others) differ from other Irregular Sea-urchins in the possession of te eth. Shell thick; mouth in the centre of the ventral surface. Rarely found in European seas.

2. Heart-urchins or Spatangidæ (genus Spatangus, etc.), toothless, shell usually thin, mouth moved forwards. Feed by ingesting material from the bottom of the sea. Several species in the North Sea, among them Brissopsis lyrifera (Fig. 90).

\section{Class 4. Holothuroidea (Sea-Cucunbers).}

In the Holothurians the principal, is always longer than the lateral, axis, usually several times as long, so that the body is cucumber-, sausage-, or worm-shaped. In correlation with this, the Holothurians 
do not rest on one pole as do other Echinoderms, but upon one side of the body; and, as a consequence, one side is often specially developed or even flattened (Fig. 93), so that external radial symmetry is more or less destroyed; the side turned downwards is termed ventral, the other dorsal.

Fig. 92.

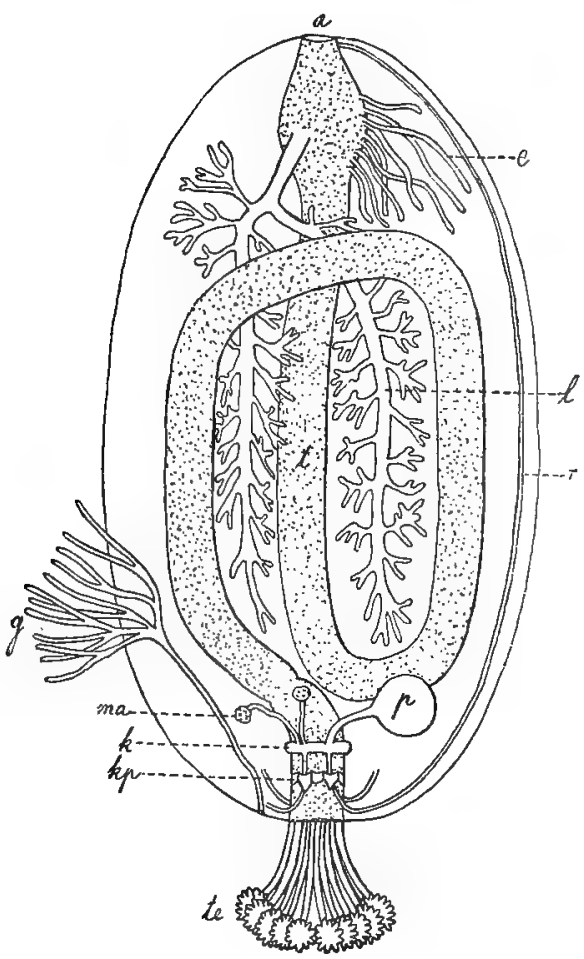

Fig. 93.

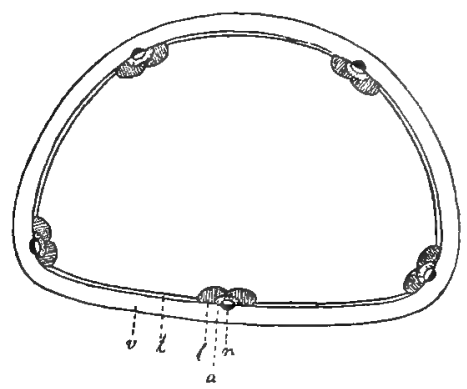

Fig. 94.

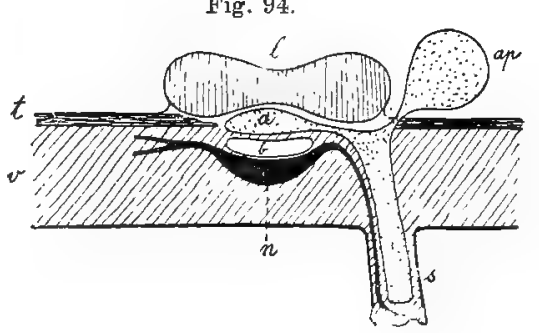

Fig. 92. Diagram of a Holothurian; the body-wall is cut through and spread out. $\alpha$ anus, $c$ Cuvierian organs, g gonad, $k$ ring canal, $k p$ calcareous ring, $l$ respiratory tree, ma madreporite, $p$ polian vesicle, $r$ radial water vessel, $t$ gut, te tentacles.-Modified from Ludwig.

Fig. 93. Transverse section of the body-wall of a Holothurian, diagrammatic. a Radial water vessel, $l$ longitudinal muscle, $n$ radial nerve (the white spot above $n$ is the radial blood vessel), $t$ transverse muscle, $v$ body-wall.-After Ludwig.

Fig. 94. Transverse section through a $\mathrm{radius}$ of the body-wall of a Holothurian. ap ampulla, $b$ radial blood vessel, $s$ tube-foot; other letters as in the preceding figure.After Ludwig.

Another feature which is very characteristic of the Holothurians is the softness of the body-wall; the wall is indeed, as in other Echinoderms, provided with calcifications, but to so slight an extent as to render it impossible to speak of a dermal skeleton. The calcifications are usually in the form of minute, often microscopic, particles 
of varied and often beautiful form, anchors, wheels, etc.; they are sometimes large, scale-like, and projecting. The anterior region of the digestive tract, is, however, surrounded by a number (usually ten) of large calcareous plates, forming a calcareous ring, from which various muscles take their origin.

The $t u b e-f e e t$ of some forms are arranged in five radial longitudinal rows (Fig. 75, 4), just as in the Sea-urchins; in others they are more irregularly distributed over the whole surface. Sometimes the dorsal tube-feet do not possess suckers, and herein differ from those on the ventral surface; or they may be wanting on the dorsal side; or again they may be altogether absent.

The mouth is surrounded by a circle of tentacles (10-30), which are usually branched (plumose or arboriform). They are hollow, and like the tube-feet, are connected with the water vascularsystem; each is traversed by a large vessel, which arises from a radial canal, or occasionally direct from the ring canal, and is usually provided with an ampulla. Probably the tentacles are to be regarded as specially modified tube-feet. In most, the stone canal (or canals, for there are often several present), is not connected with the body-wall, but opens by means of a perforated madreporite into the body cavity.

The alimentary canal is a cylindrical tabe, which is usually longer than the principal axis, and forms a large loop. The mouth and anus are situated at opposite poles.

In most Holothurians two "respiratory trees" open, either separately, or by a short, common stem, into the rectum; they are large, hollow, arboriform organs by which water is taken into the rectum and expelled again; their function is respiratory. In some forms "Cuvierian organs" are also attached to the rectum; they are saccular or racemose glandular structures of unknown function. The genitalia are only developed in one interradius: the sexual aperture is situated dorsally, usually close to the tentacles. Most Holothurians are of separate sexes, some few are hermaphrodite.

In the body-cavity of some forms (Synapta and its allies), especially on the mesenteries, there are small stalked, slipper-shaped bodies, the cavities of which are lined with long cilia: the significance of these ciliate organs is unknown.

Many forms feed, like the Spatangidæ, by ingesting sand and mud, with the contained organic particles; others remain with outstretched tentacles and from time to time draw them, one after another, into the mouth in order to obtain the small organisms which have become entangled in their branches. They crawl slowly about by means of their tube-feet; many bury themselves in the sand. They occur in all seas.

Many react to a powerful stimulus (a rough touch, or the like) by energetically contracting the body-wall, so that a large portion of the alimentary canal and 
other viscera is forced out through the anus. The lost organs are replaced by regeneration. Other forms (Synapta) when irritated, constrict transversely and break into several pieces.

The following may be cited as examples : Cucumaria, tube-feet in five double rows from the mouth to the anus, arborescent tentacles: Holothuria, with scattered tube-feet, conical on the dorsal surface, cylindrical on the ventral, tentacles peltate: $P$ solus, with the tube-feet confined to the flattened ventral sufface, calcareous scales dorsally : Synapta, without tube-feet, vermiform, with small tentacles, and microscopic calcareous anchors in the transparent skin. The genera mentioned are all found in European seas. Recently a number of peculiar abysmal forms, with flattened ventral surface, and long processes of the body, have been discovered (Elpidia and others). 


\section{Phylum 3. Platyhelminths (Flatworms).}

The Platyhelminths are bilaterally symmetrical, unsegmented, and usually almost flat. The body is soft, and appendages are wanting, though ventrally, muscular suckers are frequently present. There is no body-cavity, all the organs are imbedded in a mass of

Fig. 95 .

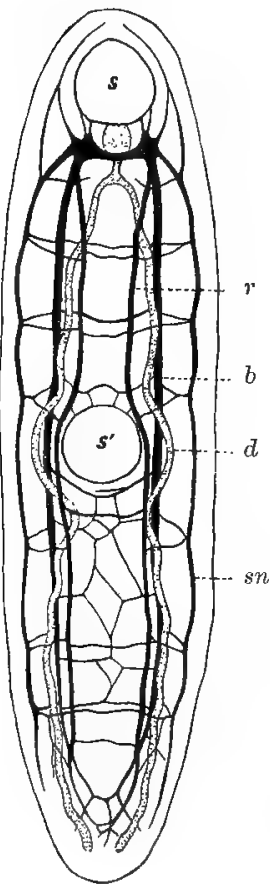

Fig. 96.

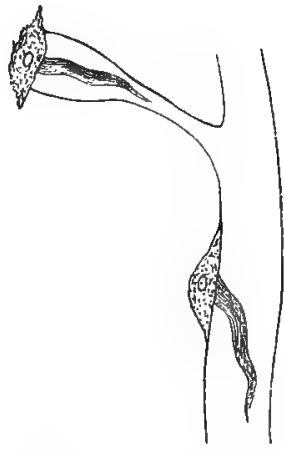

Fig. 95. Nervous system of Distomum (viewed from the dorsal side, the ventral suckers showing through). $r$ dorsal nerve, $s n$ lateral nerre, $b$ ventral nerve, $d$ digestive tube, $s$ anterior, $s^{\prime}$ posterior, sucker.-After Gaffron.

Fig. 96. Part of the excretory system of a Flatworm; diagrammatic. -Orig. soft connective tissue ; the anus, and also the vascular system are usually absent (the Nemertines excepted). The digestive tract is either a simple sac, or it is branched ; it is wanting in many parasitic, and even in some freeliving, forms. The central nervous system is represented by a paired nerve-ganglion, which lies anteriorly, and from which the nerves to the different parts of the body issue. From the hinder end of the brain, several longitudinal stems arise, and are frequently united by delicate transverse commissures. Sornetimes e y es are present, more rarely a uditory organs; they are situated anteriorly, are simple in structure, and small in size. The excretory apparatus is in the form of a much branched system of thin-walled tubes, which 
usually open in the hind region of the body by a single or double aperture: occasionally several openings are present. The principal tube sometimes exhibits a contractile enlargement just in front of the aperture (urinary bladder). The finest terminal branches of the canal-system are especially characteristic; each ends in a little cupshaped swelling, closed by a large cell (flame cell), which bears, on the side turned towards the lumen of the tube, a very powerful flagellum. Similar flame cells may also be fuund at other points in the wall of the tube. The male and female genitalia (Fig. 99) are usually present in the same individual, and are, as a rule, of a very complicated structure; testes and ovaries are often present in great numbers, and, besides these, various organs accessory to each system, as also a uterus. The genital aperture is usually ventral. Generally there is a copulatory organ.

Amongst the accessory sexual organs, the $\mathrm{yolk}-\mathrm{gland}$ (vitellarium), which is of very common occurrence, may be specially noticed. In it the so-called yolk-cells are formed, to be enclosed with the ovum in the egg membranes, and to serve later as food for the embryo. Shell.glands are generally present; their secretion, when hard, forming the firm shell surrounding the eggs of many forms.

A. Without anus or vascular system. Hermaphrodite with complicated genitalia.

1. Turbellaria. As a rule free-living, surface ciliated, alimentary canal generally present.

2. Trematoda. Parasites, without cilia. Alimentary canal present.

3. Cestoda. Parasites, without cilia. Alimentary canal'always absent. As a rule form chains.

B. With anus and vascular system. Separate sexes. Genitalia simple.

4. Nemertina.

\section{Class 1. Turbellaria.}

The Turbellaria are animals of varying, but usually small, size. The body is uniformly ciliate, which is important for locomotion as well as for respiration; some are active, many magnificently

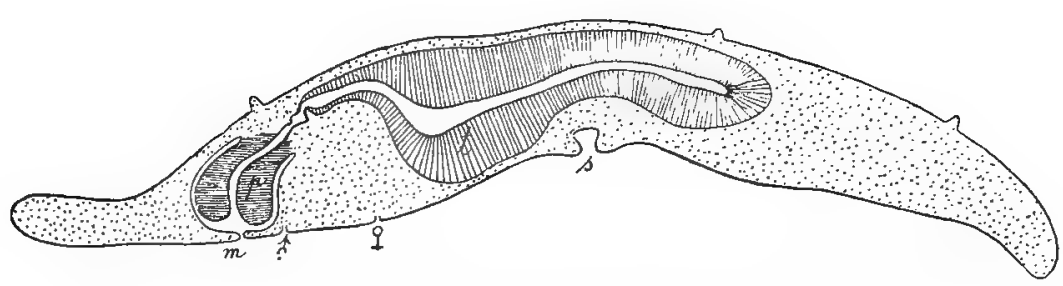

Fig. 97. Longitudinal section through a Turbellarian (Cycloporus papillosus), to show the situation of the pharynx. Of the organs caught in section only the gut is figured. $m$ mouth, $p$ retracted pharynx, $r$ dorsal papilla, s sucker, $t$ gut; $\delta^{*}$ male, $q$ female aperture, enlarged.-Adapted from Lang. 
coloured. Sometimes nematocysts, like those of the Ccelentera, are found in the skin. Eyes in varying number, and sometimes a u ditory $\circ \mathrm{rg}$ a $\mathrm{ns}$, are present; there is frequently a pair of short tentacles upon the front end, whilst the rest of the body is usually bare; a little sucker is, however, often present on the ventral surface. The mouth is ventral, sometimes near the anterior end,

Fig. 98.

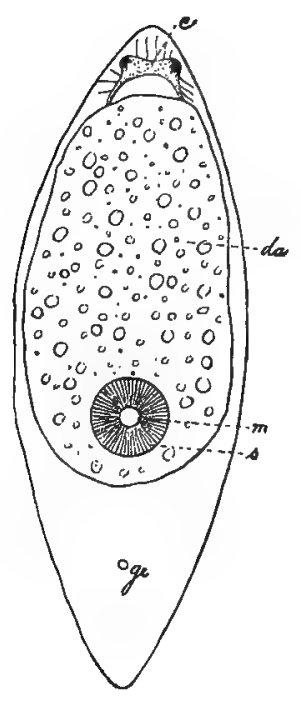

Fig. 99.

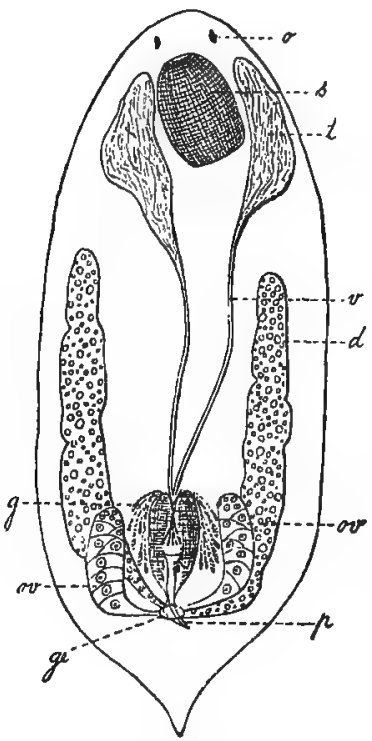

Fig. 98. Sketch of a rhabdoccelous Turbellarian (Meso. stomum splendidum) showing the digestiye tract $d a$, and the cerebral nerve mass $c$; ge genital aperture, $m$ month, $s$ pharynx. Enlarged.-After v. Graff.

Fig. 99. Sketch of a rhabdocœlous Turbellarian (Provortex affinis) with the genitalia. $d$ yolk-sac, $g$ glands in connection with the male apparatus, ge genital aperture, o eye, ov ovary, $p$ penis, $s$ pharynx (the gut is omitted), $t$ testis, $v$ vas deferens. Enlarged.-After v. Graff. sometimes in the middle, or again near the hind end; it leads into a buccal cavity, which is often furnished with a peculiar retractile, muscular pharynx. This is a longer or shorter canal, open at both ends, and firmly attached by one end to the hind part of the buccal cavity, whilst the other end can be everted through the mouth to seize the food. In other cases the so-called pharynx is simply a specially muscular portion of the walls of the buccal cavity, and is not protrusible. In a few cases even this is absent. The mouth communicates with the true $\mathrm{g} u \mathrm{t}$, which, in some forms (Rhabdocoela), is a simple sac, whilst in others it is.
branched like the veins of a leaf (Dendrocoela). Some forms (Accela) have no digestive tract, even though the mouth is still present; food is taken in and digested in the soft parenchymatous tissue of the body. The hermaphrodite genital apparatus opens on the ventral side, either by a common aperture or by separate pores. for the male or female glands. In some members of the group, reproduction by transverse fission occurs; some marine. Turbellaria undergo a metamorphosis. The larva is freeswimming, and is furnished with processes which do not appear in. the adult. 
The Turbellaria live in fresh-, or salt-water, or eren on land in damp places. By means of the ciliary morements, and by slight movements of the body-muscles, they glide through the water and

Fig. 100.

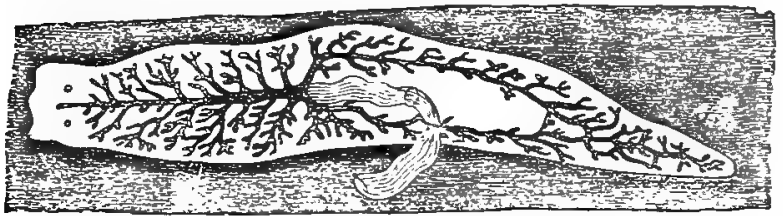

Fig. 101.

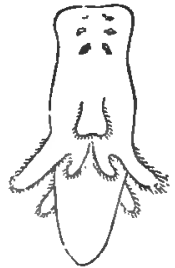

Fig. 100. Planaria lactea, a fresh-water Tarbellarian, with everted pharynx.-After 0. Smidt.

Fig. 101. Larva of a marine Turbellarian, enlarged.-After Lang.

orer any foreign objects found therein. They feed upon other animals, e.g., small Crustacea, which are seized br the pharynx and sucked out.

Both terrestrial and fresh-water forms occur in England; of the latter, species of the genus Planaria (branched gut) which sometimes attain a length of $2 \mathrm{c} / \mathrm{m}$. (Fig. 100) are the best known. Many species are found in all seas.

\section{Class 2. Trematoda.}

The Trematodes, all of which are parasitic, are nearly allied to the Turbellaria, differing from them in that the superficial cilia of the larra are lost later; they are usually pale or inconspicuous in colour. The body, which is of a firmer consistency than that of the Turbellaria, is prorided with a definite cuticle, which sometimes bears little spines, and is furnished with a varying number of small suckers, sometimes also with chitinous hooks. These adhesive organs are specially developed in ecto-parasitic forms. Eyes are wanting in the internal parasites, whilst in the external ones they are often present. The $\mathrm{mouth}$ is frequently situated at the base of a sucker, and usually at the anterior end of the body. It leads into a pharynz with muscular walls, which acts as a pumping apparatus. The pharynx is continued into the true $g u t$; this is rarely a simple sac, more often* forking into two lateral branches,

* In the asexual generations of many forms the gut is, however, only a simple sac; in others it is entirely wanting. (See the description of Distomum hepaticum given below). 
which in some forms give off more delicate branches (Fig. 102), in others are unbranched (Fig. 95). The genital openings are usually on the ventral side close together, and far forward. The Trematodes are invariably hermaphrodite, but in some (see below) female parthenogenetic generations occur.

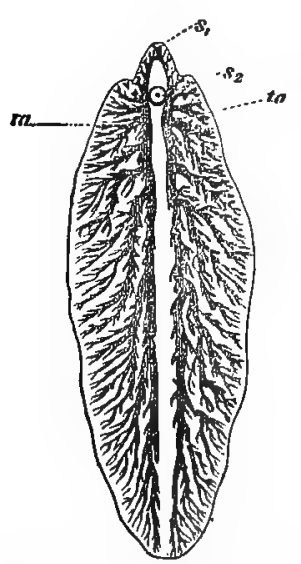

Fig. 102. Sketch of Liverfluke, showing alimentary eanal $\times 2 . s_{1}$ anterior, $s_{2}$ posterior sucker, ta gut.-After Thomas.

The ectoparasitic forms pass through a ciliated larval stage, during which they swim freely in the water. The reproduction of most of the endoparasites is far more complicated. The different generations alternate with one another, a hermaphrodite being followed by one or more parthenogenetic generations, so that a heterogony occurs. A ciliated larra hatches from the fertilised egg of the hermaphrodite generation, migrates into a lower animal, generally a Gastropod, and there develops into an imperfect female, within whose body the eggs are formed; an alimentary canal is wanting or occurs as a simple sac. The eggs produced by this generation develop without fertilisation in the body of the parent, and these individuals, in some forms, constitute the hermaphrodite generation, after they have been received into another host (a Vertebrate). In others, however, they form a second female (parthenogenetic) generation, which then produces the hermaphrodites. These are always far superior to the others in size and in complexity of organisation, and in many forms they alone are known.

\section{Order 1. Polystomeæ (Monogenetic T'rematodes).}

Almost always ectoparasitic: with usually more than two suckers, often possessing hooks: without heterogony. The majority are parasitic upon Fish (skin and gills).

1. The genus Tristomum includes large species (to almost $2 \mathrm{c} / \mathrm{m}$ long); characterised by a very large sucker at the hind end, and two smaller ones anteriorly; they are parasitic on many marine Fish.

2. Polystomum integerrimum (Fig. 105), lives in the urinary bladder of the Frog; anteriorly there are four eyes; posteriorly six large suckers, and a number of hooks of different sizes. The eggs are laid in the spring, pass to the exterior through the cloacal aperture of the host, and develop in the water in the course of some weeks. The larva is furnished with a ciliated girdle, with eyes, and with a circle of sixteen hooks upon a dise at the posterior end; the sucker of the adult is absent. The larva wanders into the gill cavity of the tadpole, where it loses the cilia and develops one or two pairs of suckers; it remains there until the gills of 
the host begin to atrophy, and then wanders (probably through the alimentary canal of the Frog) into the urinary bladder, where its further development takes place.

3. Diplozoon paradoxum, "the Double-animal," lives in the gills of different fresh-water Fish. The larva is furnished with cilia, which atrophy after it has attached itself to the gills. The young parasite is an elongate animal with two suckers in front and several behind; there is, further, a median ventral sucker, and

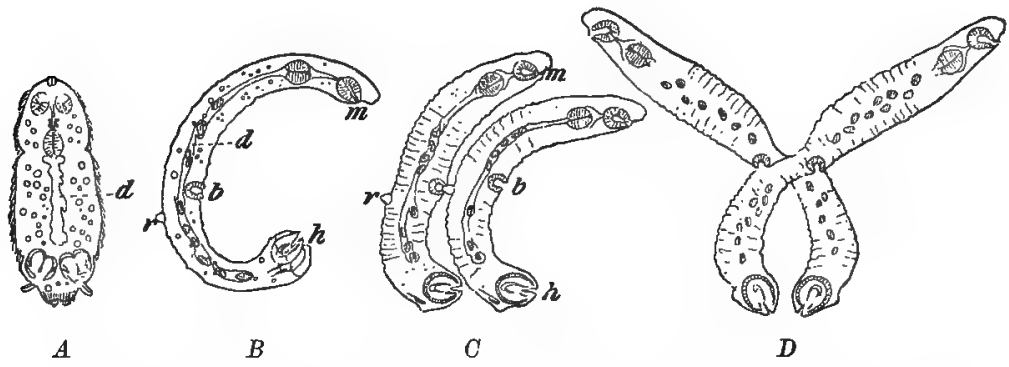

Fig. 103. Diplozoon paradoxum. $A$ free-swimming larvæ. $B$ single individual. $C$ two individuals which have begun to unite, the left has seized the dorsal papilla of the right with its ventral sucker. $D$ The same after fusion, each has seized the dorsal papilla of the other, $b$ ventral sucker, $d$ gut, $h$ adhesive apparatas of the posterior end, $m$ mouth, $r$ dorsal papilla.-After Zeller.

upon the dorsal side, just about opposite to this, a conical papilla. After some time the young animals unite in pairs, in such a way that one seizes the dorsal hump of another with its ventral sucker, and the latter turns round to clasp the dorsal hump of the former in a similar manner, so that the animals are fastened together cross-wise. They coalesce and remain attached throughout life. After the fusion, the animal still grows considerably.

\section{Order 2. Distomeæ (Digenetic Trematodes).}

Endoparasites with one or two suckers, or wholly destitute of them: with a heterogony. The hermaphrodite generation occurs in Vertebrates, the parthenogenetic in lower animals.

1. Distomum hepaticum, the Liver- $\mathbb{H}$ u ke. The hermaphrodite generation frequently occurs in the bile ducts, especially of Sheep and Cattle (more rarely of other Mammalia); length, $3 \mathrm{c} / \mathrm{m}$. Besides the anterior sucker which surrounds the mouth, there is a small ventral one at some distance from it. The animal feeds upon blood. The microscopic ova are carried by the bile into the digestive tract of the host, and are conveyed thence to the exterior. If they fall into water or some damp place, each hatches into a ciliated larva provided with two eyes; and a small papilla at the front end, by means of which it bores through the skin of a particular species of fresh-water Snail, Irimneus truncatulus; if this little Snail is not forthcoming, the larva perishes. In the Snail, the ciliated covering is lost, and numerous ova are formed in the small acolous animal, the sporocyst (first asexual generation), during its growth. The ova hatch within the sporocyst, and each develops into a small animal, a redia (second asextlal generation), differing from the parent, in the possassion 
of a pharynx and a simple sac-shaped gut. The redim break through the maternal body-wall and wander about in the Snail, consuming its liver. In these, eggr are again formed, which become "cercariæ," with a branched alimentary canal and a caudal appendage. They escape through an opening in the maternal tissues, and later from the body of the Snail also. The cercaria swims actively for some time in the water, then it attaches itself to some plant, loses its tail, and secretes a mucous-substance which hardens into a cyst round the body. If it be swallowed with the food by a Sheep or an $\mathrm{Ox}$, the capsule

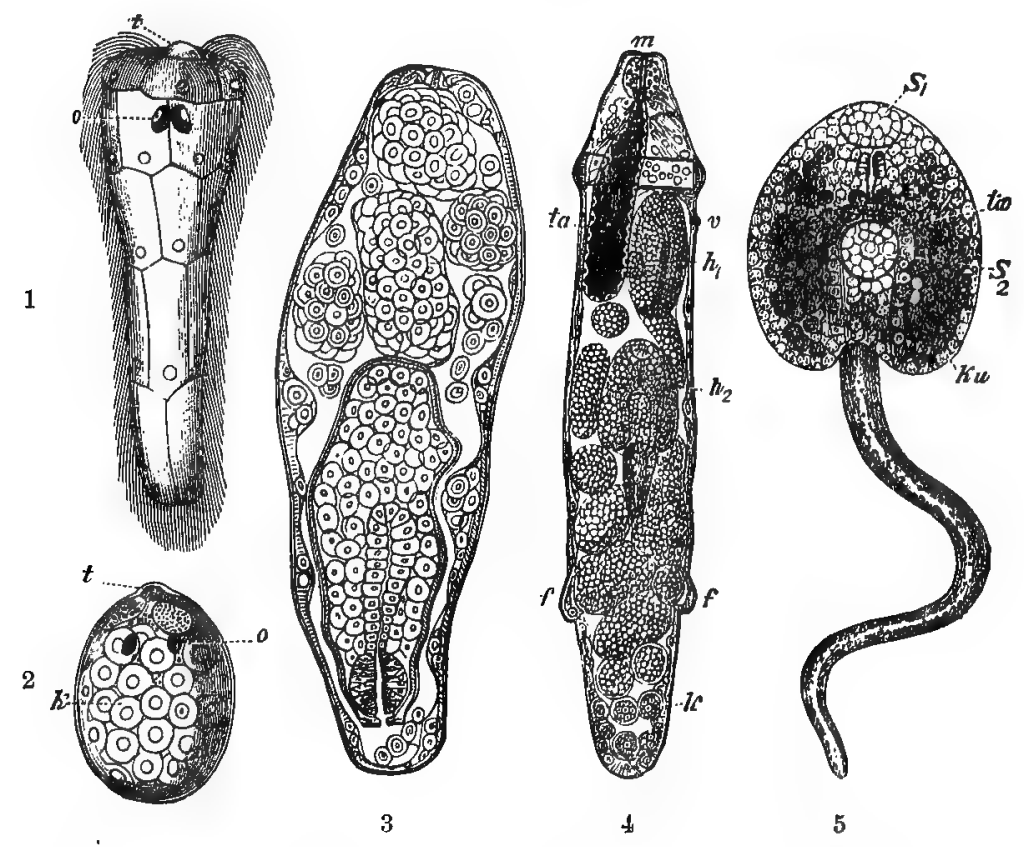

Fig. 104. Distomum hepaticum. 1 newly hatched larva. $t$ boring papilla, o e.c. 2 the same (young sporocyst of the first generation), after it has entered the snail and lost its ciliated coat. $k$ ovam. 3 adnlt sporocyst of the first generation. 4 redia of the second generation. $f$ Limb-like projection, $h_{1}-h_{2}$ cercarim developing within the redia, $k$ cercaria-embryo, $m$ mouth, $t a$ gut, $v$ genital aperture. 5 cercaria, $K u$ glande, whose secretion forms the capsule. $\boldsymbol{S}_{1}-\boldsymbol{S}_{2}$ suckers, $t a$ gut. All the figures are enlarged.-After Thomas; 4 slightly altered.

is dissolved by the gastric juice, and the young Trematode wanders into the liver, where it attains sexual maturity ( $t$ he herm aphrodite generation). Encysted Liver-flukes are found not only on water, but also on land plants, for the Snail frequently leaves the water and wanders about in the adjacent meadows.

2. Leucochloridium paradoxum is a parthenogenetic trematode, parasitic in Succinea amphibia, a Gastropod inhabiting damp places. This peculiar form has the appearance of a sac with numerous branches, some of which attain a very great development, forming thick, brightly coloured rods, which push their way into the tentacles of the Snail, and expand them enormously. Different insect. eating Birds tear the "rode" out of the Snail, which may go on living with a new branch of the Leucochloridium in its tentacle. In the digestive tract of these 
Birds, as also in that of various Marsh-birds which devour the whole Snail, the young Trematodes contained in the "rods" undergo their complete development, and become the normal hermaphrodite generation, which has been described under the name Distomum macrostomum.

Fig. 105.

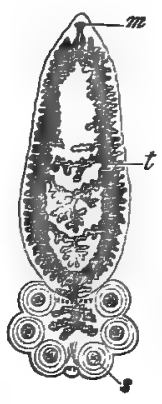

Fig. 106.

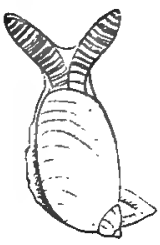

$A$
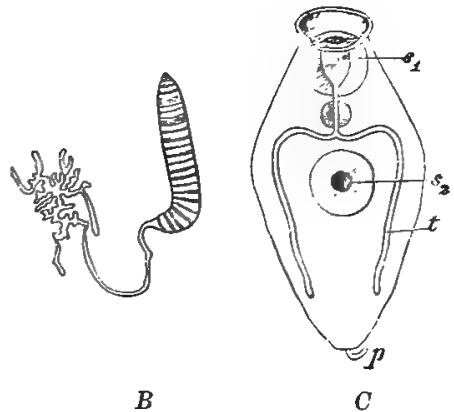

$C$

Fig. 105. Polystomum integerrimum, from the central side. $m$ Moath, s sucker, $t$ gut.-After Zeller.

Fig. 106. A Snail, with Leuchochloridium paradoxum in both tentacles. $B$ the same, taken out of the Snail. C Distomum macrostomum. $\quad p$ penis, $s_{1}$ fore-, $s_{\Omega}$ hind-sucker, $t$ gat. $A$ and $B$ natural size, $C \times 20$.-After Zeller.

\section{Class 3. Cestoda (Tapeworms).}

The Cestoda differ from the Trematoda, with which they are nearly related, in that they have no digestive tract, and almost always form $\mathrm{ch}$ ain $\mathrm{s}$ by budding.

A cestode chain consists, at first, of an asexual individual, the socalled "head" (scolex), which is provided at the anterior end with

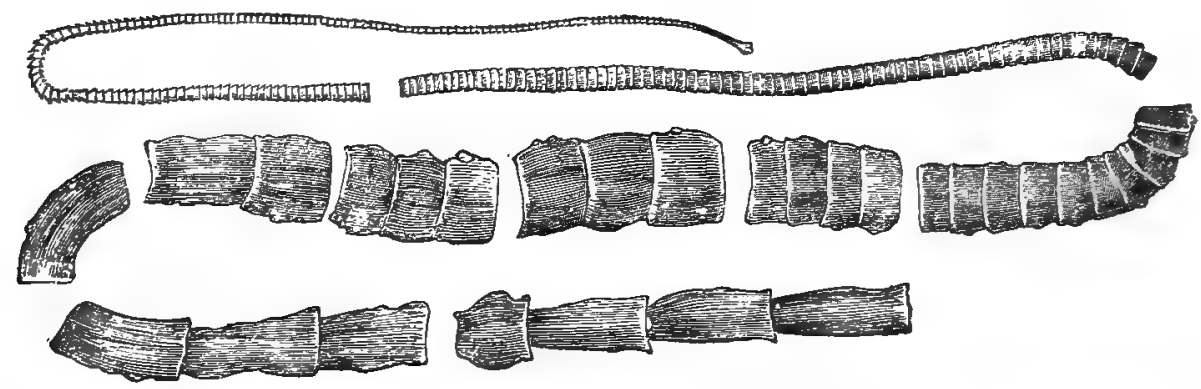

Fig. 107. Toenia mediocanellata, part of the chain, nataral size.-After Leuckart.

suckers, hooks, or other organs of adhesion. Behind the head follows a larger or smaller number of seg ments (proglottides), i.e., sexual individuals of different developmental grades, and of different sizes, 
which are separated from one another by constrictions; the segments nearest to the head are the youngest, those farthest off, the oldest and largest; new segments are constricted off from the hind part of the head. When the development of the segments bas gone so far that they contain ripe eggs in large numbers, they separate from the chain; in some cases, they break off at an earlier stage, and grow independently; in others, they remain in connection. The number of segments in a chain may increase from quite a few to many hundreds.*

The adult worm occurs exclusively in the $\mathrm{gut}$ of the Vertebrata, to the wall of which it attaches itself by the organs of adhesion on the head. It feeds by absorbing the contents of the gut through the skin. The Tapeworm does not, however, spend its whole life in the same place, or in the same host. At a younger stage, as a

Fig. 108.

Fig. $109 b$.

Fig. 110.

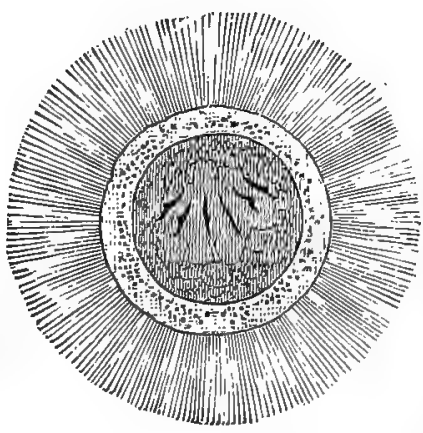

Fig. 111.

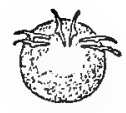

Fig. 109 a.
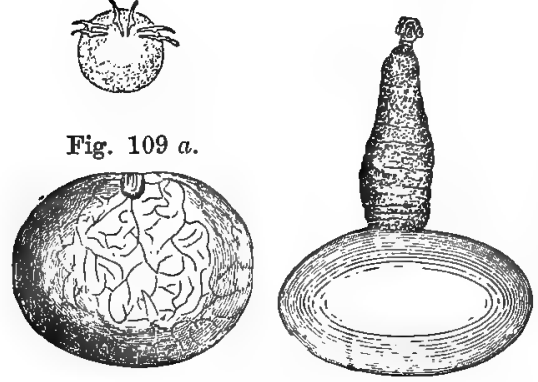

Fig. 108. Six-hooked larva of T. solium. head.

Fig. 109. Proscolex of the same Tænia, with (a) inpushed, $(b)$ evaginated

Fig. 110. Ciliated larva of Bothriocephalus latus.

Fig. 111. Proscolex of above.

All figures enlarged.-After Lenckart.

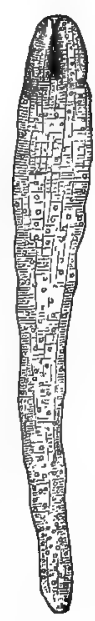

proscolex, it lives in another host, and in a different part of the body. It then often consists only of the head; in other cases, of the head and of a number of joints, which are, however, never ripe. A second host now devours the first, and thus a transference of the parasite occurs, and it attains sexual maturity. The temporary host is usually infected by taking the eggs, (or the segments containing eggs), which have passed from the permanent host with the excreta, into its alimentary canal. Each egg contains, one larva, a small round organism provided with six hooks, which,

* According to the opinion put forward by many observers in recent times, the Cestode chain is not a stock, but is a single individual, with a great number of gonads. But this view is contradicted by the fact that in some Cestoda, even after separation, the segments are capable of growth, become ripe, copulate, and thereby testify abundantly to their independent individuality. 
after its shell is dissolved by the digestive fluids, * bores through the wall of the gut and becomes a proscolex.

As regards their structure the Tapeworms are, excepting for the absence of the alimentary canal, closely connected with the Trematodes. The genitalia are hermaphrodite, of a very complicated nature; in each segment, there is a separate genital apparatus, which has no connection with those of the other segments. The genital aperture is either on the ventral side of the segment, or on its margin.

The parasitic genera, Amphilina (in the Sturgeon), and Caryophyllaus (in the Carp), seem to be a transition towards the Trematodes, for they do not form chains. The unsegmented body contains but on e reproductive apparatus; like the rest of the Cestoda, they have no gut, and agree also with them in certain special arrangements of the genitalia. Some other Tapeworms are like the above-mentioned forms, in so far as they appear to be entirely unsegmented, although they exhibit essentially different arrangements, for an inner segmentation is clearly manifest in the multiplicity of the reproductive organs. Among such forms, a separation of the reproductive individuals does not occur, and a chain of this kind may be compared with a Coral-colony, some of whose polyps are in closer connection than usual. As an example of Cestodes showing this external continuity, but internal metamerism, the Strap-worm, Iigula simplicissima, parasitic in the digestive tract of different Water-birds (in Fish in the cystic stage), may be mentioned.

Most of the 'lapeworms of Mammalia and Áves belong to the genera, T'cenia and Bothriocephalus, chiefly to the former. Numbers of other genera are known particularly in Pisces.

1. Trnia. The head is furnished with a circle of four suckers. Upon the middle of the anterior end there is, in many species, a crown of recurved hooks, which may be numerous, and sitnated upon an elevation (rostellum); in others, however, the hooks may be entirely wanting. The ripe proglottis may be elongate or short and wide; it contains a branched uterus. The genital aperture is usually at the edge of the segment.

(a) T. solium lives in the alimentary canal of Man. The scolex with a crown of hooks, the ripe proglottides considerably longer than broad; the former about as large as a pin's-head, the latter $5 \mathrm{~m} / \mathrm{m}$ broad. The chain attains a length of about 3 to $3 \frac{1}{2} \mathrm{~m}$. The ripe proglottides, and the thick-shelled eggs, containing embryos pass out with the excreta. If they be eaten by a Pig, the egg-shell dissolves, and the six-hooked larva bores through the gut into the body, where it usually takes up its abode in the muscles, more rarely in the heart or brain; there it grows considerably, and turns into a bladder-worm or proscolex (Cysticercus cellulos $x$ ), a cestode head of the same appearance as that of the adult Tapeworm, but with an appendage behind in the form of a vesicle the size of a pea, filled with fluid, in which the head is invaginated. When it is taken into the digestive tract of Man, the bladder atrophies and the head develops into the Cestode-chain. More rarely the proscolices are found in various other Mammals. They sometimes occur in Man in places where they may prove fatal, in the brain, eye, or wall of the heart. Man, like the Pig, may occasionally take in eggs containing embryos through the mouth.

(b). T. mediocanellata $(=T$. saginata, LEUCK.), also found in the digestive tract of Man; in most countries more common than T. solium. The head without

* This shell is not an egg-shell in the usual acceptation of the term, but at membrane secreted by the embryo. The "eggs" are, in reality, e n c y st ed embryos. 
rostellum, but with very powerful suckers. The branches of the uterus are more numerous than in T. solium, which this species usually resembles closely;
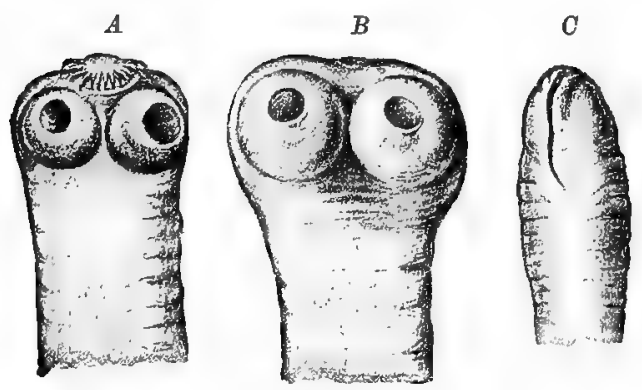

Fig. 112. "Head" of Trenia solium (A), of T. medio" canellata (B), and of Bothriocephalus latus (C).-Orig.

it attains a length of 7-8 m. The development as before. The bladder-worm, almost exclusively in the muscles of Cattle, is very much like that of $T$. solium, but a little smaller.

(c) T. conurus. With crown of hooks; in the digestive tract of the dog; 1-m. long. The bladderworm (Coenurus cerebralis), lives in the brain of Sheep, in which it induces giddiness. It becomes very large (as large as a Hen's egg or more), and produces, by budding, numerous scolices, so that there is a colony of Cestode-heads in the same vesicle.

(d) T. echinococcus. A very small Cestode (at most $5 \mathrm{~m} / \mathrm{m}$. long), with $3-4$ proglottides; in the alimentary canal of the Dog. The bladder, the Echinococcus, in the liver and other organs of Cattle, Sheep, Pigs, and Man, often attains a considerable size (that of a child's head or more), and is surrounded by a thick, stratified cuticle. Many small heads, as in T. coenurus, bud out from the vesicle, but in $T$. echinococcus are always formed as little invaginations of the wall (see Fig. $113 B$ ), the so-called brood-capsules. The Echinococcus
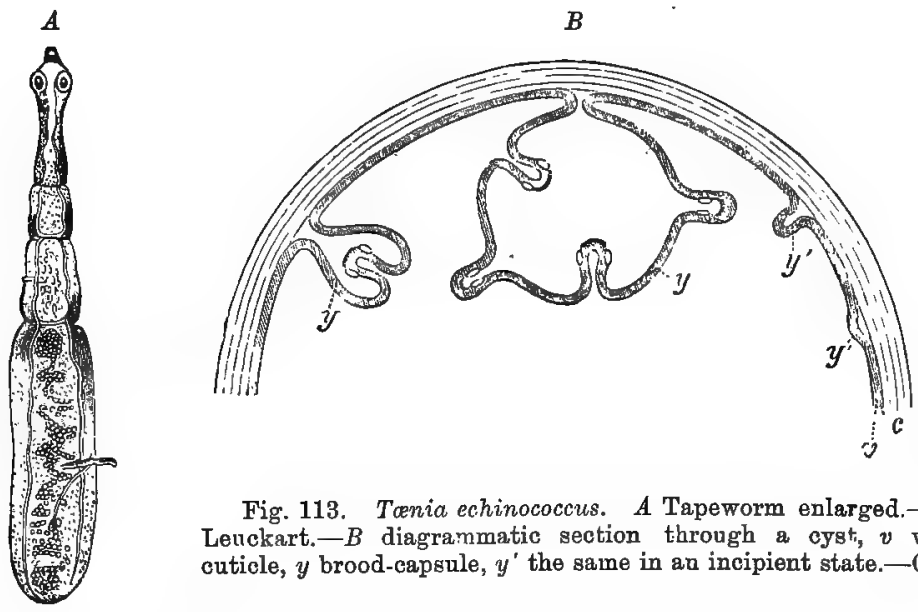

Fig. 113. Tonia echinococcus. A Tapeworm enlarged.-After Lenckart. $-B$ diagrammatic section through a cyst, $v$ wall, $c$ cuticle, $y$ brood-capsule, $y^{\prime}$ the same in an incipient state.-Orig.

occurring in Man-rare in most places, but common in Mecklenburg, Iceland, and Australia-is distinguished by often attaining an enormous size, and by having within the original bladder many smaller daughter vesicles (broodcapsules), which exhibit the same structure as the former. The brood-capsules seem to arise by a modification of heads which have broken free from the wall of the parent bladder.

(e) T. cucumerina. $75 \mathrm{~m}$. long. The head provided with a retractile proboscis-like process, with several circles of hooks. Ripe proglottides oval. 
Very common in the Dog and Cat, rare in Man (children). The proscolex, which has no bladder, lives in the Dog-lonse (Trichodectes canis); according to modern opinions, in the Dog-flea also.

2. Bothriocephalus. The head with two long sucking grooves, without true suckers and without hooks. The genital apertures are on the ventral side of the proglottides, which are always short and wide. Uterus an unbranched coiled

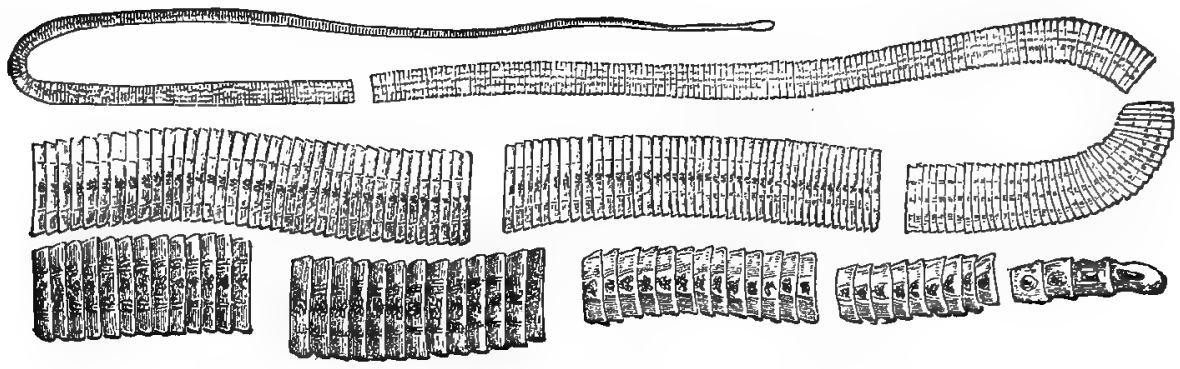

Fig. 114. B. latus, part of the chain, natural size.-After Leuckart.

canal. B. latus, the broad Tapeworm of Man, common in the Russian Baltic provinces, Finland, in W. Switzerland, though extremely rare in England, attains a length of 8-9 $\mathrm{m}$. The proscolex lives in the flesh of the Pike, and other fresh-water Fish. It is long, without a bladder $(1 \mathrm{c} / \mathrm{m}$. long). How the Fish are infected is not known. The eggs of the Tapeworm develop in water, the newly hatched larvæ are provided with a covering of cilia and within them the six hooks are found as usual. It has not, however, been found possible to infect the Fish with the larvæ, so that it is conceivable that the worm lives first in another host. On the other hand, it bas been shown by experiment that the Pikeproscolex transferred to the digestive tract of Man (and the Dog) develops into B. latus.

\section{Class 4. Nemertinea (Rliynchocoela)}

The Nemertines are, as a rule, elongate, often even ribbon-like, animals of considerable size. The skin is ciliated all over. At the front end, on each side, is a slit-like, thickly-ciliate pit, a senseorgan, which is also present in some Turbellarians; dorsally and anteriorly there is usually a number of small eyes. Quite at the front end of the animal there is an opening leading into a deep eversible blind sac, the proboscis. At the bottom of the sac, there is, in many forms, a pointed stylet, which, when the proboscis is everted, lies on its tip; frequently a poison gland opens close beside it. In others the spine is not present, but the proboscis is furnished with numerous nettle-cells. When retracted (Fig. $115 \mathrm{C}$ ) it is surrounded by a muscular proboscis - sheath, and the space between them is filled with fluid. The proboscis is everted by contractions of 
its sheath. A long muscle, the retractor, passes from its inverted end to the sheath, and this pulls in the everted organ (see Fig. $115 \mathrm{D}$ ). Its function is defensive and prehensile; it is not connected with the digestive tract. This begins in front and ventrally (behind the proboscis pore) in a slit-like mouth, and runs through the body as a canal, furnished usually with small lateral evaginations, and opening in the an us at the posterior end.

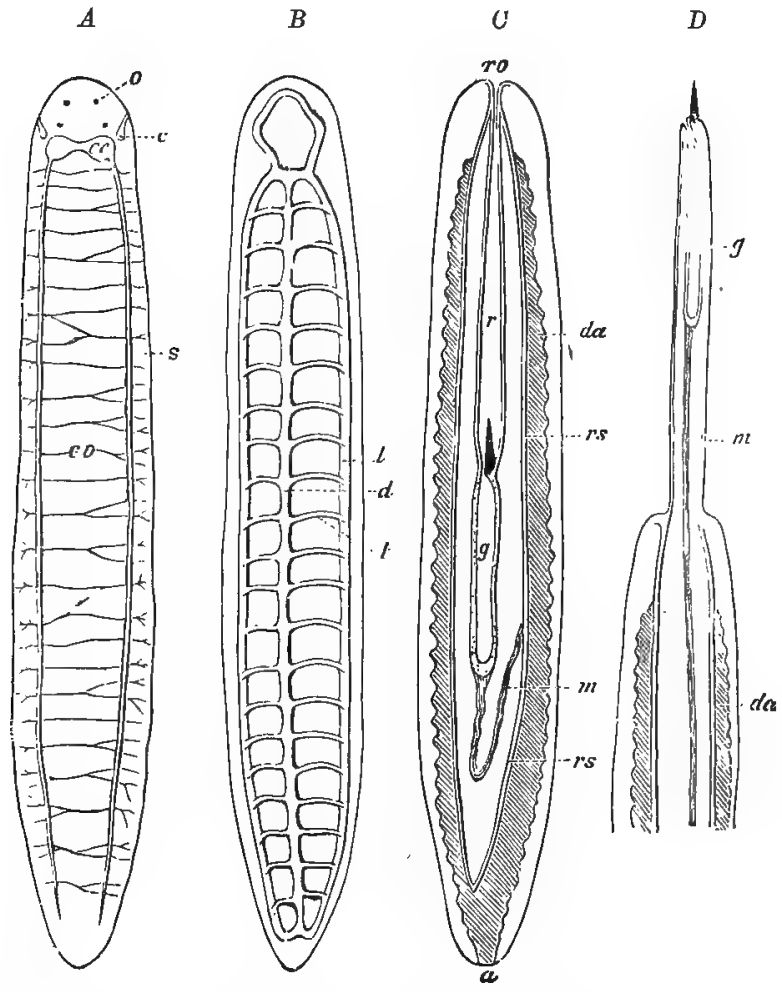

Fig. 115. A Sketch of a Nemertine, showing the nervous ssstem, $B$ do. with blood vaseular system, $C$ ditto with gut and proboscis, $D$ ditto front end with everted proboscis. Diagrammatic. a Anus, $c$ ciliated groove, $a a$ gut, $g$ poison gland, $l$ lateral vessel, $m$ retractor muscle, $r$ probscis, ro proboscis opening, $r s$ proboscis sheath, $s$ lateral nerve cord, $t$ transverse vessel.-O Orig.

The nervous system is very like the usual flatworm type; the proboscis sheath passes between the cerebral commissures. From the cerebral ganglion of either side arises a lateral nerve, running backwards, and containing numerous nerve-cells; the two cords are often connected by delicate transverse commissures, passing above and below the alimentary canal, and from them many. nerves arise. The excretory organs are similar in structure to those of other Flatworms. Besides the ciliated grooves and eyes 
already mentioned, there are auditory vesicles in a few forms. In contrast to other Flatworms, the Nemertines possess a vas cular system, usually consisting of three principal longitudinal trunks, two lateral, and one dorsal, which come into relation with one another anteriorly and posteriorly, and are besides connected by transverse vessels. The blood flows from behind forwards in the dorsal vessel, from before backwards in the lateral vessels; it is colourless or red. The Nemertines are almost always of separate sexes; the sexual organs are much simpler in structure than those of other Platyhelminths.

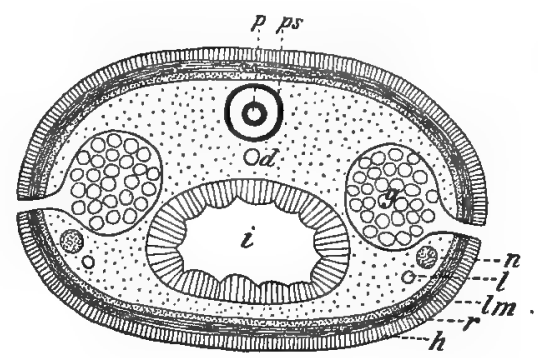

Fig. 116. Diagrammatic transverse section of a Nemertine. d Dorsal ressel, $g$ gonad, $h$ skin, $i$ gut, $l$ lateral vessel, $l m$ longitudinal muscle, $n$ lateral nerve cord, $p$ proboseis, $p s$ proboseis sheath, $r$ circular muscle. Many ovaries or testes are present, opening by one duct on the side of the body. There are no accessory and no copulatory organs.

Some Nemertines undergo a metamorphosis, which is aberrant, in that the principal part of the larval body atrophies. The larva, which is sometimes of a peculiar form (Fig. 117) is freeswimming.

$A$

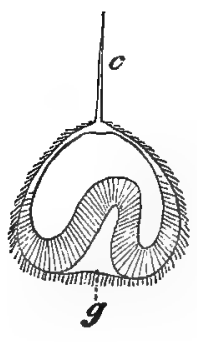

$B$

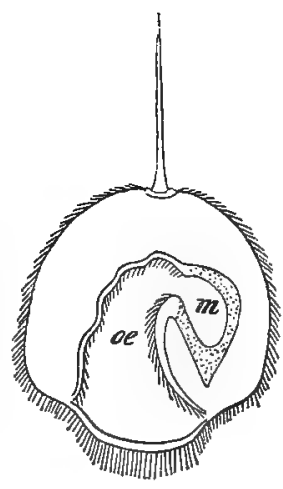

$C$

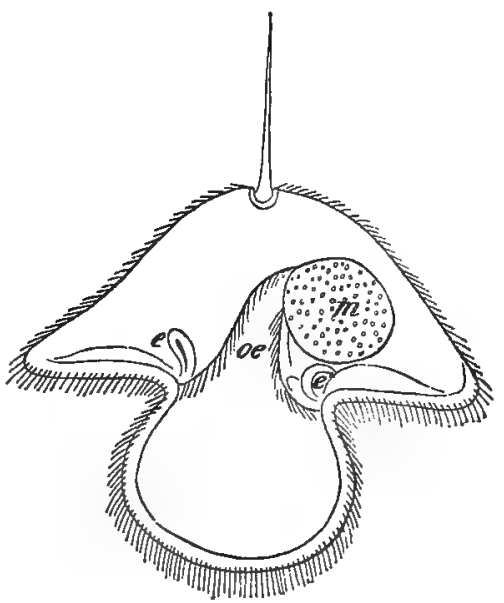

Fig. 117. Three larval stages of a Nemertine; the oldest larva (C) is the so-called pilidium. $c$ flagellum, $g$ gastrula mouth, $e$ and $e^{\prime}$ invagination of the skin, from which a great part of the final Nemertine body arises; $m$ stomach, oe osophagus.-After Metschnikoff. 
The Nemertines are for the most part marine, living usually at the bottom of the sea. Some are fresh-water or terrestrial. They feed upon other animals.

One form living in European seas, Lineus longissimus, sometimes attains a length of $13 \mathrm{~m}$., with a breadth of $8 \mathrm{~m} / \mathrm{m}$. Most species are a few centimetres, some only a few millimetres long.

\section{A pendix to the Platyhemitim.}

\section{Rotifera (Wheel Animalcules).}

The Rotifers are usually microscopic creatures, which in size, habitat, and mode of life resemble the Infusoria. The posterior part of the body, the foot, is narrowed, separated from the trunk, and provided with a pair of short appenduges or with an adhesive disc at the tip. At the anterior end, there is a more or less well-developed (sometimes scalloped) trochus, the rotato r y or ga $\mathrm{n}$, whose edge is beset with strong cilia. It serves two purposes; in the first place it is of use in swimming, and secondly, by means of the

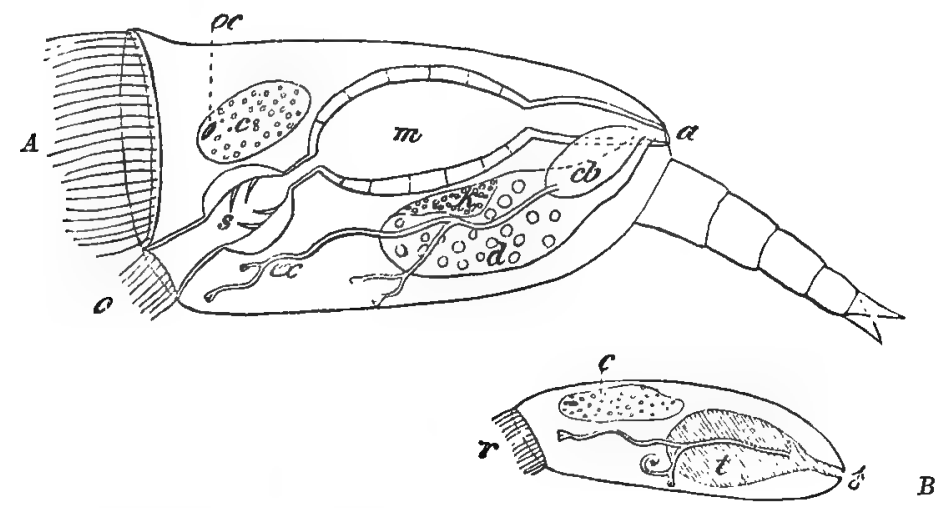

Fig. 118. A Diagram of the anatomy of a $\mathrm{f}$ e ma le $\mathbf{R}$ otif $\mathrm{r}$, lateral view. $B$ the male. $a$ anus, $c$ ganglion, $c b$ contractile bladder, $d$ yolk sac, ex excretory organ, $k$ ovary, $m$ stomach, o mouth, oc eye, $r$ wheel organ, 8 pharynx, $t$ testia, o male aperture.-Orig. (with assistance from Plate's figures).

currents set up by it, small particles are driven into the underlying mouth. The trunk, in some forms, is covered by a hard chitinous shield, the lorica (a specialised thickening of the cuticle covering the whole body), which may be armed with spines. In other cases both trunk and foot exhibit delicate constrictions which simulate a segmentation, but there is never a corresponding arrangement internally. The foot is often articulated with the body, so that by its help the animal can crawl about like 
a leech. The mouth leads into a muscular pharynx, furnished with several small jaws, which are continually being struck one against the other. The digestive tract is usually short and simple, and the anus is generally dorsal, at the base of the foot, though in some forms it is wanting. The nervous system is of the flat-worm type, cerebral ganglia anteriorly with nerves springing from them; one or two eyes are often present at the front end. The excretory organs closely resemble those of the Platyhelminths; there is a pair of principal tubes with smaller branches, whose club-shaped terminal swellings are like those of the Flat-worms. The principal ducts open, after having united to form a contractile vesicle, into the hinder part of the gut or cloaca. There is no vascular system. The Rotifera are of separate sexes. In a few forms only are the sexes alize, usually they are extraordinarily different; the males are smaller than the females, they are entirely destitute of a mouth, and the digestire tract is rudimentary, so that they can take in no nourishment; they live for only a few days; they have no lorica, even when the female has one, and the trochus is small; they are less numeruus than the females, and in many genera were long unknown. The short oviduct* opens, as a rule, into the cloaca; the vas deferens dorsally at the base of the foot. The Rotifera lay two different kinds of eggs, viz., thin-shelled, unfertilised $\mathrm{summer}$ e g g s, which develop parthenogenetically; and the thick-shelled fertilised winter eggs, which do not hatch until long after they are laid. The young ones do not undergo a metamorphosis.

In a common fresh-water Rotifer, Hydatina senta, two kinds of females are found. The one does not copulate and lays only summer eggs, which give rise to $f \in m$ ales; the other is capable of copulating, and when this takes place, lays winter eggs, or if this does not occur, summer eggs, which hatch into males. Whether similar conditions obtain for other forms is still uncertain, although probable.

Much doubt exists as to the systematic position of the Rotifers; they have been sometimes placed with the Arthropoda, sometimes with the "Vermes." From the facts which have lately come to light with regard to their structure, it is probable that they must have been derived from the Platyhelminths (see especially the excretory apparatus).

The Rotifers are usually active and free-swimming, some, however, are fixed. For the most part they live in fresh-water, a small number in the sea. Some forms which occur in damp earth or on plants (moss) can withstand complete desiccation, but this is not the case with those living in water. A few are parasitic.

* The (usually unpaired) ovary is connected with a yolk-gland which produces a mass of yolk, to be absorbed by the ovum. 


\section{Phylum 4. Nemathelminthes (Round-worms).}

The two groups of this phylum are so very different from one another, that it is a matter of great doubt whether they should be put together. Common to both groups are the elongate, cylindrical body, and the muscular body-wall which encloses a well-defined body-cavity. Cilia are absent. The sexes are always separate.

It is as yet impossible to say to which of the other phyla the Round-worms are most nearly allied. From what is known, up to the present, of their anatomy, they seem to present no characteristic affinities with any other groups.

\section{Class 1. Nematoda (Thread-worms).}

The smooth body is almost always elongate and cylindrical, often filiform, and usually pointed at both ends. It is covered with a light,

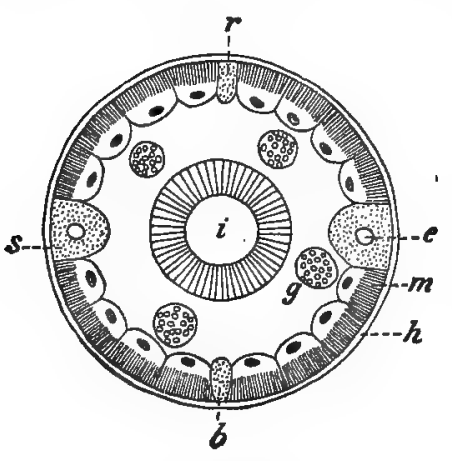

Fig. 119. Transverse section of a Nematode, diagrammatic. $b$ ventral, $i$ gut, e excretory organ, $h$ skin, $g$ gonad, $m$ muscle-cell, $r$ dorsal area, $s$ lateral area.-Orig. thick, elastic, cuticle, beneath which is a thin hypodermis, and under this is a single layer of

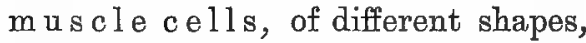
and often very large. The musculature is, however, interrupted by four so-called longitudinal areæ, ridges of the hypodermis, which traverse the length of the body, one in the mid-dorsal, one in the midventral line, and one on each side, dividing the muscular sheath into four longitudinal bands. Of the four longitudinal areæ, the lateral are the best developed. The mouth is at the front end of the body; sometimes a distinct buccal cavity lined with a stiff cuticle is present, but in most forms, this is not the case. The rest of the digestive tract, which takes a straight course through the 
body, is compused of three sections : a muscular œsophagus, which acts as a pump, a peculiar intestine devoid of muscles, and a short muscular $r$ ectum. The anus is on the ventral side, near the hind end of the body. The central n e rvous s y stem consists of a nerve-ring, provided with ganglion-cells, running round the œsophagus; several nerves are given off from this, two of which are specially important, one passing along the mid-dorsal, the other along the mid-ventral line. The ventral nerve ends posteriorly in a small ganglion. As to sense organs, the little tactile papillæ, which are always present anteriorly and posteriorly (the latter especially in the males), must be mentioned; in some freeliving Nematodes, small eyes have been found at the anterior end. The excretory apparatus seems to be represented by a pair of delicate tubes, which traverse the lateral areæ, and open anteriorly upon the under surface in a common aperture. The genitalia, in the female, consist of two long, coiled tubes, which open by a short common duct, rather anteriorly upon the ventral surface. Each canal consists of two regions, not sharply demarcated, the ovary and the oviduct; the latter is frequently much distended in the gravid female, and serves as uterus, or as brood-pouch for the numerous ova. In the male, testis and vas deferens are represented by a single, as a rule, long coiled canal opening into

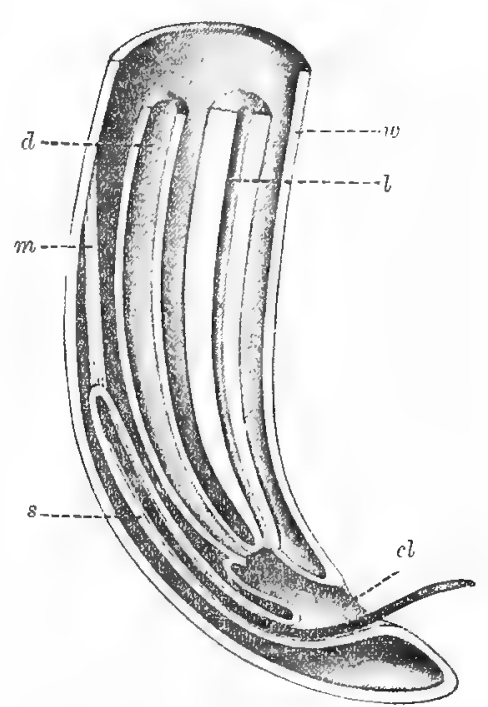

Fig. 120. Hind end of a male Nematode, longitudinal section. Diagrammatic. cl cloaca, $d$ gut, $m$ retractor muscle of the spicule, $s$ sheath of spicule, $w$ body-wall.-Orig.

the rectum, which thus represents a cloaca. The canal exhibits two parts, of which the vas deferens is the shorter and wider, the testis the longer and thinner. The male is usually provided with copulatory organs, one, or at most two, curved chitinous needles, the so-called spicula, which lie in sacs opening into the upper wall of the cloaca. In copulation, the spicula are protruded through the anus and introduced into the female genital aperture; in some forms, the cloaca is everted at the same time (see below for the special arrangements in Trichina and Strongylus). The female usually surpasses the male in size; sometimes also, other striking differences occur. The Nematodes, as a rule, lay eggs enclosed in a thick shell; frequently the development is considerably advanced when the egg is laid; not a few are viriparous. 
There is usually no pronounced metamorphosis, although the young one sometimes differs from the adult. Asexual reproduction does not occur. Most Nematodes are parasitic; some, mostly small forms, are, however, free-living, in fresh-water, damp earth, or in the sea, some in decayed substances or living plants. Many of the parasitic forms live in different hosts, at different periods, or are free for one period, parasitic for another. The habits of these animals are of peculiar interest.

. 1. The Common Round-worm (Ascaris), often of considerable size, anteriorly three prominent well-developed lips, forming a conical projection marked off from the rest of the body. The human Ascaris, A. lumbricoides is frequent in the small intestine, especially numerous in children, and then not without danger: also in Pigs. It probably feeds upon intestinal mucus, not upon blood. According to recent observations, infection is simply caused by the ova, which pass out from the host with the excreta, chancing to enter the mouth. On reaching the stomach the shell is dissolved by the gastric juice,* so that the embryos are set free. The female may attain a length of $40 \mathrm{c} / \mathrm{m}$., the male of $25 \mathrm{c} / \mathrm{m}$., both are usually about half as long. A. megalocephala of the Horse is somewhat larger than A. lumbricoides; A. mystax of the Cat and Dog is considerably smaller, the female $12 \mathrm{c} / \mathrm{m}$., the male $6 \mathrm{c} / \mathrm{m}$., and is easily recognised by the wing- or ridge-like fold of skin on either side of the anterior end.

2. The $\mathrm{Maw}$-wor $\mathrm{m}$ (Oxyuris vermicularis). With three rudimentary lips; at the anterior end, dorsal, and ventral longitudinal folds of skin. Female with thin, pointed, elongate, spike-like hind-end, $1 \mathrm{c} / \mathrm{m}$. long ; male without this " tail," smaller and less common than the female. Common in the large intestine of man (especially children), feeding on its contents, often present in very large numbers, and then causing serious suffering. Infection probably takes place in the same way as with Ascaris. A much larger species (O. curvula) in the cæcum of the horse.

3. The Strongylidæ (genera Strongylus, Eustrongylus, Dochmius), are especially characterised by the presence of a cup-shaped bursa, surrounding the cloacal aperture in the male, which serves as an organ of adhesion during copulation, and is supported by radial rib-like thickenings (Fig. $123 \mathrm{~A}$ ). Spicula are also present as usual. Most of the Strongylidæ are blood-suckers; the mouth is large, and furnished with chitinous teeth or spines.

(a) Eustrongylus gigas, the female may be $1 \mathrm{~m}$. long $(12 \mathrm{~m} / \mathrm{m}$. thick), the male $3 \mathrm{~m}$. In the pelvis of the kidney (i.e., the anterior widened part of the ureter) in the Dog, Otter, Seal, etc., very rare in Man. Life history unknown.

(b) Dochmius duodenalis (Fig. 123), the female may be $2 \mathrm{c} / \mathrm{m}$. long, the male $1 \mathrm{c} / \mathrm{m}$. Mouth with strong hooked teeth; a very dangerous blood-sucking parasite, living in the small intestine of Man. In the tropics and in warm climates (Brazil, Egypt, Italy); also farther north, e.g., in some mining districts of Germany ("Egyptian Chlorosis"). The ova leave the parent and its host, to undergo their development in damp earth or quagmires, where the larvæ live for some time. Then they encyst (the capsule has the elongate form of the animal, and is probably a loosened cuticle), and in this state are probably introduced with drinkingwater, or in some such way.

(c) Strongylus armatus, the $\mathrm{Pal}$ is a de $\mathrm{W}$ orm (the latter name comes from a row of chitinous spicules along the edge of the mouth), female to $5 \mathrm{c} / \mathrm{m}$., male 2-3 c/m. Very frequent in the large intestine (especially in the cæcum) of the

* The egg-shell, just as in most other intestinal parasites, cannot be dissolved in the intestine, the ova must pass through the stomach for this to be effected. 
Fig. 121.

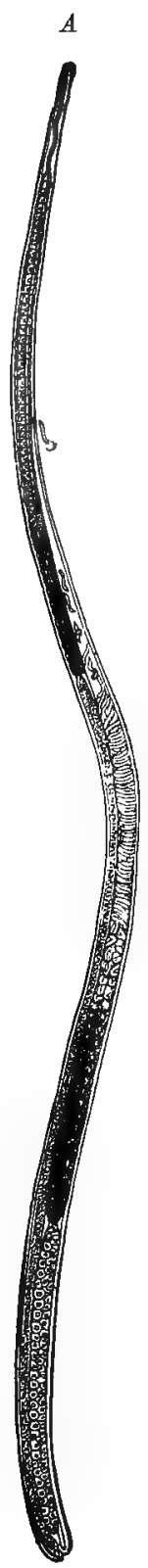

Fig. 122.

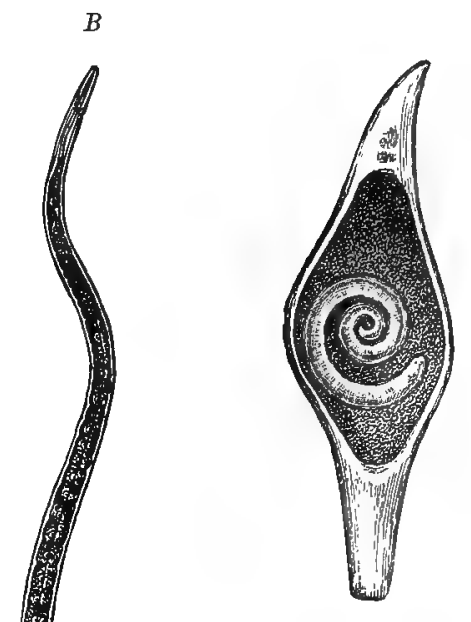

Fig. 123.

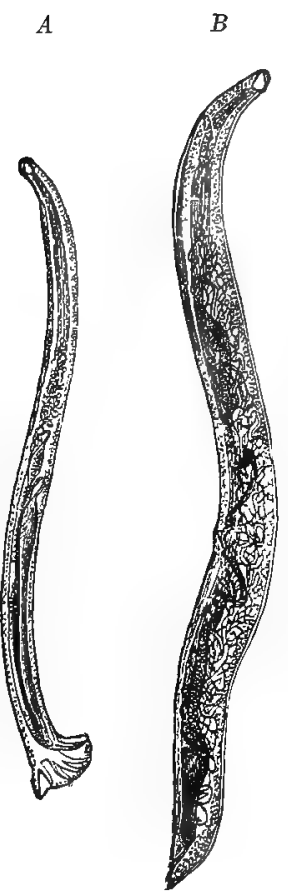

Fig. 124.

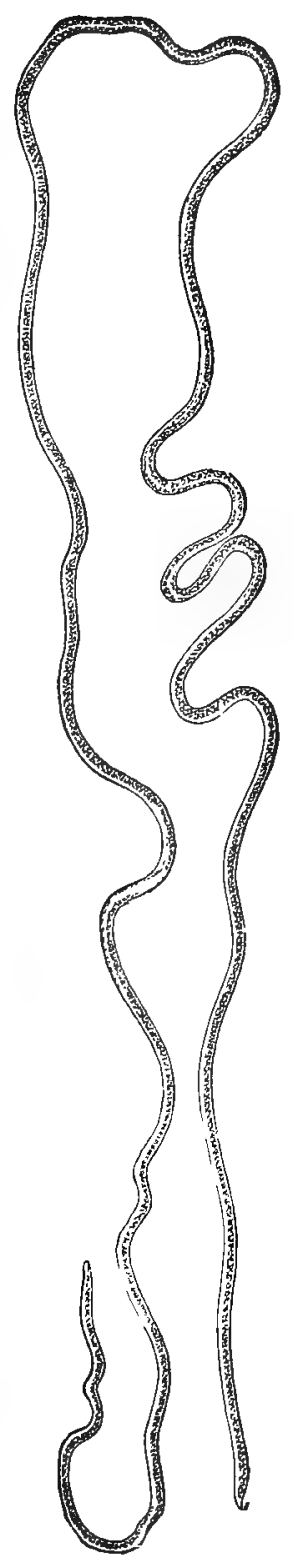

Fjg. 121. Intestinal-Trichina, $A$ 우, $B \quad$ b. -After Leuckart.

Fig. 122. M ascle-Trichina lying in its capsule-Orig.

Fig. 123. Dochmius duodenalis, A бु, B

Fig. 124. Filaria medinensis (Guinea-worm), Natural size,-After Leuckart. 
Horse. In the youngest stage it is free and is probably swallowed by the Horse with drinking'water; it lives at first in certain arteries (especially in the anterior mesenteric artery), which suffer, in consequence, a pathological change (wormanewism). Later it passes into the gut, and attains sexual maturity. How the wanderings to and from the artery occur is not yet known, Neither the presence of the worm in the artery nor in the gut seems to affect the health of the Horse directly; but a clot from the aneurism may occasion a stoppage in the intestinal vessel, and thereby a dangerous, often fatal, illness. Other Strongylide live in various domestic animals, among them the dangerous S. filaria in the lung of the Sheep.

4. Trichocephalus dispar, very frequent in the large intestine, especially the cæcum of Man, rare in England; the front part of the body is drawn out to a long, thin thread, which bores into the mucous membrane of the gut; may attain a length of $5 \mathrm{c} / \mathrm{m}$. The embryo develops within the egg-shell in damp places or in water, and is taken, still enclosed by the shell, into the digestive tract of the host, where it hatches and undergoes further development.

5. The Trichina (Trichina spiralis). The body of the sexually muture animal, the so-called Intestinal-trichina, is very thin; the female, 3 to $3.5 \mathrm{~m} / \mathrm{m}$., the male, $15 \mathrm{~m} / \mathrm{m}$., long; the female aperture lies far forwarl; the hind end of the male, with two cones, cloaca eversible, serving as a copulatory organ; spicula wanting. In the mature state in the smill intestine of Man and other Mammals, especially in the Pig and the Rat. The Intestinal-trichina produces a large number of microscopic larvæ whilst within the gut of the host (each female at least about 1500), which at once bore through the intestinal wall into the body-cavity of the same host, and thence migrate into the muscles, where each enters a muscle-fibre, causing it to swell up. The outer part of the swollen muscle-fibre hardens into a citron-shaped capsule round the young Trichina, which, meantime, has grown considerably (to $1 \mathrm{~m} / \mathrm{m}$. long), and now lies spirally coiled in the pulpy mass filling the capsule; the capsule, after some months, is infiltrated with calcareous salts, and becomes hard and opaque. If an animal, which contains such encysted $\mathrm{Muscle-trichina,} \mathrm{is} \mathrm{eaten} \mathrm{by}$ another (in which the Trichina can live), the capsules are dissolved in the stomach, the Trichinæ are freed, pass to the intestine, and attain sexual maturity in the course of a few days. They then copulate, and within a week after their entrance into a new host, the female intestinal form produces the first embryos. The female usually lives in the intestine only five or six weeks, and then dies; the adult male lives for a still shorter time. As Muscle-trichinæ, they can, however, live even several years; old forms often become calcified, and then die. Man is infected by partaking of raw pork; the Pig, by eating a Rat; the latter, probably, by devouring the sweepings of the slaughter-house, or a dearl comrade. Trichinosis is really caused by the wanderings of the young Trichina, and by its first sojourn in the muscle; when the wandering is over and the Trichina is encysted, the symptoms of disease cease, but recovery is often very gradual, and many cases terminate fatally.

6. The Threadworms (genus, Filaria, etc.) are very elongate animals, living as a rule, in parts of the host other than the gut, chiefly in connective tissue. Amongst them are :

(a) The Guinea-or $\mathrm{Med}$ in a-w or $\mathrm{m}$ (F. [Dracunculus] medinensis) living in the connective tissue under the skin or between the muscles of man; only, however, in warm regions of the old World. The female alone is known; it attains a length of $80 \mathrm{c} / \mathrm{m}$. In the adult, the digestive tract is atrophied, the anus is absent, food is taken by absorption through the body-wall. The greater purt of the body-cavity is occupied by the enormous mouthless oviduct, in which there are several million embryos. The irritation produced by the parasite, causes small abscesses through which the mature worms make their escape. The larva 
bores into a Cyclops in which it undergoes certain changes. In Man, infection is probably the result of accidentally swallowing the Cyclops in drinking water.

(b) Filaria immitis (female to 25 , male to $17 \mathrm{c} / \mathrm{m}$ ), in the heart and hypodermal connective tissue of the Dog; the young forms in the blood. Common in Eastern Asia, rare in Europe. In the tropics the young Filurix are also found in the blood of Man.

7. Mermis. Filiform aproctous Round-worms inhabiting various Insects, out of which they finally bore into damp earth, where they become sexually mature, copulate, and lay their eggs. The larræ bore their way again into the tissues of Insects. The genus Gordius, far removed in structure from the typical Nematoda, and living, in the adult state, in fresh water, presents a similar, but more complex, life-history.

8. The Anguillidie are a group of Nematodes, which are, for the most part. very small, and usually free-living, occurring in water, in different decaying substances, or in living plants. As examples, the following may be mentioned:

(a) Tylenchus tritici, the E el-w or m. In grains of wheat there is sometimes found a fibrous mass, which, upon closer examination, proves to be a number of small dried-up Nematodes; they are restored to animation by moistening. When such "ergots" are sown with sound grains, the Nematodes leave them and mount the growing wheat plants, upon which they may be met with between the glumes; later they fore into the grains, in which they become mature and lay their eggs. The young ones hatch from these and are found in the ergots arising by modification of the grains.

(b) Heterodera schachtii, another Eel-worm, produces the so-called root-knot. The larva bores into the delicate roots of the beet (and various other plants), and attains maturity there. The ripe female, which is distinguished by its short, citron-shaped form, pushes the hinder end of its body out from the root, causing the epidermis of the root to split. The elongate male, on the other hand, bores entirely out, and seeks the female for fertilization. The

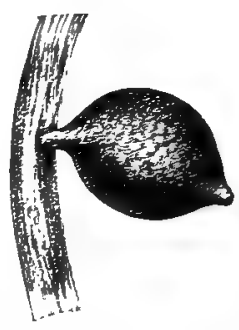

Fig. 125. Heterodera Schachtii. Female fastened to a root-fibre. Enlarged. -Orig. impregnated female later (Fig. 125), by the degeneration of the organs, becomes a brood pouch full of ova and larvæ, and finally drops off the root.

(c) Anguillula aceti, the $\mathrm{V}$ in e gar wo $\mathrm{rm}$, lives in sour paste and in vinegar.

\section{Class 2. Acanthocephala (Thorn-headed Worms).}

The body is cylindrical, elongate, often transversely wrinkled, and fairly hard. There is an eversible process, the so-called proboscis, at the anterior end, which is beset with many rows of backwardly directed chitinous hooks; the rest of the body is usually smooth. In the skin there is a peculiar vascular net-work, continued into two long bodies (the lemnisci), which spring from the bodywall, in the anterior region of the body-cavity. A digestive tract is entirely wanting. Food is absorbed through the surface, and the vascular system and the lemnisci probably carry the nutritive fluid from the skin over the rest of the body. The nervous system is represented by a ganglion lying in the forepart of the body, at the base of the proboscis, from which nerves run backwards and forwards. There are no sense organs. The Acanthocephala 
possess an exoretory apparatus, similar to that of the Platyhelminths, i.e., provided with the typical terminal branches. It opens into the oviduct or vas deferens*. O va are found free in the body-cavity of the female in different stages of development; only one oviduct is present, which, although somewhat complicated in structure, is, essentially, a canal open at both ends; the ova enter by the anterior opening: the posterior one is efferent, and opens at the hind end of the body. The male is usually smaller than the female, and possesses two t e s t es ; their efferent ducts unite to form a common vas deferens, beset with glands, and opening at the posterior end of the body in a tolerably wide eversible bursa.

All the Acanthocephala belong to the one genus Echinorhynchus; they live, in the adult state, in the alimentary canal of Vertebrata,

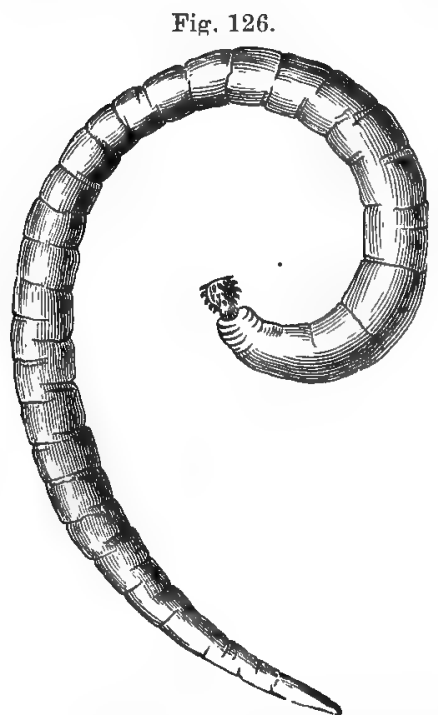

Fig. 126. Echinorhyrchus.-After Leuckart.

Fig. 127. Larva of one of the Acanthocephala.-After Kaiser.

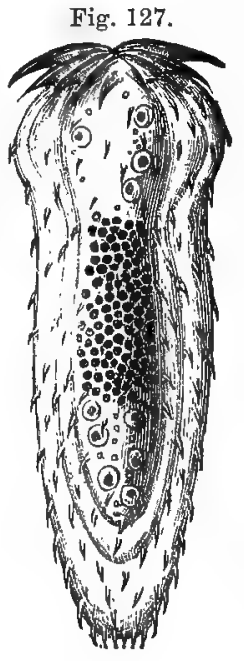

with the proboscis fixed in the mucous membrane, and they feed upon the contents of the intestine. Their development is of interest. The eggs of E. Proteus (one of the forms whose lifehistory is best known) living in the gut of different Fresh-water Fish, escape with the 'xcreta of the Fish, and are consumed by a small Crustacean, Gammarus pulex, in whose alimentary canal the elongate larvæ hatcht out. The front end of the

larva is provided with a boring apparatus consisting of ten spines (see Fig. 127), by means of which it traverses the intestinal wall into the body-cavity of the Crustacean. Here it wanders about, grows, and gradually assumes the form of the adult. If the Crustacean be eaten by a Fish, the parasite gets into the alimentary canal, and here attains sexual maturity. E. gigas, the female of which may attain a length of $50 \mathrm{c} / \mathrm{m}$. (the male only $9 \mathrm{c} / \mathrm{m}$.), lives, in the adult state, in the digestive tract of the Pig; as a larva, in the larvar of the Rose-chafer (Cetonia an*ata) and other Lamellicomia.

* The excretory organs do not, as in the Flatworms, spread over the whole body, but are limited to a small region.

+ It is noteworthy that the oviducal canal has a lateral opening, through which the unripe eggs, taken up by the oviduct, pass again into the body-cavity, whilst the ripe ones pass through the canal. 


\section{Phylum 5. Annelida.}

'The elongate, bilaterally-symmetrical body consists of a number of' somites or segments, which are separated externally by constrictions; the segments resemble each other to a certain extent both in their internal and external structure, although they are never all identical, the first or several anterior segments and the last always differing from the rest. Frequently, too, there are other differences, but, even when these variations are considerable, certain common features are always retained.

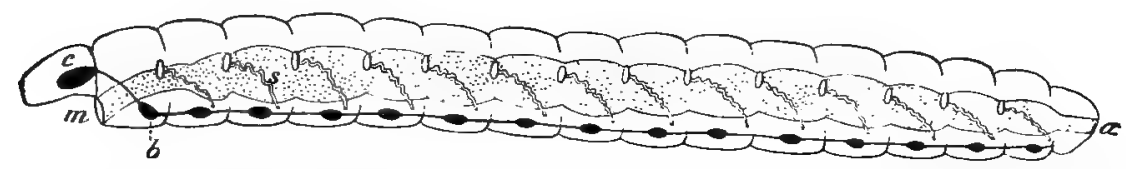

Fig. 128. An nelid seen from the side; diagram of alimentary canal, of the nervous system, and the segmental organs. $m$ mouth, $a$ anus, $c$ cerebral ganglion, $b$ sub-cesophageal ganglion, $s$ segmental organ.-Orig.

The body is covered by a thin cuticle. The month is close to the front end. The alimentary canal, consisting of several regions, usually traverses the body without convolutions, although not infrequently it is provided with lateral evaginations; the a $\mathrm{nus}$ is at the hind end. The central nervous system (Fig. 129) consists of a paired cerebral ganglion above the anterior end of the digestive tract, and two nerve cords passing from this round the buccal cavity and then running close together below the alimentary canal in the ventral body-wall. In each segment these nerves swell out into a pair of ganglia; the two ganglia of a segment are united by at longer or shorter commissure. 'The cords often lie close to one another', or are even fused, in which case the ganglia of each pair are united. By fusion of several consecutive segments, the ganglia are aggregated and even coalesced. From the cerebral and ventral ganglia nerves go to the corresponding segments. As to sense organs, tactile threads (tentacles, etc.) are often present, and also e yes: the latter, which are usually few in number and simple in structure, are 
specially found on the anterior part of the body, but sometimes also on other segments. Auditory vesicles are more rarely present. The vascular system (Fig. 130) is usually very

$A$

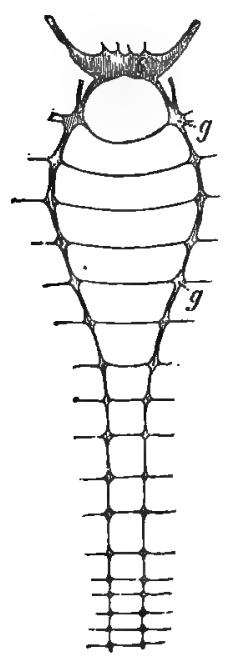

$B$

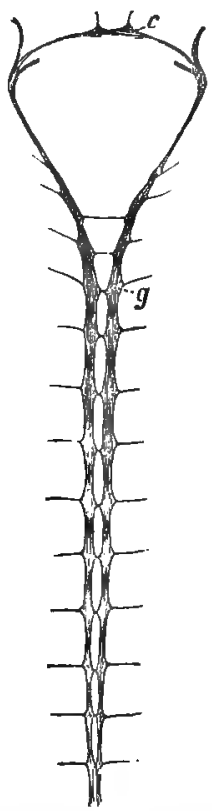

$C$

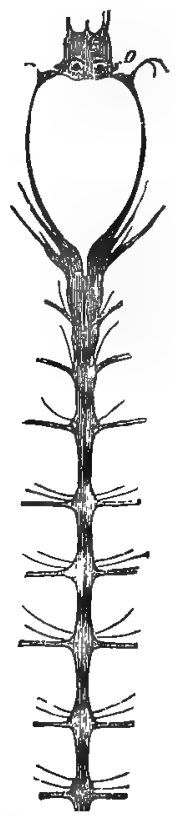

Fig. 129. Nervous system of different Chætopods ( $B$ Serpula, $C$ Aphrodite). $c$ cerebral ganglion, $g$ ventral ganglia, o eye.-After Quatrefages.

well developed; there is, as a rule, a longitudinal vessel on the dorsal side, the dor s al vessel, and a similar one on the ventral side, the ventral vessel; these are united by transverse vascular arches. The dorsal trunk, sometimes, also, some of the transverse vessels, is pulsatile, and performs the functions of a heart; the bloodstream is from behind, forwards in the dorsal vessel; in the ventral vessel in the opposite direction. From these trunks smaller branches go to different parts, to the gut, etc., also to the gills when these are present. The vascular fluid is usually coloured; as a rule red, sometimes yellow or green. The vascular system in some forms (Chætopods) is completely separated from the body-cavity which contains a special colourless fluid. In other cases, e.g., in Leeches, this system communicates with the body-cavity, which, moreover, is here of small extent, and modified to form vascular sinuses.* In cortain Chætopods the vascular system is entirely wanting.

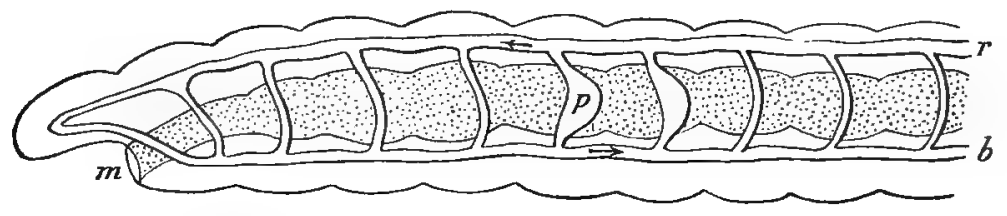

Fig. 130. Anterior end of an Annelid, with alimentary canal and vascular s y s te m figured. Diagrammatic. $m$ mouth, $r$ dorsal vessel, $b$ ventral vessel, $p$ pulsatile transverse vessel.- Orig.

* The opinion has recently been advanced, that in Leeches there is no communication between the vascular system and the spaces which represent the body-cavity. 
In most segments, there is a pair of segmental organs; each is usually a tightly-coiled glandular canal, opening at one end into the body-cavity by a ciliated funnel, and at the other, ventro-laterally, to the exterior (in Chætopoda, at the base of the ventral parapodia); the onter region, near the external pore, is often swollen into a vesicle. These are the excretory organs (nephridia) of the Annelids, ${ }^{*}$ but they often perform another function in permitting the exit of ova and spermatozoa. The sexual organs are very diversely arranged (see below); some Annelids are of separate sexes, some are hermaphrodite.

The Annelids in many réspects come rather close to the Nemertines, from which they are probably to be derived. If the two lateral nerve-cords of the latter moved ventrally and approached one another, and formed swellings at the origins of the transverse nerves, the chief portions of the Annelid nervous system would be represented. The dorsal vessel of the Nemertines corresponds entirely with that of the Annelids, the lateral vessels of the Nemertines are united to form the Annelid ventral vessel, and in both groups the arch-like transverse vessels are similar. Many Annelids possess two ciliated grooves corresponding with those of the Nemertines. It is also of great interest that many Annelid larvæ are furnished with a provisional excretory apparatus, the so-called "head-kidney," provided, at least in many chætopod larvæ, with closed end-branches, just as is the permanent excretory apparatus of the Flatworms, with which it indisputably corresponds. The segmentation of the body, the peculiar segmental organs, the formation of a body-cavity, etc., are, however, important points, distinguishing the Ammelids from all Flatworms.

\section{Class 1. Chætopoda.}

The body is divided by distinct constrictions into a large number of segments. With the exception of the anterior and posterior somites, each usually bears four so-called parapodia, two on each side (Fig. 133). These are short processes of the body-wall of different forms, each bearing a bundle of chitinous bristles (chætæ), which are sunk in deep saccular invaginations of the skin. The chæta, which is a cuticular structure, is secreted by a large cell at the bottom of the invagination. The bundle may be moved by muscles attached to the lower ends of the chæta-sacs. The chrtæ are of various and often elegant shapes; sometimes the outer moiety is jointed upon the shaft-like part; the point is often hooked, or the end may be pectinate. The chætæ may be so long as to look like long thin hairs, or they may be very short. In very many Chætopods, there is in each bundle of chætæ, a peculiarly developed thick and stiff, often dark one, the aciculum, which is implanted

* In some Chrtopods, the epithelial cells of the body-cavity also secrete waste substances, which are probably taken up by the funnels of the nephridia and carried to the exterior. 
much deeper than the others. Very frequently the two parapodia of the same side are confluent, either for their whole length, or only

Fig. 131.

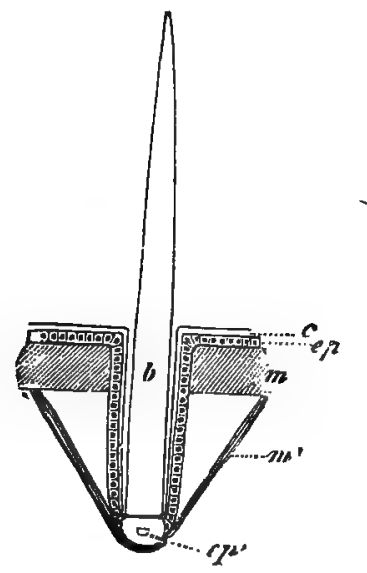

Fig. 132.

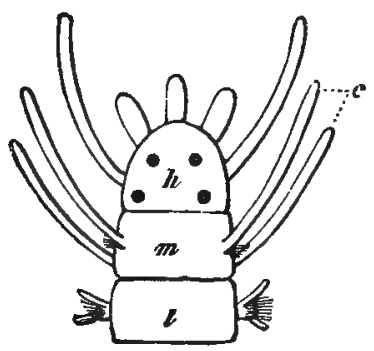

Fig. 131. Diagrammatical section through the skin of a Chætopod. c caticle, $e p$ epidermis, $e p^{\prime}$ epidermal cell, which secretes the chæta, $b, m$ muscle-sheath, $m^{\prime}$ muscle of the lower end of the chæta-sac.--Orig.

Fig. 132. Anterior end of a Chætopod (diagrammatic). $h$ prostomiam, $m$ oral segment, with $c$ the oral tentacles; $e$ the next segment.-Orig.

at the base, so that there appears to be only on e on each side, but each has still its own bundle of chætæ and its own aciculum. In other cases, the upper parapodium is rudimentary or entirely absent. The parapodia may be lobed, large, and well-developed; or they

$A$

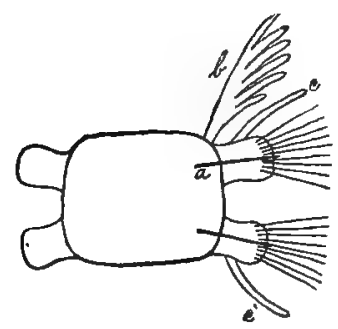

$B$

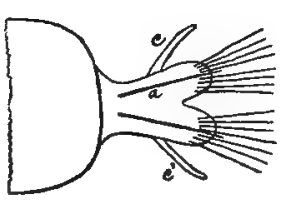

$C$

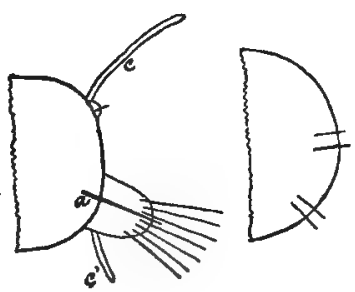

Fig. 133. Diagrammatic sections of different Chætopods. In $B$ the two parapodia are confluent, in $C$ the notopodium (with the exception of the cirrus) is rudimentary, in $D$ (Earth-worm) the parapodia are represented only by two bristles. a aciculum, $g$ gill, $c$ dorsal, $c^{\prime}$ ventral cirrus.-Orig.

are quite insignificant processes of the skin, or are merely represented by their chætæ, which then are planted directly in the 
body-wall (e.g. in Earthworms). Very rarely the parapodia are present without chætæ; in some forms they are entirely absent from certain segments. There is often a dorsal and a ventral c irrus, tentacle-like appendages, arising from the upper side of the dorsal parapodium (notopodium), and the under side of the ventral parapodium (neuropodium) respectively. In some forms, the dorsal cirri un some or all of the segments are large, and form lamellæ or elytra, covering the upper surface of the animal.

The two anterior segments differ from the others. The first segment, the prostomium, which overhangs the mouth, has no parapodia, but a number (usually $1-9$, in many tubicolous forms a much larger number, in others none at all) of thread-like appendages, the so-called palpi and tentacles. The second segment, the peristomium, which usually bears the mouth, although sometimes this is still further back, is provided with a rudimentary parapodium on each side, carrying few chætæ or none, but one or two welldeveloped, forwardly directed cirri, the so-called tentacular cirri. One or more of the ordinary segments may be fused with the peristomium, which, like the prostomium, may be destitute of appendages; in this case, their parapodia and cirri more or less resemble the oral appendages. Frequently the oral and following somites are fused and are difficult to distinguish. The terminal segment is without chætæ, and is often furnished with two long processes, the anal-cirri.

The integument is covered with a thin continuous cuticle, but, in spite of this, is often ciliated over certain limited tracts. The skin, with the underlying muscle-layer, forms a strong body-wall, which encloses a spacious body-cavity, very often divided into a series of compartments by transverse septa, corresponding to the constrictions between the segments. These septa, which are naturally traversed by the alimentary canal, and the blood vessels, are also perforated by holes to allow of the passage of the cœlomic fluid. Sometimes the septa are replaced by strands, which pass from the body-wall to the alimentary canal.

The anterior region of the digestive tract is usually a muscular pharynx, which may be everted like a proboscis. It is frequently provided with chitinous teeth, or hooks, in larger or smaller numbers. The rest of the alimentary canal is generally a straight tube, with constrictions at places where it is encroached on by the bodywall, more rarely it is coiled; in some short forms (Sea-mouse) the gut is furnished with a double row of cæca. The anus is, as a rule, situated at the posterior end of the body.

The eyes, which are, however, absent from many Chætopods, belong to the type figured in Fig. 20,5-6. They usually number from two to four, and are situated on the prostomium; in certain Tubicolæ, however, on its thread-like appendages; in a few other forms, on 
several of the body segments. One pair (or more) of auditory vesicles is present in some (e.g., Lugworm), in the neighbourhood of the cerebral ganglion.

In one division of the Chætopods, gills of different forms, tufted, pectinate, or filiform, are present on certain of the segments, one pair to each somite. They occur on the dorsal surface, at the bases of the notopodia. In many Tubicolæ (e.g., Serpula) the prostomial threads also serve as gills. Most of the Chætopods, however, possess no special respiratory apparatus.

The genital organs are very different in the Polychæta and Oligochæta, the two groups into which the Chætopods are divided. The former are almost always of separate sexes: ova or spermatozoa are as a rule formed in a great number of segments on the inner side of the body-wall, or on the septa, so that many ovaries or testes are present, which, do not appear as well-defined organs, but only as thickened spots in the wall; the genital products fall into the body-cavity, and pass out through the nephridia. The Oligochæta are, on the other hand, hermaphrodite, and the ovaries and testes, which are more definite organs, are present in only a few segments, one pair in each; there is always only one pair of ovaries, one or two of testes. The Oligochæta are further distinguished by having special oviducts and vasa deferentia, which, like the nephridia, open into the body-cavity by ciliated funnels; but there are also nephridia in these segments, so that these canals cannot be homologous with segmental organs. Instead of an oviduct, there is, in some forms, only a pair of slits in the body-wall.

In the Earth-worms (Fig. 134) the spermatozoa do not complete their development in the testis, but the cells from which they are formed break loose, and are received in a number of definite sacs (vesiculæ seminales) which are situated just within the body-wall and open by pores into the body-cavity. Here they develop into spermatozoa. In some forms there is a similar receptacle for the ova. In the Earthworms (and other Oligochæta) there are, further, sacs (spermathecæ), which open on to the surface, not into the body-cavity, and during reciprocal copulation, receive spermatozoa from the other animal.

The nervous system, vascular system, and excretory apparatus, have been referred to in the general account of the Annelida.

The development of the Polychrta is effected by a distinct metamorphosis, which is not found in the Oligochrta. The larvæ are free-swimming, and provided with cilia, which, in some forms, extend evenly over the whole body; in others, constitute a well-defined band on the often discoid anterior end, and frequently a second ring at the posterior end; or there may be a large number of ciliated bands. The body of the larva is at first short, parapodia are absent or present in small numbers; it gradually attains a considerable length, dividing into numerous segments provided with 
parapodia. Sometimes eyes and auditory organs, which do not occur in the adult, are present.

Fig. 134.

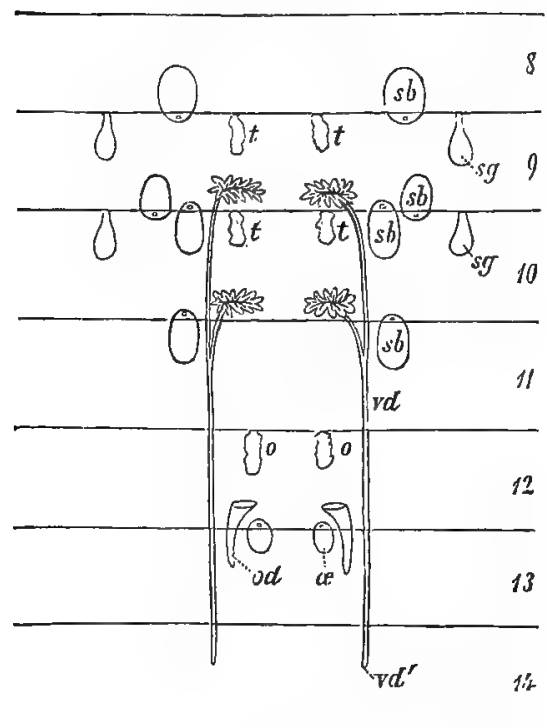

Fig. 135.

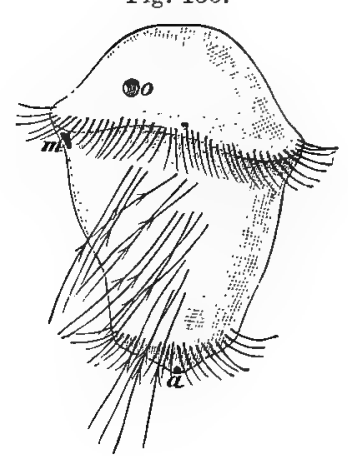

Fig. 134. Diagram of the reproductive apparatus of an Earth-worm; the animal is dissected from the mid-dorsal line and spread out. 8-14, 8th to 14th bristle-bearing segments. o ovary, od oviduct, $s b$ vesicula seminalis, $s g$ spermatheca, $t$ testis, $v d$ vas deferens, $v d^{\prime}$ its outer end, o receptaculum ovorum. The transverse lines represent the septa.-Orig.

Fig. 135. Larva of Nereis. $a$ anus, $m$ mouth, $o$ eye--After Götte.

Asexual reproduction occurs in not a few members of both groups. In some cases there is a simple transverse fission; the animal divides into two nearly equal parts, the posterior of which forms a new mouth, prostomium, etc., before the separation; whilst the anterior produces a new hind end. In other cases budding takes place; the hindmost segment (or a number of posterior segments) elongates and develops into a new individual, which then separates from the parent. Sometimes before separation, the latter begins to produce from its new posterior end, a second new individual in front of the first formed: the process may be repeated, so that a chain arises, consisting of a parent and several buds, of which the hindmost is the oldest and longest, and that nearest the parent is the youngest (Fig. 136). It will, however, easily be seen that a sharp line cannot be drawn between the fission and budding of Chrtopods; in both cases certain of the posterior segments of the original individual become a new individual; in the former a large number of somites pass over into the new animal, in the latter only 
a few, or a single one. In some forms it has been found that the individuals which produce buds develop no genitalia, whilst these

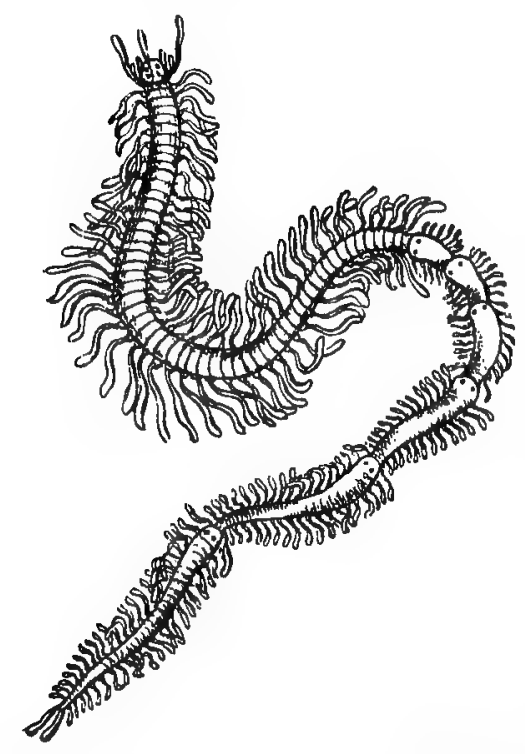

Fig. 136. Chain-f orming Chætopod (Myrianida fasciata), with very long dorsal cirri.-After H. Milne Edwards. are present in forms produced by budding, so that a regular alternation of generations occurs; in other cases, however, both kinds of individuals are sexmal.

Most Chntopods are marinc, and for the nost part cre'p) about on, or burrow into, the soft bottom*; others (Oligochntro) live in like nanner in freshwater or in damp earth; miny forms, which usially live on the bottom, are yet able to swim by serpentine movementis. A few are, however, truly pelagi ic', and like other polngic animals are transparent and provided with cyes, which for Amnolids wo of enormous size. A considerable number form $t u b e H$, consisting of foreign particles, mud, cliay, sand, small stonc', fragments of gastropod or lamellibranch shells, or rhizopod shells cemented together by the secretion of certain skin glands; the separate particles are either irregularly united or neatly fitter into one another like a mosaic. The glands often secrete a chitinous tube, on which are plastered foreign bodies: in other forms the сане consists exclusively of the hardened secretion of the skin glands, and is then either chitinous or calcareous. The tube increnses in mize as the growth of the animal advances: lines of growth may be clearly discerned just as on a snail-shell. The tube is either fastened to some foreign object or lies free; rarely the animal curries it ahout. Some Chætopods, which are provided with strong pharyngeal teoth, lead a predatory life, others feed on alga ; many ure mud and carthfeeders, living on organic particles contained in mud, sand, or wrth.

\section{Order 1. Polychæta.}

The prostomium and the peristomium are usually furnished with appendages (cirri); eyes are frequently present. The chistre are borne upon true parapodia frequently provided with cirri; gills mily

\footnotetext{
* Some species can bore into rock, stone, or clay, but how they do it is not understood.
} 
be present. Sexes separate (with some exceptions). A metamorphosis. Marine.

The following forms are given as examples of this very numerous group.

1. The Nereidæ (Nereïs) have a very elongate body. The prostomium is furnished with four small eyes. Noto- and neuro-podia fused; gills absent. The protrusible pharynx has a pair of hard chitinous jaws. One species of this genus ( $N$. diversicolor) is common on English coasts, creeping, or swimming, or boring into the sand.

2. The Polynoid exhibit a form which, in comparison with that of other Chætopods, is usually very short and broad, and is especially distinguished by having on the dorsal side a varying number of large scale-like epidermal plates; these plates are modified dorsal cirri, and are only present on a few segments, the others being provided with cirri of the ordinary form. Gills are want. ing. Polynoë squamata with rough, uneven dorsal plates; the Sea-mouse (Aphrodite aculeata), has the dorsal scales covered with the very long felt-like chota of the notopodia, forming a felted mat over the back of the animal; other dorsal chætæ are thin hairs with a metallic lustre, and others again are stiff, thick opaque spines. Both on English coasts.

3. The Lugworm (Arenicola piscatorum). Front part of cylindrical body swollen, skin rough. Prostomium and peristomium without appendages; eyes wanting. Noto- and neuro-podia separate, short; the latter a low transverse ridge with a few hooked chætæ standing from it; both without cirri. Gills present only in the middle region of the body, but here well-developed. Parapodia wanting on the hindmost third of the hody. The proboscis without teeth. The Lugworm lives in the sand, burrowing close to the shore; it swallows the sand for the sake of the contained organic particles, the excreta are deposited on the shore, above the holes, as castings. Very frequent on these coasts (used as bait for fish).

4. The Serpulidæ (Serpula) live in fixed calcareous tubes. which are either irregularly or spirally coiled. When undisturbed, the animal projects from the tube a large number of long threads provided with a double row of delicate lateral branches, which are arranged in two groups on the prostomium. These feather-like threads are respiratory, and by means of their cilia drive microscopic organisms into the mouth. One of the threads is specially strong, without lateral branches, and with a calcareous operculum of varying form at the end. When the animal is irritated it withdraws the whole bunch of threads into the tube, which it closes with the operculum. At the anterior end of the animal the notopodia are provided with hair-like chætæ, the neuropodia with hooked chrtx, whilst the converse is the case on the greater part of the posterior extremity. Several species on sea-weeds, stones, etc., on English coasts.

\section{Order 2. Oligochæta.}

The prostomium and peristomium are almost always without appendages. The parapodia are represented only by bundles of chætæ (quite a few in each bundle), no cirri ; gills wanting. Hermaphrodite. No metamorphosis.

The Oligochæta live with few exceptions in fresh water or in the earth. Compared with the Polychrta there are few species.

1. Earthworms (Lumbricus) have elongate cylindrical bodies pointed anteriorly. Each segment is provided with four bundles of chætæ, with only two 
chætæ in each bundle. Eyes are absent. Just in front of the middle is the clite $1 \mathrm{l} \mathrm{u} \mathrm{m}$, a thickened region of skin, covering several segments; and containing a large number of glands, whose mucous secretion holds the individuals together during copulation, and possibly also forms the cocoon in which the eggs are laid. In each cocoon there is generally a large number of eggs. The pharynx is not eversible; jaws are wanting. Earthworms of different species live in cultivated soil, in which they burrow, and upon which they feed. They consume dead vegetable matter, also assisting its decomposition by drawing it into their holes and pouring over it a salivary liquid. The excreta are deposited for the most part at the surface, whither the animal usually repairs only at night. In severe cold, as in very great heat, the worm leaves the surface soil, and goes into the substrata; here the holes are long, usually perpendicular, and lined with an excreted substance. There is generally an expansion at the bottom, where the animal lies in a drowsy condition, as much as 2 to $3 \mathrm{~m}$. below the ground. By these habits, especially by devouring soil and replacing it on the surface in the form of excreta, the Earthworm does more than any other animal to promote the natural elaboration of the soil, and attains thereby a paramount importance in the economy of nature. When a place is deserted by Earthworms on account, e.g., of an inadequate supply of moisture, the surface soil changes and assumes a dry turfy .character; should this occur in a forest, natural planting, by self-sowing, ceases, and unless man interfere, the wood gradually becomes a moor.

2. The Naïdx (Nais) are small (seldom more than $1 \mathrm{c} / \mathrm{m}$. long), thin, and transparent; there are usually two eyes on the prostomium. The chætæ of the dorsal bundle are long and hair-like, those of the ventral bundle short and hooked. Asexual reproduction is of frequent occurrence. The Naidæ live amongst the vegetation in fresh water. Tubifex rivulorum, a reddish worm, common in fresh water, is related to Nais. It forms burrows in the mud, from which, so long as it is undisturbed, the hinder part of its body protrudes in constant motion. Often many specimens are found close together, so that the surface of the mud seems to be coloured red in places; at the slightest movement of the water, the animals withdraw, and the red colour vanishes.

Under the term Gephyrea is usually included a number of vermiform animals, regarded as constituting a special class of the Annelids. When some forms which have proved to be Molluses, have been removed from the group, the remainder are evidently aberrant, peculiarly modified, $\mathrm{Chaetopods.}$ Some still possess $\mathrm{cha}$ et $x$ similar to those of the Chrtopods, but in small numbers, and not arranged in bundles. External segmentation is invariably wanting; instead of the double ventral ganglion chain, there is a single stout nerve cord without ganglionic swellings; it splits anteriorly into two cords encircling the buccal-cavity, and uniting with the often very slightly developed cerebral ganglion. The nephridia are very large, but few in number, at most three pairs, often only one pair, or a single one. They serve as efferent ducts for the genital products which are formed on the walls of the body-cavity. The sexes are separate; a metamorphosis occurs similar to that of the typical Chætopod. It is significant that at an early stage, segmentation of the body is sometimes indicated. Their habits resemble those of the majority of Chætopoda; they are all marine. An interesting form, Bonellia viridis, occurs in various European seas (e.g., the Mediterranean); the fe male possesses at the front end of the short saccular body, a very long tentacle-like prostomium, whose anterior end is forked (body, $5 \mathrm{c} / \mathrm{m}$.; prostomium, $1-2 \mathrm{~m}$.) ; only two chætæ are present, and one segmental organ. The pigmy $\mathrm{male}$ is quite differently proportioned; it is $1-2 \mathrm{~m} / \mathrm{m}$. long, and like a Turbellarian, uniformly ciliated, with neither mouth nor anus, and without prostomium; it lives in the nephridium of the female. 


\section{Class 2. Discophora (Leeches).}

The body is always flattened with sharp lateral edges, rarely cylindrical. The segments are externally divided, each into several small a $\mathrm{n} \mathrm{nuli}$, by transverse furrows, so that the number of segments appears many times greater than it is in reality (the same thing occurs in some Chætopods). Parapodia and chaetæ are always wanting; with few exceptions, no branchiæ are present. The posterior end of the body is modified into a sucker; around the mouth there is also an adhesive disc, which in some is cup-shaped like the hinder one, whilst in others, it consists of a long, jointed upper lip, and a shorter underlip.

The digestive tract consists of three sections: the pharynx, the crop, and the rectum. In one group, the Gnathobdellidæ,

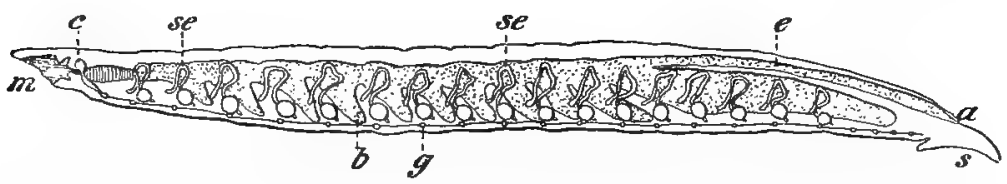

Fig. 137. Digestive tract, nervous system and excretory organs of a L ee oh in outline. $a$ anus, $b$ diverticulum, $c$ cerebral ganglion, $e$ rectum, $g$ sub-cesophageal ganglion, $m$ sucker, se nephidium.-After Lenckart.

the pharynx is muscular, and furnished in front with $\mathrm{jaws}$, three prominent, longitudinal, chitinous ridges, with teeth on their sharp edges, which work like little saws to cut holes in the skin of the prey, so that the fluids may be pumped out of its body by the pharynx. In the other division, the Rhyncobdellidæ, on the other hand, a thin, muscular tube, the proboscis, is attached to the end of the thin-walled pharynx. It may be stretched out from the mouth and pointed, so as to bore through the integument of the prey. The crop is a straight, wide tube, which is almost always provided with a number of paired diverticula; the capacity of the crop and its diverticula allows of the ingestion of a large amount of food. The intestine is narrow, and opens dorsally above the sucker.

A number of e yes is always present upon the anterior end of the animal; in some Fish-leeches on the hind margin of the posterior sucker also.

The Leeches are always he r m a p h rod ite; they possess two long or round ovaries, which open far forward on the ventral side in a common efferent duct: albumen glands open into the oviduct. The round testes are present in great numbers, 6-12 pairs, one pair in a segment; on either side there is a long vas deferens, into which all the testes of the same side open by short ducts: the two vasa deferentia 
finally unite and open by an unpaired aperture in front of the female pore. The eggs aro laid in chitinous capsules (cocoons), usually several together, with a certain amount of

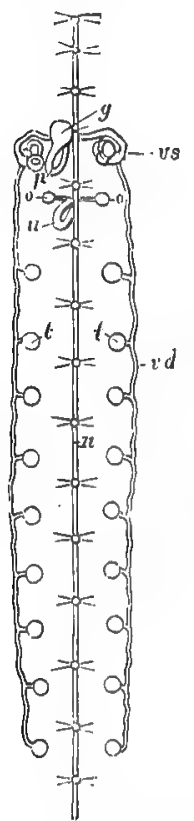

Fig. 138. Genital apparatus of a Leech. "i. ventral nerve cord, $o$ ovary, $u$ oviduct, $t$ tostio, $v d$ vas deferens, $v s$ coiled part of $v u, g$ glands, $p$ penis. -After Spengel. albumen. The capsules, which are formod by a hardened secretion of the skin-glands, have vither a sinouth surface, or are, as in the Modicinal Leoch, covered with a spongy cisse (hardened frothy mucus). 'The young' onc's leave the cocoom in the form of the adult.

Each egg is, of cour'se, covered by an egg-memlirane: the embryo of the Gnathobdellider, where the egrrys are very small, soon bursts this covering, anil lies free in the albumen, upon which it feeds, and thus grows ritpidly. It is in this stage very different from its later forms, ind possesses several provisional organs (pharynx, muscles, etc.), which atrophy, and are replaced by the permanent organs before it leaves the cocoon. The Gnathobdellidw may therefore be said to undergo a metamorphosis within the cocoon. In the Rhyncholuldlidæ, whose egges are larger, this does not occur.

The Leeches, which, compiresl with the Chaxtopoda, form a small group, arc relatively well reju'cscinted in fresh water; still a considerable number. are marine. Some are terrestrial (in the tropics), other's frequently go on shore. They are prediatory, or are temporary parasites, sucking the blood of larger animals; some arestationary parasites. They creej : ubout in the well-known mannor by ment of their suckers, but are also alle to swim by serpentine movements of the body.

1. Gnathobdellider. With jaws. Anterior : adhesive organ divided into an upper and a lower lp. Eggs small; the young ones undergo a kind of metamorphosis within the cocoon. All fresh-water or terrestrial.

(a) The Medicinal Leech (Hirudo medicinalis), a fresh-water form, varying in colour, occurring in different parts of Europe, and in. England. Its jaws are very strong and have pointed teeth. The dorsal surface is a grrenis ls grey, with reddish longitudinal stripes, flecked with black : the ventral surface is paler, but speckled. Ten eyes. The spongy egg-capsules are laid on land, in banks. To this genus belongs the well-known East Indian L a nd-le ech (H. ceylonirn). Hrmopis vorax is allied to the Medicinal-leech, which it resembles in shape and size. It is indigenous to $\mathrm{S}$. Europe and $\mathrm{N}$. Africa. It frequently enters the nostrils, pharynx, and throat of different Mammals with drinking-water, and may occasion serious inconvenience.

(b) The $\mathrm{Hor}$ se-leech (Aulastomum gulo). Very common in fresh wister. in England, of a similar size to the Medicinal-leech. It is frergnently mistaken for Hiemopis vorax. The jaws are less developed than in the Mrdicinal-leech. It attacks no Mammal, but lives on Earthworms and small aquatic animals. It is greenish-black above and yellowish-brown below. Ten eyes. The egg-capsules are like those of the Merlicinal-leech, and are laid on land. Species of the genus Nephelis are also frequently met with in freah water; they are shorter anil 
narrower, and possess only eight eyes and very weak rudimentary jaws; the cocoons are smooth, and are fastened to water plants.

2. Rhynchobdellidx. With proboscis. Anterior organ of adhesion, cup-shaped. Eggs large; no metamorphosis. Fresh-water and marine.

(a) Clepsine, a small, flattened leech, almost as hard as cartilage, which is frequently found in fresh water. The eggs, enclosed in a very thin cocoon, and the young ones, are carried about on the underside of the body of the parent, which then seems to be hollowed out like a cup.

(b) The Fish-leech (Piscicola), with cylindrical body and bell-shaped sucker at both ends; lives as a parasite upon most species of marine fish. Nearly related to this is the large Pantobdella muricata, with liurge integumentary warts; upon Skates in the North Sea.

NoTE-A little worm, parasitic upon the Crayfish (on the gills and elsewhere), Branchiobdella astaci, is usually put with the Leeches. It approaches the Chrtopoda in some points, and by some authorities is counted as one of this group. The body is cylindrical, the anterior. sucker indistinct; it possesses two jaws and a gut without diverticula. The conditions of the genitalia recall those of the Oligochæta.

\section{Class 3. Onychophora.}

This division includes only the genus Peripatus, which may be regarded as a Chrtopod adapted for terrestrial life.

In external appearance the Peripatus species are most like caterpillars. The body is elongate and cylindrical, the segments not externally demarcated. The skin is granular, and delicately striated transversely. At the anterior end there is a pair of ringed tentacles (these appendages may be ringed also in the Chætopoda), and a pair of simple eyes of the kind shown in Fig. 20, 5. In the mouth there is a pair of jawlike masticatory organs. The rest of the body consists of similar segments, each of which bears a pair of indistinctly jointed, stumpy $\operatorname{limbs}$, ending in two claws. The muscles are composed of $\mathrm{smooth}$ muscle cells. The nervous system is characterised by separation of the ventral cords, which are joined by many delicate transverse strands, whilst only feeble swellings are present in each segment. The alimentary canal is a straight tube; the anus lies at the posterior end of the body. The heart is dorsal, and is a tube provided with lateral slits; other vessels are wanting. The respiratory organs consist of a well-developed system of a ir - carrying tubes, which

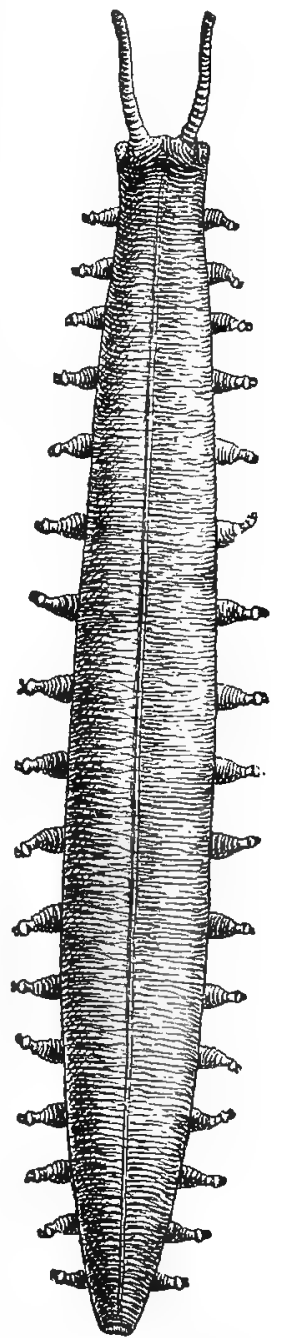

Fig. 139. Peripatus, from the dorsal side.After Balfour. ramify in the body and open upon the surface in many delicate, irregularly-distributed, respiratory apertures. In most segments there is a pair of segmental organs, similar to those of other Annelids: they 
open into the body-cavity by large funnels, , and to the exterior by delicate apertures at the bases of the limbs. The sexes are separate: the paired g on a ds open at the posterior end. The species, as a whole, is viviparous.

Recently Peripatus has been very generally classed with the Arthropoda, chiefly on account of the presence of the air-tubes mentioned above, which are like the tracheæ of Insecta, and Myriapoda. But there are weighty facts for the other side: the eyes are of the same kind as those of the Chrtopods, and quite different from the Arthropod type; a complete set of segmental organs is never found elsewhere in the Arthropoda; in the Tracheata, indeed, they are entirely wanting; also the character of the muscle cells is altogether opposed to a relationship with the Arthropods which exhibit striated muscle fibres. Under the circumstances it seems best to regard the air-tubes as merely analogous with the tracheæ, attributing their presence to a terrestrial life, whilst they are ( $c f$. Insecta) the cause of the degeneration of the vascular system.

The species of this group live exclusively in warm elimates in both hemispheres (W. Indies, Cape, and elsewhere), in damp places, in rotten wood, etc.

\section{A PPENIX TO THE ANNEIDA.}

Each of the groups now to be discussed, the Polyzoa and the Brachiopoda, occupies an isolated position in the Animal Kingdom : it is doubtless therefore most correct to treat them as two special phyla. They were formerly placed with the Mollusca, with which, however, they are not at all closely allied. From the most recent researches, it seems that their nearest relatives-though even these are sufficiently remote-are the Annelids, wherefore they are taken in this connection.

\section{Polyzoa (Moss-animals).}

With a single exception, all the Polyzoa form colonies by budding; individual members attain to only a small size, but the extent of the whole colony may be very considerable. The rather short body of each zooid is usually divided into a fore and a $\mathrm{h}$ ind portion : the latter is covered with a firm, thick, sometimes spiny, chitinous investment, the ectocyst, which is often calcified. The front part is, on the other hand, quite soft, and bears at its anterior extremity, a wreath of long ciliated tent acles (the lophophore). In the great majority of forms, this is a simple circle, but sometimes there is a large sinus on one side, which gives it a kidney, or horseshoe, shape. The whole of the front part can be withdrawn into the hinder part by means of a long muscle (Fig. 14.2). The wall of the front part is then introverted to form a sheath round the retracted tentacles (tentacle-sheath). In one section of the marine Polyzoa (the Chilostoma) there is, at the anterior

* According to some accounts the segmental organ ends in a closed, thin-walled vesicle, not in a funnel. 
end of the chitinous case, a movable, chitinised fold of the wall, which acts as an operculum to the month of the tentacle-sheath, when the soft part of the body is retracted. The mouth is at the anterior end in the midst of the circle of tentacles; the anus lies at this end
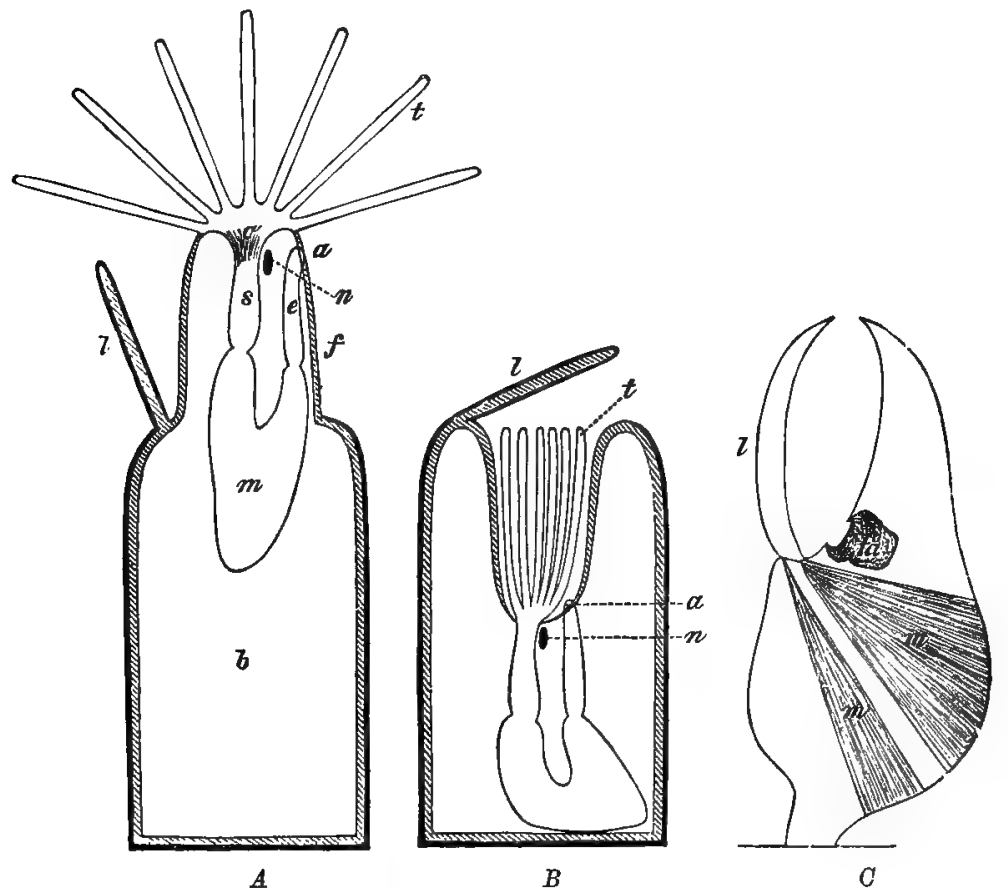

Fig. 140. $A-B$ Diagrammatic longitudinal sections of a $\mathrm{P}$ ol y z o o $\mathrm{n}, A$ expanded, $B$ retracted. $a$ anus, $b$ hind-end, e rectam, $f$ fore-end, $l$ operculum, $m$ stomach, $n$ nerve ganglion, o mouth, $s$ cesophagus, $t$ tentacle. The chitinous covering is indicated by a wide black line, the soft wall of the body is shaded, $O$ a vicularia (diagrammatic), $l$ operculum, $m$ its muscles, ta gut.-Orig.

also, not far from the mouth, and is usually just without, seldom within, the lophophore. The alimentary canal is, therefore, in the form of a loop; it is made up of an osophagus, a stomach provided with a cæecum, and a rectum. The food, consisting of microscopic particles, is driven into the mouth by the cilia of the tentacles. The central nervous system consists of a nerve ganglion, which is situate on the side of the œsophagus near the anus, and of a nerve-ring surrounding the œsophagus. Nerves from the ganglion pass to the different parts of the body. Optic and auditory organs are wanting; so are a vascular system, and special respiratory organs ; the lophophore is, however, doubtless of respiratory importance. Excret ory organs have hitherto been found in only a few Polyzoa, in the form of two short canals, opening at one end into the 
body-cavity, and at the other, to the exterior by a common aperture, near the lophophore.* The Polyzoa usually have a large body-cavity,

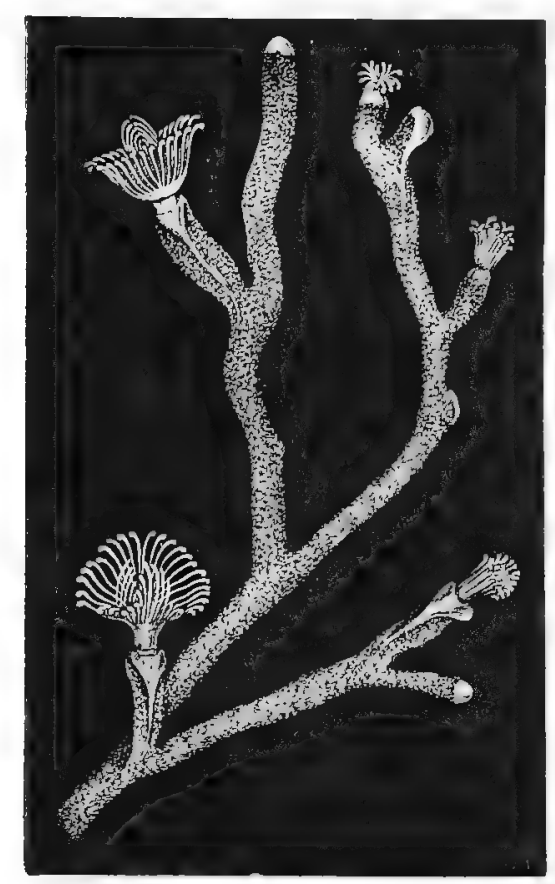

Fig. 141. Plumatella polymorpha, a freshwater Polyzoon. Enlarged.-After Kräpelin. filled with a liquid in which amœboid cells are found; it contains, besides the alimentary canal, a cord, the funiculus (Fig. 142), stretching from the stomach to the body-wall, upon which, or upon the inner side of the body-wall, ova and spermatozoa appear, both, usually, in the same individual; special sexual ducts are absent, the genital products (or embryos) pass out through holes in the body-wall, or through the excretory organs. Generally, the fertilised ovum undergoes its earliest development within the body of the parent, in many marine forms, in a special invagination of the body-wall (ocecium).

Among the freshwater Polyzoa reproduction is effected by means of statoblasts, as well as by fertilised ova. The statoblasts are small, discoid bodies arising upon the funiculus by a peculiar process of budding. They are produced chiefly towards the end of the summer, and rest during the winter, developing, in the next year, into a new colony. Each is provided with a hard ornamental shell, in whose edge there are small air cavities. The new animal is formed from a mass of cells within.

In many forms a very remarkable de generation of the lophophore and alimentary canal occurs, constituting the so-called "brown body," from which these parts are, after a time, reconstructed.

The colonies formed by the Polyzoa are of very different kinds. Some are much branched (Fig. 141), and either stand erect from, or creep over, some foreign object; others are laminate, lying upon the substratum or standing upright: or they may be more massive. The colony is almost always fixed; a single freshwater form (Cristatella) is free.

Amongst many of the Chilostoma, dimorphism, like that in the Hydrozoa, occurs. Specially common among the ordinary individuals

* These canals do not seem to form excretory products themselves, but serve as a means of exit for cells, loosened from the epithelium of the body-cavity, in whose protoplasm certain nitrogenous waste products are secreted. 
are the so-called a v i c u l a ri a (Fig. $140 \mathrm{C}$ ), small individuals, destitute (or with only rudiments) of tentacles, mouth, and digestive tract, but with a large movable operculum, which can open and shut. The best developed avicularia resemble crabs' claws or birds' beaks, for the tip of the operculum is bent like a hook, and bites upon an outgrowth of the body. They seem to be a kind of defensive person, to catch the animals crawling over the surface of the colony. More rare are the vibracula, also small reduced persons, whose operculum is developed into a long whiplike process, which sweeps over the surface of the colony.

The Polyzoa undergo a metamorphosis. There is a free-swimming larva, whose cilia are either evenly distributed over the body, or restricted to special regions (ciliated ridges or

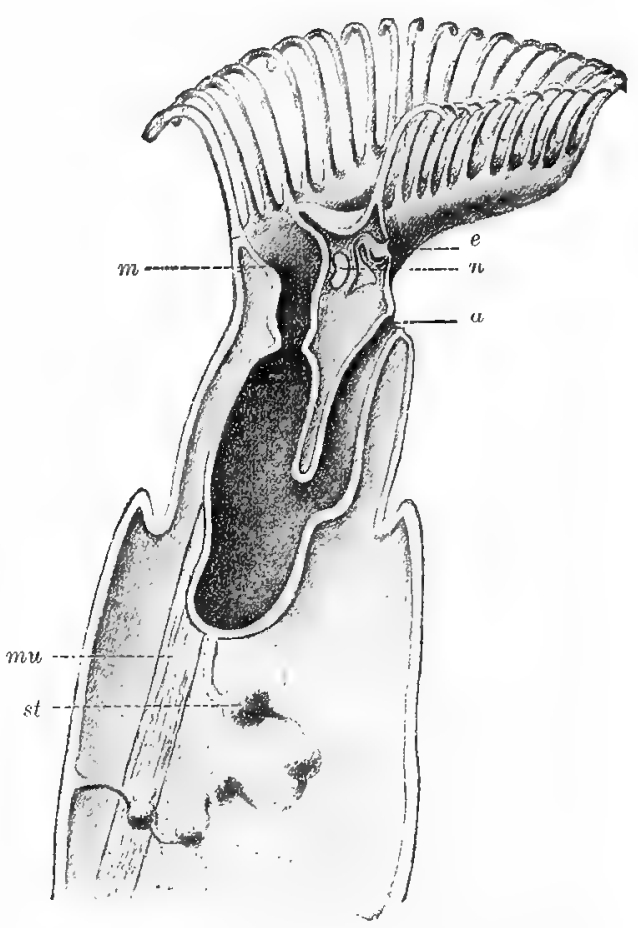

Fig. 142. Fresh-water Polyzoon, bisected. Diagrammatio. a anus, $e$ excretory aperture, $m$ mouth, mu muscle, $n$ nerve ganglion, st statoblast on the funiculus.-Orig. tufts); sometimes there is a hard cuticle or shell upon part of the body, usually it is entirely naked.

They are very numerous in all seas; a few live in fresh water.

The fresh-water forms, which are found on water plants, etc., generally have a horseshoe-shaped lophophore; and form a delicate branched colony, which is not raised much above its support: but some species grow erect, neighbouring branches supporting one another reciprocally, and thus forming large clumps. Amongst the marine forms are the Membraniporida, which may often be seen forming calcareous incrustations upon the surfaces of all large sea-weeds.

\section{Brachiopoda.}

The body is generally enclosed within two calcareous, or rarely, chitinous shells, somewhat like those of the Lamellibranchs, with which, therefore, the Brachiopoda were in timés past associated. As a matter of fact the two groups are in no.wise nearly related to one another, 
and that the presence of the shells does not denote a relationship is evident from the circumstance that those of the Brachiopoda are dorsal and ventral, whilst in the Lamellibranchs they are right and left.

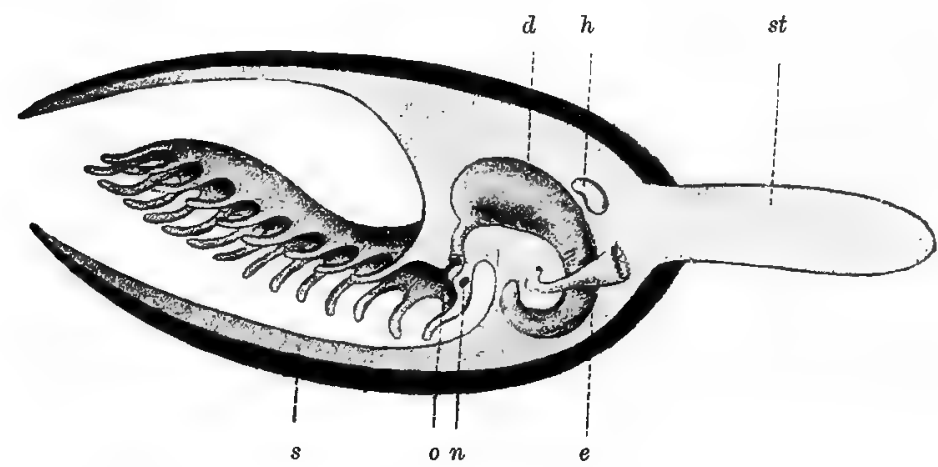

Fig. 143. Diagrammatic longitudinal section of a $\mathrm{B} \mathrm{rachi}$ opod. $d$ digestive tract, $e$ excretory organ, $h$ heart, $n$ nerve ganglion, o mouth, $s$ shell, with the mantle lying within it, st peduncle, $t$ tentacle.-Orig.

Compared with the whole extent of the animal, the actual body is of very small size, and very short. Two large mantlefolds, lining the inside of the shell, spring from it. The shells are secreted by the mantle, and are to be regarded as cuticular structures. Unlike the Lamellibranch valves they are not connected by a ligament; but in some forms they are attached by a hinge posteriorly. Chitinous bristles, implanted in pits in the skin are often present along the edge of the mantle. From the posterior end of the body there usually springs a process, the peduncle, which projects from between the valves, or from a hole in the hinder part of the dorsal shell: in some species it is longer than the rest of the body, in others it is very short. Most of these animals are fixed to foreign objects by means. of the peduncle, but some are free. In young Brachiopods a circle of tentacles surrounds the mouth, but during development, an in-pushing of the wreath occurs, which results in its becoming kidney- or horseshoe-shaped and gradually both branches of the horseshoe are drawn out into long a r m s beset with a double row of tentacles: the arms are usually spirally coiled, and lie between the mantle-lobes; they serve as a respiratory organ, and also waft food (minute organisms) into the mouth with their cilia; frequently they are supported internally by a variously shaped (e.g., ribbon-like) calcareous structure, which is connected with the dorsal valve. The alimentary canal may be short or long; curiously enough, in most Brachiopods an a $\mathrm{n} \mathrm{us}$ is wanting, when present it is on the right side of the body. There is a well-developed liver. The central nervous system is represented by a nerve-collar surrounding the cesophagus, swelling out on 
the underside into a ganglion, from which the nerves proceed. There are neither optic nor auditory organs. The vascular system is well-developed; a saccular heart lies above the digestive tract. The excretory apparatus consists of one or two pairs of tubular organs which open at one end into the body-cavity by a ciliated funnel, and to the surface at the other exhibiting a great resemblance to the segmental organs of the Annelids. They serve, at the same time, as a means of exit for the genital products, which are formed on the wall of the body-cavity. The Brachiopoda are of separate sexes. The ciliated larva swims about freely. Its body is sometimes divided (Fig. 144) by constrictions into segmentlike sections. Eyes may be present at the front end, and provisional bundles of bristles (Fig. 145) behind. (Of. the Chætopods).

The Brachiopoda are exclusively marine; they are as numerous in warm as in cold seas; there are, however, but few species. They were

Fig. 144.

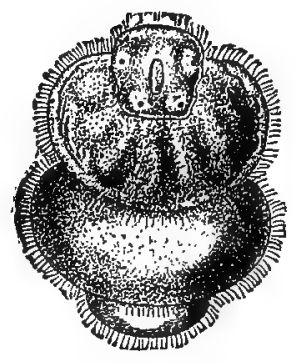

Figs. 144 and 145. Larvae of two Bracioiopods. - After Lacaze-Duthiers and Kowalevsky. very numerous in early times, and are known from the Cambrian formations. They were well represented in the Silurian, the Devonian, and the Jurassic.

As examples may be cited: Terebratula, living as well as fossil, dorsal and ventral shells convex, the former drawn out into a beak-like process, pierced by an aperture for the short peduncle, by which the animal attaches itself to stones, etc.; in other similar forms there is a notch at the same place. Dorsal valve with a loop-like brachial skeleton. Lingula, extant and fossil, two thin, flat, horny, almost equal, hingeless shells; peduncle very long, surrounded by a sandy tube. 


\section{Phylum 6. Arthropoda.}

The body is divided into a number of segments demarcated externally by constrictions, and provided with jointed limbs, which constitute efficient locomotor organs: it resembles, therefore, the Annelid body in the former respect, but differs in the latter. Moreover, there is a greater dissimilarity in the formation of the body segments than in the Annelids; among the Arthropoda, the body (exclusive of the head), is usually divided into two or more regions, which are distinguished by a special modification of the constituent segments, and the individual segments of each region often differ considerably from one another. This dissimilarity is manifest both externally and internally. Furthermore, the limits between certain of the somites are often obliterated so that they come to be more or less intimately united to form a compound structure, the origin of which can only be made out from a comparison with other forms, or from a study of the development. The most anterior region of the body, the $\mathrm{h}$ e a d, is always composed of several fused segments; some of the appendages thus brought together serve for feeding, and are called mouth-parts; there are usually, also, one or two pairs of feelers or antennæ.

In the majority of Arthropods, three pairs of mouth-parts are present; the first are the mandibles, usually strong hard-biting organs; the second and third are known respectively as the first and second maxillæ; they are almost always more feebly developed than the mandibles. These three pairs may be augumented by others called maxillipeds, when more segments are included in the head.

As in the Annelids again, the body, with its appendages, is invested by a cuticle, secreted by the epidermis. It differs in an apparently trifling, but in its results very important, respect from that of the Annelids; for it is usually of a much greater thickness and $h$ ardness than in these, forming as it were an armour for the body, an exoskeleton. Only at the constrictions between the segments, both of the body proper and of the limbs, does it retain 
a certain thinness, so that movement can take place at these points. All Arthropods moult* periodically, at least, as long as growth continues; the cuticle loosens from the underlying tissue, breaks at some point, and is cast off as a whole (i.e., the animal creeps out of it) after the epidermis has secreted a new cuticle. This is thin and soft at first, but becomes thick and hard later. Such periodic ecdyses are indispensable for growth, for the stiff, unyielding cuticle allows only of very slight increase in the size of the body. The growth of the animal would therefore cease, if the surrounding case were not now and again thrown off and replaced by a new and roomier one. Upon the body, there are larger or smaller tracts of setæ, evaginations of the cuticle, each containing a process of the soft epidermis; the cuticle at the base of the hair is thinner, so that it can move about. The cuticle consists of chitin, an organic substance, of a horny appearance, chemically however, quite different from horn. Lime salts, principally carbonate of lime, are often deposited in the chitin, especially in the Crustacea.

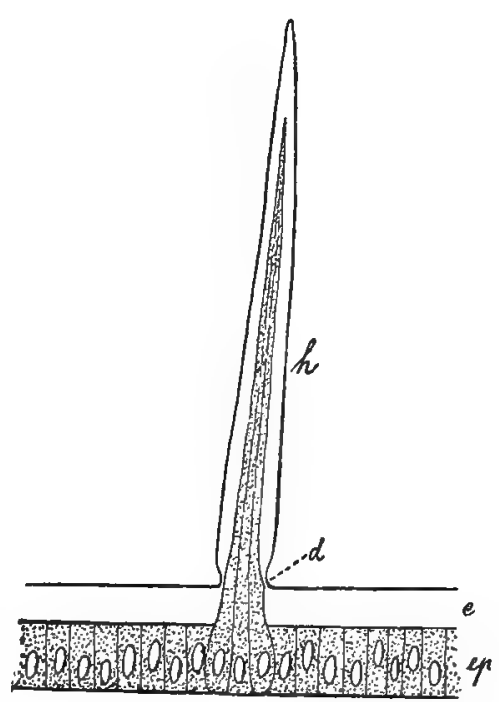

Fig. 146. Section throngh a hair and the adjacent skin of an Arthropod; diagrammatic. $c$ cuticle, $d$ thin part at the exit of the hair $h ; e p$ epidermir.-Orig. The skin is never ciliate among the Arthropoda, nor indeed is any other organ; in fact ciliated cells are entirely absent.

The muscular system is closely connected with the skin; the formation of a segmented exoskeleton, however, necessitates important deviations, from the Annelid type. Instead of a continuous musculature beneath the skin, there is usually a large number of separate muscles passing from one segment to another, and attached by their extremities to the inner side of the skin: by their contraction the segments of the body, as also the joints of the appendages, move upon one another. The muscles are often connected by the so-called tendons, which, in the Arthropods, always consists of invaginations of the cuticle, surrounded of course by a corresponding invagination of the epidermis

* In many (all?) Annelids (e.g., Leeches) and in Nematodes, a similar ecdysis occurs. 
(Fig. 148). They are thrown off with the rest of the cuticle at each moult and renewed. The muscular tissue of the Arthropods consists of striated, multinucleate muscle fibres.

Fig. 147.

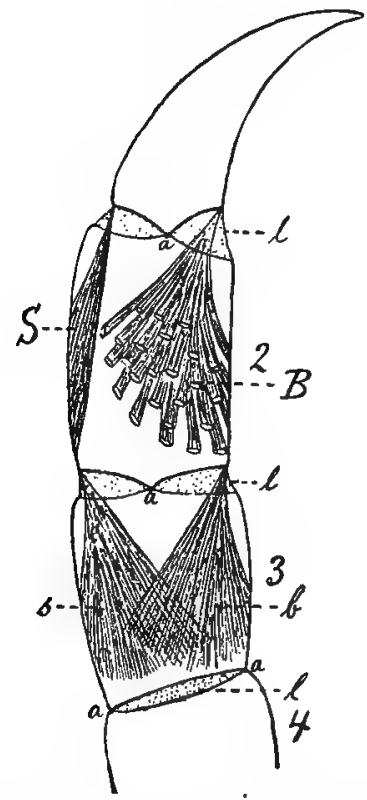

Fig. 148.

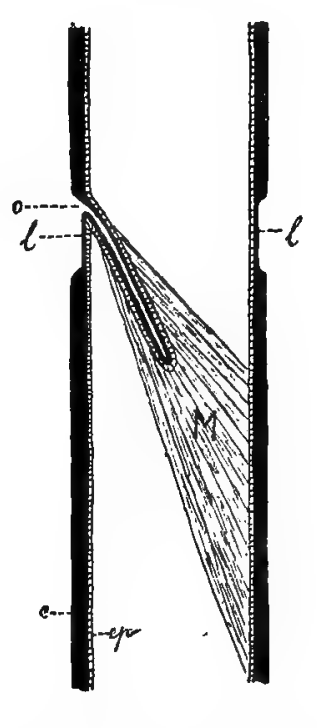

Fig. 147. The last four joints of an arthropod limb with their muscles : diagrammatic. $l$ articulation, $B$ and $b$ flexors, $S$ and $s$ extensors, $a$ places where two joints touch one another, and the articular membrane is very narrow; 1 terminal, 2 penultimate joint, etc. - Orig.

Fig. 148. Longitudinal section through a joint of an Arthropod : diagrammatic. $c$ cuticle, $e p$ epidermis, $l$ articular membrane, $M$ muscle, o opening of the tendon to which the muscle is attached.-Orig.

The nervous system agrees closely with that of the Annelids. Just as in these animals there is a pair of ventral ganglia in each segment, connected with those of the adjacent segments by a double nerve cord. From the most anterior of these ganglia spring two nerve cords, which run round the œsophagus to unite with a paired ganglion mass, the cerebral ganglion, lying in the head. This often attains to a very considerable size, which is correlated, amongst other things, with the development of certain sense organs, situate on the head, the compound eyes. The ventral ganglia often exhibit remarkable differences from those of the Annelids, differences which are due to the above-mentioned dissimilarity in the formation of the segments and their grouping into different regions. In well-developed segments for example the ganglia are large, whilst a fusion of many segments is accompanied by a fusion of their ganglia. In some cases, 
indeed, all the ventral ganglia may unite into a single unsegmented mass; this is always accompanied by the shortening of the body, as in Crabs. Sometimes ganglía are shifted during development, so that those belonging to one segment move further forward; but the nerves arising from such a pair are distributed to the segment to which they properly belong. The members of a pair are united by a commissure, which is almost always short, often so short that they appear to be fused; this is often the case, also, with the connectives of consecutive pairs.

Sense organs. The formation of a cuticular skeleton results in the restriction of the sense of touch to certain spots on the surface of the body. In particular many setæ become tactile; beneath the epidermis lie one or more sensory cells, each of which sends a filiform process into the seta from one end, and a nerve fibre from the other end, to the central nervous system. Hairs, provided with a thin cuticle, and occurring upon the first antennæ of Crustacea, act as olfactory organs; so also do the peg-shaped processes upon the antennæ of Insects (see p. 19): like the tactile structures, they are connected with sensory cells. Auditory organs are found in many Crustacea, and in some Insects; these will be considered in the several groups. O p ti c organs, which reach such a high stage of development among the Arthropoda, appear in two forms; as simple eyes, or ocelli, and as compound eyes. The most important points in the structure of these eyes have already been noticed in the General Part, pp. 21, 22. In most of these animals there is a pair of compound eyes, as well as several ocelli, but in others ocelli only are developed.

The digestive tract usually runs through the body as a tolerably straight tube; the mouth is at the anterior end, and is usually ventral; the anus

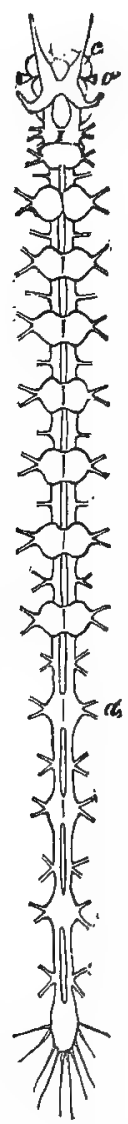

Fig. 149. Nervons system of $\mathrm{Gammarus}$. c brain, o eye, $a$ first abdominal ganglia, $I$ first thoracic ganglia. -After Sars. is posterior. Salivary glands and a liver may or may not be present.

Vascular system. The heart, which is usually tubular, corresponds to the dorsal vessel of the Annelids, and is found on the dorsal side, above the digestive tract. It is furnished with venous ostia, generally several pairs, through which the blood enters the heart from the surrounding blood-space, the pericardium: the pericardium receives the blood from the gills (lungs) when such are present, or from the body. In other respects, the vascular system of 
different Arthropods presents very considerable variations, which will be dealt with later; in a few forms (Acarines, small Crustacea), it is entirely wanting. The blood is usually a colourless fluid, with colourless, amoboid blood corpuscles.

From some small Crustacea respiratory organs are entirely absent; generally, however, there are either gills, or peculiar airbreathing organs (See special classes).

Excretory organs. The segmental organs, familiar in the Chrtopods, occur again in one division of the Arthropods, but reduced to a small number, two pairs; the antennary and shell. glands of Crustacea (see this group) are modified segmental organs. In Insecta, Myriapoda, and Arachnida there is, on the other hand, no trace of such structures; instead, they possess the so-called Malpighian tubes, long glandular canals, which open into the hind gut and perform an excretory function.

Genitalia. The Arthropoda are, with few exceptions, of separate sexes: the male and the female glands closely resemble one another. There is never more than one pair of genital glands, and this is frequently united or even fused to form an unpaired organ. From each gonad springs a duct (the oviduct or vas deferens), which opens on the ventral side, always in front of the anus; the ducts are frequently united for the last part of their course, and then there is only one aperture. Even when the glands are connected or fused there are generally two ducts.

\section{Class 1. Crustacea.}

The $\mathrm{head}$ is never sharply marked off from the rest of the body (as is the case among Insecta, for example), but some of the thoracic segments are usually fused with it. It bears, besides the eyes, which will be dealt with later, two pairs of antennæ (the antennulæ and the antennæ), and three pairs of jaws, the mandibles and the first and second $m a x i l l æ$. The antennæ are usually elongate, whip-like appendages, consisting of a short, jointed, basal piece, or peduncle, and a long, flexible end-piece, composed of many joints; or the peduncle may bear two such filaments. The most important part of the mandibles is a hard, unsegmentęd, basal piece, the true mandible, which is provided, as a rule, on the inner side with a sharp dentate edge, and often with a rough, grinding surface. The sharp edge, as well as the grinding surface, works against the corresponding parts of the other mandible. The basal part often bears a smaller jointed appendage, a "palp." The other pairs of jaws are not nearly as strong as the mandibles: they are lamellate, and the inner edge is divided into several lobes, beset with stiff setæ; 
they also often possess a small end-piece, a palp. The rest of the body bears a varying number of $\mathrm{limbs}$, arising on the ventral side, one pair to each segment. The terminal segment is frequently apodous, so also may be some of the others. In rare instances all these appendages are almost or quite identical, but usually those of the different segments are more or less dissimilar. Frequently, for instance, the foremost are nutritive in function, and are correspondingly modified, and are then called maxillipeds; the hindmost may be swimming organs, whilst others, again, are ambulatory. The limbs are, in short, highly specialised in form and function.

It is, however, possible to reduce all the limbs to a common ty pe, not only those which belong to the trunk, but also those of the head, i.e., the second antennæ,* and the three pairs of jaws. A typical crustacean limb consists of the following parts: (1) a main stem or endopod, composed of a number of joints, and constituting the chief part of the limb; (2) an outer branch or exopod springing from the second joint of the endopod; unjointed, or at least not divided into special pieces moving upon one another; usually flat, and provided with marginal setæ; (3) an epipod arising from the basal joint of the endopod, always unsegmented,

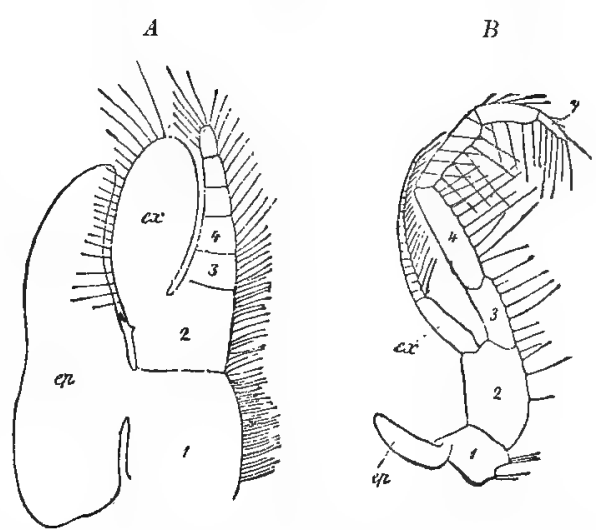

Fig. 150. Example of typical crustacean li $\mathrm{mbs}$. A thoracic limb of Nebalia. B last maxil. liped of a larval Prawn. 1--7 joints of the stalk. ex exopod, ep epipod. Enlarged.-Orig. usually sparsely setose, thin skinned, and as a rule subserving respiration. Exopod or epipod, $\uparrow$ or both, may be absent, so that the limb consists of the endopod only; and even this may be more or less degenerate. On the other hand certain joints of the endopod may be specially well-developed, as, for instance, the basal joint of the mandible.t The exopod may be flat or round, curled or uncurled, ete.

Among other cephalic structures must be noticed the carapace (or shell), a backwardly-directed, mantle-like fold, arising from the

* The first antenne, though often called limbs, do not agree with the rest of the appendages, but exhibit peculiar relations (the exopod is always absent from the second joint, etc.); like the eye-stalks they are to be regarded as special appendages, which function as the supports of special sense organs (olfactory and auditory).

+ The epipod is always absent from the appendages of the head.

I The true functional mandible is merely the basal joint developed as a cutting or grinding organ; its jointed appendage, the palp, represents the rest of the endopod. 
hind part of the head, and covering a greater or less part of the body. It is frequently concrescent with the thorax along the mid-dorsal line. Sometimes the mid-line of the shell is softer than the rest, so that it is divided into two movable halves, which surround the body like ar

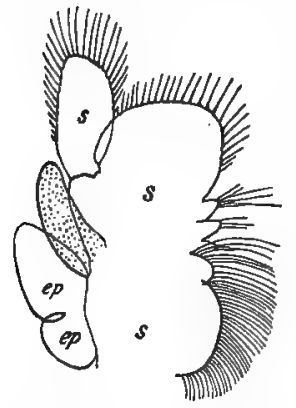

A

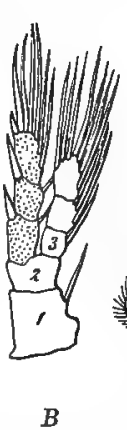

$B$

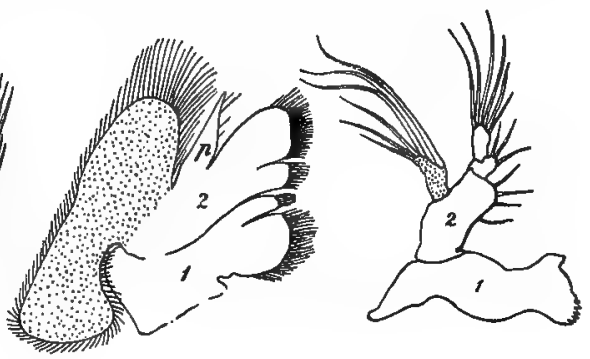

$C$

$D$

Fig. 151. Lim bs of different Crustacea; the exopod is dotted. $A$ swimming leg of Branchipus. $B$ swimming leg of a Copepod. $C$ second maxilla of a Decapod. $D$ mandible of a Copepod. $s$ endopod, 1, 2, 3 its joints, $p$ palp, ep epipod.-Orig.

lamellibranch shell. The outer surface of the shell is usually covered with a thick, hard cuticle, so that it forms a really protective covering; the inner surface, is, on the other hand, softer. The carapace is very characteristic of the Crustacea, although it is absent from many forms.

The cuticle covering the body is often of a considerable thickness and hardness, the chitin always containing lime salts (carbonate of lime) in varying quantity.

The olfactory organs are situated on the antennules, the function appearing to be restricted to long, soft, thread-like setæ. A uditory organs are known only in some Malacostraca (see Mysidæ, Decapoda). Optic organ s may be represented either by

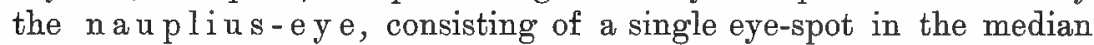
line of the head, or of a small group of them, or by a pair of large compound eyes on the sides of the head, often placed upon movable or immovable stalks. In some forms both median and paired eyes are present, in some only the former, in many only the latter. In numerous cases the nauplius-eye is present in the larva, but atrophies later.

The digestive tract begins anteriorly on the ventral surface of the head, in a mouth opening between the mandibles. It is often bounded before and behind by projecting folds of skin, the up per, or the under lip. The alimentary canal is a straight tube, which opens upon the terminal segment of the body. A liver is usually present.

Respiratory organs. The Crustacea, for the most part, breathe the oxygen dissolved in water. In many, particularly in small thin-skinned forms, special respiratory organs are wanting; then the whole surface of the body, or the greater part of it, performs 
this function. In others, again, certain parts of the body are specially developed as gills. Sometimes the thin-skinned inner side of the carapace serves as a breathing apparatus; in other cases, the flattened epipod, or some other part of the limb, acts as a gill; in yet others, the gills are special, usually branched, appendages, springing from the limbs or from the side of the body. These true gills, which are usually characterised by the possession of a close and delicate vascular network, may be supplemented by either the whole, or a part, of the surface of the body.

Some of the terrestrial Crustacea breathe atmospheric air, and here, peculiar respiratory organs are sometimes developed. This is, e.g., the case in Birgus latro, an East Indian form related to the Hermit-crab; the gills are very small, but the branchial-cavity, enclosed by the sides of the carapace, serves as an air-breathing organ, and is, therefore, provided with large vascular excrescences of the surface, arising from the inner side of the carapace. In a true hermit-crab, Coenobita, the soft skin of the abdomen serves as a respiratory organ, and is furnished with a vascular network for this purpose. In some terrestrial Isopods the abdominal limbs have branched invaginations of the skin which take in air and serve as breathing apparatus.

The vascular system exhibits a variety of modifications. In some forms, it is represented only by the heart, which drives the blood into the spaces between the organs; in a few cases, even this is wanting. A poorly-developed vascular system is usually correlated with a small body, and with the absence of special respiratory organs. When these are present, there is, as a rule, a

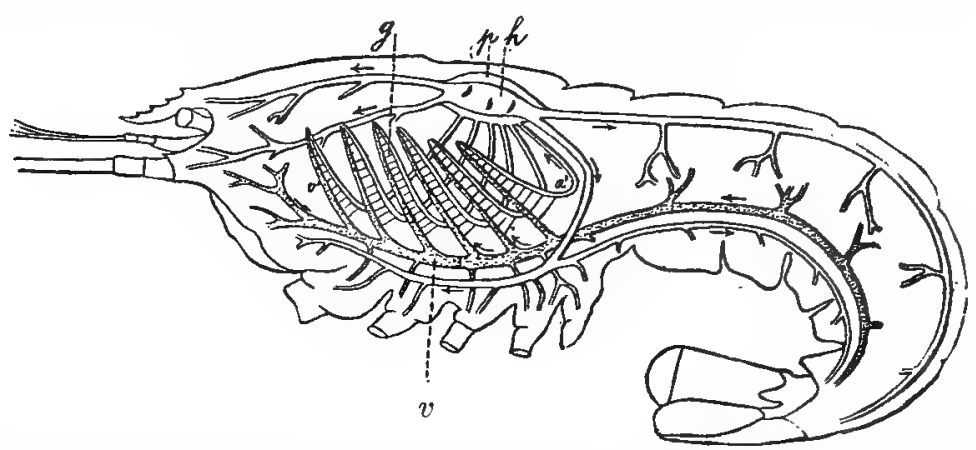

Fig. 152. Vaseular system of the Lobster, diagrammatic ; the vessels which carry arterial blood, light, the others, dark ; the arrows indicate the direction of the blood stream. $g$ gills, $h$ heart, $p$ pericardium, $v$ venous sinus, $v^{\prime}$ vessel thence to the gill, $a^{\prime}$ from the gill to the heart.-After Gegenbaur.

better-developed system, and definite vessels, although these may fail in many parts of the body, so that the blood flows in the spaces between the organs; the vascular system is consequently not entirely closed. The circulation in gill-bearing Crustacea is as follows (see Fig. 152): The blood is driven from the heart, through more or less perfect arteries into the different parts of the body; after it has received carbon-dioxide, and given up oxygen here, it collects 
in large blood reservoirs, whence it is passed on to the gills. After receiving fresh supplies of oxygen, it travels through special vessels to the pericardium, enters the heart through the ostia, and then recommences the circulation.

Excretory organs. Two pairs of tubular organs, probably representing the segmental organs of the Annelids, are found in the Crustacea. The inner opening is wanting; they are usually of considerable size and much coiled. The foremost pair, the a n ten naryglands, open on the basal joints of the second antennæ; the second, the shell-glands, ${ }^{*}$ at the base of the second maxillæ. Both pairs are seldom developed in the same animal; frequently one pair is present, and atrophies later, when the other is formed. $\dagger$ Often both are absent.

The great majority of Crustacea are of separate sexes. The genital organs open ventrally, as a rule at a considerable distance from the posterior end, and usually by two distinct openings. The apertures of the oviducts are, in many forms, further forwards than those of the vasa deferentia. Not infrequently, parthenogenesis occurs (see Branchipus, Apus, the Daphnids).

The development of the Crustacea is usually connected with a very distinct metamorphosis, for the young one,

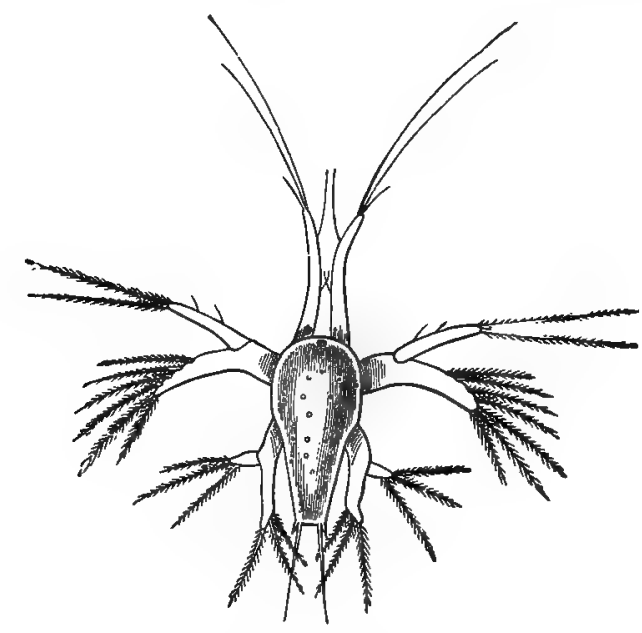

Fig. 153. Nauplius of Penceus. Enlarged.After Fr. Müller. when it leaves the egg, is essentially different from the adult. The difference depends, amongst other things, upon the smaller number of segments and limbs possessed by the larva; and further upon the different structure and even function of these limbs. A large number of Crustacea leave the egg in the so-called nauplius-state ; as small, compact creatures, furnished only with the first and second antennæ and the mandibles. These appendages are all de-

* This name is connected with the fact that the glands often (e.g., in Apus) lie for the most part in the carapace.

† Among the Entomostraca, the shell-gland is universally present in the adult, the antennary gland in the larva; whilst, conversely, the adult Mal acostrac an exhibits the antennary-gland, the larva, the shell-gland. 
veloped as tolerably powerful swimming organs, the posterior antennæ and the mandibles (which last are wholly different from their adult form) are each provided with a long setose exopod. The larva possesses only the nauplius eye, the paired (lateral) eyes are entirely wanting. It is actively free-swimming, grows gradually in length; the other limbs arise, and after a series of changes simultaneous with ecdyses, it finally attains the adult form. Amongst other Crustacea, however, the young one hatches at a more advanced stage, provided with several pairs of limbs, etc. (see below).

Most Crustacea are marine; some creep about at the bottom of the sea, others are excellent swimmers; many are pelagic; the majority are free-swimming in the larval state. A few live in fresh water, others on land or in damp places

The Crustacea are divided into two sub-classes: Entomo'straca and Malacostraca. The latter group forms a circumscribed whole, whilst the Entomostraca comprise several, and in some cases only distantly related, groups.

\section{Sub-Class 1. Entomostraca.}

\section{Order 1. Phyllopoda.}

The head is furnished with a nauplius eye, and with compound, stalked or sessile lateral eyes; the jaws are usually feebly developed, sometimes also the antennæ. The head is generally followed by a thorax composed of numerous segments; each thoracicsomite bears a pair of strong, flat, leaf-like limbs, which serve both as natatory organs and also as gills, and are about equally developed on all the segments. The terminal joints of the body are apodous (abdomen); on the most posterior is a pair of jointed or unjointed backwardly-directed appendages. In the majority of forms the body is entirely or partially covered with a carapace which arises from the head. The Phyllopoda hatch as nauplii. Most members of this small group live in fresh water, as a rule in little pools. The eggs can endure complete drought; some even do not develop until they have lain dry for some time.

1. Branchipus possesses a pair of stalked, movable lateral eyes: the carapace is wanting. The second antenna of the male is modified, to hold the female during copulation. The thorax bears eleven pairs of legs, the abdomen is nine-jointed, the caudal appendages unjointed. The organisms belonging to this genus are transparent, elongate, and minute $(1-2 \mathrm{c} / \mathrm{m}$. in length); found in fresh-water pools ; they swim about constantly with the ventral side upwards. The species of the nearly related genus Artemia (see page 67), 
live in salt lakes on flat sea coasts, or in inland seas (Utah); some of these forms reproduce parthenogenetically, males appearing only now and again (A. salina in South Europe).

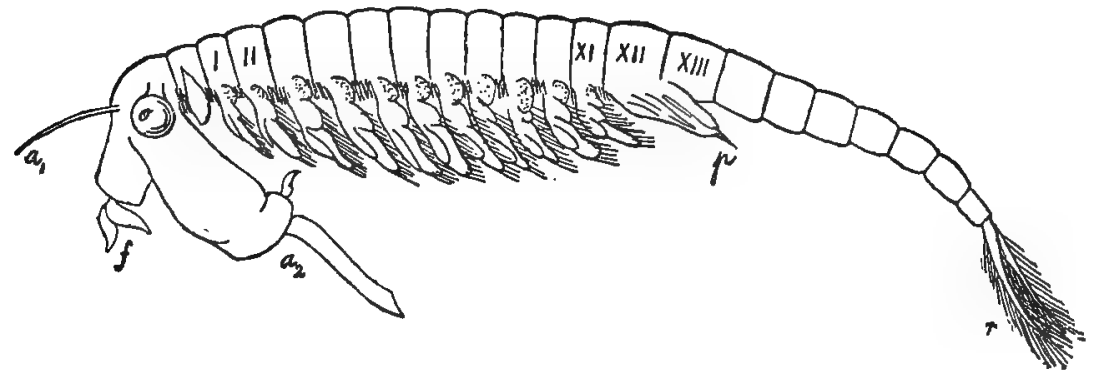

Fig. 154. Branchipus vernalis $\delta . a_{1}$ anterior, $a_{2}$ posterior antenna, $f$ rostrum, $o$ stalked-eye, $p$ penis, $r$ caudal appendage, I, II, XI : first, second, eleventh thoracic segments. XI, XIII, the two anterior abdominal segments.-After Packard.

2. Apus is provided with a broad, feebly-arched carapace, which covers the body with the exception of the hindmost part. The lateral eyes are sessile, situated close to one another and near the little nauplius eye on the

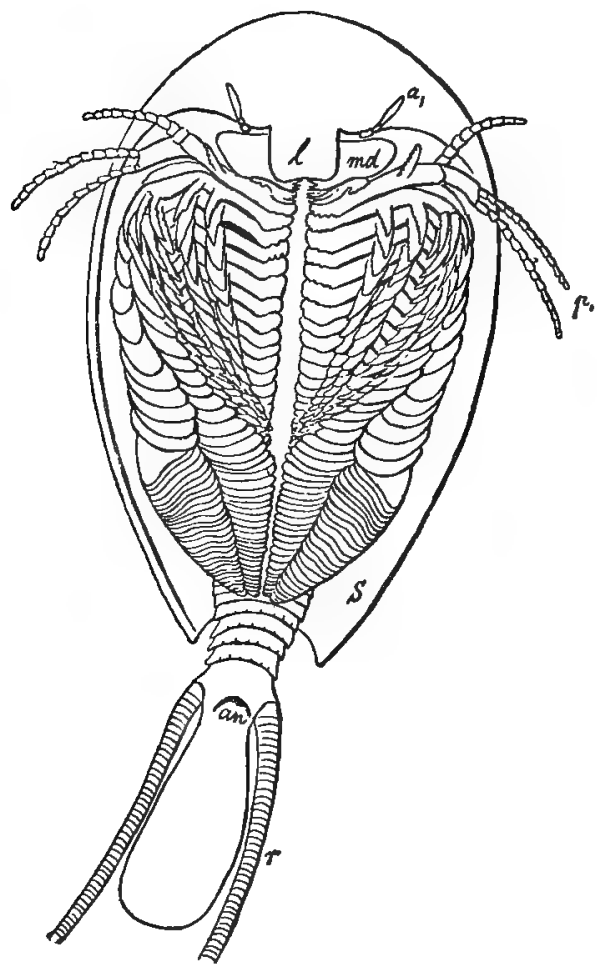

Fig. 155. Apus productus, seen from below. $a$ antenna, an anus, $l$ upper lip, $m d$ mandible, $p_{1}$ first leg, $r$ candal appendage (ends cut off), $S$ carapace.After H. Milne Edwards. dorsal side of the head. The antennæ are very small. There are about sixty pairs of leaf-like legs, whose endopod, as in other Phyllopods, is drawn out into lobes prolonged in the most anterior pair, to form long, jointed threads. In the female, the wide exopod of the eleventh pair is curved like a watchglass, and the epipod lies upon it as a cover, so that the two together form a little box, in which the eggs are carried about. The caudal appendages are long jointed threads. The shellglands lie in the carapace, and are visible through the skin. The individuals of this species are of a very considerable size (several $\mathrm{c} / \mathrm{m}$. long). They are brownish or greenish creatures with a thin exoskeleton. They occur, especially in spring, in small fresh-water lakes (often in those which dry up in the' course of the summer), swimming with the ventral surface upwards. Usually, and in some species exclusively, females only are found, males occur but seldom. Reproduction is as a rule parthenogenetic.

3. The genera Estheria, 
Limnadia, etc., afford a transition to the next order; they are distinguished by having the carapace divided into two movable halves, provided externally with a very hard cuticle, and surrounding the whole body; the valves shut together as do those of Lamellibranchs, for which they might be mistaken. Further, the lateral eyes are very near one another or even united; the second antennæ are very strong and provided with two jointed filaments, the exopod and the distal part of the endopod respectively, whilst the first antennx attain only a small size.

\section{Order 2. Cladocera.}

The Daphnids must be regarded as peculiarly developed Phyllopods with a small $\mathrm{number}$ of limbs, and a large compressed bivalve carapace enclosing the body with the appendages; upon the head there is a large compound eye, which is mounted upon a short stalk and is movable; it arises from the fusion of the lateral eyes, and is enclosed in a special socket, formed by the upgrowth of a fold of skin; there is usually also a small unpaired nauplius-eye. The first antennæ are generally short, and provided with olfactory hairs. The second antennæ are powerful biramous natatory organs. Besides the mandibles

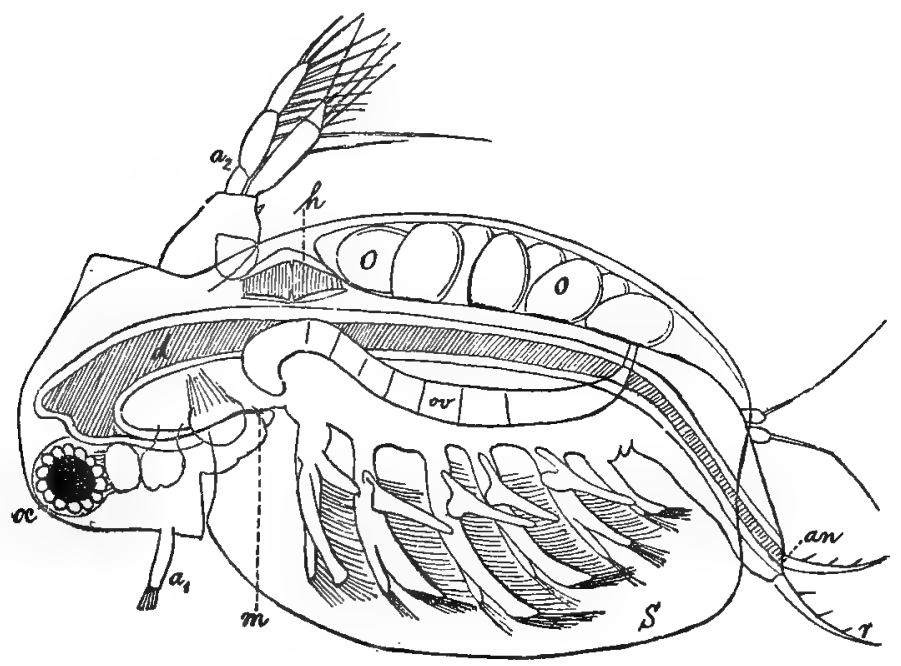

Fig. 156. A Daphnid, Sida crystallina, with eight winter eggs in the brood-ponch. $u_{1}$ first, $a_{2}$ second, antenna, an anus, $d$ gut, $h$ heart, $m$ mouth, o eggs, oe eye, ov ovary, $r$ caudal fork, $S$ carapace.-After Weismann.

there is a feebly developed pair of maxillæ. The short thorax is provided with laminate n e c to p ods like the limbs of the Phyllopoda, though there are only four to six pairs. The abdomen is curved downwards, and has two pointed, unsegmented caudal appendages at the tip. There is a powerful pulsatile heart anteriorly and dorsally, 
but special vessels are wanting. A well-developed shell-gland is present. Some Daphnids differ from the usual type now described, in that the carapace is wanting, or is only feebly developed, that the body is elongate, and that the thoracic-limbs are aberrant in form.

The Daphnids are small (at most a few $\mathrm{m} / \mathrm{m}$. long), transparent animals, which are mostly fresh-water, though a few are marine; they move through the water by jumps (Water-fleas). During the summer, usually only females are found, producing parthenogenetically large, thin-shelled "summer eggs," which are "brooded" in the cavity between the trunk and the carapace; the young ones leave the brood-pouch almost in the condition of the parent; in autumn males appear also. The fertilised ova, "winter eggs" ("resting eggs"), which are thicker-shelled than the summer

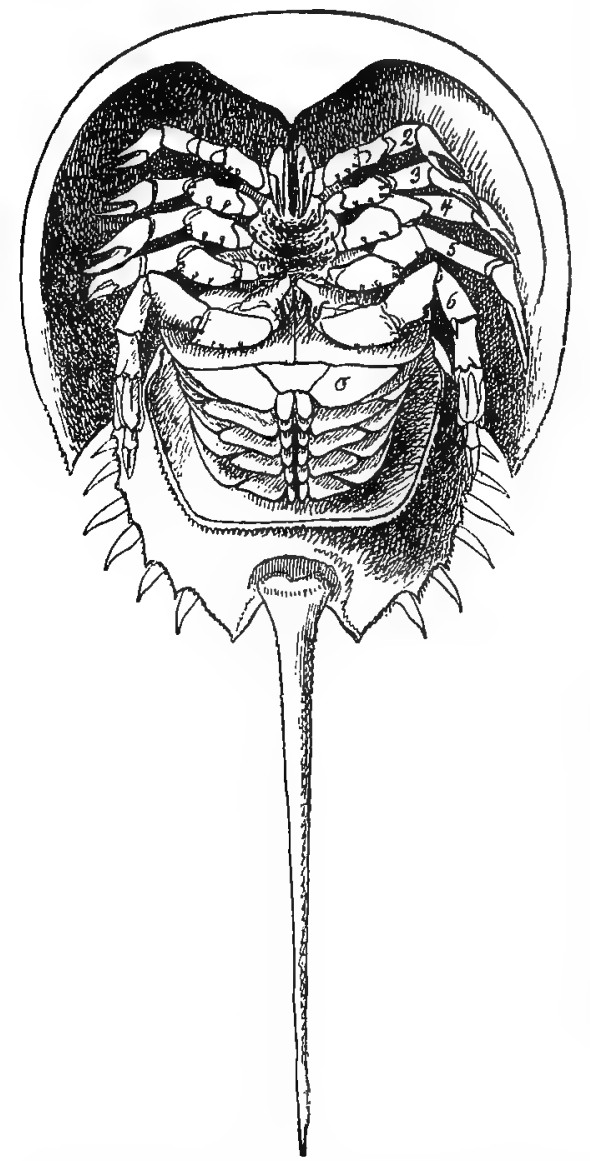

Fig. 157. Limulus polyphemus; 9 , from below : reduced. 1-6 ambulatory appendages, 0 operculum of the gill-bearing limbs, the edges of which are seen one behind the other. ones, usually pass the winter in a peculiar case ( $\boldsymbol{E}_{\text {, }}$ hippium), formed by the thickened cuticle of the whole or part of the carapace, and thrown off by the female together with the eggs. The winter eggs develop in the spring; in some forms the young ones hatch out of the winter eggs as naplii.

\section{Order 3. Xiphosura.}

In the living Xiphosura, which comprise only a single genus, Limulus (the Kingcrab), the body is divisible into two unsegmented parts, the cephalo-thorax and the $a b d o m e n$, which are movably articulated; each of these regions is composed of a number of fused segments. The cephalo-thorax is strongly arched, the sides are thin and continued down to form a shield-shaped structure, which covers in the ambulatory appendages. A carapace is wanting. Upon the dorsal surface there is a pair of large 
compound sessile eyes; the $\mathrm{nauplius}$ eye is represented by a pair of ocelli, placed near together. Antennæand jaws are entirely wanting. Ventrally there are six pairs of jointed ambulatory limbs, all of which are, in the female, furnished with chelæ ( $c f$. Decapoda), whilst in the male these are frequently wanting from some of the limbs.

The six pairs of walking legs surround the mouth, which is farr back; the first pair, which is much smaller than the others, is in front of the mouth; the basal joints are beset with spines, so as to serve also as masticatory organs. On the ventral side of the $\mathrm{abdomen}$ there are five pairs of lamellate limbs; the members of a pair are concrescent at the bases of their inner edges; and each limb bears on its posterior side a number of broad, low, bra $\mathrm{nch}$ ial lamellæ. From the hinder edge of the cephalo-thorax arises a pair of similar lamellate but more chitinised and gill-less appendages, which coalesce in the middle-line, and cover the gill-bearing limbs as an operculum. On the posterior side of the operculum, in both male and female, are the two genital apertures. The body terminates in a long, movably-jointed, pointed caudal-spine. The exoskeleton is tolerably hard, of a horny consistence and colour.

The young Limulus hatches at a comparatively advanced stage. When newly-hatched it is peculiar in having a jointed abdomen and slightly developed caudal-spine.

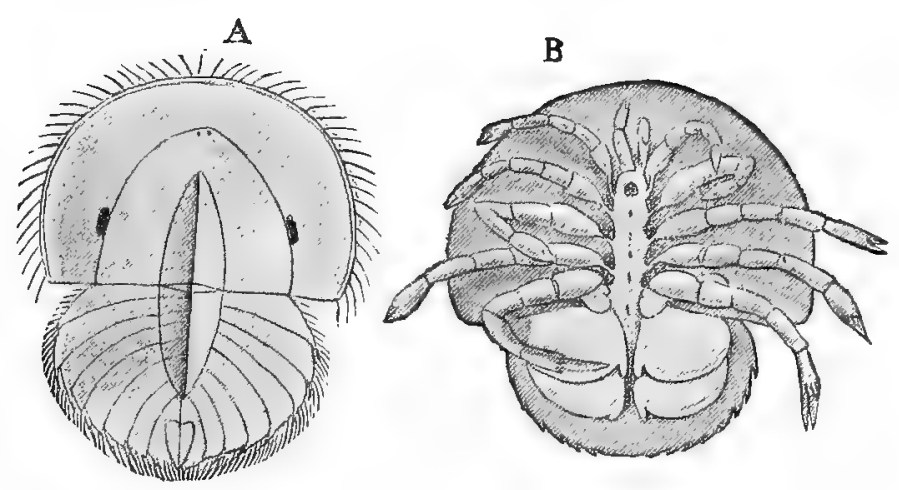

Fi. . 158. Young Limulus. A dorsal, B ventral.--After Kingsley.

The few extant species of this group are large (to over $5 \mathrm{~m}$. long), and come from the coasts of Asia and America. The caudal-spine plays a not unimportant part in locomotion, for it is used to push the body forwards. The Xiphosura are carnivorous.

Some of the extinct forms exhibit a segmented abdomen like that of the young Limulus (. .g. Belinurus, from the Carboniferous). More 
remotely related to the King-crabs are the genera Eurypteruss (Silurian) and others, with a relatively smaller cephalothorax, with five pairs of
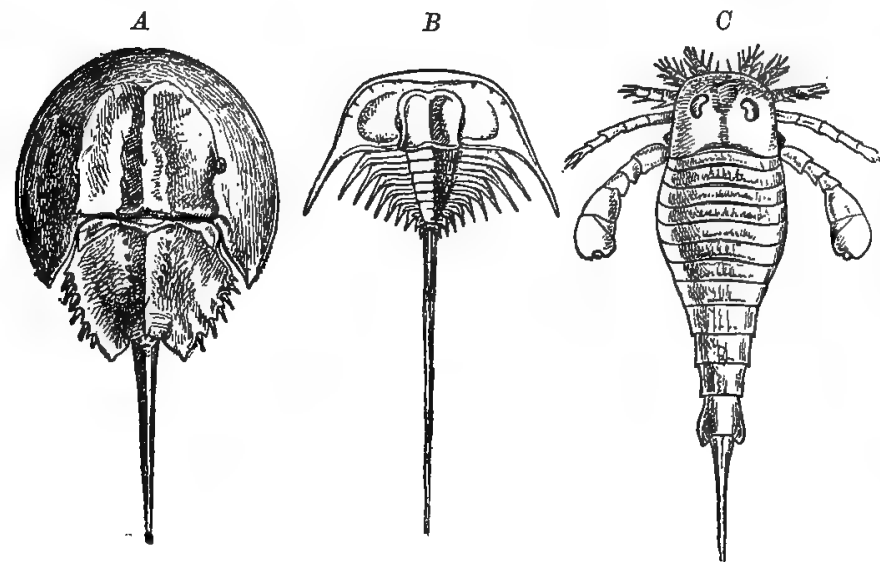

$D$

Fig. 159. A Limulus, B Belinurus, $C$ Eurypterus, $D$ a Trilobite (Dalmanites socialis).

locomotor appendages, bearing a general resemblance in size and shape to those of Limulus, and with a large jointed abdomen and a short caudal-spine.

\section{Order 4. Trilobita.}

The flat, oval body of the Trilobites is divided into three regions (see Fig. $159 \mathrm{D}$ ); cephalothorax, thorax, and abdomen, of which the thorax is usually the largest.

The cephalothorax is unsegmented, its anterior and lateral edges are curved, but its posterior one straight; the lateral angles are not infrequently prolonged into backwardly directed spines. Placed close together on the dorsal side, there is usually a pair of large, sessile, compound eyes. The thorax consists of a number (2-26) of movable, short, wide segments. The $\mathrm{abdomen}$ is formed of

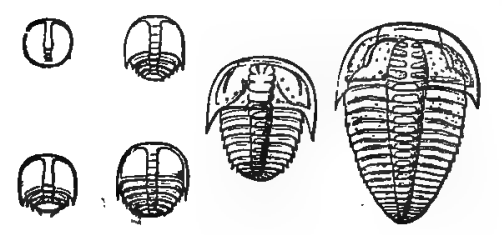

Fig. 160.-Stages in the derelopment of a Trilobite (Sao hirsuta).-After Barrande. a number of fused segments, whose limits are sometimes clear, but usually indistinguishable. Two longitudinal furrows traverse almost the entire length of the dorsal side, from one end of the body to the other, marking out the surface into a median (tergum) and two lateral areæ ( $\mathrm{p}$ le u $\mathrm{r} æ)$. The ventral surface, with the appendages, was probably very soft and thinskinned, whilst ${ }^{-}$the dorsal was hard. In only a few cases have indubitable traces of the ventral surface and the limbs been found, 
so that the structure of the appendages cannot be spoken of with certainty; they were probably soft and feeble like those of the Phyllopods. Some Trilobites had the power of rolling themselves up like Oniscus. As regards their development, it is known that they possessed a smaller number of segments in the young than in the adult state.

The order of Trilobites comprises numerous species, all of which are extinct. The group flourished especially in the Silurian, was more sparsely represented in the Devonian, and died out in the Carboniferous Period. Many specimens are of very considerable size.

\section{Order 5. Ostracoda.}

From superficial observations, the Ostracoda appear to be very like the Daphnidæ, from which, however, they prove to be very different on a closer examination. The body is short and compressed, and may, with the limbs, be entirely enclosed in the very hard shell; this is divided into two parts, which can be opened and shut like the valves of a mussel shell. In the anterior part of the animal, there is a naplius-eye, sometimes divided into two; and, in some forms, also a pair of movable lateral eyes. The first and second antennæ are much modified, and provided with long, swimming setæ; both, but especially the second pair, are natatory and locomotor. The three pairs of jaws are all well developed. Besides these appendages, only two pairs of slender, jointed thoracic legs are present. The most posterior part of the body is curved downwards, and usually terminates in two lamellate appendages. As regards the internal

Fig. 161.

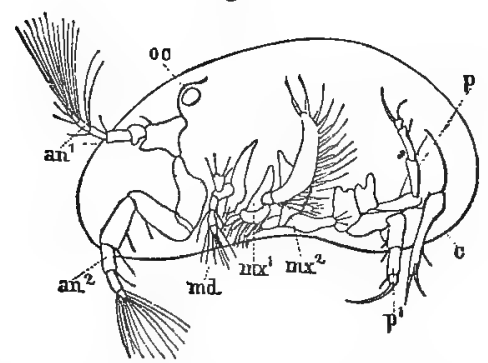

Fig. 162.

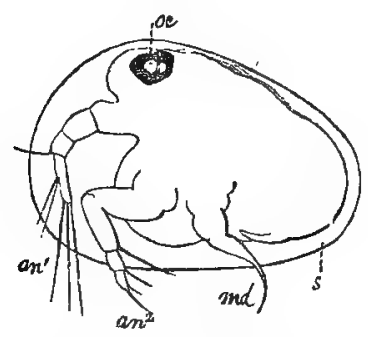

Fig. 1f1. Cypris. oc nauplius eye, $a n^{1}, a n^{2}$ first and second antennæ, $m d$ mandible, $m x^{1}, m x^{2}$ first and second maxilli, $p^{1}, p^{2}$ first and second legs, $c$ tail : enlarged.-After Zenker. Claus.

Fig. 162. Nauplius of an Ostracod. $s$ shell, the other letters as in Fig. 161.-After

structure, it must be noticed that many have no heart. Sexual dimorphism is well marked (in the structure of the limbs, etc.); the colossal size to which the spermatozoa attain is worthy of note; in the species Cypris ovum, for example, the total length is $2 \mathrm{~m} / \mathrm{m}$., i.e., more than three times as long as the whole animal. Many leave the egg in the $\mathrm{nauplius}$ state, provided only with antennæ and mandibles; the shell is usually already developed.

The Ostracoda are animals of small size, occurring both in salt and fresh water. 


\section{Order 6. Copepoda.}

The order Copepoda comprises a large number of free-living forms, and also a multitude of parasites, which conform, on the whole, to the same type, although they are often considerably modified in accordance with their peculiar mode of life.

The free-living Copepods will be first considered. The body is divided into three regions, cephalothorax, thorax, and abdomen. On the cephalothorax there is a nauplinseye, consisting of two, three, or more ocelli, and attaining a considerable size in some pelagic forms; lateral eyes, on the other hand, are always wanting. The cephalothorax bears, further, two pairs of antennæ, both of which are welldeveloped. The anterior antennæ are usually the longer,

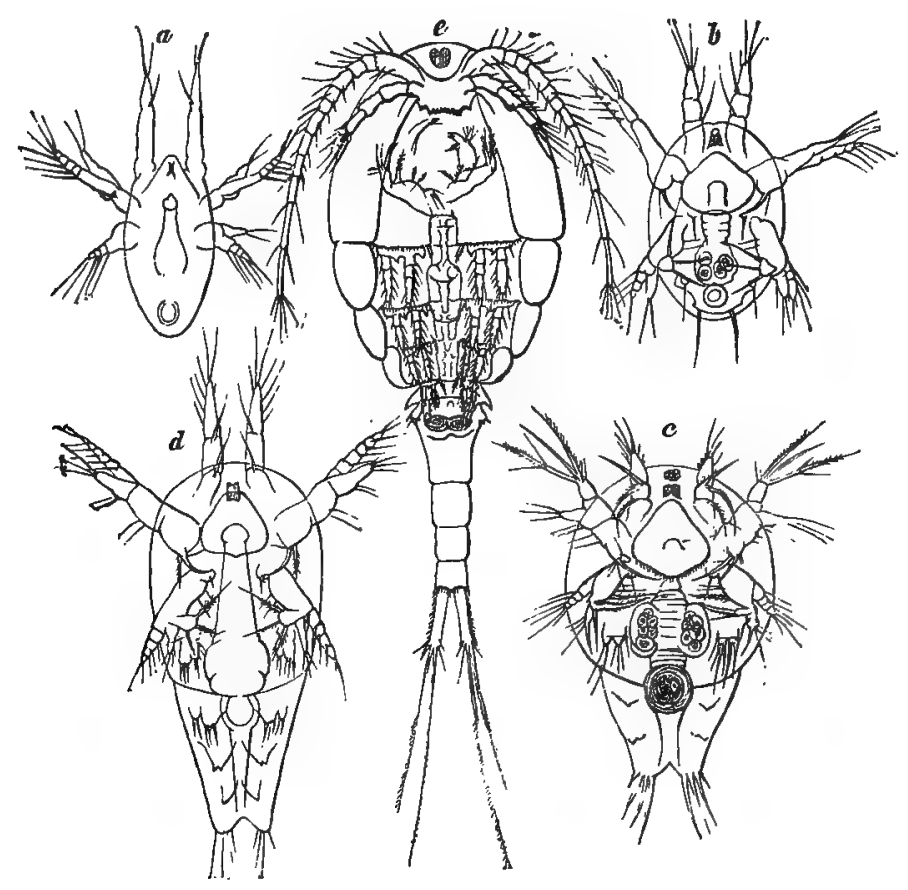

Fig. 163. Cyclops. $a$ nauplius, $b-d$ later stages of development, $e$ adult animal (only the $\mathrm{J}_{\mathrm{e}} \mathrm{f}$ mandible and maxilliped, and the $\mathrm{right}$ maxillæ are figured) : enlarged.

and serve as natatory organs; in the males they are frequently used also for holding the female during copulation; they are then bent in the middle, and the distal can close upon the proximal part. The mandibles are usually provided with palps, the palp often possesses a small exopod (Fig. $151 \mathrm{D}$ ). Behind the mandibles are the two pairs of maxillæ and a pair of maxillipeds. 
The thorax consists of five segments, of which the foremost is frequently united with the cephalothorax; each segment, or only the first four, bears a pair of swimming legs, consisting of a short peduncle with two rami; the outer ramus represents the exopod; the inner, together with the peduncle, the endopod. The abdomen is reduced and apodous, and is composed of the last five segments of the body; at its posterior end there is a pair of unjointed lamellate, or pointed, caudal appendages, between which the anus is situated. The vascular system is poorly developed; the heart usually wanting. S pecial respiratory apparatus is also absent. The eggs are carried about by the female, enclosed in one or two egg-sacs, which consist of a hardened glandular secretion, and are attached to the base of the abdomen. The egg hatches into an oval nauplius, with a nauplius-eye, and the appendages (antennæ, mandibles) characteristic of this developmental stage, by means of which it swims actively about in the water. The other limbs bud out gradually as the body increases in length.

These Copepods are small aquatic animals, which are freshwater as well as marine, and are frequently found in immense numbers; the sea may be tinted red, over large tracts, by their presence. The principal food of the great shoals of Herring consists, at least in many places, of some species of this group, which furnishes also a considerable contribution to the food of the Whalebone Whale.

Species of the genus Cyclops, carrying two egg-sacs, are common in the fresh waters of England.

The Parasitic Copepods comprise a multitude of different forms living on (seldom in) different aquatic, usually marine, animals. They are frequent upon Fish (especially on the skin and gills), also on Worms, Molluscs, etc; they often attain a larger size than the free-living forms (several c/m.). Some of them (F'ig. 164, 1-2) e.g., $\mathrm{F}$ is h-l i c e (Caligun), differ relatively little from the free-living forms. The mandibles are modified as stabbing organs (stylets), which are enclosed in a tube, the proboscis, formed by the concrescence of the upper and under lips; some of the limbs (second antennæ, second maxillæ, maxillipeds) are modified to prehensile hooks (adhesive organs); but in other respects those occurring, for instance, on the skin of fish, are not strikingly different from the free-living forms, * the sexes are not very dissimilar, and male as well as female, is locomotor, not fixed to one spot on the host. In others the alteration is greater, the adaptation to parasitic life closer; this is specially evident in the female. The modifications, which differ in extent in

* It may be noted that the first antenna, which, in free-living copepods, are generally very long, tend here to be rather short. The first maxilla is rudimentary, the eye may or may not be present. Not infrequently (e.g. in Caligus), the body is flattened, and accommodated to the surface of the host. 
different forms, are in the direction of clumsiness, deformity, and immobility; the abdomen degenerates, the segmentation is obliterated,

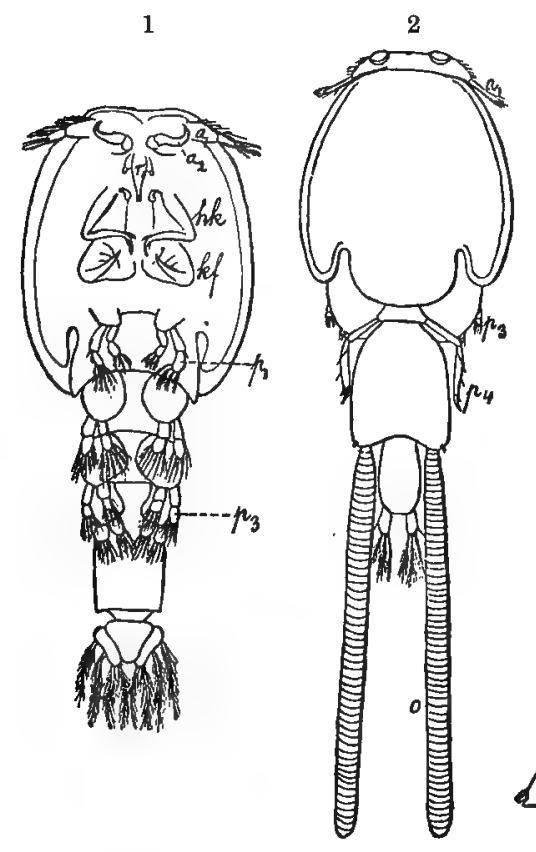

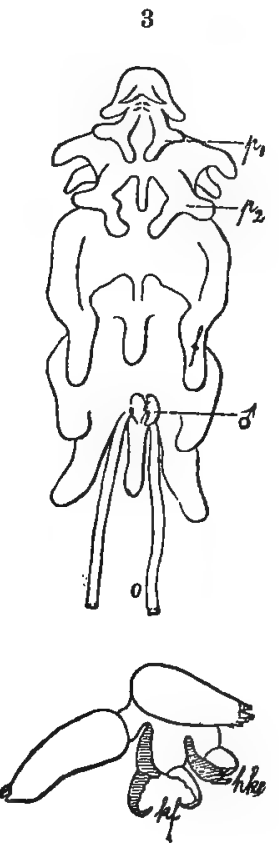

5

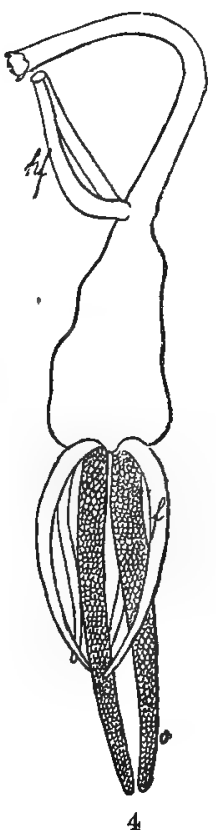

4

Fig. 164. Various parasitic Copepoda: 1 Nogagus borealis, male from the ventral side. 2 Caligus rapax, female from above. 3 Chondracanthus gibbosus, female from below ( $\delta$ the male). I Brachiella thynni, female. 5 Male of the same species (more enlarged). $\quad a_{1}-a_{2}$ first and second antennæ, $f$ caudal appendages, $h k$ second maxilla; if maxilliped, $p_{1}-p_{4}$ frst-fourth pairs of legs, o egg-sac.-1, 2, 4, 5 after St. enstrup and Lütken, 3 after Claus.

those limbs, which do not serve as organs of adhesion, atrophy, or become functionless; this applies especially to the true legs, which

$A$

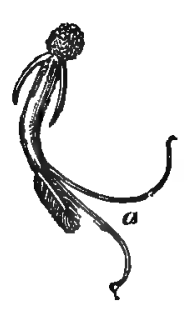

Fig. 165. A, Penella sagitta (parasitic on certain Fish), + , natural size. $B$ anterior end enlarged. $p^{1}$ first, $p^{4}$ fourth pair of legs, o egg-sac. C Herpyllobius arcticus (parasitic on Chætopods), $q$ enlarged, $o$ egg-sac. The irregularly lobed part is sunk in the body of the host.-After Steenstrup and Lütken. are either wanting, have degenerated to mere vestiges (Fig. $165 B$ ), or have become large thick appendages, without setæ, exhibiting only feeble indications of their original form. Often such parasitic Crustacea are provided with peculiar outgrowths, rendering their appearance still more striking. As a rule they are blind. Where the reduction is most advanced, the entire animal 
is a sac without limbs (Fig. $165 \mathrm{C}$ ), and with only two Ionger or shorter (often thread-like) egg-sacs. It is immovably attached to the host, in some cases by means of the second antennæ, the second maxillæ or the maxillipeds modified into long arms; in others by means of the whole front part of the animal, which is imbedded in the body of the host. The males of the more strongly modified forms are usually pigmy, attain only a small fraction of the size of the females, and as a rule are attached to them in the neighbourhood of the genital aperture; they are not usually so entirely modified as the females may be, as a rule they have several pairs of limbs, etc.

The parasitic Copepods, like the free-living ones, are hatched as na uplii, which swim freely about, and after some moults reach a state like that of the free-living adult. The parasites owe their ultimate deformity to a "retrograde metamorphosis" occurring after fixation.

In some parasitic Crustacea, e.g., Lernæa branchialis, living on the gills of the Cod, the male and female are fairly alike up to the time of copulation, presenting a tolerably normal copepod form; after pairing, however, the female grows considerably, and becomes quite distorted, whilst the male perishes; wherefore no male is found with the adult female Lernæa.

\section{Order 7. Cirripedia.}

The Cirripeds are furnished with a sort of protecting shield, the so-called mantle, which is attached to the rest of the animal only

Fig. 166.

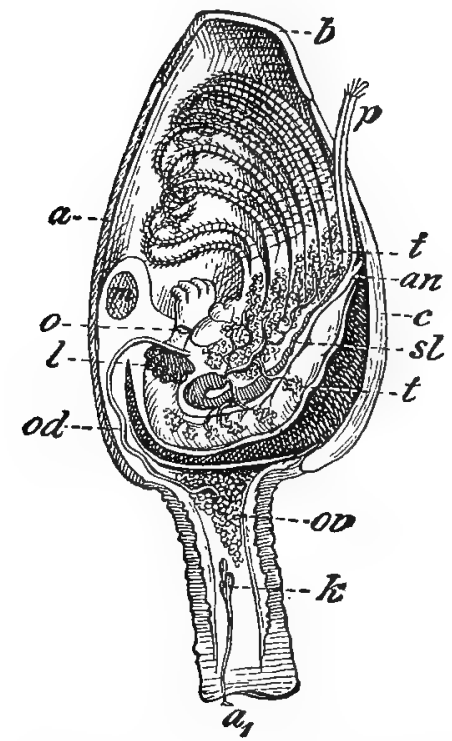

Fig. 167.

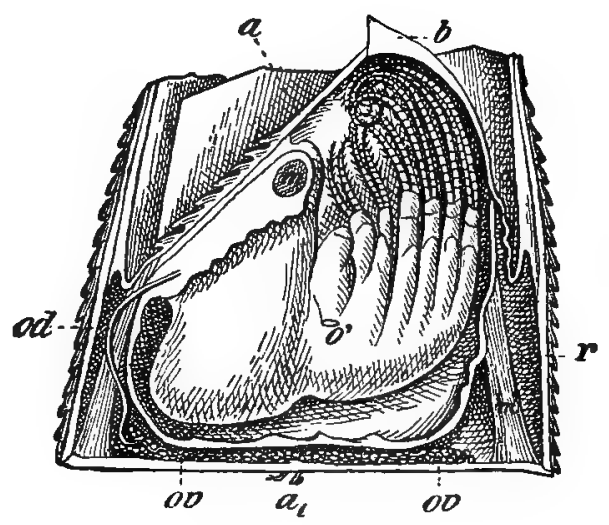

Fig. 166. Lepas. The right half of the mantle is removed, the body shuwn in longitudinal section.-After Claus.

Fig. 167. Balanus. The right half of the mantle and shell taken away.-After Darwin. $a$ and $b$ the paired valves of the mantle, $a$ scutum, $b$ tergum, $c$ unpaired dorsal valve, carina. $a_{1}$ anterior antennæ, an anus, $k$ cement gland, $l$ liver, $m$ addactor muscle of mantle, $m^{\prime}$ retractor muscle, $o^{\prime}$ female aperture, od oviduct, ov ovary, $p$ penis, $r$ shell, $s l$ vas deferens, $t$ testis. 
at the head end, whilst it covers the rest of the body loosely; the enclosed cavity communicates with the exterior only by a slit on the ventral side. In one of the chief groups of the Cirripeds, the Lepadidæ (Barnacles), the mantle is prolonged anteriorly into a thick, shorter or longer peduncle, by means of which the animal attaches itself to some foreign object. In most Lepadidæ (e.g., the genus Lepas), the mantle is provided externally with five calcareous plates or valves, of which one, the car in a, is narrow, and lies along the dorsal edge of the mantle, whilst the remaining four, scuta and terga, two on each side, cover a larger or smaller part of the lateral surface of the mantle; that part of the surface which these plates leave bare (in Lepas, only the marginal furrows between the plates, in others the greater part), is covered with a thin cuticle, which also clothes the peduncle, the inner side of the mantle, and the body; the valves are specially well-developed parts of the cuticle. In some Lepadidæ, besides these five plates, a number of large and small plates (lateralia) occurs at the edge of the peduncle and the rest of the mantle (Fig. 168 B). In the Balanidæ (Sea-acorns), another important division of the Cirripeds, the peduncle is wanting, but the animal is still fixed, and indeed by the same part of the mantle as in the Lepadidæ the adhesive surface is large and provided with a calcareous covering. The lateralia (Fig. $168 B, d$ ), are in a line with the

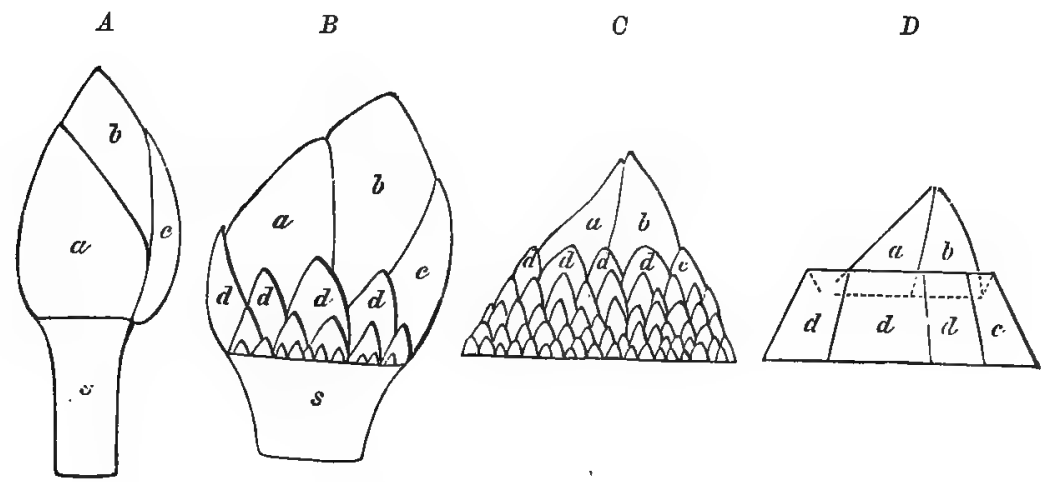

Fig. 168. Diagrammatic figures, ehcwing the trarsiticn furm Lel as to Balanus $A$ Lepas, $B$ Pollicipes, $C$ a Balanid with many lateralia, $d$ (Catophragmus), $D$ Balanus $s$ peduncle, $a-d$ valves, $a$ scutum, $b$ tergum, $c$ carina, $d$ lateralia. The lettering is the same for all the figures.-Orig.

carina (c), and are connected to form a hard shell, the testa, which surrounds the greater part of the animal like a box. The testa rarely consists of a large number of plates in several circles (Fig. $168 \mathrm{C})$, but more frequently of a smaller number (6-8) of large plates in one circle $(D, d-c)$. A lid (operculum) for the box is furnished by the rest of the mantle with the four large plates $(a, b)$ 
of Lepas, which here are relatively small, and at this point there is a narrow slit leading into the mantle-cavity.

As for the appendages, the first pair of antennæ is present in a very rudimentary state; in the Lepadidæ it occurs on the adhesive surface of the peduncle; in the Balanidæ in a corresponding position. A cement-gland opens on each antenna, and its secretion serves for the attachment of the animal. The second antennæ, on the other hand, are wanting entirely in the adult. There are usually three pairs of $\mathrm{jaws}$, none of which are welldeveloped. The ventral side of the body, which, as is evident from Figs. 166, 167, is turned upwards, bears six pairs of cirri, each consisting of a two-jointed shaft, with two multiarticulate, veryflexible, whip-like rami; the outer is the exopod, the shaft and the other branch, the endopod. The cirri, whose rami are fringed with setæ, can be extrnded through the mantle-slit and withdrawn again; they serve to waft into the mantle-cavity the little organisms which form the food of the animal, and when in motion the cirri stretch through the slit close together, then widen out like a fan, come together again, and are drawn back with a jerk. Among the Balanidæ the anterior are considerably shorter than the posterior cirri. The body is usually indistinctly segmented, and frequently bears at its tip a pair of small jointed or unjointed caudal appendages. The adult has only a double $\mathrm{n}$ auplius-eye, whilst lateral eyes are wanting. Heart and blood vessels are absent.

The ventral ganglion chain is much concentrated; in the Balanidx, the ventral ganglia are all united to a single large nerve mass. The digestive tract terminates at the end of the body. Among the Lepadidæ gills are present in the form of thin-skinned whip-like appendages, springing frow the bases of one or more of the thoracic legs. These appendages, possibly representing the epipods, are wanting in the Balanidæ, which are, however, provided with a pair of large folded gills arising within the mantle on either side. In a degenerate state they are also present in the Lepadidæ, where they have a different function, viz., that of carrying the ovigerous lamellø (see below).

In contrast to almost all other Crustacea, most of the Cirripedia are hermaphrodite. Among the Lepadidæ, the ovaries lie in the peduncle, among the Balanidæ on the adhesive surface; an oviduct opens on each side of the body; the branched testis is situate in the body proper; the seminal ducts open by a common aperture on the tip of an elongate copulatory organ, posteriorly. It is very curious that in certain Lepadidæ, besides the hermaphrodite individuals, very small $\mathrm{m}$ ales occur, attached to the former in the mantle-cavity or at its opening. These complemental males*

* In others an actual separation of sexes oxcurs; the female possesses the usual form, the nales are pigmy, like the complemental males. 
are sometimes like the hermaphrodite individuals in structure, in other cases they are very degenerate. The eggs are cemented together into large ovigerous lamellæ and remain in the mantle-cavity, until the larvæ are developed.

The Cirriped leaves the egg as a naplius of the usual kind, which after moulting acquires the so-called cypris form; the name indicates a certain resemblance to Cypris (Ostracoda). In this state, during which the animal, just as in the nauplius state, is free-swimming, the first pair of antennæ is well developed, and has an adhesive disc on the penultimate joint; the second antennæ have vanished, but six pairs of thoracic limbs are present, and resemble those of the Copepoda; besides the nauplius.eye, there is a pair of large compound lateral eyes, and a bivalve carapace surrounding the body. After a time the organism attaches itself by the antennæ, the secretion of the cement-gland flows through them, and fixes the animal permanently to the spot selected. The large eyes atrophy, though the unpaired eye remains; the swimming legs gradually change to cirri, their rami increasing in length, and by a series of modifications, the animal attains the lepas or balanus state.

\section{All the Cirripedia are marine.}

1. The Lepadidæe (Barnacles) are provided with a longer or shorter peduncle; the mantle with five (or more) valves. Many attach themselves to some object which floats in the sea (ships, floating pieces of pumice-stone, etc.); this happens, e.g. in the genus Lepas, whose five calcareous valves cover almost the whole surface of the mantle. Others, e.g., Scalpellum (like the Pollicipes figured in Fig. $168 \mathrm{~B}$ ), with numerous valves and with complemental males, attach themselves to immovable objects, generally at great depths.

The genus Lithothrya, which bores holes in chalk and coral by means of delicate chitinous spines projecting from the very thick peduncle, belongs also to the Lepadidx; and, further, the very different genus Alcippe, with distinct sexes (the female has only the first, fifth, and sixth pairs of cirri, the male is a dwarf without a. digestive tract, etc.), bores holes in dead gastropod shells. A peculiar parasitic Barnacle, Anelasma squalicola, is found embedded in the skin of certain Sharks, firmly attached by delicate branched threads which arise from its peduncle. The cirri are without setæ (recalling the limbs of certain parasitic Copepoda), the mantle destitute of calcareous plates.

2. The Balanidx (Sea-acorns) are sessile, and possess a shell, formed usually of a single circle of plates, with an operculum, which consists of four valves, and has a median slit (see above). Here belongs the genus Balanus, often occurring in great numbers on large stones on the sea-shore, where the animal is sometimes covered with water, sometimes uncovered. Other genera. are found upon the Turtle, or on the skin of the Whale (with the lower end beneath the epidermis; Coronula, and others).

3. The Rhizocephala, which are modified in correlation with a parasitic life, form the most peculiar division of the Cirripedia, and, if the adult alone were examined, would seem to be far removed from the typical members of the order. The body is divided into two regions : an anterior, consisting of much-branched threads, imbedded in the body of the host, and a posterior, sac-like part, which hangs outside the host, and is in connection with the front part by a short 
peduncle; the threads of the anterior region twine round the internal organs of the host, and absorb food by endosmosis; they are comparable, both in general appearance and in function, with the roots of a plant. The saccular part is covered by a soft mantle; the mantle cavity, in which the eggs are retained, communicates with the exterior only by a small aperture. Digestive tract and limbs are altogether wanting. The Rhizocephala undergo a metamorphosis

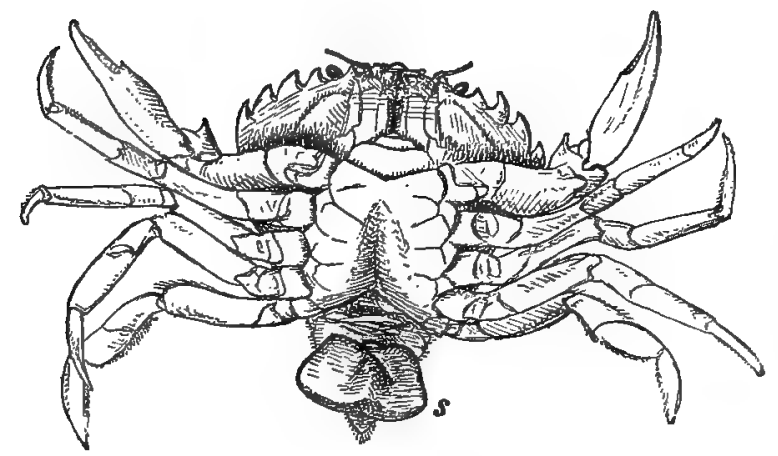

Fig. 169. A Rhizocephalon (Sacculina), $z$, on the ventral side of the abdorren of a Shore Crab; the Crab is seen from below, with the abdomen artificially stretched out.-Orig.

whose first stages are like those of a normal Cirriped (nauplius, cypris); after attachment to the host, however, the animal undergoes a modification, which results in the above-described abnormal structure. They are parasitic upon Decapoda; one species (Saceulina carcini), for instance, is very commonly found on the ventral side of the abdomen of the Common Crab (Carcinus moenas) of European coasts; another (Peltogaster paguri) on the abdomen o the Hermitcrab (the "roots" in both cases permeate the whole body of the host, whose genitalia do not ripen).

\section{Sub-Class 2. Malacostraca.}

In contrast to the Entomostraca, where the number of segments and of limbs varies within very wide limits, there is a typical $\mathrm{number}$ in the Malacostraca, which is never exceeded, but which may be reduced in some forms by the loss of some segments or pairs of appendages.

The body is divided into three regions: the head, the thorax, consisting of eight segments, and the abdomen, of seven. From the head arises in most orders a carapace, which never covers more than the thorax (often not the whole of it), and leaves the thoracic limbs and abdomen uncovered ( $c f$., the Daphuidæ, the Phyllopoda, and others); the carapace is always confluent with a certain part of the dorsal side of the thorax, whilst its sides are free; its outer surface is covered with a hard cuticle, which often attains a considerable thickness. The head bears, further, a pair of large compound lateral eyes, which are usually stalked and movable, whilst the nauplius-eye is generally absent from 
the adult animal; the antennules, each consisting of a three-jointed peduncle and two multiarticulate filaments, of which the outer bears the olfactory setæ (the inner is often absent); the antennæ, in which the peduncle is five-jointed, and produced into a multiarticulate
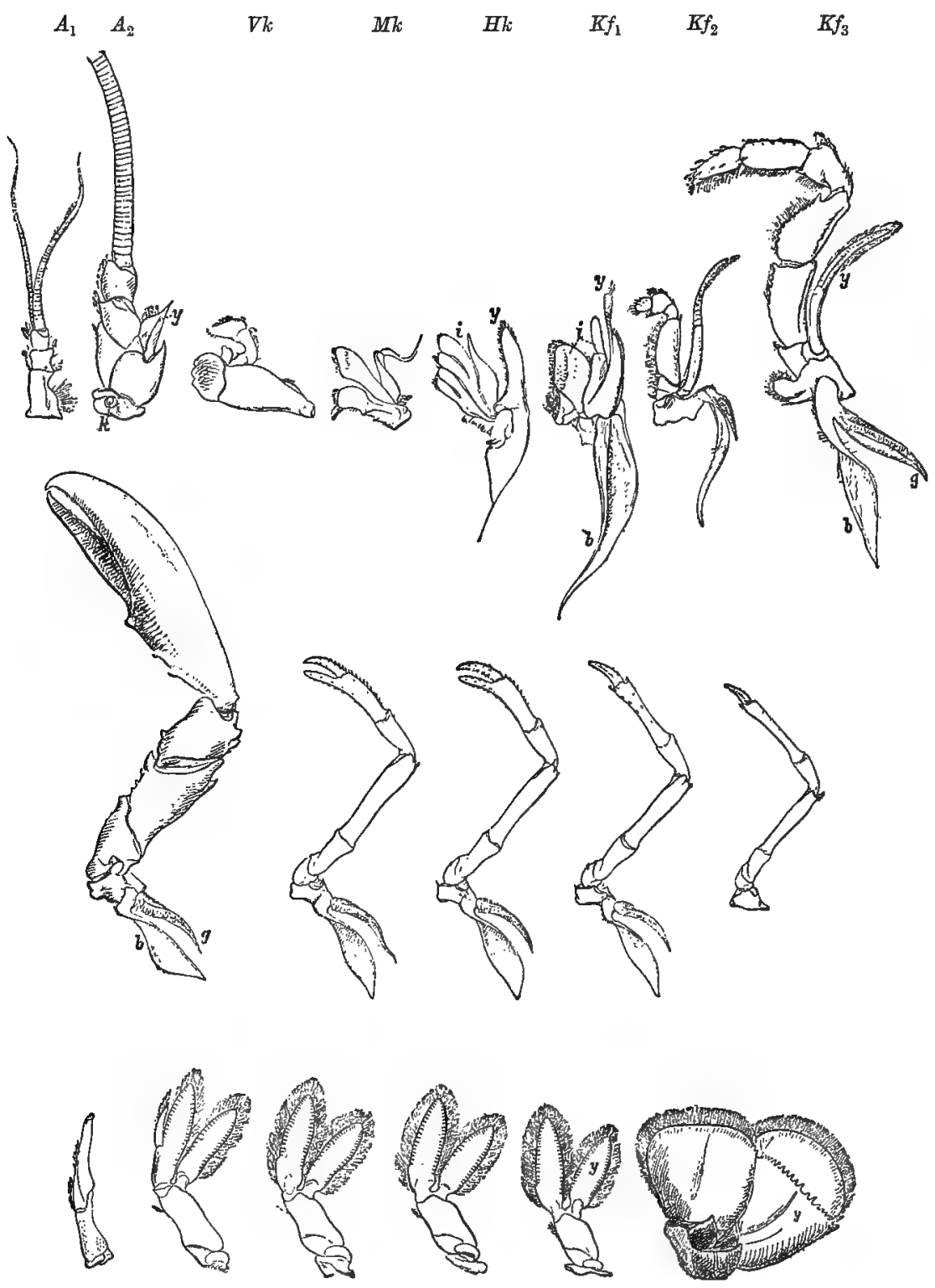

Fig. 170. The appendages of a Lobster, $\partial$; all of the left side and viewed from below. In the upper row are represented : antennule $\left(A_{1}\right)$, antenna $\left(A_{2}\right)$, mandibles, first and second maxilløe $(\nabla k, M k, H k)$, the three maxillipeds $\left(K f_{1}-{ }_{3}\right)$. In the middle row the ambulatory legs. In the lower row the abdominal appendages. $i$ endopod, $y$ exopod, $b$ epipod, 9 gill, $k$ opening of antennary gland.-Orig. 
filament, whilst a lamellate unjointed exopod very often arises from its second joint; finally, a pair of powerful mandibles, with frequently a three-jointed palp, and two pairs of maxillæ, both of a flattened form. The thor ax, which is not sharply marked off from the head, and whose segments (all, or a few) are often completely fused, bears eight pairs of limbs, consisting typically (see Fig. 150, B), of a slender seven-jointed endopod, the basal joint bearing a flat, unsegmented epipod; whilst from the second joint springs a usually narrower exopod, fringed with setæ and multiarticulate. Frequently either exopod, epipod, or both, are wanting, and as a result of concrescence, the endopod may have fewer than seven joints. The eight pairs of thoracic feet are seldom all alike; usually the first, or the first two or three, pairs are modified as maxillipeds, subserving the functions of nutrition; whilst the rest serve for locomotion, or are developed as prehensile organs. The abdomen is typically seven-jointed; it is usually filled up with powerful muscles, and forms a true locomotor apparatus, whilst the viscera are for the most part located in the thorax. Each of the six anterior segments usually bears a pair of appendages, the abdominal limbs, consisting of a two-jointed peduncle and two rami, the outer of which represents the exopod; these are usually natatory organs ( $\mathrm{swimmerets}$ ). The last pair of abdominal appendages is generally different from the rest; it is directed backwards, often broad, and with a short peduncle; with the seventh somite, which is always apodous, it frequently forms the caudal fin. Amongst other characters common to the group, the following must be noticed: the region of the fore-gut following the short wosophagus forms a gi z z ard, lined with chitin, and furnished with hard denticles and with setæ. The rest of the digestive tract is tubular; the anus is on the ventral surface of the last abdominal segment; the liver, which is composed of a number of tubes, opens into the gut bebind the gizzard; the he art is usually short and wide, sometimes more elongate, and almost always provided with three (or fewer) pairs of ostia; the ovaries are generally partly fused; the oviducts are, however, separate, and open on the under side of the ante-penultimate (sixth) thoracic segment, or on the basal joint of its appendages; the testes are usually like the ovaries; the semina] ducts open on the last (eightli) thoracic segment, or on the basal joints of the eighth thoracic limbs.

NoтE.-The genus Nebalia forms a transition from the Entomostraca, and especially the Phyllopoda, with which it should be grouped, to the Malacostraca. It lives in the Mediterranean, the North Sea, on the coast of Greenland, and elsewhere. The body is divided into head, thorax, and abdomen; the thorax is eight-jointed, with eight similar pairs of appendages, which are like those of other Phyllopoda. Each appendage (Fig. $150 \mathrm{~A}$ ) is seven-jointed, lamellate, with broad exo- and epipod. The abdomen is eight-jointed, and, as in the Phyllopoda. 
is provided with a pair of caudal appendages at the tip; the abdominal appendages (six pairs) are, however, like those of the Malacostraca. A large part of the body with the limbs (not the thorax alone, as in the Malacostraca), is covered.

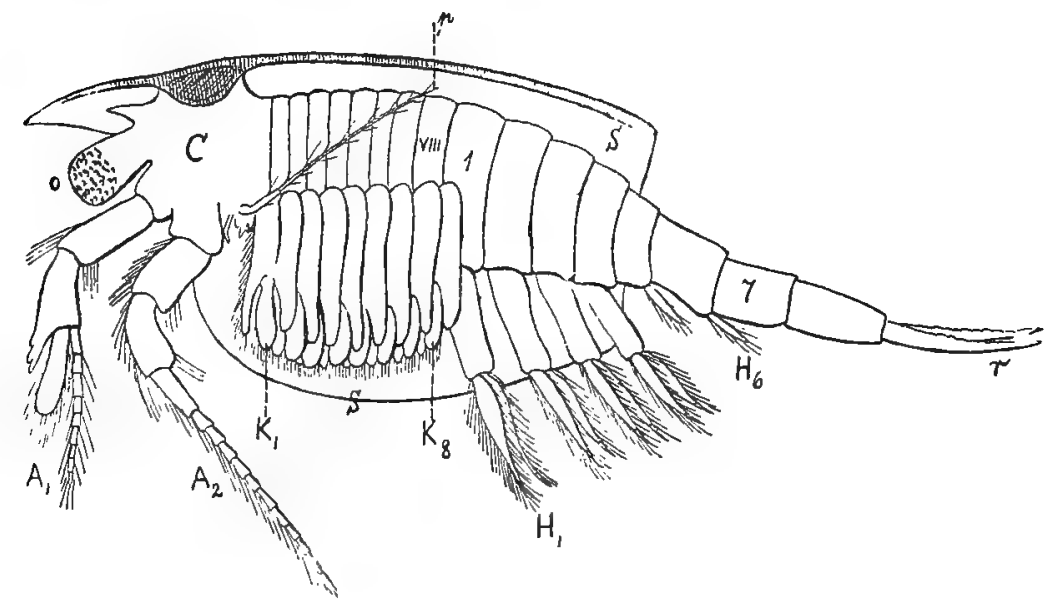

Fig. 171. Nebalia Geoffroyi. VIII Eighth thoracic segment, 1, 7 first and seventh abdominal segment; $A_{1}-A_{2}$ first and second antennæ, $C$ head; $H_{1} H_{6}$ first and sixth abdominal appendages; $K_{1}, K_{8}$ first and eighth thoracic appendages; o eje, $p$ mandibular palp, $r$ caudal appendage, $S$ carapace (left-side removed).-After H. Milne-Edwards.

by a large, compressed carapace, which lies loosely over the thorax, without undergoing concrescence with it. On the whole, the animal exhibits a curious combination of the characters of the Phyllopoda and the Malacostraca.

\section{SYNOPSIS OF ORDERS.}

Stalked eyes. developed.

Second antenna with exopod.

Sessile eyes.

Carapace small or absent.

Second antenna without exopod.

6. Decapoda

7. Stomatopoda No brood-pouch.

1. Euphausiacea

2. Mysidacea.

3. Cumacea

4. Isopoda

5. Amphipoda

$\left\{\begin{array}{l}\text { Brood-pouch } \\ \text { present. } \\ \text { One pair of } \\ \text { maxillipeds. }\end{array}\right.$

\section{Order 1. Euphausiacea.}

The Euphausiacea are transparent, prawn-like animals, a few c/m. long, which live in great numbers in the open sea. They differ from all Malacostraca in that none of the thoracic feet are modified as maxillipeds, but all the eight pairs, though the last two may be degenerate, are essentially alike, and all 
are locomotor: each consists of a seven-jointed, long and thin endopod, and a strong exopod, fringed with setæ, and serving as a natatory organ : there is also an e p i pod which is much-branched, except in the case of the first pair, and hangs free on the side of the animal, acting as a gill. Eyes, antennæ, carapace, abdomen, and swimmerets are like those of the Prawn (q.v.). The Euphausiacea are

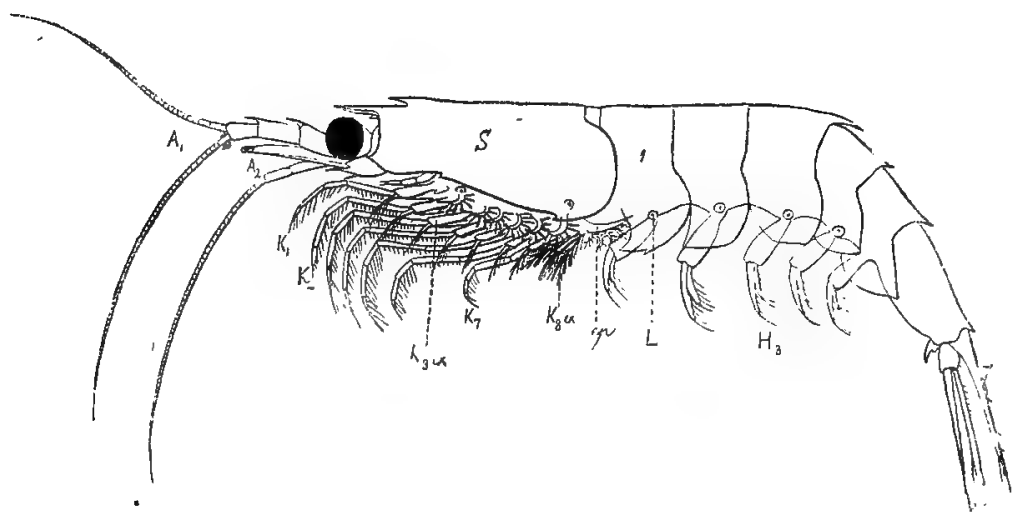

Fig. 172. Thysanopus tricuspidatus. $1-7$ first and seventh abdominal segments; $A_{2}-A_{2}$ first and second antennæ; $H_{3}$ third abdominal appendage : $K_{1}, K_{2}, K_{7}$ first, second, and seventh thoracic limbs; $K_{3} e x$ and $K_{8} e x$ exopod of the third and eighth thoracic limbs; ep epipod of the eighth thoracic limb; $L$ phosphorescent organ; $S$ carapace.-After Sars.

also characterised by the retention of the $\mathrm{n}$ a u plius eye throughout life; the possession of peculiar eye-like phosphorescent organs on the eye-stalk, on the basal joint of the second and seventh thoracic limbs, and on the ventral side of the first four abdominal somites; and by hatching as free-swimming naplii. The order, which is relatively poor in species, is represented both in warm and in cold seas (Thysanopus, Enuphausia, etc.); some species form an important part of the food of the Whale-bone Whale.

\section{Order 2. Mysidacea.}

This order is divided into two groups, the true Mysidæ and the Lophogastridæ, which latter group is confined to great depths, and comprises many curious and aberrant forms. The following account refers only to the true Mysidæ.

The general appearance of the Mysidæ, as of the Euphansiacea, is prawn-like. The body is, however, less compressed and more rounded, and the abdomen does not exhibit the very obvious bend of the Prawns (and Euphausia). Each of the thoracic limbs is furnished 
with a swimming ramus, the exopod, but only the first pair possess epipods.

The first pair of thoracic appendages is modified to form maxillipeds; the second pair is also different from the rest. The abdominal appendages, with the exception of the last pair (those of the caudal fin) are, in the females always, in the males often, feebly

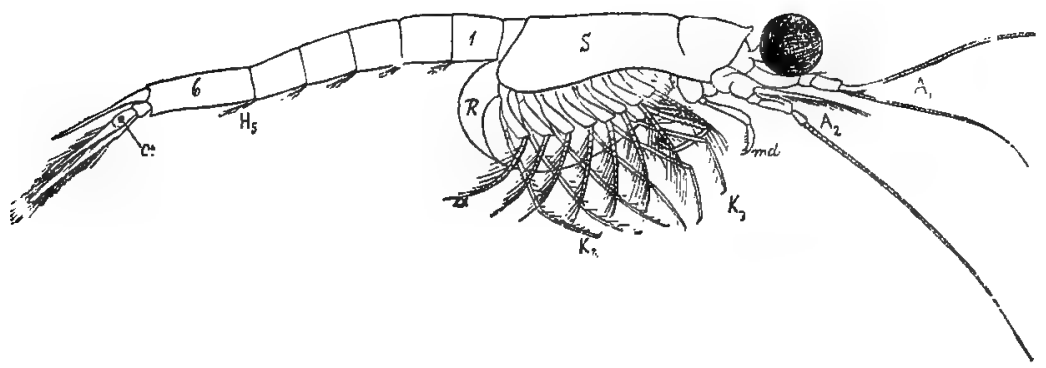

Fig. 173. Boreomysis megalops, one of the Mysidæ, + . 1, 6 first and sixth abdomina segments ; $A_{1}-A_{2}$ first and second antennw; ex exopod of the last thoracic appendage; $H_{5}$ fifth abdominal appendage; $K_{3}, K_{8}$ thoracic appendages, md mandibular palp ; Ot Otolith, $R$ brood sac, $S$ carapace.-After Sars.

developed. An auditory vesicle is found in the inner ramus of the last pair of abdominal appendages, it is furnished internally with a number of hairs supporting a large otolith (the Mysidæ are the only Crustacea possessing an auditory organ in such a position). The inner, membranous side of the carapace is provided

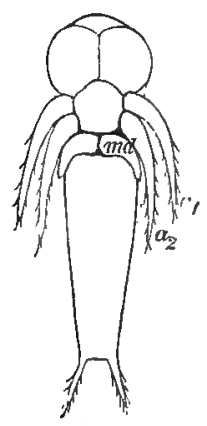

Fig. 174. M yвis. nauplins, seen from below (enlarged). $a_{1}, a_{2}$ first and second antennæ, nd mandible.--Orig. with a close vascular network, and acts as a respiratory organ. The epipod of the first thoracic limb is situated within the branchial cavity, and its movements cause a constant current of water through the chamber. From the inner sides of the basal joints of some of the thoracic appendages arise thin, curved lamellæ, which together form a ventral brood-sac (marsupium) to serve for the protection of the eggs and larvæ. The young ones leave the eggs as $n$ a u plii with the three usual pairs of appendages (antennæ and mandibles), but are incapable of free movement; they feed on the food yolk derived from the egg, and only leave the brood-pouch when they have acquired the general appearance of the adult.

Some Mysidæ are found in the open sea, others are littoral: the genus Mysis, for example, lives on the coasts of Northern Europe; it is a transparent feebly pigmented animal occurring in shoals. 


\section{Order 3. Cumacea.}

The animals of this order are indeed related to those foregoing, but they do not possess the same prawn-like appearance, and are somewhat aberrant in many respects. The dermal skeleton is hard and brittle. The carapace is so small that it only covers the anterior part of the thorax, whilst the five hindmost thoracic segments arebare.* The lateral eyes are sessile, small, usually fused into one; the antenna has no exopod. Of the thoracic appendages some have a swimming ramus, and others have not. The first is a maxilliped, and, just as in the Mysidæ, it is the only one which bears an epipod, which is here provided with a large lamellate gill; the second joint of the maxilliped is furnished with hooks, so that it can be fastened to

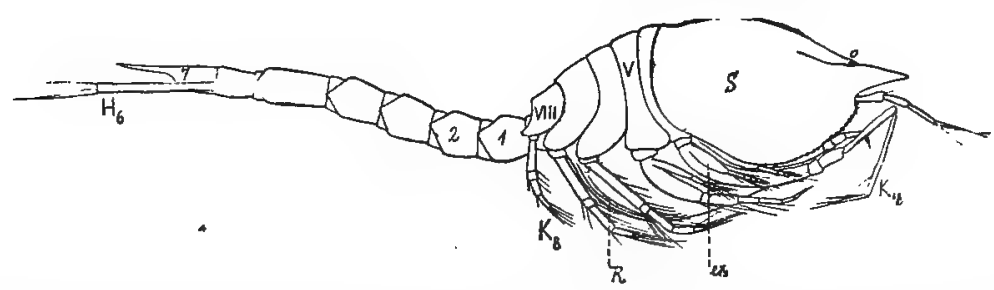

Fig. 175. Diastylis neapolitana, a Cumacean. $\nabla$ and VIII, fifth and eighth thoracic segments; 1, 2, 7 first, second, and seventh abdominal segments; ex exopod of a thoracic foot; $H_{6}$ sixth abdominal appendage; $K_{4}, K_{8}$ fourth and eighth thoracic appendages; o eye, $S$ carapace.-After Sars.

its fellow. The second thoracic legs also differ from the succeeding ones (as in the Mysidæ), the thoracic feet are, moreover-especially is this the case with the last pair-more adapted for walk ing than those of the Mysidæ and Euphausidæ. The abdomen is long, thin, straight, and very movable. Of the abdominal appendages, the female exhibits only the last, which are backwardly directed, slender, and not lamellate, and incapable of acting as a caudal fin; the males usually possess the other appendages also. The females are furnished with a brood-pouch, formed by the union of lamellate appendages of the thoracic feet just as in the female Mysis. The young ones hatch as non-motile nauplii, like those of the last mentioned order; when they leave the marsupium they are like the adult, but they are still without the last pair of thoracic legs, which are developed later (see Isupoda).

The Cumacea are small animals which live on the sea-bottom at some depth. They are met with on British coasts.

* Among the Mysidæ, too, the carapace has not coalesced with these five segments, but extends over the greater part of them (the last two segments are alone uncovered dorsally). 


\section{Order 4. Isopoda.}

The body is dorso-ventrally compressed, enclosed in a hard, often brittle, dermal skeleton; the abdomen is short, at most with six segments, for the last (seventh) is absent. Of the remaining segments the terminal one is usually large: owing to fusion there often appear to be fewer than six. The carapace is absent; the eyes (lateral) are sessile, the exopod of the second antenna is usually wanting. The first thoracic segment is fused with the head, but the remaining seven are free, movable, and well developed. The first thoracic appendage is modified as a maxilliped; its inner edge is usually provided with hooks, by means of which it may be coupled with its fellow. The other seven pairs of

1

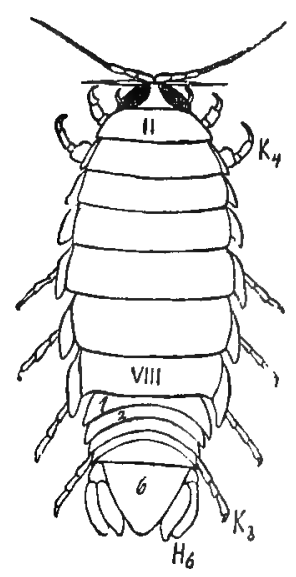

2

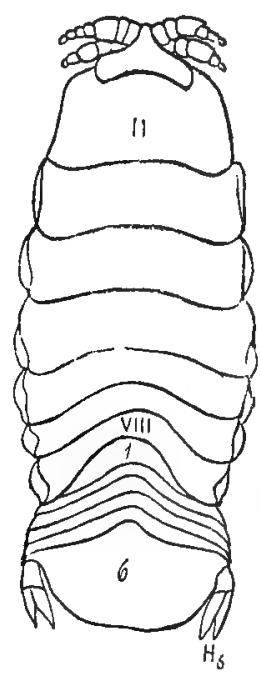

3

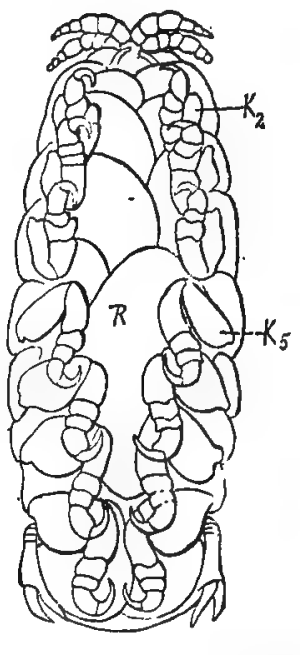

Fig. 176. 1 Aega; 1-3 Cymothoa, dorsal and ventral. II and VII second and eighth thoracic segmente; $1,2,6$ first, second, and sixth abdominal segments; $H_{6}$ sixth abdominal leg; $K_{2}, K_{4}$ etc. second, fourth, etc. thoracic limbs; $R$ plate of brood-pouch.-After H. Milne Edwards.

thoracic feet are powerful ambulatory appendages, without exopod and without epipod. The abdominal appendages are peculiar in having the inner ramus of some of their number modified as a gill; this ramus is membranous, and provided with a'delicate, close capillary net-work; as a rule, there are no other respiratory organs. The Isopoda possess a marsupium under the thorax, formed of the lamellate appendages of the basal joints of the thoracic limbs, as in the Mysidæ; the young ones leave the egg as non-motile na ulii, with three pairs of stumpy appendages; or they may be 
destitute of limbs; when they leave the brood-pouch they usually possess the general form of the perfect animal, but they still lack the last pair of thoracic legs.

Some of the Isopods are marine, some fresh-water, and others terrestrial (in damp places). They are essentially adapted for walking or for running, but some swim by means of the abdominal appendages. Many are parasitic.

1. In the North Sea live, for example, several species of Idothea, relatively elongate animals, with the last pair of abdominal appendages modified to form a valve-like operculum, covering over the others. One species of this genus (I. tricuspidata), which lives on the shore among the sea-weed, is characterised by exhibiting many different colour variations (speckled in different ways). Further, the small Gribble (Limnoria terebrans), which gnaws holes in the wood-work of harbours, etc., and is sometimes very destructive.

2. The flat, long-legged Asellus (Asellus aquaticus) is common in fresh. water lakes amongst decaying vegetation.

3. Many species of Oniscidæ are terrestrial (e.g., genus Oniscus). They are characterised by the rudimentary first antenna, and by the very minute terminal segment of the abdomen. In addition to the usual arrangement of gills, some possess a kind of lung; the outer lamina of some of the abdominal appendages encloses a branching cavity, with a slit-like aperture, which has undoubtedly a respiratory function. The Oniscidæ are light-avoiding animals, of insignificant colour; some (Armadillidium) can roll themselves up like some of the Myriapods, to which they have a superficial resemblance.

4. The numerous parasitic Isopods live principally upon Fish and Crustacea. They offer a gradation in adaption to a parasitic life, similar to that in the

1

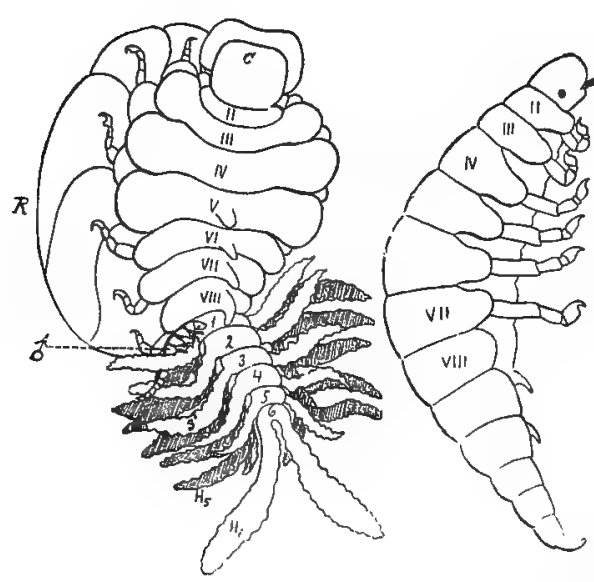

2

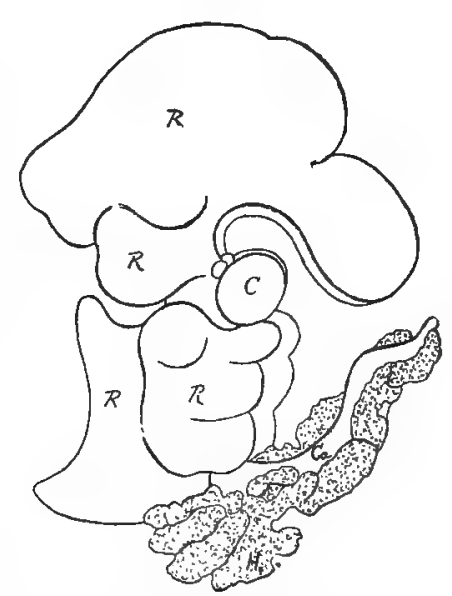

Fig. 177. 1 Cepon elegans, a Bopyride from the branchial cavity of a Crab, $q$ (the male $\delta$ is fixed to the base of the abdomen). Dorsal view. 2-3 Portunion Kossmanni, $\delta$ (from the right) and $q$ (from the left), an Entoniscide, which is parasitic in a Crab; o enlarged much more than $\%$.

$I I-V I I I$ thoracic segments, $1-6$ abdominal segments, $3^{\prime}$ lateral process of the third abdominal segment (these processes light, the abdominal appendages dark), $\mathrm{C}$ head ( + first thoracic segment), $C a$ abdomen, $H_{1}, H_{5}, H_{6}$ first, fifth, sixth abdominal appendages, $R$ marsupial lamellæ.-After Giard and Bonnier. 
parasitic Copepoda. The genus Aega, for example (Fig. 176, 1), which comprises blood-suckers living on the skin of Fish, is only slightly modified; the second, third, and fourth thoracic legs are indeed provided with hooks, and adapted for prohension, but the animal is able to move freely about, and is furnished with large eyes; sexual dimorphism is not pronounced. More adapted to the parasitic life is the clumsy Cymotho, * related to Aega (Fig. 176, 2-3), with or without small eyes, with seven pairs of hook-bearing appendages; living in the mouth and gillcavity of Fish. Still more modified are the Bopyridae (e.g., Bopyrus), parasitic in the gill-cavity of Prawns and other Decapoda; the females are symmetrical, without eyes, and with tiny hook-bearing appendages (Fig. 177, 1); the segments of the wide thorax are immovably united: the males possess a more normal Isopod form, but they are of a very small size (dwarf males), and

fixed to the abdomen of the female. The females of the Entoniscidæ (Entoniscus, etc., Fig. 177, 2-3), parasitic in certain Crustacea are almost, or entirely apodous, and altogether very remarkable in form; the pigmy males are relatively normal in structure, although somewhat reduced in size. The larvæ of the parasitic Isopoda always exhibit a normal isopod form, and are free-swimming.

5. The Tanaï æ (the genera Tanaïs, Apreudes, etc.), form a small division of the Isopoda, which differs in several respects from the foregoing, and approaches the Mysidæ and Cumacea. The organisms belonging here have only six free thoracic segments, for the first two of these (not the first only, as in most Isopods) are fused with the head. There is a small carapace, united

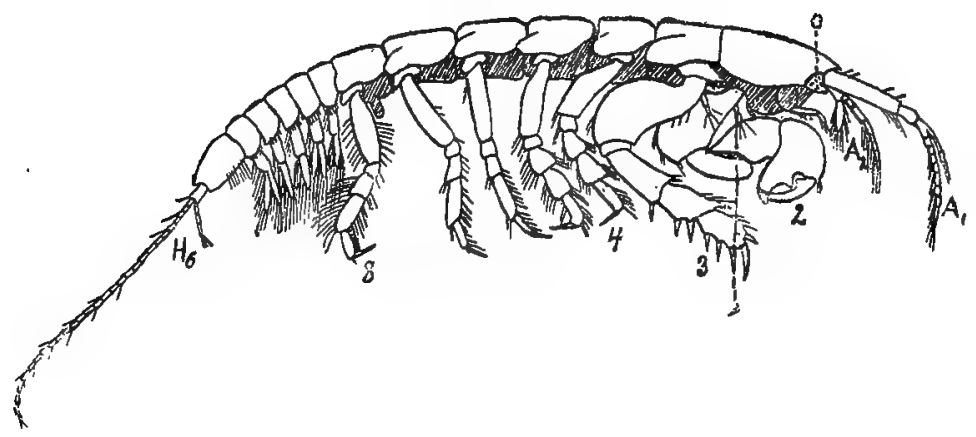

Fig. 178. Apseudes Latreillei, 2, 3, 4, 8 second, third, etc. thoracic appendages; $A_{1}-A_{2}$ first and second antennæ; ex exopod of the second thoracic appendage; $H_{6}$ sixth abdominal appendage; 0 eye.-After Sars.

dorsally with the two segments, which are fused on to the head, whilst its lateral part is free, and just as in the Myside, its inner membranous surface has a respiratory function. Below the carapace, on each side, is the soft epipod of the first thoracic appendage, which keeps up a current of water just as in the latter. The eyes are on short, fixed stalks, clearly marked off from the rest of the head. The exopod of the second antenna is sometimes present. The second and third thoracic feet, of which the first pair is modified

* An interesting observation has been made for Cymothoa and a few other parasitic Isopoda; unlike all other Malacostraca, they are hermaphrodite. During youth the individuals function for a time as males; the female genitalia are only developed later, when the male organs atrophy. 
to form the chelæ, are often provided with rudimentary, but distinct, exopods. The abdominal appendages do not serve as gills. They occur on all European coasts.

\section{Order 5. Amphipoda.}

The Amphipoda are like the Isopoda in many respects; there is no carapace, the eyes are sessile, there are seven free thoracic segments; the first thoracic appendages are maxillipeds, the others are ambulatory, consisting merely of endopod, etc. An important difference is that the abdominal appendages do not act as gills, but the organs of respiration are peculiar, lamellate, membranous processes of the inner side of the basal joint of some of the thoracic legs.* The exoskeleton is usually not so hard as in the Isopoda.

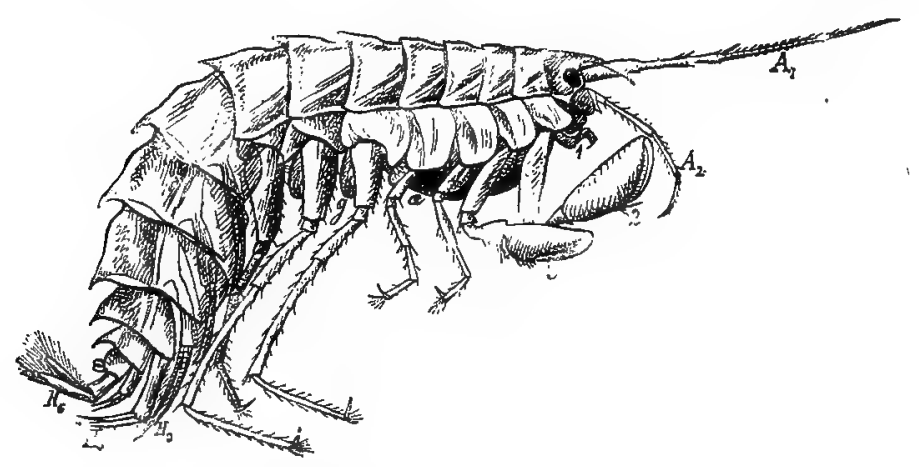

Fig. 179. An Amphipod nearly related to Gammarus (somewhat enlarged). $A_{1}-A_{2}$ first and second antennæ, 1 maxilliped, 2,3 seoond and third thoracic feet, $\infty$ brood-pouch, $g$ gill, $\boldsymbol{H}_{3}, \boldsymbol{H}_{4}, \boldsymbol{H}_{6}$ third, fourth, and sixth abdominal feet.-After Sars.

The abdomen is seven-jointed (the terminal segment small). The maxillipeds are fused at their bases. Some of the walking legs, chiefly the anterior ones, are also prehensile organs, since the terminal joint can move upon the penultimate. The basal joint of these appendages (especially of the first four pairs) is laminate and directed downwards, which gives the body a compressed appearance (the thorax itself is not compressed, Fig. 180). The first three pairs of abdominal limbs are powerful swimming legs, the three hindmost, on the other hand are smaller, somewhat stiff and turned back. The Amphipoda have just such a mars u pi um as hare the Isopoda, but the larvæ do not leave the eggs until all the limbs are developed.

* This process cannot be taken as representing the epipod, which arises from the outer side of the basal joint (see Fing. 180). 
Most of the members of this group are active organisms swimming and hopping about in the water; the former is effected by means of

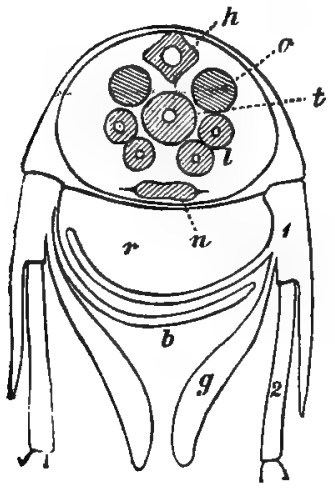
the first three pair of abdominal limbs, the latter by the flexure of the caudal appendage. Other forms (see below) are less energetic. Numerous species and individuals are marine, occurring on the shore as well as in deeper water, and in the open sea. A few are freshwater; some live among seaweed of the shore, or far from the coast on damp ground. A few are parasitic.

Fig. 180. Transverse section of the thorax of Gam. marus (enlarged). 1, 2 first and second joints of a leg, and brood pouch; $b$ one of its constituent laminæ, $g$ gill, $h$ heart, o ovary, $t$ gut, $l$ liver, $n$ ventral ganglion.-Adapted from Sars.

1. The Fresh-water Shrimp (Gammarus) may be taken as a representative of the typical Amphipod. Eyes fairly small, second and third thoracic
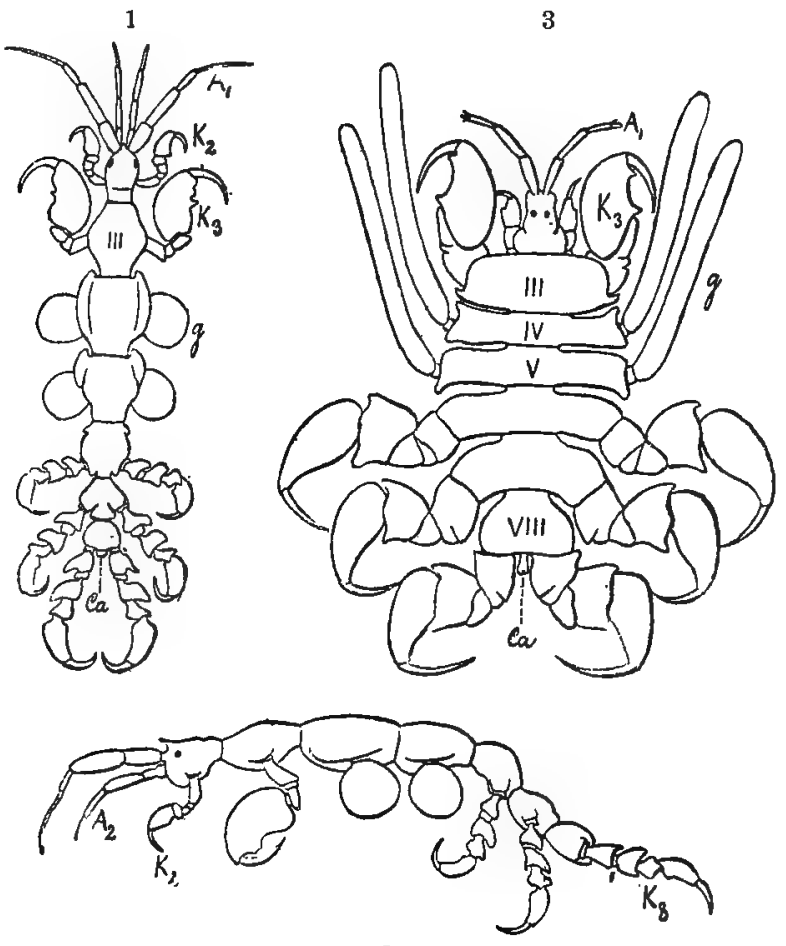

2

Fig. 181. 1-2 Caprella acutifrons, from above and from the left. 3 cyamus mysticeti, from above. III-VIII Thoracic segments. $A_{1}$ first antennæ. Ca Rudimentary abdomen. $g$ gill, $K_{2}-K_{8}$ second-eighth thoracic legs. -1, 2 after Mayer, 3 after Lütken (adapted). 
feet prehensile. Marine and fresh water : G. locusta, common on all European coasts; the nearly allied $G$. fluviatilis frequent in fresh water; the blind $G$. (Niphargus) puteanus in springs.

2. Many genera of the Hyperidze are found in the open sea; these are transparent Amphipods, with colossal eyes; some of them live in jelly fish and other transparent forms; in the common Aurelia, for instance, the species Hyperia galba is often found.

3. The genus Caprella, Skeleton Shrimp, characterised by rudimentary abdomen (reduced to a blunt process, and destitute of appendages), and the possession of only six free thoracic segments (two of them being fused with the head). The body is long and thin, almost filiform; the second and third pairs of thoracic limbs form chelæ (the first pair of these is small, the other large); of the fourth and fifth pairs only the basal joint and the gill lamella are present (gills are absent from all the other appendages), the sixth to the eighth are true ambulatory legs. Caprella is marine, and wanders about slowly over seaweeds and colonial animals. Cyamus is nearly allied; its six free thoracic segments are each produced on either side into a long process bearing a leg at the tip, so that the body is flat and isopodan in appearance; in other respects very like Caprella. Parasitic on the skin of the Whale, devouring its thick epidermis.

\section{Order 6. Decapoda.}

The well-developed carapace is fused with all eight thoracic segments dorsally, but the lateral parts are free (branchiostegites), and between them and the trunk there is, on either side, a roomy carity, the branchial chamber. The eyes are placed on morablestalks, the antennules have as a rule (excepting in Crabs) a lamellate, unjointed exopod. Of the thoracic legs (see Fig. 170, p. 208), the three anterior pairs are modified to form $m a x i l l i p e d s$; the first pair is much flattened, as are also the first and second maxillæ; the other two pairs differ but little from the rest of the thoracic feet, but are usually much shorter. The remaining five pairs of thoracic appendages are known as a mbulatory legs. They are essentially walking legs, but one or more pairs (usually the front pair) are generally modified to form claws (chelæ), the penultimate joint being elongated into a strong process, against which the terminal joint bites. Such are used either exclusively, or in addition to their normal function, as prehensile organs. The maxillipeds have, as a rule, a very well-developed, slender exopod, which is almost always absent from the ambulatory legs; an epipod may be present on both sets of thoracic limbs, and always projects into the gill-cavity. The gills arise from the epipods, from the sides of the thorax, and from the arthrodial membranes, between the thorax and its appendages. Each consists of an axis with two series of lamellæ, or with a large number of filaments; of such gills there are from five to twenty odd on each side. They are situate in the branchial cavity, into which water usually enters at the base of the thoracic feet, Hlows over the 
gills, and leaves it again at its anterior end ; the current is kept up by the constant vibrations of the large flat setose exopod of the second maxillæ. The last (sixth) pair of abdominal legs, when present, forms, with the terminal (seventh) segment, the broad caudal fin; of the other five pairs, the first and second of the male are, as a rule, partially or completely modified to form copulatory organs. The development of the abdomen is, in other respects, very different in the different forms.

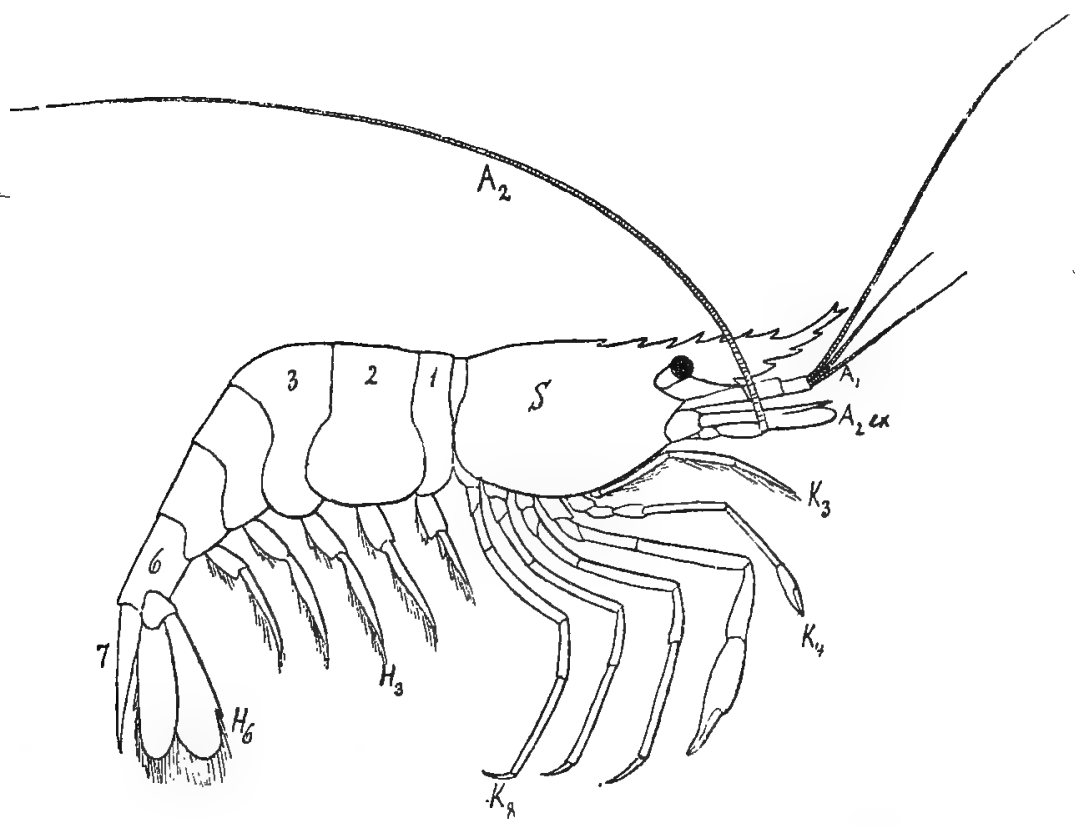

Fig. 182. Palcemon. 1-7 abdominal segments, $A_{1}-A_{2}$, first and second antennæ, $A_{2} e x$ exopod of the latter; $H_{3}, H_{6}$ third and sixth abdominal appendages; $K_{3}$ third thoracic appendage (= third maxilliped); $K_{4}$ fourth thoracic appendage (= first ambulatory limb); $K_{8}$ eighth thoracic appendage (= $=5$ th ambulatory); $S$ carapace.-After H. Milne Edwards.

The Decapoda are furnished with a pair of a u ditory organs, situated in the basal joint of the antennules. In many (Prawns, Lobsters, and others), each is a depression of the skin which opens on to the upper surface of the joint, and encloses peculiar jointed setæ (auditory hairs), which are set in motion by the sound waves. Resting upon the auditory hairs are grains of sand and the like, which are introduced into the sac by the animal, and take the place of otoliths. In others the vesicle is closed, but contains similar auditory hairs, and sometimes an otolith secreted by its walls; in yet other forms with a closed sac (Crabs), the otolith is wanting. In the simplest cases of all (certain Prawns) there is no depression at the spot corresponding with the auditory sac of other forms, but there is a number of auditory 
hairs* upon the skin. Such free auditory hairs may occur also in Crustacea possessing the vesicle, and may be present in other regions (e.g., upon the abdomen). The Decapoda possess a very strong gizzard, often with large, calcareous, masticatory teeth. In depressions in its side walls there are often to be found two rounded calcareous masses, which are absorbed before a moult ("crabs' eyes," or gastroliths). There is a large antennary gland, the "green gland," opening by a small aperture in the base of the antenna.

As the eggs leave the oviducts they are firmly fixed on to the swimmerets of the female, which never possesses a marsupium, but in spite of this almost always carries the eggs about; not, however, the larvæ, or these only for a short time. The young ones almost always undergo a complete metamorphosis. Only in a small number does a free-swimming naplius represent the first stage, e.g., in the Prawn, Pencuus (see below), and in some allied forms. The majority are further developed before hatching, having attained the so-called zoæa stage, $†$ in which condition the animal moves by the appendages which later form the maxillipeds, and at this stage are not connected with feeding, but are solely natatory in function. Swimming is chiefly effected by the exopods of these appendages. The zoæa displays further, the nauplins eye and lateral eyes, the two pairs of antennæ and the three pairs of jaws; the carapace also is present; but the ambulatory legs, and

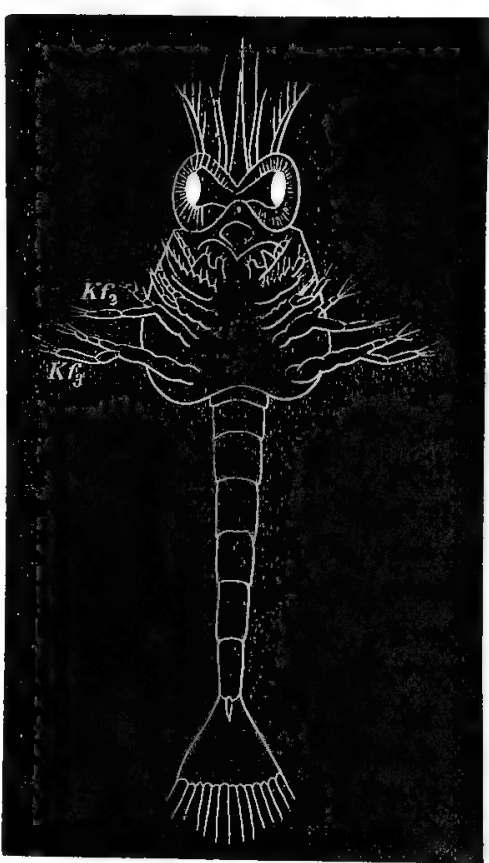

Fig. 183. Z $\mathrm{Z}$ æa of a Prawn enlarged. $K f_{2}-K f_{3}$ second and third maxillipeds.-After Claus. the swimmerets, have not yet appeared, or are only incipient, and the posterior part of the thorax, and the abdomen, are not so pronounced as they become later. Theforms, which hatch as nauplii, pass through the zoæa stage later. In many

* This is also the case in the Euphausidx.

+ Decapoda at this stage were formerly regarded as adult organisms, and described under the generic name of Zoæa; hence the name for these larvæ. 
Decapods the zora is followed by the "m $\mathrm{msis-stage"} \mathrm{(so-called}$ on account of its resemblance to the perfect Mysis), in which the development of the ambulatory limbs is completed, and the animals swim actively by means of their exopods and the posterior maxillipeds; the swimmerets are still absent, or only imperfectly developed. This stage is followed by the prawn-stage, in which the exopods of the ambulatory limbs atrophy, whilst the swimmerets are strongly developed, and form powerful swimming-organs, enabling the animal, which in this, as well as the foregoing stages, is entirely or almost transparent, to move actively about in the surface waters. For one division of the Decapoda, the Natantia, the prawn-stage corresponds with the adult; they remain throughout life in this state, the

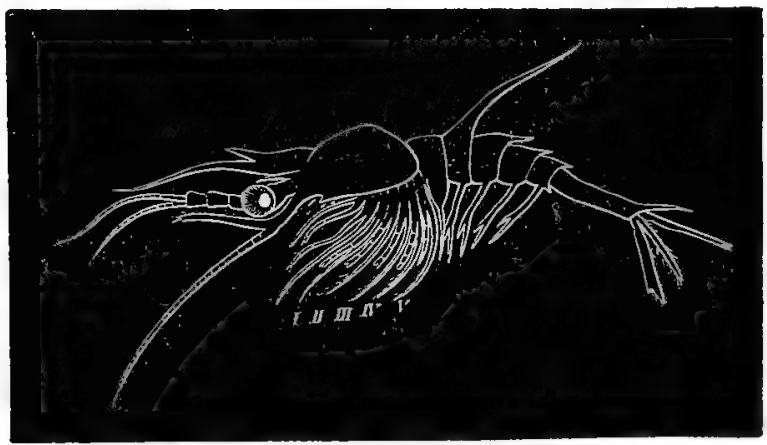

Fig. 184. Mysis-stage of Penæus (enlarged). I-V the five ambulatory limbs with long exopods and short endopods.-After Claus.

swimmerets being the permanent natatory-organs; in another division, the Reptantia, the prawn-stage is not permanent, but after some time, the abdominal limbs, with the exception of the sixth pair, atrophy; they cease to be swimming-organs, the animal becomes opaque, and the power of swimming is lost.

Some Decapoda (e.g., the Lobster), hatch at the mysis-stage, and consequently only pass through this and the prawn-stage. Others (e.g., Crabs) pass direct from the Zoæa to the prawn, missing out the mysis-stage, i.e., at no time are the ambulatory limbs provided with swimming rami.

The Decapoda constitute a very large group, including the largest forms among the Malacostraca. Most of the species are marine; a comparatively small number (Cray-fish, some Prawns) are freshwater; whilst a few are terrestrial.

\section{Sub-Order 1. Natantia (Prawns).}

The skeleton of the Prawns is not very hard, it is merely horny; the animal is transparent or semi-transparent; the body is compressed (Fig. 182); the abdomen strong and curved, and 
incapable of being straightened out. The carapace has a strong, compressed and serrated frontal spine ( $r$ ostrum). On the second antenna is a large lamellar exopod; there are long flexible flagella on both pairs of antennæ, and large eyes borne upon long stalks. The ambulatory appendages are thin and feeble; the third maxillipeds long and leg-like. The abdominal limbs, with powerful peduncle and long laminæ, are strong swimming-organs; from the inner edge of the inner ramus springs an appendage with small hooks at the tip, by means of which each swimmeret is coupled with its fellow, so that the two move in company. Prawns are for the most part active swimmers, propelling themselves forwards through the water by paddling-movements of the first five pairs of swimmerets, whilst they are able to shoot backwards by powerful flexure of the posterior portion of the abdomen and the caudal fin.

Some forms differ from the rest in that they take up their abode in Sponges, etc., and are more or less modified in correlation with this semi-parasitism; the eyes and antennæ having become small, and so on.

Of the very numerous, mostly small, forms, only a few are quoted:

1. Penæus is a genns of large Prawns reaching the size of a Cray-fish, which live only in warm seas (two species in the Mediterranean). They are compressed and elongate, with small claws on the first three pairs of ambulatory legs. Penæus, and some of its relatives, are distinguished from all other Decapods in that they hatch as $\mathrm{n}$ a u plii (Fig. 153).

2. The genus, Palæmon, is of frequent occurrence in European seas (Fig. 182). In this form only the first two pairs of ambulatory limbs are chelate; it hatches, like the great majority of Prawns, as a $\mathrm{zoma}$, and later, passes through a mysis-stage. Several species of this genus are edible, as also the Common Shrimp (Crangón vulgaris), which differs in many respects from Palzmon, and lives in the sand on the coasts of Great Britain.

\section{Sub-Order 2. Reptantia.}

The skeleton is generally thick, hard, and much calcified; the animal opaque and coloured. The body is round or flat; the abdomen, in some cases, very powerful and muscular, in others very degenerate; the rostrum short, not compressed. The exopod of the second antenna is short or absent; the antennary flagella usually feeble, the eyes $\mathrm{small}$, with shorter stalks. than in Prawns. The second to the fifth pairs of a mbulatory legs are more or less powerful walking organs; the first pair is, as a rule, much stronger than the rest, provided with large $\mathrm{claw}$. and held up during locomotion; the third maxilliped is short and not leg-like. The abdominal legs are never swimming organs in the perfect animal, with the exception of the sixth pair : they have, in the female, the primary function of carrying the eggs, whilst in the male the first two pairs serve as copulatory organs; the three following are of little importance in the males, and consequently are often absent or degenerate. The sixth pair forms, in some, a well- 
developed caudal fin, in others (Crabs), which have a feeble abdomen, it is entirely wanting. The adult Reptantia move on the sea-bottom by means of their strong ambulatory legs (which in Prawns are of quite subordinate locomotor importance), whilst they are incapable of actually swimming; $*$ those which have a muscular abdomen can shoot backwards like the Prawns.

1. The Lobster (Homarus vulgaris) is a large dark-blue Crustacean with a very muscular tail and wide fin; second antenna, with exopod, and a long, powerful flagellum. The first pair of ambulatory legs are strong chelæ, of which one (sometimes the right, sometimes the left) is the larger, and is beset with more knob-like teeth than the other; the second and third pairs are also chelate, but

Fig. 185.

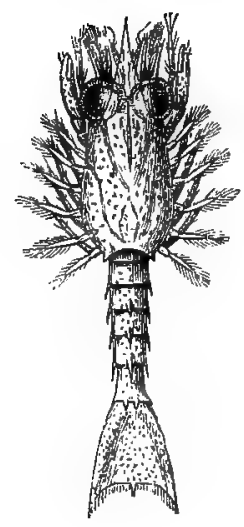

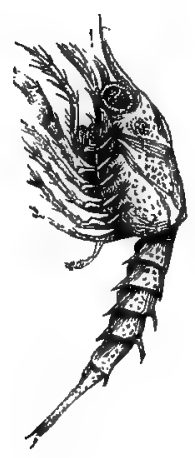

Fig. 186.

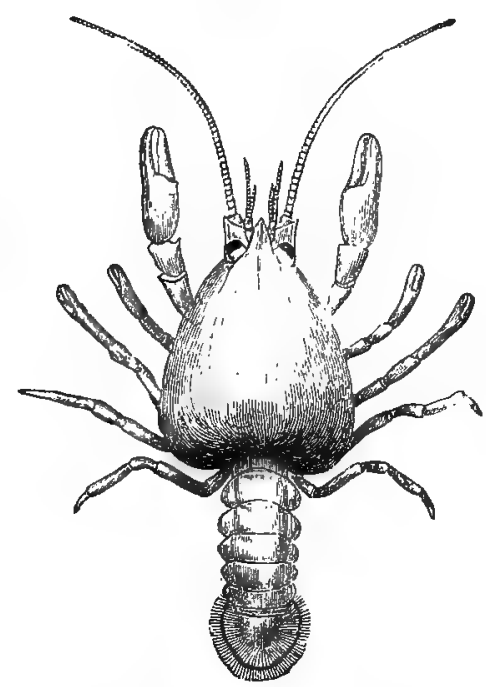

Hig. 185. Quite a young Lobster-larva (Mysis-stage), doraal and lateral views, enlarged.-After Sars.

Fig. 186.-Newly hatched $\mathrm{Cra} \mathrm{y}-\mathrm{f}$ is h, enlarged.-After Huxley.

are no stronger than the remaining two pairs. The Lobster does not pass through a zoæa stage, but at hatching is already provided with all the ambulatory limbs, which, like the third maxilliped, bear swimming rami, by means of which the almost transparent animal rows itself through the water. The mysis-stage is followed by a prawn-stage, from which, finally, the perfect animal emerges. Common on European coasts, especially on the coast of Norway; an allied species is caught in quantities on the coast of North America.

2. The Cray-fish (Astacus fuviatilis) is in most respects like the Lobster (three pairs of chelæ, etc.), but differs from it, among other things, in that the body is smaller and somewhat thicker, that the large chelæ of the first pair are equal, and that the feeler of the second antenna is shorter and weaker. With reference to the development, the Cray-fish behaves very differently, not only from the Lobster, but also from almost all Decapods. When the young one leaves the egg it is already in most respects like the adult animal; in particular, all the ambulatory legs are nearly as well developed, and have no

* Some Crabs, in which the last pair of thoracic appendages is much flattened, execute by their means a sort of swimming movement. 
exopods. Of the abdominal limbs the last pair, however, is not yet present. It is evident from this that the Cray-fish does not pass through a mysis-stage, nor, so far as is known, through a prawn-stage. The young ones cling for a time to the abdominal limbs of the parent.

3. The $\mathrm{Craw}_{\mathrm{fish}}$ (Palinurus) are large, spiny Crustaceans, which resemble Lobsters in most respects, although they differ in that none of the ambulatory limbs (all of which are of about equal strength) are modified as chela; the second antenna is provided with a very long and strong flagellum. A species living on English coasts, $P$. vulgaris, can produce a creaking sound by

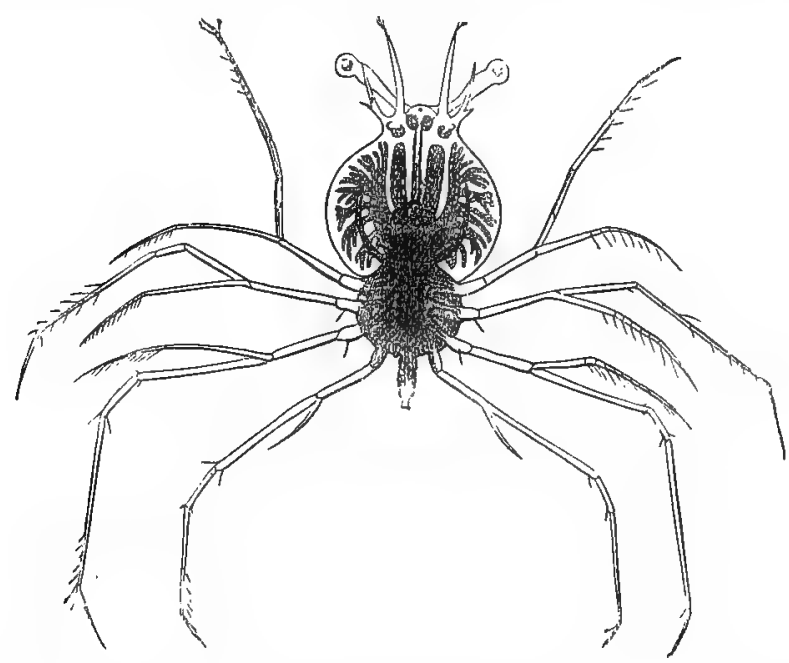

Fig. 187. Phyllozoma, slightly enlarged. The four best developed pairs of appendages are : third maxilliped, first to third ambulatory legs.

rubbing the peduncle of the second antenna against a median projection of the head. Scyllarus is nearly-related to the Craw-fish, but differs from it in that the long multiarticulate feeler is replaced by a short, broad, unsegmented plate. The larva in both genera is very singular, batching in the mysis-stage, although the hinder thoracic feet are not yet present; it is called Phyllosoma, and is chiefly characterised by its leaf-like and flattened form; the carapace, through which the branching of the liver may be seen, is a flat plate, and does not cover over the whole thorax; the latter is a roundish disc, at whose edge the long locomotor limbs (the third maxillipeds and the ambulatory legs with small swimming-rami) are articulated. The abdomen is an unimportant appendage.

4. The Hermit-crabs (Pagurus) are characterised by having the ab domen modified into a large membranous sac with hardly any muscles, but almost entirely occupied by the large liver, and the gonads, which have moved down from the thorax. The abdomen is concealed in an empty gastropod shell which the animal carries about; it is always asymmetrical; its ventral side is entirely membranous, but dorsally there are traces of the tergal portions of the abdominal segments, as thin plates separated by large soft-skinned interspaces: the last two segments alone are somewhat harder; the penultimate bears a small pair of abdominal appendages by which, with the help of the seventh segment, the animal keeps in the shell. Of the other abdominal appendages only those of the left side are present (the first pair is often entirely wanting). The ambulatory legs are also peculiar; the first pair are strong chelæ, the second and third simple walking legs, the fourth and fifth are 
very small and assist in holding the animal in its shell; the fifth have also the work of cleaning out the gill-cavity* into which they are introduced from behind. The Hermit-crabs hatch as $\mathrm{z} O æ æ$ and pass directly from this condition to the prawn-state, in which they swim about by means of the abdominal appendages; the abdomen at this time is muscular and perfectly symmetrical. At the conclusion of this stage the animal seeks a small empty shell which it later exchanges for others of a gradually increasing size. Hermit-crabs are found in all seas. $P$. Bernhardus lives in the North Sea and in the English Channel, and lodges in the shell of the Whelk.

5. Crabs (Brachyura) are a division of the Decapoda, consisting of many genera, and very rich in species, forming, as it were, the summit of this sub-order; for on the one hand the perfection of the posterior thoracic legs as ambulatory organs, on the other, the reduction of the abdomen, here reaches its climax. The body is broad (the cephalothorax frequently wider than long), the abdomen is much flattened, short, and feeble, and is turned up on the ventral side of the thorax; in the female it is wider than in the male. The antennæ are short, the second antenna. has no exopod; the last maxillipeds are laminate, covering the other mouth parts like folding-doors. Only the first pair of ambulatory appendages are chelate, the rest are strong walking legs. The sixth pair of abdominal legs (those of the caudal fin) are wanting; in the female the eggs are carried by the second to the fifth (the first pair is, as a rule, missing); in the male, only the first and second pairs, which form the copulatory organs, are present. The Crabs hatch as zoææ with the first and second maxillipeds developed as natatory organs (the third pair is not developed in this way); the crab-zoæa is often characterised by having long spines on its short carapace. There is no mysisstage, but the young one passes through a prawn-stage (the so-called megalops), in which it is in most respects like the adult animal, but the more powerful abdomen is backwardly directed, and is provided with appendages which act as swimmerets. Finally the abdomen and its appendages become reduced, the

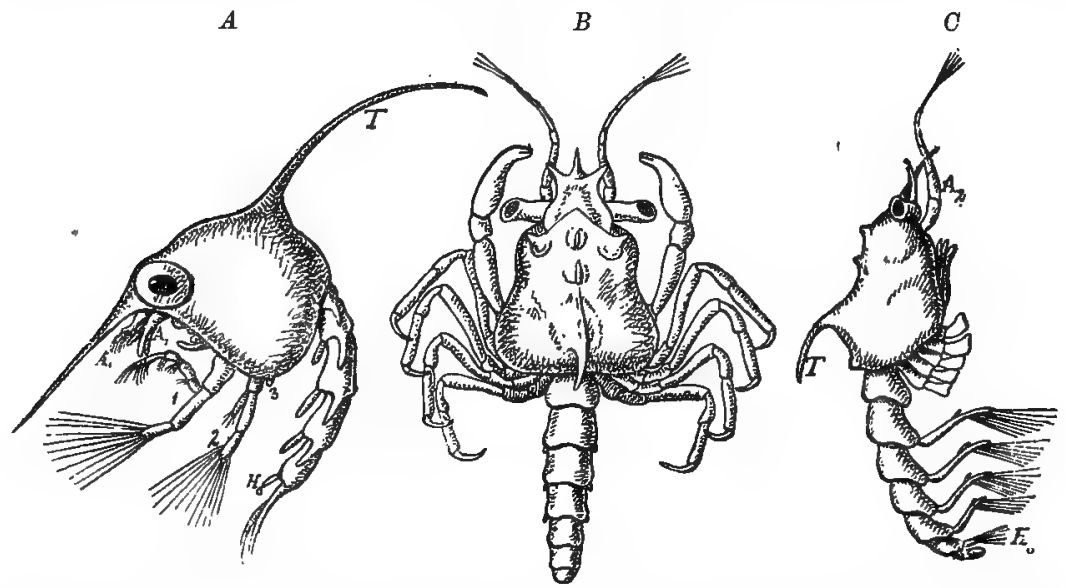

Fig. 188. $A$, z oæa of a $\mathrm{Crab}, B-C$ prawn-stage from above and from the side, enlarged (in $C$ the walking legs are for the most part cut off). $A_{1}, A_{2}$ first and second antennæ, $1-3$ maxillipeds, $H_{6}$ last abdominal leg, $T$ dorsal spine.-After Rathke.

* In some Prawns (probably many) the first, somewhat feeble, ambulatory legs are put to the same use. They are pushed into the cavity from in front and below, and brush over the gills to clean them. 
tail doubles up, and the Crab is henceforth a creeping animal. The Shore Crab (Carcinas moenas) occurs in great numbers on the coasts of England and other parts of Europe. Like other Crabs it is an active, crafty, predaceous animal, which makes strenuous resistance when attacked. The large and broad, thickshelled form, Black-clawed $\mathrm{Crab}$ or $\mathrm{Punger}$ (Cancer pagurus), also lives on English coasts in deeper water.

\section{Order 7. Stomatopoda.}

The Stomatopoda are Malacostraca with large stalked eyes, with a carapace, and with a powerful abdomen. The carapace is, however, relatively small, and the four posterior thoracic segments are free, movable, strongly built, and not covered by it. The abdomen is strong, almost straight, with the usual six pairs of appendages, of which the hindmost forms the tail fin, together with the seventh segment, whilst those of the other five pairs are all strong,

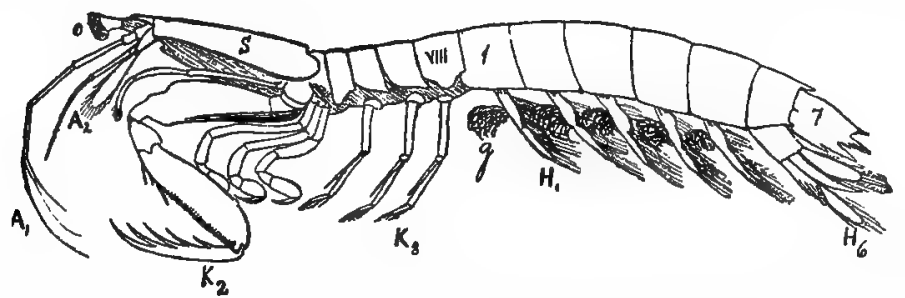

Fig. 189. Squilla. VIII eighth thoracic segment; 1,7 first and seventh abdominal segments; $A_{1}, A_{2}$ antennæ; $g$ gill; $H_{1}, H_{6}$ first and sixth abdominal appendages; $K_{2}, K_{8}$ second and eighth thoracio appendages; o eye; $S$ carapace.-After Lütken.

swimming feet, coupled together, and bearing on their outer rami, large, branching gills. Of the eight pairs of thoracic limbs, the first five are all prehensile; the last joint can be folded back upon the penultimate. The second pair is specially well developed. The last three pairs of thoracic appendages are feeble walking legs. The Stomatopoda do not carry their eggs about. The young animal passes through a metamorphosis, the first stages of which are not accurately known. The more advanced larvæ are delicate and transparent, but otherwise very like the adult; they are characteristic members of the pelagic fauna.

The group, which includes relatively few and fairly uniform members, belongs to warm seas. A noteworthy species, Squilla mantis, is found on English coasts.

\section{Class 2. Myriapoda (Centipedes).}

The multiarticulate, usually elongate body, is covered with a chitinous skin, which may, or may not, be calcified. The head is clearly defined, and is provided on either side with a group of ocelli, more rarely with true lateral compound eyes; it bears also 
one pair of antennæ, which are simply filiform or feebly clavate, and the usual three pairs of jaws, or two only. The body is not divided further into regions, but consists usually of a large number of essentially similar segments, which bear short cylindrical le gs, each composed of a simple series of joints (6-7).

The Myriapoda resemble the Insecta in their internal structure. The alimentary canal is generally straight, and is divided into a narrow osophagus, a cylindrical mesenteron, and a narrower hind-gut; salivary glands open close to the mouth; at the junction of the mesenteron and hind-gut, open two (or more rarely four) Malpighian tubules (cf. Insecta); the anus is in the terminal segment. There is no liver. The heart is a long dorsal tube with paired lateral slits, through which blood enters; it gives off an anterior, and a series of lateral arteries, whilst the

Fig. 190.

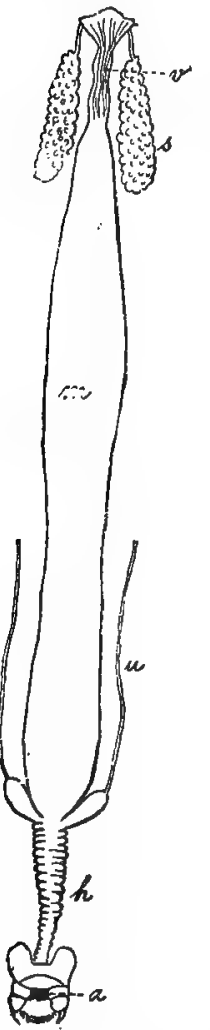
blood also flows through the various slits and spaces of the body. The Myriapods, like Insects, have a system of air-carrying tubes, a tracheal s ystem, which ramifies throughout the body, and opens by stigmata, generally at the base of certain of the pairs of legs. The nervous system is of the usual arthropod type ; the ventral nerve ganglia are generally equally developed in correspondence with the uniform development of the body segments. The ovaries or testes are always fused into an unpaired organ, which in the Chilopoda, opens

Fig. 191.

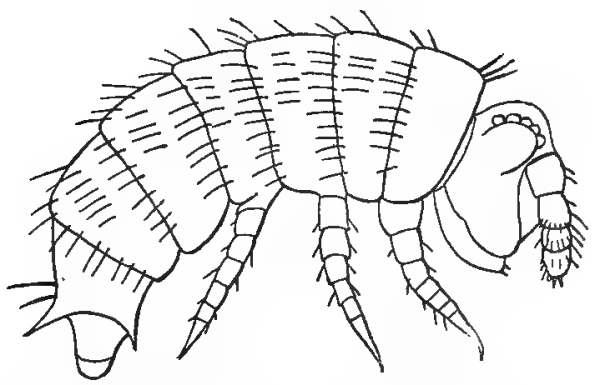

Fig. 190. Digestive tract of Lithobius (Chilopoda). $a$ anus, $h$ hind-gut, $m$ mesenteron, $s$ salivary gland, $u$ Malpighian tubule, $v$ œsophagus.-After Plateau.

Fig. 191. Newly-hatched larva of a Diplopod.-After Metschnikoff.

ventrally at the end of the body, in front of the anus, by a single aperture; whilst in the Diplopoda, a pair of genital pores lies between the second and third pairs of legs, and thus far forward on the ventral side of the body. In this last group, the limbs of the seventh segment are usually modified in the males, to form copulatory 
organs, which are filled before coitus, with spermatozoa, and inserted in the genital aperture of the female.*

In most Diplopoda (e.g. Iulus) the eggs are laid in masses, covered by a small mound, perforated at its apex, and formed of earth and a glandular secretion. Glomeris, however, surrounds each egg with a spherical covering of earth.

The Diplopoda and some Chilopoda have, when they leave the egg, fewer segments and appendages than later; in the former, the newly-hatched young ones have usually only three pairs of legs (that of Iulus is quite apodous); in the latter there are seven pairs. Other Scolopendridæ have the full number on hatching.

The Centipedes constitute a relatively small division. They are, without exception, terrestrial ; inhabiting damp, shady places, under leaves, in the soil, etc.

In most points they are so nearly allied with the large class following, the Insects, that it might be thought best to incorporate them with that group. They are, however, regarded here as a distinct class, because they offer certain peculiarities which would mark them off as very aberrant Insects, and interfere with the clear definition of that group.

\section{Order 1. Chilopoda (Scolopendras).}

The head is flat and bears three pairs of jaws, of which the first maxillæ have very often undergone concrescence in the mid-line. The basal joint of the second maxilla also coalesces with its fellow of the other side, whilst the other joints form a paip. The rest of the body, which often consists of a very large number of segments, is flattened dorso-ventrally; the legs arise far apart from one another (Fig. $193 A$ ), from the soft, lateral portions of the segment, one pair to each segment. The foremost pair of legs is very different from the rest; it is very strongly developed, and forms a pair of stout, hook-like organs, at the tip of which is the opening of a poison gland (the poison-claws). The last pair also is usually somewhat modified, being longer than the others and turned back. It has already been remarked that

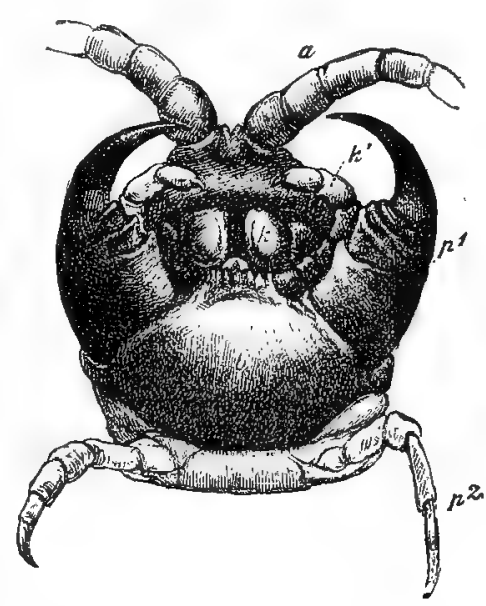

Fig. 192. Head and anterior trank segments of a Scol o pendra, from below. $a$ antenna (the greater part cut off), $k$ first maxilla (the greater part covered), $k^{\prime}$ palp of second maxilla, $p^{1}$ first pair of legs, $b$ its fnsed basal joints, $p^{2}$ second pair of legs.--Orig. the genital organs open at the posterior end.

* In one genus of the Diplopoda, Glomeris, the terminal pair of legs forms the copulatory organs. 
The Scolopendras, of which several species are luminous, are active and predaceous, killing their food with the poison-claws. In temperate zones the few species are relatively small; they attain to an important size in the Tropics (to a foot long). In England, there are several species of Lithobius, and others.

\section{Order 2. Chilognatha or Diplopoda.}

Only two pairs of jaws, generally termed mandibles and maxillæ, are present. The structure of the body is very peculiar: whilst the two legs of each pair arise far apart in the Chilopoda, separated by a wide sternal plate, here they are articulated near to one another on the ventral side. Moreover, most segments bear t w o pairs of legs, indicating that each segment has really arisen by the fusion of two. The four segments following the head have not, however, more than one pair of legs each; indeed, one of them is altogether apodous. The shape of the segments varies: in some (Fig. $193 \mathrm{~B}$ ) they are
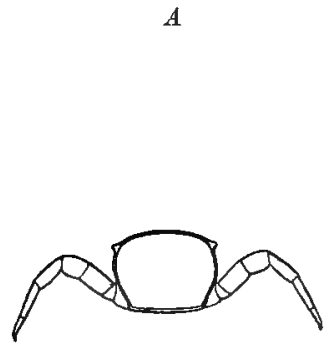

$B$

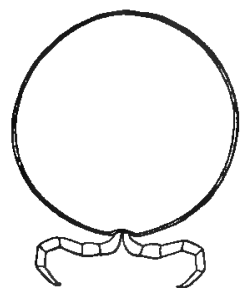

$C$

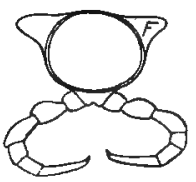

$D$

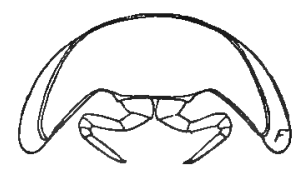

Fig. 193. Transverse sections: $A$ of a Chilopod. $B-D$ of different Diylopoda (B Iulus, $D$ Glomeris), $F$ lateral outgrowth.--Orig.

cylindrical; in others each segment is a compressed cylinder, but possesses a short lateral process, which gives the body a more flattened appearance (Fig. $193 \mathrm{C}$ ) ; in others, again, the body is itself flattened, convex dorsally and concave ventrally (Fig. $193 \mathrm{D}$ ). The legs, which are feeble and thin, are turned out; they are all essentially alike (excepting those which serve in the males as copulatory organs, see above). It has already been stated that the genital apertures are anterior and that copulatory limbs are present.

The members of this order are sluggish animals, which live on decaying or soft vegetable matter or animal remains. When disturbed, they roll themselves together.

Occurring in England are Iulus, with an elongate, cylindrical body; and Glomeris, with short, semi-cylindrical body, composed of so few segments that it bears a superficial resemblance to Armaditlidium. 


\section{Class 3. Insecta.}

The insectan body is divided into three sections: head, thorax, and abdomen. The head is sharply marked off from the thorax, and is usually freely movable: on each side there is a sessile compound eye consisting of a very large number (twenty to many thousands) of small ocelli, covered externally by convex facets; each of these is usually hexagonal in shape, and corresponds in position with an ocellus. In many Insects the eyes occupy a very large part of the head (in many Diptera, for instance, almost the whole of it). In form they are most frequently circular, but often kidney-shaped, and so on. Occasionally the compound eyes are

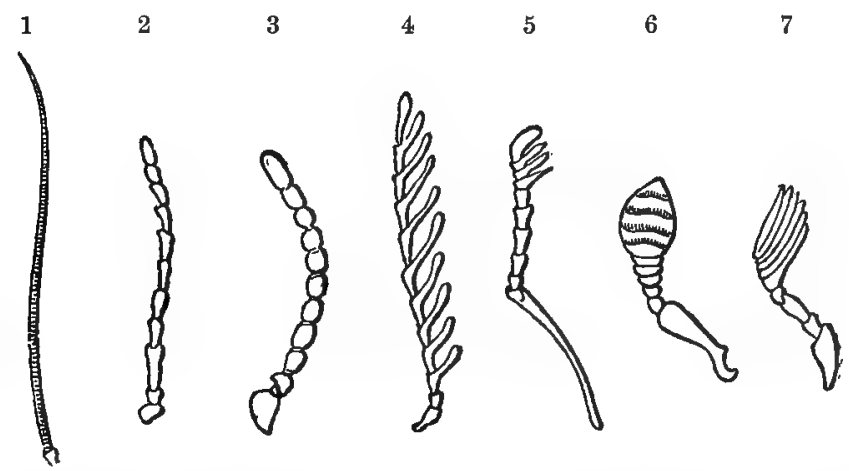

Fig. 194. Antennæ of various Insects. 1 bristle-like, 2 filiform, 3 moniliform, 4 pectinate, $5-7$ clavate ( 7 with laminate club),-After Judeich and Nitsche.

replaced by small groups of ocelli* (Collembola), or by a single ocellus on each side (Fleas and Lice). In many Insects, there are from one to three ocelli on the middle of the head, in addition to the compound eyest ( $c f$., the nauplius eye of Crustacea). From the head, arises a pair of antennæ or feelers, which either consist of a limited number of well-developed joints, or of a large number of very short ones. The form of the antennæ is very varied; at the simplest, they are filiform or bristle-like, but they are sometimes moniliform (much. constricted at the joints), pectinate (the joints being produced on one or on both sides into processes), or clavate (club-shaped).

The head also bears the mouth and the surrounding mouthparts, which vary greatly in form, though all may be referred to a common type. The simplest and most primitive condition is presented by the biting mouth-parts of the Orthoptera, the Coleoptera, the Neuroptera, and the Hymenoptera, where an upper

* Only the adult Insect is referred to here; for larval arrangements, see below.

$\uparrow$ Absent from nearly all Beetles. 
lip and three pairs of jaws occur. The upper lip (labrum) is a broad, movable unpaired plate, situated in front of the mouth. Behind the labrum are the mandibles, which are very

1
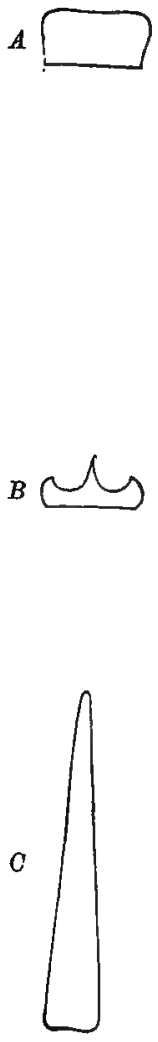

1

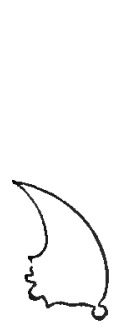

2
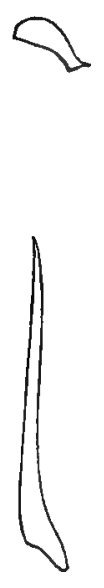

2
3
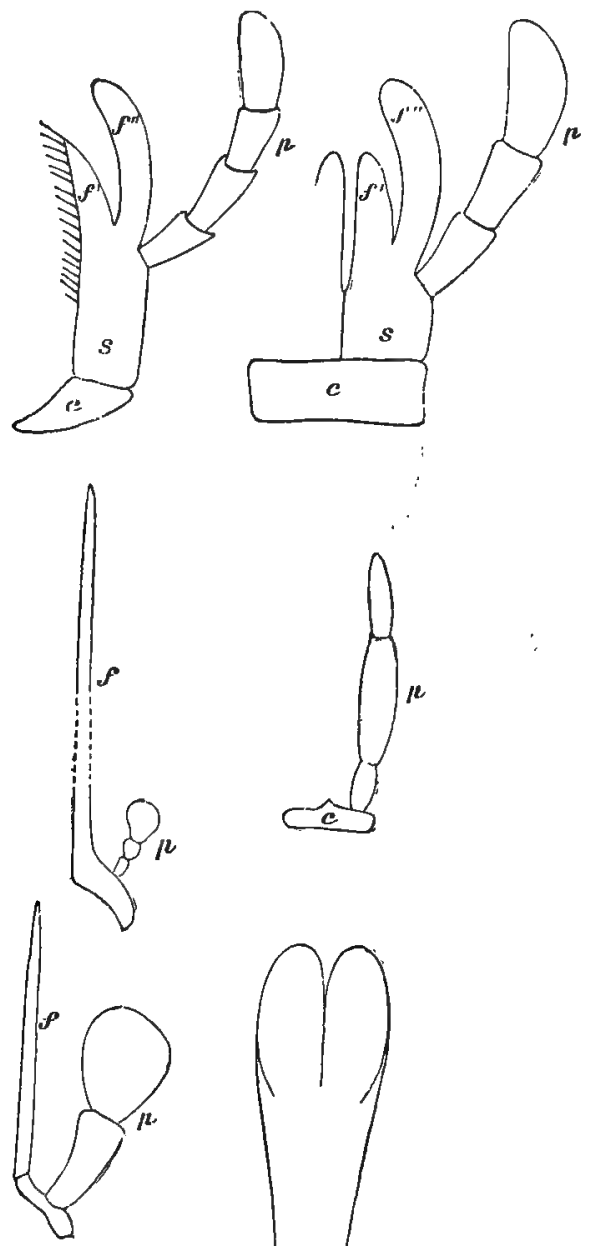

3
4
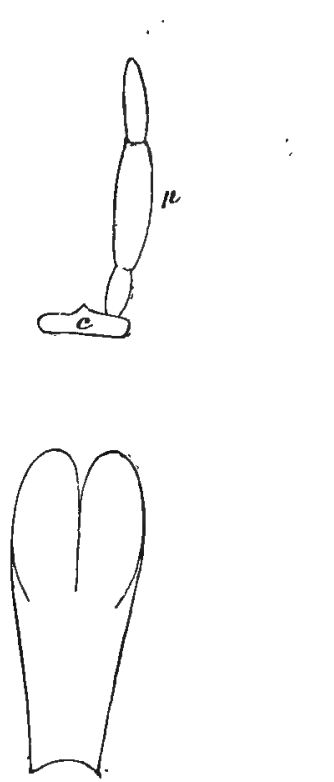

4

Fig. 195. Diagrams of the month parts of various Insects. $A$ An Insect with biting mouth-parts, B a Butterfly, C a Fly. 1 labrum, 2 mandible, 3 first maxilla. $c$ cardo, $s$ stipes, $f^{\prime}$ galea, $f^{\prime \prime}$ lacinia, $p_{1}$ palp. 4 Second maxilla=labium. $c$ submentum, $s$ mentum, $f^{\prime}$ glossa, $f^{\prime \prime}$ paraglossa of ligula, $p$ palp, $f$ proboscis of $B$ and $C$.- Orig.

much like those of the Crustacea in general structure, though as the palp is always wanting, each consists of a single, unsegmented. piece, which can be moved inwards (towards the middle line) or outwards; its inner surface forms a cutting edge, and at its base 
there is a grooved or tuberculate grinding denticle, the latter being best developed in the herbivorous forms, whilst the former is more prominent in predaceous Insects. The first maxilla usually consists of six to eight joints, of which the basal joint (cardo) is short, the second (stipes) large, and produced into two long lobes, the inner (galea) being usually fringed with stiff setæ along its outer edge; the outer (lacinia), in several forms, consisting of two joints. Sometimes only a single lobe is present. The rest of the maxilla, which usually consists of four to six joints, forms a curved palp. The first maxillæ are prehensile and gustatory in function, whilst the mandibles are masticatory; sometimes, however, the former also assist in mastication. The second maxillæ are similar to the first, but are distinguished by the fact that the two cardines are always fused to form a single plate (mentum); the stipes, too, are more or less completely fused, and the lobes are often considerably modified as compared with those of the first maxillæ; the palps are like those of the first maxillæ, but never consist of more than four joints. The second maxillæ are usually spoken of as the labium, their palps as the labial palps, the lobes as glossce and paraglossce. The labium is, of course, not comparable with the lower lip of the Crustacea; it is, like the mandible and first maxillæ, a pair of limbs, and corresponds with the second maxillæ of the Crustacea, whilst the lower lip of the latter is a membranous fold which is not represented in the Insects. The labium here forms the posterior, as the labrum forms the anterior, boundary of the mouth.

In Insects with sucking mouth-parts the same elements occur, but modified in different ways in accordance with the change of function. In the Lepidoptera, the labrum is simply a short,
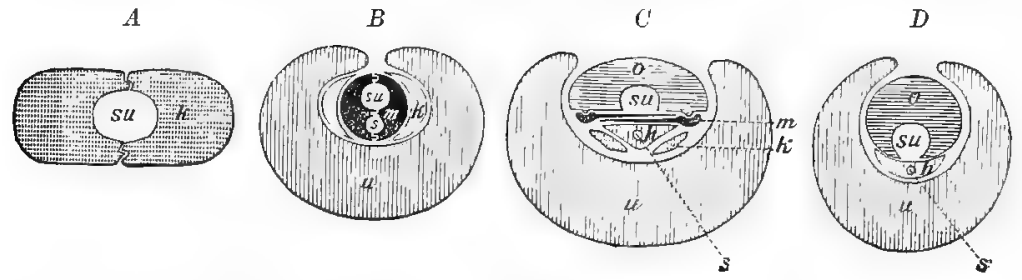

Fig. 196. Diagrammatic transverse section of the proboscis of: $A$ Butterfly, $B$ Rhynchota, C Tabanas (Gad-fiy), D Musca (another of the Diptera in which mandibles and first maxillæ are wanting). su sucking tube through which the fluid passes to the mouth; $s$ salivary tube, o labrum, $m$ mandibles, $k$ first maxillæ, $u$ labium, $h$ hypopharynx.—Orig.

broad plate with no special significance; the mandibles are rudimentary or absent. The first maxillæ on the other hand are well-developed; each possesses only a single lobe, which is elongate and gutter-like, forming, with its fellow of the other side, a long tube, open at the end; this tube is the sucking apparatus, the 
proboscis of the butterfly. Maxillary palps are present, but feeble. The unpaired portion of the labium is not well developed, but the palps are large setigerous lobes, enclosing the proboscis, which is spirally coiled when not in use. In the Rhynchota the suctorial tube is formed by the $\mathrm{mandibles}$, which are represented by two compressed blades without palps; two grooves run down the inner surfaces of the blades, which are so fitted together that they form two tubes, an upper and a lower. The proximal opening of the lower tube lies close to the opening of the duct of the salivary gland, and saliva passes down it, to be mixed with the food before it is sucked up into the mouth through the upper, wider canal. At the sides of the mandibles lie two other dagger-like organs, the modified first maxillæ, pointed like them, and thus adapted to act as stabbing weapons; their palps are wanting, both pairs of appendages are inserted in deep pits, and can be protruded or withdrawn. 'The labium is characterised by the fusion of the palps, so that the whole lower lip is an unpaired structure of three or four joints, hollowed out like a gutter, and forming a sheath surrounding the mandibles and first maxillæ. This sheath is open above, but the opening is simply a slit along most of its length, widening out only at the base, where it is covered by the triangular labrum. Formerly the labium of the Rhynchota was regarded as the functional tube, but it is now known to be simply a case for the sucking tube proper, which is formed from the mandibles. In the Dipter a the relations are as follows: In the most perfectly developed mouth parts (e.g., in the Gnat and the Gad-fly [Tabanus]) there is a labiu m of considerable length, much hollowed out on its under surface; beneath this lie the flattened, sword-like mandibles, which together with the labrum form the proboscis. Below the mandibles lies an unpaired, narrow, flattened piece, the hypopharynx, which arises posteriorly from the labium; it is traversed by the salivary tube which opens at its tip. Next below lie the first maxillæ, which, like the mandibles, are long, narrow, blade-like stabbing or cutting organs; a large palp arises from the base of each. All these parts are enclosed by a long furrowed lab i u m, which has no palp, and like that of the Rhynchota, only forms a case for the rest of the mouth parts ; the maxillary palps alone are not surrounded by the labium, but project freely at its base. In other Diptera (e.g., House-flies), the mandibles and first maxillæ (with the exception of the basal portions of the latter and their palps) are wanting; in this case, the place of the mandibles is supplied by the hypopharynx, which closes the labial groove below. In the Hymenoptera most of which possess simple biting mouth parts, these may, as in the Bees, be both biting and sucking : the mandibles performing the former, the first maxillæ and labium together subserving the latter, function. 
The thorax is composed of three segments: prothorax, mesothorax, and metathorax. Usually the last two are immovably united, the pro-thorax freely articulated. Each bears a pair of $\mathrm{leg} s$, which are divided into the following parts: coxa, trochanter, femur, tibia, and tarsus; each of the first four consists of a single joint only, whilst the tarsus is generally multiarticulate. The coxa and trochanter are usually short, the femur and tibia long, the former thicker than the latter; articulating with the lower end of the tibia there is generally a pair of movable spines (spurs). The tarsus in many Insects consists of $\mathrm{f} i \mathrm{re}$ joints (sometimes of fewer), and usually bears at its tip two movable hooks, the claws. The legs are true locomotor organs; in walking, the animal rests on the lower side of the tarsus, which is often hairy ; the distal end of the femur is turned outwards, that of the tibia downwards, the tip of the tarsus outwards; in the first pair of legs the foot is forwardly directed, in the last pair back-

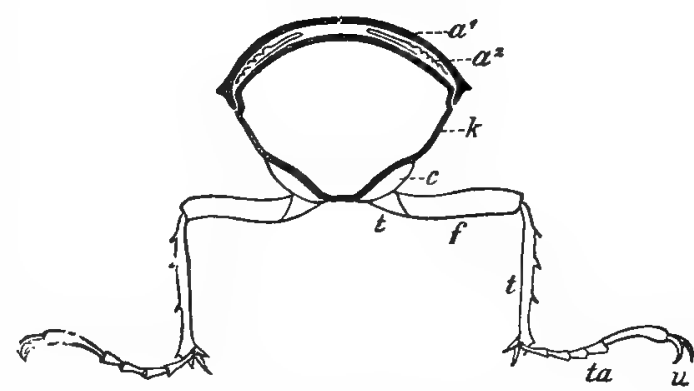

Fig. 197. Transverse section through the thor a $x$ of a Beetle (diagrammatic). $a^{1}$ elytra, $a^{2}$ wings, $k$ body-wall; $c$ coxa, $t$ (upper) trochanter, $t^{\prime}$ (lower) tibia, ta tarsus, $u$ claws.-Orig. wardly. In many forms the legs, or some of them, have another function besides that already mentioned. The front pair in the Cockchafer serve not only for ambulatory purposes but also for digging; in others the legs are so modified in connection with the secondary office, that their primitive function is lost. The first pair in the Molecricket, for instance, is only used for digging; the same pair in Water-scorpions for organs of prehension; the last pair in the Locust forms a springing apparatus, whilst in Dytiseus it is pre-eminently natatory in function.

The thorax usually bears two pairs of $w$ in $\mathrm{g} s$, which arise dorsolaterally from the meso- and meta-thorax. Each wing is a large, laminate, tegumentary outgrowth, which primitively possesses the same layers as the rest of the skin, i.e., is covered on each side with a chitinous layer (cuticle), within which, on each side, is an epidermal layer, whilst between the two epidermal layers run tracheæ, nerves, etc. When the wing is fully developed, however, the soft parts disappear, so that it then consists of little else than two closely apposed chitinous plates. The wings articulate with 
the thorax, and are moved by a muscular apparatus; they are usually thin transparent plates, in which a network of somewhat thicker, more firmly chitinised, and darker ribs occur: not infrequently they are sparsely or entirely covered with setæ (see the Lepidoptera). The two pairs are often almost identical in form and size, more frequently, as in certain Libellulidæ, they differ somewhat in these respects; sometimes the anterior, sometimes the posterior, pair is the larger. During flight, they are spread laterally, but when at rest, they turn somewhat backwards, so as to cover the abdomen, and the wings of the first pair overlie the second ones, which are then often folded like a fan.* In correlation with this, the anterior wings have been modified, in many Insects (Locusts, Beetles), to form wing-cases, or elytra; they are thicker and harder, and serve chiefly or exclusively to cover and to protect the posterior pair during rest, whilst their locomotor importance is lost; the hind wings, which are usually large, lie beneath them, folded longitudinally or transversely. The elytra attain their greatest development in Beetles (Fig. 197), where they not only protect the posterior wings most efficiently, but also the dorsal surface of the abdomen (which is, therefore, softer than the ventral side); for their inner edges are straight, and fit closely together, and their outer edges are coincident with the lateral body-wall. It results, therefore, that in many Beetles, which are apterous, or have rudimentary wings only, well-developed elytra are, nevertheless, present. Another modification of one pair of wings occurs in the Diptera, where the hind ones are developed as small club-like appendages (halteres), the significance of which is not clear, but which are certainly not organs of flight. In a number of Insects belonging to various groups, the wings are rudimentary, or altogether absent; many of these forms are parasitic.

The a b d o $\mathrm{m} e \mathrm{n}$, the posterior apodous region of the body, consists of ten or fewer segments, which are usually freely articulated, although occasionally some of them are fused; there is not generally such a deep constriction between the thorax and abdomen as between the head and thorax. In each abdominal segment a dorsal and a ventral plate (tergum and sternum) is usually distinguishable, connected by softer portions. In some Insects (Mole-cricket, Dragon-fly), the posterior end of the abdomen bears a pair of jointed or unjointed anal cerci, which turn backwards, but otherwise there are noabdominal limbs or limb-like appendages. $\dagger$

* But occasionally the first pair of wings are folded when at rest.

+ In some genera belonging to the $\mathrm{Th}$ y s a $\mathrm{n}$ u rat, a group which consists entirely of apterous Insects with biting mouth parts, there are, on the ventral side of the abdominal segments, small paired appendages, which are not jointed, but which are quite like limbs in their mode of origin. It must be mentioned, too, that in many insectan embryos, definite rudiments of limbs bud out from the first abdominal segments (sometimes from several), but these atrophy before hatching. 
The chitinous cuticle in Insects is not calcified, but, notwithstanding this, it frequently attains a very considerable firmness, and is often of great thickness; below, there is an epidermis often called hypodermis, usually a single layer of cells. In connection with the skin, there are frequently skin-glands; of these may

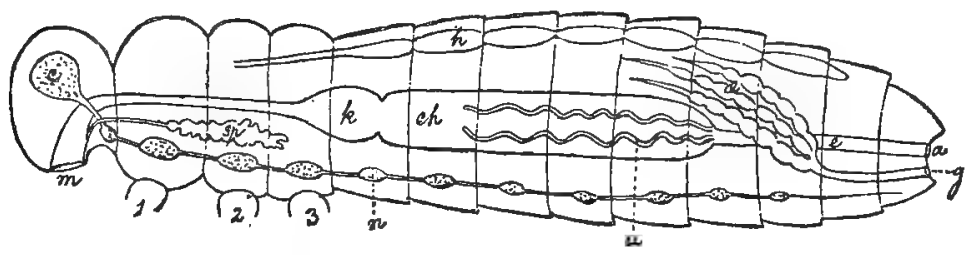

Fig. 198. Diagram of the principal anatomical points in an Insect. 1-3 first and third pairs of legs cut away. $a$ anus, $c$ cerebral ganglion, ch mesenteron, e proctodæum, $g$ genital aperture, $h$ heart, $h$ crop, $m$ mouth, $n$ ventral ganglion, $s p$ salivary gland, $u$ Malpighian tubule, $\infty$ ovary.-Orig.

be mentioned, the stink-glands on the ventral side of the thorax, in the Hemiptera; the anal-glands of the Carabidæ; the wax-glands of Apidæ and of Cocci. Some are gland-cells, some true glands; sometimes they are represented by simple, flat, thickened portions of the epidermis (wax glands of Bees).

The nervous system is characterised by the great size to which the cerebral ganglion often attains. The most anterior of the ventral series, the $\mathrm{sub}$ oes ophageal, is situated in the head, like the cerebral ganglion, and gives off branches to the mouth-parts. This is succeeded by three single or paired ganglia, one for each thoracic segment, and lastly, by a series of abdominal ganglia. Often, however, some of these fuse; the second and third thoracics may, for instance; the posterior abdominals also, or the second and third thoracics

Fig. 199. Nervons system of an ant $(A)$, a cockchafer $(B)$, and a bluebottle $(C)$. $h$ cerebral ganglion, u sub-œsophageal ganglion, $1-3$ the three thoracie ganglia, $a_{1}-a_{3}$ abdominal ganglia, $a$ fused abdominal ganglia, $s p$ passage for the cesophagus.-After Brandt.

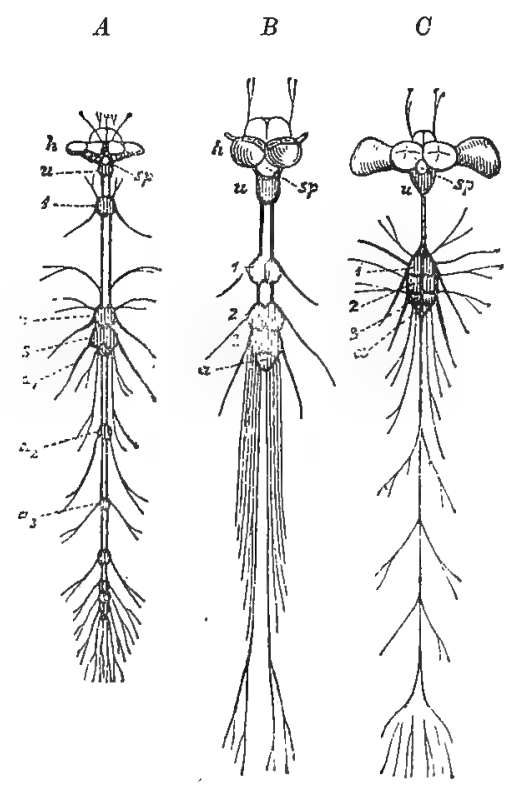


and all the abdominal ganglia may unite to form a single mass, which, in extreme cases, includes also the first thoracic ganglion.

Sense organs. Olfactory organs occur as short, delicate, thin-walled hairs, which receive filiform processes from sense-cells lying beneath them (Fig. 18); they occur on the antennæ, often situated in pits. Auditory organs probably occur in the majority of Insects, since there is direct proof that many can perceive sounds; and indirectly, it is probable that, since many can produce noises, they can also perceive them; these organs are, however, at present only known with certainty for quite a few forms. In the Grasshoppers, there is on the side of the first abdominal segment a thin membrane (the "tympanum," a specially developed portion of the skin), stretched at the bottom of a depression. Beneath the membrane there are peculiar cells, each inclosing a delicate pin-like body, and connected with a nerve fibre. It is believed that the membrane is caused to vibrate by sound waves, and that this reacts on the cells described; the sound is intensified by a tracheal bladder, which lies close to the tympanum, and serves as a resonator. Auditory organs of a somewhat different structure occur in the Locusts on the tibiæ of the first pair of legs. In other cases there are cells like those described above, but without tympanum or resonator, and it is supposed that these may also be regarded as simple auditory apparatus. For e yes, the account on p. 231, and the description of the structure of Arthropod eyes given in the General Part, may be consulted.

Alimentary canal. In Insects with sucking mouth-parts, strong muscles run from the buccal cavity to the inner side of the head: the cavity of the mouth is enlarged by their contraction, and thus the fluid into which the proboscis is dipped is drawn in. One or

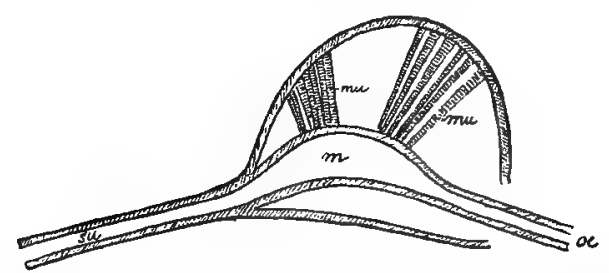

Fig. 200. Diagrammatic longitudinal section of the head of an insect with sucking mouth-parts. $s u$ sucking tube, $m$ buccal cavity, $m u$ muscles which widen the latter, $\infty$ cesophagus.-Orig. more pairs of salivary glands open into the mouth. The rest of the alimentary canal, which may be straight or looped, is made up of the osophagus, the mesenteron, and the proctodæum. The sophagus is usually narrow in front, but swells out behind into a crop, which is either a simple dilation or a special pouch-like appendage connected with the rest of the cesophagus by a narrow duct; this is the case in many suctorial Insects. The crop serves as a reservoir for the food. Occasionally the terminal part of the œsophagus is particularly muscular, provided with hard parts on the inner surface, and serves as a gizzard. The mesenteron is the essential digestive portion, though the secretion of the salivary glands assists in this 
connection; it is also the absorptive region; it is saccular, and sometimes separated into several sections. The epithelium of the alimentary canal secretes the digestive fluid; sometimes. small evaginations, which project externally from the alimentary canal as warts or papillæ, may have this function; there is never a specialised liver. The proctodæum is usually divided into an anterior narrower and a posterior wider portion; the anus is situated

Fig. 201.

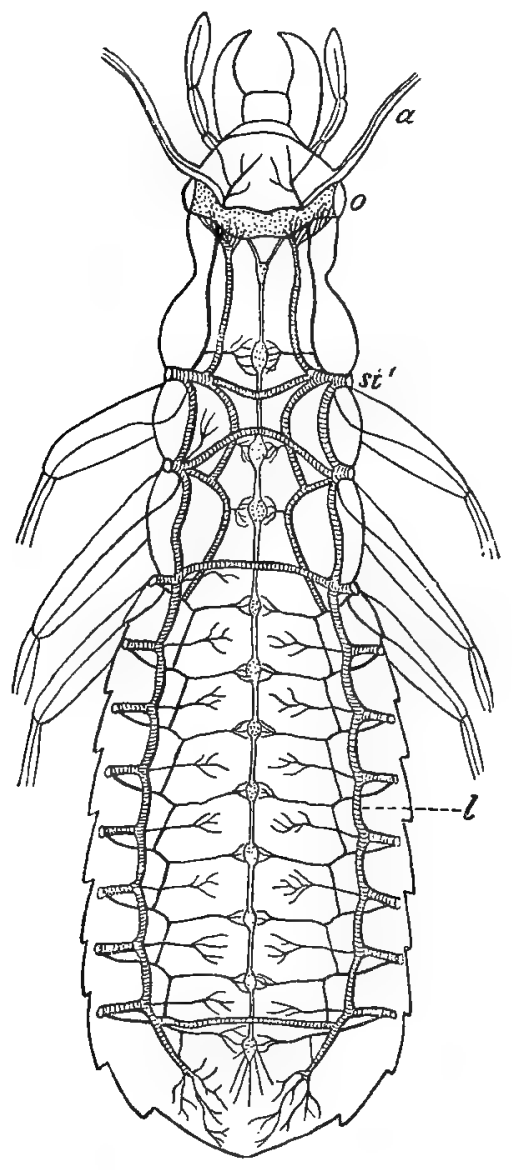

Fig. 202,

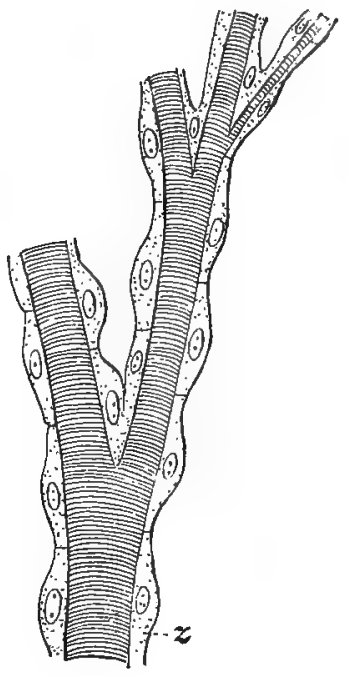

Fig. 201. Diagram of the chief trunks of the $\mathrm{trach} \theta \mathrm{al}$ system of an Insect; the central nervous system is also shown. $a$ antenna, o eye, st' anterior stigma, $l$ longitudinal trank.-After Kolbe.

Fig. 202. Portion of a trachea from a Gall - fly larva (somewhat diagrammatic). $z$ a cell of the wall.-Orig.

on the terminal segment. The Malpighian tubules, delicate unbranched, brightly-coloured (white, yellow, brown, or green) tubes, open into the proctodæum at its junction with the mesenteron. There is usually only a small number, four to six, when they reach a considerable length; in the Hymenoptera and some of the Orthoptera, however, there is a much larger number of shorter tubes. These constitute the excretory apparatus. 
The respiratory organs are represented by a system of tubes containing air, the tracheæ, which branch over the whole body, winding about among the organs and communicating with the exterior by the stigmata, which, like the whole system, are symmetrically disposed. There are at most ten pairs of stigmata, one pair on the mesothorax, one on the metathorax, and one on each of the eight anterior abdominal segments, where they lie between the sterna and terga; there are no stigmata on the head or prothorax.* The stigmata are usually slit-like apertures, which are frequently provided with marginal setæ (Fig. $203 \mathrm{~s}$ ), overlying the opening and preventing the entrance of foreign bodies; the same end may be attained in other ways. A short transverse stem usually runs in-

$A$

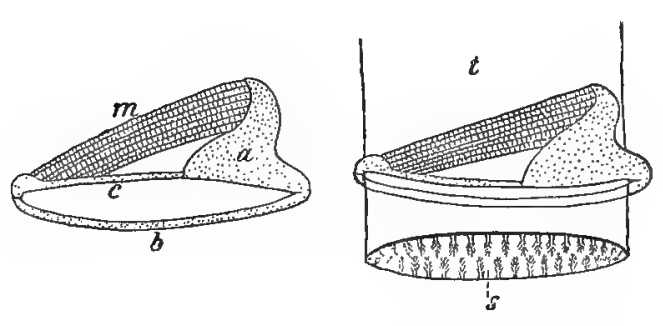

Fig. 203. Apparatus for closing the trachea of a Beetle (diagrammatic). $A$ the apparatus by itself, opened. $B$ The trachea with the apparatus closed. The apparatus consists of three chitinous pieces which surround the trachea like a ring; the piece (b) is as long as the two others together; one of these (a) sends out a process for the attachment of a muscle $(m)$, which takes its origin from the third piece (c). When the muscle contracts, $a$ and $c$ are pushed against $b$, and the trachea is clamped between the three pieces. $s$ stigma, $t$ trachea.-Orig.

wards from the stigma to open into one of the main tracheal trunks, a varying number of which traverse the whole length of the animal, connected with each other by several transverse vessels and giving off numerous branches which anastomose over the whole body. Occasionally the longitudinal trunks are absent, and the trachea arising from each stigma breaks up directly into a number of branches which are entirely independent of the rest. Some of the tracheæ may be dilated to form vesicles, which vary in size, but are sometimes quite large. These vesicles have no actual respiratory significance, but serve to decrease the specific gravity of the body, and are thus of importance in flight; in other words the tracheal system is not only respiratory, but in many forms is also a ërostatic. All the tracheæ are covered by a thin chitinous cuticle which, in the coarser tubes (but not in the vesicular dilations), is supported by a delicate spiral thickening. Respiration is effected by movements of the abdomen; by its contraction part of the air is forced out from the tracheæ, and when it expands again a fresh supply enters.

* The position of some of the stigmata may often be changed, those belonging to the mesothorax may lie between the prothorax and mesothorax (Fig. 201) or even, as in caterpillars, on the prothorax. 
In order that the air shall have access to the most distant branches of the system, the trachea arising from the stigma, possesses a peculiar apparatus by which it may be completely closed; when this is effected, and the abdomen contracts, air cannot escape from the stigma, and is, therefore, driven into the ultimate branches and into the vesicles. When the abdomen is relaxed, and the closing apparatus opened, a new supply of air streams in; then by the contraction of the abdomen, and the simultaneous closing of the stigma, this fresh supply is driven again into the finest branches and vesicles. By repetition of these processes, the tracheal system is filled with air, and the vesicles completely expanded; this is of especial significance in its function as ærostatic apparatus; and before flight, individuals may be seen expanding with air thus pumped in.

The tracheæ arise as epidermal invaginations, which branch freely, and unite to form the large longitudinal trunks; they secrete a cuticle just as does the epidermis. The apertures of the invaginations form the stigmata. At an ecdysis, the cuticle of the tracher is also renewed, the old cuticle being thrown out through the stigmata.

In a number of aquatic insectan larvæ (Dragon-flies, May-flies, Neuroptera), the tracheal system is closed, i.e. without open stigmata. The oxygen s obtained endosmotically from the water by means of the "tracheal gills," membranous appendages, with large surfaces, and containing a close trachea! network.

In addition to the respiratory and aërostatic function, the tracheal system of many Insects is also concerned in the production of sounds. Thin membranous folds often occur within the tracheæ, close to the stigmata; they are set in vibration by the air entering the trachex, and thus produce a sound (buzzing of Flies and of Cockchafers). The noises made by Insects are also brought about in many other ways. Flies, Bees, and Gnats, all of which produce sounds by the vibration of the tracheal folds, can also make humming noises by the rapid movements of the wings. Others rub various parts of the surface of the body against one another. The male Grasshopper, for instance, rubs a dentate ridge of the tibia of the last leg against the elytron of the same side; others again, strike some part of the body against a foreign object (the Fiddler-beetles for instance, knock their heads against the wall of the passages they have gnawed in wood, and thus cause the well-known ticking sound).

Some Insects are luminous in the dark. The light proceeds from large cells situated within the body beneath transparent areæ of the skin, and depends upon the oxidation of certain substances present in the cells, which are therefore surrounded by a close tracheal network.

The vascular system is but little developed, a circumistance correlated with the high specialisation of the respiratory apparatus. Since air is carried direct to all parts of the body, the importance of the blood as oxygen-carrier is necessarily limited. In the dorsal region of the abdomen there is a tubular heart, closed behind, open in front, and constricted into a series of chambers corresponding with the abdominal segments; each chamber is furnished with a pair of ostia provided with valves, and there are 
also valves at the limits of the chambers. The heart, as in other Arthropods, lies in a spacious cavity, the pericardium, which is. bounded above by the dorsal wall of the Fig. 205.

Fig. 204.

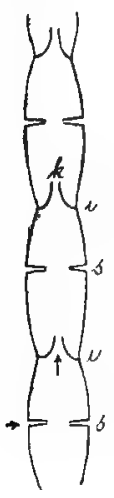
abdomen, below by a perforate plate of connective tissue interlaced with muscle fibres. The heart and a tubular extension of its anterior end, the aorta, are the only vessels, the blood circulates in spaces between the organs in a fairly regular current. After traversing the body it enters the pericardium, and from this, in consequence of the dilatation of the heart and the opening of the ostia, into the heart itself, whence it is again driven out through the aorta into the sinuses of the body.

Genital organs. The female, as in other Arthropoda, possesses a pair of ovaries. Each consists of a varying number of tubules: (ovarioles) which usually extend like fingers from the anterior end of the oviduct. Each ovariole is surrounded by a thin membrane and is immature anteriorly, consisting of small homogeneous cells; further back thereare larger cells, young ora, lying in the middle of the tube, and surrounded by smaller cells, which provide them with nutriment and also secrete the shell

Fig. 204. Portion of $t h e$ heart of an Insect, diagrammatic. $i$ constriction between two chambers, $k$ valves, $s$ venous ostia.Orig.

Fig. 205. Ovariole of an Insect, diagrammatic. a young ovum, a' mature ovum, $s$ shell, $r$ empty lower extremity of the ovarian tubule (an egg has just escaped).-Orig. (chorion), for the fully developed egg. The mature ova occupy the posterior ends of the ovarioles, and pass thence into the oviduct; when an egg passes into the latter the corresponding portion of the ovarian tubule. shrinks, and thus the egg next in front is brought nearer to the duct. The two oviducts unite to form an unpaired portion, the $\nabla$ agina, ${ }^{*}$ which opens ventral to the anus, either freely on the surface or into a cloaca, an invagination occurring at the hinder end of the body. There is usually an evagination of the vagina which serves as a receptaculum seminis and one, or a pair of accessory glands which secrete either a sticky fluid to attach the ova to foreign bodies, or the mucus surrounding them (e.g., in Insects which lay their eggs in water); sometimes there is also an evagination

* In some few Insects (Thysanura, Ephemera), the vagina is wanting, and both ducts open direct on the postero-ventral surface of the body ( $c f$., Crustacea). 
of the vagina to form the bursa copulatrix, into which the penis of the male is inserted in copulation.* Not infrequently there is at the female aperture, an ovipositor (Locusts), consisting of complicated knife-like or dagger-shaped laminæ, or a s t in g (Hymenoptera), or the last abdominal segments, which then are thin and elongate, and

Fig. 206.

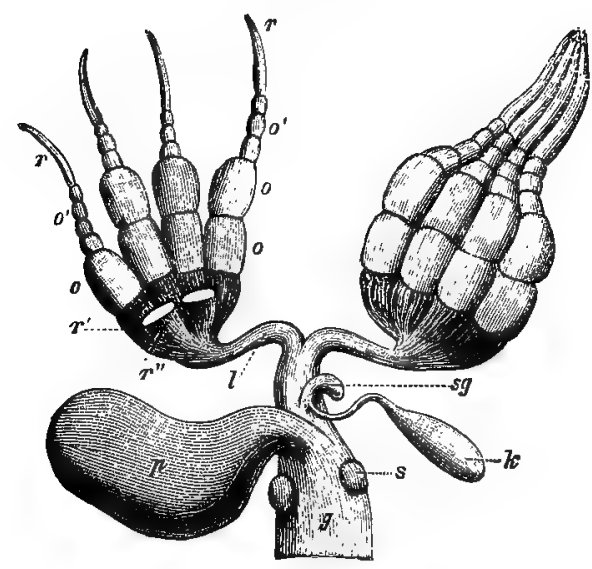

Fig. 207.

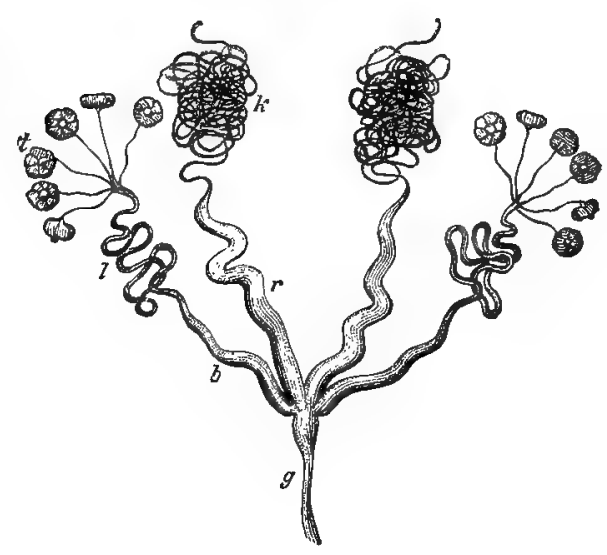

Fig. 206. Female genital organs of the Cockchafer. On the right, the ovarioles are lying together in the natural position; on the left they are separated, and two are cut away. $g$ vagina, $k$ glands which open into the receptacula, $l$ oviduct, o segments of the ovarioles containing almost ripe ova, $o^{\prime}$ regions of the same, containing immature ova, $p$ bursa copulatrix, $r$ anterior, $r^{\prime}, r^{\prime \prime}$ posterior buds of the ovarian tubules, $s$ glands, $s g$ receptacula ovorum.-Orig.

Fig. 207. Male genital organs of the same (penis not drawn). $b$ vesicula seminalis, $g$ vas deferens, $k$ glandular appendages, $r$ widened region of the duct of the same, $t$ testis, consisting of six seminal pouches.-Orig.

may be telescoped, serve in this capacity (Diptera and others). The chorion is often very hard, frequently covered with a delicate and regular sculpturing, and always provided with one or more openings, the micropyles, through which the spermatozoa may enter. The outer form of the eggs varies : it may be spherical, oval, discoid, rough, stalked, etc.

The male genitalia are for the most part a repetition of those of the female. There is a pair of testes, each consisting of several long seminal tubes or shorter seminal pouches, situated at the end of the vas deferens. The two vasa deferentia unite to form a

* In the Lepidoptera the bursa copulatrix is peculiar, in that it is not as in other forms, a simple evagination of the vagina, but is a tube, open at both ends, one end leading into the vagina, the other on to the surface of the body; so that here the female genital organs have two pores, that into the bursa serving for copulation, whilst the vaginal opening proper allows only of the escape of the eggs. 
single duct, ${ }^{*}$ which opens in a similar position to the vagina of the female. Each of the vasa deferentia widens posteriorly to form a vesicula seminalis:. Special glandular appendages frequently open into these ducts, or into their common portion. There is a more or less complicated copulatory organ, an evagination of the body-wall through which the terminal portion of the seminal duct is continued, and capable of partial or complete retraction when not in use ; in many, it may possess hard chitinous portions, and lies hidden within the cloaca, from which it may be protruded during copulation.

A fairly marked sexual dimorphism occurs very often in Insecta, due largely to the different parts played by the male and female in repro. duction. Frequently the males possess apparatus which is wanting in the females, or certain portions of the body are specially developed; for example, the large mandibles of the Stag-beetle, the huge eyes of the male Honey-bee, the well-developed antennæx of the male Cock-chafers and many Butterflies, the broad front feet of male Water-beetles; such developments, if they are in any way explicable, are attributable to the struggles carried on by the males for the possession of the female (Stag-beetle); or, in the case of special prominence of sensory organs, they result from the needs of the male in seeking for the less active female, or the parts serve as organs of retention during copulation (Water-beetles). More rarely some portion of the body of the $f \mathrm{emale}$ is

1

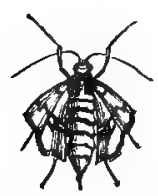

2

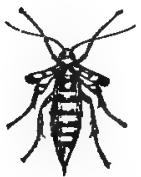

3

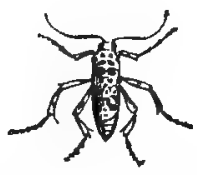

Fig. 208. Females of three allied species of Geometridæ(1 Hibernia progemmaria, $2 \mathrm{H}$. aurantiaria, 3 H. defoliaria), to show the successive stages in the degeneration of the wings.After Ratzeburg. male and female Oryctes), are, as a rule, not apparently capable of explanation. As already mentioned, the male is usually more active than the female, and in correlation with this, sexual dimorphism may be carried very far, various parts of the body of the female may be considerably modified or atrophied. In not a few Lepidoptera for instance, the wings of the female are considerably shortened, so that they have become useless as organs of flight, or they are quite rudimentary, or have vanished altogether; in some forms, indeed, degeneration goes still further, the legs are feeble, or are not developed, so that the animal sinks into a maggot-like state, which is as different from the male as possible. The converse, when the female has greater activity, may also occur, although more rarely; Fig. 209 for instance, shows a species of small Gallfly (Blastophaga grossorum), which lives as a larva in the tiny seeds of the

* This duct (like the vagina) may be absent (Ephemera, and a few others), and the vasa deferentia then open separately. 
fig; the male never leaves the fruit in which it has passed its early existence, and in consequence of this is clumsy and apterous, whilst the female must seek for young figs in which to deposit the eggs, and is active and winged.

$A$

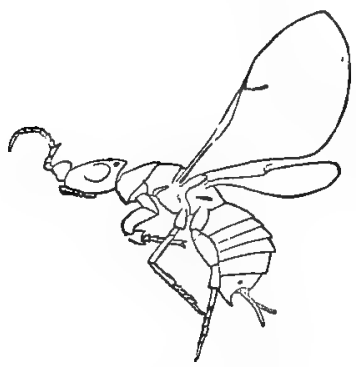

$B$

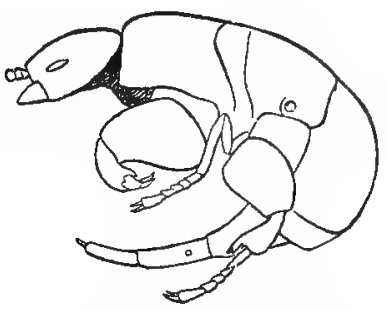

Fig. 209. Blastophaga grossorum. A $q\left(\begin{array}{c}12 \\ 1\end{array}\right) . \quad B$ d $\left(\frac{21}{1}\right)$.- - After P. Mayer.

Many species of Insects are remarkable, in that a large number of individuals remain sterile throughout life, and thus take no part in the propagation of the species; these individuals possess, as a rule, distinct rudiments of sexual organs, which, however, never develop far enough to form fertile genital products (or they are deficient in some other way, so that the individuals cannot take any part in reproduction); such sterile individuals are always, in some Insects (Bees and Ants), incompletely developed females; in others (Termites), both males and females. The occurrence of such sterile individuals depends upon the fact that these Insects are social, and form larger or smaller colonies; it is an expression of a division of labour within the colonies, the care of the brood, etc., being relegated to the sterile individuals, whilst the reproductive faculty is exercised by relatively few, which, however, produce an enormous number of offspring ( $c f$., the division of labour in Hydroid colonies).

$\mathrm{Par}$ thenogenesis has been shown to occur in many Insects. In many cases, it is an exceptional occurrence; the female of the silkworm moth for instance (Bombyx mori), if unfertilised, can still lay eggs, most of which atrophy, though they may develop in the usual way. The same thing is known for many other Lepidoptera. In other cases, parthenogenesis is of more $r e g u l a r$ occurrence; in some Insects it is the $\mathrm{rule}$, males appearing only occasionally; so in certain Lepidoptera, e.g., Psyche helix, in which the female is apterous and maggot-like, whilst the male is normal in form: or the males may appear with the females, but in small numbers, and apparently without, as a rule, copulating, as in Cynips rosce, a wellknown rose Gall-fly : or, as in some of the Saw-flies and Gall-flies, reproduction is apparently exclusively parthenogenetic, in which case the species consists entirely of females; or in certain generations only, reproduction may be exclusively parthenogenetic. Another 
regular occurrence of parthenogenesis is found in many social Hymenoptera where the males originate from unfertilised, the females from fertilised, ova. Not infrequently, parthenogenetic reproduction alternates regularly with the usual sexual mode, so that there is heterogony; either each unisexual generation alternates with a true sexual one (Gall-flies), or the sexual is followed by several parthenogenetic generations (Aphides). Usually, the parthenogenetic generations differ somewhat from the other; sometimes, if there are several such successive generations, these also differ from each other.

In some of the Diptera (Cecidomyia) eggs may arise prematurely in the larva, and develop direct without fertilisation into viviparous larvæ, the parent larva dies, whilst the young ones grow, and either give rise in the same way to another generation or become perfect insects. Thus parthenogenesis may occur precociously, when there is otherwise no sexual reproduction. This process is known as $p$ ædogenesis.

Insects are usually oviparous, but the parent is most solicitous in ensuring that the newly-hatched larvæ shall be well supplied with nourishment; this is generally accomplished by laying the eggs in places where suitable food is present, but occasionally by the collection of a supply of food where the eggs are deposited (certain Dungbeetles, Sand-wasps); more rarely the parental instinct is more highly evolved, and the female brings a fresh supply of food each day to the brood. In a few Insects the eggs are only laid when the embryo is about to be hatched; others are viviparous, embryonic development being completed within the oviduct. A peculiar arrangement is met with in the Forest-flies (Hippobosca), in which not only is the egg completely developed within the oviduct, but the larva remains there for some time feeding upon the secretion of certain glandular appendages.

The majority of Insects on leaving the egg do not resemble the adult, but undergo a metamorphosis; only in a few cases, e.g., Lice and various other apterous forms, are the changes so insignificant that it is impossible to speak of a true metamorphosis. Metamorphosis may be more or less thorough; two chief types may, therefore, be distinguished, complete or holometabolous, and incomplete or hemimetabolous.

In hemimetabolous Insects (Orthoptera, Rhynchota) the newlyhatched larva differs chiefly from the adult or i m a $\mathrm{g}$ o only in that it is apterous. In other respects the differences are slight, the number of joints in the antennæ may be fewer, the head relatively larger than in the adult and so on. The transition from the first larval stage to the adult occurs gradually; wings begin to appear,* small at first, but increasing at each ecdysis, until after the last moult they are fully formed and functional: at the same time the other portions of

* Such larvæ, possessed of incipient wings, are often termed nymphs. 
the body have attained their definite form. In some hemimetabolous Insects the differences between larva and adult are, however, much greater, attributable to essential differences in habits. These distinctions are very well marked in the Libellulidæe and the Ephemeridæ, which are aquatic during larval life, but terrestrial as adults; in the larvæ of these forms the tracheal system is closed, and they breathe by means of tracheal gills (see p. 241); in the adult, on the contrary, the usual relations obtain. Considerable differences may also be noticed in several other points, e.g., in the dispositions of the mouth-parts of the Libellulids: these differences hold throughout the whole larval life until the last ecdysis, but, just as in other forms, the wings develop gradually. At the last ecdysis all the special larval characters disappear as at one stroke, although, as a matter of fact, the changes have gone on gradually within the cuticle during the last phases. When the wings are completely developed, and functional, the insect moults no more, and growth ceases.

The Ephemeridæ, which, as already mentioned, are aquatic as larvæ, are peculiar in that the insect on leaving the water has feeble, though functional, wings, but immediately after, the final ecdysis occurs and it appears with completely developed wings. At this stage, in which it is capable of flight, but not perfectly developed, it has been termed a sub-imago.

Holometabolous Insects (Coleoptera, Hymenoptera, Lepidoptera, and Diptera) differ from the hemimetabolous forms, in that there is a complete dissimilarity between the larva and the adult; in that the larval stages exhibit, externally, no gradual approach to the adult form; lastly, and this is the most important characteristic of a complete metamorphosis, in that between the larval and imaginal stages, a special period of pupation intervenes, during which the animal doés not feed and is generally quiescent. During this restingstage a series of significant changes occurs in the body.

The larva of a holometabolous insect is distinguished from the imago in the following points : the small ocelli situated medianly on the head are absent, and the compound eyes are replaced by a group of ocelli on each side of the head (these may, however, be altogether wanting): the antennæ are almost always short, and consist of a small number of joints: the mouth-parts constitute a biting apparatus, even if those of the adult are suctorial, and if the latter has biting mouth-parts they are always essentially different in form: the legs are short, with fewer joints, and more uniform than in the adult; they have usually only a single claw: wings are altogether absent: the thorax is small, the abdomen large: the cuticle, except over the head, is generally more delicate than in the imago: the nervous system usually consists of a number of separate ganglia even in those forms in which it is later much concentrated (cf. Fig. 210); the alimentary canal is often very different, and this to a striking degree where the larval habits differ much from those 
of the adult (e.g., in Lepidoptera, Fig. 210).* In many the salivary glands are modified to form a pair of silk glands, whose secretion forms a protective covering for the larva, either alone, or with the assistance of foreign particles which it binds together. This covering or cocoon is usually developed for protection during pupation.' The tracheal system also exhibits striking deviations, for

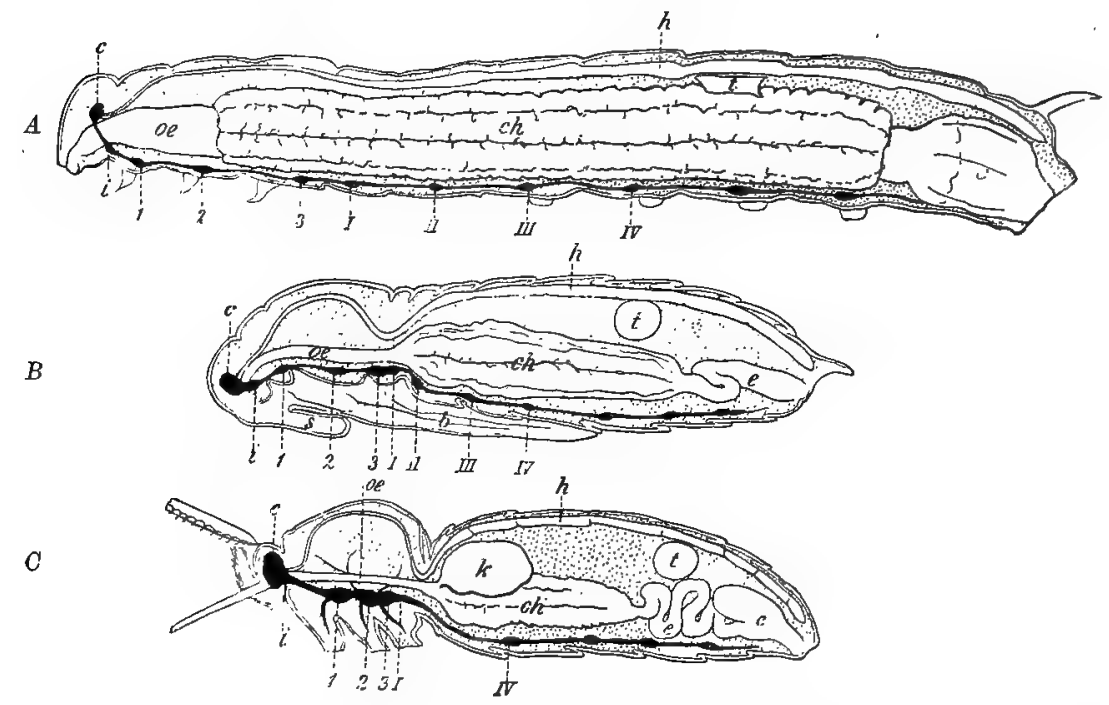

Fig. 210. Larva, pupa, and imago of a Lepidopteran (Sphinx) with various organs in situ; somewhat diagrammatic. All three figures of the same magnification. $b$ legs, $c$ brain, ch mesenteron, e proctodæum, $h$ heart, $i$ sub-csophageal ganglion, $k$ head, $a$ cesophagus, $s$ proboscrs, $t$ testis. $1-3$ three thoracic ganglia. I, II, III, IV the four first abdominal ganglia.-Adapted from Newport.

some of the stigmata which are open in the imago are closed in the larva, or conversely; the structure of the stigmata may also vary. In the bocly-cavity of the larva there are large masses of fat, the $f$ at bodies, which are to a great extent used up during metamorphosis, if they are not entirely absent from the adult. The genital organs are only incipient.

During larval life, the Insect moults repeatedly, and gradually increases in size, usually without an actual change of form. When

* In certain insectan larvæ (e.g., the larvæ of Bees and Ant-lions) a peculiar condition obtains, in that the proctodæum into which the Malpighian tubules open, is not in connection with the mesenteron; but both the posterior end of the latter and the anterior end of the former are closed. Only after metamorphosis do the two communicate.

+ In the larva of the Ant-lions (and presumably in their allies) the secretion from which the silk of the pupa-case is constructed, is probably formed in some of the Malpighian tubules (cf., the analogous conditions of some Fish where the kidneys secrete mucous threads). 
the definite size is attained, however, it appears suddenly to change its external form in a number of essential points, and then after moulting, it appears as a pupa. The pupa displays externally, a very close approach to the imaginal form; the wings are fairly prominent, and the compound eyes are also present, the legs and antennær resemble those of the adult insect, and this is also the case with the mouth-parts, but all the appendages are still somewhat. indefinite in outline, and not distinctly jointed; like the incipient limbs in the body of an embryo, they are not yet functional; they lie immorable upon the body, the general form of which is very like that of the imago (relative development of thorax and abdomen, etc.); internally, however, the pupa at the moment of the last ecdysis, is still in reality in the larval condition. The important changes. which have occurred in esternal structure, have not, of course, taken place so suddenly as they appear to have done; they have all been ready at the close of larval life; the wings may, for instance, have developed long before this as invaginations of the body-wall, and when the larval skin is thrown off for the last time, they are evaginated, and appear as outgrowths of the body; the legs have already grown within their chitinous cases, and may be seen there, folded up, towards the end of larval life. At this time, the insect is inert, and remains as quiet as possible, for the modifications have made its appendages, to a certain extent, functionless. The changes are continued during the pupal stage; the external form of the body alters underneath the protective cuticle, and within, the larval organs are gradually modified into those of the adult (see Fig. 210), so that there is a considerable difference in the organs at the beginning and end of pupation, although a p parently the aninal remains unaltered during the whole time. Many pupæ are quiescent; true locomotion occurs in only a few forms (e.g., Gnat-pupæ, which are aquatic, and must move about on the surface of the water to obtain air), and then is brought about by movements of the abdomen, which also effect respiration. When all the changes are at last concluded, the chitinous pupa-case splits, and the imago emerges. When all the appendages are unfolded, and the cuticle has hardened, the development is in all essentials complete. The adult never moults, it does not grow, or at least, not more than the chitinous coat will permit.

The changes which are undergone at the close of the larval period and during pupation, are not limited to a transformation of the parts already present, but in addition, there is a general destruction and dissolution of many larval organs; in some Insects, only a small portion of the larva actually forms the imago, whilst a larger part undergoes dissolution, and serves for the nourishment of the rest. The amoboid blood-corpuscles of the larva play an important part in this process, in that they feed upon and digest the tissues as they die, passing on the nourishment thus obtained to the actively growing organs of the animal. This process of dissolution is carried to very great lengths in a large number of Diptera, whose 
larvæ differ extraordinarily from the adult, both in appearance and in habits (e.g., the Blow-flies, and many others).

The larvæ of metabolous Insects occur in a number of different forms. Of special modifications may be mentioned the peculiar type occurring in the Lepidoptera and Tenthredinidæ, specially characterised by the presence, on the ventral side of the cylindrical body, of a number of so-called prolegs, small muscular dermal

$A$

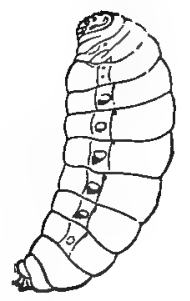

Fig. 211. A Larva (maggot) of $a \mathrm{~W}$ a s $\mathrm{p}$, seen from the left. $B$ Pupa of the same from below.-After Ratzeburg. outgrowths which play an important part in locomotion; such larvæ are termed caterpillars or (in the Tenthredinidæ) pseudo-caterpillars. In a number of larvæ of different orders, legs are altogether wanting; such forms, termed $\mathrm{mag} g$ ot $\mathrm{s}$, are usually pale, blind creatures, which are concealed in plants as parasites, etc.; occasionally they are more motile (aquatic forms, e.g., Gnat-larvæ). The most degenerate occur among the Blow-flies and other Diptera; these are termed headless maggots, since even the head is not clearly developed, whilst it is very striking in many other forms in consequence of its thick brown cuticle. Many larvæ, which lead a hidden existence in the earth, or in cavities in wood, etc., without being maggots, i.e., without loss of the thoracic limbs, have habits like those of most maggots; they are blind, or almost so, with short or feeble legs, and soft, fat bodies.

In most holometabolons Insects, the body hardly changes at all during the whole of larval life. In others, however, it has a different appearance at different ages, a fact which is chiefly connected with changed habits. This is the case in Meloë and its allies; the larvæ hatch as small active organisms, provided with legs. They crawl about on plants, and attach themselves to certain Bees, in whose dwellings, after changing into maggot-like creatures, they pass the rest of their larval life, feeding upon the stores of their hosts.

The pupæ do not exhibit such a variety of form as the larvæ, but here, too, there are many which are interesting to note. The Lepidoptera, for instance, have a peculiar pupa (chrysalis), in which the antennæ, mouth-parts, legs, and wings, lie close on to the body, and are hard and chitinous all over their outer surfaces, as is also the rest of the body where it is not covered by these parts; the limbs, therefore, appear to adhere to the sides of the body, and it looks as if they were all covered by a coat of varnish. In many of the Diptera, the chitinous cuticle is considerably hardened previous to pupation, and when it is later separated from the subjacent soft parts, it is not as usual thrown off, but remains as a hard capsule round the thin-skinned pupa, and thus serves as a protective case; it is only thrown off when the imago breaks through (coarctate pupa). 
The larva, in many species, forms a similar protective case, a cocoon, before pupating; it consists of spun silk, or of various particles bound together, and the pupa rests within it (Lepidoptera, Hymenoptera, some Coleoptera, and others).

In some of the Hymenoptera, a peculiar developmental stage is inserted between the larva and the pupa, or more correctly, there are two pupal stages. The fully-grown larva first forms a so-called $p$ seudop upa, with just the beginnings of wings, legs, and so on, and not till later does the insect enter the true pupal stage, characterised by larger wings and legs, and generally by a greater approximation to the imago.

The metamorphosis is indicative of a definite division of labour in the animal's life. The larval period is a time for eating and growing; the life of the imago is devoted to reproduction; at metamorphosis growth terminates, the animal usually takes no more food than is necessary to make good the loss due to vital activity, and it dies when reproduction has been accomplished. In some cases, the distinction between the two periods is especially well-marked; the imago takes no food at all, and thus the vegetative and reproductive periods are sharply separated. Even if the perfect form does feed, the fact that it no longer undergoes ecdyses shows that growth has really ceased. The few Insects (Termites) in which the abdomen of the adult increases enormously in size, in consequence of the great development of the ovary, form in some sort an exception to this.

According to this account the metamorphosis of Insects is in marked contrast to that of other animals, e.g., of the Crustacea, in which the conclusion of larval life, and the cessation of growth are by no means coincident.

The duration of life in Insecta is almost always sharply limited and fairly short. Usually the whole life (including the phases of egg, larva, pupa, and imago) lasts only one year; in not a few, e.g., in the Aphides it is a matter of months only: in others (many larger forms) it continues during several, usually a fairly definite number, of years (the Cockchafer, for instance, lives usually for four years). Of the whole life the larger part is generally occupied by the larval stage, and only a small portion by the imaginal period; the imago usually lives for only quite a short time, not infrequently for a few days or even for a few hours. Only exceptionally do instances of longevity (several years) in the imago occur. Honey bees have been observed to live five years in captivity, Ants even twelve.

The members of this group afford an emphatically terrestrial type of animal, their organisation being closely adapted to life on land or in the air. Not a few are, however, modified, so that they may live for their whole life, or during the larval stage only, in fresh water. Few are marine, e.g., larvæ of flies which occur in the mud on flat seashores. Some (Coleoptera, Heteroptera and others) live on the coast at spots which are only dry at ebb-tide, whilst at the flood they are 
covered with water, so that their inhabitants are excluded from the air for hours together; ${ }^{*}$ the Halobatidæ are the only actually marine forms, and these lead a life on the open sea like that of their near allies, the Hydrometridæ, in freshwater.† Various Insects (Pediculidæ, Mallophagidæ, Pulicidæ, etc.), live as imagines, or, during the whole of their life, as parasites on various Vertebrates; others are parasitic only as $l$ a $\mathbf{r}$ ₹ upon, or in, various other animals, whilst they lead a free existence as perfect-insects.

The Insecta are richer in species than any other class of animals. According to one reckoning they make up four-fifths of all species; of Insects again, one half are Beetles.

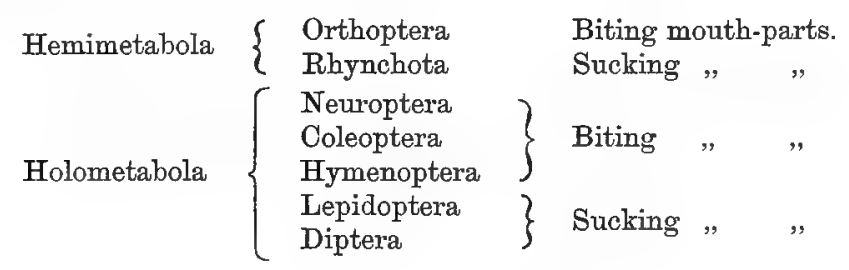

\section{Order 1. Orthoptera.}

The Orthoptera are hemimetabolous Insects with biting mouth-parts. The labium shows more clearly than in other forms that it has arisen by the fusion of a pair of jaws, the individual portions of which are usually easily recognisable. The wings are generally closely veined, but in other respects differ considerably. Frequently the number of abdominal segments is. large; the abdomen is usually furnished with two shorter or longer jointed or unjointed, anal cerci. This order includes very various forms; of the types given below, numbers one to six have. the front pair of wings modified to form leathery elytra, whilst in the rest all the wings are similar.

1. Grasshoppers (genus Acridium, and others). The limbs of the last pair are long, springing legs with thickened tibix. The front wings form long, narrow, somewhat thickened elytra, below which lie the broad hind wings folded up like fans. The prothorax is large, the antennæ short and filiform (at most only twenty-four joints), auditory apparatus (see p. 238) on the first abdominal segment. The males make a noise by rubbing a dentate ridge of the femur of the last leg against the elytron. The females have no ovipositor. Various small species are often met with in great numbers in the fields. Certain species (some large, others small) are common in warm countries as "migratory Locusts" (e.g., Acridium migratorium) ; these, after increasing enormously in some locality, migrate in incredibly large numbers, utterly destroying all vegetation in the regions through which they pass.

* This is also the case with some of the Arachnida (Mites and Pseudoscorpions) and Scolopendras.

† The Lice parasitic on Seals are also marine. 
2. Locusts (genus Locustc and others) resemble the previous group in habits, in the structure of the wings and hind legs, etc., but differ in certain other important characters. The antennæ are bristle-like, usually very long, and always composed of numerous short joints. On the tibiæ of each of the front legs there are two auditory organs (whilst there are none on the abdomen), and the males make sounds by rubbing the basal portion of one elytron, the underside of which is provided with a transversely ridged edge, over a corresponding portion of the other. The female possesses a long, sabre-like ovipositor. One of the best known species is the large bright green Locusta viridissima, which, like Locusts in general, devours both animal and vegetable food. Nearly allied to the Locusts are the Crickets (Gryllidæ), which agree with them in the possession of multiarticulate antennx, and in the position of the anditory organs and the vocal apparatus, but differ in the shorter hind legs and the long cerci (the cerci of both Locusts and Grasshoppers are very short), and generally, too, in that the posterior portions of the wings which are folded up, are not covered by the elytra, but project from these as a pair of pointed appendages. Here belong the $\mathrm{Cricket}$ (Gryllus domesticus), in bake-houses and similar warm places; and the Field-cricket (G. campestris), comomon in arid fields, and making passages in the ground; both with well-developed elytra, the female with projecting ovipositor: further the Mole-cricket or Earth-crab (Gryllotalpa vulgaris), whose front legs are developed as enormously powerful digging limbs; with very large prothorax, short elytra, and no ovipositor; they lead a subterranean existence, feeding upon plants and animals: all three in Great Britain.

3. Oockroaches (Blatta); flattened forms, with long bristle-like antennæ, and strong running legs with large femurs; the fore wings are thin elytra, partially overlapping; the abdomen has two anal cerci posteriorly; the head is covered by the anterior edge of the prothorax. Often both pairs of wings, especially in the females, are short or rudimentary. The eggs are laid in chitir us capsules, which are carried about for a long time by the female, projecting half out of the genital aperture; each' capsule contains a number of eggs lying in two rows. Two large species of this group, one of which is the well-known "Black-beetle" (B. [Periplaneta] orientalis), have been introduced from the tropies into Europe, where they live in houses; several species occur in the open in England.

4. The Mantidæ are allied to the Cockroaches, but differ in various respects. The body is on the whole more elongate, the prothorax being especially long. The first pair of legs is prehensile, with large coxæ, strong femurs with two rows of spines, and tibir also furnished with two rows of spines, which can be folded back upon the femurs; with these appendages the animal seizes its prey, which consists of other Insects. Wings well developed; in other respects like the Cockroaches; jointed cerci. The ova are attached to plants in groups, surrounded, as in Cockroaches, by a capsule formed as a glandular secretion. A large green species of this genus ( $M$. religiosa, the Praying Mantis), occurs in South Europe.

5. Earwigs (Forficula), somewhat flattened Insects, which are chiefly characterised by the condition of the wings. The elytra are quite short plates, which do not completely cover the thin hind wings, although the latter are much folded. The larger part of the abdomen is left uncovered by the wings; it is strongly chitinised, freely movable, and possesses, posteriorly, a pair of unjointed cerci, curved to form a pair of pincers. The Earwigs usually remain hidden by day; they live principally upon vegetable food. The females brood over the eggs. Several species occur in England.

6. The Stick-and Leaf-insects (Phasmidæ), form a small division of the Orthoptera, including a number of aberrant species; they are only 
indigenous to warm countries. Amongst them is the apterous genus, Bacillus, whose long body, together with the elongate legs, looks like a dry branched twig; some species in South Europe. In the East Indian, Phyllium siccifolium, the Leaf-insect, the broad abdomen and elytra are leaf-like.

7. The Termites, (genus Termes, and others) possess four large, thin wings, which are all alike, and cannot be folded up. The antennæ are short, and moniliform, the legs are like one another. The Termites are specially remarkable for living in large colonies, including, besides fertile males and females, a large number of apterous and blind individuals in which the sexual organs (in some examples male, in others, female) remain in an immature condition. Some of these wingless individuals are possessed of larger heads and more powerful mandibles, and are termed "soldiers," whilst the others are termed "workers"; the nest is made by the workers, either by gnawing passages and chambers in tree-trunks and lining them with a layer of excreta, or by constructing such dwellings out of excreta and earth. They often form extensive tunnels in the ground. The soldiers defend the nest against attack. Before pairing the males and females leave the nest, fly about for a

1

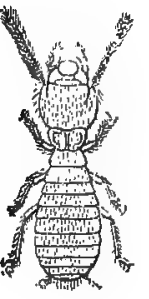

2

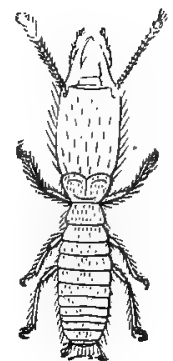

4

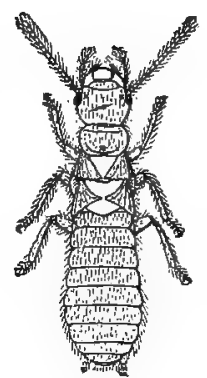

Fig. 212. Termes lucifugus. 1 Worker. 2 Soldier. 3 Male or female, with wings. 4 Female shortly after flight. 5 Female later, with enlarged abdomen.-After Lespès.

5

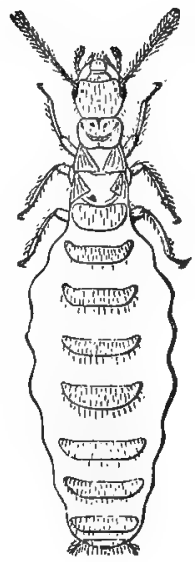

3

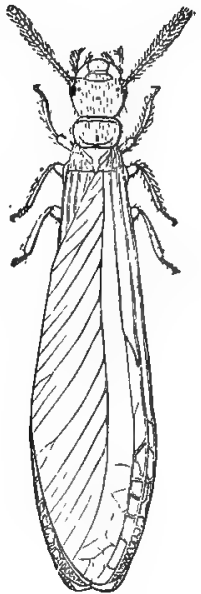

short time, lose their wings, and the majority die, only a few making their way back to the nest, where copulation occurs. Then, in many forms, the abdomen of the female enlarges to an extraordinary extent. Besides the winged males and females there are apparently others which, as regards the structure of the wings, remain at one of the older larval stages, in which merely short stumps are present; they do not leave the nest, and only become functional if none of the males or females which flew from the nest return. Some species differ in certain respects from this description; many of their habits are by no means thoroughly understood. The Termites, also called "White Ants," live chiefly in the tropics, but there are two species in South Europe (one of which is figured in Fig. 212); they often do a large amount of damage by making their nests in woodwork, and by eating clothes or furniture.

8. The Dragon-flies (Libellulidæ) possess four large wings almost equal in size, and closely veined. The head is very movable, with large compound eyes and three accessory eyes, short antennæ, strong mandibles, no first maxillæ or 
labial palps, but with a very broad labium. The legs feeble. Abdomen usually elongate with two unjointed cerci. Extremely good fliers, seizing their prey (e.g. Butterflies) upon the wing.* The larvæ inhabit fresh water, and are characterised by the modification of the labium into a long eversible prehensile organ (the $\mathrm{mask}$ ), and further by the closure of all the stigmata; respiration is effected by means of tracheal gills which in some cases are lamellate and situated at the end of the abdomen, whilst in others they are represented by a number of folds developed in the rectum; in the latter case the rectum receives and ejects water rhythmically. The larvæ of the last species move by spirting the water out. of the rectum. Here belongs the genus Libellula, in which the hind wings are broader at the base than at the tip, and the large eyes are pushed together into the middle of the head; the larva with rectal branchix: also the delicate slim Agrion-species (Demoiselle flies) in which the hind wings are narrower at their bases than at the ends, the eyes are wide apart and the larvæ possessed of three external gills.

9. May-flies (genus Ephemera and other's) are usually small insects with four thin wings, of which the hinder are smaller than the anterior. The mouth-parts of the imago are rudimentary; the abdomen with three anal filaments posteriorly. The larvæ live in water, and like the Dragon-flies possess a closed tracheal system and leaf-like or branched tracheal gills, situated in a row along each side of the abdomen; they exhibit three thread-like appendages, as does the imago. The larvæ are predaceous, and have well-developed mouth-parts; some of them dig passages in banks. The May-flies pass through a sub-imaginal stage ( $e f . p .247$ ); as imagines they take no food, and many species live for only a few hours during the night, metamorphosis occurring in the evening; others a few days; whilst the larval life, at least in some cases, lasts for two years. Several species common in England.

10. Book-lice (Troctes), are small apterous Orthoptera, which chiefly occur between old paper, in collections of Insects, and so forth; together with their winged relatives (Psocus), which live in forests, they form a special small family within the order.

11. To this order also belong the Mallophagidw, small, flat, lice-like animals, with fairly hard chitinous exoskeleton; the head is broader than the prothorax, and carries the usual biting mouth-parts, of which

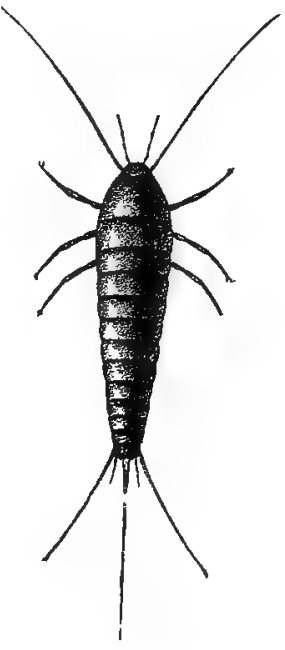

Fig. 213. Sugar-mite: (Lepisma). the mandibles are specially well developed. The antennæ have from three to five joints, there is one ocellus on each side of the head, but this may be absent. The tarsus consists of one or two joints with one or two claws; at the lower end of the tibia is a process against which the claws may be bent back, the legs are thus adapted to gripping hairs or the barbs of feathers. The numerous species of this division all live upon Mammalia and Birds, gnawing. the epidermis, hairs and feathers. The chief species occurring on Mammalia

* Copulation is very peculiar among the Dragon-flies. The second abdominal segment of the male is enlarged, and furmished with a copulatory apparatus. Before pairing, this is filled with spermatozoa from the vas deferens, which opens at the end of the body. The operation is effected by flexure of the abdomen. The male then seizes the female round the neck by means of the cerci, and the female arches its body so that the abdominal extremity reaches the male copulatory organ, and coitus is effected. 
belong to the genus Trichodectes, each of our common domestic animals possessing its own species ( $T$. canis on the $d o g$ ); on Birds, there is a number of species

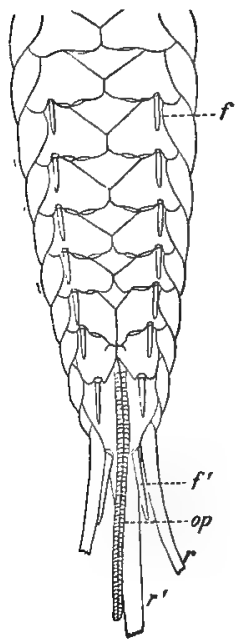

Fig. 214. Abdomen of Machilis seen from below. $f$ first, $f^{1}$ last abdominal appendage, op ovipositor, $r, r^{1}$ cerci (cutaway).--After Oudemans. of other genera (no fewer than six are known, for instance, from the Domestic Fowl).

\section{APPENDIX TO THE ORTHOPTERA.}

The Thysanura are allied to the Orthoptera; they are apterous forms, most of which are ametabolous, and possess rudimentary abdominal limbs; the mouth-parts are like those of the Oxthoptera. The Thysanura may be the most ancestral of all living Insects, the absence of wings is probably primitive, whilst in other forms it is aequired (through parasitism, etc.). Of the forms belonging here may be mentioned: Machitis, with compound lateral eyes, three ocelli, and eight pairs of rudimentary abdominal appendages; and the sugar-mite (Lepisma saccharina, Fig. 213), which, instead of the compound eye, possesses a group of ocelli on each side, and has only two pairs of ab. dominal limbs; the latter is covered with bright silvery scales (flattened hairs), and is very active; common in houses.

Allied to the Thysanura are the Collembola, genus Podura, etc., also apterous and characterised by a forked process arising from the tip of the abdomen on the ventral surface by means of which springing movements are accomplished; they are small, and are frequently found among fallen leares, or in similar places.

\section{Order 2. Rhynchota or Hemiptera.}

The Rhynchota, like the Insects of the previous order, are hemimetabolons. The mouth-parts are modified to form a suctorial apparatus, the proboscis, the structure of which has been already described ( $p .234$ ). In some, the proboscis projects in front, in others it is turned back under the body.

\section{Sub-Order 1. Homoptera.}

The fore and hind wings are usually alike, and both membranous *; the for wings larger than the hind. The head is large. The proboscis arises from its ventral side posteriorly, close to the thorax. All suck the juices of plants.

1. The Cicadas (Cicada), are large, rather bulky forms; the male makes a peculiar noise by means of the metathoracic stigmata, which are provided with vocal cords; the tone is intensified by complicated resonators. The female

\footnotetext{
* Not infrequently the fore wings are leathery over their whole extent.
} 
deposits the eggs in branches of trees by means of an ovipositor; the larvæ, whose fore legs are adapted for digging, make their way down into the earth, where they feed upon the juices of roots; they only leave the earth just before metamorphosis, climb into a tree and moult for the last time: the imagines suck young shoots. This division is confined to warm countries, but there is a single species in England. In North America the Seventeen-years Cicada (Cicada septendecim) occurs: its development lasts seventeen years (a variety of the same species has a period of development which lasts thirteen years).

2. The Frog-hopper (Aphrophora spumaria) is a small Homopteran, peculiar in that the soft, thin-skinned larva, which lives on the juices of various plants, surrounds itself with a foamy secretion (cuckoo spittle). This insect belongs to the family Cicadellidæ, of which there are several other species in Britain; most of them can spring long distances.

3. The Green-flies (Aphid:) form a large family of the Homoptera, the members of which are characterised by the bulky, thin-skinned body, feeble legs, sparse veining on the wings, and small size; very often the wings are absent, especially from the females; they are inert aninals, living together in colonies. Many of them possess glands for the secretion of delicate wax threads which surround the body as a woolly mass; in many, also, there is a pair of glands opening posteriorly on the dorsal side of the abdomen, by two apertures which are situated either on papillæ, or at the top of long projecting tubes; these glands secrete a fatty substance.* Heterogony usually occurs; several virginal generations are followed by a generation of males and females.

(a) Aphides, Green-flies in a restricted sense of the word, are green or black, soft-bodied Insects, with but little power of movement. They live in large colonies, and are extremely abundant on the leaves of all kinds of herbaceous and woody plants; they have fairly long antenne, and two long tubes on the abdomen. In the course of the summer, several successive generations of females occur, which possess no seminal pouch, and which reproduce parthenogenetically; the eggs develop in the oviducts, so that the Insects are viviparous ; some of these females have wings, but the majority are apterous. Finally, in the autumn, there is a generation of usually wingless females and winged males, which copulate, produce eggs, and die. These eggs give rise to the first female generation the following spring.

(b) The Vine-louse (Phylloxera vastatrix), famed on account of the terrible devastation it works in vineyards, especially those of France; indigenous to North America, where it does no great harm, it was accidentally introduced into Europe a few decades ago with American vines. The tubes are wanting on the abdomen, and it has shorter legs and antennæ than Aphis. In the spring, wingless females appear and feed on the roots, causing knotty swellings. Each lays about thirty or forty unfertilised eggs, from which a generation of individuals like the parent arises. In this way, from five to eight similar generations occur during the summer. At last, from the eggs of the apterous females, a generation of winged females develops. They leave the roots before metamorphosis (and, therefore, when the wings are incipient only), and betake themselves to the shoot part of the vine, where each deposits about four unfertilised eggs. These eggs are of two sizes : from the larger, females hatch out, from the smaller, males. Both sexes are small, apterous, with rudimentary mouth-parts, and no alimentary canal, so that no food can be taken. After impregnation, each female lays a single egg, which before being deposited,

* "Honeydew," a sweet sticky fluid, the excreta of the Aphides, occurs upon the plants on which they are living. 
occupies the greater portion of the small body. These eggs rest during the winter, and in spring develop into the first virginal generation. Besides the fertilised eggs, a number of apterous parthenogenetic females persist during the winter, in the larval stage, firmly attached to the roots of the vines.*

3

1
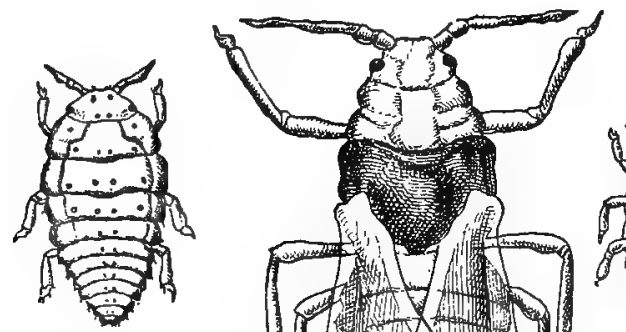

2
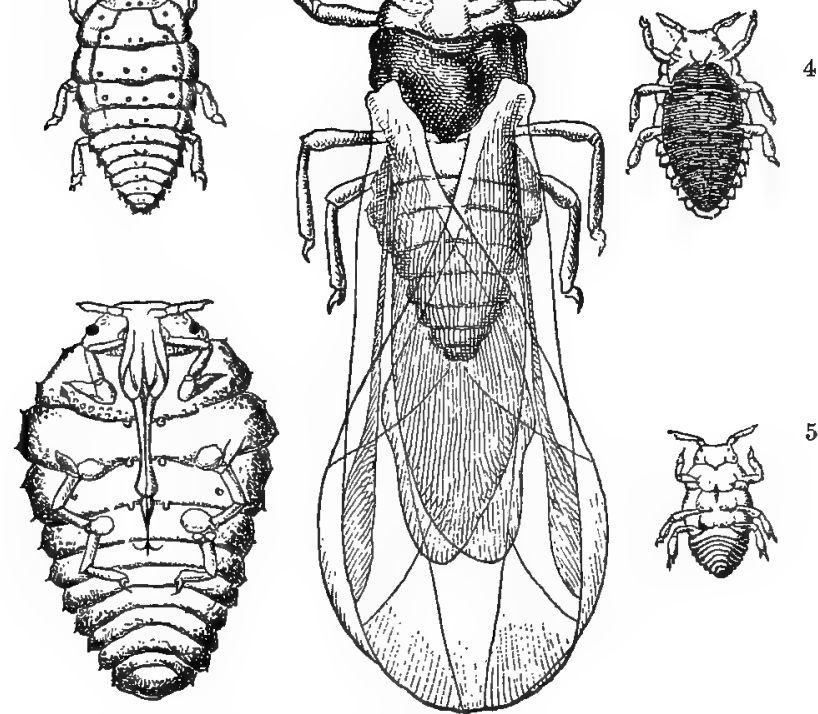

Fig. 215. Phylloxera rastatrix. 1 Young female of one of the apterous parthenogenetic generations. 2 Older ditto, from the ventral surface. 3 Adult female of the winged generation. 4 Female of true sexual generation (the orum shows through the skin). 5 Male. All the figures of equal magnifieation.--After Cornu.

(c.) Various Aphides produce characteristic galls on trees and bushes. Chermes abietis, for instance, by sucking at young pine shoots causes conelike galls by shortening and broadening the needles. Pemphigus spirothecx forms a spiral gall on the petioles of poplars; crumpled or saccular galls are caused on elm leaves by various Aphides, and so on.

4. The Scale-insects (Coccidx) are allied to the Aphides, but differfrom them in various respects. Sexual dimorphism is usually very marked. The female is cumbrous, apterous, and short-legged, and is usually somewhat motile only in youth, later becoming fixed to one place, where the eggs are laid. Soon after oviposition the coccus dies, but the body which has gradually shrunk to a thin arched shield remains as a protection to the eggs. Very often thefemale is covered dorsally by a continuons layer of wax secreted by the skin glands; sometimes the eggs are surrounded by fine wax threads. The $\mathrm{male}$ possesses well-developed fore wings (with few veins), whilst the hind wings are rudimentary and like halteres or altogether absent; the mouth-parts are rudimentary. The larve resemble young females. It is a very remarkable fact that the males (not the females) pass through a resting pupa-stage,

* In addition, it may be noticed that in exceptional cases, wingless parthenogenetic. females may occur attached to the vine leaves, where they produce galls. 
and thus, unlike all other members of the group, are holometabolous. In several of the species it has been proved that the females can reproduce parthenogenetically. Several forms differ in certain respects from this description of the Coccidæ; in some the females are locomotor throughout life and do not remain attached over the eggs; there are others, again, in which both sexes possess four wings, and which thus offer a transition to the Aphides. As examples may be mentioned: Aspidiotus nerii, the shield-like female of which is frequently met with on the oleander; similar forms are very abundant on uncultivated trees. Coccus cacti, the Cochineal-insect, lives on certain Mexican species of cactus; the males are dipterous and have long cerci. The females are wingless and bulky; they do not cover the eggs with their bodies, but surround them with wax threads as do many of the Aphides; the cochineal of commerce consists of their dried bodies. To this family belongs also the Shellac-insect (Coccus lacca), which is found in the East Indies on certain species of figs, causing the flow of a resinous substance, shellac, from the tree: and the Kermese-insect (Lecanium ilicis), which lives on a species of oaktree in South Europe; from the spherical females a dye is obtained.

\section{Sub-Order 2. Heteroptera (Bugs).}

The fore and hind wing's are dissimilar, the latter thin, membranous and adapted to flight; the former modified as elytra, which are not thickened and leathery for their whole length, but only for their basal halves or rather more, and the thin tips, when at rest, overlap; the distinction into the two regions may, however, be quite effaced. The elytra cover the greater portion of the mesothorax, the metathorax, and the abdomen, but a triangular median portion of the mesothorax (scutellum) remains uncovered. The proboscis arises anteriorly from the head, which is generally small; the prothorax is large and freely movable, the whole body is usually flattened. In the land forms a pair of stink glands opens on the ventral surface of the metathorax, and the secretion has an extremely offensive odour. The Heteroptera feed on the fluids of plants or animals (Insecta, Vertebrata).

1. Land-bug (Geocores) is the common term for a large number of bugs (forming several families), characterised by the possession of welldeveloped autennæ and a long proboscis. Most of them are terrestrial; some feed on plants, others are predaceous, sucking other Insects; some live as parasites on Vertebrata. Many are gorgeously coloured. Abundant in temperate zones, and especially so in the tropics. The Bed-bug (Cimero [Acanthia] lectularius) is a flattened, brownish, apterous (rudiments of the fore wings only are present) form, which lives as a temporary parasite upon Man. It came originally from the East Indies. Here also belongs the Hydrometra, a slim, elongate form, which runs about actively upon its middle and hind legs on the surface of freshwater; the legs of the first pair are considerably shorter than the others, but are fairly strong, and are used for catching Insects upon which it feeds; the abdomen is rather small, hardly longer than the thorax. Closely allied to the Hydrometridæ are the Halobatidæ, which run about on the open sea : they are distinguished by the extraordinarily small size of the abdomen. 
2. W ater-bugs (Hydrocores) have short antennæ and a short proboscis; they live in water, which they are, however, able to leave, in order to fly in the air. All are predaceous. Of forms found in England, the W ater-scorpion (Nepa), belongs here, a flat, darkly-coloured insect, which is very common, crawling about at the bottom of fresh water; the front legs are prehensile, the tibiæ can be folded into grooves on the femurs, posteriorly there are two filiform grooved processes, which together form a tube (the respiratory tube), at the base of which lies a pair of stigmata: also the W a ter-boatma n (Notonecta) with long, outwardly directed hind legs, covered with setæ on the tibiæ and tarsi; these are swimming organs.

3. Lice (Pediculidæ), a small group of ametabolous parasitic Insects, are probably to be regarded as peculiarly modified Heteroptera. The head is narrow, with short antennæ, and an ocellus on each side; the suctorial apparatus, which can be completely withdrawn into the head through an opening at the tip, consists essentially of a short, thick tube, provided at the end with a few hooks, through which a second thinner tube, the actual sucking tube, can be protruded; the more exact structure of the latter is not definitely known. The legs, which are short and strong, end in chelæ; the tarsus, consisting of one joint, bears a very powerful claw, which bites against a process arising from the lower end of the tibia. Wings are altogether absent, the abdomen is large, broad, and tough. The large eggs (nits) are stuck on to the hairs of the host.

1

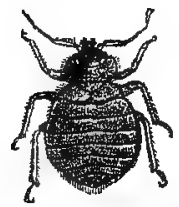

2

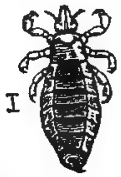

3

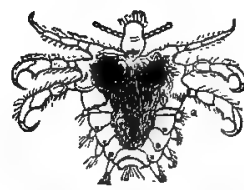

Fig. 216. 1 Cimex lectularius. 2 Pediculus capitis. 3 Phthirius pubis. All enlarged.-After Taschenberg.

Lice only occur upon Mammalia, living as stationary parasites upon the skin, sucking the blood; they grasp the hair by means of their legs. The follow. ing species occur on Man: Pediculus capitis and $P$. vestimenti, which are very similar, the former living exclusively in the hair of the head; the latter on the naked portions (or, more correctly, the sparsely hairy), of the body; also Phthirius pubis on the hairy portions of the body, with the exception of the head (in the hair of the pubic region, the beard, etc.), distinguished by the great breadth of the thorax and abdomen. Other species, on domestic Mammalia.

\section{Order 3. Neuroptera.}

The Neuroptera are holometabolous. Insects, with four similar, thin wings, and biting mouth-parts. The antennæ are usually multiarticulate; in some, the mouth-parts are well developed; in others, rudimentary. The prothorax is freely movable; the wings in some are closely veined, like those of the Libellulidæ; in others, there are fewer veins. The larvæ are provided with legs, but in other respects, are very diverse. The pupa is peculiar in seeking a convenient spot for the completion of 
metamorphosis; if it is enclosed in a cocoon, it bites its way out for this purpose. The following may be taken as examples:

1. Ant-lions (Myrmeleon). Fore and hind wings large and similar, almost of equal size, with a delicate close veining; the antennæ, fairly short and thick, somewhat club-shaped; the month-parts well developed. In their habits very like the Libellulidæ. The larva to which the name of "Ant-lions" was originally

Fig. 217.

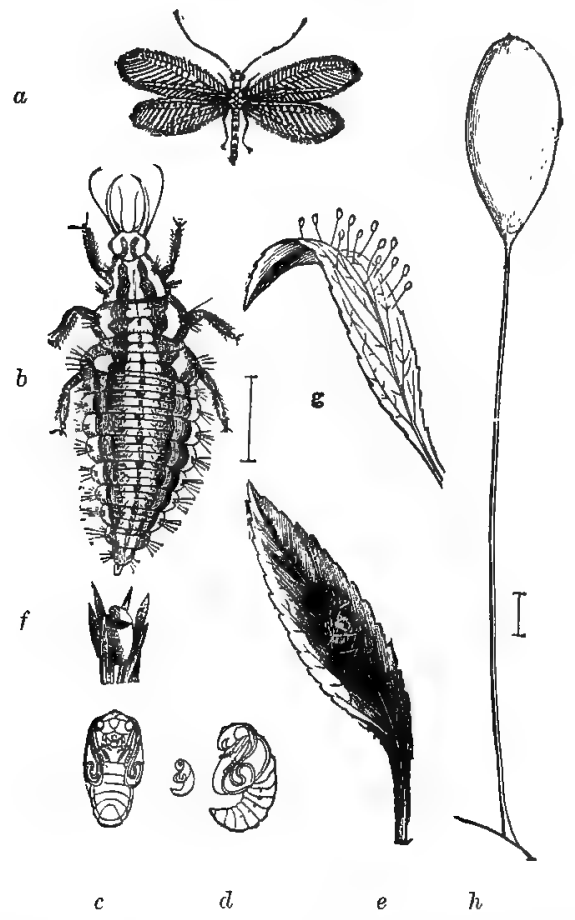

Fig. 218.

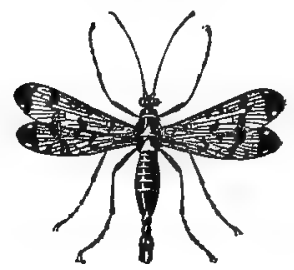

Fig. 219.

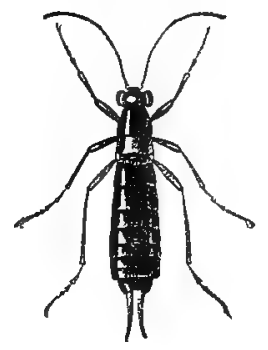

Fig. 217. Chrysopa. $a$ Imago, $b$ larva, $c-d$ pupa, $e-f$ pupa cases ( $f$ opened), $g$ eggs, $h$ egg enlarged.-After Taschenberg.

Fig. 218. Panorpa communis, ơ.

Fig. 219. Boreus hiemalis, $\frac{\mathrm{a}}{2}$.

given, have large slender mandibles, which are hollowed out ventrally; into these grooves, the elongate first maxillæ fit, so that each mandible, with its maxilla, forms a hook perforated by a canal; the canal leads into the mouth, which is closed but for this. The larva sits in a funnel-like pit in the sand, and catches passing Insects which fall in by accident, or in consequence of a shower of sand which it throws over them with its head. The prey is devoured with the help of the hooks already mentioned. Closely allied to the Ant-lions, is the Golden-eye (Chrysopa), a small delicate greenish insect, with large wings. It resembles the Ant-lions in the main points of its structure, but differs, among other things. in its long, bristle-like antennæ. The greenish larva, "Aphis-lion," is also similar to larval Ant-lions, but it moves freely about on trees and eats Aphides. The eggs are attached by long stalks to leaves. Some species are very common in Great Britain. 
2. The Scorpion-flies (Panorpa), are characterised by the snout-like elongation of the head; the males by the presence of pincers at the end of the abdomen, which like the sting of the Scorpion, may be curved upwards. Wings small and uniform, body and legs slender. Actively predaceous; length about $10 \mathrm{~m} / \mathrm{m}$. The larva (with prolegs) lives in the earth, upon decaying matters. $P$. communis abundant everywhere in the summer. Another form is the springing, apterous (possessing rudiments of wings), Boreus hiemalis, about $4 \mathrm{~m} / \mathrm{m}$. long, which occurs in the imaginal state, from October to March, sometimes even on glaciers. Larva, like that of Panorpa. In Great Britain.

3. Spring-flies or Caddis-flies (genus, Phryganea and others). Wings hairy or scaly; the hind wings, which are broader than the fore, are folded beneath the latter; the veining is less pronounced than in the Ant-lions. The antennæ are long, the mouth-parts rudimentary and functionless. The larvæ are aquatic; the abdomen is long and cylindrical, bearing thread-like tracheal gills laterally, and hidden in a tube formed of fragments of plants, snail shells, or stones, often very regular in construction; the particles of which the tube is composed, are held together by a web. When the animal moves about, the head, legs, and thorax protrude from the tube; it is attached to the tube by means of two hook-like caudal cerci, and often by stout outgrowths of the first abdominal segment. In some forms, the tube is attached to some foreign body, a large stone or the like, and, in all before pupation, it is fastened to some object in the water, and is then closed by a network of threads; the pupa, like the larva, possesses tracheal gills.

The small group, Strepsiptera, has been regarded by some authorities as belonging to the Neuroptera; its systematic position is, however, doubtful. The larvæ (genera, Xenos, Stylops, etc.), live in the larvæ, and, later, in the imagines of Bees and Wasps, the host undergoing metamorphosis in spite of the presence of the parasite. Before pupating, the strepsipteran larva pushes half-way out of the body of the host between two abdominal rings; and here the pupa may be found with one end projecting. Sexual dimorphism is well-marked; the male possesses well-developed eyes and legs (without claws), and large hind wings,

1

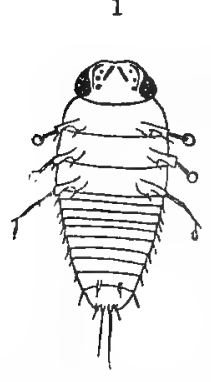

2

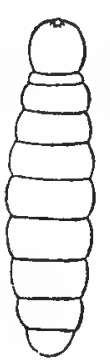

3

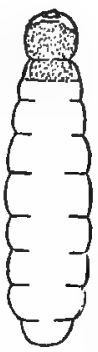

4

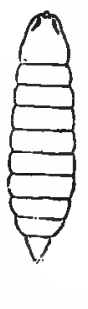

5

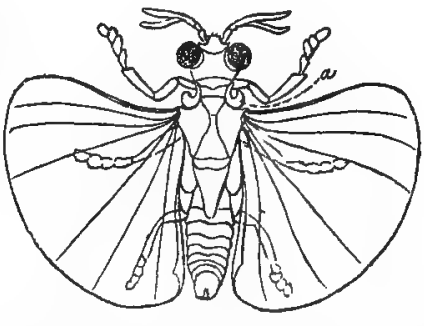

Fig. 220. 1-4 Xenos Rossii. 5 X. Peckii. 1 Newly-hatched'larva. 2 Fully grown female larva. 3 Female (imago) 4 Fully grown male larva. 5 Male (a fore-wing).1-4 after v. Siebold, 5 after Kirby.

which can be folded up lengthways, whilst the fore wings are quite rudimentary. The female is maggot-like, without limbs, wings, or eyes; it does not leave the host, but pushes out a portion of its body, and is there sought out by the male, and fertilised. The embryos are developed within the body of the parent, and hatch out as hexapod larvæ, which move actively about in the host, and later, bore into its larvæ, where they become maggot-like. In the larva, and in the adult female, an anus is wanting. 


\section{Order 4. Coleoptera (Beetles.)}

The Coleoptera are holometabolous and have biting mouth-parts; the fore wings are modified to form elrtra. The exoskeleton is usually very firm, often brightly coloured. The head, which is partially sunk into a depression of the prothorax, bears a pair of compound eyes of diverse form; sometimes they are reniform, and the inpushing on the front edge is in some cases so deep that each is divided into an upper and a lower portion, and thus there are two compound eyes on each side. Ocelli are almost always absent. The antennæ usually consist of eleven joints, but the number may be increased to about thirty, or reduced to four; in different species their form varies considerably. The mandibles differ according to the food; they are slender in predaceous forms, thicker in herbivorous; the mentum is usually a well-developed, firmly-chitinised plate, whilst the rest of the lower lip, with the exception of the palp, is usually only feebly developed. The prothorax is large, strongly-chitinised, and freely articulated with the mesothorax; between the prothorax and mesothorax there is a deep constriction. The mesothorax and metathorax, of which the latter is best developed, are immovably connected; they are covered above by the elytra so as to leave a scutellum; the most anterior portion of the mesothorax is covered by the hinder edge of the prothorax. The tarsus is usually five-jointed, but there are not a few exceptions to this. The fore wings are elytra, and during rest they meet along the mid-dorsal line or may even be folded the one over the other, whilst their lateral edges wrap round the lateral edges of the body (Fig. 197); they thus form a rery complete covering not only for the hind wings but also for the dorsal surface of the mesothorax and metathorax, and usually for most of the abdomen; they are generally very hard. More rarely they are short, so that the larger portion of the abdomen remains uncovered; in some they overlap along the mid-line. The posterior are true wings, thin and membranous, with few veins; when at rest they are usually folded not only lengthways but also transversely. In not a few they are rudimentary or they may be absent, but in spite of this, the elytra are generally just as well dereloped, since they afford a covering for the abdomen; both pairs of wings are wanting in only a very few cases. The abdominal somites are divided into tergal and sternal half-rings, which are frequently somewhat displaced; there are always fewer sterna (four to seven) than terga (usually eight); the latter are less strongly chitinised as far as they are covered by the elytra, the dorsal surface of the abdomen is thus softer than the ventral. The larvæ vary considerably in form; they usually possess legs but may be maggot-like. Only a few of the most important families of this extraordinarily large order are mentioned here. 
1. The Carabidr (genus Carabus, and many others), active, slim, usually dark-coloured beetles, with long powerful legs; antennæ filiform, mandibles slender, projecting; first maxilla with a two-jointed lacinia. The tarsi of the first pair of limbs in the males are very often broad and hairy below, enabling them to hold the females firmly; the other tarsi are long and thin. In not a few the hind wings are rudimentary. The larvæ, which, like the adults are almost always predaceous, are usually darkly-coloured, with a group of ocelli on each side, and with well-developed legs, each with two claws; in other coleopterous larvæ there is usually only a single claw on each foot. The Ti g e rbeetles (Cicindela) are small Carabids characterised by their bright colours. (green, etc.). The larva is paler than that of most of the Carabidx, and exhibits a pair of hooks on the back; it lives in a burrow in the ground, where it lies in wait for its prey. The Dytis cidæ (genus, Dytiscus, and others) are to be regarded as a type of Carabidæ specially developed for an aquatic life; in most respects they resemble the Carabids, but differ from them in the broad, oval body, and in the modification of the hind legs as natatory organs, the tarsi being broad and hairy at the edges. In the males, the first three joints of the front tarsi are still broader than in the Carabidæ, and are furnished with ventral suckers (modified setæ). They come to the surface to breathe; by night they usually leave the water and fly about. The larvæ are also aquatic, and are slender: the legs are fringed with setæ; their most striking peculiarity consists in the perforation of the thin mandibles by a fine canal, which opens at the tip and leads at the other end into the mouth (the canal is really a groove with apposed edges, $c f$., the poison tooth of snakes), whilst but for this the mouth is completely closed. The prey is sucked out by these mandibles. Allied to the Dytiscida is another group of Water-insects, the W hirligig s (Gyrinus), small forms which usually swim about actively on the surface of the water in the sunshine. They are distinguished by several features : the middle and hind legs are modified to form shor't, broad, flattened, fin-like natatory organs, whilst the longer front legs: are normal in structure, and are used as organs of attachment when the insect dives. Each eye is divided into an upper and a lower portion, of which the former looks upwards, the latter downwards. The larvæ correspond with those of the Dytiscidæ in the structure of the mandibles, etc., but differ from them in the possession of closed stigmata and a row of filiform branchix along the sides of the abdomen.

2. Staphylinidæ (genus Staphylinus, and others), distinguished by the small size of the elytra; the larger portion of the very movable abdomen is uncovered, but it is well chitinised dorsally; the hind wings are folded across twice, in order to find room under the elytra. The body is elongate, the antenne filiform, or somewhat clavate. The adult generally lives upon decaying plant and animal substances. The $l$ ar væ are like those of the Carabidæ, but possess only a single claw on each foot (or more correctly, the tarsus itself is pointed); they are provided with two jointed cerci, and the anus is situated on a tubular projection. They feed as do the adults, or are predaceous. This family is extraordinarily rich in species.

3. The Carrion-beetles (Silphidæ) have the antennæ clavate, or at least, somewhat thickened at the tips. In some forms, the elytra cover the whole of the abdomen; in others, its tip is left uncovered. They are, as a rule, carrionfeeders. The genus Silpha has slightly clavate antennæ, elytra covering the whole of the abdomen, and the body of a flat, oval form. The larve are broad and flattened, firmly chitinised, and they forage for themselves; both larvæ and adults usually feed upon dead animals, for which they seek. The Burying-beetles (Necrophorus) have markedly clavate antennæ, elongate bodies and short elytra usually coloured with black and red bands, leaving the 
hinder end of the body uncovered. They make a noise by rubbing the dorsal surface of the fifth abdominal somite, which is provided with two transversely ribbed areæ, against the hinder edges of the elytra. Several generally unite to bury small Mammalia, etc., removing the earth below the body, in which they lay their eggs. The larvæ are pale and bulky, but possess legs and eyes, and feed upon the carrion buried by the foresight of their parents; they do not forage for themselves like the larvæ of the Silphidæ.

4. Dermestidae (genus Dermestes and others), small, with clavate antennæ; the surface of the body covered over a greater or less extent with short close-set setæ. The larvæ are provided with numerous upright setæ; the pupa remains within the displaced larval skin, which thus serves as pupa-case. The Dermestidæ and their larvæ feed upon dead animal substances, and are often injurious to woollens and furs, and to museum specimens.

5. Lamellicornia or Scarabæidæ. A family of Beetles very rich in species, and comprising many beautiful and characteristic forms; each of the last three (or more) joints of the antennæ is expanded like a leaf on one side, and these laminæ, when laid upon one another, form a clavate swelling. The eye has a deep notch in front, into which the lateral edge of the head extends. The front legs are emphatically adapted for digging, with tlattened spiny tibire and cylindrical coxæ; and to the same end the prothorax is very powerfully developed. The whole body is usually fairly bulky. The males are often very unlike the females, with outgrowths on the head, prothorax, etc. The larvæ are whitish (with the exception of the much chitinised head), fat, thinskinned, sparsely setose, and usually blind; the legs are rather feeble, the abdomen curved, its end often swollen and saccular. Both larvæ and adults feed upon plants or dung. Amongst others, the following belong to this family: The C o ck c hafer. (Melolontha vulgaris), the males distinguished from the females by larger clubs to the antennæ; the larva lives upon roots, the imago on leaves; the whole duration of life extends over four years in Britain. Rose-chafers (Cetonia) are green and shiny; the elytra are notched at the lateral edges, so that after spreading the hind wings, they can be folded back along the dorsal surface, and the insects can fly with closed elytra ; the larra lives in rotten wood and in Ants' nests. Oryctes nasicornis is a large brown Lamellicorn, the males of which have large projections on the head; the larvæ occur in tan, etc. Dung-beetles (Coprophaga) live as larvæ, chiefly on the dung of Ungulata; larvæ and adults of the genus Aphodius, for instance, are found in abundance in cow dung; the female of genus Copris digs a bole in the ground, lays an egg in it, and adds a small piece of manure to serve as food for the larva when it hatches out. Geotrupes, bulky, blue insects, the eyes completely divided into upper and lower portions, strong digging legs; mode of life similar to that of the forms last mentioned. The Stag-beetle (Lucanus cervus), is the most striking of English Beetles; the male has a large square head, and huge antler-like mandibles, which are very rariable in size; the antennæ are geniculate with long basal joints, the club pectinate, the processes being not laminate, but denticulate and far apart; the larvæ live in rotten oak trees. More abundant is the allied Dorcus parallelopipedus, a small form in which the mandibles of the male are very little enlarged; the larve in rotten beechwood.

6. The Skip-jacks (Elateridx, genus Elater, etc.) are usually small with flattened bodies of an ellipsoid form. The prothorax is long, and provided, posteriorly, with a spine, which fits into a pit in the mesothorax, so that very considerable movement can take place. When the prothorax is raised the spine is supported against the edge of the depression, and on its being suddenly allowed to shoot back into the pit the animal strikes the ground with considerable force, and thus is jerked up. The leap upwards may occur 
when the animal is in the natural position, as well as when it is on its back. The head is sunk deeply into the prothorax, the antennæ are serrate or pectinate. The larvæ (Wire-worms) are long, sometimes almost wiry and firmly chitinised, with legs, but without eyes; the last segment large and varying in form. These Insects are for the most part phytophagous. The Buprestidæ (genus Buprestis and others) are allied to the Elateridæ, which they closely resemble in form, in the relations of the head, and in the serrate antennæ; they differ, however, amongst other things, in the absence of the springing apparatus. The larvæ are whitish, blind, apodous; the prothorax into which most of the head is sunk, is usually very large and broad, the abdomen nain'ow; they usually live upon wood, as do longicorn larvæ, which they closely resemble. The Buprestidx are specially abundant in the Tropics, where large and beautiful forms are met with; in temperate countries there are relatively few, and they are mostly small.

7. The Malacodermata are characterised by the unusual softness of the exoskeleton, the elytra, for instance, crumpling up when dried. The head is generally more or less hidden beneath the front edge of the broad, shield-shaped prothorax. The elytra fit closely together along the back as usual. The Glow-worms (Lampyris), represented in Britain by two species, belong here; in these the head is covered by the prothorax, the females have neither elytra nor wings, and look like larvæ; the adults of both sexes and the larvæ (which feed upon Molluscs) have phosphorescent organs on the ventral side of the abdomen. In the allied genus Telephorus (which is not luminous) the head projects freely; in the summer several species are found on flowers.

8. The Vesicantia are heteromerous, i.e.. the tarsi of the front and middle legs are five-jointed, those of the hind legs, four-jointed.* The head is constricted posteriorly to form a neck, the prothorax is narrower than the elytra, which are not so hard as in most Beetles. The claws are cleft; blistering materials are contained in the body. The ova are laid in holes in the earth; the newly-hatched larvæ possess eyes and well-developed legs, and crawl about on plants, attaching themselves to certain Bees and entering their nests with them; when the Bee lays an egg the parasite leaves its host and remains in the cell, where it first devours the egg, and then undergoes a metamorphosis, becoming a clumsy, blind, short-legged creature, which devours the supplies intended for the bee-larva. To this group belongs also the O il-beet le (Meloë), with no hind wings and with short elytra, which do not fit together, but overlap: also the Spanis $\mathrm{h}$ fly (Lytta vesicatoria), a beautiful emerald green beetle, with well-developed elytra and wings: rare in England, whilst Meloë is common. The Mealw 0 rm (Tenebrio molitor), a brown, elongate insect, like the Carabidæ, belongs to another family of Heteromera. The larva resembles that of the Elateridæ, and is well-known in meal and corn.

9. Longicorns (Cerambycidx, genus Cerambyx, etc.) The tarsi are broad, and apparently consist of only four joints, the last being short and difficult to see (Beetles with this type of foot are termed tetramerous). They are for the most part large with long heads, long antennæ in the males often strikingly developed, and emarginate eyes. The larvæ which live in wood, and especially in dead trees, gnawing out long tunnels, are whitish, long and somewhat flattened; broader in front, without (or with feebly. developed) eyes, with very small legs (or quite apodous). The Leaf.beetles (Chrysomelidæ) are apparently very different from the Cerambycidæ; they are, however, closely allied, and possess a tarsus of the same form. The body is

* The families already mentioned have usually five-jointed tarsi on all the legs, and are termed pentamerous. 
generally bulky and much arched, the head more or less covered by the prothorax, the antennæ shorter than the body, the colouring bright; but there are also more elongate forms resembling the Cerambycidæ (Doncucia). The larvo are mostly coloured, with eyes and well-developed limbs; most of them live upon leaves.

10. Weevils (Curculionidx; genus Curculio, etc.): tarsi like those of the Cerambycidæ. They are usually small with head prolonged in front into a shorter or longer probosciform process, at the tip of which are the small but well-developed mouth-parts. The antennæ are clavate and usually geniculate, with a long peduncle. The elytra bend over the edge of the abdomen; the wings are not infrequently absent; the exoskeleton usually very hard. The 1 a $r v æ$ are apodous, curved, and, with the exception of the brown head, whitish, and usually blind. Both larvæ and adults are phytophagous (eating leaves, bark, wood, roots), the former are almost always hidden. Many genera and species in Britain. Closely allied are the Tomicidx, small cylindrical forms with short head and no proboscis (this is the most important difference between the Tomicidæ and the Curculionidæ), short geniculate antennæ with thick clubs; eyes reniform; the larvæ like those of the weevils.* Before laying the eggs the female usually gnaws a longer or shorter passage, at the junction of the wood and bark, in a tree which is either sickly or has recently died, but is still fairly rich in sap; rarely is a bealthy tree bored. A number of eggs is laid along the sides of this tunnel, each in a small depression; and when the larva hatches it proceeds to make for itself a tunnel, which is gradually lengthened and widened as the creature increases in size; the larval passages branch off almost directly at right angles to the original one, and, like it, run along at the junction of the wood and bark. There are all kinds of deviations from this typical arrangement in the different forms. To the Tomicidx belong some of the worst enemies of forestry (especially pine trees), Tomicus typographus (the Printer), and many others, which may do an enormous amount of harm in the course of a short time if some accident, e.g., wreckage by wind, affords a quantity of suitable brood material, enabling the pests to increase very rapidly. Their destructiveness is due less to their normal breeding than to the fact that if they are once present in large numbers they will also lay their eggs in sound trees; moreover the adults of some species are injurious, in that they eat young buds (Hylesinus piniperda), gnaw the roots of young plants, and so on.

11. Ladybirds (Coccinellidix) have apparently only three joints in each tarsus, but actually four, of which the penultimate is very short (trimerous). They are small, often almost hemispherical, or somewhat ovate; the head is small, with short clavate antennæ, and sunk into the prothorax; the legs are short. Larvæ and imagines both resemble those of the Chrysomelidæ, but both :are predaceous, feeding upon Aphides,.

\section{Order 5. Hymenoptera.}

The Hymenoptera are holometabolous with biting mouth-parts and four membranous wings. The head is short and broad, and deeply constricted off from from the prothorax,

* The Tomicidæ are not to be confounded with the Death-watch (Anobium). The latter possesses a body similar in form and also gnaws wood, but it belongs to quite a different family and is easily distinguished by its round eyes, by the antennæ only slightly thickened at the tip, and by the possession of pentamerous tarsi; the larvæ have legs (small and like those of the Scarabæidæ) and eat irregular labyrinthine tunnels in dead, dry wood, e.g., in furniture, which is often destroyed by them. 
never sunk into it; sometimes, indeed, it is situated on a stalk-like process of the prothorax. The usual mouth-parts are present, and of these, the mandibles are powerful biting organs. In some Hymenoptera-but these are in the minority-the "tongue," which is formed by the concrescence of the ligulæ, is elongate and gutter-like ventrally, and surrounded by a tube formed of the long, flattened, labial palps and blades of the first maxillæ (only a single blade of each being present); by means of the tongue and its sheath, sweet liquids are sucked up into the mouth. The prothorax is but feebly developed, the dorsal is separated from the ventral portion, and is firmly fused on to the mesothorax, whilst the latter (with the first pair of legs) is movable. Mesothorax and metathorax are usually immovably united, but in the Sawflies and Wood-wasps they are freely articulated. The legs are characterised by the size of the coxæ; the trochanter is often divided into two. joints (in the Tenthredinidæ, Uroceridæ, Cynipidæ and Ichneumonidæ); the first joint of the pentamerous tarsus is much longer than the following (metatarsus). The front pair of wings is almost always much larger than the hind pair; both are veined, but not very closely; those of the same side are connected by a row of small hooks on the anterior edge of the hind wing, which fasten into the curved hinder edge of the front one; thus, during flight, the

$A$

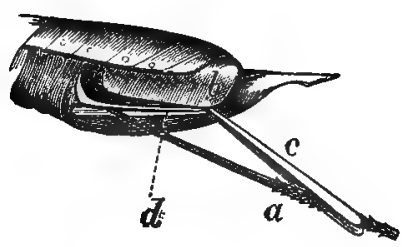

$B$

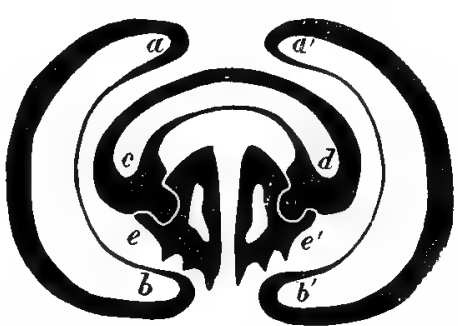

Fig. 221. A Abdomen with ovipositor of one of the Uroceridæ. The spine $(a)$ is pushed out from the groove $(d)$ in which it lies when at rest; this groove is continued from $b$ into the two long lobes $c$, which surround the terminal portion of the spine. $B$ Transverse section of the spine and the lobes, enlarged. $a b$ and $a^{\prime} b^{\prime}$ lobes $(=c$ in $A), c d, e$ and $e^{\prime}$ the three acicular pieces of the sting.-After Graber.

two act as one continuous lamina. At the base of the front wing, there is a projecting scale which covers the base of the hind wing. In all the Hymenoptera, the first abdominal segment is immovably united with the metathorax, and in the majority (i.e., in all with the exception of the Tenthredinidæ and Uroceridæ), there is a deep constriction between this and the following abdominal segments; the abdomen is thus said to be stalked, but it must not be forgotten that the constriction occurs not between the two regions, but in the abdomen itself; the segments following the constriction are usually narrower than the first. At the hinder 
end of the female, there is a hollow stabbing or boring apparatus, through which the eggs pass when being laid, and with which, in many cases, a prick or cut is made in an animal or plant, for the reception of the egg; this is the ovipositor of forms described under 1 and 2 ; in others (3 to 6 ), the spine is not simply an ovipositor, but also acts as a sting; a poison gland opens into it, and the secretion runs down the canal of the spine; with this, other animals may be pierced, either in self-defence or with other objects (see the Sand-wasps). The great majority of the larvæ are whitish, blind grubs; only in the Tenthredinidæ and Uroceridæ do they depart from this type, having legs, etc. (see below). The larvæ generally form cocoons for pupation.

1. S aw-flies (Tenthredinidx). Abdomen sessile, i.e., without constriction, broad and short; in the females, a short, serrated ovipositor, with which small cuts are made in leaves for the reception of the eggs. Mesothorax and metathorax movably articulated, trochanter two-jointed, fairly close veining in the wings. Some of the Saw-flies reproduce parthenogenetically, either exclusively (P), or in addition to reproduction by fertilised eggs. The larv æa are coloured, cylindrical, and eruciform (caterpillar-like); they usually possess six to eight pairs of prolegs without hooks, besides the thoracic legs ( $c f$., the Lepidoptera), and an ocellus on each side of the head; they live on trees and other plants, destroying the leaves. Closely allied are the Wood-wasps, genus Sirex, etc. (Uroceridæx), in which the abdomen is long, cylindrical, and provided with a longer ovipositor, whilst in other respects they resemble the Tenthredinidæ; the larvæ live in wood, in which they gnaw long winding passages; they are blind, whitish animals, with three pairs of short thoracic legs, but no prolegs.

2. Gall-flies (Cynipidæ), small Insects in which the abdomen is short, compressed, lenticular, with an ovipositor arising from the ventral surface; wings with very little veining; two-jointed trochanter. The larvæ live in galls; the female bores into living portions of plants (leaves, stems, buds) by means of her spine, and deposits an egg in the hole thus made; later, the plant tissue swells up in different ways characteristic for each species, in consequence of the presence of the larva, for it lives in, and upon, the gall thus formed. Some galls are concamerated, i.e., several eggs are introduced close together into one plant, and one continuous gall is formed round all the larvæ. In many species of Cynipidæ, which are found in great numbers on oak-trees, a regular alternation of parthenogenetic and true sexual generations is observed (one of each annually); the two generations are dissimilar, and cause galls very different in appearance. Other Oak Gall-flies are apparently all females. Allied to the Cynipidx is a very large group, the $\mathrm{I} \operatorname{chneu} \mathrm{m}$ on-flies (Ichneumonidx), usually very small in size, but often possessed of long ovipositors; their larvæ live as parasites in (rarely upon) insect larvæ, pupæ, and ova, caterpillars being especially attacked; some are parasitic in other Ichneumon larvæ. When the egg of an Ichneumon is laid in that of another insect, the parasitic larva lives and develops at the expense of the egg, and the latter degenerates. Those Ichneumons, which are parasitic in larvæ, usually complete their growth before the pupation of the host, they then break through its shin and pupate close beside it whilst it dies; or the host pupates first, and the parasite pupates within it; the former then dies, and the latter only leaves the pupal skin of the host when it has attained the imaginal state. The Ichneumon larva, which possesses very imperfeet mouthparts, apparently feeds upon the blood of its host (except when parasitic in an egg); there may be one, or several, or many in the same individual. In some 
Ichneumons, parthenogenetic reproduction has been observed; for the unst purt, males arise from the fertilised eggs.

3. Sand-wasps (Crabronidie, Pompilidie). These, like the folluwing groups, have a simple trochanter, a stalked abdomen, and a sting. They are ' active forms, and are chiefly characterised by their mode of life; they catch Insects, and their larvæ, or Spiders, paralyse them by a sting in the ventrul nerve cord, and store them in burrows, which they make in the earth or in woul; they lay one egg in each passage and then close it up; the larva feeds upon the stores thus collected. Other forms divide the burrows up into cells by means of clay partitions, and deposit one egg in each cell ; other's again form branching tunnels, and place one egg, with a supply of food, in each branch. More rarely they bring fresh supplies of food to the larva daily. The allied Golil-w as $p$ (Chrysis), is a beautiful, metallic-looking insect, with a very hard chitinous exoskeleton. This is especially firm on the abdomen, which is much arched above, but concave below, and appears to be made up of a few large segments, the last being telescoped. The antennæ are geniculate. The body may be rolled up, so as to present only the hard exoskeleton to the stings of the Sand-wasps, in whose nests they lay their eggs. The larvæ live as ecto-parasites upon the Sandwasp larvæ.

4. Ants (Formicariæ) are distinguished from other Hymenoptelit in that the second (or second and third) abdominal segment is consillerably thimer than the following, and is provided with an upright scaley, or knob-like outgrowth; the antennæ are geniculate. Ants form colonies, consisting of males, females, and workers; the latter being females with imperfectly developed sexual apparatus: both males and females have large wings, but those of the latter are thrown off after copulation: the workers, on the other hand, are quite apterous. In some species two kinds of workers are miet with, some with large heads (soldiers), others with smaller heads (true workers). Sone Ants (of course only females and workers) possess stings, other's have only the corresponding poison glands, the secretion from which is squirted into the wound made by the mandibles. The nests, which consist of irregular chambers and labyrinthine passages, are, in most cases, tunnelled in the earth, or grawed out in stumps of trees; the burrowing forms generally pile up the earth they dig out above the nest, and thus form a hillock, into which the nest is continued; others (e.g., the Red-forest ant, Formica rufa), construct mounds of pineneedles, leaves, and so on; others again, build nests in hollow trees, constructing the walls of sawdust, etc., cemented together with saliva. Ants are omnivorous; the larve are fed by the workers upon comminuted food. The haljits of these insects are of the greatest interest, and in many species the most remarkable conditions may be observed. There are, for example (occurring in England), species which steal larvæ and pupæ from the nests of others, and bring them to their own; the workers, which develop from these, form a necessary contingent of the working staff of the marauders, or may have to do all the work, even to feeding their masters. In a Mexican species the abdomen of some workers is much swollen in consequence of the enormous dilation of the crop, which is filled with a honey-like fluid ; these workers remain in the nest whilst others are out seeking for honey; on their return the latter give their booty to the inactive forms, which thus serve as regular reservoirs for the honey supplies of the nest. Besides Ants, an ant-hill harbours (just as in the case of the Termite nests) quite an insect fauna on a small scale, the members of which are known as $\mathbf{M}$ y r m e copli ilou s Insects, e.g., several small Beetles, many of which are found here exclusively. The relations between Ants and Aphides are well known, the Ants greedily sucking up the sweet excretion of the latter; many ants even carry Aphides into their nests, and keep them as "domestic animals." 
5. True wasps (Vespariæ), are characterised by geniculate antennæ, reniform eyes, long and projecting mandibles; the front wings are folded during rest. Some of them are solitary forms, leading an existence like that of the Sand-wasps; others, among them the genus Vespa (Paper-wasps, Hornets), live in large or small colonies, consisting of males, females, and workers (females with imperfect sexual apparatus, but with wings), and build ingeniously constructed nests. These consist of one or more horizontal combs, each composed of a number of closely apposed prismatic hexagonal tures closed at one end, the so-called cells, which are arranged perpendicularly with the openings downwards, and are used as dwellings for the larve and pupe; the combs may be connected by shafts, and the whole nest be surrounded by a loose or firm covering. The material chiefly used for the nest is a mass formed of finely masticated wood or bark, which, when dry, has the appearance of paper. The larra are fed upon comminuted Insects. The whole population of the nest dies in the late autumn, with the exception of the young fertilised females. They survive the winter, and in the following spring found a new colony, the completion of which is accomplished later $k y$ the workers to which they give rise; the nest, which is often large, is thus the work of a single summer.

6. Bees (Apiarix) are usually very hairy, the antennz are geniculate, the eyes not emarginate, the tongue elongate, the galex and lacinix, and the labial palps are often very long and flat; the tibia and tarsi of the hind legs are usually broad. Some Bees form colonies of males, females, and winged workers (sterile females); others are solitary. The Honey-bee (Apis mellifica) is a colonial form, and there is only a single fertile female (the queen) in each nest; the hatching of a new female is a signal for a division of the colony, to form a new swarm; the Honey-bees build combs of the wax secreted by integunentary glands upon the abdomen; the combs are arranged perpendicularly, and consist of two layers of hexagonal horizontally placed cells, closed at one end, the openings being laterally directed. The larvæ from which fertile females arise, live in special, large, roundish cells, situated at the edge of the comb; the other cells are used partly for the workers and male larvæ, partly for the storage of honey and pollen (bee-bread); the honey is carried to the hive in the crop, the

$\boldsymbol{\omega}$

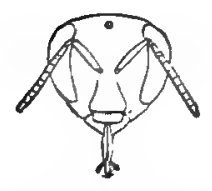

$b$

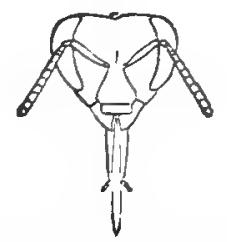

$c$

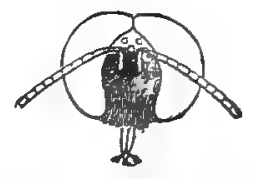

Fig. 222. Heads of Hon ey-bees. $a$ Queen, $b$ worker, $c$ male.-After Ratzeburg.

pollen is kneaded up and carried in a depression of the tibiæ, surrounded by hairs (setæ), the so-called "basket," which occurs only in the workers. The whole colony survives the winter, and this without hibernating; a fairly high temperature prevails in the nest. The male bees (drones) have very large eyes, and like the queens are much larger than the workers; they develop from unfertilised eggs. Closely allied to the Honey-bee is the Bumble-bee (Bombus), which forms small colonies, and lives in nests in holes in the ground; each colony is founded by a single large fertilised female which has survived the winter, and when complete, is made up of a few large females, some smaller females which only lay drone eggs, a number of workers, and males. Both fertile females and 
workers possess "baskets"; they do not build cells, but the eggs are laid each upon a little lump of bee-bread and honey, into which the young larva gradually eats, increasing in size by the ingestion of new material; before pupating, it spins a glossy ovate covering; this cocoon which has been wrongly regarded as a cell, may sometimes be used for the storage of food, after the Bumble-bee has crawled out of it. The females of many Solitary-bees form small cavities in the earth or in wood, or true cells of sand, loam, or pieces of leaves. Pollen or honey is stored in these cells, and one egg is laid in each, and then it is closed; the larvæ feed on the stores, the female taking no further trouble about them. The females, as well as the workers of some of the solitary Bees, possess baskets; in others, the pollen is collected on the thick hairy covering of the hind legs, or on the hairy ventral surface of the abdomen. Not a few of the solitary forms are parasitic (Cuckoo-bees), laying their eggs in the stores of other Bees, so that their larvæ may live at the expense of these supplies.

\section{Order 6. Lepidoptera.}

The Lepidoptera are holometabolous Insects, with four equally developed wings, and with sucking mouth parts. The whole animal is well covered with hairs. The head is freely movable; the multiarticulate antennæ are filiform or bristlelike, clavate, or pectinate, etc. For the structure of the mouth parts, see p. 233. The three thoracic segments are intimately connected, the prothorax small, the mesothorax large. The wings are large, covered with minute coloured imbricating scales (flattened setæ), or "dust," which usually form a complete covering over the veins and the rest of the surface; the fore wings are longer, but generally also narrower than the hind ones. The latter very often bear on the anterior margins, close to the point of origin, a strong bristle, or group of stiff bristles, (retinaculum), which fits into a small ring on the ventral surface of the fore wing; by this means the two wings of the same side are coupled. At the base of the fore wing there is a specially developed scale, just as in the Hymenoptera, but often still larger than in this group. The legs are feeble, with large coxæ and pentamerous tarsi, the basal joint being much longer than the rest ( $c f$. the Hymenoptera). There is no deep constriction between the thorax and abdomen, and the latter is therefore "sessile." The larvæ, "caterpillars," are of a very distinct type. They are cylindrical, with a long abdomen, bearing prolegs; they are almost exclusively phytophagous, and for the most part lead a free existence upon leaves, and in connection with this, and in contradistinction to most other insect larvæ, they are often brightly coloured: the exoskeleton is fairly soft, with the exception of the firmly chitinised head and prothorax. "There are five or six ocelli on each side of the head, a pair of short, three-jointed antennæ, and the usual biting mouth-parts. The thorax is provided with three pairs of short legs, each with a single claw. 
On the long abdomen there are usually five pairs of prolegs (one pair on each of the segments three to six, and one pair on segment nine), sometimes a smaller number, and then usually two pairs (in the Geometers on segments six and nine), most rarely (in a single genus of the Tineidæ) six pairs. The prolegs are provided at their lower ends reither, as in the Microlepidoptera, with a circle of movable hooks, curved outwardly, with respect to the centre of the circle; or in the Macrolepidoptera, with a $r$ ow of hooks on the inner side, and curved inwards: the prolegs are thus adapted for clasping thin branches.* Caterpillars may be distinguished from the very similar larvæ of the Tenthredinidæ by the greater number of ocelli, the smaller number of prolegs, and by the presence of hooks on the latter. The pupa is characterised by the way in which all the appendages (wings, legs, etc.) lie close to the body; all the external surfaces are firmly chitinised (whilst the surfaces lying against one another are but feebly so), so that it looks as if it had been varnished. The larvæ possess spinning-glands, which open on to the labium, and many of them before pupating either spin a complete cocoon; or form a case by binding together various particles by means of the silk; whilst others spin only a few threads; not a few surround themselves still earlier with a saccular case, open at one end, which they carry about with them.

The Lepidoptera are allied to the Hymenoptera, especially to the Tenthredinidx; they agree with the latter in the form of the legs (metatarsus, coxa), in the presence of a covering-scale at the root of the fore wing, in the feeble development of the prothorax, and in the structure of the larva.

\section{Sub-Order l. Microlepidoptera.}

The prolegs of the larvæ have a complete circle of hooks, the head is directed forwards; they live, for the most part, in concealment, either tunnelling in leaves, stems, or wood, or lying between leaves held together by the threads which they spin, etc. The pupa usually have transverse rows of spines on the dorsal side of the abdomen. The adults are, with few exceptions, of small size, with slender bodies.

1. Tineidæ, small forms with narrow wings, bordered with a fringe of hairs. The members of this group are numerous: they are often beautifully coloured, but, as a rule, very small. Among them are the Clothes. moths, Tinea pellionella, and T. tapezella; the larva of the former species lives upon fur and woollen materials; it lies in a sac, open at both ends, formed of particles gnawed off the material and spun together; and here it pupates. The

* The row of hooks corresponds with the inner portion of the circle, and the unilateral type of foot may be derived from the other by supposing the outer half of the circle to have disappeared. The last pair of prolegs in the Microlepidoptera, too, do not possess a complete circle, but a row in which the hooks are curved forward. 
larva of $T$. tapezella, which is somewhat larger, spins on the outside of the fur, a long thin-walled tube within which it can move about; the portion of fur or woollen material covered by the case, is eaten away from the surface.
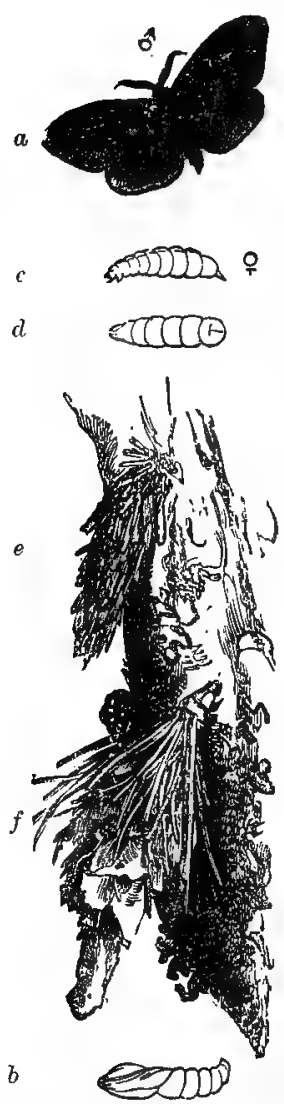

Fig. 223. Psyche. a male, $b$ male pupa, $c$ female, $d$ female pupa, $e$ sac containing female, $f$ sac containing male larva.-After Taschen. berg.

2. The "Leaf-rollers" (Tortricidæ) are, on the whole, somewhat larger than the Tineidæ, the wings broader, with a shorter marginal fringe. The larvæ very frequently-but by no means in all forms-live in and upon leaves which they have spun together. A larva, which is often found in the core of "worm-eaten " apples, belongs to one species of this division (Tortrix pomonana), other species are injurious forest pests (Tortria buoliana, etc.).

3. The Wood-borers (Xylotropha). A small family, the members of which are usually distinguishable from other Microlepidoptera by their much larger size. Here belongs the Goat-moth (Cossus ligniperda), a large, brownish-grey moth (about $80 \mathrm{~m} / \mathrm{m}$. across the wings); the larva which is almost naked, and rose-red dorsally gnaws passages in poplars, osiers, and other trees. Further, the wasp-like Clearwings (Sesia,) with transparent, almost scaleless wings, the whitish larve of which live in trees or in the stems of shrubs.

4. The Case-bearers (Psyche), are characterised by great sexual dimorphism, the females are grub-like, wings and legs are absent, whilst the males look like ordinary moths. The larva is surrounded by a sac spun out of fragments of plants or grains of sand, the female remains within this larval case. One species of the genus, $P$ syche helix, which forms a spiral case of fine particles of sand, usually reproduces parthenogenetically, males only appear now and again.

\section{Sub-Order 2. Macrolepidoptera.}

The prolegs of the larvæ have a unilateral series of hooks, the head turns downwards; they lead a free life upon plants, feeding on leaves. The pupæ have no transverse rows of spines on the abdomen. The adults are usually of considerable size.

1. The Bombycidæ are bulky forms with dull faded colours, usually of somewhat indistinct patterns; the wings are broad and overlap when at rest; the antennæ of the male are pectinate on each side, those of the female bristle-like or denticulate; the proboscis is small. The larvæ are usually hairy, often, indeed, very hairy. The pupa lies within a cocoon formed either of spun threads alone, or of these together with hairs thrown off from the larva, etc. The Bombycidæ are nocturnal, the males flying about to seek for the inactive females; in some species the latter have only rudimentary wings. To the Bombycidæ belong the Silkw orm (Bombyx mori), which came originally from China. and the cocoons of which afford the chief supply of the silk used in industries; the imago is white, the larva naked. and (unlike all other Bombycidæ) provided with a small horn at the hinder end of the body. Silk is also obtained from several other species. 
Others, again, are among the most deadly enemies to the cultivation of Pinetrees; the Pine-lappet (Bombyx pini) and the Black-arch (B. monacha). Allied to the Bombycidse are the Noctuidæ with bristle-like antennæ (often denticulate in the males), rather narrow wings, well-developed proboscis; the larvæ usually naked. Certain caterpillars of the Noctuidw (e.g., the larræ of the Rustic. Agrotis segetum), are often pests upon young plants, turnips, potatoes, etc.

2. The Loopers (Geometridæ) are somewhat like the Bombycidæ in appearance, they have broad, thin wings, bristle-like antennæ (often pectinate in the males). The almost naked caterpillar only possessing the hindmost pair of prolegs is very characteristic. It moves like a leech, by alternately straightening and arching the body (the thoracic feet and the prolegs function as do the fore and hind suckers of a Leech). In some species the female has more or less degenerate wings (Fig. 208).

3. The Hawk-moth s (Sphingidx). The body is short and spindle-shaped, with a conical pointed abdomen, long, narrow fore wings, small hind wings, long proboscis, and pointed antennæ triangular in cross-section. When at rest the wings lie horizontally. They are large excellent fliers; the larvæ are naked, and the abdomen bears a curved horn.

4. The Butterflies (Rhopalocera) have a slender body, clavate antennæ, and broad wings, which, when at rest, are held perpendicularly; they exhibit beautiful, elear colours, and fly by day. The larvæ often possess branching, spiny outgrowths, otherwise they are naked or sparsely hairy. The pupæ are characterised by their remarkably angular form; usually they are simply attached by a single silken thread round the body, more rarely they lie in a loose cocoon. Two of the best known forms may be mentioned: the $\mathrm{Cabbage-butterfly} \mathrm{(Pieris} \mathrm{brassicæ,} \mathrm{etc.),} \mathrm{with} \mathrm{white} \mathrm{wings} \mathrm{with} \mathrm{small}$ dark spots (the larvæ on cabbages), and the Small Tortoiseshell (Vanessa witicre): with reddish brown wings, flecked with black (the hairy larvæ live upon stinging nettles).

\section{Order 7. Diptera.}

The Diptera are holometabolous with reduced hind wings and sucking mouth-parts. The head bears a pair of large eres, which, in the males, where they are best developed, often touch in the mid-dorsal line. In the majority (Flies) the antennæ are short, and consist of only three well-developed joints (of which, however, the last can usually be proved to be composite), whilst in the Midges they are long and multiarticulate. The mouth-parts are used for sucking the juices of plants or animals; the chief features of their structure are given on p. 234. The three thoracic segments are fused; the prothorax is small. The rings of the first pair have few veinings, are well-developed and adapted for flight; the hind wings are reduced to halteres, which are in active motion during flight; their function is not definitely ascertained. The legs have long coxæ, long basal joints to the tarsi, and often two or three small cushions (pads) on the terminal joints. The abdomen is either sessile or separated from 
the thorax by a constriction. The larvæ are invariably $\mathrm{m}$ a g gots, i.e., the thoracic appendages are absent. Some, however, still possess a hard chitinised head furnished with eyes, antennæ, and mouth-parts. In others on the contrary, the head is not well marked, eyes are absent, the antennæ absent or very degenerate, the mouth-parts represented by a pair of darkly-coloured chitinous hooks (mandibles ?). The larvæ live in water, in decaying substances, in or upon plants, or as parasites. In those Diptera whose larvæ have well developed heads, the pupæ are like those of the Lepidoptera, the appendages lying close to the body; in those with "headless" grubs the pupæ remain within the last hardened larval skin (coarctate pupæ).

1. Midges (Nemocera) are usually slender with long antennæ, which in the males are often furnished with long hairs. The wings are narrow, the legs long and thin. To mention a few forms: Gnats (Culex) antennæx of fourteen joints, with long hairs in the male; maxillary palps in the male longer than the proboscis; the females alone possess mandibles, and stab and suck blood: the larvæ are aquatic; they have only two stigmata, situated on a terminal process (respiratory tube); the pupa is motile, and has two upright respiratory tubes at the front end of the body; both larvæ and pupæ usually hang suspended by these respiratory tubes from the surface of the water. The Daddy-long-legs or

$a$
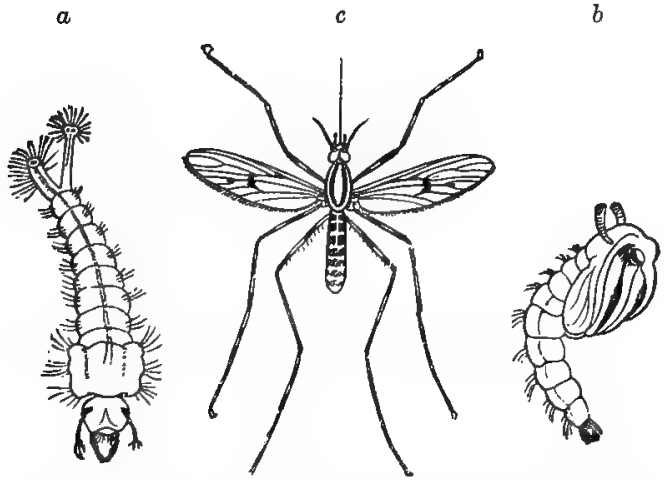

Fig. 224. Culex. $a$ larva (head downwards), $b$ pupa, $c$ perfect insect.-After Taschenberg.

Crane-flies (Tipula) are large Midges, the larvæ of which live in meadows, or in rotten wood. The Gall flies (Cecidomyia, etc.) are very small delicate forms, the larvæ of which, like the Cynipidæ, frequently live in galls (one of these for instance, C. fagi, lives in the well-known pointed gall of beech leaves) ; many species, however, do not form galls, but the larvæ are found in living or dead plants. In some species of this group, predogenesis is known to occur (see p. 246). The Sand-fly (Simulia), a

small fly-like Midge, the females of which, like Culex, are blood suckers; several of the notorious "Mosquitos" of warm countries are species of this genus; others, Black-flies, e.g., S. columbaczensis, of Hungary, are sometimes, when they occur in large numbers, a terrible plague to cattle, since they sting them in thin-skinned places, and the result of the wound is inflammation, fever, or even death. The larvæ of this genus are aquatic.

2. Gad-flies (Tabanidæ); the antenna is said to be three-jointed, but the last joint is constricted, and therefore consists of more than three joints. The head is short and broad, with very large eyes; the mandibles are only present in the female; the abdomen is flattened; the larvæ are cylindrical, living in the earth. The females suck blood from Mammalia, and are, for instance, great plagues to Horses in summer. 
the thorax by a constriction. The larvæ are invariably $\mathrm{m}$ a g g ots, i.e., the thoracic appendages are absent. Some, however, still possess a hard chitinised head furnished with eyes, antennæ, and mouth-parts. In others on the contrary, the head is not well marked, eyes are absent, the antennæ absent or very degenerate, the mouth-parts represented by a pair of darkly-coloured chitinous hooks (mandibles ?). The larvæ live in water, in decaying substances, in or upon plants, or as parasites. In those Diptera whose larvæ have well developed heads, the pupæ are like those of the Lepidoptera, the appendages lying close to the body; in those with "headless" grubs the pupæ remain within the last hardened larval skin (coarctate pupæ).

1. Midges (Nemocera) are usually slender with long antennæ, which in the males are often furnished with long hairs. The wings are narrow, the legs long and thin. To mention a few forms: G nats (Culex) antennx of fourteen joints, with long hairs in the male; maxillary palps in the male longer than the proboscis; the females alone possess mandibles, and stab and suck blood: the larvæ are aquatic ; they have only two stigmata, situated on a terminal process (respiratory tube); the pupa is motile, and has two upright respiratory tubes at the front end of the body; both larvæ and pupæ usually hang suspended by these respiratory tubes from the surface of the water. The Daddy-long-legs or

$a$
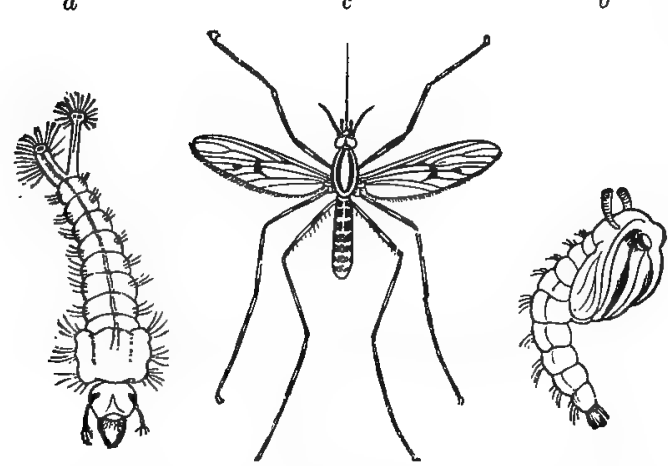

Fig. 224. Culeas. a larva (head downwards), $b$ pupa, $c$ perfect insect.-After Taschenberg.
Crane-flies (Tipula) are large Midges, the larva of which live in meadows, or in rotten wood. The Gall flies (Cecidomyia, etc.) are very small delicate forms, the larvæ of which, like the Cynipidæ, frequently live in galls (one of these for instance, C. fagi, lives in the well-known pointed gall of beech leaves); many species, however, do not form galls, but the larvæ are found in living or dead plants. In some species of this group, pædogenesis is known to occur (see p. 246). The Sand-fly (Simulia), a

small fly-like Midge, the females of which, like Culex, are blood suckers; several of the notorious "Mosquitos" of warm countries are species of this genus; others, B la ck - flies, e.g., S. columbaczensis, of Hungary, are sometimes, when they occur in large numbers, a terrible plague to cattle, since they sting them in thin-skinned places, and the result of the wound is inflammation, fever, or even death. The larvæ of this genus are aquatic.

2. Gad-flies (Tabanidæ); the antenna is said to be three-jointed, but the last joint is constricted, and therefore consists of more than three joints. The head is short and broad, with very large eyes; the mandibles are only present in the female; the abdomen is flattened; the larvæ are cylindrical, living in the earth. The females suck blood from Mammalia, and are, for instance, great plagues to Horses in summer. 
pupates immediately after birth. On the Horse (and Cow) the active, winged Horse-tick (Hippobosca equina) is found; in the wool of Sheep, the wingless Sheep-tick (Melophagus ovinus). The same mode of propagation is followed by the closely allied, small, blind, wingless Bee-lou e (Braula cæca) parasitic on Honey-bees.

The Fleas (Aphaniptera) are usually placed close to the Diptera, though probably incorrectly. The body of these Insects is compressed, the colour bright

1

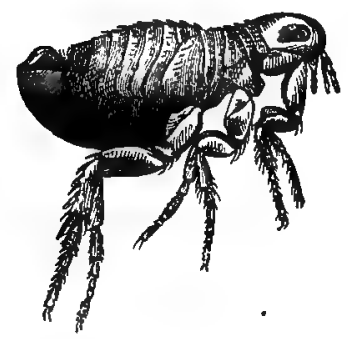

2

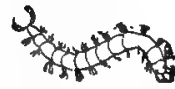

Fig. 226. Pulex irritans. 1 imago, 2 larva, 3 pupa.-After Taschenberg. yellow to dark brown, the head small with a single ocellus on each side (instead of the com. pound eye), the antennæ small, clavate, and lying in a pit behind the eye. The mouth-parts are adapted for sucking, but are very different in structure from those of the Diptera. The actual sucking-tube consists of the very long labrum which is grooved ventrally, and the two mandibles, which form a half-open tube; the first maxillæ are short, pointed, and provided with a fourjointed palp of considerable length ; they form, together with the labium which carries two threejointed palps, a kind of sheath for the true sucking-tube; a hypopharynx is absent. There are three distinct thoracic segments each bearing a pair of long powerful legs (the hind legs being somewhat stronger than the others) with very large coxæ and pentamerous tarsi; they are apterous. They live as parasites upon Mammalia and Birds. The larvæ have neither eyes nor legs; the whitish body is cylindrical, somewhat hairy; the mouth-parts are biting; before pupating they spin cocoons. They live in sweepings, etc. Pulex irritans is a parasite upon Man; and other species of the same genus also occur upon various other animals. The Chigoe or Jigger (Sarcopsylla penetrans) of the tropical regions of America sucks the blood of Man and other animals; the fertilised female bores into the skin, and as the ova develops the abdomen enlarges enormously, reaching the size of a pea; the aperture into the small cavity in the skin in which the parasite is situated is filled up by the hind end of the body, so that the egge can be conveniently deposited; after oviposition it dies.

\section{Class 4. Arachnida.}

The body is divided into a cephalothorax and an apodous abdomen. The cephalothorax is usually unsegmented; the abdomen, which is generally short, is segmented in some forms, unsegmented in others; sometimes the two regions are separated by a deep constriction (in the true Spiders), but usually there is no distinct separation: sometimes the whole body is fused into a single unsegmented mass (in the Mites). The cephalothorax is usually furnished, anteriorly, with a varying number of ocelli, grouped in different ways, compound eyes are never present. Antenn are absent. There are two pairs of jaws, termed the cheliceræ and the 
pedipalpi. The cheliceræ, which lie in front of the mouth, consist of two or three joints, and are entirely different from the mandibles of Insects and Crustacea; in many (e.g., in the Scorpions) they are in the form of small chelæ. The pedipalpi are usually leg-like, longer or shorter; the basal joint is often furnished with a

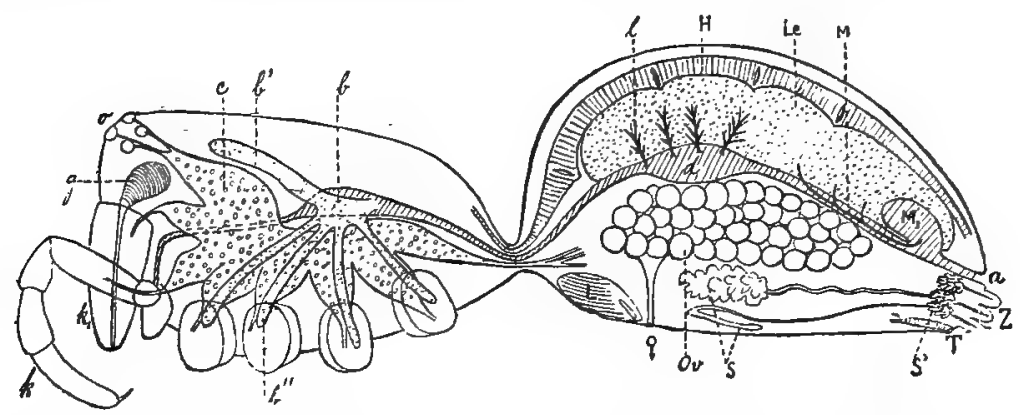

Fig. 227. Diagram of the anatomy of a Spider. $a$ anus, $b$ crcum of mesenteron, $b^{\prime}$ its anterior end, $b^{\prime \prime}$ branches of the cæcum extending into the legs which are here cut away); $c$ cerebral ganglion connected with the ventral ganglionic mass, $d$ mesenteron, $g$ poison glands, $H$ heart, $k$, cheliceræ, $k$ pedipalpi, $l$ hepatic duct, $L$ lung-sac, Le liver, $M$ Malpighian tubules, $M_{1}$ dilation of the rectum into which $M$ open, o eyes, ov ovaries, $S$ large silk glands, $S^{\prime}$ smaller do., $T$ opening of the tracheal system, $Z$ spinnerettes, + female genital aperture.-Modified from Krieger.

kind of grinding ridge, whilst the rest of the joints are either all simple, and form a strong palp, or the two distal joints are modified to form larger or smaller chelæ. Behind the pedipalpi are fou $r$ pairs of legs (ambulatory appendages), which are usually all similar, and generally consist each of seven joints.

According to the usual interpretation, the cephalothorax of the Arachnida corresponds to the head and thorax of Insecta, the cheliceræ represent the mandibles, the pedipalpi the first maxillæ, whilst the first walking legs are comparable with the second maxillæ (the labium), and the remaining legs with those of Insects. Against this view, however, may be mentioned, among other things, the structure of the cheliceræ, which are totally unlike the mandibles of Insects (consisting of several joints, etc.). Moreover, the Arachnida, on the whole, differ so essentially from the Insecta that it is impossible to make a special comparison of this nature. It would, therefore, appear very doubtful whether the jaws of the Arachnida can be compared with those of other Arthropods; more probably they are thoracic limbs, which in correlation with the degeneration of the head, have taken on the function of jaws; in this case the mouth would have moved back to lie between the thoracic limbs.**

The skin in most Arachnids is not so hard as in the Insects, usually the cuticle is leathery, often setose. Among the glands of the skin, the spinning glands, present in certain divisions

* As in Limulus, with which the Spiders are in no way allied, a view that has been incorrectly held by some authorities. 
(Spiders, Pseudoscorpions, and others) must be specially noticed. The nervous system is of the usual Arthropod type, but characterised in most forms by the

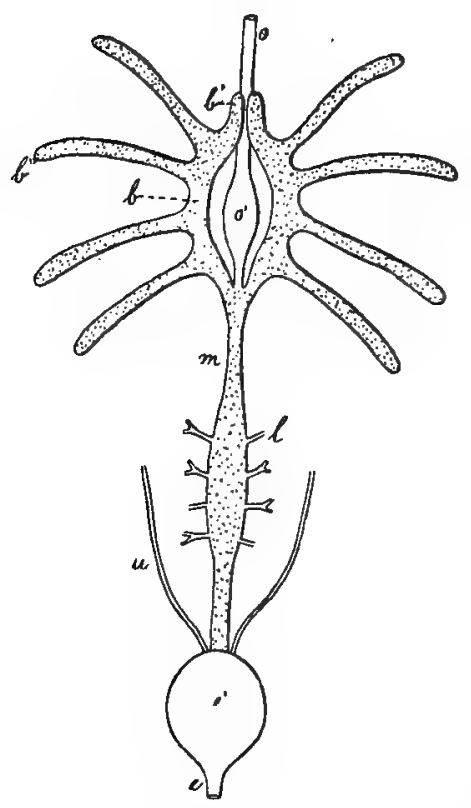

Fig. 228. Alimentary canal of a Spider, diagrammatic. $b$ cæcum, $b^{\prime}$ its anterior end, $b^{\prime \prime}$ lateral branches of the same, into which the Malpighian tubules open, $l$ hepatic duots, $m$ mesenteron, o œsophagus, $o^{\prime}$ suctorial stomach, $u$ Malpighian tubule.-Orig. fact that all the ventral ganglia are fused into a single mass; a series of distinct ganglia occurs in quite a few (e.g. the Scorpions). Of special sense organs only the eyes mentioned above are known; but since some Arachnids. can produce sounds, it is very probable that auditory organs are also present. The alimentary canal is characterised by the presence of several $\mathrm{c} æ \mathrm{ca}$ arising from the anterior portion of the mesenteron and extending some distance into the legs. In the Spiders, a single large curved cæcum arises on each side from the mesenteron, is directed forwards and gives off branches which enter the bases of the legs; the front ends of the two cæca lie close together above the fore gut (Fig. 228), and in many Spiders, unite at this point. Salivary glands are present, and, unlike Insecta, many Arachnida possess a large liver consisting of numerous tubules.

situated in the abdomen. In most Arachnida there are $\mathrm{M} \mathrm{alpighi}$ a tubules, like those of the Insecta.* The respiratory organs are represented either by $\operatorname{tracheæ}$, which open to the surface by a small number of stigmata, or by so-called $l \mathrm{ung} \mathrm{s}$; the latter are invaginations of the skin, each of which is again provided with a series of flat evaginations, lying close together like the leaves of a book; each form of respiratory organ may occur alone, or both may be present in the same individual. The vascular system is often better developed than in Insects; in the Scorpions for instance, which are provided with lungs, there is a circulation similar to that of many Crustacea; the blood flows from the heart through a number of arteries; the venous blood collects in

* In a variety of Arachnida (Scorpions, Phalangiidæ, etc.) there is, in the cephalothorax, a pair of large coxal glands which usually open at the base of the third pair of limbs. They have been regarded as excretory, and considered to be segmental organs; the correctness of this interpretation is doubtful. 
a large ventral blood sinus, and passes thence to the lungs, from which the now arterial blood returns to the pericardium, and enters. the heart through the ostia; the heart of the Scorpions is a long tube, divided, as in the Insects, into a series of chambers (eight), each provided with a pair of ostia. In other Arachnida, the heart is shorter, and has a smaller number of these ostia, the vascular system is less complete, the blood flowing into large sinuses between the organs. As in other Arthropoda, there is a pair of ovaries in the female, a pair of testes in the male; the two glands, whether ovaries or testes, are frequently partially united, and the ducts open by a common aperture, far forward on the ventral surface of the abdomen. In the Phalangiidæ and the Acarinæ, the gonads are united at one end, the other ends being - prolonged into the oviducts (or vasa deferentia). These soon unite to form a single canal, which thus arises from a circle formed by the genital glands and their two ducts. Sexual dimorphism is frequently displayed. The Arachnida only occasionally undergo a metamorphosis; the newly-hatched animals are generally like

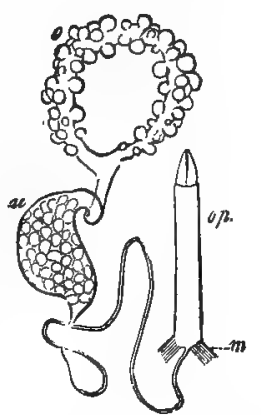

Fig. 229. Sexual apparatus of one of the Phalangiidae. $o$ ovary, $u$ swelling of the long oviduct, op ovipositor, $m$ retractor muscles of the same.After Gegenbaur. the adults, but sometimes the last pair of limbs is wanting.

Like Insecta, the Arachnida are emphatically terrestrial and fresh-water forms; many are parasitic. Besides the Pycnogonidæ, whose position here is not without some doubt, a few of the Acarinæ are marine.

\section{Order 1. Arthrogastra.}

The nembers of this order, which includes a number of very different forms, are distinguished from the two following orders, in that the abdomen is segmented. The cheliceræ are generally chelate. Respiration is effected by lungs or tracheæ.

1. The Scorpions (Scorpionidx) possess a more elongate body than the rest of the Arachnids. The eephalothorax, which is not constricted off from the abdomen, bears in the middle line, dorsally, two ocelli, and anteriorly on each side, a small group (two to five); the cheliceræ are short, strong chelæ; the pedipalpi, which forcibly recall the large chelæ of the Crayfish, are of considerable length (as long as, or longer, than the legs), and each is furnished with strong claws, the four pairs of legs are well developed. Of the thirteen abdominal segments, the last six are much narrower than the anterior, and form a very movable tail (post-abdomen), which the animal curls up over the rest of the body so as to carry it with the tip pointing forwards; this tip, the sting, bears the openings of two poison glands, which lie in the anterior swollen portion of the 
terminal joint. The anus is situated in the membrane between the last and the penultimate somites. Anteriorly, on the ventral surface of the abdomen, just behind the legs, there arises a pair of flattened, unsegmented appendages (the pectines), the posterior edges of which are toothed; their significance is

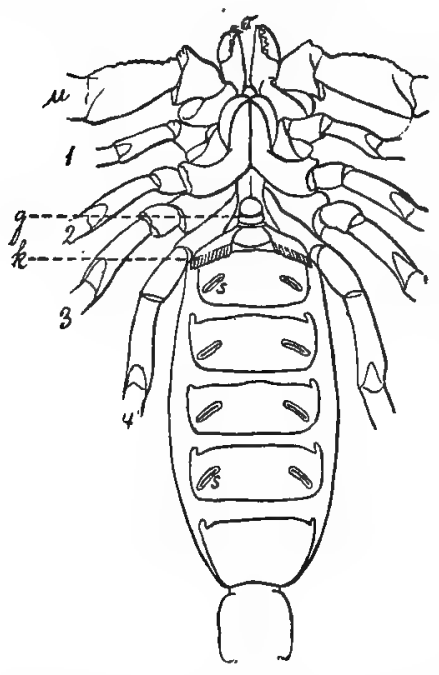

Fig. 230. Scorpion, seen ventrally; chelicerr, legs and postabdomen not completely drawn. $g$ genital aperture, $k$ pectines, o cheliceræ, $u$ pedipalpi, $s$ stigmata, $1-1$ legs.After M. Edwards. unknown. Close to them lies the genital aperture; on the broad portion of the abdomen (pre-abdomen) there are also, on the ventral surface, four pairs of slit-like stigmata, the openings of the same number of lung-sacs. The Scorpions are fairly large animals; they are viviparous, the young ones remain with the parent for the first few weeks, but the latter dies before long. They occur in the tropics and in the warmer regions of the temperate zones (two species in S. Europe). They remain in one place, feeding upon Insects and Arachnids, which they seize with their chelæ, and kill by a stab of the sting.

2. The Pseudoscorpions (genus Chelifer and others) recall at first sight the Scorpions, which they resemble in the structure of the cheliceræ and pedipalpi. They differ, however, in many respects. The abdomen consists of eleven somites; its hinder region is not developed as a post-abdomen, and a sting is absent; further,

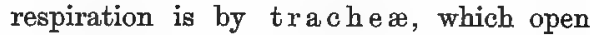
by two pairs of stigmata on the ventral surface of the abdomen. Anteriorly there are one or two eyes on each side of the

eephalothorax, but these may sometimes be absent. On the ventral surface of the abdomen, near to the genital pore, is a number of small papillæ, perforated by the apertures of the spinning glands. The ova and the larvæ, which are hatched in a very imperfect condition, are carried about on the ventral surface of the body; the former are bound together into a mass. The Pseudoscorpions are small; they live beneath bark, in moss, old books, collections of Insects, and so forth; they feed upon Mites, Book-lice, etc.

3. The Harvest Men (Phalangiidx)* have a short, arched body, which is not sharply divided into cephalothorax and abdomen. The cephalothorax, which consists of three indistinct and immovably connected segments, bears a pair of eyes dorsally, like the two median eyes of Scorpions; the cheliceræ have small chelæ, the pedipalpi are antenniform, much shorter than the extremely long legs, which are characterised by the division of the proximal joint into a number of smaller segments. The abdomen consists of eight ill-defined segments, it is provided, anteriorly, with a pair of stigmata leading into a system of tracheæ. The Phalangiidæare peculiar in that the males possess a long extensile copulatory organ, the females a long eversible ovipositor; the genital aperture is anterior. At first sight they look very like long-legged Spiders, and are chiefly met with in the dwellings of mankind.

* This description does not apply to a few aberrant forms. 


\section{Order 2. Araneïna (Spiders).}

The Spiders may be distinguished from other Arachnidas by the separation of the cephalothorax from the abdomen by a deep constriction. Both regions are unsegmented, but newly-hatched animals show indications of abdominal segmentation. Anteriorly the cephalothorax bears a group of six to eight ocelli, arranged in various ways. The cheliceræ consist of a simple, strong basal joint, and a claw-like terminal segment, at the tip of which opens a poison gland. The pedipalpi are antenniform, with broad basal joints; the terminal joint in the adult males is modified, hollowed out, etc., and thus adapted for introducing the spermatozoa into the genital aperture of the female; it is often very complicated in form. The legs are very strong, often of considerable length. Anteriorly, on the ventral surface of the abdomen, there are always two stigmata, each leading into a lung-sac; in a few Spiders, posterior to these, there is a second pair, which eith e r (Mygale) lead into a similar pair of lungsacs, or (Argyroneta) lead into t $\mathrm{r}$ a cheæ. In most of the Spiders, however, this second pair of stigmata is wanting, instead there is an unpaired stigma posteriorly, just in front of the spinnerettes, and this leads into a variously modified system of tracheæ. The majority of this group possess both lungs and tracher; a smaller number have only lungs, but in this case there are four. Posteriorly, below the anus, there are four or six spinnerettes, fairly large processes beset with a larger or smaller number of short, fine tubes (in Epeira, altogether about 700); at the apex of each of these is the aperture of a silk gland, which lies in the abdomen. These spinning glands may vary considerably in structure, even in the same animal, and may give rise to different secretions. As the substance is pressed out through the tubules it hardens to form fine threads; in many Spiders which form webs some of these threads remain sticky. By means of the feet the fine threads may be woven together into coarser ones; all Spiders spin cocoons for their eggs, many form webs or tubes, in which they live. The genital aperture lies anteriorly on the abdomen. The males are often smaller than the females, sometimes the difference is so great that, although in other respects the structure is normal, they have been termed $\mathrm{dwarf}$ males. Spiders feed chiefly upon Insects which they kill with their cheliceræ. There are very many species, but the group is very uniform, and is abundantly represented in temperate countries.

As examples may be mentioned: the Bird-spiders (Mygale), large tropical forms, thickly covered with hairs, and possessing four lung-sacs ; they will even attack and consume small Vertebrata; the common Cross-spider (Epeira diadema), which, like the House-spider (Tegenaria domestica), spins webs, and often lives in or near honses; the Water-spider (Argyroneta aquatica) 
abundant in small pieces of water, where it builds a bell-like web, the cavity of which is filled with air, carried by the animal from the surface of the water in its velvety covering of hairs.

\section{Order 3. Acarina (Mites).}

The Mites are small, frequently even microscopic Arachnids, in which the cephalothorax and abdomen are generally fused into a single mass. They possess from one to three pairs of eyes, or none at all. The mouth-parts are usually short. The pedipalpi, as well as the cheliceræ, may be chelate, they are sometimes used for biting, sometimes for stabbing. A heart has only been demonstrated in some of the Acarines; special respiratory organs are frequently absent, but in many there is a tracheal system, opening by a pair of stigmata. On hatching, the Mites possess only three pairs of legs, the fourth pair develops later; in other respects also, the larvæ may differ more or less from the adult. Many undergo a resting period before moulting.

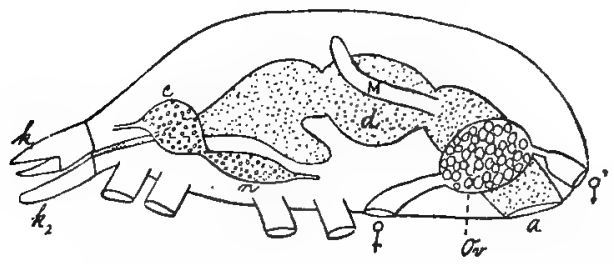

Fig. 231. Diagram of the anatomy of a Tyroglyphus; legs cut off. a anus, $c$ cerebral ganglion, $d$ mesenteron, $k_{1}$ chelicera, $k_{2}$ pedipalp, $M$ Malpighian tube, $n$ ventral ganglionic mass, $O v$ ovary; + aperture of the oviduct, $o$ ' copulatory aperture.- Orig.

The genital aperture, as in other Arachnida, is situated anteriorly on the ventral surface of the abdomen. In some females (Tyroglyphus, probably also in the Sarcoptidæ), there is, besides, the ordinary genital opening, a second one posteriorly, just in front of the anus, by which the spermatozoa are received during copulation, whilst the anterior serves as the oviducal pore.

1. Trombidiidæ, red, velvety, quadrangular animals, some of which are among the largest of the Mites. The larvæ live as parasites on Phalangium, Spiders and Insects; the adults are predaceous. The Watermites (Hydrachna and others), are roundish animals, often of a red colour, which swim about in the water by means of their hairy legs; the hexapod larvæ are parasitic on aquatic Insects, whilst the adults are usually free-living (one species of this group is in the adult stage parasitic upon the gills of the Fresh-water Mussel). The Beetle-mites (genus Gamasus) frequently occur on Beetles, Bumble-bees, etc. They are small animals, with an oval, flattened, rather hard, brownish body; they run about freely on the body of the host. An allied, but thin-skinned form, the common Bird-mite (Dermanyssus avium) occurs on Birds (Fowls, Canaries), and sucks their blood; like some of the Hemiptera, it is a temporary parasite preying upon the Birds by night. The Ticks (Ixodes) are flat, with a fairly hard, but very extensible exoskeleton; they can move about, but attach themselves to Mammals, Birds, and Reptiles, in order to suck their blood; the female increases enormously in size when gorged. The species of the Genus Tyroglyphus (Cheese-mites and Flour-mites), live in old cheese, meal, and many other half-dried organic substances; they are white, shining, almost microscopic animals. All these Mites, with the exception of Tyroglyphus, possess tracheæ. 
2. Itch-mites (Sarcoptidx) are microscopic, blind, without trachex, and generally with suckers at the tips of the feet; they live as stationary parasites on Mammals and Birds, and feed either upon the skin or upon exuded lymph. There is a marked sexual dimorphism, and it is interesting to note that copulation occurs before the females have reached the adult form, and whilst the ovary is still entirely undeveloped. Here belongs the human Itch-mite (Sarcoptes scabiei) which burrows in the epidermis; the female has suckers on the two anterior pairs of legs, the small males also have them on the fourth pair. Various nearer or more distant allies live in and on the skin of other Mammalia and Birds, causing mange. Peculiar microscopic Mites of elongate form and with short legs, destitute of suckers (Demodex folliculorum) occur in the hair follicles of the human nose; they are quite harmless, but a variety of the same species which is parasitic upon Dogs, causes a very bad skin disease in this animal.

3. Gall-mites (Phytoptus) are microscopic, with elongate bodies, and are easily distinguished from other Mites by the possession of only the two anterior pairs of legs. They suck the sap of plants and thus cause various abnormal growths of leaves and buds of many, especially woody, plants.

\section{APPENDIX TO THE ARACHNIDA.}

The three groups following are very peculiar, and their systematic position is not at all certain; they are generally, however, regarded as belonging to the Arachnida, and this is probably true for the first two. They are, however, so very aberrant in all respects that they are best considered in an appendix.

The Pentastoma (Pentastomum) live parasitically in various Vertebrata; they are animals of very considerable size, and at first sight are very like short-jointed Tapeworms. The body is elongate, usually flattened, and separated by furrows into a large number of short segments; the segmentation is, however, only external and does not affect the internal structure; the limbs are only represented by two pairs of chitinous hooks which are situated anteriorly not far from the mouth. The alimentary canal is a straight tube, the anus posterior. The male genital aperture lies far forward on the ventral surface, the female pore close to the anus; the genitalia recall those of the Arachnida. The central nervous system is reduced to a ventral ganglion below the pharynx and a ring. arising from it to run round this. Sensory, respiratory, and circulatory organs are absent. Pentastomum trniödes, when sexually mature, inhabits the nares and frontal sinuses of the Dog and the Wolf (female $8 \mathrm{c} / \mathrm{m}$. and. upwards, male $2 \mathrm{c} / \mathrm{m}$. long). The ova escape with the mucus of the

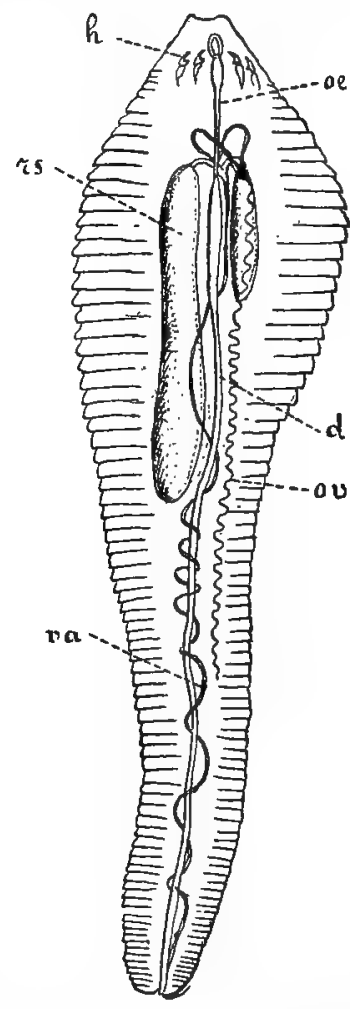

Fig. 232. Female of Pentr. stomum taeniö̈des. d gut, $h$ hooks, oe csophagus, ov ovary, rs seminal vesicle, $v a$ oviduct. After Leuckart. nose, and each contains a young Pentastomum, possessing two pairs of small hooked legs. If these ova are ingested by a Hare or Rabbit, the egg shell is dissolved in the stomach, and the small animal makes its way into the liver, where it grows considerably, but does not become sexually mature. Immature Pentastoma are also now and then found in other Mammals, 
and in Man himself; if an organ containing such parasites be devoured by a Dog, they wander into the nasal cavities and complete their development.

The Pycnogonidæ, or Crab-spidexs, have a very rudimentary $\mathrm{abdomen}$; the cephalothorax is narrow and divided into four segments. The most anterior is elongated to form a snout-like process, at the tip of which is the mouth; the cephalothorax bears four ocelli, a pair of cheliceræ which are usually clawed, and a pair of antenniform pedipalps, though both these pairs of limbs may be absent; and also four pairs of eight-jointed legs, which may be thick or very elongate, and which always make up the main mass of the body. In the males, at the base of the first pair of legs, there is a pair of jointed, leg-like appendages, to which the eggs are attached; these appendages may also sometimes occur in the females, which do not carry the eggs. The

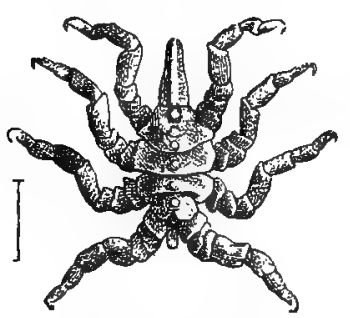

Fig. 233. Pycnogonum. cæca of the alimentary canal extend far into the legs. Respiratory organs are wanting, but a heart is present. There is a pair of ovaries, or of testes, which unite posteriorly and send branches into all the legs; ova and spermatozoa escape by apertures in the second joints of all, or of some of the legs. The newly-hatched larva are unsegmented, and possess only three pairs of limbs, of which the anterior represent small chelæ, and are modified to form the $\mathrm{chelicer}$ of the adult; the second and third pairs are short; the latter apparently degenerate, whilst the second form pedipalpi. Sometimes the larvæ are parasitic in Hydrozoa. The Pycnogonidæ are marine, crawling slowly about at the bottom of the sea. In northern seas both short-legged (Pyonogonum) and long-legged forms (Nymphon) occur.

The Tardigrada, or Bear-animalcules, are microscopic animals, which live in moss, in gutters, and in fresh water. They are elongate, indistinctly segmented, and possess four pairs of stumpy unjointed legs, which have claws at the tips, and by means of which they crawl slowly about. A pair of styletshaped stabbing organs may be protruded from the mouth. Respiratory and circulatory organs are absent; on the other hand they possess a pair of

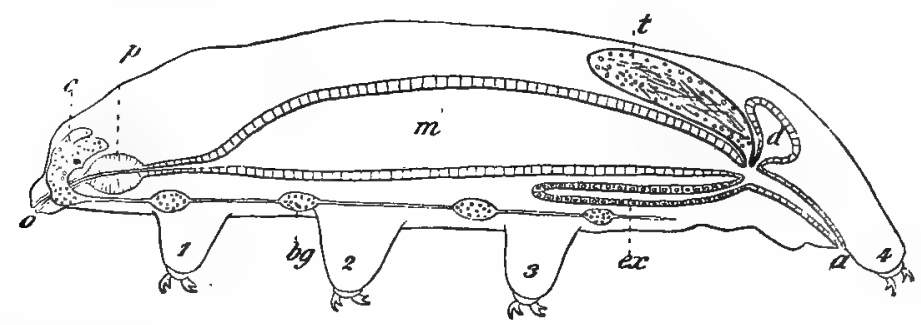

Fig. 234. Diagrammatic figure of a Tardigrade, $\delta$ viewed from the left side. $a$ anus, $b g$ ventral ganglion, $c$ cerebral ganglion, $a$ gland, ex excretory organ (i), $m$ stomach, $o$ mouth, $p$ pharynz, $t$ testis. $1-4$ four legs.-Orig. (with the use of figures by Plate.)

small eyes and a fairly well-developed nervous system, consisting of a large cerebral ganglion and several distinct ventral ganglia. The sexes are separate; the males are much more rare than the females. If the water, in which the Tardigrada are living, dries up, they shrink to small granules, and may pass years in this condition; when they are moistened again they swell out and again become active. The systematic position of this small group is still uncertain, and their location amongst the Arachnida appears to be hardly justified. 


\section{Phylum 7. Mollusca.}

The body is unsegmented, very varied in form, and without jointed appendages. The skin is soft, often ciliated over large tracts; the cuticle absent or (usually) very thin. The body-wall forms, ventrally, a muscular foot, which is either discoidal or compressed, and, on account of its great contractility, forms an important locomotor organ. Anteriorly there is a more or less well-developed $\mathrm{h}$ ead with a mouth, often also with tentacles and eyes. Above the foot and head there is a fold of skin, the mantle, which extends round the whole animal; in some instances it is narrow; in others it forms a large lamellar expansion on either side of the body (Lamellibranchs); in others again it is better developed either at the anterior or the posterior end than elsewhere (Gastropods, Cephalopods), forming a pouch, the pallial chamber, between the body and the mantle ( $k$ in Fig. 239 and $268 B$ ). In the majority, the greater part of the animal is covered by an open shell, secreted by the skin, with which it is usually only connected at isolated spots, whilst for the most part it lies free upon the upper surface of the body. The shell is never cast (like the cuticle of Arthropoda), but is continually increased in size by the formation of new material at the edge, whilst it is thickened by deposits from within; it consists of a substance, conchiolin, something like chitin, but differing from it chemically, and usually so thoroughly impregnated with calcareous salts, principally carbonate of lime, that these constitute the chief part of the shell.

The alimentary canal usually exhibits a large expansion, the stomach; the anus is either at the hind end of the animal or is moved to one side. Salivary glands opening into the mouth are generally, and a well-developed liver invariably, present. In the majority of the Mollusca (with the exception of all Lamellibranchs) there is, on the floor of the mouth a muscular pad, the tongue, covered on its upper surface with a thin, stiff membrane, the radula, or lingual ribbon, on which are arranged transverse rows. 
of delicate, chitinoid teeth of various forms, with their points projecting backwards. The teeth. in one row may be all similar, but very frequently some differ from the others; each row is definitely arranged, and a median tooth is usually present, on either side of which the other teeth are symmetrically disposed. The successive rows are generally similar. The anterior exd of the radula is continually being

Fig. 235 .

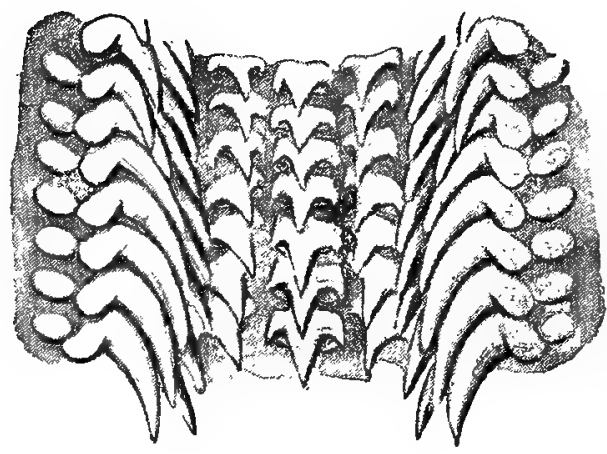

Fig. 236.

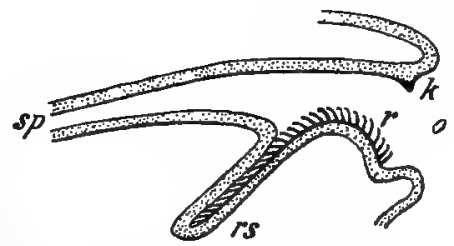

Fig. 235. Portion of the radula of a Cephalopod.-Orig.

Fig. 236. Diagrammatic longitudinal section of the mouth of a Gastropod. $k$ jaw, o mouth, $r$ radula, $r s$ radula sac, $s p$ œsophagus.-Orig.

worn down and rubbed away; the posterior end lies in a narrow sac, the radula-sac, which is frequently very deep; new teeth are formed at its end, and the radula is gradually pushed out from it. Besides this organ, which is very characteristic of the Mollusca, there occur also within the cavity of the mouth other hard structures, varying in form (also composed of a substance like chitin), which may be termed jaws.

The respiratory organs are for the most part gills of various kinds, usually occurring in the mantle cavity, which on this account may also be termed the branchial chamber. In some forms gills are wanting, and the mantle chamber may then (as in the Gastropuda) serve as a pulmonary organ; from others special respiratory organs are altogether absent. The vascular system is for the most part well developed, although the blood flows partly through spaces between the organs. The heart consists of one or two (in the Nautilus as many as four) auricles into which the blood flows from the gills (or pulmonary chamber), and a thickwalled ventricle, which receives it from the auricles, and drives it into the body. The venous blood collects in one or more large spaces which supply the respiratory apparatus. The excretory organs or kidneys are saccular, and have each two openings, of which one lies on the surface of the animali, whilst the other leads into the so-called pericardium, a portion of the body-cavity surrounding the heart. The number of the kidneys varies (one to 
four); they clearly correspond with the segmental organs of the Chætopoda.*

The nervous system is peculiar; the typical arrangement is as follows (Fig. $237 B$ ) : above the anterior portion of the alimentary canal lies a pair of cerebral ganglia, connected by a commissure; from these a nerve cord runs, on either side, round the œsophagus to a pair of pedal ganglia, lying in the foot, which are likewise connected by a commissure; behind the cerebral ganglia lie a pair of pleural ganglia, joined by one pair of connectives to the cerebral, by another to the pedal ganglia. From

$A$

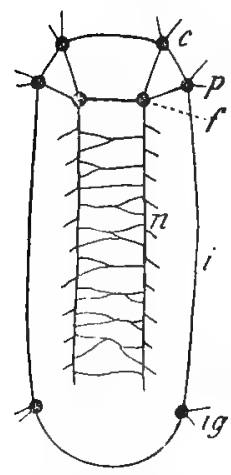

$B$

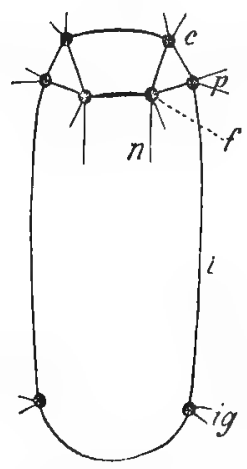

C

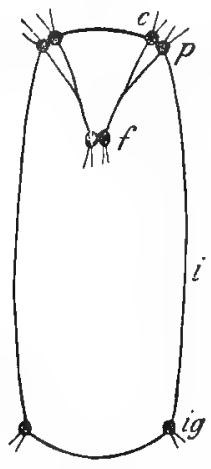

$D$

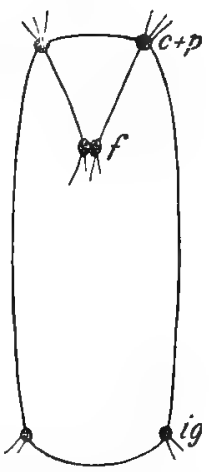

Fig. 237. Diagram of the central nervous system in various Mollusca. $A-B$ Gastropods, $C-D$ Lamellibranchs. $h$ cerebral ganglion, $f$ foot, $p$ pleural ganglion, ig visceral ganglion, $i$ visceral loop, $n$ pedal nerve.-Orig.

each pleural ganglion there arises a nerve cord, which is usually long, and runs posteriorly through the body, uniting with that of the other side to form a loop termed the visceral loop: the visceral ganglia lie upon it, posteriorly. Great differences appear in the various Molluses; the nerve cords may be long or short, sometimes so short that all the ganglia lie close together and fuse to form a single mass (Fig. $237 \mathrm{D}$ ).

The cerebral ganglia apparently correspond with the same structures in the Chætopoda, the pedal ganglia with the first ventral pair of the same; one pair of nerves, the pedal nerves, which proceed from the pedal ganglia and run posteriorly, and, in various Molluses (e.g., in Chitons and certain Gastropods, Fig. $237 A$ ), are very thick, and are connected by fine transverse cords, probably correspond with the ventral nerve cords of the Chætopods. The pleural ganglia and the visceral nerves must be regarded as new structures.

* In many Mollusca-Cephalopoda, many Lamellibranchs, several Gastropodacertain parts of the epithelial covering of the pericardium are glandular. Similar epithelium may occur upon evaginations of the auricles, or there may be true glandular evaginations of the pericardial wall. Apparently all these structures, which are called indiscriminately pericardial glands, are excretory organs. The waste substance formed in the cells, partly as concretions, is doubtless got rid of by the kidneys. 
Upon the head of the Gastropoda and Cephalopoda there is a pair of eyes, which usually conform to one of the types figured in Fig. 20, 2, 4-6 (p. 21); occasionally they are also present on other parts of the body. In Chitons and Lamellibranchs, cephalic eyes are wanting; if any are present in these animals they occur on other parts of the body. The Mollusca possess a pair of a uditory vesicles with one or more calcareous otoliths ( $c f$. Fig. 19, p. 20); the auditory vesicles are situated near the pedal ganglia, but the nerves which supply them (auditory nerves) proceed direct from the cerebral ganglia. The tentacles which are universally present in Gastropods are to be regarded as tactile organs; in other Mollusca, other appendages, papillæ, etc., have a like function. One or a pair of sensory organs (specialised portions of the skin) regarded as olfactory, occurs in the mantle cavity of most Gastropods and various Lamellibranchs; behind the eyes in the Cephalopoda there is usually a pair of pits with a similar significance.

Reproductive organs. In some Mollusca there is a pair of gonads, each with its duct; but frequently there is only a single gland and duct, or the glands are fused, the ducts, separate and so on. In other respects, also, the reproductive system offers great diversity; many forms are hermaphrodite, others are bisexual; the ducts are often furnished with accessory apparatus; usually peculiar copulatory organs of various kinds are present (see the various classes). Parthenogenesis is unknown within this group, as is also asexual reproduction.

The majority of the Mollusca undergo a metamorphosis; the larva swims by means of a velum, a discoid expansion of the head with a ciliated margin; often, however, it is represented only by a crown of cilia upon the head ( $c f$., the larva of the Chætopoda).

The Mollusca are pre-eminently aquatic, chiefly marine animals : many Gastropoda, however, live on land, but for the most part in damp places. They are not only very abundant at the present day, but in earlier periods were represented by a great diversity of forms, and their shells are among the most numerous of fossils.

\section{Class 1. Placophora (Chitons).}

The Chitons, which were formerly incorrectly placed in the next class, are a small group of Mollusca possessing a well-marked bilateral symmetry almost throughout; there is here no trace of the torsion so characteristic of Gastropods. They are rather flattened, somewhat convex, oval animals, with a large discoid foot ventrally. Dorsally there are eight transverse shell-plates, which are broad, calcareous and imbricate ; these, like the smaller calcareous 
plates, spines, and bristles, which cover the edge of the dorsal surface, are true cuticular structures. The mantle is represented only by a narrow fold which runs round the whole body, dorsal to the head and foot (Fig. $268 \mathrm{~A}$ ); it covers a series of plumose gills on either side. The head, which is not very well marked, bears neither eyes nor tentacles; on the other hand, eyes occur irregularly on the dorsal surface in several forms; they are situated at the tips of the soft projections of skin which perforate the shell-plates, and thus apparently on the shell-plates themselves. The nervous system is chiefly characterised by the fact that two of the cords which spring from the pedal ganglia and run posteriorly (see p. 289) are very strong and are connected by numerous transverse cords. A well-developed radula is present; the anus is posterior and median. The heart lies above the rectum, it is possessed of two auricles, arranged symmetrically on either side of the ventricle. There is a pair of elongate branched $\mathrm{kidneys}$, opening into the mantlegroove, one on either side, just anterior to the anus. The sexes

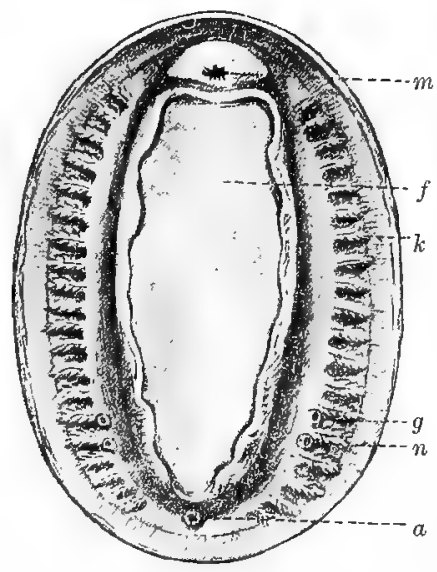

Fig. 238. Chiton, viewed from below, somewhat diagrammatic. $m$ mouth, $f$ foot, $k$ gills, $g$ genital pore, $n$ urinary aperture, $a$ anus.-Orig. are separate; ovary and testis single, but the ducts paired, and opening on either side a little in front of the excretory pores in the mantle-groove. The larvæ are oval, provided with a velum, and two eyes which later undergo degeneration. The smaller species of eyeless Chitons occur on British coasts; the larger forms live in the warmer seas.

Formerly another small group of bilaterally symmetrical Mollusca was also placed with the Gastropoda, namely, the Scaphopoda (genus Dentalium, Elephants-tusks, etc.), in which the elongate body is surrounded by a slightly curved, conical shell open at both ends. Further details of this group which is in many respects very aberrant and isolated in position, cannot, however, be given here.

\section{Class 2. Gastropoda.}

The structure of the Gastropods is most easily comprehended on the supposition that they have arisen by the modification of a Chiton in the following way (cf., Fig. $268 A$ and Fig. 239). The dorsal surface has become much arched, generally indeed drawn out into a long sac, the ventral portion of which is surrounded by the lower 
margin of the mantle-fold. At the anterior end of the sac, the mantlefurrow is much accentuated, forming a deep pouch, the mantlecavity, opening ventrally; posteriorly the furrow is shallow, just as

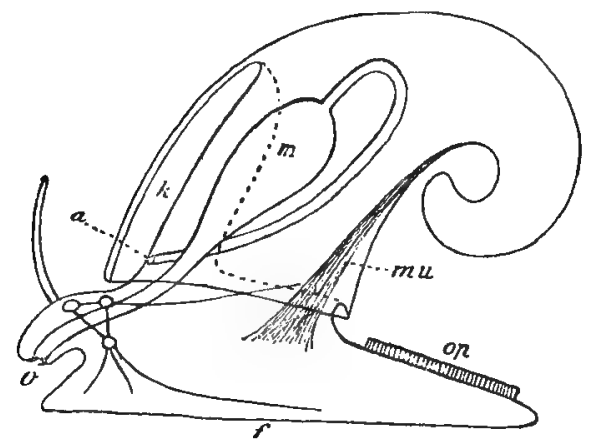

Fig. 239. Diagrammatic figure of a Gastropod seen from the left side (the shell removed). $a$ anus, $f$ foot, $k$ mantle cavity, $m$ stomach, $m u$ shell muscle, o mouth, op operculum. Besides the parts indicated by letters, certain portions of the nervous system are also drawn, viz, cerebral and pleural ganglia (seen above the cesophagus), and pedal ganglion (below the œsophagus). The dotted line indicates the boundary of the mantle chamber.-Orig. in terrestrial forms, can be invaginated like the finger of a glove, and withdrawn into the head, but in other forms, are only strongly contractile. In some Gastropods (Opisthobranchs), behind these there is another pair of tentacles, which may often be concealed within depressions on the head, and whose surface is frequently
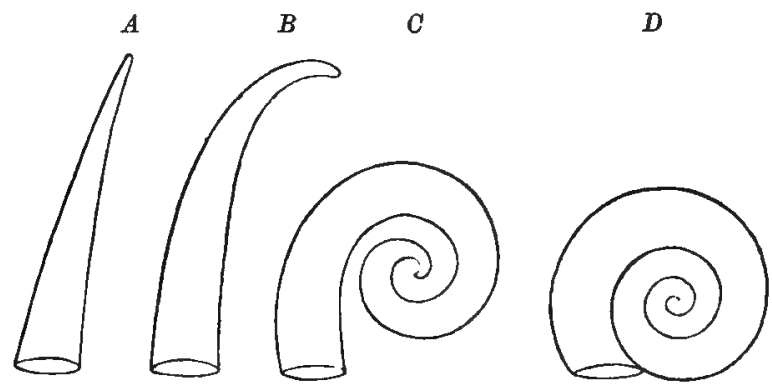

Fig. 240. Diagrams of various forms of Gastropod shells seen from the left side. $A-B$ slightly curved; $C$ spiral shell in which the successive coils do not touch; $D$ ditto, in which the coils lie closely upon each other (the common type).-Orig.

much folded; they are regarded as olfactory organs. Besides these, a pair of small eyes is generally situated upon the head; they are sometimes borne at the tips of special tentacle-like stalks (as in the Common Snail), but are usually placed directly upon the head 
itself or on the side of the tentacles. The foot is generally a flat, very contractile disc, occupying the whole veutral surface of the lower portion of the body.

The soft, thin-skinned visceral sac is covered by a tubular shell, open at one end, closed at the other, and becoming gradually wider towards the open end. Only in rare instances is the tube straight or slightly curved, usually it is a spiral, the concavity of which corresponds, in Gastropods, with the ventral side of the visceral sac (see Fig. 239). The individual coils of the shell almost invariably touch one another, and are, indeed, closely united. Some dis c o id a l shells form a flat coil, like a watch-spring; usually, however, the closed end of the tube is drawn out on one side, so that the axis of the shell describes a spiral round a cone. The form of most Gastropod shells is, therefore, conical, although many are widely aberrant from this, on account of the very different form of shelltube. If a shell be placed so that the axis of the cone stands perpendicular, with its apex (the closed end) uppermost, and the mouth towards the observer (Fig. 241), the aperture then either

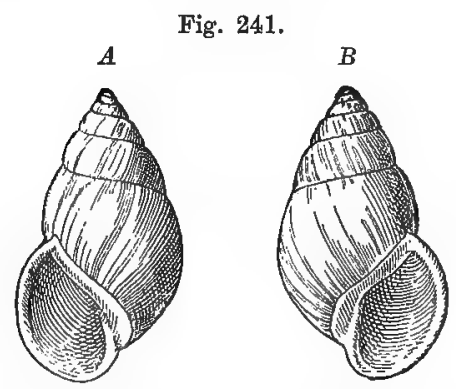

Fig. 241. The shells of two examples of a tropical land Snail (Bulimus perversus). A left-handed spiral. $B$ right-handed spiral.-After $\nabla$. Martens.

Fig. 242. Shell of a Snail (Paludina) in which a large part of the wall towards the observer is broken away. $n$ umbilicus, $s$ columella.-Orig.

Fig. 24.2.

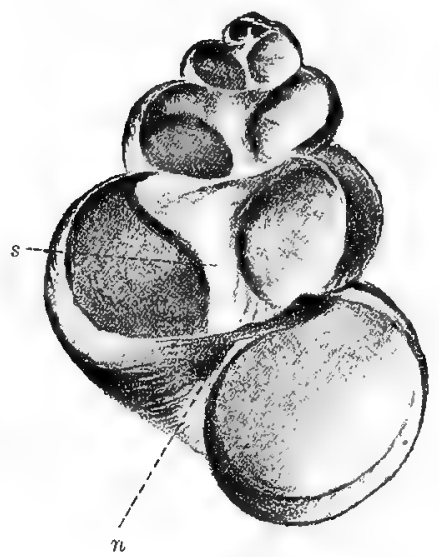

lies to the right of the axis, and the shell is said to be a right handed spiral; or it lies to the left of the axis, giving a left-handed spiral. The shell is borne by the animal in such a way, that its point, if a right-handed spiral, is towards the right (and points upwards and backwards) ; or if a left-handed spiral, towards the left (upwards and backwards). Right-banded spirals are much more common than left; in some species the one kind occurs, in others, the other ; as individual variations, examples of the left-handed spiral may be found among forms in which the right is normal; but it is very rare to find both forms equally common in the same species. In some 
Gastropods, the shell-tube is coiled in such a wry that a large cavity is left in the middle, surrounded by the turns of the shell, and open below; usually, however, the coils lie upon one another, so that this cavity, the umbilical-tube, is very narrow; and frequontly, its outer opening, the $\mathrm{umbilicus}$, is closed by the last coil. Those portions of the spiral which immediately surround the umbilical-tube, form a kind of central column, the columella.

Growth of the shell occurs as follows: new material is secreted by the thickened edge of the nantle, and is doposited at the rim of the aperture, and thus increase in length takes place; growth appears to be intermittent, in the course of a short time a large portion is added, then a longer period of rest occurs, and so on. The portion of newly-formed shell substance, which covers and grows on to the older coils, is usually thinner than the remaining free portion, and is often difficult to make out. In addition to this deposit at the mouth, calcareous material is also laid down within the shell, ov(er the whole surface of the visceral mass, and in this way an increase in thickness is effected. The small, oldest coils at the apex of the shell may, by this muns, become filled with lime: occasionally such portions get broken away. Bresides the new formation of calcareous material, a realsorption of the older part frequently occurs; for instance, it may occasionally be observed that before the period of growth begins, superficial portions of the old shell, near to the mouth, become loosened and worn away where the new shell will later be deposited; internally, too, an absorption of the concoaled portions of the shell takis place, by means of which the septa botween successive coils may be much thinned, or, completely dissolved. 'Tho Gastropod shell consists chielly of calcium carbonate, with a small amount of conchiolin; there is usually a thin, uncalcificd horny layor, superficially, which is easily removed.

Transverse sections of shells vary in form, they are rurely circular, usually somewhat compressed, occasionully even elliptical. In some instances, the younger coils may almost, or entirely, surround the older ones, so that the latter are nearly, or completely, hidden from without. The axis of the conical shell it sometimes long, sometimes short; in the latter case, the shell approaches a discoid form. In some cases the coils are very numerous, and the crons-Hecction of the tube increases gradially in size; in others there ant only a few turns, which increase rapidly in diameter. In not a few Gastropods, when growth has ceased, the mouth of the shell takes on a peculiar form, becomes thickened, and widened; in others, even in young animals, there is a peculiar rim (thickened and rough), round the mouth and at the close of ench period of growth an new rim is formed, so that the old mouths are indicated by special regions in the shell. (For. the notch in the shell for the branchial syphons, see below). The shells are often brightly coloured, and rough with finer or coarser sculpturings on the surfuce, etc. Sometimes the edge of the mantle is specially well developesl, wraps over the edge of the shell, and secretes a bright layer over the surface (e.g., in Cowries, Cyprea). 
In the majority of the Gastropods the shell is formed in the way described above; there are, however, exceptions. In some forms (e.g., Vermetus) it is coiled in quite an irregular spiral; this is correlated with its firm attachment to some foreign object; the regular spiral twisting of snail shells is connected with the fact that a regular spiral shell can be more easily carried than a long and straight or an irregular one. In some forms, which are tree-living whilst young, but sessile later, the shell is, at first, regular, but then grows straight or irregular. In others it is hardly possible to speak of a tube, the whole shell is a simple basin, and the visceral hump, a soft pad on the dorsal surface (Limpets, Patella). In many Gastropods the shell and viscera are rudimentary, or altogether absent; the viscera are then situated in the lower portion of the body. In some cases, in which the shell is rudimentary, or feebly developed, it is partially or completely enclosed in a fold of the skin.

The visceral sac, for the most part, lies freely within the shell, but it is firmly connected with it in several places, namely, where the columellar muscle arises from the columella. This muscle lies on the ventral side of the visceral sac, and thence runs into the lower portion of the body, which it withdraws into the shell when the animal is disturbed.

In many of the Gastropods there is a plate of conchiolin, or conchiolin and calcium carbonate, on the upper surface of the hinder portion of the foot. When the whole animal is retracted into the shell, the foot is drawn together in such a way that this plate lics below, forming a lid or operculum. The operculum is firmly fused to the animal beneath, and grows by the secretion of new material from the skin; sometimes growth occurs in such a way that the operculum displays a spiral line on its upper surface; this, however, is only rarely the case.

The true operculum which is firmly united to the animal, and gradually increases in size, must not be confounded with the winter operculum (epiphragma). The latter is usually as thin as paper, occasionally somewhat thicker; and occurs, e.g., in species of Helix, being very thick and firm in $H$. pomatia. When the animal is about to hibernate it withdraws into its shell and the epiphragm is formed at the mouth. It consists of a hardened calcified mucus, and is not attached to the animal, but at the end of the winter is thrown off, a new one being formed each year.

The skin is soft and slimy; the mucus is secreted by unicellular glands which open in great numbers on the surface of the body. In many of the Pulmonata, larger skin glands, which also secrete mucus, are present. In the Prosobranchiata there is a peculiar patch of glandular epithelium on the inner side of the mantle, the so-called "mucous-gland." In some Gastropoda, in addition to mucus, these glands also secrete a fluid, "purple," which, under the action of light becomes of a permanent violet colour. 
The central nervous system consists of paired cerebral, pedal, and pleural ganglia, and a varying number of visceral ganglia, which are connected with one another as shown on p. 289 . In the

$A$
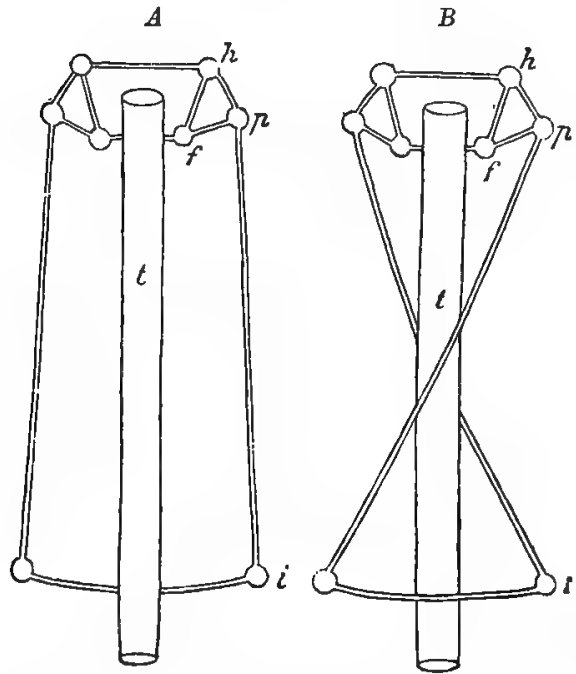

Fig. 213. Diagram of the central nervous system in relation to the alimentary canal $(A$ in an Opisthobranch, $B$ in a Prosobranch). $h$ cerebral, $p$ pleural, $f$ pedal, $i$ visceral-ganglia; $t$ alimentary canal.-Orig. position of most of the organs. Of sense-organs, the eyes have already been mentioned; for the auditory organs the account given on p. 290 for the Mollusca in general may be referred to. In most of the Gastropods there is, within the mantle-cavity, a specialised portion of skin covered with a peculiar epithelium, often folded and richly supplied with nerves; it lies near the gill, and when two gills are present two such osphradia also occur. There is no doubt that they are sense organs, and they are considered to be olfactory.

With regard to the alimentary canal, it is important to notice that the anus usually lies on the right side* of the mantlecavity quite asymmetrically; only in a few isolated forms among those Gastropods in which the shell is lost, is it symmetrical in position (see the Opisthobranchs). In the mouth there is a $\mathrm{radula}$

* Just as in other animals which are asymmetrical in some respects (e.g. Mammalia), so in Gastropoda, such a departure from the usual arrangement of organs occurs, that all which are usually right come to lie on the left side, and conversely (inversio viscerum); the anus is left instead of right and so on. This happens som etimes in the case of forms with shells in a left-handed spiral; but there are forms with such shells in which the torsion has not occurred, even the anus being on the right. 
The central nervous system consists of paired cerebral, pedal, and pleural ganglia, and a varying number of visceral ganglia, which are connected with one another as shown on p. 289. In the

$A$
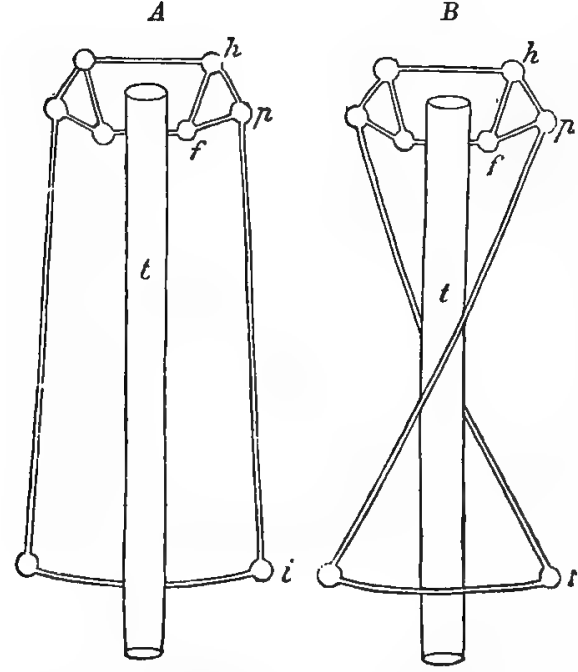

Fig. 243. Diagram of the central nervous $(A$ in an Opisthobranch, $B$ in a Prosobranch). $h$ cerebral, $p$ pleural, $f$ pedal, $i$ visceral-ganglia; $t$ alimentary canal.-Orig. system in relation to the alimentary canal

opisthobranch and pulmonate Gastropods, the visceral cord runs posteriorly, forming a loop between the two pleural ganglia and lying below the digestive tract throughout the whole of its course. In all the Prosobranchiata on the other hand, the visceral loop twists round the digestive tube in a peculiar way; the nerve cord arising from the left pleural ganglion runs below the gut, then crosses above the gut to the left side, and then again crosses this time above the gut to run anteriorly, ending in the right pleural ganglion (Fig. $243 \mathrm{~B}$ ). This peculiar arrangement of the nervous system presupposes changes in the position of most of the organs. Of sense-organs, the eyes have already been mentioned; for the auditory organs the account given on p. 290 for the Mollusca in general may be referred to. In most of the Gastropods there is, within the mantle-cavity, a specialised portion of skin covered with a peculiar epithelium, often folded and richly supplied with nerves; it lies near the gill, and when two gills are present two such osphradia also occur. There is no doubt that they are sense organs, and they are considered to be olfactory.

With regard to the alimentary canal, it is important to notice that the an us usually lies on the right side* of the mantlecavity quite asymmetrically; only in a few isolated forms among those Gastropods in which the shell is lost, is it symmetrical in position (see the. Opisthobranchs). In the mouth there is a $\mathrm{radula}$

* Just as in other animals which are asymmetrical in some respects (e.g. Mammalia), so in Gastropoda, such a departure from the usual arrangement of organs occurs, that all which are usually right come to lie on the left side, and conversely (inversio viscerum); the anus is left instead of right and so on. This happens s om etimes in the case of forms with shells in a left-handed spiral; but there are forms with such shells in which the torsion has not occurred, even the anus being on the right. 
In the Gastropods there is usually one saccular kidney, opening into the pericardium, and also to the exterior ; if a mantlecavity is present, the kidney opens into it, otherwise on the right side of the animal. It is usually much folded on its inner surface, and is sometimes much branched. Occasionally there are two kidneys.

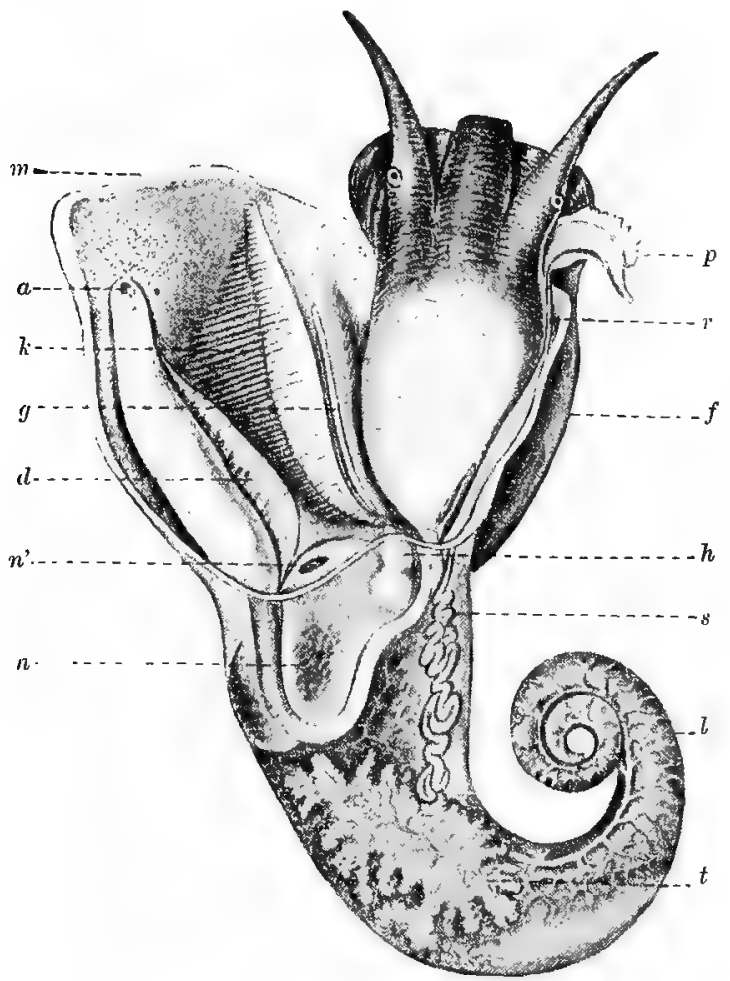

Fig. 244. Male Peri winkle (Littorina) removed from the shell and viewed from above; mantle cut along the right side and turned over to the left. $a$ anus, $d$ mucous gland, $f$ foot, $g$ osphradium, $h$ heart, $k$ gill, $l$ liver, $m$ edge of the mantle, $n$ kidney, $n$ ' urinary aperture, $p$ penis, $r$ seminal groove, $s$ seminal duct, $t$ testis.After Souleyet, modified.

The generative organs (Fig. 246) differ very considerably in the different groups ; they have, however, this in common, that the genital aperture is almost always on the right side, and usually within the mantle-chamber when this is present. The $\mathrm{Prosobranchiata,}$ which are almost invariably of separate sexes, display the simplest arrangement. In these the ovary and testis, one of each alone being present, are exactly alike externally. The oviduct is a convoluted tube, widened in one region, and opening into the mantlecavity. The seminal duct in most opens in the same position as the oviduct, and from the genital aperture a groove runs along the surface of the body to the penis, which is situated on the right side of the head; the groove runs down the copulatory organ to its apex. In others this seminal groove has become a closed canal, 
and the sperm duct opens only at the apex of the penis, which cannot be withdrawn by invagination, as in other Gastropoda. Glandular appendages of the sexual organs are usually wanting in the Prosobranchiata. The Opisthobranchiata and Pulmonata are hermaphrodite, and ova and spermatozoa are formed in one and the same organ, the hermaphrodite

Fig. 245. Female Periwinkle (Littorina) dissected as Fig. 241; some portions of the body-wall and some of the organs (kidney, pericardium), are also opened. $a$ anus, ge genital pore, $h$ ventricle, ma stomach, $n$ kidney, od oviduct, ov ovary, sd salivary gland, $s p$ cesophagus, $v$ auricle, $z$ spirally coiled radula sac.-After Souleyet, modified.

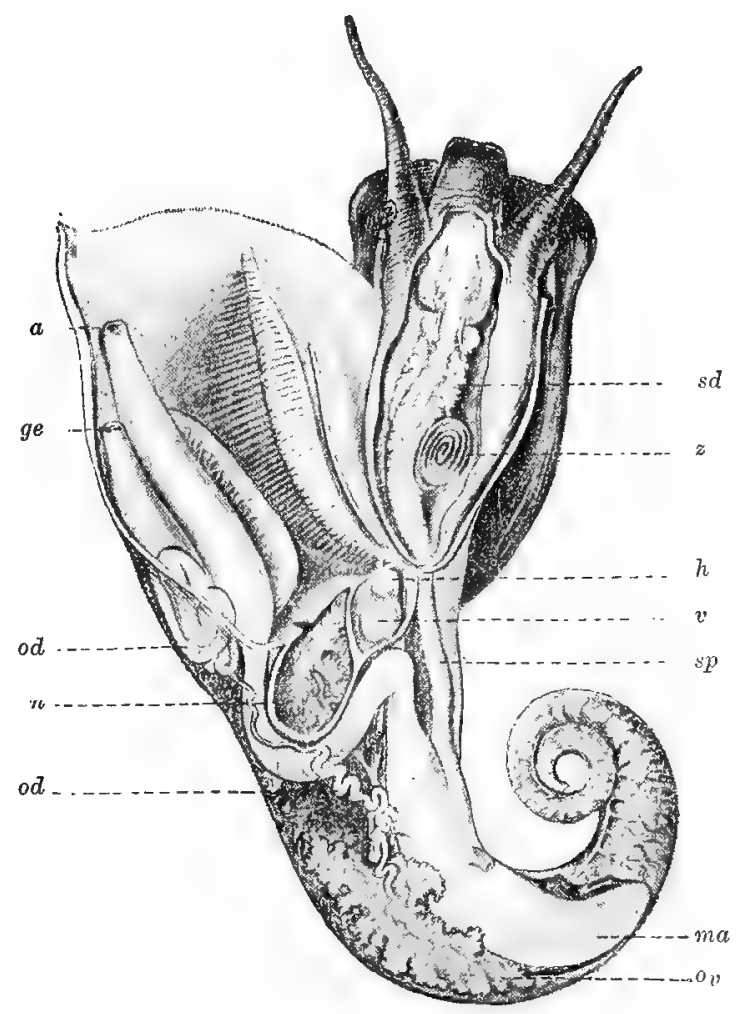

gland. The duct is in some of the Opisthobranchs common to both ova and spermatozoa, and then a common genital aperture is present; from this a groove runs along the skin to the apex of the copulatory organ. In most Opisthobranchiata and in the Pulmonata the duct is common only to a certain extent, for it splits a short distance from the hermaphrodite gland into two canals, an oviduct and a vas deferens, which usually open close together, the vas deferens at the apex of the penis, which is here furnished with an actual canal, not simply with a groove. The penis can be invaginated in all Pulmonates and Opisthobranchs. In these groups, especially in the air-breathing 
snails, the sexual apparatus possesses numerous accessory organs, albumen glands (which manufacture the albumen to surround the eggs), mucous glands (which secrete mucus during copulation), spermathecæ, etc.* Copulation in the hermaphrodite Gastropods is reciprocal.

$A$

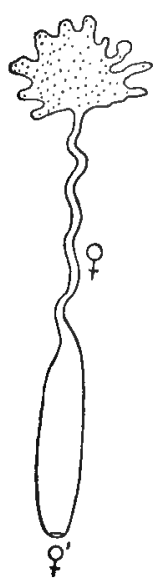

$B$

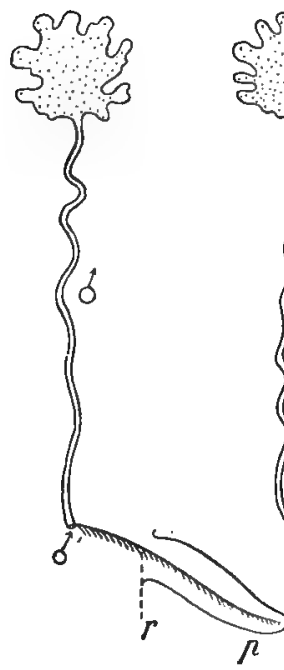

C

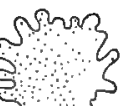

$D$

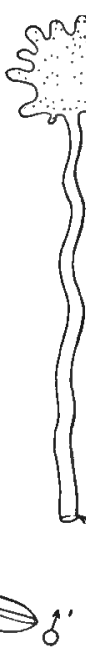

$E$

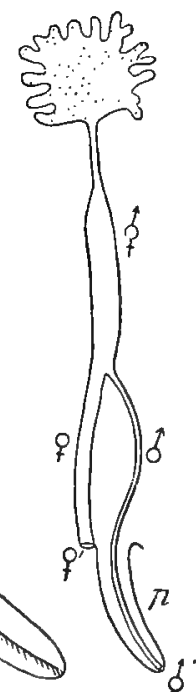

Fig. 246. Diagram of the genitalia of various Gastropoda. Sexual gland in each case dotted. $A-C$ Prosobranohiata, $A$ female, $B$ and $C$ males; $D$ Opist $h$ obran. chiata; $E$ Opisthobranchiata and Pulmonata. $\%$ oviduct, ${ }^{\prime}$ female genital aperture, $\delta$ sperm duct, $\delta^{\prime \prime}$ male genital opening, ${ }^{*}$ hemaphrodite duct, $p$ penis, $r$ seminal groove.-Orig.

The terrestrial Pulmonata lay their eggs in the earth, each egg is surrounded by a large mass of albumen which is covered by a more or less calcified, round or oval, s hell; the latter is very like that of a Bird's egg. In the freshwater Pulmonata each egg is similarly covered by a mass of albumen and a very thin transparent outer shell, but a number of such eggs lie in a common $\mathrm{m}$ a s $\mathrm{s}$ of jelly (adhering to plants, etc.); those of the Opisthobranchs, and of some of the Prosobranchs, are similarly arranged; in most Prosobranchs (e.g., the Whelk), however, several eggs lie without separate shells in a common mass of albumen, which is surrounded by a leathery capsule; the capsules, which often adhere together in groups, are of very diverse, and often strange shapes. Several of

* In some terrestrial Pulmonata (eg., the common species of Helix) there is a "dart-sac," an evagination of the oviduct close to the external genital aperture, in which a calcareous body, somewhat of the form of an arrow, the "dart," is secreted; it is extruded during copulation, and is to be regarded as an excitatory organ. 
the Prosobranchiata and Pulmonata are viviparous, the eggrs developing within the oviduct.

The eggs of Gastropods are always small, and undergo total segmentation. In the Prosobrauchs and Opisthobranchs the larva passes through a metamorphosis; when it leaves the egg it usually possesses a well-developed velum, by means of which it moves about in the water: the foot on the contrary is only feebly developed. The young Pulmonates have no velum, and undergo no such transformation.

Amongst those Prosobranchs which lay egg capsules containing many eggs, a few, or only one in each capsule develops, the undeveloped eggs are swallowed by the young animal as it swims about in the albumen. Frequently the young of such forms undergo metamorphosis within the egg-capsule, which they leave when the velum has disappeared, the foot is formed and the body has reached a considerable size (Whelk, etc.).

The Gastropods are, for the most part, creeping animals, gliding over surfaces by means of undulatory contractions of the foot; not a few (e.g., freshwater forms) have the power of at-

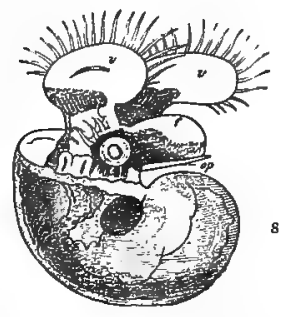

Fig. 247. Larva of a Gastropod (Opisthobranch). $f$ foot, $h$ auditory organ, op opercuculum, $s$ shell, $v$ velum. taching themselves, so to speak, to the surface of the water with the foot upwards, the visceral mass hanging below, and, in this position, they are able to move slowly along. Some smaller divisions of marine Gastropods are distinguished by the fact that they can effect actual swimming movements by means of the modified foot, or by some special organ. The majority of the Gastropods (the Opisthobranchs, and by far the greatest number of the Prosobranchs) live in the sea, not a few in fresh water (some Pulmonata and some of the Prosobranchiata), many are terrestrial (the majority of the Pulmonata and some of the Prosobranchiata).

The following table gives a summary of the chief characters of the Gastropoda:

Prosobranchiata.

Sexes separate.

Visceral loop in the form of an 8.

The auricle anterior to the ventricle.

Respiration (usually) by a gill.

Penis projecting freely.

Metamorphosis;

Opisthobranchiata.
Hermaphrodite.
Visceral loop, U-shaped.
The auricle usually poste-
rior to the ventricle.
Respiration by gills.
Penis capable of invagina-
tion.
Metamorphosis.

Pulmonata.

Hermaphrodite.

Visceral loop, U-shaped.

The auricle anterior to the ventricle.

Respiration by a pulmonary chamber.

Penis capable of invagination.

No Metamorphosis. 


\section{Order 1. Prosobranchiata.}

To this division, the essential characters of which are set forth in the preceding table, belong the majority of the shell-bearing marine Gastropoda, some of the fresh-water forms and some of the air-breathing terrestrial forms. Practically all the Prosobranchiata possess a shell, usually a well-developed spiral, with or without a notch or canal; occasionally the shell is cap-like. An operculum is usually present. There is generally a single gill within the mantle-cavity. Some are herbivorous, others feed upon living or dead animals.*

1. Marine forms. This division is represented by numerous forms; in the tropics, especially, there are many large and beautiful species; in colder seas they are also numerous, but mostly small and inconspicuous. Among those very common on British coasts may be mentioned: the Periwinkles (Littorina), small, thick-shelled Gastropods, without the syphonal noteh, which may be found in great numbers on rocks close to the shore: the $\mathrm{Whelk}$ (Buccinum undatum), a large form, with a short syphonal canal, living in somewhat deeper water, and much used for bait: the Pelican's F oot (Aphorrhais pes pelicani), the mouth of whose shell is prolonged into several claw-like processes: Limpets (Patella), forms with basin-like shells, which remain for a long time on the same spot.

2. Fresh-water forms. The following forms occur in Britain and elsewhere: the River Snail (Paludina vivipara), tolerably large (as much as $4 \mathrm{c} / \mathrm{m}$. high), with conical shell (Fig. 242), viviparous; the young when born are similar to the adults, and nearly as large as peas; the eggs are each' enclosed in a capsule containing a rich supply of albumen, and remain in the much-widened oviduct of the female; metamorphosis occurs within the egg-capsule. The allied genus Bithynia is oviparous, and comprises smaller forms. Another species, Neritinia, belongs to a family also met with in the sea; the shell is hemispherical, the inner edge of its opening flattened.

3. Cyclostoma elegans is a terrestrial form occurring in Great Britain; it breathes by means of a lung, but may easily be distinguished from the Pulmonata by the presence of an operculum.

The Heteropoda constitute a peculiar pelagic group of the Prosobranchiata. They are almost transparent and large-eyed, with a large compressed foot, by means of which they move through the water; the foot is a perpendicular muscular plate, with a sharp ventral edge, and has only retained the ordinary gastropod condition at one spot, in the form of a sucker situated at its edge (the sucker may, however, be absent). In some cases the visceral hump is very well developed, and enclosed in a compressed discoid spiral shell ; the foot carries an operculum, and can be withdrawn into the shell. In others (Fig. 248) the visceral sac is small, and provided only with a cap-shaped shell, whilst the lower portion is relatively huge, has no operculum, and naturally cannot be withdrawn into the shell. Lastly, there are some in which the visceral mass is still

* In certain Prosobranchiata (Natica) there is, on the lower side of the proboscis, a sucker-like area, the epithelium of which secretes an acid. When the animal lays this against the shell of another Molluse it forms a hole in the latter, through which the proboscis can enter to devour the soft portions of the prey. 
smaller, and which possess no shell at all. As larvæ all have shell and operculum. The Heteropoda are actively predaceous, and swim with the ventral side turned upwards; they occur in all warm seas; various forms, for instance, are found in the Mediterranean.

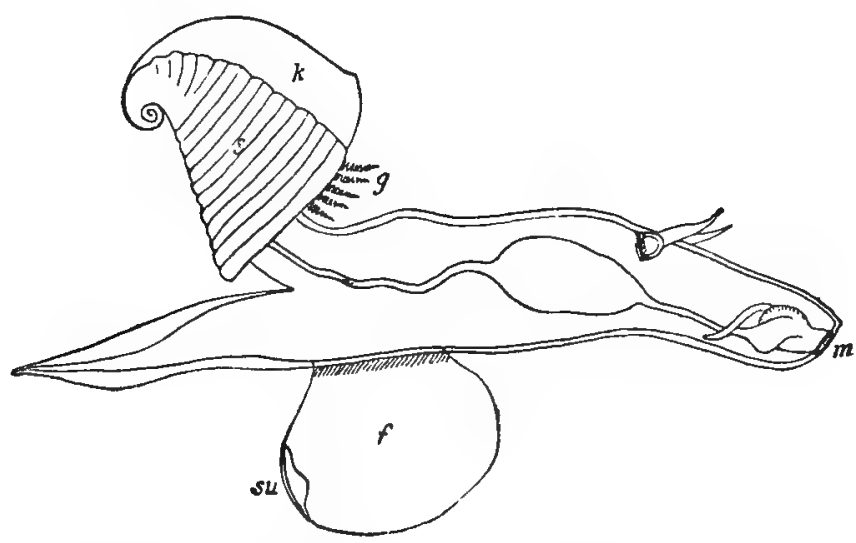

Fig. 248. One of the Heteropoda (Carinaria). $f$ foot with a posterior sucker (su), $g$ Igill projecting from the mantle eavity, $m$ mouth, s shell, $k$ keel on the shell.-After Souleyet.

\section{Order 2. Opisthobranchiata.}

Some Opisthobranchs are provided with a visceral hump ; a shell, which is usually spiral; a mantle-cavity enclosing a gill; sometimes, also, like the Prosobranchiata, with an operculum : often, however, the shell is somewhat aborted, and most of the members of the group (Nudibranchiata) have completely lost it, and with it the visceral hump and mantle-cavity; the viscera have sunk into the lower portion of the body; the nephridial and genital apertures, and frequently the anus also, are situated on the right side above the foot. In the Nudibranchs, as a rule, the ordinary gill is absent, and is then generally replaced by special outgrowths of the skin, varying in form. It is particularly interesting that the nudibranch larva is furnished with shell and operculum; both being later thrown off. All Opisthobranchiata, of which the naked forms usually display gorgeous colours, are marine, occurring in cold as well as in warm seas.

1. Of shell-bearing Opisthobranchs may be mentioned: the Bubble (Bulla), with bulging shell, the apex of which lies in a depression; common in warmer seas, allied genera in Northern seas. In some allied forms the shell is surrounded by folds of skin; these fuse with one another, and may com. pletely enclose the shell, which is then always thin (internal shell); such, for instance, is the case in the genus Aplysia (Sea-hare), which inhabits the Mediterranean and other warm seas, and is also met with on the sounth coast of England, 
2. Among the Nudibranchiata, the Doridida (genus, Doris, etc.) are peculiar in that the anus is situated posteriorly on the dorsal surface, and

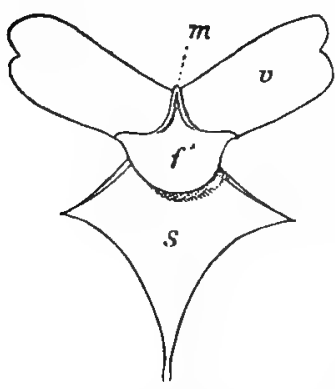

Fig. 249. A Euptero. pod (Cleodora). $m$ mouth, $f^{\prime}$ posterior portion of foot, $v$ fin, $s$ shell.-Orig. surrounded by a circle of plumose gills. The Folidr (genus, AFolis, etc.) have unbranched gills dorsally; a process extends into each from the livex, which is much branched and not sharply marked off from the gut. Many Nudibranchs are without gills (e.g., Elysia, Limapontia), and often have a striking superficial resemblance to the Flatworms. All the forms mentioned are met with in Northern seas.

To the Opisthobranchiata belong two aberrant groups of pelagic animals, which have usually, but not quite correctly, been placed together under the name, Pteropoda. The first of these divisions, the Thecosomata (Eupteropoda), is distinguished among other things by the breadth of the anterior muscular portion of the foot, which forms a pair of fin-like locomotor organs; the posterior region $\left(f^{\prime}\right.$, Fig. 249) is covered on its under surface with long close-set cilia, by which microscopic organisms are driven into the mouth. The mouth lies anteriorly between the fins, and is surrounded by a pair of lip-folds, which

Fig. 250 .

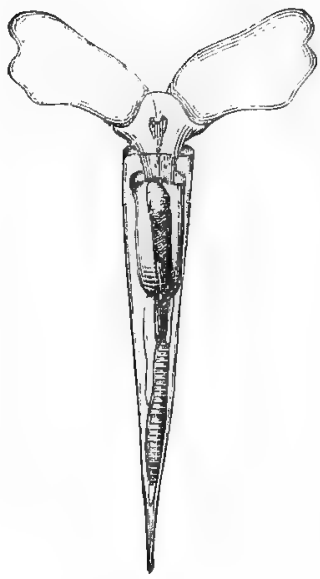

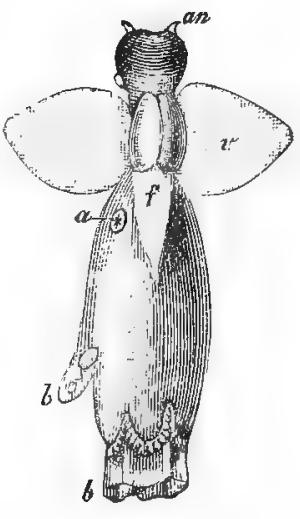

$A$
Fig. 251.

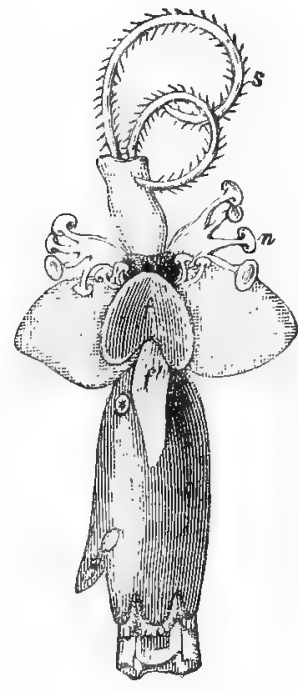

$B$

Fig. 250. One of the Eupteropoda (Cleodora) with simple tubular shell.-After Souleyet.

Fig. 251. One of the Pterota (Pneumodermon). In $B$ the arms are furnished with suckers and two hooked processes are projecting; in $A$ these are all withdrawn. $a$ anus, $a n$, tentacles, $b$ and $b^{\prime}$ gills, $f-f^{\prime}$ foot, $v$ fin.-After Souleyet.

unite in part, and thus prevent the escape of the animalculre secured by the current of the cilia. The visceral mass is well developed, and enclosed in a shell, which, in some forms, is spirally twisted (and then an operculum is usually present), but in the majority, is straight, or slightly curved and symmetrical. 
The Eupteropoda are among the most abundant and most characteristic pelagic animals; they are blind, and are specially frequent towards evening on the surfacewaters, both in warm and cold seas. The other group assigned to the Pteropoda, the Gymnosomata (Pterota), have no shell; they have a small, often almost rudimentary, foot; and move by means of two special fin-like muscular appendages, which are situated anteriorly, close to the foot, but do not represent any portion of it. Various organs of prehension, "arms" with suckers, etc., may extend from the mouth of these animals, (Fig. $251 \mathrm{~B}$ ); the Pterota are most predaceous, and attack in particular the defenceless Eupteropoda, whose distribution corresponds with their own. Clione limacina, a well-known species, occurring abundantly on the coast of Greenland, and attaining a length of as much as $4 \mathrm{c} / \mathrm{m}$., feeds upon a Eupteropod with a spiral shell, Limacina helicina.

\section{Order 3. Pulmonata.}

In the Pulmonata, just as in the Opisthobranchiata, some formsthe majority, indeed-carry shells, and possess a well-developed visceral hump, whilst others are naked, and have no such hump, the viscera being sunk into the lower portion of the body. 'The two groups differ, bowever, in that in the Pulmonata, even if the shell and visceral sac be absent, the mantle-cavity is still present on the upper surface of the animal as a pouch, covered by a shield-shaped mantle; the inuer side of the mantle in the naked, as well as in the shell-bearing, forms, is provided with a rich vascular network. The opening which leads into the mantle-chamber is not, as in other Gastropoda, a broad slit, but is merely a pore on the right side; the shell never has a syphonal notch, and an operculum is wanting. Between the shellbearing and the naked Pulmonata there is a complete series of intermediate forms; in some, the shell is not large enough to contain the whole animal, unless the air be dry, for the molluscan body increases in size in damp air ; there are others possessing a regularly formed shell, which is, however, so small, that it only covers the reduced visceral hump, whilst the rest of the animal can never be withdrawn into it; or the visceral sac has completely disappeared, and the small plate-like shell covers the mantle only; again, the shell is a small thin plate which lies beneath the mantle; ${ }^{*}$ or it may be only represented by isolated calcareous granules, which lie attached to the mantle (the latter is the case, for instance, in the Great Blackslug); or it is entirely absent. The Pulmonata live on land and in fresh water, and feed chiefly upon vegetable substances. As already mentioned, they breathe air; some Fresh-water Snails (Limnoeus), however, possess, especially in youth, the power of taking water into the mantle-chamber, and obtaining the dissolved oxygen from it.

* In one form (Parmacella), the young animal possesses a small external shell, which, later, becomes covered by the mantle, so that the older animal possesses an "internal" shell. In other forms, an "internal" shell occurs in youth, and is dependent upon similar occurrences in embryological stages; the sac in which the shell lies, is an invagination of the skin. 
1. The terrestrial Pulmonata (Stylommatophora) are characterised by the position of the eyes, which are at the tips of eye-stalks, exactly similar to the tentacles, and like these, capable of invagination into the head. To this group belong both shell-bearing and naked forms. Among the former may be mentioned the genus Helix, to which the small Garden Snail (H. hortensis), and the large Edible Snail (H. pomatia) belong; among the latter are the Great Black-slug (Arion ater), and the smaller, destructive, grey, Common Slug (Limax agrestis).

2. The freshwater Pulmonata (Basommatophora) have sessile eyes, situated at the base of the tentacles, which cannot be invaginated. To this group belong the numerous species of the Pond Snail (Limnsus), with pointed shell, and of Planorbis, with discoid shell; both genera are common everywhere in fresh water, and are represented by large and small species: some of the Limnæidx lead an amphibious life, being met with on land as well as in water.

\section{Class 3. Acephala (Lamellibranclis).}

The body of the Lamellibranchs, unlike that of the Gastropoda, is usually bilaterally symmetrical, except as regards the coils of the alimentary canal. The anus is posterior, the genital and nephridial apertures, etc., are symmetrically disposed. The following is a general account of the structure of a Lamellibranch: the body proper is rather small in comparison with the whole size of the animal; the mantle-fold is developed on either side in the form of a large lamina, dependent like a curtain. On each side, also, just below the point of origin of the mantle, two gill la $m e l l æ$ arise and hang down within it. The foot is ventral,

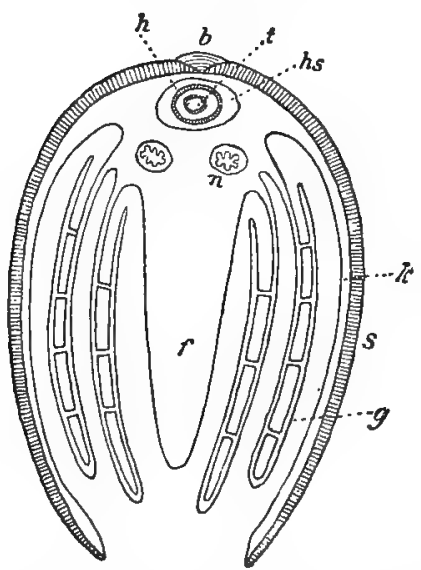

Fig. 252. A transierse sec. tion through a Lamelli. branch (diagram). b ligament, $f$ foot, $g$ gill, $h$ heart, $h s$ pericardium, is mantle, $n$ kidney, $s$ shell, $t$ gut.Orig. by means of muscles arising from the inner surface of the shell. and is usually keel-shaped. There are four large labial palps in front of the mouth. A specialised head is absent. The whole animal is generally enclosed in a symmetrical, laterally compressed, bivalve shell.

The foot is, as a rule, not very well marked off from the trunk; most tudinal keel on the ventral surface. In some forms it is longer and more projecting (sometimes geniculate) ; in a few cases it is linguiform (Mussels); in others there is a true pedal disc. It is the most important locomotor organ of the Lamellibranchs, and can be protruded from the shell by being distended with blood, whilst if the animal is disturbed, it is withdrawn often it is only a compressed longi- 
Iu a few forms (e.g., the Oyster) it is altogether wanting; in others it is rudimentary or small.

Each gill is a lamella, the upper edge of which is attached to the body; the lower portion, almost half the lamella, is bent round* and closely apposed to the upper half (just as one half of a folded sheet of paper lies upon the other); in the outer gill the reflexed portion lies external to, in the inner gill internal to, the upper portion. The edge of this reflexed portion is, in some cases, free, in others it is concrescent with the body close to the point of origin of the gill lamella, either throughout its whole extent or for part of its length only. The gill lamella consists of delicate filaments extending dorso-ventrally; they are sometimes free, only adhering to one another by "ciliated junctions" (Mytilus), but are usually firmly united by interfilamentar junctions, so that the gill lamella shows a trellis-work. The reflexed portion of the gill lamella is usually united with the upper portion by similar "ciliated junctions" (Fig. 252). On the surface of the gill lamellæ is a thick covering of cilia, by which the water is kept circulating through the meshes, and small, solid bodies are conducted towards the mouth by means of the ciliary currents on the surfaces of the gills.

In some Lamellibranchs (Fig. $253 \mathrm{~A}$ ), each gill lamella projects behind the foot in a free point; the two points of the same side have, however, undergone concrescence along their dorsal edges. $\dagger$ In others the point has become concrescent at its hindmost tip, with the posterior part of the mantle (Mussels). In others again (Fig. $253 \mathrm{~B}$ ), the right and.left inner portions of the gills coalesce behind the foot along their reflexed edges, and similarly the outer gills with the mantle; in this case, in the posterior region of the mantle-cavity, an upper portion of the cavity (suprabranchial) is separated from the rest, and extends into the cloaca.

The mantle is divided into

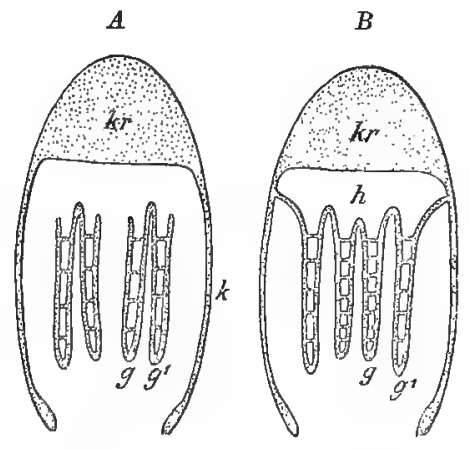

Fig. 253. Transverse sections through the posterior regions of two Lamellibranchs after the removal of the shells, to show the relations of the $h$ inder ends of the gills. $k r$ body, $k$ mantle, $g$ inner, $g^{1}$ outer gill, $h$ suprabranchial portion of the mantle cavity. -Orig. two symmetrical portions, a right and a left mantle-lobe, which lie external to the gills. Each lobe is a thin lamina, the edge of which, the pallial muscle,

* Only in a few Lamellibranchs are the gills not reflexed.

+ The two gill lamellæ of each side, in all probability, together represent a plumose gill with two series of filaments; sometimes the two lamellæ originate from a common basis. 
is, however, somewhat thicker, and provided with transverse muscle fibres. The upper ends of these fibres are firmly attached to the so-called pallial line (sue below) on the inner side of the shell, and by their contraction withdraw the margin of the mantle within its edge: along the free edge occur numerous tactile tentacles. The edges of the two mantle lobes are perfectly free from each other; in a few forms only (e.g., the Oysters), as a rule, they are partially cuncrescent, so that the large opening between them is divided into two or several divisions. In the simplest cases (e.g., in the Mussels), only the most posterior portion is separated from the rest, the edges of the mantle being connected for a short distance; in

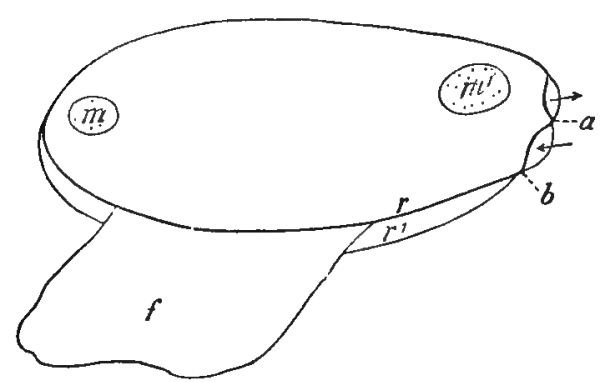

Fig. 254. A L a mell i b a $\mathrm{n}$ ch removed from the shell, seen from the left side. $a$ and $b$ points of concrescence along the edge of the mantle, $r$ left, $r^{2}$ right edge of mantle, $f$ foot, $m$ and $m^{1}$ ends of the adduetors.--Orig. water, the mantle-lobes having concresced for a longer or shorter distance; * thus the originally single slit is divided into three:

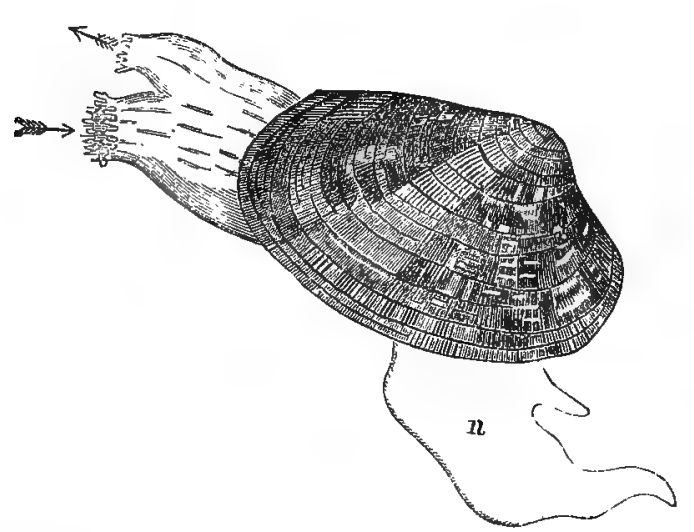

Fig. 255, A Lamellibranch (Tapes decussatus) with partially separated syphons. The arrows indicate the direction of the streams of water. $n$ foot. such there is a small posterior opening, the cloacal aper ture, through which water and excreta escape from the mantle-cavity, and a very large opening through which water streams in, and the foot can be protruded. In others (Fig. 254) the large opening just mentioned is divided into an anterior large aperture for the foot, and a posterior smaller pore for the inhalent current of posteriorly the cloacal aperture, below this the inhalent a perture, through which the water streams in, and anterior to, and below, this, the pedal or i fice, through which the foot may be protruded, the last being much larger than the other two. The cloacal and inhalent apertures are often drawn out into two tubes, the cloacal and res-

* The edges of the mantle may lie closely apposed in these regions withoụt pndergoing concresscence. 
piratory syphons, which can be protruded from the shell, and may attain a considerable length; usually they are connected together, and then appear externally like a single tube (sometimes forked at one end), but this is divided internally into two by a septum; they are rarely separated externally. In the forms with these syphons the edges of the mantle have often undergone concrescence over so large an extent that the pedal opening is considerably decreased.

The shell lies external to the mantle, but closely upon it. It is divided into two halves; connected above by a flexible band, the ligament, which will be further described below. The two halves of the shell are, as a rule, essentially similar; they are more or

$A$

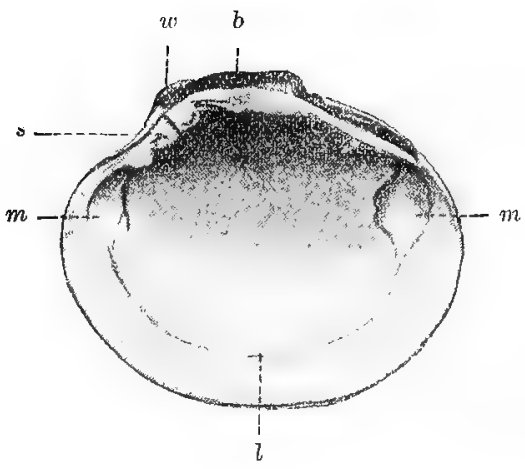

$B$

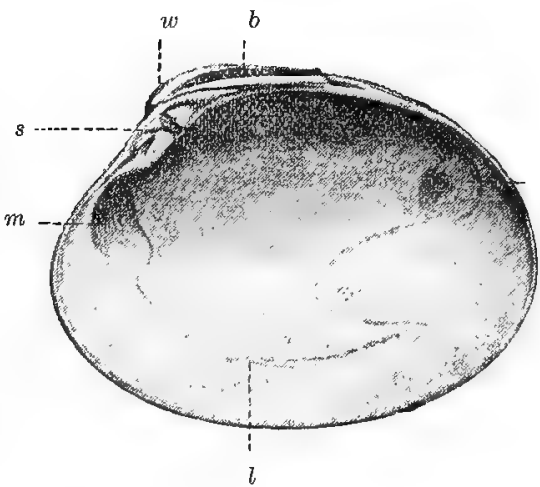

Fig. 256.-Right she 11 valves of two different Lamellibranchs, seen from within. $A$ with simple, $B$ with incurved pallial line, $b$ ligament, $l$ pallial line, $m$ impressions of adductor muscles, $w$ umbo, s hinge.-Orig.

less convex, and usually exhibit a projecting hump dorsally, the so-called $\mathrm{um} \mathrm{bo}$ (the oldest portion of the shell), which generally lies nearer to the anterior than the posterior end. The upper edge of each valve usually possesses a tooth or ledge, which fits in between corresponding portions of the other half, forming a hinge (c a r do); in not a few this is absent, or only feebly developed. When the shell is closed the edges of the valves fit closely together, so that the soft parts of the animal are completely shut in : occasionally, however, the shell is cleft in one or more places, posteriorly ; for instance, in those which are provided with large syphons, and anteriorly in those which are attached by means of a byssus (p. 311). The closure of the shell is effected by adductor muscles, usually two in number, which run transversely across the animal, one in the anterior, the 
other in the posterior, region, and are attached to the interior of the shell ; occasionally one such muscle only is present (e.g., in the Oyster). The attachment of an adductor to the shell is indicated by a sharply defined area, a muscular impression, of which there are two on the inner side of each valve. Besides these, there are frequently smaller i m pressions, which correspond with the attachments of the pedal muscles; further, the so-called pallial line, from which the muscle-fibres of the pallial muscle arise; in those Lamellibranchs which have no syphons, it runs parallel to the edge of the shell some distance from it (integripalliate) ; in those which have syphous it usually traces out a notch, extending from behind forwards (sinupalliate) (Fig. 256, B); and along this the muscles of the syphons arise, being specially developed portions of the pallial muscle; since these muscles arise more anteriorly, the syphons can be withdrawn into the shell. Three layers may be usually distinguished in the shell: an outer horny layer in some (e.g., Mussels), very definite; in others, indistinct; and two layers consisting principally of calcium carbonate, which make up the chief part of the shell; of these, the inner layer is sometimes iridescent (mother of pearl).

The flexible band which holds the two valves of the shell together, the so-called ligament, consists of an outer, flexible, but inelastic layer (an extension of the outer layer of the shell), and an inner, elastic portion of radially arranged fibres. In many forms, this ligament has a large, very convex surface, exteriorly; and is termed an external ligament: in others, it is enclosed between the upper edges, displaying a very small portion externally, whilst it is convex inwardly, and is then termed an internal ligament. Its action is essentially the same in the two cases ( $c f$. , Fig. 257). If a shell, furnished with an internal ligament, is closed by the contraction of the adductor muscles, the inner elastic portion of the ligament is compressed, and when the muscles relax, the valves of the shell are once more driven apart. In the shells provided with an external ligament, the elastic substance of the ligament undergoes a compression, or arching together, within the outer inelastic sheath, and on the relaxation of the muscles, the effect is the same as in shells with internal ligaments.* The action of the ligament is purely mechanical, and occurs after the death of the animal just as in life. It must also be mentioned that its position is either directly below the umbo, or more usually, posterior to it. Only in very incompletely developed shells (e.g., Teredo) is the ligament altogether wanting, and the shell-valves completely separate.

* The usual statement, that a stret ch in g of the external ligament must occur on closure of the shell, is incorrect. 
The shell increases in size by the addition at its margin of new material, secreted by epithelial cells at the edge of the mantle; it increases also in thickness, since the outer surface of the mantle,

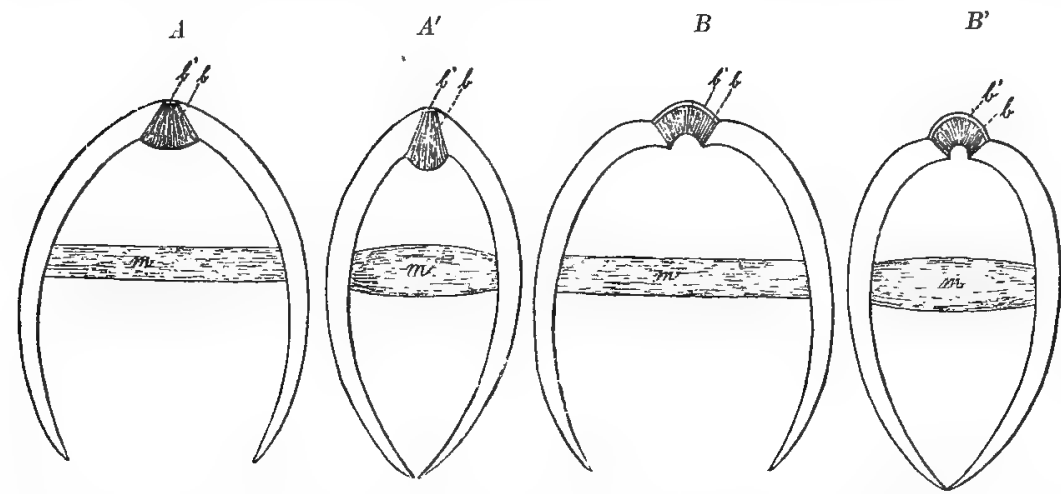

Fig 257. Diagrammatic transverse sections through the shell of Lamellibranchs with internal $\left(A, A^{\prime}\right)$ and external ligaments $\left(B, B^{\prime}\right)$. In $A$ and $B$ the shell is drawn open, in $A^{\prime}$ and $B^{\prime}$ shut, $b$ elastic portion of the ligament, $b^{\prime}$ outer inelastic portion of the same; $m$ adductor.-Orig.

and, in the upper region, the surface of the body, continually deposit new layers within it.

The shells of some Lamellibranchs differ from the usual type in being very a s y m metrical : in the Oyster and some Scallops, for instance, only one valve is convex, the other being flat. A lesser asymmetry, quite insignificant in most instances, occurs in many others, (e.g., the hinge-teeth of the two valves fit in between one another; at those places where a tooth is present in the one valve, there must be a depression in the other). Other forms are characterised by the fact that the shell covers only a small portion of the body (Teredo, and others). $P$ earls are calcareous deposits from the outer side of the mantle round foreign bodies, which bave got by accident between the mantle and the shell; they are either firmly attached to the inner side of the shell, or else lie quite freely; they occur in various forms. Some species, with elongate bodies and imperfect shells, either build a tube of small foreign bodies welded together, or (more frequently) secrete a calcareous tube, with which the small valves of the shell are sometimes united.

In connection with the skin, the formation of byssus threads, which occurs in one group, must be specially noticed. These threads are horny fibres, which are secreted by the epidermal cells in a depression of the foot, and in a groove connected with it. They constitute organs of attachment in some species (e.g., in the Mussels), one end remaining in counection with the animal, whilst the other is firmly fixed to some foreign body. Others bind small stones together by means of the byssus threads, and thus form a kind of nest, in which they take up their abode.

In many Lamellibranchs, where'no byssus is formed, a rudimentary byssus gland may nevertheless be present. Some forms possess this thread only in the larval stage, losing it when adult. 
The pleural ganglion in Lamellibranchs is almost alwars fused with the cerebral ganglion, and the connective between the pleural and pedal ganglia is fused with that between the cerebral and pedal, so that to all appearance the pleural ganglion and its cord are absent (Fig. $237 \mathrm{D}$ ). In some forms, however, the two are independent; the cord only partially so; which gives conditions closely approaching to those of the Gastropoda. Eyes are only present occasionally, and then are always situated, often in large numbers, along the edge of the mantle; in the siphonate forms, at the tips of the syphons. For example, in the Scallops (Pecten) there is along the edge of the mantle a series of eyes of somewhat complex structure. An olfactory organ, a specially modified portion of the epidermis supplied with nerves, corresponding with that of the Gastropoda, is present in many, near the anus.

The mouth is a transverse slit at the anterior end of the body, below the anterior adductor muscle. It is bounded above and below by an upper and lower lip respectively, each of which is drawn out on both sides into a usually well-developed labial palp. The labial palps are covered with numerous cilia; these serve to drive into the mouth the small particles, microscopic plants and animals, which are present in the water taken into the mantle-cavity. $\mathrm{Radula}$ and jaws are absent. A short oesophagus leads from the mouth into a $\mathrm{stomach}$, which is often provided with a $\mathrm{c} æ \mathrm{cum}$; in the latter is contained the so-called crystalline stylet, a gelatinous, transparent body which is secreted by the epithelium of the cæcum, developing when food is plentiful (in the summer), and redissolving during scarcity (in winter); it probably represents a reserve of nourishnent, and is present in almost all Lamellibranchs.* A well-developed liver surrounds the stomach, and opens into it by several apertures. The intestine is coiled several times; its posterior portion ruus along the dorsal side of the animal, and finally passes dorsal to the posterior adductor muscle to open at the hind end of the body. The ventricle is situated on the dorsal side of the animal above the rectum; it generally divides into two branches which surround the gut and unite below it, so that it is ring-shaped, "perforated by the rectum." There are two auricles, one on each side, which receive the blood from the gills and carry it to the ventricle. The vascular system is imperfect; in the gills there is, however, a rich network of capillaries. The kidneys are a pair of sac-like organs which are often more or less intimately connected with each other (the organs of Bojanus); each opens by an aperture situated laterally below the origin of the inner gills; and also into the pericardium. Most of the Lamellibranchs are of $\mathrm{separate}$ sexes, a few (e.g., the Oyster) are hermaphrodite. Ovary and

* A crystalline style is also present in some Gastropoda. 
testis are present as paired branched organs ramifying between the other viscera, in the foot, or (e.g., in the Mussel) in the mantle; they open on either side close to the nephridial aperture, or the

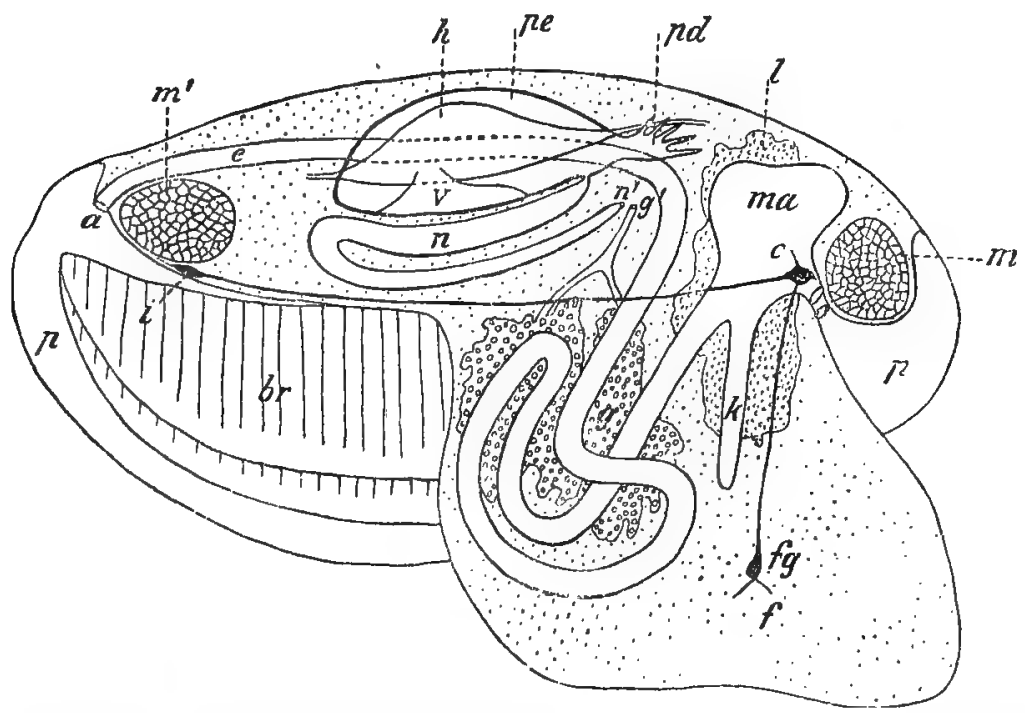

Fig. 258. Diagrammatic longitudinal section of a Lamellibranch. $a$ anus, br gill, c cerebro-pleural ganglion, $e$ rectum, $f$ foot, $f g$ pedal ganglion, $g$ generative gland, $g^{\prime}$ genital aperture, $h$ ventricle, $i$ visceral ganglion, $k$ coeum containing the crystalline stylet, $l$ liver, $m$ anterior, $m^{\prime}$ posterior adductor, $m a$ stomach, $n$ kidney, $n^{\prime}$ external renal aperture, o mouth, $p$ mantle, $p d$ pericardial gland, pe pericardiam, $v$ auricle.-Adapted from Rankin.

duct unites with that of the kidney, so that a common urino-genital aperture is present on each side.* Sometimes the fertilised eggs develop in the space between the lamellæ of the outer gills of the female.

Marine Lamellibranchs nndergo a metamorphosis similar to that of the marine Gastropods; the newly-hatched larva moves by means of a velum; eyes are frequently present anteriorly, but disappear later. Such a free-swimming larva does not occur in most fresh-water forms, and a velum is not developed or is only trausitorily present in the emoryo.

All Lamellibranchs are aquatic,

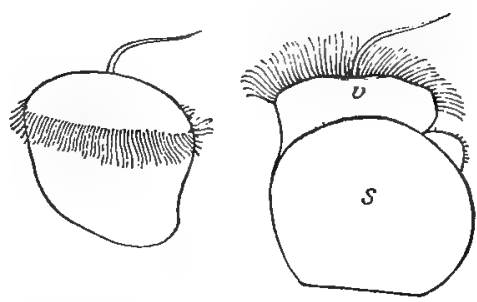

Fig. 259. Lar va e of a Cockle (Camlirm); in the older a small shell $s$ is developed, $v$ volum.-After Lovín. the majority marine. They

* In some the reproductive organ opens into the kidney itself, in certain cases even close to the reno-pericardial aperture. 
feed upon small organisms, Diatoms, etc., which are contained in the inhaled water. They can $\mathrm{cr}$ aw l slowly along by means of the foot, which they press upon or into the ground; some can take leaps by its means. In exceptional cases they can move rapidly through the water by quickly opening and shutting the shell. Some are able to work themselves into the soft sandy, or muddy ground by means of the foot, so that the cloacal and inhalent apertures alone project, and many pass most of their lives buried in this way; the formation of shorter or longer syphons is in correlation with this. Some even have the power of boring into hard substances, wood, limestone, etc., grinding the material away with the foot in which tiny silicious bodies (?) are embedded. Some forms have the shell firmly attached to the surroundings by means of a calcareous secretion, and have, of course, lost the power of locomotion. As has been mentioned abore, such an attachment may be also effected by the byssus thread; the animals so attached remain for a long time in the same place, but may leave it by throwing off the byssus; the Dreissena mentioned below is, for example, attached during summer to foreign bodies just below the surface of the water, in the autumn the byssus is discarded and the animal retreats to the bottom.

\section{As examples the following may be mentioned:}

1. The Oyster (Ostrea edulis) possesses only on e adductor ; the ligament is internal; the foot absent; the right valve flat, the left convex and adherent to various foreign bodies: on English coasts. Related to the Oysters are the Scallops (Pecten) with a radially ribbed shell, of which the anterior and posterior halves are similar. In several species the left valve is flat, the right convex; in others both are alike convex; internal ligament; eyes at the edge of the mantle; one adductor; small foot: species in the North Sea, English Channel, etc.

2. The Mussel (Mytilus edulis), characterised by the position of the umbo at the anterior end of the rather thin shell; and possessing a long internal ligament and a powerful byssus, by means of which the animal attaches itself to stones, etc.. very abundant on British coasts; if it lives in stagnant water (harbours) a poisonous substance is secreted by the liver. The allied, somewhat smaller Dreissensia polymorpha, inhabits fresh water; originally a native of South-East Europe, it has spread during the present century over the whole continent. Another allied form is the almost cylindrical, elongate Lithodomus lithophaqus, which bores into limestone; a Mediterranean form.

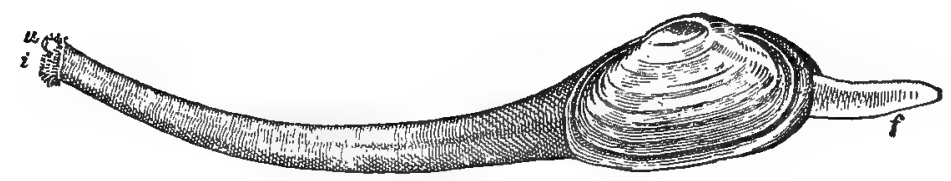

Fig. 260. Mya arenaria. $f$ foot, $i$ cloacal opening, $u$ respiratory opening.-After Meyer and Möbius. 
3. The Freshwater Mussels (Anodonta) are large, egg-shaped, thin-shelled Lamellibranchs, abundant in freshwater. The numerous eggs batch in the outer gills of the female, and the larvæ escape by the cloacal aperture. These larvæ are provided with long, sticky threads, which float in the water and adhere easily to passing Fish; when this occurs the young Mussel attaches itself firmly to the Fish by means of the teeth present on the lower edge of each valve of the shell; it is then covered by a growth of the skin, and for a time leads a parasitic life upon the Fish, which it forsakes again later. The River Mussel (Unio) and the River Pearl Mussel (Margaritana margaritifera), which are common in England, are allied forms. The latter manufactures some of the pearls of commerce. The true Pearl Mussel (Meleagrina margaritifera), which forms the best pearls, belongs to another family of Lamellibranchs; it is found in the Indian and Pacific oceans.

4. The Gaper (Mya arenaria) is distinguished by the possession of a very long syphon (formed, of course, by the fusion of two tubes), and by the way the shells gape open behind; the edges of the mantle are concrescent to a large extent. It occurs on the beach on the coast of Britain, buried more than a foot deep.

5. The Ship-worm (Teredo navalis) is a vermiform, elongate Lamellibranch, in which the edges of the mantle have undergone concrescence to a large extent; and a pair of very small shell-valves, which are not connected by a ligament, are present anteriorly; and two partially separated syphons posteriorly. It is a marine form occurring in wood (piles or ships), in which it bores long tubes, lined with a calcareous secretion; the external aperture and the portion of the tube next this are narrow, and were formed by the animal when young; further in it is wider and cylindrical: the animal is unable to leave the tube, its anterior end is in the innermost portion of the tube, the syphons at the mouth: common on the coasts of Europe: very destructive. In the allied genus, Pholas ( $\mathrm{Piddock}$ ), which bores into limestone, wood, etc., the body is shorter, the shell better developed than in Teredo; it is phosphorescent: present in European seas.

\section{Class 4. Cephalopoda.}

The body is externally, and for the most part, internally, bilaterally symmetrical. It falls naturally into two regions, the $\mathrm{head}$ and the body. The $\mathrm{head}$ is very well developed; the mouth is anterior; and in all Dibranchiata (i.e., in all Cephalopoda, except Nautilus) is surrounded by eight long muscular arm s.* In some Dibranchiata, i.e., in the Decapoda, there are

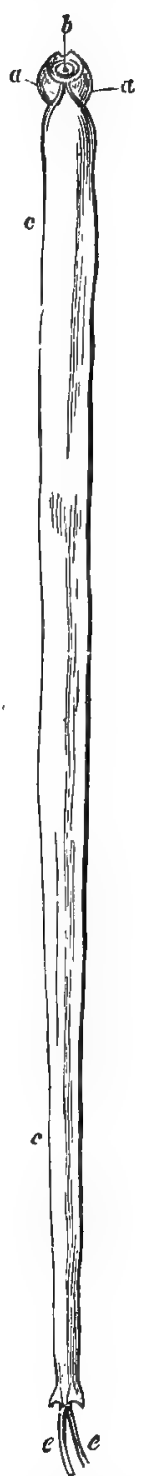

Fig. 261. Teredonavalis. $a$ shell, $b$ foot, c mantle, e syphons.

* In certain of the Cephalopods some, or all, of the eight arms are connected at their bases, or further up, by a thin web of skin (just as are the toes of many swimming Mammals and Birds). 
two more long, so-called "tentacular arms" within the other eight. All (the tentacular ones only towards the tips) are furnished with numerous muscular suckers, ranged along the

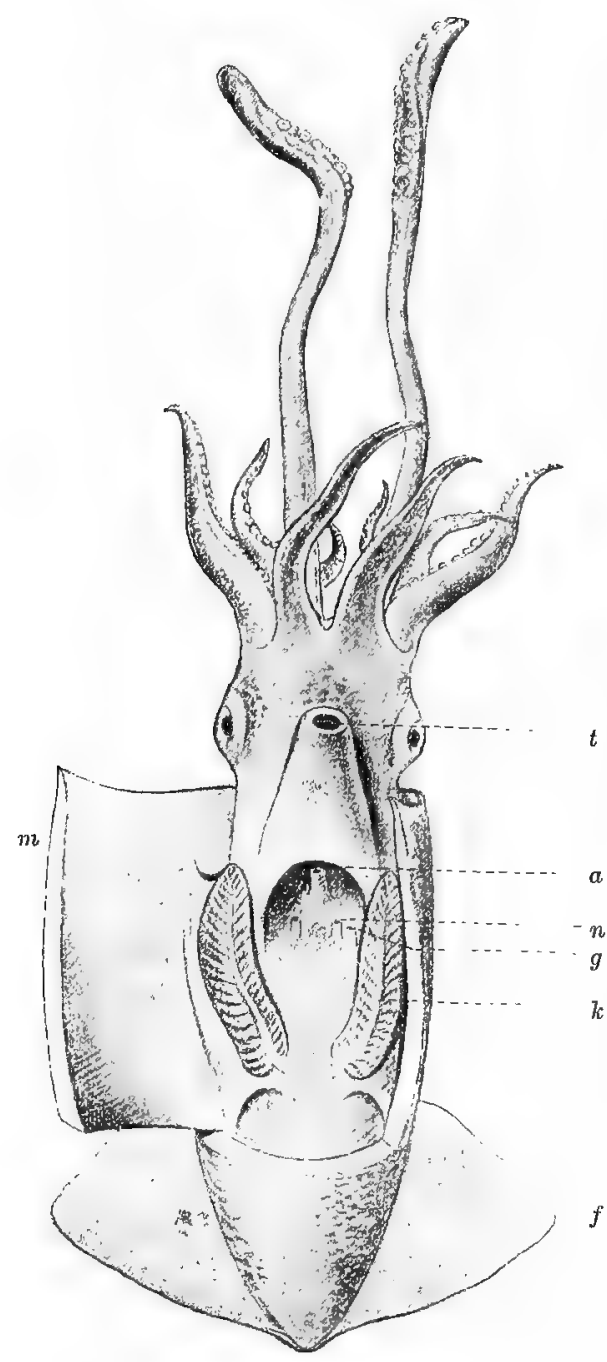

Fig. 262. Diagram of a decapodous Cephalopod viewed from below, mantle cut through down one side and turned over to the other. $a$ anus in the posterior end of the funnel, $f$ fin, $g$ genital aperture, $k$ gill, $m$ mantle, $n$ urinary aperture, $t$ anterior opening of funnel.Orig. the animal. At the junction of the head and body there is a transverse slit ventrally, leading into the spacious mantle-cavity (see Fig. 268 B), which extends over the whole length and width of 
the animal on the ventral surface. It is bounded externally by a mantle, which is usually very muscular and thick, and, in many cases, prolonged into a low fold behind the head on the upper surface. From the opening of the mantle-cavity projects the anterior end of the $f u n n \in 1$, a true open at both ends, and attached dorsally to the dorsal wall at the boundary of the head; the funnel is a true tube in the Dibranchiata only; in Nautilus it is a plate rolled up like a paper bag; it corresponds to the foot of other Mollusca. The animal takes water into the mantle-cavity by the large aperture, but expels it through the funnel by the contraction of the mantle and the pressure of its edge upon the body. In the funnel there is frequently a small tongue-like flap, which is attached posteriorly, the anterior end being free, so that it acts as a valve, preventing the entrance of water. The laminate gills lie in the mantle-cavity, one pair in the Octopods and Decapods, two pairs in Nautilus.

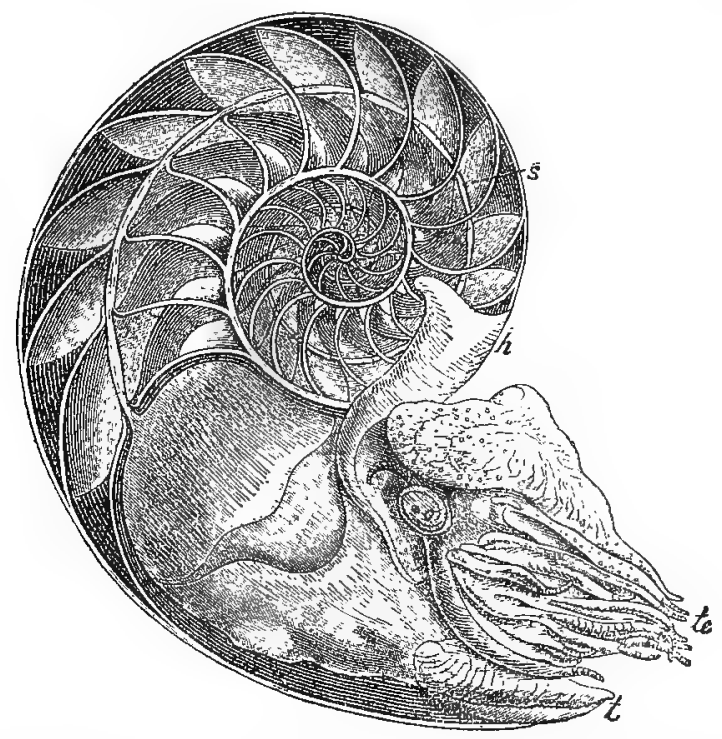

Fig. 263. Nautilus, the shell sawn through. o eye, $t$ funnel, $t e$ tentacle; $s$ the syphon, $h$ a fold of skin which overlaps the shell.-After v. Martens.

In Nautilus the body is enclosed by a shell, which, as in the Gastropods, is an epidermal secretion. It is spiral, but symmetrical ; the convexity corresponds to the ventral side of the animal (see Fig. 263); the coils touch one another. The shell is multilocular, being divided by arched transverse septa into numerous chambers, of which the outermost aud largest contains the body, whilst the others contain air ; each septum is perforaled by a hole, through which a thin tube, the siphuncle, an extension of the 
posterior end of the bods, runs through the whole length of the shell.* In some extinct nautiloid forms the shell was straight (Orthoceras); in others slightly curved or rolled into an incomplete spiral; in others it formed a perfect spiral, of which the successive coils did not touch. In the living genus Spirula, one of the decapodous Cephalopods, there is a spiral multilocular shell (Fig. 264 $A$ ) like that of the Nautilus, but the individual turns do not touch, and the shell is coiled in the opposite direction, the convexity corresponding to the dorsal surface; only a small portion of the body is contained in the shell, of which the outer chamber is small, and the shell itself is completely enclosed by folds of skin which have wrapped round and coalesced. In certain extinct decapodous forms there is also a multilocular shell (Fig. 264 $B, C$ ), but this is usually straight and drawn out into a plate-like portion anteriorly; in living Decapoda (with the exception of Spirula) this piece is almost the only part of the shell which persists; the posterior spiral, which in extinct forms was concamerated, has become quite rudimentary or is entirely absent. In these forms the

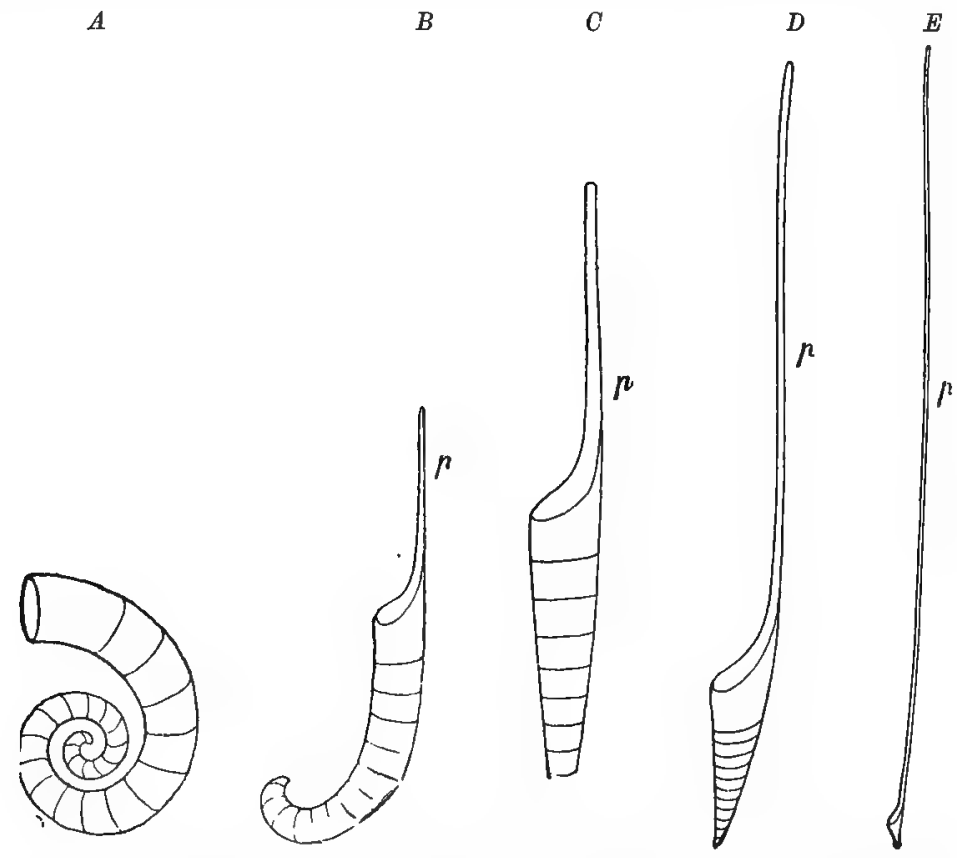

Fig. 264. Diagrammatic figures of various Cephalopod shells viewed from the side. A Spirula; B Spirulirostra (extinct) ; C Belemnites (extinct); in $B$ and $C$ the posterior portion of the shell is prolonged in a strong spiny process; $D$ Conoteuthis (extinct); $E$ Ommatostrephes (living). $p$ plate-like portion of the shell.-Orig.

* It is assumed that the septa are formed by the animal's withdrawing from the outermost as the shell grows at the mouth, and secreting a new septum in front of the last formed; the cord elongates simultaneously. 
shell is usually a thin, horny, elongate plate, occasionally it is somewhat thicker, with a calcareous layer below the horn (Sepia); it is completely enclosed in a cavity on the dorsal surface, an in $\mathrm{v}$ a gi in a tion of the outer skin. 'T'his so-called "internal shell" corresponds with the external shell of Nautilus, and like it is an epidermal secretion. In the Oetopods the shell is altogether absent. (For the very aberrant shell of Argonauta, see below, p. 323).

The skin is characterised by its constantly changing colour, due to the presence of stellate pigment cells which can contract and expand (chromatophores). The so-called in $\mathrm{k}-\mathrm{s}$ ac, a peculiar gland connected with the skin, is usually a pear-shaped bag in which an inky fluid is secreted; it opens into the mantle-cavity close behind or even into the anus, and when the animal is in danger the fluid can be poured through the funnel, rendering the water black.

The Cephalopoda possess a true, although feebly-developed, internal skeleton in the form of pieces of cartilage, of which the cartilaginous capsule in the head, surrounding the central nervous system, the auditory organs, and in part the eyes, is the most important. Besides this capsule there are usually several smaller pieces in varions regions of the body.

The nervous system is peculiar in that all the large nerve masses, cerebral, pedal, pleural, and visceral ganglia, are closely collected round the osophagus, and are directly connected by the shortening of the commissures. The eyes are large, and in many

$A$

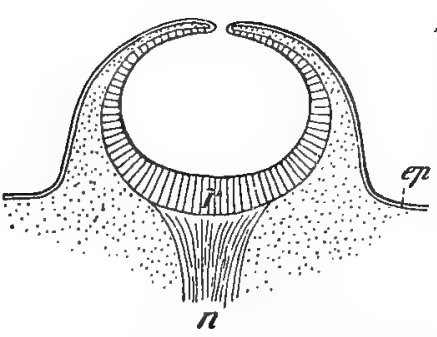

$B$

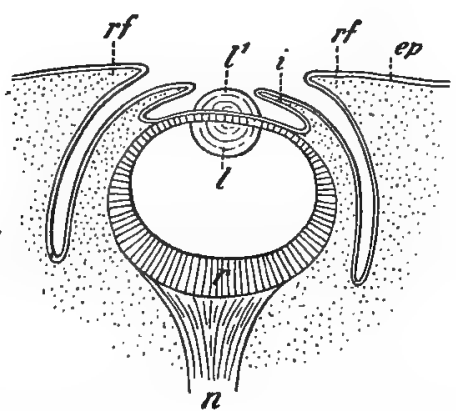

$C$

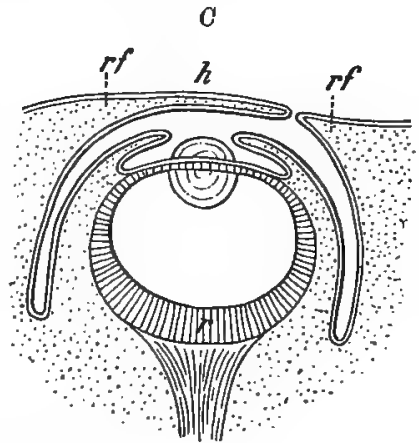

Fig. 265. Diagrammatic transverse sections through the eyes of various Cephalopoda. $A$ Nautilus, $B, C$ different Dibranchiates. $\quad e p$ epidermis, $h$ cornea, $i$ iris, $l$ inner, $l^{1}$ outer portion of the lens, $n$ optic nerve, $r$ retina, $v f$ eyelid.-Orig.

forms attain a high development. The simplest occur in $\mathrm{Nautilus}$ (Fig. $265 \mathrm{~A}$ ), where they are deep, saccular, epidermal invaginations ; the cavity communicates with the exterior by a small opening (the eye belongs to the type figured in Fig. 20, 4, but a lens is w a n t in $\mathrm{g}$ ). In the other Cephalopoda a closed optic vesicle is developed, 
and is further provided with a lens; the inner half of which is secreted by the epithelium of the optic vesicle; the outer half by the epidermis. Moreover, at the periphery of the eye, a large fold like an eyelid is present, forming a cavity round the eye; in some Decapoda ( $E$ gopsida, Fig. $265 B$ ) this cavity is widely open; in the rest (Myopsidce) and in the Octopoda (Fig. $265 \mathrm{C}$ ) the fold extends completely round the eye, and the aperture leading into the cavity is very small; where the fold lies above the lens it is transparent, and is termed the cornea. In the cavity thus formed there is a second small pigmented fold, which displays a certain resemblance to the iris of the Vertebrata, and is also designated by that name. A depression of the skin situated laterally on the head behind the eyes and supplied by a nerve from the brain, is regarded as an olfactory organ.

The mouth is surrounded by a projecting fold of skin, the lip, within which are two powerful horny $\mathrm{jaws}$, an upper and a lower; the former bites within the edge of the latter, and the two together are very similar to a parrot's beak inverted. In the mouth, which is furnished with muscular walls, there is a $\mathrm{radula}$ like that of the Gastropoda. The an us lies far forward on the ventral side of the body in the mantle-chamber, in the median line. A stomach, a large liver, and usually salivary glands are present. The beart consists of a veutricle and as many auricles as there are gills, that is,

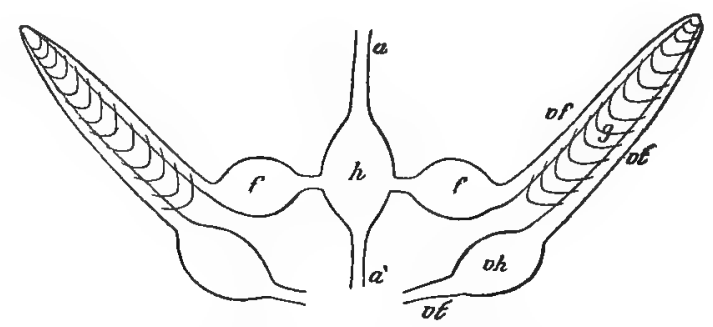

Fig. 266. Diagram of the $\mathrm{b}$ e a $\mathrm{rt}$, etc. of a Cephalopod. $h$ ventricle, $f$ auricle, $u, u$ arteries, $v h$ branchial heart, $v t^{t}$ vein to the gill, $v f$ vein from the gill, $g$ gill.-Orig.

four in Nautilus, two in other Cephalopods. In the Dibranchiata the large veins which carry the blood to the gills are known as branchial hearts; they are enlarged at the entrance of the gills and contractile. The $\mathrm{kidneys,} \mathrm{two} \mathrm{pairs} \mathrm{in} \mathrm{Nautilus,} \mathrm{one} \mathrm{pair} \mathrm{in}$ the Dibranchiata, are saccular organs opening into the pallial chamber by paired apertures. In some of the Dibranchiata the two are partially fused, but each has its own opening. The kidneys exhibit racemose evaginations of the large adjoining veins which have pushed the closely attached wall of the kidney into its cavity; these evaginations appear to hang freely into the cavity. 
The reproductive organs are similarly arranged in the male and female, the Cephalopoda being bisexual. There is a pair of ovaries or testes; neither is directly connected with the duct, but each is enclosed in a thin-walled sac, from which this arises. In some forms two symmetrical oviducts are present, and these open one on either side behind the anus into the pallial chamber; in others, one, usually the right, duct is wanting. The $\mathrm{nidamental} \mathrm{glands}$ open close to the genital aperture in many female Cephalopods; their secretion is used in the formation of the egg capsules. In some there are paired vasa deferentia, but usually one, the left, alone is present. The spermatozoa are bound into elongate, almost filiform, spermatophores, which are formed in a gland connected with the vas deferens.

Specially worthy of note is the manner in which the spermatophores of the Dibranchiata are transferred to the female; this is effected by one arm of the male, which is peculiarly modified, "hectocotylised," for this purpose. In the Decapoda, it is usually an arm of the fourth pair (occasionally of the first); in the Octopoda, one of the third pair, rarely both. The form of this arm varies; it may be spatulate at the tip, and provided with a wide ridge along the edge (Octopods), or the suckers may be absent or modified in the middle or at the base (Decapods). The modification is greatest in some of the Octopods (e.g., in the Argonauta to be mentioned below), where the arm is used exclusively for copulation; it is enclosed in a sac until needed, and in coitus is thrown off

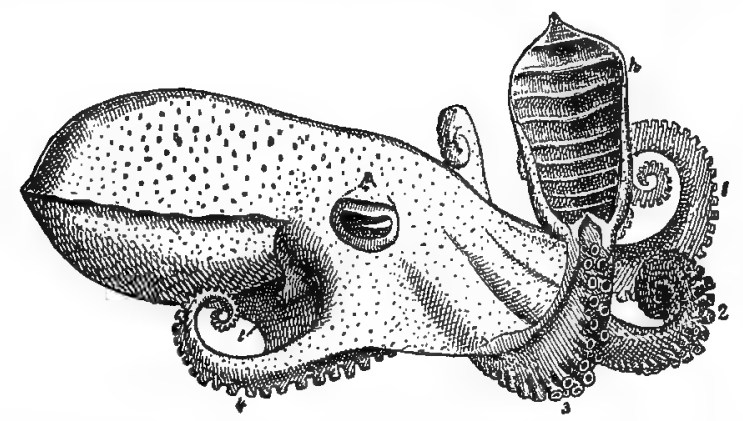

Fig. 267. An Octopod in which the hectocotylised portion $(h)$ of the right arm is very well developed. $t$ funnel, $1-4$ first and fourth arms of the right side.-After Verrill.

and remains, filled with spermatozoa, within the pallial chamber of the female. Here it may continue motile for some time, which led to its being regarded as a peculiar parasite, described under the name of Hectocotylus; later, it was considered by some observers to be a much modified male, and finally its true nature was discovered. In the males of Nautilus there is, on the left side, a 
small group of metamorphosed tentacles, which are possibly of service in copulation.

The $\theta \mathrm{ggs}$ are either heaped together in masses of mucus or are enclosed in firm capsules. They are of relatively large size; segmentation is partial, and the embryo often possesses, for a time, a large yolk sac, depending between the arms. There is no metamorphosis, the newly-hatched form resembling the adult.

The Cephalopoda, which are all marine, are for the most part, predaceous, seizing their prey (e.g., Crustacea) with their arms; the latter are also used for crawling, especially in the Octopods. They may swim by the movement of the fin backwards and forwards; a hurried flight $\mathrm{b} a \mathrm{ckw}$ a rds, usually accompanied by a discharge of ink, may be effected by the ejection of the water from the pallial chamber through the funnel. The Decapods are the best swimmers, whilst the Octopods usually crawl better. Many Cephalopods (especially the Decapods, but some Octopods too) often-occur in shoals in the open sea; others are more littoral. They are mostly found in warm seas.

$A$

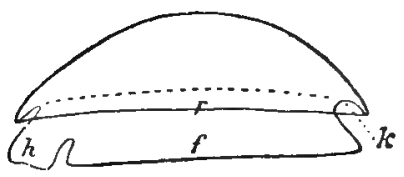

Fig. 268. Diagrammatic figures to illustrate the relations between the Chitons $(A)$ and the Cephalopods $(B)$; profile; $f$ foot (funnel), $h$ head, $k$ mantle eavity, $r$ rim of the mantle, whose upper boundary is indicated by a dotted line, $o$ eye.-Orig.

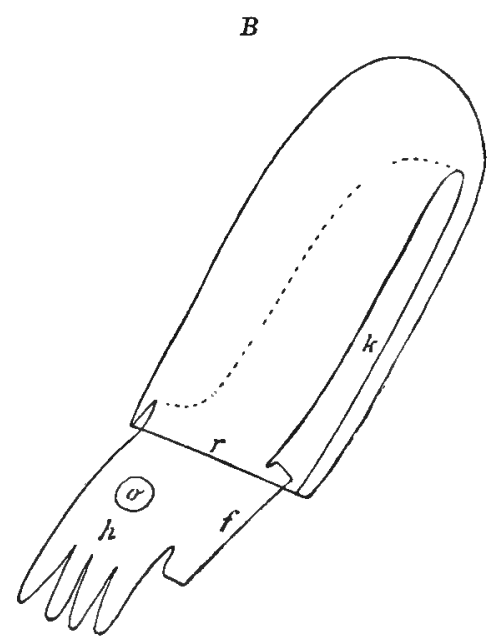

It is conceivable that the cephalopod type was derived from a chiton-like form by enormous development in the height of the dorsal surface, by the deepening of the mantle furrow on the posterior side of this upgrowth, by the great development of the head, and the reduction of the foot.

\section{Order 1. Tetrabranchiata.}

There are numerous arms (tentacles) without suckers. The funnel is a plate rolled upon itself. The eyes have no lens. There are four gills (four auricles, four kidneys); no ink sac; an external shell. 
The Tetrabranchiata are at the present day only represented by the genus Nautilus, of which there are two species in the Indian and the Pacific Oceans; they may crawl upon the ground or swim at the surface. In former times (even as early as the Silurian) this division was very numerous, partly represented by forms with straight or curved shells (ef., p. 318).

The Ammonites are a large group of extinct animals, in which the shell is multilocular like that of the Nautilus, with perforated septa, spiral or straight, curved, etc., but differing from it in that the perforations lie close to the convex side, whilst, in Nautilus, they are usually median; and also in that the septa are strongly sinuous at the fusion with the shell: many were possessed of an operculum (aptychus). The Ammonites first occur in the Silurian formations, and die out in the Cretaceous. Their systematic position is quite uncertain; they are mentioned here on account of the resemblance the shell bears to that of Nautilus, but it cannot be affirmed that they are allied to it.

\section{Order 2. Dibranchiata.}

There are eight or ten arms with suckers. The funnel is tubular; the eyes have a lens. There are two gills (two auricles, two kidneys); an ink sac; an internal shell, or, none.

1. Decapods (Decapoda). Ten arms, suckers stalked, and with chitinous rings; shell present, body elongate and with fins. To this group belong, for example: Sepia officinalis, which occurs abundantly in European seas, and whose thick shell, formed of fine calcareous laminx (os sepix of the chemist) is turned to various technical uses. Here too, belongs the Sea-clerk (Loligo vulgaris), occurring in the same places, and possessed of a thinner horny shell. Further, the gigantic Architeuthus, a pelagic animal several metres long, but not otherwise differing from the common decapod type. The so-called "thunderbolts," which are especially abundant in the Cretaceous strata, and which, on account of the displacements, consequent on the Glacial Period, occur also in the glacial deposits of North Europe, are the posterior tongue-shaped ends of the shells of certain extinct Cephalopods (Belemnites).

2. Octopods (Octopoda). Eight arms, suckers sessile and without horny rings, no shell; thick body without fins. Here belongs, for example, Octopus vulgaris, a large animal with long arms, small round body; abundant in the Mediterranean. Also Argonauta argo, the females of which are characterised by the compression of the first pair of arms, and their extension posteriorly, to form two lamellæ, reaching round the body; these secrete, on the inner side, a thin, cap-like calcareous shell, to protect the body, and to contain the eggs. It is at no point closely adherent to the upper surface of the animal. The male possesses a hectocotylus, but the first pair of arms is normal in structure, and it has no shell. Argonauta is a pelagic animal. 


\section{Group 8. Vertebrata.}

General review. The Vertebrata are bilaterally symmetrical. There is a dorsal central nervous system, of which the anterior end is usually enlarged to form the $\mathrm{brain}$, whilst the rest forms an elongate spinal cord. Below the nervous system lies another cord, the notochord or chorda dorsalis; skeletal structures are usually developed round these two. Below the notochord is the alimentary canal, the mouth is anterior, the anus ventral, usually some little distance from the posterior end. The heart lies anteriorly below the digestive tract. 'There is a pair of $\mathrm{kidneys}$ and a pair of gonads; the ducts of both these open near the anus, or into the posterior portion of the gut. Alimentary canal, heart, etc., lie in a spacious body-cavity. Optic, auditory and olfactory organs, are present anteriorly. The body is naturally divisible into the following regions: (1) the head, with the brain, senseorgans, and mouth; (2) the trunk, extending from the head to the anus, and enclosing the body-cavity with its contained organs, and usually furnished with two pairs of appendages, the limbs; these last, especially in the higher Vertebrata, play an important part in locomotion; (3) the tail, the muscular termination of the body, forming a powerful locomotor organ in the Pisces, but usually of subordinate importance in the higher Vertebrata. In the higher forms, from Reptilia upwards, the anterior portion of the trunk forms a n e ck ; that is, the body-cavity is drawn away from the anterior end of the trunk; and organs (e.g., the heart) which in other forms occur there, have also moved back, so that this portion is practically without viscera, and forms a muscular stalk-like connection between head and trunk, which is of the greatest importance in connection with the free movement of the head.

The epidermis consists, in Amphioxus, of a simple epithelium of cylindrical cells ; in other Vertebrata there is a stratified squamous epithelium of varying thickness. In Pisces all the epidermal cells 
are soft and protoplasmic, but in all other groups the outer portion of the epidermis consists of cornified cells, so that an outer stratum corneum, and an inner stratum mucosum (Rete Malpighii), in which the cells are protoplasmic, may be distinguished. In the Amphibia the stratum corneum is only one or two cells thick, in the higher Vertebrata thicker; in various regions of the body it is developed in different ways, and in certain parts may attain a very considerakle thickness and great hardness. The claws, for instance, of Reptiles, Birds, and Mammals, are thickened portions of the stratum corneum which surround the last joint of the digit. Ecdysis in the Vertebrata consists in a throwing off of the stratum corneum, either in one piece (Amphibia, some Reptilia), or bit by bit. Below the epidermis is a layer of connective tissue, the dermis, which varies in thickness and firmness, and is connected with the subjacent parts by the loose subcutaneous connective tissue; in the dermis there are numerous smooth muscle cells or transversely striped nuscle fibres, and below, but still connected with it, there are, especially in the higher Vertebrata, the continuous laminate cutaneous muscles consisting of transverse muscle fibres. In the cells of both epidermis and dermis there is frequently a deposit of pigment. Glands of many kinds belong to the skin. In the Fish, between the outermost cells of the epidermis, there are beaker-shaped cells which secrete mucus; true glands are not generally present, although in other Vertebrata they attain a great derelopment, sunk into, or below, the dermis and opening through the epidermis. In members of all the classes of Vertebrata, with the exception of the Lancelet (Amphioxus), ossifications are present in the dermis forming plates of varying thickness (scales of Pisces, etc.). Sometimes these plates attain a considerable size, and may be united to form an exoskeleton which surrounds some portion of the body (some Fish, Tortoises, a few Mammals), and are often intimately connected with the endoskeleton, especially in the head region.

The endoskeleton is, at very early stages of development, represented only by the notochord, a cord or rod of cellular connective tissue, lying below the central nervous system. In Amphioxus the adult skeleton consists almost exclusively of the notochord (Fig. 293, p. 355), but in all other Vertebrates, other skeletogenous structures develop; largely around, and in connection with, the chorda. They surpass the notochord in size, and it is, in fact, almost obliterated by the newly-developed skeleton. The latter consists partly of cartilage, partly of bone; even when the adult skeleton is principally bone, it usually consists at first of cartilage, which is gradually absorbed and replaced by bone, i.e., it os sifies: or it is covered by bony plates, membrane-bones, beneath which it may persist: or it may disappear altogether. 


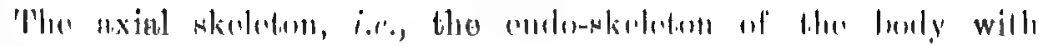

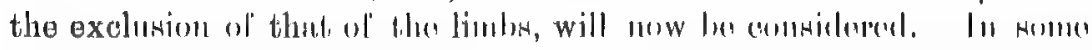

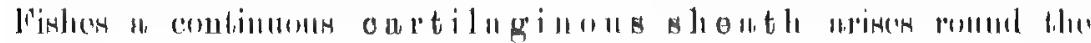

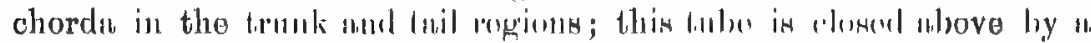

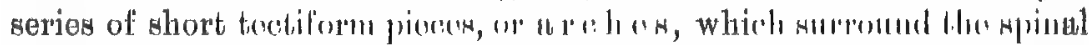

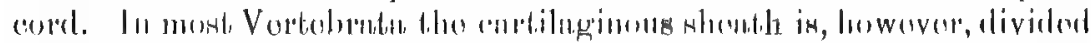

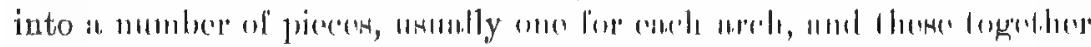

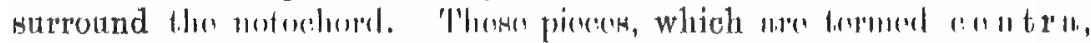

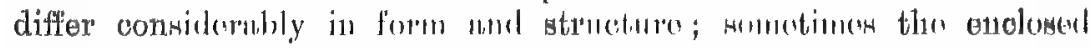

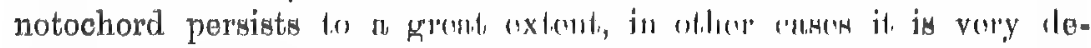

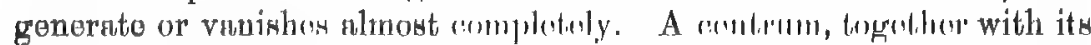

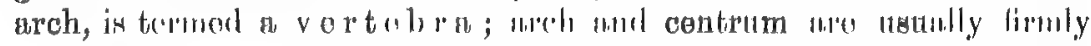

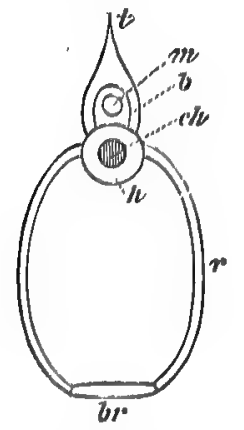

Fig. 269. Jingram of a vorto. bra sud tho parts cornected with it. r $h$ notochord, $h$ oontrum, b netral arch, 1. neural mpine, $m$ spinal cord, $r$ rit, br stornum.-Orig.

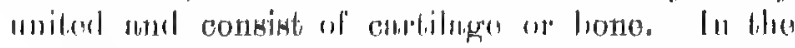

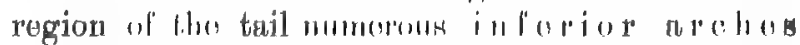

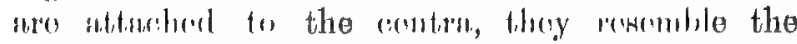

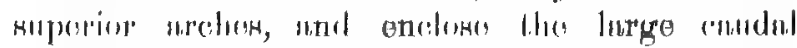

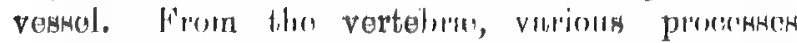

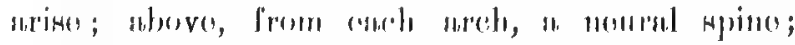

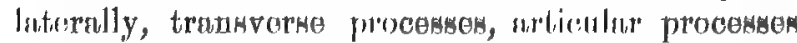
with surticular lineden, which lie uprou those of

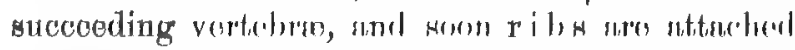
to most of the trunk vertolims, excopting thes

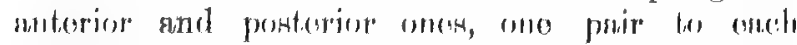

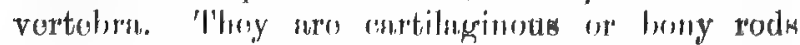

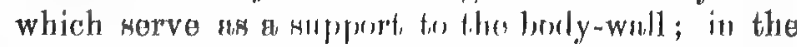

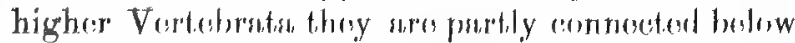

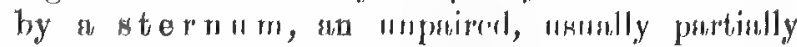

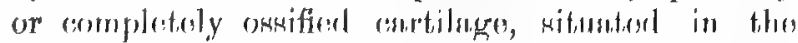
body-wall; it in absent from Fish, sund thought present in Amphihis, is not connoctsel with thes ribs. In highor Vortebrsuts, (Roptilin, Avek,

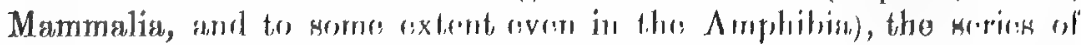

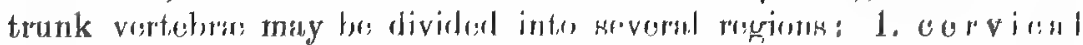
vartebra, with or withont stmall ribs; 2, thorncic vertabra,

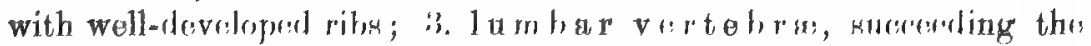

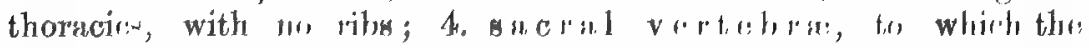

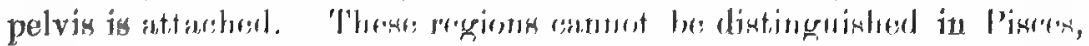
where the trank vertehess ane aswally all similar.

The primitive caphalic skrileten is st strong capsule, the

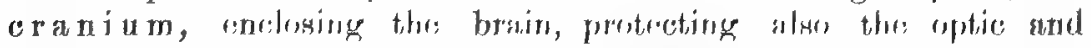
olfactory organs, whilst the anditeny anpmontas in imbedeled in its walls. It ver vertral wall is continumens with the vertebras, and surrounds the anterier end of the wetocherel-a very degentertute

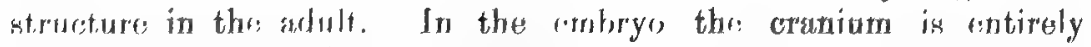

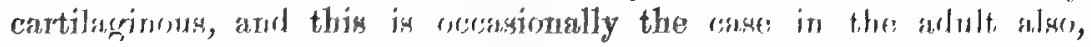
though a certain amount of filbrous tisane is present, filling in the 


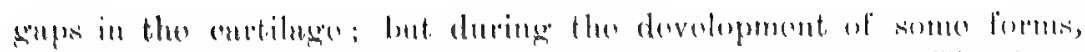

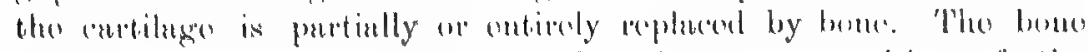

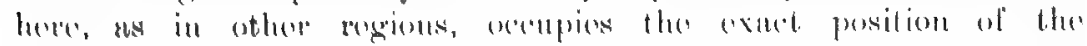

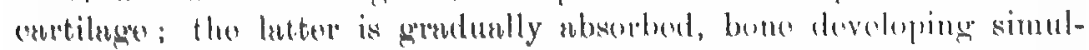

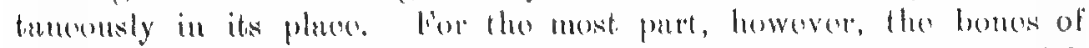

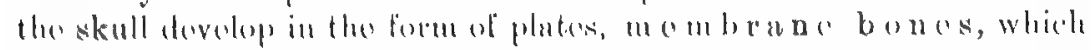

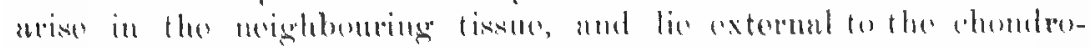

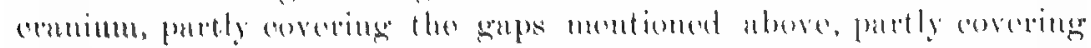

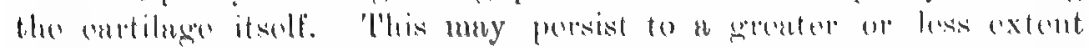

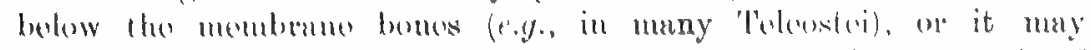

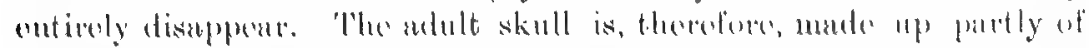

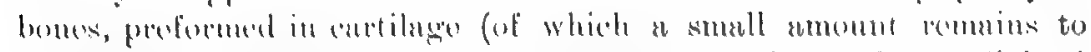

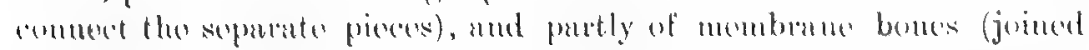

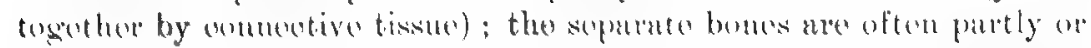

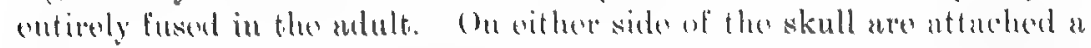

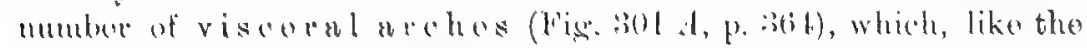

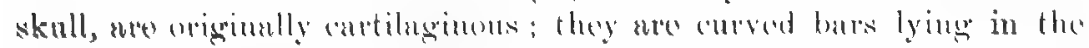
wall of the mouth; ventrully, the comesponding arehes of the two sidos

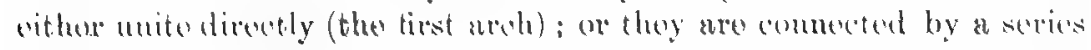

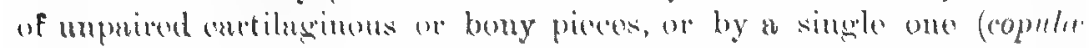

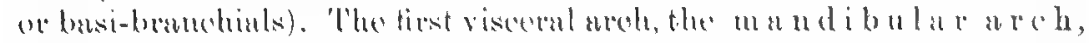

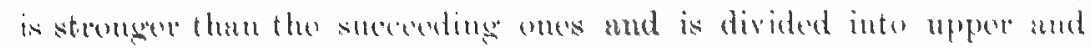

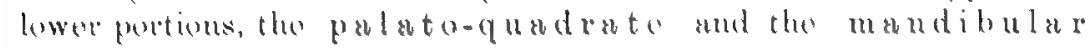

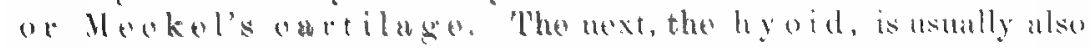

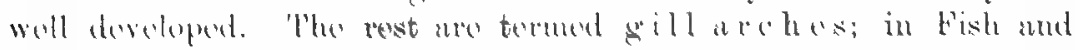

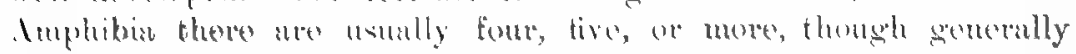

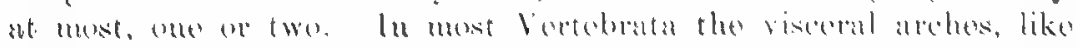

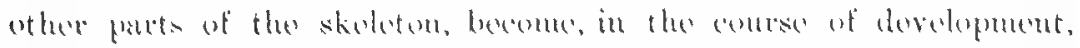

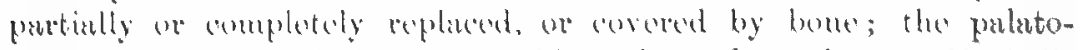

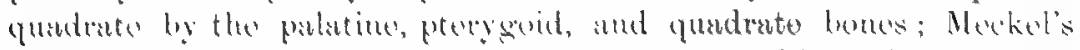

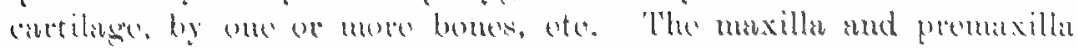

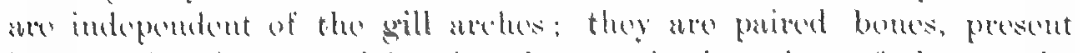

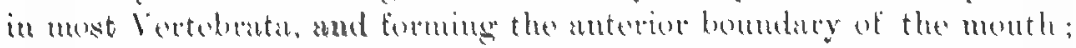

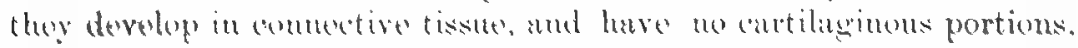

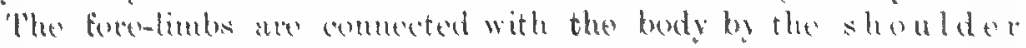

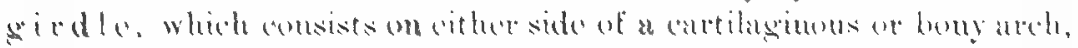

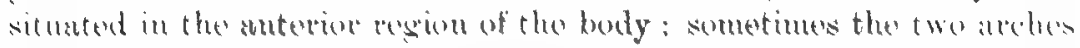

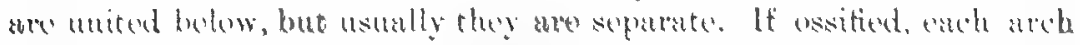

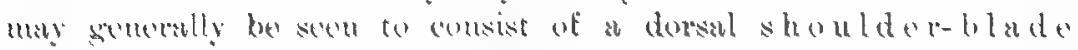

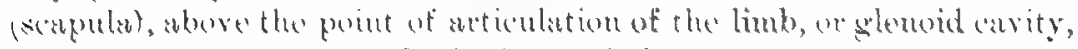

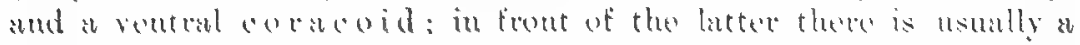

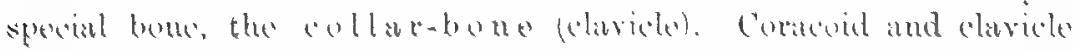

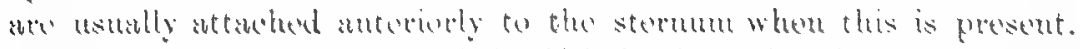

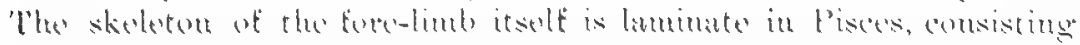

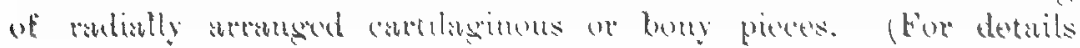

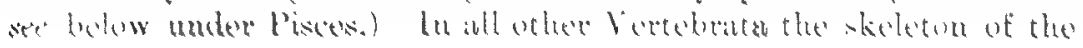


fore-limb conforms to one type: articulating with the shoulder girdle by its upper end is a long bone, the bumerus; to its lower end

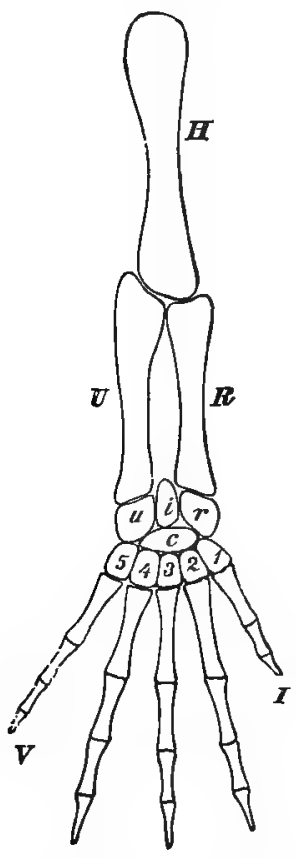

Fig. 270. Diagram of the skeleton of the fore. $1 \mathrm{im} b$ of a higher Vertebrate. $H$ humerus, $R$ radius, $U$ ulna, $u$ ulnare, $i$ intermedium, $r$ radiale, $c$ centrale, $1-5$ carpals; $I-V$ first to fifth digits.-Orig. are attached two other long bones, the radius and $u \ln a$, together forming the fore-arm; at the proximinal end of the ulna there is usually a process, the olecranon, which projects over the radius. At the lower end of the fore-arm is the carpus (wrist), consisting, when completely developed, of two transverse rows of small cartilages or bones (carpals), three in the proximal, radiale, intermedium, ulnare, and five in the distal row, one for each metacarpal : a small bone or cartilage (or sometimes two) lies between these rows, the centrale. To the carpus, which undergoes many modifications, there are attached five (or fewer, rarely more) series of cartilaginous or bony pieces, of which the proximal in each row is termed a metacarpal, the others phalanges; whilst the metacarpals usually lie close together and are enclosed in a common skin, the digits are, for the most part, free.

The pelvic girdle, like the shoulder girdle, is a paired or unpaired cartilaginous or bony arch, affording an articulation for the hind limbs. In Fish, it is independent of the vertebral column; but in other Vertebrata, it is almost invariably fused with one or more vertebræ, the sacrals, on either side. Like the shoulder girdle, each half is divisible, in all excepting Fish, into a dorsal portion, above the ac etabulum (the point of articulation of the hind limb), the ili u $\mathrm{m}$, and a ventral piece which is, however, usually divided into anterior and posterior parts, pubis and ischium; the latter is usually articulated with the corresponding bone of the other side. Ilium, ischium, and pubis are always separate bones in young animals, and are connected by cartilage, of which, indeed, the whole pelvis originally consists; later, however, the three bones more or less completely fuse with one another. The skeleton of the hind limb corresponds closely with that of the fore limb, in Fish and other Vertebrata; in the latter, there is a femur (thighbone), corresponding with the humerus; a shank corresponding with the forearm, and consisting of a tibia and a fibula; a tarsus (ankle), consisting of two rows of bones (tarsals); in the proximal row, tibiale, intermedium, fibulare; five 
in the distal; and a centrale (rarely two); five metatarsals and five toes, each composed of several phalanges.

The bony or cartilaginous pieces are united simply by a connective tissue sheath which lies between them, or more rarely by cartilage, in which case there is but little power of movement between the parts. If the movement be greater, a joint is usually present; that is, the skeletal pieces are separated at their adjacent ends by a slit-like space (the jointcavity), filled with a small amount of fluid, and are only united by a capsule of connective tissue surrounding this cavity. The apposed surfaces of these skeletal pieces, the a r tic u lar surfaces, are smooth and accommodated to one another, but are very diverse in form; they are almost always covered with a thin layer of cartilage (articular cartilages), a remnant of that of which the whole bone primitively consisted. The connective tissue in

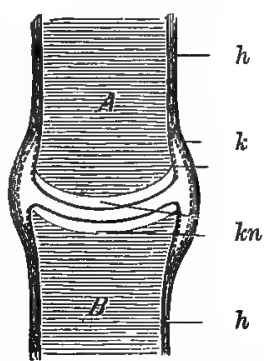

Fig. 271. Longitudimal section of a joint. $A$ and $B$ the two bones of the joint, $h$ periosteum, $k$ capsule, $k n$ articular cartilages.-AfterGegenbaur. the region of the joint is often in part modified into firmer cords or ligaments, which reach from one bone to the other. With the exception of the articular surfaces, bones are everywhere covered by a fibrous connective tissue, the periosteum cartilage is similarly covered by the perichondrium.

Bones, unless they are unusually thin, do not consist simply of osseous tissue, but have cavities within them containing connective tissue and vessels. The outer portion usually consists of a firm mass of compact bone, which is perforated only by small canals (Haversian canals); the inner portion, on the contrary, usually consists of spongy or cancellous bone in which canals and spaces (marrow spaces), preponderate, separated by fine trabeculæ and laminx. In the middle of long bones, there is frequently an expanded cavity, filled usually with adipose tissue, the marrow cavity. In cartilages, too, a smaller number of fine canals are usually present, containing connective tissue and blood vessels.

Since the skeleton of Vertebrata is an internal one, the $\mathrm{muscu}$ lature is principally external, in contrast to Arthropoda, where it lies within the skeleton. The muscles may be classified as those of the trunk and those of the limbs. In Amphioxus and Pisces the body muscles consist chiefly of large continuous masses, disposed on the sides of the trunk and tail, not closely connected with the skeleton, and divided by thin sheets of connective tissue into a rumber of segments; besides these, there are smaller muscles for the movement of the visceral skeleton, the fin rays, etc. The limb muscles are, as a rule, feebly developed in the Pisces. Similar arrangements occur, in part, in the Amphibia, whilst in the higher Vertebrata the musculature both of trunk and limbs is separated into numerous individual muscles, extending from one bone to 
another, and closely united to these at their ends; the limb muscles are usually also very powerful. They consist of striated fibres, bound together by connective tissue. Usually they terminate in $\mathrm{tendons}$, which consist of fibrous connective tissue; the tendons are not infrequently, especially in Mammalia and Aves, of considerable length. Sometimes they may be ossified to a greater or less extent, and often small "sesamoid bones" develop in those portions which pass over a bone, having cartilaginous surfaces towards the bone; the knee-cap (patella) of Birds and Mammals is a sesamoid bone.

The central nervous system arises in the Vertebrata along the dorsal surface of the animal, as a grooved infolding of the epiblast (Fig. 39, p. 48), which is later cut off from the rest of the layer, and lies as a tube below the skin. In Amphioxus it remains in this state throughout life; in others it is modified anteriorly, to form a brain, in contradistinction to the rest, the spinal cord. The lumen of the cord usually persists as a narrow canal (the central canal) in the spinal cord, in the form of larger cavities (the ventricles) in the brain. The brain is, from very early stages, divided by grooves into three regions, of which the first and last are again sub-divided into two. There are thus five sections: primary and secondary fore-brain (thalamencephalon, prosen-

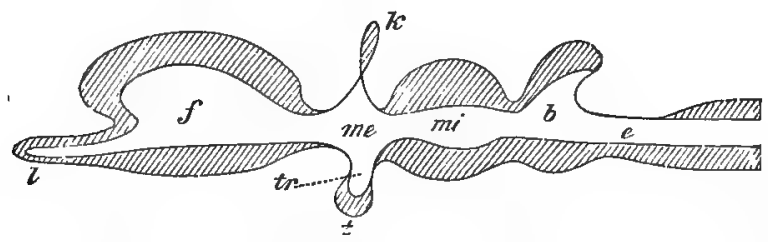

Fig. 272. Diagrammatic vertical longitudinal section through a vertebrate brain. $f$ cerebrum, me thalamencephalon, $m i$ mid-brain, $b$ cerebellum, $e$ medulla, $l$ olfactory lobes, $k$ epiphysis, $t r$ hypophysis, $t$ pituitary body.-Orig.

cephalon); mid-brain (mesencephalon); primary and secondary hind-brain (metencephalon, myelencephalon); they lie one behind the other, and can be distinguished throughout the whole series of Vertebrata, from Pisces upwards, although in other respects the structure of the brain, as a whole, and of its various parts, exhibits great variety. The most anterior section, the prosencephalon, or cerebrum, which is usually well developed, and, especially so in higher Vertebrata (Birds and Mammals), is generally divided into two halves (the cerebral hemispheres) by a longitudinal fold which dips into it from above and before, and these hemispheres are prolonged anteriorly into a pair of small hollow bodies, the olfactory lobes; the wall of the cerebrum is much thickened both above and below. The thalam- 
encephalon is always relatively small; its wall is only thickened below and at the sides, dorsally it is very thin; a small process extends from it to end above in a small body which varies considerably in form, the pineal gland (epiphysis): ventrally, the wall is evaginated to form a funnel-like pit, the inf undibulum, with a peculiar appendage, primitively an invagination of the epithelium of the mouth, the pituitary body (hypophysis). The thickened lateral portions of the thalamencephalon are termed optic thalami. The cerebrum and the thalamencephalon are separated by a deep transverse fold dorsally. The midbrain has a thickened upper wall divided into two halves (the optic lobes) by a longitudinal furrow, and in the Mammalia is also divided by a transverse groove, so that there are four lobes, hence the name, corpora quadrigemina. The cerebellum, which is specially well developed in Birds and Mammals, has, usually, a much thickened upper wall, extending posteriorly over the medulla, whose dorsal boundary is, on the contrary, very thin, whilst in other respects this portion of the brain is similar to the spinal cord, into which it is continued posteriorly without any sharp demarcation. The spinal cord extends through the vertebral column, as an almost cylindrical rod dwindling to a point posteriorly; at the two regions where the nerves for the limbs originate, it is usually somewhat enlarged. Brain and spinal cord may be seen even with the naked eye to consist of two substances, the $\mathrm{grey}$ matter and the white matter; the former consists of very numerous ganglion cells, which lie embedded in a peculiar kind of connective tissue (neuroglia), the latter consists of nerve fibrils.

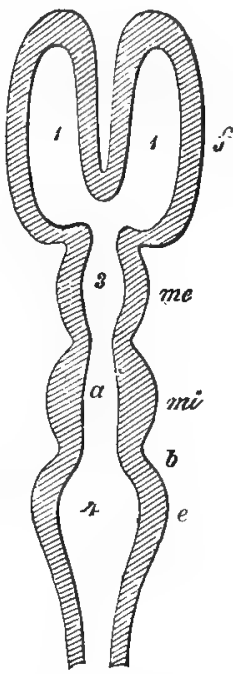

Fig. 273. Horizontal longitudinal section through the brain of a Vertebrate. Diagrammatic. cerebrum, me thalamencephalon, mi midbrain, $b$ cerebellum, $e$ medulla, 1, 1 the cavities of the cerebral hemisphere (ventriculi laterales), 3 cavity in the thalamencephalon (ventriculus tertius), a that of the mid-hrain (aquoductus Sylvii), 4 that of the hind brain (ventriculus quartus),-Orig.

Often, e.g., in Manmmalia, the primitive relations of the vertebral column and spinal canal have undergone a change, for, owing to the more rapid growth of the former, the hind end of the spinal canal is left empty. The result of this again is that the posterior spinal nerves run within the canal for some distance before making their exit.

The brain and spinal cord are surrounded by three connective tissue sheaths. Most externally lies a fibrous, hard covering, the $d$ ur a mater, which invests the inner surface of the skull closely, whilst in the Mammalia, a special portion of the periosteum lines the spinal canal; the dura mater frequently forms large folds stretching between the different sections of the brain. Next to the nervous matter lies a very vascular covering, the pia mater, and between this and the dura is the thin arachnoid membrane; this last is not 
distinguishable from the pia mater in Fish, and in other Vertebrata they are closely connected. A number of pairs of nerves arise from the brain, in part sensory (tactile, olfactory, optic, and auditory), and in part motor; they are principally distributed to the head. From the thalamencephalon and the optic lobes arise the optic nerves, which are remarkable for crossing at their point of origin; the nerve of the right eye thus arises to the left of the median line, and crosses over. The simplest case of such a crossing or chiasma occurs in many of the Teleostei, where the two nerves simply cross, without entering into any closer connection. In most other Vertebrata, on the contrary, the optic nerves exchange some fibres at the chiasma, so that, whilst the main portion of the nerve which originates on the right side runs to the left eye, a few of its fibres bend round and join the other optic nerve, which, for its part, also gives up some nerve fibres to its fellow. Of the other cranial nerves, the olfactory arise from the olfactory lobes, the others mostly from the ventral side of the medulla. One of these, the vagus, is remarkable in that it not only sends branches to the head, but also posteriorly, to supply, for example, certain portions of the alimentary canal. The $\mathrm{s} p$ in a $1 \mathrm{n}$ erves usually leave the vertebral canal laterally, one pair between every two successive vertebræ; each nerve originates from the cord in two roots, of which the dorsal is furnished with a small ganglion close to its point of origin, and contains exclusively sensory nerve fibres, whilst the ventral root consists of motor fibres only. The nerves supplying the limbs originate in a number of spinal nerves, connected together into so-called plexuses (brachial plexus and lumbar plexus, for the fore and hind limbs respectively). A peculiar system is the so-called sympathetic nervous system, the principal part of which is a pair of stout nerve cords running ventral to the vertebral column, and only connected to the spinal cord and brain by small connectives. The sympathetic nerves, which branch over the alimentary canal and other viscera, are provided with a great number of ganglia; the movements of the parts which they supply (e.g., the muscles of the alimentary canal) are involuntary.

Concerning the tactile and gustatory organs of the Vertebrata, see the General Part, pp. 18-19.

The olfactory organs are, in the Fish, a pair of large pits on the surface of the head, covered by an epithelium, in which are sensory cells. In other Vertebrata, it is only in .early embryonic stages that the olfactory organs are such superficial elongate pits (Fig. 283); gradually each pit becomes overgrown by the surrounding parts, so that it forms a tube with an anterior and a posterior orifice, of which the former opens 
freely on to the surface of the head, the latter into the mouth, within the upper jaw (the premaxilla and maxilla develop in those parts which have grown over the olfactory pits). Thus the anterior end of the head becomes perforated by two tubes which are usually close together, and separated only by a thin septum; in the tubes, there is a limited region containing the olfactory cells. The lining often projects as large folds, which may be supported within by bony or cartilaginous pieces (the turbinals). For further details, see the various classes.* In those Vertebrata, in which the olfactory apparatus consists of two tubes leading from the upper surface of the head to the mouth, they have still another function, for air for the respiratory organ enters through them.

The eye or optic bulb consists externally of the sclerotic c o at, a firm connective tissue sheath, varying in thickness and often strengthened with cartilaginous or bony plates: it becomes transparent anteriorly to form the corne.a. Within the sclerotic there lies the darkly coloured vascular coat, the choroid, and within this, again, the retina, connected with the sclerotic and choroid by the optic nerve, which perforates them. In the cavity of the eyeball, towards the exterior is the lens, consisting chiefly of long filiform cells; in aquatic animals it is usually almost round, in terrestrial forms more flattened. Behind the lens lies the vitreous humour, a gelatinous connective tissue mass; the cavity between the lens and the cornea is filled with lymph, the aqueous humour. In front of the lens there is a circular extension of the choroid, the muscular pigmented iris, whose circularly arranged muscle cells respond involuntarily to the action of the light, and so narrow the aperture, that less light enters when the illumination is brilliant. The opening of the iris, the pupil, is either round or elliptical, in the latter case either vertical or horizontal. The choroid itself is provided just behind the lens with numerous meridional folds, the ciliary processes, which are slightly developed or wanting in Pisces.

With regard to the development of the eye, it may be noted, that at an early stage of ontogeny, the fore brain forms anteriorly on either side a vesicular evagination, the primary optic vesicle, which is connected with the brain by a short stalk, whilst its distal portion lies close below the skin. Then follows an invagination of the outer part of this vesicle, so as to form a double-walled

* In some Reptiles and most Mammals, there is a peculiar saccular or tubular paired organ, Jacobson's organ, in close connection with the olfactory apparatus; its epithelium contains olfactory cells, and it receives fibres from the olfactory nerves. In Reptilia (Snakes and Lizards), it is a small sac lying below the nose, and opening anteriorly into the mouth; in Mammalia, it is a long tube, closed behind, running below the mucous membrane of the nose, on either side of the lower edge of the nasal septum; and opening usually in a fine canal (duct of Stensen), which leads into the mouth ; more rarely it opens direct into the nose, 
capsule, the secondary optic vesicle, whilst the stalk elongates; then the cavity of the stalk diminishes as does also the cavity between the two layers of the vesicle (i.e., the cavity of the primary optic vesicle). The stalk forms the optic nerve, and the wall of the vesicle forms the retin a; the outer layer becomes very thin and forms a sheath of strongly pigmented cells (the pigmented layer of the retina, tapetum nigrum), whilst the rest of the retina is formed from the thick inner layer. At the
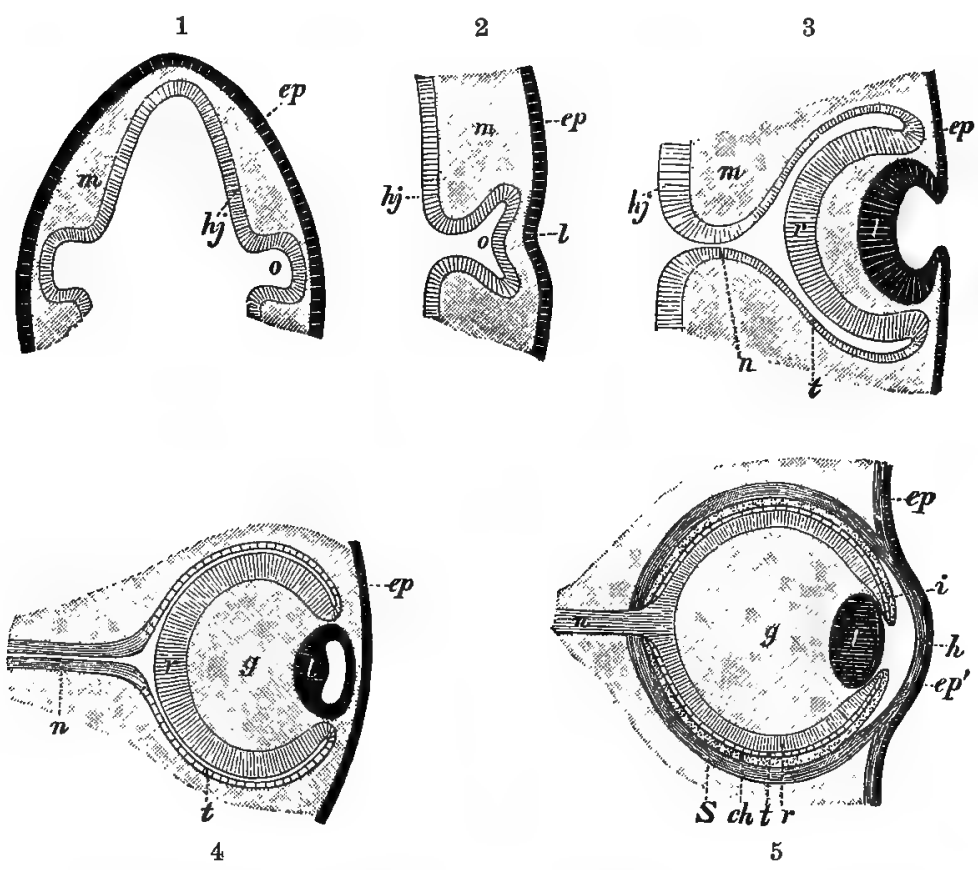

Fig. 275. Diagrammatic representation of the development of the vertebrate eye. 1 section through the head at an early stage. 2 somewhat later stage : the incipient lens, and the invagination of the optic vesicle may be seen. 3-4 further development: constriction of the lens, formation of the secondary optic vesicle. 5 the other chief parts of the eye are formed. ch choroid, ep epidermis, $e p^{\prime}$ epidermal portion of the cornea, $g$ vitreous humour, $h$ cornea, $h j$ brain, $i$ iris, $l$ lens, $m$ mesoderm, $n$ optic nerve, $n$ primary optic vesicle, $r$ retina, $S$ sclerotic, $t$ pigment layer (external sheath of the retina).-Orig.

time when the primary optic vesicle is becoming capsular, the lens begins to form. It develops as an invagination, which is finally completely cut off from the rest of the epidermis and takes up its position below this and opposite to the optic capsule. The transparent lens develops from this epithelial sac by further modification of the cells. The remaining portions of the vertebrate eye then form round this essential part: the connective tissue between the lens and the retina forms the $v$ itreous humour, the covering to the lens forms the cornea; between the latter and the lens there 
is a space in which the a queous humour is secreted; outside the retina, the choroid with the iris, and the sclerotic, develop from the surrounding connective tissue.

From this account it will be clear that the sensory, that is, the essential, part of the vertebrate eye, has a different origin from the retina of invertebrate animals. The origin of the vertebrate eye may be imagined thus : originally a vesicular evagination of the brain served for the perception of light, and later a lens, etc., developed and closed the optic vesicle, which had been modified in the meanwhile. $C f$. also the pineal eye described below, which is similarly a vesicular evagination of the brain. It must also be mentioned here that in certain lower forms from which eyes are absent, part of the central nervous system is sensitive to light (see p. 22).

The retina of the Vertebrata, like that of many other animals, possesses rod-like refringent bodies which form a special layer (rods and cones); it differs however, in that the rods are not in that part of the retina which is towards the light. They lie directly upon the pigment sheath, and in order to reach them the light has to traverse all the other layers of the retina. A considerable number of thin layers is distinguished in the fully developed retina.

The eye lies in a deep basin-like cavity on the side of the head surrounded by loose connective tissue; this depression, the or bit, is arched over above by the skull; frequently too, it is more or less completely bounded in front, below, and behind by osseous or carti-

$\boldsymbol{A}$

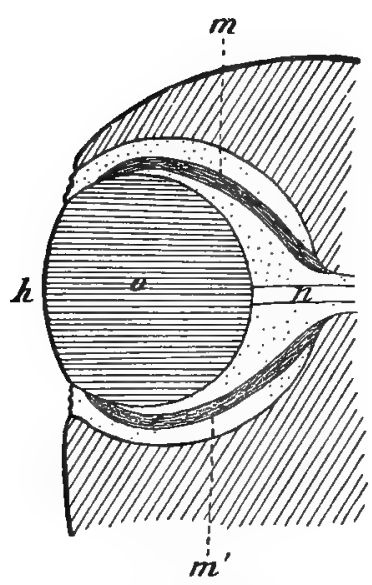

$B$

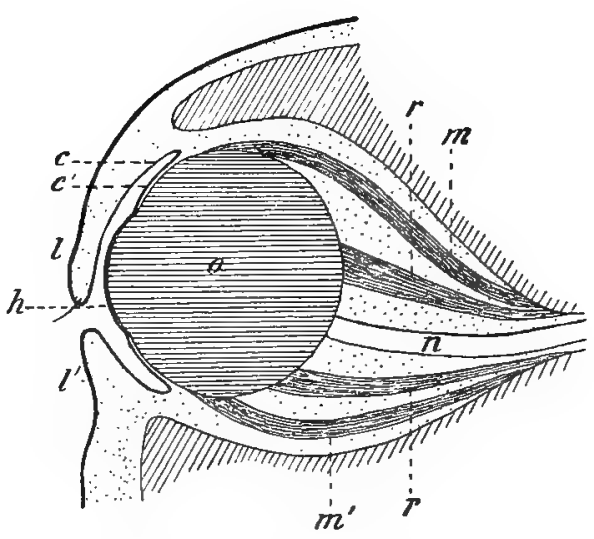

Fig. 276. Diagrammatic section through the or bit $A$ of a Fish, $B$ of a Mammal! c conjunctiva palpebrarum; $c^{\prime} c$. oculi; $h$ cornea; $l$ upper ; $l^{\prime}$ lower eyelid ; $m$ superior, $m^{\prime}$ inferior rectus muscles; $n$ optic nerve; o bulb of the eye; r retractor bulbi. The skin is represented by a thick black line, the connective tissue dotted, the wall of the orbit (consisting of bones, muscles, etc.) shaded by oblique lines).--Orig.

laginous pieces. The cornea is generally directly continuous with the skin, which is usually soft and flexible in this region, so that the eye can move within the orbit without hindrance. The movements are brought about by muscles, which arise from the skull, and are attached to the eye-ball. There are almost always four rectus eye 
muscles (musculi recti), superior, inferior, internal, and external, and two oblique muscles (musculi obliqui). The recti, which are usually attached to the skull, close to the point where the optic nerve perforates it, and to the eyeball, in a circle a short distance from the cornea, move the eye upwards, downwards, inwards, or outwards; the oblique muscles, which are usually attached to the anterior or nasal wall of the orbit, and above or below to the optic bulb, cause it to rotate on its axis which runs approximately through the middle of the cornea. Besides these, there is, in not a few animals (Amphibia, Reptilia, Mammalia), a muscle which withdraws the eye-ball (retractor bulbi); this muscle surrounds the optic nerve, and springs from a point close to the optic foramen.

Excepting in Pisces, a circular fold of skin, which can be drawn over the cornea, arises a short distance from, and almost parallel to, it. This fold consists of upper and lower portions, the upper and lower eyelids, whose edges meet when they are drawn over the eyes; in the Mammalia the upper lid is best developed; in other animals, the lower. Just below the portion of skin covered by the eyelids there is usually a thin and soft membrane, which is termed the conjunctiva bulbi.* In many Reptiles, in Birds, and in many Mammals there is a nictitating membrane, a fold of skin developed in the inner corner of the eye, within the true eyelids. In the two first-mentioned groups it is large, semi.transparent, and moved by a special muscle; in the Mammalia it is not so well-developed.t Connected with the eyes are various glands, opening below the eyelid or nictitating membrane, and serving to keep the cornea and the inner side of the eyelid damp and smooth. In Pisces such glands are altogether absent, whilst in all other Vertebrates one or more are present. There is generally a lachrymal gland which opens behind (at the outer corner of the eye), usually by several apertures, on the inner side of the lower or of the upper eyelid, $\ddagger$ and a $\mathrm{Harderian} \mathrm{gland}$, which opens anteriorly in the inner corner of the eye (usually within the nictitating membrane if this is present); the secretion of the former is usually more watery, of the latter more fatty in composition. Part of the secretion from these glands runs through a tube, the lachrymal canal, which has several openings in the inner corner of the eye and discharges into the nose. ('The lachrymal canal is primitively a trough-like epiblastic invagination which becomes constricted to form a canal; see Fig. 283 ANr).

* The soft inner covering of the eyelids is termed conjunctiva palpebrarum.

+ In many Fish i m movable folds of the skin, otherwise like eyelids, lie round the eye. In some Sharks there is a movable nictitating membrane.

I Only in Mammalia some (often the majority) of the openings of the lachrymal glands are on the upper eyelid, 
A peculiar third unpaired eye, the pineal eye (parietal eye), in connection with the pineal body, has been recently described in some $\mathrm{Lizards}$ (e.g., in the common Lacerta, Blind-worm, and others). It lies in a small perforation of the upper wall of the skull (in the parietal bone, or between this and the frontal), close below the skin, which is at this spot partially transparent. It consists of a vesicle formed of a simple epithelium, the upper portion forming a lens-like thickening, whilst the lower part is much pigmented (retina). In other Lizards the same structure occurs in the same position in a more rudimentary form, as a simple vesicle, which does not resemble an eye, but is unpigmented, and without a lens. A structure like the first described occurs also in the Cyclostomi, where, however, it is covered by the skull, which is often somewhat thin at this place, and may even, like the skin above it, be transparent. In various other Vertebrates also, facts are known which point to a

$A$

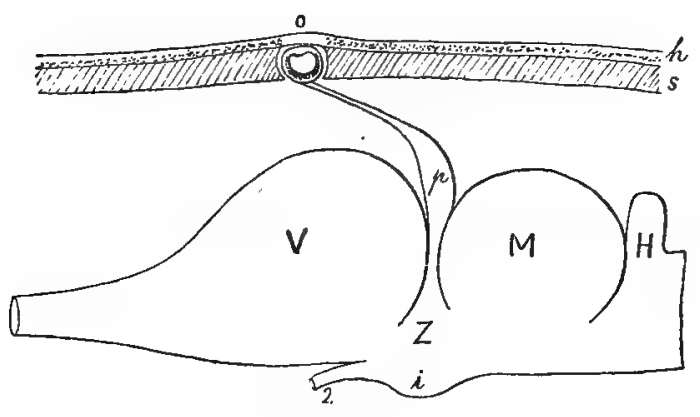

$B$

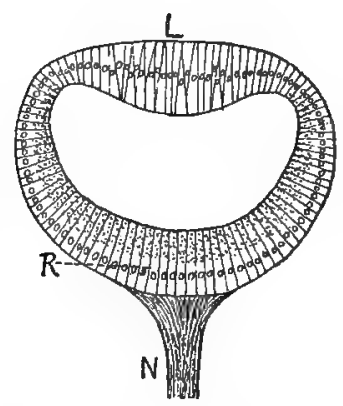

Fig. 277. Pineal eye of a Lizard; diagrammatic. A brain and upper wall of the skall, the latter cut through; $B$ pineal eye alone, in section. $\nabla, Z, M, H$ cerebrum, thalamencephalon, optic lobes, cerebellum; $h$ skin, $s$ roof of skull, o unpigmented portion of skin below which the pineal eye lies, in a hole in the roof of the skull; $p$ epiphysis, $i$ hypophysis, 2 optic nerve. $L$ lens, $R$ retina, $N$ nerve of pineal eje.-Orig. (using Spencer's figures).

connection between the pineal gland and the exterior. In the Selachians the pineal gland is filiform, and its distal end lies in a hole in the dorsal wall of the skull, covered entirely by skin; so far as is known it possesses no optic structure. In the Anura also, the epiphysis, which is short at first, elongates during larval life into a long thread, with a terminal enlargement; the thread perforates the skull-wall, and the swelling lies on the upper side of the head, directly beneath the epidermis.

'The auditory organs, one on either side, arise in the embryo as vesicular invaginations of the epidermis, which gradually sink in deeper, and become surrounded by the cartilage of the skull (liter often by bone). The invagination remains for some time connected with the exterior by means of a caual, but later is usually completely cut off from the skin, so that the developing organ is a closed sac; in some cases, however (the Selachians), the canal persists throughout life as an open tube. The vesicle does not retain its primitively simple form, but is modified, so that the auditory organ in its adult condition 
consists of a saccular chamber, and three semi-circular canals; the latter are tubes opening at both ends into the vesicle, to which they are attached like hollow handles. The sac itself is divided by a constriction into two portions, the sacculus and the utriculus; the canals open into the latter, and each possesses a swelling at one end (ampulla). The sacculus usually bears an evagination (ductus cochlearis), which is, in Fish, Amphibians, and many Reptiles, a short minute pouch; in some Reptiles (the Crocodiles) and in the Birds, a longer tube; but attains its highest development in the Mammalia, where it forms a long spiral canal. This whole vesicle, which consists of epithelium, surrounded by a thin layer of connective

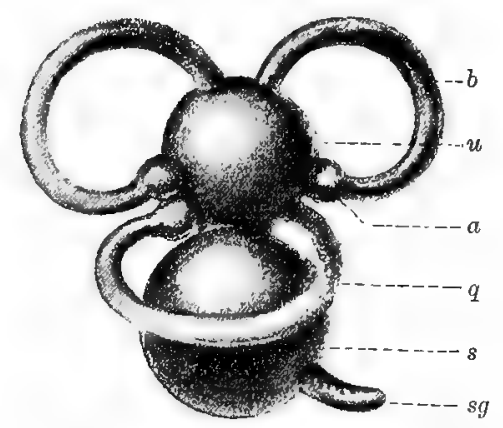

Fig. 278. Diagram of the auditory organ (membranous labyrinth) of a Vertebrate. $a$ ampulla, $b$ semicircular canal, $s$ sacculus, $u$ utriculus $(s+u$ auditory vesicle), $s g$ cochlea.-Orig. tissue, is termed the membranous labyrinth. This is the essential auditory organ which occurs in all Vertebrata, Amphioxus alone excepted, and usually includes all the parts named. There are certain cells in the epithelium provided with one or more processes, to which the branches of the auditory nerve are distributed, and which are closely connected with the sense of hearing. In the labyrinth there are otoliths, either small crystals or larger calcareous bodies (Teleostei). The membranous labyrinth, which is inclosed in the lateral wall of the skull, constitutes in Fish the whole auditory apparatus; in other Vertebrata various a c cessory structures are usually connected with it (tympanic-cavity, Eustachian tubes, tympanum, ear-bones), which will be considered in the different groups.

Those portions of the skull which closely surround the membranous labyrinth are often (e.g., in the Mammalia) more compact in structure than the rest of the bone, and may be entirely separated, giving exactly the form of the enclosed membranons labyrinth : this is termed the bony labyrinth.

The alimentary canal is divisible into the following parts: buccal cavity, œesophagus, stomach, small intestine, and rectum. Of the structures connected with the spacious buccal-cavity, the teeth will be considered first.

The teeth, both in structure and development, are essentially characteristic of the Vertebrata. They are present not only in the mouth, but in many Fish (especially in the Selachians) on the skin also. They occur within the mouth in all classes (with the exception of the Lancelet, Amphioxus), although they are frequently absent. In the simplest case (Fig. 279 A) the formation of teeth occurs 
in the following way : a papilli of the dermis, or of the corresponding connective tissue of the mucous membrane, grows into the epidermis, or what is the same thing, the epithelium of the mouth. This papilla secretes, on its upper surface, a covering of dentine, a substance as hard as bone, the structure of which will be considered later; whilst the cylindrical cells, constituting the lowest layer of the epithelium, corering the papilla, secrete on their under sides a layer of still harder material, the enamel. Between the papilla and the epithelium

A
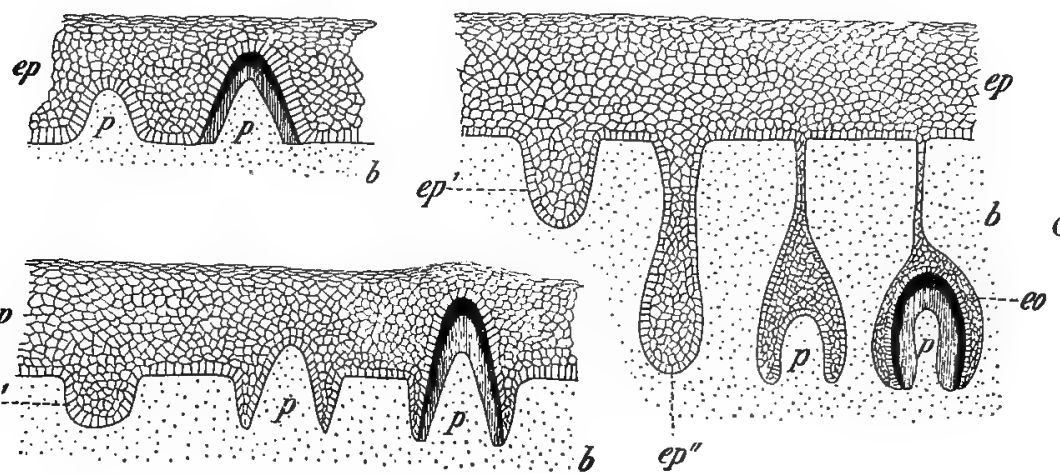

Fig. 279. Diagram of various developing teeth. To the left in each figure a very young one is represented, followed to the right by an older or several older teeth in succession. A simplest form, $B-C$ more complicated forms.. Enamel black; dentine perpendicularly shaded; connective tissue dotted. $b$ connective tissue, eo enamel organ, ep epithelium, $e p^{\prime}$ epithelial papilla (incipient enamel organ), $e p^{\prime \prime}$ older epithelial papilla, $p$ dermal papilla (pulp of tooth). - Orig.

a hard cap is thus formed, which consists within of a layer of dentine, secreted by the papilla, and externally of a layer of enamel, secreted by the epithelium; the two layers are inseparably connected, and together make up the joung tooth. The dentine is gradually thickened by the secretion of new material by the papilla, which at the same time becomes smaller, and is finally often reduced to a relatively small structure, the $p u l p$, within the tooth. The enamel is increased by the deposition of new material on its surface, it never attains so great a thickness as the dentine, and is often not distributed over the whole tooth, but occurs only at its apex. As a rule, however, development is somewhat more complicated (see Fig. $279 \mathrm{~B}-\mathrm{C}$ ) ; before the formation of the papilla a thickening of the epithelium occurs at a given spot, and in connection with it there is an ingrowth into the connective tissue $\left(e p^{\prime}\right)$, which is usually so deep that the point of the tooth does not project at all into the true layer of epithelium, but lies exclusively in the sunken portion (Fig. $279 \mathrm{C}$ ); this latter often only retains its connection with the epithelium by a thin cord, and, indeed, is often completely separat' $d$ from it. In other 
respects, however, the tooth develops in exactly the same way as in the other case : a papilla grows up to the portion of epithelium that has sunk in, and so on. Dentine has a certain resemblance to bone, consisting of cells and a calcified inter-

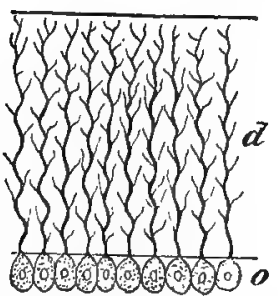

Fig. 280. Section through a tooth to show the structure of the dentine; diagram. matic. $d$ dentinal tubes, $o$ odontoblasts on the inner side of the dentine.-Orig. cellular substance. They differ, however, in that each of the cells of dentine (odontoblasts) possesses only a single long, filamentous process, provided with delicate lateral branches, which run transversely through the whole of the dentine, parallel to neighbouring processes; whilst the cell itself, with its nucleus, is never enclosed in the intercellular substance, but lies on the upper surface of the papilla. The dentine is thus provided with numerous delicate canals, each containing a cell process ; for as the dentine increases in thickness the processes gradually elongate. The enamel is a very hard substance, consisting chiefly of calcium phosphate, which in the Mammalia, at least, is composed of thread-like prisms, whilst in many lower forms it appears more homogeneous. It is a cuticular secretion of the lowest layer of epithelial cells.* The fully-formed tooth, which varies considerably in form (though all varieties are modifications of the cone), may have its tip pushed through the mucous membrane by the growth of adjoining parts, and may be attached by its lower end to the subjacent bones

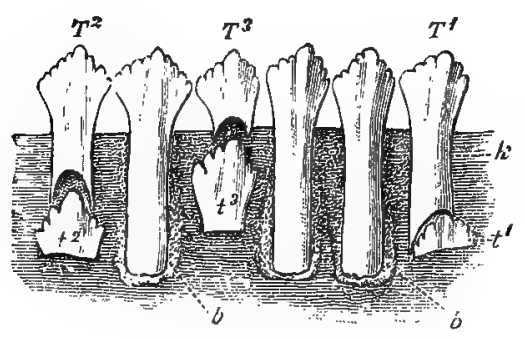

Fig. 281. Portion of upper jaw of a Lizard (Iguana) seen from the inner side; soft parts removed. $k$ jaw bone, to the inner side of which the teeth are firmly ankylosed by a porous mass of bone $b$. $T^{\mathrm{L}}-T^{3}$ three teeth which are about to fall out, the lower ends being more or less absorbed ( $T^{\mathrm{l}}$ least, $T^{3}$ most); $t^{1}-t^{3}$ the corresponding incompletely developed replacement teeth. - Orig.

(or cartilage) by a fibrous portion of connective tissue, or a small osseous mass, the socket, which develops between the tooth and the bone. In Mammalia and some others, the teeth are placed in alveoli, deep cavities in the bone, into which the lower end of the tooth is sunk. The teeth are, of course, subjected to much, and often rough, usage, and only remain for a limited time in the month. They then fall out and new ones are formed; and thus

* For the e em ent, which is only present in Mammalia, see that section, 
there is a succession of teeth; before falling out a tooth loses its connection with the bone, the socket is reabsorbed. (For the special conditions of replacement in Mammalia, see that group).

Among other hard parts connected with the oral cavity, and occasionally occurring among the Vertebrata, may be mentioned certain horny structures, locally thickened and hardened portions of the cuticle, which are developed here just as on the outer skin: the horny teeth of Cyclostomes and Monotremes, the horny jaws of Aves, Chelonia, and others.

The tongue projects from the floor of the mouth, and is intimately connected with the visceral skeleton, especially with its unpaired portions. It is a feeble structure in Fish, but in Mamamals it is well-developed, muscular, and very movable, and thus of great importance in introducing food into the mouth. It exhibits a great diversity of form, as will be noticed more particularly in the various groups: it is rarely altogether absent. Various glands are also connected with the mouth, pouring into it secretions which serve to moisten the food, etc.; they are absent from Fish, but are developed in all other groups. As a rule they are embedded in the wall of the buccal cavity, but in the Mammalia some lie apart from it, and only communicate with it by their ducts ; here they have, moreover, attained to a considerable size as "salivary glands"; this is only exceptionally the case in other groups.

Developmentally, the thy roid gland is also connected with the moutl. It arises* as one or more evaginations of the Hoor of the oral cavity, from which, however, it soon separates, and later forms a large. independent, ductless gland, which consists chiefly of epithelial sacs, filled with fluid, and united by connective tissue. The function of the thyroid was until recently a complete riddle; medical researches of the last few years, however, have demonstrated with certainty that it forms a product of vital importance, which is carried by the blood to the rest of the body. The enigmatical thymus alno arises as a series of evaginations, which separate later from the buccal cavity. It is well developed in enıbryos and young animals (in many young Mammalia it is an extensive organ, stretching far back into the thorax), but it degenerates in after life. In the adult the thymus consists chiefly of cellular connective tissue.

The esnphagus is short and wide in Fish and Amphibia, but in Reptiles and Birds may be longer, in consequence of the formation of a neck; in the Mammalia it is not only fairly long, but also rather uarrow, although capable of great distension, whilst in all the others it is very wide. The stomach is a wider region varying in form, with innumerable small tubular glands in its walls. The small intestine is a straight tube in the Cyclostomes and some other Fish, but is usually coiled. In Fish, Amphibia, and many Reptiles it is still comparatively short: in Birds and Maumals on the contrary, it attains a considerable length, several times the length of

* According to general opinion, the thyroid corresponds to the endostyle in the branchial sac of Ascidians (q.v.). 
the body. In correlation with the importance of the small intestine as un organ of absorption there are various arrangements for increasing its inner surface, folds arranged in a network, or papillæ (villi), the latter chiefly occurring in the Mammalia. At the anterior end of the small intestine opens the duct (or ducts) of the liver, a very large, often lobed gland, consisting of very numerous lobules; the duct (bile duct) is usually provided with a saccular expansion, the gall bladder, which forms a reservoir for the bepatic secretion, the bile. Close to the opening of the bile duct into the gut is the orifice of another large gland, the pancreas, which, like the liver, is

$A$

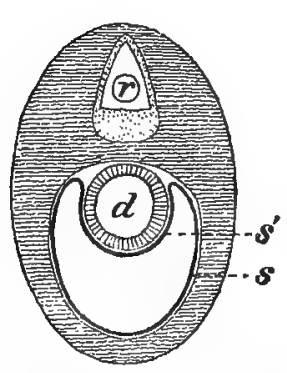

Fig. 282. Diagram to explain the structure of the mesentery; transverse section of the body. $A$ earlier, $B$ later stage. $d$ gut, $s$ peritoneum (thick line), $s^{\prime}$ the portion of this surrounding the gut: $m$ mesentery, formed of two layers ; $r$ spinal cord.-Orig. usually ertensive; it is absent from some Fish. Besides these larger glands external to the intestine, there are frequently numberless small tubular or racemose glands lying in its wall (e.g., in the Mammalia). The terminal portion of the alimentary canal is the rectum, which is wider than the small intestine. In most Vertebrata it is short, and is then straight also; it only attains a greater length in the Mammalia; here it is termed the $\mathrm{L}$ a $\mathrm{rg} \mathrm{e}$ intestine, the term rectum being reserved for the termination only. In many Vertebrata the hinder end of the rectum acts as a cloaca, the urinary and genital ducts opening into it. At its anterior end, at its junction with the small intestine, the rectum (or large intestine) is often provided in Reptiles and Mammals with one, in Birds with two, cæc a of varying length. The anus is situated ventrally at the base of the tail, it is either round or a longitudinal or transverse slit.

The alimentary canal in the embryo is for some time a straight tube running through the body-cavity along its dorsal surface, invested with a thin connective tissue membrane, the peritoneum, which covers all the organs in the body. cavity. Later it leaves the body-wall and sinks deeper into the cavity, drawing its peritoneal covering with it, so that in the adult it is suspended by a large fold of the peritoneum (Fig. 282). Where they do not surround the alimentary canal the two layers of this fold lie close together, and form the mesentery, which appears as a thin connective tissue lamina between the upper body-wall and the digestive tract. Similar mesenteries may also be formed for other organs in the body-cavity.

The respiratory organs of Vertebrata are sometimes gills, sometimes lungs, the latter occurring in almost all (with the exception of Amphioxus, the Cyclostomes, the Selachians, 
and some others), whilst the former are limited to Fish and the larvæ of Amphibia. The gills usually consist of very vascular laminæ arranged in a single series on the sides of the gill-clefts; the latter are a series of large lateral slits, which lie closely behind each other as perforations in the wall of the oral-cavity. The clefts are separated by septa, in which lie the visceral arches mentioned above (see also Pisces). It is of great interest that in the embryos of all higher Vertebrata (Reptilia, Aves, Mammalia), which at no time of their lives breathe by gills, similar visceral clefts occur; they do not, however, bear gill filaments, and they close again later.

The lungs arise as an unpaired evagination of the alimentary canal, at the junction of the buccal-cavity and œsophagus. During further development, this evagination does not usually remain simple, but divides into two sacs, right and left, communicating with the alimentary canal by a common tube. Each sac, in the simplest case, grows into a large, thin-walled bag, the wall of which is richly sup-

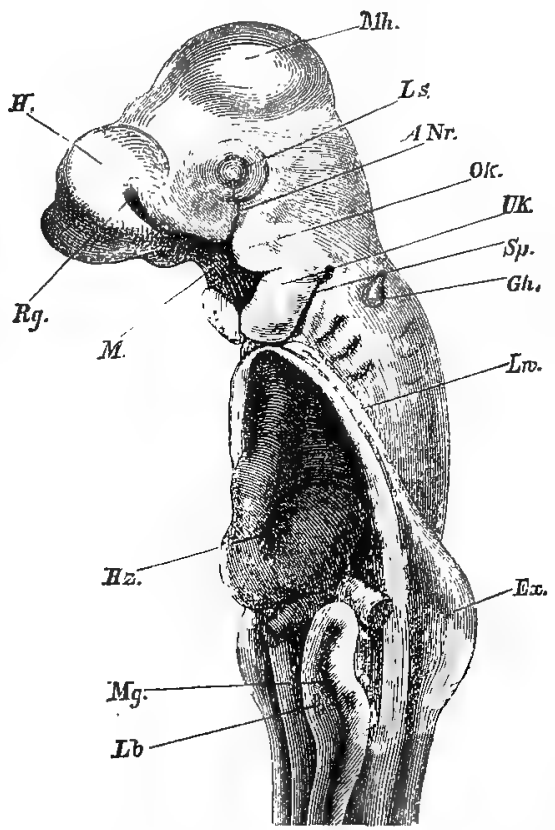

Fig. 283. Anterior portion of a $\mathrm{Chick}$ e m b r y o (four days' incuhation). $A N$ r inoipient lachrymal canal (still a groote), $E_{d}$ incipient left limb, $G h$ auditory vesicle, $H$ fore brain, $H z$ heart, $L b$ liver, $L s$ lens of eye, Lw body wall, $M$ mouth, $M g$ rudiment of stomach, $M h$ mid-brain, Ok upper jaw, $R g$ olfactory pit, $S p$ first viseeral cleft behind which three others may be observed, $0 k$ lower jaw. - After His.

plied with blood vessels (e.g., in the common Newt). Usually, however, the inner surface of the pulmonary sac becomes increased by evaginations, which remain united by connective tissue, so that the outer surface of the lung appears almost smooth. In some instances (e.g., the Frog) each lung contains a large central cavity and the evaginations are short; in others, the latter become longer and branching, whilst the central cavity is smaller (Reptilia); in the highest condition (in the Mammalia) the original sac is so much branched that it forms an arborescent organ, the finest branches of which end in tiny thin-walled vesicles. Over the walls of these minute sacs a fine capillary network is spread, whilst the trunk and the larger branches become thick-walled and firm, forming a kind of skeleton for the rest of the lung. Further, both larger and smaller 
branches are covered by connective tissue, so that the ramifications cannot be seen from without. On the inner surface of the lung there is usually, as has already been mentioned, a delicate vascular network; this is not, however, the case in most Fish; here the lung, which is usually an unpaired organ, has no such network, and therefore no respiratory function; it simply possesses the power of diminishing the specific gravity of the animal, and is termed the swim-bladder. A respiratory lung is only possessed by the Dipnoi and a few others. The unpaired, usually tubular, portion which connects the two lungs with the alimentary canal, the windpipe (trachea), varies much in length, usually in correlation with the form of the neck; it is generally strengthened by cartilaginous or bony rings, and in most Vertebrata (Amphibia, Reptilia, Aves, Mammalia, and some Pisces) it opens ventrally into the alimentary canal; in most Pisces, however, it opens dorsally. In the anterior, specially modified portion of the trachea, the larynz, there is, in many forms (Anura, Lacertilia, Crocodilia, Mammalia), a pair of projecting membranous folds, the $\nabla$ ocal cords, which are set in motion by the currents of air and thus produce the voice.

Circulatory organs. In the Fish the heart consists of three successive parts, atrium, ventricle, and con us arteriosus.* The atrium is a thin-walled sac lying above the ventricle which has thick spongy walls; the conus is tubular.

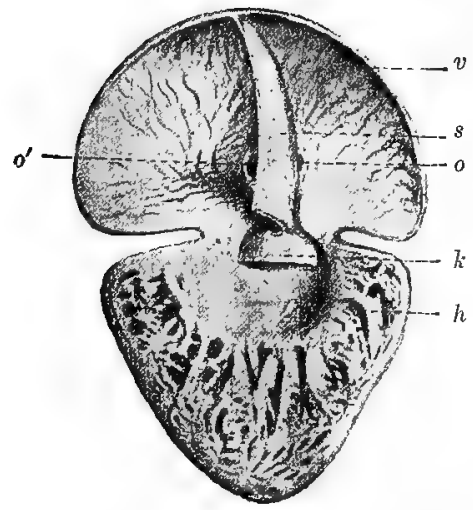

Hig. 284. Heart of an Amphibian; the ventral wall of atrium and ventricle is removed by a horizontal seotion; partly diagrammatic. $h$ spongy wall of the ventricle, $k$ valves, $o$ opening of the sinus venosus into the left auricle, $o^{\prime}$ opening of the sinus venosus into the right auriole, $s$ auricular septum, $v$ wall of auricle,-Orig.
Each portion encloses a simple cavity as does the large venous sac, the sinus $v e$ nosus, which lies in front of the auricle and brings into it the venous blood from the body. In the Am phibia the conus is well-developed, and the ventricle, with its spongy walls, is undivided, like that of Pisces. The atrium, on the other hand, is separated by a thin septum into right and left auricles; the sinus venosus is also divided. The left sides of the sinus and the corresponding auricle receive the blood from the lungs, the right that from the rest of the body (for details see Amphibia). In the Reptilia the atrium and the closely connected sinus are divided just as in the Amphibia; but the specialisation 
of the heart has proceeded further, the ventricle is at least imperfectly divided, and in the Crocodilia is completely separated by a perfect septum into right and left chambers, in communication with the corresponding auricles. The conus is rudimentary or absent. $\mathrm{Birds}$ and M ammals exhibit conditions very similar to those in Crocodiles; the auricles and ventricles are completely divided; the conus is absent. Valves which regulate the flow of the blood are always present at the junction of the auricle and ventricle and also in the couns, or when this is absent, at the limit of the ventricle. Auricles, ventricles, and conus are composed chiefly of striated muscle cells.

It must be noticed that the auricle and ventricle are simple during the embryonic life of the higher Vertebrata, the septa being formed later.

$A$

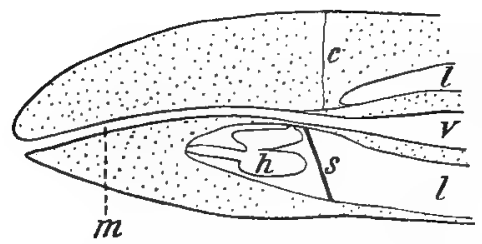

$B$

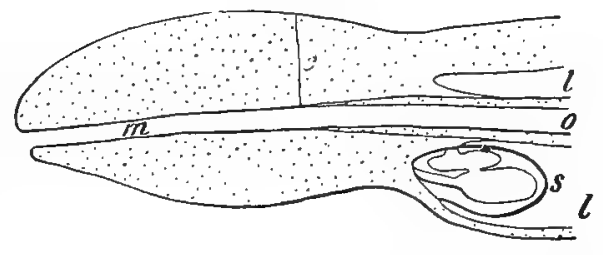

Fig. 285. Diagrammatic longitudinal section through the head and front end of the body to show the position of the heart and the pericardium. $A$ Fish, $B$ higher Vertebrate. c posterior boundary of the skull (the rest of which is not drawn), $h$ ventricle, $l$ body-cavity, $m$ buccal-cavity, o œsophagus, s septum or pericardium, $v$ stomach.-Orig.

The heart is ventral to the alimentary canal; in Pisces it lies close to the head in a special portion of the body-cavity (Fig. $285 \mathrm{~A}$ ), closed off from the rest by a transverse septum. In other Vertebrata it has moved further back, and therefore this septum is sacculated, and forms a pouch round the beart, the pericardium, which projects some way back into the general body-cavity.

A large ventral aorta springs from the heart in Fish and gives off a branch to each gill-arch, the afferent branchial arteries; there are usually fire pairs altogether in Selachians (gill-arches 2-6), and four pairs in the Teleostei (gill-arches 3-6). The blood, after passing through the capillaries of the gills of each arch, proceeds through the efferent branchial arteries to the a orta, a large unpaired artery running below the vertebral column, and giving off branches to the various organs. The large arteries of the head (carotids) arise from the first efferent branchial artery; if a functional lung is present, it usually receives blood from a branch of the last efferent branchial artery. The A m phibia generally exhibit similar arrangements during lar $\mathrm{val}$ life. Later the afferent and efferent arteries unite to form four simple arterial a r ches on either side, which run directly into the aorta. Of these, the third is usually atrophied in the adult; the 
second, on the contrary, is especially well developed, and supplies most of the blood for the aorta; the first arch practically supplies the head only; the fourth, the lungs. The second often loses its connection with the other arches, so that it alone forms the aorta. In other Vertebrata, the third arch is wanting; and it must also be noticed that the primitively simple arterial trunk is divided into two or three branches, of which one is exclusively connected with the last arch. In the Reptilia the arrangements are otherwise essentially similar to those of the Amphibia; in Birds and Mammalia, on the other hand, a further reduction has occurred, for the aorta is formed from one of the second arches only; from the right in Birds, from the left in Mammals. The connections between the first and second, and the second and fourth arches, which may still occur in the Reptiles, are absent from Birds and Mammals. (For details see the various groups.)

In Fish, the embryo develops six simple arterial arches on either side, which run along the six primary visceral arches, and unite to form the aorta; the first of these, and in Teleostei, etc., the second also, degenerates, whilst the others split into afferent and efferent branchial arteries. Further, in almost all ot h e $r$ Vertebrata these six arterial arches are present in the embryo; then some of them gradually atrophy, and thus are obtained the adult conditions already described. The appearance of arterial arches and visceral clefts in the embryos of higher Vertebrata (Reptilia, Aves, Mammalia) seems to prove that these have been derived from forms with branchial respiration.

With regard to the venous system, it must be mentioned that the venous blood from the alimentary canal, spleen, and other viscera does not go direct to the heart, but is collected into a large trunk, the portal vein, which enters the liver, and branches to form a capillary net-work. The blood is collected again into hepatic ve ins, which carry it to the heart. In Fish, Amphibia, and Reptiles such an arrangement also obtains for the kidney, the renal portal system: veins from the tail and hind limbs proceed to the kidney, and break up into capillaries, from which other veins arise and go to the heart. In Fish and Amphibia it not infrequently happens that several large veins are pulsatile (venous hearts); at these points, just as in the heart, transversely striped muscle cells are present. The veins, but not the arteries, are provided throughout with valves, which direct the current of blood. In the Vertebrata a well-developed capillary network is present, connecting the smallest arteries and veins. The blood corpuscles are of two kinds: amœboid white corpuscles, few in number; and discoid red corpuscles, constant in form, usually oval and nucleate, but in the Mammalia circular, biconcave, and without a nucleus. They impart the red colour to the blood, the plasma itself being colourless.

Occasionally an artery or vein breaks up suddenly into numerous branches, lying close together, and frequently anastomosing, and uniting again to form a single vessel. Such a network is termed a rete mirabile (swim-bladder of Fish, kidney, etc.). 

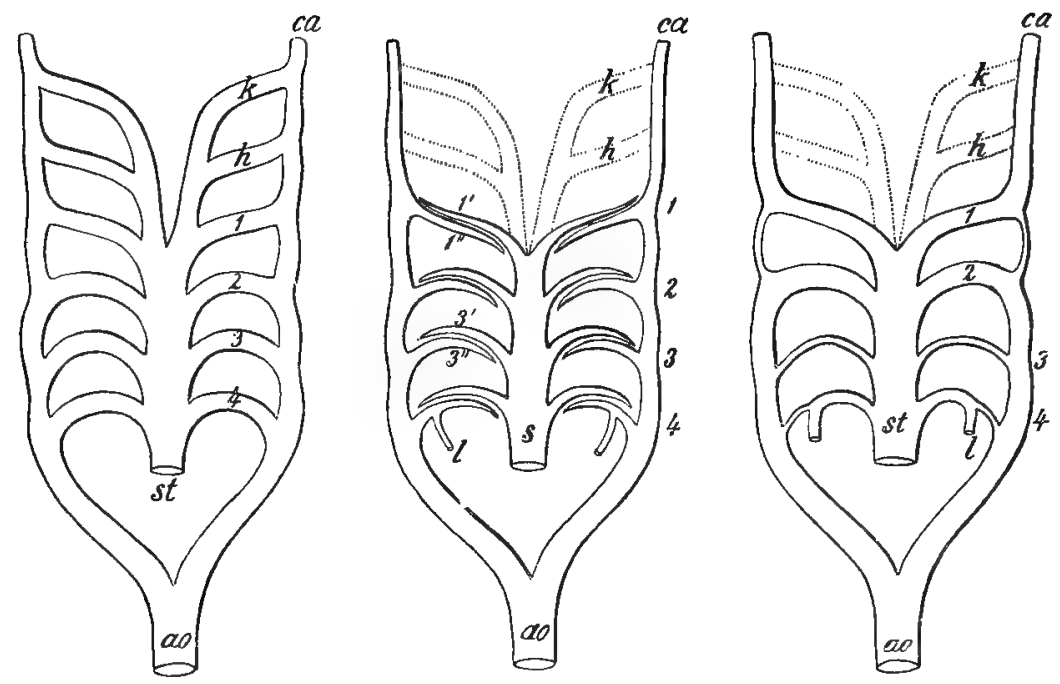

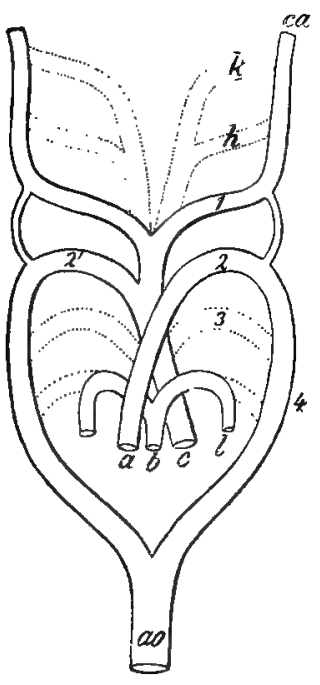

$D$

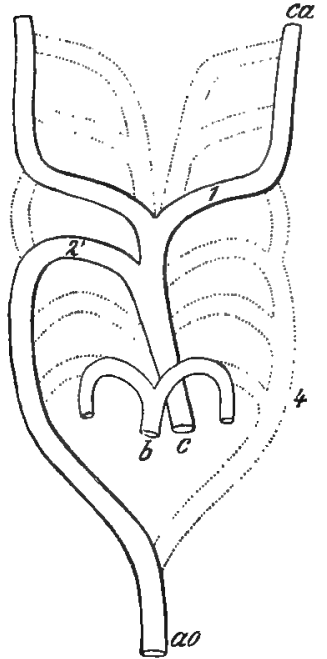

$E$

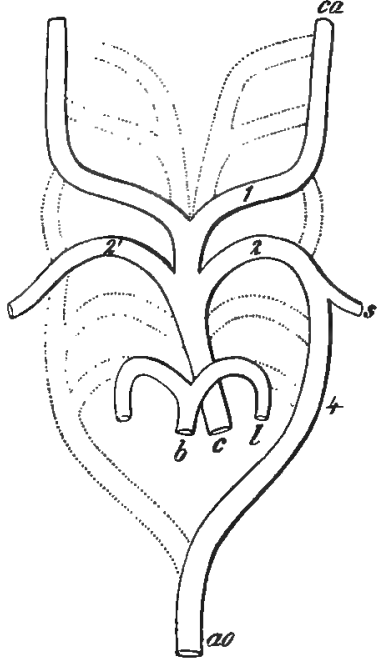

$F$

Fig. 286. Diagrams of the arterial arches of various Vertebrata. $A$ e $m$ bry on ic condition, $B$ Fish, $C$ Jrodele, $D$ Reptile (Lizard), $F$ Bird, $F$ Mammal. The atrophied vessels are represented by dotted lines. $k$ and $h$ the two first embryonic arches which almost entirely atrophy. 1-4 the four posterior arches. $1^{\prime}$ and $3^{\prime}$ the first and third afferent branchial arteries, $1^{\prime \prime}$ and $3^{\prime \prime}$ efferent branchial arteries, 2 in $D$ and $F$ second left arches, $2^{\prime}$ in $D, E$ and $F$ seoond right arches. $a, b, f$, vessels into which the ventral aorta is divided in Reptiles, Birds, and Mammals. ao aorta, ca carotid, $p$ pulmonary artery, $s$ (in $F$ ) artery of left limb, $s$ (in $B$ ) and st ventral aorta - Orig. 
Peculiar to the Vertebrata is the so-called ly mphatic system, a special system of canals and spaces, distributed over the whole of the body, and containing fluid. Its function is in part to reabsorb the plasma which has escaped from the capillaries into the tissues, in part to take up fluid nutritive substance (chyle) from the wall of the alimentary canal, and to cilury both into the blood. Its principal trunks open into certain large veins. In the lower Vertebrata (Pisces, Amphibia, Reptilia), the lymph vessels occur partly as sheaths round the arteries and veins, whilst they are elsewhere represented by special vessels which are, however, to some extent irregular in form, sometimes wide or saccular. There are often large lymph sinuses, e.g., below the skin in the Frog. Usually near the point of entrance into the veins, the large lymph vessels are rhythmically contractile, the lymph hearts; in the Frog, for instance, there is a pair posteriorly on the dorsal surface; they are absent from the Mammalia, but present in all other classes. The fluid in the lymphatics is colourless or whitish, and contains numerous leucocytes, identical with the white blood corpuscles. They are formed in very cellular portions of the connective tissue attached to the lymphatics, and, breaking loose, are carried away by the lymph; this tissue frequently forms specialised rounded bodies, the so-called lymph follicles, which, especially in the Mammalia, are collected into large lymphatic glands. An organ in connection with the true vascular system, which serves also for the production of white blood corpuscles, is the spleen, a dark-red body of considerable size, situated in the abdomen near the stomach.

The red blood corpuscles are formed chiefly in the spleen and in marrow. The epithelial lining of some of the blood

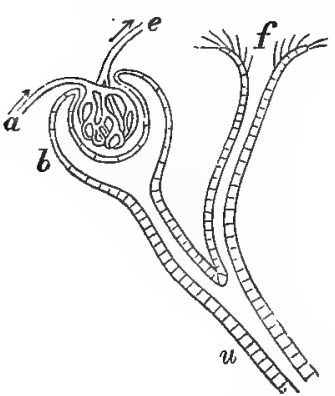

Fig. 287. Diagram of the end of a urinary $t u b u l e$ of a Vertebrate. $u$ urinary tubule, which splits into two branches, of which one ends in a ciliated funnel, $f$, the other in a Bowman's capsule, $b$. $a$ afferent, $e$ efferent arteries. -Orig. ressels in these parts is much thickened and stratified; cells of this epithelium develop into blood corpuscles, break off and enter the vascular current.

The kidneys, which lie on the dorsal wall of the body-cavity,consist of innumerable long, coiled glandular tubules, the u rinary tubules, bound together by connective tissue. The closed end of the tubule, which is somewhat expanded, is invaginated to form a capsule (Bowman's capsule), by the inpushing of a small rete mirabile, the glomerulus. This results from the breaking up of a small artery, its capillaries again uniting into a single artery, which, later, goes to form the capillary network of the kidney. The thin walls of the glomerulus appear to excrete the watery part of the urine, osmotically, whilst the salts are separated by the kidney tubules. In many Fish 
(Selachians), and in the Amphibia, branches arise from the urinary tubules and run to the surface of the kidney to open there by ciliated funnels (nephrostomes), so that they communicate directly with the body-cavity. The urine escapes through the u reters, which open either into the cloaca, or else by a single orifice near the anus. For the urinary bladder see the different groups.

In $\mathrm{Fish}{ }^{*}$ and $\mathrm{Amphibia,} \mathrm{each} \mathrm{kidney} \mathrm{is} \mathrm{connected} \mathrm{with} \mathrm{the}$ testis of the same side by fine transverse canals, so that the spermatozoa can escape through the urinary tubules and ureters. Usually the testis is only thus connected with the anterior and often narrow end. At an early stage of development a pair of embryonic kidneys is developed in Reptilia, Aves, and $\mathrm{Mammalia}$, and these for some time act as the excretory organs. Later, however, they are replaced by another pair, quite independent of the first, the adult kidneys, which are functional throughout

Fig. 288. Testis, Kidney, etc., of an Amphibian, diagrammatic. $e$ cloaca, $t$ testis, $u$ anterior, $u^{\prime}$ posterior portion of kidney, $u g$ ureter, $u g^{\prime}$ ducts of posterior portion of kidney which open into the hindmost portion of the ureter.-Orig.

Fig. 289. Testis, embryonic kidney, etc. of the embryo of a higher Vertebrate; diagrammatio. $c$ cloaca, t testis, $"$ anterior portion of embryonic kidney (which forms the epididymis), $u$ posterior portion which atrophies, $\imath g$ duct of embryonic kidney (seminal duct).--Orig.

Fig. 288.

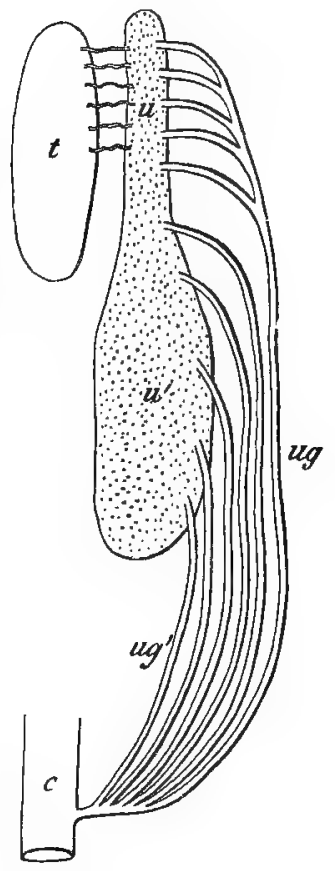

Fig. 289.

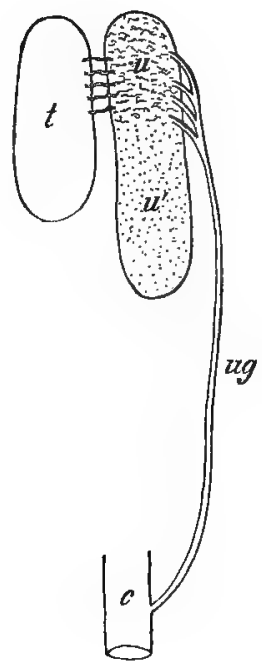

life. The embryonic kidneys and their ducts (Wolffian ducts) which open into the cloaca, are lost in the female, whilst in the male the testis becomes connected by fine tubules with the anterior portion of the embryonic kidney, and this part persists throughout life as the

* The aberrant conditions of the Teleostei are disregarded here. See Pisces. 
epididymis. This consists of numerous coiled tubes, and lies close to the testis receiving the spermatozoa from it, and carrying them to the segmental duct, which serves as the seminal duct ( $\nabla a s$ deferens) of the adult. The posterior portion of the embryonic kidney atrophies, and the epididymis loses its original excretory function. It follows, from this description, that the embryonic kidneys of Reptilia, Aves, and Mammalia correspond with the permanent organs of Pisces and Amphibia, whilst those of the adult are, on the other hand, quite new structures.

A pair of organs lying far anterior in the body-cavity is distinguished by the term head kidneys (pronephros)*: each consists of one or more glandular

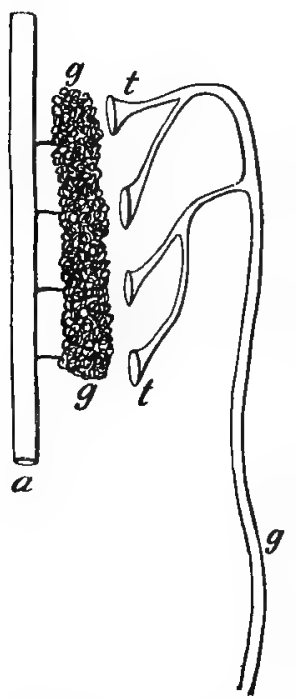

Fig. 290. Diagram of a pronephros. a aorta, $g-g$ (left) glomeruli, $g$ (below and to the right) duct of embryonic kidney, $t$ funnels.-Orig. tubes. One end of each tube opens by a funnel into the body-cavity, the other is connected with a duct, the segmental duct, opening into the cloaca. Opposite the funnels a large glomerulus projects from the body-wall. This head kidney appears in the embryo as yet another urinary organ, but before long it gradually disappears, $\dagger$ the duct, however, remains as the ureter of Fish and Amphibia, and the Wolffian 'duct of higher Vertebrata. In the latter the pronephros is rarely functional, and is very poorly developed; in Amphibia and many Fish on the other hand, it is for some time the functional excretory organ both in the embryo, and sometimes also in the young animal.

In the Vertebrata there is usually a pair of suprarenal bodies (sometimes separated into several portions), organs whose significance is still unknown. They are mentioned here since they usually lie close to the kidneys, with which, however, they do not come into any further relation. The suprarenal organs are usually yellow or brown in colour, and consist of connective tissue, in which are embedded strings of cells or vesicles. They are very vascular, and well supplied with nerves.

The Vertebrata typically possess tw o ovaries, although in many Fish they are fused, and one is generally abseut from Birds; they are firmly attached to the dorsal body-wall, and are covered by a simple epithelium, from which there are inpushings into the subjacent connective tissue eren in early developmental stages. These invaginated regions separate from the superficial epithelium as small rounder groups of cells, in which there is a central larger cell, surrounded by a sheath of smaller ones. Such a group is termed a Graafian follicle, the central cell is the young ovum, which gradually increases, and

* In relation with this, the kidney of Fish and Amphibia, and the embryonic kidney of the higher Vertebrata may be termed the mesonephris, and the adult kidney of the latter, the metanephros.

+ In certain Teleostei it persists throughout life as an excretory organ; in many others it persists in a modified condition, but in the adult no longer excrẹtęs ụrine, 
often attains an enormous size. The cells round the ovum secrete a vitelline membrane, which is sometimes very thick. The cells constitute, in all Vertebrata, a single layer round the egg, and usually remain in this condition; only in the Mammalia do they divide, so that the young egg is surrounded by several layers. Here a split appears later in the cellular mass (Fig. 291, s), and gradually enlarges, so that the ripe Graafian follicle of Mammalia looks like a hollow ball of cells, whilst the ovum, surrounded by a proliferation of cells, projects into the cavity. The ripe ova are shed into the body-cavity by the bursting of the Graafian follicles. They vary in size in the different Vertebrata; they are smallest, microscopic even, in the Mammalia, largest in Birds and Selachians. Where the ova are large, they project from the surface of the ovary, so that it looks very uneven; in Birds, it appears racemose, whilst in Mammalia, on the contrary, it is usually a small, smooth, roundish body. The ova generally escape by the Müllerian ducts, a pair of long tubes each opening at one end by a ciliated funnel into the body-cavity (usually near the ovary of the same side), at the other end into the rloaca, or to the exterior by a special aperture in the region of the anus. (For the aberrant relations of ovary and oviduct in

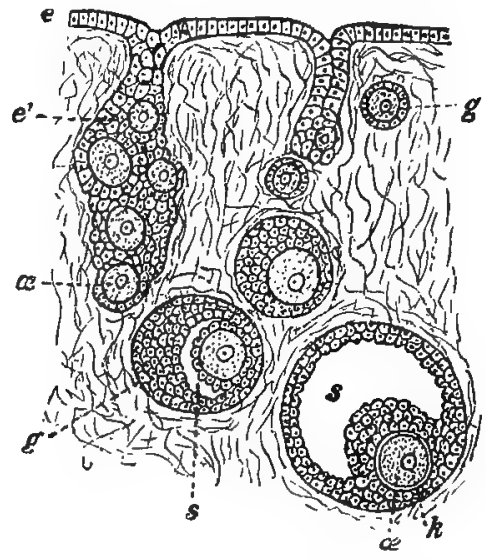

Fig. 291. Section through the ovary of a Mammal ; diagrammatic. $e$ epithelium on the surface of the ovary, $e^{\prime}$ invaginated portion of epithelium, $g^{\prime}$ young Graafian $f$ collicle, $g$ somewhat older do., $s$ split, $a$ egg, $k$ nucleus of egg.-Modified from Wiedersheim. many Pisces, see that group.)

The testes, of which there is also a pair, usually lie like the ovaries, on the dorsal wall of the body cavity (see the Mammalia for the change of position which may occur during development). They consist of numerous coiled glandular tubes (seminal tubules), which are closely packed, and in which the spermatozoa are produced by the modification of the constituent cells. For the methods by which the spermatozoa escape from the body, see above, pp. 349, 350. For copulatory organs, see the different groups.

Rudiments of the Müllerian ducts, varying in size, often occur in the male (Selachii, Amphibia, Mammalia), just as vestiges of the mesonephros (parovarium) and its duct (Gärtner's duct), may sometimes be found in the female (e.g., in the Ruminants).

The majority of the Vertebrata are of separate sexes. Only in some species of Teleostei are ova and spermatozoa formed in the same individual, i.e., there is true hermaphroditism; both genital productos develop in a common gland, 
the eggs in one region, the spermatozoa in another. In not a few others it may be noticed as a quite regular occurrence, that the sexual glands have in some measure a dual character, being, for the most part, either ovary or testis, but having a small portion of the ovary forming a testis, or of the testis forming an ovary. These small portions do not, however, form ripe sexual products. So also, a portion of the testes of the Toad (Bufo) resembles an ovary, but it does not produce ripe ova.* As rare abnormalities, such relations may also obtain in the higher Vertebrata (e.g., in Mammalia); for instance, a testis may occur on one side, an ovary on the other: or the gland of each side may possess in part, the structure of a testis ; in part, that of an ovary : but in this case ripe genital cells of both kinds are apparently not produced. More common than these true hermaphrodites are the so-called pseudo-hermaphrodites, which possess testis or ovary alone, but show the characters of the other sex in the ducts or the structure of the copulatory organs; amongst the domestic animals for instance, it is by no means rare to find males which possess very welldeveloped Müllerian ducts, like those of the female. Certain normal arrangements may be regarded as slight indications of pseudo-hermaphroditism; e.g., the presence of rudimentary Müllerian ducts in the male, which has been already mentioned, or the rudimentary copulatory organs in certain females (clitoris of Mammalia, etc.).

Most Vertebrates are oviparous. The egg, when laid, is sometimes surrounded by a gelatinous mass (Amphibia); in other cases by a horny shell (Selachii); or again, by a tough or brittle calcareous shell (Reptilia, Aves), which encloses, besides the egg-cell, a mass of albumen, which will later be gradually absorbed by the embryo; all the coverings are secreted by the glands of the oviducts. Many Vertebrata are, however, viviparous, embryonic development occurring in the oviduct of the mother (or in Teleostei in the hollow ovary). In the simplest cases, the egg, surrounded by the usual coverings (shell, etc.), develops within the body of the parent without the assistance of any additional nourishment; the ducts of the female simply afford protection to the egg (e.g., in many Reptilia): ovoviviparous animals. An approach to this condition occurs in many oviparous forms, where the egg when laid contains a more or less fally-developed embryo, the first part of development occurring within the body of the parent, the conclusion externally (e.f., in the common Ringed Snake). In other viviparous animals the embryo lies in, and is nourished by, a fluid, secreted usually by the wall of the oviduct, which it absorbs into the gut or through the skin (Zoarces, some Rays, Marsupials) ; in others, again, processes from the embryo grow into the wall of the oviduct and serve for the absorption of blood from the mother, upon which the fœetus is, as it were, parasitic (Mammalia, one Reptile, and Selachians).

\footnotetext{
* According to the interpretation of some observers, the $\mathrm{Ha} \mathrm{g}$ (Myxine) is a true hermaphrodite; which is male whilst young, later female. The correctness of this conclusion must still remain doubtful; certain it is that in some male specimens of this form, the anterior portion of the sexual gland has the character of an unripe ovary, whilst the posterior part constitutes a testis; but whether this region later develops into a ripe ovary, or whether, like the similar part in the male Toad, it remains in this condition, cannot be decided from the investigations so far made,
} 
Segmentation of the ovum is total in some of the Vertebrata-Amphioxus, Cyclostomi, Ganoidei, Amphibia (with the exception of Coecilia and several others), and most Mammalia; in others, in which the egg is large, segmentation is partial (Selachii, Teleostei, Reptilia, Aves, Monotrema). As in the lower animals a gastrula is formed, Amphioxus offering the simplest instance (see p. 43), the formation in others being more complicated ( $p$ p. 43-45); the mode of gastrula formation in Mammalia is not yet fully elucidated. Most Vertebrate embryos are for a long time provided with a yolk-sac (see p. 49), which attains a huge size in some (e.g., the Selachians), but has usually vanished or is no longer visible when the animal is born (i.e., leaves the egg-shell or the body of the parent.) In Reptilia, Aves, and Mammalia (the amniote Vertebrata), certain peculiar conditions may be observed: the embryo is surrounded by several embryonic membranes, which develop as special outgrowths of the young animal. These are embryonic organs and are thrown off at birth.

In the Hen's egg, at a very early stage of development, a fold, consisting of epiblast and the outer layer of mesoblast, is formed round that portion which will develop into the embryo itself, as distinct from the yolk-sac portion. This fold gradually grows round the whole embryo, its walls meet and fuse, and thus a cavity is formed abore. limited by the inner layer of the coalesced fold, This inner layer is now called the amnion whilst the outer layer, which is

$A$
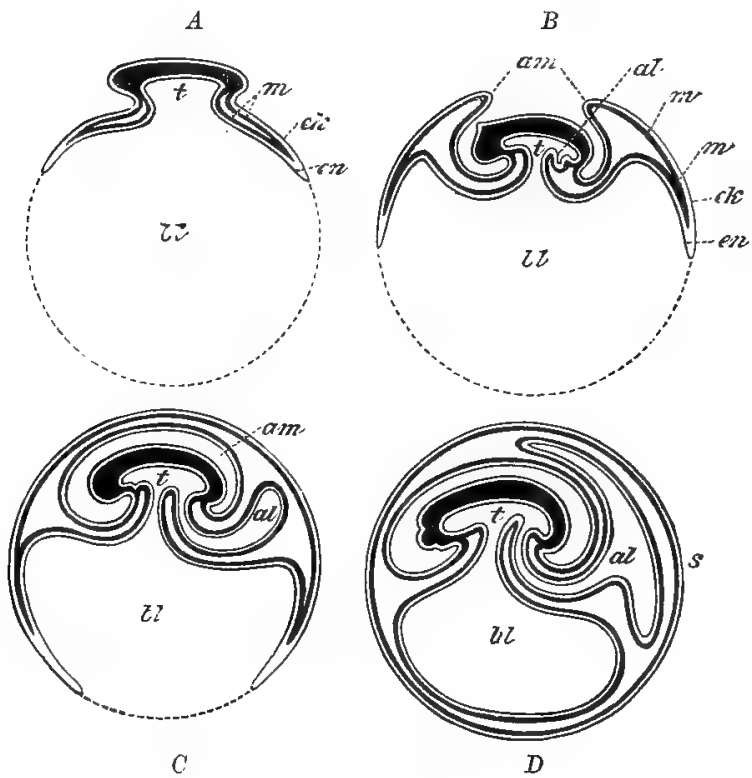

Fig. 292. Illustrating the development of the embryonic membranes in a bird embryo; diagrammatic longitudinal sections of various stages. In $A$ the development of the mombranes has not begun. ek epiblast, en hypoblast, $m$ mesoblast (broader line), am amnion, $a m^{\prime}$ folds from which the amnion and serous membrane originate, 8 sercus membrane, $a l$ allantois, $b l$ food yolk, $t$ gut.-Orig. (partly after older figures). 
continuous below with the covering of the yolk sac, is termed the serous membrane. Further, there grows into the cavity between the serous membrane and the amnion, an outgrowth from the hinder part of the gut, consisting of an inner layer of hypoblast and an outer layer of mesoblast. This outgrowth, the allantois, grows gradually into a compressed sac of considerable size, lying between the amnion and the serous coat, and communicating by a duct with the gut. The allantois is very vascular, and serves partly as a reservoir for the urinary excretions, partly as a respiratory organ. Similar conditions obtain in other Birds, Reptiles, and Mammals ; in the last-mentioned the allantois unites with the serous membrane to form vascular outgrowths, which grow into the wall of the uterus and serve as nutritive apparatus (placenta).* In Fish and Amphibia these embryonic membranes are wanting.

The Vertebrata occupy a somewhat isolated position in the Animal Kingdom, and so far no close connection with other phyla has been proved. Of the five chief divisions of the Vertebrata, excluding Amphioxus, Pisces and Amphibia contrast, in many respects (see the summary below), with the Reptilia, Aves, and Mammalia, which are likewise allied in many ways; the first two are united under the heading Anamnia, the others as Amniota. On the other hand, however, the Amphibia display many points in common with the Amniota as opposed to the Fish ( $c f$., the left of the summary).

Skeleton of limbs not divided into arm, forearm, finger, etc.

Stratum corneum absent.

Olfactory organ a pit.

Eyelids absent.

Auditory ossicles absent.

Auricle of heart not divided.

Skeleton of limbs divided into arm, fore arm, finger, etc.

Stratum corneum present. Olfactory organ with external and internal apertures.

Eyelids present.

Auditory ossicles.

Auricle of heart divided.
1. Leptocardii

3. A $m p$ hibia

2. $\mathrm{Pisces}$

4. Reptilia

5. A ves

6. Mammalia
Embryonic nembranes absent.

1st and 2nd vertebræ not specially developed.

Gills present at least in the young forms.

Mesonephros functional kidney of adult.

Ventricle not divided.

Embryonic membranes developed.

1st and 2nd vertebræ developed as atlas and axis. Gills absent.

Mesonephros replaced by metanephros.

Ventricle completely or incompletely divided.

\section{Class 1. Leptocardii (Lancelets).}

The body is elongate, compressed, and pointed at each end; along the back and the ventral side of the tail is a fin ; limbs are absent. The skeleton is represented by a well-developed notochord, running the whole length of the body, and pointed anteriorly and

- A structure similar to the embryonic membranes occurs in the Insecta and several Worms. 
posteriorly. Above this lies the central nervous system, a long cord-like organ without specialised brain; its central canal communicates with the exterior in front. There is an un paired e $\mathrm{ye}$ in the form of a pigmented spot in the anterior part of the

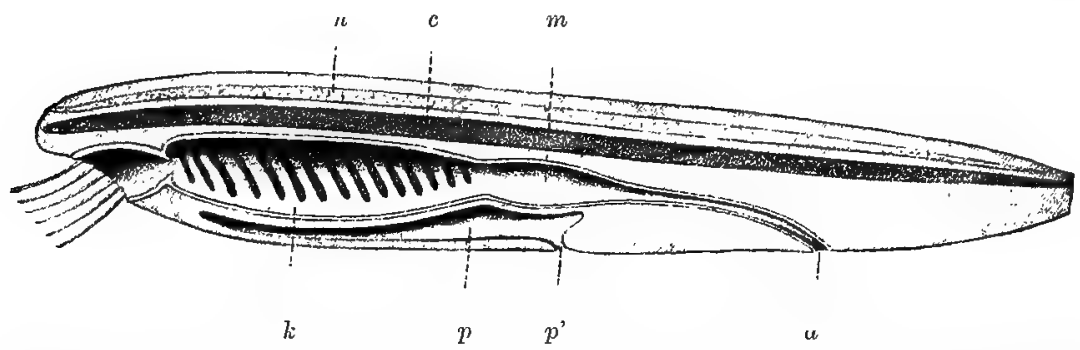

Fig. 293. Diagrammatic longitudinal section of Amphioxus. $a$ anus, $c$ notochord, $k$ gill sac, $m$ stomuch, $n$ central nervous system, $p$ atrium, $p$ atriopore.-Orig.

nervous system; paired eyes and auditory organs are absent. The musculature is arranged as in Fish, the muscle-fibres transversely striated. Below the notochord lies the alimentary canal, beginning at the anterior end of the animal with a mouth, surrounded ' by a number of projecting tentacles (cirrbi), this leads into a large pharynx, perforated by numerous transverse slits, and extending back through a large region of the body. Behind, the pharynx leads into the st o $\mathrm{mach}$, which is furnished with a large evagination, the liver; the intestine is short and straight and opens ventrally, not far from the hind end, so that the length of the tail is not great. The gillclefts do not lead direct to the exterior, but open into an atrium, surrounding the pharynx, and this again opens ventrally, anterior to the anus. In quite young animals the branchial clefts open on the surface, but later a longitudinal fold develops above them on each side, and eventually grows round them, the two uniting on the ventral surface. The vascular system is remarkable for the absence of a specialised heart, but all the vessels are pulsatile. Below the pharynx is an unpaired vessel, which receives the venous blood from the body, and sends branches to the gill bars; from the latter the blood is collected into an aorta running below the notochord. A hepatic portal system is present as in other Vertebrata. Red blood corpuscles are wanting. Numerous short ciliated tubes, arranged in a row on either side, serve for excretory organs; each tube has a single dorsal opening into the atrium, several into the body-cavity. The sexes are separate. The sexual organs are represented by several pairs of ovaries or testes, which 
lie in the body-wall on the side towards the atrium; ova and spermatozoa escape into this by the bursting of the organs, and are ejected through the mouth. Segmentation is total, a blastula is formed, the epiblast and hypoblast cells are little differentiated (gastrula formation of the type depicted in Fig. 29); the embryo hatches early, and the short larva swims by means of the cilia which cover the surface.

The class Leptocardii, which only includes the genus $A m p h$ i o x u s, occupies in many respects the most primitive position among Vertebrata (skeleton, nerrous system, development, etc.), whilst in other respects it is very peculiar and far from ancestral (atrium, etc.).

The colourless Amphioxus lanceolatus, reaching about $7 \mathrm{c} / \mathrm{m}$. long, occurs on European coasts, buried in sand.

\section{Class 2. Pisces. (Fish.)}

The body is usually compressed and spindle-shaped; head, body and tail pass gradually into each other, the last is very muscular; there is no neck, and movement of the head is usually very limited. Many Fish differ more or less considerably from this general type; for instance, some are so strongly compressed laterally that the animal resembles a perpendicular plate; in others, the head and body areflattened dorso-ventrally; in others again, the body is so elongated as to be vermiform, or, on the contrary, it may be extraordinarily short and bulky. Unpaired fins occur dorsally and ventrally upon the tail, and on the dorsal side of the body. Usually two pairs of rather feeble, flattened limbs are present; sometimes the posterior, or even both pairs, are wanting. It is characteristic of the Fish that the hind limbs, the pelvic fins, have often moved far forwards; close, or even anterior to, the fore limbs or pectoral fins.

The somewhat thin epidermis, as already mentioned, has no stratum corneum; goblet cell, are often present, and their secretion imparts to the skin its slimy character. The de $\mathrm{r}$ m is often contains ossifications, of which the best known are the so-called scales, thin, bony plates lying in cavities of the dermis; they are

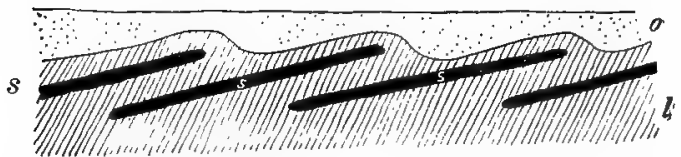

Fig. 295. Diagrammatic section of the skin of a Teleostean, to show the scales. dermis, $e$ epidermis, $s$ s c ale.Orig.

often so loosely connected with the latter, and lie so close to the surface, covered only by a thin connective tissue sheath and the epidermis, that they are easily loosened by the movements of the animal and fall off. They are usually imbricate, the overlapping edge being posterior, and are then regularly arranged in rows. Cycloid 
and ctenoid scales are distinguished; in the latter the posterior edge is finely denticulate. Scales, which are especially frequent in the Teleostei, are simply one form of dermal ossification, and are not sharply demarcated from others, bony plates, scutes, spines, etc., which are present in many Fish. The placoid scales (dermal denticles) covering the whole surface of many Selachians, and present also in various other Pisces, are entirely different. They are identical in structure and development with the buccal teeth, consisting of dentine and enamel formed in the ordinary way; they do not lie in the dermis, as do most dermal ossifications,* but their upper portion projects from the skin; they fall out and are replaced, whilst dermal ossifications usually grow with the growth of the animal, and are neither deciduous nor successional. The form of these dermal teeth varies, sometimes they have many points; usually they are small, but may reach a considerable size. In those Teleostei and Ganoidei which possess dermal denticles, their lower end is usually connected with the dermal ossifications. $\dagger$

The unpaired fins are folds of skin usually supported by hard parts. At a certain stage in development, frequently even in the newly-hatched animal, more often during embryonic life, the unpaired fins are represented by a continuous fold, which runs medianly along the dorsal surface of the body and tail, round its tip and ventral side, along part of the body. In certain cases, this ridge remains undivided throughout life, but the ventral portion in front of the anus always disappears; usually, however, it breaks into three or more sections, of which those on the dorsal surface are termed dorsalfins; that round the tail, the caudal fin; and those ventral to the tail, anal fins. When fully developed, hard portions, the so-called fin $\mathrm{rays}$, are generally present. In the Selachians, "horny rays" $\ddagger$ occur; horny, elastic, structureless fibres, stretching from the base of the fin to the edge; they lie in several layers in each fin, which is stiff and incapable of folding. $\S$ Instead of these, there are in the unpaired fins of Ganoids, Dipnoans, and Teleosteans, a series of rod-like dermal ossifications, the bony rays, which lie as supports within the fin. Of such rays, two chief forms may be distinguished, soft rays and spinose rays, between which there are, however, intermediate forms. A soft ray

* Not infrequently, however, the true dermal ossifications have a large surface, or a projecting point, bare.

$\dagger$ The fine tooth-like points along the hind border of the ctenoid scales, which are merely special portions of these, are not to be confounded with the dermal denticles.

\$The name is not a very happy one, since it is here applied to parts which develop in connective tissue, and are entirely distinct from the true horny structures of Vertebrata,

$\S$ A series of cartilaginous rays occurs in the Cyclostome fin. 
is a bony rod, which is jointed, i.e., is divided transversely into a number of short pieces connected by connective tissue; moreover, it is more or less deeply split at its apex into several branches, which are also jointed. Some, however, do not show this splitting, and in others (Fig. $296 \mathrm{w}^{\prime}$ ) the jointing is limited to the tip of

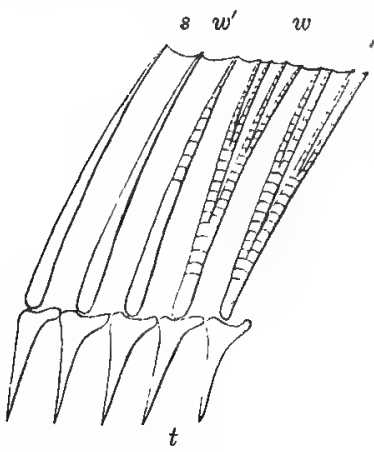

Fig. 296. Portion of a fin with spiny rays (s), and soft rays $\left(w w^{\prime}\right) ; t$ interspinal bones; diagrammatic.-Orig. the ray, or is absent altogether. Each soft ray consists of two symmetrical halves, closely apposed, and corresponding the one with the other. The spinose rays are stiff, pointed, unsegmented bony rods; they also are composed of two halves, which are either closely connected or fused. Intermediate forms also occur, which, though not jointed, are yet flexible. Whilst soft rays are present in all Pisces, and especially in those with bony rays, the spinose rays only occur in some of them, and then are almost invariably confined to the front of the fin; they are absent from the caudal fin. The bony rays are capable of depression and erection, the membrane being folded together and then expanded: Sometimes the skin is absent from several successive rays (free rays). For the endoskeleton connected with the unpaired fins, interspinal bones, etc., see below. Along the edge of the limbs, the pectoral and pelvic fins, which are usually very short, there is a $f$ in border which is of similar structure to that of the unpaired fin, furnished in the Selachians with horny rays, * in Ganoids, Dipnoans, and Teleosteans with bony rays (soft or spinose). Spiny rays are very rarely present in the pectoral, but are more abundant anteriorly in the pelvic fin, and in this case occur also in dorsal and anal fins. For the sense organs of the skin (lateral line, etc.), see below.

Phosphorescent organs occur in some Fishes; they are peculiarly modified portions of skin which look like larger or smaller spots; their structure is as yet not quite understood. Such organs are fairly common in the Deep-sea Fish, but are also present in some pelagic forms.

In many Pisces (Cyclostomes and Selachians), the skeleton, with the exception of the notochord, consists entirely of cartilage, which is, however, usually to some extent calcified, i.e., calcareous salts are absorbed by the intercellular substance ; in others (Ganoidei, Dipnoi, Teleostei), the cartilage which originally forms the whole

* The edge of the fin and the horny rays are specially well developed in the paired fins of the Sharks. In the Rajidæ, on the other hand, the cartilaginous rays belonging to the limb skeletons to be mentioned below, reach almost to the edge of the fin, and in connection with this the horny rays in the pectoral and pelvic fins are little developed, or altogether absent. 
skeleton, is more or less completely replaced by true bone. The vertebral column (Fig. 298), is not infrequently represented

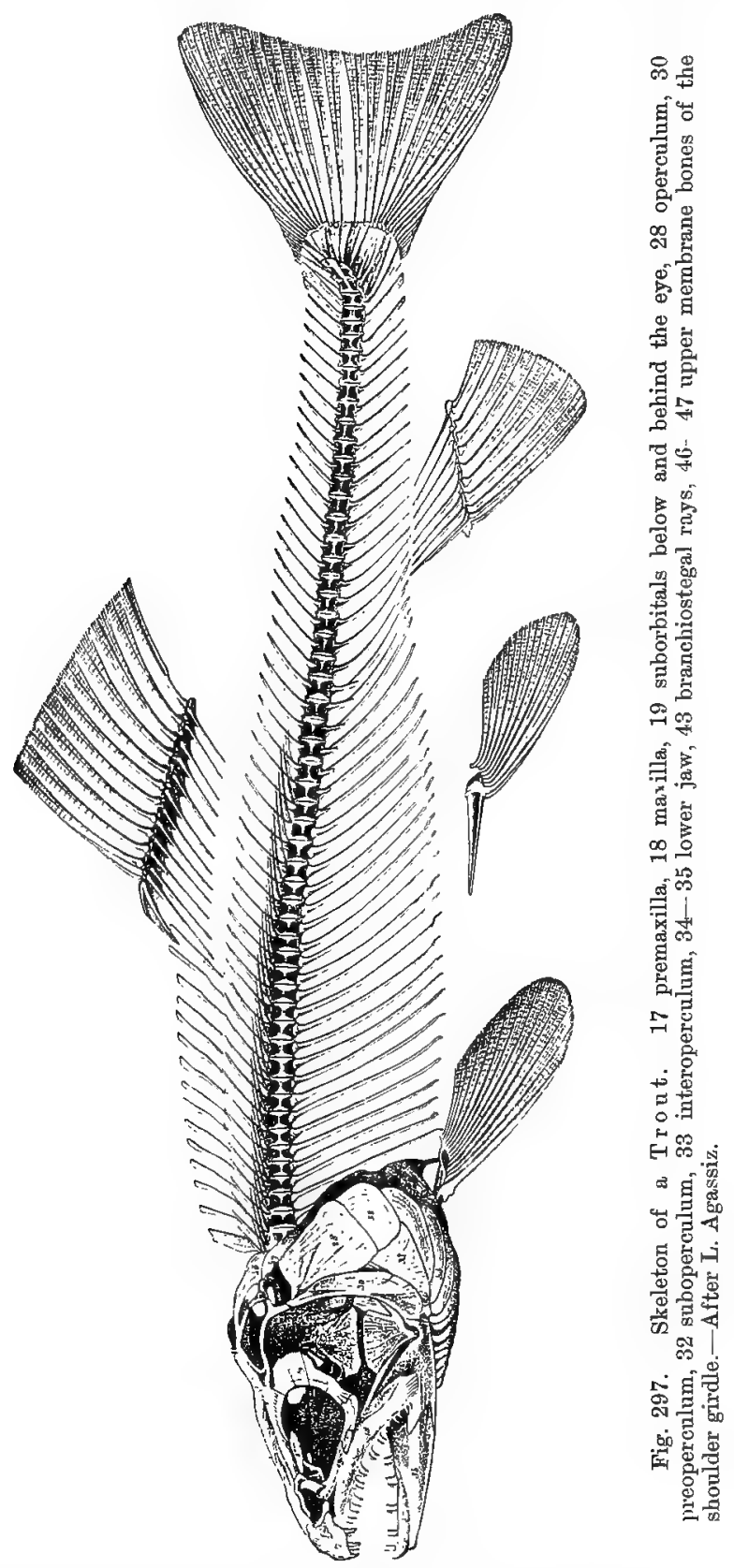

(in the Cyclostomes, some Selachians, Dipnoans, and cartilaginous Ganoids) by a continuous tube of cartilage or connective tissue which 
surrounds the chorda, is not divided into vertebræ, and carries the upper arches (the arches may even be absent, e.g., the Hag-fish Myxine). Usually, however, this tube is broken up into a number of pieces, the vertebræ, united by connective tissue. The vertebræ are short tubular bodies, thickened so that

A

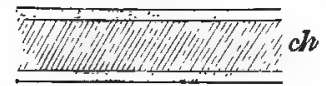

B

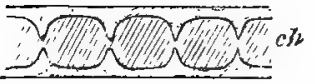

C

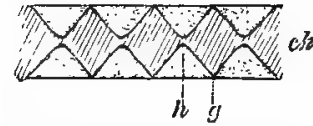

Fig. 298. Longitudinal sections through the vertebral column of various Fish ; schematic. In $A$ and $B$ a continuous cartilaginous tube is still present, in $C$ this is divided into centra (h), g boundary of two centra, ch notochord.-Orig.

the lumen is narrowed centrally and thinner at each end, where there are concavities, connected with each other by a small median opening (like that of an hour-glass): they are, therefore, biconcave (amphicœlous) vertebræ. They surround the notochord which is much constricted intravertebrally, so that it resembles a string of beads. In some Sharks, in which the vertebral column is not divided into vertebræ, the intravertebral constrictions are already indicated by ringlike thickenings on the inner side of the continuous cartilaginous sheath (Fig. $298 \mathrm{~B}$ ). Dorsally, each vertebra usually bears a neural arch* which is often produced into a neural spine; often, also, on the trunk vertebræ, there are transverse processes, which bend down at the beginning of the tail to form the $\mathrm{h} æ \mathrm{mal}$ arches, united just as are the dorsal ones. In the Selachians the vertebræ consist of cartilage often partially calcified; in the bony Ganoids and Teleosteans they consist entirely of bone; or partly of bone, partly of cartilage.

The part of the vertebral column at the end of the tail, and its relation to the candal fin, deserves special consideration. In a small number of Fish (Cyclostomi, Dipnoi) the hinder end of the vertebral column is straight and there is an almost equal portion of the tail fin above and below it, the upper portion being congruent with the lower ; the tail is then said to be diphycercal. In most Fish, on the other hand, the posterior vertebræ turn upwards; the lower portion of the fin is then usually better developed than the upper, and the tail is called heterocercal. In most Sharks, the cartilaginous Ganoids and Lepidosteus, this condition is very evident. In the Teleostei (Fig. 299 D) it also obtains, but the turned up portion consists, not of separate vertebræ, but of a single bony or cartilaginous piecet surrounding the end of the notochord, and often uniting with the last or last few lower arches, to form the most

* The spaces between the arches are filled in by cartilaginous intercalaria in Selachians and the cartilaginous Ganoids, and thus the tube round the spinal cord is completed.

+ In some Teleostei (Fig. $299 \mathrm{C}$ ) the curved portion is longer, and includes several vertebræ besides the rod-like part. 
posterior joint of the vertebral column; below this apparently last joint, there is usually a portion supported by rays, and almost congruent with its upper portion, so that the tail appears to be diphycercal; this is termed a homocercal tail. As a matter of fact, however, it is just like the heterocercal, since the end of the vertebral column is bent upwards, and the dorsal portion of the fin is smaller than the ventral (see Fig. $299 D$ ). In many, actually heterocercal, forms, in which a great length of the vertebral column is bent up, there is an approach to the same structure; since the end of the tail, as regards the exterior, is divided into two almost equal portions: a dorsal, into which the vertebral column is prolonged, and a ventral, consisting exclusively of rays (Fig. $299 \mathrm{~A}, \mathrm{~B}$ ).

$A$
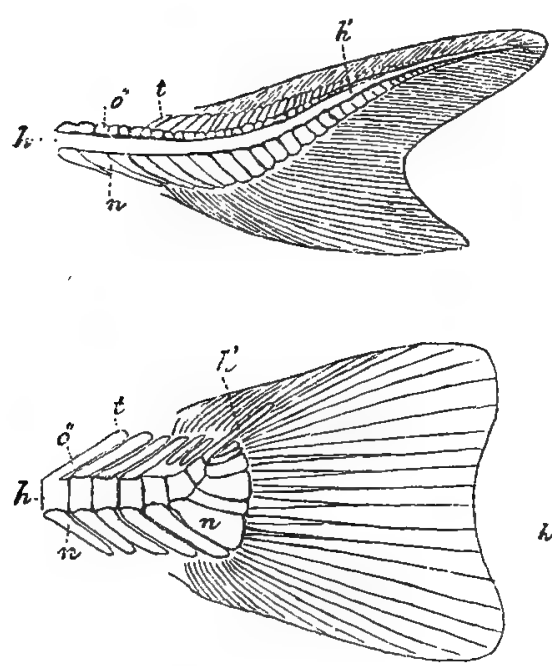

$C$
$B$
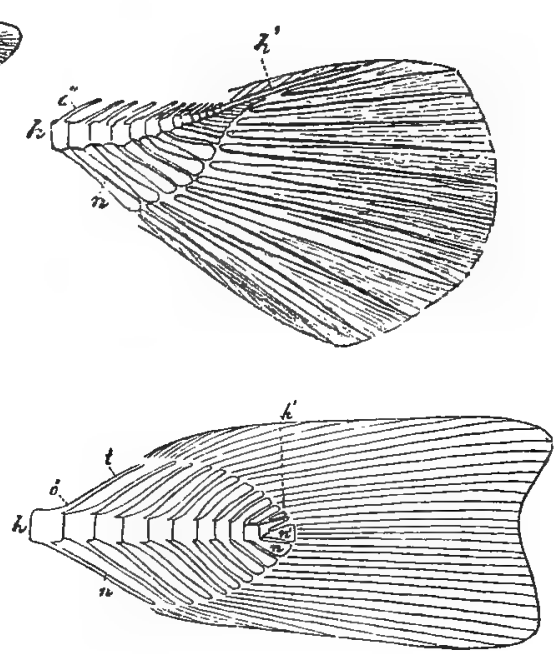

$D$

Fig. 299. End of the tail of various Fish: $A$ Sturgeon, $B$ Pike, $C$ Salmon, $D C o d$. $h$ vertebral column, $h^{\prime}$ bent up termination of the same, 0 upper arch, $t$ neural ispine, $n$ lower arch, $n^{\prime}$ last lower arch, united with $h^{\prime}$. In $C$ the bent portion of the vertebral column is still fairly well developed (it is enclosed between the two halves of the caudal fin rays of which the left are removed in the figure), in $D$, which represents the usual "Teleostean condition, it is on the contrary very small.-Partly original, partly a copy.

In the embryo, and in many cases also, in the newly-hatched animal (Teleostei), the notochord is for some time a straight rod; later the posterior end bends up, and is relatively much larger than in the adult.

Well developed, bony, or partly ossified $\mathrm{ribs}$ are attached to the transverse processes of the trunk vertebræ in most Ganoids, Teleosteans, and Dipnoans; in the Selachians the ribs are wanting or very short; they are absent also from the Cyclostomes. There is 
no sternum and the ribs do not meet ventrally. The dorsal and anal fins of Selachians are each supported by a laminate skeleton extending, on the one hand, into the base of the fin, and on the other, between lateral myotomes of the trunk and tail. Each consists of a number of cartilaginous pieces arranged like the skeleton of the pectoral and pelvic fins. In Ganoids, Dipnoans, and Teleosteans, it is replaced by the interspin ous processes, usually dagger-like bones, lying between the muscle plates, and united with the neural spines; or, on the ventral side of the tail, with the hæmal spines by connective tissue: the interspinous bones which do not extend into the fin bear each a single movably articulated ray. A pair of short bones is usually intercalated between the ray and the interspinal. The caudal fin is attached directly to the upper and lower arches which are partly separated from the vertebre at the hinder end of the tail.

The skull in Cyclostomes and Selachians is entirely cartila ginous, but in the latter is frequently calcified on the surface; in the cartilaginous Ganoids also there is a similar thick-walled cartilaginous capsule, but here it is partly covered with $\mathrm{mem} \mathrm{brane}$ bones; in bony Ganoidei, Dipnoi and Teleostei, it consists originally of cartilage, which is later not merely covered by membrane bones but is also partly os sified, i.e., replaced by bone; although some, often a considerable portion, of the cartilage is retained throughout life. The base of the skull, where it comes in contact with the spinal column, is usually hollowed like a vertebra; and on either side of the foramen magnum, there is often an articular surface, which corresponds with a similar one on the first vertebra. The eye lies in a lateral cavity protected above by a roof-like projection of the skull, which, in most Teleostei, is compressed between the eyes to a thin cartilaginous or membranous partition, with the cranial cavity prolonged as a narrow canal for the olfactory lobes above it. There is a pair of smaller cavities anteriorly for the olfactory organs.

In the Teleostei, and in the bony Ganoids as a whole, the skull consists of a larger or smaller amount of cartilage and a number of separate bones. The cartilage bones (formed by ossification of the cartilage) are: four oc cipitals (basi-, supra-, and two ex-oceipitals); of which all four, or the first and the last two alone, bound the foramen magnum; in the region of the labyrinth the most important is the petrosal or prootic, there are in addition the epiotic and opisthotic; in the basal and lateral regions in front of the parts just mentioned are the sphenoids (ali-, orbito-, and basisphenoid); dorsal ossifications anterior and posterior to the orbit of each side, the pre-and postfrontals; one or two ossifications at the anterior end of the cartilaginous skull, the et h $\mathrm{moids}$. The following are $\mathrm{membrane}$ b ones: dorsal and anterior, a pair of nasals; then a pair of frontals (sometimes united, e.g., in the Cod) behind these again a pair of parietals, lateral to which on each side is a squamosal; ventrally a long flat unpaired bone which covers the greater part of the floor of the skull, the parasphenoid; and anterior to this the similarly azygos vomer. Besides these, others 
may be present but are less constant. In the Dipnoi some of these bones occur but cartilage persists to a large extent. In the cartilaginous Ganoids, as already mentioned, only membrane bones are found, among them a parasphenoid, frontals, parietals, and several smaller bones dorsally.
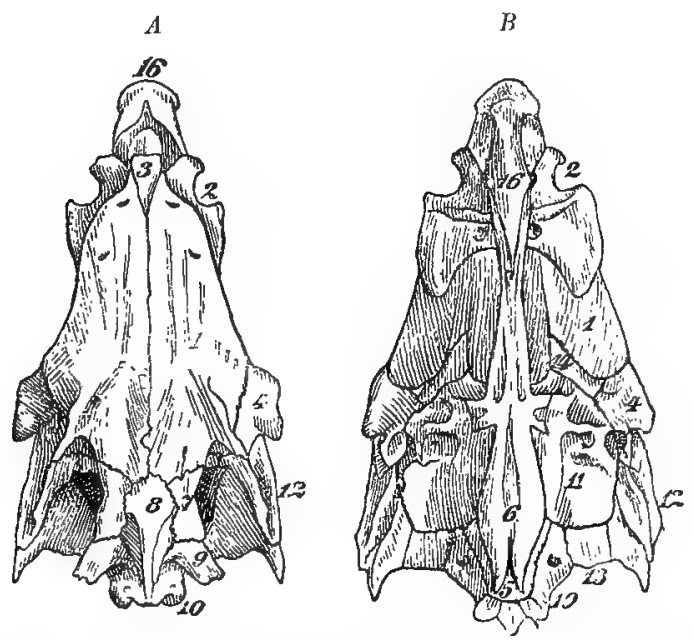

Fig. 300. Skull of a Perch, $A$ dorsal, $B$ ventral. 1 frontal, 2 prefrontal, 3 ethmoid, 4 postfrontal, 5 basioccipital, 6 parasphenoid, 7 parietal, 8 supraoccipital, 9 epiotic, 10 exoccipital, 11, prootic, 12 squamosal, 13 opisthotic, 14 alisphenoid, 16 romer.-After Curier and Valenciennes.

The dorsal membrane bones of the head are in many Fish very superficial in position, covered only by a thin layer of connective tissue and epidermis (Sturgeon, bony Ganoids, many Teleosteans); in others, the overlying connective tissue is thicker.

A large number of $\mathrm{v}$ isceral arches* usually seven pairs, occasionally more (some Sharks), are suspended from, or situated near to, the skull. The members of the first pair are united ventrally, whilst the others are attached to a series of unpaired, cartilaginous, or bony pieces (basibranchials). The most anterior, the mandibular arch, consists, in the Sharks, of an upper and a lower cartilaginous piece; the former, which is connected in front with the corresponding one of the other side, is termed the palatoquadrate, or less happily, the upper jaw; the latter, the mandibular cartilage, or lower jaw. The two portions are jointed together. The mandibular arch, the best developed, is loosely connected with the skull in the Sharks, and forms the framework of the mouth. The second, the hyoid, is similarly divided into two parts ; the upper is fastened to the skull above, whilst its lower end is attached by connective tissue to the mandibular. The other

* The Cyclostomi are excluded from this deseription of the visceral arches. The arrangements in this group are much modified and difficult to understand. 
five (occasionally six or seven) arches, the branchial arches, are each divided into several pieces, and bear on their outer borders delicate cartilaginous rays (removed in Fig. 301), which support the septa between the gill-clefts; similar rays are also present on the
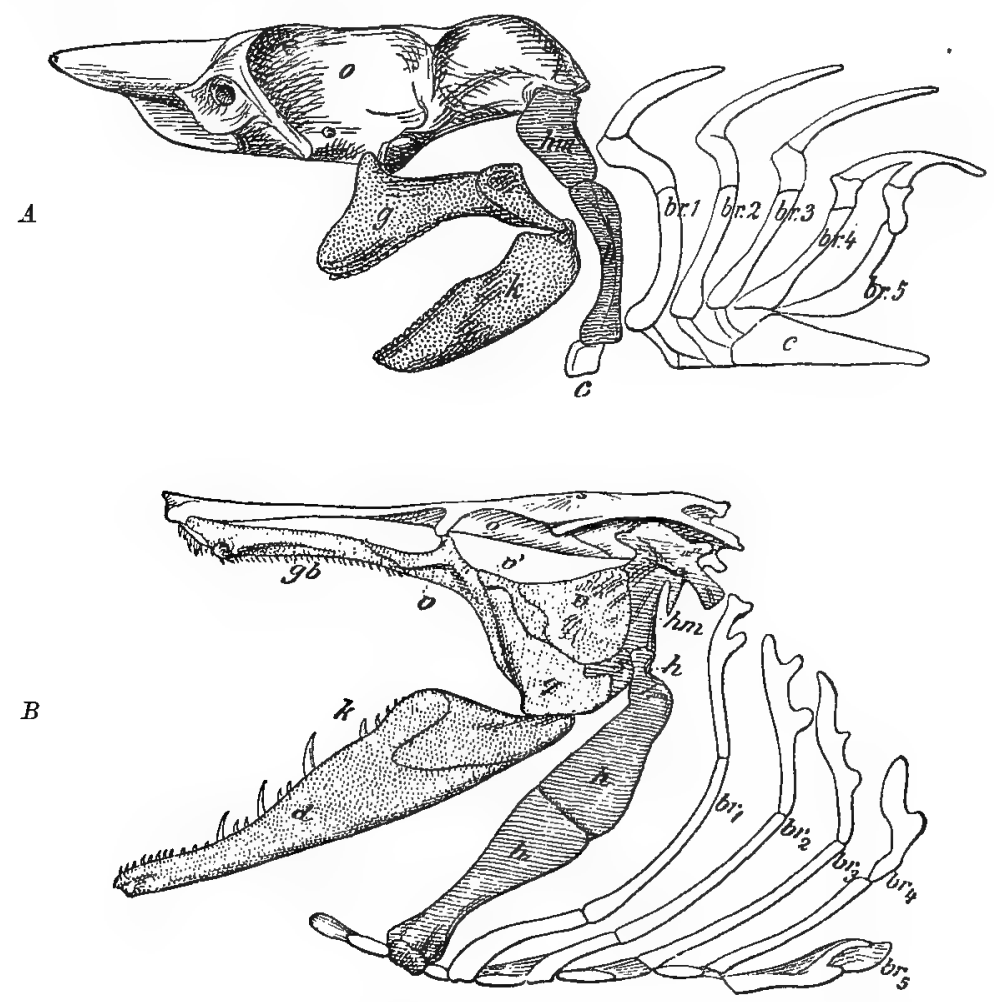

Fig. 301. A skull and $\nabla$ isceral a rches of a Shark, $B$ the same of a Pike; the arches are in part artificially separated; premaxilla and maxilla separated in $B$. First arch dotted, second shaded : $b r_{1}$ - $b r_{5}$ first to fifth branchial bars (third-seventh visceral arehes), $c$ basibranohials, $d$ dentary, $g$ palato-quadrate, $g b$ palatine, $h m$ upper portion of the hyoid arch (in $B$ hyomandibular), $h$ rest of the same, $k$ mandible, $o$ orbit, $g$ quadrate, $s$ symplectic, $v-v^{\prime \prime}$ pterggoids ( $v$ ecto-, $v$ ' endo-, $v$ " metapterygoid).-Orig.

hyoid).* In the Ganoidei, Dipnoi, and Teleostei, there are also seven visceral arches. The upper portion of the mandibular arch, the palato-quadrate, meets its fellow of the other side in the cartilaginous Ganoids, whilst in all others it remains distinct. This portion is intimately connected below, with the lower end of the upper portion of the hyoid arch, which is

* As regards visceral arches, the $\mathrm{R} a \mathrm{ys}$, on the whole, resemble the Sharks, but the hyoid shows certain peculiarities, which cannot be gone into more closely here. In many Sharks there are anteriorly, close to the gill-bars of each side, a pair of feeblydeveloped cartilaginous bars (the labial cartilages), which may perhaps be regarded as rudimentary first visceral arches. 
articulated above with the skull, and thus serves as suspensorium for the mandibular, which, therefore, possesses no direct connection with the skull. This connection of the upper portions of the mandibular and hyoid arches is specially close in the Teleosteans and bony Ganoids, forming a continuous whole, in which the cartilage is, for the most part, replaced by bone; it is less evident in the cartilaginous Ganoids, although here, also, the two portions are closely connected (for the Dipnoi, see below): the mandibular cartilage, which is now, however, more or less replaced and covered by bone, and the hyoid, which is likewise more or less ossified, are attached at the same point. Of the five branchial arches which are usually partly ossified, or covered by bony plates, the last is very short in the bony Ganoids and Teleosteans, the lowest joint alone being present. The last branchial bars, which usually carry numerous teeth, are termed the inferior pharyngeal bones. The uppermost joints of some of the other gill-bars are toothed in a similar way, and are called the superior pharyngeal bones.

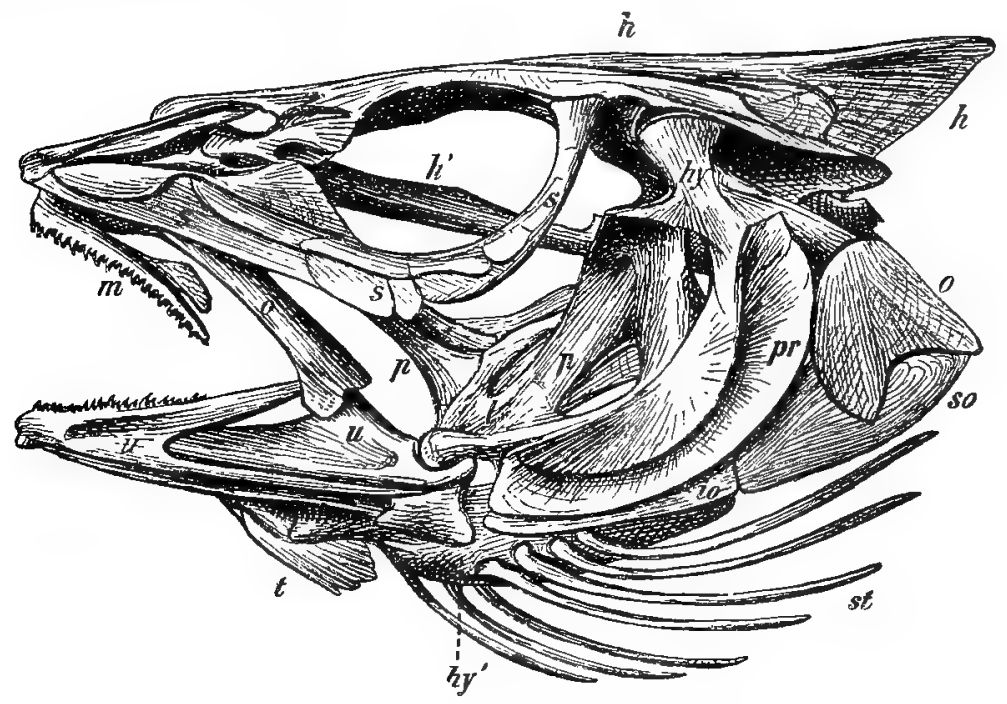

Fig. 302. Skull of a Cod. $h-i^{\prime}$ skull, $h y$ hyomandibular, $h y^{\prime}$ lower portion of the hyoid, io interoperculum (see p. 374), $l$ quadrate, $m$ premaxilla, o (left) maxilla, o (right) operculum, $p$ pterggoid, $p r$ preoperculum, $s$ suborbitals, so suboperculum, st branchi ostegal rays, (p. 374), $t$ basibranchials, $u$ mandible.

In the Teleostei, the upperportion of the mandibular arch is represented by the following bones: below and behind by the quadrate, with articular surfaces for the mandible; then by several bones, which are termed pterygoids; and anteriorly, by the palatine. The lower portion of the mandibular arch is a cartilage, the upper end of which is ossified and bears articular facets, whilst the rest is a thin curtilaginous rod (Meckel's cartilage), surrounded by membrane bones, of which the most important 
is the large dentigerous dentale, which meets its fellow of the other side anteriorly. The upper portion of the hyoid, which is united with the corresponding part of the mandibular arch, is represented by two bones; a large one, the hyomandibular, articulated with the skull, and a smaller, lower ossification, the symplectic. For the membrane bones connected with the hyoid, see below, under the gill apparatus.

In the Dipnoi, the upper portions of the mandibular and hyoid arches are concrescent, and partly ossified, thus possessing so far, the same relations as in the Teleostei ; the arcade thus formed is, however, connected immovably with the skull.

In the Ganoids and Teleosteans, two membrane bones arise anteriorly on either side of the head, quite independent of the visceral arches: an intermaxilla or premaxilla, and a maxilla, which is sometimes represented by several bones, and is situated behind, or behind and within, the former; the premaxillø are connected in the midline, and with the maxillæ, are usually somewhat loosely connected to the anterior end of the skull, forming the upper margin of the mouth, whilst the bones lying upon the mandibular cartilage form the lower edge.

The shoulder girdle is, in the Selachians, an unpaired cartilaginous arch, sometimes divided into two pieces, which lies behind

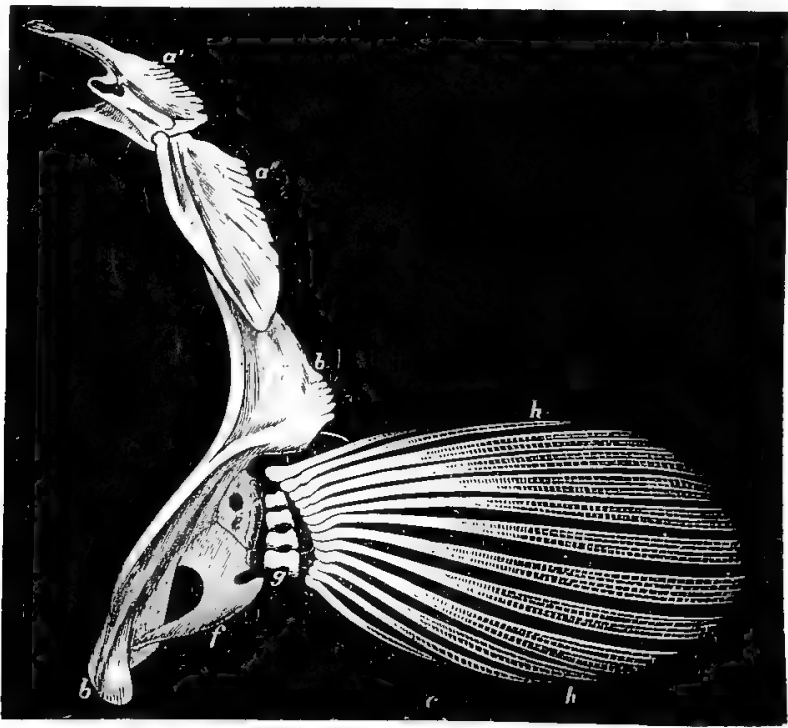

Fig. 303. Shoulder girdle and fore limb of a Perch. $a^{\prime}, a^{\prime \prime}, b, c$, membrane bones ( $b$ clavicle), $e, f$ the actual shoulder girdle (e scapula, $f$ coracoid), $g$ radialia, $h$ fin rays.

the gill bars, and reaches up on to the side of the head. In the Ganoids and Teleosteans it is divided into two halves, one on either side of the body, and closely united to each part is a series of membrane bones, of which the largest is a long, flattened, somewhat 
arched bone, the clavicle. This series of bones is fastened above to the hinder end of the skull. In the Chondrostei the cartilage of the shoulder girdle is always very well developed in spite of the presence of membrane bones; in the Holostei, and the Teleostei, the original part of the shoulder girdle is usually much reduced in size, and is represented only by a small plate, attached to each clavicle. In the Teleosteans two ossifications are present in this plate, the scapula and coracoid. In the Dipnoi relations similar to those of the Chondrostei obtain. The skeleton of the fore $1 \mathrm{imb}$ consists, in the Selachians, of a number of flattened cartilages: at the base of the limb are three large ones articulated to the shoulder girdle, the $\mathrm{b}$ as ali a, and to the edge of these is attached a larger number of jointed cartilaginous rays, the radialia. These lie close together in the Sharks, but in the Rays, where they are very long, they are somewhat further apart. In the Ganoids this skeleton is reduced in size, the radialia are shorter, the basalia

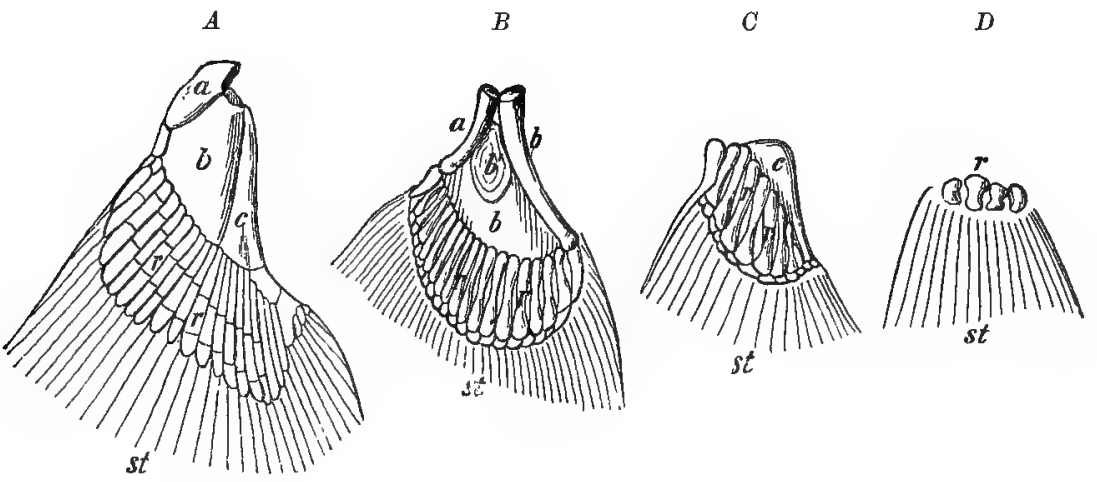

Fig. 304. Skeleton of fore limbs of $A$ Shark, $B$ Polypterus, $C$ Amia (a Ganoid), $D$ Cod. $a, b, c$ basalia ( $b^{\prime}$ ossification in $b$ ), $r$ radialia, st fin rays (not all drawn).-Chiefly after Gegenbaur.

usually less well developed, or partly absent; there is often an ossification of some parts. In the Teleostei the original skeletogenous supporting pieces are very small, the fin-border preponderating; the basalia are absent; the radialia are short and few in number : they occur only as a transverse row of four, or fewer, short rods, attaching the fin to the shoulder girdle, they are partly ossified, and have been incorrectly termed "carpals"; a few short cartilaginous pieces may also be present. The Dipnoi are very different ; there is a median, long, jointed, cartilaginous bar, bearing, in the genus Ceratodus, two series, in Protopterus, a single row only, of cartilaginous, jointed rays. (In Lepidosiren, the radialia are entirely wanting). 
The pelvis, which is not connected with the vertebral column in Fish, is, in the Selachians, an unpaired transverse ventral cartilage. In the Dipnoi, too, it is an unjointed cartilaginous plate. In the Ganoids and Teleosteans it is divided into two halves, closely apposed to one another, and except in the Chondrostei it is partially, or entirely, ossified. The hind limb closely resembles the fore limb in structure; in the Selachians there are two large basalia provided with radialia; in the Ganoids the basalia are degenerate or absent; this is also the case in the Teleostei, in which the skeleton of the hind limb is represented by a few short radialia only, it is even less developed than the fore limb. In the Dipnoi the conditions are the same for both hind and fore limbs.

The muscular system is distinguished by the feeble condition of the limb muscles, as compared with the powerful development of the trunk and tail musculature, which extends along the whole length of the body in the form of four large muscle plates, two on each side. Each of these is broken up, by transverse septa (myocommata), into a series of short segments, corresponding to the vertebræ in number (myomeres): in the dorsal muscles, and the caudal portions of the ventral muscles, the septa are bent in a peculiar way. In these muscles there are in many Fish numerous. fine rib-like bones, the epipleurals, which are attached at one end to the ribs or to the vertebræ. They are ossifications in the myocommata and serve as supports to the muscles.

The electric organs present in many Pisces generate electricity, which can be discharged at will : for instance, when the animal is caught. The essential elements of these organs are plates, modified muscle fibres, for the entire organ is a modified muscle : it is richly supplied with nerves, and fibres enter each plate and branch freely on one side. The plates are bound together by connective tissue; the organ is frequently composed of closely apposed columns, each of which consists of a number of plates. The electric organs lie in different regions of the body in different Fish; the most powerful apparatus is that of the Electric Skate (Torpedo), of the Sheath-fish or Thunderer (Malapterurus), and of the Electric Eel (Gymnotus); but feebler organs also occur in some other forms, e.g., in our native Skates (Raja), where they are elongate spindles lying one on either side of the tail.

The brain of Pisces is of small size, and does not fill the cranial cavity, which is chiefly occupied by the dura mater, a membrane of considerable thickness, consisting chiefly of adipose tissue. The olfactory lobes are usually large, and often of considerable length, since the olfactory organ lies anteriorly, far away from the brain. In many Teleostei, the fore brain is very small, smaller than the mid brain; the hind brain, on the other hand, is usually very well developed.

The olfactory organs generally occur at the anterior end of the head, as a pair of pits whose mucous membrane is usually in radial folds. The opening of each pit is single in many forms (some 
Teleosteans, Selachians); in others, a transverse membranous bridge, varying in width, divides the opening into two, anterior and posterior nares, of which the anterior may occasionally be drawn out into a narrow tube; in many Selachians, this bridge is only represented by a flap, arising from one side, and overlying the aperture without being attached to the other.

In the Dipnoi, the nasal openings are peculiar, in that both lie within the edge of the upper lip. In the Cyclostomes, the two olfactory pits are united to form a deep unpaired tubular sac, the floor of which lies closely upon the roof of the mouth; in Myxine, it perforates the roof of the mouth, and consists of a tube open at both ends, connecting the oral cavity with the exterior.

Taste buds, as already mentioned (p. 21), are present in many Fish (Teleostei), not only in the mouth, but scattered over the surface of the body.

Groups of peculiar sense-organs occur in connection with the skin; they consist of modified epidermal cells, some of which bear sensory hairs; and are thus not unlike taste-buds, from which, however, they differ in form. These sensory papillæ are supplied with nerves, and may lie free upon the surface of the body (e.g., in most Teleostei), in which case they often bear a cylindrical tube, a cuticular structure, which surrounds and protects the hairs (Fig. $305 r$ ). In other cases, those portions of the skin to which they belong, have sunk in to form small sacs, opening to the exterior (Ganoids); or the sacs have become long tubes filled with mucus, running below the skin, prorided at one end with an expansion in which the sensory cells lie, whilst at the other, they open to the surface (on the head in Selachians). Further, similar groups of cells are present in the lateral line which occurs in most Fish. This is a narrow tube (an invagination of the skiu), lying close below the surface, and extending along each side of the body. It usually divides into several branches on the head, one branch running orer the summit of the head, another above the eye,

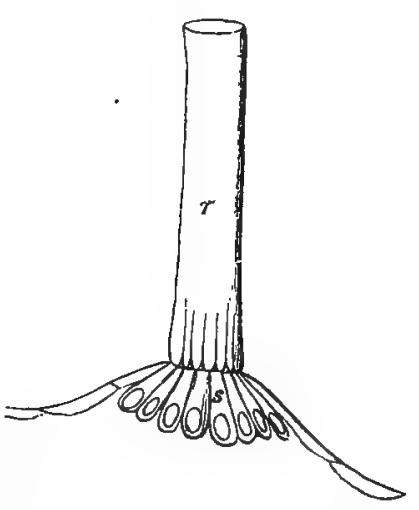

Fig. 305. Sensory papilla or a young Teleostean. $r$ the tube, $s$ cells bearing hairs.-After F. E. Schultze. a third below the eye-both the latter reaching the snout-a fourth along the lower jaw. The lateral line is richly supplied with nerves, and communicates: with the exterior by a number of openings; sometimes, indeed, it is a partially open groove; in many Teleostei, it runs along. the side of the body and tail through a series of perforated 
scales; * on the head they are partly enclosed by special, tubular, membrane bones, of which there is, for instance, usually a series below the eye (Fig. $302 s$ ), partly surrounded by the ordinary bones of the head.

The eyes of Fish are, as a rule, relatively large. The lens is spherical. Movable eyelids are absent; but the eye is often surrounded by a low circular ridge of skin, and in some species by larger, but immovable folds; in the Mackrel and the Herring, for instance, there is a transparent fold in front of, and behind, the eye, which partly covers it.

The scelerotic usually consists of an outer connective tissue layer, and an inner cartilaginous layer, varying in thickness (very thick, e.g., in the Sturgeon); in the Teleostei the cartilage, in the vicinity of the cornea, is partly replaced by two bony plates, which sometimes attain a considerable importance, and may unite to form a ring. The choroid consists of several layers: usually there is an external lustrous, tunica argentea, a thin coat of connective tissue, with an abundant deposit of crystals; the tapetum lucidum, a membrane with a metallic lustre, whose cells are filled with crystals, occurs in Selachii, and Chondrostei, though it is absent from other forms. In the Teleostei there is usually a so-called choroid gland in the choroid coat: it is a large horseshoe-shaped rete mirabile near the optic nerve; this group usually possesses also a processus falciformis, a low fold of the choroid, which runs along the inner side of the optic bulb, from the entrance of the optic nerve to the lens. In certain Squalidm there is a nictitating membrane, which can be drawn over the eye by a special muscle.

The auditory organ is only represented by the membranous labyrinth, which is enclosed in the lateral wall of the skull; within, towards the cranial capsule, the labyrinth is often not completely surrounded by cartilage or bone, but is simply separated from the brain-cavity by connective tissue. In the Selachians, the cavity of the labyrinth opens to the exterior at the surface of the head by a canal, the ductus endolymphaticus; in others this canal is present, but closed at its outer end. In the Teleostei and bony Ganoids, a large flattened otolith is present in the sacculus, a: smaller one in the evagination from the sacculus, and yet a third in the anterior portion of the utriculus. In some Fish these are replaced by bundles of delicate crystals, or by rounded bodies.

The labyrinth is in a reduced condition in the Cyclostomes, having only one or two semicircular canals, unlike all other Vertebrata.

The buccal cavity is usually provided with teeth, which in Selachians, $\dagger$ are situated upon the palato-quadrate and mandibular cartilages; in the Holostei and Teleostei upon a number of different bones: on the premaxilla, maxilla, and mandible, the palatine and

* The meaning of this is, of course, that the scales in question have developed round the tube after it was formed.

+ Besides the well-developed teeth of the jaws, the Selachians often possess numerons minute teeth on other parts of the wall of the mouth, on its roof and floor, and on the gill bars. 
pterygoid, the branchial bars (especially the superior and inferior pharyngeal bones), the basibranchials of the visceral skeleton, and the romer; they may, however, be wanting from one or other of these bones. The teeth are of somewhat diverse form: most often pointed, conical, slightly curved, and more or less powerful ; in other cases they are low, rounded, grinding teeth (Rajidæ, certain Teleostei); or compressed and triangular (Squalidæ); or chisel-shaped, resembling the incisors of Man (teeth on the premaxilla and mandible of certain Teleosteans). Very often they are extremely numerous, covering the bones like a mosaic; on the jaw there is frequently only a single row of teeth, or a row of larger, outside a row of smaller, denticles. They are either attached to the subjacent bone by connective tissue, and then often partly movable, or they are implanted in bony sockets. They are renewed throughout the whole life, the old teeth fall out as the connection between them and the cartilage or bone gives way, or if a socket is present this is absorbed. The usual conical, piscine teeth are chiefly prehensile, and the points are therefore turned backwards and inwards; they are movable so that the point may assume another position. Teeth of other forms are used for biting or masticating the food.

The œesophagus is so short and wide that the mouth passes almost directly into the stomach. In most Teleosteans a varying number of short, blind, glandular sacs $(1-100)$, the appendices pyloricce, open into the anterior part of the small intestine, close to the stomach. In Cyclostomes, Selachians, and Ganoids, there is a spiral valve in the small intestine, a large projecting fold, attached to the inner side of the gut, and almost fllling up its cavity ;* it is absent from the Teleosteans. The large intestine is quite a short tube.

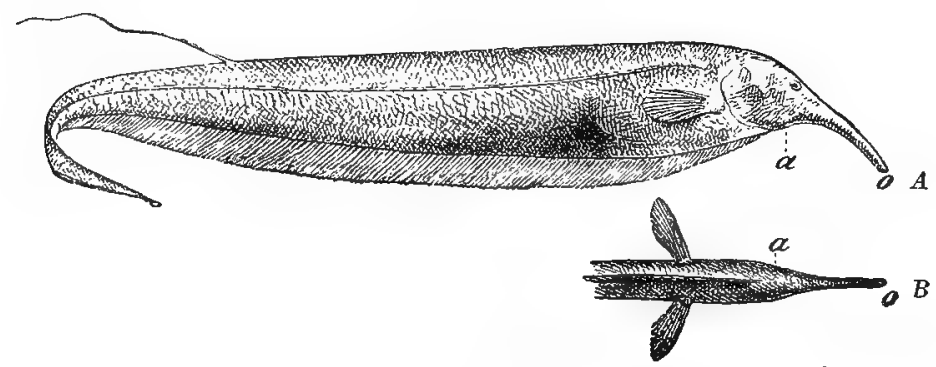

Fig. 306. A fish with pectoral anus (Sternarchus curvirostris). A lateral view, $B$ ventral view of head end. $a$ anus, o mouth,-After Boulenger.

In some Fish (e.g., the Plaice), the anus does not lie at the boundary of trunk and tail, as in all higher Vertebrata, but has moved forwards, sometimes even far on to the trunk. The anal fin in such cases follows the anus, and usually takes up its position close behind it.

* Exceptionally, in some Squalidæ, the fold springs from the gut-wall in an almost straight line, it is then broad and rolled like a piece of paper. 
The branchial apparatus. In Selachians the wall of the buccal-cavity is perforated posteriorly, by five, rarely six or seven, large oblique slits on either side; they lie in close succession,

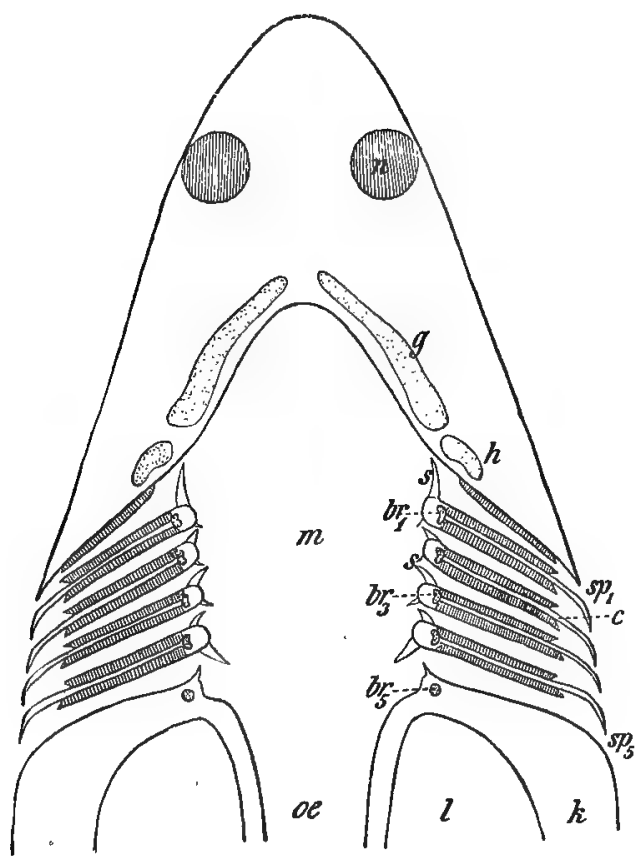

Fig. 307. Horizontal section through the head of a Shark (Acanthias) : diagrammatic. The visceral arches are dotted, the gill lamellæ shaded. $b r_{1}, b r_{3}$, $b r_{5}$ first, third, and fifth gill bars, $c$ septum, $g$ upper portion of the first visceral arch (palatoquadrate cartilage), $h$ hyoid arch, $k$ body wall, $l$ body cavity, $m$ oral cavity, $n$ olfactory pit, $a$ œsophagus, $s$ gill rakers (straining apparatus), $s p_{1}$ first, $s p_{5}$ fifth gill cleft.--Orig.

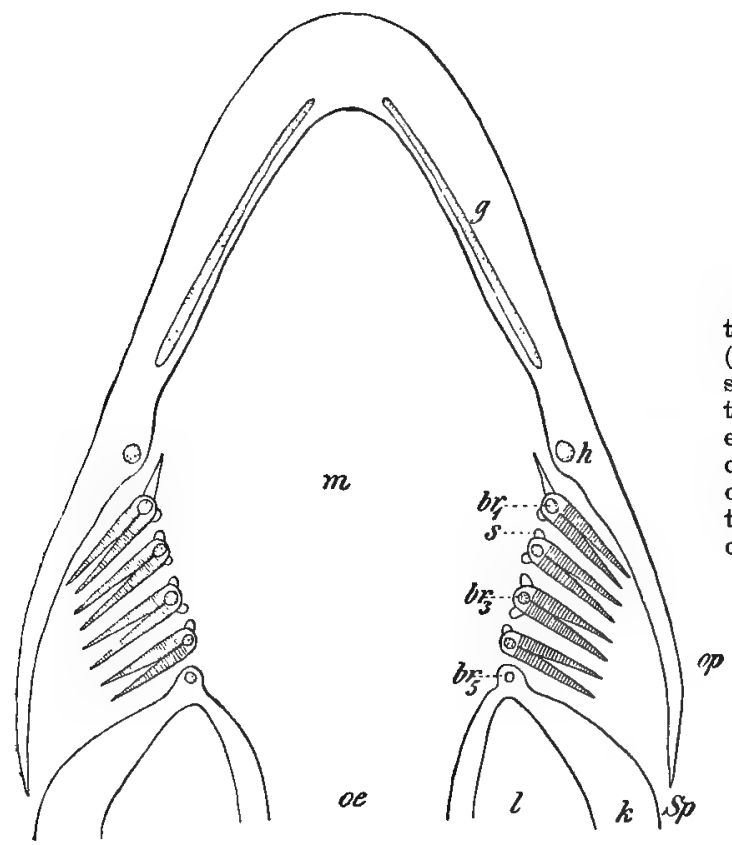

Fig. 308. Horizontal section through the head of a Teleostean (Cod), dorsal to the mouth: somewhat diagrammatic. Letters as in Fig. 307, with the exception of : $g$ upper portion of the first visceral arch (here ossified), op operculum, $s p$ external aperture of the branchial cavities.-Orig. 
and are separated by perpendicular plates. A gill bar lies at the inner or oral edge of each of these septa, which for the rest, is spread out by the cartilaginous rays arising from the bar (see p. 364). The onter opening of the cleft or branchial pouch is smaller than the inner; the first lies between the hyoid and the first branchial itch, those following between arches one and two, two and three, three and four, four and five, respectively. On both anterior and posterior walls, but in the last gill pouch only on the anterior, is a vertical row of flat, horizontal membranous folds, arranged one above the other; these are the gill lamellæ. Thus the Selachians usually possess nine rows of lamellæ on each side, the first on the posterior side of the hyoid, the other eight on the anterior and posterior sides of the first four gill bars. Each lamella is again beset with fine transverse folds. Besides these five gill pouches there is in most of the Selachians an anterior tubular pouch, the spiracle, between the upper portions of the hyoid and mandibular arches; it may contain a rudimentary series of gill lamellæ, and it opens to the exterior, by a relatively small aperture on the surface of the head. The Cyclostomes resemble the Selachians in the most important respects; the gill pouches, however, are tubular with a median enlargement; both internal and external openings are small; the lamellæ are situated in the widened part. The relations of the gill apparatus
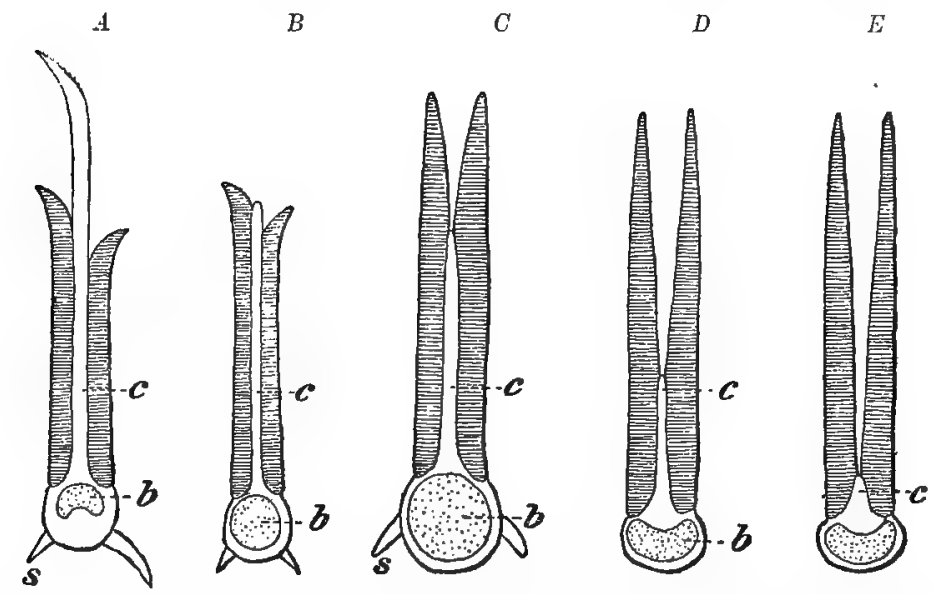

Fig. 309. Transverse section of a gill arch in various Fish. $A \mathrm{Shark}, B$ $\mathrm{Chimæra}$ (see p. 384), $C$ Sturge on, $D-E$ different Tele osteans; diagrammatic. $b$ gill bar, $c$ septum, $s$ gill rakers. Gill lamella shaded.-Orig.

in Ganoidei, Dipnoi, and Teleostei, differ considerably from those of the Selachii. In all these groups the five external openings of the gill slits are covered by the operculum, a strong membrane arising from the hyoid, and supported by bony plates and 
rods. The septa between the gill clefts have become narrower, especially in the Teleostei, whilst in the Selachians they are broad plates, not completely covered by gill lamellæ, so that there is a free edge externally; this is absent from the group just mentioned, and the outer ends of the gill-lamellæ project to a certain extent over the onter margin of the septum: this is the case in the Teleosteans, where the narrow, pointed laminæ usually arise by a short basal portion from the much reduced septum. In these groups the two series of laminæ belonging to one gill-bar constitute a gill, of which four pairs are present; the cavity within the operculum, into which the gills project, is called the branchial chamber. In Ganoids, and Dipnoans, a series of gill-lamellæ is often still present on the posterior side of the hyoid, within the operculum, the o percula gill; whilst it is rudimentary, or wanting in the Teleostei: the spiracle is also retained in the Sturgeon and Polypterus.

The operculum is attached to the hyoid, and contains flat and rod-like membrane bones (Fig. 320). In the Teleostei, along the posterior edge of the upper portion of the hyoid, is a long bone, the preoperculum, behind this are three large flat ones, operculum, sub- and interoperculum; and from the lower portion of the hyoid, arises a series of thin curred pieces, the branchiostegal rays, embedded in the lower membranous portion of the operculum. The external opening of the branchial chamber is usually a large slit, in some Fish (e.g., the Eel), however, the hinder border of the operculum is concrescent with the body to such a large extent, that only a small lateral aperture remains.

In most Pisces, water enters the buccal-cavity through the mouth, which is then closed, whilst the tongue is raised, and the operculum pressed in, so that the water is driven through the gillslits over the gill lamellæ. In the Selachians, the water is taken in, not by the mouth, but by the spiracle. In the $\mathrm{Cyclo-}$ stomes, it is generally both received and ejected by the external branchial aperture. At the inner edge of the gill-bars is a more or less well-developed straining apparatus, the gill-rakers, whose function is to prevent the solid bodies, which enter the oral-cavity with the water, from passing into the gill-sacs or branchial chamber. In the Selachians, the Dipnoans, and the cartilaginous Ganoids, this apparatus usually consists of a double series (single on the hyoid and the last gill-arch) of cartilaginous rods, on the inner edge of the gill-arch; the rods of the anterior rows on each arch dovetail with those of the posterior row on the preceding bar. In the Teleostei they are often replaced by bony outgrowths, which may be dentigerous; the anterior series of the first gill-arch is often composed of very long rods, projecting over the cleft between the first arch and the hyoid, upon which they do not occur. For the rest, they are developed to very different extents in different Teleosteans, in some, e.g., the Herring, very well developed; in others, quite insignificant. 
In Cyclostomes and Selachians, structures corresponding with the lungs of the higher Vertebrata are wanting. On the other hand, a true lung, which is not only homologous with that of Amphibia and others, but is actually functional as a respiratory organ, occurs in some bony Ganoids (Lepidosieus and Amia), in the Dipnoans, and also in a few Teleosteans. This lung is unpaired or incompletely divided into two; it lies dorsal to the alimentary canal, and opens by a wide aperture into the oesophagus. Within, it is furnished with folds, just as in the Frog's lung; air can be inhaled and exhaled through the mouth. These Fish have, in addition to the lung, gills which also serve as respiratory organs.* In the rest, a lung is also usually present; it does not act as a respiratory organ, but rather as hydrostatic apparatus, and is termed a swim-bladder. The swim-bladder is an unpaired air-containing sac, often rather thick-walled, and situated below the vertebral column, dorsal to the alimentary canal; in many Fish it communicates with the osophagust by a long, narrow tube, the pneumatic duct; in others, such a connection is present only in the embryo, closing and disappearing later. The swimbladder is sometimes incompletely divided into anterior and posterior portions (Carp), by a transverse constriction; or it may be provided with evaginations. The gas contained in the swim-bladder is not taken direct from the atmosphere, but is excreted from the vessels lying in the walls; they often form close circumscribed retia mirabilia, projecting as "red bodies" on the inner side of the bladder.

Many Fish, e.g., the common freshwater forms, in which the air-bladder is not respiratory, nevertheless come occasionally to the surface and gulp in atmospheric air through the mouth; this is soon sent out again. It probably has to do with an oral respiration of subordinate importance. In a few Fish in connection with this, a special respiratory apparatus is developed; for instance, in some Siluroids (Saccobranchus), there is a sac-like evagination on each side of the oral cavity which serves as a lung. It opens into the mouth in front of the first gill-bar, and extends far back into the body; so also in a kind of Eel (Amphipnous), an East Indian form, which lives in holes in the ground, and whose gills are very degenerate. In the East Indian Climbing Perch (Anabas), which often wanders on to land, and has similarly feebly-developed gills, there are peculiar pleated laminæ (supported by modified portions of the gill-bars), which act as respiratory organs, in the upper part of the branchial chamber. In others, e.g., the Loach (Cobitis), intestinal respiration occurs; air is inhaled through the mouth, and passed on to those parts of the alimentary canal, which are specially vascular; the air which is not absorbed escapes from the anus, together with excreted carbonic acid gas.

Many Pisces can produce sounds. The wall of the swim-bladder is made to vibrate by the action of certain skeletal muscles which are applied to it, as in the Gurnard; or particular bony surfaces may be rubbed aguinst one time.

* Some Fish can survive a drought, during which the gills are functionless for some

+ In some, the pneumatic duct opens further back, into the stomach. 
another, as in the Siluroids, where the bases of well-developed rays may play against the subjacent bones.

The heart, which is situated anteriorly (see Fig. $285 \mathrm{~A}$ ), is usually almost bilaterally symmetrical. In Selachii, Ganoidei, and Dipnoi, it consists of a large thin-walled a uricle; of a ventricle lying ventral to this, with thick walls of a spongy nature, owing to the numerous offsets passing into them from the small cavity; and lastly, of a tubular conus arteriosus, from the anterior end of which the trunk of the branchial arteries arises, and in which several rows of membranous watch-pocket valves are

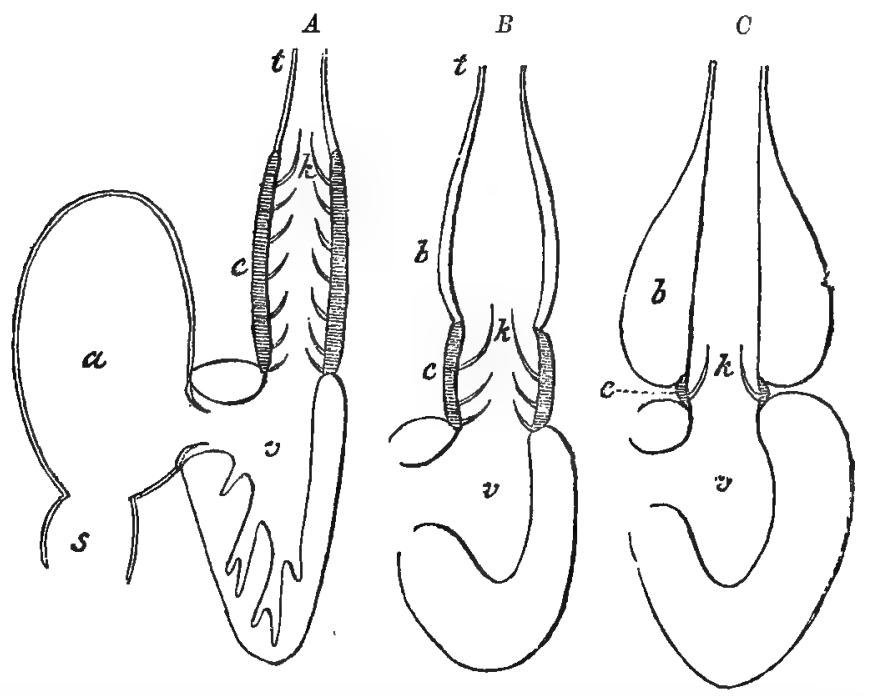

Fig. 310. Diagrammatic longitudinal section of the heart of different Fish. A of a Fish with well-dereloped conus, $B$ of $A$ mia, $C$ of a Teleostean ; in $B$ and $C$ the auricle is cut away. $a$ auricle, $b$ bulbus arteriosus, which is only just indicated in Amia, $c$ conus arteriosus, $k$ valves, $s$ sinus venosus, $t$ cardiac aorta, $v$ ventricle.-Orig.

situated. All three sections are red, and their walls are furnished with striated muscle-cells. In the Teleostei, the conus is, as a rule, quite rudimentary (extremely short and without musculature), and is provided with but two valves; only in a few cases (from the family of the Herrings) is it somewhat more significant, although still very short; and in a single genus (Butirinus) there are two rows of valves.* In the Cyclostomes a conus is wanting. In Pisces there is usually a transverse row of valves between the auricle and ventricle, and between the sinus venosus (see below) and the auricle. From the anterior end of the conus, or of the ventricle when the former is absent, arises a longer or shorter cardiac a orta, which

* In one of the Holostei, Amia, the conus is much shortened, and exhibits only three rows of valves. 
in the Teleostei, is much swollen, and provided with thick, walls just at the point of origin. This enlargement, the $\mathrm{bulbus}$ arteriosus,* is whitish like the other arteries, and contains simply smooth muscle-cells, whilst the conus, with which it was. until recently included, is red, and possesses striated musclecells. The cardiac aorta sends a branch to each gill-bearing. arch; if the opercular gill is well developed, a branch also goes to this, but not if it is rudimentary. These branches, the afferent branchial arteries, run from below upwards, along the hinder edge of the gill-bar, and give to each gill lamella a twig, which breaks into capillaries. From each lamella, there arises, again, a small vessel, which, with those like it from the same visceral arch, forms an efferentbranchial artery.t This runs near to the afferent artery, and unites dorsally with the corresponding vessels from other visceral arches, to form the a or ta, which runs backwards, just beneath the vertebral column, and gives off branches to various parts of the body. All the veins flow into the sinus venosus, which opens into the auricle. The blood entering the heart is thus venous, reaches the gills in this condition, becomes. arterialised there, and thence flows into the arteries.

There is, therefore, a complete separation of arterial, from venous, blood in Pisces, and the condition of the vascular system accords with the general plan given on pp. 28, 29. Some Fish, however, which possess other respiratory organs as well as gills, form an exception, for in them the arterial and venous blood is. more or less mixed. In Lepidosteus, for example, the lung receives from the aorta, arterial blood, to be further oxydised, whilst the pulmonary veins, which thus carry blood very rich in oxygen, unite with the large veins, bringing venous. blood from the rest of the body. The heart and gills thus receive mixed blood. In the Dipnoi, where the lung, as in higher Vertebrata, receives blood from the last arterial arch (the last efferent branchial vessel), there are special contrivances to partially remedy the defect, but these are too complicated to be gone into here.

The piscine $\mathrm{kidneys}$ are usually elongate organs, and in many Teleostei, in which they lie above the swim-bladder close against the vertebral column, extendt the whole length of the body from head to tail, and are often united§ behind. In the Selachians and

* A similar swelling is present in Amia, but in no other Pisces excepting the Teleostei ; whilst in this genus the wall is little thickened.

+ These efferent branchial vessels are frequently, but incorrectly termed, branchial veins, and the afferent vessels simply distinguished as "branchial arteries." In some Fish, instead of one, two efferent vessels may arise from each gillbar.

I The most anterior portion of this long kidney is the persistent pronephros, which is frequently very large in the adult, but has usually not an excretory function.

$\S$ A very interesting modification of the kidney occurs in the male Sea Stickleback (Spinachia vuigaris), which binds various foreign bodies together by fine mucous. threads, and thus forms a nest for the eggs. The $\mathrm{mucus}$ of which these threads. consist is manufactured in the kidneys; some of the gland cells of the urinary tubules are modified to secrete mucus, and are of different appearance from the rest of the cells. 
Dipnoans, the urinary ducts open into the cloaca; in others they unite and open behind the anus, either together with the gonaducts, or by a special aperture behind the genital pore. The last is the case in most Teleostei, where there are three openings, one behind the other; first the anus, then the genital pore, finally the urinary aperture.*

In the Teleostei the urinary (and the genital) aperture is usually situated on a small, soft process, the papilla urogenitalis. The posterior portion of the urinary duct is usually wide, and forms a bladder : in the Selachians a pair of bladders is present; in the Teleosteans one only, an expansion of the common portion of the ducts.

Female genitalia. In Selachians, Ganoids, $†$ and Dipnoans, the ovary resembles that of most other Vertebrata, and there is a pair of M üllerian ducts, each usually opening anteriorly into the body-cavity by a funnelf; in the Selachians and Dipnoi they open posteriorly into the cloaca, whilst in the Ganoids they unite with the excretory duct, and open behind the anus by an unpaired aperture. In the Selachians there is on each oviduct a swollen portion with glandular walls, which secrete the horny capsule, surrounding one or more eggs in most of these animals. In the Teleostei, Müller's ducts are altogether wanting; the ovaries are hollow and vary in form; each is prolonged into a short, tubular duct, which unites with its fellow of the other side to open behind the anus. The ovary thus displays: relations extremely different from those of all other Vertebrata, but similar to those occurring in many lower animals, e.g., the Mollusca. The ova break away from the much-folded inner wall, and fall into the cavity of the ovary, escaping to the exterior through the duct. The two ovaries are frequently fused posteriorly (e.g., in the Cod), or throughout their whole length (as in Zoarces); and the duct is then unpaired. When ripe, in the spawning season, the Teleostean ovaries are often extremely large.

Only two families differ from the condition just described. In the Salm on and the Eel the ovaries are solid, the eggs fall into the body-cavity, and escape by an unpaired opening $\S$ in the body-wall, behind the anus (porus genitalis). The Cyclostomes, which only possess a single ovary, are otherwise similar to the Salmonidæ.

* In some Fish, viz., Selachians, Ganoids, and certain Teleosteans (the Salmon family) there is in this region, on either side of the anus, a pair of small openings, the so-called abdominal pores, which perforate the body-wall, and put the body-cavity in communication with the exterior. Their significance is unknown.

† With the exception of Lepidosteus, which seems to resemble the Teleostei.

I In the Selachians the Müllerian ducts are united anteriorly, so that there is only a single funnel for the two. In some Sharks only one ovary is developed.

$\S$ Which is not to be confounded with the abdominal pores mentioned above $\left(^{*}\right)$. 
Male genitalia. In the Selachians the spermatozoa escape by the anterior portion of the kidney, often termed epididymis; this is in connection with the testis, and the duct arising from it unites posteriorly with those from the rest of the kidney, and serves really as a seminal duct, for the epididymis is of very slight importance in excretion. In the females, also, this region is very little developed. In the Ganoids, too, the sperm makes its way out through the kidney; numerous transverse canals run from the testis to the kidney (of which no part is specially modified), to communicate with the urinary tubules.* In the Teleostei such a connection with the kidney does not occur, the testis is prolonged directly into a seminal duct, $\uparrow$ and like the ovaries, the ripe testes are tolerably large, elongate, often lobed, or (e.g., in the Cod), pleated bodies; the vasa deferentia unite behind to form an unpaired duct, which has, in some forms, a special opening behind the anus, in front of the urinary aperture, whilst in other cases there is a common urino-genital opening.

In the Cyclostomes, the spermatozoa from the unpaired testis fall into the body-cavity, and escape through an opening of the abdominal wall just as do the ova.

Copulatory organs occur in all Selachians, where a portion of the hind limb in the male, is modified into a somewhat complicated rolled organ (Fig. 314), which is used in copulation ( $e f$. the copulatory organs of decapod Crustaceans). On the other hand, copulatory organs are absent from most other Fish, and the spermatozoa ("milt") are usually not introduced into the body of the female, but are poured over, or near to, the eggs when they are laid.

In certain viviparous Teleostei (Anableps) there is a long process behind the anus, with the urino-genital opening at its apex. This process, which serves as a copulatory organ, is the metamorphosed anal fin, which has fused with the urino-genital papilla. There are sinilar organs in some other viviparous Teleosteans. In all viviparous Fish there must, of course, be a direct transference of the spermatozoa from the male to the female, but copulatory organs are by no means always present.

Not a few Fish display striking sexual dimorphism; in the males certain fins may be specially well developed, or they may possess a specially brilliant colouring. Sometimes (e.g., the Stickleback) the male is distin. guished by striking colours during the reproductive season, which disappear later on. The males are usually s maller than the females (e.g., the Eel).

For hermaphroditism in Fish, see p. 351.

The eggs vary considerably in size (from the size of a pin's head to that of a Hen's egg and upwards), they are largest in the Selachians, smaller in the Teleostei, where each is covered by a thin transparent vitelline membrane sometimes furnished with a micropyle. 'The eggs

* The arrangement of the seminal ducts of the Dipnoi is not understood.

+ The Salmon and the Eel are like the others in this respect. 
of numerous marine forms (e.g., the Cod), float at the surface of the water; others are deposited at the bottom; or are attached to water plants (e.g., the Herring). In the Selachians they are
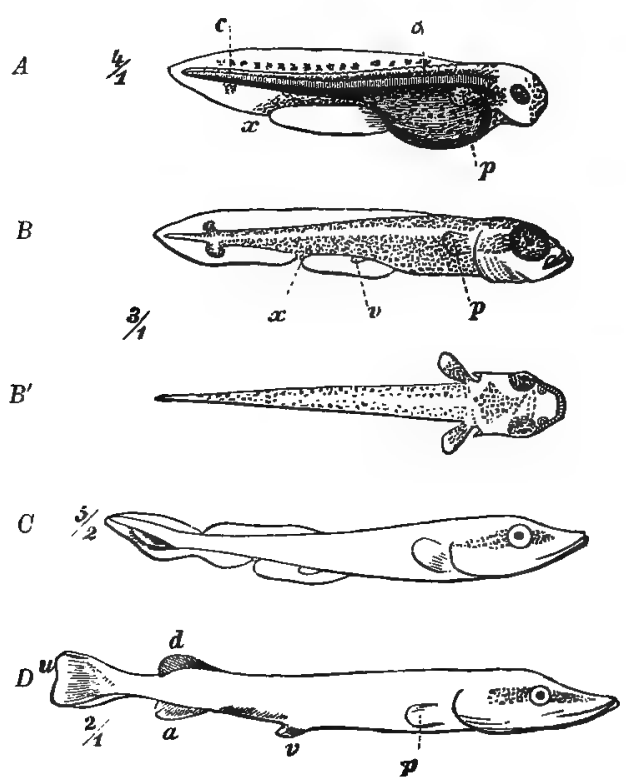

Fig. 311. Young Pike; $A$ just hatched, $B, B^{\prime}$ eleven days old, $C$ and $D$ still older. In $A$ the tail is still straight, in $C$ and $D$ maxkedly heteroceral. $a$ anal fin, $c$ chorda, $d$ dorsal-, $p$ pectoral-, $u$ caudalfin, $x$ anus.-After Sundevall. enclosed in a horny capsule, which is often flattened and quadrangular with the corners drawn out into threads. Some Fish are viviparous (e.g., most Selachians), and development takes place in a widened portion of the oviduct (uterus), which is provided with glomerular vascular folds; also some Teleosteans whose eggs develop in the cavity of the ovary (e.g., the viviparous Blenny). In some fow forms there is a special arrangement for the protection of the eggs and brood; the males of the Stickleback (and of various other Teleostei) build nests, in which the eggs are hatched; the males of the Pipe-fish carry the eggs and sometimes also the brood about with them, firmly attached to the abdomen, or enclosed in special folds of the skin. This is the case also in various Fish occurring abroad. More rarely the eggs are protected in the same way by the female.

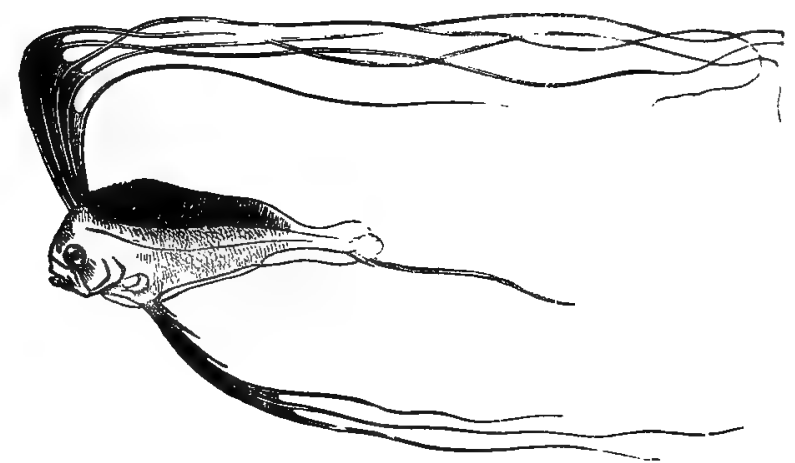

Fig. 312. L a rva of a Fish ('Trachypterus) which, in the adult, is extraordinarily long, ribbon-like, and without the fin filaments. 
It is quite correct to speak of a metamorphosis in many Teleosteans, since the young one leaves the egg in a very imperfect condition, differing much from the adult; often the caudal extremity of the vertebral column is still straight; there is a continuous dorsal and ventral fin, etc. (Fig. $311 \mathrm{~A}$ ). It may often happen that the transition from this state to the adult form is not a simple, gradually advancing development; but the larva not infrequently displays special characters for a long time after leaving the egg, which do not occur in the newly-hatched young, nor in the adult. Especially in the Pelagic Fauna, abundant large-eyed 'leleostean larvæ are met with, possessing enormous spines, and fin appendages, structures which recall those observed in many pelagic crustacean larvæ, e.g., the Crabs (Fig. 312). For the peculiar development of the Lampreys, see p. 382.

The embryos of Selachians (Fig. 313) are distinguished by the possession of a huge yolk sac, and also by the projection from the gill-slits of numerous long gill-filaments, processes from the gill-lamellæ. These filaments are embryonic organs, and atrophy before birth.

Most Fish are predaceous, only a few feed upon plants or mud. 'The majority are marine, but many are freshwater (some species are

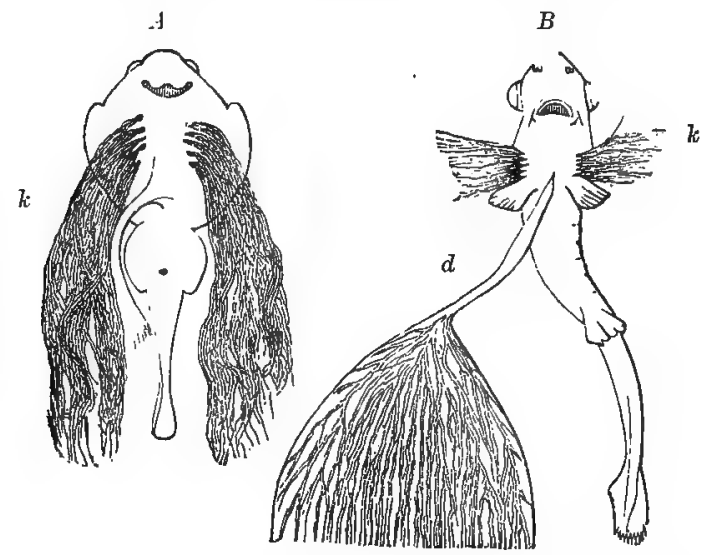

Fig. 313. A Ra y embryo, $B$ Shark embryo with external gills $(k)$. $d$ yolk sac (not completely drawn; removed in $A$ ).

both); a few wander upon land. They often migrate from one place to another in the sea, or from the sea into fresh water and back. They usually occur in shoals. Fish make their way through the water by movements of the whole body, and by lateral movements of the tail; the Teleostei can also progress slowly by undulations of all the fins (paired and unpaired).*

* A very singular movement, suggesting flight, occurs in the Rajidæ, brought about by the powerful fore limbs. 
Fish, which are represented to-day by such numerous genera and species, have also played an important part in earlier periods; the Teleosteans, which preponderate at the present time, arose comparatively late; whilst the Ganoids, which now include a few species only, were for long very abundant.

\section{Synopsis OF THE ORDERS.}

$\begin{aligned} & \text { Skeleton entirely cartila- } \\ & \text { ginous. } \\ & \text { Horny or cartilaginous rays. } \\ & \text { Scales absent. } \\ & \begin{array}{l}\text { Operculum absent. } \\ \text { Swim-bladder absent. }\end{array}\end{aligned}\left\{\begin{array}{c}\text { Cyclostomi } \\ \begin{array}{l}\text { Skeleton of cartilage and } \\ \text { bone. } \\ \text { Bony rays. } \\ \begin{array}{l}\text { Scales present. } \\ \text { Operculum present. } \\ \text { Swim-bladder or lung pre- } \\ \text { sent. }\end{array}\end{array}\left\{\begin{array}{c}\text { Ganoidei } \\ \text { Dipnoi }\end{array}\right\} \begin{array}{l}\text { Tonus arteriosus well-de- } \\ \text { veloped. } \\ \text { Müllerian ducts present. }\end{array} \\ \text { Telestei }\end{array}\right\} \begin{aligned} & \text { Oonus rudimentary. } \\ & \text { Spiral valve absent. } \\ & \text { Müllerian ducts absent. }\end{aligned}$

\section{Order 1. Cyclostomi.}

The Cyclostomes form a small group, differing in many respects from other Pisces. The body is cylindrical, vermiform and apodous; the skin is naked; the skeleton is entirely cartilaginous; the notochord is unconstricted; ribs are absent. There is a complicated oral and branchial skeleton, which can with difficulty be reduced to the common type of piscine visceral skeleton. There are usually six or seren (in a few, a still larger number) gill-pou ches on each side (see p. 373); the mouth is provided with horny teeth, but true teeth are absent; the olfactory organ is unpaired; the caudal extremity straight; and there is a continuous dorsal fin ( $c f$. in other respects, the account given for Fish in general.

The Oyclostomes are most nearly allied to the Selachians; their peculiar characters are without doubt to be attributed partly to their peculiar mode of life, as parasites or carrion-feeders.

1. The Nine-eyes or Lampreys (Petromyzon) have a circular sucking mouth with horny teeth; seven small gill-apertures on each side leading into gill-pouches; which do not open directly into the mouth, but into a short tube ventral to the cesophágus, closed behind but anteriorly in communication with the mouth. Eyes are well developed. The Lampreys attach themselves by suction to living Fish, which they devour; they also feed on smaller animals. Three species live in England; two are marine, but can make their way up into fresh water-P. Marinus, up to $1 \mathrm{~m}$. long, and the small $P$. Fluviatilis, (Pricke); whilst the third and smallest species (P. Planeri) is exclusively a freshwater form. Lampreys undergo a m e tam or phos is ; the larvæ (Ammocoetes), which in P. Planeri may be three or four years old and of a considerable size 
before a change occurs, are very different in form from the adult; horny teeth are absent, the eyes are very small, and the gill-sacs open directly into the mouth. They live in mud.

2. The Ha $\mathrm{g}-\mathrm{fish}$ (Myxine) has rudimentary eyes; the mouth is surrounded by tactile tentaicles; the gill-sacs (six on each side) are long tubes expanded in the middle, each opening direct into the pharynx, whilst the outer regions of each side unite, to open by a common aperture some way back; though in an allied foreign form, Bdellostoma, they open separately. Hag-fish, of which M. glutinosa is very common in N. European seas, and reaches as much as $30 \mathrm{c} / \mathrm{m}$. in length, bore into dead (and living ?) Fish; they secrete enormous. masses of mucus.

\section{Order 2. Selachii.}

The skeleton consists entirely of cartilage, which may, however, be partially calcified; bone is altogether wanting. A conus. arteriosus is present, and a spiral valve in the gut. There are five, rarely six or seven, gill-clefts on each side; often a spiracle, but no operculum, excepting in Chimæra. There is no swimbladder. The whole surface of the skin is often covered with teeth. In the fins, which cannot be folded together, there are horny rays. The mouth is on the ventral side of the head. Parts of the pelvic fins in the male serve as copulatory apparatus. Eggs very large. Almost exclusively marine.

1. The Sharks (Squalidæ) are animals of the ordinary piscine form, generally elongate and somewhat circular in section. The skin is usually thickly covered with small teeth. Along the edge of the jaw there are, as a rule, one or two rows of teeth, usually triangular in form, and replaced by others from the mucous membrane within the jaws: definitely heterocercal. Of the numerous forms the following may be specially mentioned: the Common Spiny Dog-fish (Acanthias vulgaris), $1 \mathrm{~m}$. long, with a spine (a strongly developed placoid scale) anteriorly on each of the dorsal fins; anal fin absent; viviparous : found in the North Sea and Baltic: the Dog-fish (Scyllium canicula), somewhat smaller; oviparous, egg-capsule quadrangular, and attached to Alga by long tendril-like appendages from the corners; common on the coasts of Britain: the Blue Shark (Carcharias glaucus), the voracious man-eating form, $3 \mathrm{~m}$. or $4 \mathrm{~m}$. long, occurring in the Mediterranean, abundant in the Tropics: the Hammerheaded Shark (Sphyrna), with each side of the head drawn out into a longer. or shorter process, at the end of which is the eye; one species in the Mediterranean: the Greenland Shark (Scymnus borealis), which reaches $8 \mathrm{~m}$. in length, is caught in great numbers for the sake of the fat liver; on the coast of Iceland: still larger (up to $12 \mathrm{~m}$.) is the Giant Shark (Selache maxima), in which the external gill-clefts are very large slits; the eyes very small; teeth small and poorly developed; the inner edge of the gill-bars, with a series of very long teeth, forming a fine comb, which acts as a straining apparatus, to retain the small. Crustacea on which this giant feeds, after the manner of the Whalebone Whale.

2. The Skates (Rajidæ) are chiefly distinguished by the flattened form of the head and body, by the thin, whip-like tail, which is often almost destitute of fins, and by the enormous development of the pectoral fins, which arise like horizontal plates from the sides of the body, so as to form a dise with it and the head, and to relegate the gill-slits, over which they lie, to the ventral surface; the eyes and spiracle are on the dorsal side. Amongst other characters it must be mentioned that the skin is usually naked over a greater or less extent; that 
some of the remaining placoid scales form large spines; and that the buccal teeth are low knobs (sometimes pointed) or plates, which are arranged in several rows and form a mosaic over the edges of the jaws. In general appearance, therefore, the Skates differ considerably from the Sharks. The skate type is not always developed to the same extent; in some forms the pectoral fins are smaller, the tail more powerful; whilst, on the other hand, there are Sharks (Squatina, the Seaangel), which are somewhat flattened, with the eyes turned upwards, and large horizontal pectoral fins reaching antero-posteriorly along the sides of the head, but not attached to it. There is indeed a complete series of transitional forms, between the usually slim shark type, to the most extreme ray type with its discoid shape, wider than it is long, and with its thin caudal whip. In British seas there are several species (chiefly of the genus Raja), all typical Rays. Of forms belonging to the Southern seas may be mentioned the Electric Skates (Torpedo), and the Saw - $\mathrm{fish}$ (Pristis); the former are well known on account of their powerful electric organs, which lie on either side of the head; in the Sawfish the snout is drawn out into a long, straight, narrow plate, with a series of large, laterally directed, teeth on each edge. Both the Electric Skates and the Saw-fish, but especially the latter, belong to the more shark-like Rays, with tolerably powerful tail. Both genera occur in the Mediterranean.

3. The Cat-fishes (Holocephali) genus Chimæra, etc., form a small division of the Selachians, which differ from their allies, and approach the following orders chiefly in the possession of an operculum (which is, however, not supported by skeletal plates); the gill lamellæ completely cover the side -of the septum, but do not project over its onter rim (Fig. 309 B). The skin is

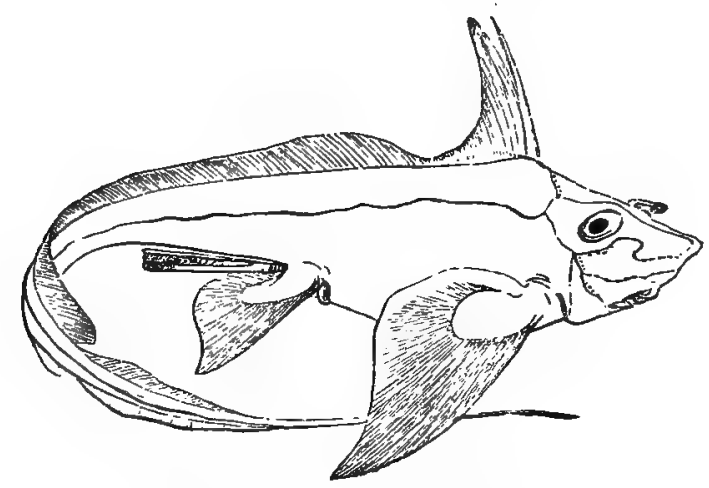

Fig. 314. Chimara monstrosa, ð.

for the most part naked, the mouth armed with a small number of large teeth. The upper portions of the mandibular and hyoid arches are attached to the skull. In other respects they exhibit for the most part the characters of other Selachians. One species, Ch. monstrosa, is abundant in the Meditenanean, on the coast of Norway. and elsewhere.

\section{Order 3. Ganoidei.}

The skeleton consists of cartilage and bone; conus arteriosus and spiral valve are present; an operculum supported by bony plates; often a spiracle; swim-bladder or true lung. The skin usually 
provided with bony plates or scales (ganoid scales); dermal denticles may also be present, but in small numbers. Bony rays occur in the folding fins.

This group was formerly very well represented; few of its members are living, however, at the present day.

\section{Sub-Order 1. Chondrostei (Cartilaginous Ganoids).}

The skeleton is, for the most part, cartilaginous; only membrane bones are present. The mouth ventral. The tail heterocercal.

1. Sturgeons (Accipenser) have five rows of large bony plates arranged along the body (one row being median) dorsally, and manył small plates irregularly placed; dorsally upon the head are large bony plates, which cover the chondrocranium : the mouth is small, edentulous (the young, however, have teeth, and sometimes small teeth occur on the gill-bars of the adult); on the ventral side of the often elongate snout there are tactile tentacles : a spiracle is present. A. sturio, which attains a length of several metres, inhabits North European seas, wandering up into the rivers to spawn; there are several other species in the Caspian and Black Seas, and in the large rivers of Russia (Sterlet, A. ruthenus, A. huso, etc.).

2. The Spoon-billed Sturgeons (Spatularia) differ from Accipenser in that the snout is prolonged into a large horizontal blade, and the skin is almost without hard parts; in the mouth weak teeth are developed. In North American and Chinese rivers.

\section{Sub-Order 2. Holostei (Bony Ganoids.)}

The skeleton is for the most part ossified. The mouth anterior. Large, rhomboidal, enamelled* scales, which may be partly dovetailed together, or, more rarely, scales like those of the Teleosteans. Usually (Lepidosteus, Amia) the respiratory organ is a true lung. All existing forms are freshwater.

1. Polypterus. Long dorsal fin, with strong fin-rays, usually fan-shaped at the tip, and not connected together; no anal fin; caudal fin rounded, feebly heterocercal (the bent-up portion of the spinal column is very small). Large rhomboidal scales. A spiracle. In Africa (e.g., in the Nile).

2. The Bony Pike (Lepidosteus). Snout much elongated; short dorsal and anal fins; well-marked heterocercality, the caudal fin being almost entirely

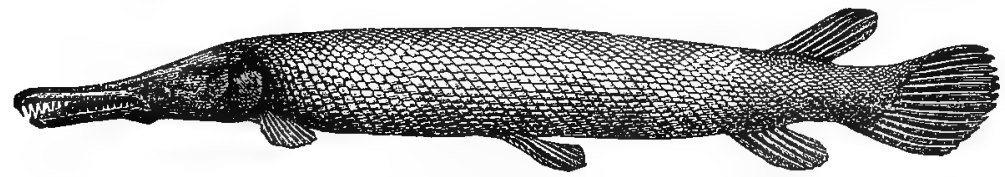

Fig. 315. Lepidosteus.

* The scales are covered externally by a smooth layer, commonly called "enamel ": but it is not true enamel, like that of the teeth, it is only an external, polished, densc layer of bone. 
ventral to the long, bent-up portion of the spinal column (Fig. $299 \mathrm{~B}$ ). Rhomboidal scales. Several species in N. America.

3. Amia. Externally almost exactly like a Teleostean; it has cycloid scales. For its chief characteristics see p. 375, foot-note * p. 377, foot-note *; Fig. 304 C ; Fig. 310 B. It occurs in North America.

\section{Order 4. Dipnoi.}

The skeleton is partially ossified; the conus arteriosus spirally coiled and provided inside with a longitudinal fold formed of modified valves; a spiral valve in the intestine; the operculum is supported by bony plates; the lung is functional; the skin provided with scales, the fins with unsegmented, soft, bony rays. Both anterior and posterior nares lie within the mouth. The limbs are either long, pointed plates, with a median, segmented, cartilaginous rod, from either side of which a series of cartilaginous rays arise; or they are filiform, with a similar, but more or less reduced skeleton. The tail is pointed and diphycercal; notochord unconstricted and well-developed; a few large teeth in the mouth. Exclusively freshwater.

This aberrant group, which at the present day is represented by a few forms only, is most nearly allied to the Ganoids, especially the Holostei. The structure of the conus is remarkable, recalling the condition in the Amphibia (q.v.); in connection with it are certain peculiarities in the structure of other parts of the heart, by which a partial separation of blood from the lung and from the rest of the body is effected. The structure of the limbs, the position of the nares, etc., is also very peculiar.

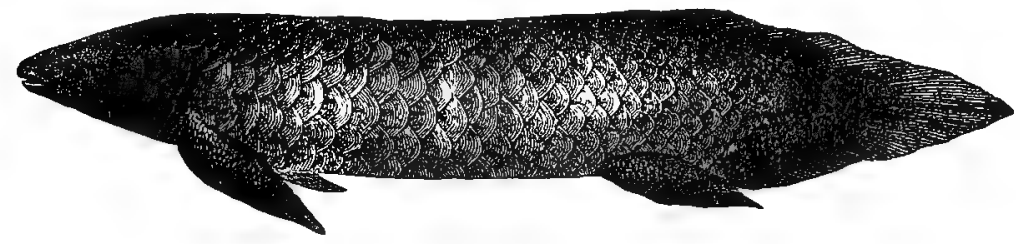

Fig. 316. Ceratodus: After Günther.

1. The Mud-fish or Barramunda (Ceratodus) is a large, elongate animal, pointed at both ends; with large scales; large, broad limbs; dorsal, caudal and anal fins continuous. It inhabits the rivers of Australia.

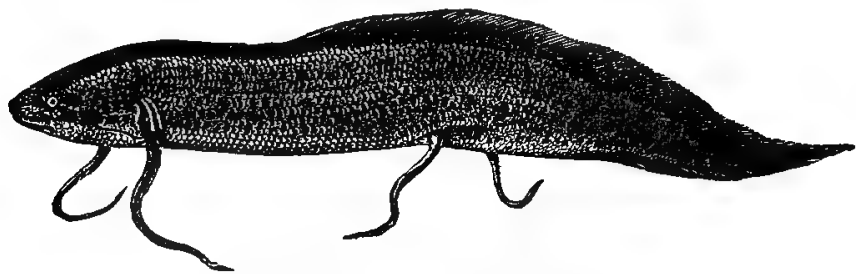

Fig. 317. Protopter'us annectens. 
2. Protopterus annectens, the African Lung-fish, has very long, thin limbs; it possesses some small, thread-like membranous appendages at the upper ends of the gill apertures; these possibly have a respiratory function; the gills of the first and second arches are absent. In other external respects it is similar to Ceratodus. If the water in which it is living dries up, it buries itself in the ground, and surrounds itself by a hard mucous capsule, in which it can remain for a long time without water (this is not the case with Ceratodus). An allied form (Lepidosiren paradoxa) occurs in S. America.

\section{Order 5. Teleostei.}

The skeleton consists of cartilage and bone; chiefly the latter. The conus arteriosus is rudimentary, a bulbus arteriosus is present. There is no spiral valve in the intestine; the operculum is bony; there is no spiracle; the skin is provided with scales or bony knobs, plates, etc.; dermal denticles are usually absent, but may be present in small numbers. The fins can be folded and are provided with bony rays.

\section{Sub-Order 1. Physostomi.}

Swim bladder connected with the alimentary canal by a pneumatic duct; pelvic fins far back, close to the anus; spinose rays usually absent; scales cycloid.

1. The Herring family (Clupeidæ). Body elongate and compressed: large, easily deciduous, cycloid scales; only one dorsal fin; teeth feeble. To this family belong: the Herring (Clupea harengus), and the. Sprat (Cl. sprattus); the Sardine (Cl. pilchardus); the Shad, (Cl. alosa) all common on British coasts; the last makes its way into rivers (e.g., the Severn) to spawn; all these very similar forms have a row of carinate scales along the ventral side. The true Anchory (Engraulis encrassicholus), without these modified scales, and with elongate snout: in the Mediterranean, occasionally in northern seas.

2. The Salmon family (Salmonidx). Scales small, or of medium size. Two dorsal fins, of which the posterior is rayless and adipose. Chiefly in freshwater. Amongst the species occurring in the British Isles: the Salmon (Salmo salar), marine, migrating into rivers to spawn; the closely-allied Common Trout (S. fario), in fresh water; the Sea- or Salmon-trout (S. trutta): the Char (sub-genus, Salvelinus), in mountain lakes. The species of Coregonus (C. thymallus, the Grayling; C. pollan, the Fresh-water Herring;) are edentulous, or have only small teeth, whilst the genus, Salmo, has large ones.

3. The Pike family (Esocidx). Small scales; dorsal fins far back; flattened, elongate snout; mouth large, with numerous teeth; some of them large. Few species; the common $\mathrm{Pike}$ (Esox lucius), abundant in fresh water.

4. The Carp family (Cyprinidæ). Body compressed, with larger or smaller scales; one dorsal fin; bones of the mouth entirely edentulous the lower pharyngeal bones are, however, provided with powerful grinding-teeth, which work against a thick horny plate on the under side of the skull; usually barbules on the edge of the mouth; freshwater fish, feeding partly upon decayed plants. Of the numerous forms, may be mentioned: the $\mathrm{C}$ a r $\mathrm{p}$ (Cyprinus 
carpio), with four barbules on the upper edge of the mouth (introduced from Asia): the Prussian Carp (Carassius vulgaris), without barbules, but otherwise similar: the Gold-fish (Car. auratus), from China; the Barbel (Barbus vulgaris), with four barbules, of which two are at the tip of the snout: the small Gudgeon (Gobio fluviatilis): the Roach (Leucisus): the Tench (Tinca vulgaris), with small scales in a thick slimy skin: the small Bitterling (Rhodeus amarus), females provided at spawning-time with a long ovipositor bearing the genital pore at the tip, by means of which the eggs are laid in the branchial chambers of the Freshwater Mussels (Unio): the Bream (Abramis brama), with high, laterally compressed body: the Loach (Cobitis), small fish with elongate, sometimes eel-like, bodies; very small scales concealed beneath the skin; six or more barbules, intestinal respiration (see p. 375). All these, except the Gold-fish and the Bitterling, are indigenous to the British Isles.

5. The Silurus family (Siluridx). The body never has the ordinary scales; it is either naked, or provided with large bony plates (dermal denticles may be present); maxillæ very poorly developed; barbules and an adipose fin usually present. Freshwater Fish, which are represented by numerous interesting tropical forms. The Sheat-fish (Silurus glanis), naked, with quite small dorsal fin far forward, long anal fin, two long and four short barbules, small eyes; as much as $4 \mathrm{~m}$. long; the only European representative of the family, occurs in England. The electric Silurid (Malapterurus electricus), with adipose fin (but otherwise without a dorsal fin), more than $1 \mathrm{~m}$. long, in Africa. The Loricaria, skin covered with large bony plates, in S. America.

6. The Eel family (Murænidx). Body snake-like, smooth or with small scales; without pelvic fins; dorsal, caudal, and anal fins continuous; small gillslits; small eyes. The Eel (Anguilla vulgaris), with scales; spawns in the sea, probably in deep water; the young, whilst still transparent, wander into fresh water, returning to the sea later. The Conger-eel (Conger vulgaris), scaleless, attains a considerable size $(2 \mathrm{~m}$.); in the North Sea. The $\mathbf{M} \mathbf{u} æ \mathbf{n}$ a (Gymnothorax muræna), apodous, even the pectoral fins being absent; in the Mediterranean. To another family of snake-like Physostomi belongs the Electric Eel (Gymnotus electricus), of S. America; anus close to the head; anal fin long; no dorsal and pelvic fins; the large electric organs along the ventral side reaching to the tip of the tail.

\section{Sub-Order 2. Aphysostomi.}

No pneumatic duct. Pelvic fins, generally moved far forwards. Spinose rays usually present (not in sub-divisions, $1-3$ ).

1. Mackrel-pikes (Scomberesocidx). Oycloid scales; dorsal fin short, far back; pelvic fins far back; no spinose rays. The Gar-pike (Belone vulgaris) has mandible and premaxilla elongated to form a beak, beset with fine teeth; body elongate; bones green; in the North and Baltic Seas. The Flying-fish (Exocoetus) distinguished by the enormous development of the pectoral fins, by means of which it can take short flights across the surface of the ocean; in tropical seas (one species in the Mediterranean).

2. The Cod-fish family (Gadidx). Body somewhat elongate with small cycloid scales; usually two or three dorsal, and one or two anal fins; pelvic in front of the pectoral-fin, no spinose rays; often a barbule on the lower jaw. To the genus Gadus, with three dorsal and two anal fins, belong: the Codfish (G. morrhua), which occurs in immense shoals in the North Atlantic, up to $1.5 \mathrm{~m}$. in length; the Ha d d ock ( $G$. æglefinus), numerous, e.g., in the North Sea; both these have barbules: the Eel-pout (Lota 
vulgaris) in fresh water, has a short anterior, and a long posterior dorsal fin (the latter corresponds to the two dorsal fins of Gadus); an anal fin, and one barbule. To an allied family $(\mathrm{Ophidiidæ)}$ belong the $\mathrm{Sand-eels} \mathrm{(Ammo-}$ dytes), small and elongate with no mandibular teeth; with projecting lower jaw; without pelvic fins; with long dorsal and anal fins; on the coasts of Britain. To the same family belongs also the genus Fierasfer, the species of which take up their abode in the cloaca of Holothurians (without being actually parasitic, since they feed upon small animals) : an allied genus, Enchelyophis, is a true parasite.

3. Flatfish (Pleuronectid:). The body is a laterally compressed dise; both eyes on the same side, in some species on the right, in others on the left (in a few species, some individuals have the eyes on the right, others on the left); the blind side is white and turned downwards, the other coloured; the mouth is often somewhat asymmetrical, being larger on the blind side where the premaxilla and maxilla, are better developed; dorsal and anal fins very long, anus far forwards; pelvic fins in front of the pectorals; no spinose rays. The lurve are perfectly symmetrical, with the eyes on either side of the body, and the animal swims with the ventral surface downwards; later, one eye moves round to the other side, and the animal lies upon one side. Amongst the forms inhabiting British seas are: the Plaice (Pleuronectes platessa), eyes right (very rarely left); scales smooth: the $\mathbf{D}$ ab ( $P l$. limanda), eyes right; scales rough: the $\mathrm{Flounder}(\boldsymbol{P l}$. flesus), with rough bony knobs; eyes usually right but frequently left; it occurs not only in the sea, but also in fresh water: the Sole (Solea vulgaris) disc not so wide as the foregoing; eyes right; the Halibut (Hippoglossus vulgaris), also with eyes on the right, attains a considerable length ( $2 \mathrm{~m}$.) : the $\mathrm{Turbot}$ (Rhombus maximus), with bony warts, and the B ill (Rh.lævis) with small smooth scales, both with the eyes on the left side.

4. The Perch family (Percidx). Scales ctenoid; two dorsal fins, which are generally, however, united, the anterior with only spinose rays; pelvic fins below the pectorals; operculum with spines. To this family belong the Common Perch (Perca fluviatilis), and the Pope (Acerina cernua) with fused dorsal fins. Both are freshwater, the former occurring also in brackish water, and are found in England. To an allied family belongs the Olim bing Perch (Anabas) mentioned before (p. 375).

5. The Wrasses (Labridx) recall the Perch in their external appearance, but are distinguished by the fusion of the lower pharyngeal bones, and especially by a pad-like thickening of skin (the lip) along the edge of the mouth. To this family, which is represented by several small species in the North Sea belongs the Parrot-fish (Scarus); in this form the edge and a portion of the front of the premaxilla and maxilla, are beset with teeth, which are connected with each other, and with the rest of the jaw by means of a bony mass, so that a continuous cutting edge is formed. Grinding teeth, united in the same way, occur on the superior and inferior pharyngeals. The Parrot-fish, which belong exclusively to warm seas (one species in the Mediterranean), can bite through even branches of Coral.

6. "Peter"s Thumb" (Trachinus draco) is a somewhat elongate form with a short head and small cycloid scales; with two dorsal fins, the posterior being long and possessing soft fin-rays, the anterior quite short and with spinose rays; pelvic fins in front of pectorals. On the operculum is a bony spine, with two poison glands, lying in grooves on its surface, and opening at its tip; similar glands in the spiny rays of the dorsal fins.* Abundant in the North Sea; usually seen with the larger part of the body buried in the sand.

* Similar poison organs occur also in a few other tropical Fish. 
7. The Squamipinnes. Fish with spiny fins, with very high, much compressed bodies, and with gorgeous colours; the scales extend some way on to the unpaired fins. In warm seas.

8. The Cataphracti. Body usually without the ordinary scales, naked or with large bony plates; one of the suborbitals is well developed, and reaches back to the preoperculum; pelvic fins below the pectorals. Here belong: the Sea Scorpion (Cottus scorpius), with a large head, naked skin, spines on the head; abundant in the North Sea: in the rivers of Great Britain is found the small River Bull-head, or Miller's Thumb (Cottus gobio), about $15 \mathrm{c} / \mathrm{m}$. in length: the small $\mathrm{Armed} B \mathrm{Bull}$ ead (Agonus cataphractus), with bony plates on the body and with numerous barbules: the Grey Gurnard (Trigla gurnardus), with mailed head; small scales; and the lowest rays of the pectoral fins free, digitiform, and used in crawling; in British seas. In the Flying Gurnard (Dactylopterus volitans) each pectoral fin is divided into two portions, one of which is very large, and by its means the animal can lift itself above the surface of the water; in other respects it resembles the two previous forms; in the Mediterranean.

9. The Sticklebacks (Gasterosteidæ) resemble the preceding family as regards the suborbital bones; the spiny-rayed portion of the dorsal fin consists of free rays; each of the pelvic fins, which are a little behind the pectorals, consists of a long spiny and of a short, soft ray; no scales, but large dermal plates; the males often build nests. The Sticklebacks (Gasterosteus) are small forms, occurring in fresh and brackish water: the $\mathrm{Three}$ spined Stickleback (G.aculeatus), with three spiny rays in the dorsal fin, and the Ten-spined Stickleback (G. pungitius), with about ten, both in Great Britain: the Sea Stickle (Spinachia vulgaris) is exclusively marine (North Sea, etc.); very elongate, with long, thin tail; and fifteen free, spiny rays.

10. Mackrel family (Scomberidæ). Spiny fins; body elongate, slightly compressed, with small scales; posterior portion of dorsal and anal fins broken up into a number of small pieces; pelvic fins below the pectorals; here belong: the Mackrel (Scomber scomber), common on Euxopean coasts, and the Tunny (Thynnus vulgaris), common in the Mediterranean, rarer in northern seas. Allied to these are the $\mathrm{Sucking}-\mathrm{fish}$ (Echeneis); the anterior. dorsal fin is modified into a suctorial apparatus, extending on to the head, and by it the animal attaches itself to large fish, ships, and so forth Further, the large $\mathrm{Sw}$ ord-fish (Xiphias gladius), with the upper jaw elongate and beak-like, and without pectoral fins; abundant in the Mediterranean, also occasionally in northern seas.

11. The Blennies (Blenniidæ). Body usually almost eel-like, with very small scales; usually a long dorsal and anal fin; * pelvic fins small, in front of the pectorals. Here belong: the Viviparous Blenny (Zoarces viviparous), very abundant in the North Sea; up to $40 \mathrm{c} / \mathrm{m}$. long: the Wolf fish (Anarrhichas lupus), large, with well-developed, strong, conical teeth in front, and grinding teeth further back in the mouth; no pelvic fins; feeds upon Lamellibranchs, etc.; in northern seas.

12. The Gobies (Gobius), small, with tolerably soft spiny rays, chiefly distinguished by the fusion of the pelvic fins, which lie below the pectorals. To another family belongs the $\mathrm{Lum} \mathrm{p}$ - fish (Cyclopterus lumpus), with the pelvic fins fused, and, moreover, modified to form a sucker; the Sea-hare is a short, clumsy form with bony spines in the skin; in British Seas.

* In Zoarces and Anarrhichas there are a few spiny rays posteriorly in the otherwise soft dorsal fin; in the genus Centronotus (Butter-fish) the whole dorsal fin consists of spiny rays; in others, again, all the rays are soft. 
- 13. The Pediculati, with bulky, naked body; head often large; gillopening small; pelvic fins in front of the pectorals, the latter stalked; the radialia, which are short in other Teleostei, are long here; the anterior portion of the dorsal fin consists of a number of free rays. The only form occurring in Northern Seas is the large Frog-fish (Lophius piscatorius), flattened; with a huge mouth; the free dorsal rays elongate, the most anterior with a soft appendage at its tip.

14. The Plectognathi are fish of very varied appearance, which agree in having the premaxillæ and maxillæ, contrary to the general rule, firmly attached to the skull; pelvic fins absent. Chiefly animals of very aberrant form inhabiting the warmer seas. The Trunk-fish (Ostracion), short, with flattened abdomen, peculiar in that most of the body is covered by a thin armowr formed of polygonal bony plates, firmly connected together; the small tail and the fins alone are movable. The Sea Hedgehog (Diodon) is beset with bony spines, which stand up when the animal puffs itself out; this is effected by filling a sac-like evagination of the cesophagus with air, which is taken in through the mouth; the creature then lies in the water with the ventral surface upwards; the dentition recalls that of the Parrot-fish. The Sun-fish (Mola or' Orthagoriscus) is a large pelagic form, much compressed and very short, the body forming a perpendicular oval disc; the caudal fin is a ridge along the hinder edge of the animal, dorsal and anal fins high.

15. The Sea-adder family (Syngnathida). Body elongate, covered with bony plates; snout drawn out into a tube, at the apex of which lies the snall edentulous mouth; pelvic fins absent; gill-lamellæ on each bar in quite small numbers, but much folded; external branchial aperture small. The eggs are carried by the males on the lower side of the body and tail, sometimes simply adhering to this; in other cases enclosed in two longitudinal folds or in a sac. The animals swim by a very rapid undulating movement of the rather short dorsal fin (or of the pectorals, if these are present). Various forms inhabit northern seas; species of the genus Syngnathus, Nerophis, etc., in the last of which only the dorsal fin is present. The Sea-horses (Hippocampus), with finless, prehensile tail; ventrally curved head; and spiny outgrowths on head and body, swim in a perpendicular position; usually in warm seas; one species, abundant in the Mediterranean,"also occurs in the North Sea.

\section{Class 3. Amphibia.}

The head, unlike that of Pisces, is, in Amphibia, generally clearly defined, and is usually capable, to some extent, of free movement, although there is no distinct neck. The head, and usually the body also, is somewhat flattened. The tail, when present, is compressed and strongly developed, though not nearly so muscular as in Fish; dorsally, it passes gradually into the trunk, but ventrally, is more sharply limited. The limbs have reached a higher stage of development than in Pisces: they are separated by joints into several regions, of which the distal is divided into digits. They have been modified to form ambulatory organs, which, compared with those of the Mammalia, at least in one of the principal groups (the Urodela), are small and feeble.

The epidermis in the adult exhibits a thin stratum c orneum, only one or two cells thick, which, as in many Reptiles, 
is periodically shed entire, and replaced by a new one (ecdysis). This layer is harder in some regions than in others, e.g., certain spots on the fore-limb of the Frog in the breeding season. $\mathrm{Claws}$ are absent. Rounded, saccular glands, opening to the surface, and distributed all over the body, occur in connection with the skin; they usually secrete a slimy fluid, which keeps it moist; in some forms, there are also small mucous glands, and larger poison glands, which may be so closely aggregated in some regions as to cause projections; such are the "parotids" behind the head in the Toad and the Land Salamander; the secretion is injurious to many animals, and thus serves as a means of defence. True scales, like those of many Fish, are present in the dermis of many Gymnophiona; in other forms, large membrane bones* may be present in certain regions of the skin; or there may be calcareous deposits in the dermis, as in old Toads. Like Pisces, the Urodela are furnished with an unpaired fin, which extends along the back for some distance, sometimes even from the head, round the tail to the ventral surface, as far as the anus; it never exhibits fin rays: it is usually better developed in the males than in the females, and here it is most prominent during the breeding season. Except during larval life (see below) it is absent from all other Amphibia.

The skeleton is for the most part ossified, although there are, as in many Fishes, considerable tracts of cartilage, especially in the skull. In the Perennibranchiata and Gymnophiona, the centra are amphicclous and the notochord is large; in others, on the

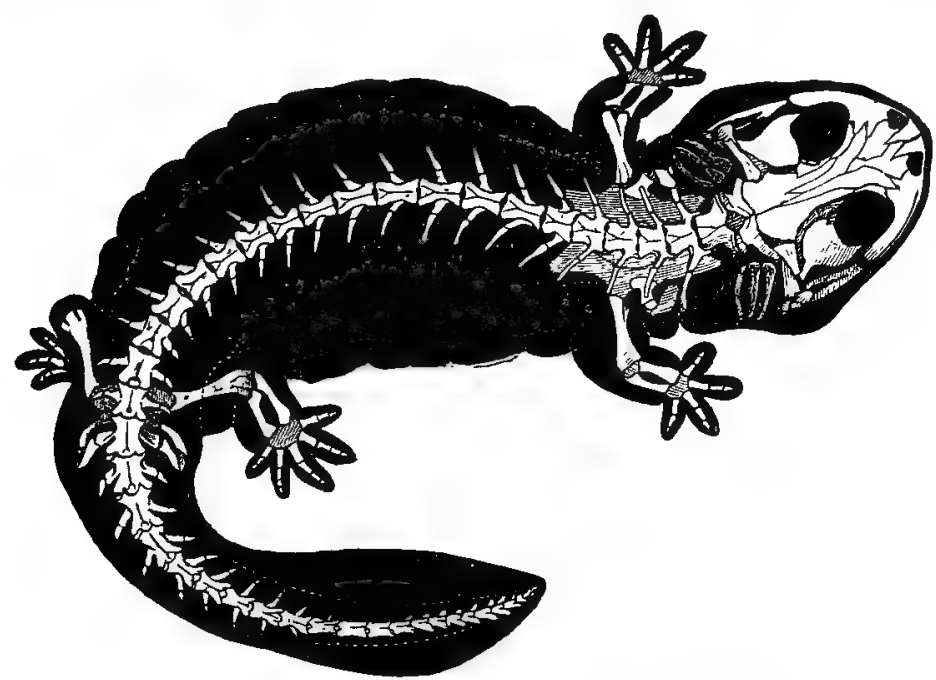

Fig. 318. Skeleton of a Urodele (Menopoma).

* Scales were also present in the dermis of many extinct Amphibia (Labyrinthodonta). 
contrary, it is intervertebrally constricted, the vertebræ articulating by joints; in the Urodela the vertebræ are opisthocelous (concave behind, convex in front); in the Anura usually procolous (convex behind, concave in front). Just as in Pisces, but in contradistinction to the classes following, the second cervical vertebra is not specially modified ( $c f$., Reptilia). The first, with which the head articulates, and the last or sacral vertebra, to which the pelvis is attached, differ from all the others. The caudal vertebræ of the Urodela are provided with hæmal arches; in the Anura, where they are twice as numerous in the larva as in the adult, they are fused into a long, unjointed bone, the urostyle* (Fig. $323 C$ ). The ribs never reach the sternum; in some extinct Amphibia (Stegocephala) they were well-developed; in all living forms they are, however, very degenerate; they are best developed in the Urodela and Gymnophiona, where they are short processes usually present on all the trunk vertebræ except the first; in the Urodela they occur on the anterior caudal vertebræ also. In the Anura the ribs are rudimentary, and in the adult usually fused with the long transverse processes. The sternum (Fig. 321-22) is not connected with the ribs, but is closely attached to the lower portion of the shoulder girdle; in the Urodela it is a short cartilaginous plate, with the insertion of the coracoid at its anterior edge; in the Anura it is often partially ossified, and closely connected with the coracoids.

The cranial skeleton is in many points very similar to that of the Ganoids and Teleostei. Considerable portions of the cartilaginous
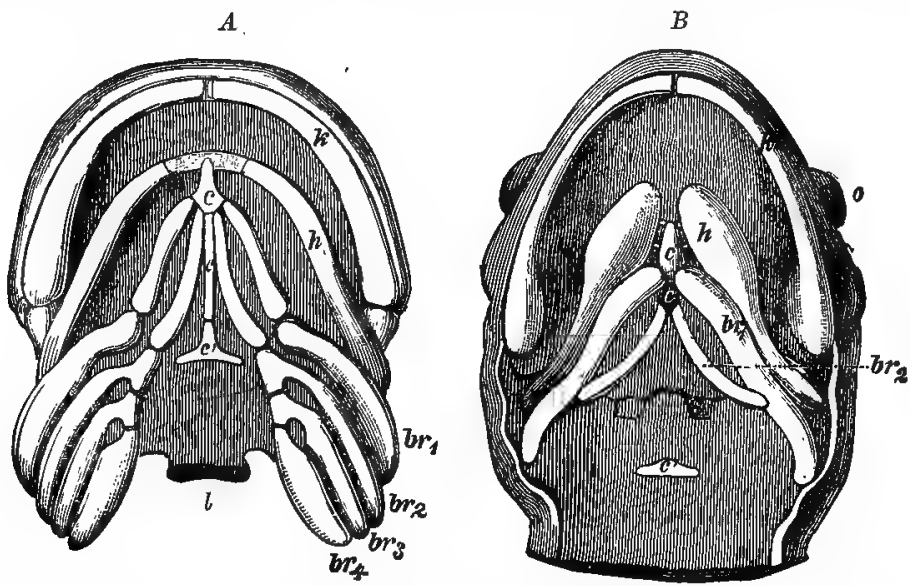

Fig. 319. The visceral arches of the Salamander, seen from below; $A$ larva, $B$ adult. $c$ basibranchials, $c^{\prime}$ the last (separated from the others in the aduit), $k$ mandible, $h$ hyoid arch, or $1-4$ first to the fourth gill-bars, $l$ occipital condyles, o eye. After Rusconi.

* The tail is not visible externally, for the long ilia, which are attached by their anterior ends to the sacral vertebra, extend backwards almost parallel to the urostyle; the latter is of about the same length as the ilia, so that their glenoid cavities are close to its tip. 
skull are retained throughout life, covered for the most part by membrane bones. There are two articular condyles on the occipital. The premaxillæ and maxillæ are closely adherent to the anterior solid portion of the skull; they are not movable as in the Teleosteans. The upper part of the mandibular arch, the palat oquadrate, is fused to the hinder part of the skull; sometimes, as in the Anura, it is also fused to the front part by its anterior end; it remains partly cartilaginous. In the larva there are usually, besides the mandibular and hyoid arches, four pairs of cartilaginous branchial arches, which degenerate to some extent in the metamorphosis; in the Urodela, the first two pairs persist. The basibranchials, hyoid, and branchial arches, are together termed the hyoid.

Of the skull bones, besides those already noticed, the following must be mentioned. In the cartilaginous cranium itself there develops a pair of exoccipitals which almost completely surround the foramen magnum, and which bear the occipital condyles; anterior to these on either side is the petrosal, and at the front end of the cranium, the sphenethmoid. The skull is covered dorsally by a pair of n a sals behind the external nares, and a pair of frontals and parietals (in the Anura those of each side fuse into a single bone); ventrally there is a p a r a s p en o id (ef., Fish) and, anterior. to this on each side, the vomer. In the palato-quadrate cartilage
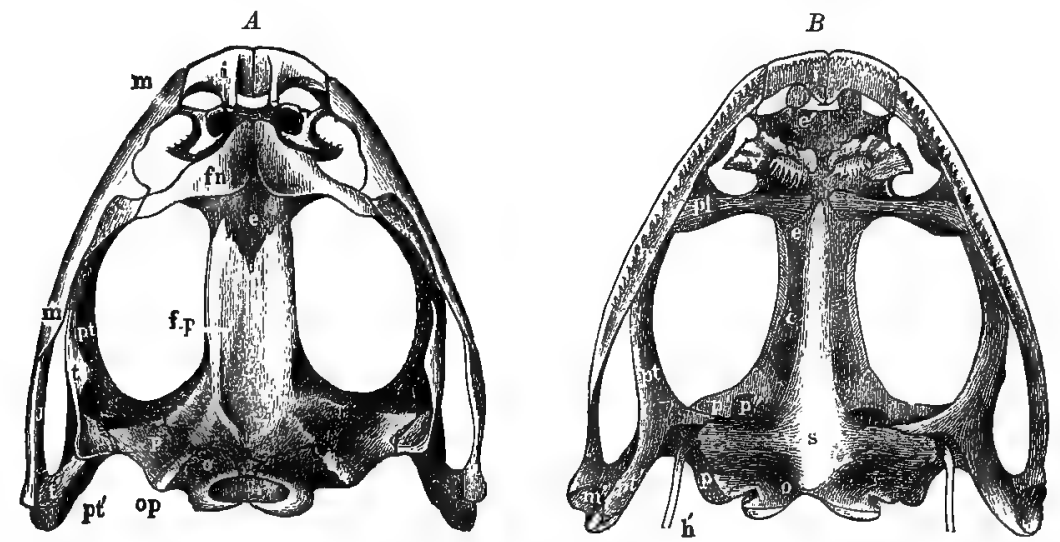

Fig. 320. Skull of a Frog (Rana esculenta). (A) from the dorsal $(B)$ from the ventral surface. $c$ cartilaginous lateral portions of the skull, $e$ sphenethmoid, $e^{\prime}$ cartilaginons nasal capsule, $f n$ nasal bone, $f p$ fronto-parietal, $h$ ' stylo-hyoid, $i$ premaxilla, $j$ quadrato-jugal, $m$ maxilla, $m^{\prime}$ quadrate, $v$ exoccipital, op cartilage between the latter and the prootic $p$, $p^{\prime}$ anterior portion of prootic, with a large nerve formmen $\left(p^{\prime \prime}\right), p l$ palatine, $p t$ pterygoid, $\mathrm{p} t$ posterior portion of the pterygoid, $s$ parasphenoid, $t-t^{\prime}$ squamosal, $v$ vomer.-After Eoker.

there is, at the point of junction with the lower jaw, an insignificant ossification, the $q u a d r a t e$, and behind, the cartilage is covered by a large membrane bone, the squamous; the pterygoid extends anteriorly, and in front of this there is in the Anura a transverse palatine attached to the skull by its inner: end. In this group, too, a thin bony rod, the jugal or quadrato-jugal, stretches from the quadrate to the maxilla. The rami of the mandible consist, as in Fish, of several bones. 
The shoulder girdle of the Urodela is represented by a cartilaginous arch on either side, in which two regions are distinguished, one dorsal the other ventral, is the glenoid for the arm. The dorsal portion, the scapula, is narrower than the ventral, the coracoid, which partially overlaps its fellow of the other side. The lower part of the scapula is ossified to a varying extent, the ossification often reaches into the coracoid region, but the upper and lower portions of the girdle remain cartilaginous (Fig. 321). In the Anura (Fig. 322), the coracoid is perforated by a large foramen, and thus separated into anterior and posterior portions, the latter ossified, the former not ossified, but covered by a membrane bone, the clavicle; the right and left coracoids either overlap or

Fig. 321.

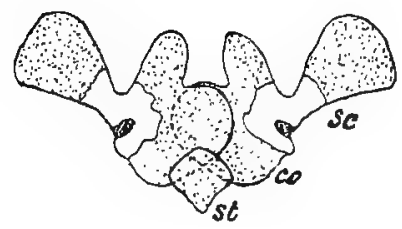

Fig. 322.

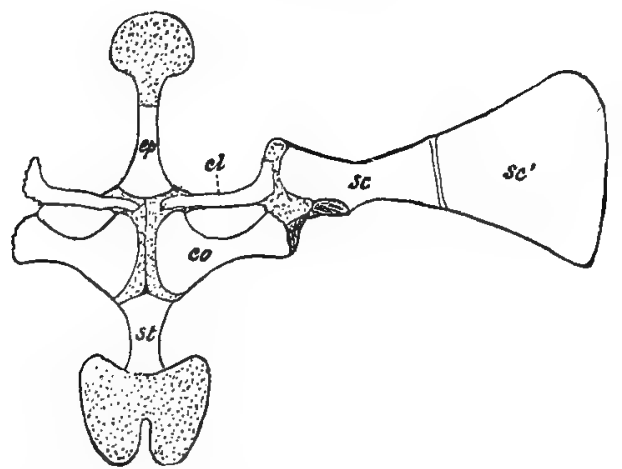

Fig. 321. Sternum and shoulder girdle of a Salamander. st sternum. co coracoid, $8 c$ scapula.

Fig. 322. The same parts of a Frog. st sternum, ep omosternum, co posterior region of coracoid, $s c$ lower portion of scapula, $s c^{\prime}$ upper portion of the same, $c l$ clavicle. The cartilaginous parts in this and the preceding figure dotted.-After Ecker.

fit close together in the median line as in the Frog.* The scapula in the Anura is divided into upper and lower parts, both ossified, but the upper only partially. The fore limb consists of the same chief parts as in the higher Vertebrata. The carpus, especially in the Anura, usually conforms closely to the typical arrangement. In extant Amphibia there are never more than four fingers; the number of phalanges varies. In the Anura the two bones of the forearm are fused.

Each half of the pelvis in the Urodela consists of a narrow upper portion, the ilium, and a lower broader part, the is chio-pub is, which is connected medianly with its fellow, and in which there is

* In some Anura (e.g., the Frog) there is, in the middle line, anterior to the coracoid, a special, partly-ossified cartilage, which has been termed the episternum, although it has no connection with the sternum, and although the episternum of other Vertebrata is purely membrane bone. It is probably to be regarded as a special development of the coracoid. 
usually a single ossification only. Anteriorly the pelvis is prolonged into a narrow, unpaired, usually $Y$-shaped, cartilage (cartilago ypsiloides. In the Anura the ilia are backwardly-directed bony rods; the ischio-pubes have fused to form a compressed vertical disc. The $\mathrm{hind}$ limb closely resembles the fore limb in structure. In the Anura the tibia and fibula are fused, and the two

Fig. 323.

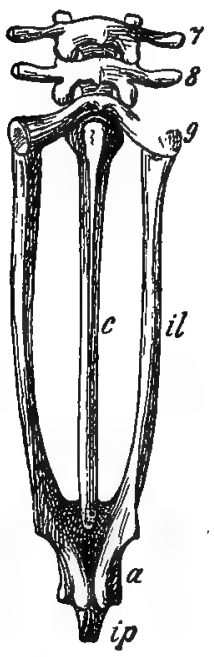

Fig. 324.

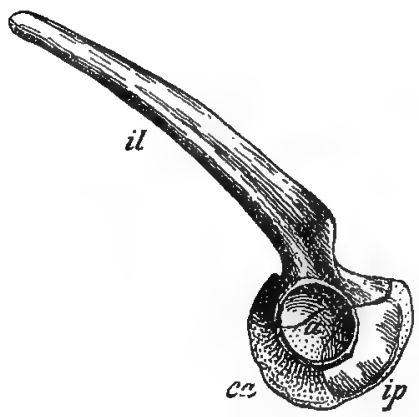

Fig. 323. P eIvis and last vertebræ of a Frog, from above. a acetabulum, $c$ urostyle, it ilium, ip ischiopubis, 7-9 vertebræ (9 sacral vertebra).—Orig.

Fig. 324. Pelvis of the same viewed from the left side. ca oartilage. The other letters as in Fig. 323. -Orig.

proximal tarsals (the third is absent) are very long and powerful. The hind limb usually has five digits.

The musculature of the body and tail, in the amphibian larva, is very similar to that of Pisces; it is separated into four

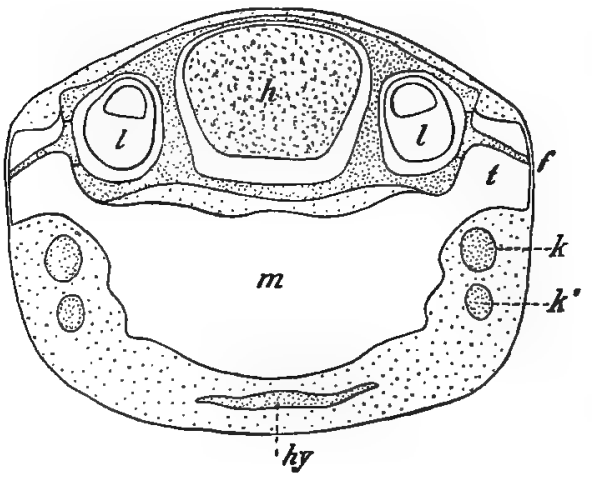

Fig. 325. Transverse section of the hind end of the head of young Frog to show the tympanic cavity; dia. grammatic. Cartilage and bone closely dotted. $f$ tympanum (at the point where the letter is situated the colu$m$ ella is attached to the tympanic membrane), $h$ brain, $h y$ hyoid, $k$ palatoquadrate cartilage, $k^{\prime}$ lower jaw, $l$ membranous labyrinth (quite diagrammatic), $m$ mouth, $t$ tympanic cavity in which the columella lies.-Orig.

longitudinal muscle plates, each of which is divided into a series of segments by thin transverse septa; in the adult Urodela the relations are little altered, whilst in the Anura great modifications occur. The brain is small, the cerebellum very little developed. 
The olfactory organs are two canals which lead from the outer side of the head into the mouth, and open there behind the edge of the jaw; the external nares can be opened and closed. Eyelids are wanting in the larvæ, in the Perennibranchiata, and in the Gymnophiona, which have rudimentary eyes; where they are present only the lower one is movable, it is often semi-transparent, and like a nictitating membrane. Lacrymal glands are absent, although a lacrymal canal occurs in the adult; a Harderian gland is, on the other hand, present.

Auditory apparatus. In most Anura, a short, wide canal; the tympanic cavity extends from the posterior region of the mouth behind the first gill-bar towards the exterior, it is not open at the surface, but is closed in by a thin membrane, the ty $\mathrm{m} p$ an $\mathrm{um}$. The canal traverses that region of the skull which encloses the membranous labyrinth, and is perforated in the region of the sacculus by a foramen (fenestra ovalis). The fenestra is covered by a small cartilaginous plate, the expanded end of a partially ossified rod, the ear-bone (columella auris), whose other end is attached to the tympanum (Fig. 325). In other A mphibia (some Anura, e.g., the Toad; all Urodela and Gymnophiona), the tympanic cavity and membrane are wanting; but all possess the fenestra ovalis and the columella.

Alimentary canal. Teeth may be present on maxillæ, premaxillæ, mandibles, vomers, and pterygoids, exceptionally also on the parasphenoid; in living Amphibia they are always small and simple in form. The tongue is better developed than in Pisces; it is attached by its under surface to the floor of the mouth, in such a way, however, that the edge is free. It is characteristic of the Anura that the posterior tip, which is free and sometimes bifid, is especially well developed; whilst the anterior edge, which is insignificant, is attached in front, so that the tongue can be flicked out of the mouth from behind forwards. In some Urodela it can be stretched out upon a kind of shaft, projecting from its ventral surface. The tongue is absent from Pipa and an allied genus. The $œ \mathrm{soph}$ a g s is short and wide, the intestine short.

The respiratory organs of Amphibia are gills or l u $\mathrm{ngs}$, the former will be considered first.

In the urodelan larva there are, on either side, four gill-s lits, the first between the hyoid and the first gill-bar, the last between the third and fourth gill-bars. Each bar bears on its outer edge a thin membranous plate, and the series is covered by a thick membranous fold without ossifications, which corresponds to the operculum of Fish. The plates correspond to the septa between the gill-clefts in Fish, but bear no gill lamellæ. At the dorsal end of each of the first th $\mathrm{r}$ e e pairs of clefts there is, however, a g ill, not covered by the oper- 
culum, and consisting of a stem and two rows of lamellæ (Fig. 326). These gills persist throughout life in the Perennibranchs, where they are somewhat more complicated (branched); the embryos of some Gymnophiona* possess similar gills (see Fig. 331). 'The lar $\mathbf{2}$ of the Anura also are furnished for a short time after hatching with three pairs of external gills, like those of the larval

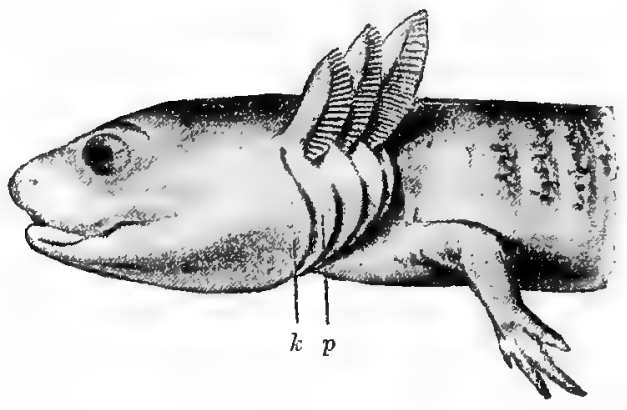

Fig. 326. Head, etc., of a Urodelan larva, diagrammatic (in the figure more of the thin plates is seen than in reality, etc.). $k$ operculum, $p$ thin plate on the first gill bar.-Orig.

Urodeles; they are, however, soon covered by the operculum, which is well developed, and covers gills and gill-clefts, concrescing

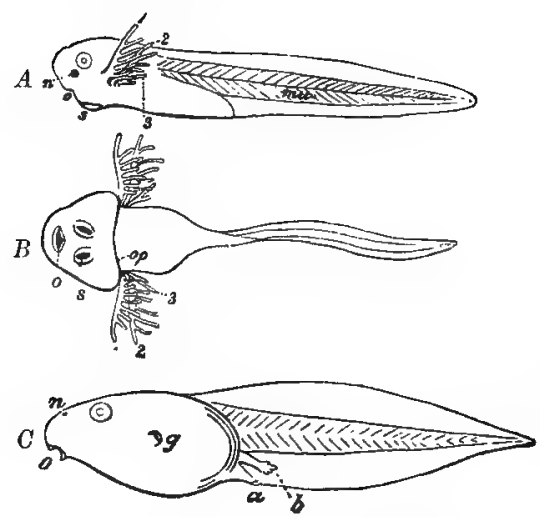

Fig. 327. A Young Tadpole of a frog (lateral view) ; $B$ somewhat older from below; $C$ still older larva with intermal gills. 1, 2, 3 the three external gills, $u$ anus, $b$ hind limb, $g$ branchial aperture, mu caudal muscles, $n$ nares, o mouth, op operculum, $s$ organ of adhesion.- $C$ orig., $A$ and $B$ with the assistance of figures by Ecker. posteriorly with the surface of the body. A large branchial cavityt results, communicating with the exterior by a single aperture, $\ddagger$ usually on the left side. The gills enclosed in this cavity atrophy, and in their place numerous branched "internal gills," structures peculiar to the anuran larræ, arise on the outer edge of all the four bars. As in Selachians, etc., there is usually an imperfect straining apparatus at the inner edge of the gill-bar, in such forms as are provided with external gills. It is represented on each arch by one or two (one on the first and fourth gill-bars, two on each of the others) rows of short processes

\footnotetext{
* In other embryos of this division there is, instead of such gills, one very vascular lamina on each side.

† The opercula of the two sides are continuous ventrally (Fig. 327 B), concrescing posteriorly to enclose a single cavity.

$\ddagger$ In Pipa, and an allied genus, there are two openings, one on either side.
} 
which dovetail with those of adjacent arches. In the larval Anura with internal gills, this straining apparatus reaches a high pitch of perfection, so that it is able to exclude even the very finest particles from the branchial-cavity and the delicate membranous gill-tufts within it. For the branchial vessels, see below.

The lungs, occurring in all Amphibia, are two saccular organs, which in some forms (e.g., Newts, Proteus) are quite simple, in others (Salamanders, Anura), are provided with short, thick-set evaginations (Fig. 345, B). In the Gymnophiona, the right lung is much shorter than the left. The trachea, which is almost always very short, opens by a longitudinal slit into the back of the mouth; it is supported by several cartilages, and in the Anura, contains vocal cords, which are absent from all the others. To effect an inspiration, the animal depresses the soft parts between the rami of the lower jaw, by shutting the mouth, and draws air into the buccal-cavity through the open nares; these are now closed and the lower wall of the buccal-cavity is raised, so that the air is forced into the trachea; it is forced out by the contraction of the body-wall and its pressure upon the elastic lungs.

In some Salamanders, e.g., two species in S. Europe, the lungs are rudimentary or entirely absent. Respiration is effected by means of the skin (of great importance in this connection in all Amphibia) and the buccal cavity, where inspiration and expiration proceed in the usual way.

The vocal cords of the Anura are made to vibrate by the expired air, and thus sounds are produced. In the males of many species, this noise may be intensified by means of evaginations of the posterior region of the buccal-cavity, which can be blown out at pleasure into thin-walled sacs of considerable size. A pair of such reson ators is present; in some (e.g., the Tree Frog), they unite to form a single unpaired resicle, which is, however, connected with the mouth by two openings. The Urodela can produce sounds, although they have no vocal cords.

The heart (Fig. 284) differs from that of most Fish in that the atrium is divided by a septum into two auricles, right and left; the latter is the smaller, and receives blood from the lungs, whilst the right receives blood from the rest of the body. The septum is often pierced by larger or smaller apertures, and is thus imperfect. The ventricle is undivided, and shows no trace of separation; as in Fish, its wall is thick and spongy, the small spaces opening into the central cavity; the auriculo-ventricular apertures are guarded by valves. The conus arteriosus, which arises in front from the right side of the ventricle, is usually a well-developed tube, somewhat spirally curved. It displays at each end a transverse row of valves, and is in addition provided with a longitudinal fold, which is connected with a valve of the anterior row, and projects into the cavity of the conus (for its significance see below).

The arterial system in amphibian $l a r \nabla æ$ is piscine in character : an afferent branchial artery, arising from a very short ventral aorta, runs to, an efferent artery runs from, each gill-bearing gill- 
bar; the efferent branchials unite to form the aorta. In urodelan larvæ a single arterial arch runs to the last branchial bar, which is destitute of gills; the pulmonary artery arises from this, or, in the larval Anura, from the last efferent branchial artery; the carotids, vessels to the head, spring from the first efferent branchial artery.

At the metamorphosis the gill vessels degenerate, and the afferent and efferent branchial arteries unite* to form simple arterial ar ches, which, like their precursors, the efferent branchial arteries,

$A$

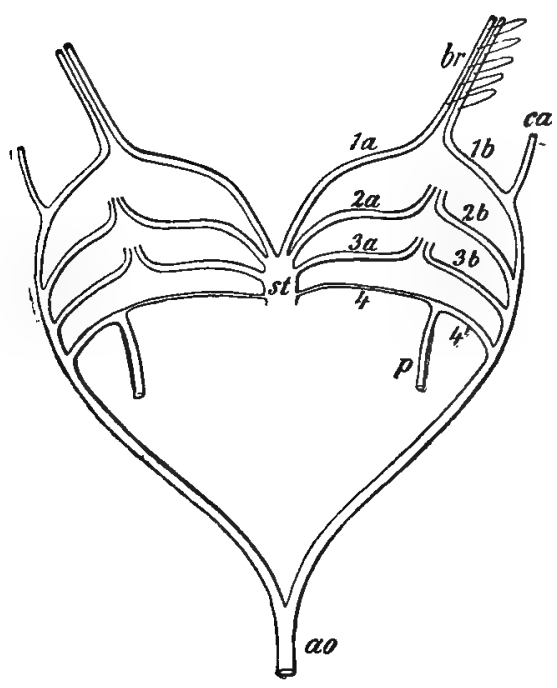

$B$

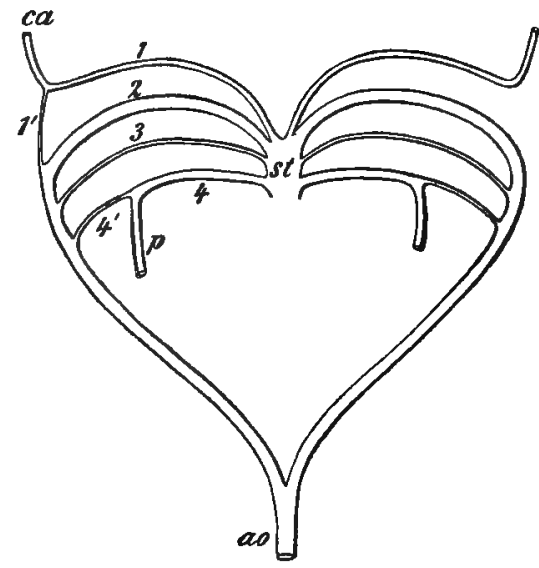

Fig. 328. Arterial arohes of the Urodela: diagrammatic. $A$ larva, $B$ adult. ao aorta, $b r$ gill (removed from the second and third arches), $c a$ carotid, $p$ pulmonary artery, st conus arteriosus ; 1-1' first, 2 second, 3 third, 4-4 $4^{\prime}$ fourth arterial arches, $1 a-3 a$ first - third afferent branchial arteries ; $1 b-3 b$ first-third efferent branchial arteries.-Orig.

unite to form the aorta. The first, however, usually lose their connection with the others, and simply supply the head with blood; the fourth also generally become independent, and form only the pulmonary arteries; and the third arterial arches, in many cases, atrophy completely. When this occurs, and if at the same time the first and fourth arches have no connection with it, the aorta is formed by the second arterial arches only, which are better developed than the others. The aorta of Amphibia is sometimes formed by a single pair of arterial arches, sometimes by several. The Gymnophiona in the adult condition are very similar to the others; the vascular system of the larva is at present unknown.

* In the larva of the Urodeles the afferent and efferent branchial arteries are connected by a small vessel at the base of the gill (anastomosis), and this enlarges at the metamorphosis. 
In the larval Urodeles that part ( 4 Fig. $328 A$ ) of the fourth arterial arch which lies between the ventral aorta and the point of origin of the pulmonary artery is much narrower than the pulmonary, whilst the rest (4') of this arterial arch is as wide as the latter: the pulmonary artery in the larva evidently receives direct the chief mass of the blood from the vessel formed by the union of the three efferent branchial arteries, i.e., arterial blood. In the adult the relative sizes of the different sections of the fourth arterial arch are exactly reversed (Fig. $328 \mathrm{~B}$ ).

In the larvæ generally the circulation is essentially piscine. In the adult, in spite of the single ventricle, the arterial blood from the lungs is to some extent separated from the venous blood; the arrangements are, however, too complicated to be more closely gone into here. Suffice it to say that by means of the spiral valve of the conus almost all the arterial blood from the left auricle flows into the first two pairs of arterial arches, whilst the venous blood from the right auricle goes partly into these, partly into the third and fourth pairs; the fourth pair, as already mentioned, gives rise to the pulmonary arteries, which receive entirely venous blood, whilst that in the systemics is "mixed."

From the fourth arterial arch, larger or smaller branches go to the $\mathrm{s} \mathbf{k}$ in ; in the Anura especially, there is a very large cutaneous artery, which therefore carries venous blood; and as a matter of fact, the skin is here of great respiratory importance; but the blood thus oxydised, mixes with that of the other veins, and goes to the right auricle. On the whole, the separation is very incomplete.

The ureters open into the cloaca, which has a ventral outgrowth serving as a urinary bladder. The latter, which is often drawn out into two points, is not directly connected with the ureters, but opens separately into the cloaca.

The ovaries vary in size, according to the time of year; in the breeding season they are very large. The $M$ üllerian ducts are long, coiled tubes, which are thickest at the breeding season, on account of the great development of the albumen glands lying in their walls; they open into the abdomen by funnels, situated quite anteriorly, and far distant from the ovaries; the ripe ova are wafted to the funnels by movements of the cilia upon a portion of the abdominal epithelium; they usually open separately into the cloaca. by their other ends. In the Anura, the hinder portion of the oviduct is expanded into a vesicle, which is filled with ova at spawning time. The testes (Fig. 288), are connected with the urinary tubules of the anterior end of the kidney, which, in the Urodela is smaller than the posterior end, and the spermatozoa pass out through the ureter; the duct of the anterior portion of the kidney is, moreover, in many cases, almost completely separated from the other renal ducts with which it unites only just before the common opening into the cloaca. In the males, there is a rudimentary Müllerian duct on each side.

Actual copulation takes place only in the G ymnophiona; the eversible cloaca of the male serving as an intromittent organ. In the Anura, the male clasps the female with the fore limbs, and as the eggs leave the cloaca, pours the sperm over them; fertilisation 
thus takes place in the water. The fore limbs are stronger in the males than in the females, and in many forms are furnished, in the breeding season, with rough horny warts, especially on the hands, so that they can grip more firmly. The male Urodele deposits the spermatophores, which consist of masses of jelly containing spermatozoa, and vary in form, at the bottom of the water. The female then moves over them, so that they become attached to the cloacal opening; they are taken into the cloaca where the spermatozoa penetrate into little sacs in the wall, which serve as receptacula seminis. Here fertilisation occurs within the body of the female.

As already mentioned, there is, in the Toads ( $B u f o)$, at the anterior end of the testis, a small body, which resembles an unripe ovary in structure. In the females of this genus, a corresponding portion of the ovary is similarly developed; it is especially noticeable in young females, but degenerates later.

A pair of yellow bodies containing fat, and often very conspicuous, is attached to the reproductive glands in Amphibia, and is frequently in close connection with them. These are the so-called fat bodies, which are digitiform in Anura, and originate by modification of a portion of the ovary or testis.

The eggs are usually laid in fresh water, surrounded by a thin albuminous coat, which swells up in the water to a thick gelatinous capsule. They are either laid singly (rarely) or in rows, strings, or masses. They vary in size from two to about ten $\mathrm{m} / \mathrm{m}$. in diameter. Segmentation is usually total, but the segmentation spheres are larger at one pole ( $c f .$, p. 45, and Fig. 34); the larger eggs, however, undergo partial segmentation. Rarely, as in the Salamander, the ovum develops within the oviduct. The eggs or brood are protected in various Amphibia: Pipa, Alytes obstetricans, Ccecilia, etc. (see below).

The metamorphosis, which all Amphibia undergo, is specially characteristic of the group. The larvæ, as already mentioned, are provided with well-developed gills; the circulation and the disposition of the vascular system are almost identical with those of Fish; lungs are already present, but have as yet no respiratory function. At the metamorphosis a significant change in structure and mode of life occurs; the gills atrophy and the lungs become functional, involving amongst other alterations, great modifications in the vascular system ( $c f$. p. 400). The differences between larva and adult are not, however, confined to these; in many other respects the former approaches the piscine type; for instance, there is no stratum corneum; lateral line organs are present; they always lie free, and even bear delicate cylinders like those of Fish.* Eyelids are absent; a continuous fin is present at first, but disappears in later larval life, and the visceral skeleton is

* The sensory papillæ also occur in adult aquatic Urodeles, but here the cylinders are wanting. 
much more piscine (p. 394) early, than in final stages. When the tadpoles hatch, they are generally somewhat unlike the fully-developed larval form; for example, the limbs are not present, or are merely indicated, and, on the head, there are frequently organs of attachment, which atrophy after a time (Fig. $327 A-B$, and $329 A$ ).

The metamorphosis itself, that is, the change from the larval to the perfect form, is completed somewhat suddenly; the changes are accomplished in quite a short time. The size attained before metamorphosis varies; closely allied species often differ considerably in this respect ; for example, within the genus Rana, the larva of the Edible Frog is very large, that of the

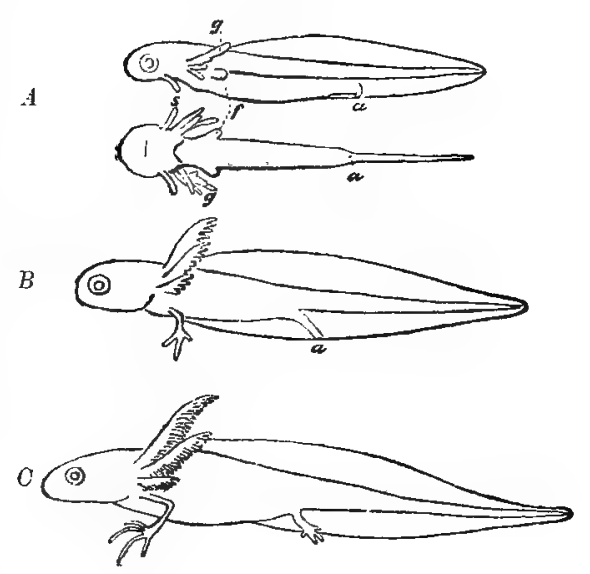

Fig. 329. Larvæ of the large Triton. A newly hatched, from the side and below. $B 12$ daye old. $C$ about 5 weeks old. (A $\times$ about $5, B 3-4$, $C$ scarcely $\times 2)$. a anus, $f$ fore limb, g gills, $s$ orgar of attachment.-After Rusconi. Grass Frog, on the other hand, rather small; growth is not usually completed at metamorphosis (as in Insects) but continues for some time.* In some Urodela, viz., in certain Tritons, it has been noticed that the larvæ sometimes grow beyond their customary size, and become sexually mature as larvæ; whether they afterwards undergo metamorphosis is unknown. The same thing occurs normally in the larva of a Mexican Salamander the Axolotl (Siredon mexicanus), at least in those individuals which have been kept in confinement; they are, a s a rule, sexually mature during the larval period, and do not undergo a metamorphosis; this happens only exceptionally, and then before sexual maturity. Lastly, there are a few Urodela, the Perennibranchiata (genus Proteus, etc.), which remain in the larval condition and never assume the adult form. These forms are like larvæ in all respects excepting the development of the reproductive organs; in some points, however, they have undergone degeneration; in Proteus, for instance, the lungs, compared with the size of the animal, are very poorly developed, and they are of just as little respiratory significance as in the larva. The retrogressions are in part of such a character as to render it possible to state

* The larvæ of a South American Frog (Pseudis paradoxa) are huge. 
definitely that these forms are no longer capable of undergoing metamorphosis.*

In two genera of the Urodela, Menopoma and Amphiuma, the gills atrophy, but the gill-slits persist, and in many respects the animals remain in a larval, or more correctly, in an intermediate condition.

All living Amphibia are freshwater or terrestrial, they are almost always small or of a medium size, and feed upon Insects and other small animals. In earlier times they were to a certain extent represented by larger forms (see below). With regard to the Geographical Distribution, the remarkable fact, that Urodela belong almost exclusively to the temperate regions of the Northern He $\mathrm{m}$ is $\mathrm{phere}$, may be noticed.

\section{Order 1. Urodela.}

The tail is well developed; the fore and hind limbs about equal, and feeble. The larva has three external gills on each side.

1. Newts (Triton) have a compressed tail, and on the dorsal side of the body, both dorsal and ventral to the tail, is a fin, which is most moarked at the breeding season, and largest in the males. At spawning time they live in water, otherwise on land (the male, however, frequently in water); the eggs are laid in the spring, singly, or in short strings on aquatic plants. The newly-hatched larva (Fig. $329 \mathrm{~A}$ ) exbibits posteriorly on the head, a pair of stalk-like processes, by means of which it attaches itself to plants ; for limbs, there are only wart-like processes, the incipient fore limbs; they develop gradually, the anterior first; the organs of attachment soon disappear. Larval life usually lasts some months. In England are found the Large Water Newt (T. cristatus), with a rough skin; the $\mathrm{Small} \mathrm{Newt} \mathrm{(T.} \mathrm{tæniatus),} \mathrm{the} \mathrm{commonest} \mathrm{species;}$ the Palmated Smooth Newt ( $T$. helveticus), with a filiform tip to its tail, rare; the last two are about the same size, the first considerably larger.

2. The Salamander (Salamandra maculosa) is an animal of considerable size (up to $18 \mathrm{c} / \mathrm{m}$ ); velvet black, with large irregular yellow spots; no trace of a fin; tail rounded. In Central and South Europe; viviparous; the young ones quite differently coloured; they are born with gills, and both pairs of limbs, and then only are aquatic. It is of interest that the embryo has much longer gill laminæ whilst still within the oviduct, than it has later. The Black Alpine Salamander (S. atra), allied to the one just described, and quite black, occurs in the Alps; viviparous, bearing only two young ones at a time, one in each oviduct ( $S$. maculosa produces a greater number at a birth). There are several eggs in the oviduct, besides the one which develops, but they merely coalesce, and furnish nutrition for the embryo. The embryo has extraordinarily large gills, which surround a great part of the body before birth, but atrophy later; metamorphosis thus occurs within the body of the parent; the Alpine Salamanderis born on dry land, and is never aquatic.

3. The Axolotl (Siredon mexicanus) is distinguished as already mentioned by the fact that, in captivity at least, it does not usually undergo a metamorphosis, but becomes sexually mature in the larval state. The form which does undergo a

* For instance, in Protems, that portion of the fourth arterial arch which lies between the ventral aorta and the point of origin of the pulmonary artery is absent, bat it is indispensable for the adult Amphibian. 
metamorphosis (Amblystoma mexicanum) is similar to a Salamander. The Axolotl, which is indigenous to Mexico, is oviparons; when first hatched, it is just like the triton larva of the same stage.

4. Under the name, Perennibranchiata, are collected all the Urodela described above (p. 403) as retaining gills and other larval characteristics throughout life. Amongst these is the blind, pale, elongate Proteus anguineus, which has rudimentary eyes, and three digits on each foot; in subterramean lakes in Austria. Furthermore, the genus, Menobranchus, less elongate, with four digits on each foot, and Siren lacertina, which may attain a length of $1 \mathrm{~m}$., with horny jaws; vermiform, without hind limbs; both in N. America. The genera, Menopoma and Amphiuma (the latter vermiform; with four very small limbs, each with two or three digits), lose their gills as already mentioned, but retain the gillslits and several other larval characters. Nearly allied to Menopoma, is the Japanese Giant Salamander (Cryptobranchus japonicus), in which the branchial clefts are closed.

Related to the living Urodela are the Stegocephala (primitive Amphibia), a large group, which lived in the Carboniferous, Permian, and Triassie periods, and of which a few were remarkable for their great size; skulls are known of $1.5 \mathrm{~m}$. long. The skull has a larger number of membrane bones than in existing Amphibia; there is, for example, a double supraoccipital and several others.* The skull bones are often scarred externally, and this signifies that they were located close below the surface, covered only by a thin skin; sometimes there are furrows on the head, for the branches of the lateral line, $\uparrow$ recalling those
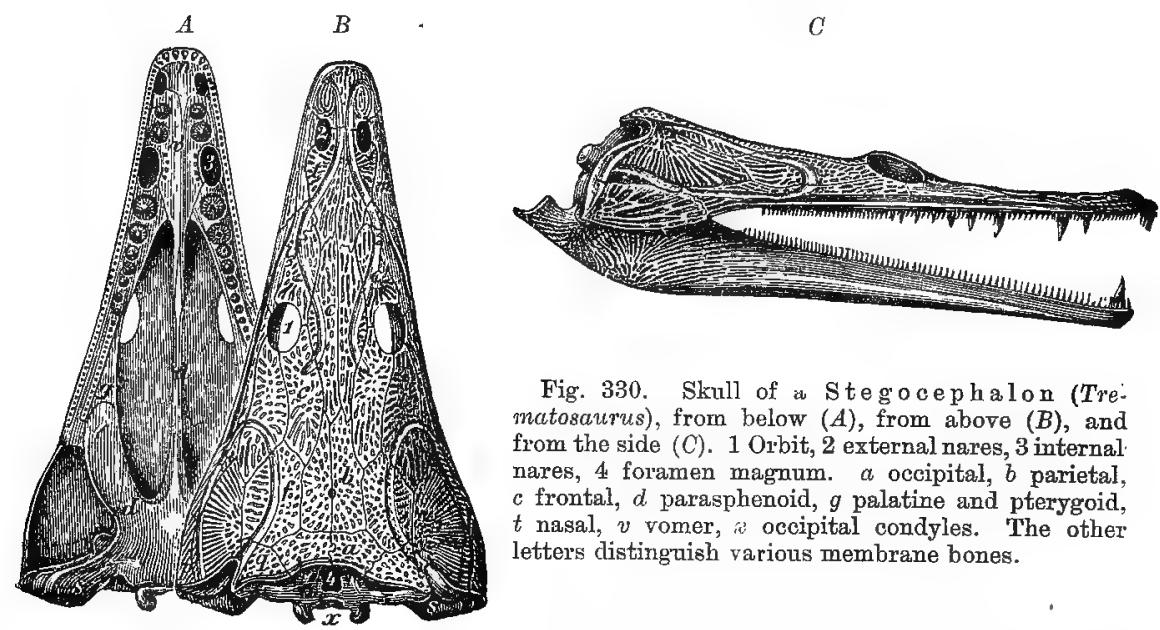

Fig. 330. Skull of a Stegocephalon (Tre: inctosaurus), from below (A), from above $(B)$, and from the side $(C) .1$ Orbit, 2 external nares, 3 internal. nares, 4 foramen magnum. a occipital, $b$ parietal, $c$ frontal, $d$ parasphenoid, $g$ palatine and pterygoid, $t$ nasal, $v$ yomer, a occipital condyles. The other letters distinguish various membrane bones.

of many Fish. As in the Amphibia of to-day there were two occipital condyles. The notochord was, to a large extent, persistent, the centra often biconcave; ribs sometimes long. Some Stegocephala have five digits on the fore limbs. The sclerotic coat of the eye (unlike that of existing Amphibia) usually had a ring of bony plates. In the skin of the ventral surface (rarely on the dorsal)

* Between the parietals there is often a fairly large parietal foramen, which indicates the presence of a parietal eye (see p. 337).

+ The head often forcibly recalls that of the bony Ganoids.

I The sternum was cartilaginous, but there was an episternum and a elavicle, like those of the Lacertilia. 
bony scales were developed. The surface of the teeth. in some of the Stegocephala, has deep, compressed spiral folds, which, especially at the bases, stretch far into the mass, and in transverse section look like curved lines; hence the name Labyrinthodonta, which has been given to this group, but which only suits one section of the members, since the rest have teeth of simple structure.

\section{Order :2. Anura.}

There is no projecting tail in the adult. The hind limbs, which are always more powerful than the anterior, are jumping or swimming legs, and have a larger or smaller web between the toes. Lower jaw edentulous. Larva at first with external, later with internal, gills.

The young larvæ (Fig. $327 \boldsymbol{A}-\boldsymbol{B}$ ) are small, elongate animals, having three external gills on each side, and a pair of sucker-like sticky organs on the head, by means of which they attach themselves firmly to plants, etc.; limbs are absent. After a few days the external gills are covered by an operculum, and atrophy, whilst internal gills arise on all the gill-bars (see above, p. 398). Simultaneously the form of the body changes, head and trunk together become almost spherical, as distinct from the powerful tail with its large fin (Fig. 327 C); the adhesive apparatus disappears. The larva (tadpole), which has horny jaws and a long coiled gut, feeds upon decayed vegetables, dead animals, or mud; it is an active swimmer. Of the gradually developing limbs the anterior lie within the branchial cavity during the whole of larval life; that is to say the points at which they project are covered, like the gill-bars, by the operculum. The fore limbs break through the outer wall of the branchial cavity, but this only occurs at the metamorphosis when the tail dwindles; the teeth develop (that is if the adult has teeth), the small mouth enlarges, etc.

1. Frogs (Rana) have teeth in the upper jaw; a smooth skin; round pupil; long, strong hind limbs, with perfect webbing between the toes. The eggs are laid in large masses. The following species inhabit the British Isles: the Common or Grass Frog (Rana temporaria), which usually lives on land, and betakes itself to the water only at the breeding season, the early spring, in contradistinction to the large Edible Frog ( $R$. esculenta), which lives the whole year through in water, and which swims and springs better than the others; it spawns later also, and its larvæ attain a considerable size.

2. Tree Frogs (Hyla, etc.), are distinguished from others in having a sucking-disc at the tip of each toe. The green Hyla arborea, which is usually found on trees, except during the breeding season, occurs over most of Europe.

3. Land Frogs, or Frog-toads (Pelobatidæ) differ from true Frogs in the short hind legs; erect pupil; and warty skin. The following European forms may be noted: the Orange-speckled Toads (Bombinator igneus and $B$. bombinus), ventral surface black and yellow; Pelobates fuscus, hind foot with a horny knob, sharp as a knife on its inner side; the larva reaches a still greater size than that of the Edible Frog; Alytes obstetricans, of which the males wrap the eggs round their hind legs and carry them about with them until the larvæ are ready to hatch, when they go into the water and the larvæ leave the egg-shells.

4. The Toads (Bufo) are edentulous, have shorter hind limbs than the Frogs, and an imperfect web between the hind toes, transverse pupils, warty skin. The eggs are laid in long strings. In England: the Common Toad 
(B. vulgaris) and the $\mathrm{Natte} \mathrm{j}$ a ok $\mathrm{Toad}$ (B. calamita), with a longitudinal yellow stripe down the back.

5. The Surinam Toad (Pipa americana), a large, flattened Amphibian, with small eyes, no tongue, no teeth, with large webs between the hind toes. With the help of the male, the fertilised eggs are placed on the back of the female : a small depression forms for each egg, in which it develops, and where metamorphosis takes place. S. America.

\section{Order 3. Gymnophiona.}

The body elongate, vermiform, and apodons; the tail rudimentary, eyes degenerate; skin with ring-like grooves on the surface, often containing bony scales.

The Gymnophiona (genus Coecilia and others) live in the earth in warm countries; they feed on Earthworms and such animals. The embryology is well known only for a single species, living in the East Indies, Epicrium glutinosum. This form lays its eggs in a hole in the ground, coils its body round them, and does not leave them until they are hatched. The completely developed embryo possesses three pairs of gills, similar to those of salamander larvæ, rudimentary hind limbs, and a short tail, provided with a fin.* It loses its gills on hatching, and betakes itself to the water, where it lives for some time.

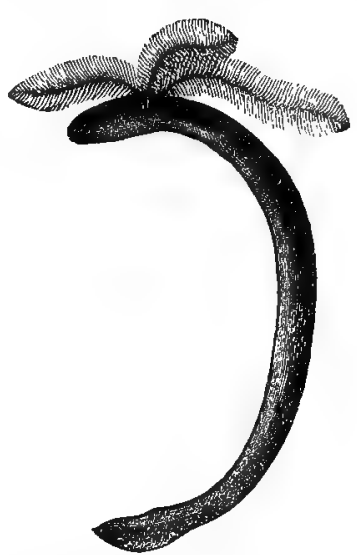

Fig. 331. Embryo of Epicrium glutinosum, removed from the egg.-After Sarasin.

\section{Class 4. Reptilia.}

As regards external form, the body is very like that of the Urodela, but differs in the presence of a more pronounced neck. The powerful tail is not sharply demarcated from the body, and is often quite round in section. The limbs are generally, as in the Urodela, small, and feeble as compared with those of the two following classes; elbow and knee are turned outwards; the tail is usually still important as a locomotor organ.

The skin is provided with a hard stratum corneum, which is shed entire at certain periods (several times a year), in Snakes and some Lizards; the former draw off the "slough" inside out, the latter crawl out of it. In the majority of Lizards, however, the horny layer is moulted in large pieces, in the Chelonia and Crocodilia in

* It is very remarkable that the egg increases in size after it is laid, until the diameter becomes twice as great, and the embryo weighs almost four times as much as the new-laid egg. This is probably consequent, to a great extent, upon an absorption of water, but possibly the egg also takes in secretion from the cutaneous glands of the female. 
quite small shreds; the very hard and thick portions of the stratum corneum are not thrown off. The surface of the body is covered with so-called scales, which are, however, quite different in structure from those of Fish. The reptilian scutes may be termed dermal warts; they are usually flattened, lie close together, and are regularly arranged. In the furrows between the scales, the corneum is thin, on their surface, thicker. In some cases, e.g. in Geckos and others, the scales are simple round warts, granular scales. On

$A$

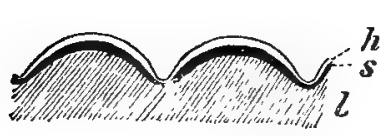

$B$

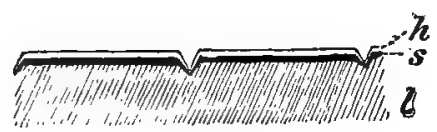

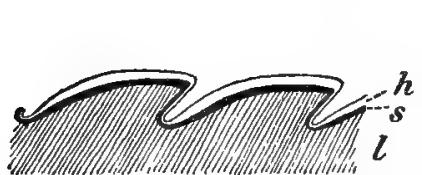

C

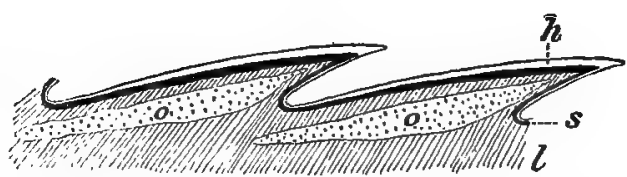

$D$

Fig. 332. Longitudinal section through various $\mathrm{s}$ cales of Reptiles; diagrammatic. $A$ grannlar scales, $B$ shield, $C$ splint scales, $D$ do. with ossifications. $h$ cuticle, $s$ mucous ayer of the epidermis, $l$ dermis, o bony plates.-Orig.

the head, sometimes also upon other parts of the body, there may be shields, i.e., large flat plates, separated from their neighbours by regular grooves. In most cases, the scale is drawn out posteriorly into a point, which overlaps the one following, true scales; if these are much broader than they are long, as on the ventral side of the body in Suakes, they are termed splints. Not infrequently the scutes are developed into longer or shorter spinose scales, as in many Ground Iguanas, and on the back of some Tree Iguanas, etc. True scales have often a small median keel (e.g., in many Snakes). Occasionally, os sifications occur in the dermis; there is, for example, in each scute of the Blind-worm, a small bony plate; in the Crocodilia, there are similar but larger plates in the dermis; and in the Chelonia they are very large, and often connected by sutures, thus making a continuous bony shell round the animal; the boundaries do not correspond with the grooves between the scutes. Skin glands are but slightly developed in the Reptilia; there is, however, e.g, in many Lizards, a row of large glands on the thigh (their openings are termed femoral pores; or, in front of the anus, pre-anal pores); in the Crocodilia also, and in many Chelonia, large isolated skin glands occur. The digits, in contradistinction to those of the Amphibia, are provided with $\mathrm{claws,}$ peculiar horny structures, covering the last phalanx like a cap; they are not affected by the ecdysis; they grow gradually from within, and are simultaneously worn away at the surface. 
In the adult, the skeleton is only to a slight extent cartilaginous; it consists almost entirely of bone. The notochord has usually disappeared; only in the Geckos does it persist as a continuous cord extending the whole length of the vertebral column. ${ }^{*}$ The centra usually articulate; they are generally procolous; in the Crocodilia, there are cartilaginous discs between them. $\dagger$ The vertebral column is usually divisible into more regions than in the Amphibia: first, there is a variable number of cervical vertebræ, without ribs or with short ones; then a number provided with longer ribs, the thoracic vertebræ; these are often followed by several ribless lumbar vertebræ; then the sacral vertebræ, usually $t w o$, to the transverse processes of which the pelvis is attached (occasionally, especially in certain extinct Reptilia, there is a larger numbers of sacrals); lastly, the ca u dal ve rte b ræ, $\ddagger$ without ribs. In the Snakes, however, in consequence of the absence of limbs, these distinctions do not hold; all the cervical and dorsal vertebræ, with the exception of the first, bear well-developed ribs; there are no sacrals, and therefore trunk and caudal vertebræ only can be distinguished. The first two cervicals, the at las and axis, are peculiar in form (Fig. 333). The centrum

Eig. 333. Diagrammatic transverse section of the atlas of one of the higher Vertebrata. $b_{1}$ arch of the atlas, 1 odontoid process, $x$ bony plate, $l$ ligament.-Orig.

Fig. 334. Diagram of axis. 1 centrum of atlas, 2 centrum of axis, $b_{2}$ arch of axis.-Orig.
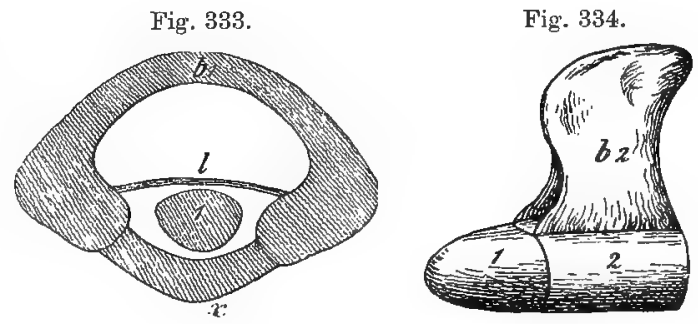

of the former is fused with the second, forming a process (the odontoid) at its anterior end. The first vertebra is, therefore, merely a bony ring formed by an arch, bridged below by a bony

\footnotetext{
* In the young Lacerta, ete., considerable portions of the chorda dorsalis are present in the centra, but they disappear later.

† Transverse processes are especially well-developed in the Crocodilia; here they are large on most of the vertebræ, although elsewhere they are most prominent in the tail. Frequently (e.g., in the Snakes) an unpaired process arises from the ventral side of the centrum of many of the vertebra, the ventral spine. Definite articular processes (zygapophyses) are present; in the Snakes and some Lizards (Iguana), besides these, there arises from the anterior side of each neural arch, a single process, with two articular facets (zygosphene), each of which fits into a pit (zygantrum) in the preceding vertebra, and thus the connection is made still firmer.
}

I In most Lizards, the tail breaks with peculiar readiness; this is correlated with the fact that in the middle of each caudal centrum, there is an uncalcified transverse disc. After fracture, the tail grows again. 
plate. The lower portion of this ring receives the odontoid process, and is separated from the upper, through which the spinal cord passes, by a broad connective tissue ligament. The thoracic $\mathrm{ribs}$ consist of an upper bony region and a lower portion, which is often cartilaginous; the latter is sometimes, e.g., in Crocodiles, divided into two parts: from the bony portion a flat lackwardly-directed process (processus uncinatus) is occasionally given off (Crocodiles). The anterior, or true, ribs are attached to the sternum, in Lacertilia, Crocodilia, and, many extinct forms; the posterior ribs are free (false ribs); no such distinction can be made in the Chelonia and Ophidia, for a sternum is not present. The ribs are partly fused to the dermal skeleton in Chelonia. In Crocodilia, small ribs, which for the most part articulate by two heads, like the thoracic ribs, occur on all the cervical vertebræ; the same points may be observed in Lizards, where, however, there are none on the atlas. The posterior cervical ribs become successively longer, so that there is a gradual transition from cervical to thoracic vertebræ.* Azygos, forked bones, the hæmal arches, occur ventrally, in Lizards and Crocodiles, between the caudal vertebræ, but have not coalesced with them. The sternum (Fig. 339) which is absent from the Chelonia and Ophidia, is usually a short rhomboidal plate, which sometimes (e.g., in Crocodiles) is prolonged posteriorly into a long narrow process; it is cartilaginous, and is usually calcified. Connected with the sternum anteriorly is a flat, longish membrane bone, the episternum, which partly covers it and is often drawn out anteriorly into two processes one on either side.

The skull consists principally of bone, and in many Reptiles is compressed between the orbits to form a perpendicular plate of cartilage or even partly of mere fibrous connective tissue, the interorbital septum; the brain is situated behind this plate; in front of it lies the olfactory organ. There is only on e occipital condyle below the foramen magnum. The premaxillæ $†$ and maxillæ are firmly attached to the skull, as are also the bones formed in the place of the palato-quadrate; viz., most posteriorly, the quadrate which bears the articular facets for the lower jaw ; in front of this the pterygoid; and still further forward the palatine; the two last bones extend forwards from the quadrate, inwards from the large maxillæ. The extraordinary mobility possessed by the palato-pterygoquadrate arcade, in connection with the maxilla, in Snakes, is remarkable; the quadrate in Lizards is also, to some extent, movable, but quite immovable in the Crocodilia and Chelonia, in which groups, the palatines, pterygoids and maxillæ are fixed. The lower jaw

* There is a number of narrow membrane bones, the so-called abdominal ribs, in the abdominal wall of Crocodiles, which must not be confused with true ribs. They have no connection with vertebræ, and are, not like ribs, preformed in cartilage.

+ In Snakes and many Lizards the premaxillæ are fused. 
consists of several bones : of these the most anterior may be anchylosed with its fellow of the other side, as in Chelonia. The hyoid
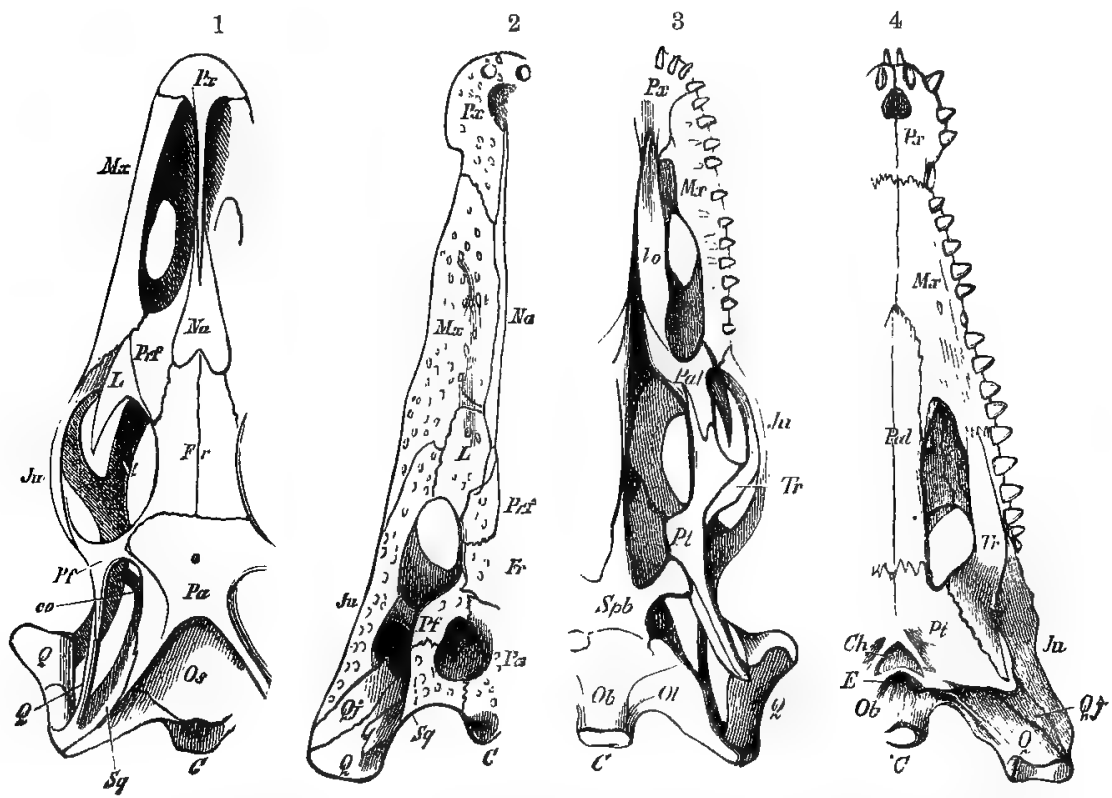

Fig. 335. 1 and 3 Skull of a $\mathrm{Li}$ a a d (Varanus). - 2 and 4 of a $\mathrm{Crocod}$ ile, dorsal and ventral. $C$ occipital condyle, $C h$ posterior nares, co columella, $E$ Eustachian tube, $F r$ frontal, $J u$ jugal, $L$ in 2 lachrymal (in 1 is membrane bone present in some forms), $M x$ maxilla, $N a$ nasal, $O b$ basi-, $O l$ ex-, Os supra-occipital, $P a$ parietal, $P a l$ palatine, $P f$ postfrontal, $\boldsymbol{P}_{\gamma f}$ prefrontal, $\boldsymbol{P} t$ pterygoid, $P x$ premaxilla. $Q$ quadrate, $Q j$ (and the lower $Q$ in 1 ) quadrato-

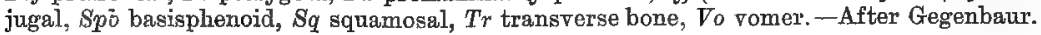

apparatus, i.e., the visceral skeleton, with the exception of the first visceral arch (the quadrate, pterygoid, palatine and mandible), consists, in the Chelonia and Lacertilia, of an unpaired portion, the body of the hyoid, corresponding to the basibranchials of Fish, and two pairs of cornua which represent the hyoid and the first gill-bar respectively ; in Crocodiles and Snakes only a single pair of horns is present, in the latter group the whole apparatus is very poorly developed.

The most important bones of the reptilian skull, besides those already mentioned, are the following: the occipitals, a supra-, basi-, and two ex-occipitals, surrounding the foramen magnum; the petrosal, in front of the

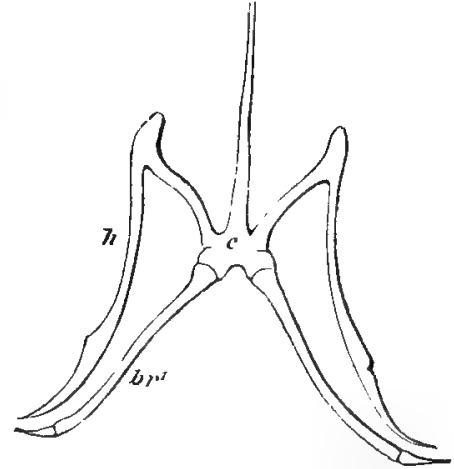

Fig. 336. Hyoid of a Lizard. $c$ body of the hyoid (copula), br ${ }_{1}$ first. gill bar.-After Walter.

\footnotetext{
* In some Lizards there are traces of two cornua representing the second gill-bar.
} 
-exoccipitals; the squamosal, in the same region, projects far forwards in Snakes, and is connected with the quadrate; the basisphenoid, in front of the basi-occipital, and like this, an ossification in the lower wall of the skull. A parasphenoid is not developed ( $c f$., Fish and Amphibia). The anterior wall of the brain-case is often unossified and membranous, sometimes with isolated ossifications. Dorsally there is a number of bones; the parietals, which are generally (Snakes, Lizards, Crocodiles) fused; the f $x$ ontals, an unpaired bone, in Crocodiles and many Lizards; the post-frontals, behind, the pre. frontals, in front of, and the lachrymals, below, the orbit (the last is present only in Lacertilia and Crocodilia); the nas als behind the external nares. Below the orbit and behind the maxilla, there is usually a $j n g a l$, and from this to the quadrate stretches the $q u$ a drat $0-j u g a l$; a paired or unpaired vomer lies ventrally, in front of the palatine. Extending from the pterygoid to the maxilla, there is in Crocodiles, Lizards, and Snakes, the peculiar transversebone which is peculiar to the Reptilia. There is yet another peculiar bone in many Lizards, the columella, extending almost perpendicularly from the parietal to the pterygoid.

The shoulder girdle of the Reptilia is very like that of the Amphibia. In Lacertilia, which will be considered first, it is

Fig. 337.
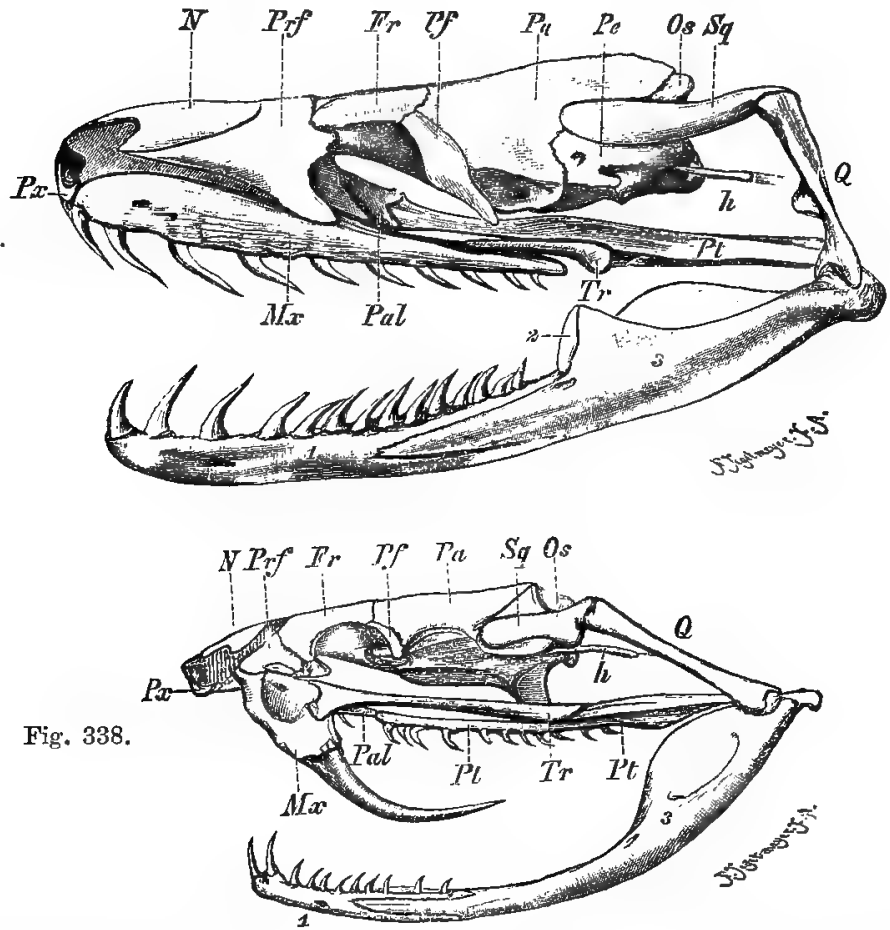

Fig. 337. Left half of the skull of a Boa constrictor, seen from the side (and somewhat from above). - Orig.

Fig. 338. Do. of a large Crotalus (Craspedocephalus atrox).-Orig.

Fr frontal, $h$ ear bone, $M x$ maxilla, $N$ nasal, Os supra-occipital, $P a$ parietal, $P a l$ palatine, $P e$ petrosal, $P f$ postfrontal, $P v f$ prefrontal, $P t$ pterygoid, $P x$ premaxilla, $Q$ quadrate, $S q$ :squamosal, $\operatorname{Tr}$ transverse bone, 1, 2, 3 bones of the lower jaw. 
represented on each side by an arched, partially ossified plate, which articulates below with the front edge of the sternum. A scapula is distinguishable above the glenoid, and below this a coracoid; which is usually divided by one or two large fenestræ into two or three regions. The scapula is divided into an upper and a lower portion, of which the former consists of calcified cartilage, the latter of bone; it is connected with the coracoid by a suture or is fused with it. A clavicle reaches from the scapula to the episternum. In the Crocodilia the shoulder blade is almost entirely ossified, only the upper edge being cartilaginous (suprascapula); the coracoid is a simple bone, and the clavicle is absent. In the Chelonia the coracoid is divided, as in the Lizards, into anterior and posterior regions, which are, however, quite separate; the former, the precoracoid, is fused with the scapula, which it meets.

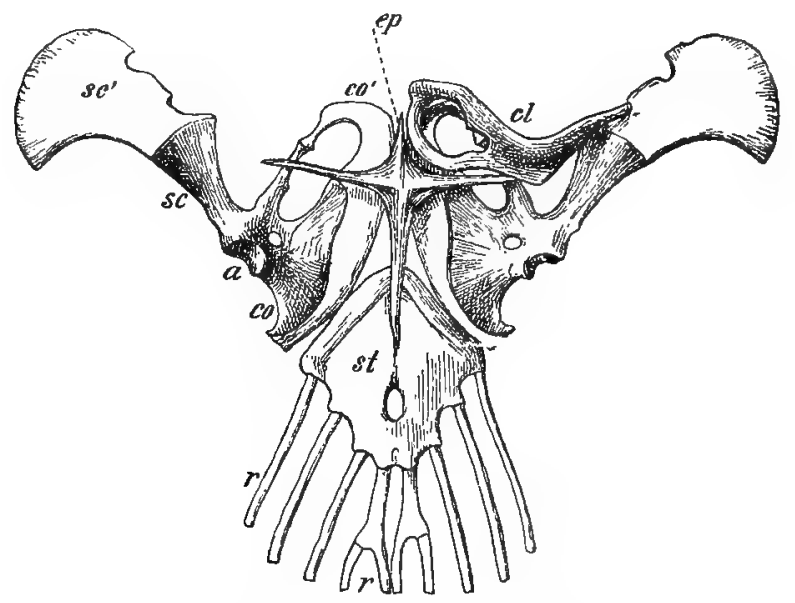

Fig. 339. Sternum and shoulder girdle of Lacerta spread out, the scapula. in reality bends upwards. The right clavicle has been removed. a glenoid for the humerus, $c l$ clavicle (in the species figured perforated by a large fenestra), co coracoid, co' cartilaginous epicoracoid, ep episternum, $r$ ribs (cut away), sc scapula (here anchylosed to the coracoid), se' eartilaginous supra-scapula, st sternum.-Orig.

at a right angle; the latter, the postcoracoid, is a separate bone. In the Ophidia the shoulder girdle is altogether wanting. With regard to the fore limb it must be noticed that the ulna is the stronger of the two bones of the forearm. In the Chelonia the nine primitive bones are present in the carpus (sometimes some are fused); in the Lizards, too, the carpus is little modified; whilst in the Crocodilia it is distinguished by the large size of the two proximal bones, and by the degeneration or fusion of the distal carpals. Attached to the outer side of the carpus there is usually a sesamoid bone, the pisiform. The number of 
fingers is usually five, but may be smaller; the phalanges vary in number; in the Lizards they are generally as follows: two in the thumb, three in the second, four in the third, five in the fourth, three in the fifth digit.

Fig. 340 .

Fig. 341.

Fig. 342.
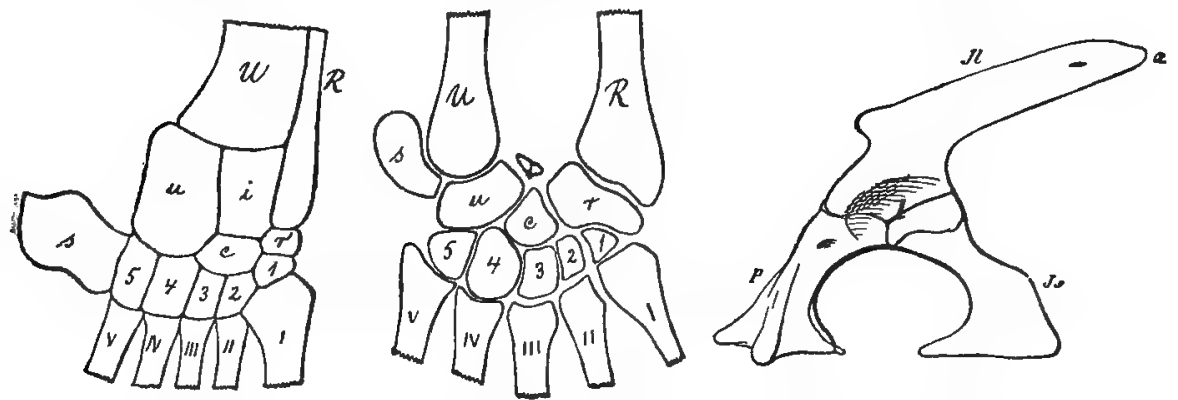

Fig. 340. Carpus of a Turtle. U lower portion of the uina, $R$ of the radius, $u$ ulnare, $i$ intermedium, $r$ radiale, $c$ centrale, $1-5$ carpals first to fifth ; $s$ pisiform $: I-V$ metacarpals.-After Gegenbaur.

Fig. 341.-Carpus of a $\mathrm{Li}$ zard (Lacerta agilis).-Modified from Gegenbaur.

Fig. 342.-Left half of pelvis of a Lizard (Varanus). $几$ Ilium, $a$ its hind end, $J s$ ischium, $P$ pubis, $l$ acetabulum.-After Gegenbaur.

The pelvis is composed of three bones on each side, the ventral portion being separated by a large fenestra into anterior and posterior parts, which ossify separately, the pubis and the ischium; they are connected medianly with their fellows of the other side; all three bones generally take part in the formation of the acetabulum. A pelvis is usually altogether absent from the Ophidia, but occasionally rudiments of it are present, e.g., in the Peropoda with rudimentary hind limbs. In the tarsus* some of the bones are always fused; it is important to note, that the proximal row of tarsals with which the centrale is also connected, is usually closely united with the distal end of the leg, and movement in the ankle occurs between the proximal and distal rows of tarsals, whilst there is very little or none between the leg and the proximal row ( $c f$. Mammalia). $\dagger$ The toes are like the fingers; five are usually present; the number of joints varies : in Lizards beginning with the hallux, as a rule, two, three, four, five, four.

The brain of Reptilia is generally rather small. In some forms, especially in Crocodiles, the cerebrum attains relatively large

* In Crocodilia the pubis is, however, excluded from the acetabulum, which is formed by ilia and ischia alone.

+ As regards special arrangements it may be mentioned that the bone of the proximal row, which corresponds with the calcaneum of Mammalia, is provided in the Crocodilia with a similar process (tuber calcis). 
proportions, just as does the e e rebellum, which in Lacertilia and Ophidia forms simply a narrow ridge in front of the medulla oblongata.

Olfactory organs, the nasal capsules, occupy the front end of the head, and are separated from one another by the nasal septum. Each capsule is a fairly roomy cavity, usually provided with a large projecting fold, the turbinal; the external nares are small, the internal usually open far forwards in the mouth, and are often prolonged into a groove on the roof; in Crocodiles, however, this groove, by the curving over and concrescence of its edges, has become a tube, opening far back in the mouth (Fig. 346), and covered below by portions of the maxilla, palatine, and pterygoid.

Optic Organs. The sclerotic is usually partly carti-
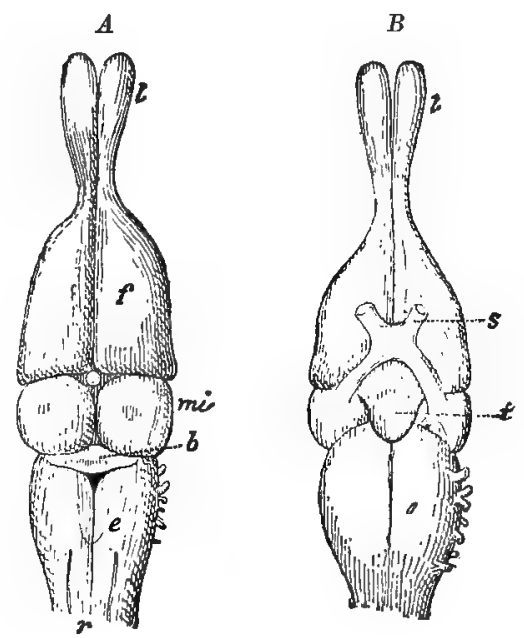

Fig. 343. Brain of a Lizard from above $(A)$ and from below $(B)$. $l$ olfactory lobes, $f$ cerebrum, $m i$ optic lobes, $b$ cerebellum, $e$ medullar $r$ spinal cord, $s$ optic nerve, $t$ hypophysis. In $A$ in front of the mid-brain may be seen the lower portion of the epiphysis.After T. Jeffery Parker. laginous, and in Lacertilia and Chelonia, though not in Ophidia and Crocodilia, it exhibits a ring of thin, bony plates, the sclerotic ring, surrounding the cornea. In Lizards, a process, projecting freely into the vitreous humour, and corresponding to the pecten of Birds, arises from

Fig. 344. A Vertical section of the eye and reyelids of a common Lizard, $B$ the same of a Snake; both diagram. matic. $h$ cornea, o upper, ulower eyelid, $a$ bulb of the -eye (in outline).-Orig.
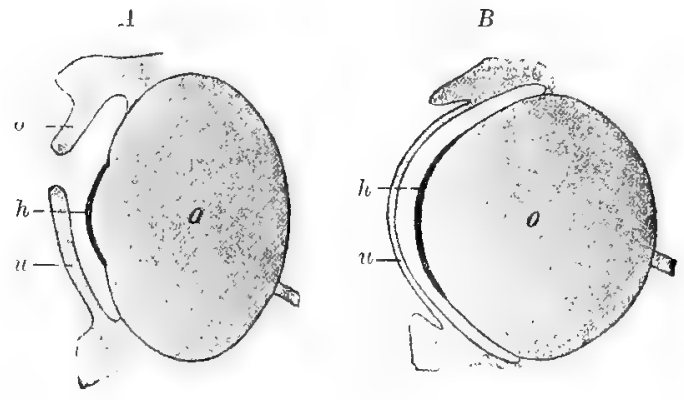

the inner wall of the optic bulb, at the entrance of the optic nerve; in others it is absent or rudimentary. An upper and a lower reyelid are present, of which the former is only slightly movable, whilst the latter can be moved across the eye as in Amphibia. The lower lid is often somewhat transparent centrally (e.g., in the common 
Lacerta); in certain other Lacertilians quite transparent. In the Ascalabotidæ, a few other Lizards, and in the Snakes, it is not only transparent, but remains drawn over the eye, with its upper edge fixed to the upper evelid, so that there is an enclosed space in front of the eye ; these animals seem to be unable to "shut" the eye, the transparent lid looking like a cornea, but as a matter of fact it is always closed. There is usually a nictitating membrane. Besides a lachrymal gland, a lachrymal duct and a Harderian gland are also present. For the parietal eye, see p. 337.

A uditory organ. The cochlea of most Reptilia is as little developed as in Fish and Amphibia, and is only a small evagination; in the Crocodilia, however, it reaches a much higher stage of development, as a fairly large, closed tube. The outer wall of the skull, which lies above the cochlea, is pierced in the Reptilia by an opening, covered by connective tissue, the fenestra rotunda; above the sacculus there is, as in Amphibia, a fenestra ovalis, which is closed by a process of the pro-otic. There is usually a ty $\mathrm{mpanic}$ cavity, closed towards the surface by a tympanic membrane,* which lies in a shallow groove, not, as in Amphibia, at the level of the rest of the skin. The tympanic cavity in the Lacertilia, as in the Amphibia, communicates directly with the mouth by a wide aperture; in the Chelonia and Crocodilia, on the other hand, by a narrow canal, the Eustachian tube. The Crocodiles are peculiar in that the tympanic cavity is connected with air spaces in the wall of the skull, and that the two Eustachian canals open by a common aperture into the mouth, not far behind the internal nares. In the Ophidia and some Lacertilia tympanic cavity and membrane are entirely absent. There is a columella, like that of the Amphibia; its flattened portion fits into the fenestra ovalis, and the other end is attached to the tympanum, when there is one. An "external ear" occurs, in the Crocodilia, as a flap or fold of the skin covering the drum externally.

Teeth occur, in most Reptiles, on premaxilla, maxilla, and mandible; in Snakes (in which the premaxilla is usually edentulous) and in Lizards, they are often present on the palatine and pterygoid also, whilst they are entirely absent from the Chelonia. They are usually attached to the bones by osseous tissue; only in the Crocodilia are they implanted in sockets. Replacement teeth are formed continuously throughout life; the old ones fall out; the osseous substance which attached them to the bones and the lower portions of the teeth themselves, being reabsorbed. The teeth are usually simple, most often conical; sometimes distally compressed and pointed; sometimes they are knob-like. Usually, all the teeth in one

* A tympanic cavity is present in Chameleons, and is closed externally by an undifferentiated portion of skin. A specially developed tympanum is wanting. 
animal are identical in form (homodont). (For the poison teeth of snakes, see p. 423, and Fig. 348). The to ngue, which is attached behind free in front, is very varied in form; in the Crocodilia and Chelonia, it is but slightly movable, with a short tip, and incapable of protrusion; whilst in the Lacertilia, it has generally a long, often very long, and bifid, tip; in the Ophidia, too, the tongue is long, narrow, and bifid, and can be stretched far out of the mouth; here, and in one division of the Lacertilia also, it can be withdrawn into a sheath on the floor of the mouth. (For the peculiar tongue of the Chameleons, see p. 422). The osophagus is long, and capable of considerable distension. The stomach of Crocodiles is very muscular, provided on each side with a tendinous disc, to which the muscle cells are attached; it suggests the gizzard of Birds. The small intestine varies in length, the rectum is short.

Respiratory organs. The trachea of Reptilia is long, and its wall is strengthened with numerous cartilaginous rings. The

$\boldsymbol{A}$

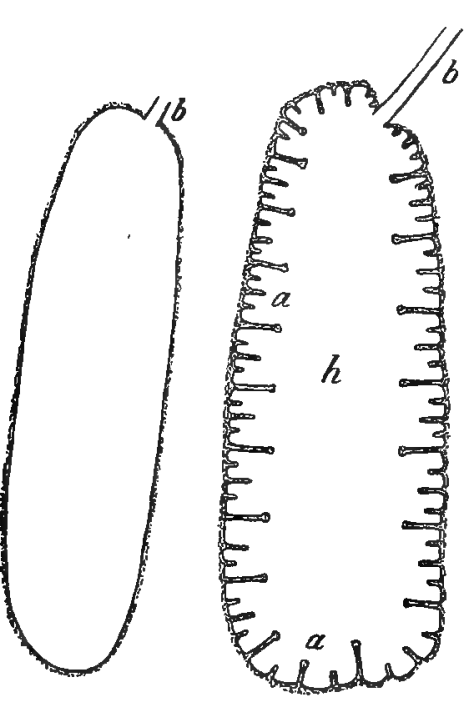

$\cdot C$

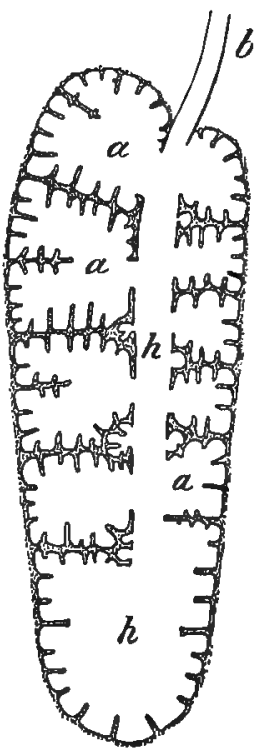

$D$

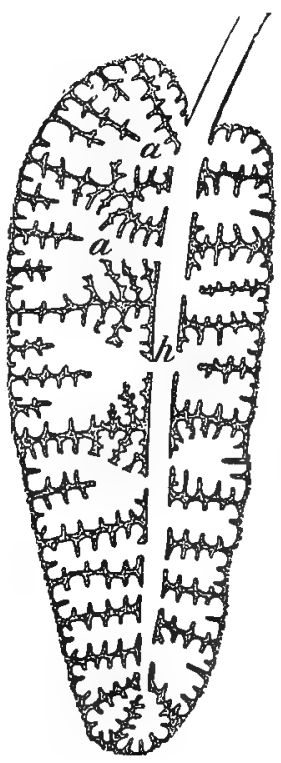

Fig. 345.-Diagrams of various lungs. $A$ Triton, $B$ Frog or small Lizard, $C$ Tortoise, $D$ Turtle. $b$ bronchus, $h$ cavity of the lung, $a$ evagination of the lung. Connective tissue dotted.-Orig.

anterior portion, the larynx, is furnished with special pieces of cartilage, and in some Lacertilia (Geckos, Chameleons), as well as in the Crocodilia, possesses a pair of vocal cords which do not occur in others. The entrance from the mouth is through a longitudinal slit behind the tongue. At the hinder end, the trachea divides into two 
shorter or longer bronchi,* one for each lung. The lungs are of very diverse forms, which may, however, all be referred to a common type. The simplest occurs in many small Lizards (e.g., Lacerta); here, just as in the Anura, there is a capacious sac, with numerous short (and very closely comnected) evaginations, which are again provided with still smaller outgrowths. In the Tortoises (Emys), the posterior region of the lung is similar to that of Lizards, but the anterior larger portion has been drawn out to a narrow tube with a number of evaginations, some very large and deep, which are again provided with smaller pits, all being bound together by connective tissue. In Turtles (Chelonia), the posterior portion of the lung has also become narrow, and is furnished with deep outgrowths ; in the anterior region, there are transverse rods of cartilage in the walls of the bronchi, not in the sacs. The arrangements in the Crocodilia are similar, but the rods of cartilage have become rings like those of the trachea. $t$ Amongst special conditions, it may be mentioned that the lungs of the elongate, apodous Lizards (e.g., the Blind-worm), are of unequal length, the right being the longer. In $\mathrm{Sn}$ akes, too, the right lung is the larger; as

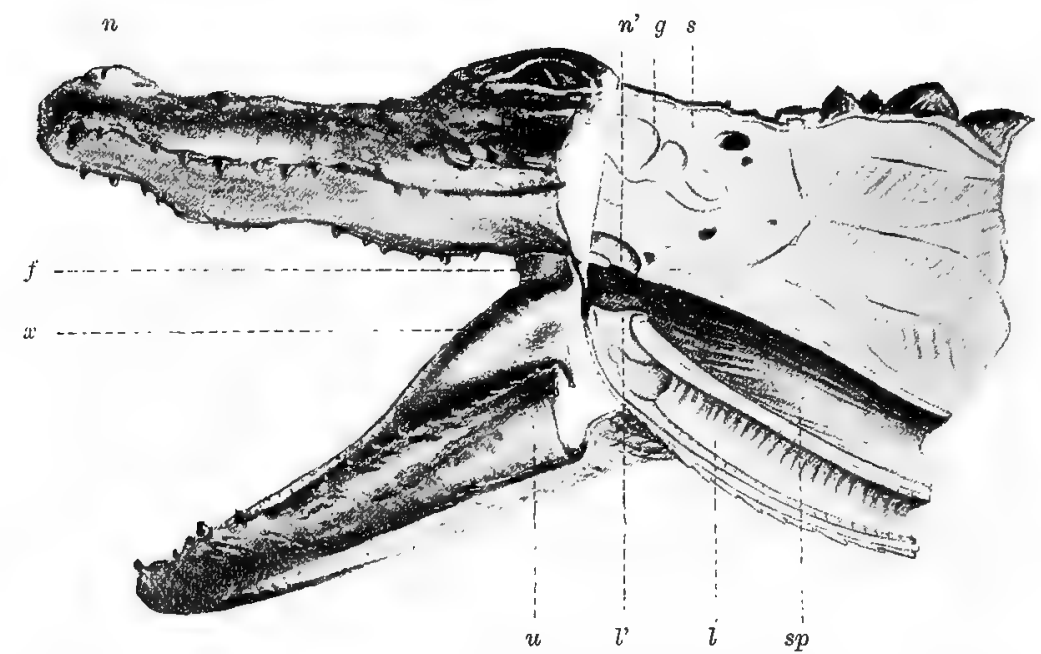

Fig. 346. Head and neck of an Alligator; posterior portion cut in longitudinal section. $f$ transverse fold behind the tongue, $g$ brain (only indicated), $l$ trachea (longitudinal section), $l^{\prime}$ its opening, $n$ left external naris, $n$ ' left internal naris (the posterior portion of the nasal tube is cut into), $s$ skull, $s p$ csephagus (opened), $u$ lower jaw, $x$ tongue.-Orig.

* Sometimes (Snakes, some Lizards) the bronchi are so short, that the two lungs open directly into the hinder end of the trachea.

t The lungs of some large Lizards are like those of the Chelonia and Crocodilia; others occupy an intermediate position. Among the Reptiles, the size of the animal has a direct influence on the structure of the lung; the most complicated structure (i.e., the relatively largest respiratory surface) is shown by the largest forms, cf., Ge-zeral Part, p. 28. 
a rule the left is rudimentary or absent. The Snakes are also peculiar, in that, whilst the anterior portion of the lung is like that of a Lizard's; posteriorly, it is simply a smooth unfolded sac which receives blood, not from the pulmonary, but from one of the systemic arteries; this portion is entirely without respiratory significance. The Chameleons (and some other Lacertilia) are characterised by the possession of thin-walled, finger-like sacs extending from the lungs between the viscera. The animal can fill these with air so that the size of the body is noticeably increased. Inspiration, in the majority of cases, is effected as follows: the capacity of the trunk is insreased by movements of the ribs, so that the air in the elastic lungs is rarified, and external air rushes in through the nares to equalise the pressure; expiration is brought about by reversed movements of the ribs. In the Chelonia, where the ribs are immovable, inspiration results from the contraction of a special diaphragm-like muscle in the ibody-cavity ( $c f$., the Mammalia).

In the Crocodilia the inner nares open, as already noticed, far back in the mouth. On the back of the tongue (Fig. 346) there is a projecting, stiff, transverse fold, which, when the mouth is opened, lies against the palate and separates a posterior cavity into which the internal nares open above, the trachea below. In consequence of this arrangement the animal can lie in the water with its month open wait ing for prey, and if only the snout with the external nares is above the surface can breathe quietly.

In consequence of the development of a neck, the heart in Reptiles is further from the head than in Amphibia and Fish. The atrium is separated into a larger right, and a smaller left, auricle, of which the latter receives the blood from the lungs, the former, that from the rest of the body. The ventricle shows only the beginning of a division into two, for the septum is still incomplete; in the Crocodilia alone the right and left ventricles are completely separated, and connected only with the right and left auricles respectively, so that the arterial pulmonary, and the venous systemic blood are entirely

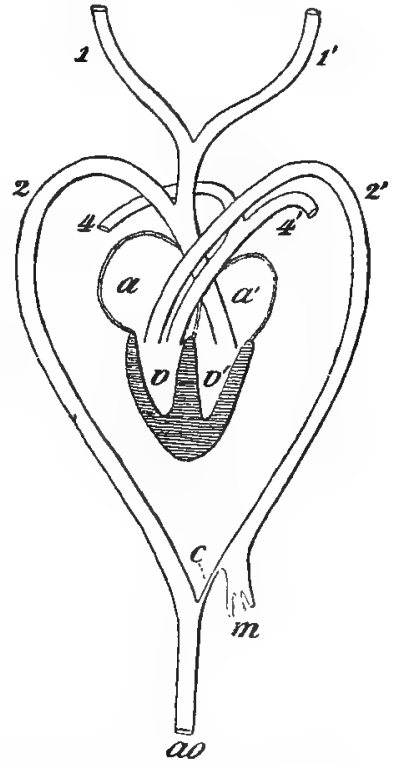

Fig. 347. Diagram of the heart and arterial arches of a Crocodile. a right, $a^{\prime}$ left auricle. $v$ and $n^{\prime}$ right and left ventricles, $1,1^{\prime}$ carotids (arterial arch 1); $2,2^{\prime}$ right anil left aortic arches (arterial arch 2 ) : cthin part of $2^{\prime}$, after giving off the vessel $m$ to the intestine ; $4,4^{\prime}$ pulmonary arteries (arterial arch 4 ), ao aorta. -Orig. separated within the heart. The conus is either rudimentary or absent, so that the arterial trunk springs direct from the ventricle. 
It is not, howerer, single, as in the Pisces and some Amphibia; but is divided into three vessels derived from $t \mathrm{hree} p$ airs of arterial arches, viz., 1, 2, and 4. The first of these vessels forms the carotids (arterial arch 1) and the right aortic arch (the right arch of the second pair); the second is prolonged into the left aortic arch (the left arch of the second pair), whilst the third forms the pulmonary arteries (arterial arch 4). At the origin of each vessel there is a transverse row of valves. The carotids and the right aortic arch arise from the left ventricle and carry arterial blood, whilst the pulmonaries and the left aortic arch are given off from the right ventricle and carry venous blood. In consequence of this arrangement, the head is supplied with pure blood, whilst the aorta carries mixed blood, for it is formed of the union of two arches, of which one contains arterial, the other venous, blood.

In the Crocodilia, the larger portion of venous blood from the left aortic arch goes direct to the alimentary canal, by a vessel $(m)$ which it gives off before it unites with the right aortic arch, so that in the aorta arterial blood preponderates.* In other Reptiles, in which the ventricular septum is quite imperfect, the blood is mixed within the heart itself, but here various arrangements prevent the mixing from being as complete as might be expected. The whole mechanism is too complicated to receive more detailed consideration here.

The kidneys are somewhat elongate, lobulated organs, lying posteriorly in the body-cavity; the urinary tubules have no open funnels as in the Amphibia. The u reters discharge separately into the cloaca, not into the urinary bladder. There is a urinary bladder in Lacertilia and Chelonia, not in Ophidia or Crocodilia; it is an evagination of the ventral wall of the cloacat; the openings of the ureters are not far from the opening of the bladder.

The two ovaries are racemose when ripe in consequence of the large size of the ova; the oviducts (Müller's ducts) are of the ordinary type, and open separately into the cloaca. In Snakes, in correspondence with the elongation of the body, the ovaries lie one in front of the other. The testis is connected, by an epididymis of finetubules, with a sperm duct which opens into the cloaca. Copulatory organs of two kinds are met with. In the Lacertilia and $O p h i d i a$ there is a pair of these organs; on each side, quite close to the anus, there is an opening leading into a sac or tube, which extends back below the skin of the tail, and is to be regarded as an invagination of the skin; this sac can be everted, and then displays on its surface a spiral groove, along which the sperm can travel when the organ (which is often provided with spines or

* The right arterial arch (and the carotids) communicates with the left arterial arch by means of an opening in the septum between the two vascular trunks; mixing of the blood, however, occurs only to a very limited extent at this spot.

$\uparrow$ In the Chelonia, besides the unpaired urinary bladder, a pair of similar sacs of unknown significance opens into the cloaca. 
folds) is inserted into the cloaca of the female; the sac is withdrawn by a muscle attached to its end. In the Crocodilia and Chelonia, the penis is, on the other hand, an unpaired, solid, linguiform organ which is attached to the ventral wall of the cloaca, and can be protruded from the anus; it has a seminal groove on its upper surface.

The e g g s of Reptiles are relatively large. During their passage through the cloaca, they are surrounded by albumen and a calcareous shell, which in Lacertilia and Ophidia, is usually leathery and tough, in Crocodilia and most Chelonia hard and brittle, like a Bird's eggshell. 'T'he shell is usually oval, occasionally round, the latter in most of the Chelonia. The eggs of not a few Ophidia and some Lacertilia remain so long in the oviducts, that the young ones are born alive. The eggs of such forms are usually provided with shells which are thrown off at birth. Segmentation is partial, food yolk abundant; the embryo is surrounded by embryonic membranes (see p. 353). The animal, when newly hatched, is in most respects like the adult.

In the fully-developed embryos of Ophidia and Lacertilia, there is a median tooth on the upper jaw (a true tooth) which is used to cut through the egg-shell. The embryos of Crocodilia and Chelonia have, at the tip of the snout, a wartlike, very cornified projection, with which to break the shell.

The Reptilia are for the most part terrestrial; but a few are amphibious, for they live partly in the water (sea or freshwater), partly on land; most of them prey on other animals (Insects; Vertebrata, etc.). They are numerous in the tropics, occur sparingly in temperate regions, and are absent from colder zones. At earlier periods of the earth's history, in Mesozoic times, this division was much better represented, and, in part, by nuch larger forms than at the present time.

\section{Synopsis of Existing ()rders of Reptilia.}

Movable quadrate. Anus a transverse slit. Paired copulatory organs.

Quadrate immovable. Anus not a transverse slit. Penis unpaired.
1. Lacertilia. Usually with limbs. Scales ventrally. Rami of lower jaw firmly anchylosed.

2. Ophidia. Without limbs. Splints ventrally. Rami of lower jaw connected by an eiastic ligement.

3. Chelonia. Edentulous. Continuous bony case round the body.

4. Crocodilia. Teeth in sockets. Heart with two ventricles.

\section{Order 1. Lacertilia (Lizards).}

For the characters of the Lacertilia, compare the above summary, and the general description of the Reptilia. Of the numerous forms, a few examples are given below. 
1. Lizards (Lacerta) have a long round tail; well-developed limbs; small dorsal scales, larger ones ventrally, usually arranged in longitudinal rows; tongue well-developed and bifid. In England, the two very similar species, the Sand $\mathrm{Lizar}$ (L. a.gilis) and the $\mathrm{C}$ o $\mathrm{m} \mathrm{m}$ on $\mathrm{Liz}$ ard (L. vivipara) are abundant. Allied are the Varanids (Varanus), large, tropical, old-world forms with long bifid tongue.

2. Ig u a nas (Iguanidæ), Lacertilia with small scales; and a thick, slightlybifid tongue; many have spines, ridges, and so forth on the skin. They fall into two natural groups, belonging to the Old and the New World; the former acrodont (i.e., with teeth fused to the edge of the jaw), the latter pleurodont (teeth on the inner side of the jaw, Fig. 281). Within both groups, there are elongate, long-legged, long-tailed forms ( $\mathrm{T}$ ree $\mathrm{Iguanas}$ ), and bulky, flat, short-tailed forms (Ground Iguanas); between these, there is no sharp distinction, as there are many intermediate forms. A peculiar genus of small tree Iguanas, the Flying Lizards or Dragons (Draco volans), have the false ribs not in the body-wall, but lying turned outwards, to form on either side supports for a large fold of the skin, which acts as a patagium. East Indies.

3. The Blind-w'orm (Anguis fraqilis), an apodous Lizard, with long tail and movable eyelids; viviparous; abundant in England. It belongs to the family of the Skinks (Scincoidei), which are characterised by smooth, flattened scales, and a short, flat tongue; within this family, there are forms with welldeveloped limbs, and a relatively short body ; forms with more or less degenerate limbs and a longer body; and lastly, apodous species like the Blind-worm. The other species belong to warmer countries; some occurring on the coasts of the Mediterranean.

4. Chameleons (Chamæleo) constitute a very peculiar group of Lizards. The slit between the eyelids is very narrow, the latter almost entirely cover the eyeball, to which they are attached, and with which they move; the tongue, which can be withdrawn into a sheath, is club-like, of considerable length, and can be projected some way from the mouth; the fingers or toes of each foot have grown together into two buadles, each consisting of two or three digits, which are united almost to the tip, but separated from the other bundle down to the tarsus; the two bundles are s: turned that they work together like the limbs of a pair of tongs, and may be used for seizing branches; body compressed; tail curled up; scales very small; the power of the Chameleons to change their colour is very well known: in warmer countries (especially Africa); one species in Andalusia,

5. The Geckos (Ascalabotæ) are characterised by the presence of suckers on the lower side of the toes, and of eyelids like those of Snakes (see p. 415); usually flattened animals, with compressed scales; in warm countries (even in S. Europe).

6. Ringed Lizards (genus Amphisbæna, etc.), short-tailed, elongate, cylindrical forms, with rery small eyes; usually apodous (or with only small fore limbs) ; scales quadrangular, not imbricating, arranged in transverse rings; mode of life like that of the Cocilias; in warm countries (one species in S. Europe).

\section{Order 2. Ophidia. (Snakes.)}

Snakes, which are nearly related to Lizards, are characterised by the absence of limbs (occasionally there are rudiments of the hind ones); the structure of the eyelids already mentioned (p. 415); 
by the absence of a tympanum and tympanic carity; the very long body, the relatively short tail; the broad scutes covering the ventral surface; the connection of the mandibular rami by an elastic ligament; the great mobility of the quadrate and the whole jaw apparatus; and by the long, bifid tongue. They are distinguished from apodous Lizards by the possession of the elastic ligament,
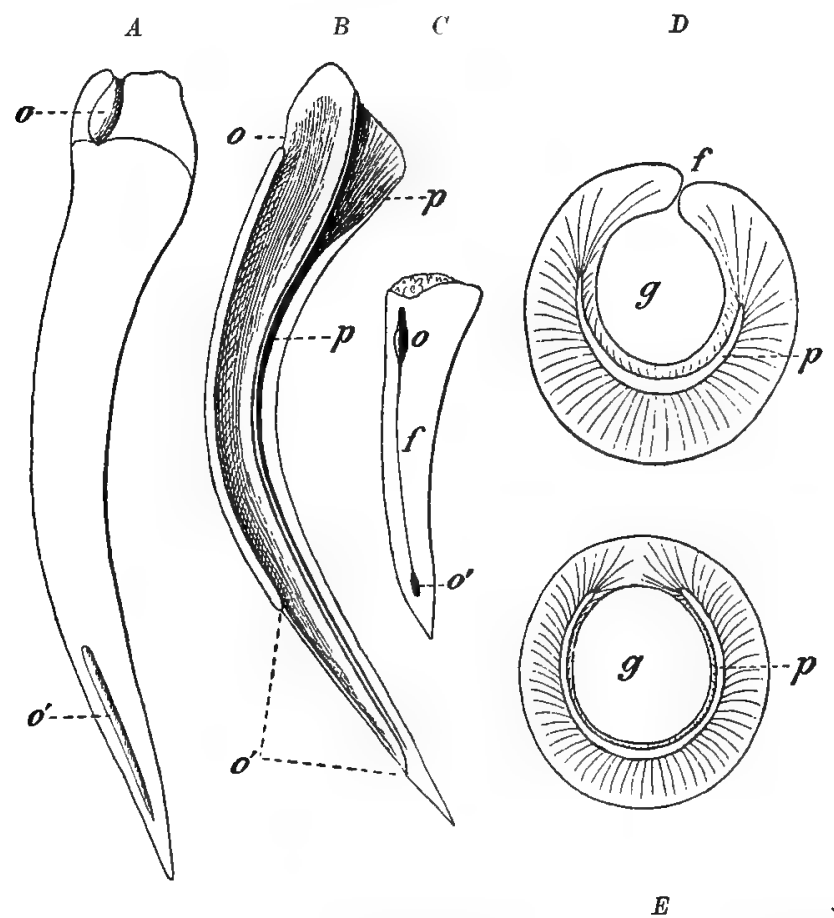

Fig. 348. A Poison tooth of a Crotalus, seen from in front and partly from the outside; $B$ the same tooth cut through longitudinally; $C$ Poison tooth of Naja twipudians, seen in similar riew; $D$ transvere section of the same; $t$ Transverse section of the tooth of a Crotalus. $f$ groore, $g$ poison canal, o upper, $o^{\prime}$ lower opening of the poison canal, $p$ pulp cavity.-Orig.

by the absence of sternum and shoulder girdle (of these parts there are at least rudiments in the Lizards), and by the rudimentary hyoid.

On account of the power of widening the buccal cavity, dependent on the great mobility of jaws and palate, and the absence of a sternum, the Snakes are able to take in very large prey; they feed at long intervals (as much as several months). Some snakes (Proteroglypha and Solenoglypha) are provided with large poison teeth, with a deep groove on the front side; the edges of the groove may be closed or even fused, but it is open at the apex of the tooth. Only one of these teeth is present at a time, anteriorly, in the maxilla of either side, and the aperture leading into its canal is connected at the 
base with a pois on gland* behind the head, which is to be regarded as a specially developed buccal gland. In the mucous membrane of the mouth, behind the poison fang, there are several

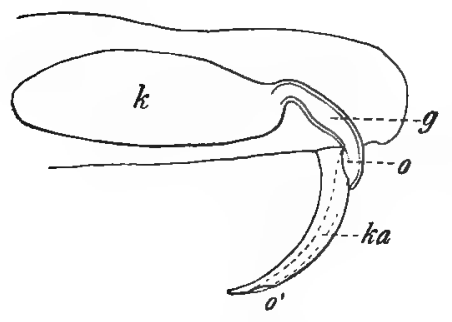

Fig. 349. Poison gland and poison fang of a Snake; diagrammatic. $k$ gland, $g$ duct of the same, $k e$ poison canal, $o$ upper, $o$ ' lower opening.--Orig.

grooved teeth. It has been stated that these teeth (always?) are likewise connected with poison glands, and that small animals die soon after being bitten by them.

A few examples of this large group are given below.

I. Giant snakes (Peropoda) exhibit rudiments of the hind limbs in the form of a small claw-like process on either side of the anus. Teeth simple. Large snakes belong here: Python (up to about $10 \mathrm{~m}$. long), several species in Asia and Africa; the females incubate their eggs (the warmth of the body while brooding considerably exceeds that of the environment): Boa constrictor, in South America, up to about $6 \mathrm{~m}$.

2. The Colubridx, like those which follow are destitute of posterior appendages; teeth simple. In Britain, the Ringed Snakes (Tropidonotus natrix), easily recognised by two large yellow patches on the back of the head: Assulapius' Snake (C. Assculapii) is probably the species venerated by the ancient Romans. Numerous forms in warm countries.

3. The Opisthoglypha possess in the posterior portion of the maxilla one or several grooved teeth. The Whip-snakes (Dryophis, etc.), a group indigenous to the Tropics; distinguished by their extraordinarily long, thin body and pointed head; they live in trees.

4. The Proteroglypha are venomous snakes, which have, anteriorly, in the maxilla a poison gland with a fine groove on the front side ( $f$. Fig. $348 \mathrm{C}-D$ ), and behind this, small simple teeth.

a. The Cobra (Naja tripudians) can spread out the skin behind the head into a wide hood, for the anterior ribs are turned outwards; a pair of spectacles is represented on this hood; $2 \mathrm{~m}$. long; India. The Coral snake (Elaps) is ringed with black and red; small; in South America. Several other genera in warm countries.

b. The Sea Snakes (genus Hydrophis, etc.), specially characterised by the very compressed tail and the very small scale-like ventral splints; numerous

* The poison gland opens quite freely on the wall of the mouth, as does the upper aperture of the poison fang; the two apertures, however, lie close together, with the edge of that of the gland round the opening in the tooth, so that the poison cannot flow into the mouth (cf. Fig. 349). 
species in the Indian and Pacific Oceans; usually small (rarely longer than the Ringed Snake, often smaller); their bite is dangerous.

5. The Solenoglypha or Vipers. The large poison fang is the only tooth in the maxilla, it has no groove anteriorly; the head is usually broad behind, and sharply marked off from the body.

a. Common Viper or Adder (Vipera berus), with a zig-zag line down the back; viviparous; abundant in England, especially upon sandy heaths. The somewhat larger $\mathrm{Sand}$ viper (Vipera ammodytes), with upwardly directed process on the snout, lives on the Mediterranean coasts, in Austria, and in South Bavaria.

b. The Pit Vipers (Crotalidx) are characterised by the possession of a deep pit on either side between the eye and the naris. To this group belong most of the dangerous snakes occurring in warm countries. The Rattle snake (Crotalus) differs from the rest, in possessing at the tail end, several loose rattling,
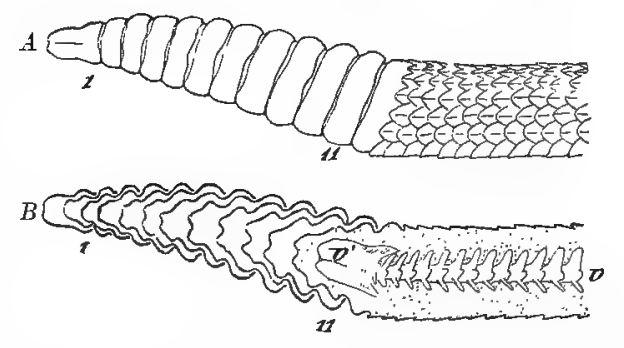

Fig. 350. Tail end of a Crotalus with eleven caps (1 the oldest, 11 the youngest). $B$ the same cut through longitudinally, $v$ vertebral column, $v^{\prime}$ several fused vertebræ, forming the last joint of the vertebral column (the surrounding soft parts dotted); the caps in sertion indicated by a thick line. As is comparison of the two figures shows, only the anterior portion of each cap, with the exception of the oldest, projects as an arched ring, the rest being covered by the next older cap.-After Garman. horny caps, fitting into one another, the remains of cast skins (see Fig. 350). Several species (to over $2 \mathrm{~m}$.) in North and South America. Species of Trigonocephalus, usually smaller, but very dangerous, live in India and America.

\section{Order 3. Chelonia (Testudinata).}

The edentulous jaws are covered by a borny sheath with a sharp edge. Large dermal plates are present, which usually fit into one another at the edges, so as to form a continuous bon y shell round that part of the body. There is a large opening in front for the head and fore limbs, and behind for the tail and hind limbs. Dorsally, there are three longitudinal rows of bony plates, of which the middle row is connected with the vertebræ (vertebral plates), whilst the two others are fused with the ribs of each side, one for each rib. Besides these three rows there is, externally, a series of small marginal plates. Ventrally, there are two rows; there is an anterior unpaired piece, probably corresponding to the episternum of other Reptiles, and in front of this a pair, which may represent the clavicles. These bony plates do not form so complete a shell in all Chelonians; in the Turtles, for instance, the plates do not fit close together, but large portions of unossified leathery skin are left between their edges. The regions in which the dermal armour lies are usually covered, externally, by large hor a y shields 
(tortoiseshell), separated by grooves; their boundaries do not correspond with those of the bony plates, although the arrangement is similar. The rest of the body is corered by small scutes; small bony plates may develop in some scales, as in certain Lacertilia.

The Chelonians feed partly on plants, partly on animals. They are dull, sluggish creatures, terrestrial, freshwater, or marine. Many are able to withdraw head and limbs within the edge of the shell.

1. Mud Turtles (Emydx), mostly flattened animals, with webbed toes; live in freshwater, but many can also walk about on land. More exclusively aquatic is the Freshwater Tortoise (genus Trionyx, etc.), with large swimming feet (each with three claws), shell without horny plates; ferocious animals; Asia, Africa, North America.

2. Tortoises (Testudinidx), closely allied to the Mud Turtles; differing ehiefly in the much arched carapace, and the very short feet, in which the toes have grown together and which possess short claws (club-footed). The Grecian Tortoise (Testudo Græca) inhabits South Europe.

3. Turtles (Cheloniæ) have a flattened carapace and immovably connected toes without claws, or with rudiments only; the fore limbs much larger than the hind, forming a strong fin-like steering apparatus. They attain a considerable size; live in the sea, but the eggs are laid on land, buried in the sand. One species in the Mediterranean.

\section{Order 4. Crocodilia. (Crocodiles).}

This order differs from other living Reptiles, and approaches Birds and Mammals in many respects: as in the divided ventricle, the structure of the brain and the cochlea, and also in the thecodont dentition (teeth in sockets). The Crocodiles have large heads with the nares on the upper side of the snout, webs between the bind toes, and a long compressed swimming tail; claws present only on the three inner toes of each foot; five toes on the forefeet, four on the hind. In the skin there are numerous bony plates (especially on the dorsal side). The anus is a longitudinal slit. For the way they breathe with the mouth open in the water, and for other peculiarities, see above.

Crocodiles, which may attain a length of about ten metres, live in warm countries in fresh water, rarely on the sea-shore, but they also frequent the land; they are rapacious beasts and carrion feeders. The eggs are laid on land, either buried in earth or among decaying plants, etc.; the female guards them, and sometimes also the young ones.

The Crocodilia of the present day may be divided into three groups; (1) Alligators (Alligator), with short snout and imperfectly webbed hind feet; the fourth tooth of the lower jaw bites into a cavity in the upper jaw; America (one species in East Asia): (2) Tru e Crocodile s (Crocodilus), with long snout and complete webbing between the hind toes; the fourth tooth of the lower jaw bites into a notch on the side of the upper jaw; in both the Old and the New World: (3) Gavialidæ (Rhamphostoma), with very long, slender snout and complete webs; the fourth tooth of the lower jaw bites into a notch; India. 
For the rest, these three groups are connected by intermediate forms; there are species of Crocodiles which approach the Alligators, and a Gavial which forms a connecting link with the Crocodiles.

The oldest Crocodiles known, from the Triassic (Belodon and others) are specially characterised by their resemblance to Lacertilia and Chelonia with

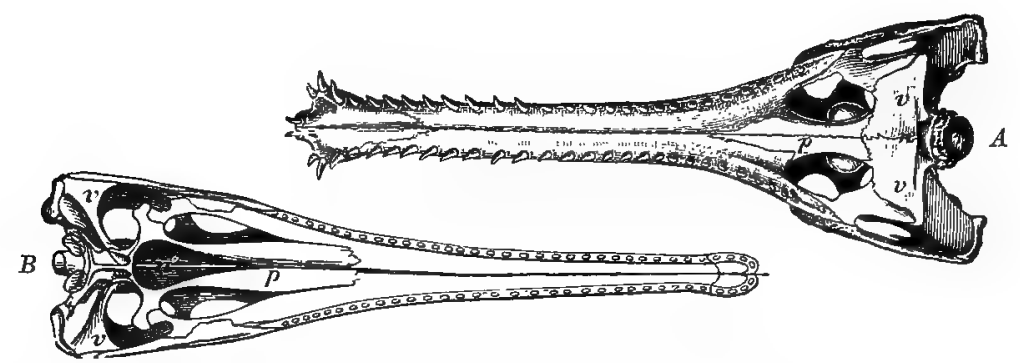

Fig. 351. A Skull of a Gavial, $B$ of a Teleoscumus. $n^{3}$ internal nares, $p$ palatine, $v$. pterygoid.

respect to the position of the internal nares: the palatines and pterygoids do not form a canal, but the internal nares open much farther forward than in those now living. The Crocodiles from the Jurassic, and some of those from the Cretaceous formations (Teleosaurus [Fig. $351 \mathrm{~B}$ ], etc.) approach the extant forms in this respect; for the palatines, but not the pterygoids, are connected to form a canal, and the nares are thus moved much further back. All these older forms differ also from those of to-day in the possession of biconcave vertebræ. On the other hand the Tertiary and some of the Cretaceous forms. exactly resemble those now existing; the pterygoids take part in the formation of the nasal canal, and the centra are procolous. The Crocodiles of different periods afford a very interesting series.

Whilst many extinct Reptiles, e.g., the Crocodiles just mentioned, as well as many others, are allied to the orders of the present day, there are also many forms constituting orders which are without living representatives. Several of these groups are of considerable interest, and the most important will now be briefly considered.

The Ichthyosaurians occupy a position among the Reptiles similar to that of the Whales among Mammals, and they are very suggestive of this group. The

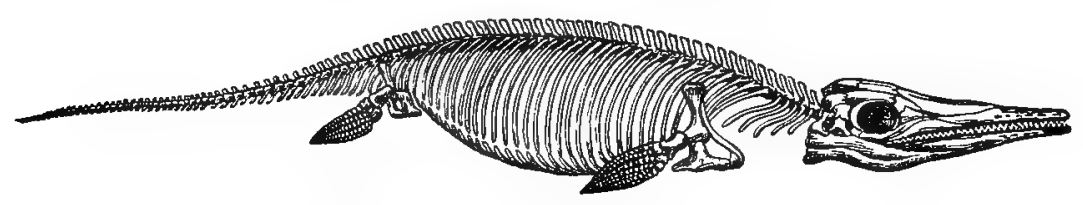

Fig. 352. An I chthyosaurian.

head, especially the snout, is of huge size, the neck uncommonly short, the tail very long and powerful; both pairs of limbs are formed like the fins of a Whale: they are short. broad plates, all the bones of which are immovably connected and much shortened; the digits, of which there were often more than five on each foot, were enclosed in a common skin, and without claws, and the number of joints to each digit was very great, though each joint was very 
short. Among other characters, it may be mentioned, that the sclerotic of the large eye was provided with a ring of bony plates; the vertebral centra were very large and strongly biconcave, the pelvis was not connected with the vertebraI column, the hind limbs weaker than the fore, the teeth in a continuous furrow in the edge of the jaw, a character which also occurs in several other animals (e.g., certain Whales). The Ichthyosatuians were marine, some of very great size (10 m. and more); they lived in Triassic, Jurassic, and Cretaceous times.

The Plesiosaurians, another extinct type of marine Reptile, are in some respects like the Ichthyosaurians, in others very different from them. The head is small, sometimes even very small; the neck, on the contrary, is long; longest in those with the smallest head. The compressed piscine form of body which obtains in the Ichthyosanrians is absent here. Fore and hind limbs,

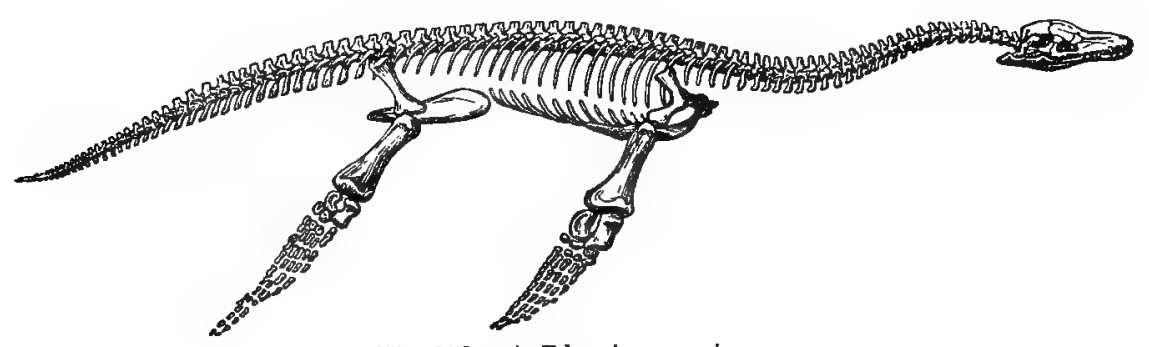

Fig. 353. A Plesiosanrian.

as in the latter, are clawless, and like the fins of a Whale; they are, however, usually larger than in the Ichthyosaurians, the bones are not so much shortened. and the number of digits does not exceed five. They attain a length equal to that of the Ichthyosaurians. Triassic, Jurassic, Cretaceous.

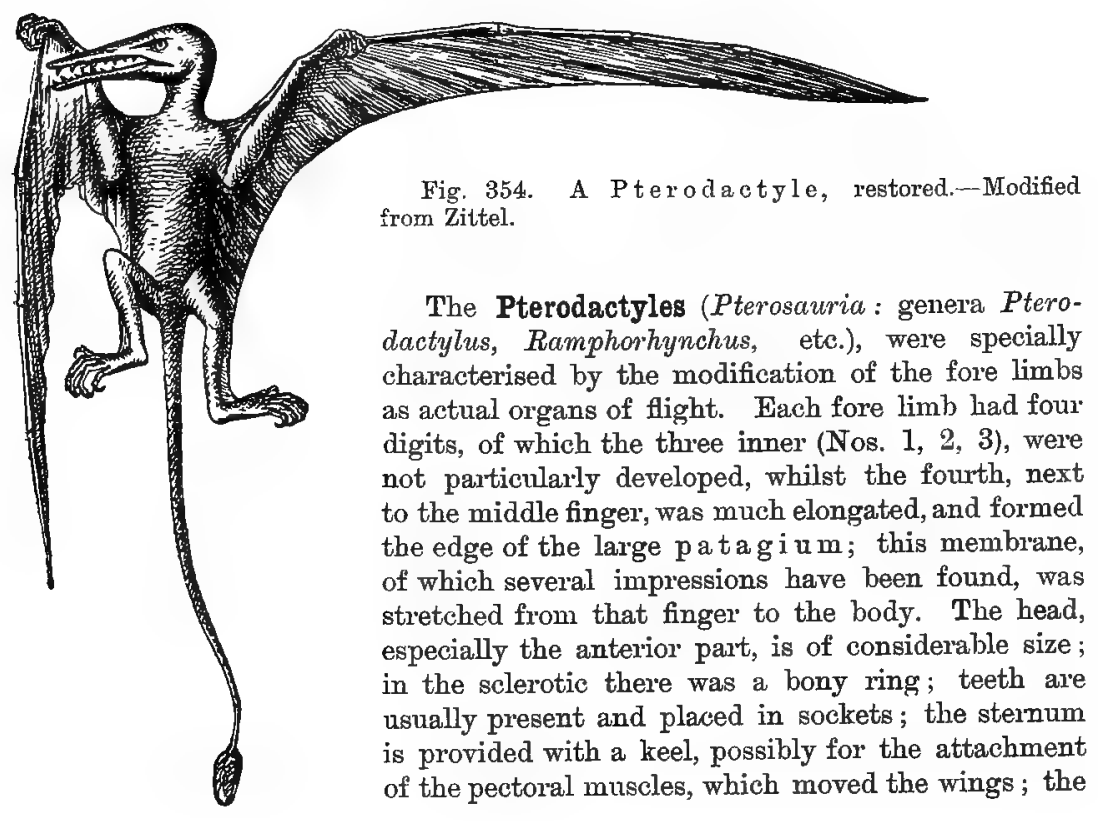


bones are pneumatic (as in Birds). The Pterodactyles, which occupy among Reptiles a position comparable with that of Bats among Mammals, were for the most part small animals, and lived in Jurassic and Cretaceous times.

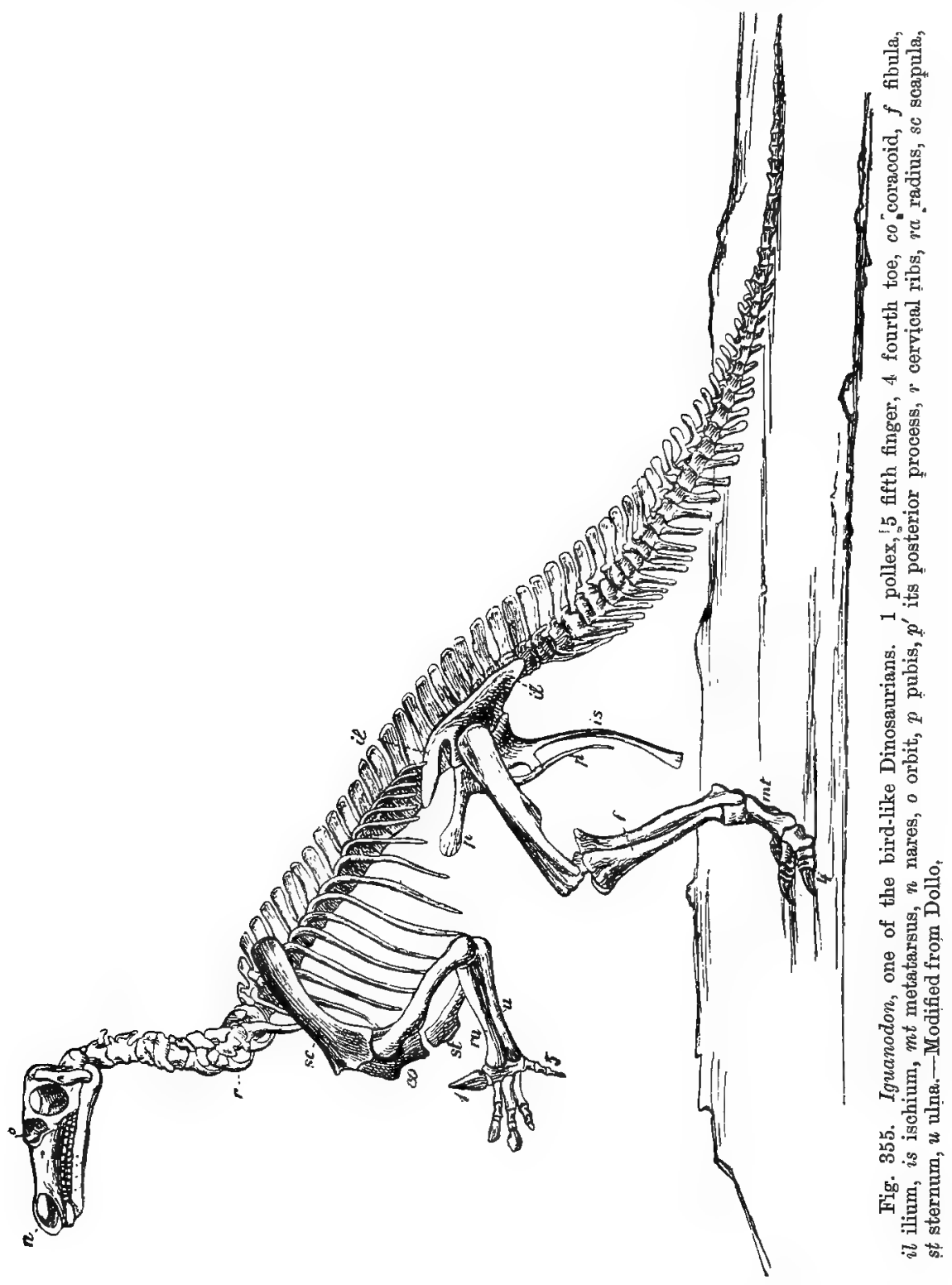

The Dinosaurians (Dinosauria) are a group of Reptiles consisting of numerous forms, which are of the greatest interest on account of their affording a link between Reptiles and Birds. Within this division there are forms which stand tolerably near to other Reptiles, and again, forms which more closely approach Birds. The Dinosaurians were torrestrial animals, chiefly of consider- 
able, some of colossal size, bigger than the largest extant terrestrial Mammals (in one species the thigh-bone is $2-3 \mathrm{~m}$. long, and very thick); though small forms also occur. The limbs are powerful; in some, fore and hind legs are about equal in length, but generally the former are smaller, sometimes even much feebler than the hind limbs, and many Dinosaurians moved almost entirely upon the latter, possibly springing along; among those with stronger hind limbs some were digitigrade, whilst other Reptiles are plantigrade; the tail is long and powerful. The pelvis is very remarkable; the ilia are much prolonged in front of the acetabulum, agreeing with Birds not with Reptiles, and in those Dinosaurians which have small fore limbs (e.g., Iguanodon, Fig. 355), the pubis also is very peculiar in form: a long thin process $\left(p^{\prime}\right)$ arises from its base, and
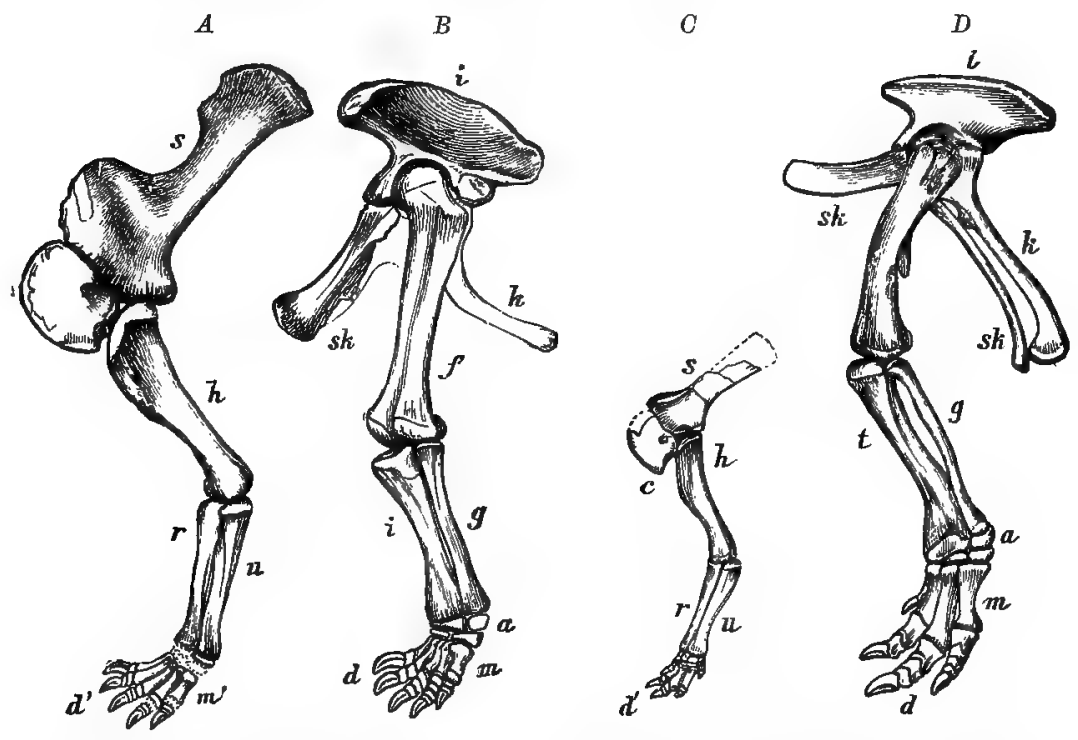

Fig. 356. $A-B$ fore and hind limbs of one of the D in os a urians, which are some way from birds (Morosaurus grandis). $C-D$ Do. of a bird-like Dinosaur (Camptonotus dispar). a tarsus, $c$ coracoid, $d$ toes, $d^{\prime}$ fingers, $f$ femur, $g$ fibula, $h$ humerus, $i$ ilium, $k$ ischium, $m$ metatarsus, $m$ ' metacarpus, $r$ radius, $s$ scapula, sh pubis, $t$ tibia, $u$ ulna.After Marsh.

reaches back, in a direction almost exactly opposite to that of the main branch, lying close against, and parallel to the ischium, which is often long and thin. There is a larger number of sacral vertebræ than in other Reptiles (four or more) and these are fused. Among other characters, it may be mentioned, that the proximal series of tarsals is in many cases immovably attached to the tibia (or fused with it); the tibia has a long projecting ridge on its front face. The forms of the femur and tibia are very different from those of other Reptiles and very birdlike. (See also the remarks made below upon avian skeletons.) The Dinosaurians lived in Triassic, Jurassic, and Cretaceous times.

\section{Class 5. Aves (Birds).}

The most striking peculiarity of the avian body lies in the structure of the $\mathrm{limbs}$, the hind are exclusively developed for walking, or hopping (sometimes, also, for swimming), whilst the fore 
limbs are never adapted for terrestrial lócornotion, but, with few exceptions, for flight. The body is usually borne upon the hind limbs in a semi-erect position; it rests only upon the toes, not on the very slender metatarsus. The neck is of considerable length and very movable, the trunk is short; the tail in all existing Birds is short, but the large steering feathers* give it an appearance of greater length. The face portion, the beak, is peculiarly modified; it is usually elongate, and covered with a horny sheath.

The skin is almost entirely covered with feathers, complicated appendages consisting of cornified epidermis cells. They arise as dermal papillæe but soon sink into sac-like pits in the skin, the feather follicles; the feather develops from the epidermal layer covering the papilla.t Feathers exhibit a great diversity of form. The contour feathers (pennce) are firm, the distal parts at least reaching the surface and forming the outer contour of the bird (in distinction to the down which lies below); they consist of the

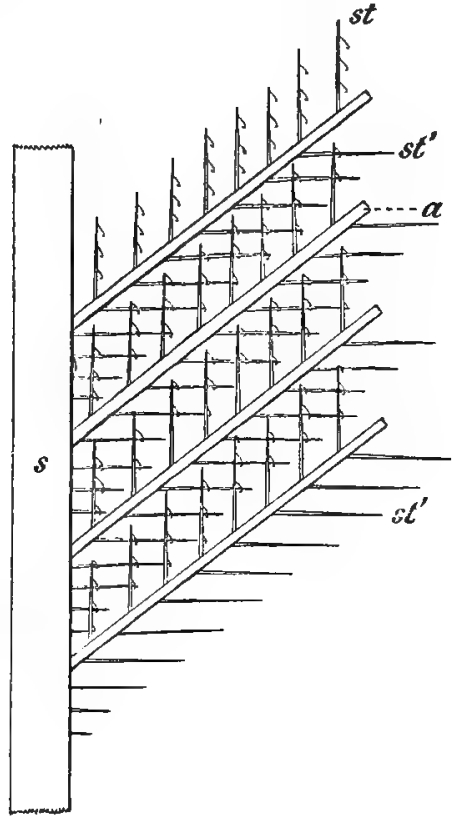

Fig. 357. Portion of a feather: diagrammatic. $s$ shaft, $\alpha$ barb, st barbules of an anterior row with hooks, st' barbules of posterior rows; the former cover the latter and seize them by means of the hooks on the edges.--Orig. following parts: the proximal portion is a short, cylindrical, hollow quill (calamus) which is imbedded in the feather-follicle, a more or less deep dermal pit. The quill is continued into the shaft (rachis) which consists externally of a hard cuticle, within of a loose horny mass; and is thinner at the tip. (Quill and shaft together form the stem [scapus] of the feather.) From the shaft there arises on each side a series of $\mathrm{b} a \mathrm{rbs}$ which are again furnished with barbules; the barbs and shaft together, are termed the $\mathrm{vane}$ (vexillum). At the $\mathrm{d}$ is $\mathrm{tal}$ portion of the shaft, and this may form a larger or smaller portion of the feather, the barbs are stiff and compressed (their flat surfaces turned towards those of neighbouring

* When, in the description of Birds, a long or short tail is mentioned, this always refers to the length of the steering feathers.

+ It is, however, only the first plumage of young Bixds, which develops as papillæ on the surface; the later feathers arise as similar papillæ, which lie, however, at the base of the follicle of the preceding feathers. 
barbs), and furnished with relatively short barbules, of which, those on the anterior row, lie obliquely above the posterior row of the preceding barb. Further, each barbule of the anterior row is provided with a series of delicate hair-like microscopic appendages, some of which are curved at their tips, to hook into the hinder row of the preceding barb; by means of these hooklets, the barbs are attached together into a continuous lamina. In the proximal

Fig 358.
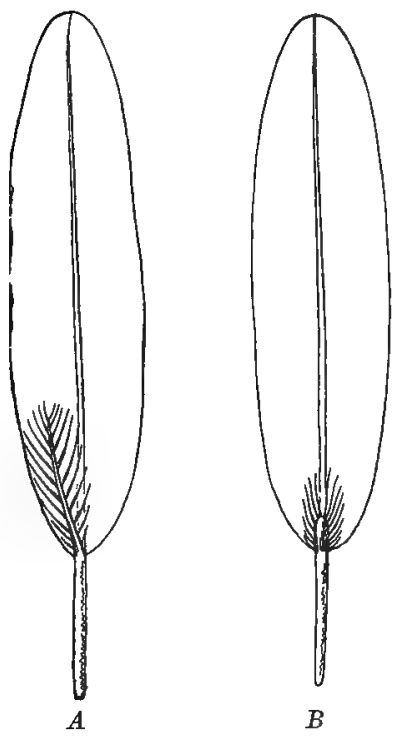

Fig. 359 .
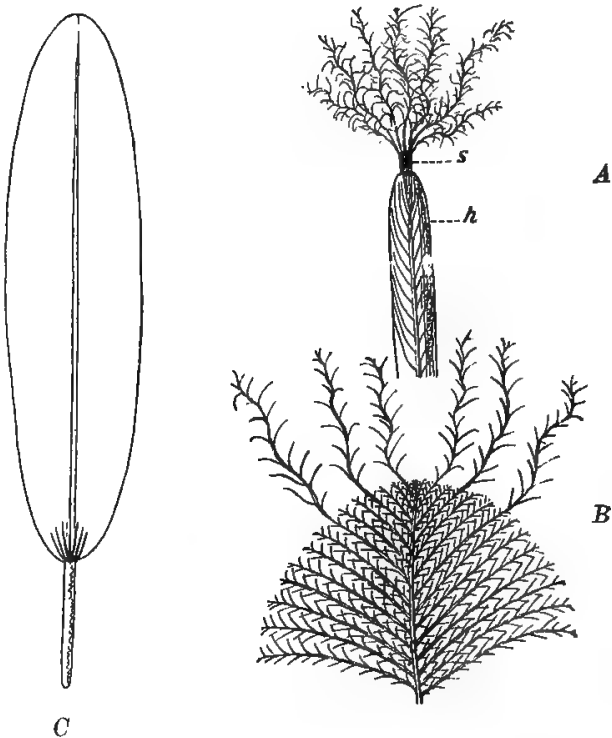

Fig. 358. Diagrams of various feathers to elucidate the varied development of the afters haft; vane only in outline.-Orig.

Fig. 359. A Down of a young Bird, resting upon the tip of the succeeding feather which is still surrounded by a horny sheath $(h) . s$ quill of the down feather. $B$ tip of a contour feather with some down barbs atill attached. Diagrammatic-Orig.

portion of the vane, the barbs are softer and thinner, the barbules long and soft, but without hooks; this portion, which is covered by other feathers, has thus a soft, loose, down y character. At the junction of quill and shaft, there usually arises from the inner side of the feather, a smaller, thinner shaft (the aftershaft), which bears a double row of soft barbs; the after shaft and its barbs may be termed the accessory vane. - This appendage is sometimes welldeveloped (e.g., Fowls, Fig. $358 \mathrm{~A}$ ) ; usually, however, it is somewhat feeble $(B)$; its shaft is often rudimentary, so that the accessory vane is only represented by a tuft of barbs arising close together $(C)$. Of the contour feathers, the flight and steering feathers (remiges, rectrices) must be noticed, the strongest, stiffest, usually 
longest feathers on the body: as a rule, they have no aftershafts, and the down-like proximal portion is very small, or is absent; they lie in very deep feather-follicles, the remiges in a row along the outer edge of the fore-arm and hand, the rectrices on the tail.

In the extinct Archæopteryx, the rectrices were arranged in two long itudinal rows, one on either side of the long tail. In some existing Birds also, they occur in two distinct oblique rows on the much shortened tail; in others, this part is so very short that the longitudinal series form a curved transverse row.

The down feathers (plumce), which are generally completely covered by the contour feathers, differ from them, in that the whole vexillum is similar to the proximal portion in the latter; they consist of soft barbs, which are often very long and beset with long barbules without hooks, the shaft is thin and feeble, often even quite rudimentary, so that the barbs arise close together at the distal end of the quill. There is often an aftershaft on the down feathers; not infrequently it is almost as large as the main shaft. The down feathers are usually whitish or grey, whilst the contour feathers vary much in colour. The two types described pass gradually into each other; there are plumæ which, in virtue of their

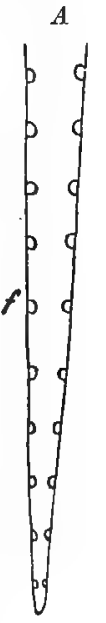
$A$

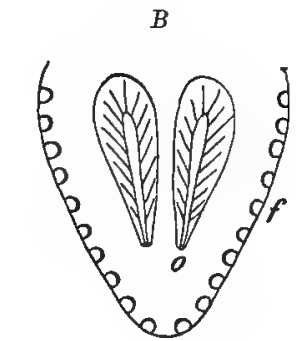

Fig. 360. Tail of: A Archoopteryx, $B \mathrm{Sw}$ an, $C$ Hen, seen from above. Diagrams to show the arrangement of the rectrices. $f$ apertures of feather follicles, 0 apertures of uropygial glands (in $B$ the latter are diagrammatically indicated).-Orig. strong shafts, etc., approach the pennæ, and modified pennæ which are so loose and soft, or which possess so small a portion with hooks, that they form a transition to the plumæ.

A special form of pluma is the so-called filoplume, a delicate feather with long thin shaft, in which the barbs are few in number, and at the tip of the shaft only; they occur in almost all Birds, arising close to the contour feathers.

Among peculiarly developed feathers, the following may be mentioned: ospreys, which grow on certain parts of the head in many Birds and have no barbs, or only in few, at the base of the shaft; the pennæ, in the Ratitæ, from which hooks are entirely absent, even from the stiff distal barbs, and which, in the Cassowaries and Emeus, are further remarkable in that the main and aftershafts are equally well developed; the remiges of the Cassowary, in which the long stiff shafts are entirely without barbs.

Newly hatched Birds are, as is well known, usually covered, over larger or smaller tracts, with down feathers, which generally consist only of a short quill and a tuft of barbs (Fig. 359 A); occasionally (Ducks, Ostriches), besides the quill, there is a thin shaft with barbs. These down-feathers are connected 
with the distal ends of the succeeding pennæ, and in many Birds the loose down quill is split by the developing feather in such a way, that the soft down barbs remain for some time at the tip of the distal barbs of the adult feather, looking like a soft external portion (Fig. $359 \mathrm{~B}$ ). The down is really to be regarded as a modified distal extremity of the feather to which it is attached.

The contour feathers are not usually distributed regularly over the whole body, but only over certain regions, the so-called feather tracts (pterylce), which are regularly, but somewhat differently, arranged in different birds; there is, for instance, a pteryla along the mid-dorsal line, another on the outer side of the thigh, etc. The intermediate tracts (apteria), bear down feathers, which are also present in the feather tracts, between the pennæ, or they are entirely destitute of feathers; the apteria are covered by the contour feathers of neighbouring tracts. There is an almost equal distribution of feathers over the whole body in the Ratitæ (where there is no down unless the entire plumage be regarded as down feathers), in the Penguins and a few others.

At regular intervals, usually once a year, all the feathers are thrown off, and are simultaneously replaced by new ones; this is known as moulting; in northern Birds it usually takes place in the course of a fow weeks in the a tumn. Besides this, a spring moult often occurs; many Birds indeed, change some of their feathers in spring, so that there is a partial (rarely a complete) ecdysis; in many cases, however, the distinction between winter and summer plumage is dependent upon this second moult. In some, the specially coloured edges of many feathers are thrown off in the spring, and the appearance of the plumage is thus considerably changed; in others there is an actual change in the colour of the feathers themselves, sometimes to a striking extent. This change of colour is the more remarkable since the feathers consist exclusively of horny material, and thus represent a dead portion of the body; the alterations are probably to be attributed to chemical changes, independent of the vital functions.

The feather is surrounded, for some time after its tip has projected from the feather-follicle, by a thin tubular horny sheath, which binds the barbs together; this sheath is gradually thrown off. The so-called "powder down," which is found on some Birds, e.g., the Herons, is composed of feathers which grow continuously from the feather-follicle (just as the teeth of certain Mammalia grow from persistent pulps), whilst at the same time their free ends are worn off; the powder comes from the crumbling away of the homy sheath, which is constantly renewed at the proximal end.

Attached to the follicles of the larger feathers there are ustally small muscles, by which, for example, the rectrices can be spread out like a fan, the ordinary pennæ raised, etc.

Feathers are probably to be regarded as modified reptilian scales; indications of this are afforded by their origin from papillæ. Birds also possess $t r u e s c a l e s$, exactly like those of the Reptiles, but occurring only on the hind feet; these scales are of various forms: 
knobs, plates, splints. The spur of the Cock and other male gallinaceous Birds, which is provided with an internal ossification firmly attached to the metatarsus, is a peculiar, large, conical scale; this spur is present in the Hen also, but usually as a simple, wart-like scale.* Claws are present on the toes of the hind foot; in perching forms they are long, curved, and pointed; in ground Birds, shorter and thicker. 'They are often entirely absent from the fore limbs; but there is frequently a small, often rudimentary, claw on the pollex, and not seldom there is another such claw on the second digit, but this is generally rudimentary; it may sometimes be present in the young one, and lost later; in the Ostriches of Africa both claws are of fair size and well developed. In all living forms the claw of the third finger is wanting; but in Archæopteryx well-developed claws are present on all three digits, a fact which can be determined with certainty from the form of the last finger (Fig. 378). The edges of the jaw and the adjacent parts of the head, the beak, are usually covered by a thick, hard mass of horn, often with sharp edges; occasionally this is partly or entirely replaced by a thinner and softer horny sheath. An e cd ysis of the whole skin like that of many Reptiles, does not occur in Birds; the stratum corneum is thrown off in small portions.

Birds usually possess only on e pair of integumentary glands, the large, round u rop y gial glands, which are situated dorsally on the short tail; they lie close together, and their apertures are near each other, generally on a small papilla. Each gland consists of numerous tubes, opening into a large central cavity, which is continued into the duct; in some each gland has several ducts. They secrete a lubricating fluid, which the Birds remove with their bills for preening their feathers; they are largest in aquatic species, and are absent from the Ostriches, some Parrots, and a few others.

The skeleton. The vertebral column may be divided into regions similar to those of Reptiles. The cervical vertebræ are numerous; there may be as many as twenty, very loosely articulated with each other. The first and second vertebræ, as in the Reptilia, are developed as atlas and axis (the centrum of the atlas is fused with that of the axis, etc.). Further, the articular surfaces of the cervical vertebræ are saddle-shaped (each centrum is, anteriorly, concave from right to left, convex dorsoventrally, and, posteriorly, convex from right to left, concave dorsoventrally); in Archæopteryx and some of the Odontornithes (Ichthyornis) the surfaces of the centra were flat or feebly biconcave. For the cervical ribs see below. The thoracic vertebræ, in contradistinction to the cervicals, are somewhat few in number, and but slightly

* On the fore limbs too, similar spurs may also occur, but these must not be confused with the claws mentioned above. 
movable; sometimes, even, fused together; the articular surfaces of the centra are usually like those of the cervicals. The last, or the last two or three thoracics, the lumbar, sacral, and some of the

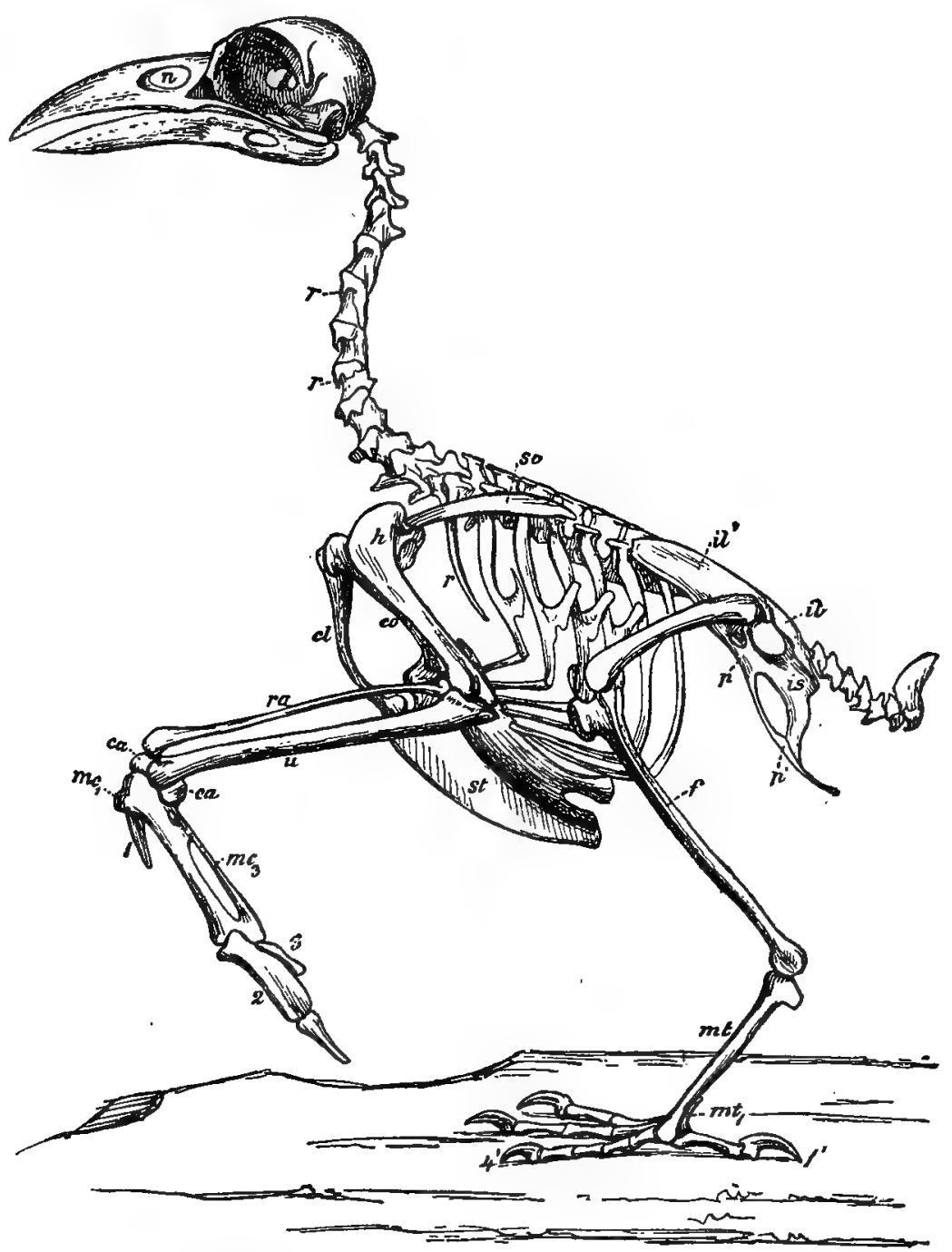

Fig. 361. Skeleton of a $\mathrm{r}$ a $v$ en. 1, 2, 3 first-third fingers, 1 ' and 4' first and fourth toes, ca carpus, $c l$ clavicle, co coracoid (nearly covered by $h$ ), $f$ fibula, $h$ humerus, $i l$ ilium, $i l^{\prime}$ anterior portion of this, is ischium, $m r_{1}$ and $m c_{3}$ first and third metacarpals, $m t$ large metatarsals (consisting of the fused 'metatarsals 2-4), $m$ t first metatarsus, n nares, $p^{\prime}$ pubis, $r$ cervical ribs, $r a$ radius, $s c$ scapula, st sternum, $u$ ulna.-Orig.

caudal vertebræ, are fused together and furnish an attachment for the pelvis, whilst a distinct lumbar region is wanting, and the pelvis lies immediately behind the rib-bearing region. In all existing Birds 
the number of free caudal vertebræ* is small, usually from six to eight; the terminal bone, however, which is much compressed, is a fusion of several short vertebræ, separate in young Birds. The tail vertebræ are, like the tail itself, short; though in Archæopteryx (Fig. 378) there was a long, thin tail of a reptilian type, and consisting of a number of vertebræ, some of which were elongate. Short ribs are attached to the cervical vertebræ, and like the thoracic ribs have two articular processes; they are fused in the adult, though separate in young forms. The cervical ribs gradually increase in length posteriorly, and remain separate throughout life, thus affording a transition to the thoracic ribs, $\uparrow$ which consist of two bony portions connected at an angle; the ventral portion is attached to the sternum, and from the hinder edge of the dorsal part arjses a narrow oblique process (processus uncinatus), which overlaps the next rib; in young Birds it is a separate bone. The sternum is completely ossified and very large, covering the greater part (or at least a large part) of the ventral region of the body. It is almost always furnished with a large projecting keel, to which some of the muscles of flight are attached, and which is only absent from certain forms with rudimentary wings (e.g., Ratitæ); here the sternum itself is smaller than usual. Posteriorly it is often perforated or notched on either side, the gaps being covered with connective tissue. There is no episternum.

The skull usually resembles that of the Reptiles very closely; among living forms, especially that of the Lizards; there is only one occipital condyle; the quadrate is reptilian, as also the conditions of the palatine and pterygoid; the region between the large orbits is compressed into a perpendicular bony lamina, the interorbital or orbital plate, which may be partially membranous. The prominent premaxillæ, which fuse early to form a single bone, are characteristic of Birds; they form the entire edge of the beak, and also send a long branch, almost to the frontals, between the external nares. The maxillæ, on the other hand, are relatively small, and lie within the posterior portions of the premaxillæ. The lower end of the large, very movable quadrate is connected with the beak (the premaxilla and maxilla) by a bony bridge formed of the pterygoid posteriorly, the palatine anteriorly; the pterygoid and palatine are both elongate bones, and in most Birds they slide upon the thickened lower rim of the orbital plate mentioned above at their point of contact. From the lower end of the quadrate to the beak there runs yet another bony bridge, the

* The caudal centra are biplanar.

+ Usually, the first vertebra bearing ribs attached to the sternum, is termed the first thoracic; the transition from cervicals to thoracics is, however, quite gradual, and this distinction is artificial. 
jugal, external to that already noticed; it is a thin rod of bone, and is formed posteriorly by the quadratojugal, medianly by the jugal, and anteriorly by a process of the maxilla; in the adult these bones are often anchylosed. The beak is attacbed above to the rest of the skull, by the upper portion of the pre-

Fig. 362.

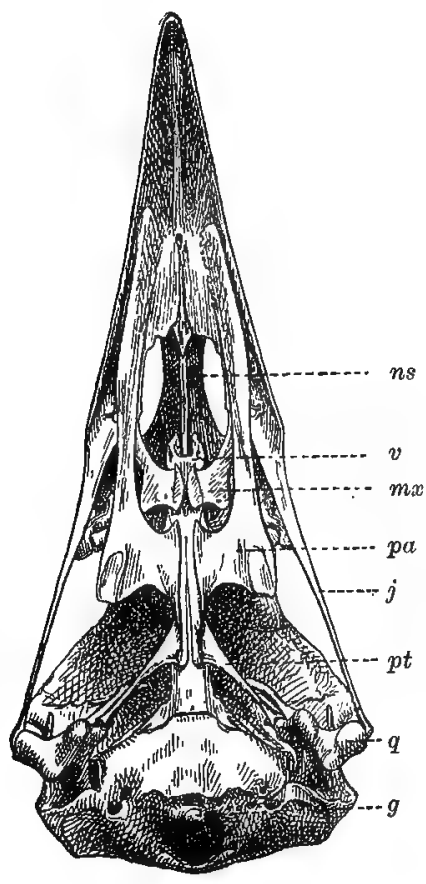

Fig. 363.

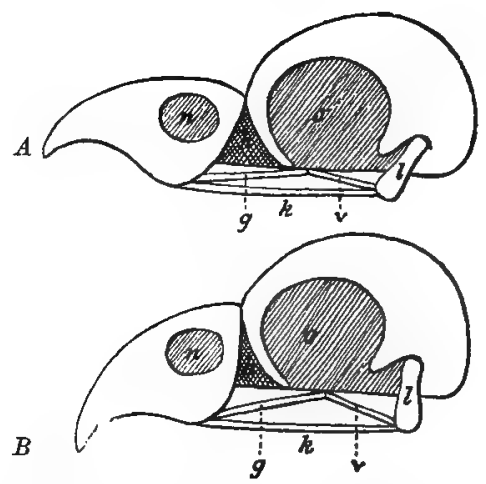

Fig. 362. Skull of a Raven from the ventral side. $g$ foramen magnum, $j$ jugal, $m x$ maxilla, $n s$ nasal septum, $p a$ palatine, $p t$ pterygoid, $q$ quadrate, $v$ vomer. - Orig.

Fig. 363. Diagrammatic figures to illustrate the movement of the beak in Birds. $n$ nasal septum, $h$ posterior membranous portion of this, $o$ orbit, $l$ quadrate, $k$ jugal, $v$ pterygoid, $g$ palatine. In $A$ the bill is raised, in $B$ lowered.-Orig.

maxilla, and by the nasal, which lies behind the nares; the posterior portions of these bones (premaxillæ and nasals) are, howerer, flattened, thin, and elastic,* and since the lower part of the nasal septum is membranous, Birds are able to move the beak up and down. The movement upwards is effected by the forward motion of the lower end of the quadrate, by which the two bony bridges are pushed forwards, and pressed against the lower, hinder portion of the beak, so as to send its point upwards; the movement downwards, on the other hand, is the result of the retraction of the quadrate. As for other characters, it may be noticed, that most of the sutures have disappeared, even in the young animal, owing to the fusion of the bones; further the cranial-cavity is very large as compared with that of most Reptiles.

* In the Parrots and some others which have a specially movable beak, this bon $\bar{y}$ mass is interrupted by a narrow strip of connective tissue. 
The skull consists of almost the same bones as that of Reptiles (but preand post-frontals, transverse bone, and columella are always wanting). The vomer is an unpaired bone of varied form, sometimes compressed, sometimes fairly broad, etc., lying below the hinder portion of the nasal septum, and connected behind with the palatine, in the movement of which it takes part. The lachrymal lies at the anterior edge of the orbit, and in many Birds remains distinct throughout life. The low er $\mathrm{j}$ a w consists of several bones on each side, of which the anterior (dentary), in all living Birds, fuses very early with its fellow of the other side; in extinct toothed forms (Odontornithes), on the contrary, they remained separate.

Fig. 364.

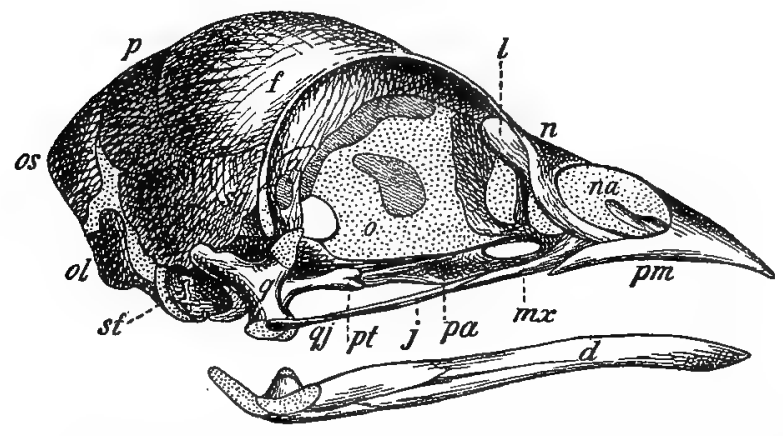

Fig. 364. Skull of two days old Chick. $a$ alisphenoid, $d$ dentary, $f$ frontal, $j$ jugal, $l$ lachrymal, $m x$ maxilla, $n$ nasal, $n a$ outer cartilaginous wall of the nasal cavity, o orbital plate, of ex-, os supraoccipital, $p$ parietal, $p a$ palatine, $p m$ premaxilla, pt pterygoid, $q$ quadrate, $q j$ quadratojugal, $s q$ squamosal, st ear bones. The parts which are still cartilaginous are dotted, the membranous portions shaded.-After K. Parker.

Fig. 365. Hyoid of the Fow 1 . $h$ hyoid (anterior horn), $b r$ first branchial arch (posterior horn).-After K. Parker.
Fig. 365.

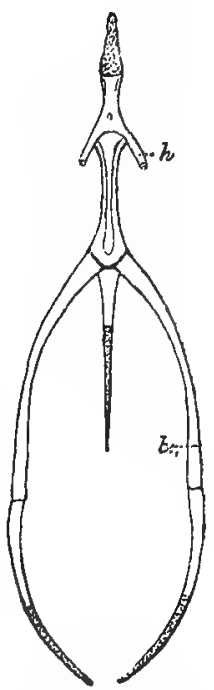

The hyoid consists of an unpaired, partially ossified median rod (copulæ), to which is added (not invariably) a pair of very short anterior cornua, and a pair of long posterior cornua, the first branchial arch. The median rod is usually divided into two or three pieces, lying one behind the other. The cornua are not closely connected with the skull; the posterior pair bend round the back part of the skull, but in the Woodpeckers, where the tongue is specially protrusible, they curve up more anteriorly, even as far forward as the base of the beak.

The shoulder girdle (Fig. 366) consists of the usual elements. Both scapula and coracoid are completely ossified; the former is a flat, narrow, sabre-shaped bone, which is generally connected with the coracoid at an acute or right angle; the coracoid is, as a rule, rather long and narrow as compared with that of the Reptiles, but yet very strong; its lower broad end articulates with the anterior edge of the sternum. In Ratitæ, and in some Odontornithes, which were also incapable of light, the two bones lie more 
nearly in a line, and fuse with age; in these forms, the coracoid is short and broad. The clavicles are two long thin bones, fused ventrally (in most Birds) to form the merrythought (furcula), which is attached to the anterior end of the sternal keel, by a ligament, passing from the point of fusion. Each of the other extremities of the fork is attached to the dorsal end of one of the coracoids, from which it is otherwise separated by a large fenestra. In the Ratitæ and some others, the clavicles are rudimentary or

Fig. 366.

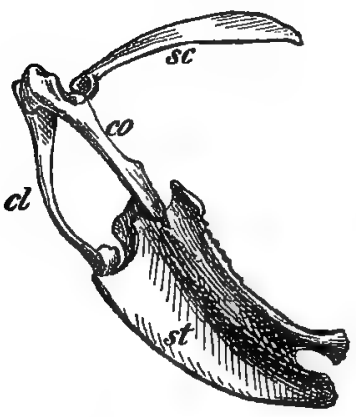

Fig. 366. Sternum and shoulder girdle of the Raven, viewed from the left side. $c l$ clavicle, $c o$ coracoid, sc scapula, st sternum.Orig.

Fig. 367. Manus of a young Ostrich (Struthio). $a, b$ carpals which remain separate, $c$ carpals of distal row (already united, later to be fused with the metacarpals), $m^{1}, m^{2}, m^{3}$ first- third metacarpals (still distinct), $r$ radius, $u$ ulna, $1 a$ and $1 b$ phalanges of the pollex, $2 a-2 c$ second digits, 3 third digit--Orig.

Fig. 367.

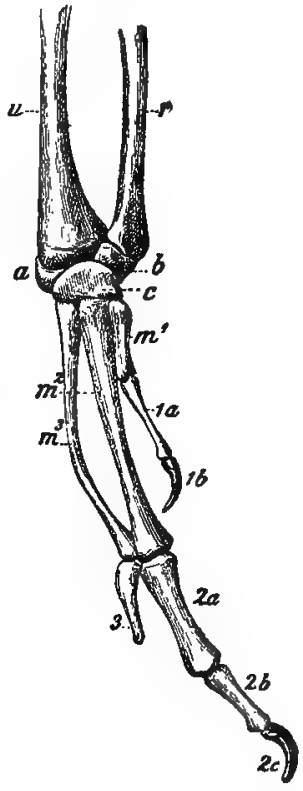

Fig. 368. Foot of a young $\mathrm{Chick}, m^{1-3}$ first-
Fig. 368.

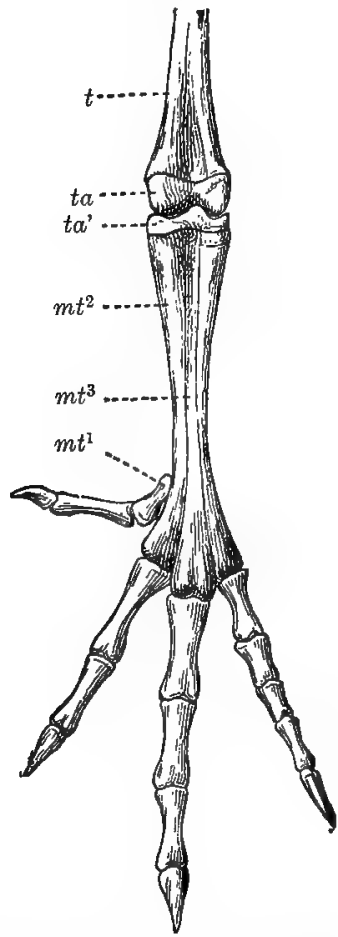
third metatarsals (the metatarsals $2-4$ are already fused, but there are traces of their original distinctness), $t$ tibia, $t a$ and $t a^{\prime}$ proximinal distal portion of the tarsus. - Orig.

absent. The fore $l i m b$ is usually very long. The $u l n a$ is much more powerful than the radius; the carpus of the adult consists of two separate bones only.* The manus is very slim, and never exhibits more than three fingers with their metacarpals ; the fourth and fifth fingers of Reptiles are absent. Of the three metacarpals, the first is short, the two others much longer; all three are fused in the adult, the second and third, however, only at the two ends, not

* One of these is the radiale, the other corresponds to the ulnare and intermedium. The carpals of the distal row are represented by two bones which fuse with the metacarpus. 
for the whole length; only in Archæopteryx were they separate. The pollex has one or two joints, the second digit two or three, the third only one (rarely two); in Archæopteryx alone, the third possessed three or perhaps four joints. In Birds which are capable of flight, the arm when at rest lies along the body with the elbow backwards; the forearm is directed anteriorly, and lies along the arm, the ulna outwards, and the hand is curved at the wrist, so that it lies along, and external to, the forearm, with the point backwards, the inner edge (with the pollex) outwards.

The pelvis closely resembles that of the Dinosaurians, but it is further specialised (cf., p. 430). The ilium is elongate, and is fused with a number of vertebræ ( $f f .$, p. 436$)$; it is connected with the other two bones at the acetabulum, which lies at its lower edge, and in the formation of which, all three take part; they are all fused in the adult. The ischium, a strong bone directed backwards, -runs almost parallel to the posterior portion of the ilium; in most

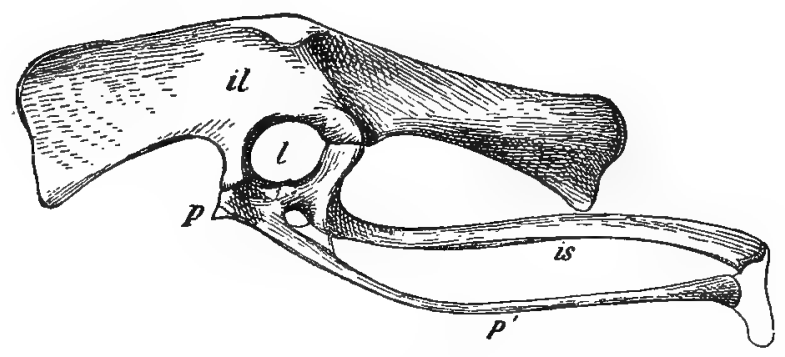

Fig. 369. Pelvis of a young American Ostrich (Rhea). il ilium, is ischium, $l$ acetabulum (with large perforation), $p-p^{\prime}$ pubis.-Orig.

Birds, its hinder portion is firmly anchylosed in old age with the ilium, whilst in Odontornithes and Struthious Birds it is either quite free or only fused with the ilium posteriorly. The most remarkable part of the avian pelvis is, however, the $\mathrm{pubis}$. This is a long thin bone stretching backwards parallel to the ischium, with which it usually partially anchylosed. In some Birds (Odontornithes, Ratitæ, Gallinaceæ), there is a short process ( $p$, Fig. 369) at the upper end of the pelvis, just in front of the acetabulum; but it is usually absent. It corresponds to the chief part of the pubis of Dinosaurians, and to that bone itself in other Reptiles, whilst the rest of the pubis $\left(p^{\prime}\right)$ corresponds with the posterior pubic process of Dinosaurians. Further, it must be noticed that the pelvis of Birds is almost always quite open below, since neither the ischium nor the pubis is fused with its fellow. In the hind limb, the femur is relatively short; the fibula thin, imperfect, and pointed at its lower end (except in Archæopteryx) ; the tibia is long and strong, and is furnished above, on the anterior surface, with a 
well-developed crest (a similar crest occurs in the Dinosaurians, but is very feeble in other Reptiles). There is usually a patella. The structure of the tarsus is interesting. As in the Reptiles, it is divided into proximal and distal portions, between which there is a very perfect joint; the tarsals of the proximal row are distinct from the tibia in the young animal, but in the adult are fused so as to leave no trace. In the same way, the distal portion fuses with the metatarsus, so that in the adult there is apparently no tarsus. The foot never consists of more than four toes, there is never any trace of the fifth or of its metatarsal. Metatarsals two, three, and four, are long, and only separate in the embryo; later they fuse almost duwn to the toes, furming a long thin bone (the tarso-metatarsus); on the other hand, the first metatarsal is separate, but much shorter than the others, and attached to these at its distal end. The hallux which is usually directed backwards, consists of two phalanges, the second digit of three, the third of four, and the fourth of five. (as a rule); all as in Lacertilia and Dinosauria. Of the forwardly directed toes the middle one (No. 3) is usually the longest; the hallux may be large, but is often rudimentary or absent.

The brain, in comparison with that of the Reptiles, is large. The hemispheres, especially, are well developed; the cere-
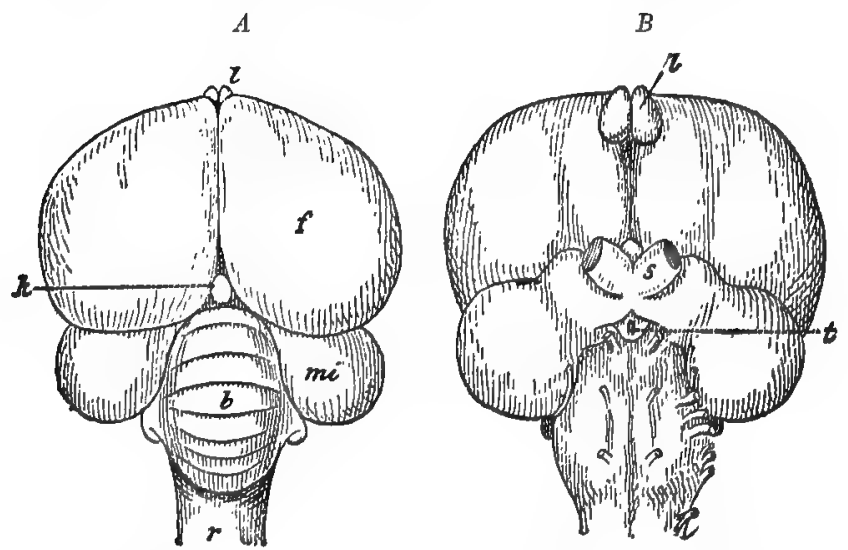

Fig. 370. Brain of a Pigeon, dorsal $(A)$, and ventral $(B) . \quad b$ cerebellum, $f$ cerebrum, $l$ epiphysis, $l$ olfactory lobes, mi mid-brain, $r$ spinal cord, $s$ optic nerve, $t$ hypophysis.-After Jeffery Parker.

$\mathrm{bellum}$ is likewise large; its median portion, which is elongate, and furnished with deep transverse furrows, covers both the medulla and the middle portion of the mid-brain, the two lobes of which are pushed out to the sides. Of living Reptiles, the Crocodiles come nearest to the Birds in regard to the development of the brain.

The olfactory organ is very like that of Lizards; the external nares, on account of the length of the premaxillæ, are usually 
some distance from the tip of the beak, and may even be at its base; the interual nares open into the mouth, rather far forwards, in a groove, which is partly covered by lateral longitudinal folds (cf. Reptilia). On the outer wall of the nasal-cavity there is a cartilaginous projection often spirally folded; which corresponds with the $t$ urbinal of Reptilia; there are also two other more or less well-developed folds.* The " $\mathrm{y} \theta$ and its accessory structures are also very like those of Reptiles. The optic bulb is of very considerable size. The front wall of the sclerotic, in which there is a circle of bony plates (the sclerotic ring), has the form of a frustrum of a cone; the absent apical portion of the cone is replaced by the cornea, which is often rery convex, whilst its base is formed by the posterior curved portion of the sclerotic; if the frustrum is long, and its wall, as sometimes happens (e.g., in the Owls, Fig. 371, B) is somewhat arched inwards, the form of the eyeball differs much from the

$A$

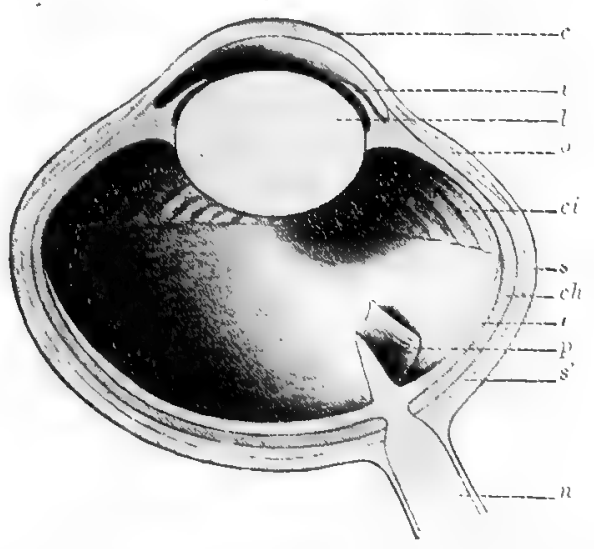

$B$

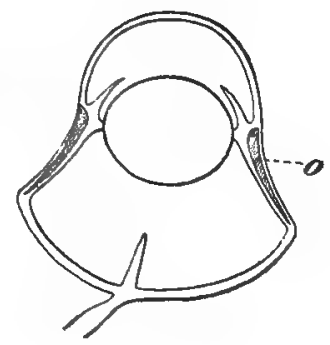

Fig. 371.- A E ye of a Bird, dingrammatio transverse section. e cornea, ch choroid, ci viliary processes, $i$ iris, $l$ lens, $n$ optic nerve, o transrerse section of bony plates, $p$ peoten, $r$ retina: $s$ external conneotive tissue portion, $s$ internal cartilaginous portion of the solerotio. $B$ Seotion through the eye of an $\mathrm{OWl}$, in order to show its peculiar shape.-Orig.

ordinary spherical type, whilst in others it deviates but little from this. A large membranous process, which is folded and pigmented, the pecten, arises from the retina at the point of entrance of the optic nerve, and projects freely into the vitreous humour. Of the two eyelids, the lower is much larger and more movable than the upper (as in Reptiles); there is a well-developed nictitating membrane, which can be flicked across the eye by a special muscle. The Harderian gland opens at the anterior corner of the

* Into the nasal cavity opens the duct of a large $n$ a a $l$ gland, which usually lies abre the frontal bone (in Sea-gulls and others, in a longitudinal depression at the edge of the orbit); this gland ocours in many Reptiles, but is situated in a different region of the head. 
eye; at the posterior, a small lachrymal gland. As to the a u ditory organ, the membranous labyrinth, especially as regards the $\operatorname{cochlea}$, is very like that of the Crocodilia. There is a short external auditory meatus, at the base of which lies the tympanum (cf., Reptilia); its opening is covered by regularly-arranged feathers (a pinna, i.e., a movable flap of skin covering the opening, only

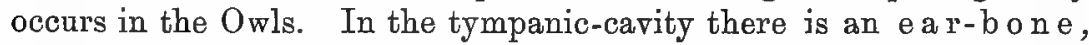
like that of Reptiles: it consists of a long rod, expanding at one end into a dise, which fits into the fenestra ovalis; whilst by the other end, which is provided with two or three cartilaginous processes, it is attached to the tympanum. A fenestra rotunda is present. The Eustachiantubes, which are partially enclosed in the wall of the skull (sphenoid bone), are united with each other, and open into the mouth by a single aperture.

Alimentary canal. There are no traces of $\mathrm{teeth}^{*}$ in any living Birds, but in Archæopteryx and the Odontornithes, there were simple conical teeth, placed in sockets on the edges of the jaws as in the Crocodiles; in some of the Odontornithes, the sockets become confluent, so as to form a continuous groove in each jaw, a condition which may also be observed in some Mammalia. The roof of the $\mathrm{mouth}$ is usually provided with backwardly directed spiny processes. The tongue is generally flattened, narrow, stiff, and hard, and covered with a thick, hard cuticle, which is especially well-developed at the anterior, usually pointed, end; occasionally it is thick and soft, as in the Parrots and the Flamingo; not infrequently it is rough or prickly. The œsophagus is of considerable length and fairly wide. In many, but by no means in all Birds, it widens out at the base of the neck to form a crop, in some a simple expansion of the osophagus, from which it is not sharply marked off; in others, a more definite sac opening into the œesophagus. It usually serves merely as a storage for food, but in Pigeons, it has another function: here, during the breeding season (in cock as well as in hen), the stratified epithelium lining it becomes thickened, the superficial cells filled with oil globules break away, and form a crumby liquid with which the fledglings are fed. The $\mathrm{stomach}$ of Birds is divided into two, usually rather sharply defined, chambers, a glandular and a muscular portion. The $\mathrm{glandular}$ sto $\mathrm{mach}$ is a short tube which appears as a direct, somewhat widened continuation of the osophagus; embedded in its walls are numerous glands of two kinds: (1) large, close-set glands secreting a digestive fluid, either distributed over the whole wall, or limited to definite regions; and (2) quite small tubular glands, which secrete a mucous covering for the whole inner surface of this region. Lower down, at its junction with the next

* The "teeth" along the edge of the beak in, for instance, Merganser (Mergus), are dentiform processes of the edge of the beak, and are thus horny structures. 
part, the large glands are wanting, the lining becomes firmer, and gradually passes into that of the muscular stomach. 'L'his is short and saccular, and has muscular walls; it possesses glands of one kind only, namely, simple, close-set glandular pits, like those which secrete the mucous layer of the other chamber. Their secretion is, however, very peculiar; each gland forms a hard, horny thread, which projects from its mouth, and adhering to neighbouring threads, forms a horny lining; ${ }^{*}$ it is continually being worn away at its surface, and renewed by fresh secretion at the base of the threads. On the outer surface of the muscular stomach, there is a dorsal and a ventral tendinous disc, from which the muscular elements arise. The muscles of the wall are especially strong in herbivorous, notably in graminivorous species (e.g., Fowls and Ducks), in which this organ is provided with a large muscle mass, planoconvex from within outwards; whilst its cavity is very small. In such, the muscular stomach forms a true gizzard, in which the food may be ground by the two muscles just mentioned; the horny lining is very thick and hard, and sand and stones are swallowed to assist in trituration. In insectivorous and predaceous forms the muscular stomach, on the other hand, is thin walled, the musculature feeble, and the cavity large. Its openings into the small intestine, and into the glandular stomach, are close together. The small intestine is well-developed, and longest in the herbivorous forms; it is continued into a $\mathrm{rectum}$ which is almost invariably short, and which has a posterior widened portion, the cloaca. Two cæca usually open at the junction of the small intestine with the rectum, and in many herbivorous and omnivorous Birds they are of considerable length, whilst in the carnivorous forms they are usually quite short or rudimentary (this may also be the case in others; Pigeons, for instance, have quite short cæca).' 'There is a large brownish-red liver provided with a gall bladder, and an elongate whitish pancreas lying in the first loop of the small intestine. $\dagger$

Many Birds disgorge the indigestible portions of their food, bones, hairs, feathers, insect skeletons, etc., in small masses, the so-called pellets; the best known of such pellets are thrown up by the Owls, and usually consist of skin and bones of Mice, but various others disgorge similar ones: Swifts (insect remains), Kingfishers (fish bones), Ravens, etc.

Respiratory:organs. A. longitudinal slit in the mouth close behind the tongue leads into the larynx, which is continued

\footnotetext{
* In Birds of Prey and other carnivorous forms, the lining of the muscular stomach hàs a softer character.

+ In most young Birds, there opens on the dorsal wall of the cloaca, a small unpaired-sac-with-a-narrow-aperture, the-bursa-Fabricii, in the-wall of which lie small portions of epithelium, which are indeed constricted evaginations of the epithelium of the sac". "In older forms, "the bursa, of which the significance is unknown, is usually degenerate or absent In $^{\text {in }}$ Struthious Birds, instead of this, there is a large, undefined outgrowth of the cloaca.
} 
into a trachea of considerable length, furnished with numerous cartilaginous or bony rings, and dividing below into two branches, one for each lung. Unlike most other air-breathing Vertebrata there are no vocal cords in the larynx ; but most Birds possess a peculiar $10 \mathrm{w}$ e $\mathrm{x}$ larynx (syrinx) at the junction of the trachea, with the upper ends of the two large bronchi; and here the walls of the bronchi form membranous folds ( $m e$ and mi, Fig. 374), which are caused to vibrate by the exhalent current of air (for details see below). The lungs are spongy organs, closely apposed to the dorsal wall of the bodycavity ; in structure, they resemble very closely those of most Reptiles. A tubular continuation of the bronchus runs through the lung,

Fig. 372.

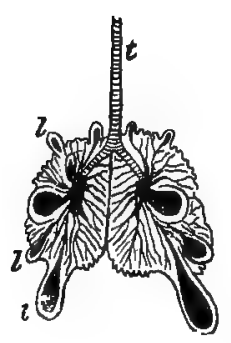

Fig. 373.

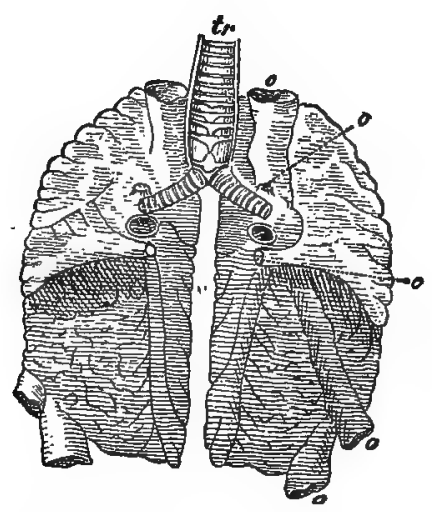

Fig. 372. Lungs of an eleven days Chick embryo. $t$ trachea, $l$ rudiments of branchial sacs.--After Selenka.

Fig. 373. Lungs of a Pigeon. $t r$ trachea, o apertures from the lungs into the sacs which have been removed.-After J. Parker.

dividing and sub-dividing on its way; the delicate terminal branches are the actual respiratory organs. All these branches are closely bound together by connective tissue; some of them are continued into large thin-walled air sinuses, which extend back between the viscera, between certain of the subcutaneous muscles, and even send long processes into many of the bones, e.g., the limb bones, in which they occupy the position of the marrow cavities; the bones are thus to a large extent $\mathrm{pneumatic}$. This development of air sacs is of significance, from the small specific gravity that the body thus acquires, in other words, the lungs of Birds, like the tracheæ of

* It may be mentioned here, that Birds are not the only animals with pneamatic bones; the Pterodactyles, and many Dinosaurians had them, which leads to the conclusion that they, also, were provided with air sacs. 
Insects, constitute not only a respiratory, but also an ärostatic apparatus.

The syrinx has usually the following structure: the two bronchi are separated at their upper ends, where they pass into the trachea, by a median bony rod, or septum, connected with the last tracheal ring. The inner wall of the anterior portion of each bronchus is membranous, and is termed the internal tympanic membrane. The outer side of the wall of the bronchus is strengthened by semicircles of cartilage or bone, and there is often here also a membranous portion, the external tympanic membrane, which, in other cases, may be replaced by a thickening of connective tissue, projecting into the lumen from one of the half hoops of cartilage. The lower end of the trachea itself, which may be termed the tympanum, is usually modified for the production of voice, the last rings, for instance, may be fused, or this portion is compressed or widened, etc. In the males of Merganser (Mergus) and most Ducks, the tympanum possesses a lateral saccular outgrowth with ossified walls, the res onator (or the labyrinth).

To these structures accessory to the respiratory system, the following may be added: in the Whistling Swan, the Crane, and others, the keel of the sternum is thick and hollowed, with an opening above; within this cavity the trachea makes a long loop before passing back into the body-cavity; in some other birds similar coils of the trachea lie below the skin or in the body-cavity: the tubular continuation of the bronchus

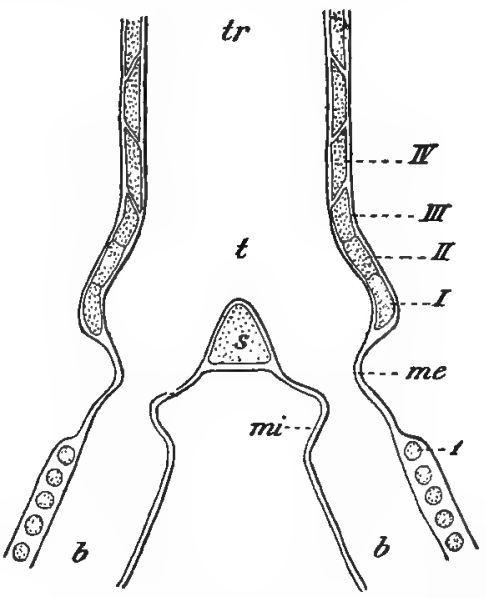

Fig. 374. Section through the lower end of the trachea and the upper ends of the two large bronchi of a Bird; diagrammatic. $b$ bronchi, me external, mi internal tympanic membranes, $s$ bony septum, $t$ drum, $t r$ trachea. I-IV the four lower rings of the trachea; 1 uppermost half-hoop of a bronchus.-Orig. which runs through the lung (see above), gives off branches from which numerous long parallel tubes arise, from these are given off close-set radial, somewhat racemose, tubules, which end blindly; these are all bound together by connective tissue, and thus form a thick layer round each of the parallel tubes: the air-sacs in the bones of the skull are extensions from the tympanic and nasal cavities: several other air-sacs of the head are connected with the latter (e.g., one in the orbit below the eye), these reach back into the neck, and, in some birds, communicate with the pulmonary air-sacs. Inspiration is brought about by movements of the ribs, resulting in a forward motion of the sternum and the widening of the bodycavity. Certain muscles, which arise from the inner side of the wall of the body-cavity (from the ribs and sternum), and are attached to a membrane which extends over the ventral surface of the lung, assist in the process, since by their contraction, the lungs are expanded.

The heart and the large arterial trunks arising from it, offer relations which may be shown to be a modification of those obtaining in the Crocodiles. Both auricle and ventricle are completely divided into right and left halves. The conus is wanting. The left aortic 
arch (left arterial arch of the second pair), which arises in Crocodiles from the right ventricle, is here altogether absent; the aorta is thus exclusively formed by the right arch arising from the left ventricle; in other respects the relations are like those of the Crocodiles. No mixing of arterial and venous blood occurs in Birds;

$A$

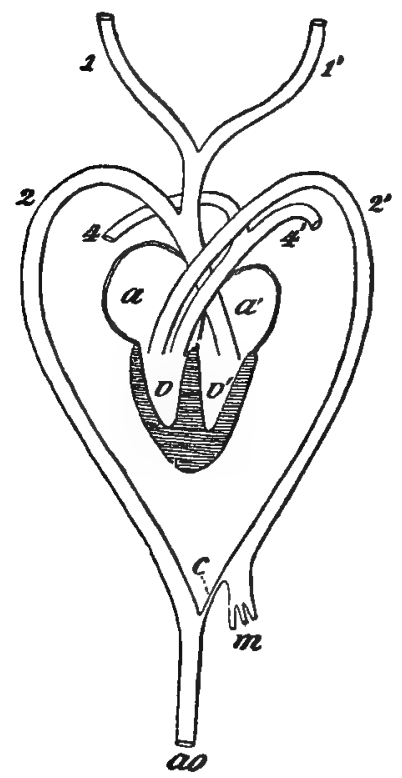

$B$

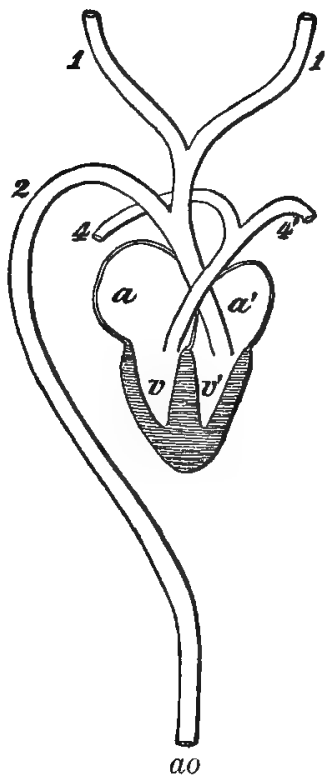

Fig, 375. Diagram of the heart and arterial arches of a Crocodile $(A)$ and of a B ird (B). a right, $a^{\prime}$ left auricle; $v$ right, $v^{\prime}$ left ventricle; ao aorta. 1,2, 41 st, 2 nd, and 4th arterial arches of the right side, $1^{\prime} 2^{\prime} 4^{\prime}$ the same of the left side ( $c$ and $m$ see Fig. 347).-Orig.

the venous blood enters the right auricle, goes thence into the right ventricle, and from the latter to the lungs; the arterial blood from the lungs goes to the left auricle, thence to the left ventricle, and so into the body.

The kidneys are long, dark-red bodies lying in the pelvic region, just below the vertebral column; they occupy the spaces between the transverse processes, and are divided ventrally by transverse constrictions into several (usually three) lobes. Sometimes the two glands fuse, to a greater or less extent, along their inner edges. The ureters open separately into the cloaca; a urinary bladder is absent. The urine is semi-solid and whitish.

Of the ovaries, only the left is developed; exceptionally there is howevel a rudimentary right one; in many diurnal Birds of Prey (Falcon, Sparrow-hawk, Buzzard) such a rudiment is large 
and fairly constant. On account of the size of the eggs the Graafian follicles project from the surface of the ovary, giving it a racemose appearance. The left oviduct (Müllerian duct), also, is alone well-developed, though a rudiment of the right may be present; during the breeding season the oviduct is long and wide, at other

Fig. 376.

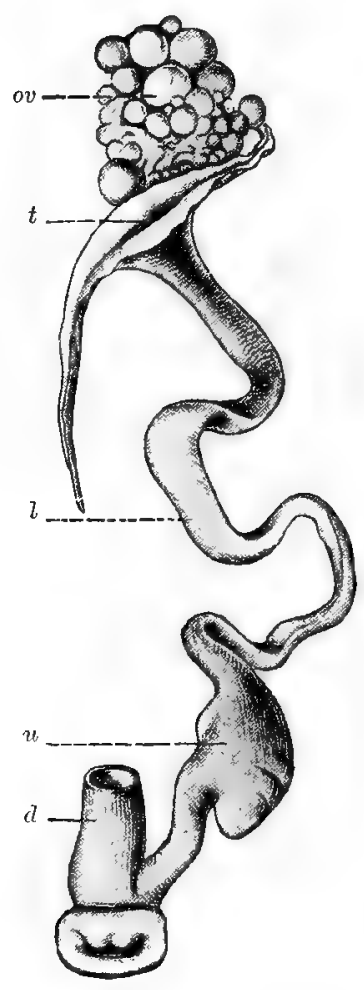

Fig. 377.

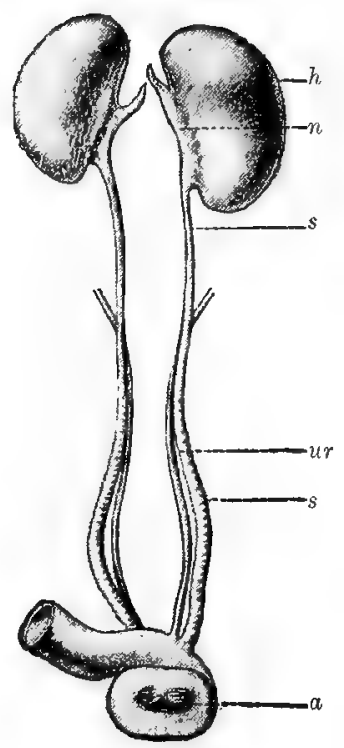

Fig. 376. Reproductive organs of a $H \in \mathbf{H}$. $a$ rectum, $l$ oviduct, ov ovary, $t$ funnel, $u$ uterus.Orig.

Fig. 377. Reproductive organs (and ureter) of a Cock; rectum turned to one side to show the place where the ureters and vasa deferentia open into the cloaca. $a$ anus, $h$ testis, $n$ epididymis, $s$ vas deferens, $u r$ ureter.-Orig.

times it is a narrow tube opening, by a large funnel, into the body-cavity; not far from the opening into the cloaca it widens out to form the uterus, where the shell is secreted. The testes, both of which are well developed (although sometimes the left is larger), lie in front of the kidneys; the vasa deferentia, each of which arises from a small epididymis, have a convoluted course, and open separately into the cloaca, usually on small papillæ. The testes are very small, except at the breeding season, when they attain a considerable size; this holds also for the ovaries. A definite $\mathrm{pen}$ is occurs only in the males of a small number, in Struthious Birds, Ducks, and some others; in the rest it is rudimentary or absent. 
It is homologous with the copulatory organ of Chelonia and Crocodilia; it is situated on the rentral wall of the cloaca; the free tip is directed backwards, and is provided with a superficial groove, at the anterior end of which the vas deferens opens, and along which the spermatozoa pass during coitus. In Ducks the penis is spiral, in others, linguiform, or rod-like; the tip is usually invaginable. Where the cock-birds have a penis, the hens usually have a rudimentary copulatory organ (clitoris).

Very generally larger or smaller external (secondary) s e x u a l d if f e r e n ces are noticeable: usually the males are somewhat larger (in Fowls, etc.), rarely smaller than the females (in Birds of Prey); the cocks are often distinguished by the special development of certain feathers (Peacock, Birds of Paradise), by peculiar cuticular processes (spur"of the Cock), or by virid colouring.

Most Birds breed only once a year (usually in the spring), others several times (e.g., the Honse Sparrow). As a rule, they live in pairs during the breeding season, i.e., they are monogamous; occasionally the male has several hens, or is polygamous.

The e g g $\mathrm{s}$ of Birds are of very considerable size, and contain a large amount of food yolk. As they pass down the oviducts they are covered first by a mass of albumen, then by a shell-membrane, and finally, in the uterus, by a hard calcareous shell; all the coverings are secreted by glands in the wall of the oviduct. The eggs are incubated by the females alone, or by the males and females together, rarely by the males only, this occurs in the African Ostrich and the Phalarope (Phalaropus); usually the sitting Bird is provided with brood spots, regions from which the feathers have fallen off, so that the eggs may come into direct contact with the warm skin. Most Birds build n est s for the reception of their eggs, but occasionally they are laid upon the bare ground. In the simplest cases the Birds drag together a scanty collection of twigs, straws, feathers, etc.; in others similar materials are woven into a basketshaped or spherical nest; occasionally the nest is built of clay, dirt, or the like, and saliva (Swallows and others), or from saliva alone (Salangane). The nests of some forms are built upon the ground, and others in excavations or in natural holes in the earth (Sandmartins, Puffins), in holes in trees (Woodpecker), on trees, etc. Usually the males and females build the nest together. As a rule the young Birds do not leave the nest immediately after hatching, but remain in it for some time, and are fed by the parents ("altrices"); occasionally ("præcoces") they are immediately able to feed themselves (usually, however, under the protection of the hen). The newly-hatched young one generally differs considerably from the adult; it is either covered with down or is almost naked, and differs in colour, and usually in the form of the beak (e.g., in many singing Birds) ; the food, too, is often different from that of the adult (for instance, many grain-eating Birds feed their young ones upon Insects). 
The plumage, which replaces the down feathers, is usually essentially different from that of the parent.

Many young Birds, like young Chelonia and Crocodilia, possess, on the upper side of the beak, a small hard cuticularised knob, with which they break through the egg (neb).

Whilst some Birds inhabit the same confined locality throughout the year, and may therefore be called residents, others undertake longer or shorter excursions or true migrations. Some wander about through a large district in search of food, and are most like the residents; others, according to the season, leave the mountains and betake themselves to the neighbouring valleys, or, impelled by necessity, exchange the forest for the open country, etc. The more conspicuous migrants travel greater distances, for they breed every year in a cold climate, and spend the winter in a warm country some way off. They follow special routes, which are arranged so that, as far as possible, only those countries which resemble the native habitat sball be touched at. Coast-birds usually take a course along the sea-coast, or, if necessary, along rivers; Marsh-birds go over marshy-ground, along rivers, etc. The same route is generally followed, whether going or returning. Most travel in large flocks, sometimes several species in company, and as old and young $f$ y together, the knowledge of the way is always handed on to, and preserved by, successive generations; Birds cannot find the way "instinctively," although in Birds of Passage an inberited indefinite instinct to wander may be noticed, which shows itself in restlessness in young caged individuals, at the time when migration occurs. Migrations from colder regions take place at different, but for each species definite, times, usually in the autumn, for some species even in August and July; the return occurs from February to May, those forms which are among the earliest to go away, returning last. Most of the Birds of Passage, which breed in Britain, winter in South Europe or North Africa.

It is easy to understand that wanderings in general, and also true migration, originated in the need for food; it has been noticed that certain forms which do not usually migrate, journey South in severe winters in search of food, whilst, in mild winters, some Birds of Passage remain in their breeding place: on the other hand, it may be observed, that migration is undertaken so instinctively by most Birds that they will start even in time of plenty, so that their wandering is no longer directly dependent upon the food supply.

Birds occur wherever there is life, although they are most abundant in the Tropics, and constitute at the present time a very numerous but fairly uniform class. Geologically this is the youngest of all vertebrate classes, since the oldest avian form known, a single species only, comes from the Jurassic, so that they were certainly very scarce at that time; a larger number occurs in the Cretaceous (all Odontornithes) and many in Tertiary formations. 


\section{Synopsis of the ORders of Aves.*}

1. Saururæ: caudal region of vertebral column longer than trunk; teeth present.

2. Odontornithes: caudal region shorter than trunk; teeth present.

The young ones are covered at first with a thick coat of down.

The young ones on hatching are almost naked and very helpless.
3. Ratitæ: wings not functional; pedes cursorii.

4. Rasores: short, slightly-curved beak; pedes gradarii; wing short and curved.

5. Natatores: pedes palmati.

6. Grallatores: pedes vadantes.

7. Raptatores: beak powerful and hooked; pedes insidentes.

8. Oscines: pedes fissi, ambulatorii, ad. hamantes.

9. Clamatores: pedes adhamantes, ambulatorii, fissi or gressorii.

10. Scans ores : pedes scansorii.
Hind toe usually small.

Hind toe usually well-developed

\section{Order 1. Saururæ.}

Of this order only a single species is known, Archoeopteryx lithographica, from the Jurassic (lithographic slates). Archæopteryx,

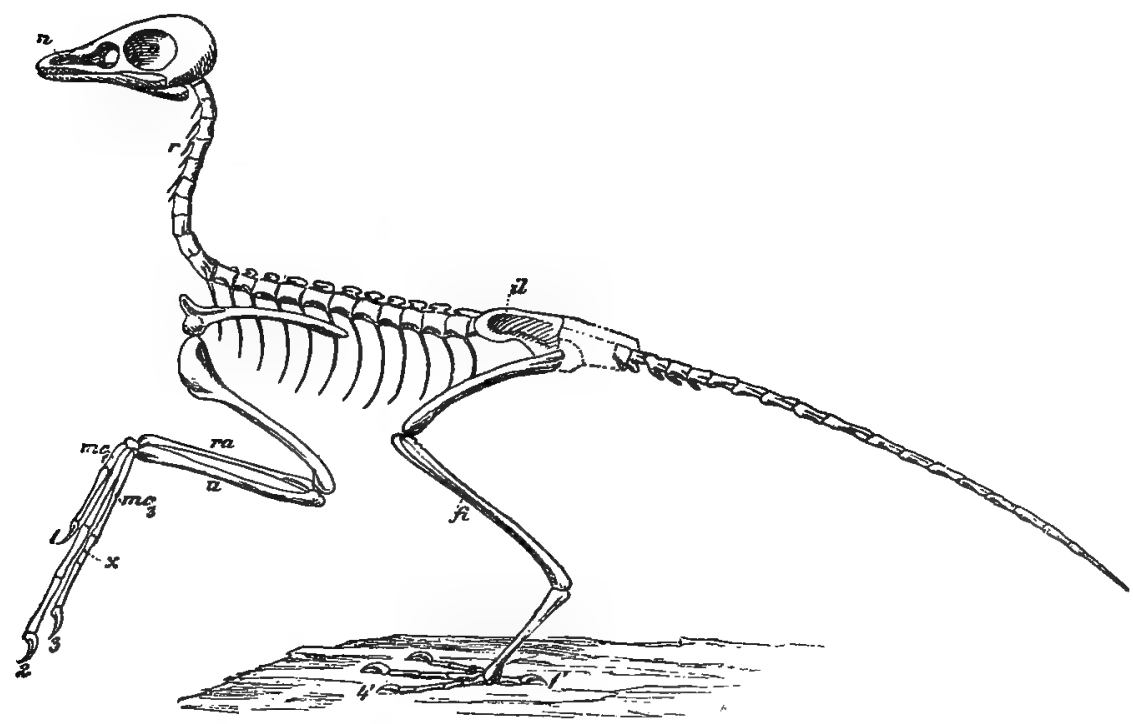

Fig. 378. Archeopteryx. 1-3 first to third fingers, $1^{\prime}$ first, $4^{\prime}$ fourth toe, $f$ fibula, il ilium, $m e_{1}$ first, $m e_{3}$ third metacaxpal, $n$ nares, o orbit, $r$ cervical rib, ra radius, $u$ ulna, $x$ is possibly a joint, but perhaps due to injury (according to the second interpretation the third finger has three joints, according to the former, four).- Orig. (with the use of figures by Dames).

* The systematic arrangement of Aves offers considerable difficulties, on account of their great uniformity; several of the orders given here are not natural groups.

+ For the significance of these terms, see the descriptions of the various orders. 
of all known Birds, stands nearest to Reptiles. It is characterised firstly by the very long tail consisting of twenty elongate vertebræ, to which the rectrices, known from impressions on the slates, were attached in a single row on either side: and further, by the separation of the metacarpals: by the three well-developed fingers furnished with claws (a point which may be recognised from the form of the last phalanx): and by the presence of conical teeth on the edges of the jaws. Among other characters it may be noticed that the somewhat thin thoracic ribs have apparently no uncinate processes; that the cervical ribs are longer; that the neck and pelvic regions are shorter; the sternal region, on the other hand, more extensive than in Aves in general (the thoracic vertebræ also appear to have been more freely articulated than is usual); that the surfaces of the centra are apparently biplanar (not saddle-shaped); and that the lower end of the fibula is complete, not simply ending in a point; it is even somewhat widened below. From the wellpreserved impressions of the large remiges it is proved that Archæopteryx must have been a good flier. Its size was about that of a Pigeon, but it is only known from two specimens, both incomplete ; and the sternum, pelvis, and coracoid are not made out, or only imperfectly.

\section{Order 2. Odontornithes (Toothed Birds).}

The Toothed Birds, of which several species are known from the Cretaceous of North America, are on the whole very like existing forms, though differing in the possession of teeth upon the edges of the jaws. Some of them (Ichthyornis) possess slightly biconcave centra; in others (Hesperornis), the vertebræ are similar to those of living Birds. The mandibular rami are not anchylosed anteriorly. The pelvis is characterised by the fact that the ilium and ischium are not fused posteriorly. For the rest, a considerable variety of forms is grouped under this name: some were able to fly, others possessed rudimentary wings like the Ratitæ.

\section{Order 3. Ratitæ (Strutlious Birds).}

The most prominent character of this order is the degenerate condition of the wings, which can never be used for flight, and, indeed, are often quite rudimentary. The sternum has no keel. The hind limbs, on which there is usually no hind toe, are generally very powerful, and are used for running; the claws are short and stumpy. The feathers are not arranged in tracts, but are fairly regularly distributed over the whole body (there are, however, naked portions, e.g., on the inner side of the fore limbs in the Ostrich and 
Rhea); there is no down between the feathers; remiges and retrices generally differ but little from the other feathers. There are no uropygial glands.

The Ratitæ are placed directly after Archæopteryx and the Odontornithes because, in several respects, they show more primitive characters than other living forms. For instance, the palatines do not touch the lower wall of the skull, but lie a little distance from the middle line, as in Lizards; a character which they have in common only with a small isolated group of the Rasores * (in Archæopteryx and the Odontornithes this portion is not preserved); the bones of the skull remain separate longer than in Carinates, as do also the cervical ribs and tarsal bones; the ischium does not fuse with the ilium (as in Odontornithes) or only quite posteriorly; the second digit of the fore limb possesses a fairly welldeveloped claw. In various other points, however, they are considerably specialised; the condition of the wings is clearly secondary (i.e., the Ratitre are descended from flying birds); the absence of the keel is a consequence of the loss of the power of flight and the degeneration of the pectoral muscles; so with the feathers, etc.

Most of the Ratites are of very considerable size; they are true prairie animals inhabiting the warmer portions of the Southern Hemisphere. They prefer a vegetable diet, but also eat small animals. The males undertake the whole or most of the brooding. The young ones are covered with down and are able to run about directly they are hatched.

1. Ostriches (Struthionidx). Beak short and broad, feathers without aftershaft; wings relatively well developed, with pollex and large feathers; smaller or larger feathers on the tail. Belonging to this family are: the American Ostrich or Nandu (Rhea) with three toes; in South America: the African Ostrich (Struthio Camelus) with only two toes (3 and 4) of which the inner bears a large claw, whilst the outer has no claw or only a small one; wings with very large, and tail also with fair-sized, feathers; in Africa and West Asia.

2. Cassowaries (Dromsidx). Beak short, main-shaft and aftershaft of equal size: wings very feeble, pollex absent, tail scarcely distinguishable; three toes. The Cassowary (Casuarius) with a bony ridge covered with horn on the top of the head; a compressed beak; and on each wing five long strong feathers without barbs : inhabiting New Guinea, the Moluccas, and the north of Australia. The Emeu (Dromzus) with flat beak; without crest and without naked feather shafts, occurring in Australia. To the same family belong the extinct Moas, some of which had a hind toe. They flourished in New Zealand several centuries ago, and some of them were of gigantic size (Dinornis and others).

3. K i w is (Apteryx) are small, short-legged, and short-necked birds, (about the size of a fowl) with long thin beak, on which the nares lie close to the tip; feathers without after-shafts; wings quite rudimentary ; hind limbs with a small hind toe. Their food consists essentially of Earthworms; in places where they live, the earth is riddled with borings made with the beak. They are nocturnal animals and brood in holes in the earth, which they dig out themselves; New Zealand.

* Namely, the Tinamous (Crypturidæ), a division of Rasores distinguished by a long beak; very short rectrices, if any (so that they appear short tailed or tailless); and a very small hind toe, if present at all. They inhabit South America. 


\section{Order 4. Rasores (Gallinaceous birds).}

Beak short, slightly curved at the tip; pedes g r a d a ri i, strong feet with small hind toe, which is articulated at a higher level than the rest of the toes, and has a slightly curved, short compressed claw; occasionally the hind toe is large. The wings are usually short, rounded, curved. The Rasores are, as a rule, of medium size, they are not very good fliers and generally remain on the ground; they are mostly omnivorous, scraping up seeds, larvæ, worms, etc., with their claws. Not a few are polygamous, when the males are usually larger and more gorgeously coloured than the females. The eggs are, as a rule, laid on the ground and brooded by the hens; the newly-hatched chicks are stronger than those of most other birds, and are able to run about immediately.

1. Tetraonomorphx. The nares and the base of the beak covered with thick feathers. Metatarsus more or less feathered, without a spur. Here belong: the Capercaillie (Tetrao urogallus) occurring in Scotland; and the Black Grouse (T. tetrix); magnificent birds, the former the larger: in both cases the metatarsus is completely feathered, the toes naked; polygamous; the cocks much larger than the hens; the latter brown, the former blackish. In the genus Lagopus, the whole foot is feathered; they are brown in summer, usually white in winter; the European species live only in cold regions, the Alps and elsewhere; two species are met with in Scotland, the Red grouse (L. scoticus), which is brown all the year round, and the Ptarmigan ( $L$. mutus), the former occurring also in the Orkney Isles. The Sand Grouse (Syrrhaptes paradoxus) is characterised by long wings and short feathered feet, from which the hind toe is wanting, the fore toes being fused; it is indigenous to the Steppes of Western Asia, but in recent years has several times wandered in large flocks into Europe, and has even been known to breed in Scotland.

2. Phasianomorphx. The naris naked, covered with a small arched scale. Metatarsus of the male usually furnished with a spur (occasionally with two), which is rudimentary in the female.

(a) The Pheasant Family (Phasianidx). Tail feathers sloped from a median plane like a roof: naked outgrowths usually present on the head: spur present: sexual dimorphism well marked: South Asia. The Domestic Fowl (Gallus domesticus), with a naked comb on its head; cock with long curved tail coverts; descended from Jungle-fowl (G. bankiva). Further, the Pheasant (Phasianus), of which one species (Ph. colchicus) occurs in many places in England in a half wild condition; distinguished by their long pointed tail (the rectrices themselves are lengthened).

(b) The Peacock Family (Pavonida). Tail flattened and fairly long; spur present. The Peacock (Pavo cristatus), with a tuft of feathers on the head; male with extraordinarily long tail feathers, which can be spread out: East Indies. The Turkey (Meleagris gallopavo), head and neck naked, in the male a soft process of skin depends from the dorsal side of the head at the base of the beak: North America.

(c) The Partridge Family (Perdicidx). Tail flattened, short; spur often absent. In England there occur, the common Partridge (Perdix cinerea) and the common $\mathrm{Quail}$ (Coturnix communis), of which the latter is a Bird of Passage and polygamous; both have a naked patch of skin behind the eye; the spurs absent; male and female fairly similar. The Guinea- 
fowl (Numida meleagris), with naked head, which bears a large bony process; grey, with white spots; without spur: indigenous to Africa.

3. The Curassows (Cracidre: genus Crax, etc.). Large, with fairly long metatarsus, curved and pointed claws; long tail; beak covered at the base with soft naked skin (cere), where there is often a large knob; an upright crest of feathers, frequently curves forwards from the top of the head: breed in trees : Mexico and South America.

4. Mound-birds or Talegallas (genus Megapodius, etc.), distinguished by the length of the claws and the powerful structure of the hind toe, which is articulated at the level of the other toes. They are specially remarkable in that they do not brood over their very large eggs, but deposit them in a mound of vegetable matter, which they have collected, in a sand heap, or in a pit dug in the sand; the eggs are then either incubated by the warmth resulting from the fermentation of the regetable matter, or simply by the heat of the sun. The young ones lose the covering of down whilst still within the egg, and hatch out with the adult plumage. Australia, the Philippines.

\section{Order 5. Natatores (Swimming Birds).}

The feet are generally pedes palmati, i.e., a nembrane is stretched between the front toes almost to their tips. As a rule the feet are short, the claws short and flattened, the hind toe generally very small, the lower end of the fore leg bare and scaly. The tail is usually short; the plumage thick and elastic. The Natatores are able to swim by means of their hind limbs, and not a few of them can dive, when they often use the wings as swimming organs; others can only bring the head, neck, and fore limbs below the water, the rest of the body remaining above. Usually they cannot walk well; the power of flight is considerable in some forms, in others it may be lost.

1. The Gulls (Longipennes). Long, pointed wings; short hind toe; lateral slit-like nares; tail well-developed. Most are coast-birds (some may also live near - fresh water), feeding on Pisces and other marine animals, for which they plunge into the sea; excellent fliers. The Gulls (Larus) are large and light-coloured, with the tip of the beak curved; and a stumpy tail ; numerous species on the coast of Great Britain: the Com mon Gull (L. canus), the B lack-headed Gull ( $L$. ridibundus), the Laughing Gull ( $L$. atricilla), the Herring Gull (L. argentus), and others. The Terns (Sterna) differ from the Gulls in their long, straight, pointed beaks, and their forked tails. Out of eight or nine species occurring on English coasts, the Common Tern (S. hirundo), and the Lesser Tern (S. minuta), may be noted. An interesting form is Buffon's $\mathrm{Sku}$ a (Lestris), dark in colour, with the two median rectrices longer than the others, and the beak grooved. The Skuas follow other marine forms which have secured any prey, seizing upon it if the possessors allow it to fall; they also fish for themselves, and behave as true Birds of Prey, since they hunt small Birds and Mammals. They are northern, and are met with in the Orkneys.

2. The Petrels (Tubinares). Chiefly distinguished from the preceding groups, in that the nares are situated at the ends of two tubes lying above the beak; usually met with in the open sea. Among those on British coasts, may be noted, the Fulmar Petrel (Fulmarus glacialis) of the Orkneys' and St. Kilda's, and the small, dark-coloured Stormy Petrel (Procellaria 
pelagica) of the English Channel, the Atlantic, and elsewhere. The group is represented in the Southern Hemisphere, at the Cape, etc., by the large Albatross (Diomedea exulans), in which the hind toe is wanting.

3. Steganopodes. A large, often backwardly directed, hind toe, which is connected by a web with the other toes, so that there is here a web between all four ( $p$. stegani) ; beak long, straight, the tip usually curved downwards. The Cormorant (Graculus carbo) is dark-coloured, with a narrow beak hooked at the tip; it breeds in flocks in trees near the sea or by fresh water; feeds upon fish; almost throughout the whole of Europe, in Great Britain, Asia, North America (in the winter, also in Africa). The Pelican (Pelecanus), white, with reddish or yellow touches, the beak long, straight, and broad, hooked at the tip; the skin between the mandibular rami capable of great distension, to form a large sac for the reception of the prey; tongue rudimentary; indigenous to warm countries; two species in South Europe. The Frigate Bird (Tachypetes aquila), with long, pointed wings, forked tail, and feeblydeveloped web, lives in the open sea within the Tropics; feeds chiefly upon Flying Fish, which it catches as they fly. The Gannet (Sula bassana), with long wings, and a long powerful, pointed beak, plunges deep into the water after its prey; common in Iceland and the Pharoe Islands, also met with in Devonshire.

4. Pygopodes. Wings weakly developed, but with the ordinary joints; beak of diverse form; leg, and most of the foreleg, enclosed in the body-wall, from which the lower end projects close to the anus; tail very short; the body can be held upright whilst walking. They dive after Fish, Shell-fish, and such like; belong to the Northern Frigid Zones.

(a) The Divers (Colymbus) usually possess pedes palmati, with a small hind toe; a long beak, pointed and straight. Northern birds, building their nests close to fresh water; several in England: the Great Northern Diver (C. glacialis), the Black-throated $\mathrm{Diver}$ (C. arcticus), and the Red-throated or Speckled Diver (C. septentrionalis). Grebes or Dabchicks (Podiceps) are like the Divers, but differ in that they have no continuous web, each fore toe having on either side a broad ridge (pedes fissipalmati); they build a floating nest on stagnant water; several species in Great Britain: the Red-necked Grebe (P. grisegena), the Eared Grebe ( $P$. auritus), the Little Grebe ( $P$. minor), and others.

(b) The Auk Family (Alcidæx) is distinguished from the preceding group by the absence of a hind toe. They breed in flocks by the sea. The Guillemots (Uria), with fairly long, straight, pointed beak, breed chiefly on Northern seas; three species abundant on the shores of North Britain, the Orkneys and Shetlands. Only one species of Auk, the Little Auk (Alca alle) breeds in Great Britain; the Razor-bill (Alca tonda) breeds in colder countries, but is occasionally found in the North Sea in winter; related to it is the extinct Great Auk (Alca impennis), in which the degenerate wings were quite useless for flight. It inhabited Iceland, Newfoundland, etc. The Puff in (Mormon fratercula) has the beak much compressed laterally and grooved; it digs out tunnels in the earth and nests in them; breeding chiefly on the Northern Coasts (Iceland, etc.).

5. The Penguins (Impennes). A very aberrant group, chiefly characterised by the small fore limbs, which move only from the shoulder, and are covered with small scale-like feathers (no specially developed remiges); they are of course useless as organs of flight, but are used for swimming.* Like the Auks, the Penguins walk upright; the metatarsus is short

* Indeed, the Bird swims almost entirely by means of the fore limbs, the feet being stretched backwards with the soles upwards, and serving as steering organs. 
and broad, the small hind toe turned forwards: the tail is very short: the feathers are evenly distributed over the body. They live in the Southern Hemisphere.

6. Anseres (Lamellirostres). Large, usually broad, bill, high at the base, flattened at the tip; most of the beak is covered by soft skin, only at the tip is there a hard, horny plate; along the edges of the jaw there is a series of small, usually laminate, processes; thick, soft tongue; small hind toe.

(a) D u cks (Anatinæ). Small anserine forms with short neck and broad, flat bill with a small horny plate; the male more brightly coloured than the female; undergo a seasonal change of colour. Birds of Passage. Among a number of Ducks occurring in Great Britain the following may be noted: the Wild D u ck (Anas boschas), ancestor of the Domestic Duck; the Common Shieldrake or Stock Annet (A.tadorna); the $\mathrm{Pintail} \mathrm{Duck}$ (A. acuta), a regular winter visitor; the Teal ( $A$. crecca); the Garganey (A. querquedula); the Shoveller or Spoon-bill Duck (A. clypeata), in which the bill is very large and provided with long laminæ at its edge. Many Ducks breed in England, but others, e.g., the Wid ge on (A.penelope), only, or usually, in more Northern Countries. The Fuligulinx differ from the Ducks in the possession of a small flap of skin, which projects from the hind toe; further in the power of diving: they are mostly northern forms, several occur on the English coasts : the Tufted Duck (Fuligula cristata); the Staup Duck (F. marila). To this division belong the Eider Ducks (Somateria mollissima), which breed on the Faroes, in Iceland and Greenland in great numbers. The Mergansers (Merginæ, genus Mergus and others), differ from the Fuligulinæ in the narrow beak, hooked at the tip and furnished with dentiform processes at the edge. Several species in England.

(b) Geese (Anserinæ). Large, fairly long-necked, and long-legged, without flaps of skin on the hind toe, beak high at the base and with a large horny plate at the tip. In contradistinction to other Lamellirostres, which feed either upon animals or are omnivorous, Geese feed chiefly on plants, grazing with their bills; they live upon land much more than do the others. Usually there is no striking sexual dimorphism. The Grey Goose (Anser cinereus), the ancestor of the Domestic Goose; the Red-breasted Goose (A. ruficollis); the Bean Goose (A. segetum), and others occur in Britain.

(c) Swans (Cygnus). Large, very long-necked, but short-legged; the hind toe without a flap of skin, bill bigh at the base, flattened at the tip. In Temperate and Frigid Zones; those of the Northern Hemisphere, white; those of the Southern, partially or entirely, black. Amongst others the W histling $\mathrm{Swan}$ or Wild $\mathrm{Swan}$ (C. musicus), and the Mute $\mathrm{Swan}$ (C. olor), occur in England; the latter is frequently kept as a tame animal. The Black Swan (C. atratus) inhabits Australia.

(d) Flamingoes (Phonicopterus) are, with regard to their very long forelegs and metatarsals, like the Grallatores; the neck is extraordinarily long; the beak looks as if broken, otherwise like that of the Ducks; tongue soft and large; web present. One species of this large Bird inhabits Mediterranean lands, wading about on the coasts.

\section{Order 6. Grallatores (Wading birds).}

The members of this group have pedes grallarii; the lower portion of the leg is naked, scutellate, the metatarsus long; there is usually no web, although this is present in exceptional cases. The head is small, the beak usually long and narrow. The neck long, 
much curved, and sigmoid; the feathers which cover the curves are often long, making the whole neck look short and thick. The food is usually of an animal nature.

1. Altinares. Beak large and strong, much longer than the rest of the head, with firm, horny sheath, small basal nares, wings large. Birds of considerable size, which build their nests high above the ground (in trees, etc.) and foster the young ones.

(a) Herons (Herodii). Hind toe long, with a large claw, resting its whole length upon the ground. In Britain there are: the $\mathrm{Com}$ mon Her on (Ardea cinerea), abundant, nests in flocks in trees; the $\mathrm{Common} \mathrm{Bittern} \mathrm{(Botaurus}$ stellaris), with soft plumage, brownish in colour, nocturnal; the $\mathbb{N}$ ig h t Her on (Nyeticorax griseus) with thick bill, an occasional visitor; and others.

(b) Storks (Pelargi). Hind toe short, with small claw articulated at a higher level than the other toes. Here belong the White Stork (Ciconia $a l b a)$, and the Black Stork (C. nigra) both of which occasionally visit England. The Adjutant or Marabou (Leptoptilus) with very powerful beak; bare neck and head; a carrion feeder, in Africa and the East Indies: the White Spoonbill (Platalea leucorodia) with much-flattened bill broad at the tip, in South Europe, rare in England: the white Sacred Ibis (Ibis religiosa) of ancient Egypt, distinguished by the thin, soft, curved beak, and the naked head and neck; now rare in Egypt but abundant in the Soudan and Southern Nubia.

2. Brevirostres. Bill short, usually fairly thick, with firm, horny sheath and nares sub-basal. Most of them are small or of medium size and nest on the ground; the young ones can run from the first.

(a) Plovers (Charadriidæ). Small birds with or without a small hind toe. Among those occurring in England the following may be mentioned: the Peewit (Vanellus cristatus), with a crest of feathers on the head, hind toe present, nests on meadow land; the Turnstone (Strepsilas interpres), with hind toe, the short beak somewhat arched upwards, with a world-wide distribution on sea-coasts; the Oyster Catcher (Hæmatopus ostralegus) without hind toe and with a long beak; the Golden Plover, (Charadrius pluvialis), without hind toe; with short beak clubbed at the tip : an inland form, on moors, etc. Of these, the Turnstone is only a seasonal visitor, the others are indigenous.

(b) The Bustards'(Otidæ). Large hen-like forms with short conical bill; and short powerful toes; the hind toe absent; inhabiting dry treeless plains The Large Bustard (Otis tarda) and the Small Bustard (O. tetrax) occasionally visit England; the latter is a native of Mediterranean coasts.

(c) Water-fowls, Rails (Rallidæ). Toes long, hind toe well developed; beak varying in length. As examples may be mentioned : the Water Rails (Rallus aquaticus), beals straight, larger than the rest of the head; the Corn Crake or Land Rail (Crex pratensis), a migratory form; the Moor-ben (Gallinula chloropus); and the $\mathrm{Common} \operatorname{Coot}$ (Fulica atra), with a ridge o skin along each side of the fore toes; both of the latter with a naked, horny frontal plate above the beak.

(d) Cranes (Gruidæ). Large, with fairly large, straight, pointed beak; legs very long, toes short, hind toe small, neck long. The Common Crane (Grus cinerea) was formerly a native of England, but long ago ceased breeding here, and now only appears at uncertain intervals.

3. Debilirostres. Bill long and thin, often flexible, and with a soft skin otherwise like the Brevirostres; in mode of life, true Wading Birds. The Snipes (Scolopax), with long, straight, soft beak (Woodeock [S. rusticola], Great Snipe [S. major], Common snipe [S. gallinago], Jack Snipe [S. gallinula]) : 
the Sandpipers (Tringa); some indigenous, some seasonal visitors: the Ruff (Machetes pugnax): the Redshank (Totanus): the Godwits (Limosa): the Curle ws (Numenius arcuata), with very long, arched downwardly curved beak: the Avoset (Recurvirostra avocetta), with very long, upwardly curved beak, and incomplete webbing between the toes. All those mentioned (and others besides) occur in England; some are Birds of Passage, others indigenous. The Phalaropes (Phalaropus), with ridge of skin along the toes, are Northern forms (Iceland, etc.), which occasionally stray into England. The males alone incubate the eggs.

\section{Order 7. Accipitres or Rapaces (Birds of Prey).}

Beak short and strong, thick at the base, which is provided with a cere, much curved and with its point directed downwards. The feet are powerful; the strong claws are of an elongate, conical shape, pointed and curved, forming the talons; the hind toe is usually very strong; pedes raptatorii. Wings large. For the most part, majestic Birds, feeding upon their prey or upon carrion. The females are larger than the males. The newly-hatched young ones, although well covered with down, remain for a long time within the nest, and are fed by the parents.

1. Diurnal birds of prey (Hemeroharpages). Head and neck feathered; hind toe large, articulated at the level of the fore toes, and bearing a very short claw. They prey upon living animals.

(a) $\mathrm{Hawks}$ (Asturidæ). Lower side of the metatarsus covered with large horny plates; wings of medium length. Amongst British species are the Goshawk (Astur palumbarius) and the small Sparrow-hawk (A. nisus). The Secretary (Gypogeranus secretarius), an extraordinarily long-legged bird, with a very long metatarsus and short toes, recalling a Wading Bird; lives in the deserts of Africa, feeding chiefly upon Reptiles. The Buzzards (Buteo) differ from the Hawks principally in the greater length of wing. The H a r riers (Circus), also with long wings, are characterised by the possession of a facial disc, like that of the Owls.

(b) Falcons (Falconidx). The hinder side of the metatarsus, with numerous small scales. Short, powerful beak, curved from the base, and with a large tooth-like projection on the edge, near the tip. Wings usually long. The most important occurring in England are the $\mathrm{Kestrel}$ (Falco tinnunculus), the Peregrine Falcon ( $F$. peregrinus), the Hobby (F. subbuteo), and the Merlin (F. zsalon): F. subbuteo, a migratory form wintering in South Africa; the others indigenous. The Gyrfalcon (F.gyrfalco) is an Arctic bird, which occasionally visits England.

(c) Eagles (Aquilidæ). The metatarsus like that of Falcons, but often feathered; beak usually longer, only curved at the tip, very strong, without the dentiform projection; large with long wings; the Eagles (Aquila), characterised by the well-feathered metatarsus, only occur as stragglers in England, but are more common in the Highlands. The only two British species are the Golden or Mountain Eagle (A. chrysaëtus), and the large Sea Eagle or White tailed Eagle (Haliaëtus albicilla), in which the metatarsus is only feathered over its upper half. This form feeds both upon land animals and fish; it occurs in all parts of Europe, and wanders south to breed. The Osprey or Fishing $\mathrm{Hawk}$ (Pandion haliaëtus), distinguished by the 
short beak, and by the reversible onter toe, feeds on fish; and is very cosmopolitan, being met with in all five continents. The Kite or Glede (Milvus regalis) is distinguished from the Eagles proper by its smaller beak and its forked tail; abundant in England and Europe generally. To the Eagles belongs also the Bearded $V$ ulture (Gypaëtus barbatus), in the mountainous regions of South Europe and South Asia (at one time in the Alps). It was formerly elassed with the Vultures, which it resembles in its mode of life (feeding chiefly upon carrion), but the head and neck are covered with true feathers.

2. Vultures of the Old World (Saproharpages). Head and upper part of the neck bare or covered with down; hind toe large, articulated at the same level as the others; talons less powerful, somewhat depressed ; wings large ; numerous small scales on the posterior side of the metatarsus. Large forms, fèeding chiefly upon carrion, and inhabiting the hotter parts of the Old World. The large White-headed Vulture (Vultur fulvus), with the head and neck covered with whitish down; and the small Alp ine Vulture (Neophron percnopterus), with naked head and very long, thin beak; live in countries bordering the Mediterranean and in Africa.

3. Vultures of the New World (Necroharpages). Head and upper portion of the neck usually naked; hind toe small, articulated above the level of the rest; nasal septum perforate; very large wings; carrion feeders; in America, especially South America. The largest species is the Condor (Sarcorhamphus gryphus); another large form is the $\mathrm{King} \mathrm{Vulture} \mathrm{(S.} \mathrm{papa),} \mathrm{with} \mathrm{brightly-coloured}$ head and neck; and the smaller Carrion Vulture (Cathartes).

4. Ow Is (Nyctharpages). The back part of the head so broad that the eyes look forwards (in other Birds of Prey they look sideways). The face is surrounded by a circle of peculiar short feathers, the facial disc; there is also a circle of feathers round each eye; between these rings is the large auditory opening. Bristle feathers surround the base of the beak; the plumage is soft, usually mottled brown; the outer toe (fourth) is reversible, i.e., may be turned backwards; the hind toe is articulated somewhat above the others; the foot and toes usually feathered.

(a) Diurnal Owls (Striges diumz). The ear simple, without an operculum; facial disc incomplete above. They hunt both by day and in the evenings. Diurnal Owls are rare in England. The Snow y Owl (Nyctea nivea) has been found off the Shetlands; and the Hawk Owl (Surnia nisoria) at sea, off Cornwall, but both these are more Northern (Scandinavia). Others which are Continental birds, occasionally visiting the British Isles, are the G r e a t - E a r ed Owl (Bubo maximus), and the small Scops Eared Owl (Ephialtes scops), both with two tufts of feathers on the head; and the Little $\mathrm{Owl}$ (Athene noctua).

(b) Nocturnal Owls (Striges nocturnæ). Ear very large, with an operculum; facial disc complete. In England there are the Taw n y Owl (Syrnium aluco), the Long-Eared Owl (Otus vulgaris), the Short-Eared OwI (O. brachyotus), the almost cosmopolitan Barn Owl (Strix flammea), and as an occasional visitor, Tengmalm's Owl (Nyctale funerea).

\section{Order 8. Oscines. (Singing Birds).}

The feet are thin and delicately formed. The hind toe, which is strong and provided with a larger claw than the others, can be moved by itself, whilst in all other Birds it can only be moved with the other toes, since the tendon of the hind toe is generally 
connected with that of the fore toes, though in the Oscines it is independent. The contour feathers of the wing are small and few in number. In the majority the back of the metatarsus is almost covered by two long, narrow plates, instead of being scutellate. At the lower end of the trachea there are generally several small muscles, which are absent from other forms (singing muscles). The nest is often rather ingenious in construction. The Oscines feed, for the most part, upon grain, berries, or Insects.

1. Turdiformes. Beak usually straight or slightly curved at the tip, often with a marginal notch in front; nares bas al.

(a) Singers (Sylviadx). Beak somewhat feeble, compressed, of medium length with a shallow notch : small or medium-sized some of them noted singers : feeding upon Insects and berries. The following English Birds, among others, belong here: many of the Turdidx; B la ckbird: (Turdus merula); the Ring Ouzel (T. torquatus), the Song Thrush (T. musicus), etc. The Common Dipper (Cinclus aquaticus), almost the same size as the Thrushes, dives into running water; a sedentary Bird; the Nightingale (Inscinia philomela); and the $\mathrm{Redbreast} \mathrm{(L.} \mathrm{rubecola);} \mathrm{the} \mathrm{Redstart}$ (Ruticilla); the Wheatear (Saxicola); the genus Sylvia (Sedge Warblers, Reed Warblers, and Willow Warblers) dainty little forms, usually inconspicuous in colour. The Gold-crested Regulus (Regulus), and the Wrens, (Troglodytes parvulus) the smallest British Birds. The Wagtails (Motacilla) with long dipping tail, near small pieces of water. The Pipit (Anthus) with long hind claw, like the Larks.

(b) Shrikes (Laniad:e) differ from the Sylviadx in the strong beak, at the edge of which is a dentiform process on either side just within the curved tip. They catch Insects and small Vertebrates and spike them on thorns. Several species occur in Europe, the largest, the Great Grey Shrike or Butcherbird (Lanius excubitor), which is as big as a Thrush, is occasionally met with in England.

(c) The Tits (Paridæ) small, with soft plumage; the beak is short, fairly thick, straight, and without a notch; the nares covered by bristles. Insecteaters, which breed chiefly in hollow trees and such places. Amongst English forms are the Great Tit (Parus major), the Blue Tit (P. coeruleus), the Long-tailed Tit (P. caudatus), etc.

(d) The Fly-catchers (Muscicapidæ) have short, straight, flattened beaks, broad at the base and with stiff bristles at the root. The Spotted Fly-catcher (M. grisola) is a seasonal visitor to England; two other species are occasionally met with.

(e) The Bohemian Waxwing (Ampelis garrulus) has a rathex short beuk somewhat broad at the base, and soft plumage. The most remarkable peculiarity of these creatures is that on the remiges, and sometimes also the rectrices, some of the branches have united with the tip of the shaft to form a spatulate lamina. They breed in Scandinavia, but in the winter occur in great numbers on the continent and occasionally visit England. To an allied group belongs the Golden Oriole (Oriolus galbula), which is of a beautiful yellow, of the size of a thrush; rare in Britain.

2. Conirostres. Beak short, thick, and conical, with nares high up. The food consists chiefly of seeds, but the young ones are fed upon Insects.

(a) Finches (Fringilla), beak thick, without a hooked tip; the $\mathrm{Haw}$ finch ( $F$. coccothraustes), the large English Finch, beak extraordinarily thick and strong; the Chaffinch (F. coelebs); the Mountain Finch (F. monti- 
fringilla); the Greenfinch (F. chloris); the Serin Finch (F. serinus); the Common Linnet ( $F$. cannabina); the Mountain Linnet ( $F$. montium); the Goldfinch ( $F$. carduelis); the small yellowish-green $\mathrm{Sis}$ in (F. spinus); the Lesser Redpole (F. linaria); the House sparrow (F. domestica) occurs in Europe, Asia, and North Africa, and has also been introduced into America and Australia; has increased enormously in North America; the Tree sparrow ( $\boldsymbol{F}$. montana); the Bullfinch ( $\boldsymbol{F}$. pyrrhula): all these occur in England, but the Mountain and. Serin Finches and the Siskin are rare. Among foreign forms may be mentioned the $\mathrm{Canar}$ ies ( $\boldsymbol{F}^{\top}$. canaria) from the Canary Islands.

(b) Buntings (Emberiza), beak compressed at the tip, narrower and lower than the mandible, the edge arched; there is usually a hard knob on the palate. The Snow Bunting ( $E$. nivalis), which nests in the north, and in the winter comes to England, has no palatine knob. The Common Bunting ( $E$. miliaria); the Yellow Bunting or Yellow Hammer (E. citrinella); the Black-headed.Bunting (E. schoniclus); the Ortolan Bunting (E. hortulana), all occur in England, but the last is rare.

(c) Cross bills (Loxia) are chiefly characterised by the crossing of the tips of the beak and mandible; pine-tree birds : L. curvirostra and $L$. pytiopsittacus occasionally visit England. Allied is the Pine Grosbeak (Pinicola [Pyrrhula] enucleator), about the size of a Thrush, with hooked tip to the beak; a northern form of rare occurrence in England.

3. Corviformes. Strong, fairly large, almost straight beak; fairly strong feet. For the most part large, social, and omnivorous.

(a) Starlings (Sturnus vulgaris), medium-sized, with long, straight, compressed beak; the nares not covered with feathers. They breed in holes, are insectivorous and indigenous. Allied is the Rose-coloured Pastor (Pastor roseus), in which the top of the beak is feebly curved; occasionally strays into England.

(b) Raven family (Corvidi), with very strong, anteriorly compressed, somewhat curved beak ; the nares are covered with bristles. Large. The Raven (Corvus corax), the largest of English Oscines, quite black in colour and not very abundant. The black Carrion Crow (C. corone), and the partially black Hooded Crow (C. cornix) are not separate species, but only geographical varieties; there is a series of transitions between the two, and they are quite fertile together. The Rook (C.frugilegus), quite black, the bristle feathers at the base of the beak wanting in the adult. The $\mathrm{Jackdaw}$ (C. monedula), slaty black, beak shorter than in the others mentinned. The Magp i ( ica caudata), with long tail, black and white. The Jays (Garrulus glandarius), brightly-coloured, with short beak, the tip of which is hooked. The Nutcracker (Nucifraga caryocatactes), with long, almost straight beak. Rare in England.

(c) Birds of Paradise (Paradiseidz), distinguished by their gorgeous colouring and the peculiar structure of the feathers, but these points characterise the males only, the females being more modestly arrayed; large, with strong, compressed beak, and nares covered by feathers; New Guinea and adjacent islands.

4. Swallows (Longipennes). Wings very long, feet short, beak short; broad at the base; the angles of the mouth reach far back. Small migratory Birds, excellent fliers; insectivorous. In England occur the Swallows (Hirundo rustica), with brownish red throats, and the House Martins (H. urbica), both of which build the well-known nests of mud and saliva; the brownish-grey Sand Martins ( $H$. riparia), which dig horizontal nesting 
tunnels, $1 \mathrm{~m}$. to $1.5 \mathrm{~m}$. long in perpendicular walls of sand; and breed in the innermost, somewhat widened portion.

5. The Common Creeper (Certhia familiaris), the $\mathrm{Nuthatch}$ (Sitta crsia), and the Wall Creeper (Tichodroma muraria) belong to a special group of the Oscines, and are characterised by the very great size of the hind toe, whilst the fore toes are enclosed at their roots in a common skin. The claws are much compressed and very pointed. They are all found in England, but the Wall Creepers are very rare. They run up the trunks of trees or up rocks. All the Creepers have long, thin, arched beaks (longest in the Wall Creepers); the Nuthatch has a straight, strong, pointed beak. The Wall Creepers are Alpine.

6. Larks (Alaudidæ), distinguished from all the other Oscines so far mentioned in that the metatarsus is covered posteriorly by several small plates. The hind toe has a long, straight claw; the beak is of medium length, fairly strong and almost straight, the summit arched. They feed chiefly on seeds, and nest on the ground; the following occur in England: the Sky Lark (Alauda arvensis), the Wood Lark (A. arborea), and the Crested Lark (A. cristata), the last is rare. The $\mathrm{Shore} \mathrm{Lark}$ (Otocoris alpestris) is of striking appearance, it inhabits the Northern parts of Asia and Europe, and not infrequently visits the East coast of Britain. Allied to the Larks is the Hoopoe (Upupa epops), with long, thin, arched beak, a hind claw like theirs, and an upright tuft of feathers on the head. Rather rare in England. Insectivorous, migratory.

7. The Lyre Birds (Menura), fowl-like, with short, straight beak. They are characterised especially by the peculiar, long, lyre-shaped tail of the males. The innermost and the outermost rectrices are curved outwards, the rest are thinly furnished with long barbs, which possess no barbules: Australia.

\section{Order 9. Clamatores (Shrieking birds).}

Distinguished from the Singing Birds in that the hind toe, and especially its claw, is less powerful and that it cannot be moved by itself. The singing muscles are not developed.

1. The Roller (Coracias garrula). Beak of medium length, compressed in front, broad at the base, slightly arched at the tip. Gorgeous blue-green; about the size of a Thrush; comparatively rare in England; nests in holes in trees; insectivorous and migratory.

2. Swifts (Cypselidx). The mouth very large, extending back behind the eye; beak short and weak; broad at the base and flattened; uncommonly long wings, very small feet. Insectivorous forms like Swallows. In England occurs the Common Swift (Cypselus apus), in which all four toes are directed forwards; the dish-like nest is formed in holes in walls, etc., and is built of straws and feathers, which are bound together by saliva. The Swifts are represented in the Alps and on Mediterranean coasts by the very similar, somewhat larger, Alpine Swift (Cypselus melba), with white abdomen; it occasionally visits England. The Salanganes (Collocalia), with normal feet, but in other respects like the foregoing species, live in the East Indies, and construct their nests of saliva (edible birds-nests). To an allied family belongs the $\mathrm{Nightjar} \mathrm{(Caprimulgus} \mathrm{europæus),} \mathrm{which} \mathrm{is} \mathrm{large,} \mathrm{brown,} \mathrm{and} \mathrm{coloured} \mathrm{like}$ an Owl; feather bristles at the base of the beak; nocturnal; the eggs are laid on the bare ground. A summer visitant to Britain.

3. Humming Birds (Trochilidx). The beak is long, thin, and tubular; the tongue is deeply cleft and can be greatly protruded. The wings are long; 
the feet short. Gorgeously coloured, especially the males; often, too, some of the feathers are of a peculiar structure. Insectivorous. To this family, which is only met with in the warmer parts of America, belong the smallest of all Birds.

4. The Kingfishers (Alcedinidx) have a straight, strong, angular beak; external and middle fore toes united up to the second joint, the middle internal ones to the first pedes gressorii. Brightly coloured; mostly natives of 'warm countries. In England the long-beaked $\mathrm{K}$ in $\mathrm{f} \mathrm{f}$ is $\mathrm{h}$ e $\mathrm{r}^{\circ}$ (Alcedo ispida), which lives upon Pisces and is often very destructive of the fry. The same type of foot is found in the Bee-eater (Merops apiaster), with long, very-pointed, slightly-arched beak; winters in South Africa, migrating into South Europe in the summer, and occasionally flying into England. In the Rhinoceros Birds (Bucerotidx) too, the fore toes are connected at their roots; they are further distinguished by their very long, thick. somewhat-curved beak, which usually bears a large process at the root. Africa and East Indies.

5. Pigeons (Columbidx), characterised by the fact that the ratner short beak has a horny covering only at the tip, being soft at the base. The $\mathbf{R}$ ing . dove (Columba palumbus), the Stock Dove (C. cenas), which nests in high trees, and the Turtle Dove (Turtur auritus)* occur in England, the last as a seasonal visitor. The Rock Pigeon (C. livia), on the coasts of the Mediterranean, in England, etc., is the ancestor of the numerous races of $\mathrm{ta} \mathrm{m} e$ Pigeons. The Migratory Pigeon (C. migratoria), of North America. wanders in immense flocks through large districts in search of food. Numerons other Pigeons occur in various parts of the world. An aberrant form is Didunculus strigirostris of the Samoan Isles, distinguished by its short, istrong beak, hooked at the tip, and by the presence of two dentiform processes on each side of the lower jaw. The extinct Dodo (Didus ineptus) was about the size of a Swan, very clumsy, with strong legs and strong beak; on account of the very small size of the wings it was unable to tly (sternal keel absent); the tail, too, was very degenerate. It lived in the Mauritius, and was extinct by the close of the seventeenth century.

\section{Order 10. Scansores (Climbing Birds).}

Distinguished from the Clamatores in that the fourth toe is turned lack, so that they possess two fore and two hind toes (pedes -scansorii).

1. Cuckoos (Cuculidis) have a beak of medium Jength and somewhat hooked; the fourth toe can be turned to the side. Here belings the $\mathrm{Gomm}$ on Cuckoo (Cuculus canorus), an insectivorous, migratory form. which lays its -eggs in the nests of other Birds (Oscines) in order that they may do the brooding.

2. Woodpeckers (Picidix) have a rery strong, straight, angu'ar beak, compressed at the tip and keel-like; the tongue, which "an be stretched out a very long way, is provided at the edges with delicate bukwarlly-directed processes; the tail feathers are very stiff, and support the loin in climbing. They are Forest-birds, feeding upon wood-boring larre and other Insects, hut also upon seeds; they breed in holes in trees, which they chisel nut fur themselves; they are "res dents," $0:$ wander about in a limited locality. In Fngland are found the Great Black Woodpecker (Picus martius), the Green Wood.

\footnotetext{
* The Collard or Barbary Dove (T. risorius), which is often lept tame, lives wild in Asia and Africa.
} 
pecker ( $P$. viridis); and the Spotted Woodpeckers, the Large $(P$. major), the Medium (P. medius), and the Small ( $P$. minor). The Three-toed Woodpecker (P. tridactylus), in which the inner hind toe (hallux) is lost, occurs in the Alps. The Wryneck (Iynx torquilla), is a near ally; beak conical, not keel-like; the tail feathers are not stiff enough to act as a support; migratory: visiting England in the spring.

3. Parrots (Psittacidx) have an uncommonly short, thick, much arched beak, overhanging a short mandible; the beak is freely movable; the tongue, thick and soft. They are brightly-coloured (green, red, etc.) and plant-eating; they are found in the Tropics. They may be divided into several groups: (1) Cockatoos (Plictolophinx), of Asia and Australia, with an upright tuft of feathers on the head, often brightly-coloured plumage; (2) Parrakeets (Sittacinæ), with long tails; (3) True Parrots (Psittacinæ), with short tails, and without the crest of feathers on the head; (4) Lories (Trichoglossinz), of Australia, the tip of the tongue is brush-like in consequence of numerous long, filiform, horny papillæ; (5) Stringopina, owl-like Parrots, of which there is only a single genus in New Zealand (Stringops habroptilus). The last are nocturnal, with soft, dark greenish plumage, living by day in holes in the ground, where also they nest; they fly very little, or not at all (the sternal keel is reduced) and for the most part move about on the ground.

4. Toucans (Rhamphastidæ) have a very large, thick, somewhat curved beak, which is often notched at its edges, and which is almost as long as the whole body; the tongue is narrow, horny, and frayed out at its margin. They are South American forms, of medium size, and with gorgeous colouring.

\section{Class 6. Mammalia.}

As regards external configuration, the Mammalia are usually characterised by the possession of a very pronounced $\mathrm{neck}$, varying considerably in length; of a much reduced tail, with no locomotor significance, and of little use; of $\operatorname{limbs}$, so well-developed, on the other hand, that the body is raised some distance off the ground: the elbows are turned backwards; the knees, fingers, and toes forwards. In many cases the animal does not rest upon the whole foot, but only on the toes, or even their tips, whilst the rest is raised, and contributes. to the lengthening of the limb.* Within the class, besides the ordinary walking type, various others are specialised, such as springing, flying, swimming forms (ef., Reptilia). When the body is. adapted to a peculiar mode of life, the outer form may be very aberrant. This is especially noticeable in certain aquatic Mammalia (Whales), where the neck is reduced to a minimum; the limbs are very degenerate, whilst the tail attains enormous proportions, so that the appearance is in the highest degree piscine.

The $\mathrm{sk}$ in consists of the usual layers (dermis, and epidermis with stratum corneum and stratum mucosum or Malpighii); on the surface of the dermis there are papillæ extending into the Malpighian layer. Pigment may be present in the epidermis (in both layers), and

* The limbs are longest, and the surfaces touching the ground smallest in the swiftest animals (Ungulata) 
also in the dermis. The stratum corneum is not shed all at once, but in minute portions.

As a rule the greater part of the skin is covered with hair, a very characteristic feature, absent in very few cases. The hairs consist almost entirely of cornified cells, and each is inserted in an invagination of the skin, the hair follicle. At the base of each follicle there is a small hair papilla, covered by an outgrowth of the stratum mucosum, by the cornification of which the overlying hair is formed. The rest of the hair follicle is covered by outgrowths of the mucous and horny layers of the ordinary epidermis, the outer and inner root-sheaths; the latter is continued below into the hair, the former into the stratum mucosum of the papilla; the hairs are nothing, then, but well-defined, enormouslydeveloped portions of the stratum corneum. In many of the thicker hairs there is an inner medulla of loosely-packed cells, surrounded by the harder cortex; externally there is a layer of thin, flattened cells, the epidermis; many, especially thin, hairs consist simply of cortex and epidermis. In many Mammals two kinds may be distinguished, contour hairs and woolly hairs; the latter finer and covered by the others. Peculiar long stiff "tactile hairs" or "whiskers" (vibriss $x$ ) are often inserted on certain regions of the head, especially on the upper lip; they are well-developed and regularly arranged; their follicles lie in a blood space, which is in communication with blood vessels. Other peculiar stiff hairs, the eyelashes, are

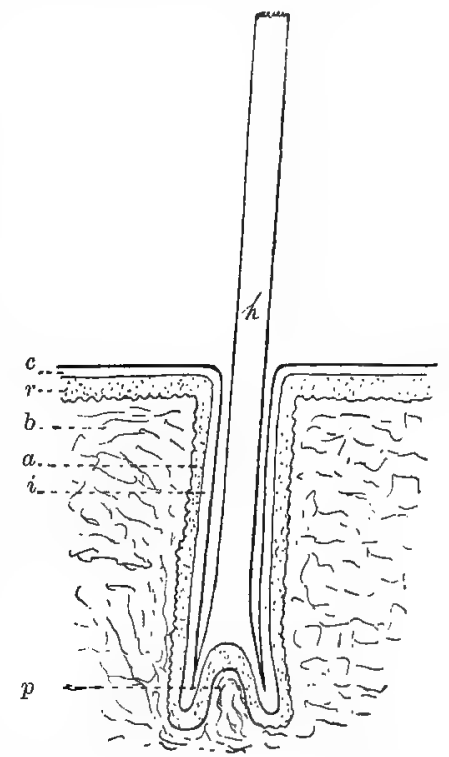

Fig. 379. Longitudinal section of a hair, and the connected hair follicle; diagrammatic. $a$ outer root sheath, $b$ connective tissue, $c$ stratum corneum, $h$ hair, $i$ inner root sheath, $r$ stratum Malpighii, $p$ hair papilla.-Orig. often present along the edges of the eyelids. Sometimes certain of the hairs attain immense proportions, such as the spines of the Hedgehog and Porcupine. The hairs are, for the most part, obliquely implanted in the skin, and are regularly arranged, usually in small groups or tufts. Smooth muscle fibres are attached to the base of the follicle, they arise from the dermis, and by their contraction the hair can be erected. Nerves also run to the hairs (or more correctly to the hair follicles), especially to the whiskers mentioned above, which are true tactile organs. 
Like the feathers of Birds, hairs are shed at regular intervals and replaced by new ones; each is detached from the papilla, and a new one arises from the base of the follicle. In some Mammalia (Man and the Apes) this moulting goes on gradually throughout the whole year, now one hair, now another being thrown off and replaced by a new one. In others, however, it is confined to a short period recurring annually; and usually, for northern forms, in the spring,* when a total ecdysis takes place, both wool and hair being thrown off. Simultaneously new hairs arise, and the tips of the wool hairs bud out, but complete their development later in the year. In some animals which are dark in summer, white in winter, there is a colour change $\dagger$ of the summer coat in late autumn, in the Alpine Hares, for instance. In others, in which the summer and winter coats are different, a change of hair occurs in the autumn, as well as in the spring, e.g., in Stags.

In many Mammals some portion of the body is covered by scales or plates like those of the Reptiles (Manis, Dasypus, tails of Mice). Sometimes, e.g., in Dasypus, the dermal part of each scale contains an ossification; and besides this there are in several Mammalia, smaller or larger independent ossifications in the dermis.

Small glands in connection with the skin are usually distributed over almost the whole surface; two principal kinds may be distinguished, sebaceous and sudoriparous. The sebaceous glands are small and racemose, and usually open into the hair follicles; rarely directly on to the surface; they are therefore generally absent from hairless tracts, they secrete a fatty substance. The sudoriparous glands are simple and tubular, with the lower portion, usually lying in the loose layer below the skin, coiled into a knot. They also often discharge their secretion into the hair follicles, but closer to the surface than the sebaceous glands; many open quite independently, in great numbers, for instance, in certain naked tracks. Like the sebaceous glands, too, they occur on various regions, in various numbers, and are of various sizes. Most of them secrete $\mathrm{s} w \mathrm{e}$ at ; the secretion may, however, be more fatty; for instance, the wax-glands of the ear are peculiar sweat-glands.

The mammary glands, common to all Mammalia, are peculiarly modifed sudoriparous glands. Those of the Monotremes are of the most primitive type; here, there is, on either side of the abdomen, a small hairy pit into which a number of large brauched sudoriparous glands open; they secrete milk. In other Mammalia they open upon somewhat projecting papillæ, the $m a m m i l l æ$, or nipples, usually several upon each (about twenty in Man, five to eight in the Dog, two in the Horse), seldom there is only

* But a change of some hairs may also occur at other times.

$+C f$, change of colour in feathers, p. 434 . 
one (Ruminants). The glands or mammæ, which vary in number (one to seven, most in those animals which produce many young ones at a time), lie in two rows on the ventral aspect of the body. They are of considerable size and much branched; the terminations are vesicular, and for this reason the glands were formerly regarded as sebaceous; comparison with those of Monotremes demonstrates, however, that they are really sudoriparous. During gestation the mammary glands increase in size and complexity, aud are functional for some time after parturition. Milk is a watery liquid in which are suspended numerous oil globules; these impart its white appearance. At the close of lactation the glands become simpler again; they are usually rudimentary in the males.

Specially modified skin glands, isolated or in patches occur more rarely, but still fairly frequently. As a rule the skin is invaginated to form a pit, covered with hairs, and in this region the glands are specially prominent: such are the interdigital glands of Sheep and other Ruminants; the so-called lachrymal pits in front of the eyes of the Red Deer; the perinæal pouches opening, in the Dog and other Carnivora, at the sides of the anus; the musk sac in the Musk deer, etc. In other cases the highly-developed skin glands open freely on to the surface; a very large one opens on the back of the Peccary; in certain Shrews there is a region of the skin with numerous specially modified glands, etc.

On the lower side of the foot there is usually a naked elastic tract of skin, the sole, covered by a thick but soft stratum corneum, and provided with numerous sweat glands. In some cases it extends over the whole ventral surface, or it is limited to certain regions, namely, to the toes.

Like Reptiles and Birds, Mammals have cap-shaped claws at the tips of the digits, and here also they are differentiated into two parts,

Fig. $380 . \quad A \mathrm{Claw}$ of a Mammal (removed from the subjacentskin), $B$ Nail of a monkey, $C$ hoof; diagrammatic. $s$ sole (of the claw); the rest, wall.
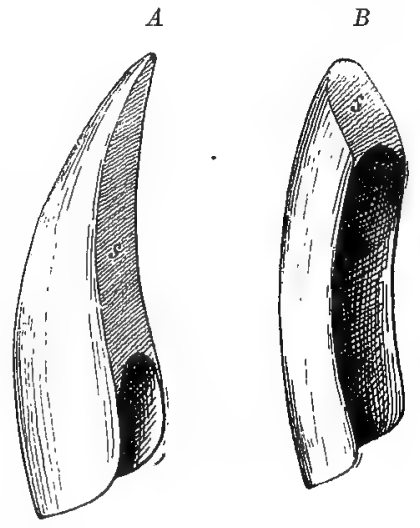

C

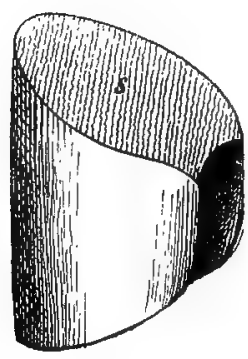

a harder dorsal (and lateral) wall, and a ventral horny sole, consisting of looser horu; the base of the claw is oblique, so that the sole is shorter than the wall; the latter forms a horny plate, arched longitudinally and transversely, whilst the former is flattened 
or concave below. The wall is nsually covered at the base by a fold of skin. The nails of Monkeys differ from most claws in that the sole is very short and consists of very soft horn (this may also be the case in many true claws), and that the wall is but slightly curved, either longitudinally or transversely; in Man the sole has practically vanished, merely an insignificant remnant of it lies below the edge of the wall, and the "nail" is the wall only. The modifications termed hoofs, are peculiar in that they are short with blunt ends, and the thick wall is convex transversely, but not longitudinally (or very slightly); and in that the horny sole is thick and hard, and the fold of skin at the base very poorly developed. The peculiarity of the hoof is correlated with its function of supporting the animal when walking, whilst in other forms the sole of the foot serves this purpose, and the claws are used for climbing, digging, etc., and in this connection, among other points, a solid attachment by means of a deep fold of skin appears of special importance.

In most Ungulata (excepting the Tapir and the Rhinoceros) there is an intimate connection between the hoof and the sole of the foot, which is usually

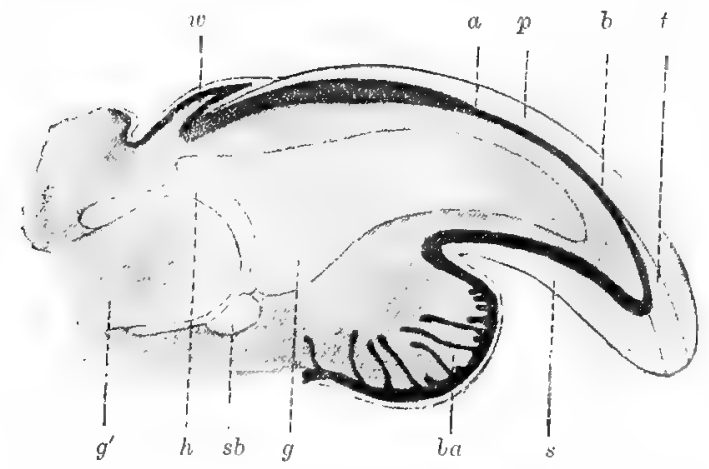

Fig. 381. Longitudinal section of the tip of a Mammalian digit. Malpighian layer dark, $a$ and $b$ see p. $471, b a$ ball of the foot with sweat glands, $g$ last phalanx of the digit, $g^{\prime}$ next phalanx (not completely drawn), $h$ cavity of the joint, $p$ and $t$ wall, $s$ sole, $s b$ sesamoid bone, $w$ fold of skin at the base of the claw.-Orig.

very small here, and confined to the distal portion of the toes. In the Horse (Fig. $382 D$ ) the hoof is, so to speak, arched round the very small sole of the foot (the "frog"), so that the latter lies at the back of the hoof; relations

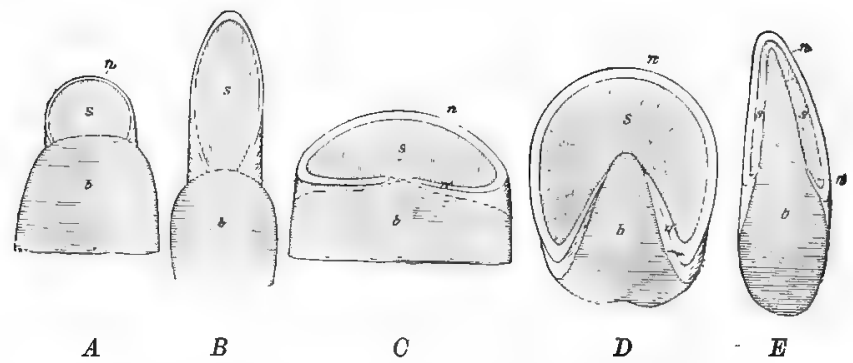

Fig. 382. Tip of toes seen from below : $A$ Monkey, $B$ a clawed animal, $C$ Rhinoceros, $D$ Horse, $E$ EIk; diagrammatic. $\quad b$ sole of the foot, $n$ edge of wall, s horny sole.-Orig. 
somewhat similar obtain in the Pig, where, however, the sole of the foot reaches farther back than in the Horse. Ruminants (Fig. $382 E$ ) display an advance on the Pig, since the true sole reaches far forwards, and the horny sole is largely suppressed, and only represented by a narrow rim along the lower edge of the horny wall: further, the most anterior portion of the true sole in many Ruminants (Red-deer, Oxen, etc.) has attained greater firmness than in others, becoming like the horny sole, whilst in others (e.g., the Roe and the Elk) it is as soft as usual.

As in the Crocodiles, Chelonians, and Birds, growth of the claws is the result of the formation of a new thin stratum corneum over the whole surface of the subjacent Malpighian layer; thus the cap-shaped claw is pushed forwards (just as in the growth of the horns of Ruminants, see below). In Mammals (and Lizards), on the other hand, a large portion of the subjacent Malpighian layer is sterile (Fig. $381 a-b$ ), i.e., is not concerned in strengthening the wall which is formed at the proximal margin of the layer (left of $a$ ), and pushed over sterile part; at the tip (right of $b$ ) a formation of horny substance $(t)$ again occurs. The horny wall, therefore, increases in thickness from the base up to $a$, retains the same thickness up to $b$, thence increasing in thickness again, except in so far as it is prevented by wear.* The horny sole, on the other hand, becomes continuously thicker from the base to the tip.

The horn of the Rhinoceros is an enormous local thickening of the stratum corneum; into it extends a papilla from the dermis, covered of course with the stratum Malpighii. The horns of Ruminants are of quite a different structure; each is to be regarded as a large, naked projection of skin, which is internally ossified, and covered superficially with a firm, thick layer of horn; the structure therefore consists of a bony mass within, the core, fused to the frontal bone; outside this, there is a layer of connective tissue and of the stratum Malpighii, and externally, the stratum corneum, which increases by new deposits from within, and is thus pushed out distally; the basal edges of the individual layers of horn appear as rings on the surface. The antlers of the Stag are very like, but they differ in having a comparatively thin layer of horn, and a covering of hair ("velvet"). In the Giraffe, where the antlers are of small size, the soft parts persist round the core; in other animals, however, when they are fully developed, the velvet shrivels over most of the surface and is rubbed off; only the basal region, the pedicle, retains the integumentary covering. The bare bony mass, the true antler, is loosened annually from the pedicle, and is thrown off; the adjacent skin then grows over the bare edges, and a new antler develops at the same spot, covered at first with velvet. In the Giraffe, no shedding occurs.

The vertebræ are usually biplanar, rarely opisthocœlous; they are connected by thick ligamentous discs of fibrous connective tissue, which contain a remnant of the notochord, the so-called nucleus pulposus, centrally. The vertebral column is composed of the same sections as in Reptilia. There are almost always seven cervical vertebræ, $\dagger$ regardless of the length of the neck. The first two of these are, as in the Reptilia, developed as atlas and axis. Monotremes alone possess separate cervical ribs (on the

* In the transparent nail of Man, the bright basal portion ("lunula") corresponds to the portion $a$ in Fig. 381 ; a bright line near the free edge corresponds with the spot $b$.

+ Exceptions: the Manatee has only six, so has one of the Sloths '(Cholopus Hoffmani), whilst another of the same genus (Ch. didactylus) has seven, and yet another (genus .Bradypus), nine. 
last six cervical vertebræ), and even here they are only separate in early life, fusing later with the vertebræ; the posterior ones are not, as in Reptiles and Birds, longer than the anterior. In other Mammalia, cervical ribs are, indeed, present, the so-called transverse processes of the cervical vertebræ; but they are at no time separated from the vertebræ, so that their identity can only be recognised by comparison with other forms. The thoracic vertebræ are more sharply marked off from the cervicals than in Reptiles and Birds, since the first bears a movable rib, articulating with the sternum. In the $\mathrm{lumb}$ a r e gion there are usually fairly large transverse processes. There are, as a rule, twenty thoracic and lumbar vertebre (the number may, however, sink to fourteen, or rise to thirty); the thoracics are twelve or thirteen, but may rise to upwards of twenty. Of true sacral vertebræ, i.e., those to which the ilia are attached; there are, as in Reptiles, usually only $t w o$ fused together in the adult, but in most Mammals one or more of the anterior caudal vertebræ (the false sacral vertebræ) assist in forming the sacrum. The caudal vertebræ vary considerably in number
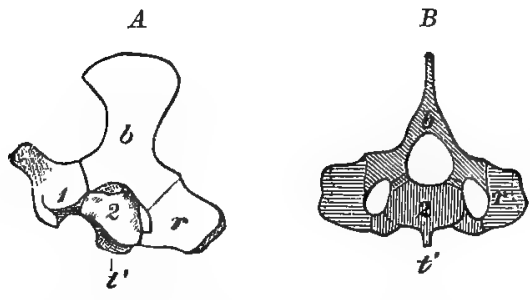

Fig. 383. Axis of a young Platy pu (Ornithorhynchus) from the left side $(A)$, and from behind $(B)$. 1 centrum of the first cervical vertebra, 2 do. of the second, $b$ arch, $r$ rib, $t^{\prime}$ inferior spine. In $B$ the arches, centrum, and ribs are shaded in different ways.-Orig.

the anterior ones usually have well-developed transverse processes, and often bear $\mathrm{V}$-shaped bones, like those of many Reptiles, on the ventral side; the posterior tail vertebræ are always more or less imperfectly developed, especially the last (arches and processes degenerate).

The ribs always consist of an upper and a lower portion, of which the latter is usually cartilaginous or only partially ossified. In the Monotremes, yet a third portion is intercalated ( $c f$., the Crocodilia) between these two. The majority of the ribs, the anterior so-called true ribs, are attached to the sternum, whilst the posterior, or so-called false ribs, are attached to one another and to the last true rib, or terminate quite freely (floating ribs). The rib articulates with the transverse process of the corresponding thoracic vertebra by. an external outgrowth, the tuberculum (generally absent from the posterior ribs); and with the centrum by the capitulum, the true dorsal end of the rib. The articular facet lies upon one centrum, or between that centrum and the one in front of it. The true ribs, of which the first is usually especially strong, become longer towards the 
sacrum; the false ribs, shorter. The sternum, which is almost always long and narrow, consists at first of a cartilaginous mass, in which a series of ossifications appears later; the latter usually remain separate throughout life, so that the adult sternum retains a jointed appearance; occasionally they fuse to a great extent (as in Man). The most anterior joint, the manubrium, is generally somewhat broader than the succeeding ones; to this the first pair of ribs are attached, whilst the other true ribs are attached at the junctions of the other joints. The last joint, the xiphisternum (processus xiphoides), with which no ribs are connected, usually ends in a broad cartilaginous plate. Only in the Monotremes is there an episternum corresponding with that of Reptiles, as in many Lizards it is here a large T-shaped bone.

The skull of the adult consists chiefly of bone, and exhibits but little cartilage. Not only the small premaxilla and the large maxilla, but also the bones belonging to the upper portion of the gill-bars, are fused with the skull. Of these only the palatine, which is attached anteriorly to the premaxilla, and the somewhat small pterygoid are present, whilst the quadrate has disappeared (or at least in its usual form, see below under the ear) ; the lower jaw, which consists of a single bone on each side, articulates directly with the skull. The two

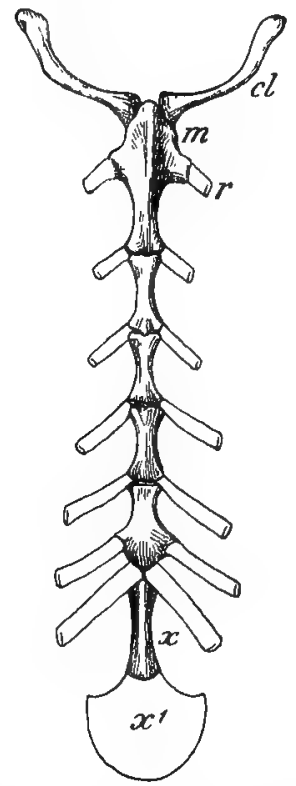

Fig. 384. Sternum and clavicle of a Kangaroo. cl clavicle, $m$ manubrium, ? ribs (cut off), $x$ xiphisternum, $x^{1}$ its cartilaginous terminal plate.-Orig.

rami are either united anteriorly by means of connective tissue, or (in the adult) are anchylosed (Horse, Man, and others). There are two occipital condyles instead of one as in Reptiles and Birds. There is no interorbital septum as in many Reptiles, etc.; the cranial cavity extends forwards as far as the nasal cavities. The latter are usually very well developed; they are separated by a plate, at first cartilaginous, later partially replaced by bone, which arises from the anterior wall of the skull, and projects forwards. They are also at first surrounded laterally and dorsally by outgrowths from the anterior region of the cartilaginous cranium, but after a time these ossify, or are covered in by membrane bones, and then dwindle away; those portions which surround the external nares and the adjacent regions persist, however, throughout life. In the adult the nares are surrounded by various bones, laterally chielly by the maxillæ, dorsally by the well-developed, flattened nasals, which tourh in the median line; ventrally by the palate 


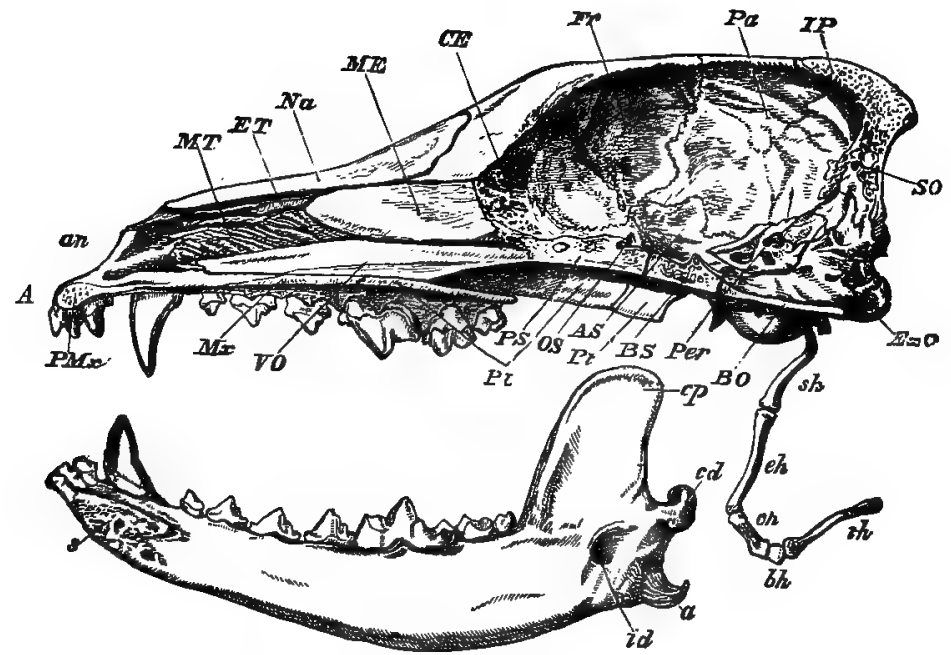

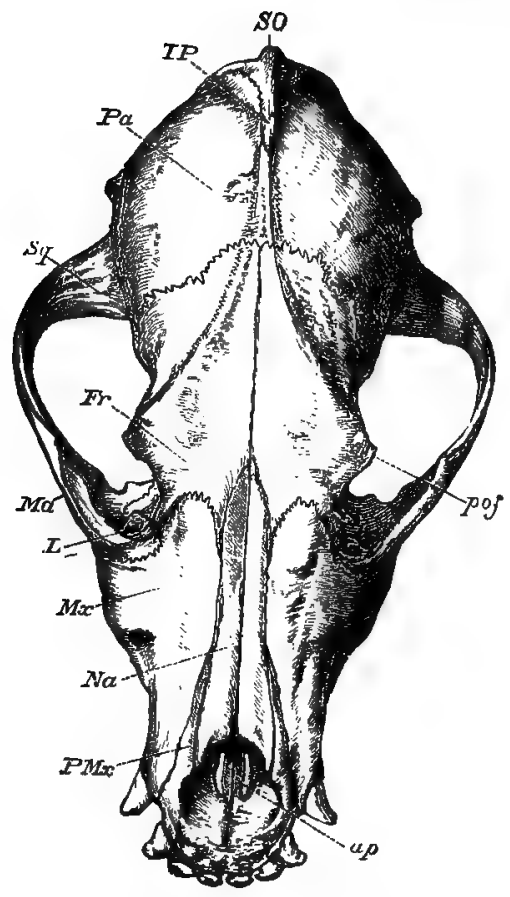

B

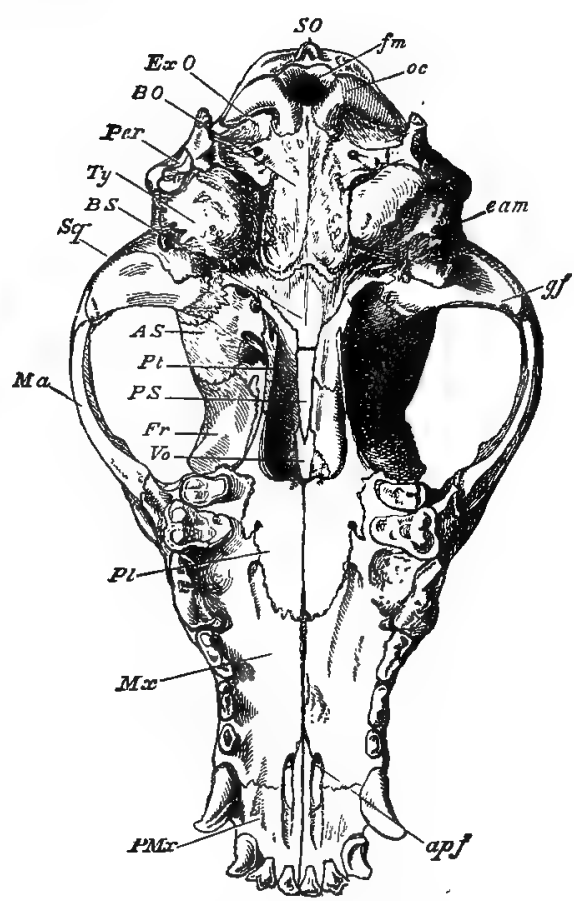

C

Fig. 385. Skull of a Dog. A sawn through longitudinally; $B$ dorsal, $C$ ventral. The cartilaginous parts are removed. $A B$ lateral parts (wings) of the sphenoid, $B O$ basioccipital, $B S$ basisphenoid, $C E$ cribriform plate, $E T$ ethmo-turbinal, $E x O$ exoccipital, $F r$ frontal, IP interparietal, $L$ lachrymal, $M a$ jugal, $M E$ bony portion of the nasal septum (connected behind with the cribriform plate), $M t$ maxillo-turbinal, $M x$ maxilla, $N a$ nasal, $O S$ orbitosphenoid, $P a$ parietal, $P e r$ petrosal, $P l$ palatine, $P M x$ premaxilla, $P S$ presphenoid, $P t$ pterygoid, $S O$ supraoccipital, $S q$ squamosal, Ty tympanic bulla, $\nabla_{0}$ vomer, $c h, e h, s h$ joints of the anterior cornua of the hyoid, $b h$ body of the hyoid, $t h$ posterior cornua, an external nares, ap and apf canalis incisivus, cd articular facets of the mandible, eam external auditory meatus, $f m$ foramen magnum, $g f$ articular facet on the skull for the lower jaw, oc occipital facets, $s$ symphysis of the mandible.-After Flnwer. 
(hard palate), which is formed of horizontal medianly apposed portions of the premaxillæ, maxillæ and palatines.* Behind, in the septum between the cranium and the nasal cavities, which was originally cartilaginous, there is a bone with numerous fine perforations for the olfactory nerves, the cribriform plate. From the front side of this thin, folded, bony lamellæ arise, covered by a thin membrane and projecting far into the nasal cavity ; they are known as the ethmoturbinal. Further forward on the outer wall, there is a bone composed of a varying number of delicate bone lamellæ, the maxilloturbinal, so that the greater part of the cavity is filled up. There are larger or smaller air spaces (Fig. 386) in certain bones of the head in connection with the nasal cavities of the Mammalia; especially in the maxilla (maxillary sinus) and in the frontal (frontal sinus); sometimes (in the Ox, Elephant, etc.) these sinuses are

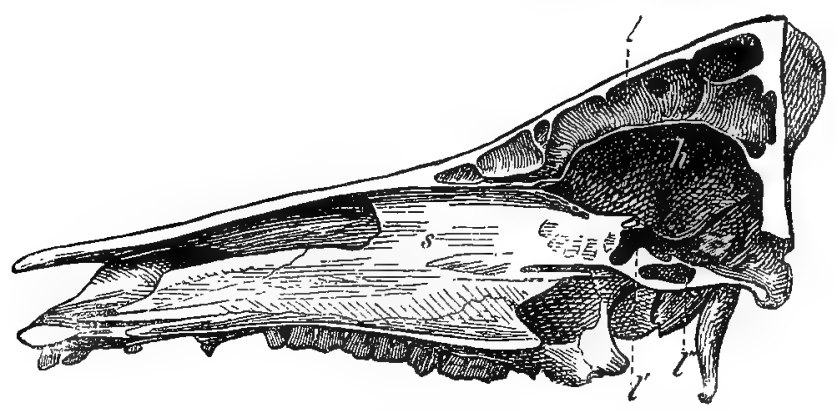

Fig. 386. Skull of an old Pig, sawn through longitudinally, in order to show the large a $\mathrm{r}$ sinuses. $h$ cranial cavity, $l l^{\prime} l^{\prime \prime}$ air sinuses partially ( $l^{\prime}$ the frontal sinus) divided by bony plates, s bony nasal septum.-After Bendz.

of considerable size, extend into other bones, and are divided by incomplete septa into a number of small cavities. Amongst other characteristics of the skull it may be mentioned here, that a bony bridge, the zygomatic arch, $\uparrow$ runs from the articulation of the mandible to the maxillæ; it is formed by a process of the squamous (see below), of the jugal, and sometimes a process of the maxilla ( $c f$., the similar bony bridge in Reptiles and Birds which is formed of the quadratojugal and jugal). The hyoid consists of an unpaired body and two horns on each side. The anterior horn, which corresponds to the hyoid of Fish, is usually the longer, and consists of three movable joints; it is attached by its upper end to the skull (prootic).

* The palate is perforated in front at the junction of premaxillæ and maxillæ by two openings (canales incisivi), through which the Stensen's ducts, mentioned on p. 333 , pass.

f In some Mammals there is a process near the middle of the jugal which meets a similar one from the frontal and forms with this a bony bridge behind the eye. 
The posterior horn, which corresponds to the first gill bar, is short and unsegmented.

The foramen magnum is surrounded by four bones, the supra- basi- and two ex-occipitals; the latter bear the condyles, which may, however, extend on to the basi-occipital. In front of this bone lies the basisphenoid, in front of this again, the presphenoid, both developed in the ventral region of the cartilaginous cranium, and both provided with wing-like lateral processes (aliand orbito-sphenoids) which assist in forming the cranial boundaries; in front of the sphenoids lies the cribriform plate. The periotic (petrosal) within which the auditory organ is situated, lies in front of the ex-occipital: the squamous, from which the jugal arises, is attached externally to this; and the tympanic, a circular bone over which the tympanum is stretched, lies upon it: in many Mammalia these three bones fuse at a very early period, and are termed together the temporal. A single or paired bone, the interparietal, lies above the supraocipital, and in many cases (e.g., Man) fuses with it even in embryonic life. The parietals lie in front of the interparietal, and anterior to these again are the frontals, overlapping the lachrymals, upon which the lachrymal duct opens in front of the orbit on either side. The posterior region of the nasal septum is ossified, and forms a plate, the lamina perpendicularis, perpendicular to and fused with the cribriform plate, whilst the anterior portion remains cartilaginous; the lower part is formed by an unpaired compressed bone in the form of a trough, the v o mer, (difficult to homologise with the bone so-called in the lower Vertebrata). The pre- and post-frontals, the quadratojugal, the transverse bone, and the columella, besides the quadrate, all well-known in reptilian skulls, have disappeared. In general, the bones of the mammalian skull are only separate during youth, in later life they fuse entirely or to a great estent.

The great diversity of external form displayed by mammalian skulls is directly dependent on the varying development of the organs within and upon it. In this connection, the brain is of great significance; with a great development. of this, as compared with the other organs of the head, the posterior region of the skull preponderates over the anterior (face portion); this is the case, for instance, in Man. The heterogeneity of the teeth has also considerable influence upon the form of the skull; their great development leads to a corresponding hypertrophy of the bones in which they are implanted, and also of the parts upon which the masticatory muscles are inserted. The development. of the organs of the nasal cavity, and also the varying size of the eye, are of great importance, whilst the presence of horns or antlers brings about an increased development of that part of the skull to which they are attached. In large skulls of large animals with large teeth, horns, etc., the air-sinuses often occupy a considerable space: the large bony masses necessary to support these parts, or to afford attachment for the muscles, are hollowed out, a point of very great importance to the animal (e.g., Horse, Ox, Elephant). It may also be mentioned here that the skull of the young animal often differs considerably in external form from that of the adult: the brain is proportionately larger; the teeth and masticatory muscles feebler; the face portion therefore small; the air sinuses little developed; the projections from which the masticatory muscles arise, small or absent, etc. The skull of smaller (adult) Mammals bears in many respects the same relation to the skulls of larger allied forms that the young one does to the adult of the same species; the cranial portion is larger, the muscular ridges smaller.

The shoulder girdle of the Monotremes is similar to that of the Reptilia; both scapula and coracoid are well developed; the latter is broad and flattened, divided into anterior 
and posterior portions, and attached by its anterior end to the sternum; a clavicle also occurs, extending from the edge of the scapula to the episternum, just as in Reptiles. In all other Mammalia, however, there is a considerable modification; the coracoid* has become rudimentary, and does not reach the sternum: it fuses early in life with the scapula, and is represented only by a projection from the ventral end of this, the coracoid process. The scapula is usually a broad plate, the upper part of which generally remains cartilaginous; it is provided on its outer

Fig. 387.

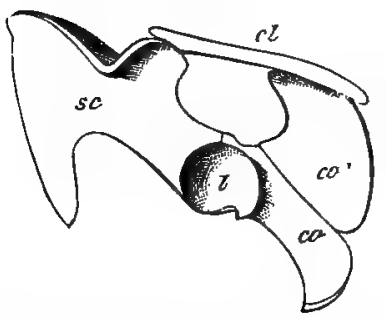

Fig. 388.

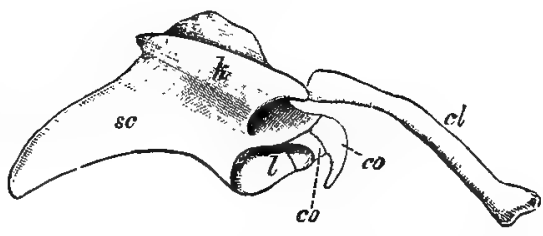

Fig. 387. Right half of the ahoulder girdle of a young Platypus. $c$ l clavicle, co' anterior, co posterior portion of the coracoid, $l$ glenoid cavity, sc scapula.Orig.

Fig. 388. Right half of the ghouldex girdle of a young Ape; shoulder blade much foreshortened. $k$ spine of the scapula. Other letters as in the preceding figure.-Orig.

surface with an erect longitudinal "spine" with a ventral projection, the acromion, to which the outer end of the clavicle is attached; whilst its inner end is connected with the manubrium. In many Mammals, the clavicle is wanting (e.g., in all the Ungulata), or is rudiment ry (Dog), and in these cases the shoulder girdle has no direct connection with the axial skeleton; in others, e.1/, in digging, climbing, and flying forms, the clavicle is a strong rodlike bone.

The skeleton of the for e limb consists of the usual parts. 'l'he bones of the forearm are usually either about equal in size, or the radius is stronger, at least, at its lower end, whilst the lower portiou of the ulna is often rudimentary, though its upper end, which bears the projecting olecranon, is usually well developed. The two bones often $\mathrm{cross}$, since the radius is articulated above to the outer, the ulna to the inner side of the humerus, whilst below, the radius is connected with the inner, the ulna with the outer portion of the carpus : in other cases, however, the distal end of the ulna is pressed right behind the radius, so that no true crossing occurs. The two

\footnotetext{
* In young animals, the coracoid is represented by two separate ossifications, which fuse later with the scapula (cf., Fig. 388.)
} 
bones are either movable, or are immovably bound together; in the latter case they often fuse with increasing age. The carpus consists of two transverse rows of bones; in the proximal row there are usually tbree bones, in the distal, four, the two outer bones of the typical five (fourth and fifth distal carpals) being fused. $\dagger$ At the outer edge there is a rather large sesamoid bone, the $p$ is if $0 \mathrm{rm}$. Of the five digits, the pollex (first) has two phalanges, the others each three; only in certain, much modified forms (Whales), is the number increased. In certain Mammalia the pollex is more freely movable than the other fingers, so that the hand is a prehensile organ; where it has not this function it is usually reduced, or altogether absent. Other fingers also may dwindle or vanish, particularly if the limbs are specially adapted for walking or running (Ungulata): with the decrease in number, there is an increase in power on the part of the remainder; in such cases a fusion of metacarpals may occur. The structure of the fore limb shows great variety in correlation with the varied function (digging, climbing, flying). (See also the special descriptions).

The pelvis is peculiar in that the ilia are directed backwards; the point of attachment to the sacral vertebræ lies towards its anterior,

Fig. 389.

Fig. 390.
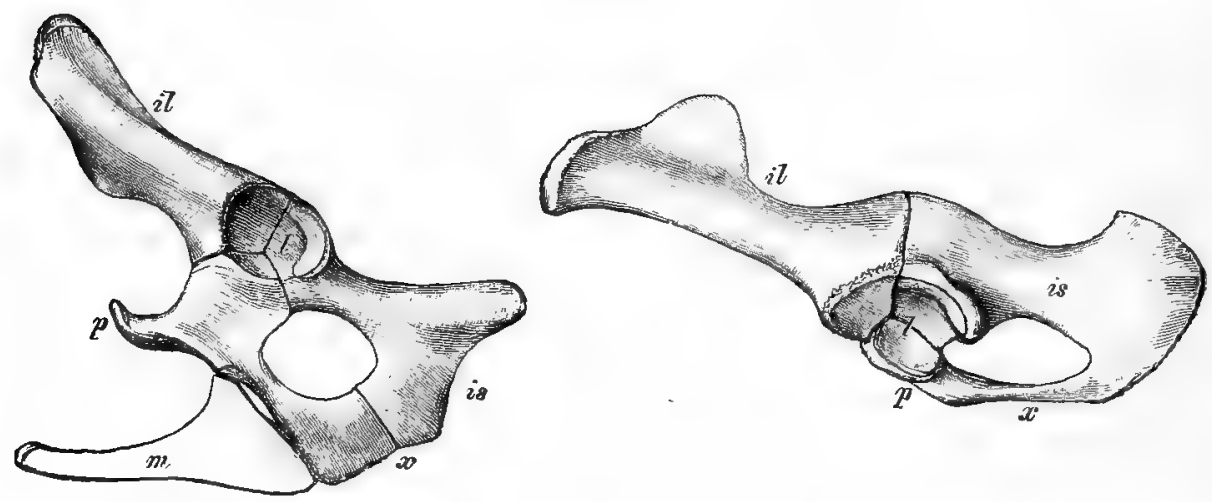

Fig. 389. Left half of the pelvis of a young Ornithorynchus. $l$ acetabulum, il ilium, is ischium, ' $p$ pubis, $x$ point at which the ischium and pubis unite behind. $m$ marsupial bone.-Orig.

Fig. 390. Left half of the pelvis of a new born Calf. oc point at which ischium and pubis unite with one another below; the other letters as in the preceding figure.--Orig.

* The lower end of the radius, with the hand (which is only connnected at a definite spot with the ulna, elsewhere with the radius), can then swing outwards; this is especially the case in Man.

$\dagger$ The carpals of Mammalia are usually distinguished by the following names : the proximals from within outwards, scaphoid, lunar, and cuneiform; in the distal row, trapezium, trapezoid, magnum, and unciform. In some cases (by reduction of the number of metacarpals), some of these bones may be absent; or fusions may occur. Occasionally a centrale is developed between the rows. 
the acetabulum at the posterior end (whilst in Reptilia the ilium is directed forwards or antero-ventrally). The ischia and pubes of each side fuse, and the pubes also anchylose in the mid line; as may also the ischia; occasionally there is no connection between the two halves of the pelvis (e.g., certain Insectivora). In the adult all three bones of each side are completely fused.*

In the Monotremes and the Marsupials, attached to the anterior edge of the pubis, is a pair of forwardly-directed bones, the so-called marr u pial bones; they may be regarded as ossifications in the tendons of the abdominal muscles.

Hind limbs. The tibia is always stronger than the fibula, which is. often very thin, or indeed imperfect at its lower. end, where it is usually fused with the tibia. A large patella lies over the knee-joint, anteriorly. There are only two bones in the proximal row of the tarsus; the astra$\mathrm{g}$ alus within, and the calcaneum, with the much projecting heel, postero-externally. Movement occurs between the lower end of the fore-leg and the astragalus (or sometimes the calcaneum), whilst movement between the tarsals themselves is usually much limited ( $c f$. , the very different conditions in Reptiles and Birds). In the distal row there are fou r bones, $\uparrow$ as in the band; between the two rows on the inner side there is a c e n $\mathrm{trale}$ (naviculare). Metatarsus and toes as regardsthe number of phalanges, etc., agree with the metacarpus and fingers; and with regard to special developments, such as reduction in the number of toes, the relations are usually similar. Sometimes, however, the hand is modified differently from the foot (e.g., in leaping or digging animals).

Other sesamoid bones are found besides these already mentioned (pisiform, patella). namely, below the joints between each metacarpal and the first phalanx (also between each metatarsal and the first phalanx of each toe), there are two small bones;

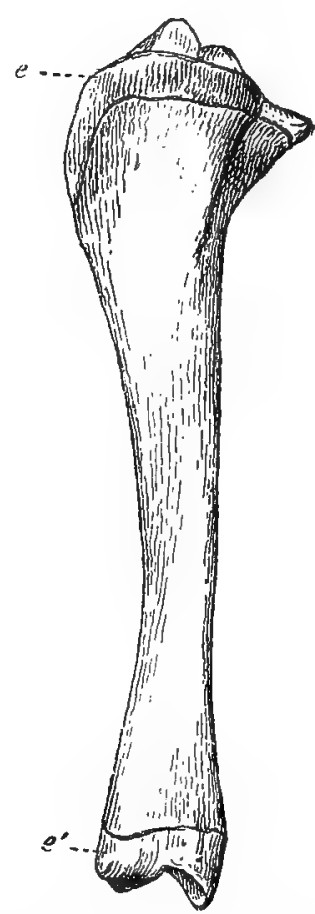

Fig. 391. Tibia of a one year old Horse to show the epiphyses $e$ and $e^{\prime}$.- Orig. and below the joint, between the last and the penultimate phalanx of the finger and toe, one sesamoid bone; other smaller ones may also occur, but less frequently.

* The two halves may be anchylosed in the mid-ventral line, and the ilia may also fuse with the sacral vertebræ. In some forms (eg., certain Edentata), the ischia may be fused with the posterior false sacral vertebræ.

$\dagger$ Cuneiforms 1, 2, and 3, and the cuboid, the latter consisting of distal tarsals, 4 and 5. 
A general characteristic of the mammalian skeleton consists in the occurrence of special ossifications for the end portions of many bones, especially of long bones, and also of many processes, so that in young animals many bones consist of several pieces, which fuse later. These special terminal ossifications are termed epiphyses.

The brain is characteristic in many respects. The cerebrum is of considerable size; its surface is marked by deep labyrinthine furrows, the sulci, separated from each other by ridges, the gy r i : occasionally the surface is smooth, or almost smooth, as in the Rodents. 'The hemispheres cover not only the thalamencephalon, but usually the mid-brain also, and sometimes part of the cerebellum. Peculiar to the Mammalia is the so-called corpus callosum, an important system of transverse nerve fibres, which pass from one hemisphere to the other; these fibres are least developed in the Monotremes and Marsupials. The $\mathrm{mid}-\mathrm{brain}$ is divided not only by a longitudinal, but also by a transverse furrow, so that it forms four dorsal lobes (corpora quadrigemina). The cerebell $\mathrm{um}$ is well developed, its much thickened dorsal wall is divided into two median and two lateral portions, and is transversely folded.

The size of the brain, as compared with that of the rest of the body, is closely correlated with the intellectual level of the species under consideration (see, for instance, the enormous development in Man). There are, however, other circumstances which are of great importance in this connection : noticeably it is a rule that small Mammals have relatively larger brains than have their nearest allies; it may be said that, on the whole, the size of the brain varies inversely as the bulk of the animal; the Elephant, for instance, in spite of its striking intellectual qualities, has a relatively minute brain. It may also be noticed here that the brain of $\mathrm{y} \circ \mathrm{ung}$ animals is relatively larger than that of the adult.

Olfactory organs. Prominent folds, the turbinals, project into the nasal cavities, which are usually of considerable size. The turbinals arise from the postero-external wall, developing as large lamellæ, which become folded and coiled so as to form very complex structures. They are supported at first by cartilage, which becomes partly or entirely ossified, to form the maxillo- and the ethmoturbinals. The olfactory epithelium is situated in that part of the mucous membrane which lines the region of the nasal carity nearest the cribriform plate; it is yellowish-brown in colour. 'I'he rest of the cavity has no olfactory significance; in addition to mucu secreting glands it has a rich vascular network which, according to some authorities, serves to warm the air entering the lungs. The air sacs of some of the skull bones mentioned above (p. 475-6) are outgrow ths from the nasal cavity, and are lined with a continuation of its mucous membrane.

Optic Organs. In contrast to other Vertebrata the upper eyelid is larger and more movable than the lower. A nictitating me mbrane is always present but less well developed than in Birds and Reptiles, and usually not provided with special muscles; it slips 
over the outer surface of the eyeball when this is withdrawn into the orbit and covers it partially or entirely. Sometimes (e.g., in Man) it is rudimentary.

The sclerotic consists of connective tissue without cartilage* or bone; in some Mammalia, especially in the Whales, it is very thick. In the choroid coat there is frequently a peculiar greenish, blue, or whitish, shimmering membrane varying somewhat in structure, the tapetum (e.g., in the Horse, Ruminants, Carnivora). The form of the pupil varies, it is either circular (e.g., in Man,), or a perpendicular (Cat, Fox), or horizontal slit (Horse, Ruminants).

Auditory organ. The cochlea of Monotremes is like that of Crocodiles and Birds; in all other Mammalia, however, it is much longer and is spirally coiled. As in Reptiles there is a fenestra ovalis and a fenestra rotunda. The single ear-

Fig. 392. Diagrammatic transverse section of the head of a Mammal, to show the relations of the auditory organ; (the labyrinth is drawn proportionally much too large, etc.). $a$ ampulla, $b$ semi-circular canal (only one is represented) $c$ cochiea; $s a$ sacculus, $u$ utriculus (together forming the vestibule); round the labyrinth the cavim perilymphaticum, black in the figure. $h$ bones of the skull, $h$ malleus, am incus, $s$ stapes, $t$ tympanic cavity, $r$ fenestra rotunda, e Eustachian tube, tr tympanic membrane, $g$ external auditory meatus, $\ddot{o}$ external ear. Orig. (with partial use of older figures).

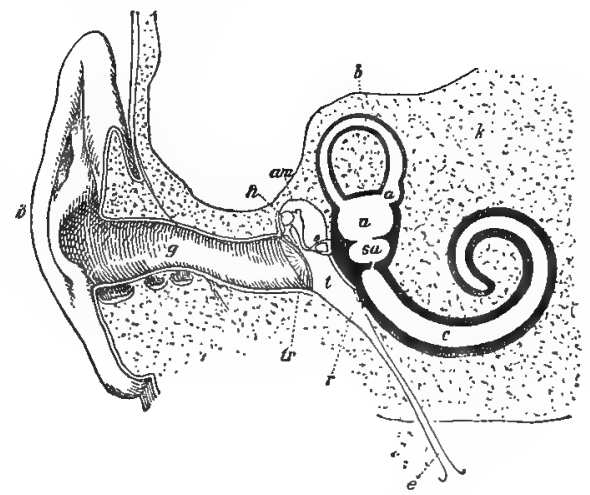

bone of Reptiles is broken into three, the $\mathrm{malle} \mathrm{u} \mathrm{s}$, which is connected with the tympanum, the in cus; and the stapes, the terminal disc of which closes the fenestra ovalis ; $t$ in the Monotremes it consists of a plate and a single shaft; which is usually broader and perforate in other orders, so that the ossicle becomes like a stirrup. The presence of an external meatus is characteristic of the Mammalia; the pit, at the base of which the tympanum is situated in Reptiles, has become a long tube here; the inner part is often ossified (a tubular elongation of the tympanic bone), whilst the external portion is supported by cartilages. The external auditory meatus is usually surrounded by the pinna, a large fold of skin varying in form and containing a considerable amount of elastic cartilage.

* In the Monotremes, the sclerotic is partly cartilaginous.

† According to another interpretation the malleus is homologous with the quadrate of Reptiles, whilst the incus is to be regarded as representing the outer portion of the reptilian columella auris; others again regard the incus as corresponding to the quadrate, the malleus to the upper posterior bone of the mandible of Reptiles (articulare). 
The tympanic carity which lies enclosed in the temporal bone, is often of considerable size, so that the surrounding bony portions (especially the tympanic bone) are swollen to form a vesicle (bulla); sometimes the tympanic cavity is connected with air sinuses in the neighbouring bones (cf. Crocodilia and Aves). The walls of the eustachian tubes are usually partly ossified; they open separately into the pharynx. In the Horse and Tapir, each Eustachian canal bas a very large thin-walled, saccular extension.

The buccal cavity in young embryos, as in most Reptiles, is undivided. Quite soon, however, a ledge develops laterally and above, and unites with its fellow of the other side to form a horizontal septum, the anterior end of which (covered, of course, on both sides with mucous membrane) is the $\mathrm{h}$ a $\mathrm{rd} \mathrm{palate,} \mathrm{whilst}$ the hinder portion remains soft, and forms the muscular soft palate. The cavity above the hard palate unites with the nasal cavities (the nasal septum growing down and becoming commected with the hard palate); the cavity above the soft palate, which communicates freely at its front end with the nasal cavities, remains single, and is termed the pharynx; it includes also the posterior region of the primitive buccal cavity. The eustachian canals open above, the trachea below into the pharynx (Fig. 396). 'The buccal cavity lies below both hard and soft palates and encloses the teeth, tongue, etc.

The teeth of Mammalia are chiefly remarkable in that their number is small and fairly constant for a given species; that their form is usually relatively complex; that they are implanted in sockets; and especially that the mode of replacement is peculiar, for the teeth are not, as in other Vertebrata, replaced continuously throughout life, but only two series, the milk and the permanent dentitions, are present, following each other in regular sequence. It may be noticed further that the teeth are used not only for the prehension of food, but also very largely for its mastication. In addition to the two ordinary components of teeth yet a third is present, the cement, occurring chiefly at the root (see below). It is simply a sheath of osseous matter deposited by the connective tissue surrounding the tooth; it lies external to the rest of the tooth, and is formed last: it is not as hard as dentine.

Two parts may be distinguished in a mammalian tooth, the crow $n$ and the root. The root is the lower, ${ }^{*}$ usually narrower part, and is often split into several branches; it is destitute of enamel, but is covered with cement. The crown is the upper enamelled portion, which generally projects quite freelr, and is usually clearly demarcated from the root; for instance, by a constriction. Cement does not occur in this region, excepting as an occasional layer, varying in thickness, upon the surface of the enamel. The crown exhibits a great diversity of for m; it may be simply conical or chisel shaped,

* The free end of the tooth is termed the upper, the opposite the lower, end; although this terminology is actually correct only for the teeth of the lower jaw, 
it may be low and broad, provided with rounded or pointed tubercles, or it may be much compressed and serrate at the edge; or again, it may be traversed by marked transverse or longitudinal ridges separated by valleys, which may be so deep as to reach the base of the crown (e.g., in the molar teeth of the Elephant). Perpendicular furrows may also occur on the sides of the teeth; usually the deeper folds are partially or completely filled with cement (in the Horse and Elephant). During use, the enamel, especially in plicate teeth, is very frequently worn away at all the projecting points, and the subjacent dentine is thus laid bare; at the grinding surface, therefore, may be seen islands of dentine surrounded by slightly elevated enamel borders, and the latter are often again surrounded by cement (especially in teeth of herbivorous animals). The crowns and roots of many teeth are of almost equal length; sometimes one, sometimes the other may, however, be the longer. The former especially the case with much folded teeth, which are subjected to considerable wear and tear; in these the root (or roots) is often very short; the crown, on the contrary, is very long, but it projects from the jaw for only part of its length, and gradually, as the free end is woru away, the tooth is pushed out (e.g., in the molars of the Horse). The crown has often begun to wear down before the root is formed; in other cases there is actually no development of a root; as the crown is worn away above, growth takes place below, and never ceases; such teeth are said to grow from persistent pulps (the incisors of Rodents, the molars of many of the same group, the canines of the Boar, etc.).

The teeth are arranged in a single row along the edge of premaxilla, maxilla, and mandible; those of the premaxilla are designated in $\mathrm{c}$ i s o r s, the anterior teeth of the maxilla, next to these, canines; the rest, molars; in the lower jaw the teeth which bite just in front of the upper canines are known by that name also; those anterior, the incisors; those behind, the molars. In most placental* Mammals the number of teeth on each side of the jaw, $\dagger$ in the second or permanent dentition, is not more than eleven, three incisors $\left(i^{1}, i^{2}, i^{3}\right)$, one canine (c), seven molars, of which the four
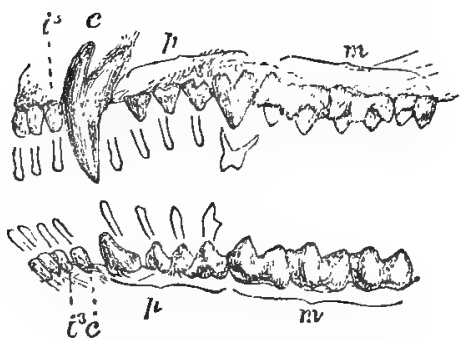

Fig. 393. Dentition of a $\mathrm{Mol} \theta$ (complete dental formula); the milk teeth are drawn in outline above or below the corresponding permanent ones. $i^{3}$ third incisor, $c$ canine, $p$ premolar, $m$ molar.After Ch. Tomes. anterior are termed premolars

* The placental Mammals include all Mammalia excepting Monotremes and Marsupials (see p. 493).

† One premaxilla and one maxilla are regarded as half of the upper jaw. 
$\left(p^{1}-p^{4}\right)$; the three posterior, molars $\left(m^{1}-m^{3}\right) *^{*}$ In the complete first dentition, the milkdentition, which is present in the young animal, but is lost after a time, there are eight teeth in each half of the jaw; three incisors $\left(d i^{1}-d i^{3}\right)$, one canine $(d c)$, and four molars $\left(d p^{1}-d p^{4}\right)$, occupying the places in the jaw which will be filled afterwards by the corresponding permanent incisors, canines, and premolars, whilst the true molars have no predecessors. The number of teeth is, however, reduced in many forms, and the reduction affects not only molars, but even incisors and canines. Usually it is not difficult to determine, by comparison, which are the missing teeth. In the molar series the reduction usually begins either at the anterior or the posterior end, so

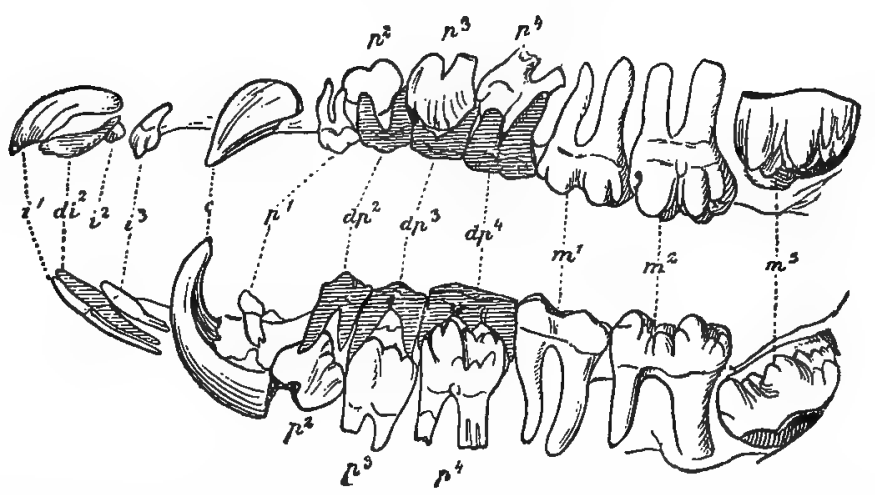

Fig. 394. The teeth of a $P i g$ showing the $x \in p l a c e m e n t$, the jaws cut away. $i^{1}-i^{3}$ first to third incisors, $c$ canine, $p^{1}-p^{4}$ premolars, $m^{1}-m^{3}$ molars, $d i^{2}$ second deciduous incisor, $d p^{2}-d p^{4}$ deciduous molars. (milk teeth shaded). Of the deciduous teeth $d i^{1}, d i^{3}, d c$ have already fallen out; $d p^{1}$ is wanting in the Pig.-Orig.

that if only six molars are present the absent tooth is either the first premolar or the last molar; if only five are present the missing teeth will be $p^{1}$ and $p^{2}$, or $m^{2}$ and $m^{3}$, or again, $p^{1}$ and $m^{3}$. In some groups, the molars disappear first (in the Seals); in others, the premolars (e.g., in the Rodents); in yet others (e.g., the Cat), teeth are missing from both ends of the series. The number of milk teeth may be similarly reduced; if a tooth of the permanent dentition is wanting, as a rule the corresponding milk tooth has also disappeared; but there are several exceptions. Of the typical deciduous molars, however, the first $\left(d p^{1}\right)$ is usually absent, even when the corresponding permanent tooth $\left(p^{1}\right)$ is present; occasionally other milk teeth are wanting, although the permanent ones are present; sometimes (e.g., in the Seals) they are absorbed during embryonic life, or fall out at birth, and they are then

\footnotetext{
.$^{*} i^{1}$ is the most anterior (the innermost) incisor, $p^{1}$ the most anterior premolar, $m^{1}$ the most anterior molar, etc,
} 
very poorly developed, or even rudimentary. The molars are usually the most complicated teeth, whilst the incisors and canines are simpler; the canines are generally conical, the incisors, for the most part, chisel-shaped. The teeth of the milk dentition usually resemble those of the permanent set; but a given milk tooth is not always exactly like that which is to take its place: in the Carnivora, for instance, each milk molar is very like the permanent molar, one place further back in the series. In some placental Mammals, there may be conditions very different from those just described, in that a la $\mathrm{rg}$ e r $\mathrm{number}$ of teeth may be present. This is especially the case in forms which, in correlation with peculiar habits, have in some respects, descended, so to speak, to a lower zoological grade; for instance, in the Toothed Whales, whose mode of life closely resembles that of Fish, the teeth are uniform, usually conical in shape (bomodont), and very numerous; in animals, too, whose teeth are of subordinate importance, there may be an increase in number accompanying a degeneration in form and structure (e.g., in the Dasypodidæ). Where there is so aberrant a condition of the permanent teeth, there is frequently an entire absence of milk dentition. 'The permanent dentition of the Marsupials differs from that of the Placentalia in that it is composed of a larger number of teeth, and also, that the milk dentition is represented by a single molar; for details see this group.

The following points may be added to the description of the mammalian dentition just given. The enamel is frequently thinner in some parts of the

Fig. 395. A Incis or of a Dog, shortly after it has come into use; $B$ the sam e tooth of an old Dog; longitudinal section. In the young tooth the pulp cavity is very large; there is as yet no cement (or very little); in the old tooth the upper portion of the primitive pulp cavity is entirely filled with dentine, and the rest is very narrow, the cement abundant, the tip of the tooth worn away. $c$ cement, $d$ dentine, $p$ pulp cavity, $s$ enamel.-Orig.

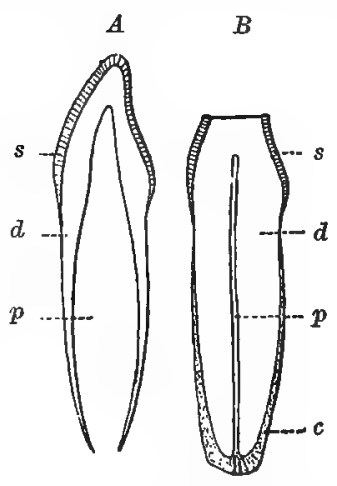

crown than in others; or it may be absent from certain regions (e.g., from the posterior side of the incisors of the Rodents); from almost the whole tooth (in the incisors of Elephants, enamel occur's only upon the tip of the tooth before it is cut); or it may be completely wanting (as in many Whales). When the tooth is cut and comes into use, it is not, as a rule, completely developed; the root is frequently not yet fully formed; the dentine has not attained its greatest thickness; the pulp-cavity is large, and decreases gradually as the bulk of the dentine increases; the cement at the root of the tooth also continues to be deposited, and in very old animals, is often of considerable thickness; whilst in 
young animals it is scarcely indicated; the enamel (except in teeth growing from persistent pulps) appears to be completely developed only when the tooth comes into use. Before falling out, the milk teeth are usually absorbed to a certain extent in the lower portions by special cells lying in the surrounding connective tissue.

The number of teeth will be indicated here according to the following plan: $i \frac{s}{2}$ (= 3 incisors above, 2 below on each side), $c \frac{1}{0}$ ( $=1$ canine above, 0 below), $m \frac{6}{5}$ (=6 molars above, 5 below), or $p m \frac{3}{3}$ ( $=3$ premolars above, 3 below), $m \frac{3}{2}$ (= 3 molars above, 2 below). If the actual teeth present are to be expressed, the following formula is used, the signs above the line indicating the teeth of the upper jaw, those below, those of the lower jaw :

$$
\frac{\mathrm{i}^{1} \mathbf{i}^{2} \mathrm{i}^{3}}{\mathrm{i}^{2} \mathrm{i}^{3}} \stackrel{\mathrm{c}}{\mathrm{c}} \frac{\mathrm{p}^{2} \mathrm{p}^{3} \mathrm{p}^{4}}{\mathrm{p}^{3} \mathrm{p}^{4}} \frac{\mathrm{m}^{1} \mathrm{~m}^{2}}{\mathrm{~m}^{1}} \text {. }
$$

Some of the Mammalia are altogether edentulous, but where this is the case with the adult (e.g., Whale-bone Whale) teeth may have been present in embryonic life; or even in youth, such are, however, never cut; they are absorbed.

The presence of an uper and a low er lip is characteristic of the Mammalia. These are large, muscular folds of skin covering the edges of the jaws and continuous with each other laterally;

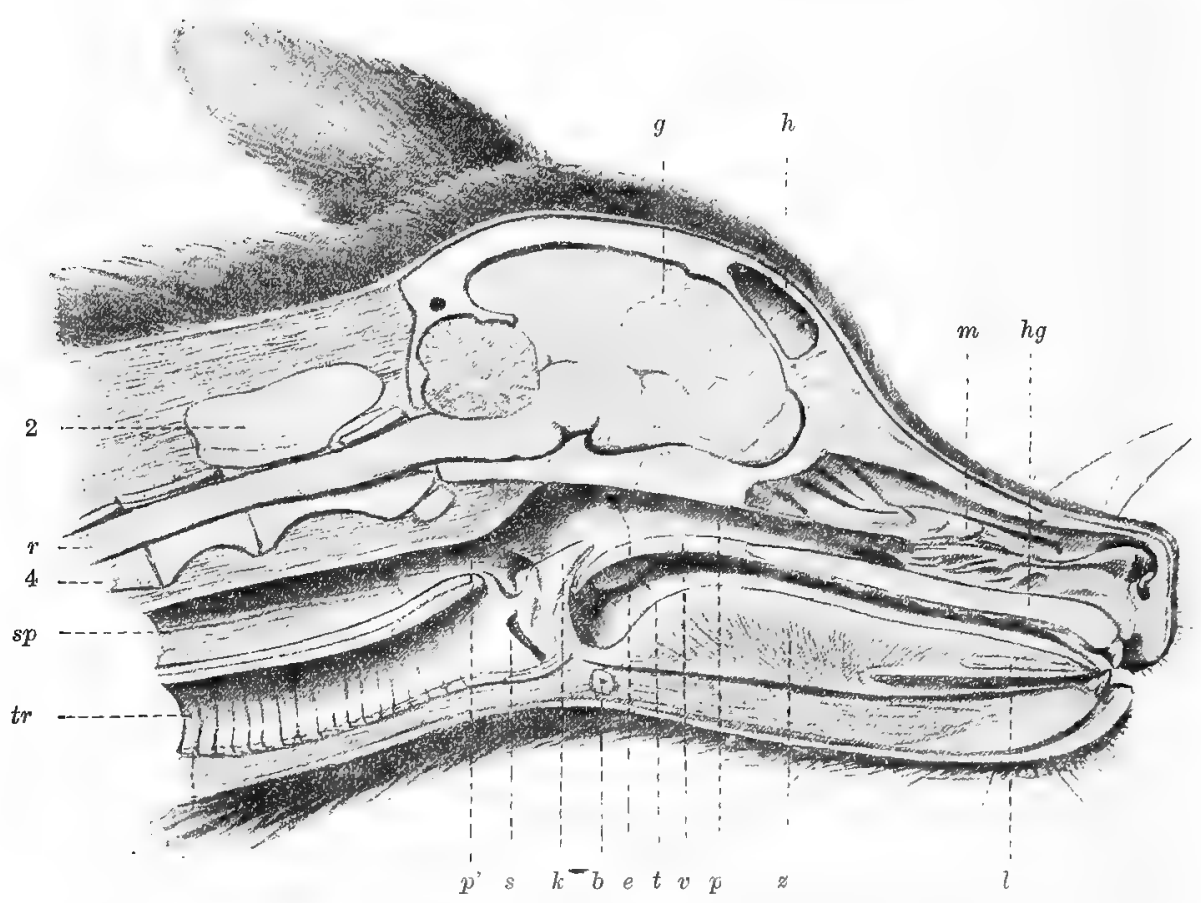

Fig. 396. Longitudinal section through the head and neck of a Dog, decreased. $b$ hyoid, $e$ opening of eustachian canal into the pharynx, $g$ brain (only suggested), $h$ frontal sinus, $h g$ hard palate, $k$ epiglottis (lies above the edge of the soft palate), $l$ Iyssa ( $c f$. foot note, p. 487)), $m$ turbinals, $p$ anterior, $p$ ' posterior edge of the pharynx, $r$ spinal cord, $s$ vocal cords, $s p$ cosophagus, $t$ tonsil (see foot note, p. 487), $t r$ trachea, $v$ soft palate, $z$ tongue, 2 axis, 4 fourth cervioal vertebra.-Orig. 
they form the cheeks and are absent unly in rare cases. In some Mammalia (e.g., many Apes, Rodents) there are cheek pouches which serve as reservoirs for food. The to $\mathrm{g} u$ u is very muscular, strong, and movable, and is thus vory useful in bringing food into the mouth; it is covered on its upper surface with small, pointed processes (papillee filiformes) which are sometimes much cornified (in the Cat); there is also a small number of various other processes ( $p$. fungiformes, circumvallatre, and foliat(' $)^{\prime}$ ), which bear taste buds.* On the hard palate there is usually a double series of fairly hard transverse folds, the palatal ridges, which often, e.g., in cattle, project considerably; or are almost or quite effaced (Man); for the peculiar development of the palatine ridges in the Whale-bone Whale, see Cetacea. Besides small glands embedded in the wall, several large salivary glands open into the buccal cavity: viz.. the parotid, submaxillary, and sublingual. $t$

The pharynx is continued into the $œ s o p h a g u s$ which is usually long and narrow. The stomach is generally a short, wide, somewhat curved tube, provided, close to the entrance of the œsophagus, with a short, blind sac which, however, passes quite gradually into the general cavity. In some Mammalia the stomach is compound, i.e., is divided by constrictions into several regions (in certain Rodents, the Whales, Ruminants, etc.) : or it is characterised by the possession of several short, blind sacs (in the pig): or it differs from the ordinary type in yet other ways, e.y., in being elongate and intestine-like (Kangaroo). Usually it is entirely lined by a cylindrical epithelium and its walls are furnished with uumerous glands (gastric and mucous): sometimes, however, the epithelium of the cesophagus, which is stratified like that of the moutb, reaches some way into the stomach, and often it may extend over a very considerable area, in the Horse about half: in most Ruminants the rumen, reticulum, and psalterium are lined with stratified epithelium. The sinall intestine is of considerable length, longest in herbivorous forms. That portion of the alimentary canal which is designated rectum in Vertebrates, is generally of considerable length in the Mammalia, usually fairly wide also, and is known as the

* On each side of the ventral surfike of the tongue, there is a fold which often unites with that of the other side; it is termed the "sub-lingua," and attains its highest development in the Prosimii, where it forms a linguiform appendage of the true tongue. In the anterior region of the tongue (Fig. 396) there is, close to the lower side in many Mammalia, an elongate structure, the so-called worm (lyssa); it is surrounded by loose connective tissue, and consists of museular and connective tissue; sometines it contains a cartilaginous portion, which apparently corresponds to the anterior end of the hyoid of Lizards (Fig. 336). Behind, at the base of the tongue, on each side, is the tonsil (tonsilla) (Fig. $396 t$ ), a region of the mucous membrane in which there are numerous lymph follicles. Such follicles are also embedded in other portions of the mucous membrane of the mouth. its duct. 
large intestine, the end portion alone being termed rectum. A $c æ c u m$ almost always arises from the large intestine at its junction with the small intestine; in some animals, (e.g., the Horse), it attains an enormous length, whilst in others (e.g., Man), it is small, or even rudimentary.* The liver, which is situated behind the diaphragm, is usually, but not invariably, provided with a gall bladder (it is wanting in, e.g., the Horse), The pancreas has generally on e duct, $\dagger$ which opens into the anterior portion of the small intestine, either together with, or independently of, the bile duct. $f$

Respiratory Organs. The entrance to the larynx is a longitudinal slit behind the tongue, anterior to the entrance into the cesophagus. In front of the opening, there is a peculiar flap, the e p i g l o t t is, which contains a large elastic cartilage; under ordinary conditions it is directed forwards, often reaching even up over the edge of the soft palate (Fig. 396), but when food passes from the mouth through the pharynx into the oesophagus, the epiglottis is let down over the glottis. The walls of the larynx are supported by large cartilages, viz., the cricoid behind, the large thyroid below; in front and above, the two arytenoids. The rest of the $\mathrm{trachea}$ is usually strengthened for some distance by cartilaginous rings; it branches posteriorly into two large bronchi, which branch again; each lung, as already stated, is an arboriform organ, of which both the larger and finer branches are hollow. Only the peripheral branches, which have thin walls, and are furnished with small dilations (alveoli) are respiratory; in

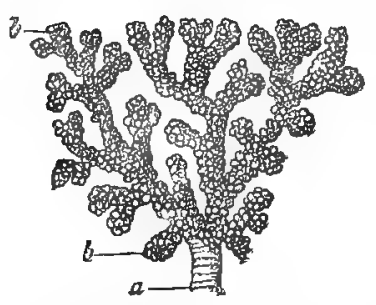

Fig. 397. Small portion of a Mammalian lung filled with mercury. $a$ finest bronchial tube, $b$ respiratory portion of the lung.-After Frey. other regions the tubes have thicker walls, provided in the larger branches with cartilaginous rings like those of the trachea, or with small cartilaginous plates. All the branches are held together by connective tissue. The lungs, with the heart, are situated in the anterior portion of the body-cavity, the thorax, along the dorsal wall of which the cesophagus passes; whilst the rest of the alimentary canal, with the kidneys and reproductive organs, lies in the posterior portion, the abdomen. A large septum, the dia$\mathrm{phragm}$, divides the thorax from the abdomen; it is tendinous

* In Man and some other Mammalia, the cæcum is continued into a thin narrow appendage, the processus vermiformis.

+ Occasionally the pancreas has two ducts, which either open both direct into the gut, or one unites with the bile duct.

¥ The great omentum is a specially developed portion of the mesentery, depending in many forms over the ventral side of the stomsch and the intestine. 
centrally, elsewhere muscular: when the muscular portion contracts, the diaphragm is flattened so as to enlarge the thorax, the very elastic lungs are expanded, and consequently air rushes in: when the diaphragm is relaxed, it is arched up into the thorax, the lungs are compressed, and the air is partially expelled. The diaphragm is thus an apparatus for effecting respiration. The muscles which move the ribs are also of importance in this connection, since, when the lower ends of the ribs are moved forwards, the thoracic cavity is widened.

In many Mammals which hibernate (Bats, Hedgehogs, Marmots, Hamsters, Myoxus), and in some others (Mole, Shrew-mouse, Mouse, Rabbit), there is a so-called hibernating gland, a lobed mass, reaching from the thorax to the neck, over a portion of the back; it is largest in autumn, and decreases in size during winter. The organ consists of connective tissue with numerous large cells containing oil-globules, and is to be regarded, like other fatty masses, solely as a reserve of nourishment.

Vascular System. As in Crocodiles and Birds, both atriu m and ventricle are completely divided, the left auricle receives blood from the lungs, the right from the rest of the body. The pulmonary arteries (the arterial arches of the fourth pair), arise by a common stem from the right ventricle. The arterial arches of the first and second pairs arise together by a common trunk, the innominateartery, from the left ventricle, and thus carry simply arterial blood; the right arterial arch of the second pair only gives off the artery for the right limb; the aorta (in contrast to that of Birds) is formed by the left arch of the same pair; the first pair of arterial arches forms the carotids as usual. Thus in Mammalia we have, as in Aves, a complete separation of arterial and venous blood. A conus arteriosus is absent.

In the new-born Mammal there is a large opening (foramen ovale) in the septum between the two auricles, which is, however, soon closed. In the same way there is an open duct (the ductus

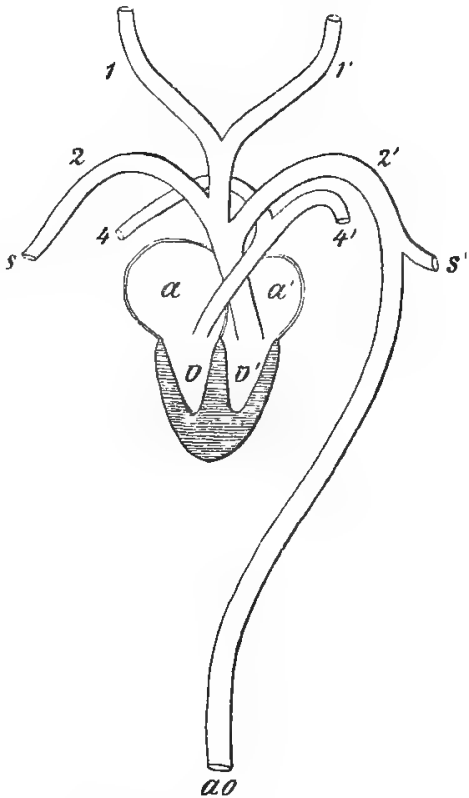

398. Heart and arterial arches of Mammalia, diagrammatic. a right, $r^{\prime}$ left auricle, ao aorta, $s$ and $s^{\prime}$ sub. clavian arteries, $v$ right, $v^{\prime}$ left ventricles, 1, 1', 2, 2', 4, 4' arterial arches.-Orig.

Botalli) between the second left arterial arch and the pulmonary artery (the fourth arterial arch); after birth it degenerates to a solid cord. 
The kidneys are short, roundish organs, with a laroe cavity, the pelvis of the lidney, into the inner side of which the urinary tubules open; several large papillæ project into the pelvis from the substance of the kidney (consisting of urinary tubuless) surrounding it; the pelvis is continued into the ureter. A urinary bladder is present; for its opening and for that of the ureters, see the reproductive organs.

In most Mammalia the surface of the kidney is smooth; in others (e.g., Cattle) lobed, in others again (Bear, Seal, Whales, etc.), the kidney is branched, and consists of numerous lobules, exch of which is mounted upon a branch of the proximal end of the ureter, whilst they are only held together by loose connective tissue.

The ovaries are comparatively small, usually with a flat or somewhat lobed surface; in a few Mammals only (Monotremes, the Pig, etc.) the ovary, in consequence of the projecting Graaffian follicles, has a racemose appearance.* The Graaffian follicles as

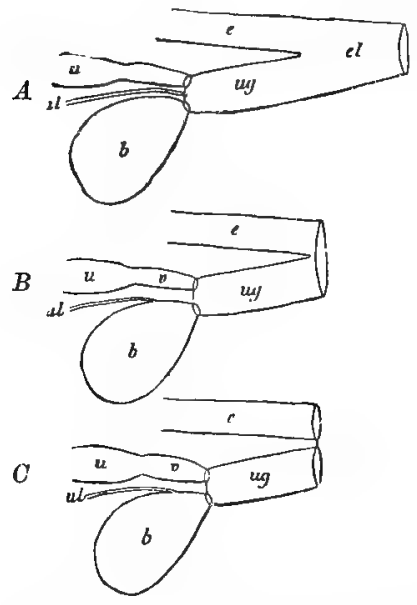

Fig. 399. The terminal portions of the gut, of the urinary and generative apparatus in the females of various Mammalia viewed from the side, diagrammatic. A Monotreme, $B$ Marsupial, $C$ other Mammalia. $b$ bladder, $c l$ cloaca, e reotum, $u$ aterus, $u g$ urinogenital duct, $u$ ureter, $v$ vagina.Orig.

into which the rectum opens above, and the urinogenital sinus below; the two vaginæ open separately on the floor of the urinoof other Vertebrata in that they are provided with a large number of follicle cells, and contain a large cavity; in the Monotremes, however, just as in the lower Vertebrates there is only one layer of cells round the ovum, and there is no cavity. Each oviduct in the Mammalia in general is composed of three regions; an anterior, usually very narrow, portion, the fallopi a $\mathrm{n} t \mathrm{ube}$, with a funnel opening into the abdominal cavity; a median wider part, the uterus; and a terminal section, the vagina; in the Monotremes the division between uterus and vagina is not demarcated, and the oviducts open separately into a saccular dilation of the ventral wall of the cloaca, the urinogenital sinus, into which the urinary bladder of the two ureters also opens; all five apertures are close to its base. In the Marsupials the cloaca is shortened, so that it forms simply a shallow pit, pens above, and the urinogenital sinus described above, p. 351, differ from those

* When the ovum leaves the follicle the cavity is filled with very cellular connective tissue, which in some Mammalia increases so much in size that the follicle becomes many times as large as it was previously; as large indeed as the whole of the rest of the ovary. Later it degenerates to form the corpus $1 \mathrm{uteum}$. 
genital sinus, which contains also the aperture of the urinary bladder, whilst those of the ureters lie on the dorsal wall of the bladder.* In the placental Mammals there is no cloaca in the adult; the urinogenital sinus (vestibule) and the rectum open independently, either close together or separated by a considerable interval. The posterior ends of the oviducts are almost always united for a greater or less extent: either the vaginæ simply; or the posterior portions, or even the whole length of the uteri also: in the first case there are thus two entirely separate uteri (uterus duplex), as in Rabbits; in the second, there

A

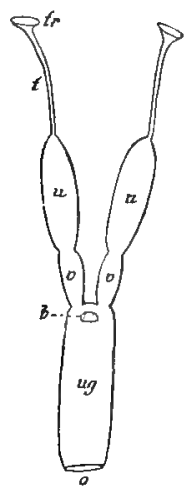

$B$

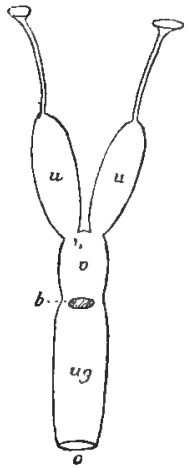

$C$

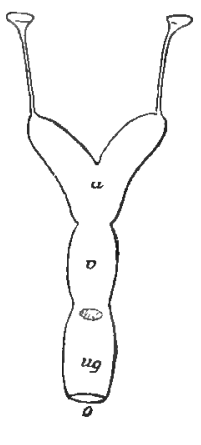

$D$

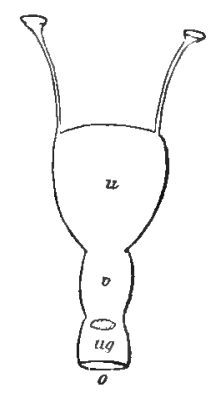

Fig, 400. The Müllerian ducts and urinogenital sinus of various Mammalia. $A$ of a Marsupial, $B$ uterus duplex, $O$ bicornuate, $D$ simplex, $b$ opening of the bladder into the front end of the urinogenital sinus, o external opening of the sinus, $t$ fallopian tube, $t r$ funnel, $u$ uterus, $v$ vagina.-Orig.

is a bicornuate uterus ( $u$. bicomis), as in the Horse; in the last, a simple uterus (u. simplex), as in Man. The ureters and the bladder are arranged as in the Marsupials.

At the junction of the vagina and the vestibule there is in many Mammalia a thin membranous septum (hymen), perforated by a small pore, and broken down at the first coitus. The vestibule varies very much in length; in some forms it is very long (e.g., in Hares), in others, very short, hardly apparent (e.g., in Man). Very frequently, a clit or is, a rudimentary organ homologous with the penis of the male, is present in the female; it is usually a papilla, rarely elongate, and lies on the ventral side of the external aperture of the urinogenital sinus. Further, there may be rudiments of the mesonephros (epoophoron, for instance, in man) and of its duct (Gärtner's duct in the Ruminants).

Male Genitalia. In all Mammalian embryos the testest lie close to the dorsal wall of the abdomen, just as in Reptiles and Birds,

* In some Marsupials the two oviducts are separate throughout their length, in others, the vaginxe are united for a certain distance, but open separately in to the urinogenital sinus.

+ In many Mammalia, as in Aves (see p. 419), the testis diminishes in size after the breeding season (Roebuck, Hedgehog, ctc). 
and in some (Monotremes, Cetacea, Proboscidians, etc.), they retain this position throughout life. In most species, however, at the close of foetal life, or in early youth, each descends into an evagination of the ventral abdominal wall; usually the two evaginations are united externally to form a pouch-like appendage (scrotum), divided by a septum into two compartments, each containing one of the testes;

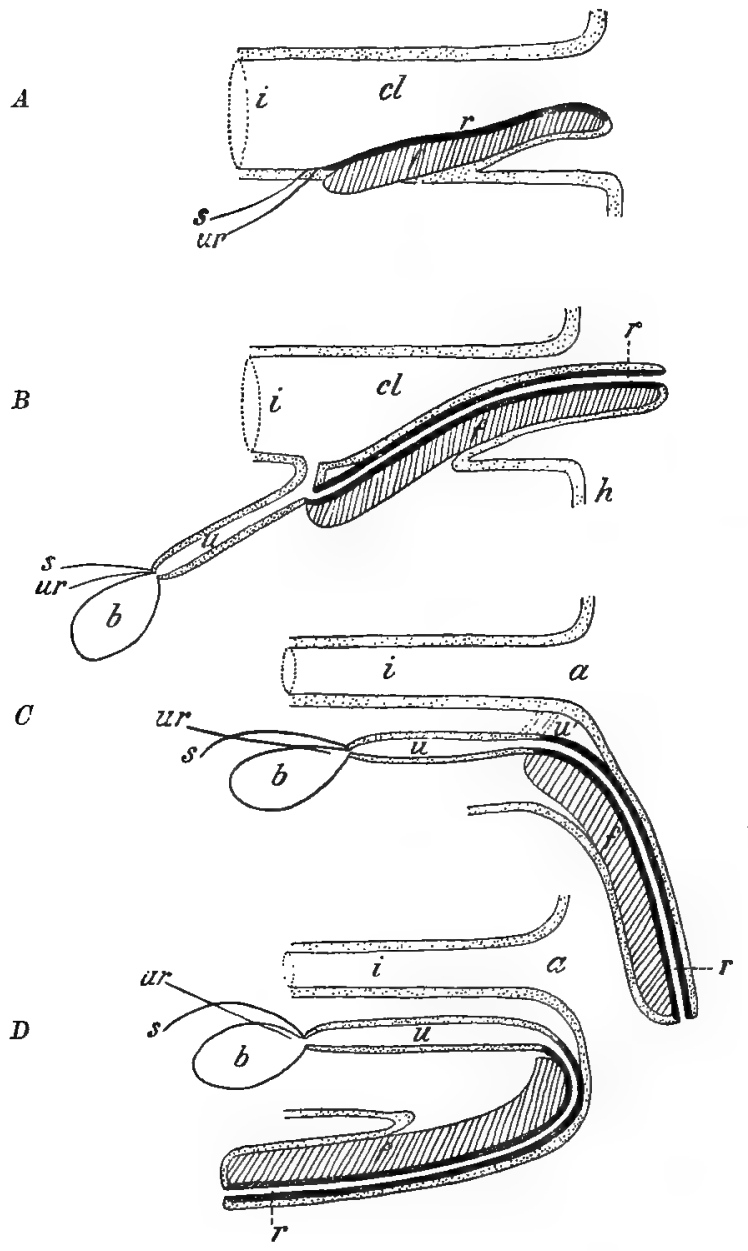

Fig. 401. Diagrammatic longitudinal section of the cloaca (or rectum) and the copulatory organs, $A$ of a Crocodile, $B$ of a Monotreme, $C-D$ of various other Ma m malia. $a$ anus, $b$ urinary bladder, $c l$ cloaca, $f$ corpus fibrosum, $h$ skin, $i$ gut, $r$ (in $A$ ) seminal groove, in $(B-D)$ seminal tube, $s$ vas deferens, $u$ urinogenital sinus (in $C$ the degenerated portion of the sinus is dotted: $u^{\prime}$ ), ur ureter.-Orig.

whilst its cavity communicates with the abdomen by a duct varying in width, which is often closed in the adult.* In the Monotremes the vasa deferentia open, together with the ureters and urinary bladder, into a deep, though narrow outgrowth of the ventral wall

* In some Mammalia (Insectivora, Rodentia) the testes only lie in the scrotum during the breeding season (when they are much enlarged); at other times, in the abdomen. 
of the cloaca, the urinogenital sinus, corresponding to the same organ in the female. In connection with the ventral wall of the cloaca there is a $p \in \mathrm{nis}$, which differs from the homologous organ of the Chelonia, Crocodilia, and Aves, in the closure of the groove on the dorsal side, to form a tube, the seminal tube, opening anteriorly into the urinogenital sinus, at the other end to the exterior (Fig. 401, B). An elongate mass of fibrous connective tissue, the corpus fibrosum, lies ventral to the seminal canal. In all other male Mammalia, including the Marsupials, there is no cloaca; the original opening of the urinogenital sinus into it has closed (Fig. $401, O)$, and the sinus itself only opens to the exterior by the seminal tube, through which the excretory products must also pass. The copulatory organ is ventral to the anus, but in many Mammalia it is connected with the abdominal wall $(D)$, so that the tip is directed forwards.

The seminal tube is surrounded by a vascular network, and one is contained in the corpus fibrosum. The dilation of the ressels effects the erection of the penis. An ossification (os penis) is often developed in the copulatory organ (Carnivora, Apes). Various glands open into the urinogenital canal and into the seminal tube, and their secretion escapes with the spermatozoa; among these the prostate glands and Cowper's glands are the most constant. A vesicula seminalis, a saccular or branched hollow organ, which serves both as a seminal reservoir, and also as an organ of secretion, opens, in many Mammals, into each vas deferens, close to its union with the urinogenital sinus; or independently into the sinus. A larger or smaller rudiment of the oviduct (uterus masculinus) is often present.

Of the Mammalia, the Monotremes alone are oviparous; here the egg is relatively large, ${ }^{*}$ and segmentation is partial; as in many Reptiles the egg is surrounded by a parchment-like shell. All other forms are viviparous; an egg-shell is always absent, the ovum is microscopic, segmentation total. In the Marsupials the embryo lies in the uterus surrounded by the embryonic membranes, is nourished and grows by the absorption of a fluid secreted by the uterine glands; there is not a close connection between the embryo and the uterine wall, and the young one is born in a condition which, in comparison with that of the new-born placental Mammal must be considered very undeveloped; it is nourished by the milk of the parent for a long time after birth. In the placental Mammals the outer embryonic membrane comes into close connection with the uterine wall; delicate vascular branched villi are developed on its surface and fit into corresponding crypts in the vascular wall of the uterus, serving as organs for the absorption of the maternal plasma. The villi are either uniformly distributed over the whole surface, as in the Horse, Pig, Camel, Whale, or are chiefly or exclusively developed in one region, which is then

* In Echidna the egg with its shell has a longitudinal diameter of $15 \mathrm{~m} / \mathrm{m}$. , a transverse diameter of $13 \mathrm{~m}, \mathrm{~m}$; ; that of Ornithorynchus is similar. 
termed the placent " fuetalis, * or there are several regions upon which the villi are well-developed. This is the case in most Ruminants, which possess a great number of small, very prominent placentæ (c oty led ons); elsewhere a large continuous placenta, either zonary (Carnivora, Seal, Elephant) or discoidal (in Man and others $\dagger$ ), occurs. That portion of the uterine wall which is connected with the placenta is termed the uterine placenta, p. uterina. In some cases the villi are simply withdrawn from the pits in the uterine wall at birth (Horse, Ruminants); in others (in all with zonary or discoidal placenta) a portion of the mucous nembrane of the uterus remains attached to the embryonic membranes and is thrown off with them (decidua), so that the former has, to a great extent, to be regenerated.

In the placental Mammals the serous membrane (cf., p. 354) is closely connected with the allantois, and partially fuses with it; the vascular membrane thus formed is termed the chorion, and gives rise to the vascular.

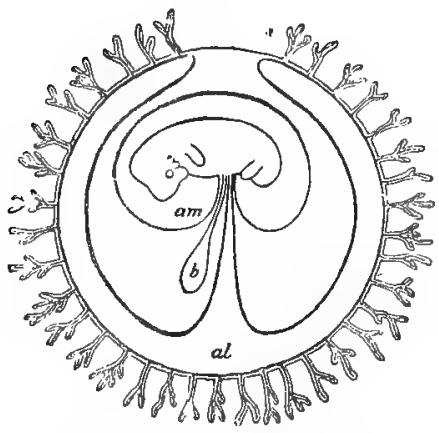

Fig. 402. Pl a centa of a Mammal, diagrammatic. am amnion, al allantois, $b$ yolk sac; the outermost line is the serous membrane. The external layer of the allantois is fused with the serous membrane forming the chorion which is beset with branched processes.-Orig.

papillæ already mentioned. In older Mammalian embryos the amnion is much extended, and often lies close to the allantois, and then it immediately surrounds the tubular peduncles of the allantois and of the yolk sac, as with a sheath. These peduncles (see Fig. 402), together with the sheath, are termed the umbilical cord.

The circulation in an advanced embryo is in several respects very different from that of the adult, the lungs of course are not yet functional; the oxygen which the embryo needs is received with the plasma from the parent. The chief points of the circulation are the following: the arterial blood from the placenta mixes with the venous blood from the posterior part of the body and flows into the right auricle, which also receives venous blood from the anterior regions. Part of the blood from the right auricle flows into the right ventricle, thence to the pulmonary artery, and so partly into the lungs, partly through the ductus Botalli into the aorta; an other portion of blood from the right auricle goes through the opening in the auricular septum into the left auricle, and from this through the left ventricle into the main arterial trunk. A very considerable mixing of arterial and venous blood, therefore, occurs in the embryo.

The length of time during which the placental embryo remains in the uterus (uterine gestation) varies considerably for different forms, thuth it is fairly constant for each species. As a general rule large Mammals have a long gestation (a year or more), and

* If the villi are uniformly distributed over the whole membrane, the animal (e.g., the Horse) is said to possess a diffuse placenta; strietly speaking there is none.

† Apes, Bats, Insẹtịivores, and Rodents, 
only produce one or quite a few offspring at a birth; whilst in small Mammalia the time is short, and several or many young ones are born at the same time. If there are several embryos in the uterus at once, the ova from which they develop have all been fertilised at the same time; they are, therefore, all at about the same stage of development, and are born in immediate succession. In some placental forms the offspring at birth are very helpless, naked, with closed eyes (the eyelids adhering together); whilst others are more advanced, or are immediately capable of independent movement. They are all at first nourished by milk from the parent.

The Mammalia are grouped in the following way:-

A. Oviparous Mammalia. Egg laid, large, surrounded by a shell. Cloaca long. One order only, the Monotremes.

A. Aplacental Mammalia. Egg small, develops in the oviduct, whose walls secrete a fluid for the nourishment of the embryo. This is very small and imperfectly developed at birth. Cloaca rudimentary (only present in the female). One order only, the Marsupials.

C. Placental Mammals. The embryo develops from a small egg; the outer embryonic membrane is closely connected by villi with the wall of the oviduct. Cloaca absent. All other orders of Mammalia belong here.

\section{Order 1. Monotremata (Monotremes).}

This swall group differs from other Mammalia in a number of characteristics which bring it near the Reptilia. It is especially remarkable that Monotremes are oviparous; that the egg is relatively large, and is surrounded by a leathery shell; that they possess a well-developed cloaca. Other points tending in the same direction, are the presence of well-developed cervical ribs, a large coracoid, a very reptilian episternum, the absence of a scapular spine, the form of the stapes, the straight cochlea, the presence of cartilage in the sclerotic of the eye, the feeble development of the corpus callos um, the whole relations of urinary and genital organs, as already noticed. It is interesting that the temperature of the body* is lower than in other Mammals.

That they are not incorrectly placed with the Mammalia may be seen, however, from the fact that they accord with them, and differ from Reptiles in the following respects: they are covered with hair, possess sebaceous and sudoriparous glands, have a long, jointed sternum, no quadrate, two occipital condyles, three ear bones; whilst the $\mathrm{mid-brain}$ is divided into four lobes, the penis is tubular, etc.

It may also be mentioned that Monotremes possess marsupial bones, like those of Marsupials, connected with the pelvis; further,

* In Echnidna under usual conditions $28^{\circ} \mathrm{C}$, in Ornithorhynchus $25^{\circ} \mathrm{C}$. (c). In other Mammalia as a rule $38-39^{\circ} \mathrm{C}$. 
it must be remembered that mammillæare absent, and that the mammary glands are primitive in character. The few known forms are edentulous when adult, but horny teeth may be present. Recently it has been demonstrated that the young Ornithorhynchus has true teeth, but that they are lost later. There is no definite external ear. The males have a horny s p u r on the heel, perforated by the duct of a gland.

There are only three living species of Monotremes known, they are mentioned below. They are of medium size, and are confined to Australia, New Guinea, and Tasmania. Very little is known definitely concerning the fossil remains of this group.

1. The Duck-billed Platypus (Ornithorynchus paradoxus). The snout is flattened, broad, and covered with a naked skin; at the back of the mouth on either side of each jaw, there is a large horny tooth, and a smaller horny ridge in front; the tail is powerful and flattened; the feet webbed; the fur soft. They feed upon small aquatic animals. The eggs are laid two at a time, in holes dug in the ground; the young ones, when hatched, are fed with milk by the mother. East Australia, Tasmania.

2. The Spiny Anteater (Echidna aculeata). The snout is narrowed, especially towards the tip, and covered with naked skin; the mouth small, the tongue long and sticky; the body covered with hairs and spines; the tail very short; strong digging claws. The food consists of Ants, Termites, etc. The egg (only one is laid at a time) is placed in an unpaired saccular depression on the ventral surface, and there incubated; the temperature of the sac rises several degrees above that of the body. It serves later to protect the young one, and then atrophies, forming anew before the next oviposition. The brood pouch is absent from Ornithorynchus. Different varieties inhabit New Guinea, Australia, and Tasmania. The three-toed Echidna (E. [Proechidna] Bruijnii), is a near relative; it has a longer and curved bill, and only three toes on each foot, both hind and fore, whilst $\mathbb{E}$. aculeata has five. New Guinea.

\section{Order 2. Marsupialia (Marsupials).}

The leading characteristic of this group is the absence of a true placenta; the outer embryonic membrane does not project as villi into the uterine wall, but the embryo is nourished by a secretion of uterine glands, and is born in a very immature and imperfect condition. Other characters also show the low grade of the Marsupials, as compared with all subsequent orders of Mammalia: for instance, the corpus callosum is feebly developed; in the female, there is a cloaca, but it is a mere pit, and the two oviducts open separately into the urinogenital sinus. Like the Monotremes, and unlike all other Mammals, the Marsupials have marsupial bones, a pair of peculiar ossifications connected with the pubes and extending forwards in the abdominal wall. They have nothing to do with the marsupium usually present in females. This is an open saccular cavity on the ventral side of the animal, limited by a large fold of skin; it covers the mammillæ, and the young ones are placed in it 
immediately after birth; they are each attached immovably by suction to one of the mammillæ for a long time.

The dentition of the Marsupials on the whole resembles that of other Mammals; they differ, however, in some points. The molars vary within the number seven in each half of the jaw. Only a single form with degenerate teeth has a larger number; the incisors may, however, be as many as five abore, four below. Of the seven molars the third alone bas a predecessor, the only milk tooth met with so far in this group. The form of the teeth, especially of the molars, varies considerably in correlation with very diverse habits.

In most points of structure the Marsupials stand nearer the placental Mammals than do the Monotremes; mammilla are present, the coracoid is rudimentary, there is no episternum, the cochlea is spirally coiled, the penis is essentially like that of the placental Mammals, the testes move back into a scrotum, the ovum is very small, and segmentation is total, etc.

Most existing Marsupials live in the Australian region, the Opossums* alone inhabit America. In earlier geological times, however, Marsupials occurred also in other parts of the world.

At least three small similar incisors on each side of the lower jaw. The canines larger than the incisors.

Only one large incisor in each ramus of the lower jaw. The canine small or absent.
Opossums.

Bandicoots.

Kangaroos.
None of the toes of the hind leg united.

Toes 2 and 3 thinner than 4 and 5 , and united together.

1. Opossums (Polyprotodontia). Four or five incisors in the upper, three or four in the lower jaw. Well developed conical canines. Molars with cusps or tubercles. The second and third digits of the hind foot are like the fourth and fifth, and are not united.

(a) The True Opossums (Didelphyidi) have, on the hind foot, a welldeveloped, but clawless, hallux which can be opposed to the rest of the toes; $i \frac{5}{4}, c \frac{7}{1}, m \frac{7}{7}$. Tail long and prehensile, almost naked, but scaly. The pouch is well developed in some forms, rudimentary or wanting in others. They feed upon Insects, and are small animals, living exclusively in the New World, chiefly in South America.

(b) Native Cats, etc. (Dasyuridx). Hallux rudimentary or absent. $i \frac{4}{3}$. Tail not developed as a prehensile organ, Carnivorous or insectivorous. Among them the peculiar Native Cat (Dasyurus), and the long-legged, wolf-like Tasmanian Wolf (Thylacinus), occurring only in Tasmania. Further, the small squirrel-like Marsupial Ant-eater (Myrmecobius), with $\frac{9}{8}$ small molars, and with long, smooth protrusible tongue.

2. The Bandicoots (Peramelina) resemble the preceding group as regards the teeth, whilst they agree with that following in the structure of the hind feet, for the second and third toes are thin and enclosed in a common

* The forms cited below, which are not specially commented on, live in Australia (some of them also in New Guinea, Tasmania, etc.). 
membrane; the hallux is wanting or rudimentary. $i \frac{5}{3}$." Whilst in other Marsupials the fore limb has five well-developed digits, in one of the two genera of Bandicoots (Perameles) the first and fifth fingers are very degenerate and destitute of claws, in the other (Choeropus) they are completely absent, and the fourth has also become rudimentary. In the True Bandicoot (Perameles), a rudiment of the hallux is present, the fourth is the best developed toe; in the Pig-footed Bandicoot (Chroropus) (Fig. 403 c), the rudiment of the hallux is wanting, and the second, third, and fifth are very thin, almost rudimentary.

3. Kangaroos (Diprotodontia) have usually three incisor teeth in the upper, one in the lower jaw. The canines are absent or small, the molars with rough cusps or transverse ridges. Of the toes of the hind foot the second and third are weaker than the fourth or fifth, and are surrounded by a common membrane (syndactylous). Herbivorous.

(a) Australian Opossums (Phalangistidx). The hind, are little longer than the fore, limbs. Hallux well-developed, without a claw; opposable (Fig. $403 A$ ). $i \frac{3}{1}$. Climbing animals. The following may be noted: the
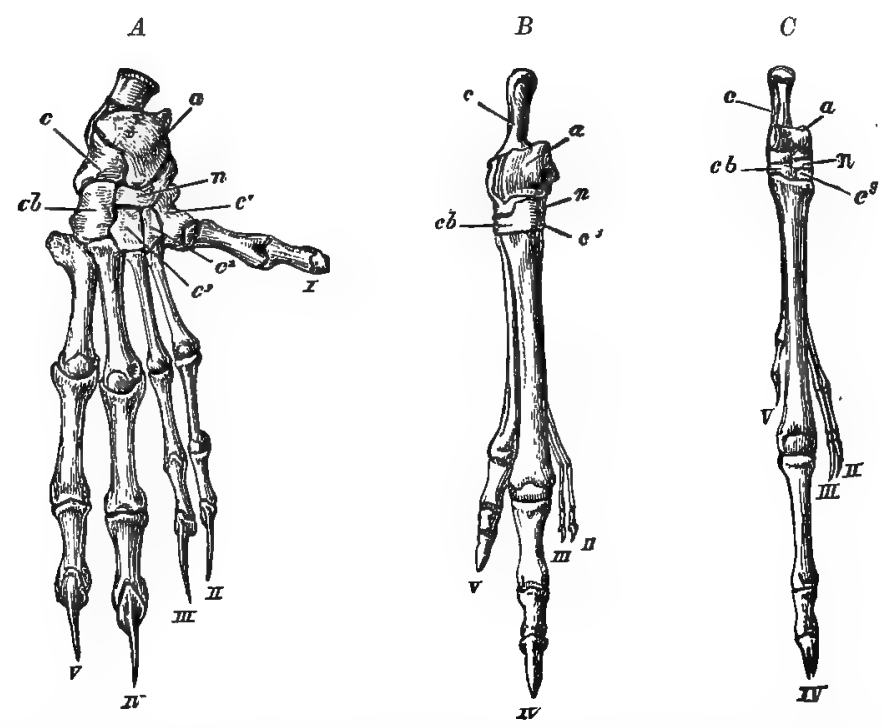

Fig. 403. Right hind foot: $A$ of Phalangista, $B$ of the $\mathrm{Kangaroo,} C$ of Choropus. $a$ astragalus, $c$ calcaneum, $n$ centrale (naviculare), $c^{1}-c^{3}$ cuneiforms, $c b$ cuboid $I-V$ first to fifth toes.-After Flower.

Cuscus (Phalangista), with long prehensile tail: the Fly ing $\mathrm{Phal}$ angers (Petaurus), with a large membrane (patagium) stretched between the fore and hind limbs: the Koala or Australian Bear (Phascolarctos), a clumsy ecaudate creature; in which the fingers, like those of the Chameleon, are united in two bundles (the first and second may be opposed against the third, fourth, and fifth) : and the small, aberrant, insectivorous, Long-snouted Phalanger (Tarsipes) with long protrusible tongue; few rudimentary molars $\left(\frac{4}{3}\right)$; and rudimentary claws on all the fingers and toes with the exception of the second and third toes of the hind foot.

(b) Kangaxoos (Macropodida). Hind legs very long, adapted for jumping; hallux absent; toes, second and third, very thin, fourth and fifth, 
strong (Fig. $403 \quad B$ ); fore limbs small; tail very strong, used as a support in sitting. $i \frac{s}{1}$. Large and small forms (Halmaturus, Hypsiprymnus, etc.) in Australia and adjacent islands.

(c) Wombats (Phascolomys). Distinguished in that they have only a single incisor on each side, above and below (like the Rodents); all the teeth grow from persistent pulps; the second and third hind toes only slightly weaker than the others; tail very short; clumsy nocturnal animals.

Note.-The recently discovered Australian Mammal, Notoryctes typhlops, with habits like those of the Mole, is also a Marsupial. The third and fourth digits of the fore limbs are provided with strong, compressed, digging claws (the rest of the claws are smaller); a hard, horny plate is developed on the dorsal side of the snout, and the head is used in digging; the eyes are rudimentary ; pinnæ are absent. Claws are developed on all five digits of the hind foot, whilst in other Marsupials the hallux, if present, has no claw. $i \frac{3}{2}$.

\section{Order 3. Insectivora.}

The Insectivora are small, short legged, placental Mammals; with the snout elongated, more or less like a proboscis; and with multicuspidate molar teeth, the front ones being usually small and unicuspidate. They generally walk on the whole foot (plantigrade) ; both fore and hind feet have usually five similar toes.

The canines are frequently small, some of the incisors large; clavicles are present; eyes and pinnæ are usually little developed; mammillæ abdominal.

The Insectivora feed chiefly upon Insects, Worms, etc., rarely on vegetable substances. They are entirely absent from Australia and South America.

1. The Hedgehog (Erinaceus). Spines (very thick, stiff hairs) dorsally, usually finer or coarser hair ventrally; feet simple, tail short. It can roll itself into a ball by the ventral flexure of the head, limbs and tail, the spiny dorsal surface being drawn down by the contraction of the large skin muscles. Canines absent; first incisor, above and below, larger than the rest of the incisors; the cusps of the molars blunter than in other Insectivora; altogether, ten teeth above, eight below, on each side. The Common Hedgehog ( . europæus) is distributed almost throughout the whole of Europe ; it lives both upon plant and animal food; it hibernates for the winter.

2. The Mole (Talpa). The fore limbs are developed into very strong digging organs; the hand is broad, with long, powerful, and almost straight, claws, and so compressed, that the inner edge, supported by a peculiar sickle-shaped bone, is turned downwards, whilst the palm is turned outwards ; the clavicle is extremely short and powerful, pre-sternum with a keel; the eyes are rudimentary; pinnæ absent; tail short; fur soft; dental formula complete, $\frac{21}{11}$; canines of the lower jaw like the incisors, which are small and simple (Fig. 393). The Talpidæ live exclusively upon animal food, chiefly Earthworms. The English species is the Oommon Mole (T.europa). The Golden or Cape Moles (Chrysochloris) constitute another group of fossorial Insectivora. They are blind, subterranean animals with velvety coat; the claws and last phalanges of the second, and especially of the third, fingers, are very powerful, the first and fourth fingers are small, the fifth absent; the hand is not compressed. South Africa.

3. The Shrew (Sorex) is a small Insectivore, with a long tail; pointed proboscis; feet of simple structure; and soft fur. There is only one incisor in each 
ramus of the mandible, it is very large, and projects forwards; the first upper incisor is similar in form; the canines small; the cusps are in many cases reddish-brown. The Shrews, which feed upon Insects and Worms, are represented in Britain by the following species: the $\mathrm{Com}$ m on $\mathrm{S} \mathrm{hrew} \mathrm{(Sorex} \mathrm{vulgaris),} \mathrm{the}$ Lesser Shrew (S. pygmzus), comparatively rare in England, but taking the place of the Common Shrew in Ireland; the W at er Shrew (Crossopus fodiens), distributed over England and the South of Scotland; all with brown teetb. Two Musk Shrews (C. aranea and C. suavolens) occur on the continent of Europe, but not in Britain.

4. Of foreign Insectivora, besides the Cape Moles, the following may be mentioned: The Desmans (Myogale), aquatic forms with webbed feet; proboscis long, tail long and scaly; possessed of musk glands : a large species, the Russian Desman ( $M$. moschata), with compressed tail, occurs in South Russia; another smaller species (M. pyrænica), with cylindrical tail, in the Pyrenees. The Jumping S hrew s (Macroseelides) are saltatorial animals, with elongate metatarsus; long proboscis; large pinnæ: in Africa. Cladobates, with powerful tail; furnished with long laterally-directed hairs; squirrel-like animals, living in trees in Africa. The $\mathrm{Cobego}$ or $\mathrm{Kaguan}$ (Galeopithecus volans) is in many respects an aberrant form; a patagium is stretched between the fore. limbs, the body and the hind limbs; the edges of the lower incisors are pectinate. It is about the size of a Cat and is herbivorous, inhabiting the South Sea islands, the Moluccas and Philippines.

Note.-The genus Hyrax may be mentioned here. It was formerly regarded as an Ungulate, but appears to the author to be allied to the Insectivora; its systematic position is, however, still very doubtful. The few species of the genus are small rodent-like animals with soft fur; pointed snout; quite short tail; legs of medium length: there are four well-developed digits on the fore feet (the pollex is rudimentary), the hind foot has only three toes; all the digits are provided with flat nails (not hoofs), except the inner toe of the hind foot which bears a claw; there is a large sole to the foot. $i \frac{2}{2}, c \frac{0}{0}, p \frac{4}{4}, m \frac{3}{3}$; the grinding surface of the molars is very like that of the Rhinoceros; the inner incisors are large, so that the dentition is somewhat rodent-like: herbivorous; Africa and West Asia.

\section{Order 4. Chiroptera (Bats).}

The Bats are remarkable, chiefly on account of the peculiar modification of the fore limb. With the exception of the first, all the metatarsals and their corresponding digits are much elongated, and a naked patagium* is stretched between them. It reaches from the fifth finger along the arm and forearm, to the body and hind libsm; in front a similar membrane is stretched in the angle between the arm and forearm, and there is frequently another between the hind. limbs and the tail. The hind feet and the short pollex are free; of the fingers, the third and fifth are without claws; in the small Bats, the second also; but the thumbs and the five digits of the hind feet possess curved claws: the terminal phalanx of the clawless digits is absent: besides the metatarsus and the digits, the arm and

" The patagium is not absoluteiy naked, for there are very fine hairs scuttered about on it. 
forcarm are also elongate, although to a relatively small extent. The hind legs are turned outwards in a peculiar way; they are thin and feeble : a long, thin bone or cartilage, the s p u r, arises from the ankle; it lies on the edge of the membrane extending between the hind limbs. The patagium can be folded up like an umbrella, and laid along the body. The clavicles are long and powerful, the presternum provided with a long keel. The mammillæ, one or two pairs, are thoracic.* The Bats are nocturnal or crepuscular. Their best mode of locomotion is flight, but they can manage to crawl on their hind limbs and thumbs. They rest suspended by the hind feet.

1. Large Bats (Megachiroptera: genus Pteropus, the Fruit-Bats or Flying Foxes, etc.). Claws on the first and second digits; head long; molars with two longitudinal ridges; pinna simple: chiefly frugivorous. Large forms inhabiting the warmer parts of the Old World and Australia.

2. Small Bats (Microchiroptera). There is no claw on the second finger; head short; molars with several tubercles (like those of the Insectivora); opening into the external auditory meatus more or less completely covered by a membranous flap of the pinna, the antitragus. They feed principally on Insects, which they catch as they fly, discovering them by the tactile sense located in the skin, especially of the patagium, of the pinna, which is sometimes very large, and of peculiar outgrowths (nasal processes), which are frequently present on the head. Some South American forms ( $\nabla$ ampire, Desmodus) suck the blood of other living Mammals. The group is very rich in species, distributed over the whole world, especially abundant in the tropics; mostly small animals. A fairly large number of diverse forms occurs in England; among them may be noted: several species of the genus Vesperugo, of which the Pipistrelle (V.pipistrellus) is the common English one. This genus is characterised by the short antitragus, and by the possession of $\frac{4-5}{5}$ cheek teeth: of the genus Vespertilio, Daubenton's Bat (V. daubentoni), is a well-known British species. with a large antitragus and $\frac{6}{6}$ cheek teeth: of the Horse-shoe Bats (Rhinolophus), the greater ( $R$. ferrum-equinum), and the lesser (R. hipposiderus), both occur in England although they are not very common; they are distinguished by the complicated nose-piece. All the British species hibernate, passing the winter suspended in hollow trees and elsewhere.

\section{Order อ̆. Ungulata.}

The limbs are elongate and specially adapted for walking or running, the trunk is raised well above the ground. The metacarpus and metatarsus are usually of considerable length; the digits are more or less completely enclosed in a common skin (with the exclusion, however, of the last) ; the animal usually steps upon the last phalanx only, chiefly upon the surrounding hoof (p. 4.70); the rest of the foot does not touch the ground, but assists in lengthening the limb. 'The first digit and the corresponding meta-carpal or tarsal is absent from all four limbs of all existing Ungulata. Clavicles are also wanting.

* Many indigenous Bats are very remarkable in that whilst copulation occurs in. the autumn, fertilisation of the ovum does not take place till the following spring; the spermatozoa are stored in the uterus of the female during hibernation. 
Herbivorous animals, generally of considerable size, with plicate or tuberculate molars, and usually with a long cæcum.

\section{Sub-Order 1. Perissodactyla.}

The third digit is almost symmetrical, stronger than the rest, and the median plane of the foot passes through the middle of it; the fifth is usually absent. The femur has on its outer edge a process (trochanter tertius), which is wanting in the Artiodactyles. On the distal surface of the astragalus there is a large, flat, articular facet for the naviculare, and a smaller one for the cuboid. The rami of the lower jaw are anchylosed. The molars are folded, and, with the exception of the first premolar, of about equal size. Stomach simple; cæcum colossal; placenta diffuse.*

1. The Tapir (Tapirus). Fore foot with four toes (all but the pollex), hind foot with three (hallux and minimus wanting); the third is not much stronger
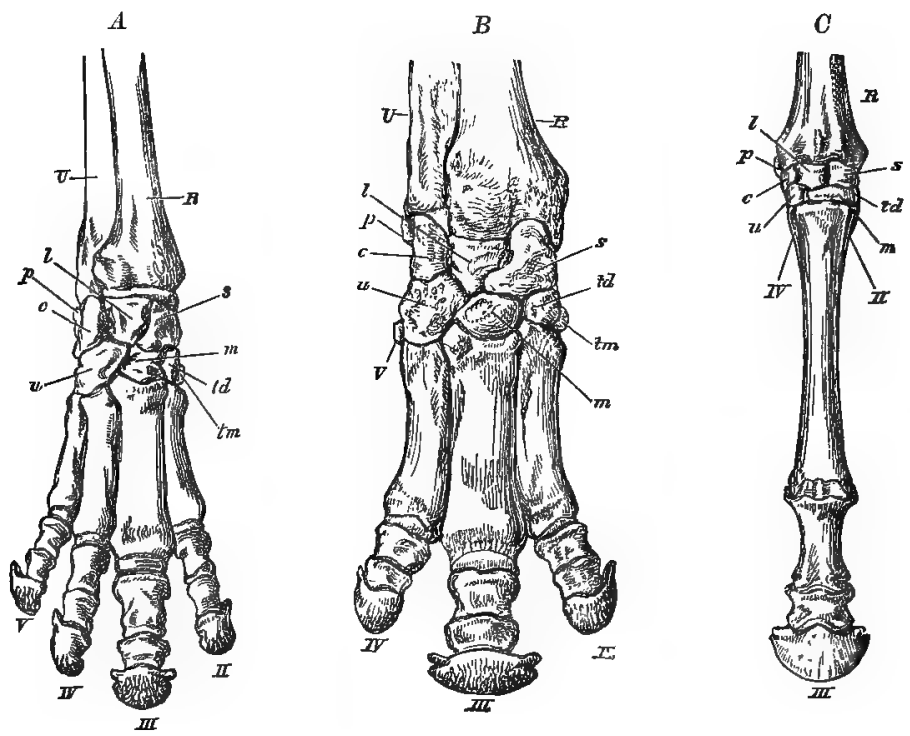

Fig. 404. Hand (fore foot) of : $A$ Tapir, $B$ Rhinoceros, $C$ Horse. $R$ radius, $u$ ulna $s, l, c$ proximal row of carpals (naviculare, lunare, cuneiform); $p$ pisiform ; $t m, t d, m, u$ distal row of carpals (trapezium, trapezoid, os magnum, unciform); II-V second-fifth fingers (in $B, V$ is the rudimentary fifth metacarpal, in $C, I I$ and $I V$ denote the second and fourth metacarpals).-After Flower.

than the second and fourth; digitigrade. $i_{3}^{3}, c \frac{1}{1}, p \frac{4}{3}, m \frac{3}{3}$; the molars have each two transverse ridges; the snout is elongated to form a short proboscis; the skin is well covered with hair. One species in the East Indies, another in South America. The extinct (Eocene) genus, Palæotherium, is somewhat closely

* Gall-bladder absent. Two abdominal mammæ. At least twenty-two dorsal vertebræ. 
related to the Tapirs; it had, however, only three toes on each fore-foot, and the molar's were like those of the Rhinoceros.

2. The Rhinoceros. Both fore and hind limbs symmetrical and threetoed; the median toe (third) somewhat stronger than the other two (second and fourth) ; digitigrade. $i \frac{2-0}{2-0}, c \frac{0}{0}, p \frac{4}{4}, m \frac{3}{3}$; incisors more or less degenerate, no canines, strong plicate molars. Antero-dorsally in the median line of the head one or two horns (cf. p. 471). The skin is thick, inflexible, and very sparsely covered with hairs; the upper lip very mobile. Limited to the warmer parts of Asia and Africa. In Africa there are two species with smooth skin and with two horns (Rh. bicornis and simus); in Asia a two-horned species and also a one-horned species with large deep skin folds (Rh. unicornis, etc.). The Woolly Rhinoceros (Rh. tichorinus), with ossified nasal septum, two horns and an abundant covering of hair, lived in Quarternary times in Central Europe and Siberia, together with the Mammoth.

3. The Horse Family (Equidæ) is characterised by the great development of the middle (third) as compared with the lateral toes, and by the great length of the metatarsus. Fore and hind limbs similar. They tread upon the hoof. Dental formula complete : $i \frac{3}{3}, c \frac{1}{1}, p \frac{4}{4}, m \frac{3}{3}$. Bony orbit complete (i.e., a process from the frontal has united with a process of the zygoma behind the eye.

(a) All Horses now living belong to the genus Equus. The second and fourth toes are altogether absent, so that each foot has a single toe only, the third, which with the corresponding metacarpal or metatarsal (cannon bone) is extremely well-developed; the second and fourth metacarpals (or metatarsals) are present in the form of long, thin bones (splint bones) on the sides of the cannon bone. The animals tread only on the hoof upon the last phalanx; this hoof surrounds the very small sole of the foot (cf. p. 470 , Fig. $382 D$ ). The incisors are characterised by the possession of a large pit partially filled with cement (the mark); the canines are well developed in the male, rudimentary in the female; the germ of the first premolar is present in both upper and lower jaws, but usually only develops in the upper; even there it is rudimentary, and generally falls out early (wolf tooth); the other molars of both jaws are of about equal size (broader in the upper than in the lower jaw); they have very long crowns and short roots; the crowns exhibit folds and pits; these reach to the roots and are filled with cement, which is extraordinarily well-developed here, surrounding the crown with a thick coat; very soon after the tooth comes into use, streaks of enamel appear upon the grinding surface, and the crown becomes gradually worn away, so that in old Horses it is very short. In contrast to the conditions in the Tapir and Rhinoceros, the lower portion of the ulna (or fibula) is very weak, in part only represented by a ligament. To this genus belong: the Zebras (E. zebra, quagga, Burchelli, and others), with dark transverse stripes; small hoofs; bovine tail : in South Africa. The Ass (E. asinus), with a black stripe along the middle of the back, and a similar transverse one across the shoulders; small hoof; tail bovine : wild in North Africa. Two allied forms (E. hemionus, the Dschiggetai, and E. onager, the Kulan, in Asia). The Hor s e (E. caballus) is usually larger than those just mentioned: it has larger hoofs; the tail is covered with a large tuft of long hairs : there are naked, horny patches on the skin both of the fore and the hind limbs, the so-called chestnuts and ergots (in others, only on the fore limbs); native place not definitely known.

(b) Some of the Quaternary and Pliocene Horses also belong to the genus Equus, and occurred not only in the Old World, but also in the New. Other Pliocene forms belong to the genus Hipparion, a small form which resembles Equus in most respects, but differs from it in that the second and fourth digits were present on all four limbs, although only as poorly developed 
"accessory toes," which did not touch the ground in walking.* Hipparion lived in recent Miocene times as well as in the Pliocene. The Genus Anchitherium is more remote from Equus; it has toes like Hipparion, but the second and fourth are considerably stronger than in the latter, although still much weaker than the third; the crowns of the molars are shorter than in Equus, the folding more like that of the Rhinoceros (or Palæotherium), the cement little developed; the wolf

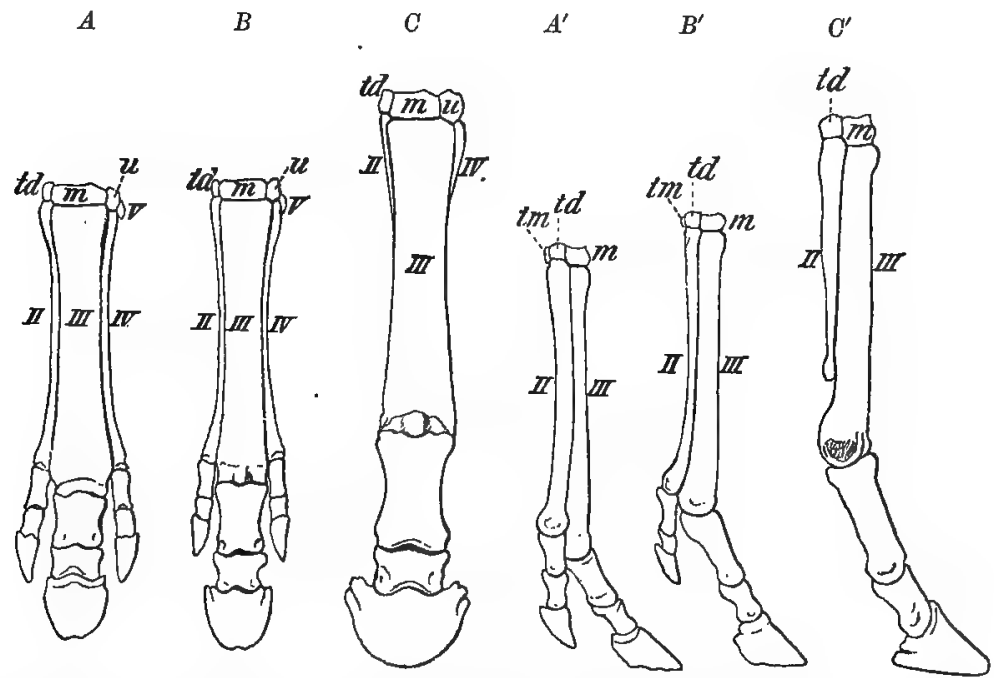

Fig. 405. Left fore foot of Anchitherium $\left(A, A^{\prime}\right)$, Hipparion $\left(B, B^{\prime}\right)$, H o r s e $\left(C, C^{\prime}\right)$, from in front and from within. All similarly decreased (about $\frac{2}{7}$ ). $t m$ trapezium, $t h$ trapezoid, $m$ os magnum, $u$ unciform. $I I, I I I, I V$, second-fourth metacarpals, $v$ rudimentary fifth metacarpal.-After Gaudry.

tooth (first premolar) is larger, and present also in the lower jaw ; the front teeth without the mark; the ulna and fibula better developed than in Equus. This genus, which lived in Miocene times, is very like the Eocene Palæotherium mentioned above.

\section{Sub-Order 2. Artiodactyla.}

The third and fourth digits on both fore and hind feet are each asymmetrical in themselves, but the two correspond, as does an object with its image in a mirror; the median plane of the foot passes between these two toes. The third and fifth are smaller, do not usually touch the ground in walking, and are situated somewhat behind the others; indeed they are often rudimentary or absent. The trochanter tertius is wanting. The two articular facets on the distal edge of the astragalus, for the naviculare and cuboid, respectively, are of about equal size, and both are much arched from before backwards ; the stomach is more or less compli-

* The fifth metacarpal is present (Fig. $405 \mathrm{~B}$ ), but quite rudimentary; it may also be present in the horse. 
cated, the cxecum smaller than in the Perissodactyles; the molars folded or tuberculate, the premolars smaller than the true molars.*

\section{Group 1. Non-Ruminantia.}

Incisors well developed; the third and fourth metacarpals or metatarsals are almost always separate, the second and fifth digits and the corresponding bones usually comparatively well developed; ulna and fibula strong; mandibular rami anchylosed; stomach less complicated than in the Ruminants; in some forms of the group (e.g., the Common Pig), fairly simple; in others (e.g., the Hippopotamus) with definite indications of division into several sections; there is no rumination; mammæ. often along the whole ventral side; placenta diffuse.

$A$

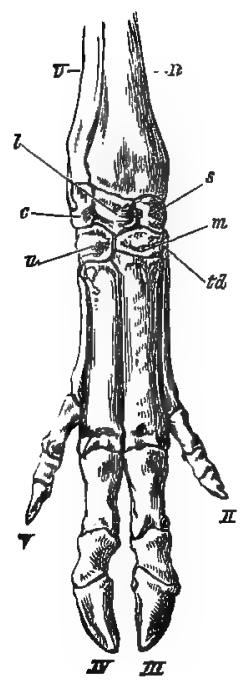

$B$

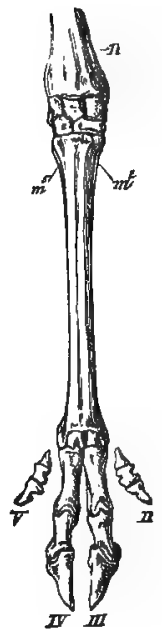

$C$

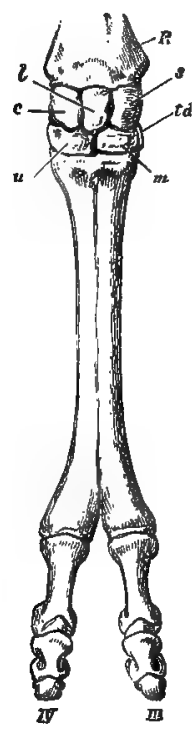

Fig. 406. Manus of : A Pig, $B$ Stag, $C \mathrm{Camcl} R$ radius, $U$ ulna; $s$ naviculare, $l$ lunar, $c$ cuneiform, $t d$ trapezoid, $m$ magnum, $u$ unciform; $m^{2}$ and $m^{5}$ second and fifth (rudimentary) metacarpals; $I I \cdot V$ second to fifth fingers.--After Flower.

1. Pig family (Suidæ). Limbs slender; digits two and five are considerably shorter than three and four, are situated somewhat behind them, and do not usually touch the ground in walking; ball of the foot small and soft; molars tuberculate; there is a short proboscis; skin with hairy covering.

(a) The Pigs (Sus) are confined to the Old World, and represented by various species : $i \frac{3}{3}, c \frac{1}{1}, p \frac{4}{4}, m \frac{3}{3}$; the incisors of the lower jaw are directed forwards, those of the upper jaw downwards; the upper canines turned outwards and upwards, the lower ones much arched (those of the male grow from persistent

* Gall bladder usually present. The number of dorsal vertebre less than twentytwo (rarely more than nineteen). 
pulps and are more curved than those of the female); the premolars are compressed, the molars have broad tuberculate grinding surfaces. Here belong the European Wild Hog (Sus Scrofa), the ancestor of the old race of the North European Domestic Pig; most existing English breeds are hybrids of the latter and of the Chinese pig, which was derived from one or more species of Asiatic Wild Pigs, and differs in several respects (in the skeleton) from the old race and from the European Wild Hog.

(b) Among other Suidæ the following may be noticed: the Peccaries (Dicotyles), small forms, with a large skin gland dorsally; the fifth hind toe is wanting; the canine in the upper jaw is directed downwards, but neither it nor that of the lower jaw is of striking size; South America. In the Babyrusa of Celebes (Porcus babyrusa) the canines of the upper jaw are turned upwards and much curved, in the male they are enormously elongated. The Wart-hog (Phacochorus) is chiefly distinguished by the extraordinary development of the last molar, this tooth is also the largest in Sus ; in very old animals it is the only persisting molar; canines much like those of Sus; South Africa.

2. Hippopotami (Hippopotamidx) are huge animals with thick limbs; the second and fifth digits are very powerful; the animals place all four toes on the ground and the sole of the foot is large; molars plicate and tuberculate; incisors and canines very powerful; head very large, without a proboscis, with very broad snout, sparsely covered with hairs: only two living species; the best known is the Hippopotamus (H. amphibius), which is distributed over large tracts in Africa; another smaller species (Chceropsis liberiensis), which approaches the Suidæ in some respects, inhabits West Africa.

3. There are also many extinct $\mathrm{Non}-\mathrm{ruminants,}$ which are in some points like the Pigs and Hippopotami, but in others differ considerably from them. There are, for example, various forms with molars like those of the Ruminants, whilst in other respects they stand fairly close to the Suidæ; others, like the Anoplotherium of Eocene and Miocene times, with long neck and long legs, offer a superficial resemblance to the Ruminants, but are distinguished from them by the possession of a complete dentition $\left(\frac{11}{11}\right)$; by the well-developed upper incisors; and by the separation of the metatarsals.

\section{Group 2. Ruminantia.}

Incisors are absent from the premaxillæ (or the third is alone developed) ; canine of the lower jaw usually (but not in the Camels) like the incisors, so that there appear to be four of these in each ramus. The molars, and to some extent the premolars also, have each four curved longitudinal ridges, two external and two internal. On all four limbs, the third and fourth metatarsals (or metacarpals) are almost invariably fused, forming a single long bone (cannon bone), whilst the second and fifth are incomplete or absent*; in the Tragulidæ alone are they complete. Digits two and five are small or absent. For the structure of the hoof, see p. 470 and. Fig. $382 \mathrm{E}$. Ulna and fibula are poorly developed; the lower end of the latter is separate, and resembles a tarsal bone. Rami of the lower jaw

* The upper ends of the second and fifth are, at least in the hind foot, fused with the third and fourth respectively, but the rest, if present, remain as small separate bones. 
anchylosed only in the Camels, elsewhere separate. The stomach is constricted into several portions, and after the food has been there for some time, it is regurgitated and masticated anew. There is usually a number of cotyledons; but in Camels, the placenta is diffuse like that of the Pigs and the Perissodactyles. The mammæ are abdominal.

In the majority of Ruminants (Cavicornia, Stags, Giraffes), the stomach is divided into three sharply-defined portions. The first compartment follows the esophagus, and from the junction a deep groove runs along the anterior side of the chamber, to its opening into the second region, the manyplies (psalterium or omasum.) The first part, which attains a considerable size, is furnished with several ingrowths, one of which is very large, and divides the cavity into two incom. pletely separated subsections, the large pauch (rumen), and the smaller honey comb bag (reticulum); the latter is furnished internally with a projecting network of folds; the former with villi. The manyplies is furnished within with numerous large longitudinal laminæ, which lie closely together and fill up the greater part of the eavity. The last portion, the reed (abomasum) is almost tubular. The rumen, reticulum, and manyplies are lined by a stratified epithelium like that of the œsophagus and the

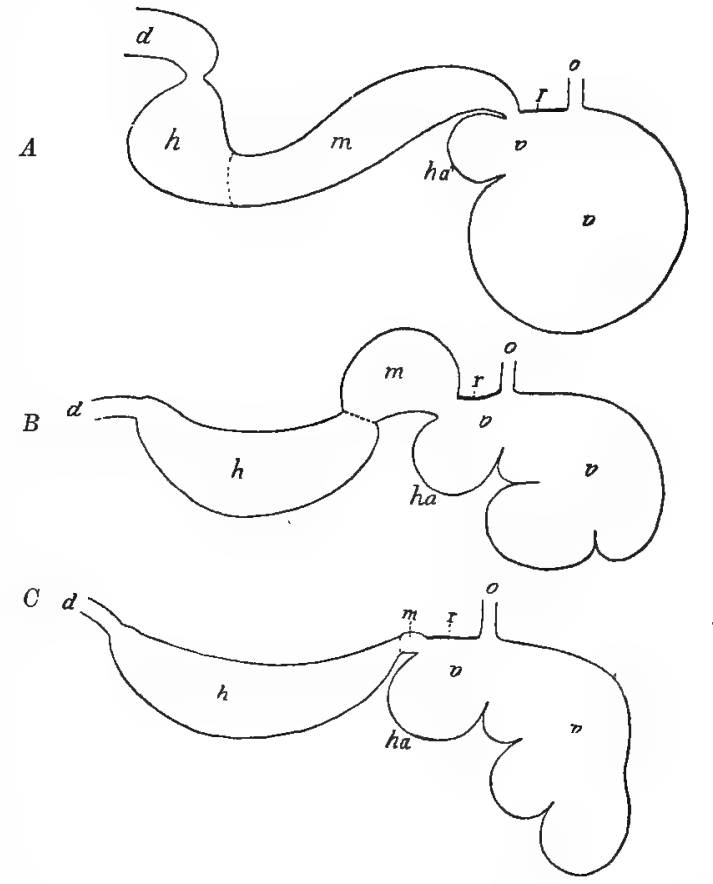

Fig. 407. Diagrammatic longitudinal section of the stomach: $A$ of a CameI, $B$ of an ordinary Ruminant, $O$ of a Tragulus. $d$ small intestine, $h$ abomasum $h a$ retioulum ( $h a^{\prime}$ portion of the rumen of a Camel, which may be falsely compared with the reticulum of others), $m$ manyplies, o cesophagus, $r$ groove in the rumen, $v$ first chamber.-Orig. buccal cavity, and are non-glandular; the abomasum is lined with a cylindrical epithelium, and is furnished with glands. The food, which is not much masticated in the mouth, forms a large bolus, passes through the cesophagus, dilating it as it goes, and reaches the rumen, where it undergoes a kind of fermentation or maceration, until it is again brought up in small quantities into the mouth, to be masticated and mixed with saliva. Then it passes a second time, but in a viscid condition, through the cesophagus, running, however, along the groove of the rumen, and so reaches the psalterium, whose laminæ absorb some of the fluid, and lastly it enters the reed. Fluid substances apparently pass direct from sthe cesophagus into the 
manyplies through the groove of the rumen. In $\mathrm{Camels}$ the stomach consists of the same compartments as in most other Ruminants, but the manyplies is a long tube with low, longitudinal folds, and is not externally separated from the short abomasum; further, the abomasum is lined with cylindrical epithelium and provided with s hort tubular glands.* The abomasum is furnished with long, well-developed glandular pits, and thus an internal distinction is made between the two chambers, the mucous membrane of the latter being of a much

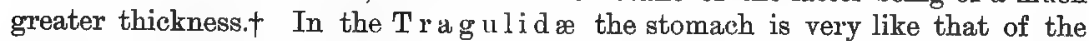
majority of Ruminants; but it is different in that the manyplies, though clearly demarcated, is rudimentary. A transition to this condition is afforded by several other small Ruminants, in which the manyplies is very short and little developed.

1. Camels (Camelidx). In contradistinction to other Ruminants the last upper incisor is present and caniniform ; the canine of the lower jaw, like that of the upper, is caniniform (conical); the stomach is aberrant (see above); the placenta diffuse; $\S$ horns are absent; there are only two digits on each foot; the hoof is small and curved, the sole is large and soft (in contradistinction to all other Ruminants); they are plantigrade. The Camel genus (Camelus) includes long-legged animals with a large fatty hump on the back; dental formula, $\frac{i^{3}}{i^{1} i^{2} z^{3}} \frac{c}{c} \frac{p^{1} p^{3} p^{4}}{p^{1} p^{4}} \frac{m^{1} m^{2} m^{3}}{m^{1} m^{2} m^{3}}$; the first premolar of both jaws is caniniform and separated from its fellows by a diastema. In the Bactrian Camel (C. bactrianus) of Asia, the hump is divided into two, anterior and posterior: in the Dromedary (C. dromedarius), of Africa, Arabia, Persia, etc., it is simple: these two essentially desert animals are only known in the tame condition (except when they have run wild). The Llamas (Auchenia) are smaller, without humps, and without the caniniform molar $\left(p^{1}\right)$; several species, both tame and wild, occur in western South America.

2. Giraffes (Camelopardalis giraffa) possess two hairy, internally ossified, outgrowths on the head; very long legs, fore legs longer than the hind; neck long; Africa.

3. Stags (Cervidix) constitute a large group of mostly slim, thin-legged, short-tailed Ruminants; the males (and occasionally the females also) usually bear antlers; in the fully developed condition (for structure and development see p. 471), they are naked bony processes; at the base of each is a slightly widened portion, the "burr" (above the lower portion, the pedicle, which remains covered with skin). The first antlers borne by young Stags are simple, unbranched and small; later they are larger and usually branched. The dental formula is: $i \frac{0}{3}, c \frac{1 \text { or } 0}{1}, p \frac{3}{3}, m \frac{s}{3} . \|$ The following occur in Britain; the Roe (Cervus capreolus), smaller than other European forms; the antlers of the adult with rarely more than three tines: the $\mathrm{Red} \mathrm{Deer}(C$. elaphus), only in the Highlands: and the Fallow Deer (C. dama), a native of the countries bordering the Mediterranean, whence it was introduced into Central Europe and Britain

* Cylindrical epithelium and short glands are also present on the floor of the "water cells" of the first chamber (cf. foot note $\dagger)$ : for the rest these portions are lined with non-glandular stratified epithelium.

+ In Camels the first compartment is incompletely separated by constrictions into several parts ; the sub-sections thus formed are not, however, comparable with those of other Ruminants. Some portions are provided with a net-work of deep folds bounding small prismatic or honey-comb-like cavities, the so-called "water cells."

I In the rudimentary condition $i^{2}$ may also sometimes be present, whilst in the milk dentition both $d i^{2}$ and $d i^{3}$ are always developed.

$\S$ Blood corpuscles, unlike those of all other Mammalia, oval.

II A canine may or may not be present in the upper jaw (e.g., in the Red Deer). The premolar which is absent is $p^{2}$. 
probably several centuries ago. Remains of a large extinct form, the $I_{r}$ ish Deer (C. euryceros), characterised by the colossal size of its antlers, are found on the Continent and in Scotland as well as in Ireland, where this animal must have lived into the Middle Ages. The Reindeer (C.tarandus), the does of which are characterised by the possession of small antlers, inhabits the circum. polar lands of the Northern Hemisphere, and fossil remains are found in the Quarternary formations of Central Europe. The Moose Deer (C. alces) is a clumsy long-legged animal with very spreading antlers; its range is almost coincident with that of the Reindeer, although not extending so far north; in former times it, too, was met with in Central Europe, but its distribution now does not reach further west than Prussia. The Wapiti (C. canadensis) must be mentioned as the New World representative of the closely allied Red Deer. Further, there is the Musk Deer (Moschus moschiferus) of Asia, without antlers, the males of which have very long canines projecting from the mouth, and have a ventro-abdominal dermal pouch in which musk is secreted.

4. The Tragulids (Tragulidæ) form a circumscribed group of small Ruminants without antlers; in external form they are very like Stags, and are indeed, in most respects nearly allied to the Cervidæ. They are characterised chiefly by the fact that the third and fourth metatarsals and carpals fuse late or not at all, and that the second and fifth are complete. The manyplies is rudimentary (see above). East Indies; Africa.

5. The Cavicornia have two horns, which, instead of being hirsute, are covered externally by a hard thick horny layer; they are ossified internally (cf. p. 471). They are usually developed in both sexes, though occasionally rudimentary or wanting in the female: $i \frac{0}{3}, c \frac{0}{1}, p \frac{3}{3}, m \frac{3}{3}$; the absent premolar is the first.

(a) Antelopes (Antilopinæ) is the common term for a large number of Ruminants which are for the most part cervine in appearance, though some resemble Cattle. The horns are of very diverse form; in some cases they are absent from the females. Especially abundant in Africa.*

(b) Sheep (Ovis). Snout hairy, horns transversely wrinkled, thick, pointed, often much curved backwards and outwards; a dermal invagination between the two large toes (interdigital pouch); two mammillæ. The D o mes t i c Sheep (Ovis aries) belongs here; the ewes have rudimentary horns or none: of unknown descent. Among wild species may be mentioned the Mouflon (O. musimon) of Corsica and Sardinia, and the Argali (O. ammon) of Central Asia, besides several other Asiatic species. All wild Sheep are mountain animals. Closely allied are the Goats (Capra) with compressed, less-curred horns, and without interdigital sacs; mountain animals. The descent of the Domestic Goat (C.hircus) is unknown. Among wild forms may be noted: the Steinbock $(C, i b e x)$ of the Alps and other mountains of South Europe, and the Bezoar Goat (C. ægagrus) in Asia Minor, Crete, etc. The $\mathrm{Cbamo}$ is (Capella rupicapra) is allied to the Goats; it has small upright horns curved only at the tips; in the Alps, Pyrenees, etc. The Musk Ox (Ovilos moschatus) is also related to the Sheep; it is a large long-haired Ruminant; with horns recalling those of the Buffalo; with hairy snout ; and only two mammillæ; it inhabits Arctic North America and also occurs in the Quaternary formations of Europe.

(c) Cattle (Bovinæ). Large, bulky animals, with broad naked nose; long tail, with terminal tuft; no interdigital sacs; often a dewlap (dependent fold of skin)

* The Prong-buck or Prong-horned Antelope (Antilocapra amiricana) is usually regarded as an Antelope: it is remarkable because the horns, each of which in the adult possesses two tines, are thrown off and replaced annually. When the horny cap is cast, bony cores are seen covered with a hairy skin, but later, a thick horny layer is formed above the hair: when the horns are thrown off the hair goes also. On the prairies of Western North America. 
on the neck and chest; four mammillæ; the horns usually round and smooth curved outwards at the base, upwards at the tip. The Domestic Ox (Bos taurus), with flat forehead, is probably descended from several wild species; one of its ancestors is the now extinct gigantic A urochs or Ure Ox $(B$. primigenius), which lived in Britain and many parts of the continent in early times. Nearly allied to the Domestic Cow is the tame $\mathrm{Zebu}$ (B. indicus), with a fatty hump; occurring in Asia and Africa. Somewhat more remote is the long-haired $\mathrm{Y}$ a $\mathrm{k}$ (B. grunniens), of which both wild and tame forms inhabit the mountain districts of Central Asia. The Bis on (Bison) has an arched forehead and fairly small horns, which are far apart at the base, just as in the genus Bos; the anterior portion of the body is humped in consequence of the great development of several of the neural spines; the European Bison* (Bison europæus) is now almost exterminated (only persisting in Lithuania and the Caucasus); formerly it was widely distributed throughout Central Europe. The nearly allied American species (B. americanus, "Buffalo") was common in large herds in North America some time ago, but is now rarely seen. The Buffal os (Bubalus) are distinguished by the horns, which are very flat at the buse, and sometimes almost touch in the median line; they are poorly covered with hair; beasts of burden, of which, among others, a tame species descended from an Indian form (Bub. vulgaris), is kept in South Europe.

\section{Order 6. Proboscidea (Elephants).}

Existing Elephants (Elephas) are large, bulky, longlegged animals, with little hair. The feet, including the metacarpals

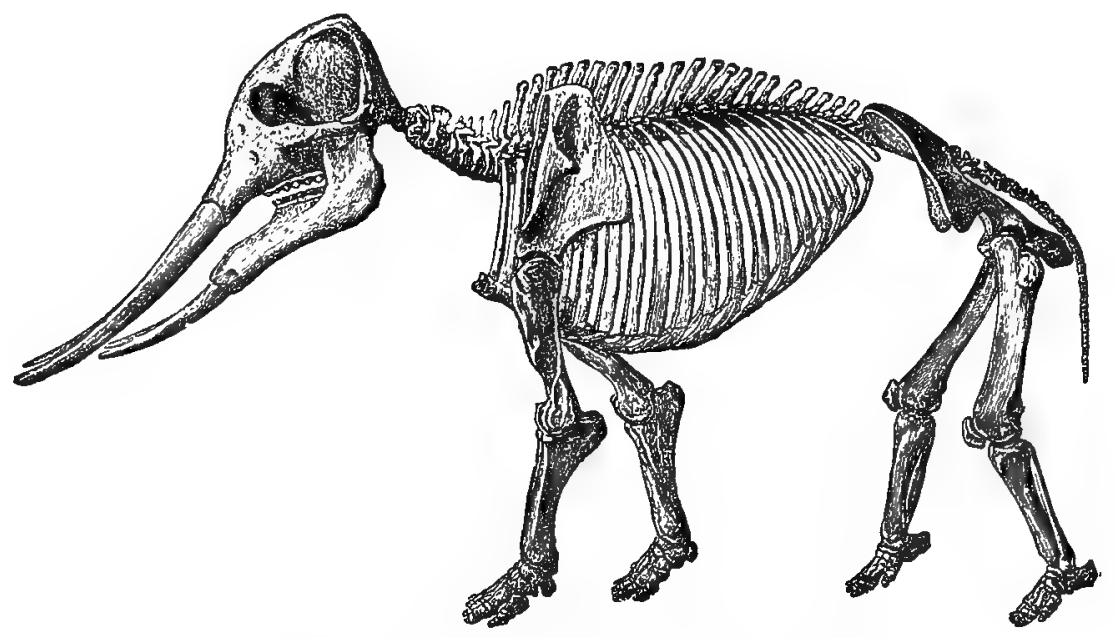

Fig. 408. Skeleton of a Mastodon.-After Gaudry.

or metatarsals are short, and each has five toes bearing short hoofs; $\dagger$ there is a large sole ventral to the toes, which are enclosed in a

\footnotetext{
* The name Aurochs is used for this as well as for Bos primigenius.

$\dagger$ Hoofs may be absent from one or two digits,
} 
common membrane. The snout is produced into a long $t r u n k$, bearing the nares and, in the Indian Elephant, a finger-like process at the tip; it is a prehensile organ, and conveys food (plants) to the mouth; water is sucked up into it and squirted into the mouth, towards which the tip can be directed. The pinnæ are large dependent flaps. The mammæ (two) are close to the forelegs. The head is borne upon a short thick neck, and is of colossal size; the cranium small; extensive air sacs in the bones of the head. Incisors absent from the lower, one on each side in the upper jaw; this, in the males especially, is modified to form a long tusk which is curved forwards, and is practically devoid of enamel; it projects some distance from the mouth, and grows continuously throughout life. Canines are absent. The molars are large, with high crowns and short roots; the crown is made up of a varying number of compressed trunsverse plates coated with enamel, and

$A$

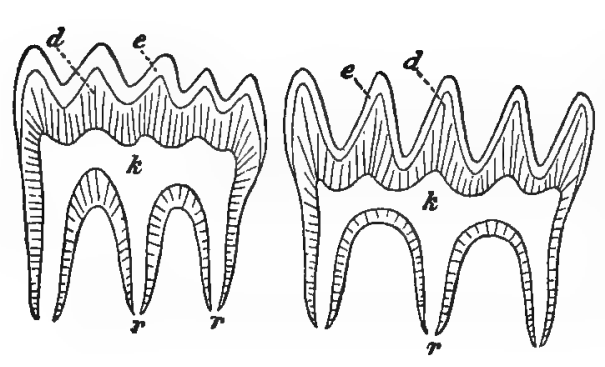

$C$

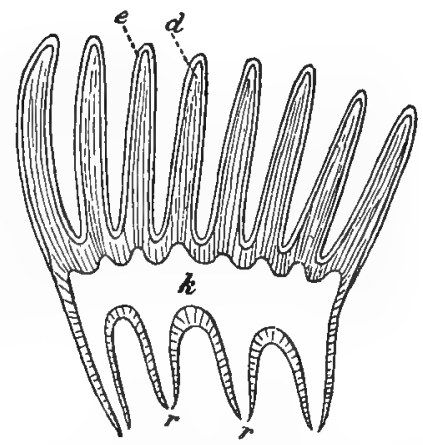

Fig. 409. Molar teeth in longitudinal section. $A, B$ different species of Mastodon, $C$ Elephant; diagrammatic, cement removed; $d$ dentine, e enamel, $k$ pulp cavity, $r$ root.-Orig.

bound together with an abundant supply of cement. There is never more than one, or at most, two, teeth in use at the same time in each half of the jaw : as one tooth is worn away another comes forward and gradually takes its place; the anterior end comes into use, whilst the posterior part is still within the jaw: it is therefore worn away first, so that at last only the posterior end remains. Altogether six molars appear in this way on each side; the first to arise being the smallest.* There are only two living species: the Indian Elephant ( $E$. indicus), with the molar-plates numerous and much compressed; with relatively small pinnæ; it is both wild and

* The six molars of the Elephant are: $d p^{2}, d x^{3}, d p^{4}, m^{1}, m^{2}, m^{3}$; the premolars are wanting in living forms, but rudiments occeur in an extinct species, and also in Mastodon, 
domesticated: the African Elephant (E. africanus), with fewer, thicker plates; and very large ears.

The extinct forms are numerous. The $\mathrm{M}$ a $\mathrm{m} m$ oth (E. primigenius), from the Quarternaries of Siberia and Europe, ap-

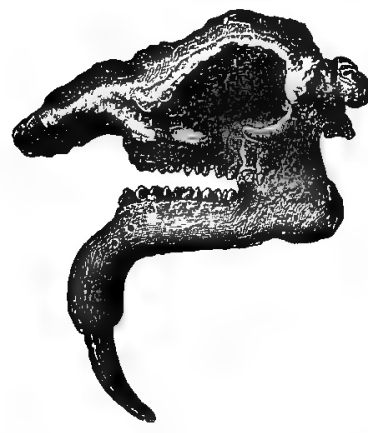

Fig. 410. Skull of Dinotherivm. proached the Indian Elephant in build, but had a thick coat of hair to fit it for the raw climate. The Mastod on s were aberrant forms, with several molars in use at once; the molar ridges are few, and not connected by cement. Someforms had a large incisor in the mandibular ramus turned forwards and downwards; they also had upper incisors like those of the Elephant. The two genera, Mastodon and Elephas, are closely connected by their outlying forms: Tertiary. The Miocene genus, Dinotherium, had relatively small molars, like those of the Tapir; the upper incisors were absent, but lin each jaw there is a single downwardly-directed incisor.

The genus Dinoceras, recalls the Elephants in size and form, and is associated with them : it differs, however, in many respects; there are no incisors in the premaxilla (six in the lower jaw), but there are very large upper canines; the molars are small : Miocene of North America.

\section{Order 7. Sirenia (Sea Cows).}

The Sirenia, a small group of marine Mammalia, were formerly classed with the Whales ; they bave, however, absolutely no connection with them. The resemblance in certain structural points, is to be regarded as dependent upon adaptations to a similar mode of life. In some respects this group recalls the Ungulata.

The body has a sparse covering of bristles. The head is borne upon a very short neck, but is well marked off from the trunk; the nares lie at the end of the snout; the lips are large and thick; pinnæ are absent. The trunk passes gradually into a powerful tail, at the end of which there is a large horizontal fold of skin on each side, the two folds together forming the "caudal fin." The fore limbs are short and fin-like, with the digits enclosed in a common membrane; the thumbs are rudimentary; the others, three-jointed (in contradistinction to the Whales); the arm is not movable at the shoulder only, as in Whales, but also at the elbow; in the Manatee, rudimentary hoof-like nails occur. Hind limbs are altogether wanting in all living Sirenia, and the pelvis is vestigial; but in the Miocene genus, Halitherium, traces of the hind limbs in the form of rudimentary femurs have been found; the two mammæ lie between the fore limbs. In young forms, upper and lower incisors are present, but they usually fall out, so that the adult is edentulous anteriorly; in the male Dugong alone, a pair of upper incisors develops into tusks; in 
the females they are never cut, but a horny plate covers the jaw above and below; canines are absent; the molars are small with transverse ridges; the Manatee has about ten in each half of the jaw, the Dugong fewer: the stomach is complicated in structure.*

The Sirenia are herbivorous and feed upon algæ; they are of considerable size (existing forms $3 \mathrm{~m}$. to $5 \mathrm{~m}$.), and are found on sea coasts and in rivers. The only representatives now living are the $\mathrm{M}$ a natee (Manatus), on the Atlantic coasts of Africa and America (and the adjacent rivers); and the Dugong (Halicore dugong), in the Indian Ocean. The gigantic, edentulous Rhytina, Steller's Cow (Rhytina stelleri), is now exterminated. Until the last century it was found in the North Pacific.

\section{Order 8. Carnivora.}

The Carnivora constitute a large group, including numerous genera and species, which exhibit great diversity both in structure and also in habits; certain characteristic features run through the whole group, and all the members are reducible to a common ty $\mathrm{pe}$.

This is particularly evident with regard to the dentition, which is most easily comprehended if that of the $\mathrm{Dog}$ is considered first; for that of other members of the group may be regarded as

Fig. 411.

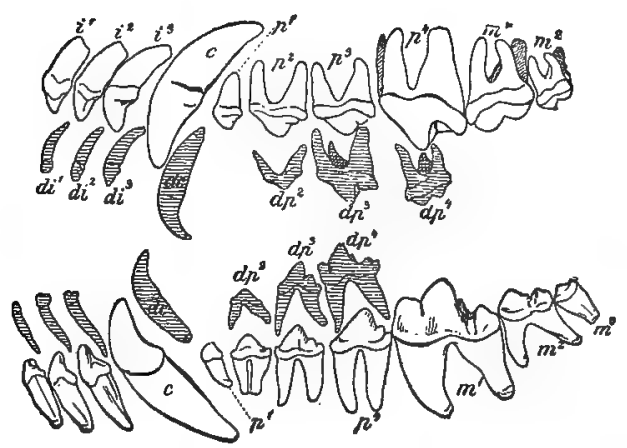

Fig. 412.

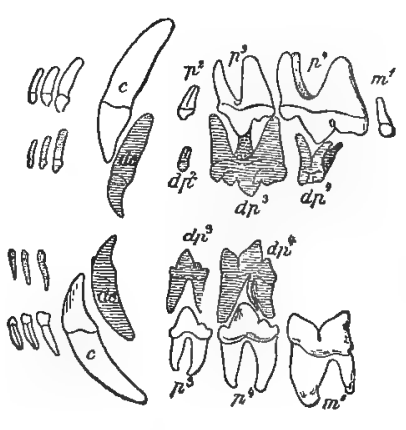

Fig. 411. The teeth of the permanent dentition of the left half of the skull of a D og, and the mill dentition of the same, the latter shaded.-Orig.

Fig. 412. The same of a Cat.-Orig.

variously modified from it. There are, then, on each side of the upper jaw of the Dog, three incisors (of which the third and outermost is larger than the others), one conical curved canine and six molars (four premolars and two molars). The three anterior upper

* With regard to the skeleton it may be noticed that the lower jaw is very large and heavy, very unlike that of the Whale's, and this is true also for the rest of the skull. 
premolars may be termed interdigitating teeth (for they usually interlock with those of the lower jaw), each has a compressed triangular pointed crown, and one or two smaller cusps on the posterior edge of the triangle, the anterior one being the smallest. The fourth premolar $\left(p^{4}\right)$, the sectorial or carnassial tooth, is similarly compressed; behind the apex there is a narrow notch in the edge; and on the inner side a small tubercle: next to the sectorial there follow two broad trituberculate teeth $\left(m^{1}\right.$ and $\left.m^{2}\right)$, of which the anterior is the larger. The incisors and canines of the lower jaw are like those of the upper; there are, however, seven molirs $(p 4, m 3)$, the first four resembling the interdigitating teeth; the filth $\left(\mathrm{m}^{1}\right)$, the largest tooth of the lower jaw, is something like the fourth premolar of the upper, and is also termed a sectorial; its anterior portion, which is situated below the upper sectorial, is compressed and provided with two cusps, of

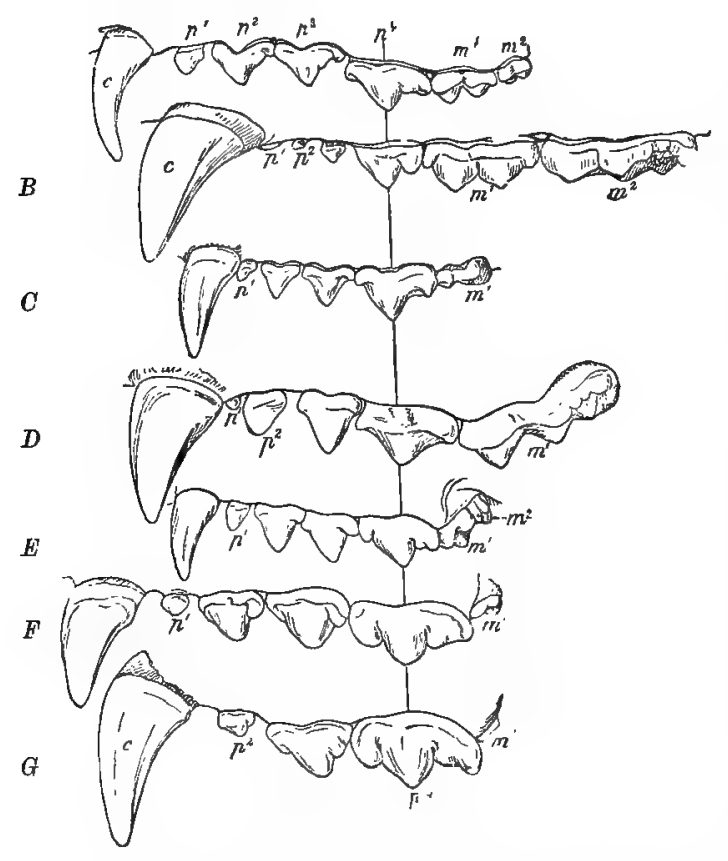

Fig. 413. The teeth of the left half of the upper jaws of: $A$ Dog, $B$ Bear, $C$ Marten, $D$ Badger, $E$ Viverid (Herpestes), F Hyæna, $G$ Lion. The chief point to notice is the great development of the tubercular portion $\left(m^{1}-m^{2}\right)$ in $B$ and $D$, and the degeneration of this in $E-G$.-Orig. in all. To give examples of this : in the $\mathrm{C}$ at (Fig. 412), the dentition is degenerate as compared with that of the Dog; of the six upper molars of the latter, the first and the last have disappeared, and of the four remaining, the first and the last are almost rudimentary; as a little higher than the anterior, whilst the small posterior portion of the tooth is low and tuberculate. The last two molars $\left(m^{2}\right.$ and $\left.m^{3}\right)$ are tritubercular, like those of the upper jaw, but are smaller.

The modification of the dental system occurring in other Carnivora tends partly towards a reduction of the molar series proceeding from both ends, partly to a hypertrophy either of the tubercular or of the sectoria] portion, whilst the incisors and canines as regards number and form are practically the same which the posterior is 
to the seven lower molars, both the first two and the last two are absent. The tubercular part of the series is almost completely atrophied, for not only are the molars (with the exception of the rudimentary one of the upper jaw) absent, but the tubercular part of the lower sectorial has also disappeared. In B e ars, the opposite extreme is reached; the tubercular teeth are all present, and like the posterior (tubercular) portion of the lower sectorial, extraordinarily well developed, whilst the first three premolars are small, and some fall out with maturity. For other groups see the special descriptions and Fig. 413.

The milk dentitions are still more nearly coincident, since with one exception (see below), three premolars are present in each jaw, viz,, the second, third, and fourth; the second upper interdigitates with the second and third lower, the third is exactly like the upper permanent sectorial, the fourth in the upper jaw is a tubercular tooth, in the lower jaw, a sectorial.* It is remarkable that the sectorial of the permanent dentition does not occupy the same position as does the milk sectorial, but in both upper and lower jaws, is one place further back. Only where the number of premolars is less than three (in the lower jaw of the Cat), is the number of milk molars less than the typical number; the milk molar corresponding with the absent premolar (the second) is then also wanting.

The last phalanx of each toe bears a claw which is often much curved and can be turned upwards by means of an elastic ligament passing from its phalanx to the penultimate; so that in some animals (e.g., the Cat) it does not touch the ground in walking (retractile claws). The first digit is usually smaller than the others, and is frequently absent from the hind limb. These animals are either plantigrade, or digitigrade. The clavicle is poorly developed or absent. Placentation zonary. In many forms there are, in the region of the anus, glands or glandular pits, whose secretion has an offensive odour.

Most of the Carnivora are of medium size, and feed either upon other animals, or upon plants (succulent roots, berries, etc.). 'They occur all the world over (with the exception of Australia), and are most abundant in the Tropics.

The Carnivora form three large natural groups, one of which (Cynoidea) includes the Dogs, another (Arctoidea) the Bears, Raccoons and Martens, a third (Aluroidea) the Cats, Viverras, and Hywnas. The differentiation is shown chiefly in numerous minute points in the skull, a full account of which would, however, involve so much detail, that this bare statement must suffice.

1. The Dog Family (Canidæ). $p \frac{4}{4}, m \frac{2}{3}$; the tubercular portions of the molars are of medium strength. Head long, tail long, legs long, with five toes in front, four behind; digitigrade. To this family belong the Fox (Canis vulpes), the Polar Fox $\uparrow$ (C. lagopus), both with perpendicular pupils; the latter an inhabitant of the Arctic regions: the Wolf (C. lupus), with round

* In the milk dentition of Carnivora there is therefore the same number of tubercular teeth as in the permanent dentition of the Cat, $i e, \frac{1}{v}$.

$\dagger$ In the Fox there are usually $m_{2}^{2}$. 
pupil; in Europe, North Asia and North America, exterminated in England: the Jackal ( $\mathrm{C}$. aureus), nearly allied to the Wolf; in Asia, North Africa, and the Balkan Peninsula: the Domestic Dog (C. familiaris) probably a descendant of the Jackal or its naer relations. An isolated canine form, the genus Icticyon of Brazil, is distinguished by the possession of $m \frac{1}{2}$ only, in other respects, near to the rest of the Canidx. Another, the South African Otocyon caffer has very large pinnæ; and is vulpine, with a pointed nose, and with more than the typical number of molars, viz. $p \frac{4}{4}, m \frac{4}{4}$.

2. The Bear family (Ursidæ). $p \frac{1}{4}, m \frac{2}{3}$; tubercular region of the molar series principally developed, sectorial portion degenerate (usually some of the premolars are absent from the adult). Elongate head, very short tail; each foot has five toes armed with very strong claws; plantigrade; animals of considerable size, feeding chiefly upon plants. Here belong: the Common Bear (Ursus arctos), which occurs on the continent, e.g., in Switzerland, Hungary, Russia, Scandinavia; it hibernates: the American B lack Bear (U. americanus) in North America, where is also the Grizzly Bear (U. cinereus): the Sloth Bear (U. labiatus) of India, with very projecting lips, and unusually long claws; the incisors are generally lost early : the Pol a r Bear (U. maritimus), white, with hairy soles, belongs to the Aretic regions. The Quaternary Cave Bear ( $U$. spelæus) was larger than living forms; its remains are frequently met with in the bone caves of Europe.

3. 'R accoons (Procyonidx). $p \frac{4}{4}, m \frac{2}{2}$; tubercular portion of the molar series not so pronounced as in the Bears; head longish, tail long, five toes on each foot; plantigrade; small forms; omnivorous. Here belong the W a shing Racoon (Procyon) and the Coatimondi (Nasua); both in America.

4. The Marten family (Mustelidæ). $p \frac{3 \text { or } \frac{4}{3} \text { or }}{4}, m \frac{1}{2}$; in some, the carnassial region of the molar series (i.e., the interdigitating teeth, the sectorial of the upper jaw, the anterior portion of the lower sectorial); in others, the tubercular portion, is most pronounced. The tail is usually well-developed; legs short, five toes, digitigrade or plantigrade.

(a) Martens (Mustela). Small, very elongate, thin forms, prey chiefly upon warm-blooded Vertebrates; digitigrade; tubercular region rather small. The following occur in Europe: the Pin e-marten ( $M$. foin $a$ ), a large species with white throat: the Polecat ( $M$. putorius), brown; the Ferret (M. furo) is a paler, degenerate, domesticated breed of the Polecat: the Ermine ( $M$. erminea), becomes white in winter; the short-tailed Weazel (M. vulgaris), the smallest species, occurs in the British Isles : the European Mink (M. lutreola), of the size of the Polecat, is uniformly brown, and has webbed toes; it is aquatic, and common in Russia; it recalls the Otter. The Sable ( $M$. zibellina) of Siberia, stands near the Pine-marten. Allied to the Martens, but larger and clumsier, is the Glutton (Gulo borealis), with a very short, bushy tail, plantigrade; in Scandinavia, Russia, Siberia, North America.

(b) The Otters (Lutra) are larger, and characterised by the long, powerful tail, webbed toes, blunt nose, and very short pinnæ. They are extremely good swimmers, and feed upon fish. The Common Otter (L. vulgaris) of Britain and other parts of Europe, inhabits fresh water as well as the sea. Allied to it is the Sea Otter (Enhydra marina), with $i \frac{3}{2}$; the hind limbs resemble those of Seals : on the cousts of the North Pacific.

(c) B adgers (Meles taxus), characterised by the great development of the molars, and of the hinder portion of the lower sectorial; plantigrade forms, with strong digging claws on the fore limbs; omnivorous. The Sk unks (Mephitis) are allied to the Badyers, and occur in North and South America, Africa, and Asia Minor, 
5. The Givet Family (Vivervidx). $p \frac{4}{4}, m_{\frac{2}{2}}^{2}$; sectorial portion of the molar series preponderatingly developed. Small animals, resembling the Martens, with elongate body and short legs. In the warmer parts of the old World. The following may be noted: the Civet $\mathrm{Cat}$ (Viverra), one species of which, the Genet ( $V$. genetta), inhabits South Europe and North Africa; and the Mongoos e (Herpestes ichneumon), of Africa and Indiá.

6. The Hyæna Family (Hyænidæ). $p \frac{4}{3}, m \frac{1}{1}$. Large, long-legged, wolf-like, fairly long-tailed animals ; digitigrade. In the Old World, The species of the genus Hyæna are carrion feeders; the genus Proteles of South Africa, with very feeble, small, cusped molar's, preys chiefly upon lambs.

7. The Cat Family (Felidix). $p \frac{3}{2}, m \frac{1}{2}$; tubercular portion of the molar series rudimentary. Slim, elongate animals, with roundish head, long tail; four toes on the hind foot, very much curved, compressed and pointed claws; digitigrade; feed almost entirely on warm-blooded animals. The Li on (Felis leo), of a uniform, tawny colour ; male with mane; Africa, West Asia, formerly in SouthEast Europe: the extinct (Quarternary) Cave Lion, (F. spelaxa), is a near relative. The Tiger (F. tigris), with transverse stripes; Asia. The Jaguar ( $F$. onca), in the Southern districts of America; and the Leopard or Panther (F.pardus) of which there are several varieties in Africa and Southern Asia, large, with circular spots. The $\mathrm{Puma}$ or $\mathrm{Cougar}$ ( $F$. concolor) of median size and uniform colour; in South America and in most of North America (the "Panther" of the Americans). Smaller forms are: the Tiger Cats, various small spotted forms (F. tigrina and others); the Wild Cats (F. catus), in Central and South Europe, similar in colour to the grey Domestic Cat, but shorter tailed; the Domestic Cat ( $F$. domestica), which is apparently a descendant of the $\mathrm{Nubian} \mathrm{Wild} \mathrm{Cat}$ ( $F$. maniculata). The following are aberrant forms : the G u e parde (F. [Cynailurus] jubata), a large-spotted, longlegged form with claws less retractile than in other Felidæ; Africa and Asia; may also be tamed: the Ly $\mathrm{nx}$ ( $F$. lynx or Lynx vulgaris) distinguished by its long legs, short tail, and the pencils of hairs on its ears (in the Lynx the first interdigitating tooth of other Felidæ is generally wanting, the dental formula being $\left.p \frac{2}{2}, m \frac{1}{2}\right)$; Scandinavia, Russia, etc.; formerly also in Germany. The extinct Sabre-toothed Cats (Macharodus) have $p \frac{1}{1 \text { or } 2}, m \frac{1}{1}$, and thus the molar series is still more degenerate than in living Cats, to which, in other respects, they are allied; the canine of the upper jaw is extremely powerful and very long. In another extinct group, the genus Dinictis, there is, on the other hand, a larger number of teeth than in the living forms, one additional interdigitating tooth and a small tubercular tooth in the lower jaw (the teeth of the upper jaw being as in Felis): $p \frac{3}{3}, m \frac{1}{2}$.

\section{Order 9. Pinnipedia.}

The Pinnipedia are nearly related to the preceding group, with which they have many characters in common; indeed they may be regarded as Carnivora, which have been adapted to a marine life.

The limbs are short and broad and are turned back; the proximal part of the fore limb is concealed beneath the skin of the trunk, the free portion bears a superficial resemblance to the pectoral tin of a Fish: the hind limbs lie close to the trunk, with the tips of the feet pointing backwards; they are enclosed for the greater part of their length within the general skin; in the true Seals they are fixed in this position, but in the Walrus and Eared Seals they ma: be turned so far 
forwards, that the animal can walk upon them. Each foot has five digits provided with straight claws; all are webbed, and the membrane is prolonged beyond the tips of the toes as a more or less welldeveloped ridge of skin. The digits of the fore limb decrease in length and strength from the first to the fifth (the first and second, however, are about equally strong); on the hind limb the tirst and fifth are stronger and usually also longer than the other three; the tail is short; the pinnæ small or absent; the eyes large; the nares mere slits, which close spontaneously by the elasticity of their walls, but are opened by a muscular apparatus; the hair is usually closeset, smooth, and sleek; with, sometimes, a thick covering of wool below; the new-born animal is generally covered with a woolly fur which is sometimes shed before birth: the vibrisse are very strong. Below the skin there is a thick layer of adipose tissue (blubber).

Fig. 414 .

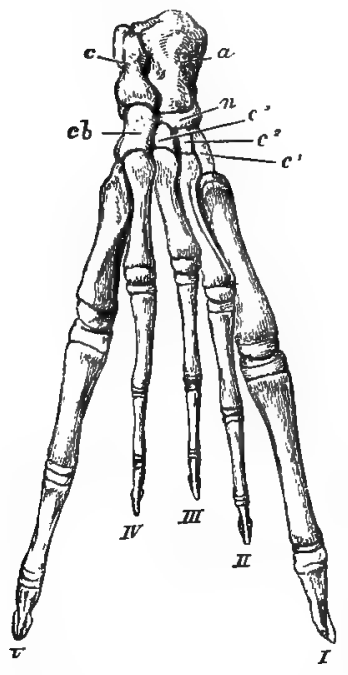

Fig. 415 .

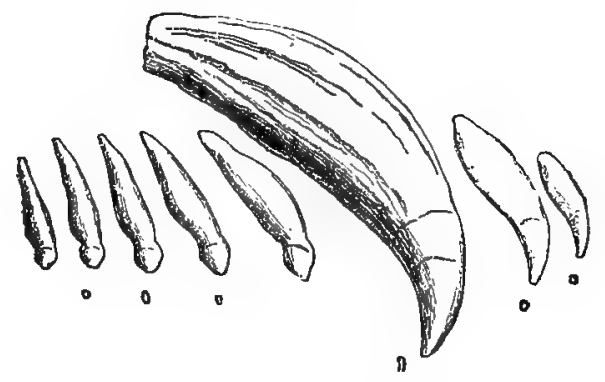

Fig. 414. Pes of a young S ea Elephant. a astram galus, $c$ calcaneum, $n$ centrale, $c^{1}-c^{3}$ cuneiforms, $c b$ cuboid ; I- $\mathrm{P}$ first-fifth toes.-After Flower.

Fig. 415. Upper teeth of the Sea Elephant; the milk teeth are drawn below the teeth of the permanent set. -After Flower.

There are usually $\frac{3}{2}$ incisors (or a smaller number, rarely $\frac{3}{3}$ ) ; they are more conical than those of the Carnivora, and do not form a continuous cutting edge in closing: the canines are usually feebler than in Carnivora, but otherwise similar. Of the molars, $p \frac{4}{4}$ and $m \frac{1}{1}$, are usually present; they are practically all alike, usually similar to the interdigitating teeth of the Carnivores or simple and conical; they are relatively feeble. The milk teeth are rudimentary, they are either absorbed before, or shed quite soon after, birth.

It may also be mentioned that the hindermost portion of the skull is very broad, whilst the interorbital region is usually much compressed. The lower jaw is generally feeble; lachrymal bone and duct are absent; the lachrymal gland is small, the Harderian gland well developed : the placenta, as in the Carnivores, is zonary. 
The Pinnipedia are of considerable size, sometimes even gigantic ; they are marine (a few live in large lakes, e.g., the Caspian Sea), moving with the greatest activity by means of the very flexible posterior portion of the body, whilst the large, backwardly-directed, hind feet function as the caudal fin of a fish. They usually come on shore to rest, to breed, etc. ; they are littoral forms, but can only move slowly upon land. Eared Seals and Walruses are able to walk on all four legs; the true Seals hop along with great difficulty, arching their backs and pushing themselves forward by means of the tail end; they rest with the ventral surface on the ground; the fore limbs are not generally used in locomotion. The food consists of Fish and marine Invertebrates (Crustacea, Mollusca). They are usually polygamous; the males are frequently, as in many other polygamous animals, considerably larger than the females. They belo $\mathrm{n}$ principally to cold and temperate regions.

1. Eared Seals (Otariidæ). With small pinnæ; long neck; fore limbs large; they can walk on the feet which are naked below; there is a large ridge on all four feet, lobed on the hind ones; claws in part rudimentary or very small (this holds for all the claws of the fore limb, and for the first and fifth of the hind, whilst that of the middle toe of the hind limb is well developed). The males are always much larger than the females. This group comes nearest to the Carnivora, and many of the characteristic peculiarities of the Pinnipedia are not well marked. Among these are the so-called SeaLions or Sea-Bears, whose skins afford the well-known sealskin.* They inhabit the southern waters of the South Hemisphere, and the northern regions of the Pacific.

2. The Walrus (Trichechus [Odobænus] rosmarus) is devoid of pinnæ, but in most respects is closely allied to the Otariidæ, though very peculiar as regards the dentition. Like the Otariidæ this animal can support itself on its fins, which have large borders, the ventral surface of the feet is naked; the structure of the claw as in the Otariidx.' The young animal has $i \frac{3}{3}, c \frac{1}{1}$, $m$, but several of the teeth are small and soon fall out or are never cut, so that the adult usually possesses the following functional teeth: $i \frac{1}{0}, c \frac{1}{2}, m \frac{3}{3}$. The upper canine is a long tusk, and continues to grow throughout life; the other teeth are conical at first, but later worn right away. The Walrus feeds on bivalves, worms, etc, which it grubs up with its long tusks from the bottom of the sea. Fairly large; indigenous to the Arctic regions.

3. True Seals (Phocidr). Pinnæ wanting; neck short; fore limbs small. The ventral sides of the feet are hairy, and the limbs are absolutely useless for walking; border of the feet narrow; claws for the most part well developed. Chiefly in the Aretic regions.

(a) The genus Phoca with three upper, two lower incisors, and compressed, multitubercular molars. The Common Seal (Ph. vitulina), occasionally the Ring.ed or Marble Seal ( $P h$. foetida), and the Greenland Seal ( $P$. greenlandica), occur on the shores of Britain. Allied to Phoca is the Grey Seal (Halichorus grypus), with conical molars, occurring on the shores of Northern Europe, including Britain.

* The sealskin of commerce is deprived of the contour hairs, so that the woolly fur alone remains; and it has, therefore, a very different appearance from the fresh skin. 
(b) The Hooded or Bladder-nose Seal (Cystophora cristata), with two upper, one lower incisor. The male is characterised by the possession of a flexible proboscis, which it dilates when angry. Polar seas. The Sea Elephants (C. proboscidea) are somewhat similar forms. In the Indian and Pacific Oceans, chiefly in the southern regions.

\section{Order 10. Cetacea (Whales).}

The Whales are superficially much more like Fish than Mammals; as they have been completely adapted to an exclusively marine

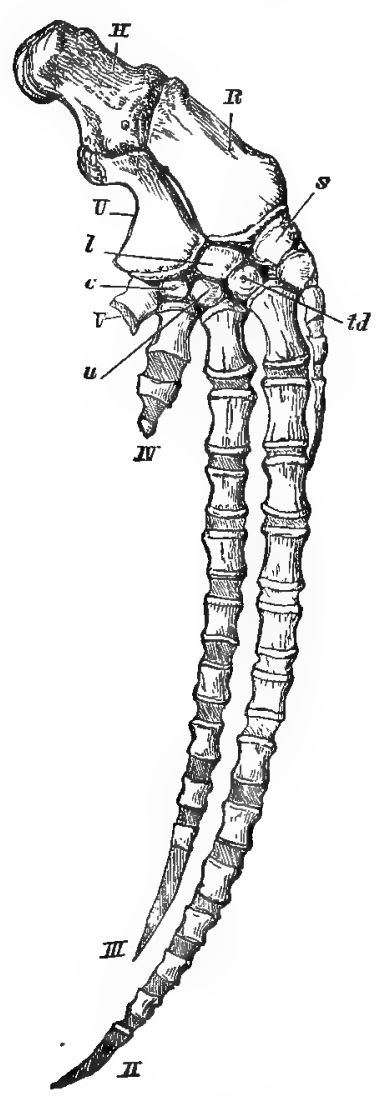

H'ig. 416. Right anterior appendage of a Pilot Whale. $H$ humerus, $R$ radius, $U$ ulna; $s$ scaphoid, l lunar, $c$ cuneiform; $t d$ trapezoid, $\dot{u}$ unciform; $I-$ $I V$ first to fourth fingers, $V$ fifth metacarpal.-After Flower. existence, for which they are much better suited than are other marine Mammalia (Pinnipedia, Sirenia).

The body is piscine in form and pointed at both ends; head, trunk, and tail, are evenly continuous; externally there is no trace of a neck; the tail is compressed, extraordinarily powerful for a Mammal, and very muscular. There is a horizontal tail fin, a broad, stiff, bilateral expansion of skin, at the end of the tail. Dorsally, there is usually a short, upright, compressed dermal process, the dorsal fin. The skin is smooth and shiny; hair and skin glands are generally wanting in the aduilt, at most there are a few hairs in certain regions of the head, especially near the edges of the mouth ;* the dermis is very thick, and contains an extraordinary amount of fat (blubber). Lips are absent. The anterior limbs only are well developed (for the rudimentary hind limbs see below); they are modified into stiff, clawless plates, only movable from the shoulder; the fingers are enclosed in a common skin, and their limits are not recognisable externally. The nares open high upon the head, and are often united into a single aperture; the eyes are small; the external auditory opening extremely small, and pinnæ are wanting. The mammillæ, one on each side, are situated in pits near the anus.

* Only some of the Mystacoceti (and a South American river Dolphin Inia) have hairs when adult. On the other hand, the embry os of almost all Whales (both Mystacoceti and Odontoceti) have a few hairs; in the Odontoceti these only occur above the upper jaw. 
The cervical region of the vertebral column is very short, but, consists of the usual seven vertebræ, several of which are generally fused; sometimes, as in the Arctic Right Whale, they are all fused

Fig. 417.

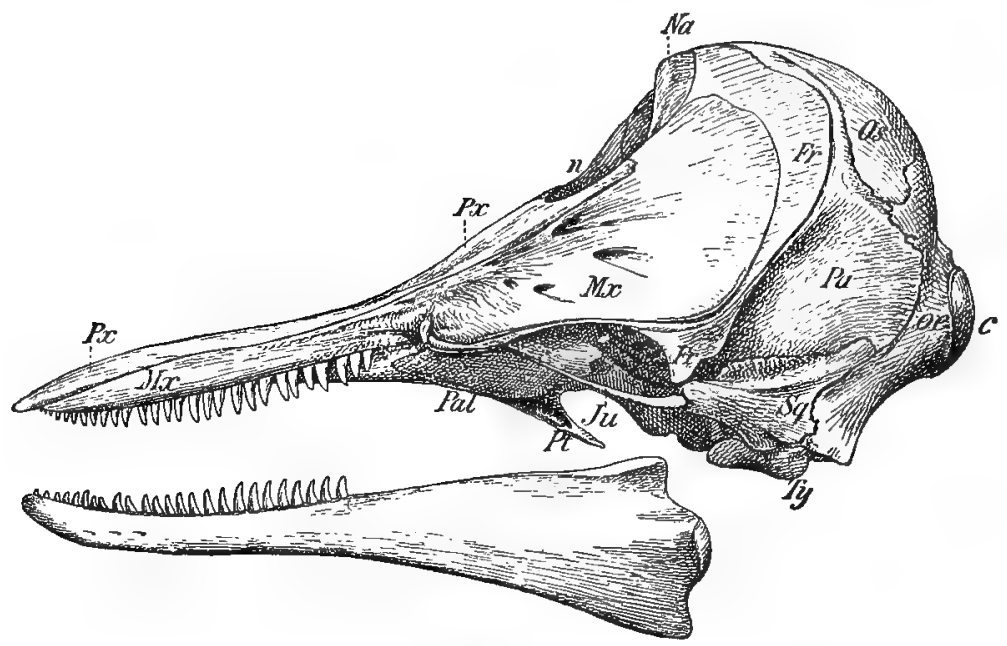

Fig. 418

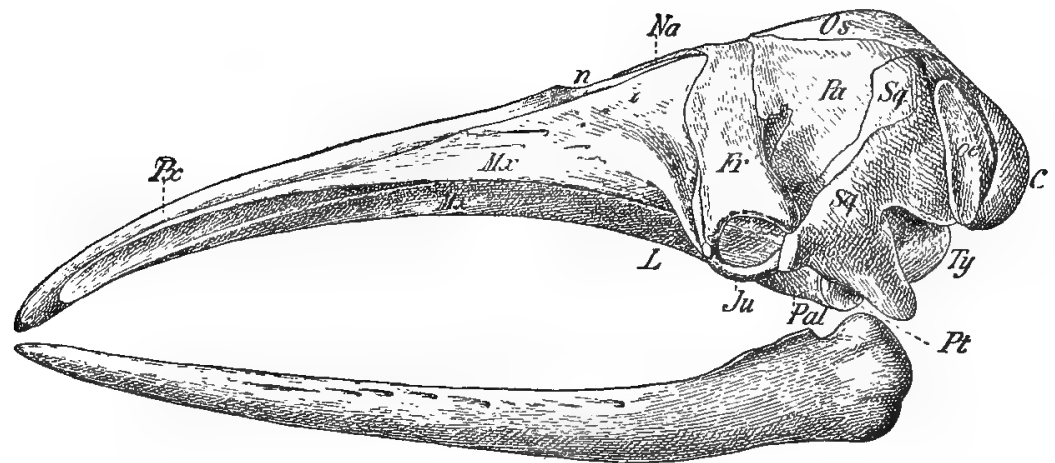

Fig. 417. Skull of a Dolphin (Delphinus) from the side. Decreased.-Orig.

Fig. 418. Skull of a Mystacocete (Balaena japonica), foetus. Decreased.-After Eschricht.

$C$ occipital condyle, Fr frontal, Ju jugal, $L$ lachrymal, $M x$ maxilla, $n$ naris, $N a$ nasal, oe exoccipital, $O s$ supraoccipital, $P a$ parietal, $P a l$ palatine, $P t$ pterygoid, $P x$ premazilla, $S q$ squamosal, $T y$ tympanic bulla.

in the Balænopteridæ and some Odontoceti, on the other hand, they are all separate, the centra are flattened discs. Very few of the ribs are attached to the short sternum. The lumbar region is characterised by its great length; sacral vertebræ are not dis- 
tinguishable: the entire vertebral column, with the exception of the cervical region, is very flexible, the intervertebral discs thick. The jaws are much elongated; the jugal in the Odontoceti is very thin; the nasal very short, often rudimentary (best developed in the Mystacoceti). The scapula without a spine; clavicles absent. As was mentioned above, the bones of the fore limb are immorably connected; there are four or five fingers; it is interesting to note that some of these have more than three joints. There is a vestigial pelvis in the form of two bones, one on each side, which are neither connected with each other nor with the vertebral column: in some of the Mystacoceti rudiments of the hind limb, the femur and tibia, are also present, but embedded in the muscle. Lachrymal glands and ducts are absent, but the Harderian gland is present, and, indeed, well developed, the secretion having a fatty consistency. The nasal cavities are a pair of tubes, oblique in the Mystacoceti, almost perpendicular in the Odontoceti; in the Mystacoceti, rudimentary turbinals and small olfactory nerves are present; in the Odontoceti the turbinals are wanting, whilst olfactory nerves may or may not be present. In the Odontoceti the teeth are usually very numerous, generally homodont and conical; there is no replacement. The Mystacuceti have teeth in the embryonic condition (similar in form to those of the Odontoceti), but they are small and are never

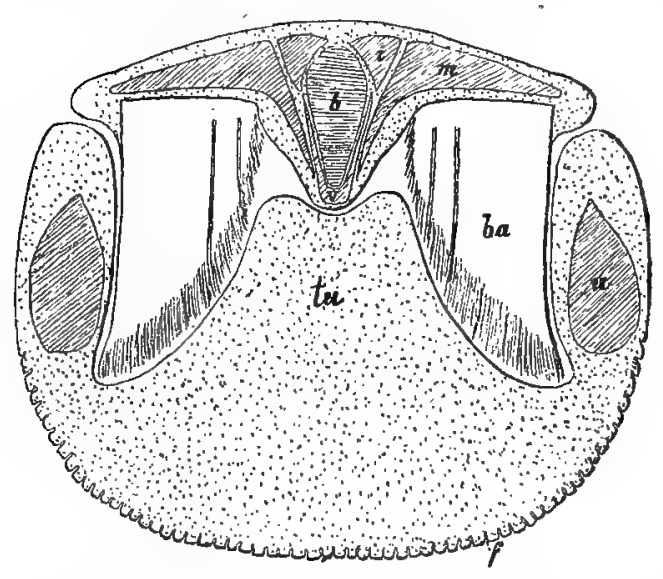

Fig. 419. Diagrammatic transverse section of the anterior portion of the head of Balaenopterid. $b$ cartilage, corresponding to the nasal septum of other Mammalia; $b a$ whalebone, $f$ grooves of the skin, $i$ premaxilla, $m$ maxilla, $t u$ tongue, $u$ mandible, $v$ vomer.After Yves Delage. cut. The baleen or whalebone in the mouths of these Whales, consists of two longitudinal rows of strong transverse folds of skin depending perpendicularly from the palate, and corered with a welldeveloped horny layer which constitutes the chief mass. The whalebone, therefore, is a stiff three-cornered plate of horn, which is, for the most part, solid, but has a cavity at the base, wherein is the soft part, consisting of connective tissue and mucous membrane. The whalebone has three edges: a shorter, dorsal one, connected with the palate; an outer, smooth, straight one; and an inner, oblique edge, which is the longest of the three, and much frayed out; in the inner 
portion there are several perpendicular notches reaching to the base. 'The plates, of which the tirst and last of each row are the smallest, lie fairly close together on each side, and fill up a large portion of the buccal-cavity, in the middle of which, however, a space, triangular in transverse section, persists. When the mouth is closed, the whalebone is covered by the lower jaw: it serves as a filtering apparatus; the Mystacnceti swim for some distance with the mouth gaping open, then close it, and the water trickles out between the baleen, leaving the contained organisms imprisoned by the fibrous inner edge of the blades. The whalebone is to be regarded as extraordinarily welldeveloped palatal ridges (see p. 487). The salivary glands are rudimentary or absent, the stomach is complex. The larynx extends forwards as a tubular prolongation, surrounded by the well-developed soft palate; thus a continuous passage between nostrils and trachea is formed, on either side of which the food passes into the oesophagus. The testes are retained in the abdominal cavity.

The Whales are almost all marine; some few occur in rivers. Like Fish, they move by undulations of the tail, and never voluntarily come on to land. They are able to remain for considerable periods below the water (half an hour or more). Their food consists chiefly of Fish and marine Invertebrata. They frequent all seas, the large forms, however, occur chiefly in the colder regions of the world. 'The largest animals known belong to this group.

\section{Sub-Order 1. Mystacoceti (Whale-bone Whales).}

Edentulous, but provided with whale-bone. Two external nares, placed more anteriorly than in the Odontoceti. Only one pair of true ribs. The skull is extraordinarily large and bilaterally symmetrical; nasals relatively well-developed. The Mystacoceti feed on various small marine animals, which live in shoals (Euphausidæ, Copepoda, etc.) ; many of the Balænoptera also feed upon small Fish. This suborder includes the largest Whales.

1. The Fin-whales (Balænopteridx). With dorsal fin. On the ventral surface of the head and body, there are numerous deep longitudinal grooves. Elongate animals, with relatively small head and short whalebone; narrow pectoral fins. The Blue-whale or Sibbald's $\mathbf{E}^{\prime}$ in-whale (Balinoptera Sibbaldii), which attains a length of about $30 \mathrm{~m}$.; and the rather smaller Rorqual (B. musculus), which is abundant in North European seas, are not uncommon off British coasts. Both of these are the objects of a regular fishery on the coasts of northern Norway. The Lesser Fin-whale ( $B$. rostrata) is much smaller (the largest only $10 \mathrm{~m}$.) and also occurs in the North Atlantic. The very large $\mathrm{H} u \mathrm{mp}-\mathrm{back} \mathrm{Whale}$ (Megaptera böops), with low hump-like dorsal fins and with very long pectoral fins, is less elongate than most other Balænoptera; a few have been taken off British coasts.

2. Balænidæ. No dorsal fin; no ventral furrows; body less elongate; heal relatively very large; whalebone long and narrow; pectoral fius broad. The 
huge Greenland or Polar Whale (Balæna mysticetus), up to $20 \mathrm{~m}$. long: in Greenland, etc.; now much reduced in numbers. $B$. biscayensis, very like the Greenland form, occurs somewhat further south (in the North Atlantic).

\section{Sub-Order 2. Odontoceti (Toothed Whales).}

Furnished with teeth, but no whalebone. External nares united, to form a single aperture* situated dorsally and far back on the head; several true ribs. The face portion of the skull is distinctly asymmetrical; nasal bones rudimentary. Feed chiefly upon fish.

1. Dolphins (Delphinus) have a pointed, beak-like snout, marked off from the forehead by a groove; numerous (twenty or more) small conical teeth in each half of the jaw; a high dorsal fin. Animals of about $3 \mathrm{~m}$. Iong, several species occur on British coasts. Allied is the Sea-hog or Por pois e (Phocæna communis), $1.5 \mathrm{~m}$. long, with short blunt snout, compressed teeth (about twenty-five in each half of the jaw); abundant in European seas. The Pilotwhale or B lack - whale (Globiocephalus melas), has teeth only in the anterior'

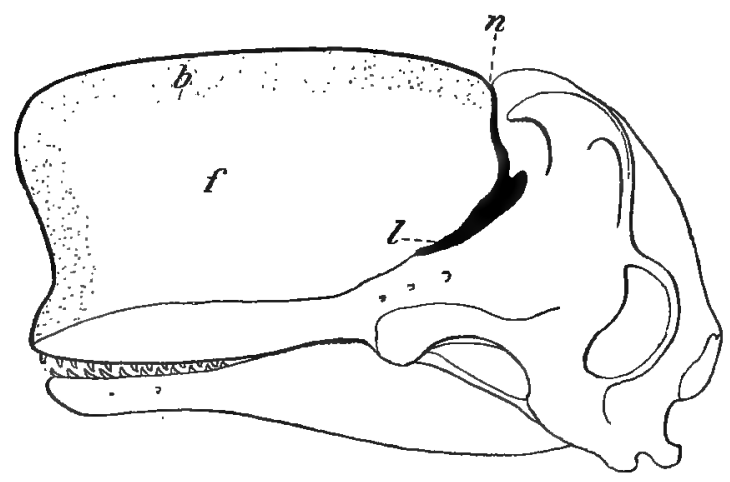

Fig. 420. Skull of a Pil ot W hale, from the left side, with large mass of blubber lying upon the snout; this mass is divided medianly. $f$ blubber, $b$ firm layer of connective tissue below the epidermis, which is indicated by a thick black line, $n$ nares, $l$ air sinus continued on from the nasal duct.-After Murie.

portion of each jaw; the head is thick and rounded in front, with quite a short projecting pointed snout; up to $6 \mathrm{~m}$. long. It is regularly caught off the Faroe Islands, and is a frequent, though irregular, visitor to British coasts. It feeds principally upon Cuttle-fish. The "Grampus" or "Killer" (Orca gladiator), somewhat larger than the Pilot-whale, with very high dorsal fin (whence it is sometimes termed the Sword-fish), and about twelve powerful conical teeth in each half of the jaw ; feeds upon Porpoises, Seals, and Fish; very widely distributed; occurs on the west coasts of Britain.

2. Of the more aberrant Odontoceti may be mentioned, the Cachalot or Sperm.whale (Physeter macrocephalus), a large form with a huge head, on the flattened snout-like portion of which, there lies an immense

* In the Odontoceti, but not in the Mystacoceti, there are saccular outgrowths, both from the short unpaired and also from the upper portion of the paired external nasal duct. 
mass of blubber* (yielding spermaceti); strong, conical teeth in the lower, rudimentary, in the upper jaw. Widely distributed; stragglers have been caught several times off the coasts of Britain. The Bottle-nose Whale (Hyperoodon rostratus), with narrow, pointed snout, and the head much arched behind this; almost edentulous (only one larger and one smaller tooth anteriorly in each half of the lower jaw ; besides this, sevaral rudimentary teeth above and below); in the North Atlantic, fairly abundant, e.g., off the Faroes, and one of the most common Cetaceans; stranded on British shores. The Narwal (Monodon monoceros) is characterised by the possession of a long, spirallytwisted tusk, which sticks straight out from the mouth on the left side; in the right side of the upper jaw, there is a similar, but much smaller tooth, which remains within the bone; there are no other teeth; $\uparrow$ in the female, both are enclosed in the jaw. As an example of an Odontocete living in fresh water, the Ganges Dolphin (Platanista gangetica), which possesses long, thin jaws with numerous pointed teeth, may be mentioned; the eyes are rudimentary, and have no lens; the skeleton is in many respects peculiar. The animal, which is only 2 to $3 \mathrm{~m}$. long, lives in the Ganges, Indus, etc. Two allied forms in rivers of South America.

\section{Order 11. Bruta (Edentata).}

The animals belonging to this group are peculiar in that the teeth, when present, are imperfectly developed, and do not form a continuous series; they are devoid of enamel, are usually all approximately alike, and grow from persistent pulps: incisors are absent (in one of the Dasypodidæ alone, the last incisor of the upper jaw is present) : there is, as a rule, no replacement. The claws are generally long, curved, and very powerful. A number of fairly diverse forms belong to this order; most of them are natives of hot countries.

1. Sloths (Bradypodidx: genus Bradypus, etc.). The body is covered with long coarse hair; the head is round; the pinne very small; ${ }_{4}^{5}$ cylindrical teeth; fore longer than hind limbs; three fingers (the second, third, and fourth), or only two (the second and third); on the hind limbs there are always three toes (second, third, and fourth); both fingers and toes are enclosed in a common skin up to the terminal phalanx, which can be opposed to the palm or sole; the claws are falciform and extremely long and powerful; tail rudimentary; exclusively arboreal, feeding upon leaves; South and Central America.

2. The extinct Megatheria or Giant Sloths (Megatheriidx: genus Megatherium, Mylodon, etc.) occupy an approximately intermediate position between the foregoing and the succeeding divisions, for they resemble the Sloths in respect of the head and teeth, whilst the vertebral column, the limbs (of which the posterior are about the same length as the anterior) and the long powerful tail are more like the corresponding. parts in Myrmecophaga. They were herbivorous animals, generally of considerable size (the largest bigger than the Rhinoceros), of extremely bulky structure, and with very massive bones;

* In the same region of other Odontoceti, there is a thinner or thicker layer of blubber, which in, e.g., the Pilot Whale, is well developed, and gives the head its arched form (Fig. 420).

† There may be a few.rudimentary teeth in the upper jaw, posterior to the tusk, 
some had small bony knobs in the skin. The remains have been found in the Quarternary strata in various parts of America.

3. Ant-eaters (Myrmecophaga) are covered with fine or coarse hair, the head is more or less elongate, sometimes very long; teeth are absent, the mouth is very small, the tongue vermiform, the submaxillary glands of unusual length; the third fingers are very large, with long falciform claws, the other fingers are smaller or even atrophied; the animal rests upon the outer edge of the hand in walking; the hind feet have four or five almost equal toes with powerful claws; the tail is long. This group is insectivorous, feeding, e.g., upon Termites which adhere to the long tongue by means of the sticky saliva: South America. The Great Ant-eater (M. jubata), with coarse hair and bushy tail, lives upon the ground, whilst the other species are, for the most part, or exclusively, arboreal. e.g., the Little or Two-toed Ant-eater (M. didactyla), which has a short snout; fine. soft fur; a prehensile tail; and only two claw-bearing digits on each of the fore limbs.

$A$

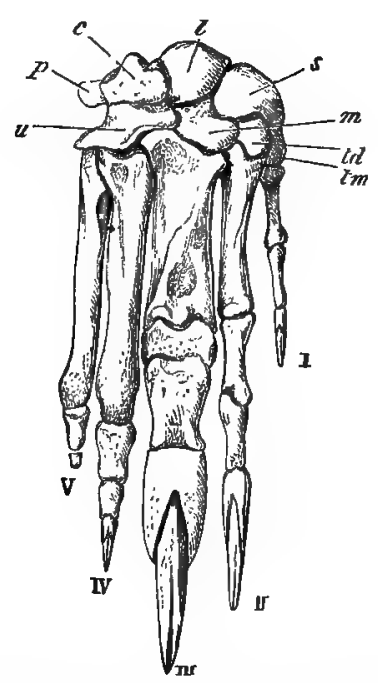

$B$

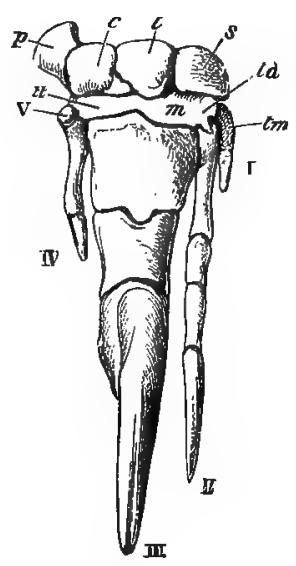

Fig. 421. A manus of the Great Anteater, B of the Two-toed Anteater, $s$ scaphoid, $l$ lunar, $c$ cuneiform, $p$ pisiform, $t m$ trapezium, $t d$ trapezoid, $m$ os magnum, $u$ unciform. $I--V$ digits.-After Flower.

4. Armadillos (Dasypodidx) are characterised throughout by a dorsal covering of large flattened scales, like those of Reptiles; these scales or plates are separated from one another by grooves, the outer surface being very horny, whilst within each scale there is a large ossification. They lie in several transverse rows, separated by soft skin in the median region of the back, but in front and behind they are close together; here the ossifications are firmly fused, and this is also the case with those of each transverse row, so that the back is covered by large bony shields in front and behind, and in the middle with a varying number (three to twelve) of half hoops of bone. There are similar scales on the dorsal side of the head, on the limbs, and on the tail, but these are wanting from the hairy ventral surface. The teeth are cylindrical, often fairly numerous; the head is long with well-developed pinnæ; the legs short with strong claws, The animal is plantigrade ; it is fossorial, essentially insec- 
tivorous. wather small or of medium size; some forws can roll themselves up: South America and the southern parts of North America. Allied to them are the extinct Glyptodons, in which all the dorsal plates were immovably united to form a large, thick, arched coat of mail; large portions of the vertebral column were also fused; they were extremely clumsy animals and of considerable size. Quaternary of South America.

5. The Cape Ant-eaters or Aardvarks (Orycteropus) are animals of fair size; sparsely haired; with long snout and tongue; small mouth; large pinnæ; powerful tail; strong, but not very long claws; possessing teeth: Africa.

6. Pangolins or Scaly Anteaters (Manis) are specially characterised by having the dorsal region of the body covered with large, very horny, imbricating scales, between which a few hairs appear. The head is long; pinnæ are absent; the mouth is small and edentulous; the tongue long; the tail is powerful; claws long and falciform. Insectivorous and representing the Ant-eaters (which they resemble in many respects) in the tropical regions of the old World.

\section{Order 1:. Rodentia (Rodents).}

The Rodents are primarily characterised by the peculiar dentition. Cauines are absent; there is only a single incisor in each side of the lower jaw, situated anteriorly and close to its fellow; there is usually also only one in the premaxilla, placed as in the lower jaw; the incisors are long and grow from persistent pulps, they are almost quadrangular prisms and are curved; the enamel only covers the front and the lateral edges, its surface is sometimes reddish brown; the free end of the tooth is cut away obliquely like a chisel. The upper incisors have a greater curvature than have the lower ones, in both cases the portion within the jaw extends far back, in the lower usually even to the most posterior extremity below all the molars. In the Leporida there is a smaller incisor behind the large one in the upper jaw ; it may also be noticed that here the lower incisor only extends back as far as the front end of the molar series (Fig. $422 \mathrm{~A}$ ). There is always a large diastema between the incisors and molars: the molars exhibit a great

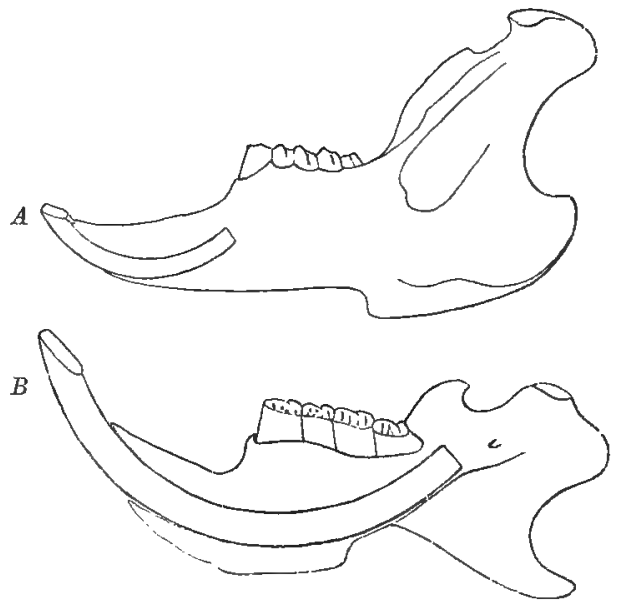

Fig. 422. Right ramus of the mandible, $A$ of a Rabbit, $B$ of an Agouti, from the inner side. Sockets of the incisors removed for their whole length to show the different lengths of the tooth. $B$ gives the usual rodent condition.-Orig. 
diversity of form; the crown is short and tuberculate; or furnished with low transverse ridges (Mouse, Rat) ; or each has two fangs, but the crown is longer and is folded both from above downward and also laterally; or again, the roots are quite short as compared with the long plicate crowns; lastly, they often grow from persistent pulps, and are provided on each side with deep, perpendicular folds

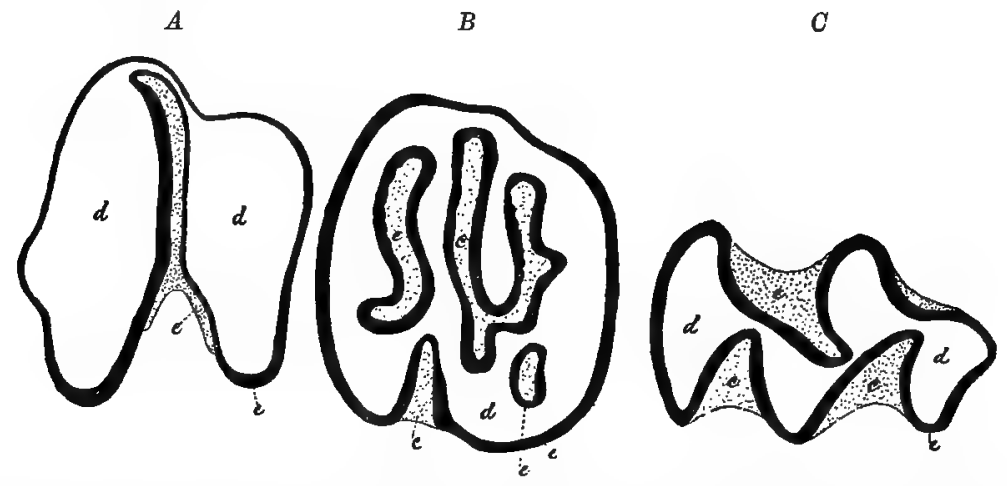

Fig. 428. Transverse sections of molars of various Rodents (at about a similar stage of attrition). 4 Hare, $B$ Beaver, $C$ Field Mouse. $c$ cement, $d$ dentine, e enamel.-After Owen.

which extend for some distance into the tooth, and are partially or entirely filled with cement. On the grinding surface, therefore, there are transverse or oblique stripes of enamel with cement and dentine between. Occasionally the molars with persistent pulps ( $c f$. , the molars of Elephants) are even divided into a series of perpendicular transverse plates with cement between them. This variety in the form of the teeth is correlated with a diversity of habit. The molars with short crowns are relatively little used, the others more or very much. The number of teeth is greatest in the Hares, $p \frac{3}{2}, m \frac{3}{3}$; in others it is more or

Dental Formulæ. Hare . . . $p \frac{3}{2}, m \frac{3}{3}$ Pika . . . , , $\frac{2}{2}, \frac{3}{3}$ Squirrel . . , , , , $\frac{3}{3}$ Beaver . . , , , $\frac{1}{1}$ Sminthus . , $, \frac{1}{0}, \frac{3}{3}$ Mouse . . , , $\frac{0}{0} \frac{3}{3}$ Australian

Rat . . ,,$\frac{0}{0}$ less reduced from the anterior end of the series; even, as may be seen from the accompanying list, to the exclusion of all the premolars;* only from quite a few forms (e.g., the Australian rat, Hydromys, belonging to the Muridæ), one of the molars, namely, the last $m^{3}$, is also absent.

Whilst the articular facets for the lower jaw in most Mammalia are in the form of transverse surfaces or pits, in most Rodents there

\footnotetext{
* As the corresponding millk teeth are also generally absent, and as (with the exception of the Hares) the incisors have no predecessors, there is abbolutely no replacement in forms deștitute of premolars;
} 
is a longitudinal groove, so that the mandible possesses considerable mobility from before backwards' (in masticating, the lower jaw moves backwards and forwards; the enamel stripes upon the molar run in the opposite direction, i.e., transversely). The feet are usually small and bear claws, and the animals are generally plantigrade. The first digits of the manus are rudimentary or absent, whilst the other fingers and toes are usually all present. In several forms there are internal cheek-pouches, outgrowths of the cheeks, connected with the buccal cavity; in some, in approximately the same position, there are ingrowths of the skin covered with hairs (external cheekpouches).*

The Rodents are widely distributed and rich in species, including for the most part small forms which are almost exclusively herbivorous.

1. The Rabbit family (Leporidx). $i \frac{2}{1}, m \frac{5 \text { or } 6}{5}$, the large upper incisors are grooved, the molars are plicate and grow from persistent pulps. The genus Lepus, with $m \frac{6}{5}$, long pinnæ; very short tail; long hind limbs ; comprises the Hare (L. Europæus), distributed throughout the greater part of Europe: and the Polar or Variable Hare (L timidus or variabilis), in the Northern parts of Europe and Asia, in Ireland, the Alps and the Pyrenees; it is white during winter in the colder regions : also the shorter-legged burrowing $\mathbf{R}$ abbit (L.cuniculus), indigenous to South Europe; the Pika (Lagomys), with $m \frac{5}{5}$; short pinnæ; shorter limbs than the Hares; ecaudate; in Siberia and North America.

2. The Squirrel family (Sciuridx). $m \frac{5}{4}$, tuberculate or plicate; the anterior molars of the upper jaw very small; pollex rudimentary; tail hairy: the Squirrel (Sciurus vulgaris), with long, bushy tail; arboreal: the Flying Squirrel (Pteromys), characterised by the possession of a patagium between the fore and hind limbs (one European species, Pt. volans, in North Russia). The Marmots (Arctomys) are fossorial, hibernating animals; they are thick-set, with short pinnæ and a short tail: there are two European species, the Alpine Marmot (A. marmota), and an allied form, the Pouched Marmot (Spermophilus citillus) of East Europe. Allied to the Squirrels is the Beaver (Castor fiber), a fair-sized animal with $\frac{4}{4}$ plicate molars; short ears; large, flattened, scaly tail; and webbed toes on the hind feet: they are excellent swimmers and diggers, and feed upon bark. Beavers were formerly abundant in the British Isles, but are now quite extinct; they occur on the Continent, and are, for instance, still fairly abundant in the Elbe: an allied species (C. canadensis) occurs in North America.

3. Dormice (Myoxidx). $M \frac{4}{4}$, with transverse bands of enamel; pollex rudimentary ; tail long and hairy: superficially, they somewhat resemble Squirrels or Mice. The small, mouse-like Common Dormouse (M. avellanarius)

* In the maxillm of Mammals generally, there is a shorter or longer canal, the canalis infraorbitalis, through which a large nerve (the maxillary branch of the trigeminus) runs. The canal opens in front of the orbit by a perforation termed the infraorbital foramen. In the Rodents the infraorbital canal is quite short, and usually very wide, and a portion of the masseter muscle passes through it.

+ The soles of the feet appear to have a complete covering of hair, but as a matter of fact there are small, naked, plantar cushions, which are, however, covered by the hair of adjacent regions.

$\ddagger$ In many books this is incorrectly termed $L$. timidus. 
occurs in Britain, and is widely distributed over the Continent. Several other species are met with in southern, or southern and eastern Europe: the S q u i r r e ltailed Dormouse (M. glis), the largest species: the Garden Dormouse (M. nitela): the Tree Dormouse (M. dryas). Allied is the Sminthus betulinus or vagus, in North and East Europe, very like a Mouse externally, with $m \frac{4}{3}$. The Sminthus is closely allied to the Jerboa (Dipus), which is chiefly characterised by the great length of the hind foot; this especially affects the second to the fourth metatarsals, which are fused (the first to the fifth toes are small or absent); the animals hop along, stepping only upon the second, third, and fourth toes of the hind foot; the tail is long, with a tuft of hairs at the end : desert animals; South Russia, Asia, Africa. Also allied to the Sminthus is the blind, earless, and tailless Mole-rat (Spalan typhlus), whose habits are somewhat similar to those of the Mole; Sonth-East Europe (e.g., South Russia) and Western Asia.

4. Mouse Family (Muridæ). $M \frac{3}{3}$ (occasionally $\frac{2}{2}$, of., p. 528); very varied in structure; tail longer or shorter, scaly ; pollex rudimentary. Usually of small size.

(a) Rats and Mice (Mus). Molars tuberculate, with short crowns and with roots; tail long, slightly hairy; pinnæ fairly well developed. In England occur: the Wood Mouse, or Long-tailed Field Mouse (M. sylvaticus), and the Harvest $\mathrm{M}$ ouse ( $M$. minutus): the following have invaded and live in human dwellings: the House Mouse ( $M$. musculus), the Black Rat ( $M$. rattus), now rare, having been almost completely exterminated by the more recent immigrant, the $\mathrm{Brown} \mathbf{R}$ at (M. decumanus). Allied to the Mice is the brightly-coloured $\mathrm{Hamster}$ (Cricetus frumentarius), with cheek pouches and short tail ; somewhat larger than a Rat; Central Europe.

(b) Field Mice (Arvicola). Molars long, growing from persistent pulps. with deep grooves on each side (grinding surface with loops of enamel); occasionally there are short roots; tail shorter and more hairy than in the Mice; pinnx short. They burrow in the ground, and are more exclusively herbivorous (feeding upon roots, bark, etc.) than the true Mice. The following species occur in England; the Bank-vole (A. glareola), which affords a transition to true Mice; the molars with short roots; pinnæ and tail somewhat longer than in. the rest: the Field Mouse (A. agrestis and arvalis): the Water Rat (A. amphibius); the latter is the size of a Brown Rat, the others about as large as the House Mice. Closely allied is the Le m ming (Myodes lemmus), with very short tail, and strong claws on the fore limbs; Scandinavia ; famed on account of its migrations. Another form allied to the Field Mice is the $\mathbf{M u s q u a s h}$ or Musk Rat (Fiber zibethicus), with long, compressed tail; the toes with stiff hairs at the edges; furry animals of fuilly large size, inhabiting northern North America, and by their mode of life recalling the Beaver.

5. Hystricomorpha, a group consisting of numerous forms, differing very much externally, but agreeing chiefly in the characteristics of the skull.* Molars $\frac{1}{4}$, banded, with roots, or growing from persistent pulps.

(a) The Coypu (Myopotamus coypu), an aquatic animal of beaver-like appearance, but smaller, and with a rounded tail; the toes of the hind foot are webbed; South America.

(b) Porcupines (Hystricidx), characterised by the modification of some of the hairs into stiff spines, often of enormous thickness and considerable length; animals of considerable size. The Common Porcupine (Hystrix cristata), in South Europe; lives in holes in the ground; tail short. In America, there are various arboreal forms, (Cercolabes) with prehensile tails.

* E.g., the infraorbital foramen is huge, and the mandiblr is peculiar in form. 
(c) Subungulata. Claws short, hoof-like, legs for the most part long, usually digitigrade; the number of toes on the hind foot varies; forefoot, with four well-developed digits, and with or without a rudimentary pollex; tail small or absent: all in South or Central America. The Pacas (Coelogenys paca) with five toes: the Agoutis (Dasyprocta): the Guinea-pig (Cavia cobaya): the Capybara (Hydrochoerus capybara); all with three toes; the last-named is the largest of all living Rodents; in South American rivers.

\section{Order 13. Prosimiæ (Lemurs).}

As in the Apes, with which the Lemurs were formerly grouped, the first digit on both fore and hind limbs is separated from the others, and is opposable. On the hind foot, usually only the second toe has a claw, the other fingers and toes are provided with flat $\mathrm{n}$ ails. The fore limbs are shorter than the hind. Of teeth, there are at most $i \frac{2}{2}, c \frac{1}{1}, p \frac{3}{3}, m \frac{3}{3}$; often, however, the number is smaller. The upper incisors are generally small, and there is usually a median diastema; the lower incisors and canines are all alike, narrow and directed obliquely forwards; the upper canines are caniniform; all the premolars (or the anterior ones alon $\theta$ ) are compressed and triangular (the first of the lower jaw is caniniform); the other molars tubercular, or each with two transverse ridges.

Most of the Lemurs are very hairy, many have long tails. The skeleton differs in many respects from that of Apes; for instance, the orbit is incomplete behind, and as in ather Mammalia, remains in widely open communication with the temporal fossa (there is, however, as in various other Mammals, a closed ring of bone round the orbit); the rami of the mandible are usually sepa-

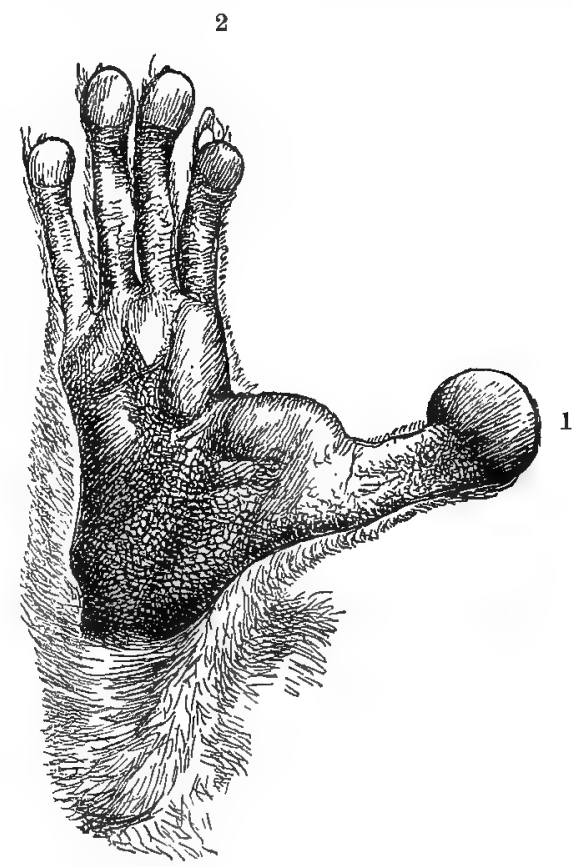

Fig. 424. Left hind foot of a M a ki, ventral view. 1 first digit, 2 second ditto (with elaw).Orig.

rate; the facial region of

the skull is larger in proportion to the cranial part than in most Monkeys. The uterus has two long horns (uterus bicornis). There is a pair of thoracic, sometimes also a pair of abdominal, 
mammæ. The Lemurs are arboreal animals feeding on fruits, Insects, and small Vertebrates; and are usually nocturnal. They occur only in the Old World, a considerable number in Madagascar.

1. The Makis (Lemurs). Muzzle pointed and vulpine; tail long; $i \frac{2}{2}, c \frac{1}{1}$, $m \frac{6}{6}$; Madagasear. Allied to these are the Loris (Stenops), with short muzzle; large eyes; tail small or absent; India.

2. The Tarsiex (Tarsius spectrum) characterised by the great elongation* of some of the tarsals (calcaneum and naricular) so that the foot appears to have a handle; broad soft pads below the tips of the toes; toes two and three with claws; tail long and tufted; eyes huge. Nocturnal springing animals; in the Malay Archipelago.

3. The Aye-Aye (Chiromys madagascariensis) is peculiar in several respects. Anteriorly, both in upper and lower jaws, there is a large tooth growing from a persistent pulp, which recalls the incisors of the Rodents; that in the upper jaw is an incisor, that in the lower apparently corresponds with the outermost forwardly directed tooth of other Lemurs, i.e., seems to be a canine (therefore the following may be given as the dental formula: $i \frac{1}{0}, c \frac{n}{1}, m \frac{4}{3}$ ). Hallux with nail, all the other digits with claws; the third finger exceptionally thin (used for pulling Insects out of holes or crevices); Madagascar.

\section{Order 14. Primates.}

In the members of this order, Monkeys and Man, both pollex and $h$ allux are separated from the rest of the digits, and are more freely movable than the latter, being usually more or less perfectly opposable, so that the limbs may serve as organs of prehension; the hallux in particular is usually free and movable (except in Man). As a rule all the digits are furnished with rather feebly arched nails. The facial region is generally short and small in comparison with that of other Mammals, and with the cranium. There is usually not much hair on the face. The eyes look forwards, and are placed close together. Unlike all other Mammals, the or bit is separated from the temporal fossa by a bony transverse septum (consisting of portions of the jugal, the frontal, and the alisphenoid). The teeth of the upper and lower jaw are similar, both as regards number and structure; in each half of the jaw there are two chisel-shaped incisors, one canine of the usual form, two or three premolars, and, as a rule, three (occasionally two) molars; all the molars are tuberculate, and have short crowns. There are always two $\mathrm{ma} m \mathrm{~m} \approx$ only, and these are thoracic. Of other characters it may be noticed that the anterior cornua of the hyoid are shorter than the posterior, and that the hyoid is not directly connected with the skull. The uterus is simplex.

The Primates are, for the most part, essentially adapted to an arboreal life; many of them can, however, move along the ground

* Such an elongation of the ankle is almost unique among Mammalia; in some allied Lemurs there is, however, an approach to this condition. (Cf. the tarsus of the Anura.) 
with ease, using all four limbs, the whole of the foot or hand resting upon the ground. In Man alone the hind limbs exclusively are used, and are very strongly developed for terrestrial locomotion. They are almost all tropical animals, feeding principally upon fruit. Hallux capable of Hind limbs little or no stronger than the fore.

\section{Hallux only capable} of slight movement. Hind limbs extraordinarily well-developed.
Distance between the external nares fairly large. External auditory meatus unossified. A small foramen in the septum between the orbit and the temporal fossa. $p \frac{\mathrm{a}}{\mathrm{3}}$.

\section{Catarrhinæ}

Distance between the nares small. External auditory meatus partially ossified. No Anthropidx foramen in the septum between the orbit and the temporal fossa. $p \frac{2}{2}$.

\section{Sub-Order 1. Platyrrhinæ.}

The external nares are separated by a broad membranous bridge. Three premolars are present both above and below; usual dental formula : $i \frac{2}{2}, c \frac{1}{1}, p \frac{3}{3}, m \frac{3}{3}$. No portion of the external auditory meatus ossified. In the posterior border of the jugal there is a tiny perforation of the septum between the orbit and the temporal fossa, i.e., the septum is not quite complete. Cæcum relatively large. Cheek-pouches and ischial callosities absent. Fore limbs usually somewhat shorter than hind. Tail well developed, sometimes prehensile. Confined to South and Central America.

1. Sapajous or Capuchin Monkeys (Cebus) have a long tail covered with hair, and capable of being rolled up like a watch spring and coiled round branches of trees. In the Howling Monkeys (Mycetes) the tail is very powerful, its tip is naked on the ventral side and sensory, and it is developed as an organ of attachment (the animal can even hang by it alone), i.e., is a genuine prehensile tail; the hyoid is large and hollowed out to receive an outgrowth of the larynx. The Spider Monkeys (Ateles), with similar tails, are characterised by the rudimentary nature or absence of the pollex.

2. The Marmosets (Hapalid:x) have a flattened nail only on the hallux; on all the other digits on the contrary, the nails are so much arched as to be claw-like. This small group may also be distinguished from other Platyrrhines in that $m \frac{2}{2}$ only are present. The tail is hairy, and cannot be coiled up; the pollex has but little power of independent movement. In other respects the Hapalidæ come near to the other Platyrrhines.

\section{Sub-Order :. Catarrhinæ.}

The external nares are close together. Two premolars (dental formula always: $\left.i \frac{2}{2}, c \frac{1}{1}, p \frac{2}{2}, m \frac{3}{3}\right)$. Proximal portion of the external auditory meatus ossified over a considerable extent. 
No perforation in the septum between the orbit and the temporal fossa. Cæcum small. Cheek-pouches frequently, ischial callosities usually present; tail never developed into an organ of prehension, often absent. Occur exclusively in the Old World.

1. Cynomorphx. Below each of the thick, broad ischia, there is a naked coloured portion of the skin, an ischial callosity. Nails relatively much arched. Tail usually present. Hind somewhat longer than fore limbs. Ch e ek p o u ches usually present. External nncisor of the lower jaw narrower than (or the same breadth as) the inner; first molar of the lower jaw with four tubercles. The thorax compressed (as is usual in the Mammalia); the manubrium of the sternum broad, the rest very narrow. Pelvis long and narrow; the symphysis (the line of junction of the two halves) long, the ilia long and narrow. The sacrum consists of three vertebræ.

(a) Cercopithecidx. Tail long; muzzle short; cheek pouches present; several African species. Closely allied is the $\mathrm{M} \mathrm{a} \mathrm{got} \mathrm{or} \mathrm{Barbary} \mathrm{Maca} \mathrm{qu} \mathrm{e}$ (Inuus ecaudatus); with rudimentary knob-like tail; occurring in North Africa and Gibraltar (the only European Ape). The Baboons (Cynocephalus), distinguished from the Cercopithecidæ by the very long, canine muzzle; tail long or short; cheek pouches present. They nsually remain on the ground, are only occasionally seen in trees. Africa and Arabia.

(b) Semnopitheciræx, characterised by the absence of cheek pouches; and the division of the stomach into several sections (whilst in other Apes it is simple). Amongst forms belonging here is $S$. nasicus, of Borneo, with very long nose. Colobus, in which the pollex is wanting, is nearly allied; Africa.

2. Anthropoid Apes (Anthropomorpha). Ischial callosities absent or small. Nails arched in the Gibbons, more flattened in other forms. Gibbons well clothed with hair; in the others, certain regions sparsely covered. Tail absent (rudimentary caudal region of the vertebral column consisting of four or five small vertebræ); fore limbs longer than hind; no cheek pouches; outer lower incisor broader than the inner, first molar of the lower jaw with five tubercles; thorax broader than in the Cynomorphe; pelvis in the Gibbons like that of the Cynomorphæ; in others, the ilia are broader, the symphysis is short; the sacrum consists of five vertebra.* The Anthropoid Apes are more exclusively arboreal than are the other Catarrhinæ; they do not walk like ordinary Mammalia (as the Platyrrhinæ do), but upon their hind legs, supporting themselves by the knuckles of the fore limbs, or they move in other aberrant ways. The largest Monkeys belong here.

(a) Gibbons (Hylobates) come nearest to the Cynomorphr; they possess small ischial callosities ; the nails are strongly arched; and the pelvis is long and narrow, like that of the Cynomorphæ; they are covered with thick hair, and have extraordinarily long arms, which they swing as they walk on their hind limbs. Smaller than those following: several species in Asia.

(b) Orang-Utang (Pithecus satyrus). Head almost conically arched above, face very projecting, nose flattened; fore limbs very long, reaching to the ankles when the animal stands upright; hand and foot long and narrow; hallux fairly small; reddish brown; height up to $1.5 \mathrm{~m}$. (measured in the upright position) : Sumatra and Borneo.

* In the Orang, the Chimpanzee, and the Gorilla (and in one of the Gibbons Hylobates syndactylus), just below the skin, there are one or two large air sacs; they proceed from the larynx, and extend on to the neck and thorax; they may be inflated with air and enormously expanded. 
(c) The Chimpanzee* (Simia troglodytes or Troglodytes niger) and the Gorilla* (S. or T. gorilla) correspond in most points. The forehead slopes back, the nose is broad and flat, but projects further than in the Orang; the fore limbs also are shorter, the hands and feet broader, the hallux large and well-developed; both are black. The Gorilla attains a height of $1.7 \mathrm{~m}$.; the Chimpanzee is somewhat smaller. Both occur in the tropical parts of West Africa.

\section{Sub-Order 3. Anthropidæ. (Man.)}

In contrast to other Primates, the Anthropidæ are characterised by the specialisation of the hind limbs as organs of locomotion adapted for supporting the body in an upright position without assistance from the fore limbs. In correlation with this they are very powerful, much longer than the fore limbs, and extremely muscular. The hallux is but little more separated from the other toes than these from one another, $\dagger$ possesses only a slight power of independent movement and is not opposable; it is somewhat longer than, or about.equal in length to, the second digit, or a very little shorter (in other Primates much shorter); the other' four toes are short, the metatarsus long. The pelvis is very short and broad, the ilia in particular are very short, broad, and strongly curved; the symphysis is short. The fore limbs, which are very like those of the Anthropoid Apes, are relatively weaker than in the latter; they are extremely well developed as prehensile organs, but are of no importance for the ordinary mode of locomotion. Another characteristic point is the enormous development of the $\mathrm{brain}, \ddagger$ and the consequent abnormal preponderance of the cranial capsule over the slightly developed facial region: in other respects the structure of the brain, even as regards points of detail, is very like that of the Anthropoid Apes. Further peculiarities are the slight covering of hair over the greater portion of the body; the small size of the canine tooth; and a feature which is closely connected with this, the absence of a diastema between the external incisor and the canine of the upper jaw larger than between the other teeth (in other Primates the canine of the lower jaw bites into this space) ; lastly it must be mentioned that the thoracic cavity is still broader and flatter than in the Anthropoid Apes.

* Possibly several closely allied species are included under each of these titles.

t The gap, however, is clearly deeper, and the distance between the hallux and the second toe is greater than between the other toes; still greater and more distinct in the embryo and young child than in the adult.

$\ddagger$ Man, however, does not possess the largest brain as compared with the whole weight of the body; even among the Primates, a relatively larger brain occurs in some small forms (in one of the Hapalidm, the weight of the brain, when compared with that of the whole body, is as 1:20, in Man it averages 1: 40). On the other hand, the brain of Man is much larger than in any other animal of similar size (the brain of the Gorilla, relatively to the weight of the body, is $1: 200$ ). 
For the rest, Man agrees in all the chief points of structure with the Catarrhinæ, particularly with the Anthropomorphæ. This coincidence obtains in all those characters by which the Catarrhines are separated from the Platyrrhines: in the position of the nares, the number of premolars (the dental formula of the Anthropidæ is identical with that of the Catarrhinæ); the ossified external auditory meatus; the absence of a foramen in the septum between the orbital and the temporal fossæ; the small cæcum; etc. In particular the Anthropidæ agree with the Anthropomorphæ, especially the larger forms (Orang, Chimpanzee, Gorilla), in the following points: the absence of ischial callosities, cheek pouches, and tail; there is a rudimentary caudal region (consisting of four or five fused vertebræ); the nails are flattened; the external incisor of the lower jaw is broader than the inner; the first lower molar has five cusps; the thoracic cavity is broad; the sternum broad and flattened; the broad pelvis of the large Anthropoids a p proaches that of Man; the sacrum consists in both of five vertebræ. There are also numerous other points of agreement. On the whole this group stands extraordinarily close to the Anthropoid Apes, the differences are almost all such as may be attributed to the adaptation to an upright gait; the great development of the brain; and the relative weakness of some portion of the musculature, e.g., the jaw muscles. The intimate correspondence may be to some extent masked by the development of subordinate parts; the skull of the Gorilla, for instance, which is more human than that of any other Anthropoid, is at first sight very unlike that of Man, e.g., in the presence of projecting bony ridges, which are wanting here; but the appearance of these ridges is directly correlated with the great development of the jaw and neck musculature, ${ }^{*}$ whilst careful and detailed consideration demonstrates the closest correspondence in most points.

All Men are usually regarded as one species, Homo sapiens, divided into a number of races. These differ, however, in some respects, quite as much as do other species of many other groups of animals. They are considered to belong to on e species chiefly from their perfect fertility inter se ( $c f . \mathrm{p}$. 36), and this often renders their division into races extremely difficult, for hybridisation has occurred to a great extent. The more detailed study of the various races constitutes, however, a special science, Ethnology, whose province must not be trespassed upon here. It must, however, just be mentioned that certain races come nearer to the Anthropoid Apes than others, although in no case is the approximation very close. The Negro Race, for instance, is distinguished by the broad flat nose; projecting (strongly-developed) facial region; large teeth, obliquely set incisors; receding chin; long, narrow thoracic cavity; deep narrow pelvis; long digits : characters which as a whole lead back to the Apes.

* Among other nearly allied Mammalia such ridges may be present in one form, ahsent from another (Badger, Marten). 


\section{A PPENDIX TO THE VERTEBRATA.}

\section{Tunicata (Sea-Squirts).}

The Tunicata are a small group of marine animals, which were formerly usually regarded as Mollusca, or placed with some other division of the Invertebrata; only recently has it been demonstrated that they are most nearly allied to the Vertebrata, a relationship which is made specially clear by a consideration of their ontogeny. In particular it has been shown that in early life at least they agree with the Vertebrata in the possession of a notochord, and in the position of the central nervous system, both fundamental points. In spite of this, they are not, however, incorporated with the Vertebrates, but treated of in an appendix, because the majority undergo so peculiar a metamorphosis that the Vertebrate characters
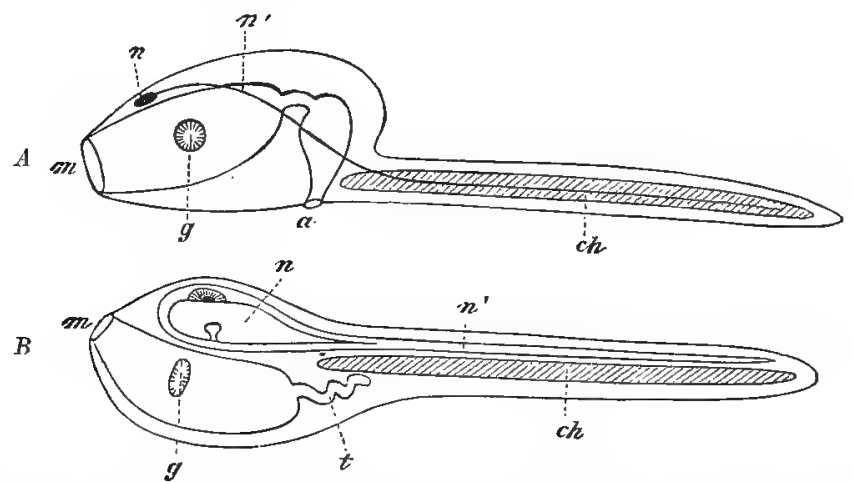

Fig. 425. A diagram of one of the Appendicularia, viewed from the side, stretched out straight. $B$ ditto of an $A$ scidian larva. $a$ anus, $c h$ chorda, $g$ branchial chamber, $m$ mouth, $n$ brain, $n^{\prime}$ nerve cord, $t$ gut.-Orig.

have completely disappeared in the adult, which has received an entirely different impress: thus it is more convenient to consider them separately.

It may also be noted here that the Tunicata, like the true Vertebrata, do not exhibit definite affinities with any of the Invertebrata.

Insight into the characteristics of this group is best attained by a separate consideration of its various sub-divisions belonging to it. The following general characteristics may, however, be noticed: the skeleton is at best only represented by the notochord, the nervous system is feebly developed, so also are the sense organs. The Tunicata are hermaphrodite, ovaries and testes are continued directly into their ducts. Reproduction by budding frequently occurs.

The Appendicularia possess the simplest and most easily comprehended organisation. They are tiny, transparent, free-swimming, marine forms, with some resemblance to tadpoles. The body is 
divisible into a roundish trunk and a flattened tail, which is folded on to the ventral side. The wall of the capacious pharynx is perforated on each side by a ciliated aperture, the gill-slit, which opens on to the surface; the rest of the alimentary canal is short, the anus is ventral. The notochord only occurs in the tail, where, however, it is well developed. The central nervous system is represented by a cord swollen into a bulb (the brain) dorsal to the pharynx, and provided with smaller swellings on the rest of its course; it is continued down the tail on the left side of the notochord, so that the tail is really laterally compressed and has undergone a rotation of $90^{\circ}$. The simple heart lies below the alimentary canal. An otocyst is present,

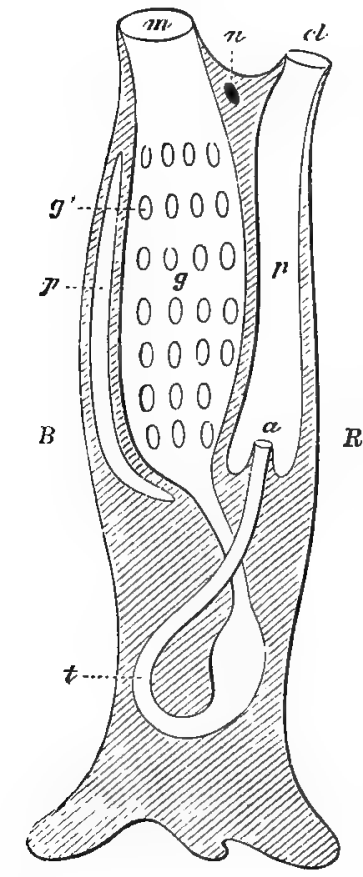

Fig. 426. Diagrammatic longitudinal section of an Ascidian; the section is not quite median, but somewhat lateral. $a$ anus, $c l$ cloacal aperture, $g$ branchial sac, $g^{\prime}$ apertures in its wall, $m$ mouth, $n$ nerve ganglion, $p$ peribranchial cavity, $t$ gut, $B$ ventral, $R$ dorsal surface--Orig. but eyes are wanting.

The simple Ascidians (i.e., those which do not form colonies, genus Ascidia, etc.) are apparently of quite a different structure. They are barrel-shaped, round, or of some other form, and, for the most part, gelatinous; they are fixed by one end or by one side. At the free end two openings are present; one of these, the mouth, leads into a very roomy pharynx or $\mathrm{b} r \mathrm{r} \mathbf{n} \mathrm{chial}$ $\mathrm{sac}$, the walls of which are perforated by numerous ciliated clefts; these do not lead directly on to the surface, but into a large peribranchial cavity surrounding the branchial sac, and communicating with the exterior by the second opening, the cloacal aperture. The branchial sac is fused along one side with the outer wall of the peribranchial cavity, and along this line there is a longitudinal furrow, the ventral furrow or endostyle, with large mucus-secreting and ciliated cells; along the opposite side runs a dorsal lamina, often considerably folded, and connected anteriorly with the endostyle by a ciliated band on each side (the peripharyngeal bands). The pharynx leads below into a rather short intestine, which bends upon itself and opens into the peribranchial cavity. The heart lies below the alimentary canal; the current of blood is remarkable in that it flows alternately in opposite directions through it; there is a fairly well-developed system of vessels, the branchial sac being especially well provided; the blood corpuscles are all amœboid and 
colourless. The central nervous system is reduced to a nerve ganglion situated between the mouth and the cloacal aperture. Ductless vesicles varying in size perform the function of excret or y organs; they excrete hard particles, in which the presence of uric acid has been demonstrated. The reproductive organs (an ovary and a testis) open into the peribranchial cavity close to the anus. The body is entirely covered with a thick gelatinous or leathery coat, the "mantle," a product of the epidermis.*

The difference between the Appendicularia and the adult Ascidian is thus very great. But a comparison of the la rval forms reveals quite other relations; there is a close agreement in almost every respect. The Ascidian larva are still more like Tadpoles than are the Appendicularia; they have a rounded trunk, and a long compressed tail with a notochord, which extends a short distance into the trunk. Dorsal to the notochord lies the central nervous system, which extends along the whole tail, and has a swelling, the brain, anteriorly; in connection with the latter, there is an eye and an organ which is regarded as auditory; both are remarkable in that they lie within the cavity of the brain, and are specially developed portions of its wall. There is as yet no peribranchial cavity, but two simple gill slits lead from the pharynx to the surface. As may be seen, therefore, there is very great resemblance between these larvæ and the Appendicularia. Before long, however, the larva attaches itself, the tail dwindles; the notochord, the sense organs, etc., degenerate; and the animal gradually attains to the very aberrant form of the adult Ascidian.

It is easy to see how closely the type of structure of this larva corresponds with that of the Vertebrata (as regards the position of the nervous system, the notochord, and the alimentary canal); the relation is still more obvious than in the adult Appendicularia, where the rotation of the tail, etc., to some extent, masks the conformity. It must also be noticed here that the notochord arises in exactly the same way as in the true Vertebrata.

Some of the Tunicates (Compound Ascidians) form colonies, throwing off thread-like outgrowths, from which new individuals develop, in other respects independent of each other. In other colonial forms, Ascidice compositce, there is a common test: in this case the colonies are firmly attached to some foreign body, and form soft spongy masses, in which the individuals are embedded, often on a stellate plan. The members of each group have usually a common cloacal opening, but each has its own mouth. The pelagic, free-swimming, phosphorescent Pyrosoma, also belongs here. This colony has the form of a thick-walled tube, open at one end, closed at the other, walls being formed by the tiny animals which are arranged close together, their mouths opening at the surface of the

* The mantle is to be regarded as a much thickened cuticle. It is interesting to note that it contains scattered cells, which are amoboid, and have migrated from the mesoblast through the epidermis into the mantle. 
tube, their cloacal apertures into the cavity; the water which is taken in at the mouth thus passes into the tube, from the open end of which it is expelled; by means of this exhalent current the colony is driven through the water, with the closed end forwards.

The free-swimming Salpa offers a remarkable modification of the Ascidian type. The buccal and cloacal apertures are almost at opposite poles of the body. The branchial sac is, however, very degenerate; its lateral walls are absent, so that, with the exception of the ventral region with the endostyle, only the dorsal portion remains as a band stretching across the united branchial and peribranchial cavities ( $r$, Fig. 427). The visceral mass is of insignificant size as compared with that of the whole animal, the cavity just mentioned constitutes the greater part of the animal (in most Tunicata the branchial cavity is very extensive). In the transparent body-wall, beautiful circular muscle-bands are seen, by the contrac-

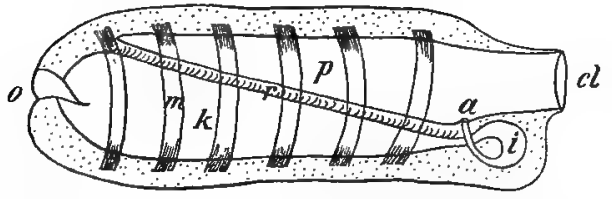

Fig. 427. Diagram of a S a lpa, $a$ anus, $c l$ oloacal opening, $k$ branchial cavity, $i$ gut, $m$ muscle bands, o mouth, $p$ peribranchial cavity, $r$ dorsal lamina.-Orig.

tions of which water is expelled; they are homologous with a continuous layer of muscle which is present in the body-wall of Ascidia. The Salpidæ are not only remarkable in structure, but also in affording an instance of a regular alternation of generations. There are both solitary asexual forms and $\mathrm{chains}$ consisting of a larger or smaller number of somewhat loosely connected sexual individuals. The asexual generation forms chains by budding; the chains remain within the body-wall of the solitary salp until they have attained a certain development, when they break free and swim about independently. The solitary and colonial individuals differ somewhat from each other. The colonial forms are remarkable in that they first give rise to eggs which are fertilised by spermatozoa from another chain, whilst later on they themselves produce spermatozoa. Each individual usually produces only a single egg, which undergoes development in the body of the parent.

Of the forms mentioned above, various species of simple Ascidians are widely distributed in European seas; they are attached to seaweed, stones, piles, etc. There are species of Compositæ and of Appendicularia, also in northern seas; Pyrosoma and Salpa are pelagic, and occur in the large oceans as well as in the Mediterranean. All the Tunicata feed upon microscopic organisms, which they take into the branchial cavity with the water. 


\section{N D E X .}

Aardvark, 527

Abdomen, 193

Abdominal limbs, 209

" pores, 378

" ribs, 410

Abomasum, 507

Abramis brama, 388

Abysmal fauna, 61, 75

Acalepha, 109

Acanthia lectularius, 259

Acanthias vulgaris, 383

Acanthocephala, 163

Acarina, 284

Accessory intestine, 137

Accipenser, 385

$$
\begin{array}{ll}
\text { " } & \text { huso, } 385 \\
\text { " } & \text { ruthenus, } 385 \\
\text { " } & \text { sturio, } 385
\end{array}
$$

Accipitres, 460

Acephala, 306

Acerina cernua, 389

Acetabulum, 328

Achromatin, 5

Aciculum, 167

Acola, 144.

Acontia, 112

Acraspeda, 109

Acridium, 252

Acromion, 477 migratorium, 252

Actiniaria, 118

Actinosphærium eichhornit, 91

Adaptation, 66

Adder, 425

Adductor muscles, 309

Adhesive organs, 14

Adipose tissue, 10

Adjutant, 459

Fluroidea, 515

妮olidæ, 304

Ális, 304

Affinities of animals, 53

African Elephant, 512

$$
\text { " Ostrich, } 450
$$

Agonus cataphractus, 390
Agouti, 531

Agrion, 255

Agrotis segetum, 275

Air sinuses, 475-6

, frontal sinus, 475

" maxillary sinus, $\mathbf{4 7 5}$

Alaudidæ, 464

Albatross, 457

Alca alle, 457

, impennis, 457

, tonda, 457

Alcedinidæ, 465

Alcedo, 465

Alcidx, 457

Alcippe, 206

Alcyonaria, 113

Alcyonium digitatum, 114

Alga, 216

Alimentary canal, 23

Alisphenoid, 362

Allantois, 354

Alligators, 426

Alpine Marmot, 529

Alternation of Generations, 37

Altinares, 459

Altrices, 450

Alytes obstetricans, 406

Amblystoma Mexicanum, 405

Ambulacral groove, 129

$$
\text { , plates, } 134
$$

American Black Bear, 516

Amia, 385, 386

Ammocotes, 382

Ammodytes, 389

Ammonites, 323

Amnion, 353

Amniota, 354

Amcba, 3-5, 89

Ampelis garrulus, 462

Amphicolous, 360

Amphilina, 151

Amphipnous, 375

Amphioxus lanceolatus, 356
Amphipoda, 210

Amphisbrna, 4:22

Amphiuma, 404-5

Ampulla, 124

Anabas, 375, 389

Anamnia, 354

Anal area, 136

, cerci, 236

" cirrus, 169

"glands, 237

Analogy, 57

Anarrhicas lupus, 390

Anas, 458

Anas acuta, 458

, boschas, 458

" clypeata, 458

" crecca, 458

" penelope, 458

, querquedula, 458

" sadorna, 458

Anchitherium, 503

Anchovy, 387

Anelasma squalicola, 206

Anguilla valgaris, 388

Anguillidm, 163

Anguillula aceti, 163

Anguis fragilis, 422

Ankle, 328

Annelida, 83, 165

Annuli, 175

Anobium, 267

Anodonta, 315

Anoplotherium, 506

Anser cinereus, 458

, ruficollis, 458

" segetum, 458

Anseres, 458

Ant-eaters, 526

Antedon, 130

Antenna, 184

Antennary gland, 192

Anthozoa, 111

Anthropidæ, 533, 535

Anthropoid Apes, 534

Anthropomorpha, 534.

Anthus, 462 
Antilocapra Americana, 509

Antilope, 509

Antilopinæ, 509

Antimeres, 40, 122

Antitragus, 501

Antlers, 471

Ant-lions, 261

Ants, 270

Anura, 406

Anus, 23

Aorta, 29

Aphaniptera, 278

Aphidæ, 257

Aphides, 257

Aphis, 257

Aphis-lion, 261

Aphodius, 265

Aphorrhais pes pelicani, B02

Aphrodite, 173

Aphrophora spumaria, 257

Aphysostomi, 388

Apiariæ, 271

Apical plates, 135

Apis mellifica, 271

Aplysia, 303

Appendices pyloricæ, 371

Appendicularia, 537

Apseudes, 216

Apteria, 434

Apteryx, 454

Aptychus, 323

Apus, 194

Aqueous humour, 333

Aquila chrysaëtus, 460

Aquilidæ, 460

Arachnida, 278

Arachnoid membrane, 331

Araneina, 283

Archæopteryx, 444

Archenteron, 43

Arches, 326

Architeuthus, 323

Arctoidea, 515

Aretomys, 529 marmota, 529

Ardea cinerea, 459

Arenicola, 173

Argali, 509

Argonauta argo, 323

Argyroneta aquatica, 283

Arion ater, 306

Aristotle's Lantern, 137

Armadillidium, 230

Armadillo, 526

Artemia, 193

$$
\text { , salina, } 194
$$

, milhausenia, 194

Arterial blood, 28

, heart, 29

Arteries, 26

Arthrogastra, 281

Arthropoda, 83, 184

Articular cartilages, 329

Artiodactyla, 504

Arvicola, 530

, agrestis, 530
Arvicola, amphibius, $\mathbf{5 3 0}$

, arvalis, 530

" glareola, 530

Arytenoid cartilages, 488

Ascalabotidæ, 422

Ascaris, 422

Ascidiæ compositæ, 539

Ascidians, 538

Ásellus, 215

, aquaticus, 215

Asexual reproduction, 29

Aspidiotus nerii, 259

Ass, 503

Astacus fluviatilis, 224

Asterias rubens, 133

Asterida, 131

Asteroidea, 130

Asthenosoma, 138

Astrophyton, 134

Asturidæ, 460

Astur nisus, 460

, palumbarius, 460

Atavism, 39

Ateles, 533

Athene noctua, 461

Atlas, 409

Atrium, 344

Auchenia, 508

Auditory hairs, 221

Auditory organs, 19

Auk, 457

$\Rightarrow$ Great, 457

Little, 457

Aülastomum gulo, 176

Aurelia aurita, 111

Auricle, 26

Aurochs, 510

Australian Bear, 498 , Opossums, 498

Autozooids, 114 Region, 74

Aves, 430

Avicularia, 181

Avoset, 460

Axial skeleton, 113

Axis, 409

Axolotl, 403, 404

Aye-aye, 532

Baboon, 534

Babyrusa, 506

Bacillus, 254

Badgers, 516

Balæna biscayensis, 324

Balæna mysticetus, 324

Balænidæ, 523

Balænoptera musculus, 523 rostrata, 523

Balænopteridæ, 523 Sibbaldii, 523

Balanidæ, 206

Balaninus nuoum, 244.

Balantidium, 94.

Balanus, 206

Baleen, 522

Bandicoots, 497

Bank-vole, 530

Barbary Macaque, 534
Barbel, 388

Barbus, 388

Barnacles, 206

Barramunda, 389

Basal plate, 115

Basi-branchials, 327

Basi-occipital, 362

Basi-sphenoid, 362

Basket, 271, 272

Basommatophora, 306

Bath Sponge, 121

Bats, 500

Bdellostoma, 383

Bean Goose, 458

Bear animalcules, 286

Beaver, 529

Bed-bug, 259

Bee-eater, 465

Bee-louse, 278

Bees, 271

Beetles, 263

Beetle-mite, 284

Belemnites, 323

Belinurus, 197

Bell-animalcule, 94

Belodon, 427

Belone valgaris, 388

Beroë, 118

Bezoar Goat, 509

Bilateral symmetry, 41, 126

Biology, 58

Bird-mite, 284

Birds, 430

" of Paradise, 450, 463

, of Passage, 451

" of Prey, 461

, Climbing, 465

" Gallinaceous, 455

" Shrieking, 464

"Struthious, 464

" Swimming, 456

"Toothed, 453

Wading, 458

Bird-spiders, 283

Bison, 510

, Europæus, 510

Americanus, 510

Bithynia, 302

Bitterling, 388

Bittern, 459

Black-arch, 275

Black-beetle, 263

Blackbird, 462

Black-clawed Crab, 227

Black Flies, 276

, Rat, 530

Whale, 524

Bladder-nosed Seal, 520

Blastophaga grossorum, 244

Blastula, 43

Blatta, 253

, orientalis, 253

Blenniidæ, 390

Blenny, 390

Blind-worm, 418, 422

Blood, 26

Blood corpuscles, 25 
Blood plasma, 25

Blubber, 518, 520

Blue-bottle, 277

Blue Whale, 525

Boa Constrictor, 424

Body cavity, 39

Bohemian Waxwing, 462

Bombinator bombinus, 406

Bombus, 271 igneus, 406

Bombycidø, 274

Bombyx monacha, 275

, mori, 275

, pini, 274

Bone, 10

Bonellia viridis, 174

Bony labyrinth of the ear, 338

Bony Pike, 385

Book-lice, 255

Bopyridæ, 216

Bopyrus, 216

Boring Sponges, 121

Bos grunniens, 510

, indicus, 510

" primigenius, 510

", taurus, 510

Botaurus stellaris, 459

Bot-flies, 277

Bothriocephalus, 151, 153

Bottle-nosed Whale, 525

Bovinæ, 509

Bowman's capsule, 348

Brachiopoda, 83, 178

Brachial plexus, 332

Brachyura, 226

Bradypodidæ, 525

Bradypus, 525

Brain, 324, 330

Branchial arteries, 345

$\begin{array}{ll}\text {, } & \text { chamber, } 26 \\ \text { " } & \text { hearts, } 320 \\ \text { ", } & \text { lamellæ, } 197 \\ \text { sac, } 538\end{array}$

Branchiobdella astaci, 177

Branchiostegite, 219

Branchipus, 193

Braula cæca, 278

Bream, 388

Breeding season, 69

Brevirostres, 459

Brill, 389

Brissopsis lyrifera, 138

Brittle-stars, 133

Bronchi, 418

Brown body, 180

Bruta, 525

$$
\text { Rat, } 530
$$

Bubalus, 510

Bubble, 303 vulgaris, 510

Bubo maximus, 461

Buccal cavity, 23

Buccinum undatum, 302

Bucerotidx, 465

Buffalo, 510

Buffon's Skua, 456

Bufo, 406
Bufo, calamita, $40 \%$ , vulgaris, 407

Bugs, 259

Bulbus arteriosus, 377

Bulla, 303

Bullfinch, 462

Bull-head, 390

Bumble Bee, 2 个 1

Buntings, 463

Buprestidæ, 266

Buprestis, 266

Bursa copulatrix, 243

, Fabricii, 445

Bursæ (respiratory), 134

Burying-beetles, 264

Bustards, 4.59 " Small, 459 Large, 459

Butcher Bird, 462

Buteo, 460

Butirimus, 376

Butterflies, 275

Byssus, 308

, $\quad$ gland, 311

Buzzards, 460

Cabbage Butterfly, 275

Cachalot, 524.

Caddis- $\sharp$ lies, 262

Cæca, 25

Cæcilia, 402, 407

Calcareous valves, 204.

Caligus, 201

Camelidæ, 508

Camels, 508

Camelus, 508 „ bactrianus, 508 , dromedarius, 508

Camelopardalis giraffa, 508

Canales incisivi, 475

Canaries, 463

Cancer pagurus, 22ł

Canidæ, 515

Canines, $48:$

Canis aureus, 516

, familiaris, 516

, lagopus, 515

"lupus, 515

"vulpes, 515

Cape Ant-eater, 527

Capella rupicapra, 509

Cape Mole, 499

Capercaillie, 455

Capillaries, 26

Capitulum, 472

Capra, 509

$\begin{array}{ll}" & \text { ægagrus, } 509 \\ \Rightarrow & \text { hircus, } 509 \\ \text {, ibex, } 509 & \end{array}$

Caprimulgus europæus, 464

Capuchin Monkey, 533

Capybara, 531

Carabidæ, 264

Carabus, 264

Carapace, 189

Carassius auratus, 388

vulgaris, 388
Carcharias glancus, 383

Carcinus moenas, 227

Cardo, 233, 309

Carina, 204.

Carnassial tooth, 514

Carnivora, 513

Carp, 375, 387

Carpals, 328

Carpus, 328

Carrion-beetles, 264

Cartilaga ypsiloides, 396

Cartilage, 10

Cartilaginous sheath, 326

Caryophyllæus, 151

Case-bearers, 274.

Cassowaries, 454

Castor canadensis, 529 , fiber, 529

Casuarius, 454

Cataphracti, 390

Catarrhinæ, 533

Caterpillar, 250,272

Cat-fishes, 384 fly, 277

Cathartes, 461

Cattle, 509

Caudal appendages, 195 , fin, 209 , spine, 197

Cave Bear, 516

, Lion, 517

Cavernicolous fauna, 61.

Cavia cobaya, 531

Cavicornia, 509

Cecidomyia, 246, 276

Cells, 5

" ciliate, 8

, epidermal, 99

, epithelio-muscle, 100

" fixed, 7

, flagellate, 8

, glandular, 9

, goblet, 9

, nerve, 120

" nettle, 100

"pigment, 31

, sensory, 13

, simple, 85

, wandering, 6,31

Cement, 482

'gland, 205

Cestipedes, 227

Centrale, 328, 329

Centrosome, 5

Centrum, 326

Cephalopoda, 315

Cephalothorax, 198

Cerambycidæ, 266

Cerambyx, 266

Ceratodus, 367, 386

Cercaria, 148

Cercolabes, 530

Cercopithecidæe, 534

Cerebellum, 331

Cerebral ganglia, 289

Cerebrum, 330 hemispheres, 330

Certhia familiaris, 464 
Cervical ribs, 437,471 vertebræ, 326

Cervidæ, 508

Cervus alces, 509 , canadensis, 509

" capreolus, 508

, dama, 508

" elephus, 508

" euryceros, 509

, tarandus, 509

Cestoda, 143, 149

Cestus veneris, 118

Cetacea, 520

Cetonia, 265

Chrta, 167

Chætopoda, 167

Chaffinch, 462

Chamæleo, 422

Chameleon, 422

Chamois, 509

Char, 387

Charadriidæe, 459

Charadrius pluvialis, 459

Cheek pouches, 485

Cheese-fly, 277

Chelæ, 219

Cheliceræ, 279

Chelifer, 282

Chelonia, 425

Cheloniæ, 426

Chequered Meat- $\mathrm{fly}, 277$

Chermes abietis, 258

Chestnuts, 503

Chigoe, 278

Chilognatha, 230

Chilopoda, 229

Chimera monstrosa, 384

Chimpanzee, 535

Chiromys madagascariensis, 532

Chiroptera, 500

Chitin, 185

Chitons, 290

Chlorophyll, 81, 95

Chœropsis liberiensis, 506

Choeropus, 498

Chondocranium, 327

Chondrostei, 385

Chorda dorsalis, 324

Chorion, 242

Choroid, 333

$$
\text { " gland, } 370
$$

Chromatin, 5

Chromatophores, 10

Chromosomes, 5

Chrysalis, 250

Chrysis, 270

Chrysochloris, 499

Chrysomelidæ, 266

Chrysopa, 261

Chyle, 348

Cicada, 256 " septendecim, 257

Cicadellidæ, 257

Cicindela, 264

Ciconia alba, 459 " nigra, 459
Cidaris, 138

Cilia, 5, 16, 92, 95

Ciliary processes, 333

Ciliated junction, 307

Ciliate organs, 146

Cimex lectularius, 259

Cinglus aquaticus, 462

Circus, 460

Cirrhus, 129, 169, 205

Cirripedia, 203

Civet Cat, 517

Cladobates, 500

Cladocera, 195

Clamatores, 464

Class, 55

Classification, 53

Clavicle, 327

Claws, 235, 408, 435, 469

Clear wings, 274

Clepsine, 177

Climbing Perch, 389

Clione limacina, 305

Clitellum, 174

Clitoris, 450

Cloaca, 159, 342

Cloacal aperture, 308 , syphon, 308

Clothes-moths, 273

Clupea alosa, 387

$$
\begin{array}{ll}
\text { " } & \text { harengus, } 387 \\
\text { ", } & \text { pilchardus, } 387 \\
\text { " } & \text { sprattus, } 387
\end{array}
$$

Clupeidæ, 387

Clypeaster, 138

Clypeastridæ, 138

Coarctate pupa, 250

Coatimondi, 516

Cobego, 500

Cobitis, 388

Cobra, 424

Coccidium oviforme, 96

Coccidæ, 258

Coccinellidæ, 267

Coccus cacti, 259

, lacca, 259

Cochineal Insect, 259

Cochlea, 444

Cockatoos, 465

Cockchafer, 265

Cockroaches, 253

Cocoons, 269

Cod, 388

Coelentera, 83, 98

Cologenys paca, 531

Coenenchyme, 113

Ccenurus cerebralis, 152

Coleoptera, 252, 263

Collar-bonè, 327

Collar-cells, 119

Collembola, 256

Collocalia, 464

Colobus, 534

Colony, 32, 66

Colour change, 434, 468

Colubridæ, 424

Columba, 465

$$
\begin{aligned}
& \text { " livia, } 465 \\
& \text { " migratoria, } 465
\end{aligned}
$$

Columba, œnas, 465 palumbus, 465

Columbidæ, 465

Columella, 116, 294, 412

$$
\text { , auris, } 397,416
$$

Columellar muscle, 295

\begin{tabular}{|c|c|}
\hline & \\
\hline , & Bittern, 459 \\
\hline "פ & Coot, 459 \\
\hline צ' & Crane, 459 \\
\hline$\Rightarrow$ & Creeper, 464 \\
\hline , & Dipper, 462 \\
\hline ", & Hedgehog, 499 \\
\hline פו & Mole, 499 \\
\hline , & Mouse, 529 \\
\hline " & Porcupine, 530 \\
\hline , & Round-worm, 160 \\
\hline, & Seal, 519 \\
\hline , & Shrew, 500 \\
\hline , & Shrimp, 223 \\
\hline & Slug, 306 \\
\hline
\end{tabular}

Colymbus, 457

$$
\begin{array}{ll}
" & \text { arcticus, } 457 \\
" & \text { glacialis, } 457 \\
& \text { septentrionalis, } \\
& 457
\end{array}
$$

Comatula, 130

Combustion, 4,30

Commensalism, 65

Common Bear, 516

Comparative Anatomy, 58

Complemental males, 205

Compositæe, 540

Compound Ascidians, 539

, eye, 22

$$
\text { " stomach, } 487
$$

Conchiolin, 287

Condor, 461

Conger-eel, 388

$$
\text { " valgaris, } 388
$$

Conirostres, 462

Conjunctiva bulbi, 336

$$
\text { " palpebrarum, }
$$

Conjugation, 86, 96

Connective tissue, 10

Conus arteriosus, 344

Coot, 459

Copepoda, 200

Copris, 265

Coprophaga, 265

Copulæ, 327

Copulation, 36

Copulatory organs, 34

Coracias. garrula, 464 .

Coracoid, 327

Corallium rubrum, 115

Coral reefs, 117

Coregonus, 387

Cormorant, 457

Corn Crake, 459

Cornea, 333

Coronula, 206

Corpora quadrigemina, 331

Corpus callosum, 480

"fibrosum, 493

Corvidæ, 463 
Corviformes, 463

Corvus corax, 463

, cornix, 463

" corone, 463

" frugilegus, 463

" monedula, 463

Cossus ligniperda, 274

Cottus gobio, 390

, scorpius, 390

Coturnix communis, 455

Cotyledons, 494

Cougar, 517

Covering pieces, 108

Cowper's glands, 493

Coxa, 235

Coxal glands, 380

Coypu, 530

Crabronidæ, 270

Crabs, 226

Crab-spiders, 286

Cracidæ, 456

Craneflies, 276

Cranes, 459

Crangon vulgaris, 223

Cranium, 326

Craspedota, 103

Crawfish, 227

Crax, 456

Creeper, 464

, Common, 464

Wall, 464

Cribriform plate, 475

Cricetas frumentarius, 530

Cricket, 253

Cricoid, 4.88

Crinoidea, 127

Crocodilia, 426

Crocodilus, 426

Crop, 24

Cross bills, 463

Crossopus aranea, 500 , fodiens, 500 , suavolens, 500

Cross-spiders, 283

Crotalidæ, 425

Crotalus, 425

Crow, 463

Crustacea, 190

Cryptobranchus japonicus, 405

Crystalline cone, 22

Ctenophora, 118

Cuboid, 479

Cuckoo Bees, 272

Cuckoos, 465

Cuckoo-spittle, 257

Cuculidx, 465

Cuculus canorus, 465

Cucumaria, 141

Culex, 276

Cumacea, 210, 213

Cuneiform, 478, 479

Curassows, 456

Curculio, 267

Curculionidæ, 267

Curlews, 460

Cuscus, 498

Cuvierian organs, 140
Cyamus, 219

Cyanea capillatat, 111

Cyclops, 201

Cyclopterus lumpus, 390

Cyclostoma elegans, 302

Cyclostomi, 382

Cydippe, 118

Cygnus, 458

, atratus, 458

" musicus, 458

, olor, 458

Cymothoa, 216

Cynailurus jubata, 517

Cynipidæ, 269

Cynips rosæ, 245

Cynocephalus, 534

Cynoidea, 515

Cynomorpha, 534

Cyprinidæ, 387

Cyprinus carpio, 387

Cypris (larva), 207

Cypris ovum, 199

Cypselidx, 464

Cypselus, 4.64

Cysticercus cellulosæ, 151

Cystophora cristata, 520

$$
\text { " proboscidea, }
$$

Dab, 389

Dabchicks, 457

Dactylopterus volitans, 390

Dactylozooid, 105, 107

Daddy-long-legs, 276

Daphnia, 195

Dasypodidæ, 526

Dasyprocta, 531

Dasyuridæ, 497

Dasyurus, 497

Daubenton's Bat, 501

Dead Men's Fingers, 114

Death, 69

Death-watch, 267

Debilirostres, 459

Decapoda, 210, 219, 323

Decapods, 323

Deep sea fauna, 59

Defensive polyps, 105

Delphinus, 524

Demoiselle Fly, 255

Denclrocoela, 144.

Dental formula, 486

Dentalium, 291

Dentary, 439

Denticles, 133

Dentine, 339,340

Dentition, 483 , Milk, 484

, Permanent, 483

Dermal branchix, 125, 137

Dermanyssus avium, 284

Dermestes, 265

Dermestidse, 265

Desmans, 500

Desmodex folliculorum, 285

Desmodus, 501

Deutoplasm, 32
Diaphragm, 37, 488

Dibranchiata, 323

Dicotyles, 506

Didelphyidæ, 497

Didus ineptus, 465

Digenetic Trematodes, 147

Dimorphism, 54,

Dinictis, 517

Dinoceras, 512

Dinornis, 454

Dinosaurians, 429

Dinotherium, 512

Diodon, 391

Diomedea exulans, 457

Diplopoda, 230

Diplozoon paradoxum, 147

Dipnoi, 386

Dipper, 462

Diprotodontia, 498

Diptera, 252, 275

Dipus, 530

Discophora, 175

Dispersal of Animals, 58

Distal, 42

Distomea, 147

Distomum hepaticum, 147

Divers, 457

, Black-throated, 457

"Great Northern, 457

, Red-throated or speckled, 457

Diverticula, 24,

Division, 5 nuclear, 5

$$
\text { " } " \text { direct, } 5
$$

Dochmius, 160 indirect, 5

Doctrine of Descent, 53

Dodo, 465

Dog-fish, 383

Dolphin, 524

Domestic Cat, 517

Dog, 516

, Goat, 509

Ox, 510

, Pig, 506

"Sheep, 509

Donacia, 267

Dorcus parallelopipedus, 265

Dorididæe, 304

Doris, 304

Dormice, 529

Dorsal, 39

Dorsal lamina, 538

Double animal, 147

Doves, 465

Draco volans, 422

Dracunculus, 162

Dragon-flies, 254:

Dragons, 422

Dreissensia polymorpha, 314

Dromæidæ, 454

Dromæus, 454.

Drones, 424

Dschiggetai, 503 
Ducks, 458

Duck-billed Platypus, 496

Ductus Botalli, 489

, cochlearis, 338

" endolymphaticus, 370

Dugong, 513

Dung-beetles, 265

Dura mater. 331

Duration of life, 68,70

Dwarf males, 216

Dytiscidæe, 264

Dytiscus, 264

Eagles, 460

, Golden or Mountain, 460

" Sea or White-

Ear-bones, 481 tailed, 460

Eared Seals, 519

Earth Crab, 253

, Worms, 173

Earwigs, 253

Ecdysis, 31, 185

Echeneis, 390

Echidna, 496

Echinoderma, 83, 122

Echinoidea, 134

Echinometra, 138

Echinus, 138

Ectocyst, 178

Ectoderm, 30

Ectoparasites, 63

Ectosarc, 91

Edentata, 525

Edible Snail, 306

Eel, 388

Eel-pout, 388

, -worm, 163

Egg-sacs, 201

Elater, 265

Elateridæ, 265

Electric Eels, 388

$$
\text { , organs, } 368
$$

, Skates, 384

Elephants, 510

$$
\text { " Tusks, } 291
$$

Elephas africanus 512

Elpidia, 14i

primigenius, 512

Elysia, 304

Elytra, 236

Emberiza, 463

Embryo, 50

Embryology, 42

Embryonic membranes, 353

Imeu, 454

Emdyæ, 426

Enamel, 339, 340

Enchelyophis, 389

Encystation, 86

Endoderm, 98

Endoparasites, 63

Fndopod, 189

Endosarc, 91

Endoskeleton, 16

Endosmosis, 26, 96

Endostyle, 538

Engraulis encrassicholus, 387

Enhydra marina, 516

Enteric cavity, 111

Entomostraca, 193

Entoniscidæ, 216

Entoniscus, 216

Entozoa, 64

Environment, 66

Epeira diadema, 283

Ephemera, 255

Ephialtes scops, 461

Ephippium, 196

Epiblast, 43

Epibolic gastrula, 44

Epicrium glutinosum, 407

Epididymis, 350

Epiglottis, 488

Epiotic, 362

Epiphragm, 295

Epiphyses, 480

Epiphysis, 331

Epipod, 189

Epitheca, 116

Epithelial tissues, 7

Epithelium, 7

Epoophoron, 491

s,

, simple, 7 stratified, 7

Equidæ, 503

Equus asinus, 503

, Burchelli, 503

, caballus, 503

„hemionus, 503

, onager, 503

" quagga, 503

"zebra, 503

Ergots, 163, 503

Erinaceus, 499

Ermine, 516

$$
\text { europæus, } 499
$$

Esocidæ, 387

Esox lucius, 387

Estheria, 194:

Ethiopian region, 74 .

Ethmoid, 362

Euphausia, 211

Euphausiacea, 210

Euplectella aspergillum, 121

Eupteropoda, 304

European sub-region, 74

Eurypterus, 198

Euspongia, 121

Eustachian tube, 416

Eustrongylus, 160

Excretory organs, 30

Exhalent canal, 119

Exoccipital, 362

Exocotus, 388

Exopod, 189

Exoskeleton, 15, 184

External auditory meatus, 444

ear, 416
External ligament, 310 mouth, 111

Eyelashes, 467

Eyelids, 336

Eye-muscles, 335-6

.spots, 132

„stalk, 211

Falciform young, 96

Falco xsalon, 460

"gyrfalco, 460

" peregrinus, 460

¿ subbuteo, 460

tinnunculus, 460

Falconidæ, 460

Falcons, 460

". Peregrine, 460

Fallopian tube, 490

Fallow Deer, 508

False ribs, 472

Family, 55

Fat bodies, 248, 402

Feathers, 431

Feather-follicle, 431

F'eelers, 107, 184

Felidx, 517

Felis cattus, 517

, concolor, 517

, domestica, 517

, jubata, 517

, leo, 517

" lynx, 517

, maniculata, 517

" onca, 517

, pardus, 517

spelæa, 517

tigrina, 517

, tigris, 517

Femoral pores, 408

Femur, 235, 328

Fenestra ovalis, 397,416 rotunda, 416

Ferret, 516

Fertilisation, 32

Fiber zibethicus, 530

Fibula, 328

Fibulare, 328

Fiddler-beetle, 241

Field-cricket, 253

, -mice, 530

Fierasfer, 389

Filaria, 162

Filoplume, 433

Finches, 462

, Mountain, 462

"Serin, 463

Fin Whales, 523

Fish, 356

Fishing Hawk, 460

Fish-leech, 177

, -lice, 201

Fission, 4, 31

Flagellata, 95

Flagellate chambers, 119

Flagellum, 33, 95, 119

Flame cell, 14.3

Flamingoes, 458

Flat-fish, 389 
Flat-worms, 142

Fleas, 278

Floating ribs, 472

Filounder, 389

Flour-mites, 284

Fly-catchers, 462

Flying Fish, 388

¿ Phalangers, 498

$\Rightarrow \quad$ Foxes, 501

" Squirrel, 529

Fotus, 50

Food-yolk, 45

Foot, 293

Foramen magnum, 362 " ovale, 4.89

Fore-arm, 328 , -gut, 23

Forest-flies, 277

Forficula, 253

Formicariæ, 270

Formica rufa, 270

Fossils, 76

Fowls, 450

$\begin{array}{ll}\text { " } & \text { Domestic, 455 } \\ \text {, Jungea, 455 } \\ \end{array}$

Fox, 515

Free-living Copepods, 200

Freshwater Fauna, 59

$$
\begin{array}{ll}
\text { " } & \text { Mussels, 315 } \\
\text { " } & \text { Shrimps, 218 } \\
\text { " } & \text { Sponges, 121 } \\
\text { Tortoise, 426 }
\end{array}
$$

Frigate Bird, 457

(2) 426

Fringilla, 462

Frog of foot, 470

Frog, Edible, 406

Grass, 406

, Land, 406

Tree, 406

Frog-fish, 391

„-hopper, 257

, Toads, 406

Frontal, 362

Fruit Bats, 501

Fulica atra, 459

Fuligulinæ, 458

Fuligula cristata, 458 marita, 458

Fulmarus glacialis, 458

Fungia, 117

Funiculus, 317

Funnel, 317

Furcula, 440

Gadflies, 276

Gadidæ, 388

Gadus, 388

Galeopithecus volans, 500

" æglefinus, 388

, morrhua, 388

Gall-bladder, 342

Gall-fly, 269

Gallinula chloropus, 459

Gall-mites, 285

Gallus bankiva, 455

" domestious, 455
Gamasus, 284

Gammarus, 218

$$
\text { " Aluviatilis, } 219
$$

"locusta, 219

" puteanus, 219

Ganges Dolphin, 525

Ganglion cells, 10

Gannet, 457

Ganoidei, 384

Gaper, 315

Garden Dormonse, 530

, Snail, 306

Gar-pike, 388

Garrulus glandarius, 463

Gärtoner's duct, 351, 491

Gasterosteus, 390

$$
\begin{array}{cc}
\text { acuteatus, } \\
& 390 \\
& \text { pungitius, } \\
& 390
\end{array}
$$

Gasterosteidæ, 390

Gastric cavity, 109 pouch, 109

Gastropoda, 291

Gastrozooids, 107

Gastrula, 4.3, 98

$$
\text { , mouth, } 43
$$

Gastrus equi, 277

Gavialidæ, 426

Gecko, 417, 422

Geese, 458

Gemmation, 31, 86

Gemmulæ, 120

Genet, $\mathbf{5 1 7}$

Genital organs, 38

" plates, 136

Genus, 55

Geocores, 259

Geographical Distribution, 78

Geology, 76

Geological Distribution, 76

Geometridæ, 275

Geotrupes, 265

Gephyrea, 174

Germinal spot, 32

$$
\text { , vesicle, } 32
$$

Germ-layers, 47

Gestation, 494

Giant-Sloth, 525

Gibbons, 534

Gills, 538

Gill arches, 327

, clefts, 343

" lamellæ, 306

rakers, 374

Giraffe, 508

Gizzard, 24, 445

Glands, 14, 24

Glandular cells, 9

Glede, 461

Glenoid cavity, 327

Globigerina, 89

Globiocephalus melas, 524

Glomeris, 230

Glomerulus, 348

Glossæ, 233

Glow-worms, 266
Glutton, 516

Glyptodon, 527

Gnathobdellidæ, 176

Gnats, 276

Goat, 509

$$
\text { -moth, } 274
$$

Gobio fluvialis, 388

Gobius, 390

Godwits, 460

Gold-crested Regulus, 462

Golden-eye, 261 " Mole, 499

" Oriole, 462

Goldfinch, 462

Goldfish, 388

Gold Wasp, 270

Gonozooid, 107

Goose, 458

$$
\text { " Grey, } 458
$$

" Red-breasted, 458

Gordius, 163

Gorgonia, 114

Gorgonidæ, 114

Gorilla, 535

Goshawk, 460

Graafian follicle, 350

Graculus carbo, 457

Grallatores, 458

Grampus, 524.

Grasshoppers, 252

Grayling, 387

Great Ant-eater, 526

" Black Slug, 306

Crebes, 457

$$
\begin{array}{ll}
\Rightarrow & \text { Eared, } 457 \\
\Rightarrow & \text { Little, 457 } \\
\text { Red-necked, 4.57 }
\end{array}
$$

Green-finch, 462

Green-flies, 257

, gland, 221

Greenland Whale, 521.

Gregarina, 87

Gregarinida, 95

Grey Seal, 519

Gribble, 215

Grizzly Bear, 516

Grooved Teeth, 424

Grouse, 455

$$
\begin{array}{ll}
" & \text { Black, } 455 \\
" & \text { Red, 455 } \\
\text { " Sand, 455 }
\end{array}
$$

Gruidæ, 459

Grus cinerea, 459

Gryllidæ, 253

Gryllotalpa vulgaris, 253

Gryllus campestris, 253

" domesticus, 253

Gudgeon, 388

Gueparde, 517

Guinea-pig, 531 -worm, 162

„ Black-headed, 456

"Common, 456

" Herring, 456

, Laughing, 456

Gulo borealis, 516

Gurnard, 390 
Gurnard, Flying, 390

Grey, 390

Gustatory organs, 19

Gut, 23

Gymnomyxa, 87

Gymnophiona, 407

Gymnosomata, 305

Gymnothorax muræna, 388

Gymnotus, 368 " electricus, 388

Gypaëtus barbatus, 461

Gypogeranus secretarius, 460

Gyrfalcon, 466

Gyri, 480

Gyrimus, 264

Haddock, 388

Hæmatopus ostralegus, 459

Hrmocyanin, 30

Hæmoglobin, 29

Hæmopis vorax, 176

Hag-fish, 383

Hair, 467

, follicle, 467

" papilla, 467

Hairs, contour, 467

" tactile, 467

, woolly, 467

Halibut, 389

Halichoerus grypus, 519

Halicore dugong, 513

Halmaturus, 499

Halobatidæ, 259

Hamster, 530

Hapalidr, 533

Harderian gland, 336

Hare, 529

Harriers, 460

Harvest-men, 282 Mouse, 530

Haversian canal, 329

Hawfinch, 462

Hawk, 460

"Fishing, 460

Sparrow, 460

Hawk-moths, 275

Head, 41, 324.

Head-kidney, 167, 350

Heart, 26 , Urchins, 138

Hectocotylised arm, 321

Hectocotylus, 321

Hedgehog, 499

Heliozoa, 91

Helix, 306

, hortensis, 306

, pomatia, 306

Hemerohaxpages, 460

Hemimetabola, 250

Hemimetabolous, 246

Hemiptera, 256

Heredity, 39

Hermaphrodite, 34

gland, 35, 299

Hermaphrodites, protandrous, 35
Hermaphrodites, protogynous, 35

Hermit Crab, 225

Herodii, 459

Heron, Common, 459

, Night, 459

Herons, 459

Herpestes ichneumon, 517

Herring, 387

Heterodera schachtii, 163

Heterogony, 38, 146

Heteropoda, 302

Heteroptera, 259

Hexactinellidæ, 121

Hibernation, 69

Hibernating gland, 489

Hind gut, 23

Hipparion, 503

Hippobosea equina, 278

Hippoboscidæ, 277

Hippocampus, 391

Hippoglossus vulgaris, 389

Hippopotamidæ, 506

Hippopotamus, 506 amphibius,

Hirudo, 176 506

Hirundo, 463

Hobby, 460

Holocephali, 384

Holometabola, 250

Holometabolous, 246

Holostei, 385

Holothuria, 14.1

Holothuroidea, 138

Homarus vulgaris, 224

Homodont teeth, 485

Homology, 57

Homoptera, 256

Homo sapiens, 536

Honey-bee, 271 "-dew, 257 , -comb-bag, 507

Hooded Seal, 520

Hoofs, 470

Hoopoe, 464

Hornets, 271

Horns, 471

Horny fibres, 120

Horse, 503

$$
\begin{array}{ll}
" & \text { Bot-fly, 277 } \\
" & \text { Leeoh, 176 } \\
" & \text {-shoe Bats, 501 } \\
\text { " } & \text {-tick, 278 }
\end{array}
$$

Hosts, 63

House-fly, 277

, Mouse, 530

Spider, 283

Howling Monkey, 533

Humerus, 328

Humming Birds, 464

Hump-back Whale, 523

Hunger, 73

Hyæna, 517

Hyænidæ, 517

Hybrid, 36

Hybridisation, 386

Hydatina senta, 157
Hydra, 106

Hydrachna, 284.

Hydrochorus capybara, 531

Hydrocores, 260

Hydrocysts, 107

Hydroid, 103

Hydromedusæ, 103

Hydrometra, 257

Hydrometridæ, 259

Hydrophyllia, 108

Hydrophis, 424

Hydrosoma, 107

Hydrozoa, 102

Hyla arborea, 406

Hylesinus piniperda, 267

Hylobates, 534

Hymen, 491

Hymenoptera, 252, 267

Hyoid, 327

Hyperidæ, 219

Hyperoodon rostratus, $\mathbf{5 2 5}$

Hypoblast, 43

Hypoderma bovis, 277

Hypopharynx, 234

Hypophysis, 331

Hypsiprymnus, 4.99

Hyrax, 500

Hystricomorpha, $\mathbf{5 3 0}$

Hystrix cristata, 530

Tbis religiosa, 459

Ichneumon-flies, 269

Ichneumonidæ, 269

Ichthyornis, 453

Iehthyosaurians, 427

Icticyon, 516

Idothea, 215 , tricuspidata, 215

Iguanas, 422

$$
\text { , Ground, } 422
$$

" Tree, 422

Iguanidæ, 422

Iguanodon, 430

Ilium, 328

Imago, 249

Impennes, 457

Imperforate Rhizopoda, 88

Impressions, 77

Incisors, 4.83

Incus, 481

Inferior arches, 326

Infundibulum, 118, 331

Infusoria, 89, 91

Inhalent aperture, 308

Ink-sac, 317

$$
\text { " canal, } 119
$$

Inner germ-layer, 43 , root-sheath, 467

Insecta, 231

Insectivora, 499

Integripalliate, 310

Interambulacral plates, 134

Interdigital glands, 469

Interfilamentar junctions, 307

Intermedium, 328

Internal ligament, 310 
Internal mouth, 111

, shell, 305, 319

Interparietal, 476

Interradii, 123

Interspinous processes, 362

Intestinal Trichina, 162

Intestine, 23

Inuus ecaudatus, 534

Invagination, 43

Iris, 333

Irish Deer, 509

Irregular Sea-urchins, 138

Irritability, 4

Ischium, 328

Isis, 115

Isopoda, 210, 213

Itch-mites, 285

Iulus, 230

Ixodes, 284

Iynx torquilla, 466

Jackal, 517

Jackdaw, 463

Jacobson's organ, 333

Jaguar, 517

Jaws, 176, 205

Jays, 4,63

Jelly-fish, 111 veil, 90

Jerboa, 530

Jigger, 278

Joint, 329

Jugal, 394, 412

Jumping Shrews, 500

Kaguan, 500

Kangaroos, 498

Kermese-insect, 259

Kestrel, 460

Kidneys, 31

Killer, 524

King-crab, 196

Kite, 461

Kiwi, 454

Knee-cap, 330

Koala, 498

Kulan, 503

Labial cartilages, 364 palps, 233, 306

Labium, 233

Labridæ, 389

Labrum, 232

Lacerta, 422

$$
\text { " agilis, } 422
$$

, vivipara, 422

Lacertilia, 421

Lachrymal bone, 439

Lacinia, 233 gland, 336

Ladybirds, 267

Lagomys, 529

Lagopus, 455

" mutus, 455

" scoticus, 455
Lamellibranchs, 306

Lamellicornia, 265

Lamellirostres, 458

Lamina perpendicularis, 476

Lampreys, 382

Lampyris, 266

Lancelets, 354

Land-bug, 259

,leech, 176

Laniadæ, 462

Lanius excubitor, 462

Large Bats, 501

Larks, 464

Larus, 456

, argentus, 456

, atricilla, 456

, canis, 456

ridibundus, 456

Larva, 247

Larvæ, 50

Larynx, 344

Lateralia, 204

Lateral-line organs, 369

Leaf-beetles, 266

, -insects, 253

, -rollers, 274

Lecanium ilicis, 259

Leeches, 175

Lemnisci, 163

Lemming, 530

Lemurs, 531, 532

Lens, 20, 333

Leopard, 517

Lepadidø, 206

Lepas, 206

Lepidoptera, 252, 272

Lepidosiren paradoxa, 367, 387

Lepidosteus, 375, 378, 385

Lepisma saccharina, 255

Leporidæ, 529

Leptocardii, 354

Leptoptilus, 459

Lepus, 529

cuniculus, 529

europæus, 529

timidus, 529

variabilis, 529

Lernea branchialis, 203

Lesser Fin-whale, 523 , Shrew, 500

Lestris, 456

Leueiscus, 388

Leucochloridium paradoxum, 148

Libellula, 255

Libellulidæ, 254

Lice, 160

Ligament, 310, 329

Ligula simplicissima, 151

Limacina helicina, 305

Limapontia, 304

Limax agrestis, 306

Limbs, 42

Limnadia, 195

Limnæus, 306

Limnoria terebrans, 215
Limosa, 460

Limpets, 302

Limulus, 196

Lineus longissimus, 156

Lingual ribbon, 287

Lingula, 183

Linnet, 468

Lion, 517

, Common, 463

Lip, 486

Lithobius, 230

Lithodomus lithophagus, 314

Lithophrya, 206

Little Ant-eater, 526

Littoral fauna, 38

Littorina, 302

Liver, 24,

Liver-fluke, 147

Lizards, 422

$$
\begin{array}{ll}
" & \text { Common, 422 } \\
" & \text { Flying, 422 } \\
\text { " } & \text { Ringed, 422 } \\
\text { Sand, 422 }
\end{array}
$$

Llamas, 508

Loach, 388

Lobster, 224

Locomotion, 65

Locomotor organs, 63

Locusta, 253 viridissima, 253

Locusts, 253

Loligo vulgaris, 323

Longicorns, 266

Longipennes, 456, 463

Longitudinal areæ, 158

Long-tailed Field Mouse, 530

Loopers, 276

Lophius piscatorius, 391

Lophogastridæ, 211

Lophophore, 178

Lorica, 156

Lories, 465

Loris, 532

Lota vulgaris, 388

Lucanus cervus, 265

Lugworm, 173

Lumbar plexus, 332

$$
\text { , vertebræ, } 326
$$

Lumbricus, 173

Lump-fish, 390

Lunar, 4i78

Lung-sacs, 282

Luscinea philomela, 462 ubecola, 462

Lutra, 516

, vulgaris, 516

Lymphatic system, 347

Lymph follicles, 348

, glands, 26

Lynx, 517

hearts, 348

, vulgaris, 517

Lyre Birds, 464.

Lyssa, 487

Lytta vesicatoria, 266 
Machærodus, 517

Machetes pugnax, 4,60

Machilis, 256

Mackerel, 390 , pikes, 388

Macrolepidoptera, 274

Macronucleus, 92

Macropidæ, 498

Macroscelides, 500

Madreporaria, 118

Madreporite, 124, 131

Maggots, 250

Magnum, 478

Magot, 534

Magpie, 463

Makis, 532

Malacodermata, 266

Malacostraca, 193, 207

Malapterurus, 368, 388 $" \quad$ electricus, 388

Malleus, 481

Mallophagidæ, 255

Malpighian tubes, 188

Mammæ, 469

Mammalia, 466

Mammary glands, 468

Mammillw, 468

Mammoth, 512

Man, 535

Manatee, 513

Manatus, 513

Manchurian sub-region, 74 Mandibles, 184

Mandibular arch, 327

Manis, 527

Mantidæ, 253

Mantis religiosa, 253

Mantle, 203, 287 , folds, 182 " lobe, 307

Manubrium, 102, 105, 473

Manyplies, 507

Marabou, 459

Marble Seal, 519

Margaritina margaritifera, 315

Marine fauna, 59

Marmosets, 533

Marmots, 529

Marsupial Ant-eater, 497

Marsupials, 496

Marsupial bones, 479

Marsupium, 214, 217

Martens, 516

Martins, 463

Mask, 255

" House, 463

Sand, 463

Mastodons, 512

Maw-worm, 160

Maxilla, 410

Maxillæ, 184

Maxillipeds, 184

Maxillo-turbinal, 475

May-flies, 255

Meal-worm, 266

Meckel's cartilage, 327
Medicinal Leech, 176

Medina Worm, 162

Mediterranean sub-region, 74

Medusa, 99

Medusoid generation, 102

Megachiroptera, 501

Megapodius, 456

Megaptera böops, 523

Megatheria, 525

Megatheriidæ, 525

Meleagrina margaritifera, 315

Meleagris gallopavo, 455

Meles taxus, 516

Meloë, 266

Melolontha vulgaris, 265

Melophagus ovinus, 278

Membrane bones, 325

Membranellæ, 92

Membraniporidæ, 181

Membranous labyrinth, 338

Menobranchus, 405

Menopoma, 405

Mentum, 233

Menura, 464

Mephitis, 516

Mergansers, 458

Merginw, 458

Mergus, 458

Merlin, 460

Mermis, 163

Merops apiastes, 465

Merrythought, 4,40

Mesencephalon, 330

Mesenterial filaments, 112

Mesenteron, 23

Mesentery, 39, 111, 342

Mesoblast, 47

Mesogloea, 98

Mesonephros, 350

Mesothorax, 235

Metacarpal, 328

Metameres, 41

Metamorphosis, 50

Metanephros, 350

Metatarsal, 329

Metathorax, 235

Metazoa, 5, 85, 98

Metencephalon, 330

Mice, 530

Microchiroptera, 501

Microlepidoptera, 273

Micronucleus, 92

Micropyle, 243

Mid-brain, 330

Middle germ-layer, 45

Midges, 276

Mid gut, 23

Miescher's corpuscles, 91

Migrations, 62

Migratory Locusts, 252

Mill, 469

Millepora, 106

Miller's Thumb, 390

Milvus regalis, 461

Mimicry, 71
Mink, 516

Mites, 284

Mola, 391

Molars, 483

Mole, 499

Mole-cricket, 253

, -rat, 530

Mollusca, 84, 287

Monadinidæ, 95

Mongoose, 517

Monodon monoceros, $\mathbf{5 2 5}$

Monogenetic Trematodea, 146

Monotremes, 495

Moor-hen, 459

Moose-deer, 509

Mormon fratercula, $\mathbf{4 5 7}$

Morphology, 57

Moschus moschiferus, 509

Mosquitos, 276

Moss-animals, 178

Motacilla, 462

Mother-of-pearl, 310

Mouflon, 509

Moulting, 15, 31

Mound-birds, 456

Mouth, 23

Mouth-parts, 184

Mucous gland, 295

Mud̈-fish, 386 membrane, 24

"turtles, 426

Müllerian ducts, 351

Muridæ, 530

Muræna, 388

Murænidæ, 388

Mus, 530

" decumanus, 530'

, minutus, 530

, musculus, 530

, sylvaticus, 530

Musca domestica, 277

" vomitoria, 277

Muscicapidæ, 462

Muscidæ, 277

Muscle-cells, 11

$$
\text { , smooth, } 11
$$$$
\text { "striated, } 12
$$

Muscle fibrillæ, 92 -trichina, 162

Muscular impression, 310

Museular system, 16

Muscular tissues, 7

Mushroom coral, 117

Musk Deer, 509

, Rat, 530

, Shrew, 500

Musquash, 530

Mussel, 314

Mustela, 516

" erminea, 516

, foina, 516

, furo, 516

, Iutreola, 516

putorius, 516

" vulgaris, 516

" zibellina, 516

Mustelidæ, 516 
Mya arenarja, 315

Mycetes, 533

Myelencephalon, 330

Mygale, 283

Mylodon, 525

Myodes lemmus, 530

Myogale, 500

, moschata, 500

, pyrænica, 500

Myopotamus coypu, 530

Myoxidæ, 529

Myoxus avellanarius, 530

" dryas, 530

, glis, 530

" nitela, 530

Myriapoda, 227

Myrmecobius, 497

Myrmecophaga, 526

didactyla, 526 jubata, 526

Myrmecophilous Insects, 270

Myrmeleon, 261

Mysidacea, 210, 211

Mysidæ, 211

Mysis, 212

Mysis-stage, 222

Mystacoceti, 523

Mytilus edulis, 314

Myxine, 383

, glutinosa, 383

Naïdæ, 174

Nails, 470

Naîs, 174

Naja tripudians, 424

Narwhal, 525

Nasal, 362

" gland, 443

Nasua, 516

Natantia, 222

Natatores, 456

Natica, 302

Native Cats, 497

Natural selection, 57

Nauplius, 192

Nautilus, 323 eye, 190

Naviculare, 479

Nearctic region, 74

Neb, $45 \mathrm{I}$

Nebalia, 207

Neck, 324

Necrophorus, 264

Nectocalyces, 108

Negro, 536

Nemathelminthia, 83, 158

Nematoda, 158

Nematocyst, 100

Nemertinea, 153

Nemocera, 276

Neophron percnopterus, 461

Neotropical region, 74

Nepa, 160

Nephelis, 176

Nephridia, 167

Nephroharpages, 461
Nereidæ, 173

Nereis, 173

Neritina, 302

Nerophis, 391

Nerve fibres, 13

, ring, 102

Nervous system, 17 , central, 17

" peripheral, 17

"sympathetic, 18 ", tissues, 7

Neural spine, 326

Neurilemma, 13

Neuroglia, 331

Neuroptera, 252, 260

Neuropodium, 169

Newts, 404

$$
\begin{gathered}
\text { Large Water, } 404 \\
\text { Palmated-smooth, } \\
404
\end{gathered}
$$$$
\text { Small, 404 }
$$

Nictitating membrane, 336

Nidamental glands, 321

Nightingale, 462

Nightjar, 464

Nine-eyes, 382

Niphargus puteanus, 219

Nits, 260

Noctuidæ, 275

Nocturnal animals, 71

Non-ruminantia, 505

Notochord, 324

Notonecta, 160

Notopodium, 169

Notoryctes typhlops, 499

Nubian Wild Cat, 517

Nucifraga caryocatactes, 463

Nucleolus, 3, 85

Nucleus, 3, 85, 96 pulposus, 471

Nudibranchiata, 303

Numenius arcuata, 460

Numida meleagris, 456

Nummulites, 89

Nutcracker, 463

Nuthatch, 464,

Nutritive polyps, 104, 107

Nut Weevil, 244

Nyctale funerea, 461

Nyctea nivea, 461

Nyctharpages, 461

Nycticorax griseus, 459

Nymphon, 286

Occipital, 362

Ocelli, 187

condyles, 394

Octactinia, 113

Octopoda, 323

Octopods, 323

Octopus vulgaris, 323

Ocular plates, 136

Odobænus rosmarus, 519

Odontoblasts, 340

Odontoceti, 524

Odontoid, 409

Odontornithes, 453
Esophagus, 23

Estridæ, 277

Estrus, 277

Oil-beetle, 266 globules, 10, 85

Olecranon, 328

Olfactory lobes, 330

", , organs, 18

Oniscidæ, 215

Oniscus, 215

Ontogeny, 40

Onychophora, 177

Oceium, 180

Operculum, 179, 197. 204, $295,373,374$

Ophidia, 422

Ophidiidæ, 389

Ophiura, 134

Ophiuridæ, 133

Opisthobranchiata, 301,303

Opisthoglypha, 424,

Opisthotic, 362

Opossums, 4.97

Optic chiasma, 332

, lobes, 331

" organs, 20

" thalami, 331

Oral disc, 112

„, tentacles, 109

Orang-utang, 534

Orbit, 333

Orbitosphenoid, 362

Orca gladiator, 524

Order, 55

Organ-pipe coral, 114

Organs, 13 , of Bojanus, 312

Oriental region, 74

Oriolus galbula, 462

Ornithorynchus, 496

Orthagoriscus, 391

Orthoptera, 252

Orycteropus, 527

Oryctes, 244

Oscines, 461

Osculum, 119

Os penis, 493

Osphradia, 296

Osprey, 433, 460

Os sepire, 323

Ossicles, 129, 133

Ossifications, 325

Ostracion, 391

Ostracoda, 199

Ostrea edulis, 314

Ostrich, 453, 454.

Otariidæ, 519

Otidæ, 459

Otis tarda, 459

tetrax, 459

Otocoris alpestris, 464

Otocyon caffer, 516

Otocysts, 19

Otoliths, 20

Otters, 516 
Otus brachyotus, 461 , vulgaris, 461

Outer germ layer, 43 , root sheath, 467

Ovarioles, 242

Ovary, 34

Ovibos moschatus, 509

Oviduct, 33

Ovigerous lamellæ, 206

Oviparous, 47

Ovipositor, 243, 269

Ovis, 509

, ammon, 509

, aries, 509

, musimon, 509

Ovum, 5, 32, 34

OwIs, 461

" (a) Diurnal, 461

, Great Fared, 461

, Hawk, 461

" Little, 461

, Scops Eared, 461

Snowy, 461

(b) Nocturnal, 461

Barn, 461

Long-eared, 461

Short-eared, 461

Tawny, 461

"Tengmalm's, 461

Oxyuris vermicularis, 160

Oyster, 314

. catcher, 459

Paca, 531

Prodogenesis, 118

Pagurus, 225

Berhardus, 226

Palæarctic region, 74

Palæmon, 223

Palæontology, 77

Palæotherium, 502

Palatal ridges, 487

Palate, 482

, hard, 482

"soft, 48

Palato-quadrate, 327

Palinurus, 225 , vulgaris, 225

Palisade Worm, 160

Pallial chamber, 287

, line, 308

" muscle, 307

Palp, 169, 188

Paludina vivipara, 302

Pancreas, 342

Pandion haliaëtus, 460

Pangolin, 527

Panorpa, 262

Panther, 517

communis, 262

Paper-wasps, 271

Papillæ circumvallatæ, 487

, filiformes, 487

" foliatæ, 487

, fungiformes, 487

Paradiseidæ, 463

Paraglossæ, 233
Paramœcium, 94

Parasites, 24, 63

Parasitic Copepods, 201

Parasitism, 62

Parasphenoid, 363, 394

Parental instinct, 52

Paridø, 462

Parietal, 362

Parovarium, 351

Parrakeets, 465

Parrots, 446

Parrot-fish, 389

Parthenogenesis, 37

Partial segmentation, 45

Partridge, 455

Parus caudatus, 462

, coruleus, 462

, major, 462

Pastor, 463

Patagium, 428

Patella, 302, 330

Paunch, 507

Pavo cristatus, 455

Pavonidæ, 455

Paxillæ, 123

Peacock, 455

Pearl Mussel, 315

Pearls, 311

Peccaries, 506

Pecten, 314, 443

Pedal disc, 112

, ganglia, 289

, nerves, 289

" orifice, 308

Pedes adhamantes, 452

, ambulatorii, 452

" cursorii, 452, 453

" fissi, 452

" fissipalmati, 457

" gradarii, 452

, grallarii, 458

, gressorii, 452, 465

" insidentes, 452

" palmati, 452, 456

, raptorii, 460

$\Rightarrow$ scansorii, 452, 465

, stegani, 457

" vadantes, 452

Pedicellariæ, 123, 132, 137

Pediculati, 391

Pediculatidæ, 160

Pediculus capitis, 260

"vestimenti, 260

Peduncle, 182, 188, 204

Peewit, 459

Pelagic fauna, 61

Pelargi, 459

Pelecanus, 457

Pelican, 457

Pelican's foot, 302

Pelobates fuscus, 406

Pelobatidæ, 406

Peltogaster paguri, 207

Pelvic girdle, 328

Pemphigus spirothecæ, 258

Penæus, 223

Penguins, 457
Penis, 34

Pennæ, 431

Pennatula, 115

Pentacrinus, 130

Pentastoma, 285

Perameles, 498

Peramelina, 497

Perca fluviatilis, 389

Perceptive cells, 21

Perch, 389

Percidæ, 389

Perdicidæ, 455

Perdix cinerea, 455

Perforate Rhizopoda, 88

Perennibranchiata, 405

Peribranchial cavity, 538

Pericardium, 187, 345

Perichondrium, 329

Perinæal pouches, 469

Periosteum, 329

Peripatus, 177

Periplaneta orientalis, 253

Perissodactyla, 502

Peristome, 136, 138

Peristomium, 169

Peritoneum, 342

Periwinkles, 302

Peropoda, 424

Peter's Thumb, 389

Petrel, Fulmar, 456

, Stormy, 456

Petrels, 456

Petrifactions, 76

Petromyzon, 382

$\begin{array}{ll}\text { " } & \text { fluviatilis, 382 } \\ \text { " } & \text { marinus, 382 } \\ \text { planeri, 382 }\end{array}$

Petrosal, 362

Phacochorus, 506

Phalanges, 328

Phalangiidæ, 282

Phalangista, 498

Phalangistidæ, 498

Phalaropes, 460

Phalaropus, 460

PharyngeaI bones, 365

Phascolarctos, 498

Phascolomys, 499

Phasianidæ, 455

Phasianus, 455 , colchicus, 455

Phasianomorphæ, 455

Phasmidæ, 253

Pheasants, 455

Phoca, 519

" fœtida, 519

" greenlandica, 519

" vitulina, 519

Phocæna communis, 524

Phocidæ, 519

Phoenicopterus, 458

Pholas, 315

Phosphorescent organs, 28, 211

Phryganea, 262

Phthirius pubis, 266

Phyllium siccifolium, 254

Phyllopoda, 193 
Phyllosoma, 225

Phylloxera vastatrix, 257

Phylum, 55

Physalia, 108

Physetes macrocephalus, 524

Physiology, 58

Physophora, 108

Physostomi, 387

Phytoptus, 285

Pia mater, 331

Pica caudata, 463

Picidæ, 465

Picus martius, 465

, viridis, 466

Piddock, 315

Pieris brassicæ, 275

Pig, 505

Pigeons, 465

Pigment, 31

, granules, 10,85

Pigmy male, 201, 205

Pika, 529

Pike, 387

Pilot Whale, 524

Pineal eye, 337

, gland, 331

Pine Grosbeak, 463

$\therefore$ Lappet, 275

, Marten, 516

Pinicola enucleator, 463

Pinna, 481

Pinnipedia, 517

Pinnules, 128

Piophila casei, 277

Pipa, 398, 402

"s americana, 407

Pipistrelle, 501

Pipit, 462

Pisees, 356

Piscicola, 177

Pisiform, 414

Pithecus satyrus, 534

Pituitary body, 331

Placenta, 354. , fcetalis, 354 , uterina, 354

Placophora, 290

Plaice, 389

Planaria, 145

Planorbis, 300

Platalea leucorodia, 459

Platanista gangetica, 525

Platyhelminthia, 83, 142

Platyrrhinæ, 533

Plectognathi, 391

Plesiosaurians, 428

Pleura, 198

Pleúral ganglia, 289

Pleuronectes flesus, 389

limanda, 389

$$
\text { . } \quad \text { limanda, } 389
$$

Pleuronectidæ, 389

Plictolophinæ, 465

Plovers, 459

Plumæe, 433

$$
\text { Golden, } 459
$$

Pneumatic duct, 375
Pneumatocyst, 107

Podiceps, 457

$$
\begin{array}{ll}
\Rightarrow \quad & \text { auritus, } 457 \\
\Rightarrow & \text { grisegena, } 457 \\
\text { minor, } 457
\end{array}
$$

Podura, 256

Poison-claws, 229

, glands, 392, 424

, teeth, 423

Polar Bear, 516

"Fox, 515

"Hare, 529

, Whale, 523

Polecat, 516

Polian vesicle, 124

Pollex, 478

Pollicipes, 206

Polyactinia, 115

Polychæta, 172

Polymorphism, 54

Polynoë, 173

Polynoidæ, 173

Polyp, 98

Polypoid generation, 102

Polyprotodontia, 497

Polypterus, 374, 385

Polystomeæ, 146

Polystomum intergerrimum, 146

Polyzoa, 83, 178

Pompilidæ, 270

Pond-snail, 306

Pontobdella muricata, 177

Pope, 389

Porcupines, 530

Porcus babyrusa, 506

Pores, 119

Pore plates, 134

Porifera, 118

Porpita, 108

Porpoise, 524

Portal vein, 346

Porus genitalis, 378

Post coracoid, 413

, frontal, 362

Pouched Marmot, 529

Powder-down, 434

Præcoces, 450

Prawns, 222

Prawn-stage, 222

Praying Mantis, 253

Preanal pores, 408

Precious Coral, 115

Precoracoid, 413

Prefrontal, 362

Prehensile organs, 63

Premaxilla, 410

Premolars, 483

Primates, 532

Printer, 267

Pristis, 384

Proboscidea, 510

Proboscis, 153, 201, 234, 256

Proboscis-sheath, 153

Procellaria pelagica, 456

Processus falciformis, 370

"uncinatus, 410,
Processus, vermiformis, 488

Proctodæum, 23 xiphoides, 473

Procyon, 516

Procyonidæ, 516

Proechidna, 496

Proglottides, 149

Prolegs, 250

Pronephros, 350

Prongbuck, 509

Prong-horned Antelope, 509

Prootic, 362

Proscolex, 150

Prosencephalon, 330

Prosimiæ, 531

Prosobranchiata, 301, 302

Prostate glands, 493

Prostomium, 169

Protective adaptations, 70

Proteles, 517

Proteroglypha, 424

Proteus, 399, 403 , anguineus, 404

Prothorax, 235

Protoplasm, 3, 85

Protopterus annectens, 387

Protozoa, 3, 83, 85

Provisional larval organs, 51

Proximal, 42

Psalterium, 507

Pseudis paradoxa, 403

Pseudo-caterpillars, 250 , -navicellæ, 96 . " -pupa, 251

Pseudopodia, 3, 25, 85, 87, 90

Pseudo-scorpions, 282

Psittacidæ, 466

Psittacinæ, 465

Psocus, 255

Psolus, 141

Psorospora gigantea, 96

Ptarmigan, 455

Pterodactyles, 4,28

Pterodactylus, 428

Pteromys, 529

Pteropoda, 304.

Pteropus, 501

Pterosauria, 428

Pterota, 305

Pterygoid, 394

Pterylæ, 434

Pubis, 328

Puffin, 457

Pulex irritans, 278

Pulmonary syphon, 297

Pulmonata, 301, 305

Pulp, 339

Puma, 517

Punger, 227

Pupa, 249

Pupil, 333

Purple, 295

Pyche, 274

, helix, 245 
Pycnogonidæ, 286

Pycnogonum, 286

Pygopodes, 457

Pyrosoma, 540

Pyrrhula, 463

Python, 424

Quadrate, 394

Quadrato-jugal, 394

Quail, 455

Queen-bee, 271

Rabbit, 529

Racoons, 516

Radial canal, 102, 109

Radiale, 328

Radial symmetry, 41, 99, 122

Radiata, 122

Radii, 122

Radiolaria, 89

Radius, 329

Radula, 287

Rail's, 459 sac, 288

" Land, 459

"Water, 459

Rainey's corpuscles, 97

Raia, 368

Raiidæ, 383

Ramphorhynchus, 428

Rana, 406 , esculenta, 406 " temporaria, 406

Rapaces, 460

Rallidæ, 459

Rallus aquatious, 459

Rasores, 455

Ratitæ, 453

Rats, 530

Rattle-snake, 425

Raven, 463

Rays, 40

Razor-bill, 457

Receptacula seminis, 34, 242

\section{Rectrices, 432}

Recurvirostra avocetta, 460

Red bodies, 375

Redbreast, 462

Red corpuseles, 25

Red Deer, 508

Red Forest Ant, 270

Redia, 147

Redpole, Lesser, 463

Redshank, 460

Redstart, 462

Reed, 507

Regeneration, 32

Regular Sea-urchins, 138

Regulus, 462

Reindeer, 509

Remiges, 432

Replacement of teeth, 482

Reproduction, 31, 86

Reproductive organs, 31

Reptantia, 223

Reptilia, 40 '
Resonators, 399

Respiration, 26

Respiratory organs, 27

$$
\text { , syphon, } 309
$$

"trees, 125, 140

Rete malpighii, 325

" mirabile, 346

Retina, 21

Retinaculum, 272

Retinal cells, 2 I

Rhabdocola, 144

Rhamphastidæ, 465

Rhamphostoma, 426

Rhinoceros, 503

$\begin{array}{ll}\Rightarrow & \text { bicornis, } 503 \\ \Rightarrow & \text { simus, } 503 \\ \Rightarrow & \text { tichorinus, } 503 \\ \Rightarrow & \text { unicornis, } 503 \\ & \text { Birds, } 465\end{array}$

Rhinolophus, 501 501
ferrum-equi-
num, 501

hipposiderus, 501

Rhizocephala, 206

Rhizocrinus lofotensis, 130

Rhizopoda, 87

Rhodeus amarus, 388

Rhombus lævis, 389

" maximus, 389

Rhopalocera, 275

Rhynchobdellidæ, 17 7

Rhyncocola, 153

Rhyncota, 252, 256

Rhytina, 513

, stelleri, 513

Ribs, 118, 326

Ring canal, 124

Ringed Seal, 519

Ring Ouzel, 462

River Pearl-mussel, 315

, Mussel, 315

, Snail, 302

Roach, 388

Rodentia, 527

Rodents, 527

Roe, 508

Roller, 464

Rook, 463

Rorqual, 523

Rosechafers, 265

Rostrum, 223

Rotatory organ, 156

Rotifera, 83, 156

Rudimentary organs, 40

Ruff, 460

Rumen, 507

Ruminantia, 506

Russian Desman, 500

Rustic, 275

Ruticilla, 462

Sable, 516

Sabre-toothed Cats, 517

Saccobranchus, 375

Sacculina carcini, 207

Sacculus, 338

Sacral vertebræ, 326
Salamander, 402

$\begin{array}{ll}\text { " } & \text { Black Alpine, } \\ & 404 \\ & \text { Giant, } 405 \\ \text { " } & \text { maculosa, 404 }\end{array}$

Salanganes, 464

Salinity, 60, 67

Salivary glands, 24, 341

Salmon, 387

, fario, 387

, salar, 387

" trutta, 387

Salmon-trout, 387

Salpa, 546

Salvelinus, 387

Sand-eels, 389

Sand-fly, 276

Sand-pipers, 460

Sand-wasps, 270

Sapajou, 533

Saproharpages, 461

Sarcocystis, 97

Sarcodina, 87

Sarcolemma, 12

Sarcophaga carnaria, 277

Sarcopsylla penetrans, 278

Sarcoptes scabieia, 285

Sarcoptidæ, 285

Sarcorhamphus gryphus, 461

Sardine, 387

papa, 461

Sauruxæ, 452

Saw-fish, 384

, -flies, 269

Saxicola, 462

Scale-insects, 258

Scales, ctenoid, 356

, cycloid, 357

, placoid, 357

" reptilian, 408

Scallops, 314

Scalpellum, 206

Scaly Ant-eater, 527

Scansores, 465

Scaphoid, 478

Seaphopoda, 291

Scapula, 327

Scarabæidæ, 265

Scarus, 389

Scincoidei, 422

Sciuridæ, 529

Sciurus vulgaris, 529

Sclerotic coat, 333

Scolex, 149

Scolopax gallinago, 459

$$
\begin{array}{ll}
\text { gallinula, } 459 \\
\text { major, } 459 \\
\text { rusticola, } 459
\end{array}
$$

Scolopendras, 229

Scomberesocidæ, 385

Scomberidæ, 390

Scomber scomber, 390

Scorpion-flies, 262

Scorpionidæ, 211

Scorpions, 281

Scrotum, 492

Scuta, 204 
Scutellum, 259

Scyllarus, 225

Scyllium canicula, 383

Scymnus borealis, 383

Scyphomedusæ, 109

Sea-acorns, 206

Anemones, 118

, Adder, 391

Angel, 384.

, Bear, 519

, Clerk, 323

Cows, 512

"Cucumbers, 138

"Elephants, 526

"Feathers, 115

"Gulls, 443

Hare, 303, 390

", Hedgehog, 391

" Hog, 524

"Horse, 391

"Lilies, 127

"Lion, 519

"Mouse, 173

" Otter, 515

, Palms, 130

"Roses, 118

", Scorpion, 390

"Squirts, 537

"Stickle, 377, 390

"Urchins, 134

Seasonal dimorphism, 38

Sebaceous glands, 468

Secondary sexual characters, 35

Secretary, 460

Sectorial tooth, 514

Segmentation, 156

Segments, 41, 184 cavity, 43

Selache maxima, 383

Selachii, 383

Self fertilisation, 36

Semicircular canals, 338

Seminal duct, 34

Seminal tubes, 243

Semnopithecidx, 534

Semnopithecus nasicus, 534

Sense of equilibrium, 20

Sense organs, 18

Sensory cells, 13

Sepia officinalis, 323

Serous membrane, 354

Serpula, 173

Serpulidæ, 173

Sesamoid bones, 330

Sesia, 274

Sessile forms, 65,66

Set", 185 medusa, 106

Seventeen-years Cicada, 257

Sexual reproduction, 31

Shad, 387

Shark, 328

Sharks, 383

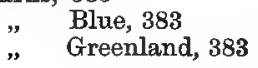

Sharks, Giant, 383

n Hammer-headed, 383

Sheat-flsh, 388

Sheath of Schwann, 13

Sheep, 509

Sheep Bot-fly, 277

Tick, 278

Shell, 92, 182, 287

Shellac-insect, 259

Shell-gland, 143, 192

Shield-urchins, 138

Ship-worm, 315

Shore-crab, 227

Shoulder-blade, 327

Shrew, 499 girdle, 327

Shrikes, 462

Great Grey, 462

Sib"bald's Fin-whale, 523

Siberian sub-region, 74

Siliceous fibres, 120

Silk glands, 248

, -worm Moth, 245, 274

Silpha, 264

Silphidæ, 264

Siluridæ, 388

Silurus glanis, 388

Simia Gorilla, 535

" Troglodytes, 535

Simulia, 276

$$
\text { " columbaczensis, }
$$
276

Sinupalliate, 310

Sinus venosus, 344

Siphon, 137

Siphonophora, 107

Siphonozooids, 114

Siphuncle, 317

Siredon mexicanus, 403, 404

Sirenia, 512

Siren lacertina, 405

Sirex, 269

Siskin, 463

Sitta cæsia, 464

Sittacinæ, 465

Skates, 383

Skeletal tissues, 7,9

Skeleton, 4, 15, 85

Skeleton Shrimp, 219

Skin, 14

Skin glands, 237

Skinks, 422

Skip-jacks, 265

Skull, 327

Skunks, 516

Sleep, 69

Sloth Bear, 516

Sloths, 525

Slough, 407

Smail Bats, 501

Small Tortoiseshell, 275

Sminthus betulinus or vagus, 530

Snakes, 422 , Asculapius, 424

"Giant, 424
Snakes, Ringed, 424

" Sea, 424

"Whip, 424

Snipe, Common, 459

Great, 459

, Jack, 459

Snipes, 459

Solaster, 133

Soldiers, 254, 270

Sole, 389

, (of foot), 469

Solea vulgaris, 389

Solenoglypha, 425

Solitary Bees, 272

, Corals, 117

Somateria mollissima, 458

Sorex, 499

" pygmæus, 500

"vulgaris, 500

Sound-producing organs, 30

Spalax typhlus, 530

Spanish-fly, 266

Sparrow, 463

" House, 463

, Tree, 463

Spatangidæ, 138

Spatangus, 138

Spatularia, 385

Species, 53

Spermatophore, 34

Spermatozoon, 32, 33

Spermophilus citillus, 529

Sperm Whale, 524

Sphenoid, 362

Sphenethmoid, 394

Spheridia, 138

Sphingidæ, 275

Sphyrna, 383

Spicula, 159

Spider Monkey, 533

Spiders, 283

Spinachia vulgaris, 390

Spinal cord, 324, 330, 331,

Spines, 123, 137

Spinnerettes, 283

Spinning glands, 279

Spiny Ant-eater, 496

, Dog-fish, 383

Spiracle, 373

Spiral valve of intestine, 371

Spleen, 348

Sponges, 83, 118

Spongiæ, 118

Spongilla fluviatilis, 121

Spontaneous movement, 3

Spoon-billed Sturgeon, 385

Spores, 96

Sporocyst, 147

Sprat, 387

Spring-flies, 262

Spur, 235, 435

Squalidæ, 383

Squamipennes, 390

Squamosal, 362

Squatina, 384.

Squilla mantis, 227

Squirrel, 529 
Squirrel-tailed Dormouse, 530

Stag, 508

Stag-beetle, 265

Stages of life, 68

Stapes, 481

Staphylinidæ, 264

Staphylinus, 264

Starfish, 131

Starlings, 463

Stationary parasites, 61

Statoblast, 180

Steganopodes, 457

Stegocephala, 405

Steinbock, 509

Steller's Cow, 513

Stenops, 532

Stensen's duct, 333

Sterlet, 385

Sterna, 456

$$
\Rightarrow \quad \text { hirundo, } 456
$$

" minuta, 456

Sternum, 326

Stick-insects, 253

Stickleback, Ten-spined, 390

”

Three-spined, 390

Sticklebacks, 379, 390

Stigmata, 240

Sting, 243, 269

Stink-glands, 237

Stipes, 233

Stock, 32

Stolon, 103

Stomach, 23

Stomapoda, 210, 227

Stomodæum, 23

Stone canal, 124.

Stork, Black, 459

"White, 459

Storks, 459

Strap-worm, 151

Stratum corneum, 325, 466

, Malpighii, 466

mucosum, 325,466

Strepsilas interpres, 459

Strepsiptera, 262

Striges nocturnæ, 4.61

Stringopinæ, 465

Stringops habroptilus, 465

Strix flammea, 461

Strongylidæ, 160

Strongylus, 160

Struthionidæe, 454

Sturgeons, 363, 374, 385

i.Sturnus vulgaris, 463

Stylets, 201

Stylommatophora, 306

Stylonychia, 94,

Stylops, 262

Subcortical crypts, 120

Subimago, 247

Sublingua, 487

Sub-regions, 74

Subungulata, 521

Succinea amphibia, 148

Suckers, 14
Sucking-fish, 390

Sudoriparous glands, ;468

Suidæe, 505

Sula bassana, 457

Sulci, 480

Summer sleep, 69

Sun-fish, 391

Supporting cells, 21

Suprabranchial cavities, 307

Supraoccipital, 362

Suprarenal body, 350

Suprascapula, 413

Surnia nisoria, 4.61

Sus, 505

, scrofa, 505

Swallows, 463

Swan, Black, 458

, Mute, 4.58

"Wild or Whistling,

Swans, 458 458

Swarm-spores, 90

Swift, Alpine, 464

, Common, 464

Swifts, 464

Swim-bladder, 344,

Swimmerets, 209

Swimming bells, 108

Sword Fish, 390

Sylvia, 462

Sylviadæ, 462

Symbiosis, 91

Sympathetic nervous system, 332

Synapta, 141

Synapticula, 116

Syngnathidæ, 391

Synovial cavities, 40

Syrinx, 446, 447

Syrnium aluco, 461

Syrrhaptes paradoxus, 455

Tabanidæ, 276

Tachina, 277

Tachypetes aquila, 457

Tænia, 151

" ccenurus, 151

" cucumerina, 151

" echinococcus, 151

, mediocanellata, 151

Tail, 4i, 324 saginata, 151

"diphycercal, 360

" heterocercal, 360

"homocercal, 361

Talegallas, 456

Talpa, 499

" europæa, 449

Tanaïdæ, 216

Tapetum, 481

, lucidum, 370

, nigrum, 333

Tapeworms, 149

Tapir, 502

Tapirus, 502

Tardigrada, 286

Tarsals, 328
Tarsier, 532

Tarsipes, 498

Tarsus, 235, 328

Tarsius spectrum, 532

Tasmanian Wolf, 497

Taste-buds, 19

Teeth, 23, 338

Tegenaria domestica, 283

Teleosaurus, 427

Teleostei, 387

Telephorus, 266

Temporal, 476

Temperature, 60

Temporary conjugation, 92

Tench, 388

Tendons, 185, 330

Tenebrio molitor, 266

Tentacles, 103, 111, 140, 178, 292,316

Tentacle-sheath, 316

Tentacular axms, 316

" cirrus, 169

Tentaculocyst, 111

Tenthredinidæe, 269

Terebratula, 183

Teredo navalis, 315

Terga, 204

Tergum, 198

Termes, 254

Termites, 254

Tern, Common, 456

" Lesser, 456

Terns, 456

Terrestrial, 58

Testis, 34

Testudinata, $\$ 25$

Testudinida, 426

Testudo græca, 4,26

Tetrabranchiata, 322

Tetraonomorphæ, 455

Tetrao tetrix, 455 , urogallus, 455

Thalamencephalon, 330

Theca, 115

Thecosomata, 304

Theory of Descent, 56

Thigh bone, 328

Thoracic segments, 188

, verteloræ, 326

Thorax, 190

Thorm-headed Worm, 163

Thread-worms, 158, 162

Thrush, 462

Thunderbolts, 323

Thylacinus, 497

Thymus, 341

Thynnus vulgaris, 390

Thyroid gland, 341, 488

Thysanopus, 211

Thysanura, 250

Tibia, 235, 328

Tibiale, 328

Tichodroma muraria, 464

Ticks, 284

Tiger, 517

Tiger-beetles, 264 
Tinamou, 454

Tinca rulgaris, 388

Tinea pellionella, 273 , tapezella, 273

Tineidæ, 273

Tintinnus, 94

Tipula, 276

Tissues, 7

epithelial, 7
muscular, 7
nervous, 7
skeletal, 7

Tit, Blue, 462

,Great, 462

, Long-tailed, 462

Tits, 462

Toad, 406, 407

, Common, 406

, Natterjack, 407

, Orange-speckled, 406

, Surinam, 406

Tonnicidæ, 267

Tomicus typographus, 267

Tongue, 287, 341

Tonsil, 487

Toothed Whales, 524

Torpedo, 368, 384

Tortoises, 426 , Grecian, 426

Tortricidæ, 274

Tortrix buoliana, 274: " pomonana, 274

Totanus, 460

Toucans, 465

Toxopneustes, 138

Trachea, 417

Tracher, 240

Tracheal bladder, 238

$$
\begin{aligned}
& \Rightarrow \quad \text { gills, } 241 \\
& \Rightarrow \quad \text { system, } 228
\end{aligned}
$$

Trachinus draco, 389

Tragulidæ, 509

Transverse bone, 412

Trapezium, 478

Trematoda, 143, 145

Trichechus rosmarus, 519

Trichina spiralis, 162

Trichodectes, 256

Trichoglossinæ, 465 canis, 153,256

Trigla gurnardus, 390

Trigonocephalus, 4,25

Trilobita, 198

Trilobites, 198

Tringa, 460

Trionyx, 426

Tristomum, 146

Triton, 403, 4.04.

" cristatus, 404

" helveticus, 4.04

, tæmiatus, 404

Trochanter, 235

Trochilidx, 464

$$
\text { tertius, } 502
$$

Troctes, 250

Troglodytes gorilla, 535

$$
\text { , niger, } 535
$$

, parvulus, 462
Trombidiidæ, 284

Tropidonotus natrix, 424

Trout, 387

True Seals, 519

,Wasps, 271

Trunk, 324,

, -fish, 391

Tube-feet, 122

Tuber calcis, 414

Tuberculum, 472

Tubifex rivulorum, 174.

Tubipora, 114

Tunica argentea, 370

Tunicata, 84, 537

Tunny, 390

Turbellaria, 143

Turbinals, 333

Turbot, 389

Turdidæ, 462

Turdiformes, 462

Turdus merula, 462 , musicus, 462 "torquatus, 462

Turkey, 455

Turnstone, 459

Turtles, 426

Turtur auritus, 465 risorius, 465

Two-toed Anteater, 526

Tylenchus tritici, 163

Tympanic cavity, 397 " membrane, 447

Tympanic membrane, internal, 447

Tympanic membrane, external, 44.4

Tympanum, 238, 397

Tyroglyphus, 284

Ulna, 328

Ulnare, 328

Umbilical cord, 494

" tube, 294

Umbilicus, 294

Umbo, 309

Umbrella, 102

Unciform, 478

Undulating membrane, 92

Ungulata, 501

Unio, 315

Upupa epops, 464

Urea, 30, 31

Ure ox, 510

Uric acid, 4, 30

Urinary bladder, 31

Urine, 31

organs, 30

Uroceridæ, 269

Urodela, 404

Uropygial glands, 435

Ursidæ, 516

Ursus americanus, 516

, aretos, 516

" cinereus, 516

" labiatus, 516

" maritimus, 516

spelæus, 516

Uterus, 490
Uterus, bicornis, 490

"duplex, 490

, maseulinus, 493

, simplex, 490

Utriculus, 338

Vacuole, 4, 85, 92

Vagina, 242, 490

Valves, 26

Vampire, 501

Vanellus cristatus, 459

Vanessa urticæ, 275

Varanids, 422

Varanus, 422

Variable Hare, 529

Varieties, 54

Vascular system, 25

Vascular system in connection with respiratory apparatus, 28

Vas deferens, 34

Vegetable Kingdom, 80

Veins, 26

Velella, 109

Velum, 105, 107, 290

Velvet, 471

Venus's Flower-basket, 121 , Girdle, 118

Venous blood, 28 heart, 29

Ventral, 41

Ventricle, 26

Vertebra, 326

Vertebrata, 84, 324:

Vesicantia, 266

Vesicles (tracheal), 240

Vesicula seminalis, 244, 4.93

Vespa, 271

Vespariæ, 271

Vespertilio, 501 , Daubentoni, 501

Vesperugo, 501

Vestibule, $49 \mathrm{I}$ pipistrellus,501

Vibracula, 181

Vibrissæ, 467

Vili, 342

Vinegar-worm, 163

Vine-louse, 257

Vioa, 121

Vipera, 425

" ammodytes, 4,25

, berus, 425

Viper, Common, 425

, Pit, 425

Vipers, 425

Viscera, 39

Visceral arches, 327

$$
\begin{array}{ll}
\Rightarrow & \text { ganglia, } 289 \\
\Rightarrow & \text { hump, 292 } \\
y & \text { loop, 289 }
\end{array}
$$

Vitellarium, 143

Vitelline membrane, 32

Vitreous body, 21

" humour, 333

", Sponges, 121 
Viverra, 517

3) genetta, 517

Viverridæ, 517

Viviparous, 47, 126

Vocal cords, 30, 417

Vomer, 362

Vorticella, 94

Vultur fulvus, 461

Vulture, Alpine, 461

, Bearded, 461

, Carrion, 461

, King, 461

" White-headed,461

Wagtails, 462

Walrus, 519

Wapiti, 509

Warblers, 462

$$
\begin{array}{ll} 
& \text { Reed, 462 } \\
\text { " } & \text { Sedge, 462 } \\
\text { Willow, } 462
\end{array}
$$

Warm-blooded animals, 30

Wart-hog, 506

Washing Racoon, 516

Water Boatman, 160

, Bugs, 260

" cells, 508

" Flea, 196

" Fowls, 4.59

" Mites, 284

" Rat, 530
Water Scorpion, 260

"Shrew, 500

" Spider, 283

, vascular system, 124

Wax glands, 237

Weazel, 516

Weevils, 267

Whale-bone, 522

Wheatear, 462

Whales, 523

Wheel Animalcules, 156

Whelk, 302

Whirligigs, 264

White Ants, 254

"Spoonbill, 459

Widgeon, 458

Wild Cat, 517

, Hog, 506

W'ing, 235

" cases, 236

Winter sleep, 69

Wire-worms, 266

Wolf, 515

Wolffian ducts, 349

Wolf-fish, 390

Wombats, 499

Wood-borers, 274

Woodcock, 459

Wood Mouse, 530

s -peckers, 465

" Wasps, 269
Woolly Rhinoceros, 503

Workers, 254, 270

Wrass, 389

Wrens, 462

Wrist, 328

Wryneck, 466

Xenos, 262

Xiphias gladius, 390

Xiphisternum, 473

Xiphosura, 196

Xylotropa, 274

Yellow cells, 90

Yolk gland, 143

" granules, 32

, sac, 49

Zebra, 503

Zoantharia, 115

Zoæa, 221

Zoarces, 390

, vivipara, 390

Zoogeographical regions, 74

Zooid, 178

Zygantrum, 409

Zygapophysis, 409

Zygosphene, 409 


\section{E R R A T A.}

Page 118, line 42, delete "structure."

"149, Fig. 105, for "central" read "ventral."

"175, Fig. 137, for " subossophageal" read " ventral."

"274, line 19, insert, after dorsally.

" 283, line 1, for "Arachnidas" read "Arachnida."

" ] 289, Fig. 237, for "foot" read "pedal ganglion."

" 314, line 18, for "Dreissena" read "Dreissensia."

" 317, Fig. 263, for "syphon" read "siphuncle."

" 351, Fig. 291, for " $f$ collicle" read "follicle."

" 355, Fig 293, for "gill-sac" read "pharynx."

" 388, line 37, and page 390, lines 29, 32, for "Mackrel read

"Mackerel."

"478, Fig. 390, for " oc" read " $\mathrm{x}$."

" 500, line 36, for " metatarsals" read "metacarpals."

N.B.-The term Raptatores, used p. 452, is interchangeable with the terms Rapaces or Accipitres, used p. 460. 




